\title{
STORM WATER POLLUTION PREVENTION PLAN FOR THE OAK RIDGE Y-12 PLANT
}

\author{
Prepared by the \\ Water Compliance Program Section \\ of the \\ Environmental Management Department \\ Health, Safety, Environment, and Accountability \\ Organization
}

September 1995

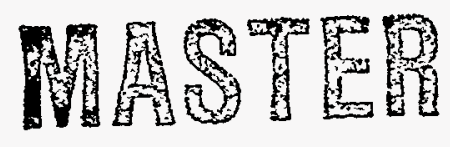

\author{
Prepared for the \\ Oak Ridge Y-12 Plant \\ Oak Ridge, Tennessee 37831 \\ managed by \\ LOCKHEED MARTIN ENERGY SYSTEMS, INC. \\ for the \\ U.S. DEPARTMENT OF ENERGY \\ under contract \\ DE-AC05-840R21400
}

MAHAGED BY

MARTIH MARIETTA ENERGY SYSTEMS, INC.

FOR THE UNITED STATES

DEPARTMENT OF EMERGY 


\section{DISCLAIMER}

This report was prepared as an account of work sponsored by an agency of the United States Government. Neither the United States Government nor any agency thereof, nor any of their employees, makes any warranty, express or implied, or assumes any legal liability or responsibility for the accuracy, completeness, or usefulness of any information, apparatus, product, or process disclosed, or represents that its use would not infringe privately owned rights. Reference herein to any specific commercial product, process, or service by trade name, trademark, manufacturer, or otherwise, does not necessarily constitute or imply its endorsement, recommendation, or favoring by the United States Government or any agency thereof. The views and opinions of authors expressed herein do not necessarily state or reflect those of the United States Government or any agency thereof. 


\section{STORM WATER POLLUTION PREVENTION PLAN FOR THE OAK RIDGE Y-12 PLANT}

September 1995

Prepared by the

Water Compliance Program Section of the

Environmental Management Department

Health, Safety, Environment, and Accountability Organization

Prepared for the

Oak Ridge Y-12 Plant

Oak Ridge, Tennessee 37831

managed by

LOCKHEED MARTIN ENERGY SYSTEMS, INC.

for the

U.S. DEPARTMENT OF ENERGY

under contract

DE-AC05-84OR21400

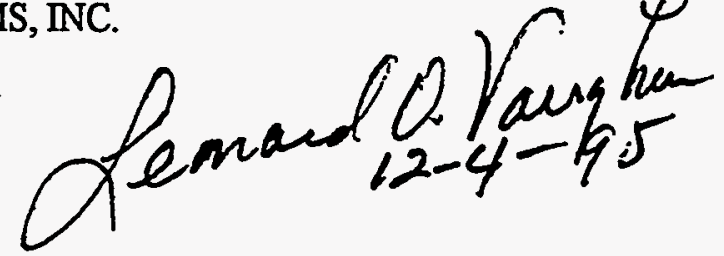

DISTAIBUTION OF THIS DOCUMENT IS UNLIMITED

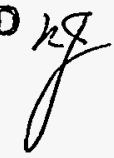




\section{DISCLAIMER}

Portions of this document may be illegible in electronic image products. Images are produced from the best available original document. 


\title{
Y-12 STORM WATER POLLUTION PREVENTION PLAN (SWP3)
}

\author{
Table of Contents
}

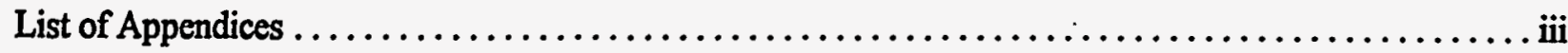

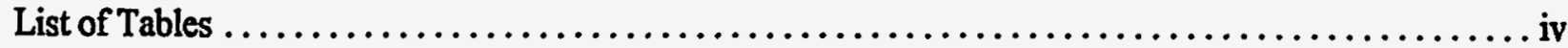

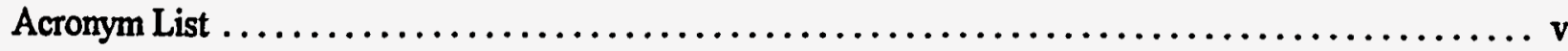

Section 1 Introduction of Y-12 Storm Water and General Information $\ldots \ldots \ldots \ldots \ldots \ldots \ldots \ldots$

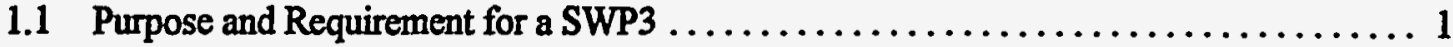

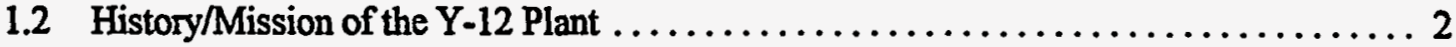

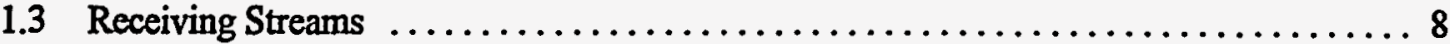

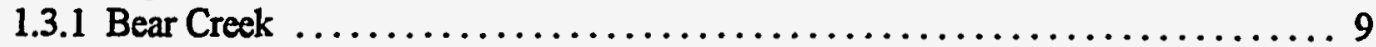

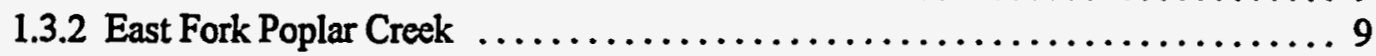

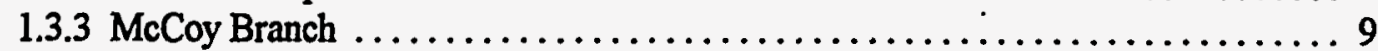

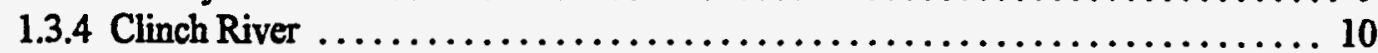

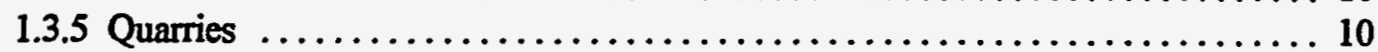

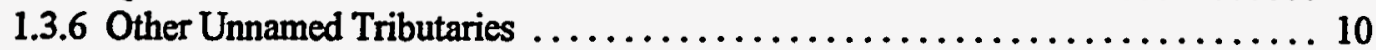

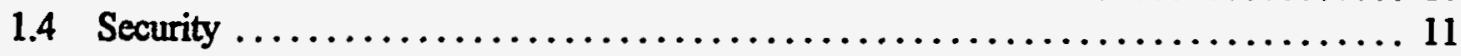

Section 2 Storm Water Controls (Programmatic and Physical) $\ldots \ldots \ldots \ldots \ldots \ldots \ldots \ldots \ldots \ldots$

2.1 Best Management Practices $\ldots \ldots \ldots \ldots \ldots \ldots \ldots \ldots \ldots \ldots \ldots \ldots \ldots \ldots$

2.1.1 Plantwide Best Management Practices (BMP) Plan $\ldots \ldots \ldots \ldots \ldots \ldots \ldots 1$

2.1.2 Site/Project Specific BMPs ......................... 1

2.1.3 Feasibility Study for the BMP to Control Area Source

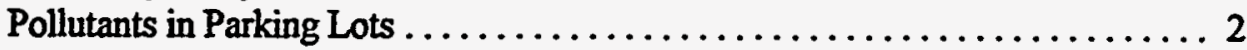

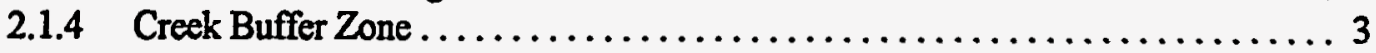

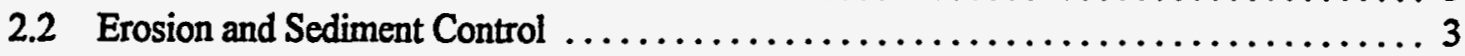

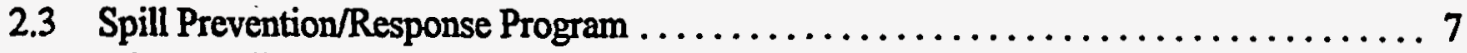

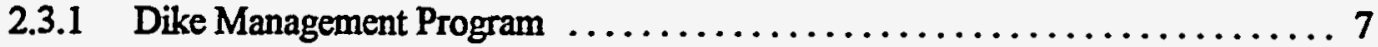

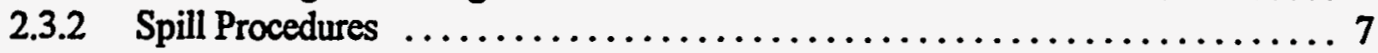

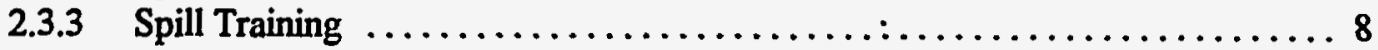

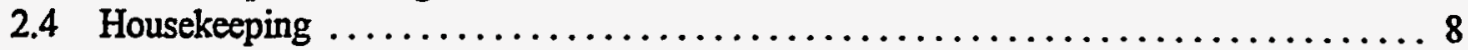

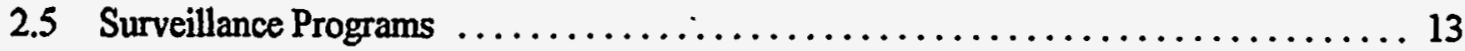

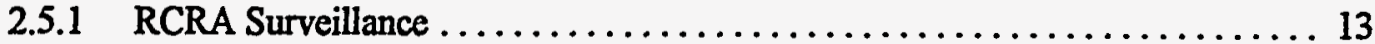

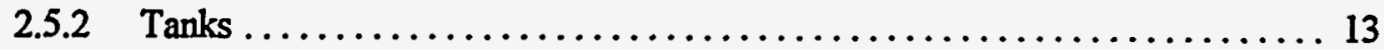

2.5.3 Dike Surveillances ................................... 14

2.5.4 Construction Projects (Pre, During \& Post) ................... 14

2.5.5 Housekeeping (Plant Appearance) Surveillance .................. 15

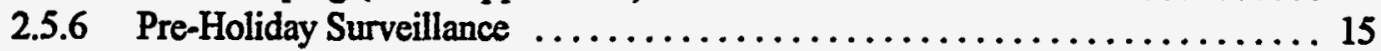

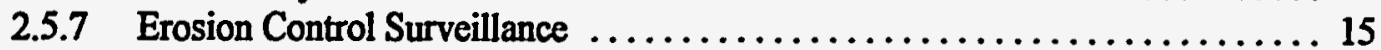

2.5.8 Spill Response/Prevention Inspections ........................ 15

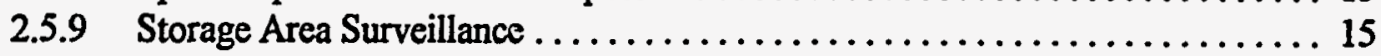

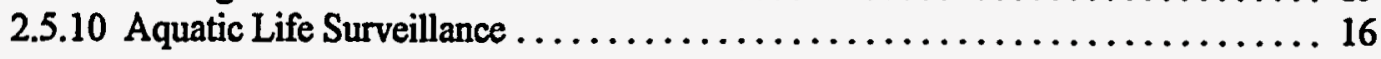




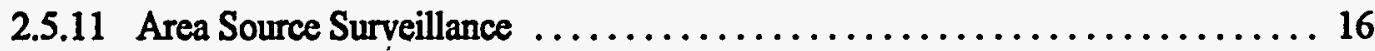

2.5.12 Creek Surveillance $\ldots \ldots \ldots \ldots \ldots \ldots \ldots \ldots \ldots \ldots \ldots \ldots \ldots \ldots \ldots \ldots \ldots \ldots, 16$

2.5.13 Documentation of Concerns and

Tracking of Corrective Actions ............................ 17

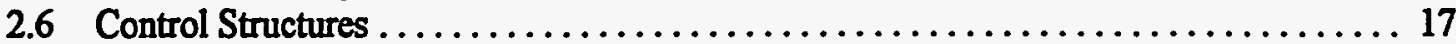

2.6.1 Coal Pile Runoff Collection System with Treatment ................ 17

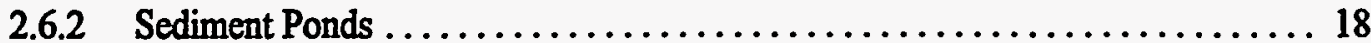

2.6.3 Oil/Water Separator $\ldots \ldots \ldots \ldots \ldots \ldots \ldots \ldots \ldots \ldots \ldots \ldots \ldots \ldots \ldots \ldots \ldots, 18$

$2.6 .4 \quad$ Lake Reality $\ldots \ldots \ldots \ldots \ldots \ldots \ldots \ldots \ldots \ldots \ldots \ldots \ldots \ldots \ldots \ldots \ldots \ldots \ldots, 18$

2.7 Toxicity Reduction Evaluation $\ldots \ldots \ldots \ldots \ldots \ldots \ldots \ldots \ldots \ldots \ldots \ldots \ldots \ldots \ldots \ldots \ldots \ldots \ldots, 20$

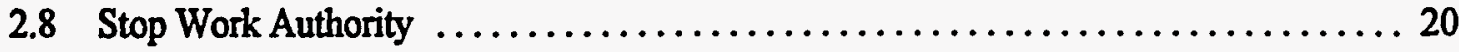

Section 3 Potential Pollutant Sources ..................................... 1

3.1 Toxic Substances Control ActPolychlorinated Biphenyls $\ldots \ldots \ldots \ldots \ldots \ldots \ldots \ldots 1$

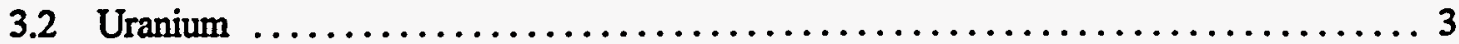

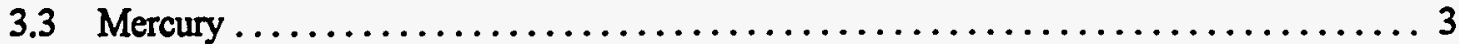

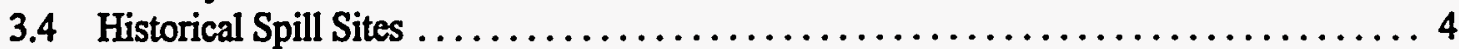

3.5 Salvage Yard $\ldots \ldots \ldots \ldots \ldots \ldots \ldots \ldots \ldots \ldots \ldots \ldots \ldots \ldots \ldots \ldots, 4$

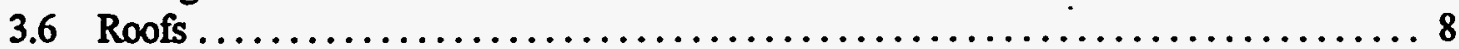

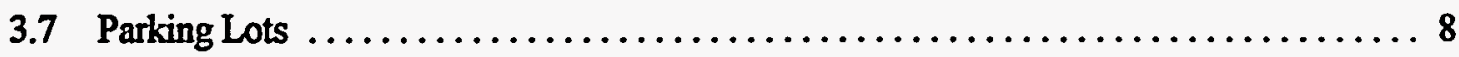

3.8 Solid Waste Management Units (SWMUs) $\ldots \ldots \ldots \ldots \ldots \ldots \ldots \ldots \ldots \ldots \ldots, 8$

3.9 Areas Where Significant Materials are Stored, Treated, or Disposed ............. 20

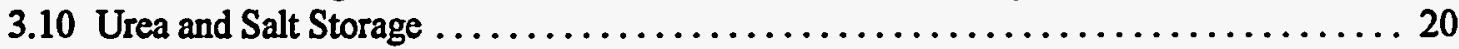

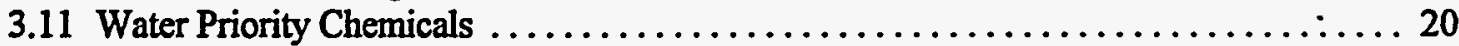

3.11.1 Storage of Water Priority Chemicals (WPCs) $\ldots \ldots \ldots \ldots \ldots \ldots \ldots, 21$

3.11.2 Inspection of WPC Storage Areas Exposed to Storm Water ........... 21

3.11.3 Training for Personnel Managing WPCs ...................... 21

Section 4 Outfall Specific Information ....................................... 1

4.1 Category I Storm Water Outfalls and Monitoring Points $\ldots \ldots \ldots \ldots \ldots \ldots \ldots \ldots \ldots$

4.2 Category II Storm Water Outfalls and Monitoring Points .................... 4

4.3 Category III Storm Water Outfalls and Monitoring Points .................. 10

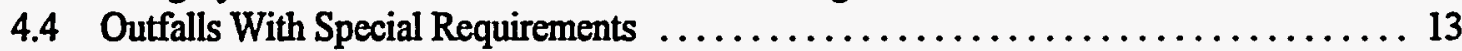

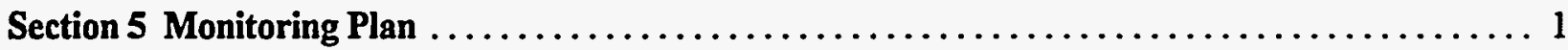

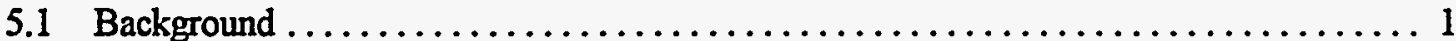

5.2 Requirements ........................................... 1

5.2.1 Category I Storm Water Outfalls and Monitoring Points ............... I

5.2.2 Category II Storm Water Outfalls and Monitoring Points .............. 1

5.2.3 Category III Storm Water Outfalls and Monitoring Points ............... 2

5.2.4 Storm Water Outfalls of Special Interest $\ldots \ldots \ldots \ldots \ldots \ldots \ldots \ldots \ldots 2$

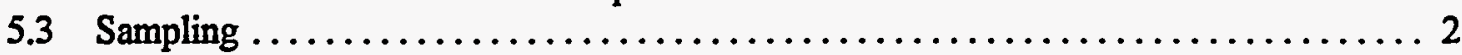

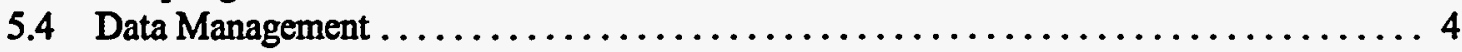

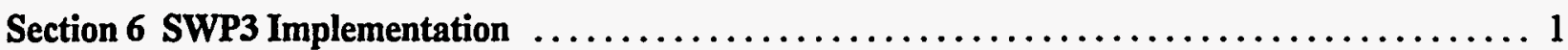

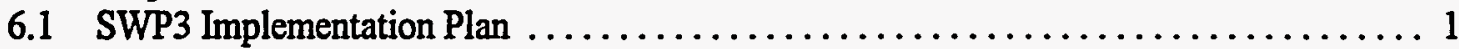

6.2 Storm Water Working Group for Construction Activities ................... 1

6.3 Employee Training/Education of SWP3 Requirements .................... 2 
6.4 Semi-Annual Inspections to Ensure Effectiveness of the SWP3 $\ldots \ldots \ldots \ldots \ldots \ldots . \ldots$

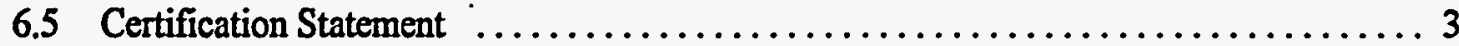

\section{List of Appendices}

A: Analytical Data From Storm Water Monitoring Points

B: Y-12 Site Maps

C: Y-12 Plant National Pollutant Discharge Elimination System Permit 


\section{List of Tables}

Section 1 Introduction of Y-12 Storm Water and General Information

1.1 Storm Water Outfalls for East Fork Poplar Creek ...................... 3

1.2 Storm Water Outfalls and Monitoring Points for Bear Creek and

Clinch River Tributaries ................................ 6

Section 2 Storm Water Controls (Programmatic and Physical)

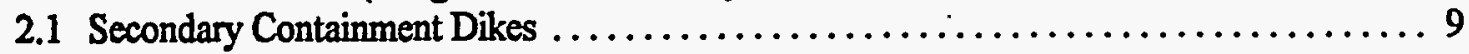

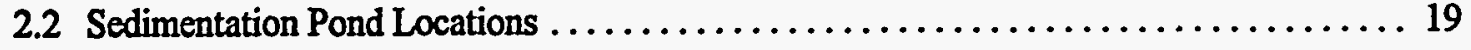

Section 3 Potential Pollutant Sources

3.1 Historical Spill Sites . . . . . . . . . . . . . . . . . . . . . . . . . . . . 5

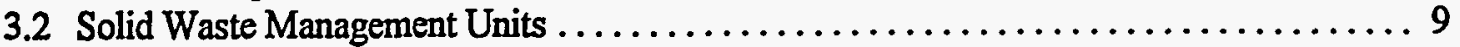

3.3 Water Priority Chemical Storage Locations ....................... 22

Section 5 Monitoring Plan

5.1.A Category I Storm Water Outfalls and Monitoring Points $\ldots \ldots \ldots \ldots \ldots \ldots \ldots \ldots$

5.1.B Category II Storm Water Outfalls and Monitoring Points $\ldots \ldots \ldots \ldots \ldots \ldots \ldots 7$

5.1.C Category III Storm Water Outfalls and Monitoring Points $\ldots \ldots \ldots \ldots \ldots \ldots \ldots 9$

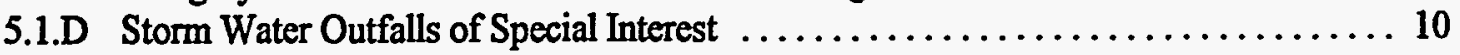

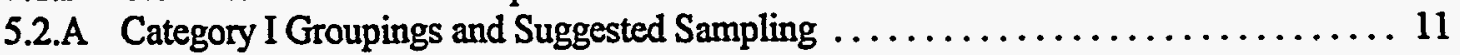

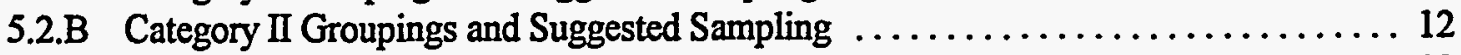

5.2.C Category III Groupings and Suggested Sampling $\ldots \ldots \ldots \ldots \ldots \ldots \ldots \ldots \ldots \ldots \ldots$

5.2.D Suggested Sampling for Outfalls of Special Interest $\ldots \ldots \ldots \ldots \ldots \ldots \ldots \ldots \ldots$

5.3 Suggested Sampling for All Storm Water Monitoring Locations $\ldots \ldots \ldots \ldots \ldots \ldots$

5.4 Additional Tests Specified for Certain Groups of Outfalls $\ldots \ldots \ldots \ldots \ldots \ldots \ldots 17$

5.5 List of Parameters Included Under Each Test ..................... 18 


\section{List of Acronyms}

AGSP

ARAP

BCBG

BMAP

BMP

BOD

CERCLA

CFR

COD

CWA

CWSA

DARA

DOE

EFK

EFPC

EMD

EO

EPA

ER

ET\&I

ICP

LSF

MBAS

MKF

NOI

NPDES

ORNL

PCB

PSS

RCRA

SPCC

SWMU

SWCP

SWP3

TDEC

TRE

TSCA

TSS

WCS

WTLO
Above Ground Storage Pads Facility

Aquatic Resources Alteration Permit

Bear Creek Burial Grounds

Biological Monitoring and Abatement Program

Best Management Practices

Biochemical Oxygen Demand

Comprehensive Environmental Response, Compensation, and Liability Act

Code of Federal Regulations

Chemical Oxygen Demand

Clean Water Act

Containerized Waste Storage Area

Disposal Area Remedial Action

U.S. Department of Energy

East Fork Kilometer

East Fork Poplar Creek

Environmental Management Department

Environmental Officer

Environmental Protection Agency

Environmental Restoration

Equipment Testing and Inspection

Inductively-Coupled Plasma

Liquid Storage Facility

Methylene Blue Activated Substances

MK-Ferguson

Notice of Intent

National Pollutant Discharge Elimination System

Oak Ridge National Laboratory

Polychlorinated Biphenyls

Plant Shift Superintendent

Resource Conservation and Recovery Act

Spill Prevention, Control, and Countermeasures

Solid Waste Management Unit

Storm Water Control Plan

Storm Water Pollution Prevention Plan

Tennessee Department of Environment and Conservation

Toxicity Reduction Evaluation

Toxic Substances Control Act

Total Suspended Solids

Water Compliance Section

Waste Transportation and Landfill Operations 


\section{SECTION 1}

\section{INTRODUCTION OF Y-12 PLANT STORM WATER AND GENERAL INFORMATION}

\subsection{Purpose and Requirement for a Storm Water Pollution Prevention Plan}

The Environmental Protection Agency (EPA) published the final storm water regulation on November 16, 1990. The storm water regulation is included in the National Pollutant Discharge Elimination System (NPDES) regulations. It deals with permit application requirements for certain storm water discharges such as municipal separate storm water sewers serving populations of 100,000 or greater or industrial activity. Storm water discharge associated with industrial activity is defined as discharge from any conveyance which is used for collecting and conveying storm water which is directly related to manufacturing, processing, or raw material storage areas located at an industrial plant. Quantitative testing data was required for these discharges to characterize the pollutants of concern. The Y-12 Plant submitted a storm water permit application to the Tennessee Department of Environment and Conservation (TDEC) in October 1992 to satisfy the regulatory requirement that all industries with the potential to impact storm water quality submit storm water permit applications by October 1992. An NPDES permit was issued for the Y-12 Plant on April 28, 1995, and was effective on July 1, 1995. The permit requires that a Storm Water Pollution Prevention Plan (SWP3) be developed by December 28, 1995, and be fully implemented by July 1, 1996; this plan has been developed to fulfill that requirement. The outfalls and monitoring points described in this plan contain storm water discharges associated with industrial activities as defined in the NPDES regulations.

For storm water discharges associated with industrial activity, including storm water discharges associated with construction activity, that are not specifically monitored or limited in this permit, Y-12 Plant personnel will meet conditions of the General Storm Water Rule 1200-4-10. A Notice of Intent to comply with general storm water requirements will be sent to the TDEC, Division of Water Pollution Control, if outfalls that are not specifically listed in this permit are planned.

This document presents the programs and physical controls that are in place to achieve the following objectives:

- ensure compliance with Section 1200-4-10-.04(5) of the TDEC Water Quality Control Regulations and Part IV of the Y-12 Plant NPDES Permit (TN0002968),

- provide operating personnel with guidance relevant to storm water pollution prevention and control requirements for their facility and/or project, and

- prevent or reduce pollutant discharge to the environment, in accordance with the Clean Water Act (CWA) and the Tennessee Water Quality Control Act.

Section 1.2 of this document provides background information necessary for an understanding of the physical layout of the Y-12 Plant site. Section 1.3 contains a description of the surface waters receiving storm water runoff from the Y-12 Plant and its related operations/activities.

Section 2 describes in detail the storm water pollution controls (physical and programmatic) that are in place to prevent and/or reduce pollutants from entering storm water associated with the Y-12 Plant.

Section 3 describes pollutant sources that could potentially contact storm water. These sources include parking lots; roofs; historical spill sites; areas where significant materials are or have been treated, stored, or disposed of; and the plant salvage yard. 
Section 4 provides a detailed look at each storm water monitoring location. Tables 1.1 and 1.2 provide a listing and brief description of these monitoring sites. The information includes narrative descriptions of the area drained by the outfall, potential pollutant sources in that area, and the storm water controls specific to the area drained by the outfall. Analytical data obtained from sampling storm water discharges from each outfall or monitoring point in Categories I, II, III, and four other outfalls is presented in Appendix A.

Section 5 presents the monitoring plan for each storm water outfall and/or monitoring point. This section includes information related to the parameters that will be monitored at each point, the frequency that the point will be sampled, and which storm events will be considered as meeting the requirements for a storm water characterization.

Section 6 details the implementation plan of the SWP3, which includes the training program that will be implemented to ensure appropriate personnel receive the necessary training to successfully implement this SWP3.

A copy of this SWP3 will be submitted to TDEC, Division of Water Pollution Control, personnel within ten working days of the request. This plan will be available on site at Building 9116 and will be maintained by personnel of the Water Compliance Section (WCS) of the Environmental Management Department (EMD).

\subsection{History/Mission of the Y-12 Plant}

The Oak Ridge Y-12 Plant was originally constructed in 1943 by the U.S. Army Corps of Engineers as a part of the classified Manhattan Project. The plant was one of three constructed in the Oak Ridge area, the other two being the Oak Ridge National Laboratory (ORNL) and the K-25 Site.

The original mission of the Y-12 Plant was to separate fissionable isotopes of uranium by the electromagnetic process. It has progressed from this single mission to become a highly sophisticated weapons component manufacturing and development engineering organization. Since 1984, it has been managed by Lockheed Martin Energy Systems, Inc., under contract with the U.S. Department of Energy (DOE).

The primary mission of the Oak Ridge Y-12 Plant is to serve as a key manufacturing technology center for the development and demonstration of unique materials, components, and services of importance to the DOE and the nation. This is accomplished through reclamation and storage of nuclear materials, manufacture of nuclear materials, manufacture of components for the nation's defense capabilities, support to national security programs, and services provided to other customers as approved by DOE.

The Y-12 Plant goal is to be innovative, responsive, and responsible to its personnel, the community, and customers. It is considered a leader in worker health and safety, environmental protection, and stewardship of our national resources.

Activities conducted to accomplish this mission include:

- weapons dismantlement and storage,

- enriched uranium material warehousing and management,

- nuclear weapons process technology and development support,

- management/landlord activities including renovating standby or shutdown facilities by decontamination and decommissioning, 
TABLE 1.1

STORM WATER OUTFALLS

FOR THE OAK RIDGE Y-12 PLANT

EAST FORK POPLAR CREEK

\begin{tabular}{|c|c|c|c|c|}
\hline Outfall & Category & Size/Description & Latitude & Longitude \\
\hline 002 & III & 48-in. RCP & N 35⒌ $59^{\prime} 46^{\prime \prime}$ & $W 84^{\circ} 14^{\prime} 27^{\prime \prime}$ \\
\hline 003 & I & CT & N 3559'44" & W $84^{\circ} 14^{\prime} 23^{\prime \prime}$ \\
\hline 004 & II & 54-in. CMP & N 35॰59'39" & $\mathrm{W} 84^{\circ} 14^{\prime} 21^{\prime \prime}$ \\
\hline 006 & $\mathrm{I}$ & 24-in. CMP & N 3559'33" & W $84^{\circ} 14^{\prime} 18^{\prime \prime}$ \\
\hline 007 & I & 24-in. RCP & N 3559'36" & $\mathrm{W} 84^{\circ} 14^{\prime} 15^{\prime \prime}$ \\
\hline 008 & I & 36-in. RCP & N 3559'30" & $\mathrm{W} 84^{\circ} 14^{\prime} 13^{\prime \prime}$ \\
\hline 009 & I & 14-in. CS & N 35⒌'27" & W $84^{\circ} 14^{\prime} 25^{\prime \prime}$ \\
\hline 010 & II & 24-in. RCP & N $35^{\circ} 59^{\prime} 25^{\prime \prime}$ & $\mathrm{W} 84^{\circ} 14^{\prime} 31^{\prime \prime}$ \\
\hline 011 & I & 16-in. RCP & N 35⒌'25" & $\mathrm{W} 84^{\circ} 14^{\prime} 29^{\prime \prime}$ \\
\hline 014 & II & 36-in. RCP & N 35⒌'23" & W $84^{\circ} 14^{\prime} 33^{\prime \prime}$ \\
\hline 015 & I & 6-in. CS & N 35⒌'23" & $\mathrm{W} 84^{\circ} 14^{\prime} 35^{\prime \prime}$ \\
\hline 016 & II & 48-in. RCP & N 35⒌'22" & W $84^{\circ} 14^{\prime} 34^{\prime \prime}$ \\
\hline 017 & Special Interest & 16-in. RCP & N 35⒌'21" & $\mathrm{W} 84^{\circ} 14^{\prime} 56^{\prime \prime}$ \\
\hline 018 & I & 12-in. CMP & N 3559'21" & W $84^{\circ} 14^{\prime} 37^{\prime \prime}$ \\
\hline 019 & II & 15-in. RCP & N 35⒌'19" & $\mathrm{W} 84^{\circ} 14^{\prime} 40^{\prime \prime}$ \\
\hline 020 & II & 24-in. RCP & N 35⒌'18" & $\mathrm{W} 84^{\circ} 14^{\prime} 45^{\prime \prime}$ \\
\hline 021 & Special Interest & 48-in. RCP & N 35'59'18" & W $84^{\circ} 14^{\prime} 44^{\prime \prime}$ \\
\hline 033 & I & 36-in. TC & N 35⒌'16" & $\mathrm{W} 84^{\circ} 14^{\prime} 49^{\prime \prime}$ \\
\hline 034 & III & 15-in. VC & N 35 $59^{\prime} 15^{\prime \prime}$ & $\mathrm{W} 84^{\circ} 15^{\prime} 00^{\prime \prime}$ \\
\hline 041 & II & 15-in. RCP & N 35⒌'16" & $\mathrm{W} 84^{\circ} 14^{\prime} 51^{\prime \prime}$ \\
\hline 042 & III & 16-in. VC & N 35⒌'16" & W $84^{\circ} 14^{\prime} 53^{\prime \prime}$ \\
\hline 044 & II & 12-in. C & N 35'59'16" & W $84^{\circ} 14^{\prime} 53^{\prime \prime}$ \\
\hline 045 & I & 15-in. C & N 35⒌'16" & W $84^{\circ} 14^{\prime} 53^{\prime \prime}$ \\
\hline 046 & I & 6-in. TC & N 3559'15" & $\mathrm{W} 84^{\circ} 15^{\prime} 00^{\prime \prime}$ \\
\hline
\end{tabular}


TABLE 1.1

\section{STORM WATER OUTFALLS \\ FOR THE OAK RIDGE Y-12 PLANT}

EAST FORK POPLAR CREEK

\begin{tabular}{|c|c|c|c|c|}
\hline Outfall & Category & Size/Description & Latitude & Longitude \\
\hline 047 & III & 24-in. RCP & N 355' $18^{\prime \prime}$ & $W 84^{\circ} 15^{\prime} 05^{\prime \prime}$ \\
\hline 048 & III & 24-in. RCP & N 35'59'18" & $W 84^{\circ} 15^{\prime} 05^{\prime \prime}$ \\
\hline 054 & III & 12-in. VC & N 35 $55^{\circ} 15^{\prime \prime}$ & W $84^{\circ} 14^{\prime} 56^{\prime \prime}$ \\
\hline 057 & II & 10-in. ST & N 3559'14" & W $84^{\circ} 14^{\prime} 58^{\prime \prime}$ \\
\hline 058 & I & 36-in. TC & N 35 $59^{\prime} 14^{\prime \prime}$ & W $84^{\circ} 14^{\prime} 58^{\prime \prime}$ \\
\hline 062 & I & 18-in. RCP & N 3559'13" & $\mathrm{W} 84^{\circ} 15^{\prime} 10^{\prime \prime}$ \\
\hline 063 & II & 12-in. RCP & 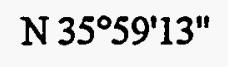 & W $84^{\circ} 15^{\prime} 01^{\prime \prime}$ \\
\hline 064 & III & 12-in. RCP & N 35'58'13" & $\mathrm{W} 84^{\circ} 15^{\prime} 10^{\prime \prime}$ \\
\hline 067 & II & 24-in. RCP & N 35'59'12" & W $84^{\circ} 15^{\prime} 04^{\prime \prime}$ \\
\hline 071 & III & 12-in. CI & N 35०59'11" & W $84^{\circ} 15^{\prime} 05^{\prime \prime}$ \\
\hline 083 & II & 15-in. VC & N 35⒌'11" & W $84^{\circ} 15^{\prime} 06^{\prime \prime}$ \\
\hline 086 & I & 18-in. CI & N 355' $10^{\prime \prime}$ & $\mathrm{W} 84^{\circ} 15^{\prime} 06^{\prime \prime}$ \\
\hline 087 & I & 36-in. VC & N 35⒌'10" & $\mathrm{W} 84^{\circ} 15^{\prime} 06^{\prime \prime}$ \\
\hline 088 & II & 10-in. CI & N 35⒌ $59^{\prime} 10^{\prime \prime}$ & $\mathrm{W} 84^{\circ} 15^{\prime} 07^{\prime \prime}$ \\
\hline 098 & I & 6-in. C & N 35⒌ $59^{\prime} 09^{\prime \prime}$ & W $84^{\circ} 15^{\prime} 08^{\prime \prime}$ \\
\hline 099 & II & 12-in. CI & N 35⒌ $59^{\prime} 95^{\prime \prime}$ & W $84^{\circ} 15^{\prime} 09^{\prime \prime}$ \\
\hline 109 & III & 54-in. RCP & N 35⒌ $59^{\prime} 09^{\prime \prime}$ & W $84^{\circ} 15^{\prime} 09^{\prime \prime}$ \\
\hline 110 & I & 15-in. RCP & N 3559'09" & W $84^{\circ} 15^{\prime} 11^{\prime \prime}$ \\
\hline 113 & III & 18-in. RCP & N 35 $59^{\circ} 07^{\prime \prime}$ & W $84^{\circ} 15^{\prime} 14^{\prime \prime}$ \\
\hline 114 & III & 18-in. RCP & N 35 $59^{\circ} 06^{\prime \prime}$ & $W 84^{\circ} 15^{\prime} 17^{\prime \prime}$ \\
\hline 125 & III & 22-in. CS & N 35'59'05" & $W 84^{\circ} 15^{\prime} 18^{\prime \prime}$ \\
\hline 126 & II & 18-in. RCP & N 35'59'14" & $\mathrm{W} 84^{\circ} 14^{\prime} 57^{\prime \prime}$ \\
\hline 134 & I & 24-in. CS & N 35 $59^{\circ} 04^{\prime \prime}$ & $\mathrm{W} 84^{\circ} 15^{\prime} 22^{\prime \prime}$ \\
\hline 135 & Special Interest & 36-in. RCP & N 35 $59^{\circ} 03^{\prime \prime}$ & $\mathrm{W} 84^{\circ} 15^{\prime} 21^{\prime \prime}$ \\
\hline
\end{tabular}


TABLE 1.1

STORM WATER OUTFALLS

FOR THE OAK RIDGE Y-12 PLANT

EAST FORK POPLAR CREEK

\begin{tabular}{|c|c|c|c|c|}
\hline Outfall & Category & Size/Description & Latitude & Longitude \\
\hline 200 & Special Interest & 76-in. RCP & N 35 $59^{\circ} 03^{\prime \prime}$ & $W 84^{\circ} 15^{\prime} 22^{\prime \prime}$ \\
\hline 213 & $\mathrm{I}$ & 6-in. VC & N 35'59'13" & $W 84^{\circ} 15^{\prime} 01^{\prime \prime}$ \\
\hline
\end{tabular}


TABLE 1.2

STORM WATER OUTFALLS AND MONITORING POINTS FOR THE OAK RIDGE Y-12 PLANT

BEAR CREEK AND CLINCH RIVER TRIBUTARIES

\begin{tabular}{|c|c|c|c|c|c|}
\hline $\begin{array}{c}\text { Monitoring } \\
\text { Point }\end{array}$ & Category & Size/Description & Drainage Area. & Latitude & Longitude \\
\hline S01 & I & 36-in. RCP & S-3 Parking Lot & N 355'ㄴㄱ" & W $84^{\circ} 16^{\prime} 19^{\prime \prime}$ \\
\hline $\mathrm{S} 02$ & II & 48-in. CMP & Headwaters to $B C$ & N 35 $58^{\prime} 29^{\prime \prime}$ & W $84^{\circ} 16^{\prime} 32^{\prime \prime}$ \\
\hline S03 & I & 18-in. RCP & Building 9624 Area & N 35'58'29" & W $84^{\circ} 16^{\prime} 32^{\prime \prime}$ \\
\hline S04 & I & 18-in. RCP & OD-9 Area & N 3558'29" & W $84^{\circ} 16^{\prime} 32^{\prime \prime}$ \\
\hline S05 & III & In-stream Point & Tributary 1 & N 355'ㅇ' & W $84^{\circ} 16^{\prime} 36^{\prime \prime}$ \\
\hline S06 & I & In-stream Point & Bear Creek & & \\
\hline S07 & I & In-stream Point & Tributary 2 & N 35 $58^{\prime} 21^{\prime \prime}$ & W $84^{\circ} 16^{\prime} 48^{\prime \prime}$ \\
\hline S08 & II & In-stream Point & Tributary 3 & & \\
\hline S09 & I & In-stream Point & Tributary 4 & N 3558'04" & W 84 $4^{\circ} 17^{\prime} 12^{\prime \prime}$ \\
\hline S10 & H & In-stream Point & Tributary 5 & N 3557'56" & W $84^{\circ} 17^{\prime} 25^{\prime \prime}$ \\
\hline S11 & II & In-stream Point & Tributary 6 & N 35०57'50" & W $84^{\circ} 17^{\prime} 37^{\prime \prime}$ \\
\hline $\mathrm{S} 12$ & II & 24-in. CI & Riprap Channel & N 35'57'45" & W $84^{\circ} 17^{\prime} 48^{\prime \prime}$ \\
\hline S13 & II & In-stream Point & Tributary 7 & N 3557'43" & W $84^{\circ} 17^{\prime} 50^{\prime \prime}$ \\
\hline S14 & III & In-stream Point & Tributary 8 & N 35 $57^{\prime} 29^{\prime \prime}$ & $\mathrm{W} 84^{\circ} 18^{\prime} 02^{\prime \prime}$ \\
\hline S15 & I & Sedimentation Basin & West Borrow Area & N $35^{\circ} 56^{\prime} 21^{\prime \prime}$ & W $84^{\circ} 19^{\prime} 15^{\prime \prime}$ \\
\hline S16 & I & Sedimentation Basin & East Borrow Area & N 35 $59^{\circ} 21^{\prime \prime}$ & W $84^{\circ} 13^{\prime} 58^{\prime \prime}$ \\
\hline S17 & II & In-stream Point & Kerr Hollow Quarry & N $35^{\circ} 58^{\prime} 47^{\prime \prime}$ & W $84^{\circ} 13^{\prime} 52^{\prime \prime}$ \\
\hline S18 & I & In-stream Point & $\begin{array}{l}\text { Sludge Application } \\
\text { Area }\end{array}$ & N 3558'44" & W $84^{\circ} 14^{\prime} 14^{\prime \prime}$ \\
\hline S19 & II & 24-in. CI & Rogers Quarry & N $35^{\circ} 58^{\prime} 07^{\prime \prime}$ & W $84^{\circ} 14^{\prime} 56^{\prime \prime}$ \\
\hline S20 & II & 48-in. CMP & Building 9213 & N $35^{\circ} 58^{\prime} 24^{\prime \prime}$ & W $84^{\circ} 14^{\prime} 56^{\prime \prime}$ \\
\hline S21 & II & Sedimentation Basin & Landfill II & 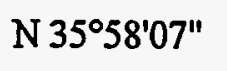 & W $84^{\circ} 15^{\prime} 42^{\prime \prime}$ \\
\hline S22 & II & Sedimentation Basin & Landfill IV & N 355'ㄹ' & W $84^{\circ} 16^{\prime} 13^{\prime \prime}$ \\
\hline
\end{tabular}




\section{TABLE 1.2}

STORM WATER OUTFALLS AND MONTTORING POINTS FOR THE OAK RIDGE Y-12 PLANT

\section{BEAR CREEK AND CLINCH RIVER TRIBUTARIES}

\begin{tabular}{|c|c|c|c|c|c|}
\hline $\begin{array}{c}\text { Monitoring } \\
\text { Point }\end{array}$ & Category & Size/Description & Drainage Area & Latitude & Longitude \\
\hline $\mathbf{S 2 4}$ & II & In-stream Point & Bear Creek & N 35 $57^{\circ} 28^{\prime \prime}$ & W $84^{\circ} 18^{\prime} 03^{\prime \prime}$ \\
\hline $\mathrm{S} 25$ & II & Sedimentation Basin & Landfill VI & N 355' $11^{\prime \prime}$ & W 84ำ $16^{\prime} 06^{\prime \prime}$ \\
\hline S26 & II & Sedimentation Basin & Landfill V (West) & N 35'58'49" & $\mathrm{W} 84^{\circ} 15^{\prime} 05^{\prime \prime}$ \\
\hline S27 & II & Sedimentation Basin & Landfill V (East) & N $35^{\circ} 58^{\prime} 60^{\prime \prime}$ & $\mathrm{W} 84^{\circ} 14^{\prime} 41^{\prime \prime}$ \\
\hline $\mathbf{S} 28$ & II & Sedimentation Basin & Landfill VII (North) & 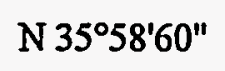 & $W 84^{\circ} 14^{\prime} 41^{\prime \prime}$ \\
\hline $\mathbf{S} 29$ & II & Sedimentation Basin & Landfill VII (South) & Future & Future \\
\hline
\end{tabular}


- providing capabilities and technologies not found in the private sector to DOE-approved tasks,

- transferring technology developed at DOE facilities to enhance the nation's industrial competitive edge in worldwide markets,

- maintaining and supporting the National Security Program Office for DOE, and

- providing support to other government agencies.

The Y-12 Plant lies in the Valley and Ridge physiographic province of East Tennessee. This province is characterized by alternating northeast-southwest trending ridges and valleys formed by severely folded and faulted sedimentary and metamorphic rock. It is bounded by the Cumberland Plateau province $(3,000+\mathrm{ft}$ above mean sea level) 10 miles to the northwest of the Y-12 Plant and by the Blue Ridge province $(6,000+\mathrm{ft}$ above mean sea level) 70 miles to the southeast of the plant.

The Y-12 Plant, ORNL, and the K-25 Site are situated on portions of a 37,000-acre federal reservation in the Clinch River drainage basin. Located 3 miles from the population center of the city of Oak Ridge, the main Y-12 Plant site occupies an area of 811 acres in the eastem end of Bear Creek Valley and at the headwaters of the East Fork Poplar Creek (EFPC). Approximately 600 of these acres are enclosed by perimeter security fencing. The main plant site is $2 / 3$ of a mile wide by 3.2 miles long, at elevations of 920 to $1,000 \mathrm{ft}$ above mean sea level. Bear Creek Road, the principal access to the plant site, runs northeast-southwest along its northern perimeter. Scarboro Road marks the eastern boundary of the plant.

The Y-12 Plant site and its environs support a predominantly hardwood (oak-hickory) forest. This forest and numerous cleared areas provide protected homes for a variety of native plants and animals. Parts of the federal reservation are managed as the Oak Ridge National Environmental Research Park.

\subsection{Receiving Streams}

The Clinch River is the major hydrologic feature of the Y-12 Plant site. Headwaters of the Clinch River are. in Tazewell County, Virginia. From the headwaters, the Clinch River flows approximately 350 miles southwest to its confluence with the Tennessee River near Kingston, Tennessee. The northwestern boundary of the basin is formed by the Cumberland Mountains. The southeastern boundary follows Clinch Mountain and Black Oak Ridge. The Oak Ridge area is drained by the Clinch River and some smaller creeks, which are tributaries of the Clinch River. These tributaries include, but are not limited to, White Oak Creek, Poplar Creek, EFPC, and Bear Creek.

The Y-12 Plant discharges various types of wastewater into EFPC, which originates within the plant. Bear Creek also originates within the Y-12 Plant; however, it is mostly affected by storm water runoff and tributaries that drain areas previously remediated or that are undergoing remedial activity. In addition, runoff from waste management areas on the southern slopes of Chestnut Ridge (which borders the south side of the plant) enters the Clinch River through a number of branches including (1) McCoy Branch, which enters and exits Rogers Quarry, an area previously used for the disposal of fly ash and bottom ash from the Y-12 Steam Plant; (2) Walker Branch embayment area, which may receive some runoff from the Y-12 Sanitary Landfill and from ORNL research areas; (3) Kerr Hollow Branch, which feeds and exits the quarry once used for disposal of reactive waste; and (4) Scarboro Creek embayment area, which could receive runoff from other waste management areas on the east end of Chestnut Ridge. 


\subsubsection{Bear Creek}

Bear Creek drains a small portion of the west end of the Y-12 Plant and drains all of the Bear Creek Burial Grounds. Bear Creek flows southwest from the Y-12 Plant through second-growth hardwood forests and late-successional old fields. The headwaters for Bear Creek are near the area known as the S-3 Ponds (now filled in and capped), where the creek flows southwest. The upper reach of EFPC begins near the same area and flows northeast along the south side of the Y-12 Plant.

At White Wing Road (State Route 95), Bear Creek turns northwest for the final $3.2 \mathrm{~km}$ of its approximately $12.5 \mathrm{~km}$ and converges with EFPC at Kilometer 2.4. Stream width and depth from the Y-12 Plant to the mouth of Bear Creek increase from 0.9 to $4.6 \mathrm{~m}$ and 0.1 to $0.9 \mathrm{~m}$, respectively. About $65 \%$ of the basin is wooded. The average flow in Bear Creek at White Wing Road is approximately 2.4 MGD. Except for those areas impacted by man, Bear Creek contains a relatively small amount of sediment and is made up of many riffles and pools. However, sediment is a special concern in the Bear Creek watershed due to the presence of Tennessee Dace in the creek. A Best Management Practices (BMP) plan is required for construction projects or other activities that may impact Bear Creek.

\subsubsection{East Fork Poplar Creek}

The headwaters of EFPC originate on the northwestern slopes of Chestnut Ridge in the vicinity of the Y-12 Plant. Stream flow was controlled until November 1988 by New Hope Pond, now filled in and capped, which was a settling basin of approximately 5 acres on the east side of the Y-12 Plant. The average flow through New Hope Pond was approximately 10 to 15 MGD. The replacement basin, Lake Reality, reportedly has a decreased flow of approximately 2.5 to 4.0 MGD, due to a reduction in plant operations. The lake functions primarily as an emergency spill containment basin with an approximate 8-hr retention time.

Most outfalls from the Y-12 Plant enter EFPC between Lake Reality and the North/South Pipes upstream at Creek Kilometer 25.4. Most of this reach is confined by 2.4-m-high overgrown, riprap stream banks of limestone rock. Stream substrate also consists of limestone rocks with some interspersed gravel. Stream width varies from 1 to $4.6 \mathrm{~m}$ within the reach between the North/South Pipes and Lake Reality. Much of the flow in this reach originates from the North/South Pipes, which is approximately $1.2 \mathrm{~km}$ above Lake Reality and fed by the Y-12 Plant storm sewer system. The storm sewer system extends west for approximately 2 $\mathrm{km}$ above the North/South Pipes.

After leaving the Y-12 Plant area, EFPC flows northwest through densely forested secondary-growth hardwoods, although small portions of it flow through urban areas of Oak Ridge where no forest canopy is present. Unlike Bear Creek, it is a sediment-rich stream. The predominant substrate is $2.5-$ to $10.2-\mathrm{cm}$ rocks. Stream width varies from 3 to $7.7 \mathrm{~m}$. Average stream gradient is about $4 \mathrm{~m} / \mathrm{km}$. After approximately $25 \mathrm{~km}$, EFPC enters Poplar Creek, which ultimately flows into the Clinch River. The EFPC is impacted by urban runoff from the city of Oak Ridge for approximately $12 \mathrm{~km}$ after it leaves Lake Reality, and the city of Oak Ridge Sewage Treatment Plant discharges into EFPC at River Mile 7.5.

\subsubsection{McCoy Branch}

McCoy Branch feeds into and drains Rogers Quarry. The headwaters to McCoy Branch begin near the top of Chestnut Ridge, just south of the Y-12 Plant. A dam was constructed at the headwaters of McCoy Branch to create a pond for settling ash once sluiced over Chestnut Ridge from the Y-12 Steam Plant. As a directive from TDEC, ash could no longer be sluiced directly to McCoy Branch. Under a temporary arrangement 
approved by TDEC, a pipeline was constructed to carry sluiced coal ash directly to Rogers Quarry. A dry ash handling system and a bottom ash handling facility have been constructed to process ash, and Rogers Quarry no longer receives any process discharges from the Y-12 Plant.

The lower reach of McCoy Branch converges with the Clinch River in a recreational area frequently used for swimming and fishing (e.g., Clark Center Recreational Park). In addition, the drinking water supply used at the park is withdrawn from Melton Hill reservoir within the McCoy Branch embayment.

Rogers Quarry and McCoy Branch were impacted by Y-12 Plant activities due to coal ash disposal practices. The reach below Rogers Quarry is quite short before it enters the embayment area $(1 / 3 \mathrm{~km})$. This stretch of the stream was also impacted by Y-12 Plant activities and, as a result, is sediment rich. Disposal practices have been discontinued at Rogers Quarry, and an abundance of fish and aquatic life are present.

\subsubsection{Clinch River}

The Clinch River drains an area of 2,857,415 acres and is the source of most of the potable water used in Oak Ridge. Supplies are drawn for DOE facilities, Clinton, Oak Ridge, and surrounding communities from the Clinch River. Water pumped by the Oak Ridge pumping station is delivered to ORNL, the Y-12 Plant, and the city of Oak Ridge. Treated wastewater is returned to the Clinch River from ORNL via White Oak Creek, from the Y-12 Plant via EFPC, and from the city of Oak Ridge via EFPC. Domestic wastewater (i.e., sanitary sewage) generated at the Y-12 Plant is discharged to the city of Oak Ridge Sewage Treatment Plant and undergoes treatment prior to being discharged to the EFPC at Creek Kilometer 13.4.

The Clinch River is a highly turbid, hard water system. The flow in the river is controlled through dam releases at Norris, Melton Hill, and Watts Bar reservoirs. Water supplies are taken from Melton Hill reservoir. Releases via Bear Creek and EFPC enter the Clinch River in the upper reaches of Watts Bar reservoir (i.e., below Melton Hill Dam). The 3-consecutive-day average, minimum flow rate expected to recur once every 20 years (3Q20) of the Clinch River has been calculated to be 96.3 MGD near its confluence with Poplar Creek. Norris, Melton Hill, and Watts Bar reservoirs are used extensively for recreation (e.g., fishing, boating, and swimming).

\subsubsection{Quarries}

Rogers Quarry is approximately 11.5 acres and was used as a settling basin for fly ash and bottom ash from the steam plant. Fly ash and bottom ash disposal has been discontinued from this quarry. Rogers Quarry regularly discharges to McCoy Branch, which enters the Clinch River at River Mile 41. Kerr Hollow Quarry is approximately 1.7 acres and was used for disposal of reactive wastes. Disposal was discontinued, and remediation activities have been completed. Rogers and Kerr Hollow Quarries are currently inactive.

\subsubsection{Other Unnamed Tributaries}

Several other unnamed tributaries on the south side of Chestnut Ridge are impacted by Y-12 Plant activities, including:

- unnamed tributary to Walker Branch at River Mile 0.6,

- unnamed tributary to McCoy Branch at River Mile 0.8, 
- unnamed Tributary 1 to Scarboro Creek at River Mile 0.7, and

- unnamed Tributary 2 to unnamed Tributary 1 at River Mile 0.5 .

The unnamed tributary to Walker Branch and the unnamed tributary to McCoy Branch could be impacted by the Y-12 Centralized Sanitary Landfill II. In addition, the unnamed tributary to McCoy Branch could be impacted by past operations at the Criticality Lab Building 9213, which is now inactive. No known disposal operations or other activities could have impacted the unnamed tributary 1 to Scarboro Creek. However, this tributary is fed by unnamed tributary 2, which may be impacted by Kerr Hollow Quarry and Chestnut Ridge Sediment Disposal Basin; both are past disposal sites which are now closed.

\subsection{Security}

The Oak Ridge Y-12 Plant is a DOE facility that produces and handles special nuclear materials. It is a fully secure facility surrounded by a barbed wire-topped chain-linked fence $8 \mathrm{ft}$ in height. A large security force and centralized security operations oversee the entire plant. The facility is continuously manned $24 \mathrm{hr} / \mathrm{day}$, 7 day/wk by armed guards from the Y-12 Plant Security Department. The perimeter of the facility is patrolled at intervals day and night via a system of patrol roads.

Most gates for pedestrian and vehicular access are manned by armed guards, and only authorized personnel are admitted. All individuals entering the Y-12 Plant must be authorized by the Y-12 Plant Security Department. These security procedures and equipment prevent accidental or intentional entry. 


\section{SECTION 2}

\section{STORM WATER CONTROLS (PROGRAMMATIC AND PHYSICAL)}

\subsection{Best Management Practices (BMPs)}

Best Management Practices apply to all water discharges, including storm water, and provide for overall protection of surface waters as required by Title 40, Code of Federal Regulations (CFR), Part 125, Subpart K (Criteria and Standards for Best Management Practices authorized Under Section 304(e) of the CWA). The objectives of the CWA are to restore and maintain the chemical, physical, and biological integrity of the nation's waters. In addition, all facilities subject to the requirements of the NPDES must maintain and implement a BMP plan prepared for that facility.

\subsubsection{Y-12 Plantwide BMP Plan}

The BMP plan for the Y-12 Plant specifies measures designed to prevent contaminants from entering EFPC, Bear Creek, Clinch River, and other tributaries that Y-12 Plant activities could affect. Water discharges emphasized in the plantwide BMP plan include precipitation runoff, groundwater, noncontact process waters, and other ancillary activities. The major objectives of the BMP plan are to:

- ensure compliance with various NPDES permit requirements,

- provide operating personnel with guidance relevant to NPDES permit requirements for their facility and/or project, and

- prevent or reduce pollutant discharge to the environment in accordance with the CWA and the Tennessee Water Quality Control Act.

\subsubsection{Site-/Project-Specific BMPs}

Many site-specific BMP plans are only in effect during the construction phase of the project to ensure that such concerns as site characterization, spill prevention and control, protective buffer zones, erosion/sediment control, and storm water control are a part of the preplanning process. However, site-specific BMP plans are also written as operational guidance for use by facility operators to ensure that pollution is prevented or minimized as required by the Y-12 Plant NPDES permit and the CWA. Surveillances are performed in accordance with TDEC storm water regulations by construction personnel, and oversight surveillances are conducted by Y-12 EMD personnel to ensure that project activities, including storm water management, do not negatively impact area streams.

Of particular importance regarding storm water controls in Bear Creek Valley is the protection of a minnowsized fish, the Tennessee Dace. Although not yet on the endangered species list, the state of Tennessee has placed this fish in the category of "in need of management." The Tennessee Dace is present in Bear Creek and many of its tributaries. This species is very susceptible to siltation caused by erosion/storm water, especially during the spawning period of late March through early July. Personnel involved with construction projects in the vicinity of the Bear Creek watershed will consider scheduling work around this period or provide state-of-the-art erosion/storm water controls before, during, and after any disturbance is made. 
The following is a list of projects that have site-specific BMP plans that are still in effect because the facility/site is still active or may be reactivated and are associated with storm water management:

- Steam Plant Ash Disposal - This BMP plan is primarily devoted to the construction of the Steam Plant Ash Disposal landfills, specifically, Industrial Landfill V and Construction/Demolition Landfill VII and supporting facilities. Located adjacent to one another, a total of four sedimentation ponds will be built to serve these landfills being built within a 210-acre tract of land drained by $\mathrm{McCoy}$ Branch and an unnamed tributary to the Clinch River. In addition, one off-site runoff pond has been constructed as part of Landfill $V$ to intercept storm water runon and to provide water for area wildlife. Many different types of storm water controls, both temporary and permanent, have been installed as part of this project, with many oversight surveillances to ensure compliance. Construction at Landfills V and VII is expected to continue into 1999.

- Elza 1 Site Work and Control Building (Electrical Supply) - This 4-acre construction site is located at the east end of the Y-12 Plant and drains to EFPC. Temporary storm water controls such as silt fences and staked straw bales have been installed. Surveillances have been performed during rain events to ensure that these controls remain functional. This project is expected to be completed in late 1995.

\subsubsection{Feasibility Study for the BMP to Control Area Source Pollutants in Parking Lots}

The report, "Feasibility Study for the Best Management Practices (BMP) to Control Area Source Pollution Derived from Parking Lots at the DOE Y-12 Plant," was issued in September 1992 and is based on data accumulated from five storm events over the period of November 1988 to April 1989. Major outfalls to EFPC were sampled during these storm events and analysis conducted to identify contaminates and their concentrations. The outfalls analyzed for this study were 4, 109, and 200 (the North/South Pipes), all of which constitute approximately $75 \%$ of the total flow of EFPC.

The major parameters of concern were identified as biochemical oxygen demand (BOD), chemical oxygen demand (COD), total suspended solids (TSS), fecal coliform, aluminum, copper, iron, lead, mercury, and zinc. Only TSS, aluminum, and iron were estimated to exceed anticipated regulatory storm water criteria. Note that the possibly anticipated parameter of oil and grease did not exceed water quality criteria.

The BMPs recommended for further evaluation consisted of nonstructural and structural controls. Nonstructural options consisted of sweeping and vacuum cleaning parking lots, use of absorbents, and education/training. Structural BMPs consisted of runon diversion, sedimentation basins, suspended solids separators, structural surface tiles, and chemical treatment. An oil/water separator is also in operation on EFPC just prior to the Lake Reality inlet.

Parking lot sweeping/cleaning and surveillance for leaking vehicles is a regular part of the Y-12 Plant practices. Parking lots are swept periodically, and surveillances for leaking vehicles are made daily. Major parking lots used by employees for personal vehicles are aligned the length of and just south of Bear Creek Road. These parking lots are designated East Portal, North Portal, Central Portal, West Portal, Bear Creek Portal, and S-3 Ponds Parking Lot. When gasoline or oil leaks are identified, the Plant Shift Superintendent (PSS) is notified, and the Spill Response Team is summoned to contain and remove the spill. Vehicle owners are notified that they need to take corrective actions to prevent further occurrences.

Additional sampling and studies were conducted in late 1993 to help determine if parking lots, specifically, were contaminate contributors to storm water runoff. Three parking lots were selected, and storm water runoff samples collected. The analytical data from these three lots was compared with the previous outfall 
sampling, which represented runoff from many different use areas besides parking lots. Research was also made of national studies on parking lots in order to provide a comparison of Y-12 Plant conditions to typical residential, commercial, or industrial locations. Data obtained in the Y-12 study was compared with information reported by the EPA, NPDES Storm Water: Group Application Data Base for Various Industrial Types. The data demonstrates that Y-12 Plant outfalls and the sampled parking lot runoff are less contaminated than similar employee vehicle parking lots in various industrial sectors. Comparisons were made to Industrial Sector 29--fabricated metal products; Industrial Sector 30--industrial/commercial machinery; Industrial Sector 31--electronics; and Industrial Sector 33--industrial activities on military facilities. This information is provided in a report entitled, "Storm Water Runoff for the Y-12 Plant and Selected Parking Lots."

Parking "areas" within the Y-12 Plant fence are not routinely swept, but are maintained clean by exercise of various housekeeping and BMP practices. All spills/leaks are reported to the PSS and corrected.

\subsubsection{Creek Buffer Zone}

A protective buffer zone has been established along the banks of EFPC. The buffer zone is an area on either bank of EFPC where activities such as vehicle parking, material storage, etc., are restricted in order to reduce the potential for pollution. The buffer zone is marked by signs, posts, and chains approximately $25 \mathrm{ft}$ from the top edge of the creek bank. The advantages of maintaining a buffer zone are:

- reduces the risk of spills and area-source pollution;

- improves surveillance along the creek;

- reduces the risk of flooding due to blockage of culverts, etc.; and

- improves the appearance of the creek and allows for improved habitat for fish and aquatic life.

\subsection{Erosion and Sediment Control}

The Y-12 Plant has prepared a guidance document, "Guidance for Erosion and Sediment Control Measures Applicable to Construction Projects at the Y-12 Plant," which, as the title suggests, provides guidance information to construction personnel for implementing storm water controls. In November 1994 a training course for engineering and construction personnel on implementation of effective erosion control measures was conducted.

General criteria are considered minimum requirements for controlling erosion and sediment from landdisturbing activities conducted at the Y-12 Plant. These general criteria establish the minimum standards of soil-conservation practices that apply to all land-disturbing projects.

Applicable general criteria will be satisfied in each approved erosion and sediment control plan; however, if a plan is found inadequate (with respect to the general criteria) in the field, the general criteria apply in addition to the provisions of the approved plan unless a variance has been granted.

\section{Conservation Practices}

The general criteria relate to the application of erosion- and sediment-control practices specified in this section; however, other innovative practices or modifications to standard practices may be used if approved by the EMD personnel prior to installation. Such practices will be thoroughly described and detailed to the satisfaction of the EMD personnel. 


\section{General Criteria}

\section{Stabilization of Denuded Areas and Soil Stockpiles}

Permanent or temporary soil stabilization will be applied to denuded areas after a final grade is reached on any portion of the site. Soil stabilization will also be applied to denuded areas which may not be at a final grade but will remain dormant (undisturbed) for longer than 60 days.

Soil stabilization refers to measures which protect soil from the erosive forces of raindrop impact and flowing water. Applicable practices include vegetative establishment, mulching, and the early application of a gravel base on areas to be paved. Soil stabilization measures will be selected to be appropriate for the time of year, site conditions, and estimated duration of use.

Soil stockpiles will be stabilized or protected with sediment-trapping measures or covered with impermeable materials to prevent soil loss.

\section{Establishment of Permanent Vegetation}

A permanent vegetative cover will be established on denuded areas not otherwise permanently stabilized. Permanent vegetation will not be considered established until a ground cover is achieved which, in the opinion of EMD personnel, is mature enough to control soil erosion satisfactorily.

\section{Protection of Adjacent Properties}

Properties adjacent to the site of land disturbance will be protected from sediment deposition. This may be accomplished by preserving a well-vegetated buffer strip around the lower perimeter of the land disturbance by installing perimeter controls such as sediment barriers, filters or dikes, sediment basins, or by a combination of such measures.

Vegetated buffer strips may be used alone only where runoff in sheet flow is expected. Buffer strips will be at least $20 \mathrm{ft}$ in width. If at any time it is found that a vegetated buffer strip alone is ineffective in stopping sediment movement onto adjacent property, additional perimeter controls will be provided.

\section{Stabilization of Sediment-Trapping Measures}

Sediment basins and traps, perimeter dikes, sediment barriers, and other measures intended to trap sediment on site will be constructed as a first step in grading and be made functional before up-slope land disturbance takes place. Earthen structures such as dams, dikes, and diversions will be seeded and mulched after installation.

\section{Sediment Basins}

Storm water runoff from drainage areas with 2 acres or greater disturbed area will pass through a temporary sediment basin or other suitable-sediment trapping facility with equivalent or greater storage capacity. The EMD staff may require sediment basins or traps for smaller disturbed areas where deemed necessary. The sediment basin requirement may also be waived, by variance, if the EMD staff agrees that site conditions do not warrant its construction. Water discharge permit applications will be submitted 180 days before basin completion. 


\section{Cut and Fill Slopes}

Cut and fill slopes will be designed and constructed in a way that will minimize erosion. Consideration will be given to the length and steepness of the slope, the soil type, up-slope drainage area, groundwater conditions, and other applicable factors. Slopes which are eroding excessively within 1 yr of construction will be provided with additional slope stabilizing measures until the problem is corrected. The following guidelines are provided to aid site planners and plan reviewers in developing an adequate design:

- Roughened soil surfaces are generally preferred to smooth surfaces on slopes.

- Diversions will be constructed at the top of long, steep slopes which have significant drainage areas above the slope. Diversions or terraces may also be used to reduce slope length.

- Concentrated storm water will not be allowed to flow down cut or fill slopes unless contained within an adequate temporary or permanent channel or slope drain structure.

- Wherever a slope face crosses a water seepage plane which endangers the stability of the slope, adequate drainage or other protection will be provided.

\section{Storm Water Management Criteria for Controlling Off-Site Erosion}

Properties and waterways downstream from construction sites will be protected from erosion due to increases in the volume, velocity, and peak flow rate of storm water runoff.

Concentrated storm water runoff leaving a construction site will be discharged directly into a well-defined, natural or manmade off-site receiving channel or pipe. If a well-defined off-site receiving channel or pipe does not exist, one will be constructed to convey storm water to the nearest adequate channel. Newly constructed channels will be designed as adequate channels.

An adequate channel is defined as a natural or manmade channel or pipe which could convey the runoff from a 2-yr storm without overtopping its banks or eroding after development of the site in question. A receiving channel may also be considered adequate at any point where the total contributing drainage is at least 100 times greater than the drainage area of the development site in question or if it can be shown that the peak rate of runoff for the site for a 2-yr storm will not be increased after development.

If an existing off-site receiving channel is not an adequate channel, one of the following options will be chosen:

1. Develop a site design that will not cause the predevelopment peak runoff rate from a 2-yr storm to increase. Such a design may be accomplished by enhancing the infiltration capability of the site or by providing on-site storm water detention measures.

or

2. Provide a combination of channel improvement, storm water detention, or other measures which are satisfactory to the EMD staff to prevent downstream channel erosion. 
All channel improvements or modifications will comply with all applicable laws and regulations. Prior to making any modifications to a flowing stream, a site review will be conducted by EMD personnel for approval to proceed.

Increased volumes of sheet flows which will cause erosion or sedimentation on adjacent property will be diverted to a stable outlet or detention facility.

\section{Stabilization of Waterways and Outlets}

All on-site storm water conveyance channels will be designed and constructed to withstand the expected velocity of flow from a 2-yr frequency storm without erosion. Stabilization adequate to prevent erosion will also be provided at the outlets of all pipes and paved channels.

\section{Storm Sewer Inlet Protection}

All storm sewer inlets which are made operable during construction will be protected so that sediment-laden water will not enter the conveyance system without first being filtered or otherwise treated to remove sediment.

\section{Working In or Crossing Watercourses}

Construction vehicles will be kept out of watercourses to the extent possible. Where in-channel work is necessary, precautions will be taken to stabilize the work areas during construction to minimize erosion. The channel (including bed and banks) will always be restabilized immediately after in-channel work is completed.

Where a live (wet) watercourse must be crossed by construction vehicles regularly during construction, a temporary stream crossing will be provided. Personnel from the WCS will determine if an Aquatic Resources Alteration Permit (ARAP) is needed.

\section{Construction Access Routes}

Wherever construction vehicle access routes intersect paved public roads, provisions will be made to minimize the transport of sediment (mud) by runoff or vehicle tracking onto the paved surface. Where sediment is transported onto a road surface, the roads will be cleaned thoroughly at the end of each day. Sediment will be removed from roads by shoveling or sweeping and be transported to a sediment-controlled disposal area. Street washing will be allowed only after sediment is removed in this manner.

\section{Disposition of Temporary Measures}

All temporary erosion- and sediment-control measures will be disposed of after final site stabilization is achieved or after the temporary measures are no longer needed, unless otherwise authorized by the local program administrator. Trapped sediment and other disturbed soil areas resulting from the disposition of temporary measures will be permanently stabilized with a vegetative cover to prevent further erosion and sedimentation. 
Maintenance

All temporary and permanent erosion- and sediment-control practices will be maintained and repaired as needed to ensure continued performance of their intended function.

\subsection{Spill Prevention/Response Program}

\subsubsection{Dike Management Program}

A dike is an embankment or ridge of either natural or manmade materials used to prevent the movement of liquids, sludges, solids, or other materials. As used in this plan, the embankments or ridges are typically greater than 6 in. in height. The dike is the most numerous type of secondary containment structure used at the Y-12 Plant. Three types of dikes are used: poured concrete dikes, excavated earthen dikes, and portable dikes.

The Y-12 Plant developed a dike management program based upon Procedure Y70-909, “Dike Management." This procedure underwent an extensive revision in 1991 after a team completed a survey of all secondary containment structures throughout the plant. Revisions to the procedure included directions on discharge of rain water that accumulates in dikes. Sampling parameters and discharge limits were established. The NPDES permit limits, average concentration values of water quality parameters in the EFPC, and other regulatory guidelines were used in establishing the discharge limits. The dike survey included a review of the contents of each tank in each dike; from this information a dike-specific list of parameters to be sampled for in accumulated rainwater was developed. Specific field sampling parameters, such as $\mathrm{pH}$, conductivity, and visual inspection for sheen or floating solids, were developed that are tested before each discharge. Field parameters such as nitrates and chlorine and other dike-specific sample parameters are sampled every $6 \mathrm{mo}$. The results of the $6 \mathrm{mo}$. sample results, the field measurements, along with the process knowledge that no leaks have occurred in the last 6 mo. support the conclusion that the concentrations of pollutants in the rainwater in a particular dike are minimal. Dikes in radiological areas are sampled before each discharge for total uranium and percent uranium-235.

When rainwater accumulates in a dike the operator notifies personnel in the WCS of the EMD; if necessary, a technician is sent to the field to perform the environmental monitoring. The data is returned to personnel in the WCS, and results are compared against discharge limitations. Dependent upon whether the results meet the established criteria, the water may be discharged to the storm sewer system, discharged to the sanitary sewer system; or collected and transported for on-site treatment at one of the four wastewater treatment facilities located within the Y-12 Plant. Records of each discharge are maintained by the dike operator and personnel in the WCS. Sample results are maintained in a data base, and trend analysis is periodically performed to identify and/or potentially circumvent any potential problems with contaminating storm water that collects in a particular dike.

Table 2.1 lists the secondary containment dikes in the Y-12 Plant exposed to storm water such that rainwater can accumulate in the dike. The table lists the storm water outfall or monitoring point that drains the area in which each dike is located.

\subsubsection{Spill Procedures}

Employees are required to immediately report all spills and releases to the Y-12 PSS. The PSS and the Emergency Preparedness Organization have developed a set of site-specific reference books for the Y-12 Plant to be utilized by the spill response team during a spill or release emergency. These reference 
books include the $Y-12$ Emergency Operations Plan and the Y-12 General Emergency Action Levels and Protective Action Guides. These reference books provide information on chemicals used and stored at the Y-12 Plant and actions to be taken in the event of a spill or release. The Spill Prevention, Control, and Countermeasures (SPCC) Plan and the Y-12 Guidance for Spill Response and Reporting provide guidance on spills or releases to the environment, federal agencies to be notified, and cleanup standards to be achieved. The Y-12 Plant procedures adopted to address responses to spills or releases both on and off the plant site are Y-12 Plant Procedures 40-007 and 40-008. These plant procedures address the response of Y-12 Plant Fire Department personnel, who are responsible for mitigation of the spill; Waste Transportation and Landfill Operations (WTLO) Department personnel, who are responsible for the cleanup of hazardous materials spills or releases; and Enriched Uranium Operations Decontamination Organization personnel, who are responsible for the cleanup of hazardous materials spills or releases in radioactive contamination areas.

\subsubsection{Spill Training}

The Y-12 Plant provides the necessary level of training for spill response personnel as mandated by the Occupational Safety and Health Act in 29 CFR, Part 1910. Under 29 CFR, Part 1910, all employees responsible for mitigation of a spill or supervising the response to a hazardous material spill or release have been trained to the level of Hazardous Material Technician $(80 \mathrm{hr})$. This level of training is obtained by the Y-12 Plant Spill Response Coordinator and members of the Y-12 Plant Fire Department. For hazardous material cleanups, 29 CFR, Part 1910, mandates that all employees be trained to the Hazardous Material Operations level ( $40 \mathrm{hr}$ ). The Y-12 Plant provides this level of training for those employees of WTLO Department and Enriched Uranium Operations Decontamination Organization responsible for the cleanup of hazardous materials spills. In conjunction to the hazardous material training mandated by $29 \mathrm{CFR}$, Part 1910, the Y-12 Plant provides additional training for personnel involved in spill response on Incident Command Structure (response procedures).

\subsection{Housekeeping}

The Y-12 Plant has established extensive housekeeping standards for building interiors and exterior areas. Each building or area responsible manager typically assigns housekeeping responsibilities to individual employees. Those individuals will periodically inspect their areas to ensure compliance and ensure that actions are taken to correct any unsatisfactory situations. The major items of concern are leaking containers, open containers, and trash that present a noncompliance or unsightly situation.

Housekeeping has been formalized by dividing the plant into 23 zones; select members from each area organization are assigned to the areas and are responsible for conducting quarterly inspections (see Section 2.5.5). Inspections are conducted quarterly, and results reported plantwide and to DOE. Items needing correction are identified, and lists maintained for follow-up to ensure action has been taken. The Plant Housekeeping and Site Appearance Committee additionally inspects some zones monthly as a validation of the quarterly zone inspections. Again, items needing correction are identified and logged for follow-up. 
TABLE 2.1

SECONDARY CONTAINMENT DIKES

\begin{tabular}{|c|c|c|c|c|l|}
\hline Outfall & $\begin{array}{c}\text { Dike } \\
9500\end{array}$ & Location & Volume (ft) & $\begin{array}{c}\text { Capacity } \\
\text { (gal) }\end{array}$ & Comments \\
\hline 002 & 0030 & 9712 North & 603 & 4,511 & \\
\hline 004 & 0031 & $9754-2$ West (car wash) & 9,600 & 71,813 & \\
\hline 016 & 0111 & 9737 SE & 230.40 & 1,724 & \\
\hline $021 / 022$ & 0028 & $9620-2$ East & 1,210 & 9,051 & Out-of-service, tank empty \\
\hline $021 / 022$ & 0107 & 9769 West & 201.67 & 1509 & \\
\hline $021 / 022$ & 0112 & 9224 South & 8,400 & 62,836 & \\
\hline $021 / 022$ & 0139 & $9417-9$ South & 270 & 2,020 & \\
\hline 033 & 0027 & $9404-5$ SE & $26,868.36$ & 200,989 & Earthen dike, tank empty \\
\hline 044 & 0025 & $9404-5$ SW & & Dike has been breached, no tanks present \\
\hline 044 & 0026 & $9404-5$ SW & & Dike has been breached, no tanks present \\
\hline $047 / 048$ & 0014 & $9201-2$ South & $1,852.5$ & 13,858 & \\
\hline $047 / 048$ & 0032 & $9401-1$ NW & 201.25 & 1505 & \\
\hline 057 & 0120 & Chestnut Ridge, CWSA & 8,500 & 63,634 & Under a roof \\
\hline 057 & 0121 & Chestnut Ridge, CWSA & 8,500 & 63,634 & Under a roof \\
\hline 062 & 0056 & $9720-44$ (Chestnut Ridge) & 1,400 & 10,473 & \\
\hline 109 & 0012 & 9995 East & 74.33 & 556 & Under a roof \\
\hline 109 & 0059 & 9212 NE & 2,880 & 21,544 & Out of service, tank empty \\
\hline 109 & 0068 & 9995 North (Alley) & 777.78 & 5,818 & Out of service, tank empty \\
\hline 113 & 0129 & $9204-1$ East & 20.8 & 156 & Out of service, with roof and \\
\hline 115 & 0024 & $9204-1$ SE & 1,600 & 11,969 & \\
\hline 135 & 0004 & $9204-2$ SE & 1,512 & 11,311 & \\
\hline 135 & 0005 & 9206 West & $5,331.38$ & 39,881 & \\
\hline 135 & 0065 & 9206 West & $1,162.02$ & 8,693 & Out of service, tank empty \\
\hline 135 & 0066 & 9206 West & 175 & 1,309 & Out of service, tank empty \\
\hline 200 & 0001 & 9818 South & $3,786.33$ & 28,324 & \\
\hline & & & & \\
\hline & & & & & \\
\hline & & & & \\
\hline
\end{tabular}


TABLE 2.1

SECONDARY CONTAINMENT DIKES

\begin{tabular}{|c|c|c|c|c|c|}
\hline Outfall & $\begin{array}{c}\text { Dike } \\
9500\end{array}$ & Location & Volume (ft3) & $\begin{array}{c}\text { Capacity } \\
\text { (gal) }\end{array}$ & Comments \\
\hline 200 & $0002 \mathrm{E}$ & $9204-2$ West & $1,843.44$ & 13,790 & \\
\hline 200 & $0002 \mathrm{~W}$ & $9204-2$ West & 600.42 & 4,491 & \\
\hline 200 & 0003 & $9201-5$ SE & \multicolumn{3}{|c|}{ Out of service, temporary dike in use } \\
\hline 200 & 0006 & $9204-2$ West & $5,426.67$ & 40,594 & \\
\hline 200 & 0007 & $9204-2$ West & 2,840 & 21,245 & \\
\hline 200 & 0008 & 9818 West & $3,863.33$ & 28,900 & \\
\hline 200 & $0009 \mathrm{~N}$ & $9201-5 \mathrm{~N}$ West & 335.52 & 2,510 & \\
\hline 200 & 00095 & $9201-5 \mathrm{~N}$ West & 111.84 & 837 & \\
\hline 200 & 0010 & $9401-3$ North & 945 & 7,069 & Out of service, condensate tank \\
\hline 200 & 0011 & $9811-1$ East & $6,951.58$ & 52,001 & \\
\hline 200 & 0013 & $9201-5$ East & 292.74 & 2,190 & \\
\hline 200 & 0015 & $9720-13$ South & $2,823.8$ & 21,123 & \\
\hline 200 & 0016 & $9204-4$ South & $5,818.08$ & 43,522 & \\
\hline 200 & 0017 & $9204-4$ South & $2,142.25$ & 16,025 & \\
\hline 200 & 0018 & $9204-4$ South & 528.42 & 3,953 & \\
\hline 200 & 0019 & $9204-4$ South & \multicolumn{2}{|c|}{ Dike has been breached, no tanks present } \\
\hline 200 & 0020 & $9204-4$ South & 661.89 & 4,951 & \\
\hline 200 & 0021 & 9215 West & $1,747.46$ & 13,072 & \\
\hline 200 & 0022 & $9204-3$ South & 1,728 & 12,926 & Earthen dike, empty tank \\
\hline 200 & 0023 & $9204-3$ South & 1,728 & 12,926 & Earthen dike, empty tank \\
\hline 200 & 0052 & $9811-1$ East & 133.33 & 997 & \\
\hline 200 & 0053 & $9401-3$ North & 64 & 479 & \\
\hline 200 & 0054 & $9401-3$ North & 349.84 & 2,617 & \\
\hline 200 & $0055 E$ & $9204-4$ East & $1,367.95$ & 10,233 & \\
\hline 200 & $0055 \mathrm{~W}$ & $9204-4$ East & 257.89 & 1,929 & \\
\hline & & & & & \\
\hline
\end{tabular}


TABLE 2.1

SECONDARY CONTAINMENT DIKES

\begin{tabular}{|c|c|c|c|c|c|}
\hline Outfall & $\begin{array}{c}\text { Dike } \\
9500\end{array}$ & Location & Volume (fi') & $\begin{array}{c}\text { Capacity } \\
\text { (gal) }\end{array}$ & Comments \\
\hline 200 & 0057 & $9204-2$ E North & 777 & 5,812 & \\
\hline 200 & 0058 & $9401-3$ North & 322.67 & 2,414 & \\
\hline 200 & 0064 & 9623 West & $8,043.35$ & 60,168 & \\
\hline 200 & 0067 & $9204-2$ West (trailer) & \multicolumn{2}{|c|}{ Dike has been breached, flows to dike 0090 } \\
\hline 200 & 0069 & $9201-5$ North & 777.78 & 5,818 & \\
\hline 200 & 0084 & $9204-4$ North & 217.5 & 1,627 & \\
\hline 200 & 0087 & 9999 West & $1,493.5$ & 11,172 & \\
\hline 200 & 0088 & 9999 West & $1,797.13$ & 13,443 & \\
\hline 200 & 0089 & 9999 West & $1,493.5$ & 11,172 & \\
\hline 200 & 0090 & $9204-2$ West & 2,160 & 16,158 & \\
\hline 200 & 0091 & 9215 SW & 612 & 4,578 & \\
\hline 200 & 0092 & $9201-5$ W South & 328.67 & 2,459 & \\
\hline 200 & 0093 & $9204-4$ SW & 825 & 6,171 & \\
\hline 200 & 0094 & $9201-4$ SW & 16 & 120 & \\
\hline 200 & 0096 & $9720-47$ Trailer & 986.94 & 7383 & \\
\hline 200 & 0097 & $9720-47$ Tanks & $2,748.01$ & 20,557 & \\
\hline 200 & 0098 & $9401-2$ North & 342.83 & 2,565 & \\
\hline 200 & 0099 & $9401-2$ North & 987.5 & 7,387 & \\
\hline 200 & 0100 & $9201-4$ SW & 4,400 & 32,914 & \\
\hline 200 & 0102 & $9204-4$ South & 117 & 875 & \\
\hline 200 & 0106 & $9204-4$ NE & 783 & 5,857 & \\
\hline 200 & $0110 E$ & 9818 West & $1,459.1$ & 10,915 & \\
\hline 200 & 0110 W & 9818 West & 806.42 & 6,032 & \\
\hline 200 & 0138 & $9417-8$ North & 270 & 2,020 & \\
\hline S02 & 0045 & $9616-7$ Tank Farm \#1 & 45,000 & 336,623 & \\
\hline & & & & & \\
\hline
\end{tabular}


TABLE 2.1

SECONDARY CONTAINMENT DIKES

\begin{tabular}{|c|c|c|c|c|c|}
\hline Outfall & $\begin{array}{c}\text { Dike } \\
9500-\end{array}$ & Location & Volume (ft $)$ & $\begin{array}{c}\text { Capacity } \\
\text { (gal) }\end{array}$ & Comments \\
\hline S02 & 0119 & Johnson Controls garage & 130 & 972 & \\
\hline S04 & 0046 & $9616-6$ North & \multicolumn{3}{|c|}{ Dike has been covered, no tanks present } \\
\hline S04 & 0047 & $9616-7$ Tank Farm \# 2 & 45,000 & 336,623 & \\
\hline S04 & 0048 & $9616-7$ Tank Farm \# 3 & 45,000 & 336,623 & \\
\hline S04 & 0108 & $9616-7$ Tank Farm \# 4 & 115,200 & 861,756 & \\
\hline S05 & 0086 & $9811-8$ South & 9,827 & 73,511 & \\
\hline S08 & 0101 & $9720-41$ North & 5,940 & 44,340 & \\
\hline S08 & 0103 & $9720-41$ North & 900 & 6,732 & \\
\hline S08 & 0104 & $9720-41$ North & 1,102 & 8,244 & \\
\hline S09 & 0128 & $9983-79$ North (BCBG) & 431.38 & 3,227 & \\
\hline S13 & 0109 & $9720-60$ DARA & 15,552 & 116,337 & \\
\hline S18 & 0137 & Landfill V & 18,095 & 135,336 & \\
\hline
\end{tabular}


Good housekeeping is also an important and required part of all construction and work site-specific BMPs. Housekeeping practices include keeping the site clean of trash and neatly organized. Examples of good housekeeping activities emphasized are:

- keeping paper and other trash contained,

- keeping portable toilets clean and contained,

- containerizing any rinse waters,

- not overfilling trucks, fuel tanks, etc.,

- keeping hazardous materials (i.e., oil, gas, etc.) in a safe and out-of-the-way area, and

- installation and maintenance of erosion-control features.

\subsection{Surveillance Programs}

A variety of surveillance programs are conducted at the Y-12 Plant. These surveillances serve as a programmatic control to reduce and/or eliminate contaminants from entering storm water on the plant site. Surveillance records for the programs detailed below are kept for at least $3 \mathrm{yr}$.

\subsubsection{Resource Conservation and Recovery Act (RCRA) Surveillances}

A high level of oversight for all RCRA hazardous waste management areas within the plant are maintained by EMD personnel. Surveillances are scheduled weekly at RCRA 90-day accumulation areas, permitted areas, interim status areas, and Toxic Substances Control Act (TSCA) facilities. Surveillances of satellite accumulation areas are typically conducted once a month. Results of each surveillance are entered into a computer data base maintained by the EMD at the Y-12 Plant. Memos are sent to waste generators to keep them informed of reported deficiencies, changes to regulations, upcoming audits, and the general condition of their facilities on an as-needed basis. Although the primary focus of these surveillances is compliance with the RCRA regulations, they also include a check for corrosion, leaks, spills, etc. Many aspects of the surveillances also prove to be useful in identifying potential problems associated with preventing pollutants from entering storm water.

Approximately 300 site reviews are conducted weekly at RCRA and TSCA facilities, but only a small portion of these areas are exposed to storm water. These surveillances are conducted by personnel from the EMD who have been trained regarding RCRA and TSCA regulations.

\subsubsection{Tanks .}

The Y-12 Plant has a recall inspection program managed by the Equipment, Testing, and Inspection (ET\&I) Department. Tank inspections are performed periodically using documented procedures and checklists to determine structural integrity and the condition of ancillary equipment. The results of all inspections are sent to the operators of the tanks, and a file copy is maintained in the ET\&I document center. The EMD staff also performs periodic surveillances during updating of the Y-12 Plant SPCC Plan.

During these inspections, emphasis is placed on spill-containment methods that are in place to prevent migration of spills and/or leaks to the Y-12 Plant storm sewer and the EFPC. 


\subsubsection{Dike Surveillances}

A surveillance program has also been developed for secondary containment dikes in the Y-12 Plant. Two types of surveillances are conducted. The ET\&I staff conducts the more technical inspection. When a new dike is installed on site, ET\&I conducts a hydrostatic (leak) test before the dike is put into service and schedules periodic retests. In addition, ET\&I conducts a visual review of every in-service dike on the site. The ET\&I inspectors look for cracks in floors and walls, peeling protective coatings, and other structural concerns. Upon completion of the inspection/test, an ET\&I Inspection Tag is placed in a visible place on the dike. This tag shows the date, and results of the latest inspection/test.

Personnel from the WCS schedule an annual visual surveillance of every outside dike on the site. The Y-12 Site is divided into five areas, based on clusters of dikes, and each area is reviewed once per year. Surveillances typically take place in March, May, July, September, and November. The focus of this surveillance is to look for environmental concerns; structural concerns are also noted. The EMD inspector looks for the ET\&I inspection tag on the dike as well as water, dirt, debris, trash, etc., contained within the dike. Upon completion of the surveillance, dike owners are contacted concerning the results of the surveillance and any corrective actions that may be necessary. Completed check sheets are maintained in the Water Compliance Inspection Notebooks.

\subsubsection{Construction Projects (Pre-, During, and Post-)}

Construction projects reviews, conducted by WCS personnel, may be conducted during all phases of the project. Initially, a preconstruction site review is conducted prior to an area being disturbed. These surveillances help determine if an NPDES, ARAP, U.S. Army Corps of Engineers permit, other water discharge permit, or BMP plan is needed for the project. This type of surveillance can also document preconstruction conditions and help establish protective buffer zones.

During construction, reviews are conducted to ensure permit conditions, the BMP plan, procedure, or guidance is being followed, and no obvious or potential environmental problems (water-related) exist. Environmental protection is the primary responsibility of the construction manager who conducts routine surveillances and maintains a $\log$ which is available for review by the EMD. During the surveillance, WCS personnel focus on items such as spill prevention and control, erosion-control measures, and surveillance of buffer zones. Permit conditions that are a part of an ARAP, Notice of Intent (NOI) to Construct, or other water quality permit can also be checked under this type of surveillance.

After construction is complete for a project covered by a storm water general permit or NOI, a sitestabilization surveillance is conducted to review and document when construction is complete and stable. At this time in the project, responsibilities for maintaining the site (e.g., storm water control, erosion/sediment control, revegetation) are transferred from the construction manager to the facility owner when the site is stable. This site-stabilization surveillance and accompanying checklist will be completed by WCS personnel.

\subsubsection{Housekeeping (Plant Appearance) Inspection}

The Y-12 Plant Housekeeping and Site Appearance inspections are conducted quarterly for each of the 23 zones (see Section 2.4). Preprinted forms are used to inspect building exteriors, construction sites, grounds and paved areas, and warehouse/storage areas. A standards document has been published that provides assistance in ensuring uniform criteria is applied across the Y-12 Plant. 
Quarterly results are tabulated and summarized for distribution. The individual zone managers select qualified personnel to conduct the inspections, maintain and follow deficiency items, and ensure that corrective actions have been taken. One goal of this inspection program is to ensure clean and trash-free areas, especially along EFPC and all drainage areas of the plant. These activities directly contribute to the reduction, if not elimination, of trash in storm water runoff.

\subsubsection{Pre-Holiday Surveillances}

Pre-holiday surveillances are conducted plantwide before a company holiday to identify potential environmental hazards. Emphasis is given to potential spill hazards that could go unnoticed while many employees are absent from work. This surveillance is normally performed the day before a 3-day weekend by the WCS staff. If a concern is noted, the project supervisor or department manager is contacted to correct the matter by close of business that day. Special attention is given to storm water control devices and potential storm water concerns.

\subsubsection{Erosion Control Surveillances}

Erosion control surveillances are conducted at all times of the year for active and inactive sites that can potentially cause siltation of waterways and/or deterioration of the Y-12 Plant property. Emphasis is placed on surveillance of inactive sites in the spring and fall during optimum revegetation periods. Erosion-control surveillances are also conducted during rain events to discover possible heavy contributions of sedimentation. Surveillances during rain events are usually conducted to investigate specific areas that could potentially contribute sediment or area-source pollutants to streams. Typically, these surveillances involve construction sites and are performed when the soil is saturated due to previous storm events. These surveillances are performed by WCS personnel trained in proper erosion-control techniques.

\subsubsection{Spill Response/Prevention Surveillances}

Each time the Spill Response Coordinator is notified of a potential spill, he or she completes a spill response report and maintains the report to document the response to the incident. The information collected includes:

- time/date and location of the spill,

- material and quantity spilled,

- responsible division,

- reason/cause of the spill,

- cleanup/corrective actions taken, and

- whether a reportable quantity has been exceeded.

Frequency analysis is periodically performed on the spill response reports to determine if particular types of incidents are recurring or to identify any other trends that may be occurring.

\subsubsection{Storage Area Surveillances}

In 1992 the WCS staff divided the Y-12 Plant into 12 zones, using fences and roads as boundary lines. One zone is reviewed each month by WCS personnel so that all outside storage areas are reviewed at least once per year. 
Surveillances are conducted using a standard Storage Area Inspection Checklist. Personnel from the WCS visit the zone looking for drums and/or polytanks. A checklist is completed for each drum/polytank or group of drums/polytanks. Each drum/polytank is reviewed for proper labeling, proximity to the storm sewer/creek, and the need for secondary containment. In addition, drums are observed for structural condition, visible corrosion, or other flaws that might inhibit their ability to contain materials; polytanks are inspected for leaking valves or fittings.

Upon completion of the surveillance, responsible individuals are contacted concerning deficiencies and necessary corrective actions. Deficiencies are logged in a notebook, and corrective actions are tracked. Completed forms are maintained by the WCS.

\subsubsection{Aquatic Life Surveys}

Semiannual fish population surveys are conducted in the EFPC in the Y-12 Plant boundary. These surveys have been conducted for years and are for tracking the increase in population of fish over time. The surveys are conducted by aquatic biologists from the Environmental Sciences Division at ORNL. Three locations in the EFPC--just below Lake Reality [East Fork kilometer (EFK) 23.4], behind the truck scales (EFK 24.7), and at the North/South Pipe (EFK 25.4)--are sampled every spring and fall using electroshocking wethooks. Elimination of untreated process discharges coupled with the closure of New Hope Pond in 1988, which removed the physical barrier and allowed fish migration upstream, the fish population in the upper reaches of the EFPC began to increase. Additionally, after January 1993, with the installation of the Systems I and II Dechlorination Units, the fish population in the uppermost portions of the EFPC has increased.

\subsubsection{Area Source Surveillance}

The Y-12 Plant NPDES permit requires that surveillances of the facility be conducted at least semiannually. This requirement is met through the semiannual surveillance of the effectiveness of the SWP3, as described in Section 6.4. As part of implementing this SWP3 an on site, outside area surveillance will be conducted along with review of available records and monitoring data to evaluate the accuracy of the plans and maps and to examine the adequacy of BMP measures in reducing pollutants in storm water runoff. This surveillance will be conducted by WCS personnel.

Associated with this surveillance, inspections will be conducted of Y-12 Plant parking lots to evaluate effectiveness of BMP measures. The Y-12 Plant Security personnel routinely patrol parking areas for spills of vehicle fluids. Automotive repairs are not permitted in employee parking lots. If a vehicle is observed leaking, the owner of the vehicle is contacted so the vehicle can be removed and repaired. Leaks are handled as spills, and immediately cleaned up. In addition to plant public address system announcements to inform employees that repairing vehicles on site is not allowed, personnel also sweep major parking areas periodically. Parking lot surveillances made as part of the SWP3 (at least semiannually) will be conducted during off-shift hours and focus on appearance, problem oil-spill spots, and debris which may be collected in storm catch basins.

\subsubsection{Creek Surveillance}

In 1993, the Facilities Management Organization assigned one employee on each of the 12-hr rotating shifts to observe EFPC for unusual conditions. At least one surveillance per shift is made of EFPC and Lake Reality. In addition, dechlorination systems are operated and maintained by shift employees and inspected at least once per shift. Numerous operational readings and samples are taken to ensure compliance. A 
procedure on the operation and maintenance of the dechlorinators is currently in draft and will be published soon.

Regular surveillance is also achieved as part of the Y-12 Plant Biological Monitoring and Abatement Program (BMAP) conducted under the NPDES permit. Biologists spend many hours on EFPC, Bear Creek, and other area streams, sampling water and conducting studies on fish and aquatic life as part of BMAP.

Surveillance has also been improved by implementing BMPs in the vicinity of the creek and the storm drains. See discussion of creek buffer zone in Section 2.1.4. Some of the most effective creek surveillance tools, however, are the real-time monitoring stations on EFPC, which can detect immediate changes such as $\mathrm{pH}$, conductivity, and flow. These monitoring stations are linked to the Y-12 Utility Management System office which triggers an alarm if set parameters are exceeded.

\subsubsection{Documentation of Concerns and Tracking of Corrective Actions}

The WCS staff of the EMD has a formalized program which includes surveillances of the areas mentioned in Sections 2.5.2 through 2.5.12. Each type of surveillance has a checklist developed to aid the inspector in conducting a complete and thorough review of the area of concern. Concerns are noted on the checklists which are kept in notebooks maintained by the WCS staff. The concerns are relayed to the responsible individual for follow-up and correction. Personnel review the notebooks for status of corrective actions and follow-up on a frequency dependent upon the severity of the concern.

The Y-12 Plant NPDES permit requires that inspections of the facility will be conducted at least semiannually to check the accuracy of the plan and maps and determine whether measures in the plan to reduce pollutants in storm water runoff are adequate and properly implemented or whether additional controls are needed. This requirement will be met through the execution of the various programs detailed in Section 2.5 of this plan. The storm water pollution prevention team will evaluate the effectiveness and completeness of the SWP3 semiannually by the assessment of all the surveillance programs.

\subsection{Control Structures}

A variety of control structures are in place at the Y-12 Plant. These serve as physical controls to reduce and/or eliminate contaminants from entering storm water on the plant site.

\subsubsection{Coal Pile Runoff Collection System with Treatment}

The Y-12 Plant coal pile (approximately 2.5 acres), located along the west side of the steam plant (Building 9401-3), is surrounded by a concrete collection trench which captures storm water runoff from the coal pile and directs it to a 400,000-gal, polyethylene-lined equalization basin. During and after rain events, acidic runoff is generated from the percolation of rainfall through the coal pile. This waste stream contains elevated levels of aluminum, iron, and zinc. The metals concentration in the runoff is significantly reduced through chemical precipitation at the Steam Plant Wastewater Treatment Facility prior to discharge to either the sanitary sewer system or the storm sewer system through NPDES Outfall 503.

\subsubsection{Sediment Ponds}

Sediment ponds have been constructed at the borrow areas and landfills on the Y-12 Plant site to control the sediment load. All storm water runoff from the borrow areas and landfills is directed through sedimentation 
ponds which provide a means to reduce the sediment load to area receiving streams. The sedimentation basins are designed to allow a significant portion of the sediment to settle out of the runoff. Accumulated sedimentation from the ponds is cleaned out regularly and disposed of at approved areas. Table 2.2 summarizes the locations of sediment ponds on the Y-12 Plant site.

\subsubsection{Oil Water Separator}

The Oil Water Separator is located on EFPC at the east end of the Y-12 Plant immediately south of the, now closed, New Hope Pond (see Y-12 Plant Storm Sewer Map A). This structure consists of a floating boom across the top of EFPC that diverts all floating debris into a side channel for collection and subsequent removal by Waste Management or Facilities Management Organization personnel. The diverted water is returned to EFPC by way of an inverted syphon. The side channel is a concrete structure that directs incoming debris and water in a circular flow to an inner area. This arrangement prevents backup and reintroduction of debris to EFPC. The separator was constructed in 1988, and the boom modified in 1990 for better performance during storm events.

Typically, collected debris consists of packing foam pieces, cups, grass/weeds, and wood; debris could contain any floating chemicals such as oil and gasoline. Waste Management and Facilities Management Organization personnel conduct periodic surveillances of the separator to ensure proper operation and timely removal of collected debris.

\subsubsection{Lake Reality}

Lake Reality is a hypolon-lined basin of approximately 2.7 acres in area with a maximum depth of $16 \mathrm{ft}$. Storm water runoff from the Y-12 Plant and adjacent areas enters EFPC at various points within the plant and flows through Lake Reality before exiting Bear Creek Valley and the DOE reservation. The drainage area extends north to the topographical peak of Pine Ridge, south to the topographical peak of Chestnut Ridge, and west to a north-south line that bisects the S-3 Ponds Parking Lot.

Water enters Lake Reality from the concrete-lined distribution channel through eighteen, 24-in. diameter corrugated metal dispersion pipes. A sluice gate is located approximately at the midpoint of each pipe in the berm between the channel and the basin. Water exits Lake Reality through a primary discharge structure at the west side of the facility. The discharge structure consists of a combination of Cipolletti and V-notch weirs; four, 48-in. reinforced concrete outlet pipes; and four fabricated slide gates.

Lake Reality serves as a sediment-collection basin and diversion system that, in the event of an uncontrolled spill, could contain the spilled material. The estimated retention time for Lake Reality is approximately $8 \mathrm{hr}$, but is dependent upon the rate of influent. Regular maintenance is performed on all sluice gates, housekeeping inspections are made, and the area is monitored by the PSS using nearby television cameras. Safety equipment consisting of life lines, a row boat, barriers, and access restriction signs are in place.

Lake Reality, at normal pool level, can accept an additional volume of approximately 20 acre-ft. As such, it serves as an excellent means for containment of any materials that might be spilled into EFPC. Procedures are maintained for collection of any spilled contaminant in Lake Reality; these procedures have been exercised twice.

During a spill containment event, the discharge slide gates are closed when the spilled material is observed about to exit Lake Reality. The spill will continue to be collected until the level of Lake Reality reaches the 
TABLE 2.2

\section{SEDIMENT POND LOCATIONS AT THE Y-12 PLANT}

\begin{tabular}{|c|c|c|}
\hline Pond Location & Area Land Use & $\begin{array}{c}\text { Approximate Area of } \\
\text { Disturbed Soil } \\
\end{array}$ \\
\hline East Borrow Area & $\begin{array}{l}\text { Clean soil borrow area for } \\
\text { Chestnut Ridge and east end } \\
\text { construction projects }\end{array}$ & 15 acres \\
\hline West Borrow Area & $\begin{array}{l}\text { Clean soil borrow area for Bear } \\
\text { Creek Burial Grounds and west } \\
\text { end construction projects }\end{array}$ & 40 acres \\
\hline Sanitary Landfill II & $\begin{array}{l}\text { Class II waste (e.g., industrial } \\
\text { waste) }\end{array}$ & 10 acres \\
\hline Industrial Landfill IV & $\begin{array}{l}\text { Class II waste (e.g., } \\
\text { nonhazardous, nonradioactive, } \\
\text { solid industrial) }\end{array}$ & 4 acres \\
\hline Landfill V & $\begin{array}{l}\text { Class II waste (e.g., industrial } \\
\text { waste, special waste, asbestos, } \\
\text { sludge) }\end{array}$ & $\begin{array}{l}25 \text { acres (entire area may not be } \\
\text { disturbed at the same time) }\end{array}$ \\
\hline Landfill VI & $\begin{array}{l}\text { Class IV waste (e.g., } \\
\text { construction/demolition waste, } \\
\text { fly ash, bottom ash from steam } \\
\text { plant, nonfriable asbestos) }\end{array}$ & 4 acres \\
\hline Landfill VII & $\begin{array}{l}\text { Class IV waste (e.g., } \\
\text { construction/demolition waste, } \\
\text { fly ash, bottom ash from steam } \\
\text { plant) }\end{array}$ & $\begin{array}{l}30 \text { acres (entire area may not be } \\
\text { disturbed at the same time) }\end{array}$ \\
\hline
\end{tabular}


bottom elevation of the 18 inlet pipes. At that time, the inlet pipe sluice gates are shut, and spill cleanup and removal can begin. Water will continue to fill the concrete distribution channel until it overflows through controlled discharge at the north end of Lake Reality. The EFPC, immediately below Lake Reality, will be essentially dry during the time between shut off of Lake Reality discharge and the diversion channel overflowing. This time duration will vary between $4-12 \mathrm{hr}$ depending on the flow rate in EFPC and will be much shorter during a rain event. The further downstream reaches of EFPC will not be totally dry because other tributaries will maintain a reduced flow considered sufficient to preclude any significant impact on fish population. There may be significant impact on fish populations in the Y-12 Plant area of EFPC, Lake Reality, and possibly even downstream depending upon the toxicity of the spilled material, quantity of material, duration of a continuing spill, and the flow rate of EFPC.

\subsection{Toxicity Reduction Evaluation}

The Y-12 Plant NPDES permit requires plant personnel to initiate a Toxicity Reduction Evaluation (TRE) study within $\mathbf{3 0}$ days of two consecutive significant toxicity test failures, or three significant toxicity test failures within a 12-month period, as specified in the biomonitoring requirements section of the permit (Part III-C, page 39). Although it was not required, a TRE was initiated in November 1994.

The Y-12 Plant TRE is a systematic evaluation intended to identify potential causes of toxicity in EFPC at the North/South Pipes and to determine the actions necessary to eliminate toxicity as defined in the Y-12 Plant NPDES permit. Because the Y-12 Plant is a complex facility with multiple wastewater treatment plant, process, and legacy discharges, the TRE involves an extensive investigation to identify toxicants of concern and cost-effective treatment or source-reduction options.

The Y-12 Plant TRE has four components and is modeled after guidance provided in the EPA publication Generalized Methodology for Conducting Industrial Toxicity Reduction Evaluations (TREs). The first component or tier of the Y-12 Plant TRE is the Toxicity Identification Evaluation. The second tier of the Y-12 Plant TRE will involve a Source Identification Evaluation. The third tier of the Y-12 Plant TRE is an evaluation of toxicity reduction methods, followed by the last tier which is a follow-up and confirmation that methods have reduced toxicity.

\subsection{Stop Work Authority}

All personnel at the $\mathrm{Y}-12$ Plant, including subcontractors and visitors, have the authority and responsibility to stop work considered to be an imminent danger to the safety or health of workers or other personnel, or to the environment. In such instances, employees are empowered to remove themselves and others from the hazard or take immediate actions to eliminate the hazard. Additionally, employees are not required to participate in work that they believe is a threat to their safety or health or to the environment. This policy is described in detail in Lockheed Martin Energy Systems, Inc., (Energy Systems) procedures. 


\section{SECTION 3}

\section{POTENTIAL POLLUTANT SOURCES}

\subsection{Toxic Substances Control Act (TSCA)/Polychlorinated Biphenyls (PCBs)}

At the Y-12 Plant, PCBs have been used in transformers, capacitors, heat-transfer systems, hydraulic systems, etc. Polychlorinated biphenyls and items containing PCBs (PCB items) are also stored awaiting offsite disposal. Consequently, PCBs and PCB items are closely regulated under TSCA. Existing Y-12 Plant procedures control PCB management at the plant. The procedures apply to management issues such as disposal of PCB light ballasts, control of PCBs in the laboratory, and PCB storage area management.

\section{Transformers}

Transformers are used throughout the Y-12 Plant. These electrical devices contain varying quantities of oil. Presently, the Y-12 Plant has approximately 161 pole-mounted PCB transformers in the power-distribution system. These transformers have not been sampled for PCB content; however, due to regulation, they will be assumed to contain greater than or equal to $500 \mathrm{ppm}$ PCB. A plan has been initiated to replace these transformers with non-PCB transformers. In addition, several high-voltage and pole-mounted transformers exist in the power distribution system with less than $50 \mathrm{ppm}$ but greater than or equal to $2 \mathrm{ppm}$ PCB. These transformers are not regulated for use under $40 \mathrm{CFR}$, Part 761. All transformers with a PCB concentration of greater than or equal to $50 \mathrm{ppm}$ are inspected quarterly, and those with concentrations between 2 and $50 \mathrm{ppm}$ are inspected annually.

\section{Transformer Pads}

In the late 1980s, all 89 PCB Askerel transformers were removed from the high-voltage system, and most were replaced with dry type equipment. The transformer pads for these Askerel transformers remain in place. Many pads are PCB-contaminated from historical leaks or spills of the Askerel fluid. These pads are cordoned off, and PCB spill signs are at each site.

Complications are incurred when attempting to decontaminate a porous material. A plan is being pursued to fit a steel plate over the PCB-contaminated transformer pads to ensure that PCB tracking does not take place.

\section{Z-Oil}

An active portion of the Y-12 Plant Z-Oil system (a massive heat-transfer system that once connected several buildings) uses mineral oil as a coolant. Levels of PCBs were inadvertently added to this portion of the Z-Oil system at some unknown time during past operations. The size of the Z-Oil system has been significantly reduced to the point where spills are highly unlikely from the active portion.

The active portion of the Z-Oil system is contained in Building 9204-3. The active portion is a closed system, completely disconnected from the inactive portion. The active portion contains approximately $30,000 \mathrm{gal}$ of oil, as well as aboveground piping and system components. The average concentration of PCBs in the Z-Oil is currently $14 \mathrm{ppm}$, well below the $50 \mathrm{ppm}$ ceiling set by the TSCA regulations. Walk down inspections are periodically performed on the system by Utilities Department personnel. 
The inactive portion of the Z-Oil system has been drained, the storage house disconnected, and lines capped or blank-flanged. No components have been removed at this time. It is assumed that residual oil remains in the active system. The active portion of the system receives a periodic walk down inspection.

Spills from the Z-Oil system are handled by spill response personnel. One of the first priorities in a spill response situation at this facility is to shut down the system's pumps. This will decrease the outflow rate of Z-Oil from a leak point, facilitating closure of the leak and reducing the amount of spilled oil to be controlled and cleaned up.

\section{PCB Storage Areas}

Polychlorinated biphenyl waste is temporarily stored at the Y-12 Plant prior to shipment off site for disposal. Storage for disposal, in regards to TSCA, refers to the "temporary" (i.e., less than $12 \mathrm{mo}$ ) storage of PCBs designated for disposal. Uranium-contaminated PCB wastes are being stored in excess of the $1-y r$ limit imposed by TSCA because of the lack of treatment and disposal capabilities. A compliance agreement with EPA Region IV is being pursued. Polychlorinated biphenyl waste is stored in either a PCB 1-yr storage area or a PCB 30-day storage area. The Y-12 Plant has four liquid PCB storage tank locations which include the Waste Oil Solvent Storage Facility, the Disposal Area Remedial Action Liquid Storage Facility, the Flammable Mixed Waste Storage Facility, and the Transformer Oil Storage Facility.

Some PCB items may be stored for up to $\mathbf{3 0}$ days from date of removal from service, provided a notation is attached to the PCB item indicating the date the item was removed from service. These PCB 30-day areas are sometimes located outside; however, the containers will be covered and will be placed on all-weather surfaces, such as asphalt or concrete. To ensure protection of the PCB containers, all 30-day areas are inspected weekly.

\section{Drained PCB-Contaminated Equipment}

Polychlorinated biphenyl-contaminated transformers and other PCB-contaminated electrical equipment (between 50 and $500 \mathrm{ppm}$ PCB) drained of free-flowing dielectric fluid are not subject to PCB storage requirements. Several drained PCB-contaminated and PCB-detectable (between 2 and $50 \mathrm{ppm} \mathrm{PCB)}$ transformers are located outside at the Y-12 Plant. In addition, several undrained PCB-detectable transformers are located in the line yard east of Building 9201-3. These pieces of equipment are not regulated for storage under $40 \mathrm{CFR}$, Part 761; however, the storage location is fenced and locked to ensure protection. The area is regularly inspected by electrical maintenance personnel. Should leaks be detected, spill response and cleanup are immediately undertaken.

\section{PCB Spills}

The cleanup of new PCB spills from a source of $50 \mathrm{ppm}$ or greater will begin as quickly as possible and no more than $24 \mathrm{hr}$ from the time of discovery, except that the cleanup of PCB transformers will begin within $48 \mathrm{hr}$. Any delays in the initiation of a cleanup will be justified (e.g., a civil emergency, adverse weather conditions such as a tornado, or emergency operating conditions) and documented. The cleanup will be given priority and will not be placed on indefinite hold. Spill areas that are inactive during "waiting periods" (i.e., awaiting sampling or analysis) will be cordoned off to restrict access. Response procedures for PCB spills are in accordance with the SPCC Plan for the Y-12 Plant. 


\subsection{Uranium}

The production of weapons components primarily involves metallurgical and machine shop operations rather than chemical process activity. Although recycling/recovery of special nuclear material and metal cleaning processes have involved the use of acids, organic solvents, and other hazardous materials, environmental regulations have promoted minimal use and close tracking of these materials. The long half-life of ${ }^{238} \mathrm{U}$ $\left(10^{9} \mathrm{yr}\right)$, however, requires continued effort to mitigate the effects of past practices that did not focus on containment of contamination.

Depleted uranium ${ }^{238} U$ ) and enriched uranium $\left({ }^{235} U\right)$ are the primary isotopes handled at the Y-12 Plant. The extremely valuable ${ }^{235} \mathrm{U}$ is an accountable material handled in exclusive areas, which drastically reduces the possibility of inadvertent or intentional loss of significant amounts of the material. Depleted uranium in trace quantities is widespread in certain areas of the plant. A possible source of uranium in precipitation runoff may be fallout from stack emissions prior to the required use of high efficiency particulate air filters on all uranium stacks.

Because uranium is primarily considered an inhalation or ingestion hazard due to alpha activity, it is not perceived as an element posing significant risk in water that eventually flows off-site. However, since 1980, many actions have been taken to improve uranium isolation and minimize the potential for release into the environment. Besides the additional emissions controls and monitoring (with associated alarms), material that can be tracked out on shoes from uranium-processing areas is mitigated by the use of shoe scuffs and boundary control stations.

Current BMPs prohibit the unsheltered storage of uranium billets and address uranium as a potential contaminant to surface water by diverting runon and runoff from areas that contain materials suspected or known to be radioactively contaminated.

Uranium in wastewater is removed at the Y-12 Plant West End Treatment Facility via hydroxide precipitation. Sludge from this process is accumulated in large storage tanks near the facility. Some uranium-contaminated metal and equipment is stored at the Y-12 Scrap Yard (see Section 3.5). BMPs emphasize contingency control of uranium contamination due to accidental releases and minimization of overland runoff through contaminated areas.

Uranium concentrations measured in EFPC, just prior to it leaving the site (Station 17), consistently demonstrate activities below the derived concentration guidelines established in DOE Order 5400.5 .

\subsection{Mercury}

The major source of mercury presently entering EFPC is through residual materials from the lithium isotope separation operations conducted at the Y-12 Plant from 1955 to 1963. Although these operations were conducted in closed systems (very little mercury was intentionally discarded as a process waste), significant losses did occur through mechanisms including process losses, leaks, spills, and trackout in equipment during maintenance operations. Most of the mercury lost to EFPC occurred between 1955 and 1959. About $192,500 \mathrm{lb}$ of the total $239,000 \mathrm{lb}$ of mercury lost to the creek occurred during that 8-yr period. Approximately $25 \mathrm{~g} /$ day of mercury residual is currently detected in EFPC at the Y-12 Plant daily.

By 1966, production activities requiring mercury had ceased, and all but one building (9201-4) formerly containing mercury had been stripped, decontaminated, and converted for other uses. Although 
Building 9201-4 was decommissioned and mercury was drained from the equipment in the early 1980 s, all mercury-contaminated equipment has not been removed from the building. Building 9201-4 remains out of service and closed except for decontamination efforts.

In 1985, an investigation was launched to determine the chronic and episodic sources of mercury that contaminate the Y-12 Plant drainage waters and to determine the impact that New Hope Pond had on effluent released to EFPC. The study showed that New Hope Pond acted as a very effective trap for mercury received from plant drainage waters. Several individual continuing sources of mercury were identified, and recommendations were made to monitor various locations within the plant and isolate mercury-contaminated streams from clean water streams. In addition, a task team equipped with vacuum-type equipment is trained to remove mercury from storm drains when visible mercury is discovered.

The NPDES permit requires the completion of projects which are designed to reduce mercury loading in EFPC to less than 5 g/day by December 31, 1998; however, this limit for mercury is under appeal by DOE. Mercury concentrations in the effluent from the Y-12 Plant have dropped considerably, and routine monitoring at Station 17 continues.

\subsection{Historical Spill Sites}

Table 3.1 is a listing of significant spills that have occurred at the Y-12 Plant in the past $3 \mathrm{yr}$. The events included are considered significant spills or releases with an identifiable source which occurred over a short period of time. This list does not include all incidents reported to the National Response Center as required under 40 CFR, Part 302.6, nor does it include minor oil sheens or discoveries of previously reported releases of mercury.

\subsection{Salvage Yard}

The Salvage Yard is an open and uncovered area in the western portion of the Y-12 Plant used for the storage of clean and radiologically contaminated scrap metals surplused from plant operations and construction/demolition work (see Y-12 Plant Storm Sewer Site Map C). The original storage area is now bisected (north-south) by a security zone that has been cleared of all materials. The result is an eastern and western area, both of which are maintained and operated by the Waste Management Organization.

Scrap metal is stored in both piles and SeaLand-type containers. Neither the piles nor containers are covered. Runoff from the areas is channeled into the adjacent plant storm water sewers. Runon from some adjacent areas is evident. All hazardous materials, including oils, greases, etc., have been removed from the scrap metal prior to arrival at the Salvage Yard. Waste Management personnel are in the ongoing process of containerizing radiological contaminated materials and shipping them off site for further processing. It is the intent to continue utilizing the Salvage Yard for storage and eventually remove all scrap metals.

The Salvage Yard is considered a source of metal and possibly radiological contamination for storm water runoff. A feasibility study was completed September 1993 that outlines measures that could be taken to control runon and runoff and provide for runoff collection in sediment basins. Runoff sampling and analysis for contaminates was conducted in early 1994 to determining actual contaminates and levels and thus, better define the extent of the potential problem. 
TABLE 3.1

HISTORICAL SPILL SITES AT THE OAK RIDGE Y-12 PLANT

\begin{tabular}{|c|c|c|c|}
\hline Date & Location & Quantity & Material \\
\hline $1 / 14 / 92$ & $\begin{array}{l}\text { Believed to have originated } \\
\text { at Building } 9212 / 9818\end{array}$ & $10-20 \mathrm{gal}$ & aluminum nitrate \\
\hline $1 / 24 / 92$ & Building 9212 & $600 \mathrm{lb}$ & hydrogen fluoride \\
\hline $2 / 16 / 92$ & EFPC; North/South Pipes & $<20 \mathrm{gal}$ & oil \\
\hline $4 / 3 / 92$ & Central Post & $\cdot 1.5 \mathrm{gal}$ & ethylene glycol \\
\hline $5 / 12 / 92$ & Building 9712 & $5 \mathrm{gal}$ & ethylene glycol \\
\hline 6/8/92 & Building 9401-2 & $20-50 \mathrm{gai}$ & chromated rinse water \\
\hline $7 / 20 / 92$ & Building 9204-2E & $200 \mathrm{lb}$ & aluminum oxide powder \\
\hline $8 / 19 / 92$ & EFPC; North/South Pipes & $\sim 10 \mathrm{lb}$ & sodium aluminum silicate \\
\hline 9/21/92 & Bethel Valley Road & $50-60 \mathrm{gal}$ & ethylene glycol \\
\hline $11 / 17 / 92$ & Building 9201-1 & $2 \mathrm{gal}$ & $\begin{array}{c}\text { xylene dimethyl benzene } \\
\text { (RCRA waste) }\end{array}$ \\
\hline $11 / 24 / 92$ & EFPC & 1 gal & hydraulic oil \\
\hline $12 / 1 / 92$ & Building 9206 & $2 \mathrm{gal}$ & ethylene glycol \\
\hline $12 / 2 / 92$ & West End Treatment Facility & $185 \mathrm{gal}$ & treated effluent \\
\hline $12 / 15 / 92$ & Building 9204-2 & $10-15 \mathrm{gal}$ & hydrochloric acid \\
\hline $2 / 3 / 93$ & Building 9204-4 & $200 \mathrm{gal}$ & raw sewage \\
\hline $2 / 10 / 93$ & Building 9809 & Unknown & uranium smoke \\
\hline $2 / 17 / 93$ & Building 9212 & $30 \mathrm{gal}$ & caustic solution \\
\hline $2 / 22 / 93$ & Building 9616-3TK3 & $200-250 \mathrm{gal}$ & process water \\
\hline $2 / 27 / 93$ & Building 9204-3 & $100-200 \mathrm{gal}$ & raw sewage \\
\hline $3 / 25 / 93$ & Building 9404-3 & $25 \mathrm{gal}$ & PCB oil \\
\hline 4/7/93 & Building $9720-8$ & $50 \mathrm{gal}$ & diesel fuel \\
\hline $4 / 24 / 93$ & Building 9204-2 & $15-20 \mathrm{gal}$ & hydrochloric acid \\
\hline $5 / 7 / 93$ & North Portal & $\sim 8 \mathrm{lb}$ & ethylene glycol \\
\hline $5 / 11 / 93$ & Building 9404-12 & $350 \mathrm{gal}$ & sulfuric acid \\
\hline
\end{tabular}


TABLE 3.1

HISTORICAL SPILL SITES AT THE OAK RIDGE Y-12 PLANT

\begin{tabular}{|c|c|c|c|}
\hline Date & Location & Quantity & Material \\
\hline $7 / 25 / 93$ & Bear Creek Burial Grounds & $1 \mathrm{gal}$ & PCB-contaminated \\
\hline 9/24/93 & Building 9998 & $2 \mathrm{gal}$ & ethylene glycol \\
\hline $11 / 20 / 93$ & Building 9204-2 & $5,000-6,000 \mathrm{gal}$ & $\begin{array}{l}\text { sodium hypochlorite, spill was } \\
\text { contained in dike }\end{array}$ \\
\hline $12 / 17 / 93$ & Building 9204-2 & $1,000 \mathrm{gal}$ & sodium hypochlorite \\
\hline $12 / 29 / 93$ & Building 9204-4 & $1,150 \mathrm{gal}$ & $\begin{array}{l}\text { rad-contaminated mop water, } \\
\text { spill contained in dike }\end{array}$ \\
\hline $1 / 20 / 94$ & Building 9201-5E & $6,000 \mathrm{gal}$ & potable water \\
\hline $1 / 26 / 94$ & Building 9204-4 & $2,000 \mathrm{gal}$ & $\begin{array}{l}\text { chromated rinse water, spill } \\
\text { contained in dike }\end{array}$ \\
\hline $2 / 15 / 94$ & Building $9401-2$ & $1,700 \mathrm{gal}$ & $\begin{array}{l}\text { chromated rinse water, spill } \\
\text { contained in dike. }\end{array}$ \\
\hline $2 / 18 / 94$ & Mount Vernon Road & $9 \mathrm{lb}$ & ethylene glycol \\
\hline $4 / 14 / 94$ & Building 9995 & $350 \mathrm{gal}$ & $\begin{array}{l}\text { hazardous waste acid from } \\
\text { analytical chemistry } \\
\text { operations, spill contained in } \\
\text { dike }\end{array}$ \\
\hline $4 / 18 / 94$ & Liquid Storage Facility & $88 \mathrm{gal}$ & $\begin{array}{l}\text { PCB leachate, spill contained } \\
\text { in dike }\end{array}$ \\
\hline 4/21/94. & Building $9767-3$ & $3,000 \mathrm{lb}$ & R-114 Freon \\
\hline $4 / 22 / 94$ & $\begin{array}{l}\text { Waste Coolant Processing } \\
\text { Facility }\end{array}$ & $700 \mathrm{gal}$ & $\begin{array}{c}\text { waste coolant, spill contained } \\
\text { in dike }\end{array}$ \\
\hline $5 / 17 / 94$ & Building 9404-18 & $150 \mathrm{gal}$ & acid waste \\
\hline 6/8/94 & Elza Switchyard & $20,000 \mathrm{gal}$ & potable water \\
\hline $6 / 21 / 94$ & Liquid Storage Facility & $3,300 \mathrm{gal}$ & $\begin{array}{l}\text { PCB leachate, spill contained } \\
\text { in dike }\end{array}$ \\
\hline $6 / 21 / 94$ & Building 9723-25 & $4.5 \mathrm{lb}$ & ethylene glycol \\
\hline 9/7/94 & Building 9404-20 & $16 \mathrm{lb}$ & ethylene glycol \\
\hline
\end{tabular}


TABLE 3.1

HISTORICAL SPILL SITES AT THE OAK RIDGE Y-12 PLANT

\begin{tabular}{|c|c|c|c|}
\hline Date & Location & Quantity & Material \\
\hline $10 / 12 / 94$ & Building 9723-11 & $9 \mathrm{lb}$ & ethylene glycol \\
\hline $1 / 1 / 95$ & Biology Area & $20,000 \mathrm{gal}$ & potable water \\
\hline $2 / 15 / 95$ & Liquid Storage Facility & $20 \mathrm{gal}$ & $\begin{array}{c}\text { PCB leachate, spill contained } \\
\text { in dike }\end{array}$ \\
\hline $2 / 27 / 95$ & Building 9818 & $5,000 \mathrm{gal}$ & $\begin{array}{c}\text { nitrate waste, spill contained in } \\
\text { dike }\end{array}$ \\
\hline $4 / 28 / 95$ & Building 9404-18 & $300 \mathrm{gal}$ & $\begin{array}{c}\text { softener water for } \\
\text { demineralizer facility }\end{array}$ \\
\hline $4 / 30 / 95$ & Building 9401-3 & $100 \mathrm{gal}$ & boiler blow-down \\
\hline $6 / 7 / 95$ & Second Street & $1.5 \mathrm{lb}$ & mercury \\
\hline $6 / 22 / 95$ & Bear Creek & $2,000 \mathrm{gal}$ & potable water \\
\hline $6 / 28 / 95$ & Landfills V \& VII & $4,500 \mathrm{gal}$ & potable water \\
\hline $7 / 19 / 95$ & Building 9212 & $300-600 \mathrm{gal}$ & brine solution \\
\hline $7 / 21 / 95$ & Building 9706-1A & $79 \mathrm{lb}$ & R-12 Freon \\
\hline $7 / 28 / 95$ & Liquid Storage Facility & $15 \mathrm{gal}$ & $\begin{array}{c}\text { PCB wastewater, spill } \\
\text { contained in dike }\end{array}$ \\
\hline $8 / 2 / 95$ & Building 9831; Manhole & $200 \mathrm{gal}$ & raw sewage \\
\hline $8 / 3 / 95$ & EFPC, Outfall 109 & $<5 \mathrm{gal}$ & $40 \%$ Styrene mixture \\
\hline
\end{tabular}




\subsection{Roofs}

There are almost 600 buildings or trailers at the Y-12 Plant with approximately 100 being considered major facilities based solely on size. Roof surfaces, therefore, constitute considerable area subject to possibly unique contaminate concentrations from numerous exhaust systems. Additionally, most roofs are the primary locations for electrical and heating/cooling systems that can be the source of oils and greases. Roofs are also a potential collection area for bird droppings. Therefore, during storm events, plant roof areas can contribute a significant portion of the total plant runoff to EFPC.

Sampling and analysis of major roof drain runoff was conducted in 1994. This data did not indicate that roofs are significant sources of contaminates; therefore, no corrective actions are planned.

\subsection{Parking Lots}

See discussion presented in Section 2:1.3

\subsection{Solid Waste Management Units (SWMUs)}

For purposes of this plan, SWMUs include any discernable units that have ever accumulated, treated, stored, or disposed of solid wastes (regardless of whether the units were intended for waste management). Such units may include landfills, surface impoundments, waste piles, land treatment units, incinerators, injection wells, tanks (including storage, treatment, and accumulation tanks), container storage units, and recycling units. SWMUs include any units subject to $40 \mathrm{CFR}$, Part 264, plus the units not subject to such regulations on the DOE Oak Ridge Y-12 Plant site. The definition also includes areas contaminated by routine, deliberate, or systematic discharges from process components.

The SWMU definition also includes (1) RCRA Interim Status area or RCRA-permitted facilities that are exposed to storm water runoff, including completed closures that have not yet received state acceptance of submitted closure certifications, and (2) RCRA 90-day accumulation areas used for accumulating large amounts of RCRA hazardous wastes for no longer than 90 days, after which the wastes will be sent for treatment, storage, or disposal.

Not included in the SWMU definition (for this plan) are (1) areas or facilities physically located totally within buildings which preclude their coming in contact with storm water, and (2) RCRA satellite accumulation areas, both inside and outside buildings, which can accumulate a maximum 55 gal of waste (or 1 qt of P-listed acutely toxic waste). Satellite areas are judged not to represent a significant pollutant source.

Those SWMUs that may represent a significant potential storm water pollutant source are listed in Table 3.2. The Environmental Restoration (ER) organization is tasked with the responsibility of remediation of SWMUs under the RCRA and Comprehensive Environmental Response, Compensation, and Liability Act (CERCLA) regulations. Site investigation, remediation decision making, and actual remediation are ongoing activities being conducted by ER personnel. The SWMU list is currently being updated and revised by ER personnel. Annual funding authorizations and risk-based prioritization determine specifically which SWMUs are being addressed at any one time.

The priority Y-12 Plant ER project is Reduction of Mercury in Plant Effluent Phase II. The goal of this program is to achieve compliance with the NPDES limits for mercury. The initial actions of this program have been the removal of known sources of mercury (selected sumps in Building 9202-1) from EFPC by the 
TABLE 3.2 Y-12 PLANT SOLID WASTE MANAGEMENT UNITS (SWMUs) SUBJECT TO CONTACT WITH STORM WATER

\begin{tabular}{|c|c|c|c|c|}
\hline SWMU Name & SWMU No. & Unit Location & $\begin{array}{l}\text { Outfall or } \\
\text { Storm Water } \\
\text { Monitoring } \\
\text { Point } \\
\end{array}$ & Description of Wastes \\
\hline Chestnut Ridge Security Pits & YD.023 & Chestnut Ridge & $062, \$ 18$ & $\begin{array}{l}\text { Classified wastes including machining fixtures, parts, shipping } \\
\text { containers, badges, computer tapes, trash; small quantities of } \\
\text { lithium hydride, beryllium oxide, hydrochloric acid, mineral oil, } \\
\text { organics, and lead. Unit has been closed. }\end{array}$ \\
\hline Bear Creek Burial Grounds & YD.024 & $\begin{array}{l}\text { Bear Creek Burial } \\
\text { Grounds }\end{array}$ & $\mathbf{S 1 3}$ & $\begin{array}{l}\text { Heavy metals, oils, coolants, salts, debris, solvents, asbestos, } \\
\text { mop water, material contaminated with radioisotopes. Units } \\
\text { have been closed. }\end{array}$ \\
\hline $\begin{array}{l}\text { Hazardous Chemical Disposal Area-- } \\
\text { Burn Yand-Bone Yard }\end{array}$ & YD.024.HC & $\begin{array}{l}\text { Bear Creek Burial } \\
\text { Grounds }\end{array}$ & SO8 & $\begin{array}{l}\text { Reactive and explosive laboratory chemicals, gas cyllinders, } \\
\text { sanitary trash, metal shavings, solvents, oils, and laboratory } \\
\text { chemicals, organics, metals, debris, acids, and beryllium. Area is } \\
\text { inactive. }\end{array}$ \\
\hline $\begin{array}{l}\text { Chestnut Ridge Sediment Disposal } \\
\text { Basin }\end{array}$ & $\mathrm{YD}-025$ & Chestnut Ridge & $\mathbf{S 1 7}$ & $\begin{array}{l}\text { Dredged sediments from New Hope Pond. Unit has been } \\
\text { closed. }\end{array}$ \\
\hline United Nuclear Landfill & YD.026 & Chestnut Ridge & $\mathbf{S 2 0}$ & $\begin{array}{l}\text { Drums of uranium-contaminated soil and waste sludges fixed in } \\
\text { concrete, boxes of equipment and soil, nitrate- and uranium- } \\
\text { contaminated wastes. Unit is inactive. }\end{array}$ \\
\hline Beta-4 Security Pits & YD.100 & $\mathrm{N}$ of $9204-4$ & 200 & $\begin{array}{l}\text { Uranium and uranium alloys, depleted and enriched uranium- } \\
\text { contaminated metal, organic compounds, acids, and } \\
\text { miscellaneous waste and debris. Unit is inactive. }\end{array}$ \\
\hline Sanitary Landfill I & YD.101 & \begin{tabular}{|l} 
Bear Creek Burial \\
Grounds
\end{tabular} & SO8 & Sanitary trash. Unit has been closed. \\
\hline Sanitary Landfill II & YD.102 & S of 9213 & $\mathbf{S 2 1}$ & $\begin{array}{l}\text { Sanitary industrial trash, beryllium oxide, asbestos, spoil } \\
\text { materials, and other special wastes. Unit has been closed. }\end{array}$ \\
\hline S-2 Site & YD. 103 & Third Street & 200 & $\begin{array}{l}\text { Corrosive and toxic aqueous wastes, possibly halogenated and } \\
\text { nonhalogenated solvents. Unit is inactive. }\end{array}$ \\
\hline Coal Pile Trench & YD.104 & W of 9401.3 & 503 & $\begin{array}{l}\text { Depleted uranium and depleted uranium alloys. Unit is } \\
\text { inactive. }\end{array}$ \\
\hline Ravine Disposal Site & YD.105 & $\mathrm{N}$ of 9712 & 002 & $\begin{array}{l}\text { Primarily construction spoil and possibly a small amount of } \\
\text { uranium. Unit is inactive. }\end{array}$ \\
\hline Rust Spoil Area & YD.106 & $\begin{array}{l}\text { W of West End } \\
\text { Treatment Facility }\end{array}$ & $\mathbf{S 2 4}$ & $\begin{array}{l}\text { Nonuranium-contaminated construction debris and spoil. Unit } \\
\text { is inactive. }\end{array}$ \\
\hline Spoil Area I & YD.107 & $\begin{array}{l}\text { S of Old Bear Creek } \\
\text { Road near West End } \\
\text { Treatment Facility }\end{array}$ & so6 & $\begin{array}{l}\text { Construction debris (e.g., concrete, asphalt, brick, brush, rock, } \\
\text { tile). Unit is inactive. }\end{array}$ \\
\hline
\end{tabular}


TABLE 3.2 Y-12 PLANT SOLID WASTE MANAGEMENT UNITS (SWMUs) SUBJECT TO CONTACT WITH STORM WATER

\begin{tabular}{|c|c|c|c|c|}
\hline SWMU Name & SWMU No. & Unit Location & $\begin{array}{c}\text { Outfall or } \\
\text { Storm Water } \\
\text { Monitoring } \\
\text { Point } \\
\end{array}$ & Description of Wastes \\
\hline Rogers Quarry & YD.108 & Bethel Valley Road & S19 & $\begin{array}{l}\text { Fly ash slurry from steam plant; also some ferrous metals and } \\
\text { small amounts of heavy metals, acids, and bases. Unit is } \\
\text { inactive. }\end{array}$ \\
\hline Filled Coal Ash Pond & YD.112 & Near McCoy Branch & S19 & Fly ash from steam plant. Unit is inactive. \\
\hline Bear Creek Road Debris Burial & YD.873 & $\begin{array}{l}\text { SE quadrant of } \\
\text { intersection of Bear } \\
\text { Creek Rd and Old Bear } \\
\text { Creek Rd, immediately } \\
\text { W of Rust Spoil Area }\end{array}$ & S24 & $\begin{array}{l}\text { Soil mixed with debris. Beta/Gamma counts up to } 250 \mathrm{cpm} \text { are } \\
\text { present in some areas. Unit is inactive. }\end{array}$ \\
\hline Creekside Debris Burial & YD.874 & $\begin{array}{l}\text { Wooded area on N side } \\
\text { of Bear Creek where } \\
\text { Spring SS-I flows into } \\
\text { Bear Creek }\end{array}$ & S06 & $\begin{array}{l}\text { Soil mixed with debris. Beta/gamma up to } 300 \mathrm{cpm} \text { are present } \\
\text { at some locations. Unit is inactive. }\end{array}$ \\
\hline ACN Drum Yand & YS.015 & W of 9720.13 & 200 & $\begin{array}{l}\text { Acetonitrile from } 1986 \text { to } 1985 \text {; area previously stored } \\
\text { equipment associated with an adjacent maintenance building. } \\
\text { Unit is inactive. }\end{array}$ \\
\hline $\begin{array}{l}\text { 9409-5 Storage Facility (IS-31); TS- } \\
07 ; \text { TS-08 }\end{array}$ & YS-017 & $\begin{array}{l}\text { 9409-5; includes IS-31, } \\
\text { TS.07, TS.08 }\end{array}$ & 033 & Waste oll/solvent mixtures. Unit is inactive. \\
\hline Salvage Yard Oil Storage Tanks & YS.018 & & 200 & PCB-contaminated oils. Unit is inactive. \\
\hline Garage Underground Tanks (IS-03) & YS-019 & $\begin{array}{l}\text { W of } 9712 ; \text { includes IS- } \\
03 \text { and TS.25 }\end{array}$ & 002 & $\begin{array}{l}\text { Waste oils, some of which contain chlorinated organics, PCBs, } \\
\text { and uranium. Unit has been closed. }\end{array}$ \\
\hline $\begin{array}{l}\text { Salvage Yard Oil/Solvent Drum } \\
\text { Storage Area }\end{array}$ & YS.020 & & 200 & $\begin{array}{l}\text { Waste oils containing chlorinated organics, uranium, and/or } \\
\text { beryllium; chlorinated organic solvents; nonchlorinated } \\
\text { lammable solvents. Unit is inactive. }\end{array}$ \\
\hline $\begin{array}{l}\text { Building } 9811-1 \text { Tank Storage Unit } \\
\text { (OD-7) (IS-23) }\end{array}$ & YS.021 & 9811.1 & 200 & $\begin{array}{l}\text { Waste oil/solvent/water mixtures. This unit operates under a } \\
\text { RCRA Permit. }\end{array}$ \\
\hline Interim Drum Yand (IS-20) & YS.030 & $W$ of $9720-32$ & 200 & $\begin{array}{l}\text { Various hazardous, mixed, and nonhazardous wastes including } \\
\text { chromium-containing sludge, mercury-contaminated wastes, } \\
\text { chlorinated and nonchlorinated organics, plating solutions, and } \\
\text { PCB-contaminated materials. Unit is inactive. }\end{array}$ \\
\hline Building 9215 Tank & YS.032 & sketch included & 200 & Mop water \\
\hline Building 9808 Tank & YS.033 & 9808 outside & 200 & Rinse water from pump shop. \\
\hline Building 9201-2 Tank & YS.034 & S side of $9201-2$ & 064 & Demineralizer regeneration wastewater. \\
\hline
\end{tabular}


TABLE 3.2 Y-12 PLANT SOLD WASTE MANAGEMENT UNITS (SWMUs) SUBJECT TO CONTACT WITH STORM WATER

\begin{tabular}{|c|c|c|c|c|}
\hline SWMU Name & SWMU No. & Unit Location & $\begin{array}{c}\text { Outfall or } \\
\text { Storm Water } \\
\text { Monitoring } \\
\text { Point } \\
\end{array}$ & Description of Wastes \\
\hline Building 9720.13 Tank & YS.035 & S side of 9720.13 & 200 & Rinse water from purmp shop. \\
\hline $\begin{array}{l}\text { Waste Oil/Solvent Storage Facility } \\
\text { (OD-9) (IS-28) }\end{array}$ & YS.039 & 9811.8 & S04 & $\begin{array}{l}\text { PCB-contaminated waste oil containing various concentrations } \\
\text { of organic solvents and uranium. }\end{array}$ \\
\hline $\begin{array}{l}\text { Liquid Organic Waste Storage } \\
\text { Facility (OD-10) (IS-30) }\end{array}$ & YS.040 & $9720-45$ & SO7 & $\begin{array}{l}\text { Chlorinated and nonchlorinated organics. This unit operates } \\
\text { under a RCRA Permit. }\end{array}$ \\
\hline Building 9212 Tank Farm & YS-041 & $\mathrm{N}$ of $9723-25$ & 200 & Nitric acid and caustic wastes. \\
\hline $\begin{array}{l}\text { Chestnut Ridge Borrow Area Waste } \\
\text { Pile }\end{array}$ & YS.042 & Chestnut Ridge & S18 & Mercury-contaminated soil. Unit is inactive. \\
\hline $\begin{array}{l}\text { East Chestnut Ridge Waste Pile (IS- } \\
\text { 32) }\end{array}$ & YS-043 & Chestnut Ridge & 017 & Contaminated soil. Unit is inactive. \\
\hline $\begin{array}{l}\text { Disposal Area Remedial Action } \\
\text { Liquid Storage and Treatment Unit }\end{array}$ & YS.052 & Adjacent to 9720.60 & $S 13$ & Groundwater contaminated with organics, PCBs, and oil. \\
\hline $\begin{array}{l}\text { Salvage Yard Scrap Metal Storage } \\
\text { Area }\end{array}$ & YS.111 & Salvage Yard & 200 & $\begin{array}{l}\text { Scrap metal contaminated with low levels of depleted or } \\
\text { enriched uranium. }\end{array}$ \\
\hline $\begin{array}{l}\text { Chesnut Ridge Uranium Oxide } \\
\text { Vaults }\end{array}$ & YS-114 & $\begin{array}{l}\text { Intersection of Third St. } \\
\text { and E Patrol Rd. }\end{array}$ & 019 & Uranium oxide. Unit is inactive. \\
\hline Thind Street Soil Pile & YS.116 & S of $9404 \cdot 3$ & 098 & Mercury-contaminated soil. Unit is inactive. \\
\hline $81-10$ Soil Pile & YS-117 & $81-10$ & 200 & Mercury-contaminated soil. Unit is inactive. \\
\hline Line Yard & YS-120 & W of 9720.8 & 019 & Electrical equipment. \\
\hline Roofing Waste Pile & YS-122 & S of $9204-4$ & 200 & Mercury-contaminated roofing waste. Unit is inactive. \\
\hline SY-200 Yand & YS.125 & $\begin{array}{l}\text { Adjacent to Old Bear } \\
\text { Creek Road }\end{array}$ & S06 & $\begin{array}{l}\text { PCB-, lead-, and radioactive-contaminated waste. Unit is } \\
\text { inactive. }\end{array}$ \\
\hline Mercury Contaminated Areas & YS.127 & numerous & 200 & Mercury. \\
\hline $\begin{array}{l}\text { Building 9201-2 Transformer \& } \\
\text { Capacitor Storage Area }\end{array}$ & YŚ-128 & $9201-2$ & 064 & PCB-contaminated oil. \\
\hline $\begin{array}{l}\text { Building 9204-1 Transformer } \\
\text { Storage Area }\end{array}$ & YS-129 & $9204-1$ & 114 & PCB-contaminated oll. \\
\hline $\begin{array}{l}\text { Building 9204.3 Transformer } \\
\text { Storage Area }\end{array}$ & YS-130 & $9204-3$ & 200 & PCB-contaminated oil. \\
\hline Tank & YS.215 & 9720-22 Tank & 200 & Wastewater and oil from press. \\
\hline Tank & YS-216 & & & Mop water. \\
\hline Tank & YS-217 & NE comer 9204.4 & 200 & Dilute caustic cleaner. \\
\hline Tank & YS-218 & NE corner 9204.4 & 200 & Ferric chloride/nitric acid. \\
\hline
\end{tabular}


TABLE 3.2 Y-12 PLANT SOLID WASTE MANAGEMENT UNITS (SWMUs) SUBJECT TO CONTACT WITH STORM WATER

\begin{tabular}{|c|c|c|c|c|}
\hline SWMU Name & SWMU No. & Unit Location & $\begin{array}{l}\text { Outfall or } \\
\text { Storm Water } \\
\text { Monitoring } \\
\text { Point }\end{array}$ & Description of Wastes \\
\hline Tank & YS.219 & $\begin{array}{l}\text { Between } 9622 \text { and First } \\
\text { Street; adjacent to } 9201 \\
\text { SE }\end{array}$ & 200 & Electrochemical machining wastewater contains chromium. \\
\hline Tank & YS-220 & $9204-2 \mathrm{E} / 9215$ alley & 200 & Dilute phosphoric/chromic acid. \\
\hline Tank & YS-221 & $9401-2$ & 200 & Dilute plating rinse solutions and acids. \\
\hline Tank & YS-222 & $9204-2 \mathrm{E} / 9215$ alley & 200 & Dilute chromic/phosphoric acid and mopwater. \\
\hline Tank & YS-223 & 9204-2E/9215 alley & 200 & Dilute wastewater. \\
\hline Tank & YS-224 & 9204-2E/9215 alley & 200 & Mopwater, caustic and acid waste. \\
\hline Tank & YS-225 & E side of 9202 & 047 & Laboratory wastewater and mop water. \\
\hline Tank & YS-226 & 9204- & 200 & Etching waste and mopwater. \\
\hline Tank & YS-227 & $9204-2$ & 200 & Caustic cleaning solutions. \\
\hline Tank & YS-228 & $9204-2$ & 200 & Sodium hydroxide. \\
\hline Tank & YS-229 & S of $9201.5 \mathrm{~N}$ & 200 & Detergent solution. \\
\hline Tank & YS-230 & S of $9201-5 \mathrm{~N}$ & 200 & Dilute plating rinsewater. \\
\hline Tank & YS-231 & NW corner $9204-3$ & 200 & Isotope separation waste. \\
\hline Tank & YS-232 & $\begin{array}{l}\text { SE corner } 9204.4 ; \\
\text { adjacent to } S W \text { corner } \\
\text { of } 9201-5\end{array}$ & 200 & Mop water and coolant. \\
\hline Tank & YS-240 & NE corner $9204-4$ & 200 & Dilute acid. \\
\hline Tank & YS-241 & NE corner $9204-4$ & 200 & Dilute cyanide rinse water. \\
\hline Tank & YS-243 & $\begin{array}{l}\text { Between } 9720.1 \text { and } \\
9201-1 \text { Tank }\end{array}$ & 109 & Mop waters and waste machine coolant. \\
\hline Dock 6 & YS-300 & E of 9212, Dock 6 & 109 & Uranium-contaminated trash, scrap metal and asbestos. \\
\hline Building 9995, Dock 12, AA-28 & YS-301 & E of 9995 & 109 & $\begin{array}{l}\text { Uncontaminated and uranium-contaminated asbestos, onganic } \\
\text { solvents, beryllium waste, used oil, uranium metal, and } \\
\text { laboratory wastewaters }\end{array}$ \\
\hline Dock 13 & YS.302 & SW comer 9995 & 109 & $\begin{array}{l}\text { Organic solvents, beryllium-, uranium-, and plutonium- } \\
\text { contaminated trash, and used oll. }\end{array}$ \\
\hline Dock 14 (includes AA-23) & YS.303 & $\begin{array}{l}\text { SE side of } 9212, \text { Dock } \\
14\end{array}$ & 200 & $\begin{array}{l}\text { Uranium machine turnings, mixed metal machine tumings, } \\
\text { mop water, waste machine coolant, uranium-contaminated } \\
\text { trash, used oil, organic solvents, and scrap metal. }\end{array}$ \\
\hline Dock 16 & YS.304 & \begin{tabular}{|l} 
SW side of 9996, Dock \\
16
\end{tabular} & 109 & Scrap metal. \\
\hline
\end{tabular}


TABLE 3.2 Y-12 PLANT SOLID WASTE MANAGEMENT UNIIS (SWMUs) SUBJECT TO CONTACT WITH STORM WATER

\begin{tabular}{|c|c|c|c|c|}
\hline SWMU Name & SWMU No. & Unit Location & $\begin{array}{l}\text { Outfall or } \\
\text { Storm Water } \\
\text { Monitoring } \\
\text { Point }\end{array}$ & Description of Wastes \\
\hline Dock 17 & YS-30 $\dot{5}$ & $\begin{array}{l}\text { SE side of } 9998, \text { Dock } \\
17\end{array}$ & 200 & $\begin{array}{l}\text { Uranium-contaminated trash and scrap, uranium oxide, and } \\
\text { carbon scrap. }\end{array}$ \\
\hline Dock 18 & YS-306 & E side 9215 & 200 & $\begin{array}{l}\text { Uranium-contaminated trash, metal scrap, and solvents, used } \\
\text { oil, waste acetic acid, and waste machine coolant. }\end{array}$ \\
\hline Building 9201-5 Southwest Yard & YS-307 & 9201-5 SW Yand & 200 & Penchloroethylene, methanol, and emulsifiers. \\
\hline Dock 20 & YS.308 & W side 9998 & 200 & Uranium-contaminated trash and scrap metal. \\
\hline Dock 50 & YS.309 & SE corner 9728 & 021 & $\begin{array}{l}\text { Uranium-contaminated clothing and wastewater from respirator } \\
\text { cleaning. }\end{array}$ \\
\hline Dock 125 & YS-310 & 9977-1, Dock 125 & & Used oil. \\
\hline Dock 147 & YS-311 & E side $9204-2$ & 200 & $\begin{array}{l}\text { Uranium-contaminated trash, photographic solutions, organic } \\
\text { solvents, and used oil. }\end{array}$ \\
\hline Dock 155 & YS.312 & $N$ side 9201.4 & 200 & $\begin{array}{l}\text { Uranium-contaminated trash and mercury-contaminated scrap } \\
\text { and trash. }\end{array}$ \\
\hline Dock 164 & YS-313 & E side 9808 & 200 & Uranium-contaminated trash, used oil, and paint thinner. \\
\hline Dock 167 & XS-314 & $\begin{array}{l}\text { NE corner 9201-5, } \\
\text { Dock } 167\end{array}$ & 200 & $\begin{array}{l}\text { Uranium-contaminated trash, beryllium-contaminated oils and } \\
\text { solvents, photographic and other testing solutions, and used oil. }\end{array}$ \\
\hline Dock 169 & YS.316 & NW side $9201-5$ & 200 & Uranium metal scrap, used oil, epoxies, and solvents. \\
\hline Dock 212 & YS-317 & NE side $9204-2 \mathrm{E}$ & 200 & $\begin{array}{l}\text { Uranium-contaminated trash, scrap metal and machine } \\
\text { turnings, and beryllium- and solvent-contaminated trash. }\end{array}$ \\
\hline Dock 213 & YS-318 & SW side $9204.2 \mathrm{E}$ & 200 & $\begin{array}{l}\text { Photographic solutions, uranium-contaminated scrap metal, and } \\
\text { epoxies. }\end{array}$ \\
\hline Dock 214 & YS-319 & SE corner 9204.4 & 200 & Photographic solutions, scrap metal, and epoxies. \\
\hline Dock 216 (includes AA-22) & YS-320 & $\begin{array}{l}\text { SW side } 9201-5 \mathrm{~N} \\
\text { Dock } 216\end{array}$ & 200 & $\begin{array}{l}\text { Uranium-contaminated trash, scrap metal, metal machine } \\
\text { turnings, photographic and metal plating solutions, uranium } \\
\text { machine turnings, and used oil. }\end{array}$ \\
\hline $\begin{array}{l}\text { Building 9201.1 West Yard } \\
\text { (includes AA-72) }\end{array}$ & YS-32I & 9201.1 W Yand & 099 & $\begin{array}{l}\text { Metal machine turnings, used oil, graphite scrap, sanitary trash, } \\
\text { and mop water. }\end{array}$ \\
\hline Building 9201-5E, Northeast Yard & YS-322 & $9201 \cdot 5 E, N E$ Yard & 200 & $\begin{array}{l}\text { Beryllium-contaminated solvents, uranium-contaminated } \\
\text { liquids, and used oil. }\end{array}$ \\
\hline Building 9201-5E South Yard & YS-323 & 9201-5E S Yard & 200 & Scrap metal, carbon foam, and beryllium-contaminated trash. \\
\hline Building 9201.5W South Yard & YS-324 & 9201-5W S Yard & 200 & $\begin{array}{l}\text { Uranium-contaminated trash, uranium and metal machine } \\
\text { tumings, used oil, and other miscellaneous wastes. }\end{array}$ \\
\hline Building 9202 East Dumpsters & YS-325 & E side 9202 & 047 & Uranium-contaminated trash. \\
\hline
\end{tabular}


TABLE 3.2 Y-12 PLANT SOLID WASTE MANAGEMENT UNI'SS (SWMUs) SUBJECT TO CONTACT WITH STORM WATER

\begin{tabular}{|c|c|c|c|c|}
\hline SWMU Name & SWMU No. & Unit Location & $\begin{array}{l}\text { Outfall or } \\
\text { Storm Water } \\
\text { Monitoring } \\
\text { Point }\end{array}$ & Description of Wastes \\
\hline Building 9202, East Pad, AA-31 & YS.326 & 9202 E pad & 047 & $\begin{array}{l}\text { Laboratory chemicals, and uranium- and beryllium- } \\
\text { contaminated trash. }\end{array}$ \\
\hline Building 9202 West Pad & YS-327 & $9202 \mathrm{~W}$ pad & 047 & Uranium-contaminated trash. \\
\hline $\begin{array}{l}\text { Building 9204-2 Hypochlorite } \\
\text { Tanker Station }\end{array}$ & YS.328 & SW corner $9204-2$ & 200 & Sodium hypochlorite wastewater. \\
\hline Building 9204-2 West Yard, AA.12 & YS.329 & 9204.2 W Yand & 200 & Drums of sludge, mineral oil, and various production wastes. \\
\hline Building 9204-4 East Pad & YS.330 & 9204.4 E pad & 200 & Uranium-contaminated trash. \\
\hline Building 9204-4 SW Yard & YS-331 & 9204.4 SW yard & 200 & $\begin{array}{l}\text { Uranium-contaminated trash, uranium and metal machine } \\
\text { tumings, uranium oxide, used oil, and uranium-contaminated } \\
\text { metal scrap. }\end{array}$ \\
\hline $\begin{array}{l}\text { Building } 9206 \text { Southeast Dock } \\
\text { (includes AA-13) }\end{array}$ & YS.332 & 9206 Dock 215 & 135 & $\begin{array}{l}\text { Scrap metal, uranium-contaminated trash, asbestos, and } \\
\text { uranium-contaminated organic solvents. }\end{array}$ \\
\hline Building 9215 West Pad & YS.333 & 9215 Dock 19E & 200 & Used oil and scrap metal. \\
\hline Building 9401-2, AA-17 & YS-334 & $\begin{array}{l}9401-2 \text { polytank } \\
\text { station }\end{array}$ & 200 & Electroless nickel bath and nitric acid. \\
\hline Building 9401.3 East Yand & YS-335 & 9401.3 E yand & 200 & Used oil. \\
\hline Building 9404-11, AA-11 & YS.336 & 9404.11 W yand & 200 & Used acetonitrile and rinse water. \\
\hline Building 9620-2 West Yard & YS.337 & $9620.2 \mathrm{~W}$ yard & 021 & Used oil. \\
\hline $\begin{array}{l}\text { Building } 9712 \text { Northeast Yard } \\
\text { (includes AA-03) }\end{array}$ & YS.338 & E side 9712 & 002 & Used oil and solvents. \\
\hline Building 9720.3 North Yard & YS-339 & $9720.3 \mathrm{~N}$ Yard & 200 & Uranium-contaminated trash. \\
\hline $\begin{array}{l}\text { Building 9720.6 North Polytank } \\
\text { Stations }\end{array}$ & YS-340 & $\mathrm{N}$ side 9720.6 & 010 & Laboratory and miscellaneous wastewater. \\
\hline Building 9720.13 West Yand & YS-341 & W side 9720.13 & 200 & Uranium-contaminated trash and used oil. \\
\hline Building 9744 North Dock & YS-342 & 9744 N dock & 200 & Uranium-contaminated filters. \\
\hline SID 50/51 Tanker Station & YS-344 & & & Waste nitrate solution and waste caustic. \\
\hline Building 9995, AA-28 & YS-345 & E side 9995, Dock 12 & 109 & Miscellaneous waste laboratory acids and wastewaters. \\
\hline Building 9204-2, AA-12 & YS-346 & SW side $9204-2$ & 200 & $\begin{array}{l}\text { Used oil, solvents, anisole, batteries, aerosol cans, and chemical } \\
\text { products. }\end{array}$ \\
\hline $\begin{array}{l}\text { Building } 9731 \text { South Pad (includes } \\
\text { AA-33) }\end{array}$ & YS-347 & $9731 \mathrm{~S}$ pad & 114 & Nitric acid and photographic solutions. \\
\hline Dock 119 & \begin{tabular}{|l|} 
YS.348 \\
\end{tabular} & SE side 9204.1 & 114 & Copy machine toner. \\
\hline Building 9733.2 Dock & YS.349 & $9733-2$ & 109 & Copy machine toner. \\
\hline
\end{tabular}




\begin{tabular}{|c|c|c|c|c|}
\hline 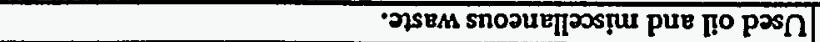 & $\angle 90$ & preX II-I0Z6 & $9 \varepsilon L \cdot S X$ & $\nabla Z \cdot V V ! I-I 0 Z 6$ su!pl!ng \\
\hline 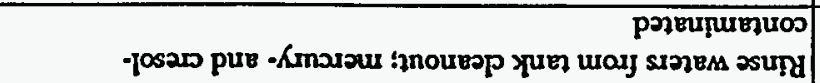 & 002 & 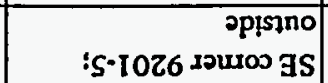 & $\exists \varepsilon L-S X$ & 00IVV:S-I 0z6 Su!p!ng \\
\hline 's2qunz jusosalonแ & 002 & $9 \mathrm{I}^{\circ} 0 \mathrm{~L} \angle 6 \mathrm{~J}^{\circ} \mathrm{MN}$ & $8 Z L \cdot S \lambda$ & $06 \cdot V V: 91-0 Z \angle 6$ \&u!p!!ng \\
\hline 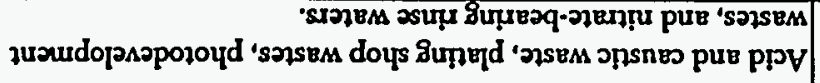 & SOS & 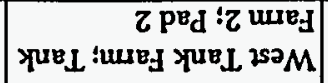 & $\angle Z L \cdot S X$ & 68-VF :L-9196 8uाp!!ng \\
\hline 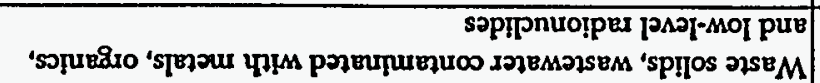 & EIS & 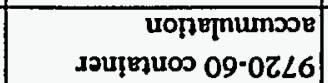 & $S Z L-S X$ & 98-VV :09-0ZL6 8u!p!ng \\
\hline 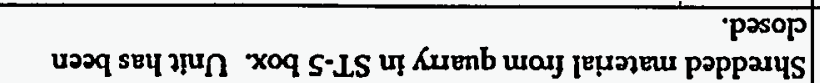 & LIS & 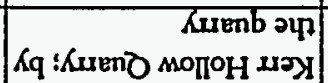 & $\varepsilon Z \angle-S \Lambda$ & 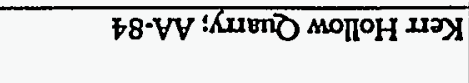 \\
\hline 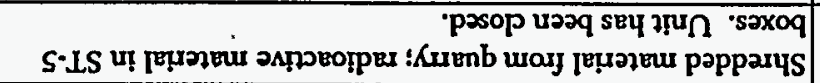 & LIS & 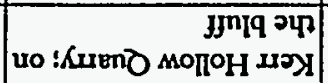 & $z \tau L \cdot S \lambda$ & 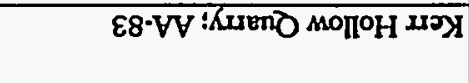 \\
\hline 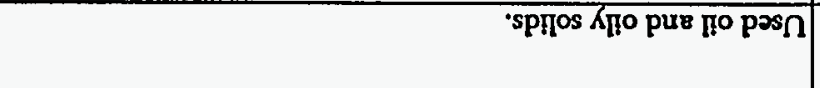 & 980 & 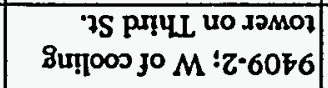 & $0 Z L \cdot S \lambda$ & $8 Z I \cdot V V: 8 L \cdot V V: Z \cdot 6076^{8}$ 8u!PI!ng \\
\hline 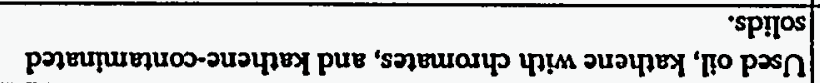 & 002 & 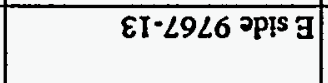 & $61 \angle-S X$ & $\angle L-V V$ 'EI-L9L6 \&u!p!!ng \\
\hline 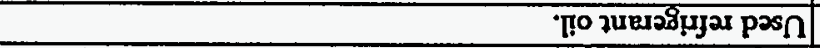 & 010 & 9.0ZL6 गp!s S & $8 I \angle-S \lambda$ & SL-VV :9-0ZL6 8utp!!ng \\
\hline 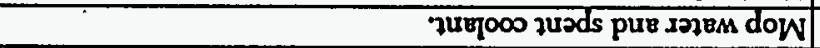 & 660 & pie $\lambda M ! I \cdot 10 Z 6$ & $9 I \angle-S \lambda$ & $Z L-V V ! I-I 0 Z 6$ \&u!p! \\
\hline 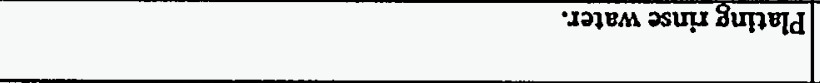 & 002 & 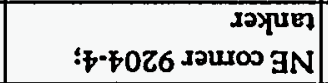 & SIL-SX & $0 L-V V: 7-70 Z 6$ \&u!PI!ng \\
\hline & 002 & 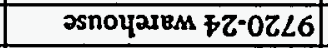 & $0 I \angle-S X$ & $76-V V: 7 Z-0 Z L 6$ \&u!p!!ng \\
\hline & $\angle F O$ & 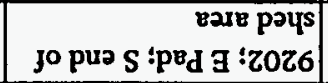 & $80 L-5 \lambda$ & I6-VV:Z0Z6 8ับ?p!ng \\
\hline & 002 & $8666^{\text {IวUนOO }} \mathrm{MN}$ & $\angle O L \cdot S X$ & L8-VV ‘8666 \&u!p!!ng \\
\hline 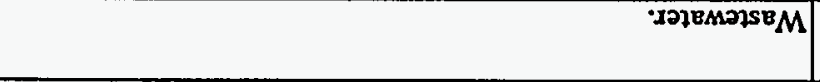 & 002 & 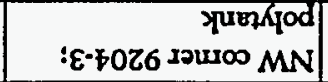 & SOL-SX & Z8-VV $: \varepsilon-70 Z 6$ \&u!PI!ng \\
\hline 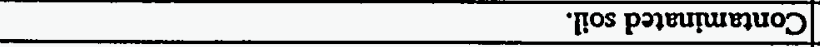 & 002 & OI-I8 & $00 \angle-S x$ & 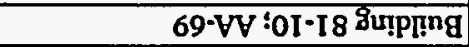 \\
\hline 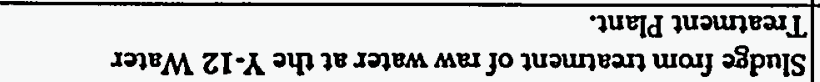 & & & $207-5 x$ & 28ిยง \\
\hline 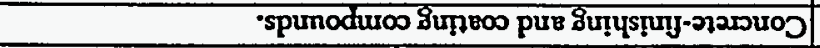 & 90S & & $\operatorname{IOF}-\mathrm{S} \alpha$ & 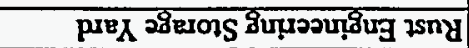 \\
\hline 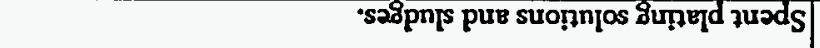 & 002 & prex g z:I076 & ISE-S $\lambda$ & 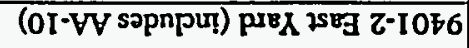 \\
\hline 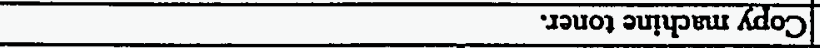 & 002 & & OSE-S $\lambda$ & 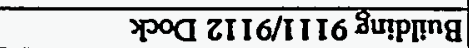 \\
\hline sə7se $M$ Jo uond dỵsad & 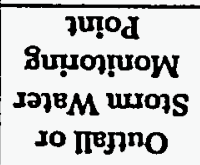 & uopteso' 7!บ $\Omega$ & -ON OWMS & วU8N NWMS \\
\hline
\end{tabular}

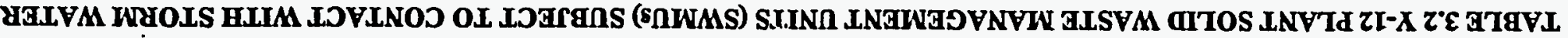


TABLE 3.2 Y-12 PLANT SOLID WASTE MANAGEMENT UNITS (SWMUs) SUBJECT TO CONTACT WITH STORM WATER

\begin{tabular}{|c|c|c|c|c|}
\hline SWMU Name & SWMU No. & Unit Location & $\begin{array}{c}\text { Outfall or } \\
\text { Storm Water } \\
\text { Monitoring } \\
\text { Point }\end{array}$ & Description of Wastes \\
\hline Building 9201-4; AA102 & YS.740 & $\begin{array}{l}\text { SE corner 9201-4; } \\
\text { outside near tank } \\
2100 \mathrm{U}\end{array}$ & 200 & Lead-, mercury-, and chromium-contaminated sludge and water. \\
\hline Building 9201.4; AA104 & XS.741 & $\begin{array}{l}\text { SE corner 9201-4; } \\
\text { outside near tank } \\
2100 U\end{array}$ & 200 & Lead-, mercury-, and chromium-contaminated sludge and water. \\
\hline Building 9201-5; AA-09 & YS.742 & S side 9201.5 & 200 & \\
\hline Building 9201-5; AA-20; AA.64 & $\begin{array}{l}\text { YS-743; YS. } \\
749\end{array}$ & $\begin{array}{l}\text { E side 9201-5; Dock } \\
167\end{array}$ & 200 & Used oil and beryllium oxdde oil filters. \\
\hline Building 9201.5; AA-21 & YS.744 & $\begin{array}{l}\text { 9201-5; W Shop; SW } \\
\text { Yard }\end{array}$ & 200 & Used oil and miscellaneous wastes. \\
\hline Building 9201-5E; AA-55 & YS-748 & NE comer $9201-5 E$ & 200 & Mop water and spent coolant. \\
\hline Building $9201-5 N_{;}$AA-16 & YS-750 & $\begin{array}{l}9201.5 N_{;} W \text { end } \\
\text { polytank }\end{array}$ & 200 & Wastewater. \\
\hline Building $9201-5 N_{i}$ AA-30 & YS-751 & \begin{tabular}{|l|} 
9201-5N; Tanker SE of \\
Dock 216
\end{tabular} & 200 & Mop water and spent coolant. \\
\hline Building 9201.5W; AA.54 & YS.754 & $\begin{array}{l}\text { SW comer } 9201-5 W \\
\text { mopwater tank }\end{array}$ & 200 & Mop water and spent coolant. \\
\hline Building 9203; AA120 & YS-757 & 9203; S Pad & 047 & Miscellaneous waste. \\
\hline Building 9204-2; AA1 17; AA127 & YS.758 & W of $9204-2$ & 200 & Sodium hydroxdde and sodium hypochlorite. \\
\hline Building 9204.4; AA-41 & YS.760 & NE corner $9204-4$ & 200 & \\
\hline Building 9206; AA-13 & YS-763 & 9206 E Side & 135 & $\begin{array}{l}\text { Distillates, organics, mop water, coolant, freon, combustibles, } \\
\text { acids, oil, aerosol cans, batteries, and light bulbs. }\end{array}$ \\
\hline Building $9401-2 ;$ AA-10 & YS.774 & W side 9401-2; outside & 200 & $\begin{array}{l}\text { Acid baths, nickel-chloride plating solution, lead plating } \\
\text { solution, and nickel strip solution. }\end{array}$ \\
\hline Building $9616.6 ;$ AA-18 & YS.780 & 9616.6 & S05 & \\
\hline Building 9616-7; AA-26 & YS.781 & 9616-7; E Side WETF & sos & Miscellaneous wastewater. \\
\hline Building 9616-7; AA-38 & YS-782 & 9616-7; WETF; outside & S03 & Contaminated solids, spent filters, and spent carbon. \\
\hline Building 9616.7; AA.39 & YS-783 & $\begin{array}{l}\text { 9616-7; SW of tank } \\
\text { farm }\end{array}$ & sos & Miscellaneous wastewater. \\
\hline
\end{tabular}


TABLE 3.2 Y-12 PLANT SOLID WASTE MANAGEMENT UNITS (SWMUs) SUBJECT TO CONTACT WITH STORM WATER

\begin{tabular}{|c|c|c|c|c|}
\hline SWMU Name & SWMU No. & Unit Location & $\begin{array}{c}\text { Outfall or } \\
\text { Storm Water } \\
\text { Monitoring } \\
\text { Point } \\
\end{array}$ & Description of Wastes \\
\hline Building 9616-7; AA122 & YS-784 & $\begin{array}{l}\text { West Tank Farm \#1; } \\
\text { Unloading Pad }\end{array}$ & S02 & Metal sludge. \\
\hline Building 9616-7; AA125 & YS-785 & W of $9616-7$ & SO5 & Contamianted solids, spent filters, and spent carbon. \\
\hline Building 9623; AA107 & YS-787 & $\begin{array}{l}9623 ; \text { outside near tank } \\
21010\end{array}$ & 200 & Lead., mercury-, and chromium-contaminated sludge and water. \\
\hline Building 9712; AA112 & YS.789 & W of Building 9712 & 002 & Organic wastes and personal protective equipment. \\
\hline Building 9720-13; AA.05 & YS.791 & 9720.13 Pump Shop & 200 & Used oil, oily rags, and petroleum solvents. \\
\hline Building 9720.6; AA.06 & YS-798 & S side 9720.6 & 010 & \\
\hline Building 9720.6; AA.43 & YS-799 & $\begin{array}{l}\text { SE corner } 9720.6 ; \\
\text { polytank }\end{array}$ & 010 & \\
\hline Building 9731; AA-33 & YS-802 & 9731 S Pad & 114 & Miscellaneous wastes. \\
\hline Building 9754; AA-49 & YS.804 & $\begin{array}{l}9754 ; \text { tanks directly } \mathrm{N} \\
\text { of building }\end{array}$ & 004 & \\
\hline Building 9754; AA.50 & YS.805 & $\begin{array}{l}9754 ; \mathrm{N} \text { of } \\
\text { miscellaneous waste } \\
\text { from tank excavation }\end{array}$ & 004 & \\
\hline Building 9767-13; AA-40 & YS-806 & $\begin{array}{l}9767-13 ; \text { polytank } \\
\text { section of tank transfer } \\
\text { area }\end{array}$ & 200 & \\
\hline Building 9769; AA-15 & YS-807 & $\begin{array}{l}\text { 9769; Dock } 251 \\
\text { (Outside) }\end{array}$ & 021 & $\begin{array}{l}\text { Acids, hexane, PCBs, miscellaneous organics, and methylene } \\
\text { chloride. }\end{array}$ \\
\hline Building $9818 ;$ AA-19 & YS.811 & $\begin{array}{l}9818 \text { Tanker Loading } \\
\text { station; NW comer of } \\
9212\end{array}$ & 200 & $\begin{array}{l}\text { Nitrate waste, blodenitrification sludge waste, and caustic } \\
\text { waste. }\end{array}$ \\
\hline Building 9983.47; AA129 & YS.812 & 9983.47 & & Sodium hydroxide and sodium hypochlorite. \\
\hline Kerr Hollow Quarry; AA.61 & YS.815 & $\begin{array}{l}\text { Kerr Hollow Quarry; } \\
\text { near front access gate }\end{array}$ & S17 & $\begin{array}{l}\text { Absorbent materia, rubber gloves, oil- and grease-contaminated } \\
\text { tyvek suits, bulk oil, and grease. Unit has been closed. }\end{array}$ \\
\hline Building 9212; AA-53 & YS.818 & 9212; alley & 200 & Mop water and spent coolant. \\
\hline 9818, NW Side, AA130 & YS.819 & 9818 & 200 & Light bulbs, aerosol cans, scrap metal, and starters. \\
\hline LSF Diked Area; AA137 & YS.826 & Building 9720.60 & S13 & $\begin{array}{l}\text { Contaminated personal protective equipment, solids, and spent } \\
\text { filter drums. }\end{array}$ \\
\hline $9103 ;$ AA-45 & YS.831 & Building 9103, W Dock & 200 & Photographic fixer, and spent batteries. \\
\hline
\end{tabular}


TABLE 3.2 Y-12 PLANT SOLID WASTE MANAGEMENT UNITS (SWMUs) SUBJECT TO CONTACT WITH STORM WATER

\begin{tabular}{|c|c|c|c|c|}
\hline SWMU Name & SWMU No. & Unit Location & $\begin{array}{c}\text { Outfall or } \\
\text { Storm Water } \\
\text { Monitoring } \\
\text { Point }\end{array}$ & Description of Wastes \\
\hline 9207; AA.59 & YS.832 & $\begin{array}{l}\text { 9207, N Dock, Room } \\
1019\end{array}$ & 021 & \\
\hline Urea Storage Site & YS-834 & Chestnut Ridge & 017 & Not waste storage. Unit is inactive. \\
\hline 9202; AA143 & YS.835 & $\begin{array}{l}\text { 9202, E Pad, SE End of } \\
\text { Pad }\end{array}$ & 047 & Radioactive-contaminated lead and ferrous metal. \\
\hline 9998, Beside Dock 20 (TS-34) & YS.849 & 9998, Beside Dock 20 & 200 & PCB and Capsur combustibles and Capsur liquids. \\
\hline Y-12 Plant Pistol Range & YS.860 & S of New Hope Pond & 007 & Lead. \\
\hline Old Fuel Station Site & YS.861 & Scarboro Road & 9422-1 & Fuel. \\
\hline $\begin{array}{l}\text { 9616-6, Outside, North of Building } \\
\text { (AA-147) }\end{array}$ & YS.863 & $\mathrm{N}$ of 9616.6, Outside & $\mathrm{SO2}$ & Corrosive wastewater samples. \\
\hline 9720.47, Hypo Station (AA.149) & YS.866 & 9720.47; Hypo Station & 200 & Silver-contamianted rain water. \\
\hline $\begin{array}{l}9818 \text {, Northwest of Building (AA- } \\
\text { 152) }\end{array}$ & YS-868 & 9818 & 200 & Raffinate. \\
\hline 9983-BZ, NE of Bldg. (AA-153) & YS.869 & NE of 9983-BZ & & Solids and liquids from dike decontamination. \\
\hline $\begin{array}{l}\text { 9204-3, Northwest Corner, Dike } 40 \\
\text { (AA-155) }\end{array}$ & YS.872 & $\begin{array}{l}\text { NW corner 9204-3; } \\
\text { Dike 40 }\end{array}$ & 200 & Corrosive TCLP wastewater. \\
\hline Biodenitrification Unit & YT.002 & 9818 & 200 & Nitrate-bearing uranium recovery wastes. \\
\hline $\begin{array}{l}\text { Waste Machine Coolant } \\
\text { Biodegredation Facility }\end{array}$ & YT.003 & & 200 & $\begin{array}{l}\text { Waste machine collants containing organic carbon, chlorinated } \\
\text { organics, methyl ethyl ketone, some EP toxic metals, and } \\
\text { depleted uranium. Unit is inactive. }\end{array}$ \\
\hline Oil Retention Pond No. 1 & YT.008 & $\begin{array}{l}\text { Bear Creek Burial } \\
\text { Ground }\end{array}$ & S13 & Seepage from burial trenches. Unit has been closed. \\
\hline Oil Retention Pond No. 2 & YT.009 & $\begin{array}{l}\text { Bear Creek Burial } \\
\text { Ground }\end{array}$ & S13 & Seepage from burial trenches. Unit has been closed. \\
\hline New Hope Pond & YT-010 & & 9422-1 & $\begin{array}{l}\text { Discharges to New Hope Pond have contained plating } \\
\text { rinsewaters, acids, caustics, and various organics. Unit has been } \\
\text { closed. }\end{array}$ \\
\hline Kerr Hollow Quarry (IS.02) & YT.012 & & S17 & $\begin{array}{l}\text { Water-reactive and potentially explosive wastes, including alkali } \\
\text { metals, metal hydrides, unstable organics, chlorides, and } \\
\text { materials with dangerous properties. Unit has been closed. }\end{array}$ \\
\hline
\end{tabular}


TABLE 3.2 Y-12 PLANT SOLID WASTE MANAGEMENT UNITS (SWMUs) SUBJECT TO CONTACT WITH STORM WATER

\begin{tabular}{|c|c|c|c|c|}
\hline SWMU Name & SWMU No. & Unit Location & $\begin{array}{c}\text { Outfall or } \\
\text { Storm Water } \\
\text { Monitoring } \\
\text { Point }\end{array}$ & Description of Wastes \\
\hline Waste Material Preparation Facility & YT-013 & $9720-28,9401.4$ & 200 & $\begin{array}{l}\text { General plant trash, light gauge ferrous metols, and wooden } \\
\text { pallets, all of which are contaminated with low levels of } \\
\text { uranium. Unit is inactive. }\end{array}$ \\
\hline Oil Landfarm & YT-014 & $\begin{array}{l}\text { Bear Creek Burial } \\
\text { Ground }\end{array}$ & S08 & Used oil and machine coolant. Unit has been closed. \\
\hline Waste Coolant Processing Facility & YT.038 & S of 9204.4 & 200 & $\begin{array}{l}\text { Waste machine coolants containing organic carbon, chlorinated } \\
\text { organics, methyl ethyl ketone, some EP toxic metals, and } \\
\text { depleted uranium. Unit is inactive. }\end{array}$ \\
\hline Decant Treatment Facility & YT.044 & $\begin{array}{l}\text { Formerly S-3 Liquid } \\
\text { Treatment Facility }\end{array}$ & SO2 & $\begin{array}{l}\text { Denitrified supernatant from S-3 Ponds and West End } \\
\text { Treatment Facility tanks. Unit is inactive. }\end{array}$ \\
\hline Sewer Sediment Drying Facility & YT-118 & $\begin{array}{l}\text { S Patrol Road, next to } \\
\text { Containerized Waste } \\
\text { Storage Area }\end{array}$ & 062 & Mercury-contaminated storm sewer sediments. Unit is inactive. \\
\hline $\begin{array}{l}\text { Interim Reactive Waste Treatment } \\
\text { Area (IS-42) }\end{array}$ & YT-150 & W of 9830.1 & S10 & Sodium.potassium alloy. Unit is inactive. \\
\hline Uranium Treatment Unit (IS-48) & YT-151 & 9206; E dock & 135 & Uranium-contaminated solutions. Unit is inactive. \\
\hline
\end{tabular}


construction and operation of an Interim Mercury Treatment Unit. The Central Mercury Treatment Unit is being designed for handling mercury-contaminated sump flows from other buildings. Personnel from ER are currently conducting investigations (sampling and analysis) of runoff for the upper EFPC, Bear Creek, and $\mathrm{McCoy}$ Branch drainage areas. Four dry-weather sampling events for upper EFPC were completed in 1993, and several storm water sampling events were completed in 1994. This data may assist in identification of which specific SWMUs are potential contributors to runoff contaminates. This type of investigation may continue until specific remediation actions are identified and completed.

\subsection{Areas Where Significant Materials are Stored, Treated, or Disposed}

For the purpose of this SWP3, significant materials include but are not limited to the following types of materials:

- raw materials

- solvents

- fuels

- detergents

- metallic products

- CERCLA hazardous substances

- Superfund Amendments Reauthorization Act extremely hazardous substances

- fertilizers

- herbicides

- pesticides

- waste products

- scrap metal

A detailed discussion is provided in Section 4, "Outfall Specific Information," of the significant materials stored in the areas drained by the storm water outfalls and monitoring points.

\subsection{Urea and Salt Storage}

Urea is purchased in bags rather than bulk and is stored in a semienclosed, roofed area on the east side of Building 9720-2. Because of these changes, the potential that a urea release could reach a surface stream is remote. The Salt Storage Area is used to store road salt and gravel. It is located just west of Building 9993. The facility consists of a row of three plywood bins, each measuring $20 \mathrm{ft}$ wide by $45 \mathrm{ft}$ long by $6 \mathrm{ft}$ high on an asphalt-paved lot that slopes to the north. Road gravel is stored in the center bin, and road salt is contained in the two adjacent bins. A plastic covering on each of the salt bins protects the contents from precipitation and wind, as do the bin walls. The asphalt pavement under the bins protects against leaching.

\subsection{Water Priority Chemicals (WPCs)}

Water priority chemicals (WPCs) are those chemicals which meet the following criteria:

1. Listed at $40 \mathrm{CFR}$, Part 372.65 ; and

2. Manufactured, processed, or used above threshold levels; and

3. Meet one of the following:

a. Listed in 40 CFR, Part 122, Appendix D, Tables II, III, or V;

b. Listed as a "hazardous substance" at 40 CFR, Part 116.4; or

c. EPA listed pollutants for which acute or chronic toxicity criteria have been published. 


\subsubsection{Storage of WPCs}

The WPCs in use at the Y-12 Plant include hydrochloric acid, methanol, and nitric acid. This list will be updated each year. Table 3.3 contains a list of the locations where WPCs are stored. These locations provide one or more of the following storm water protection measures: curbing, gutters, or other drainage control, roofs, covers, or other protection from storm water or wind.

The WPCs are stored in containers (i.e., tanks, drums) and are conveyed in overhead piping which are compatible with their contents. Most WPCs are stored indoors or under a roof or cover to prevent contact with storm water. If the WPC container must be exposed to storm water (i.e., bulk storage tanks, rail car unloading areas), secondary containment is provided, and accumulated storm water is not released to the environment until sampling has confirmed that a leak has not occurred. All secondary containment units are manually drained to prevent accidental discharge.

\subsubsection{Surveillance of WPC Storage Areas Exposed to Storm Water}

See Section 2.5.3 for a description of secondary containment dike surveillances.

Surveillance frequency of WPC storage areas exposed to storm water varies from daily to monthly depending on the quantity and frequency of use of the chemical, as well as operational experience. The surveillances are conducted by a designee of the facility manager and are designed to identify leaks or conditions which could lead to direct contact of storm water these WPCs. Visual inspections are conducted of any overhead piping conveying WPCs to ensure that unintentional discharges are minimized. Personnel in the WCS also conduct a surveillance of these areas annually. The WPC storage areas are listed in Table 3.3.

\subsubsection{Training for Personnel Managing WPCs}

See Section 6.3 for a description of the storm water pollution prevention training program. 
TABLE 3.3 STORAGE LOCATIONS OF WATER PRIORITY CHEMICALS

\begin{tabular}{|c|c|c|c|}
\hline Chemical Name & $\begin{array}{c}\text { Container Type } \\
:\end{array}$ & $\begin{array}{l}\text { Building } \\
\text { No. }\end{array}$ & Storage Location \\
\hline Hydrochloric Acid & Glass Bottles & 1405 & Inside \\
\hline Hydrochloric Acid & Glass Bottles & 9206 & Inside, Second Floor, Room 100E \\
\hline Nitric Acid & Tank Inside Building & 9206 & Inside, Second Floor, Room 100E \\
\hline Nitric Acid & Aboveground Tank & 9206 & Outside, West Side \\
\hline Hydrochloric Acid & Glass Bottles & 9207 & Inside, First Floor \\
\hline Hydrochloric Acid & Glass Bottles & 9207 & Inside, Fourth Floor \\
\hline Methanol & Glass Bottles & 9207 & Inside, Fifth Floor \\
\hline Methanol & Glass Bottles & 9207 & Inside, Thind Floor \\
\hline Nitric Acid & Plastic Bottles & 9207 & Inside, Annex \\
\hline Nitric Acid & Plastic Bottles & 9207 & Inside, Fifth Floor \\
\hline Nitric Acid & Plastic Bottles & 9207 & Inside, First Floor \\
\hline Nitric Acid & Plastic Bottles & 9207 & Inside, Fourth Floor \\
\hline Nitric Acid & Tank Inside Building & 9212 & Inside, B-1 Wing, Penthouse \\
\hline Nitric Acid & Glass Bottles & 9212 & Inside, E-Wing, Room 1021 \\
\hline Methanol & Glass Bottles & 9215 & Inside, Basement \\
\hline Methanol & Glass Bottles & 9766 & Inside, First Floor, Room 18 \\
\hline Hydrochloric Acid & Glass Bottles & 9769 & Inside, Second Floor \\
\hline Methanol & Glass Bottles & 9769 & Inside, Second Floor. \\
\hline Methanol & Glass Bottles & 9769 & Inside, Third Floor \\
\hline Nitric Acid & Aboveground Tank. & 9815 & Outside \\
\hline Hydrochloric Acid & Glass Bottles & 9995 & Inside, Basement \\
\hline Hydrochloric Acid & Glass Bottles & 9995 & Inside, First Floor \\
\hline Methanol & Glass Bottles & 9995 & Inside, Basement \\
\hline Methanol & Glass Bottles & 9995 & Inside, First Floor \\
\hline Hydrochloric Acid & Plastic Drum & $9201-5$ & Inside \\
\hline Hydrochloric Acid & Glass Bottles & $9201-5$ & Inside \\
\hline Methanol & Can & $9201-5$ & Inside, First Floor \\
\hline Nitric Acid & Glass Bottles & $9201-5$ & Inside, Second Floor \\
\hline Hydrochloric Acid & Glass Bottles & $9201-5 \mathrm{~N}$ & Inside, First Floor, Room 133 \\
\hline Methanol & Can & $9201-5 \mathrm{~N}$ & Inside \\
\hline Nitric Acid & Glass Bottles & $9201.5 \mathrm{~N}$ & Inside, First Floor, Room 133 \\
\hline Methanol & Plastic Bottles & $9204-1$ & Inside \\
\hline Hydrochloric Acid & Aboveground Tank & $9204-2$ & Outside, West Side \\
\hline Methanol & Can & $9204-2$ & Inside, Basement \\
\hline Methanol & Can & $9204-2$ & Inside, First Floor \\
\hline Methanol & Can & $9204-2$ & Inside, Second Floor \\
\hline Hydrochloric Acid & Glass Bottles & $9204-2 \mathrm{E}$ & Inside, Third Floor \\
\hline Methanol & Glass Bottles & $9204-2 \mathrm{E}$ & Inside, Third Floor \\
\hline Nitric Acid & Glass Bottles & $9204-2 E$ & Inside, Third Floor \\
\hline Hydrochloric Acid & Glass Bottles & $9204-3$ & Inside, First Floor \\
\hline Nitric Acid & Glass Bottles & $9204-3$ & Inside, First Floor \\
\hline Nitric Acid & Steel Drum & $9204-3$ & Inside, Second Floor \\
\hline Hydrochloric Acid & Glass Bottles & $9204-4$ & Inside, Second Floor \\
\hline Methanol & Glass Bottles & 9204.4 & Inside, Basement \\
\hline Methanol & Glass Bottles & $9204-4$ & Inside, First Floor \\
\hline Methanol & Can & $9204-4$ & Inside, Second Floor \\
\hline Methanol & Glass Bottles & $9204-4$ & Inside, Second Floor \\
\hline Nitric Acid & Glass Bottles & 9204.4 & Inside, Second Floor \\
\hline Hydrochloric Acid & Plastic Drum & $9401-2$ & Inside, Plating Shop, Annex \\
\hline Hydrochloric Acid & Glass Bottles & $9401-2$ & Inside, Plating Shop, Annex \\
\hline Methanol & Plastic Bottles & $9401-2$ & Inside, Plating Shop, Annex \\
\hline Nitric Acid & Glass Bottles & $9401-2$ & Inside, Plating Shop, Annex \\
\hline
\end{tabular}


TABLE 3.3 STORAGE LOCATIONS OF WATER PRIORITY CHEMICALS

\begin{tabular}{|l|l|l|l|}
\hline \multicolumn{1}{|c|}{ Chemical Name } & \multicolumn{1}{|c|}{$\begin{array}{c}\text { Container Type } \\
:\end{array}$} & $\begin{array}{c}\text { Building } \\
\text { No. }\end{array}$ & \multicolumn{1}{|c|}{ Storage Location } \\
\hline Hydrochloric Acid & Glass Bottles & $9401-3$ & Inside, First Floor \\
\hline Methanol & Steel Drum & $9720-2$ & Inside, Bay 43 Flammable Storage \\
\hline Nitric Acid & Glass Bottles & $9720-2$ & Inside, Bay 10 \\
\hline Nitric Acid & Glass Bottles & $9720-2$ & Inside, R36 \\
\hline Methanol & Steel Drum & $9767-4$ & Inside, Compressor Area \\
\hline
\end{tabular}




\section{: $\quad$ NOTICE}

Section 4 and Maps B and C of this document have been determined to contain Unclassified Controlled Nuclear Information per Section 148 of the Atomic Energy Act of 1954, as amended (42 U.S.C. 2168).

In order to protect this information as required in $10 \mathrm{CFR}$, Part 1017, this information has been included in a separate attachment, "Storm Water Pollution Prevention Plan for the Oak Ridge Y-12 Plant, Addendum." For access to this attachment, please contact B. E. Skaggs at (423) 241-2582. 


\section{SECTION 5}

\section{STORM WATER MONITORING PLAN FOR THE Y-12 PLANT}

\subsection{Background}

Initial quantitative testing on a number of discharges characteristic of plant drainage areas was performed, and the results were provided to the TDEC in the form of a storm water permit application (see Appendix A). The NPDES permit for the Y-12 Plant requires further characterization of these and other storm water discharges.

The NPDES permit also requires the permittee to make a minimum of 25 storm water characterizations per year under this monitoring plan including both grab and composite sampling in each characterization. The storm water characterization results will be compared to the results submitted in the storm water permit application. A follow-up investigation will be conducted if pollutant levels indicate pollutants are getting into the storm water runoff. Such pollutant levels do not have to be a threat to water quality before the investigation is begun. For example, elevated chloride, sulfate, or fecal coliforms may serve as indicators of other pollutants without constituting a threat to water quality in and of themselves.

\section{Project Objective}

The objective of the storm water characterization effort is to comply with the storm water characterization requirements of the Y-12 Plant NPDES permit.

\subsection{Requirements}

\subsubsection{Category I Storm Water Outfalls and Monitoring Points}

The permit states that storm runoff from Category I outfalls will be monitored by a characterization at least twice during the permit period using flow-weighted or time-weighted composites. Characterization of a single outfall may be made where several outfalls are very similar, rather than characterization of each of the outfalls in this group of similar outfalls. Approval of this SWP3 by the Division of Water Pollution Control of the TDEC will serve as approval of the various outfall groupings for monitoring purposes.

Characterization will be conducted at Outfalls 007 and 033 during the first year of the permit; Outfall 032 has been eliminated. Others to be characterized at least twice during the permit period include the following: 003, 006, 008, 009, 011, 015, 018, 045, 046, 058, 062, 086, 087, 098, 110, 134, 213, S01, S03, S04, S06, S07, S09, S15, S16, and S18.

\subsubsection{Category II Storm Water Outfalls and Monitoring Points}

Category II outfalls will have representative storm flow characterizations made at least once per year. Storm water composite samples will be either flow weighted or time weighted. Characterization of one outfall may be made where several outfalls are very similar, rather than characterization of each of the individual outfalls in this group of similar outfalls. Approval of this SWP3 by the Division of Water Pollution Control of the TDEC will serve as approval of the various outfall groupings for monitoring purposes.

Storm water sampling that is made of ponds or quarries where runoff collects may be sampled by other than an initial grab sample and a flow-weighted composite where an alternative sampling scheme seems justified. 
Such alternative sampling for these situations may be made if the Division of Water Pollution Control of the TDEC approves of the sampling methods. :The following outfalls and monitoring points are Category II: 004, 010, 014, 016, 019, 020, 041, 044, 057, 063, 064, 067, 083, 088, 099, 126, S02, S08, S10, S11, S12, S13, S17, S19, S20, S21, S22, S24, S25, S26, S27, S28, and S29.

\subsubsection{Category III Storm Water Outfalls and Monitoring Points}

Category III outfalls will have representative storm flow characterizations made according to the SWP3 at least once per year. Storm water composite samples will be either flow weighted or time weighted. Characterization of one outfall may be made where several outfalls are very similar, rather than characterization of each outfall in this group of similar outfalls. However, such groupings will be listed in the SWP3 and be approved by the TDEC, Division of Water Pollution Control. The following outfalls and monitoring points are Category III: 002, 034, 042, 047, 048, 054, 071, 109, 113, 114, 125, S05, and S14.

\subsubsection{Storm Water Outfalls of Special Interest}

No specific requirement exists in the NPDES permit to perform a storm water characterization at the following four outfalls: $017,021,135$, and 200.

Due to the magnitude of the flows from each of these outfalls and, consequently, their potential for positive or negative impact on the water quality of the EFPC, Y-12 Plant personnel have chosen to include them in this SWP3. A storm water characterization will be conducted at each of these four outfalls once per year.

\subsection{Sampling}

Sites listed under each permit category (I, II, and III listed in Tables 5.1A, 5.1B, and 5.1C) will be grouped (see Tables 5.2A, 5.2B, and 5.2C) within their respective categories based on their similarity as to land use and possible pollutants. Only one site in the grouping will be sampled during the specified time period, and a different site will be chosen each time period. If only one site constitutes a grouping, then that site will be sampled every time period. If there is more than one site in a grouping, and all sites have been sampled, sampling will continue one site per time period throughout the life of the permit. Grab and composite samples will be taken from each sampling site (listed in Tables 5.1A, 5.1B, 5.1C, and 5.1D) using the groupings and schedule detailed in Tables 5.2A, 5.2B, 5.2C, and 5.2D. When sampling quarries, ponds, or other impoundments, only grab samples will be collected. Sampling will begin with the onset of a storm event after $48 \mathrm{hr}$ of less than $0.1 \mathrm{in}$. of precipitation. Grab samples will be obtained during the first $30 \mathrm{~min}$ of the discharge or as soon after as practicable. Automatic composite samplers will be started by a rain gauge, and sampling will continue for $3 \mathrm{hr}$ or for the entire event. The storm event will be at least $0.1 \mathrm{in}$. in magnitude. Where feasible, the variance in the duration of the event and the total rainfall of the event will not exceed $50 \%$ from the average or median rainfall event in this area. According to Scientific Application International Corporation, an average rainfall event, for the Central U. S. Rain Zone (which contains East Tennessee), has a duration of $9.2 \mathrm{hr}$ and a magnitude $0.62 \mathrm{in}$. This translates to a rainfall that has a magnitude between 0.31 and 0.93 in. with a duration between 4.6 to $13.8 \mathrm{hr}$. Rainfall magnitude and duration along with total discharge flow for the sampling period will be recorded by the automatic sampling equipment and downloaded to a portable personal computer. Flow measurements will be taken by flow meters programmed for the particular site configuration, such as round pipes, weirs, or flumes. Ditches and streams will be measured using a portable flume with a suitably programmed meter. However, if a stream is heavily lined with large gravel, dimensions of the channel will be recorded and flows will be estimated from level measurements taken by the flow meter and the dimensions of the channel. All sites will be sampled for 
heavily lined with large gravel, dimensions of the channel will be recorded and flows will be estimated from

level measurements taken by the flow meter and the dimensions of the channel. All sites will be sampled for a common listing of analytical tests. The tests to be performed and procedures used for analysis are detailed in Table 5.3. Additional tests are required at some sites; these are detailed in Table 5.4.

Each year, every effort will be made to complete the storm water sampling schedule in accordance with the requirements outlined herein. Collection of samples and reporting of the data to satisfy the plan requirement, if accomplished outside of the rainfall guidelines, will not necessarily be construed as a nonconformance to this plan.

\section{Parameter Selection Criteria}

The following parameters are required when applying for a storm water discharge permit:

$\begin{array}{ll}\text { BOD } & \text { Nitrites } \\ \text { COD } & \text { Total phosphorus } \\ \text { Total suspended solids } & \text { Oil and grease } \\ \text { Total Kjeldahl nitrogen } & \text { pH }\end{array}$

The following parameters are suspected present at the Y-12 Plant or were found in the previous storm water characterizations:

$\begin{array}{ll}\text { Mercury } & \text { Some inductively-coupled plasma (ICP)-determined metals } \\ \text { Sulfate } & \text { Surfactants/methylene blue activated substances (MBAS) } \\ \text { Fecal coliform } & \text { Alpha activity } \\ \text { Uranium } & \text { Beta activity } \\ \text { Phenols } & \text { Gamma activity }\end{array}$

The following parameters were found at some sites and will be monitored at those monitoring points and others within the same grouping:
Volatile organics
PCBs
Pesticides
Base neutrals and acid extractables
Cyanide

\section{Quality Control}

Approximately one sample out of every twenty will be taken in duplicate, and the results compared for sampling consistency. Due to inadequate sample volumes, some parameters may have to be taken in duplicate at different monitoring points. A blank of laboratory water will also be prepared and sent to the laboratory for analysis for every twenty samples. All samples will be kept under chain of custody at all times according to procedure ESP-500. All equipment will be decontaminated between samples in accordance with ESP-900. All grab and composite samples will be taken in accordance with ESP-301-1 and ESP-301-3, respectively. All analyses, with the exception of alpha, beta, and gamma activities and uranium, will be conducted in accordance with NPDES-accepted methodology. 


\section{Safety Concerns}

The sampling technicians will wear safety glasses, gloves, and company clothing as the minimum personal protective equipment. Due to inclement weather conditions, sampling personnel will work in teams of at least two persons at all times. Some larger outfalls may require confined space entries to install flow-monitoring equipment.

\subsection{Data Management}

Data will be stored and managed in the Environmental Monitoring Management Information System. Data will be supplied, as it is acquired, to the TDEC as an attachment to the monthly NPDES Discharge Monitoring Report. 
TABLE 5.1.A

\section{CATEGORY I STORM WATER OUTFALLS AND MONITORING POINTS}

\begin{tabular}{|c|c|c|}
\hline Site & Description & Grouping \\
\hline 003 & Concrete trench to EFPC & $\mathbf{A}$ \\
\hline 006 & 24-in. CMP outfall to EFPC & B \\
\hline $007 *$ & 24-in. RCP outfall to EFPC & B \\
\hline 008 & 36-in. RCP outfall to EFPC & C \\
\hline 009 & 14-in. CS outfall to EFPC & $\mathrm{C}$ \\
\hline 011 & 16-in. RCP outfall to EFPC & $\mathrm{A}$ \\
\hline 015 & 6-in. CS outfall to EFPC & C \\
\hline 018 & 12-in. CMP outfall to EFPC & $\mathrm{C}$ \\
\hline 033* & 36-in. TC outfall to EFPC & E \\
\hline 045 & 15-in. C outfall to EFPC & $\mathrm{D}$ \\
\hline 046 & 6-in. TC outfall to EFPC & $\mathrm{E}^{\cdot}$ \\
\hline 058 & 36-in. TC outfall to EFPC & $\mathrm{E}$ \\
\hline 062 & 18-in. RCP outfall to EFPC & E \\
\hline 086 & 18-in. CI outfall to EFPC & $\mathrm{E}$ \\
\hline 087 & 36-in. VC outfall to EFPC & $E$ \\
\hline 098 & 6-in. Coutfall to EFPC & $\mathrm{E}$ \\
\hline 110 & 15-in. RCP outfall to EFPC & $\mathrm{D}$ \\
\hline 134 & 24-in. CS outfall to EFPC & D \\
\hline 213 & 6-in. VC outfall to EFPC & $\mathrm{E}$ \\
\hline $\mathrm{S} 01$ & 36-in. $\mathrm{RCP}$ to $\mathrm{BC}$ & $\mathrm{F}$ \\
\hline $\mathrm{S} 03$ & 18-in. RCP outfall to BC & $\mathrm{F}$ \\
\hline $\mathrm{S} 04$ & 18-in. RCP outfall to $B C$ & F \\
\hline S06 & BC In-stream Point & $\mathrm{F}$ \\
\hline S07 & Tributary 2 to $\mathrm{BC}$ & G \\
\hline S09 & Tributary 4 to $\mathrm{BC}$ & G \\
\hline
\end{tabular}

$\mathrm{BC}=$ Bear Creek; C = Clay; CI = Cast Iron; CMP = Corrugated Metal Pipe; CS = Carbon Steel; EFPC = East Fork Poplar Creek; RCP $=$ Reinforced Concrete Pipe; ST = Steel; TC = Tile Clay; VC = Vitreous Clay 
TABLE 5.1.A

CATEGORY I STORM WATER OUTFALLS AND MONITORING POINTS

\begin{tabular}{|c|c|c|}
\hline Site & Description & Grouping \\
\hline S15 & West Borrow Area Sediment Pond & H \\
\hline S16 & East Borrow Area Sediment Pond & H \\
\hline S18 & Sludge Application Area Site 2 & I \\
\hline * These sites will be monitored during the first year of the permit. & \\
\hline
\end{tabular}

$\mathrm{BC}=\mathrm{Bear}$ Creck; $\mathrm{C}=$ Clay; $\mathrm{CI}=$ Cast Iron; CMP = Corrugated Metal Pipe; $\mathrm{CS}=$ Carbon Steel; EFPC = East Fork Poplar Creek; RCP = Reinforced Concrete Pipe; ST = Steel; TC = Tile Clay; VC = Vitreous Clay 
TABLE 5.1.B

\section{CATEGORY II STORM WATER OUTFALLS AND MONITORING POINTS}

\begin{tabular}{|c|c|c|}
\hline Station & Description/Location & Grouping \\
\hline 004 & 54-in. CMP outfall to EFPC & $\mathbf{A}$ \\
\hline 010 & 24-in. RCP outfall to EFPC & A \\
\hline 014 & 36-in. RCP outfall to EFPC & A \\
\hline 016 & 48-in. RCP outfall to EFPC & $\mathrm{C}$ \\
\hline 019 & 15-in. RCP outfall to EFPC & A \\
\hline 020 & 24-in. RCP outfall to EFPC & A \\
\hline 041 & 15-in. RCP outfall to EFPC & $\mathrm{B}$ \\
\hline 044 & 12-in. C outfall to EFPC & B \\
\hline 057 & 10-in. ST outfall to EFPC & $\mathrm{B}$ \\
\hline 063 & 12-in. RCP outfall to EFPC & B \\
\hline 064 & 12-in. RCP outfall to EFPC & $\mathrm{B}^{-}$ \\
\hline 067 & 24-in. RCP outfall to EFPC & $\mathrm{C}$ \\
\hline 083 & 15-in. VC outfall to EFPC & $\mathrm{C}$ \\
\hline 088 & 10-in. CI outfall to EFPC & $\mathrm{C}$ \\
\hline 099 & 12-in. CI outfall to EFPC & $\mathrm{C}$ \\
\hline 126 & 18-in. RCP to EFPC & A \\
\hline $\mathrm{SO} 2$ & 48-in. CMP headwaters to $B C$ & $\mathrm{D}$ \\
\hline S08 & Tributary 3 to $B C$ & E \\
\hline $\mathbf{S 1 0}$ & Tributary 5 to $\mathrm{BC}$ & $\mathrm{E}$ \\
\hline $\mathrm{S} 11$ & Tributary 6 to $\mathrm{BC}$ & $\mathrm{E}$ \\
\hline $\mathrm{S} 12$ & Riprap Drainage Channel & $\mathrm{E}$ \\
\hline S13 & Tributary 7 to $B C$ & $\mathrm{E}$ \\
\hline S17 & Tributary from Kerr Hollow Quarry & $\mathrm{H}$ \\
\hline $\mathbf{S 1 9}$ & Outfall from Rogers Quarry & I \\
\hline $\mathbf{S 2 0}$ & 48-in. CMP from Building 9213 & $\mathbf{F}$ \\
\hline
\end{tabular}


- TABLE 5.1.B

CATEGORY II STORM WATER OUTFALLS AND MONITORING POINTS

\begin{tabular}{|c|c|c|}
\hline Station & Description/Location & Grouping \\
\hline S21 & Sanitary Landfill II Sediment Pond & $\mathrm{J}$ \\
\hline S22 & Industrial Landfill IV Sediment Pond & $\mathrm{J}$ \\
\hline S24 & In stream near BC Kilometer 9.4 & $\mathrm{G}$ \\
\hline S25 & Construction/Demolition Landfill VI Sediment Pond & $\mathrm{J}$ \\
\hline S26 & Industrial Landfill V Sediment Pond & $\mathrm{K}$ \\
\hline S27 & Industrial Landfill V Sediment Pond & $\mathrm{K}$ \\
\hline S28 & Construction/Demolition Landfill VII Sediment Pond & $\mathrm{K}$ \\
\hline S29 & Construction/Demolition Landfill VII Sediment Pond (Future) & $\mathrm{K}$ \\
\hline
\end{tabular}


TABLE 5.1.C

CATEGORY III STORM WATER OUTFALLS AND MONITORING POINTS

\begin{tabular}{|c|c|c|}
\hline Site & Description & Grouping \\
\hline 002 & 48-in. RCP outfall to EFPC & A \\
\hline 034 & 15-in. VC outfall to EFPC & B \\
\hline 042 & 16-in. VC outfall to EFPC & B \\
\hline 047 & 24-in. RCP outfall to EFPC & B \\
\hline 048 & 24-in. RCP outfall to EFPC & B \\
\hline 054 & 12-in. VC outfall to EFPC & C \\
\hline 071 & 12-in. Cl outfall to EFPC & D \\
\hline 109 & 54-in. RCP outfall to EFPC & E \\
\hline 113 & 18-in. RCP outfall to EFPC & D \\
\hline 114 & 18-in. RCP outfall to EFPC & D \\
\hline 125 & 22-in. CS outfall to EFPC & E \\
\hline S05 & Tributary 1 to BC & A \\
\hline S14 & Tributary 8 to BC & F \\
\hline
\end{tabular}


TABLE 5.1.D

STORM WATER OUTFALLS OF SPECIAL INTEREST

\begin{tabular}{|c|c|c|}
\hline Site & Description & Grouping \\
\hline 017 & 16-in. RCP outfall to EFPC & J \\
\hline 021 & 48-in. RCP outfall to EFPC & I \\
\hline 135 & 36-in. RCP outfall to EFPC & H \\
\hline 200 & 76-in. RCP Headwaters of EFPC (North/South Pipes) & G \\
\hline
\end{tabular}


TABLE 5.2.A

CATEGORY I OUTFALL GROUPINGS AND SUGGESTED SAMPLING

\begin{tabular}{|c|c|c|}
\hline Grouping & Sites & Sampling \\
\hline A & 003,011 & $\begin{array}{c}\text { Table 5.3 + Table 5.4 (Cyanide }+ \\
\text { Pesticides only) }\end{array}$ \\
\hline B & 006,007 & $\begin{array}{c}\text { Table 5.3 + Table 5.4 (Cyanide + } \\
\text { BNA only) }\end{array}$ \\
\hline C & $008,009,015,018$ & $\begin{array}{c}\text { Table 5.3 + Table 5.4 (Cyanide + } \\
\text { Pesticides only) }\end{array}$ \\
\hline D & $045,110,134$ & $\begin{array}{c}\text { Table 5.3 + Table 5.4 (Pesticides } \\
\text { and BNA only) }\end{array}$ \\
\hline E & $033,046,058,062,086,087,098,213$ & $\begin{array}{c}\text { Table 5.3 + Table 5.4 (Pesticides } \\
\text { and BNA only) }\end{array}$ \\
\hline F & S01, S03,S04, S06 & Table 5.3 + Table 5.4 (BNA only) \\
\hline G & S07, S09 & Table 5.3 only \\
\hline H & S15, S16 & Table 5.3 only \\
\hline I & S18 & \begin{tabular}{c} 
Table 5.3 only \\
\hline
\end{tabular} \\
\hline
\end{tabular}


TABLE 5.2.B

CATEGORY II OUTFALL GROUPINGS AND SUGGESTED SAMPLING

\begin{tabular}{|c|c|c|}
\hline Grouping & Sites & Sampling \\
\hline A & $004,010,014,019,020,126$ & $\begin{array}{c}\text { Table 5.3 + Table 5.4 (BNA, PCB, } \\
\text { Cyanide + Pesticides only) }\end{array}$ \\
\hline B & $041,044,057,063,064$ & $\begin{array}{c}\text { Table } 5.3+\text { Table } 5.4 \text { (Pesticides } \\
\text { only) }\end{array}$ \\
\hline C & $016,067,083,088,099$ & $\begin{array}{c}\text { Table } 5.3+\text { Table } 5.4 \text { (Pesticides } \\
\text { only) }\end{array}$ \\
\hline D & S02 & Table 5.3 only \\
\hline E & S08, S10, S11, S12, S13 & $\begin{array}{c}\text { Table 5.3 + Table 5.4 (Pesticides } \\
\text { and BNA only) }\end{array}$ \\
\hline F & S20 & Table 5.3 only \\
\hline G & S24 & Table 5.3 only \\
\hline H & S17 & Table 5.3 only \\
\hline I & S19 & $\begin{array}{c}\text { Table 5.3 + Table 5.4 (Cyanide and } \\
\text { Pesticides only) }\end{array}$ \\
\hline J & S21, S22, S25 & Table 5.3 only \\
\hline K & S26, S27, S28, S29 & Table 5.3 only \\
\hline
\end{tabular}


TABLE 5.2.C

CATEGORY III OUTFALL GROUPINGS AND SUGGESTED SAMPLING

\begin{tabular}{|c|c|c|}
\hline Grouping & Sites & Sampling \\
\hline A & 002, S05 & Table 5.3 + Table 5.4 (BNA only) \\
\hline B & $034,042,047,048$ & $\begin{array}{c}\text { Table 5.3 + Table 5.4 (Pesticides, } \\
\text { Cyanide, and BNA only) }\end{array}$ \\
\hline C & 054 & Table 5.3 only \\
\hline D & $071,113,114$ & Table 5.3 + Table 5.4 \\
\hline E & 109,125 & Table 5.3 + Table 5.4 (BNA only) \\
\hline F & S14 & Table 5.3 + Table 5.4 (PCB and \\
VOA only)
\end{tabular}


TABLE 5.2.D

SUGGESTED SAMPLING FOR OUTFALLS OF SPECIAL INTEREST

\begin{tabular}{|c|c|}
\hline Storm Water Outfalls & Suggested Sampling \\
\hline 017 & Table 5.3 only \\
\hline 021 & $\begin{array}{c}\text { Table 5.3 + Table 5.4 (PCB and } \\
\text { BNA only) }\end{array}$ \\
\hline 135 & $\begin{array}{c}\text { Table 5.3 + Table 5.4 (Cyanide and } \\
\text { BNA only) }\end{array}$ \\
\hline 200 & Table 5.3 only \\
\hline
\end{tabular}


TABLE 5.3

SUGGESTED SAMPLING FOR ALL STORM WATER MONITORING LOCATIONS

Composite Samples Will Include:

\begin{tabular}{|c|c|c|}
\hline Analytical Parameter & Lab Test ID & $\begin{array}{l}\text { Method } \\
\text { Number }\end{array}$ \\
\hline Biochemical oxygen demand & $\mathrm{BOD}$ & EPA-405.1 \\
\hline Chemical oxygen demand & COD & EPA-410.4 \\
\hline Total suspended solids & TSS & EPA-160.2 \\
\hline Total Kjeldahl nitrogen & TKN & EPA-351.3 \\
\hline Total phosphorus & TOTP & EPA-365.2 \\
\hline $\begin{array}{l}\text { Inductively-coupled plasma (ICP) } \\
\text { sweep* }\end{array}$ & ICPEPA & EPA-200.7 \\
\hline Mercury & AA HG & EPA-245.1 \\
\hline Radiological parameters & $\begin{array}{c}\text { ALPBET } \\
\text { GR_GAM } \\
\text { NDGAMMA } \\
\text { CT_AM } \\
\text { CT_NP } \\
\text { CT_PU } \\
\text { CT_RA } \\
\text { CT_TH } \\
\text { CT_U } \\
\text { TC99 } \\
\text { TOT_SR } \\
\text { TOT_TH } \\
\end{array}$ & $\begin{array}{l}\text { Internal } \\
\text { Procedures }\end{array}$ \\
\hline Uranium and percent uranium-235 & MS_U & $\begin{array}{c}\text { Internal } \\
\text { Procedures } \\
\end{array}$ \\
\hline Nitrites, Sulfate, Nitrates & $\begin{array}{c}\text { ICPNPD (SO4, } \\
\text { NO2, NO3) }\end{array}$ & EPA-300.0 \\
\hline Surfactants & MBAS & EPA-425.1 \\
\hline
\end{tabular}

- See Table 5.5 for a list of specific parameters. 
TABLE 5.3

\section{SUGGESTED SAMPLING FOR ALL STORM WATER MONITORING LOCATIONS}

Grab Samples Will Include:

\begin{tabular}{|c|c|c|}
\hline Analytical Parameter & Lab Test ID & $\begin{array}{l}\text { Method } \\
\text { Number }\end{array}$ \\
\hline $\mathrm{pH}$ & Not applicable & ESP-307.2 \\
\hline Oil and grease & OIL GR & EPA-413.1 \\
\hline Biochemical oxygen demand & BOD & EPA-405.1 \\
\hline Chemical oxygen demand & $\mathrm{COD}$ & EPA-410.4 \\
\hline Total suspended solids & TSS & EPA-160.2 \\
\hline Total Kjeldahl nitrogen & TKN & EPA-351.3 \\
\hline Total phosphorus & TOTP & EPA-365.2 \\
\hline $\begin{array}{c}\text { Inductively-coupled plasma (ICP) } \\
\text { sweep* }^{*}\end{array}$ & ICPEPA & EPA-200.7 \\
\hline Mercury & $\mathrm{AA} \mathrm{HG}$ & EPA-245.1 \\
\hline Phenols & PHENOL & EPA-420.1 \\
\hline Fecal coliform & FCOLI & EPA-909 \\
\hline Radiological parameters & $\begin{array}{c}\text { ALPBET } \\
\text { GR_GAM } \\
\text { NDGAMMA } \\
\text { CT_AM } \\
\text { CT_NP } \\
\text { CT_PU } \\
\text { CT_RA } \\
\text { CT_TH } \\
\text { CT_U } \\
\text { TC99 } \\
\text { TOT_SR } \\
\text { TOT_TH } \\
\end{array}$ & $\begin{array}{l}\text { Internal } \\
\text { Procedures }\end{array}$ \\
\hline Uranium and percent uranium-235 & MS_U & $\begin{array}{c}\text { Internal } \\
\text { Procedures } \\
\end{array}$ \\
\hline Nitrites, Sulfate, Nitrates & $\begin{array}{l}\text { ICNPD (SO4, } \\
\text { NO3, NO2) }\end{array}$ & EPA-300.0 \\
\hline Surfactants & MBAS & EPA-425.1 \\
\hline
\end{tabular}

\footnotetext{
- See Table 5.5 for a specific list of parameters.
}

Note - Grab samples for $\mathrm{pH}$, oil and grease, phenols, and fecal coliform will be taken manually. The other parameters can be taken by the automatic camplers, either in a separate sampler connected to the same flow meter or in a different bottle in the same sampler. 
TABLE 5.4

\section{ADDITIONAL TESTS FOR CERTAIN GROUPS OF OUTFALLS}

Some composites samples will include:

\begin{tabular}{|c|c|c|}
\hline Analytical Parameter & Lab Test ID & Method Number \\
\hline $\begin{array}{c}\text { Base Neutrals* and Acid } \\
\text { Extractable Organics* (BNA) }\end{array}$ & BNA625 & EPA-625 \\
\hline Pesticides* & PESH20 & EPA-608 \\
\hline $\begin{array}{c}\text { Polychlorinated biphenyl } \\
\text { (PCB)* }\end{array}$ & PCBH20 & EPA-608 \\
\hline
\end{tabular}

*See Table 5.5 for a list of specific parameters.

Some grab samples will include:

\begin{tabular}{|c|c|c|}
\hline Analytical Parameter & Lab Test ID & Method Number \\
\hline Volatile Organics* (VOA) & VOA624 & EPA-624 \\
\hline Cyanide & CYANIDE & EPA-335.2 \\
\hline $\begin{array}{c}\text { Base Neutrals* and Acid } \\
\text { Extractable Organics* (BNA) }\end{array}$ & BNA625 & EPA-625 \\
\hline Pesticides* & PESH20 & EPA-608 \\
\hline $\begin{array}{c}\text { Polychlorinated biphenyl } \\
\text { (PCB)* }\end{array}$ & PCBH20 & EPA-608 \\
\hline
\end{tabular}

- See Table 5.5 for a list of specific parameters.

Note - Grab samples for volatile organics and cyanide will be taken manually. The other parameters can be taken by the automatic samplers, either in a separate sampler connected to the same flow meter or in a different bottle in the same sampler. 


\section{LIST OF PARAMETERS INCLUDED UNDER EACH TEST}

\section{Acid Extractable Parameters}

4-Nitrophenol

2-Methyl-4,6-dinitrophenol

4-Chloro-3-methylphenol

Pentachlorophenol

Phenol

2-Chlorophenol

2-Nitrophenol

2,4-Dichlorophenol

2,4-Dimethylphenol

2,4-Dinitrophenol

2,4,6-Trichlorophenol

Cresols*

\section{Base Neutral Extractable Parameters}

Acenaphthene

Acenaphthylene

Anthracene

Benzidine

Benzo(a)anthracene

Benzo(a)pyrene

Benzo(ghi)perylene

Benzo(k) fluoranthene

Bis(2-chloroethoxy) methane

Bis(2-chloroethyl) ether

Bis(2-chloroisopropyl) ether

Bis(2-ethylhexyl) phthalate

Benzyl butyl phthalate

Chrysene

Di-n-butyl phthalate

Di-n-octyl phthalate

Dibenzo(a,h)anthracene

Diethyl phthalate

Dimethyl phthalate

Fluoranthene

Fluorene

4-Bromophenylphenyl ether

4-Chlorophenylphenyl ether
Hexachlorobenzene

Hexachlorobutadiene

Hexachlorocyclopentadiene

Hexachloroethane

Indeno(1,2,3-cd)pyrene

Isophorone

N-Nitrosodi-n-propylamine

$\mathrm{N}$-Nitrosodimethylamine

$\mathrm{N}$-Nitrosodiphenylamine

Naphthalene

Nitrobenzene

1,2-Dichlorobenzene

Benzo(b)fluoranthene

1,2,4-Trichlorobenzene

1,3-Dichlorobenzene

1,4-Dichlorobenzene

Phenanthrene

Pyrene

3,3-Dichlorobenzidine

2-Chloronaphthalene

2,4-Dinitrotoluene

2,6-Dinitrotoluene

1,2-diphenylhydrazine*

(as azobenzene) 
TABLE 5.5

LIST OF PARAMETERS INCLUDED UNDER EACH TEST

Inductively-Coupled Plasma (ICP) Sweep Parameters

$\begin{array}{ll}\text { Aluminum } & \text { Molybdenum } \\ \text { Arsenic } & \text { Nickel } \\ \text { Barium } & \text { Niobium } \\ \text { Beryllium } & \text { Phosphorus } \\ \text { Boron } & \text { Potassium } \\ \text { Cadmium } & \text { Scandium } \\ \text { Calcium } & \text { Silicon } \\ \text { Cerium } & \text { Silver } \\ \text { Chromium } & \text { Sodium } \\ \text { Cobalt } & \text { Strontium } \\ \text { Copper } & \text { Thallium } \\ \text { Gallium } & \text { Thorium } \\ \text { Iron } & \text { Titanium } \\ \text { Lead } & \text { Vanadium } \\ \text { Lithium } & \text { Zinc } \\ \text { Magnesium } & \text { Zirconium } \\ \text { Manganese } & \end{array}$

Pesticide and Polychlorinated Biphenyl (PCB) Parameters

Aldrin alpha-BHC

Endosulfan I

beta-BHC

Endosulfan II

Chlordane

delta-BHC

Dieldrin

Endosulfan sulfate
Endrin

Endrin aldehyde

4,4--DDD

4,4--DDE

4,4--DDT

gamma-BHC (Lindane)

Heptachlor

Heptachlor epoxide

PCB-1016
PCB-1221

PCB-1232

PCB-1242

PCB-1248

PCB-1254

PCB-1260

Toxaphene 
TABLE 5.5

\section{LIST OF PARAMETERS INCLUDED UNDER EACH TEST}

\section{Volatile Organic Parameters}

Acrolein*
Acrylonitrile*
Benzene
Bromoform
Carbon tetrachloride
Chlorobenzene
Bromodichloromethane
Chloroethane
Chloroform
cis-1,3-Dichloropropene
Dibromochloromethane

Ethylbenzene

Bromomethane

Chloromethane

Methylene chloride

1,1-Dichloroethane

1,1-Dichloroethene

1,1,1-Trichloroethane

1,1,2-Trichloroethane

1,1,2,2-Tetrachloroethane

1,2-Dichloroethane
1,2-Dichloropropane

Tetrachloroethene

Tóluene

trans-1,2-Dichloroethene

trans-1,3-Dichloropropene

Trichloroethene

Trichlorofluoromethane

2-Chloroethylvinyl ether

Vinyl chloride

Xylenes*

* Standards for these parameters are not routinely analyzed for; however, they will be detected if,present. 


\section{SECTION 6}

\section{SWP3 IMPLEMENTATION}

\subsection{SWP3 Implementation Plan}

Personnel in the WCS are responsible for the preparation and maintenance of the SWP3. The programmatic components of the plan, such as preparation of BMPs, spill response coordination, spill prevention, surveillance programs, evaluation of analytical data, and in many cases oversight of projects for improved water pollution control is directed by personnel in this section. Monitoring of storm water is a function of the Compliance Monitoring Services in the EMD. A team of individuals assigned to the WCS, with responsibility for various components of the plan, have been selected to provide compliance oversight of plan requirements, data evaluation, plan update, and continued guidance to Y-12 Plant management personnel for plan implementation. This team by job description is as follows:

\section{STORM WATER POLLUTION PREVENTION/PERMITTING TEAM}
B. E. Skaggs
Team Leader
K. G. Hanzelka
Data Coordination
R. H. Harden
Field Surveillance/Dike Management
M. C. Wiest
Best Management Practices
R. M. Shedden
Spill Response Coordinator
S. E. Poligone
Waste Management, Pollution Prevention

\subsection{Storm Water Working Group for Construction Activities}

Energy Systems has established a Construction Storm Water Working Group. The group--consisting of representatives from the Legal Department, Project and Construction Engineering, the Environmental Compliance Departments from the three Oak Ridge sites, DOE, and MK-Ferguson (MKF) (the construction subcontractor)--was formed so there would be consistency among the sites in compliance to the regulations governing storm water permitting associated with construction activities. This group examined implementation of permitting for construction activities involving land disturbances of greater than 5 acres. The main product of the group is the generation of a three-site (Oak Ridge Reservation) procedure governing NOI forms and Storm Water Control Plans (SWCPs). This procedure will initially be a three-site procedure with the possible expansion to a five-site (Oak Ridge sites plus Paducah and Portsmouth) procedure in the future. Ohio and Kentucky state law varies from Tennessee state law in some areas and may inhibit the fivesite expansion.

A standard NOI form has been generated and is currently under review by the parties involved. The current plan is that Energy Systems, MKF, and DOE representatives will sign each permit. The NOI will be prepared by the site Environmental Department (WCS at the Y-12 Plant), with input from Engineering and MKF. It will be issued to DOE, along with the signed SWCP, for signature and submittal to the TDEC. 
Each specific SWCP will be prepared by Engineering personnel (or a contractor to Engineering) and reviewed by representatives from the site Environmental Compliance group and MKF. The responsible employees from Energy Systems and MKF will sign the SWCP indicating approval, and it will then be included with the NOI.

In addition, a subgroup was formed to develop a Site Stabilization Checklist. The purpose of the checklist is to establish uniformity throughout the sites in acceptance criteria for site stabilization activities such as vegetation. This subgroup consisted of representatives from the Construction Engineering Department, the Y-12 Plant WCS and MKF. The Y-12 Plant WCS Storm Water Pollution Prevention/Permitting Team will review the SWCP prior to issuance and perform surveillances for compliance oversight.

\subsection{Employee Training/Education of SWP3 Requirements}

The Y-12 Plant NPDES permit, issued by the state of Tennessee, requires that employees at all levels of responsibility be trained in the components of the SWP3. Awareness training sessions for employees regarding environmental compliance, including storm water regulations and storm water pollution prevention, is predominately accomplished through the Y-12 Training Program. A person in the EMD is assigned the responsibility for determining the regulatory requirements and serves as the interface between training personnel and subject matter experts. Monthly meetings are held with the Environmental Officers (EOs) to. provide awareness training on environmental regulatory requirements, compliance strategies such as appropriate BMPs, and to disseminate information on changes in regulations, procedures, or other reference materials. Personnel in the WCS of the EMD serve as experts on various aspects of storm water pollution prevention and provide information, make presentations, and assist in developing training materials for the EOs and other employees. The EOs will communicate changes in the regulatory requirements, disseminate information, and conduct informational meetings in their respective organization for employees. The EOs will consult directly with subject matter experts in developing environmental regulatory guidance and for planning/implementing environmental programs.

Employee training on storm water pollution prevention at the Y-12 Plant will be planned and conducted to accomplish the following objectives:

- provide relevant employees with a basic understanding of pollution control laws and regulations, and information on pollution prevention measures available at the plant;

- enable relevant employees to identify the main components of the Y-12 Plant Storm Water Pollution Prevention Plan;

- provide information on BMPs and physical controls that are in place or can be implemented to reduce discharges of pollutants in storm water runoff from the Y-12 Plant; and

- enable relevant employees to understand the importance of proper maintenance of existing practices and controls and to identify improvements which can be recommended for implementation.

Special training has been offered to Y-12 Plant personnel involved in certain key jobs which relate to storm water pollution prevention. Spill prevention and spill response training are provided to employees with responsibilities involving hazardous materials. Additional training is required for employees who are responsible for response and control of spills. Three major groups that participate in spill response training include the Spill Response Coordinator and alternates, the Fire Department and Emergency Squad, and Waste Storage and Shipping Operations Department personnel. Additional pollution prevention training is also 
required annually for employees who manage water priority chemicals. Storm water pollution control plans are specifically prepared for all construction projects which disturb five or more acres of land. Also, all programs or projects which could have an adverse impact on surface water are required to prepare a BMP plan. Experts on matters such as water pollution control or soil erosion prevention from the WCS of the Y-12 Plant EMD provide guidance to project employees, operational or construction personnel, as well as EOs through on-site surveillances or informational meetings. In general, the many surveillance programs conducted at the Y-12 Plant, including those presented in Section 2.5 , constitute a valuable form of on-thejob training. For instance, guidance provided to dike and tank managers as part of the yearly surveillance and direction provided regarding review of water analysis for approval of BMP discharges offers an excellent training tool for operations personnel.

Other training or formats for information dissemination are planned. Internal memorandum and information bulletins on such topics as the renewed NPDES permit, storm water pollution prevention requirements, and BMP plans will be prepared and distributed to appropriate personnel. Informational meetings with responsible area managers and personnel to review identified problem areas or discuss analytical data obtained as part of this program will be held as a means to implement improvements.

A clean water computer-based training course designed for the EOs, but useable by other personnel, has also been developed. This training includes a module for storm water.

\subsection{Semiannual Surveillances to Ensure the Effectiveness of the SWP3}

The Y-12 Plant NPDES permit requires that surveillances of the facility will be conducted at least semiannually to check the accuracy of the plan and maps, and to examine whether measures in the plan to reduce pollutants in storm water runoff are adequate and properly implemented or whether additional controls are needed. This requirement will be met through the execution of a specific facility surveillance to meet this requirement of the permit.

The Storm Water Pollution Prevention/Permitting Team will evaluate the effectiveness and completeness of the SWP3 semiannually by this assessment.

\subsection{Certification Statement}

The Y-12 Plant NPDES permit Part IV, Storm Water Pollution Prevention Plan, requires that the plan be signed by a principal executive officer. The permit, Part IV additionally requires:

The plan shall contain a certification that the discharge has been tested for the presence of non-storm water discharges. The certification shall include a description of the results of any tests for the presence of non-storm water discharges, the method used, the date of any testing, and the on-site drainage points that were directly observed during the test.

The Y-12 Plant, through the oversight of the WCS of EMD, has surveyed all surface water outfalls. This effort has been accomplished by the use of engineering drawing reviews, tracing of lines by field survey crews, smoke or dye testing storm drain and sanitary sewer lines and many water sources in all major plant buildings, smoke testing of certain outfalls, and observation of local surface streams by compliance monitoring personnel and creek surveillance personnel. In addition, analytical testing of water in the outfalls, such as monitoring for chlorine, has been successfully used to indicate the possible presence of process wastewater. Information regarding analytical test methods, dates, and observations during testing is contained on field sampling or laboratory sheets. Stream observations are recorded on spill response forms 
maintained by EMD personnel. Information on sources tied to building drains and stream outfalls is contained on the data base and drawings prepared and maintained in the Y-12 Drain Identification Data Base for Buildings, Point Source Information and Outfall Characterization Program (Y/TS-612). 


\section{STORM WATER POLLUTION PREVENTION PLAN • FOR THE OAK RIDGE Y-12 PLANT}

\section{CERTIFICATION STATEMENT}

I certify that the storm water discharges described by this plan have been tested for the presence of non-storm water discharges. Testing was accomplished by the use of engineering drawing reviews, tracing of lines by field survey crews, smoke or dye tracing storm drain and sanitary sewer lines and many water sources in all major plant buildings, and observation of local surface streams by compliance monitoring personnel and creek surveillance personnel. In addition, analytical testing of water in the outfalls, such as monitoring for chlorine, has been successfully used to indicate the possible presence of process wastewater. Testing was conducted between May 1989 and June 1995. The results of the tests are included in the appropriate EPA Form 2C applications, Appendix A of this plan, the Y-12 Drain Identification Data Base for Buildings, Point Source Information and Outfall Characterization Program (Y/TS-612), and field sampling or laboratory sheets. More detailed information on the types of testing, the results of the tests, methods used, dates, and on-site drainage points observed are contained in the documents referenced above, which are available for review by TDEC personnel.
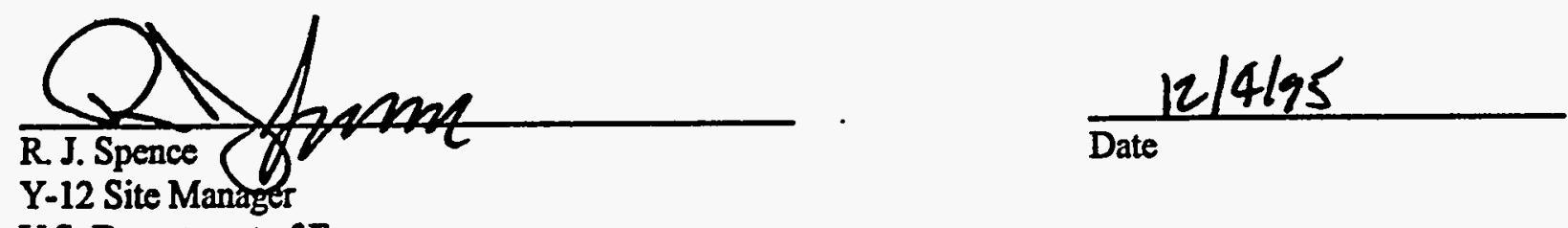

U.S. Department of Energy 
APPENDIX A

ANALYTICAL DATA FROM STORM WATER MONITORING POINTS 
VI. Disctargo Information (continued from page 3 of Form 2A)

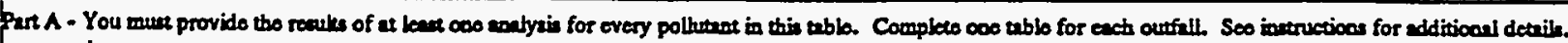

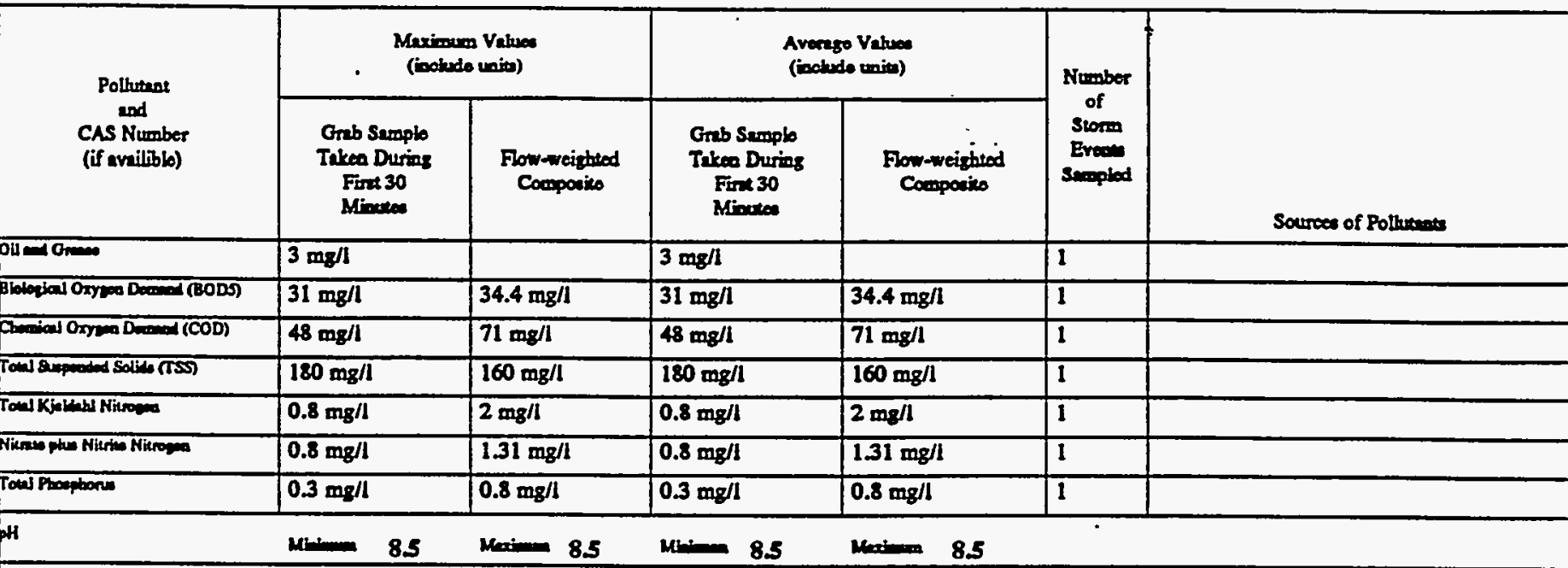

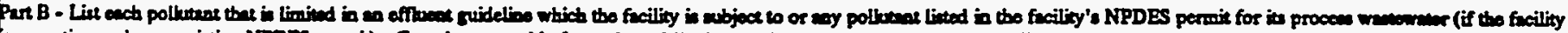

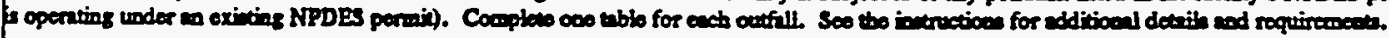

\begin{tabular}{|c|c|c|c|c|c|c|}
\hline \multirow{2}{*}{$\begin{array}{l}\text { Pollumat } \\
\text { and } \\
\text { CAs Number } \\
\text { (if muilibb) }\end{array}$} & \multicolumn{2}{|c|}{ 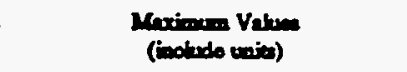 } & \multicolumn{2}{|c|}{ Armares Valenes } & \multirow{2}{*}{$\begin{array}{l}\text { Number } \\
\text { of } \\
\text { storm } \\
\text { Eveon } \\
\text { Simpled }\end{array}$} & \multirow[b]{2}{*}{ Sources of Polberese } \\
\hline & 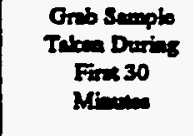 & $\begin{array}{l}\text { Fon-maighed } \\
\text { Compocing }\end{array}$ & $\begin{array}{c}\text { Grib Samplo } \\
\text { Tabu Durias } \\
\text { Fin } 30 \\
\text { Min }\end{array}$ & $\begin{array}{l}\text { Fommoizhed } \\
\text { Compotices }\end{array}$ & & \\
\hline prisonoy $7400-360$ & $<0.04 \mathrm{mg} / 1$ & $<0.04 \mathrm{mg} / \mathrm{l}$ & $<0.04 \mathrm{mg} / 1$ & $<0.04 \mathrm{mg} / \mathrm{l}$ & 1 & \\
\hline $\operatorname{sen}$ & $<0.002 \mathrm{mg} / 1$ & & $<0.002 \mathrm{mg} / 1$ & & 1 & \\
\hline Mowany 7 639-976 & $0.0003 \mathrm{mg} / \mathrm{l}$ & $0.0004 \mathrm{mg} / \mathrm{l}$ & $0.0003 \mathrm{mg} / 1$ & $0.0004 \mathrm{mg} / 1$ & 1 & \\
\hline romots & $0.003 \mathrm{mg} / \mathrm{l}$ & & $0.003 \mathrm{mg} / \mathrm{l}$ & & 1 & \\
\hline 50 ind $772-692$ & $<0.002 \mathrm{mg} / 1$ & $<0.002 \mathrm{mg} / 1$ & $<0.002 \mathrm{mg} / \mathrm{l}$ & $<0.002 \mathrm{mg} / \mathrm{l}$ & 1 & \\
\hline Penten & $16 \mathrm{mg} / \mathrm{l}$ & $24 \mathrm{mg} / 1$ & $16 \mathrm{mg} / \mathrm{l}$ & $24 \mathrm{mg} / \mathrm{l}$ & 1 & \\
\hline Purtacomes & $0.06 \mathrm{mg} / \mathrm{l}$ & $<0.05 \mathrm{mg} / \mathrm{l}$ & $0.06 \mathrm{mg} / \mathrm{l}$ & $<0.05 \mathrm{mg} / 1$ & 1 & \\
\hline thminim 7429-50-5 & $2.64 \mathrm{mg} / \mathrm{l}$ & $2.78 \mathrm{mg} / \mathrm{l}$ & $2.64 \mathrm{mg} / \mathrm{l}$ & $2.78 \mathrm{mg} / 1$ & 1 & \\
\hline $4=-107400-34-2$ & $<0.04 \mathrm{mg} / \mathrm{l}$ & $<0.04 \mathrm{mg} / \mathrm{l}$ & $<0.04 \mathrm{mg} / \mathrm{l}$ & $<0.04 \mathrm{mg} / 1$ & 1 & \\
\hline $3000007400-128$ & $0.013 \mathrm{mg} / 1$ & $0.022 \mathrm{mg} / \mathrm{l}$ & $0.013 \mathrm{mg} / \mathrm{l}$ & $0.022 \mathrm{mg} / \mathrm{l}$ & 1 & \\
\hline Berglinen 746-4t-7 & $<0.0004 \mathrm{mg} / \mathrm{l}$ & $<0.0004 \mathrm{mg} / \mathrm{l}$ & $<0.0004 \mathrm{mg} / \mathrm{l}$ & $<0.0004 \mathrm{mg} / 1$ & 1 & \\
\hline $0 \min 7400430$ & $<0.004 \mathrm{mg} / \mathrm{l}$ & $<0.004 \mathrm{mg} / \mathrm{l}$ & $<0.004 \mathrm{mg} / 1$ & $<0.004 \mathrm{mg} / \mathrm{ll}$ & 1 & \\
\hline Coldand 7400-70-2 & $152 \mathrm{mg} / \mathrm{l}$ & 20.1 mg/l & $15.2 \mathrm{mg} / 1$ & $20.1 \mathrm{mg} / 1$ & 1 & \\
\hline 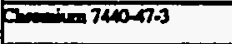 & $<0.006 \mathrm{mg} / \mathrm{l}$ & $<0.006 \mathrm{mg} / 1$ & $<0.006 \mathrm{mg} / \mathrm{l}$ & $<0.006 \mathrm{mg} / \mathrm{l}$ & 1 & \\
\hline Conall 740-84 & $0.004 \mathrm{mg} / \mathrm{l}$ & $0.003 \mathrm{mg} / \mathrm{l}$ & $0.004 \mathrm{mg} / \mathrm{l}$ & $0.003 \mathrm{mg} / \mathrm{l}$ & 1 & \\
\hline Carmerabas & $0.009 \mathrm{mg} / 1$ & $0.009 \mathrm{mg} / \mathrm{l}$ & $0.009 \mathrm{mg} / \mathrm{l}$ & $0.009 \mathrm{mg} / 1$ & 1 & \\
\hline 50074392986 & $7.01 \mathrm{me} / 1$ & $5.07 \mathrm{mg} / 1$ & $7.01 \mathrm{mg} / 1$ & $5.07 \mathrm{mg} / 1$ & 1 & \\
\hline m 7030201 & $<0.02 \mathrm{mg} / 1$ & $<0.02 \mathrm{mg} / 1$ & $<0.02 \mathrm{mg} / 1$ & $<0.02 \mathrm{mg} / 1$ & 1 & \\
\hline 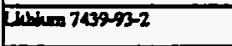 & $<0.02 \mathrm{mg} / \mathrm{l}$ & $<0.02 \mathrm{mg} / \mathrm{l}$ & $<0.02 \mathrm{mg} / \mathrm{l}$ & $<0.02 \mathrm{mg} / \mathrm{l}$ & 1 & \\
\hline hapoinor 733999 & $2.53 \mathrm{mg} / 1$ & $3.59 \mathrm{mg} / \mathrm{l}$ & $2.53 \mathrm{mg} / 1$ & $3.59 \mathrm{mg} / \mathrm{l}$ & 1 & \\
\hline 700000014396.5 & $1.28 \mathrm{mg} / \mathrm{l}$ & $0.812 \mathrm{mg} / \mathrm{l}$ & $1.28 \mathrm{mg} / 1$ & $0.812 \mathrm{mg} / \mathrm{l}$ & 1 & \\
\hline 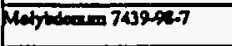 & $<0.006 \mathrm{mg} / \mathrm{l}$ & $<0.006 \mathrm{mg} / \mathrm{l}$ & $<0.006 \mathrm{mg} / \mathrm{l}$ & $<0.006 \mathrm{mg} / 1$ & 1 & \\
\hline$\sqrt{150017400000}$ & $<0.008 \mathrm{mg} / \mathrm{l}$ & $<0.008 \mathrm{mg} / \mathrm{l}$ & $<0.008 \mathrm{mg} / \mathrm{l}$ & $<0.008 \mathrm{mg} / 1$ & 1 & \\
\hline Pomainer $740-09-7$ & $2.3 \mathrm{mg} / 1$ & $7.7 \mathrm{mg} / 1$ & $2.3 \mathrm{mg} / 1$ & $7.7 \mathrm{mg} / \mathrm{l}$ & 1 & \\
\hline 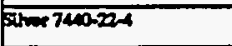 & $<0.006 \mathrm{mg} / \mathrm{l}$ & $<0.006 \mathrm{mg} / \mathrm{l}$ & $<0.006 \mathrm{mg} / \mathrm{l}$ & $<0.006 \mathrm{mg} / 1$ & 1 & \\
\hline $30 \mathrm{ken} 7400225$ & $0.60 \mathrm{mg} / \mathrm{f}$ & $1.62 \mathrm{mg} / 1$ & $0.60 \mathrm{mg} / 1$ & $1.62 \mathrm{mg} / 1$ & 1 & F \\
\hline $7 \operatorname{singman} 740-32-6$ & $0.04 \mathrm{mg} / \mathrm{l}$ & $0.04 \mathrm{mg} / \mathrm{l}$ & $0.04 \mathrm{mg} / \mathrm{l}$ & $0.04 \mathrm{mg} / 1$ & 1 & \\
\hline $2=74006$ & $0.09 \mathrm{mg} / \mathrm{h}$ & $0.08 \mathrm{mg} / \mathrm{l}$ & $0.09 \mathrm{mg} / \mathrm{l}$ & $0.08 \mathrm{mg} / \mathrm{l}$ & 1 & \\
\hline
\end{tabular}


Cootinued from frove.

Part C- List each pollutant shown in Tablea 2F-2. 2F-3, and 2F4 that you know or beve resson to believo is present. See the instructions for additional derails and requireaneats. Cor one uable for each outfill.

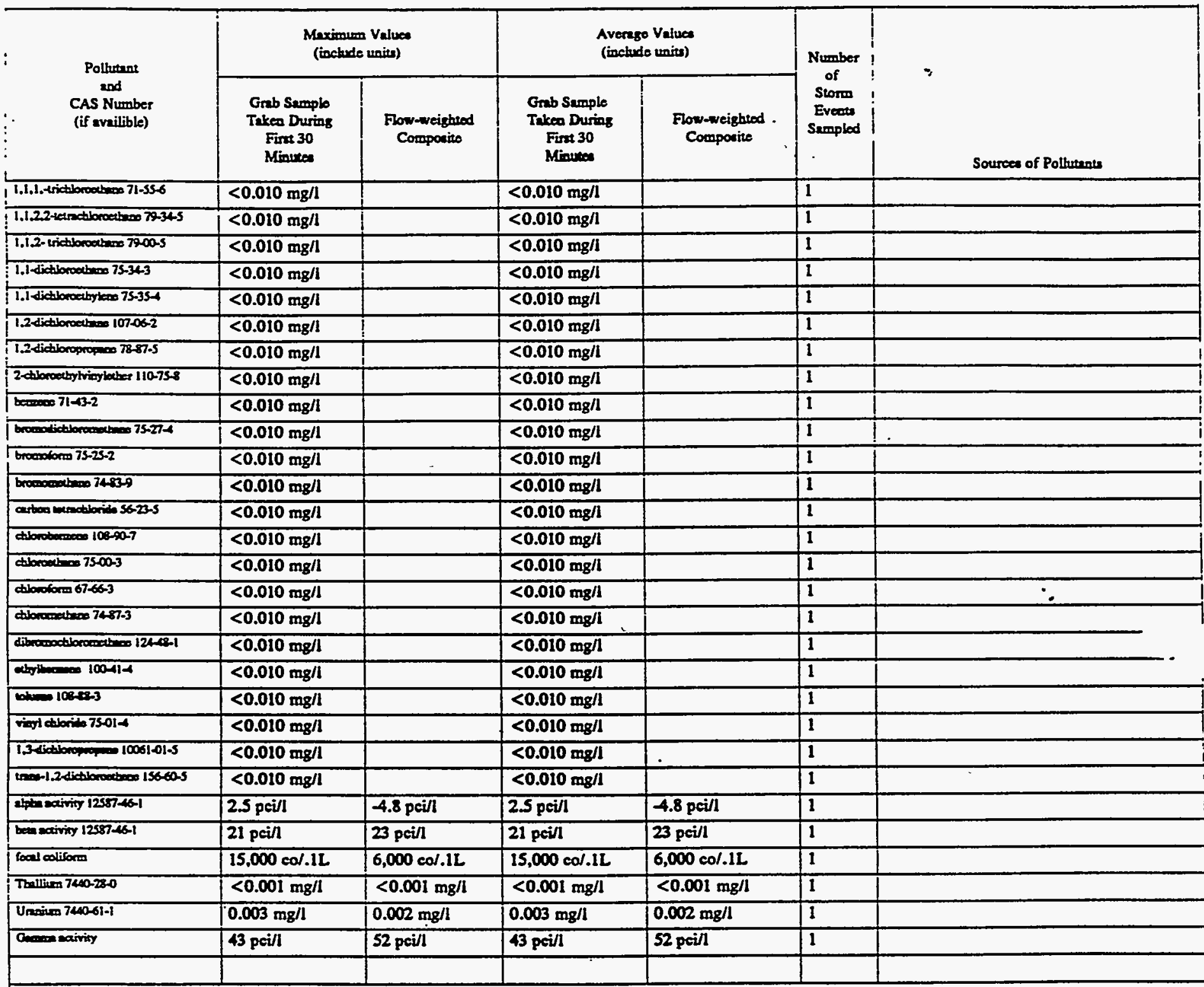

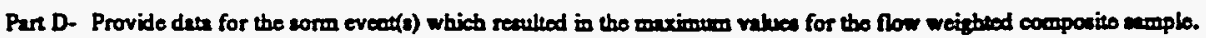

\begin{tabular}{|c|c|c|c|c|c|c|c|}
\hline $\begin{array}{l}\text { I. } \\
\text { Date of } \\
\text { Storm } \\
\text { Event }\end{array}$ & $\begin{array}{l}2 . \\
\text { Durition } \\
\text { of Seorm } \\
\text { (in } \\
\text { minutes) }\end{array}$ & $\begin{array}{l}3 . \\
\text { Toul minfill } \\
\text { during storm ovent } \\
\text { (in ioches) }\end{array}$ & $\begin{array}{l}4 . \\
\text { Number of bours between } \\
\text { beginning of morm mene- } \\
\text { urad and cod of provious } \\
\text { memurablo nin oveat }\end{array}$ & $\begin{array}{c}\text { S. } \\
\text { Maximum flow raso } \\
\text { during rnin eveat } \\
\text { (allosimin or spocify } \\
\text { unis) }\end{array}$ & $\begin{array}{l}6 . \\
\text { Total flow from } \\
\text { nin ovent } \\
\text { (ollowe or } \\
\text { epeoify unit) }\end{array}$ & $\begin{array}{c}7 . \\
\text { Seseon } \\
\text { anple was } \\
\text { tuken }\end{array}$ & $\begin{array}{l}8 . \\
\text { Form of } \\
\text { Precipitution } \\
\text { (riafill, } \\
\text { nowmeli) }\end{array}$ \\
\hline $7 / 24 / 91$ & 185 & 0.8 & 154.2 & 19,810 & 723,900 & Summer & Ruinfall \\
\hline
\end{tabular}

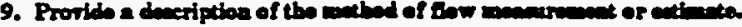

An ISCO 3230 Flow meter was used to obtain water leved readings. This instrument was calibrated with the normal dry weather flow, if preseat, as level zero. The flow meter was programmed with the characteristics of the converance so that flow rates and total flows conld be calculated by the zneter. 
Outfall 2

\begin{tabular}{|c|c|c|c|c|c|c|}
\hline \multicolumn{7}{|l|}{ Parse, B and C Continened } \\
\hline \multirow{2}{*}{$\begin{array}{l}\text { Polbuand } \\
\text { and } \\
\text { CAS Number } \\
\text { (if availiblo) }\end{array}$} & \multicolumn{2}{|c|}{$\begin{array}{l}\text { Marionem Vahuea } \\
\text { (ibcholo unity) }\end{array}$} & \multicolumn{2}{|c|}{$\begin{array}{l}\text { Aversos Values } \\
\text { (includo unio) }\end{array}$} & \multirow{2}{*}{$\begin{array}{l}\text { Nomber } \\
\text { of } \\
\text { Storm: } \\
\text { Eveo } \\
\text { Sompled }\end{array}$} & \multirow[b]{2}{*}{ Sourece of Polke } \\
\hline & 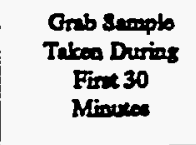 & $\begin{array}{l}\text { Fonverviphed } \\
\text { Compocies }\end{array}$ & $\begin{array}{l}\text { Grbb Samplo } \\
\text { Trbea During } \\
\text { Fira } 30 \\
\text { Minuteo }\end{array}$ & $\begin{array}{l}\text { Flow-wrighbed } \\
\text { Coppotite }\end{array}$ & & \\
\hline Bashem 740-32-3 & $0.0938 \mathrm{mg} / \mathrm{l}$ & $0.0926 \mathrm{mg} / \mathrm{l}$ & $0.0938 \mathrm{mg} / 1$ & $0.0926 \mathrm{mg} / 1$ & 1 & \\
\hline 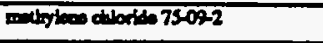 & $<0.010 \mathrm{mg} / 1$ & & $<0.010 \mathrm{mg} / \mathrm{l}$ & & 1 & \\
\hline 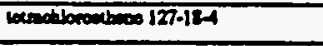 & $<0.010 \mathrm{mg} / \mathrm{l}$ & & $<0.010 \mathrm{mg} / \mathrm{I}$ & & 1 & \\
\hline 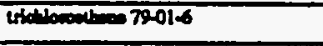 & $<0.010 \mathrm{mg} / \mathrm{l}$ & & $<0.010 \mathrm{mg} / \mathrm{l}$ & & 1 & \\
\hline xyines & $<0.010 \mathrm{mg} / \mathrm{l}$ & & $<0.010 \mathrm{mg} / \mathrm{l}$ & & 1 & \\
\hline 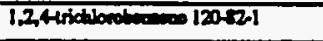 & $<0.010 \mathrm{mg} / \mathrm{l}$ & $<0.100 \mathrm{mg} / 1$ & $<0.010 \mathrm{mg} / 1$ & $<0.100 \mathrm{mg} / 1$ & 1 & \\
\hline 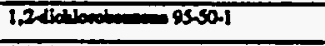 & $<0.010 \mathrm{mg} / \mathrm{l}$ & $<0.100 \mathrm{mg} / \mathrm{l}$ & $<0.010 \mathrm{mg} / \mathrm{l}$ & $<0.100 \mathrm{mg} / 1$ & 1 & \\
\hline 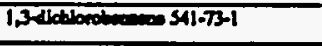 & $<0.010 \mathrm{mg} / 1$ & $<0.100 \mathrm{mg} / \mathrm{l}$ & $<0.010 \mathrm{mg} / \mathrm{l}$ & $<0.100 \mathrm{mg} / 1$ & 1 & \\
\hline 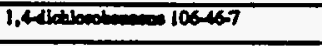 & $<0.010 \mathrm{mg} / \mathrm{I}$ & $<0.100 \mathrm{mg} / 1$ & $<0.010 \mathrm{mg} / \mathrm{l}$ & $<0.100 \mathrm{mg} / 1$ & 1 & \\
\hline 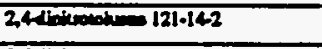 & $<0.010 \mathrm{mg} / \mathrm{l}$ & $<0.100 \mathrm{mg} / 1$ & $<0.010 \mathrm{mg} / \mathrm{l}$ & $<0.100 \mathrm{mg} / \mathrm{l}$ & 1 & \\
\hline 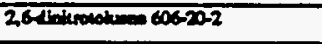 & $<0.010 \mathrm{mg} / \mathrm{l}$ & $<0.100 \mathrm{mg} / \mathrm{l}$ & $<0.010 \mathrm{mg} / 1$ & $<0.100 \mathrm{mg} / \mathrm{l}$ & 1 & \\
\hline 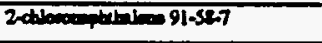 & $<0.010 \mathrm{mg} / \mathrm{l}$ & $<0.100 \mathrm{mg} / \mathrm{l}$ & $<0.010 \mathrm{mg} / \mathrm{I}$ & $<0.100 \mathrm{mg} / 1$ & 1. & \\
\hline 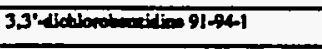 & $<0.010 \mathrm{mg} / \mathrm{l}$ & $<0.100 \mathrm{mg} / \mathrm{h}$ & $<0.010 \mathrm{mg} / \mathrm{l}$ & $<0.100 \mathrm{mg} / \mathrm{l}$ & 1 & \\
\hline 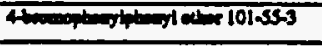 & $<0.010 \mathrm{mg} / \mathrm{l}$ & $<0.100 \mathrm{mg} / \mathrm{l}$ & $<0.010 \mathrm{mg} / \mathrm{l}$ & $<0.100 \mathrm{mg} / 1$ & 1 & \\
\hline 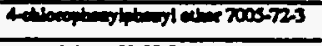 & $<0.010 \mathrm{mg} / \mathrm{h}$ & $<0.100 \mathrm{mg} / \mathrm{l}$ & $<0.010 \mathrm{mg} / \mathrm{l}$ & $<0.100 \mathrm{mg} / 1$ & 1 & \\
\hline 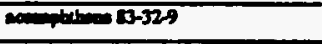 & $<0.010 \mathrm{mg} / \mathrm{l}$ & $<0.100 \mathrm{mg} / \mathrm{l}$ & $<0.010 \mathrm{mg} / \mathrm{g}$ & $<0.100 \mathrm{mg} / \mathrm{l}$ & 1 & \\
\hline 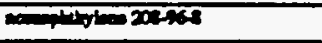 & $<0.010 \mathrm{mg} / \mathrm{l}$ & $<0.100 \mathrm{mg} / \mathrm{l}$ & $<0.010 \mathrm{mg} / \mathrm{l}$ & $<0.100 \mathrm{mg} / \mathrm{l}$ & 1 & \\
\hline $120-127$ & $<0.010 \mathrm{mg} / \mathrm{l}$ & $<0.100 \mathrm{mg} / 1$ & $<0.010 \mathrm{mg} / 1$ & $<0.100 \mathrm{mg} / 1$ & 1 & \\
\hline mings.5 & $<0.030 \mathrm{mg} / \mathrm{l}$ & $<0.300 \mathrm{mg} / \mathrm{h}$ & $<0.030 \mathrm{mg} / 1$ & $<0.300 \mathrm{mg} / \mathrm{l}$ & 1 & 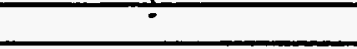 \\
\hline 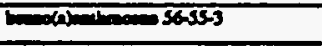 & $<0.010 \mathrm{mg} / \mathrm{t}$ & $<0.100 \mathrm{mg} / 1$ & $<0.010 \mathrm{mg} / \mathrm{h}$ & $<0.100 \mathrm{mg} / \mathrm{l}$ & 1 & \\
\hline 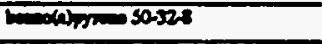 & $<0.010 \mathrm{mg} / 1$ & $<0.100 \mathrm{mg} / \mathrm{l}$ & $<0.010 \mathrm{mg} / 1$ & $<0.100 \mathrm{mg} / \mathrm{l}$ & 1 & \\
\hline 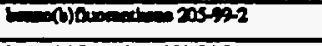 & $<0.010 \mathrm{mg} / 1$ & $<0.100 \mathrm{mg} / 1$ & $<0.010 \mathrm{mg} / 1$ & $<0.100 \mathrm{mg} / 1$ & 1 & \\
\hline 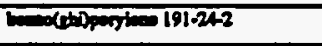 & $<0.010 \mathrm{mg} / \mathrm{l}$ & $<0.100 \mathrm{mg} / \mathrm{h}$ & $<0.010 \mathrm{mg} / 1$ & $<0.100 \mathrm{mg} / 1$ & 1 & \\
\hline 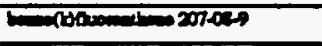 & $<0.010 \mathrm{mg} / 1$ & $<0.100 \mathrm{mg} / 1$ & $<0.010 \mathrm{mq} / 1$ & $<0.100 \mathrm{mg} / \mathrm{l}$ & 1 & \\
\hline 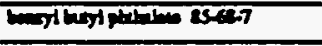 & $<0.010 \mathrm{mg} / \mathrm{l}$ & $<0.100 \mathrm{~m} / \mathrm{h}$ & $<0.010 \mathrm{mg} / 1$ & $<0.100 \mathrm{mg} / \mathrm{h}$ & 1 & \\
\hline 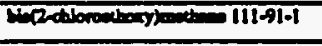 & $<0.010 \mathrm{mg} / \mathrm{l}$ & $<0.100 \mathrm{mg} / \mathrm{l}$ & $<0.010 \mathrm{mg} / \mathrm{l}$ & $<0.100 \mathrm{mg} / \mathrm{l}$ & 1 & - \\
\hline 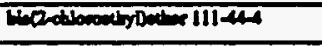 & $<0.010 \mathrm{mg} / \mathrm{l}$ & $<0.100 \mathrm{mg} /$ & $<0.010 \mathrm{mg} / \mathrm{l}$ & $<0.100 \mathrm{mg} / \mathrm{l}$ & 1 & \\
\hline 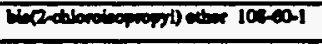 & $<0.010 \mathrm{mg} / \mathrm{l}$ & $<0.100 \mathrm{mg} / 1$ & $<0.010 \mathrm{mg} / 1$ & $<0.100 \mathrm{mg} / 1$ & 1 & \\
\hline 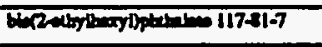 & $<0.010 \mathrm{mg} / \mathrm{l}$ & $<0.100 \mathrm{mg} / \mathrm{l}$ & $<0.010 \mathrm{mg} / \mathrm{l}$ & $<0.100 \mathrm{mg} / \mathrm{l}$ & 1 & \\
\hline dispoune 21501-9 & $<0.010 \mathrm{mg} / \mathrm{l}$ & $<0.100 \mathrm{mg} / \mathrm{l}$ & $<0.010 \mathrm{mg} / \mathrm{l}$ & $<0.100 \mathrm{mg} / \mathrm{l}$ & 1 & \\
\hline 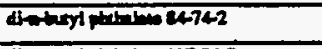 & $0.036 \mathrm{mg} / \mathrm{I}$ & $<0.100 \mathrm{mg} / \mathrm{l}$ & $0.036 \mathrm{mg} / \mathrm{l}$ & $<0.100 \mathrm{mg} / \mathrm{l}$ & 1 & \\
\hline 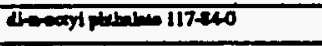 & $<0.010 \mathrm{mg} / 1$ & $<0.100 \mathrm{mg} / \mathrm{l}$ & $<0.010 \mathrm{mg} / \mathrm{l}$ & $<0.100 \mathrm{mg} / 1$ & 1 & \\
\hline 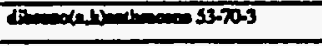 & $<0.010 \mathrm{mg} / \mathrm{l}$ & $<0.100 \mathrm{mg} / 1$ & $<0.010 \mathrm{mg} / 1$ & $<0.100 \mathrm{mg} / \mathrm{l}$ & 1 & \\
\hline 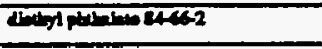 & $<0.010 \mathrm{mg} / 1$ & $<0.100 \mathrm{mg} / 1$ & $<0.010 \mathrm{mg} / \mathrm{I}$ & $<0.100 \mathrm{mg} / \mathrm{l}$ & 1 & \\
\hline 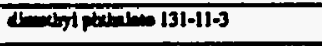 & $<0.010 \mathrm{mg} / \mathrm{l}$ & $<0.100 \mathrm{mg} / \mathrm{l}$ & $<0.010 \mathrm{mg} / \mathrm{l}$ & $<0.100 \mathrm{mg} / \mathrm{l}$ & 1 & \\
\hline 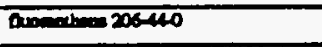 & $0.012 \mathrm{mg} / \mathrm{l}$ & $<0.100 \mathrm{mg} / \mathrm{l}$ & $0.012 \mathrm{mg} / 1$ & $<0.100 \mathrm{mg} / \mathrm{l}$ & 1 & \\
\hline 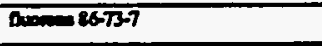 & $<0.010 \mathrm{mg} / 1$ & $<0.100 \mathrm{mg} / \mathrm{l}$ & $<0.010 \mathrm{mg} / \mathrm{l}$ & $<0.100 \mathrm{mg} / \mathrm{I}$ & 1 & \\
\hline 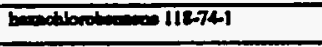 & $<0.010 \mathrm{mg} / \mathrm{l}$ & $<0.100 \mathrm{mg} h$ & $<0.010 \mathrm{mg} / \mathrm{l}$ & $<0.100 \mathrm{mg} / \mathrm{l}$ & 1 & \\
\hline 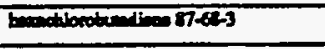 & $<0.010 \mathrm{mg} / \mathrm{l}$ & $<0.100 \mathrm{mg} / \mathrm{l}$ & $<0.010 \mathrm{mg} / 1$ & $<0.100 \mathrm{mg} / 1$ & 1 & \\
\hline 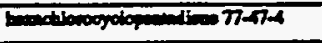 & $<0.010 \mathrm{mg} / \mathrm{l}$ & $<0.100 \mathrm{mg} / \mathrm{h}$ & $<0.010 \mathrm{mg} / \mathrm{h}$ & $<0.100 \mathrm{mg} / \mathrm{l}$ & 1 & \\
\hline $67-72-1$ & $<0.010 \mathrm{mg} / \mathrm{l}$ & $<0.100 \mathrm{mg} / \mathrm{l}$ & $<0.010 \mathrm{mg} / 1$ & $<0.100 \mathrm{mg} / 1$ & 1 & \\
\hline 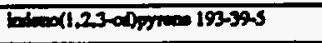 & $<0.010 \mathrm{mg} / \mathrm{l}$ & $<0.100 \mathrm{mg} / \mathrm{l}$ & $<0.010 \mathrm{mg} / \mathrm{l}$ & $<0.100 \mathrm{mg} / \mathrm{I}$ & 1 & \\
\hline Eopherisel & $<0.010 \mathrm{mg} / \mathrm{l}$ & $<0.100 \mathrm{mg} / 1$ & $<0.010 \mathrm{mg} / 1$ & $<0.100 \mathrm{mg} / 1$ & 1 & \\
\hline 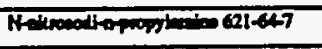 & $<0.010 \mathrm{mg} / \mathrm{l}$ & $<0.100 \mathrm{mg} / 1$ & $<0.010 \mathrm{mg} / 1$ & $<0.100 \mathrm{mg} / \mathrm{l}$ & 1 & \\
\hline 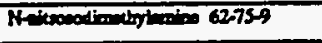 & $<0.010 \mathrm{mg} / \mathrm{l}$ & $<0.100 \mathrm{mg} / \mathrm{l}$ & $<0.010 \mathrm{mg} / \mathrm{h}$ & $<0.100 \mathrm{mg} / \mathrm{l}$ & 1 & \\
\hline 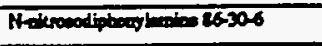 & $<0.010 \mathrm{mg} / \mathrm{h}$ & $<0.100 \mathrm{mg} / \mathrm{l}$ & $<0.010 \mathrm{mg} / 1$ & $<0.100 \mathrm{mg} / \mathrm{l}$ & 1 & \\
\hline 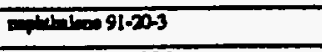 & $<0.010 \mathrm{mg} / \mathrm{l}$ & $<0.100 \mathrm{mg} / 1$ & $<0.010 \mathrm{mg} / 1$ & $<0.100 \mathrm{mg} /$ & 1 & \\
\hline 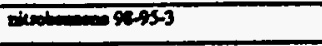 & $<0.010 \mathrm{mg} / \mathrm{l}$ & $<0.100 \mathrm{mg} / 1$ & $<0.010 \mathrm{mg} / 1$ & $<0.100 \mathrm{mg} / \mathrm{l}$ & 1 & \\
\hline 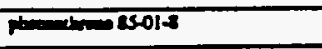 & $<0.010 \mathrm{mg} / 1$ & $<0.100 \mathrm{mg} / 1$ & $<0.010 \mathrm{mg} / \mathrm{i}$ & $<0.100 \mathrm{mg} /$ & 1 & \\
\hline prone 1220000 & $<0.010 \mathrm{mg} / \mathrm{l}$ & $<0.100 \mathrm{mg} / \mathrm{l}$ & $<0.010 \mathrm{mg} / \mathrm{l}$ & $<0.100 \mathrm{mg} / \mathrm{h}$ & 1 & \\
\hline 24,6 thethenowhed $2506-2$ & $<0.030 \mathrm{mg} / \mathrm{h}$ & $<0.300 \mathrm{mg} / 1$ & $<0.030 \mathrm{mg} / 1$ & $<0.300 \mathrm{mg} / \mathrm{l}$ & 1 & \\
\hline 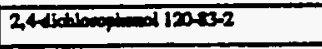 & $<0.030 \mathrm{mg} / \mathrm{l}$ & $<0.300 \mathrm{mg} / \mathrm{I}$ & $<0.030 \mathrm{mg} / 1$ & $<0.300 \mathrm{mg} / \mathrm{h}$ & 1 & \\
\hline 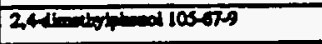 & $<0.030 \mathrm{mg} / 1$ & $<0.300 \mathrm{mg} / 1$ & $<0.030 \mathrm{mg} / 1$ & $<0.300 \mathrm{mg} / 1$ & 1 & \\
\hline 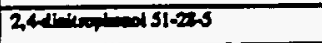 & $<0.030 \mathrm{mg} / \mathrm{l}$ & $<0.300 \mathrm{mg} / \mathrm{l}$ & $<0.030 \mathrm{mg} / 1$ & $<0.300 \mathrm{mg} / 1$ & 1 & \\
\hline
\end{tabular}


Outfall 2

\begin{tabular}{|c|c|c|c|c|c|c|}
\hline Parts B and C Continued & & & & & & \\
\hline ת & $\begin{array}{l}\text { Meximur } \\
\text { (inchud }\end{array}$ & $\begin{array}{l}\text { Values } \\
\text { units) }\end{array}$ & $\begin{array}{l}\text { Avens: } \\
\text { (incher }\end{array}$ & $\begin{array}{l}\text { Values } \\
\text { units) }\end{array}$ & Nurober & \\
\hline $\begin{array}{l}\text { CAS Number } \\
\text { (if availible) }\end{array}$ & $\begin{array}{l}\text { Greb Sumple } \\
\text { Tiken During } \\
\text { First } 30 \\
\text { Minuese }\end{array}$ & $\begin{array}{l}\text { Flow-weighted } \\
\text { Comporite }\end{array}$ & $\begin{array}{l}\text { Grab Sumple } \\
\text { Taken During } \\
\text { Firat } 30 \\
\text { Minutea }\end{array}$ & $\begin{array}{c}\text { Forv-weighed } \\
\text { Composits }\end{array}$ & $\begin{array}{l}\text { Storm } \\
\text { Eveotit } \\
\text { Sempled }\end{array}$ & Sourcese of Polturentes \\
\hline 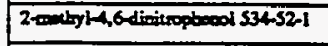 & $<0.030 \mathrm{mg} / 1$ & $<0.300 \mathrm{mg} / \mathrm{l}$ & $<0.030 \mathrm{mg} / 1$ & $<0.300 \mathrm{mg} / \mathrm{l}$ & 1 & \\
\hline 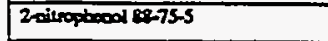 & $<0.030 \mathrm{mg} / \mathrm{l}$ & $<0.300 \mathrm{mg} / \mathrm{l}$ & $<0.030 \mathrm{mg} / \mathrm{l}$ & $<0.300 \mathrm{mg} / \mathrm{l}$ & 1 & \\
\hline 4cthoro-3 - wabyl pheol $59.50-7$ & $<0.030 \mathrm{mg} / \mathrm{l}$ & $<0.300 \mathrm{mg} / \mathrm{l}$ & $<0.030 \mathrm{mg} / \mathrm{h}$ & $<0.300 \mathrm{mg} / 1$ & 1 & \\
\hline Solecopbeod $100-02.7$ & $<0.030 \mathrm{mg} / \mathrm{l}$ & $<0.300 \mathrm{mg} / \mathrm{l}$ & $<0.030 \mathrm{mg} / 1$ & $<0.300 \mathrm{mg} / \mathrm{h}$ & 1 & \\
\hline peousctionopheopol $8786-5$ & $<0.030 \mathrm{mg} / 1$ & $<0.300 \mathrm{mg} / 1$ & $<0.030 \mathrm{mg} / \mathrm{l}$ & $<0.300 \mathrm{mg} / 1$ & 1 & \\
\hline$p \operatorname{poc} 100-95-2$ & $<0.030 \mathrm{mg} / \mathrm{l}$ & $<0.300 \mathrm{mg} / 1$ & $<0.030 \mathrm{mg} / \mathrm{l}$ & $<0.300 \mathrm{mg} / 1$ & 1 & \\
\hline $4,4^{\circ}-D D D 72-548$ & $<0.000004 \mathrm{mg} / \mathrm{l}$ & $<0.000004 \mathrm{mg} / \mathrm{I}$ & $<0.000004 \mathrm{mg} / 1$ & $<0.000004 \mathrm{mg} / \mathrm{l}$ & 1 & \\
\hline 4.6-DDE $2.55-9$ & $<0.000004 \mathrm{mg} / \mathrm{l}$ & $<0.000004 \mathrm{mg} / \mathrm{l}$ & $<0.000004 \mathrm{mg} / \mathrm{l}$ & $<0.000004 \mathrm{mg} / \mathrm{l}$ & 1 & \\
\hline 4,4-DDT $50-29-3$ & $<0.000004 \mathrm{mg} / \mathrm{l}$ & $<0.000004 \mathrm{mg} / \mathrm{l}$ & $<0.000004 \mathrm{mg} / \mathrm{l}$ & $<0.000004 \mathrm{mg} / \mathrm{l}$ & 1 & \\
\hline $2 \sin 30200-2$ & $<0.000004 \mathrm{mg} / \mathrm{l}$ & $<0.000004 \mathrm{mg} / \mathrm{l}$ & $<0.000004 \mathrm{mg} / \mathrm{l}$ & $<0.000004 \mathrm{mg} / \mathrm{l}$ & 1 & \\
\hline thendere 57-74 & $<0.00002 \mathrm{mg} / \mathrm{l}$ & $<0.00002 \mathrm{mg} / \mathrm{l}$ & $<0.00002 \mathrm{mg} / !$ & $<0.00002 \mathrm{mg} / \mathrm{l}$ & 1 & \\
\hline diedria $60-57-1$ & $<0.000004 \mathrm{mg} / \mathrm{l}$ & $<0.000004 \mathrm{mg} / \mathrm{l}$ & $<0.000004 \mathrm{mg} / \mathrm{l}$ & $<0.000004 \mathrm{mg} / \mathrm{l}$ & 1 & \\
\hline cosonulfen $1959-96$ & $<0.000004 \mathrm{mg} / 1$ & $<0.000004 \mathrm{mg} / 1$ & $<0.000004 \mathrm{mg} / \mathrm{l}$ & $<0.000004 \mathrm{mg} / \mathrm{l}$ & 1 & \\
\hline atcoulten 1133213655 & $<0.000004 \mathrm{mg} / \mathrm{l}$ & $<0.000004 \mathrm{mg} / \mathrm{l}$ & $<0.000004 \mathrm{mg} / \mathrm{l}$ & $<0.000004 \mathrm{mg} / \mathrm{l}$ & 1 & \\
\hline 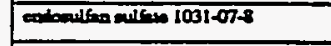 & $<0.000004 \mathrm{mg} / \mathrm{l}$ & $<0.000004 \mathrm{mg} / \mathrm{l}$ & $<0.000004 \mathrm{mg} / \mathrm{l}$ & $<0.000004 \mathrm{mg} / 1$ & 1 & , \\
\hline $\operatorname{cosin} 72-208$ & $<0.00005 \mathrm{mg} / \mathrm{l}$ & $<0.00005 \mathrm{mg} / \mathrm{l}$ & $<0.00005 \mathrm{mg} / \mathrm{l}$ & $<0.00005 \mathrm{mg} / \mathrm{l}$ & 1 & \\
\hline condrin eldeby do $7421-934$ & $<0.000004 \mathrm{mg} / \mathrm{l}$ & $<0.000004 \mathrm{mg} / \mathrm{I}$ & $<0.000004 \mathrm{mg} / \mathrm{l}$ & $<0.000004 \mathrm{mg} / \mathrm{h}$ & 1 & \\
\hline bepectbor $76-448$ & $<0.000004 \mathrm{mg} / 1$ & $<0.000004 \mathrm{mg} / \mathrm{l}$ & $<0.000004 \mathrm{mg} / \mathrm{l}$ & $<0.000004 \mathrm{mg} / 1$ & 1 & - \\
\hline 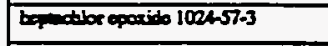 & $<0.000004 \mathrm{mg} / \mathrm{l}$ & $<0.000004 \mathrm{mg} / \mathrm{l}$ & $<0.000004 \mathrm{mg} / \mathrm{l}$ & $<0.000004 \mathrm{mg} / \mathrm{h}$ & 1 & \\
\hline $000001-35-2$ & $<0.0002 \mathrm{mg} / \mathrm{l}$ & $<0.0002 \mathrm{mg} / \mathrm{l}$ & $<0.0002 \mathrm{mg} / \mathrm{l}$ & $<0.0002 \mathrm{mg} / \mathrm{l}$ & 1 & \\
\hline 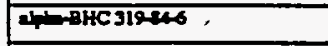 & $<0.000004 \mathrm{mg} / 1$ & $<0.000004 \mathrm{mg} / \mathrm{l}$ & $<0.000004 \mathrm{mg} / 1$ & $<0.000004 \mathrm{mg} / \mathrm{l}$ & 1 & \\
\hline DHC 319-85-7 & $<0.000004 \mathrm{mg} / 1$ & $<0.000004 \mathrm{mg} / \mathrm{l}$ & $<0.000004 \mathrm{mg} / \mathrm{l}$ & $<0.000004 \mathrm{mg} / \mathrm{l}$ & 1 & \\
\hline delw-8KC 319868 & $<0.000004 \mathrm{mg} / \mathrm{l}$ & $<0.000004 \mathrm{mg} / \mathrm{h}$ & $<0.000004 \mathrm{mg} / \mathrm{h}$ & $<0.000004 \mathrm{mg} / \mathrm{l}$ & 1 & \\
\hline $8000-B H C 58398$ & $<0.00001 \mathrm{mg} / \mathrm{l}$ & $<0.00001 \mathrm{mg} / \mathrm{l}$ & $<0.00001 \mathrm{mg} / \mathrm{l}$ & $<0.00001 \mathrm{mg} / 1$ & $\overline{1}$ & \\
\hline 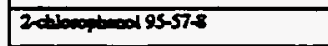 & $<0.030 \mathrm{mg} / \mathrm{l}$ & $<0.300 \mathrm{mg} / \mathrm{l}$ & $<0.030 \mathrm{mg} / \mathrm{l}$ & $<0.300 \mathrm{mg} / \mathrm{l}$ & 1 & \\
\hline Arodon $12120253605-21-9$ & $<0.0005 \mathrm{mg} / 1$ & $<0.0005 \mathrm{mg} / \mathrm{l}$ & $<0.0005 \mathrm{mg} / \mathrm{l}$ & $<0.0005 \mathrm{mg} / \mathrm{l}$ & 1 & \\
\hline Arodon-12561109769-1 & $<0.0005 \mathrm{mg} / \mathrm{h}$ & $<0.0005 \mathrm{mg} / \mathrm{l}$ & $<0.0005 \mathrm{mg} / \mathrm{l}$ & $<0.0005 \mathrm{mg} / 1$ & 1 & \\
\hline Arodom-121 $1110+22-2$ & $<0.0005 \mathrm{mg} / \mathrm{l}$ & $<0.0005 \mathrm{mg} / 1$ & $<0.0005 \mathrm{mg} / 1$ & $<0.0005 \mathrm{mg} / \mathrm{ll}$ & 1 & \\
\hline Arodon-1232 III\&1-16.5 & $<0.0005 \mathrm{mg} / \mathrm{l}$ & $<0.0005 \mathrm{mg} / \mathrm{l}$ & $<0.0005 \mathrm{mg} / \mathrm{l}$ & $<0.0005 \mathrm{mg} / \mathrm{l}$ & 1 & \\
\hline Arodor-1248 12072-296 & $<0.0005 \mathrm{mg} / 1$ & $<0.0005 \mathrm{mg} / \mathrm{l}$ & $<0.0005 \mathrm{mg} / \mathrm{l}$ & $<0.0005 \mathrm{mg} / 1$ & 1 & \\
\hline 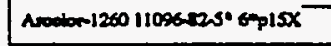 & $<0.0005 \mathrm{mg} / 1$ & $<0.0005 \mathrm{mg} / 1$ & $<0.0005 \mathrm{mg} / \mathrm{l}$ & $<0.0005 \mathrm{mg} / \mathrm{l}$ & 1 & \\
\hline Aredon-1016 12074-11-2 & $<0.0005 \mathrm{mg} / \mathrm{h}$ & $<0.0005 \mathrm{mg} / 1$ & $<0.0005 \mathrm{mg} / \mathrm{h}$ & $<0.0005 \mathrm{mg} / \mathrm{h}$ & 1 & \\
\hline Crecoles & $<0.030 \mathrm{mg} / \mathrm{l}$ & $<0.300 \mathrm{mg} / \mathrm{l}$ & $<0.030 \mathrm{mg} / 1$ & $<0.300 \mathrm{mg} / \mathrm{h}$ & 1 & \\
\hline Anoticio & $<0.010 \mathrm{mg} / \mathrm{l}$ & $<0.010 \mathrm{mg} / \mathrm{I}$ & $<0.010 \mathrm{mg} / \mathrm{l}$ & $<0.010 \mathrm{mg} / \mathrm{l}$ & 1 & \\
\hline Aastaciactib & $<0.010 \mathrm{mg} / \mathrm{l}$ & $<0.010 \mathrm{mg} / \mathrm{l}$ & $<0.010 \mathrm{mg} / \mathrm{h}$ & $<0.010 \mathrm{mg} / \mathrm{l}$ & 1 & \\
\hline 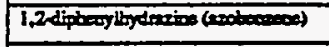 & Reaulte pending & Reanlts pending & Results peoding & Rezults pending & 1 & \\
\hline & & & & & & \\
\hline & & & & & & \\
\hline & & & & & & \\
\hline & & & & & & \\
\hline & & & & & & \\
\hline & & & & & & \\
\hline & & & & & & \\
\hline & & & & & & \\
\hline & & & & & & \\
\hline & & & & & & \\
\hline & & & & & & \\
\hline & & & & & & \\
\hline & & & & & & \\
\hline & & & & & & \\
\hline
\end{tabular}




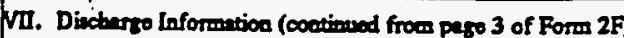

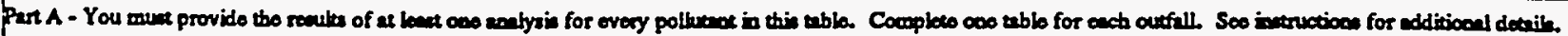

\begin{tabular}{|c|c|c|c|c|c|c|}
\hline \multirow{2}{*}{$\begin{array}{l}\text { Pollutuat } \\
\text { and } \\
\text { CAs Number } \\
\text { (if avilibis) }\end{array}$} & \multicolumn{2}{|c|}{ 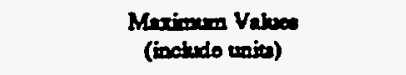 } & \multicolumn{2}{|c|}{$\begin{array}{l}\text { Avernge Valume } \\
\text { (inclodo unial) }\end{array}$} & \multirow{2}{*}{$\begin{array}{l}\text { Number } \\
\text { of } \\
\text { Storm } \\
\text { Everes } \\
\text { Sempled }\end{array}$} & \multirow[b]{2}{*}{ Sourcese of Pollentron } \\
\hline & 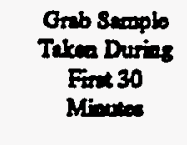 & $\begin{array}{l}\text { Fow-woighed } \\
\text { Compocies }\end{array}$ & 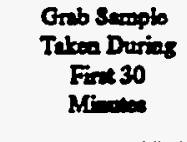 & $\begin{array}{l}\text { Fow-wrizted } \\
\text { Couposits }\end{array}$ & & \\
\hline pil nat ones & $<2 m g / l$ & & $<2 \mathrm{mg} / 1$ & & 1 & \\
\hline 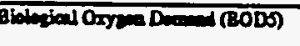 & $<5 \mathrm{mg} / \mathrm{l}$ & $<5 \mathrm{mg} / \mathrm{l}$ & $<5 \mathrm{mg} / 1$ & $<5 m g / 1$ & 1 & \\
\hline$C=10 x=1(C O D)$ & $41.7 \mathrm{mg} / \mathrm{l}$ & $27 \mathrm{mg} / \mathrm{l}$ & $41.7 \mathrm{mg} / 1$ & $27 \mathrm{mg} / 1$ & 1 & \\
\hline 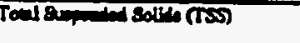 & $28 m g / 1$ & $<5 \mathrm{mg} / 1$ & $28 \mathrm{mg} / 1$ & $<5 m g / 1$ & 1 & \\
\hline 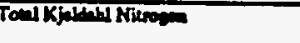 & $1.2 \mathrm{mg} / \mathrm{l}$ & $0.88 \mathrm{mg} / \mathrm{l}$ & $1.2 \mathrm{mg} / \mathrm{l}$ & $0.88 \mathrm{mg} / \mathrm{l}$ & 1 & \\
\hline 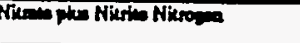 & $0.14 \mathrm{mg} / \mathrm{l}$ & $0.14 \mathrm{mg} / \mathrm{l}$ & $0.14 \mathrm{mg} / 1$ & $0.14 \mathrm{mg} / 1$ & 1 & - \\
\hline Toul phemborse & $0.25 \mathrm{mg} / 1$ & $0.21 \mathrm{mg} / \mathrm{l}$ & $0.25 \mathrm{mg} / 1$ & $0.12 \mathrm{mg} / 1$ & 1 & \\
\hline H & Mth- 7.8 & $M=7.8$ & $M=7.8$ & $\begin{array}{l}M \\
M\end{array}$ & & \\
\hline
\end{tabular}

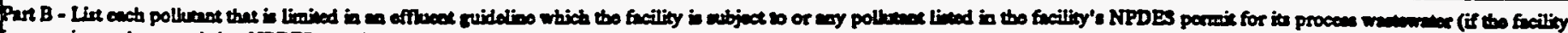

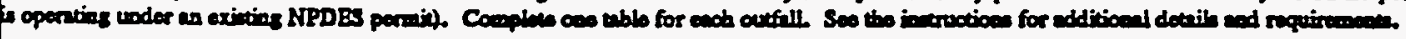

\begin{tabular}{|c|c|c|c|c|c|c|}
\hline \multirow{2}{*}{ 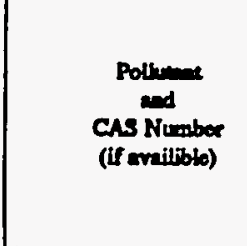 } & \multicolumn{2}{|c|}{ 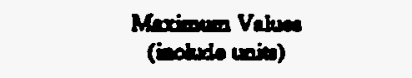 } & \multicolumn{2}{|c|}{ 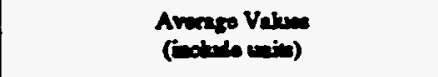 } & \multirow{2}{*}{$\begin{array}{l}\text { Nember } \\
\text { of } \\
\text { stores } \\
\text { Eveces } \\
\text { Serpied }\end{array}$} & \multirow{2}{*}{ 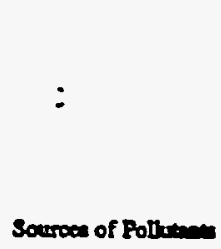 } \\
\hline & 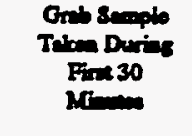 & 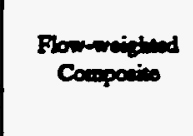 & 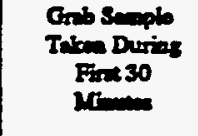 & Flow-moighnd & & \\
\hline $10=7800-360$ & $<0.01 \mathrm{mg} / \mathrm{l}$ & $<0.01 \mathrm{mg} / \mathrm{l}$ & $<0.01 \mathrm{mg} / 1$ & $<0.01 \mathrm{mg} / 1$ & 1 & \\
\hline 20 & $<0.002 \mathrm{mg} / 1$ & & $<0.002 \mathrm{mg} / 1$ & & 1 & \\
\hline 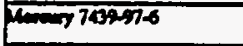 & $<0.0002 \mathrm{men}$ & $0.0002 \mathrm{mg} / \mathrm{l}$ & $<0.0002 \mathrm{mg} / 1$. & $0.0002 \mathrm{mg} / 1$ & 1 & \\
\hline h & $0.005 \mathrm{mg} / \mathrm{l}$ & & $0.005 \mathrm{mz} / 1$ & & 1 & \\
\hline alminan $7 m 2-2$ & $<0.002 \mathrm{mg} / \mathrm{l}$ & $<0.002 \mathrm{mg} / \mathrm{l}$ & $<0.002 \mathrm{mg} / \mathrm{l}$ & $<0.002 \mathrm{mg} / \mathrm{h}$ & 1 & \\
\hline sum & $18 \mathrm{mg} / \mathrm{l}$ & $18 \mathrm{mg} / \mathrm{l}$ & $.18 \mathrm{mg} / 1$ & $18 \mathrm{mg} / 1$ & 1 & \\
\hline Surtionamens & $<0.05 \mathrm{mg} / \mathrm{l}$ & $<0.05 \mathrm{mg} / \mathrm{l}$ & $<0.05 \mathrm{mg} / 1$ & $<0.05 \mathrm{mg} / 1$ & 1 & \\
\hline 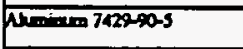 & $0.25 \mathrm{mg} / 1$ & $0.14 \mathrm{mg} / \mathrm{l}$ & $0.25 \mathrm{mg} / \mathrm{l}$ & $0.14 \mathrm{mg} / \mathrm{l}$ & 1 & \\
\hline $10 \log 740-32-2$ & $<0.04 \mathrm{mpll}$ & $<0.04$ mgll & $<0.04 \mathrm{mg} / \mathrm{l}$ & $<0.04$ mog/1 & 1 & \\
\hline 0074002 & $0.022 \mathrm{mpl} / 1$ & $0.020 \mathrm{mg} / 1$ & $0.002 \mathrm{mg} / 1$ & $0.020 \mathrm{mg} / \mathrm{l}$ & 1 & \\
\hline Borflimen 74001-7 & $<0.0004 \mathrm{~m} \& / 1$ & $<0.0004 \mathrm{mg} / \mathrm{l}$ & $<0.0004 \mathrm{mg} / \mathrm{I}$ & $<0.0004 \mathrm{mg} / \mathrm{l}$ & 1 & \\
\hline $20=740-139$ & $<0.004 \mathrm{mg} / \mathrm{l}$ & $<0.004 \mathrm{mg} / \mathrm{l}$ & $<0.004 \mathrm{mg} / \mathrm{l}$ & $<0.004 \mathrm{mg} / \mathrm{l}$ & 1 & \\
\hline andele 7400.70 .2 & $42.6 \mathrm{mgg} / 1$ & $45 \mathrm{mg} / \mathrm{l}$ & $42.6 \mathrm{mg} / \mathrm{l}$ & $45 \mathrm{mg} / \mathrm{l}$ & 1 & \\
\hline 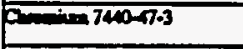 & $<0.006 \mathrm{mg} / \mathrm{l}$ & $<0.006 \mathrm{mg} / \mathrm{l}$ & $<0.006 \mathrm{mg} / \mathrm{h}$ & $<0.006 \mathrm{mg} / \mathrm{l}$ & 1 & \\
\hline 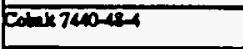 & $<0.002 \mathrm{mg} / \mathrm{l}$ & $<0.002 \mathrm{mg} / \mathrm{l}$ & $<0.002 \mathrm{mg} / \mathrm{l}$ & $<0.002 \mathrm{mg} / \mathrm{l}$ & 1 & \\
\hline Eamp 7400.502 & $<0.006 \mathrm{mg} / \mathrm{l}$ & $<0.006 \mathrm{mg} / \mathrm{l}$ & $<0.006 \mathrm{mg} / \mathrm{l}$ & $<0.006 \mathrm{mg} / \mathrm{l}$ & 1 & \\
\hline$\overline{70096}$ & $0.44 \mathrm{mg} / \mathrm{I}$ & $0.32 \mathrm{mg} / \mathrm{h}$ & $0.44 \mathrm{mg} / \mathrm{l}$ & $0.32 \mathrm{mg} / \mathrm{l}$ & 1 & \\
\hline 1704392,1 & $<0.02 \mathrm{mg} / \mathrm{l}$ & $<0.02 \mathrm{mg} / 1$ & $<0.02 \mathrm{mg} / \mathrm{l}$ & $<0.02 \mathrm{mg} / 1$ & 1 & \\
\hline 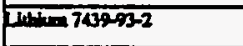 & $<0.02 \mathrm{mg} / 1$ & $<0.02 \mathrm{mg} / 1$ & $<0.02 \mathrm{mg} / \mathrm{l}$ & $<0.02 \mathrm{mg} / 1$ & 1 & \\
\hline 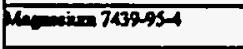 & $7.31 \mathrm{mg} / \mathrm{l}$ & $7.66 \mathrm{mg} / \mathrm{l}$ & $7.31 \mathrm{mg} / 1$ & $7.66 \mathrm{mg} / 1$ & 1 & \\
\hline 700965 & $0.154 \mathrm{mg} / 1$ & $0.134 \mathrm{mg} / \mathrm{l}$ & $0.154 \mathrm{mg} / \mathrm{h}$ & $0.134 \mathrm{mg} / \mathrm{l}$ & 1 & \\
\hline 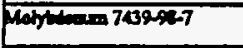 & $<0.006 \mathrm{mg} / \mathrm{l}$ & $<0.006 \mathrm{mg} / \mathrm{l}$ & $<0.006 \mathrm{mg} / 1$ & $<0.006 \mathrm{mg} / \mathrm{l}$ & 1 & \\
\hline 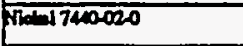 & $<0.003 \mathrm{mg} / 1$ & $<0.008 \mathrm{mg} / \mathrm{l}$ & $<0.008 \mathrm{mg} / \mathrm{l}$ & $<0.008 \mathrm{mg} / \mathrm{l}$ & 1 & \\
\hline 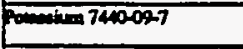 & $6.6 \mathrm{mg} / 1$ & $5.1 \mathrm{mg} / \mathrm{l}$ & $6.6 \mathrm{mg} / \mathrm{l}$ & $5.1 \mathrm{mg} / 1$ & 1 & \\
\hline (5) 7400-2at & $<0.006 \mathrm{mg} / \mathrm{l}$ & $<0.006 \mathrm{mg} / \mathrm{l}$ & $<0.006 \mathrm{mg} / \mathrm{l}$ & $<0.006 \mathrm{mg} / \mathrm{l}$ & 1 & \\
\hline sodion-7400235 & $2.72 \mathrm{mgll}$ & $3.07 \mathrm{mg} / \mathrm{l}$ & $2.72 \mathrm{mg} / 1$ & $3.07 \mathrm{mg} / 1$ & 1 & \\
\hline $7=740-2526$ & $<0.02 \mathrm{mg} / \mathrm{l}$ & $<0.02 \mathrm{mg} / 1$ & $<0.02 \mathrm{mg} / 1$ & $<0.02 \mathrm{mg} / 1$ & 1 & \\
\hline 20074006 & $0.01 \mathrm{mg} / 1$ & $<0.01 \mathrm{mg} / \mathrm{l}$ & $0.01 \mathrm{mg} / 1$ & $<0.01 \mathrm{mg} / \mathrm{l}$ & 1 & \\
\hline
\end{tabular}


Continend from front.

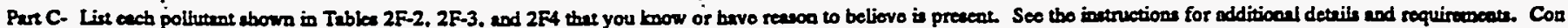
ose tublo for coch outill.

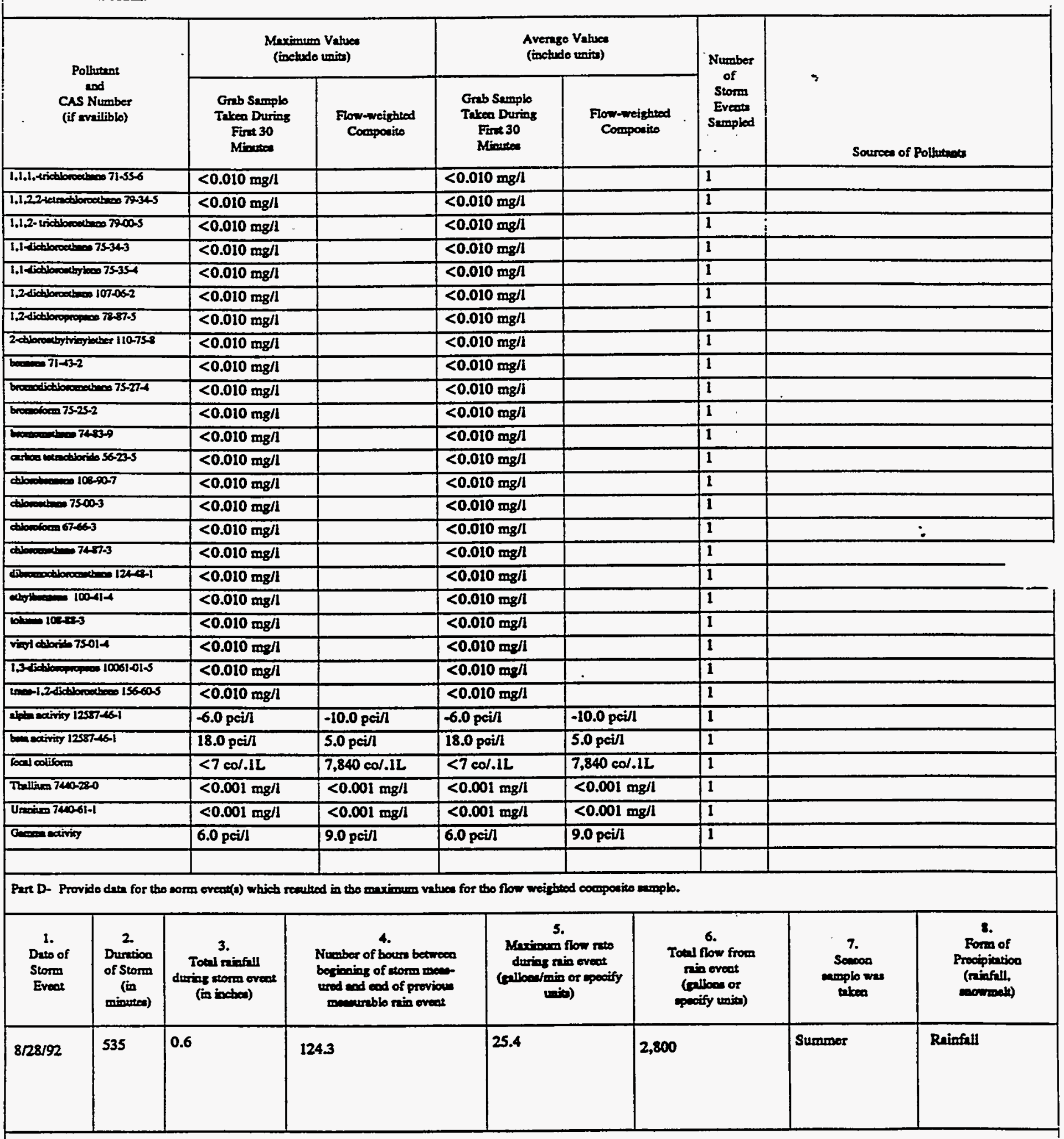

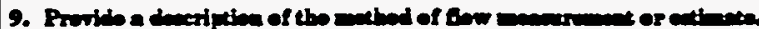


Outfall 3

Purte B and C Coatioused

\begin{tabular}{|c|c|c|c|c|c|c|}
\hline \multirow{2}{*}{$\begin{array}{l}\text { Pollunant } \\
\text { and } \\
\text { CAs Number } \\
\text { (if availible) }\end{array}$} & \multicolumn{2}{|c|}{$\begin{array}{l}\text { Marimanom Valuses } \\
\text { (inclede units) }\end{array}$} & \multicolumn{2}{|c|}{$\begin{array}{l}\text { Avernge Vihine } \\
\text { (inchude unios) }\end{array}$} & \multirow{2}{*}{$\begin{array}{c}\text { Number } \\
\text { of } \\
\text { storm } \\
\text { Evens } \\
\text { Sorspled }\end{array}$} & \multirow[b]{2}{*}{ Sourrose of Pollhomen } \\
\hline & $\begin{array}{l}\text { Onab Samplo } \\
\text { Taken During } \\
\text { Firs } 30 \\
\text { Minuteces }\end{array}$ & $\begin{array}{l}\text { Flow-wroighted } \\
\text { Cospocito }\end{array}$ & $\begin{array}{l}\text { Onab Semplo } \\
\text { Taka Durias } \\
\text { Firs } 30 \\
\text { Minume }\end{array}$ & $\begin{array}{c}\text { Flow-woighted } \\
\text { Componies }\end{array}$ & & \\
\hline Rerthen $740-32,3$ & $0.0466 \mathrm{mg} / 1$ & $0.0472 \mathrm{mg} / 1$ & $0.0466 \mathrm{~g} / 1$ & $0.0472 \mathrm{mg} / 1$ & 1 & \\
\hline 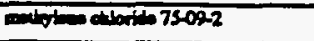 & $<0.010 \mathrm{mg} / \mathrm{l}$ & & $<0.010 \mathrm{mg} / 1$ & & 1 & \\
\hline 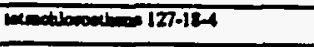 & $<0.010 \mathrm{mg} / \mathrm{l}$ & & $<0.010 \mathrm{mg} / \mathrm{l}$ & & 1 & \\
\hline 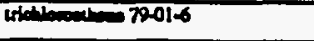 & $<0.010 \mathrm{mg} / \mathrm{l}$ & & $<0.010 \mathrm{mg} / 1$ & & 1 & \\
\hline Xylex & $<0.010 \mathrm{mg} / \mathrm{l}$ & & $<0.010 \mathrm{mg} / \mathrm{l}$ & & 1 & \\
\hline 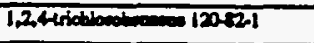 & $<0.010 \mathrm{mg} / \mathrm{l}$ & $<0.010 \mathrm{mg} / 1$ & $<0.010 \mathrm{mg} / \mathrm{l}$ & $<0.010 \mathrm{mg} / 1$ & 1 & \\
\hline 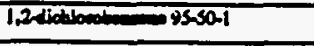 & $<0.010 \mathrm{mg} / \mathrm{l}$ & $<0.010 \mathrm{mg} / \mathrm{l}$ & $<0.010 \mathrm{mg} / \mathrm{l}$ & $<0.010 \mathrm{mg} / 1$ & 1 & \\
\hline 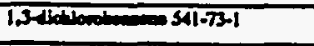 & $<0.010 \mathrm{mg} / 1$ & $<0.010 \mathrm{mg} / \mathrm{l}$ & $<0.010 \mathrm{mg} / 1$ & $<0.010 \mathrm{mg} / 1$ & 1 & \\
\hline 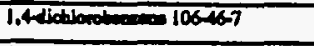 & $<0.010 \mathrm{mg} / \mathrm{l}$ & $<0.010 \mathrm{mg} / 1$ & $<0.010 \mathrm{mg} / \mathrm{h}$ & $<0.010 \mathrm{mg} / \mathrm{l}$ & 1 & \\
\hline 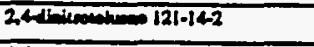 & $<0.010 \mathrm{mg} / \mathrm{l}$ & $<0.010 \mathrm{mg} / \mathrm{l}$ & $<0.010 \mathrm{mg} / \mathrm{l}$ & $<0.010 \mathrm{mg} / 1$ & 1 & \\
\hline 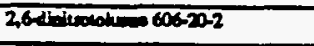 & $<0.010 \mathrm{mg} / \mathrm{l}$ & $<0.010 \mathrm{mg} / \mathrm{l}$ & $<0.010 \mathrm{mg} / \mathrm{l}$ & $<0.010 \mathrm{mg} / 1$ & 1 & - \\
\hline 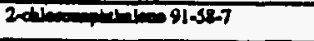 & $<0.010 \mathrm{mg} / \mathrm{l}$ & $<0.010 \mathrm{mg} / 1$ & $<0.010 \mathrm{mg} / \mathrm{l}$ & $<0.010 \mathrm{mg} / \mathrm{I}$ & 1 & \\
\hline 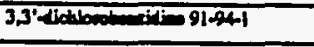 & $<0.010 \mathrm{mg} / \mathrm{l}$ & $<0.010 \mathrm{mg} / \mathrm{l}$ & $<0.010 \mathrm{mg} / \mathrm{l}$ & $<0.010 \mathrm{mg} / \mathrm{l}$ & 1 & \\
\hline 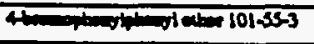 & $<0.010 \mathrm{mg} / \mathrm{l}$ & $<0.010 \mathrm{mg} / \mathrm{l}$ & $<0.010 \mathrm{mg} / \mathrm{l}$ & $<0.010 \mathrm{mg} / 1$ & 1 & \\
\hline 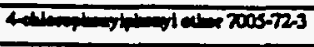 & $<0.010 \mathrm{mg} / 1$ & $<0.010 \mathrm{mg} / \mathrm{l}$ & $<0.010 \mathrm{mg} / \mathrm{h}$ & $<0.010 \mathrm{mg} / 1$ & 1 & \\
\hline $20=123-329$ & $<0.010 \mathrm{mg} / 1$ & $<0.010 \mathrm{mg} / \mathrm{l}$ & $<0.010 \mathrm{mg} / \mathrm{l}$ & $<0.010 \mathrm{mg} / 1$ & 1 & \\
\hline 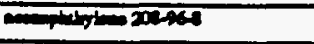 & $<0.010 \mathrm{mg} / \mathrm{h}$ & $<0.010 \mathrm{mg} / \mathrm{h}$ & $<0.010 \mathrm{mg} / 1$ & $<0.010 \mathrm{mg} / 1$ & 1 & \\
\hline 120,127 & $<0.010 \mathrm{mg} / \mathrm{l}$ & $<0.010 \mathrm{mg} / 1$ & $<0.010 \mathrm{mg} / \mathrm{h}$ & $<0.010 \mathrm{mg} / 1$ & 1 & \\
\hline midi-92075 & $<0.030 \mathrm{mg} / \mathrm{l}$ & $<0.030 \mathrm{mg} / 1$ & $<0.030 \mathrm{mg} / \mathrm{l}$ & $<0.030 \mathrm{mg} / 1$ & 1 & $=$ \\
\hline 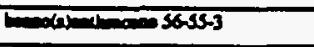 & $<0.010 \mathrm{mg} / \mathrm{l}$ & $<0.010 \mathrm{mg} / 1$ & $<0.010 \mathrm{mg} / \mathrm{l}$ & $<0.010 \mathrm{mg} / \mathrm{l}$ & 1 & \\
\hline$=502325$ & $<0.010 \mathrm{me} / \mathrm{l}$ & $<0.010 \mathrm{me} / \mathrm{l}$ & $<0.010 \mathrm{mg} / 1$ & $<0.010 \mathrm{mg} / 1$ & 1 & \\
\hline 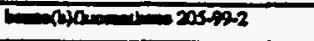 & $<0.010 \mathrm{mg} / \mathrm{l}$ & $<0.010 \mathrm{mg} / \mathrm{l}$ & $<0.010 \mathrm{mg} / 1$ & $<0.010 \mathrm{mg} / 1$ & 1 & \\
\hline 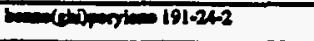 & $<0.010 \mathrm{mg} / \mathrm{l}$ & $<0.010 \mathrm{mg} / 1$ & $<0.010 \mathrm{mg} / 1$ & $<0.010 \mathrm{mg} / \mathrm{l}$ & 1 & \\
\hline 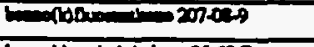 & $<0.010 \mathrm{mg} / 1$ & $<0.010 \mathrm{mg} / 1$ & $<0.010 \mathrm{mg} / 1$ & $<0.010 \mathrm{mg} / 1$ & 1 & \\
\hline 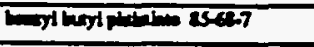 & $<0.010 \mathrm{mg} / \mathrm{l}$ & $<0.010 \mathrm{mg} / \mathrm{l}$ & $<0.010 m, h$ & $<0.010 \mathrm{mg} / \mathrm{l}$ & 1 & \\
\hline 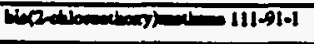 & $<0.010 \mathrm{mg} / \mathrm{ll}$ & $<0.010 \mathrm{mg} / 1$ & $<0.010 \mathrm{mg} / 1$ & $<0.010 \mathrm{mg} / 1$ & 1 & \\
\hline 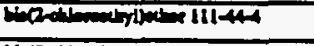 & $<0.010 \mathrm{mg} / 1$ & $<0.010 \mathrm{mg} / \mathrm{l}$ & $<0.010 \mathrm{mg} / 1$ & $<0.010 \mathrm{mg} / \mathrm{h}$ & 1 & \\
\hline 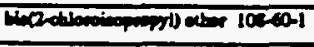 & $<0.010 \mathrm{mg} / \mathrm{l}$ & $<0.010 \mathrm{mg} / \mathrm{l}$ & $<0.010 \mathrm{mg} / \mathrm{l}$ & $<0.010 \mathrm{mg} / \mathrm{l}$ & 1 & \\
\hline 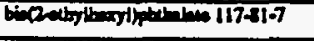 & $<0.010 \mathrm{mg} / \mathrm{l}$ & $<0.010 \mathrm{mg} / \mathrm{l}$ & $<0.010 \mathrm{mg} / 1$ & $<0.010 \mathrm{mg} / 1$ & 1 & \\
\hline dormen-21201न & $<0.010 \mathrm{mg} / \mathrm{l}$ & $<0.010 \mathrm{mg} / \mathrm{l}$ & $<0.010 \mathrm{mg} / \mathrm{l}$ & $<0.010 \mathrm{mg} / 1$ & 1 & \\
\hline 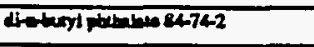 & $<0.010 \mathrm{mg} / \mathrm{l}$ & $<0.010 \mathrm{mg} / \mathrm{l}$ & $<0.010 \mathrm{mg} / \mathrm{l}$ & $<0.010 \mathrm{mg} / \mathrm{l}$ & 1 & \\
\hline 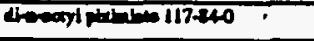 & $<0.010 \mathrm{mg} / \mathrm{l}$ & $<0.010 \mathrm{mg} / \mathrm{h}$ & $<0.010 \mathrm{mg} / 1$ & $<0.010 \mathrm{mg} / 1$ & 1 & \\
\hline 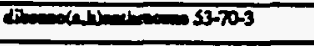 & $<0.010 \mathrm{mg} / \mathrm{l}$ & $<0.010 \mathrm{mg} / \mathrm{l}$ & $<0.010 \mathrm{mg} / \mathrm{l}$ & $<0.010 \mathrm{mg} / \mathrm{l}$ & 1 & \\
\hline 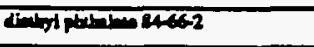 & $<0.010 \mathrm{mg} / 1$ & $<0.010 \mathrm{mg} / \mathrm{l}$ & $<0.010 \mathrm{mg} / \mathrm{l}$ & $<0.010 \mathrm{mg} / \mathrm{l}$ & 1 & \\
\hline 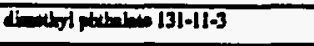 & $<0.010 \mathrm{mg} / \mathrm{l}$ & $<0.010 \mathrm{~m} / \mathrm{l}$ & $<0.010 \mathrm{mg} / 1$ & $<0.010 \mathrm{mg} / 1$ & 1 & \\
\hline 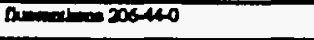 & $<0.010 \mathrm{mg} / \mathrm{l}$ & $<0.010 \mathrm{mg} / 1$ & $<0.010 \mathrm{mg} / 1$ & $<0.010 \mathrm{mg} / \mathrm{l}$ & 1 & \\
\hline $0=26-73-7$ & $<0.010 \mathrm{mg} / \mathrm{l}$ & $<0.010 \mathrm{mg} / 1$ & $<0.010 \mathrm{mg} / 1$ & $<0.010 \mathrm{mg} / \mathrm{l}$ & 1 & \\
\hline 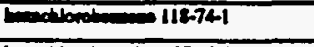 & $<0.010 \mathrm{mg} / 1$ & $<0.010 \mathrm{mg} / \mathrm{l}$ & $<0.010 \mathrm{mg} / \mathrm{l}$ & $<0.010 \mathrm{mg} / \mathrm{l}$ & 1 & \\
\hline 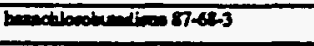 & $<0.010 \mathrm{mg} / \mathrm{l}$ & $<0.010 \mathrm{mg} / \mathrm{l}$ & $<0.010 \mathrm{mg} / 1$ & $<0.010 \mathrm{mg} / \mathrm{l}$ & 1 & \\
\hline 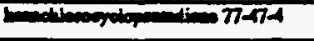 & $<0.010 \mathrm{mg} / 1$ & $<0.010 \mathrm{mg} / 1$ & $<0.010 \mathrm{mg} / 1$ & $<0.010 \mathrm{mg} / 1$ & 1 & \\
\hline $7=0.721$ & $<0.010 \mathrm{mg} / \mathrm{l}$ & $<0.010 \mathrm{mg} / \mathrm{l}$ & $<0.010 \mathrm{mg} / 1$ & $<0.010 \mathrm{mg} / 1$ & 1 & \\
\hline 193.39 .5 & $<0.010 \mathrm{mg} / 1$ & $<0.010 \mathrm{mg} / 1$ & $<0.010 \mathrm{mg} / \mathrm{l}$ & $<0.010 \mathrm{mg} / \mathrm{l}$ & 1 & \\
\hline 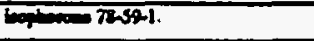 & $<0.010 \mathrm{mg} / \mathrm{l}$ & $<0.010 \mathrm{mg} / \mathrm{l}$ & $<0.010 \mathrm{mg} / \mathrm{l}$ & $<0.010 \mathrm{mg} / \mathrm{l}$ & 1 & \\
\hline 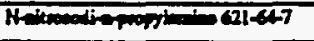 & $<0.010 \mathrm{mg} / \mathrm{l}$ & $<0.010 \mathrm{mg} / 1$ & $<0.010 \mathrm{mg} / 1$ & $<0.010 \mathrm{mg} / 1$ & 1 & \\
\hline 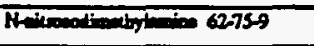 & $<0.010 \mathrm{mg} / 1$ & $<0.010 \mathrm{mg} / 1$ & $<0.010 \mathrm{mg} / 1$ & $<0.010 \mathrm{mg} / \mathrm{l}$ & 1 & \\
\hline 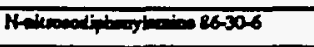 & $<0.010 \mathrm{mg} / \mathrm{l}$ & $<0.010 \mathrm{mg} / \mathrm{l}$ & $<0.010 \mathrm{mg} / 1$ & $<0.010 \mathrm{mg} / \mathrm{l}$ & 1 & \\
\hline 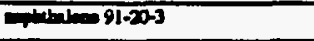 & $<0.010 \mathrm{mg} / \mathrm{l}$ & $<0.010 \mathrm{mg} / \mathrm{l}$ & $<0.010 \mathrm{mg} / 1$ & $<0.010 \mathrm{mg} / 1$ & 1 & \\
\hline 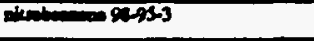 & $<0.910 \mathrm{mg} / 1$ & $<0.010 \mathrm{mg} / \mathrm{l}$ & $<0.010 \mathrm{mg} / \mathrm{l}$ & $<0.010 \mathrm{mg} / \mathrm{l}$ & 1 & \\
\hline 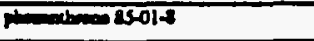 & $<0.010 \mathrm{mg} / 1$ & $<0.010 \mathrm{mg} / 1$ & $<0.010 \mathrm{mg} / 1$ & $<0.010 \mathrm{mg} / 1$ & 1 & \\
\hline $50=129000$ & $<0.010 \mathrm{mg} / 1$ & $<0.010 \mathrm{mg} / 1$ & $<0.010 \mathrm{mg} / 1$ & $<0.010 \mathrm{mg} h$ & 1 & \\
\hline 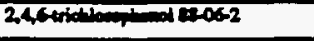 & $<0.030 \mathrm{mg} / 1$ & $<0.030 \mathrm{mgll}$ & $<0.030 \mathrm{mg} / 1$ & $<0.030 \mathrm{mg} / 1$ & 1 & \\
\hline 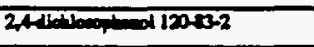 & $<0.030 \mathrm{mg} / \mathrm{l}$ & $<0.030 \mathrm{mg} / \mathrm{l}$ & $<0.030 \mathrm{mg} / \mathrm{l}$ & $<0.030 \mathrm{mg} / 1$ & 1 & \\
\hline 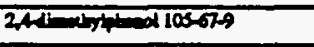 & $<0.030 \mathrm{mg} / \mathrm{l}$ & $<0.030 \mathrm{mg} / \mathrm{h}$ & $<0.030 \mathrm{mg} / \mathrm{l}$ & $<0.030 \mathrm{mg} / \mathrm{l}$ & 1 & \\
\hline 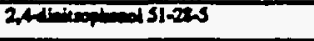 & $<0.030 \mathrm{mg} / \mathrm{l}$ & $<0.030 \mathrm{mg} / \mathrm{l}$ & $<0.030 \mathrm{mg} / 1$ & $<0.030 \mathrm{mg} / \mathrm{l}$ & 1 & \\
\hline
\end{tabular}


Outfall 3

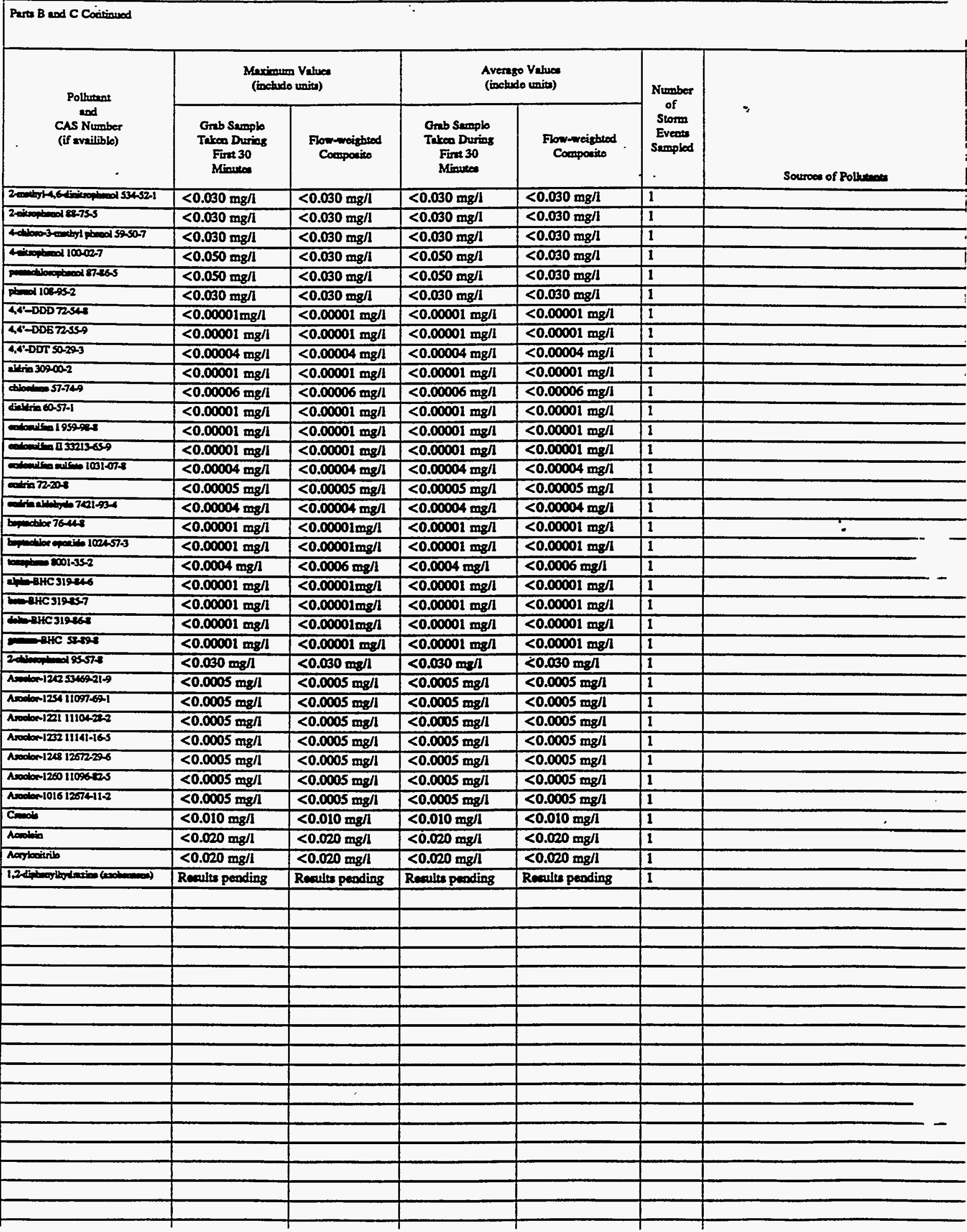


VII. Dischurge Loformation (cootinued froes page 3 of Form $2 F$ )

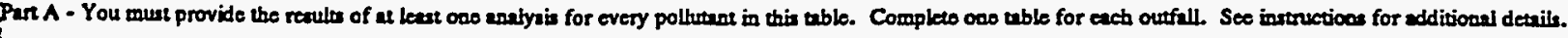

\begin{tabular}{|c|c|c|c|c|c|c|}
\hline \multirow{2}{*}{$\begin{array}{l}\text { Pollunent } \\
\text { and } \\
\text { CAS Number } \\
\text { (if avnilible) }\end{array}$} & \multicolumn{2}{|c|}{ 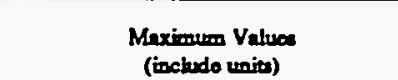 } & \multicolumn{2}{|c|}{$\begin{array}{l}\text { Averase Valuce } \\
\text { (includo uniza) }\end{array}$} & \multirow{2}{*}{$\begin{array}{l}\text { Number } \\
\text { of } \\
\text { Storm } \\
\text { Eveoses } \\
\text { Sempled }\end{array}$} & \multirow[b]{2}{*}{ Soureces of Pollutanses } \\
\hline & $\begin{array}{l}\text { Gab Semplo } \\
\text { Taken During } \\
\text { Firse } 30 \\
\text { Minutes }\end{array}$ & $\begin{array}{l}\text { Flow-weighted } \\
\text { Compocito }\end{array}$ & $\begin{array}{c}\text { Grab Semple } \\
\text { Takeo During } \\
\text { Fins } 30 \\
\text { Minuece }\end{array}$ & $\begin{array}{l}\text { Flow weighted } \\
\text { Compocito }\end{array}$ & & \\
\hline pil mad Gomen & $7 \mathrm{mg} / \mathrm{l}$ & & $7 \mathrm{mg} / \mathrm{l}$ & & 1 & \\
\hline 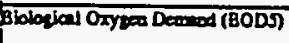 & $55.1 \mathrm{mg} / \mathrm{l}$ & $31.4 \mathrm{mg} / \mathrm{l}$ & $55.1 \mathrm{mg} / \mathrm{l}$ & $31.4 \mathrm{mg} / \mathrm{l}$ & I & \\
\hline Cremical Oxylar Doond (COD) & $150 \mathrm{mg} / 1$ & $61 \mathrm{mg} / \mathrm{l}$ & $150 \mathrm{mg} / 1$ & $61 \mathrm{mg} / 1$ & 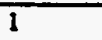 & \\
\hline Troul Sarpoded salide (TSS) & $150 \mathrm{mg} / \mathrm{I}$ & $20 \mathrm{mg} / \mathrm{l}$ & $150 \mathrm{mg} / 1$ & $20 \mathrm{mg} / \mathrm{l}$ & 1 & \\
\hline Toul Kjothat Nitroses & $1 \mathrm{mg} / \mathrm{l}$ & $2 \mathrm{mg} / \mathrm{l}$ & $1 \mathrm{mg} / \mathrm{l}$ & $2 \mathrm{mg} / \mathrm{l}$ & 1 & \\
\hline 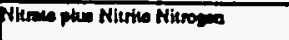 & $1.52 \mathrm{mg} / \mathrm{l}$ & $1.13 \mathrm{mg} / \mathrm{l}$ & $1.52 \mathrm{mg} / \mathrm{t}$ & $1.13 \mathrm{mg} / \mathrm{l}$ & 1 & \\
\hline Toual Phoophorm & $0.2 \mathrm{mg} / \mathrm{l}$ & $0.8 \mathrm{mg} / \mathrm{l}$ & $0.2 \mathrm{mg} / \mathrm{l}$ & $0.8 \mathrm{mg} / \mathrm{l}$ & 1 & \\
\hline PH & Minim & Meriman 78 & Mi:ines 78 & Marimen & & \\
\hline
\end{tabular}

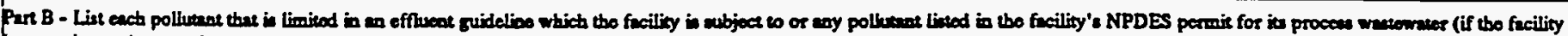

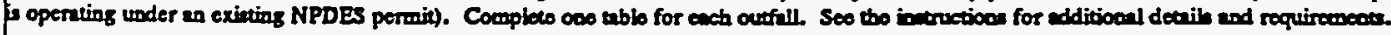

\begin{tabular}{|c|c|c|c|c|c|c|c|}
\hline \multirow{2}{*}{$\begin{array}{l}\text { Pollutent } \\
\text { and } \\
\text { CAs Nurmber } \\
\text { (if availible) }\end{array}$} & \multirow[b]{2}{*}{1} & \multicolumn{2}{|c|}{ 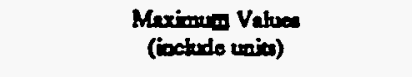 } & \multicolumn{2}{|c|}{ 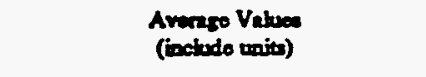 } & \multirow{2}{*}{$\begin{array}{c}\text { Numbor } \\
\text { of } \\
\text { Storm } \\
\text { Eveots } \\
\text { Serspled }\end{array}$} & \multirow{2}{*}{$\begin{array}{c}: \\
\text { Sources of Polleweses }\end{array}$} \\
\hline & & $\begin{array}{l}\text { Gab Sampib } \\
\text { Taken Duries } \\
\text { Firs } 30 \\
\text { Misedes }\end{array}$ & $\begin{array}{l}\text { Flow-weightod } \\
\text { Compocico }\end{array}$ & 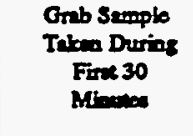 & $\begin{array}{l}\text { Fonmoighted } \\
\text { Compocito }\end{array}$ & & \\
\hline $7400-360$ & & $<0.04 \mathrm{mg} / \mathrm{l}$ & $<0.04 \mathrm{mg} / \mathrm{l}$ & $<0.04 \mathrm{mg} / \mathrm{l}$ & $<0.04 \mathrm{mg} / 1$ & 1 & \\
\hline sris & & $0.002 \mathrm{mg} / \mathrm{l}$ & & $0.002 \mathrm{mg} / \mathrm{l}$ & & 1 & \\
\hline Merary 74329016 & & $0.0003 \mathrm{mg} / \mathrm{l}$ & $<0.0002 \mathrm{mg} / \mathrm{l}$ & $0.0003 \mathrm{mg} / \mathrm{l}$ & $<0.0002 \mathrm{mg} / 1$ & 1 & \\
\hline Procole & & $0.009 \mathrm{mg} / \mathrm{l}$ & & $0.009 \mathrm{mg} / \mathrm{l}$ & & 1 & \\
\hline 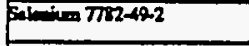 & & $<0.002 \mathrm{mg} / \mathrm{l}$ & $<0.002 \mathrm{mg} / \mathrm{l}$ & $<0.002 \mathrm{mg} / \mathrm{l}$ & $<0.002 \mathrm{mg} / \mathrm{l}$ & 1 & \\
\hline Pillan & & $26 \mathrm{mg} / 1$ & $27 \mathrm{mg} / \mathrm{l}$ & $26 \mathrm{mg} / \mathrm{t}$ & $27 \mathrm{mg} / \mathrm{l}$ & 1 & \\
\hline Purteraves & & $0.20 \mathrm{mg} / \mathrm{l}$ & $<0.05 \mathrm{mg} / \mathrm{l}$ & $0.20 \mathrm{mg} / 1$ & $<0.05 \mathrm{mg} / 1$ & 1 & \\
\hline Numinm 742900.5 & & $1.98 \mathrm{mg} / \mathrm{l}$ & $0.85 \mathrm{mg} / \mathrm{l}$ & $1.98 \mathrm{mg} / \mathrm{I}$ & $0.85 \mathrm{mg} / \mathrm{l}$ & 1 & \\
\hline $100007460-35-2$ & & $<0.04 \mathrm{mg} / \mathrm{l}$ & $<0.04 \mathrm{mg} / \mathrm{l}$ & $<0.04 \mathrm{mg} / \mathrm{l}$ & $<0.04 \mathrm{mg} / \mathrm{l}$ & 1 & \\
\hline $30000740-129$ & & $0.022 \mathrm{mg} / \mathrm{l}$ & $0.036 \mathrm{mg} / \mathrm{l}$ & $0.022 \mathrm{mg} / \mathrm{l}$ & $0.036 \mathrm{mg} / \mathrm{l}$ & 1 & \\
\hline Benglingon 7400-1.7 & & $<0.0004 \mathrm{mg} / \mathrm{l}$ & $<0.0004 \mathrm{mg} / \mathrm{l}$ & $<0.0004 \mathrm{mg} / 1$ & $<0.0004 \mathrm{mg} / \mathrm{l}$ & 1 & \\
\hline 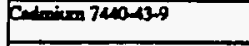 & & $<0.004 \mathrm{mg} / \mathrm{l}$ & $0.053 \mathrm{mg} / \mathrm{l}$ & $<0.004 \mathrm{mg} / \mathrm{l}$ & $0.053 \mathrm{mg} / 1$ & 1 & \\
\hline 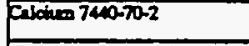 & & $24.0 \mathrm{mg} / \mathrm{l}$ & $27.7 \mathrm{mg} / \mathrm{l}$ & $24.0 \mathrm{mg} / \mathrm{l}$ & $27.7 \mathrm{mg} / \mathrm{l}$ & 1 & \\
\hline Droming $7400-7.3$ & & $0.006 \mathrm{mg} / \mathrm{l}$ & $<0.006 \mathrm{mg} / \mathrm{l}$ & $0.006 \mathrm{mg} / \mathrm{h}$ & $<0.006 \mathrm{mg} / \mathrm{l}$ & 1 & \\
\hline $\cos 2 \times 740004$ & & $0.003 \mathrm{mg} / \mathrm{l}$ & $<0.002 \mathrm{mg} / \mathrm{l}$ & $0.003 \mathrm{mg} / \mathrm{h}$ & $<0.002 \mathrm{mg} / 1$ & 1 & \\
\hline Carper 740a-sos & & $0.016 \mathrm{mg} / \mathrm{l}$ & $0.007 \mathrm{mg} / \mathrm{l}$ & $0.016 \mathrm{mg} / \mathrm{l}$ & $0.007 \mathrm{mg} / \mathrm{l}$ & 1 & \\
\hline 100703596 & & $3.58 \mathrm{mg} / \mathrm{l}$ & $1.00 \mathrm{mg} / 1$ & $3.58 \mathrm{mg} / 1$ & $1.00 \mathrm{mg} / \mathrm{I}$ & 1 & \\
\hline Ind 7432021 & & $0.03 \mathrm{mg} / 1$ & $<0.02 \mathrm{mg} / \mathrm{l}$ & $0.03 \mathrm{mg} / 1$ & $<0.02 \mathrm{mg} / \mathrm{l}$ & 1 & \\
\hline Tibiuon 7439-93-2 & & $<0.02 \mathrm{mg} / \mathrm{l}$ & $<0.02 \mathrm{mg} / \mathrm{l}$ & $<0.02 \mathrm{mg} / 1$ & $<0.02 \mathrm{mg} / \mathrm{l}$ & 1 & \\
\hline M1200010 7439-934 & & $3.91 \mathrm{mg} / 1$ & $3.35 \mathrm{mg} / 1$ & $3.91 \mathrm{mg} / \mathrm{l}$ & $3.35 \mathrm{mg} / 1$ & 1 & \\
\hline 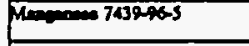 & & $0.531 \mathrm{mg} / \mathrm{l}$ & $0.058 \mathrm{mg} / \mathrm{l}$ & $0.531 \mathrm{mg} / 1$ & $0.058 \mathrm{mg} / \mathrm{l}$ & 1 & \\
\hline 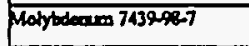 & & $<0.006 \mathrm{mg} / \mathrm{l}$ & $<0.006 \mathrm{mg} / \mathrm{l}$ & $<0.006 \mathrm{mg} / \mathrm{l}$ & $<0.006 \mathrm{mg} / \mathrm{l}$ & 1 & \\
\hline 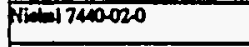 & & $0.014 \mathrm{mg} / \mathrm{l}$ & $<0.008 \mathrm{mg} / \mathrm{l}$ & $0.014 \mathrm{mg} / \mathrm{l}$ & $<0.008 \mathrm{mg} / \mathrm{l}$ & 1 & \\
\hline Poncinom 740097 & & $2.6 \mathrm{mg} / 1$ & $7.8 \mathrm{mg} / \mathrm{l}$ & $2.6 \mathrm{mg} / \mathrm{l}$ & $7.8 \mathrm{mg} / 1$ & 1 & \\
\hline siven 7400-20- & & $<0.006 \mathrm{mg} / \mathrm{l}$ & $<0.006 \mathrm{mg} / \mathrm{l}$ & $<0.006 \mathrm{mg} / \mathrm{l}$ & $<0.006 \mathrm{mg} / 1$ & 1 & \\
\hline $7400-23-5$ & & $1.68 \mathrm{mg} / 1$ & $3.04 \mathrm{mg} / \mathrm{l}$ & $1.68 \mathrm{mg} / \mathrm{l}$ & $3.04 \mathrm{mg} / \mathrm{l}$ & 1 & \\
\hline Trancon 740-32-6 & & $0.03 \mathrm{mg} / 1$ & $<0.01 \mathrm{mg} / \mathrm{l}$ & $0.03 \mathrm{mg} / 1$ & $<0.01 \mathrm{mg} / 1$ & 1 & \\
\hline $2 \ln 740-66$ & & $0.21 \mathrm{mg} / \mathrm{l}$ & $0.05 \mathrm{mg} / 1$ & $0.21 \mathrm{mg} / \mathrm{l}$ & $0.05 \mathrm{mg} / \mathrm{l}$ & 1 & \\
\hline
\end{tabular}


Contenoud froen frope.

\begin{tabular}{|c|c|c|c|c|c|c|}
\hline \multirow{2}{*}{$\begin{array}{l}\text { Pollurunt } \\
\text { and } \\
\text { CAS Number } \\
\text { (if availible) }\end{array}$} & \multicolumn{2}{|c|}{$\begin{array}{l}\text { Maximusm Valuea } \\
\text { (inchude units) }\end{array}$} & \multicolumn{2}{|c|}{$\begin{array}{l}\text { Average Values } \\
\text { (include unitu) }\end{array}$} & \multirow{2}{*}{$\begin{array}{c}\text { Number } \\
\text { of } \\
\text { Storm } \\
\text { Evenses } \\
\text { Sempled }\end{array}$} & \multirow[b]{2}{*}{ Sources of Pollutunts } \\
\hline & $\begin{array}{c}\text { Grab Sumple } \\
\text { Taken During } \\
\text { Firnt } 30 \\
\text { Minurea }\end{array}$ & $\begin{array}{l}\text { Flow-weigbted } \\
\text { Comporite }\end{array}$ & $\begin{array}{l}\text { Grab Semple } \\
\text { Taken During } \\
\text { Firn } 30 \\
\text { Minutes }\end{array}$ & $\begin{array}{l}\text { Flow-weighted } \\
\text { Compotite }\end{array}$ & & \\
\hline 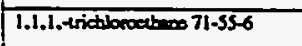 & $<0.010 \mathrm{mg} / \mathrm{l}$ & & $<0.010 \mathrm{mg} / \mathrm{l}$ & & 1 & \\
\hline 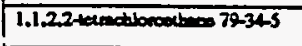 & $<0.010 \mathrm{mg} / \mathrm{l}$ & & $<0.010 \mathrm{mg} / \mathrm{l}$ & & 1 & \\
\hline 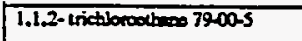 & $<0.010 \mathrm{mg} / \mathrm{l}$ & & $<0.010 \mathrm{mg} / \mathrm{l}$ & & 1 & \\
\hline 1,1-dichloroutberes 75-34-3 & $<0.010 \mathrm{mg} / \mathrm{l}$ & & $<0.010 \mathrm{mg} / \mathrm{l}$ & & 1 & \\
\hline 1.1-dicthorectylibeo 75-35-4 & $<0.010 \mathrm{mg} / 1$ & & $<0.010 \mathrm{mg} / 1$ & & 1 & \\
\hline 1.2-dictibroction $107.06-2$ & $<0.010 \mathrm{mg} / \mathrm{l}$ & & $<0.010 \mathrm{mg} / 1$ & & 1 & \\
\hline 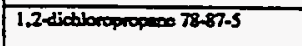 & $<0.010 \mathrm{mg} / 1$ & & $<0.010 \mathrm{mg} / \mathrm{l}$ & & 1 & \\
\hline 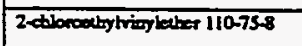 & $<0.010 \mathrm{mg} / \mathrm{l}$ & & $<0.010 \mathrm{mg} / \mathrm{l}$ & & 1 & \\
\hline $71<3-2$ & $<0.010 \mathrm{mg} / \mathrm{l}$ & & $<0.010 \mathrm{mg} / \mathrm{l}$ & & 1 & \\
\hline 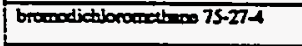 & $<0.010 \mathrm{mg} / \mathrm{l}$ & & $<0.010 \mathrm{mg} / \mathrm{l}$ & & 1 & \\
\hline broentoom 75-25-2 & $<0.010 \mathrm{mg} / \mathrm{l}$ & & $<0.010 \mathrm{mg} / \mathrm{l}$ & & 1 & \\
\hline bromonecteos 74839 & $<0.010 \mathrm{mg} / 1$ & & $<0.010 \mathrm{mg} / \mathrm{l}$ & & 1 & \\
\hline artoon lanactioride 56.23 .5 & $<0.010 \mathrm{mg} / \mathrm{l}$ & & $<0.010 \mathrm{mg} / \mathrm{l}$ & & 1 & \\
\hline \begin{tabular}{|l|l} 
chlog-90-7 & $108-7$
\end{tabular} & $<0.010 \mathrm{mg} / \mathrm{l}$ & & $<0.010 \mathrm{mg} / \mathrm{l}$ & & 1 & \\
\hline abouculberen $75-00-3$ & $<0.010 \mathrm{mg} / 1$ & & $<0.010 \mathrm{mg} / \mathrm{l}$ & & 1 & \\
\hline ctboroform $6766-3$ & $<0.010 \mathrm{mg} / \mathrm{l}$ & & $<0.010 \mathrm{mg} / \mathrm{l}$ & & 1 & $\because$ \\
\hline 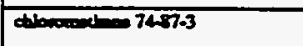 & $<0.010 \mathrm{mg} / \mathrm{l}$ & & $<0.010 \mathrm{mg} / \mathrm{l}$ & & 1 & \\
\hline 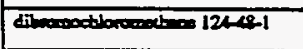 & $<0.010 \mathrm{mg} / \mathrm{l}$ & & $<0.010 \mathrm{mg} / \mathrm{l}$ & & 1 & \\
\hline 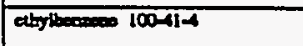 & $<0.010 \mathrm{mg} / \mathrm{l}$ & & $<0.010 \mathrm{mg} / \mathrm{l}$ & & 1 & \\
\hline 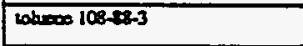 & $<0.010 \mathrm{mg} / \mathrm{l}$ & & $<0.010 \mathrm{mg} / 1$ & & 1 & \\
\hline vingl ctibride 75-014 & $<0.010 \mathrm{mg} / \mathrm{l}$ & & $<0.010 \mathrm{mg} / \mathrm{l}$ & & 1 & \\
\hline 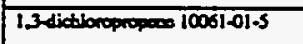 & $<0.010 \mathrm{mg} / \mathrm{l}$ & & $<0.010 \mathrm{mg} / \mathrm{l}$ & & 1 & \\
\hline 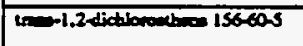 & $<0.010 \mathrm{mg} / 1$ & & $<0.010 \mathrm{mg} / 1$ & & 1 & \\
\hline 21pheativity $1258746-1$ & $2.5 \mathrm{pci} / \mathrm{l}$ & $1.9 \mathrm{pci} / \mathrm{l}$ & $2.5 \mathrm{pei} / \mathrm{l}$ & $1.9 \mathrm{pei} / 1$ & 1 & . \\
\hline $12587-46-1$ & 18 peill & $11 \mathrm{pci} / 1$ & $18 \mathrm{pci} / 1$ & 11 peill & 1 & \\
\hline fool colitions & $25,000 \mathrm{co} / .1 \mathrm{~L}$ & $<1 \mathrm{col} .1 \mathrm{~L}$ & $25,000 \mathrm{co} / .1 \mathrm{~L}$ & $<1$ col.1L & 1 & \\
\hline Thallines $7440-250$ & $<0.001 \mathrm{mg} / \mathrm{l}$ & $<0.001 \mathrm{mg} h$ & $<0.001 \mathrm{mg} / \mathrm{l}$ & $<0.001 \mathrm{mg} / \mathrm{l}$ & 1 & \\
\hline Unasisen 740061-1 & $<0.001 \mathrm{mg} / 1$ & $0.002 \mathrm{mg} / \mathrm{l}$ & $<0.001 \mathrm{mg} / \mathrm{l}$ & $0.002 \mathrm{mg} / \mathrm{L}$ & 1 & \\
\hline Gompenesivis & 61 peill & $56 \mathrm{pei} / 1$ & $61 \mathrm{peil}$ & 56 pcill & 1 & \\
\hline
\end{tabular}

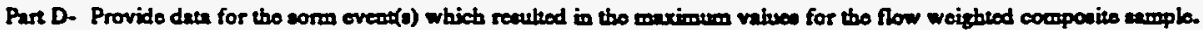

\begin{tabular}{|c|c|c|c|c|c|c|c|}
\hline $\begin{array}{l}1 . \\
\text { Date of } \\
\text { Storm } \\
\text { Eveal }\end{array}$ & $\begin{array}{l}2 . \\
\text { Duration } \\
\text { of Storm } \\
\text { (in } \\
\text { minutea) }\end{array}$ & $\begin{array}{l}3 . \\
\text { Toul rinfill } \\
\text { durieg worm ovent } \\
\text { (in inctea) }\end{array}$ & 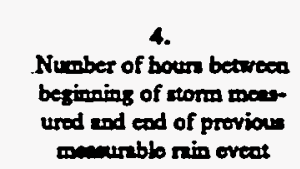 & $\begin{array}{l}\text { S. } \\
\text { Maximum flow mito } \\
\text { during nin cveat } \\
\text { (allong/min or specify } \\
\text { unitu) }\end{array}$ & $\begin{array}{l}6 . \\
\text { Total flow from } \\
\text { min ovent } \\
\text { (ealloes or } \\
\text { spocify units) }\end{array}$ & $\begin{array}{l}7 . \\
\text { Semon } \\
\text { anmplo was } \\
\text { tuken }\end{array}$ & $\begin{array}{l}8 . \\
\text { Form of } \\
\text { Precipitution } \\
\text { (nimfill. } \\
\text { anderell) }\end{array}$ \\
\hline $7 / 24 / 91$ & 185 & 0.8 & 154.2 & 5,082 & 230,851 & Summer & Rainfall \\
\hline
\end{tabular}

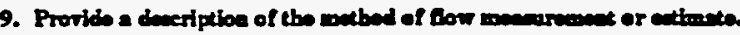

An ISCO 3230 Flow meter was used to obtain water level readings. This instrument was catibrated with the normal dry weather Ilow, if present, as level zero. The flow meter was programmed with the characteristios of the conveyance so that flow rates and total flows conid be calculnted by the meter. 
Outfoll 4

Parte B and C Continued

\begin{tabular}{|c|c|c|c|c|c|c|}
\hline \multirow{2}{*}{$\begin{array}{l}\text { Poltutual } \\
\text { ood } \\
\text { CAS Number } \\
\text { (if aveiliblo) }\end{array}$} & \multicolumn{2}{|c|}{$\begin{array}{l}\text { Meximum Values } \\
\text { (ivectude units) }\end{array}$} & \multicolumn{2}{|c|}{$\begin{array}{l}\text { Avernge Values } \\
\text { (inchedo units) }\end{array}$} & \multirow{2}{*}{$\begin{array}{l}\text { Number } \\
\text { of } \\
\text { Stormen } \\
\text { Eveses } \\
\text { Sempled }\end{array}$} & \multirow[b]{2}{*}{ Sourcee of Polbutents } \\
\hline & 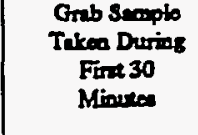 & $\begin{array}{l}\text { Flow-meiztitod } \\
\text { Compoutice }\end{array}$ & $\begin{array}{l}\text { Grab Sempio } \\
\text { Taken During } \\
\text { Fint } 30 \\
\text { Minute }\end{array}$ & $\begin{array}{l}\text { Flow-weithtiod } \\
\text { Composits }\end{array}$ & & \\
\hline Buriven 740-39-3 & $0.0613 \mathrm{mg} / 1$ & $0.0371 \mathrm{mg} / 1$ & $0.0613 \mathrm{mg} / 1$ & $0.0371 \mathrm{mg} / 1$ & 1 & \\
\hline mobylemes ctibrido 750092 & $<0.010 \mathrm{mg} / 1$ & & $<0.010 \mathrm{mg} / \mathrm{L}$ & & 1 & \\
\hline 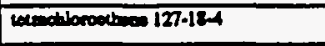 & $<0.010 \mathrm{mg} / \mathrm{l}$ & & $<0.010 \mathrm{mg} / \mathrm{l}$ & & 1 & \\
\hline 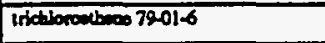 & $<0.010 \mathrm{mg} / \mathrm{l}$ & & $<0.010 \mathrm{mg} / \mathrm{l}$ & & 1 & \\
\hline Xylowe & $<0.010 \mathrm{mg} / \mathrm{l}$ & & $<0.010 \mathrm{mg} / \mathrm{l}$ & & 1 & \\
\hline 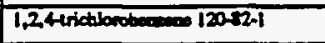 & $<0,010 \mathrm{mg} / \mathrm{h}$ & $<0.010 \mathrm{mg} / \mathrm{l}$ & $<0.010 \mathrm{mg} / \mathrm{l}$ & $<0.010 \mathrm{mg} / \mathrm{l}$ & 1 & \\
\hline 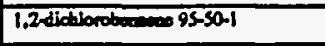 & $<0.010 \mathrm{mg} / \mathrm{l}$ & $<0.010 \mathrm{mg} / 1$ & $<0.010 \mathrm{mg} / 1$ & $<0.010 \mathrm{mg} / \mathrm{l}$ & 1 & \\
\hline 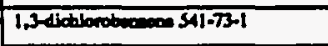 & $<0.010 \mathrm{mg} / 1$ & $<0.010 \mathrm{mg} / 1$ & $<0.010 \mathrm{mg} / \mathrm{l}$ & $<0.010 \mathrm{mg} / \mathrm{l}$ & 1 & \\
\hline 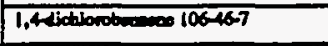 & $<0.010 \mathrm{mg} / \mathrm{t}$ & $<0.010 \mathrm{mg} / \mathrm{l}$ & $<0.010 \mathrm{mg} / 1$ & $<0.010 \mathrm{mg} / \mathrm{l}$ & 1 & \\
\hline 2,4 dinisolothere $121 \cdot 14-2$ & $<0.010 \mathrm{mg} / 1$ & $<0.010 \mathrm{mg} / \mathrm{l}$ & $<0.010 \mathrm{mg} / 1$ & $<0.010 \mathrm{mg} / 1$ & 1 & \\
\hline 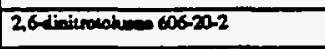 & $<0.010 \mathrm{mg} / \mathrm{l}$ & $<0.010 \mathrm{mg} / \mathrm{l}$ & $<0.010 \mathrm{mg} / \mathrm{l}$ & $<0.010 \mathrm{mg} / 1$ & 1 & \\
\hline 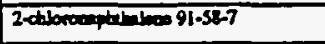 & $<0.010 \mathrm{mg} / \mathrm{l}$ & $<0.010 \mathrm{mg} / \mathrm{l}$ & $<0.010 \mathrm{mg} / \mathrm{l}$ & $<0.010 \mathrm{mg} / \mathrm{l}$ & 1 & \\
\hline 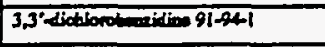 & $<0.010 \mathrm{mg} / \mathrm{l}$ & $<0.010 \mathrm{mg} / \mathrm{l}$ & $<0.010 \mathrm{mg} / 1$ & $<0.010 \mathrm{mg} / 1$ & 1 & \\
\hline 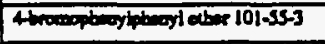 & $<0.010 \mathrm{mg} / \mathrm{l}$ & $<0.010 \mathrm{mg} / \mathrm{l}$ & $<0.010 \mathrm{mg} / \mathrm{l}$ & $<0.010 \mathrm{mg} / 1$ & 1 & \\
\hline 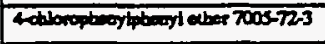 & $<0.010 \mathrm{mg} / \mathrm{l}$ & $<0.010 \mathrm{mg} / \mathrm{l}$ & $<0.010 \mathrm{mg} / \mathrm{l}$ & $<0.010 \mathrm{mg} / 1$ & 1 & \\
\hline 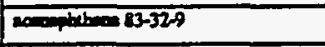 & $<0.010 \mathrm{mg} / 1$ & $<0.010 \mathrm{mq} / 1$ & $<0.010 \mathrm{mg} / \mathrm{h}$ & $<0.010 \mathrm{mg} / \mathrm{l}$ & 1 & \\
\hline 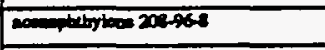 & $<0.010 \mathrm{mg} / \mathrm{h}$ & $<0.010 \mathrm{mg} / \mathrm{l}$ & $<0.010 \mathrm{mg} / \mathrm{l}$ & $<0.010 \mathrm{mg} / \mathrm{l}$ & 1 & \\
\hline 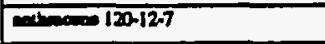 & $<0.010 \mathrm{mg} / \mathrm{t}$ & $<0.010 \mathrm{mg} / 1$ & $<0.010 \mathrm{mg} / \mathrm{L}$ & $<0.010 \mathrm{mg} / \mathrm{l}$ & 1 & \\
\hline milim 927.5 & $<0.030 \mathrm{mg} / \mathrm{l}$ & $<0.030 \mathrm{mg} / 1$ & $<0.030 \mathrm{mg} / \mathrm{l}$ & $<0.030 \mathrm{mg} / 1$ & 1 & $\because$ \\
\hline 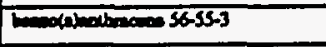 & $<0.010 \mathrm{mg} / 1$ & $<0.010 \mathrm{mg} / \mathrm{l}$ & $<0.010 \mathrm{mg} / \mathrm{l}$ & $<0.010 \mathrm{mg} / \mathrm{l}$ & 1 & \\
\hline 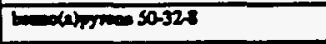 & $<0.010 \mathrm{mg} / 1$ & $<0.010 \mathrm{mg} / 1$ & $<0.010 \mathrm{mg} / \mathrm{l}$ & $<0.010 \mathrm{mg} / 1$ & 1 & \\
\hline 205992 & $0.017 \mathrm{mg} / \mathrm{h}$ & $<0.010 \mathrm{mg} / 1$ & $0.017 \mathrm{mg} / 1$ & $<0.010 \mathrm{mg} / \mathrm{l}$ & 1 & \\
\hline 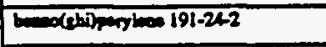 & $<0.010 \mathrm{mg} / \mathrm{l}$ & $<0.010 \mathrm{mg} / \mathrm{l}$ & $<0.010 \mathrm{mg} / \mathrm{l}$ & $<0.010 \mathrm{mg} / 1$ & 1 & \\
\hline 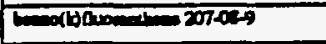 & $<0.010 \mathrm{mg} / 1$ & $<0.010 \mathrm{mg} / \mathrm{l}$ & $<0.010 \mathrm{mg} / 1$ & $<0.010 \mathrm{mg} / \mathrm{l}$ & 1 & \\
\hline 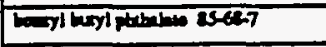 & $<0.010 \mathrm{mg} / \mathrm{l}$ & $<0.010 \mathrm{mg} / 1$ & $<0.010 \mathrm{mg} / 1$ & $<0.010 \mathrm{mg} / \mathrm{l}$ & 1 & \\
\hline 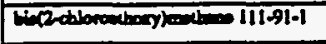 & $<0.010 \mathrm{mg} / \mathrm{l}$ & $<0.010 \mathrm{mg} / \mathrm{l}$ & $<0.010 \mathrm{mg} / 1$ & $<0.010 \mathrm{mg} / 1$ & 1 & \\
\hline 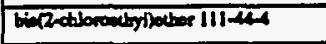 & $<0.010 \mathrm{mg} / \mathrm{l}$ & $<0.010 \mathrm{mg} / \mathrm{l}$ & $<0.010 \mathrm{mg} / \mathrm{l}$ & $<0.010 \mathrm{mg} / \mathrm{l}$ & 1 & \\
\hline 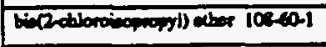 & $<0.010 \mathrm{mg} / 1$ & $<0.010 \mathrm{mg} / 1$ & $<0.010 \mathrm{mg} / \mathrm{l}$ & $<0.010 \mathrm{mg} / \mathrm{l}$ & 1 & \\
\hline 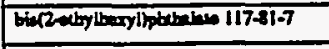 & $<0.010 \mathrm{mg} / 1$ & $<0.010 \mathrm{mg} / \mathrm{l}$ & $<0.010 \mathrm{mg} / \mathrm{l}$ & $<0.010 \mathrm{mg} / 1$ & 1 & \\
\hline 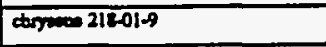 & $<0.010 \mathrm{mg} / \mathrm{l}$ & $<0.010 \mathrm{mg} / \mathrm{l}$ & $<0.010 \mathrm{mg} / \mathrm{l}$ & $<0.010 \mathrm{mg} / \mathrm{l}$ & 1 & \\
\hline 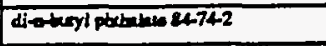 & $0.034 \mathrm{mg} / \mathrm{l}$ & $0.058 \mathrm{mg} / \mathrm{l}$ & $0.034 \mathrm{mg} / \mathrm{l}$ & $0.058 \mathrm{mg} / \mathrm{l}$ & 1 & \\
\hline 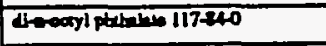 & $<0.010 \mathrm{mg} / \mathrm{h}$ & $<0.010 \mathrm{mg} / \mathrm{l}$ & $<0.010 \mathrm{mg} / 1$ & $<0.010 \mathrm{mg} / \mathrm{l}$ & 1 & \\
\hline 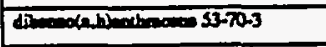 & $<0.010 \mathrm{mg} / \mathrm{l}$ & $<0.010 \mathrm{mg} / \mathrm{l}$ & $<0.010 \mathrm{mg} / 1$ & $<0.010$ mg $/ 1$ & 1 & \\
\hline 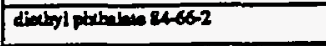 & $<0.010 \mathrm{mg} / \mathrm{l}$ & $<0.010 \mathrm{mg} / \mathrm{I}$ & $<0.010 \mathrm{mg} / 1$ & $<0.010 \mathrm{mg} / 1$ & 1 & \\
\hline 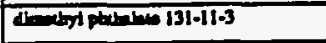 & $<0.010 \mathrm{mg} / 1$ & $<0.010 \mathrm{mg} / \mathrm{l}$ & $<0.010 \mathrm{mg} / \mathrm{l}$ & $<0.010 \mathrm{mg} / \mathrm{l}$ & 1 & \\
\hline 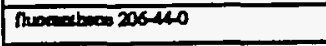 & $0.040 \mathrm{mg} / \mathrm{l}$ & $<0.010 \mathrm{mg} / 1$ & $0.040 \mathrm{mg} / \mathrm{l}$ & $<0.010 \mathrm{mg} / 1$ & 1 & \\
\hline thenes 26.73-7 & $<0.010 \mathrm{mg} / \mathrm{l}$ & $<0.010 \mathrm{mg} / \mathrm{l}$ & $<0.010 \mathrm{mg} / 1$ & $<0.010 \mathrm{mg} / 1$ & 1 & \\
\hline 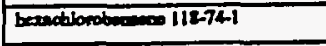 & $<0.010 \mathrm{mg} / \mathrm{l}$ & $<0.010 \mathrm{mg} / \mathrm{l}$ & $<0.010 \mathrm{mg} / \mathrm{h}$ & $<0.010 \mathrm{mg} / \mathrm{l}$ & 1 & \\
\hline 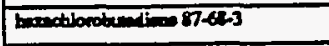 & $<0.010 \mathrm{mg} / 1$ & $<0.010 \mathrm{mg} / \mathrm{l}$ & $<0.010 \mathrm{mg} / \mathrm{l}$ & $<0.010 \mathrm{mg} / \mathrm{l}$ & 1 & \\
\hline 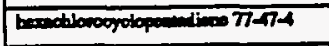 & $<0.010 \mathrm{mg} / \mathrm{l}$ & $<0.010 \mathrm{mg} / \mathrm{l}$ & $<0.010 \mathrm{mg} / 1$ & $<0.010 \mathrm{mg} / \mathrm{l}$ & 1 & \\
\hline 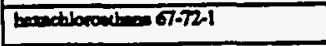 & $<0.010 \mathrm{mg} / \mathrm{l}$ & $<0.010 \mathrm{mg} / 1$ & $<0.010 \mathrm{mg} / \mathrm{l}$ & $<0.010 \mathrm{mg} / \mathrm{l}$ & 1 & \\
\hline 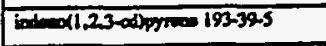 & $<0.010 \mathrm{mg} / \mathrm{l}$ & $<0.010 \mathrm{mg} / 1$ & $<0.010 \mathrm{mg} / \mathrm{l}$ & $<0.010 \mathrm{mg} / 1$ & 1 & \\
\hline Eoptoren $7259-1$ & $<0.010 \mathrm{mg} / \mathrm{l}$ & $<0.010 \mathrm{mg} / \mathrm{t}$ & $<0.010 \mathrm{mg} / \mathrm{l}$ & $<0.010 \mathrm{mg} / \mathrm{l}$ & 1 & \\
\hline 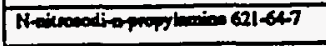 & $<0.010 \mathrm{mg} / \mathrm{h}$ & $<0.010 \mathrm{mg} / \mathrm{i}$ & $<0.010 \mathrm{mg} / 1$ & $<0.010 \mathrm{mg} / \mathrm{l}$ & 1 & \\
\hline 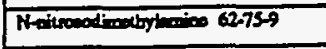 & $<0.010 \mathrm{mg} / \mathrm{l}$ & $<0.010 \mathrm{mg} / \mathrm{l}$ & $<0.010 \mathrm{mg} / \mathrm{l}$ & $<0.010 \mathrm{mg} / \mathrm{l}$ & 1 & \\
\hline 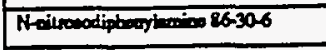 & $<0.010 \mathrm{mg} / \mathrm{l}$ & $<0.010 \mathrm{mg} / \mathrm{l}$ & $<0.010 \mathrm{mg} / 1$ & $<0.010 \mathrm{mg} / 1$ & 1 & \\
\hline $91-20-3$ & $<0.010 \mathrm{mg} / 1$ & $<0.010 \mathrm{mg} / \mathrm{l}$ & $<0.010 \mathrm{mg} / 1$ & $<0.010 \mathrm{mg} / \mathrm{l}$ & 1 & \\
\hline 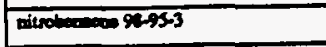 & $<0.010 \mathrm{mg} / \mathrm{l}$ & $<0.010 \mathrm{mg} / \mathrm{l}$ & $<0.010 \mathrm{mg} / \mathrm{l}$ & $<0.010 \mathrm{mg} / \mathrm{l}$ & 1 & \\
\hline 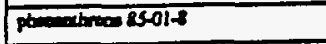 & $<0.010 \mathrm{mg} / 1$ & $<0.010 \mathrm{mg} / \mathrm{l}$ & $<0.010 \mathrm{mog} / 1$ & $<0.010 \mathrm{mg} / 1$ & 1 & \\
\hline$m=122000$ & $0.031 \mathrm{mg} / 1$ & $<0.010 \mathrm{mg} / \mathrm{l}$ & $0.031 \mathrm{mg} / \mathrm{l}$ & $<0.010 \mathrm{mg} / \mathrm{l}$ & 1 & \\
\hline 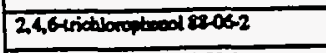 & $<0.030 \mathrm{mg} / \mathrm{l}$ & $<0.030 \mathrm{mg} / \mathrm{l}$ & $<0.030 \mathrm{mg} / \mathrm{l}$ & $<0.030 \mathrm{mg} / \mathrm{h}$ & 1 & \\
\hline 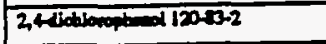 & $<0.030 \mathrm{mg} /$ & $<0.030 \mathrm{mg} / \mathrm{l}$ & $<0.030 \mathrm{mg} / \mathrm{l}$ & $<0.030 \mathrm{mg} / 1$ & 1 & \\
\hline 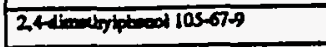 & $<0.030 \mathrm{mg} / \mathrm{l}$ & $<0.030 \mathrm{mg} / \mathrm{l}$ & $<0.030 \mathrm{mg} / \mathrm{l}$ & $<0.030 \mathrm{mg} / \mathrm{I}$ & 1 & \\
\hline 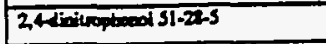 & $<0.030 \mathrm{mg} / \mathrm{l}$ & $<0.030 \mathrm{mg} / 1$ & $<0.030 \mathrm{mg} / \mathrm{h}$ & $<0.030 \mathrm{mg} / \mathrm{l}$ & 1 & \\
\hline
\end{tabular}


Outfall 4

Parse B and C Continied

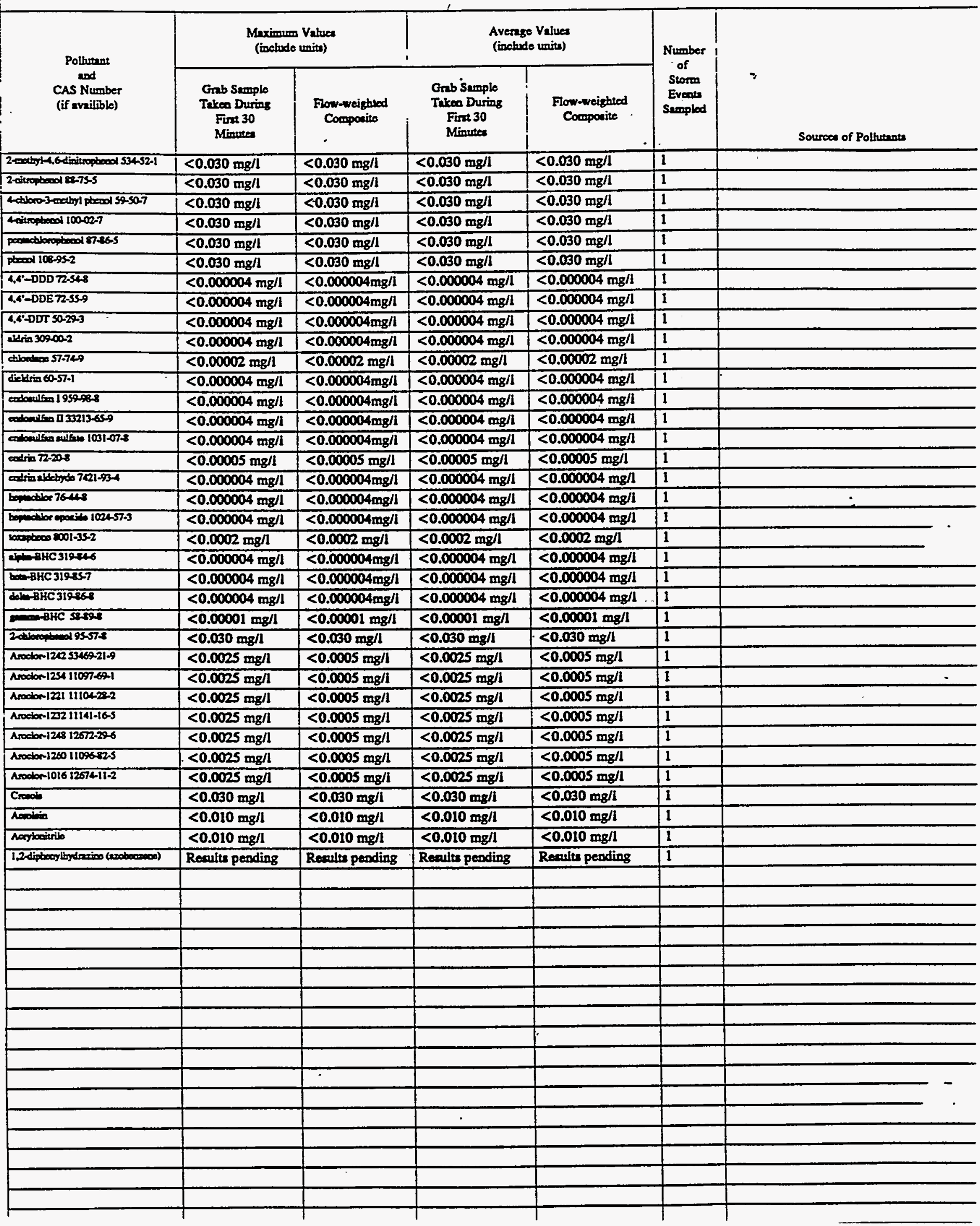


VII. Dischargo Information (continuod from page 3 of Form 2F)

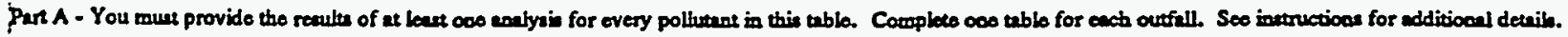

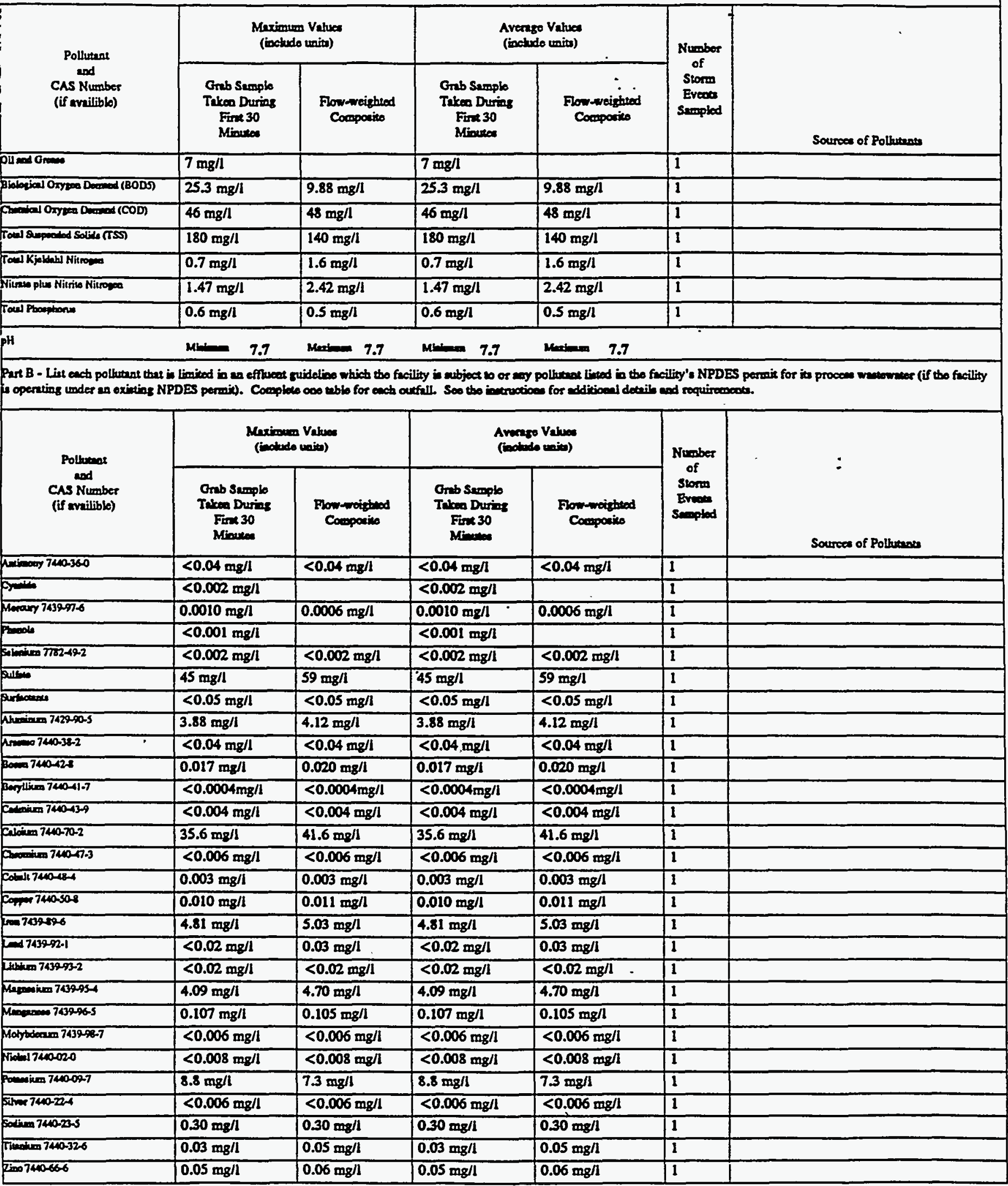


Part C. List each pollutent ahown in Tables 2F-2, 2F-3. and 2F4 that you know or have reason to believe is preaent. See the inntructions for additional detrils and requiremeats. Con one tuble for eacb outell.

\begin{tabular}{|c|c|c|c|c|c|c|c|c|c|}
\hline \multirow{2}{*}{\multicolumn{2}{|c|}{$\begin{array}{l}\text { Pollutant } \\
\text { and } \\
\text { CAS Number } \\
\text { (if availible) }\end{array}$}} & \multicolumn{2}{|c|}{$\begin{array}{l}\text { Maximum Valuea } \\
\text { (include units) }\end{array}$} & \multicolumn{3}{|c|}{$\begin{array}{l}\text { Avernge Veluce } \\
\text { (inchide units) }\end{array}$} & \multirow{2}{*}{$\begin{array}{l}\text { Number } \\
\text { of } \\
\text { Storm } \\
\text { Events } \\
\text { Srmplod } \\
\text {. }\end{array}$} & \multirow[b]{2}{*}{$\therefore$ ce } & \\
\hline & & $\begin{array}{c}\text { Grab Sumplo } \\
\text { Taken During } \\
\text { Fint } 30 \\
\text { Minutes }\end{array}$ & $\begin{array}{l}\text { Flow-weighred } \\
\text { Comporive }\end{array}$ & $\begin{array}{l}\text { Grib Semplo } \\
\text { Taken During } \\
\text { Find } 30 \\
\text { Minuted }\end{array}$ & \multicolumn{2}{|c|}{$\begin{array}{c}\text { i Flow-weighted } \\
\text { Comporito }\end{array}$} & & & ollutanests \\
\hline \multicolumn{2}{|c|}{ 1.1.1.-trictboroethes 71.556} & $<0.010 \mathrm{mg} / 1$ & $!$ & $<0.010 \mathrm{mg} / 1$ & \multicolumn{2}{|l|}{; } & 1 & & \\
\hline \multicolumn{2}{|c|}{ 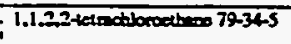 } & $<0.010 \mathrm{mg} / \mathrm{l}$ & & \multicolumn{3}{|l|}{$<0.010 \mathrm{mg} / \mathrm{l}$} & 1 & & \\
\hline \multicolumn{2}{|c|}{1.1 .2 -trichloroethere $7900-5$} & $<0.010 \mathrm{mg} / \mathrm{l}$ & & $<0.010 \mathrm{mg} / \mathrm{l}$ & & & T & & \\
\hline \multicolumn{2}{|c|}{ 1.1-dictionceateres 75-36-3 } & $<0.010 \mathrm{mg} / \mathrm{l}$ & & $<0.010 \mathrm{mg} / \mathrm{l}$ & 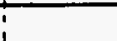 & & 1 & & \\
\hline \multicolumn{2}{|c|}{ 1.1-didtaroedbykace 75-35-4 } & $<0.010 \mathrm{mg} / \mathrm{l}$ & & $<0.010 \mathrm{mg} / 1$ & & & 1 & & \\
\hline \multicolumn{2}{|c|}{1.2 dichlorcthersion 10706-2 } & $<0.010 \mathrm{mg} / \mathrm{l}$ & & $<0.010 \mathrm{mg} / \mathrm{l}$ & & & 1 & & \\
\hline \multicolumn{2}{|c|}{ 1.2-dictiorepropenos 78.87.5 } & $<0.010 \mathrm{mg} / 1$ & & $<0.010 \mathrm{mg} / \mathrm{l}$ & & & 1 & & \\
\hline 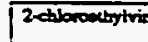 & $110-75-8$ & $<0.010 \mathrm{mg} / 1$ & & $<0.010 \mathrm{mg} / \mathrm{l}$ & & & 1 & & \\
\hline $71-43-2$ & & $<0.010 \mathrm{mg} / \mathrm{l}$ & & $<0.010 \mathrm{mg} / \mathrm{l}$ & & & 1 & & \\
\hline broweodictionet & $75-274$ & $<0.010 \mathrm{mg} / \mathrm{l}$ & & $<0.010 \mathrm{mg} / \mathrm{l}$ & & & 1 & 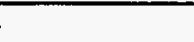 & \\
\hline bromolores 75-9 & & $<0.010 \mathrm{mg} / \mathrm{l}$ & & $<0.010 \mathrm{mg} / \mathrm{l}$ & & & I & & \\
\hline 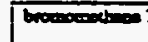 & & $<0.010 \mathrm{mg} / 1$ & & $<0.010 \mathrm{mg} / \mathrm{l}$ & & & 1 & & \\
\hline arteo vetentive & $56-22-5$ & $<0.010 \mathrm{mg} / 1$ & & $<0.010 \mathrm{mg} / \mathrm{l}$ & & & 1 & & \\
\hline chlorobareseres & & $<0.010 \mathrm{mg} / \mathrm{l}$ & & $<0.010 \mathrm{mg} / \mathrm{l}$ & & & 1 & & \\
\hline chlorcethere 75 & & $<0.010 \mathrm{mg} / 1$ & & $<0.010 \mathrm{mg} / \mathrm{l}$ & & & 1 & & \\
\hline chloroform 67 & & $<0.010 \mathrm{mg} / 1$ & & $<0.010 \mathrm{mg} / \mathrm{l}$ & & & 1 & & 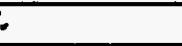 \\
\hline 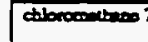 & & $<0.010 \mathrm{mg} / 1$ & & $<0.010 \mathrm{mg} / 1$ & & & 1 & & \\
\hline & & $<0.010 \mathrm{mg} / \mathrm{l}$ & & $<0.010 \mathrm{mg} / \mathrm{l}$ & & & 1 & & \\
\hline ethyiberoces ic & & $<0.010 \mathrm{mg} / \mathrm{l}$ & & $<0.010 \mathrm{mg} / \mathrm{l}$ & & & 1 & & \\
\hline loken 108-08: & & $<0.010 \mathrm{mg} / \mathrm{l}$ & & $<0.010 \mathrm{mg} / 1$ & & & 1 & & \\
\hline vinglationibe 7 & & $<0.010 \mathrm{mg} / 1$ & & $<0.010 \mathrm{mg} / \mathrm{l}$ & & & 1 & & \\
\hline 1.3-diatlonoping & 10051.01 .5 & $<0.010 \mathrm{mg} / 1$ & & $<0.010 \mathrm{mg} / 1$ & $\cdot$ & & 1 & & \\
\hline Irom-1.2-dictio & $-00156-605$ & $<0.010 \mathrm{mg} / \mathrm{l}$ & & $<0.010 \mathrm{mg} / \mathrm{l}$ & & & 1 & & \\
\hline alpinextivity 12 & $-16-1$ & $3.3 \mathrm{pci} / 1$ & $1.2 \mathrm{pci} / \mathrm{l}$ & $3.3 \mathrm{pci} / 1$ & 12 pcin & & 1 & & \\
\hline beonecivity 12 & $\overline{Q-1}$ & 17 pcill & $21 \mathrm{pci} / \mathrm{l}$ & 17 pcit & 21 pcill & & 1 & & \\
\hline foal coliform & & TNTC col.1L & $>4,000 \mathrm{co} / .1 \mathrm{~L}$ & TNTC co/.1L & $>4,000$ & $0 / .1 \mathrm{~L}$ & 1 & & \\
\hline Thellitum 7400-2 & & $<0.001 \mathrm{mg} / \mathrm{l}$ & $<0.001 \mathrm{mg} / \mathrm{l}$ & $<0.001 \mathrm{mg} / \mathrm{l}$ & $<0.001$ & $g / 1$ & 1 & & \\
\hline Unaming 7400 & & $0.001 \mathrm{mg} / \mathrm{l}$ & $0.002 \mathrm{mg} / \mathrm{l}$ & $0.001 \mathrm{mg} / 1$ & $0.002 \mathrm{~ms}$ & & 1 & & \\
\hline Ganemexivity & & $14 \mathrm{pei} / \mathrm{l}$ & 44 pcill & $14 \mathrm{pci} / 1$ & $44 \mathrm{pci} / \mathrm{l}$ & & 1 & & \\
\hline Part D. Pror & for the & Im eveats(o) which & ind in the maximut & for the flow & comp & ? & & & \\
\hline $\begin{array}{l}1 . \\
\text { Date of } \\
\text { Storm } \\
\text { Eveat }\end{array}$ & $\begin{array}{l}2 . \\
\text { Duration } \\
\text { of Storns } \\
\text { (in } \\
\text { minutes) }\end{array}$ & $\begin{array}{l}3 . \\
\text { Totel rinfall } \\
\text { during storm event } \\
\text { (in incos) }\end{array}$ & $\begin{array}{l}4 . \\
\text { Number of boun be } \\
\text { beginning of atom } \\
\text { ured and end of pre } \\
\text { monarable min }\end{array}$ & $\begin{array}{l}\text { Mexim } \\
\text { durio } \\
\text { (alloow }\end{array}$ & $\begin{array}{l}\text { fow mito } \\
\text { in ovent } \\
\text { or specify } \\
\text { (1) }\end{array}$ & & $\begin{array}{l}6 . \\
\text { flow from } \\
\text { ovent } \\
\text { Iloas or } \\
\text { ify unita) }\end{array}$ & $\begin{array}{c}7 . \\
\text { Secion } \\
\text { semplo was } \\
\text { taken }\end{array}$ & $\begin{array}{l}\text { 8. } \\
\text { Form of } \\
\text { Procipitation } \\
\text { (ninfill, } \\
\text { eacmmell) }\end{array}$ \\
\hline $7 / 24 / 91$ & 185 & 1.0 & 154.2 & 257 & & 11,462 & & Summer & Rainfall \\
\hline
\end{tabular}

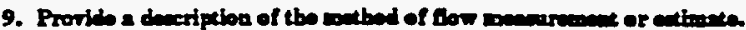

An ISCO 3230 Flow meter was used to obtain water level readings. This instrument was calibrated with the normal dry weather flow, if present, as level zero. The Aow meter was programmed with the characteristica of the convey ance so that flow rates and total flows conid be calculated by the meter. 


\begin{tabular}{|c|c|c|c|c|c|c|}
\hline \multirow{2}{*}{$\begin{array}{l}\text { Pollutent } \\
\text { and } \\
\text { CAS Number } \\
\text { (if availiblo) }\end{array}$} & \multicolumn{2}{|c|}{$\begin{array}{l}\text { Meximum Vahues } \\
\text { (includo unite) }\end{array}$} & \multicolumn{2}{|c|}{$\begin{array}{l}\text { Avorago Vahuce } \\
\text { (ibetudo units) }\end{array}$} & \multirow{2}{*}{$\begin{array}{l}\text { Number } \\
\text { of } \\
\text { Storm, } \\
\text { Evecte } \\
\text { Sampled }\end{array}$} & \multirow[b]{2}{*}{ Sourcen of Polturtans } \\
\hline & $\begin{array}{c}\text { Grab Semplo } \\
\text { Taken Durims } \\
\text { Firne } 30 \\
\text { Minuree }\end{array}$ & $\begin{array}{l}\text { Flow wreighed } \\
\text { Compotixo }\end{array}$ & $\begin{array}{c}\text { Gabb Semplo } \\
\text { Trkea During } \\
\text { First } 30 \\
\text { Minereo }\end{array}$ & $\begin{array}{l}\text { Flow-weighted } \\
\text { Componics }\end{array}$ & & \\
\hline Berien 7400-39-3 & $0.0403 \mathrm{mg} / \mathrm{l}$ & $0.0441 \mathrm{mg} / \mathrm{l}$ & $0.0403 \mathrm{mg} / 1$ & $0.0441 \mathrm{mg} / 1$ & 1 & \\
\hline 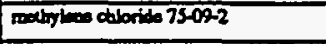 & $<0.010 \mathrm{mg} / 1$ & & $<0.010 \mathrm{mg} / 1$ & & 1 & \\
\hline 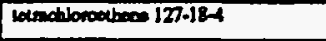 & $<0.010 \mathrm{mg} / \mathrm{l}$ & & $<0.010 \mathrm{mg} / \mathrm{l}$ & I & 1 & \\
\hline 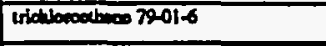 & $<0.010 \mathrm{mg} / \mathrm{l}$ & & $<0.010 \mathrm{mg} / \mathrm{l}$ & & 1 & \\
\hline Xyleoses & $<0.010 \mathrm{mg} / \mathrm{l}$ & & $<0.010 \mathrm{mg} / \mathrm{l}$ & & 1 & \\
\hline 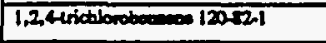 & $<0.010 \mathrm{mg} / \mathrm{l}$ & $<0.010 \mathrm{mg} / 1$ & $<0.010 \mathrm{mg} / \mathrm{l}$ & $<0.010 \mathrm{mg} / 1$ & 1 & \\
\hline 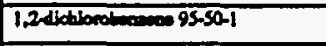 & $<0.010 \mathrm{mg} / \mathrm{l}$ & $<0.010 \mathrm{mg} / \mathrm{l}$ & $<0.010 \mathrm{mg} / \mathrm{l}$ & $<0.010 \mathrm{mg} / 1$ & 1 & \\
\hline 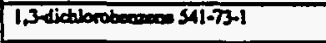 & $<0.010 \mathrm{mg} / \mathrm{l}$ & $<0.010 \mathrm{mg} / \mathrm{l}$ & $<0.010 \mathrm{mg} / \mathrm{l}$ & $<0.010 \mathrm{mg} / 1$ & 1 & \\
\hline 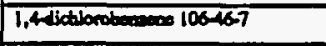 & $<0.010 \mathrm{mg} / \mathrm{l}$ & $<0.010 \mathrm{mg} / \mathrm{l}$ & $<0.010 \mathrm{mg} / \mathrm{l}$ & $<0.010 \mathrm{mg} / \mathrm{l}$ & 1 & \\
\hline 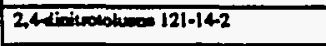 & $<0.010 \mathrm{mg} / \mathrm{l}$ & $<0.010 \mathrm{mg} / 1$ & $<0.010 \mathrm{mg} / \mathrm{l}$ & $<0.010 \mathrm{mg} / \mathrm{l}$ & 1 & \\
\hline 26-1initrodolumecos $606-20-2$ & $<0.010 \mathrm{mg} / \mathrm{l}$ & $<0.010 \mathrm{mg} / \mathrm{l}$ & $<0.010 \mathrm{mg} / \mathrm{l}$ & $<0.010 \mathrm{mg} / \mathrm{l}$ & 1 & \\
\hline 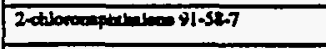 & $<0.010 \mathrm{mg} / \mathrm{l}$ & $<0.010 \mathrm{mg} / \mathrm{l}$ & $<0.010 \mathrm{mg} / 1$ & $<0.010 \mathrm{mg} / 1$ & 1. & \\
\hline 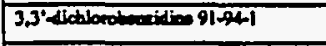 & $<0.010 \mathrm{mg} / \mathrm{l}$ & $<0.010 \mathrm{mg} / 1$ & $<0.010 \mathrm{mg} / \mathrm{l}$ & $<0.010 \mathrm{mg} / 1$ & 1 & \\
\hline 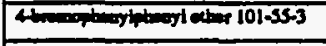 & $<0.010 \mathrm{mg} / \mathrm{l}$ & $<0.010 \mathrm{mg} / \mathrm{h}$ & $<0.010 \mathrm{mg} / \mathrm{l}$ & $<0.010 \mathrm{mg} / 1$ & 1 & \\
\hline 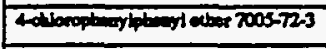 & $<0.010 \mathrm{mg} / \mathrm{h}$ & $<0.010 \mathrm{mg} / 1$ & $<0.010 \mathrm{mg} / \mathrm{l}$ & $<0.010 \mathrm{mg} / \mathrm{l}$ & 1 & \\
\hline anderes-32-9 & $<0.010 \mathrm{mg} / 1$ & $<0.010 \mathrm{mg} / 1$ & $<0.010 \mathrm{mg} / \mathrm{l}$ & $<0.010 \mathrm{mg} / \mathrm{l}$ & 1 & \\
\hline 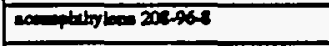 & $<0.010 \mathrm{mg} / \mathrm{l}$ & $<0.010 \mathrm{mg} / 1$ & $<0.010 \mathrm{mg} / \mathrm{l}$ & $<0.010 \mathrm{mg} / 1$ & 1 & \\
\hline $120-12-7$ & $<0.010 \mathrm{mg} / \mathrm{l}$ & $<0.010 \mathrm{mg} / 1$ & $<0.010 \mathrm{mg} / \mathrm{l}$ & $<0.010 \mathrm{mg} / \mathrm{l}$ & 1 & \\
\hline mailien 9257.5 & $<0.030 \mathrm{mg} / \mathrm{h}$ & $<0.030 \mathrm{mg} / \mathrm{l}$ & $<0.030 \mathrm{mg} / \mathrm{l}$ & $<0.030 \mathrm{mg} / 1$ & 1 & $\vdots$ \\
\hline 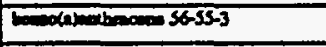 & $<0.010 \mathrm{mg} / \mathrm{l}$ & $<0.010 \mathrm{mg} / 1$ & $<0.010 \mathrm{mg} / \mathrm{l}$ & $<0.010 \mathrm{mg} / \mathrm{l}$ & 1 & \\
\hline 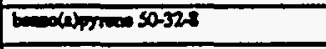 & $<0.010 \mathrm{mg} / \mathrm{l}$ & $<0.010 \mathrm{mg} / \mathrm{I}$ & $<0.010 \mathrm{mg} / \mathrm{l}$ & $<0.010 \mathrm{mg} / \mathrm{l}$ & 1 & \\
\hline 200392 & $<0.010 \mathrm{mg} / 1$ & $<0.010 \mathrm{mg} / \mathrm{l}$ & $<0.010 \mathrm{mg} / 1$ & $<0.010 \mathrm{mg} / \mathrm{l}$ & 1 & \\
\hline 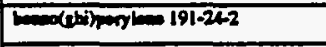 & $<0.010 \mathrm{mg} / \mathrm{l}$ & $<0.010 \mathrm{mg} / 1$ & $<0.010 \mathrm{mg} / \mathrm{l}$ & $<0.010 \mathrm{mg} / \mathrm{l}$ & 1 & \\
\hline 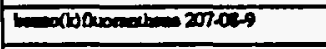 & $<0.010 \mathrm{cog} / 1$ & $<0.010 \mathrm{mg} / 1$ & $<0.010 \mathrm{mg} / \mathrm{l}$ & $<0.010 \mathrm{mg} / \mathrm{l}$ & 1 & \\
\hline 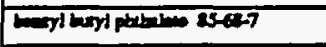 & $<0.010 \mathrm{mg} / 1$ & $<0.010 \mathrm{mg} / 1$ & $<0.010 \mathrm{mg} / 1$ & $<0.010 \mathrm{mg} / \mathrm{l}$ & 1 & \\
\hline 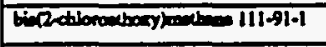 & $<0.010 \mathrm{mg} / 1$ & $<0.010 \mathrm{mg} / \mathrm{l}$ & $<0.010 \mathrm{mg} / \mathrm{l}$ & $<0.010 \mathrm{mg} / \mathrm{l}$ & 1 & \\
\hline 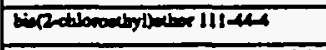 & $<0.010 \mathrm{mgg} / \mathrm{l}$ & $<0.010 \mathrm{mg} / \mathrm{l}$ & $<0.010 \mathrm{mg} / 1$ & $<0.010 \mathrm{mg} / 1$ & 1 & \\
\hline 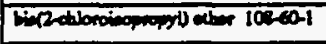 & $<0.010 \mathrm{mg} / 1$ & $<0.010 \mathrm{mg} / 1$ & $<0.010 \mathrm{mg} / 1$ & $<0.010 \mathrm{mg} / \mathrm{l}$ & 1 & \\
\hline 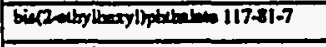 & $<0.010 \mathrm{mg} / \mathrm{l}$ & $<0.010 \mathrm{mg} / 1$ & $<0.010 \mathrm{mg} / \mathrm{l}$ & $<0.010 \mathrm{mg} / \mathrm{l}$ & 1 & \\
\hline \begin{tabular}{|l} 
aryoue $21801-9$ \\
\end{tabular} & $<0.010 \mathrm{mg} / \mathrm{l}$ & $<0.010 \mathrm{mg} / \mathrm{l}$ & $<0.010 \mathrm{mg} / \mathrm{l}$ & $<0.010 \mathrm{mg} / \mathrm{l}$ & 1 & \\
\hline 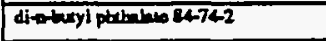 & $0.033 \mathrm{mg} / 1$ & $0.049 \mathrm{mg} / \mathrm{l}$ & $0.033 \mathrm{mg} / \mathrm{l}$ & $0.049 \mathrm{mg} / \mathrm{l}$ & 1 & \\
\hline 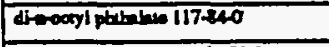 & $<0.010 \mathrm{mg} / \mathrm{l}$ & $<0.010 \mathrm{mg} / \mathrm{l}$ & $<0.010 \mathrm{mg} / 1$ & $<0.010 \mathrm{mg} / \mathrm{l}$ & 1 & \\
\hline $53.70-3$ & $<0.010 \mathrm{mg} / 1$ & $<0.010 \mathrm{mg} / \mathrm{l}$ & $<0.010 \mathrm{mg} / \mathrm{l}$ & $<0.010 \mathrm{mg} / 1$ & 1 & \\
\hline 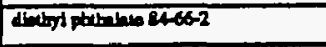 & $<0.010 \mathrm{mg} / \mathrm{l}$ & $<0.010 \mathrm{mg} / \mathrm{h}$ & $<0.010 \mathrm{mg} / \mathrm{l}$ & $<0.010 \mathrm{mg} / 1$ & 1 & \\
\hline 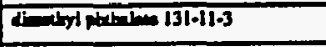 & $<0.010 \mathrm{mg} / 1$ & $<0.010 \mathrm{mg} / \mathrm{l}$ & $<0.010 \mathrm{mg} / \mathrm{L}$ & $<0.010 \mathrm{mg} / \mathrm{l}$ & 1 & \\
\hline 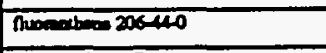 & $<0.010 \mathrm{mg} / \mathrm{l}$ & $<0.010 \mathrm{mg} / 1$ & $<0.010 \mathrm{mg} / \mathrm{l}$ & $<0.010 \mathrm{mg} / \mathrm{l}$ & 1 & \\
\hline nuosose- 26-73-7 & $<0.010 \mathrm{mg} / 1$ & $<0.010 \mathrm{mg} / 1$ & $<0.010 \mathrm{mg} / 1$ & $<0.010 \mathrm{mg} / \mathrm{l}$ & 1 & \\
\hline 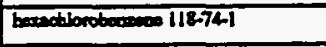 & $<0.010 \mathrm{mg} / \mathrm{l}$ & $<0.010 \mathrm{mg} / 1$ & $<0.010 \mathrm{mg} / 1$ & $<0.010 \mathrm{mg} / \mathrm{l}$ & 1 & \\
\hline 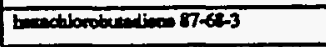 & $<0.010 \mathrm{mg} / 1$ & $<0.010 \mathrm{mg} / \mathrm{l}$ & $<0.010 \mathrm{mg} / 1$ & $<0.010 \mathrm{mg} / \mathrm{l}$ & 1 & \\
\hline 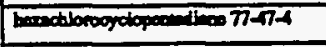 & $<0.010 \mathrm{mg} / 1$ & $<0.010 \mathrm{mg} / 1$ & $<0.010 \mathrm{mg} / \mathrm{l}$ & $<0.010 \mathrm{mg} / \mathrm{l}$ & 1 & \\
\hline - 67.72-1 & $<0.010 \mathrm{mg} / \mathrm{h}$ & $<0.010 \mathrm{mg} / 1$ & $<0.010 \mathrm{mg} / 1$ & $<0.010 \mathrm{mg} / 1$ & 1 & \\
\hline 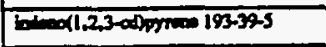 & $<0.010 \mathrm{mg} / 1$ & $<0.010 \mathrm{mg} / 1$ & $<0.010 \mathrm{mg} / \mathrm{l}$ & $<0.010 \mathrm{mg} / \mathrm{l}$ & 1 & \\
\hline 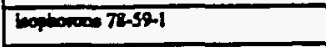 & $<0.010 \mathrm{mg} / 1$ & $<0.010 \mathrm{mg} / 1$ & $<0.010 \mathrm{mg} / \mathrm{l}$ & $<0.010 \mathrm{mg} / \mathrm{l}$ & 1 & \\
\hline 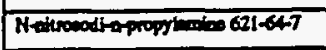 & $<0.010 \mathrm{mg} / \mathrm{t}$ & $<0.010 \mathrm{mg} / \mathrm{l}$ & $<0.010 \mathrm{mg} / 1$ & $<0.010 \mathrm{mg} /$ & 1 & \\
\hline 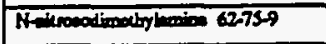 & $<0.010 \mathrm{mg} / 1$ & $<0.010 \mathrm{mg} / 1$ & $<0.010 \mathrm{mg} / 1$ & $<0.010 \mathrm{mg} / \mathrm{l}$ & 1 & \\
\hline 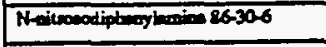 & $<0.010 \mathrm{mg} / \mathrm{l}$ & $<0.010 \mathrm{mg} / \mathrm{l}$ & $<0.010 \mathrm{mg} / \mathrm{l}$ & $<0.010 \mathrm{mg} / 1$ & 1 & \\
\hline 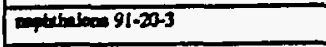 & $<0.010 \mathrm{mg} / \mathrm{l}$ & $<0.010 \mathrm{mg} / 1$ & $<0.010 \mathrm{mg} / 1$ & $<0.010 \mathrm{mg} / 1$ & 1 & \\
\hline tivolimenesesess-3 & $<0.010 \mathrm{mg} / \mathrm{l}$ & $<0.010 \mathrm{mg} / \mathrm{l}$ & $<0.010 \mathrm{mg} / \mathrm{l}$ & $<0.010 \mathrm{mg} / 1$ & 1 & \\
\hline 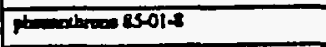 & $<0.010 \mathrm{mg} / 1$ & $<0.010 \mathrm{mg} / 1$ & $<0.010 \mathrm{mg} / \mathrm{l}$ & $<0.010 \mathrm{mg} / \mathrm{l}$ & 1 & \\
\hline rmon 129000 & $<0.010 \mathrm{mg} / 1$ & $<0.010 \mathrm{mg} / \mathrm{l}$ & $<0.010 \mathrm{mg} / 1$ & $<0.010 \mathrm{mg} / \mathrm{l}$ & 1 & \\
\hline 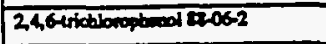 & $<0.030 \mathrm{mg} / \mathrm{l}$ & $<0.030 \mathrm{mg} / \mathrm{l}$ & $<0.030 \mathrm{mg} / 1$ & $<0.030 \mathrm{mg} / \mathrm{l}$ & 1 & \\
\hline 2,4 dichlorophed $120,13-2$ & $<0.030 \mathrm{mg} / \mathrm{l}$ & $<0.030 \mathrm{mg} / 1$ & $<0.030 \mathrm{mg} / 1$ & $<0.030 \mathrm{mg} / 1$ & 1 & \\
\hline 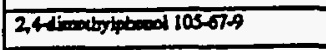 & $<0.030 \mathrm{mg} / 1$ & $<0.030 \mathrm{mg} / 1$ & $<0.030 \mathrm{mg} / \mathrm{l}$ & $<0.030 \mathrm{mg} / \mathrm{l}$ & 1 & \\
\hline 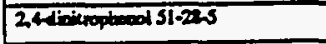 & $<0.030 \mathrm{mg} / 1$ & $<0.030 \mathrm{mg} / \mathrm{h}$ & $<0.030 \mathrm{mg} / \mathrm{l}$ & $<0.030 \mathrm{mg} / 1$ & 1 & \\
\hline 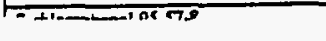 & $\cdots$ & $\cdots$ & anma & $\operatorname{lnmnn-nt}$ & $T$ & \\
\hline
\end{tabular}


Outfoll 6

Pars B and C Concinued

\begin{tabular}{|c|c|c|c|c|c|c|}
\hline \multirow{2}{*}{$\begin{array}{l}\text { Pollutuat } \\
\text { end } \\
\text { CAs Number } \\
\text { (if availible) }\end{array}$} & \multicolumn{2}{|c|}{ 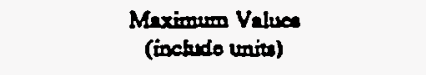 } & \multicolumn{2}{|c|}{$\begin{array}{l}\text { Averase Vilues } \\
\text { (ioclude writs) }\end{array}$} & \multirow{2}{*}{$\begin{array}{l}\text { Number } \\
\text { of } \\
\text { Storm } \\
\text { Eveals } \\
\text { Sempied }\end{array}$} & \multirow[b]{2}{*}{ Sourcese of Pollutuats } \\
\hline & $\begin{array}{c}\text { Grab Sample } \\
\text { Taken Duriag } \\
\text { Firnt } 30 \\
\text { Minutes }\end{array}$ & $\begin{array}{c}\text { Flow-woighted } \\
\text { Composite }\end{array}$ & $\begin{array}{l}\text { Grab Sumplo } \\
\text { Takeo During } \\
\text { Fint } 30 \\
\text { Minutea }\end{array}$ & $\begin{array}{l}\text { Flow-weighted } \\
\text { Componice }\end{array}$ & & \\
\hline 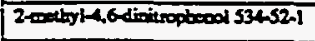 & $<0.030 \mathrm{mg} / \mathrm{h}$ & $<0.030 \mathrm{mg} / \mathrm{l}$ & $<0.030 \mathrm{mg} / 1$ & $<0.030 \mathrm{mg} / 1$ & 1 & \\
\hline 20 illoptool $28-75-5$ & $<0.030 \mathrm{mg} / \mathrm{l}$ & $<0.030 \mathrm{mg} / \mathrm{l}$ & $<0.030 \mathrm{mg} / \mathrm{l}$ & $<0.030 \mathrm{mg} / 1$ & 11 & \\
\hline 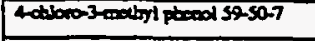 & $<0.030 \mathrm{mg} / 1$ & $<0.030 \mathrm{mg} / \mathrm{l}$ & $<0.030 \mathrm{mg} / \mathrm{l}$ & $<0.030 \mathrm{mg} / \mathrm{l}$ & 1 & \\
\hline Adisopheod 100027 & $<0.030 \mathrm{mg} / 1$ & $<0.030 \mathrm{mg} / 1$ & $<0.030 \mathrm{mg} / \mathrm{l}$ & $<0.030 \mathrm{mg} / 1$ & 1 & \\
\hline 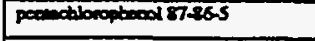 & $<0.030 \mathrm{mg} / \mathrm{l}$ & $<0.030 \mathrm{mg} / \mathrm{l}$ & $<0.030 \mathrm{mg} / 1$ & $<0.030 \mathrm{mg} / \mathrm{l}$ & 1 & \\
\hline phomed 100-9s-2 & $<0.030 \mathrm{mg} / \mathrm{l}$ & $<0.030 \mathrm{mg} / \mathrm{l}$ & $<0.030 \mathrm{mg} / \mathrm{l}$ & $<0.030 \mathrm{mg} / 1$ & 1 & \\
\hline 4.4-DDD 72-548 & $<0.000004 \mathrm{mg} / \mathrm{l}$ & $<0.000004 \mathrm{mg} / 1$ & $<0.000004 \mathrm{mg} / \mathrm{l}$ & $<0.000004 \mathrm{mg} / \mathrm{l}$ & 1 & \\
\hline 4,4-DDE72-35-9 & $<0.000004 \mathrm{mg} / \mathrm{l}$ & $<0.000004 \mathrm{mg} / \mathrm{l}$ & $<0.000004 \mathrm{mg} / \mathrm{l}$ & $<0.000004 \mathrm{mg} / \mathrm{h}$ & 1 & \\
\hline 4.4'-DOT SO-29-3 & $<0.000004 \mathrm{mg} / \mathrm{l}$ & $<0.000004 \mathrm{mg} / \mathrm{l}$ & $<0.000004 \mathrm{mg} / \mathrm{l}$ & $<0.000004 \mathrm{mg} / 1$ & 1 & \\
\hline attria $302-00-2$ & $<0.000004 \mathrm{mg} / \mathrm{l}$ & $<0.000004 \mathrm{mg} / 1$ & $<0.000004 \mathrm{mg} / \mathrm{l}$ & $<0.000004 \mathrm{mg} / \mathrm{l}$ & 1 & \\
\hline 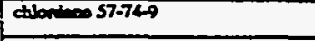 & $<0.00002 \mathrm{mg} / 1$ & $<0.00002 \mathrm{mg} / \mathrm{l}$ & $<0.00002 \mathrm{mg} / 1$ & $<0.00002 \mathrm{mg} / 1$ & 1 & \\
\hline dieldrin 60-57-1 & $<0.000004 \mathrm{mg} / \mathrm{l}$ & $<0.000004 \mathrm{mg} / 1$ & $<0.000004 \mathrm{mg} / \mathrm{l}$ & $<0.000004 \mathrm{mg} / \mathrm{l}$ & 1 & \\
\hline codosullom I 959-968 & $<0.000004 \mathrm{mg} / \mathrm{l}$ & $<0.000004 \mathrm{mg} / \mathrm{l}$ & $<0.000004 \mathrm{mg} / \mathrm{l}$ & $<0.000004 \mathrm{mg} / \mathrm{t}$ & 1 & \\
\hline 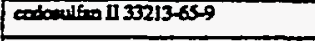 & $<0.000004 \mathrm{mg} / \mathrm{m}$ & $<0.000004 \mathrm{mg} / \mathrm{l}$ & $<0.000004 \mathrm{mg} / \mathrm{l}$ & $<0.000004 \mathrm{mg} / 1$ & 1 & \\
\hline 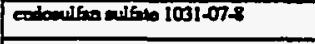 & $<0.000004 \mathrm{mg} / \mathrm{l}$ & $<0.000004 \mathrm{mg} / \mathrm{l}$ & $<0.000004 \mathrm{mg} / \mathrm{l}$ & $<0.000004 \mathrm{mg} / \mathrm{l}$ & 1 & \\
\hline 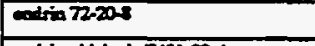 & $<0.00005 \mathrm{mg} / \mathrm{l}$ & $<0.00005 \mathrm{mg} / 1$ & $<0.00005 \mathrm{mg} / \mathrm{l}$ & $<0.00005 \mathrm{mg} / \mathrm{l}$ & 1 & \\
\hline colrin ablety $7621-93-4$ & $<0.000004 \mathrm{mg} / \mathrm{l}$ & $<0.000004 \mathrm{mg} / \mathrm{l}$ & $<0.000004 \mathrm{mg} / \mathrm{l}$ & $<0.000004 \mathrm{mg} / 1$ & 1 & \\
\hline bopection $76-148$ & $<0.000004 \mathrm{mg} / \mathrm{l}$ & $<0.000004 \mathrm{mg} / \mathrm{l}$ & $<0.000004 \mathrm{mg} / \mathrm{l}$ & $<0.000004 \mathrm{mg} / \mathrm{l}$ & 1 & \\
\hline topation epocido 102157.3 & $<0.000004 \mathrm{mg} / \mathrm{l}$ & $<0.000004 m g / 1$ & $<0.000004 \mathrm{mg} / \mathrm{l}$ & $<0.000004 \mathrm{mg} / 1$ & 1 & \\
\hline 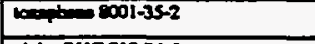 & $<0.0002 \mathrm{mg} / 1$ & $<0.0002 \mathrm{mgl}$ & $<0.0002 \mathrm{mg} / 1$ & $<0.0002 \mathrm{mg} / 1$ & 1 & \\
\hline 4to-BHC 31976 & $<0.000004 \mathrm{mg} / \mathrm{l}$ & $<0.000004 \mathrm{mg} / \mathrm{l}$ & $<0.000004 \mathrm{mg} / \mathrm{l}$ & $<0.000004 \mathrm{mg} / 1$ & 1 & \\
\hline BHC 319857 & $<0.000004 \mathrm{mg} / \mathrm{h}$ & $<0.000004 \mathrm{mg} / \mathrm{l}$ & $<0.000004 \mathrm{mg} / 1$ & $<0.000004 \mathrm{mg} / \mathrm{l}$ & $\mathrm{I}$ & \\
\hline 6h-8HC 319868 & $<0.000004 \mathrm{mg} / \mathrm{I}$ & $<0.000004 \mathrm{mg} / 1$ & $<0.000004 \mathrm{mg} / \mathrm{l}$ & $<0.000004 \mathrm{mg} / 1$ & 1 & \\
\hline 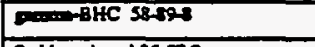 & $<0.00001 \mathrm{mg} / \mathrm{l}$ & $<0.00001 \mathrm{mg} / 1$ & $<0.00001 \mathrm{mg} / 1$ & $<0.00001 \mathrm{mg} / \mathrm{l}$ & 1 & \\
\hline 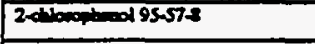 & $<0.030 \mathrm{mg} / \mathrm{h}$ & $<0.030 \mathrm{mg} / \mathrm{h}$ & $<0.030 \mathrm{mg} / \mathrm{l}$ & $<0.030 \mathrm{mg} / 1$ & 1 & \\
\hline Arodoen-121253601-21-9 & $<0.0005 \mathrm{mg} / \mathrm{l}$ & $<0.0005 \mathrm{mg} / 1$ & $<0.0005 \mathrm{mg} / \mathrm{l}$ & $<0.0005 \mathrm{mg} / \mathrm{l}$ & $I$ & \\
\hline 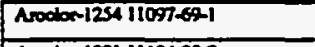 & $<0.0005 \mathrm{mg} / \mathrm{l}$ & $<0.0005 \mathrm{mg} / 1$ & $<0.0005 \mathrm{mg} / 1$ & $<0.0005 \mathrm{mg} / 1$ & 1 & \\
\hline Aroch-121 11104-28-2 & $<0.0005 \mathrm{mg} / 1$ & $<0.0005 \mathrm{mg} / 1$ & $<0.0005 \mathrm{mg} / \mathrm{l}$ & $<0.0005 \mathrm{mg} / \mathrm{l}$ & 1 & \\
\hline Aroction-1232 111141-16-5 & $<0.0005 \mathrm{mg} / \mathrm{l}$ & $<0.0005 \mathrm{mg} / \mathrm{l}$ & $<0.0005 \mathrm{mg} / \mathrm{h}$ & $<0.0005 \mathrm{mg} / 1$ & 1 & \\
\hline Aroctor $124812572-29-6$ & $<0.0005 \mathrm{mg} / \mathrm{l}$ & $<0.0005 \mathrm{mg} / \mathrm{l}$ & $<0.0005 \mathrm{mg} / \mathrm{l}$ & $<0.0005 \mathrm{mg} / \mathrm{l}$ & 1 & \\
\hline Arocior-1260 110\%6-82-5 & $<0.0005 \mathrm{mg} / 1$ & $<0.0005 \mathrm{mg} / \mathrm{l}$ & $<0.0005 \mathrm{mg} / \mathrm{l}$ & $<0.0005 \mathrm{mg} / \mathrm{l}$ & 1 & \\
\hline Aroctor-1016 12674-11-2 & $<0.0005 \mathrm{mg} / \mathrm{l}$ & $<0.0005 \mathrm{mg} / \mathrm{l}$ & $<0.0005 \mathrm{mg} / 1$ & $<0.0005 \mathrm{mg} / 1$ & 1 & \\
\hline \begin{tabular}{|c|c|} 
Cranols \\
\end{tabular} & $<0.030 \mathrm{mg} / 1$ & $<0.030 \mathrm{mg} / \mathrm{l}$ & $<0.030 \mathrm{mg} / 1$ & $<0.030 \mathrm{mg} / \mathrm{l}$ & 1 & \\
\hline Arothis & $<0.010 \mathrm{mg} / 1$ & $<0.010 \mathrm{mg} / \mathrm{l}$ & $<0.010 \mathrm{mg} / \mathrm{l}$ & $<0.010 \mathrm{mg} / \mathrm{l}$ & 1 & \\
\hline Anglooitrik & $<0.010 \mathrm{mg} / \mathrm{l}$ & $<0.010 \mathrm{mg} / \mathrm{l}$ & $<0.010 \mathrm{mg} / \mathrm{l}$ & $<0.010 \mathrm{mg} / \mathrm{l}$ & 1 & \\
\hline 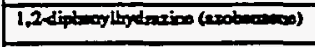 & Recults perding & Recults perding & Reaulus pending & Recults pending & 1 & \\
\hline & & & & & & \\
\hline & & & & & & \\
\hline & & & & & & \\
\hline & & & & & & \\
\hline & & & & & & \\
\hline & & & & & & \\
\hline & & & & & & \\
\hline & & & & & & \\
\hline & & & & & . & \\
\hline & & & & & & \\
\hline & & & & & & \\
\hline & & & & & & \\
\hline & & & & & & \\
\hline & & & & & & \\
\hline & & & & & & \\
\hline & & $-\infty$ & $-\quad \therefore, \therefore$ & $\because \cdots$ & & \\
\hline
\end{tabular}


VI. Discharse Information (coneinued from page 3 of Form 2F)

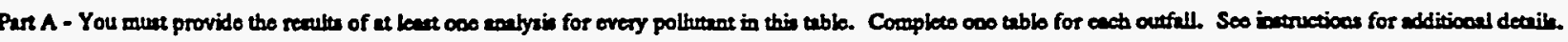

\begin{tabular}{|c|c|c|c|c|c|c|}
\hline \multirow{2}{*}{$\begin{array}{l}\text { Pollutense } \\
\text { and } \\
\text { CAs Numbor } \\
\text { (if aveilible) }\end{array}$} & \multicolumn{2}{|c|}{$\begin{array}{l}\text { Maximum Value } \\
\text { (ixclude unitu) }\end{array}$} & \multicolumn{2}{|c|}{$\begin{array}{l}\text { Averego Veluee } \\
\text { (includo unita) }\end{array}$} & \multirow{2}{*}{$\begin{array}{l}\text { Number } \\
\text { of } \\
\text { Storm } \\
\text { Eveoss } \\
\text { Sempled }\end{array}$} & \multirow[b]{2}{*}{ Sourcese of Polluewe } \\
\hline & $\begin{array}{c}\text { Orab Semplo } \\
\text { Takes During } \\
\text { Fint } 30 \\
\text { Minueare }\end{array}$ & $\begin{array}{l}\text { Flow-wrighted } \\
\text { Compocico }\end{array}$ & $\begin{array}{l}\text { Grab Sample } \\
\text { Taken During } \\
\text { Firnt } 30 \\
\text { Mincteo }\end{array}$ & $\begin{array}{l}\text { Frow-weightiod } \\
\text { Compoxito }\end{array}$ & & \\
\hline Pit and Gom & $<2 \mathrm{mg} / \mathrm{I}$ & & $<2 \mathrm{mg} / \mathrm{l}$ & & 1 & \\
\hline 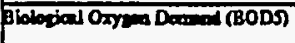 & $<5 \mathrm{mg} / 1$ & $6.3 \mathrm{mg} / \mathrm{l}$ & $<5 \mathrm{mg} / \mathrm{l}$ & $6.3 \mathrm{mg} / \mathrm{l}$ & 1 & \\
\hline 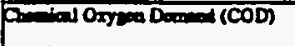 & $62 \mathrm{mg} / \mathrm{l}$ & $47 \mathrm{mg} / \mathrm{l}$ & $62 \mathrm{mg} / \mathrm{l}$ & $47 \mathrm{mg} / 1$ & 1 & \\
\hline Toal Singend Solthe (TSS) & $1300 \mathrm{mg} / \mathrm{l}$ & $430 \mathrm{mg} / 1$ & $1300 \mathrm{mg} / \mathrm{l}$ & $430 \mathrm{mg} / \mathrm{l}$ & 1 & \\
\hline Tonl Kjolthel Nitroger & $1.4 \mathrm{mg} / 1$ & $1.6 \mathrm{mg} / \mathrm{l}$ & $1.4 \mathrm{mg} / \mathrm{l}$ & $1.6 \mathrm{mg} / \mathrm{I}$ & 1 & \\
\hline 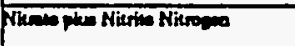 & $1.51 \mathrm{mg} / 1$ & $1.32 \mathrm{mg} / \mathrm{h}$ & $1.51 \mathrm{mg} / 1$ & $1.32 \mathrm{mg} / \mathrm{l}$ & 1 & \\
\hline Toul proponters & $4.2 \mathrm{mg} / \mathrm{l}$ & $1.6 \mathrm{mg} / \mathrm{l}$ & $4.2 \mathrm{mg} / 1$ & $1.6 \mathrm{mg} / \mathrm{l}$ & 1 & \\
\hline PH & Minim 8.0 & Merimin 8.0 & Minimen 8.0 & Meximen 8.0 & & \\
\hline
\end{tabular}

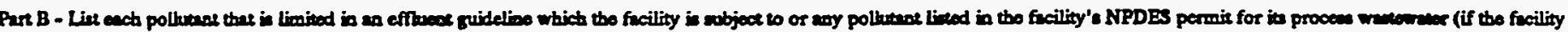

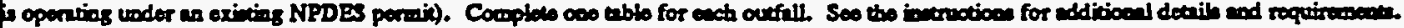

\begin{tabular}{|c|c|c|c|c|c|c|}
\hline \multirow{2}{*}{$\begin{array}{l}\text { Pollunast } \\
\text { nod } \\
\text { CAs Numbor } \\
\text { (if aviliblo) }\end{array}$} & \multicolumn{2}{|c|}{ 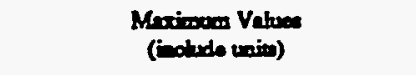 } & \multicolumn{2}{|c|}{ 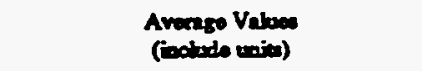 } & \multirow{2}{*}{$\begin{array}{l}\text { Nembue } \\
\text { of } \\
\text { Storm } \\
\text { Eveoce } \\
\text { Sampied }\end{array}$} & \multirow{2}{*}{$\begin{array}{c}: \\
\text { Sources of Pollowes }\end{array}$} \\
\hline & 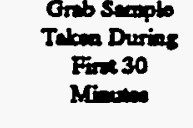 & $\begin{array}{l}\text { Flow-migited } \\
\text { Compoies }\end{array}$ & 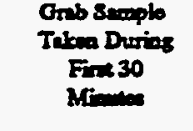 & $\begin{array}{l}\text { Flomerroighted } \\
\text { Composico }\end{array}$ & & \\
\hline 740.360 & $<0.003 \mathrm{mg} / 1$ & $<0.003 \mathrm{mg} / \mathrm{l}$ & $<0.003 \mathrm{mg} / \mathrm{l}$ & $<0.003 \mathrm{mg} / 1$ & 1 & \\
\hline 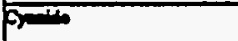 & $0.003 \mathrm{mg} / \mathrm{l}$ & & $0.003 \mathrm{mg} / \mathrm{l}$ & & 1 & \\
\hline romand 7039016 & $0.0011 \mathrm{mg} / 1$ & $0.0014 \mathrm{mg} / \mathrm{l}$ & $0.0011 \mathrm{mg} / \mathrm{l}$ & $0.0014 \mathrm{mg} / \mathrm{l}$ & 1 & \\
\hline ponde & $0.003 \mathrm{mg} / \mathrm{l}$ & & $0.003 \mathrm{mg} / \mathrm{l}$ & & 1 & \\
\hline 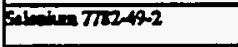 & $<0.002 \mathrm{mg} / \mathrm{l}$ & $<0.002 \mathrm{mg} / \mathrm{l}$ & $<0.002 \mathrm{mg} / \mathrm{l}$ & $<0.002 \mathrm{mg} / \mathrm{l}$ & 1 & \\
\hline Pालme & $<10 \mathrm{mg} / 1$ & $<10 \mathrm{mg} / \mathrm{l}$ & $<10 \mathrm{mg} / \mathrm{l}$ & $<10 \mathrm{mg} / 1$ & 1 & \\
\hline surtronese & $<0.05 \mathrm{mg} / \mathrm{l}$ & $<0.05 \mathrm{mg} / \mathrm{l}$ & $<0.05 \mathrm{mg} / \mathrm{l}$ & $<0.05 \mathrm{mg} / \mathrm{l}$ & 1 & \\
\hline 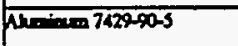 & $17.2 \mathrm{mg} / \mathrm{l}$ & $8.38 \mathrm{mg} / \mathrm{h}$ & $17.2 \mathrm{mg} / \mathrm{l}$ & $8.38 \mathrm{mg} / \mathrm{l}$ & 1 & \\
\hline 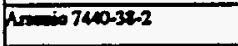 & $<0.04 \mathrm{mg} / 1$ & $<0.04 \mathrm{mg} / \mathrm{l}$ & $<0.04 \mathrm{mg} / \mathrm{l}$ & $<0.04 \mathrm{mg} / \mathrm{l}$ & 1 & \\
\hline $30009740-128$ & $0.025 \mathrm{mg} / \mathrm{l}$ & $0.019 \mathrm{mg} / \mathrm{l}$ & $0.025 \mathrm{mg} / \mathrm{l}$ & $0.019 \mathrm{mg} / \mathrm{l}$ & 1 & \\
\hline Berplitum 7440-11-7 & $0.0011 \mathrm{mg} / 1$ & $0.0005 \mathrm{mg} / \mathrm{l}$ & $0.0011 \mathrm{mg} / 1$ & $0.0005 \mathrm{mg} / 1$ & 1 & \\
\hline anding 740-13-9 & $<0.004 \mathrm{mg} / \mathrm{l}$ & $<0.004 \mathrm{mg} / 1$ & $<0.004 \mathrm{mg} / \mathrm{l}$ & $<0.004 \mathrm{mg} / \mathrm{l}$ & 1 & \\
\hline alach $7400-\pi, 2$ & $266 \mathrm{mg} / 1$ & $104 \mathrm{mg} / \mathrm{l}$ & $266 \mathrm{mg} / \mathrm{l}$ & $104 \mathrm{mg} / \mathrm{l}$ & 1 & \\
\hline and $740-17-3$ & $0.028 \mathrm{mg} / 1$ & $0.014 \mathrm{mg} / \mathrm{l}$ & $0.028 \mathrm{mg} / 1$ & $0.014 \mathrm{mg} / \mathrm{l}$ & 1 & \\
\hline 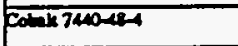 & $0.015 \mathrm{mg} / \mathrm{l}$ & $0.007 \mathrm{mg} / \mathrm{l}$ & $0.015 \mathrm{mg} / \mathrm{l}$ & $0.007 \mathrm{mg} / \mathrm{l}$ & 1 & \\
\hline comprasasos & $0.034 \mathrm{mg} / \mathrm{h}$ & $0.019 \mathrm{mg} / \mathrm{l}$ & $0.034 \mathrm{mg} / \mathrm{l}$ & $0.019 \mathrm{mg} / \mathrm{l}$ & 1 & \\
\hline 1007420296 & $21.1 \mathrm{mg} / 1$ & $10.4 \mathrm{mg} / \mathrm{h}$ & $21.1 \mathrm{mg} / 1$ & $10.4 \mathrm{mg} / \mathrm{l}$ & 1 & \\
\hline Im 7032-21 & $0.05 \mathrm{mg} / 1$ & $0.02 \mathrm{mg} / 1$ & $0.05 \mathrm{mg} / \mathrm{l}$ & $0.02 \mathrm{mg} / 1$ & 1 & \\
\hline 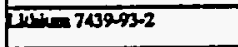 & $0.03 \mathrm{mg} / \mathrm{l}$ & $<0.02 \mathrm{mg} / \mathrm{l}$ & $0.03 \mathrm{mg} / \mathrm{l}$ & $<0.02 \mathrm{mg} / \mathrm{l}$ & 1 & \\
\hline 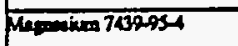 & $13.4 \mathrm{mg} / 1$ & $7.38 \mathrm{mg} / 1$ & $13.4 \mathrm{mg} / 1$ & $7.38 \mathrm{mg} / \mathrm{l}$ & 1 & \\
\hline Mencosen 720996.5 & $0.412 \mathrm{mg} / \mathrm{l}$ & $0.217 \mathrm{mg} / 1$ & $0.412 \mathrm{mg} / 1$ & $0.217 \mathrm{mg} / 1$ & 1 & \\
\hline Mobfdeam 7439969 & $<0.006 \mathrm{mg} / \mathrm{l}$ & $<0.006 \mathrm{mg} / \mathrm{l}$ & $<0.006 \mathrm{mg} / \mathrm{l}$ & $<0.006 \mathrm{mg} / \mathrm{l}$ & 1 & \\
\hline$\sqrt{104} 17400020$ & $0.029 \mathrm{mg} / \mathrm{l}$ & $0.014 \mathrm{mg} / \mathrm{l}$ & $0.029 \mathrm{mg} / \mathrm{l}$ & $0.014 \mathrm{mg} / \mathrm{l}$ & 1 & \\
\hline 740027 & $11.8 \mathrm{mg} / 1$ & $9.3 \mathrm{mg} / \mathrm{l}$ & $11.8 \mathrm{mg} / \mathrm{h}$ & $9.3 \mathrm{mg} / \mathrm{h}$ & 1 & \\
\hline $30=740-204$ & $<0.006 \mathrm{mg} / \mathrm{l}$ & $<0.006 \mathrm{mg} / 1$ & $<0.006 \mathrm{mg} / \mathrm{l}$ & $<0.006 \mathrm{mg} / \mathrm{l}$ & 1 & \\
\hline 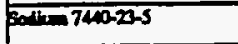 & $0.51 \mathrm{mg} / 1$ & $0.76 \mathrm{mg} / \mathrm{l}$ & $0.51 \mathrm{mg} / \mathrm{l}$ & $0.76 \mathrm{mg} / \mathrm{h}$ & 1 & \\
\hline $740-326$ & $0.14 \mathrm{mg} / \mathrm{I}$ & $0.08 \mathrm{mg} / \mathrm{l}$ & $0.14 \mathrm{mg} / \mathrm{l}$ & $0.08 \mathrm{mg} / \mathrm{l}$ & 1 & \\
\hline 2100740666 & $0.15 \mathrm{mg} / \mathrm{l}$ & $0.09 \mathrm{mg} / \mathrm{l}$ & $0.15 \mathrm{mg} / \mathrm{l}$ & $0.09 \mathrm{mg} / \mathrm{l}$ & 1 & \\
\hline
\end{tabular}




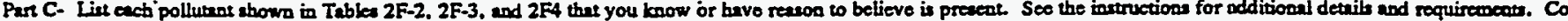
ono teble for each outfill.

\begin{tabular}{|c|c|c|c|c|c|c|}
\hline \multirow{2}{*}{$\begin{array}{l}\text { Polhume } \\
\text { and } \\
\text { CAS Number } \\
\text { (if availible) }\end{array}$} & \multicolumn{2}{|c|}{$\begin{array}{l}\text { Meximinn Values } \\
\text { (include unite) }\end{array}$} & \multicolumn{2}{|c|}{$\begin{array}{l}\text { Avernge Values } \\
\text { (inctude unitu) }\end{array}$} & \multirow{2}{*}{$\begin{array}{l}\text { Number } \\
\text { of } \\
\text { Storm } \\
\text { Events } \\
\text { Sumpled }\end{array}$} & \multirow[b]{2}{*}{ Sources of Pollutants } \\
\hline & $\begin{array}{c}\text { Grab Sumple } \\
\text { Taken During } \\
\text { Fint } 30 \\
\text { Minutes }\end{array}$ & $\begin{array}{l}\text { Flow-weighted } \\
\text { Comporite }\end{array}$ & $\begin{array}{l}\text { Grab Semple } \\
\text { Takea During } \\
\text { Fint } 30 \\
\text { Minutea }\end{array}$ & $\begin{array}{c}\text { Flow-weighted } \\
\text { Componito }\end{array}$ & & \\
\hline 1.1.1.-tnctiorocthen 71-55-6 & $<0.010 \mathrm{mg} / \mathrm{l}$ & & $<0.010 \mathrm{mg} / 1$ & 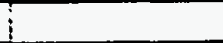 & 1 & \\
\hline 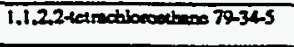 & $<0.010 \mathrm{mg} / 1$ & & $<0.010 \mathrm{mg} / 1$ & ! & 1 & \\
\hline 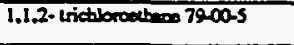 & $<0.010 \mathrm{mg} / \mathrm{l}$ & & $<0.010 \mathrm{mg} / \mathrm{l}$ & & 1 & \\
\hline 1,1 dictioncedtem $75-34-3$ & $<0.010 \mathrm{mg} / 1$ & & $<0.010 \mathrm{mg} / 1$ & & 1 & \\
\hline 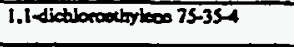 & $<0.010 \mathrm{mg} / \mathrm{l}$ & & $<0.010 \mathrm{mg} / \mathrm{l}$ & & 1 & \\
\hline 1.2 dietbroet $107-06-2$ & $<0.010 \mathrm{mg} / \mathrm{l}$ & & $<0.010 \mathrm{mg} / \mathrm{l}$ & & 1 & \\
\hline 1.2 dictloropioperes 78-87-5 & $<0.010 \mathrm{mg} / \mathrm{l}$ & & $<0.010 \mathrm{mg} / 1$ & 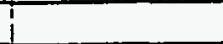 & 1 & \\
\hline 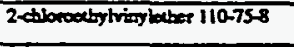 & $<0.010 \mathrm{mg} / \mathrm{l}$ & & $<0.010 \mathrm{mg} / 1$ & 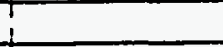 & 1 & \\
\hline $600000000413-2$ & $<0.010 \mathrm{mg} / \mathrm{l}$ & & $<0.010 \mathrm{mg} / \mathrm{l}$ & & 1 & \\
\hline browodichlorematume 75-274 & $<0.010 \mathrm{mg} / 1$ & & $<0.010 \mathrm{mg} / 1$ & i & 1 & \\
\hline brocosiond $75-25-2$ & $<0.010 \mathrm{mg} / \mathrm{l}$ & & $<0.010 \mathrm{mg} / 1$ & $!$ & 1 & \\
\hline 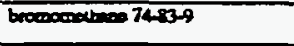 & $<0.010 \mathrm{mg} / \mathrm{l}$ & & $<0.010 \mathrm{mg} / \mathrm{l}$ & & 1 & \\
\hline 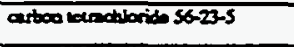 & $<0.010 \mathrm{mg} / \mathrm{l}$ & & $<0.010 \mathrm{mg} / 1$ & & 1 & \\
\hline chlonotacosocos $10890-7$ & $<0.010 \mathrm{mg} / 1$ & & $<0.010 \mathrm{mg} / \mathrm{l}$ & 1 & 1 & \\
\hline chacoutane 75003 & $<0.010 \mathrm{mg} / \mathrm{l}$ & & $<0.010 \mathrm{mg} / \mathrm{l}$ & 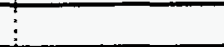 & 1 & \\
\hline chloroform $67.66-3$ & $<0.010 \mathrm{mg} / \mathrm{I}$ & & $<0.010 \mathrm{mg} / 1$ & 1 & 1 & i \\
\hline $7487+3$ & $<0.010 \mathrm{mg} / \mathrm{l}$ & & $<0.010 \mathrm{mg} / 1$ & & 1 & \\
\hline 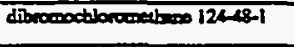 & $<0.010 \mathrm{mg} / 1$ & & $<0.010 \mathrm{mg} / \mathrm{l}$ & & 1 & \\
\hline 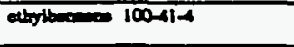 & $<0.010 \mathrm{mg} / \mathrm{l}$ & & $<0.010 \mathrm{mg} / 1$ & & 1 & \\
\hline bluesos $108-823$ & $<0.010 \mathrm{mg} / \mathrm{l}$ & & $<0.010 \mathrm{mg} / \mathrm{l}$ & & 1 & \\
\hline vish chbile 73014 & $<0.010 \mathrm{mg} / \mathrm{l}$ & & $<0.010 \mathrm{mg} / 1$ & & 1 & \\
\hline 1.3-dichionopicpere 10061-01-5 & $<0.010 \mathrm{mg} / 1$ & & $<0.010 \mathrm{mg} / \mathrm{l}$ & & 1 & \\
\hline 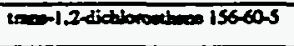 & $<0.010 \mathrm{mg} / \mathrm{l}$ & & $<0.010 \mathrm{mg} / 1$ & & 1 & \\
\hline uphe sativity $12557-46-1$ & $69 \mathrm{poi} / \mathrm{l}$ & 17 peill & 69 peill & 17 peill & 1 & \\
\hline bedivity $1258746-1$ & $76 \mathrm{pcill}$ & 33 pcill & 76 peill & 33 peill & 1 & \\
\hline focal ooliform & $102,000 \mathrm{co} / .1 \mathrm{~L}$ & $165,000 \mathrm{co} / .1 \mathrm{~L}$ & $102,000 \mathrm{col} .1 \mathrm{~L}$ & $165,000 \mathrm{col} .1 \mathrm{~L}$ & 1 & \\
\hline Thallinen 7400-280 & $<0.001 \mathrm{mg} / !$ & $<0.001 \mathrm{mg} / \mathrm{l}$ & $<0.001 \mathrm{mg} / \mathrm{l}$ & $<0.001 \mathrm{mg} / \mathrm{l}$ & 1 & \\
\hline Unasing 74na-61-1 & $0.008 \mathrm{mg} / \mathrm{l}$ & $0.005 \mathrm{mg} / \mathrm{l}$ & $0.008 \mathrm{mg} / \mathrm{l}$ & $0.005 \mathrm{mg} / \mathrm{l}$ & 1 & \\
\hline Geromedinity & $38 \mathrm{pci} / 1$ & 23 pcill & $38 \mathrm{pci} / 1$ & 23 pcill & 1 & \\
\hline
\end{tabular}

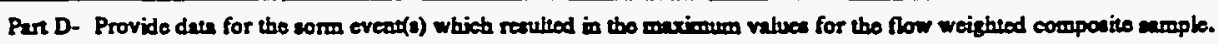

\begin{tabular}{|c|c|c|c|c|c|c|c|}
\hline $\begin{array}{l}1 . \\
\text { Deto of } \\
\text { Storm } \\
\text { Eveat }\end{array}$ & $\begin{array}{l}2 . \\
\text { Durntion } \\
\text { of Storm } \\
\text { (in } \\
\text { minutea) }\end{array}$ & $\begin{array}{l}3 . \\
\text { Toul rainfill } \\
\text { during stom eveat } \\
\text { (in iobed) }\end{array}$ & $\begin{array}{l}\text { N. } \\
\text { Number of bour between } \\
\text { begingins of atom mean } \\
\text { ured and end of previous } \\
\text { menurable nin event }\end{array}$ & $\begin{array}{l}\text { S. } \\
\text { Merimum flow nte } \\
\text { during min event } \\
\text { (oallocu/min or specify } \\
\text { unim) }\end{array}$ & $\begin{array}{l}6 . \\
\text { Total flow from } \\
\text { nin avent } \\
\text { (olloos or } \\
\text { ppeoify units) }\end{array}$ & $\begin{array}{c}7 . \\
\text { Senson } \\
\text { semplo was } \\
\text { taked }\end{array}$ & $\begin{array}{l}\text { 8. } \\
\text { Fonm of } \\
\text { Procipitution } \\
\text { (rinfill. } \\
\text { soowroll) }\end{array}$ \\
\hline $6 / 4 / 92$ & 210 & 1 & 128.8 & 48.4 & 1,497 & Spring & Rainfall \\
\hline
\end{tabular}

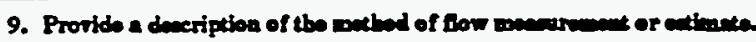

An ISCO 3230 Flow meter wes used to obtain water level readings. This instrument was calibrated with the normal iry weather flow, if preant, as lev6 zero. The flow meter was programmed with the characteristics of the conveyance so that flow rates and total Ilows conld be colculated by the meter. 
Outfall 7

Parte B and C Contioned

\begin{tabular}{|c|c|c|c|c|c|c|}
\hline \multirow{2}{*}{$\begin{array}{l}\text { Polluanse } \\
\text { and } \\
\text { CAs Number } \\
\text { (if avilibie) }\end{array}$} & \multicolumn{2}{|c|}{$\begin{array}{l}\text { Meximum Valuce } \\
\text { (includo uniw) }\end{array}$} & \multicolumn{2}{|c|}{$\begin{array}{l}\text { Averago Valuce } \\
\text { (inctude units) }\end{array}$} & \multirow{2}{*}{$\begin{array}{l}\text { Number } \\
\text { of } \\
\text { Storm: } \\
\text { Eveous } \\
\text { Sempled }\end{array}$} & \multirow[b]{2}{*}{ Sourcese of Polhutante } \\
\hline & $\begin{array}{l}\text { Grab Semplo } \\
\text { Taken Durizs } \\
\text { Firat } 30 \\
\text { Minuteo }\end{array}$ & $\begin{array}{c}\text { Fonv-weighted } \\
\text { Compocits }\end{array}$ & $\begin{array}{l}\text { Grab Semple } \\
\text { Takeo Duriag } \\
\text { Firse } 30 \\
\text { Minerce }\end{array}$ & $\begin{array}{c}\text { Flow-weighted } \\
\text { Comporito }\end{array}$ & & \\
\hline Berium 740-39-3 & $0.179 \mathrm{mg} / \mathrm{l}$ & $0.0859 \mathrm{mg} / 1$ & $0.179 \mathrm{mg} / 1$ & $0.0859 \mathrm{mg} / \mathrm{I}$ & 1 & \\
\hline 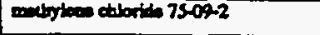 & $<0.010 \mathrm{mg} / \mathrm{ll}$ & & $<0.010 \mathrm{mg} / \mathrm{l}$ & & 1 & \\
\hline 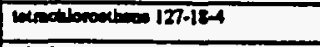 & $<0.010 \mathrm{mg} / 1$ & & $<0.010 \mathrm{mg} / 1$ & & 1 & \\
\hline 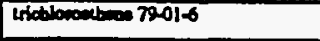 & $<0.010 \mathrm{mg} / \mathrm{l}$ & & $<0.010 \mathrm{mg} / 1$ & & 1 & \\
\hline Xylemen & $<0.010 \mathrm{mg} / \mathrm{l}$ & & $<0.010 \mathrm{mg} / \mathrm{l}$ & & 1 & \\
\hline 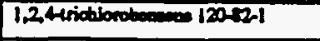 & $<0.010 \mathrm{mg} / 1$ & $<0.010 \mathrm{mg} / \mathrm{l}$ & $<0.010 \mathrm{mg} / \mathrm{l}$ & $<0.010 \mathrm{mg} / 1$ & 1 & \\
\hline 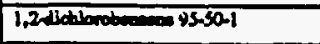 & $<0.010 \mathrm{mg} / \mathrm{l}$ & $<0.010 \mathrm{mg} / \mathrm{l}$ & $<0.010 \mathrm{mg} / \mathrm{l}$ & $<0.010 \mathrm{mg} / 1$ & 1 & \\
\hline 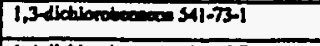 & $<0.010 \mathrm{mg} / \mathrm{h}$ & $<0.010 \mathrm{mg} / 1$ & $<0.010 \mathrm{mg} / \mathrm{l}$ & $<0.010 \mathrm{mg} / \mathrm{l}$ & 1 & \\
\hline 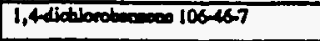 & $<0.010 \mathrm{mg} / 1$ & $<0.010 \mathrm{mg} / 1$ & $<0.010 \mathrm{mg} / \mathrm{l}$ & $<0.010 \mathrm{mg} / 1$ & 1 & \\
\hline 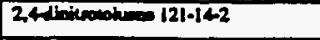 & $<0.010 \mathrm{mg} / \mathrm{l}$ & $<0.010 \mathrm{mg} / \mathrm{l}$ & $<0.010 \mathrm{mg} / \mathrm{t}$ & $<0.010 \mathrm{mg} / \mathrm{l}$ & 1 & \\
\hline 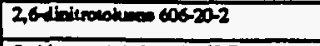 & $<0.010 \mathrm{mg} / \mathrm{l}$ & $<0.010 \mathrm{mg} / \mathrm{l}$ & $<0.010 \mathrm{mg} / \mathrm{t}$ & $<0.010 \mathrm{mg} / \mathrm{l}$ & 1 & \\
\hline 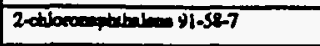 & $<0.010 \mathrm{mg} / 1$ & $<0.010 \mathrm{mg} / 1$ & $<0.010 \mathrm{mg} / \mathrm{l}$ & $<0.010 \mathrm{mg} / \mathrm{l}$ & $1 \cdot$ & \\
\hline 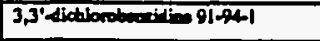 & $<0.010 \mathrm{mg} / 1$ & $<0.010 \mathrm{mg} / 1$ & $<0.010 \mathrm{mg} / 1$ & $<0.010 \mathrm{mg} / 1$ & 1 & \\
\hline 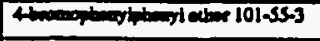 & $<0.010 \mathrm{mg} / 1$ & $<0.010 \mathrm{mg} / 1$ & $<0.010 \mathrm{mg} / 1$ & $<0.010 \mathrm{mg} / \mathrm{l}$ & 1 & \\
\hline 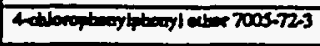 & $<0.010 \mathrm{mg} / 1$ & $<0.010 \mathrm{mg} / 1$ & $<0.010 \mathrm{mg} / \mathrm{l}$ & $<0.010 \mathrm{mg} / 1$ & $I$ & \\
\hline 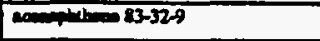 & $<0.010 \mathrm{mg} / 1$ & $<0.010 \mathrm{mg} / 1$ & $<0.010 \mathrm{mg} / 1$ & $<0.010 \mathrm{mg} / 1$ & 1 & \\
\hline 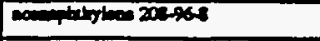 & $<0.010 \mathrm{mg} / 1$ & $<0.010 \mathrm{mg} / 1$ & $<0.010 \mathrm{mg} / \mathrm{l}$ & $<0.010 \mathrm{mg} / \mathrm{l}$ & 1 & \\
\hline $120,12.7$ & $<0.010 \mathrm{mg} / \mathrm{l}$ & $<0.010 \mathrm{mg} / 1$ & $<0.010 \mathrm{mg} / 1$ & $<0.010 \mathrm{mg} / \mathrm{h}$ & 1 & $\cdot$ \\
\hline millowas.5 & $<0.030 \mathrm{mg} / \mathrm{l}$ & $<0.030 \mathrm{mg} / \mathrm{l}$ & $<0.030 \mathrm{mg} / \mathrm{l}$ & $<0.030 \mathrm{mg} / \mathrm{ll}$ & 1 & \\
\hline 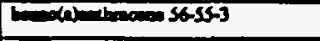 & $<0.010 \mathrm{mg} / \mathrm{h}$ & $<0.010 \mathrm{mg} / \mathrm{l}$ & $<0.010 \mathrm{mg} / 1$ & $<0.010 \mathrm{mg} / 1$ & 1 & \\
\hline 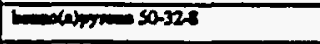 & $<0.010 \mathrm{mg} / 1$ & $<0.010 \mathrm{mg} / 1$ & $<0.010 \mathrm{mg} / 1$ & $<0.010 \mathrm{mg} / 1$ & 1 & \\
\hline 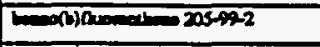 & $<0.010 \mathrm{mg} / 1$ & $<0.010 \mathrm{mg} / 1$ & $<0.010 \mathrm{mg} / \mathrm{l}$ & $<0.010 \mathrm{mg} / \mathrm{l}$ & 1 & \\
\hline heo(chi)porybes 191.242 & $<0.010 \mathrm{mg} / \mathrm{l}$ & $<0.010 \mathrm{mg} / \mathrm{L}$ & $<0.010 \mathrm{mg} / 1$ & $<0.010 \mathrm{mg} / 1$ & 1 & \\
\hline 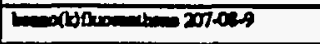 & $<0.010 \mathrm{mg} / \mathrm{ll}$ & $<0.010 \mathrm{mg} / 1$ & $<0.010 \mathrm{mg} / \mathrm{l}$ & $<0.010 \mathrm{mg} / \mathrm{l}$ & 1 & \\
\hline 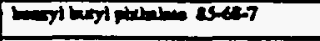 & $<0.010 \mathrm{mg} / 1$ & $<0.010 \mathrm{mg} / 1$ & $<0.010 \mathrm{mg} / \mathrm{l}$ & $<0.010 \mathrm{mg} / 1$ & 1 & \\
\hline 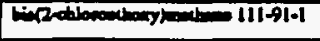 & $<0.010 \mathrm{mg} / \mathrm{l}$ & $<0.010 \mathrm{mg} / 1$ & $<0.010 \mathrm{mg} / 1$ & $<0.010 \mathrm{mg} / 1$ & 1 & \\
\hline 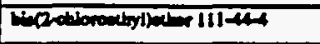 & $<0.010 \mathrm{mg} / \mathrm{t}$ & $<0.010 \mathrm{mg} / \mathrm{h}$ & $<0.010 \mathrm{mg} / \mathrm{l}$ & $<0.010 \mathrm{mg} / \mathrm{l}$ & 1 & \\
\hline 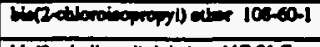 & $<0.010 \mathrm{mg} / \mathrm{l}$ & $<0.010 \mathrm{mg} / \mathrm{l}$ & $<0.010 \mathrm{mg} / \mathrm{I}$ & $<0.010 \mathrm{mg} / \mathrm{l}$ & 1 & \\
\hline 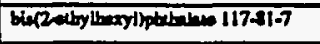 & $<0.010 \mathrm{mg} / \mathrm{l}$ & $<0.010 \mathrm{mg} / \mathrm{l}$ & $<0.010 \mathrm{mg} / 1$ & $<0.010 \mathrm{mg} / \mathrm{l}$ & 1 & \\
\hline argenes 21801-9 & $<0.010 \mathrm{mg} / \mathrm{l}$ & $<0.010 \mathrm{mg} / \mathrm{l}$ & $<0.010 \mathrm{mg} / 1$ & $<0.010 \mathrm{mg} / 1$ & 1 & \\
\hline 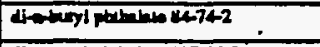 & $<0.010 \mathrm{mg} / \mathrm{l}$ & $<0.010 \mathrm{mg} / 1$ & $<0.010 \mathrm{mg} / \mathrm{l}$ & $<0.010 \mathrm{mg} / \mathrm{h}$ & 1 & \\
\hline 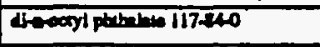 & $<0.010 \mathrm{mg} / \mathrm{l}$ & $<0.010 \mathrm{mg} / \mathrm{h}$ & $<0.010 \mathrm{mg} / 1$ & $<0.010 \mathrm{mg} / 1$ & 1 & \\
\hline 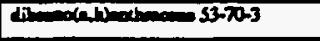 & $<0.010 \mathrm{mg} / 1$ & $<0.010 \mathrm{mg} / \mathrm{l}$ & $<0.010 \mathrm{mg} / \mathrm{h}$ & $<0.010 \mathrm{mg} / 1$ & 1 & \\
\hline 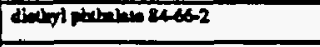 & $<0.010 \mathrm{mg} / \mathrm{h}$ & $<0.010 \mathrm{mg} / \mathrm{l}$ & $<0.010 \mathrm{mg} / 1$ & $<0.010 \mathrm{mg} / 1$ & 1 & \\
\hline 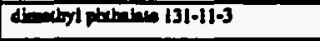 & $<0.010 \mathrm{mg} / \mathrm{l}$ & $<0.010 \mathrm{mg} / 1$ & $<0.010 \mathrm{mg} / 1$ & $<0.010 \mathrm{mg} / \mathrm{l}$ & 1 & \\
\hline Anowion $20 \times 160$ & $<0.010 \mathrm{mg} / \mathrm{l}$ & $<0.010 \mathrm{mg} / 1$ & $<0.010 \mathrm{mg} / 1$ & $<0.010 \mathrm{mg} / 1$ & 1 & \\
\hline $260=00-73-7$ & $<0.010 \mathrm{mg} / \mathrm{l}$ & $<0.010 \mathrm{mg} / 1$ & $<0.010 \mathrm{mg} / 1$ & $<0.010 \mathrm{mg} / 1$ & 1 & \\
\hline 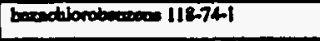 & $<0.010 \mathrm{mg} / \mathrm{h}$ & $<0.010 \mathrm{mg} / \mathrm{l}$ & $<0.010 \mathrm{mg} / \mathrm{l}$ & $<0.010 \mathrm{mg} / l$ & 1 & \\
\hline 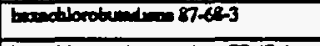 & $<0.010 \mathrm{mg} / 1$ & $<0.010 \mathrm{mg} / \mathrm{l}$ & $<0.010 \mathrm{mg} / 1$ & $<0.010 \mathrm{mg} / \mathrm{l}$ & 1 & \\
\hline 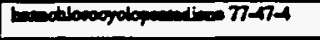 & $<0.010 \mathrm{mg} / 1$ & $<0.010 \mathrm{mg} / 1$ & $<0.010 \mathrm{mg} / 1$ & $<0.010 \mathrm{mg} / \mathrm{I}$ & 1 & \\
\hline 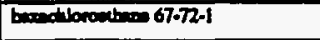 & $<0.010 \mathrm{mg} / 1$ & $<0.010 \mathrm{mg} / 1$ & $<0.010 \mathrm{mg} / \mathrm{l}$ & $<0.010 \mathrm{mg} / 1$ & 1 & \\
\hline 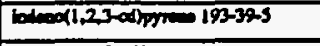 & $<0.010 \mathrm{mg} / \mathrm{t}$ & $<0.010 \mathrm{mg} / 1$ & $<0.010 \mathrm{mg} / \mathrm{l}$ & $<0.010 \mathrm{mg} / 1$ & 1 & \\
\hline Leplonocos 72-59-1 & $<0.010 \mathrm{mg} / \mathrm{l}$ & $<0.010 \mathrm{mg} / \mathrm{l}$ & $<0.010 \mathrm{mg} / \mathrm{l}$ & $<0.010 \mathrm{mg} / 1$ & 1 & \\
\hline 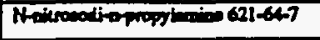 & $<0.010 \mathrm{mg} / 1$ & $<0.010 \mathrm{mg} / 1$ & $<0.010 \mathrm{mg} / \mathrm{l}$ & $<0.010 \mathrm{mg} / 1$ & 1 & \\
\hline 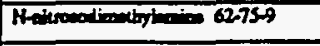 & $<0.010 \mathrm{mg} / \mathrm{l}$ & $<0.010 \mathrm{mg} / \mathrm{l}$ & $<0.010 \mathrm{mg} / \mathrm{I}$ & $<0.010 \mathrm{mg} / 1$ & 1 & \\
\hline 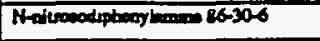 & $<0.010 \mathrm{mg} / \mathrm{l}$ & $<0.010 \mathrm{mg} / \mathrm{l}$ & $<0.010 \mathrm{mg} / \mathrm{l}$ & $<0.010 \mathrm{mg} / 1$ & 1 & \\
\hline $91-20.3$ & $<0.010 \mathrm{mg} / \mathrm{ll}$ & $<0.010 \mathrm{mg} / \mathrm{ll}$ & $<0.010 \mathrm{mg} / \mathrm{l}$ & $<0.010 \mathrm{mg} / \mathrm{l}$ & 1 & \\
\hline 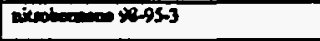 & $<0.010 \mathrm{mg} / \mathrm{l}$ & $<0.010 \mathrm{mg} / \mathrm{t}$ & $<0.010 \mathrm{mg} / \mathrm{l}$ & $<0.010 \mathrm{mg} / 1$ & 1 & \\
\hline 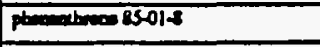 & $<0.010 \mathrm{mg} / 1$ & $<0.010 \mathrm{mg} / \mathrm{l}$ & $<0.010 \mathrm{mg} / 1$ & $<0.010 \mathrm{mg} / \mathrm{l}$ & 1 & \\
\hline $770=129000$ & $<0.010 \mathrm{mg} / \mathrm{l}$ & $<0.010 \mathrm{mg} / 1$ & $<0.010 \mathrm{mg} / \mathrm{l}$ & $<0.010 \mathrm{mg} / \mathrm{l}$ & 1 & \\
\hline 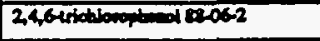 & $<0.030 \mathrm{mg} / 1$ & $<0.030 \mathrm{mg} / \mathrm{l}$ & $<0.030 \mathrm{mg} / \mathrm{l}$ & $<0.030 \mathrm{mg} / 1$ & 1 & \\
\hline 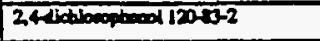 & $<0.030 \mathrm{mg} / \mathrm{l}$ & $<0.030 \mathrm{mg} / 1$ & $<0.030 \mathrm{mg} / 1$ & $<0.030 \mathrm{mg} / \mathrm{l}$ & 1 & \\
\hline 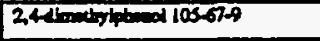 & $<0.030 \mathrm{mg} / 1$ & $<0.030 \mathrm{mg} / 1$ & $<0.030 \mathrm{mg} / 1$ & $<0.030 \mathrm{mg} / 1$ & 1 & \\
\hline 2,4tiolisophead S1-225s & $<0.030 \mathrm{mg} / \mathrm{l}$ & $<0.030 \mathrm{mg} / \mathrm{l}$ & $<0.030 \mathrm{mg} / \mathrm{l}$ & $<0.030 \mathrm{mg} / 1$ & 1 & \\
\hline
\end{tabular}


Outfall 7

Part B and C Continued

\begin{tabular}{|c|c|c|c|c|c|c|}
\hline \multirow{2}{*}{$\begin{array}{l}\text { Pollutunt } \\
\text { and } \\
\text { CAs Number } \\
\text { (if nvailiblo) }\end{array}$} & \multicolumn{2}{|c|}{$\begin{array}{l}\text { Maximan Values } \\
\text { (inchudo unite) }\end{array}$} & \multicolumn{2}{|c|}{$\begin{array}{l}\text { Avengo Vabue } \\
\text { (iveludo unito) }\end{array}$} & \multirow{2}{*}{$\begin{array}{l}\text { Nurober } \\
\text { of } \\
\text { Stonm } \\
\text { Events } \\
\text { Seropled }\end{array}$} & \multirow[b]{2}{*}{ Soursoen of Polknemen } \\
\hline & $\begin{array}{l}\text { Oreb Sample } \\
\text { Theon During } \\
\text { Fint } 30 \\
\text { Minute }\end{array}$ & $\begin{array}{l}\text { Flowmeighed } \\
\text { Compotito }\end{array}$ & $\begin{array}{l}\text { Grab Semplo } \\
\text { Takeo Durings } \\
\text { Firnt } 30 \\
\text { Minutes }\end{array}$ & $\begin{array}{l}\text { Flom-maighted } \\
\text { Compotins }\end{array}$ & & \\
\hline 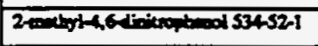 & $<0.030 \mathrm{mg} / \mathrm{l}$ & $<0.030 \mathrm{mg} / \mathrm{l}$ & $<0.030 \mathrm{mg} / \mathrm{l}$ & $<0.030 \mathrm{mg} / \mathrm{l}$ & 1 & \\
\hline 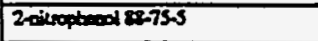 & $<0.030 \mathrm{mg} / \mathrm{l}$ & $<0.030 \mathrm{mg} / \mathrm{l}$ & $<0.030 \mathrm{mg} / 1$ & $<0.030 \mathrm{mg} / \mathrm{l}$ & 1 & \\
\hline 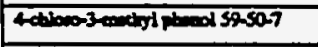 & $<0.030 \mathrm{mg} / \mathrm{h}$ & $<0.030 \mathrm{mg} / \mathrm{h}$ & $<0.030 \mathrm{mg} / \mathrm{h}$ & $<0.030 \mathrm{mg} / \mathrm{l}$ & 1 & \\
\hline 4ricopolined 1000007 & $<0.030 \mathrm{mg} / 1$ & $<0.030 \mathrm{mg} / \mathrm{l}$ & $<0.030 \mathrm{mg} / \mathrm{t}$ & $<0.030 \mathrm{mg} / \mathrm{h}$ & 1 & \\
\hline 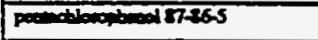 & $<0.030 \mathrm{mg} / 1$ & $<0.030 \mathrm{mg} / \mathrm{l}$ & $<0.030 \mathrm{mg} / \mathrm{l}$ & $<0.030 \mathrm{mg} / \mathrm{l}$ & 1 & \\
\hline Pbed los-85-2 & $<0.030 \mathrm{mg} / \mathrm{l}$ & $<0,030 \mathrm{mg} / \mathrm{l}$ & $<0.030 \mathrm{mg} / \mathrm{h}$ & $<0.030 \mathrm{mg} / \mathrm{l}$ & $I$ & \\
\hline 4,400007254 & $<0.00001 \mathrm{mg} / \mathrm{l}$ & $<0.00001 \mathrm{mg} / \mathrm{l}$ & $<0.00001 \mathrm{mg} /$ & $<0.00001 \mathrm{mg} / \mathrm{l}$ & 1 & \\
\hline 4,4'DDEE 72-S5-9 & $<0.00001 \mathrm{mg} / \mathrm{l}$ & $<0.00001 \mathrm{mg} / 1$ & $<0.00001 \mathrm{mg} / \mathrm{l}$ & $<0.00001 \mathrm{mg} / \mathrm{l}$ & 1 & \\
\hline 4,4-DDT $50-28-3$ & $<0.00001 \mathrm{mg} / 1$ & $<0.00001 \mathrm{mg} / \mathrm{I}$ & $<0.00001 \mathrm{mg} / \mathrm{l}$ & $<0.00001 \mathrm{mg} / \mathrm{l}$ & 1 & \\
\hline aldin 30900-2 & $<0.00001 \mathrm{mg} / \mathrm{l}$ & $<0.00001 \mathrm{mg} / \mathrm{l}$ & $<0.00001 \mathrm{mg} / \mathrm{l}$ & $<0.00001 \mathrm{mg} / \mathrm{l}$ & 1 & \\
\hline chedine 57.749 & $<0.00004 \mathrm{mg} / \mathrm{l}$ & $<0.00004 \mathrm{mg} / \mathrm{l}$ & $<0.00004 \mathrm{mg} / \mathrm{l}$ & $<0.00004 \mathrm{mg} / 1$ & 1 & \\
\hline dentrie 60.57-1 & $<0.00001 \mathrm{mg} / \mathrm{l}$ & $<0.00001 \mathrm{mg} / 1$ & $<0.00001 \mathrm{mg} / \mathrm{l}$ & $<0.00001 \mathrm{mg} / \mathrm{l}$ & 1 & \\
\hline 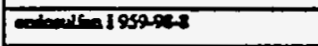 & $<0.00001 \mathrm{mg} / \mathrm{l}$ & $<0.00001 \mathrm{mg} / 1$ & $<0.00001 \mathrm{mg} / \mathrm{l}$ & $<0.00001 \mathrm{mg} / \mathrm{h}$ & 1 & \\
\hline andina 33213-65-9 & $<0.00001 \mathrm{mg} / \mathrm{l}$ & $<0.00001 \mathrm{mg} / 1$ & $<0.00001 \mathrm{mg} / 1$ & $<0.00001 \mathrm{mg} / \mathrm{l}$ & 1 & \\
\hline 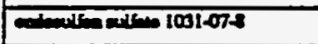 & $<0.00001 \mathrm{mg} / \mathrm{l}$ & $<0.00001 m g / 1$ & $<0.00001 \mathrm{mg} / \mathrm{h}$ & $<0.00001 \mathrm{mg} / \mathrm{l}$ & 1 & \\
\hline colitin 7200 & $<0.00005 \mathrm{mg} / 1$ & $<0.00005 \mathrm{mg} / \mathrm{l}$ & $<0.00005 \mathrm{mg} / \mathrm{l}$ & $<0.00005 \mathrm{mg} / \mathrm{l}$ & 1 & \\
\hline 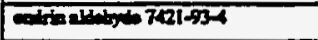 & $<0.00001 \mathrm{mg} / \mathrm{l}$ & $<0.00001 \mathrm{mg} / 1$ & $<0.00001 \mathrm{mg} / 1$ & $<0.00001 \mathrm{mg} / \mathrm{l}$ & 1 & \\
\hline 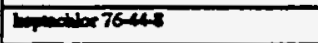 & $<0.00001 \mathrm{mg} / \mathrm{h}$ & $<0.0000 \mathrm{img} / \mathrm{l}$ & $<0.00001 \mathrm{mg} / 1$ & $<0.00001 \mathrm{mg} /$ & 1 & $\div$ \\
\hline 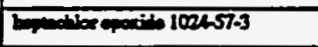 & $<0.00001 \mathrm{mg} / \mathrm{l}$ & $<0.00001 \mathrm{mg} / 1$ & $<0.00001 \mathrm{mg} / \mathrm{l}$ & $<0.00001 \mathrm{mg} / \mathrm{l}$ & 1 & \\
\hline$-1001-35.2$ & $<0.0004 \mathrm{mg} / 1$ & $<0.0004 \mathrm{mg} / \mathrm{l}$ & $<0.0004 \mathrm{mg} / 1$ & $<0.0004 \mathrm{mg} / 1$ & 1 & \\
\hline 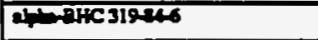 & $<0.00001 \mathrm{mg} / \mathrm{l}$ & $<0.00001 \mathrm{mg} / \mathrm{l}$ & $<0.00001 \mathrm{mg} / 1$ & $<0.00001 \mathrm{mg} / \mathrm{h}$ & 1 & \\
\hline 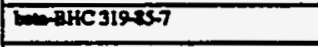 & $<0.00001 \mathrm{mg} / \mathrm{l}$ & $<0.00001 \mathrm{mg} / \mathrm{g}$ & $<0.00001 \mathrm{mg} / \mathrm{l}$ & $<0.00001 \mathrm{mg} / \mathrm{l}$ & 1 & \\
\hline 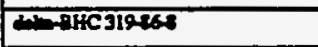 & $<0.00001 \mathrm{mg} / \mathrm{t}$ & $<0.00001 \mathrm{mg} / \mathrm{h}$ & $<0.00001 \mathrm{mg} / \mathrm{l}$ & $<0.00001 \mathrm{mg} / \mathrm{h}$ & 1 & \\
\hline 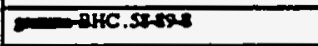 & $<0.00001 \mathrm{mg} / \mathrm{l}$ & $<0.00001 \mathrm{mg} / 1$ & $<0.00001 \mathrm{mg} / 1$ & $<0.00001 \mathrm{mg} / \mathrm{h}$ & 1 & \\
\hline 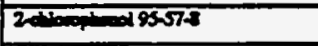 & $<0.030 \mathrm{mg} / 1$ & $<0.030 \mathrm{mg} / \mathrm{l}$ & $<0.030 \mathrm{mg} / \mathrm{l}$ & $<0.030 \mathrm{mg} / \mathrm{l}$ & 1 & \\
\hline$A=12025369219$ & $<0.0005 \mathrm{mg} / \mathrm{l}$ & $<0.0005 \mathrm{mg} / 1$ & $<0.0005 \mathrm{mg} / 1$ & $<0.0005 \mathrm{mg} / \mathrm{l}$ & 1 & \\
\hline Andon-12911097-69) & $<0.0005 \mathrm{mg} / 1$ & $<0.0005 \mathrm{mg} / \mathrm{l}$ & $<0.0005 \mathrm{mg} / \mathrm{h}$ & $<0.0005 \mathrm{mg} / \mathrm{l}$ & 1 & \\
\hline$A-1211110420-2$ & $<0.0005 \mathrm{mg} / \mathrm{l}$ & $<0.0005 \mathrm{mg} / \mathrm{l}$ & $<0.0005 \mathrm{mg} / 1$ & $<0.0005 \mathrm{mg} / \mathrm{l}$ & 1 & \\
\hline Arockon $123211141-165$ & $<0.0005 \mathrm{mg} / \mathrm{l}$ & $<0.0005 \mathrm{mg} / \mathrm{l}$ & $<0.0005 \mathrm{mg} / 1$ & $<0.0005 \mathrm{mg} / \mathrm{l}$ & 1 & \\
\hline 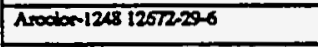 & $<0.0005 \mathrm{mg} / \mathrm{l}$ & $<0.0005 \mathrm{mg} / \mathrm{i}$ & $<0.0005 \mathrm{mg} / \mathrm{I}$ & $<0.0005 \mathrm{mg} /$ & 1 & \\
\hline A & $<0.0005 \mathrm{mg} / \mathrm{l}$ & $<0.0005 \mathrm{mg} / 1$ & $<0.0005 \mathrm{mg} / 1$ & $<0.0005 \mathrm{mg} / \mathrm{l}$ & 1 & \\
\hline Acodon-1016 12674-11-2 & $<0.0005 \mathrm{mg} / 1$ & $<0.0005 \mathrm{mg} / 1$ & $<0.0005 \mathrm{mg} / 1$ & $<0.0005 \mathrm{mg} / \mathrm{l}$ & 1 & \\
\hline Canols & $<0.010 \mathrm{mg} / 1$ & $<0.010 \mathrm{mg} / \mathrm{I}$ & $<0.010 \mathrm{mg} / 1$ & $<0.010 \mathrm{mg} / \mathrm{l}$ & 1 & \\
\hline Anolis: & $<0.010 \mathrm{mg} / \mathrm{l}$ & $<0.010 \mathrm{mg} / \mathrm{l}$ & $<0.010 \mathrm{mg} / \mathrm{l}$ & $<0.010 \mathrm{mg} / 1$ & 1 & \\
\hline Anostacikib & $<0.010 \mathrm{mg} / 1$ & $<0.010 \mathrm{mg} / \mathrm{l}$ & $<0.010 \mathrm{mg} / \mathrm{l}$ & $<0.010 \mathrm{mg} / \mathrm{l}$ & 1 & \\
\hline 1,2 - & Reculte peoding & Reaulte peading & Reaults pending & Reaults peoding & 1 & \\
\hline & & & & . & & \\
\hline & & & & & & \\
\hline & & & & & & \\
\hline & & & & & & \\
\hline & & & & & & \\
\hline & & & & & & \\
\hline & & & & & & \\
\hline & & & & & & \\
\hline & & & & & & \\
\hline & & & & & & \\
\hline & & & & & & \\
\hline & & & & & & \\
\hline & & & & & & \\
\hline & & & & & & \\
\hline
\end{tabular}


VII. Dicherge inforrantion (coctinued from page 3 of Form 2F)

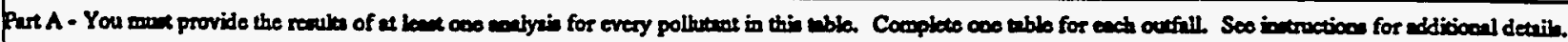

\begin{tabular}{|c|c|c|c|c|c|c|}
\hline \multirow{2}{*}{$\begin{array}{l}\text { Pollumat } \\
\text { and } \\
\text { CAS Nimber } \\
\text { (if evilible) }\end{array}$} & \multicolumn{2}{|c|}{$\begin{array}{l}\text { Mocinem Values } \\
\text { (ivclude mits) }\end{array}$} & \multicolumn{2}{|c|}{$\begin{array}{l}\text { Avense Vabee } \\
\text { (include unita) }\end{array}$} & \multirow{2}{*}{$\begin{array}{c}\text { Nomber } \\
\text { of } \\
\text { Stoces } \\
\text { Evere } \\
\text { Serepled }\end{array}$} & \multirow[b]{2}{*}{ Sourees of Pollewn } \\
\hline & 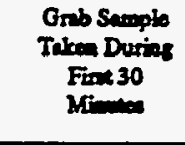 & $\begin{array}{l}\text { Flow-weifhed } \\
\text { Cooponice }\end{array}$ & 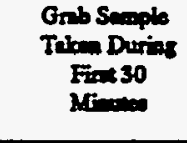 & $\begin{array}{l}\text { Flow-meigitiod } \\
\text { Componious }\end{array}$ & & \\
\hline pil anomeses & $<2 \mathrm{mg} / 1$ & & $<2 m g / 1$ & & 1 & \\
\hline Bladodal Oxym Dened (BODS) & $<5 \mathrm{mg} / \mathrm{l}$ & $<5 \mathrm{mg} / \mathrm{l}$ & $<5 \mathrm{mg} / 1$ & $<5 \mathrm{mg} / 1$ & 1 & \\
\hline 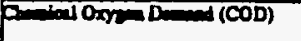 & $46 \mathrm{mg} / \mathrm{h}$ & $28 \mathrm{mg} / 1$ & $46 \mathrm{mp} / \mathrm{l}$ & $28 \mathrm{mg} / \mathrm{l}$ & 1 & \\
\hline raw supoud Solin (TS) & $65 \mathrm{mp} / 1$ & $54 \mathrm{mg} / 1$ & $65 \mathrm{mg} / 1$ & $54 \mathrm{mg} / \mathrm{l}$ & 1 & \\
\hline 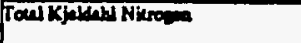 & $0.70 \mathrm{mg} / 1$ & $0.50 \mathrm{mg} / \mathrm{l}$ & $0.70 \mathrm{mg} / 1$ & $0.50 \mathrm{mg} / \mathrm{l}$ & 1 & \\
\hline 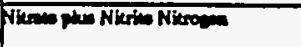 & $0.017 \mathrm{mg} / 1$ & $0.47 \mathrm{mg} / \mathrm{l}$ & $0.017 \mathrm{mg} / \mathrm{h}$ & $0.47 \mathrm{mg} / \mathrm{l}$ & 1 & \\
\hline Taed Prophore & $0.14 \mathrm{mgh}$ & $<0.1 \mathrm{mg} / 1$ & $0.14 \mathrm{mg} / 1$ & $<0.1 \mathrm{mg} / 1$ & 1 & \\
\hline $\mathrm{pH}$ & 82 & Menim 8.2 & Mind 8.2 & $\mathrm{Med}=8.2$ & & \\
\hline
\end{tabular}

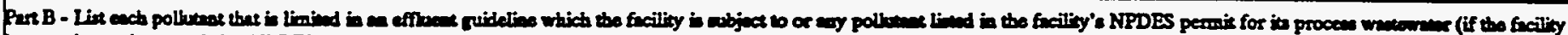

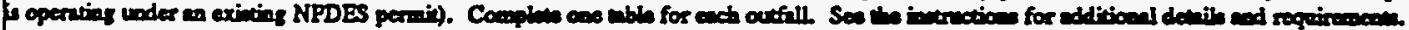

\begin{tabular}{|c|c|c|c|c|c|c|}
\hline \multirow{2}{*}{$\begin{array}{l}\text { Pollewate } \\
\text { CAs Number } \\
\text { (if milbile) }\end{array}$} & \multicolumn{2}{|c|}{ 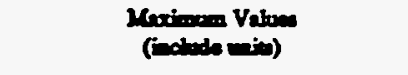 } & \multicolumn{2}{|c|}{ Averise Vabus } & \multirow{2}{*}{ 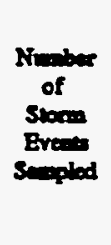 } & \multirow{2}{*}{$\begin{array}{c}: \\
\text { Sormece of Pollon }\end{array}$} \\
\hline & $\begin{array}{c}\text { Grib Sample } \\
\text { Tabo Durint } \\
\text { Fine } 30 \\
\text { Mom }\end{array}$ & $\begin{array}{l}\text { Flom-wrighend } \\
\text { Compocien }\end{array}$ & 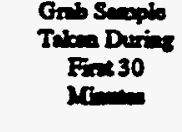 & $\begin{array}{l}\text { Plom-weipted } \\
\text { Conpocines }\end{array}$ & & \\
\hline$n=0+240-360$ & $<0.04 \mathrm{mp} / \mathrm{l}$ & $<0.04 \mathrm{mg} / \mathrm{l}$ & $<0.04 \mathrm{mg} / 1$ & $<0.04 \mathrm{mg} / \mathrm{l}$ & 1 & \\
\hline $2 y=$ & $0.004 \mathrm{mg} / 1$ & & $0.004 \mathrm{me} / \mathrm{l}$ & & 1 & \\
\hline 700906 & $<0.0002 \mathrm{mg} / 1$ & $<0.0002 \mathrm{mg} h$ & $<0.0002 \mathrm{mg} / \mathrm{t}$ & $<0.0002 \mathrm{mg} / 1$ & 1 & \\
\hline$=\infty$ & $0.004 \mathrm{mg} / \mathrm{h}$ & & $0.004 \mathrm{mg} / 1$ & & 1 & \\
\hline 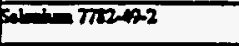 & $<0.002 \mathrm{mg} / 1$ & $<0.002 \mathrm{mg} / \mathrm{l}$ & $<0.002 \mathrm{mq} / 1$ & $<0.002 \mathrm{mg} / \mathrm{l}$ & 1 & \\
\hline 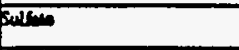 & $24 \mathrm{mg} / \mathrm{l}$ & 11 men & $24 \mathrm{mg} /$ & $11 \mathrm{mg} /$ & 1 & \\
\hline Serfocenes & $<0.05 \mathrm{mg} / 1$ & $<0.05 \mathrm{mg} / 1$ & $<0.05 \mathrm{mg} / \mathrm{l}$ & $<0.05 \mathrm{mg} / \mathrm{l}$ & 1 & \\
\hline the $742900-5$ & $3.27 \mathrm{mg} / \mathrm{l}$ & $2.92 \mathrm{mg} / 1$ & $3.27 \mathrm{mg} / \mathrm{l}$ & $2.92 \mathrm{mg} / 1$ & 1 & \\
\hline $5=1000-35-2$ & $<0.04 \mathrm{mg} / \mathrm{h}$ & $<0.04 \mathrm{mg} / \mathrm{l}$ & $<0.04 \mathrm{mg} / \mathrm{l}$ & $<0.04$ men/ & 1 & \\
\hline $300007460-25$ & $0.012 \mathrm{mq} / \mathrm{l}$ & $0.010 \mathrm{mg} / \mathrm{l}$ & $0.012 \mathrm{mg} / \mathrm{l}$ & $0.010 m \pi$ & 1 & \\
\hline ing yilen 740-11.7 & $<0.0004 m y / 1$ & $<0.0004 \mathrm{mq} / \mathrm{l}$ & $<0.0004 \mathrm{mg} / 1$ & $<0.0004 m g h$ & 1 & \\
\hline 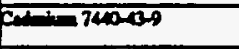 & $<0.004 \mathrm{mg} / 1$ & $<0.004 \mathrm{mg} / \mathrm{l}$ & $<0.004 \mathrm{mg} / \mathrm{l}$ & $<0.004 \mathrm{mg} / \mathrm{l}$ & 1 & \\
\hline 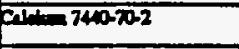 & $12.3 \mathrm{mg} / \mathrm{h}$ & $10.7 \mathrm{mg} / \mathrm{l}$ & $12.3 \mathrm{mg} / \mathrm{l}$ & $10.7 \mathrm{mg} / 1$ & 1 & \\
\hline 50127007.3 & $<0.006 \mathrm{mg} / 1$ & $<0.006 \mathrm{mg} / \mathrm{I}$ & $<0.006 \mathrm{mg} / 1$ & $<0.006 \mathrm{mg} / \mathrm{l}$ & 1 & \\
\hline Catak 7400-404 & $0.003 \mathrm{mg} / \mathrm{l}$ & $0.002 \mathrm{mg} / 1$ & $0.003 \mathrm{mg} / \mathrm{l}$ & $0.002 \mathrm{mg} / 1$ & 1 & \\
\hline 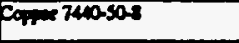 & $<0.006 \mathrm{mg} / 1$ & $<0.006 \mathrm{mg} / 1$ & $<0.006 \mathrm{mg} / \mathrm{l}$ & $<0.006 \mathrm{men} / \mathrm{n}$ & 1 & \\
\hline 50103206 & $4.84 \mathrm{mell}$ & $2.82 \mathrm{mg} / 1$ & $4.84 \mathrm{mg} / \mathrm{l}$ & $2.82 \mathrm{mg} / 1$ & 1 & \\
\hline 1001702921 & $<0.02 \mathrm{mg} / \mathrm{l}$ & $<0.02 \mathrm{mg} / 1$ & $<0.02 \mathrm{mg} / 1$ & $<0.02 \mathrm{mg} / \mathrm{l}$ & 1 & \\
\hline $70+1=703293-2$ & $<0.02 \mathrm{mg} / \mathrm{h}$ & $<0.02 \mathrm{mg} / 1$ & $<0.02 \mathrm{mg} / 1$ & $<0.02 \mathrm{mg} / 1$ & 1 & \\
\hline 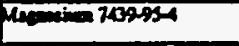 & $2.29 \mathrm{mg} / 1$ & $1.67 \mathrm{mg} / 1$ & $2.29 \mathrm{mg} / \mathrm{f}$ & $1.67 \mathrm{mg} / 1$ & 1 & \\
\hline 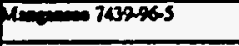 & $0.101 \mathrm{mg} / \mathrm{l}$ & $0.061 \mathrm{mg} / 1$ & $0.101 \mathrm{mg} / 1$ & $0.061 \mathrm{mg} / \mathrm{l}$ & 1 & \\
\hline 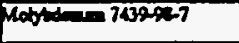 & $<0.006 \mathrm{mg} / \mathrm{h}$ & $<0.006 \mathrm{mg} / \mathrm{h}$ & $<0.006 \mathrm{mg} / \mathrm{l}$ & $<0.006 \mathrm{mg} / \mathrm{l}$ & 1 & \\
\hline$\sqrt{160+1740000}$ & $<0.008 \mathrm{mg} / \mathrm{l}$ & $<0.008 \mathrm{mg} / \mathrm{l}$ & $<0.008 \mathrm{mg} / 1$ & $<0.008 \mathrm{mg} / \mathrm{h}$ & 1 & \\
\hline rancine 740097 & $1.4 \mathrm{mg} / 1$ & $1.3 \mathrm{mg} / 1$ & $1.4 \mathrm{mg} / \mathrm{l}$ & $1.3 \mathrm{mg} / \mathrm{l}$ & 1 & \\
\hline stron $740-204$ & $<0.006 \mathrm{mg} / \mathrm{l}$ & $<0.006 \mathrm{mg} / \mathrm{l}$ & $<0.006 \mathrm{mg} / \mathrm{I}$ & $<0.006 \mathrm{mg} / \mathrm{l}$ & 1 & \\
\hline 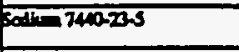 & $313 \mathrm{mg} / 1$ & $326 \mathrm{mg} / \mathrm{l}$ & $313 \mathrm{mg} / 1$ & $326 \mathrm{mg} /$ & 1 & \\
\hline Fumban 740-326 & $0.04 \mathrm{mg} / \mathrm{l}$ & $0.03 \mathrm{mg} / \mathrm{l}$ & $0.04 \mathrm{mg} / \mathrm{l}$ & $0.03 \mathrm{mg} / \mathrm{l}$ & 1 & \\
\hline 20240666 & $0.12 \mathrm{mg} / 1$ & $0.09 \mathrm{mg} / 1$ & $0.12 \mathrm{mg} / 1$ & $0.09 \mathrm{mz} / 1$ & 1 & \\
\hline
\end{tabular}


Continged from from.

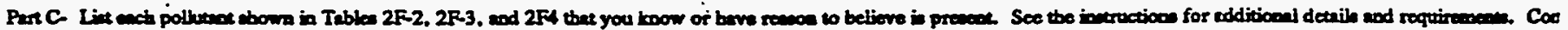
oos tuble for ench oufall.

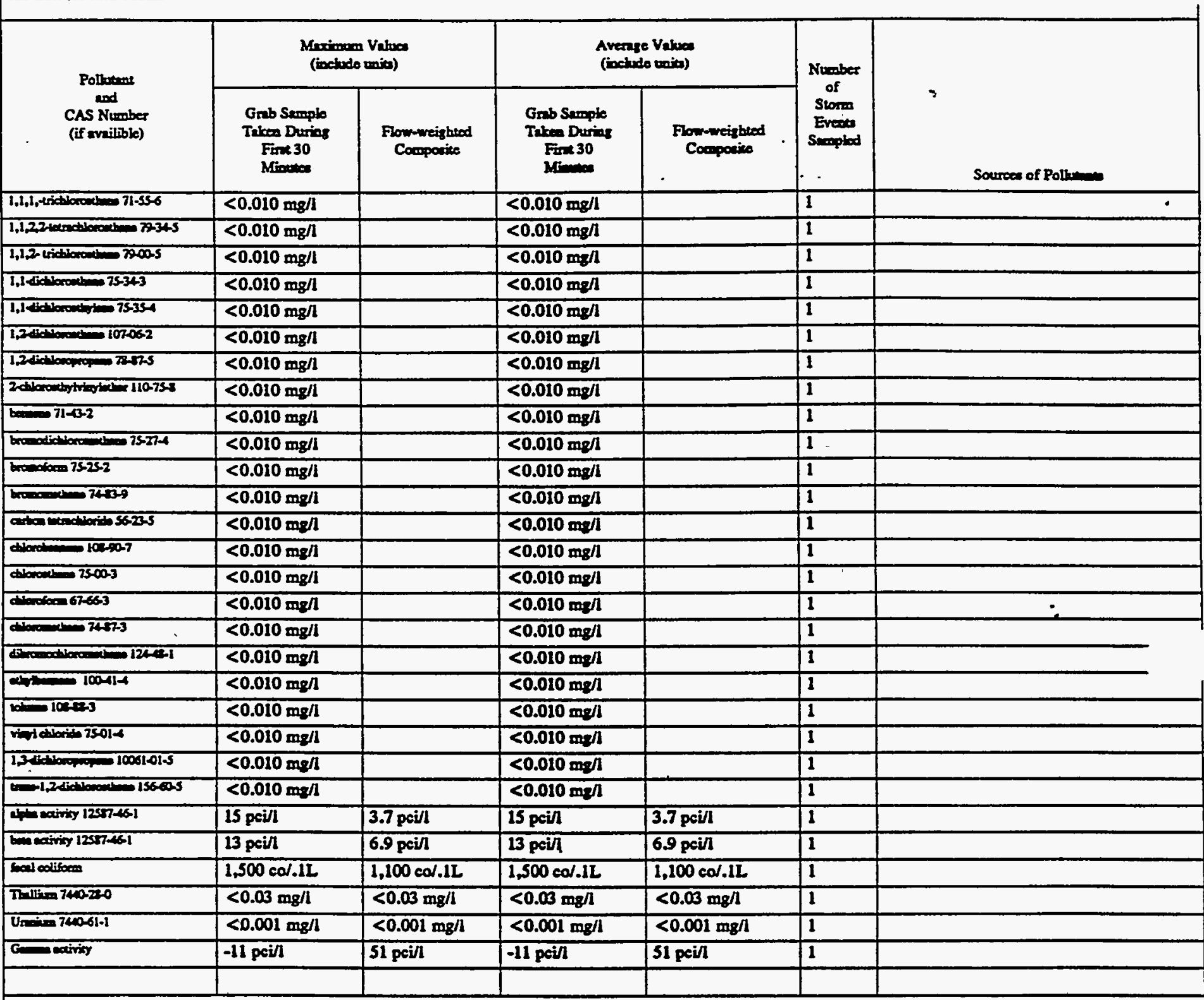

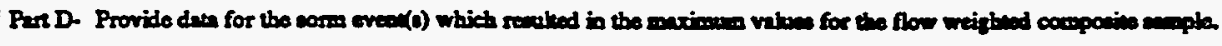

\begin{tabular}{|c|c|c|c|c|c|c|c|}
\hline $\begin{array}{l}1 . \\
\text { Dew of } \\
\text { Storm } \\
\text { Erant }\end{array}$ & $\begin{array}{l}2 . \\
\text { Durion } \\
\text { of Stonm } \\
\text { (i. }\end{array}$ & 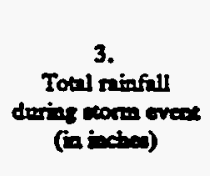 & 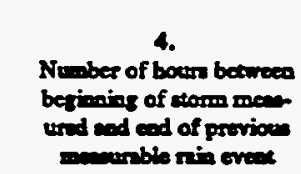 & 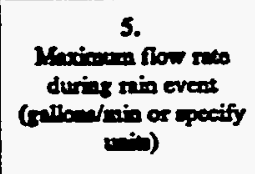 & 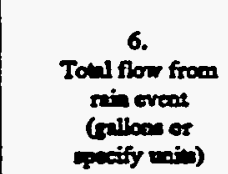 & 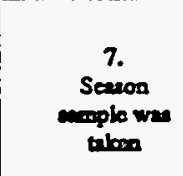 & $\begin{array}{l}8 . \\
\text { Eorin of } \\
\text { Precipiation } \\
\text { (ainfill, }\end{array}$ \\
\hline $12 / 9 / 91$ & 530 & 1.1 & 148.6 & 64 & 58,775 & Fall & Reinfall \\
\hline
\end{tabular}

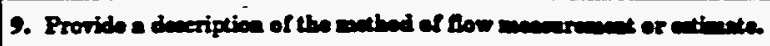

An ISCO 3230 Flow meter was wed to obtiin water level readinge. This instrument was calibrated with tho sormal dry weather flow, if present, as bevel

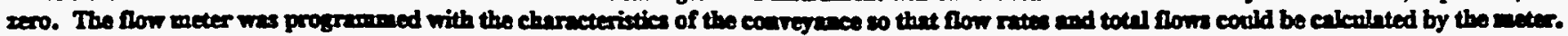


Outfall 11

Parta B and C Coationad

\begin{tabular}{|c|c|c|c|c|c|c|}
\hline \multirow{2}{*}{$\begin{array}{l}\text { Poltuenat } \\
\text { and } \\
\text { CAs Number } \\
\text { (if muilible) }\end{array}$} & \multicolumn{2}{|c|}{$\begin{array}{l}\text { Meximum Vaheen } \\
\text { (inctado uniou) }\end{array}$} & \multicolumn{2}{|c|}{$\begin{array}{l}\text { Averase Valuee } \\
\text { (ibeludo unita) }\end{array}$} & \multirow{2}{*}{$\begin{array}{c}\text { Number } \\
\text { of } \\
\text { Stomen } \\
\text { Evectid } \\
\text { Sempled }\end{array}$} & \multirow[b]{2}{*}{ Soureses of Polbreaces } \\
\hline & $\begin{array}{l}\text { Orab Sumpto } \\
\text { Taken Durins } \\
\text { Fins } 30 \\
\text { Minuea }\end{array}$ & $\begin{array}{l}\text { Flow-weighted } \\
\text { Comporive }\end{array}$ & $\begin{array}{l}\text { Grab Semplo } \\
\text { Tikeon During } \\
\text { Firs } 30 \\
\text { Micuto }\end{array}$ & $\begin{array}{c}\text { Flow-woighed } \\
\text { Conpoice } \\
\text {. }\end{array}$ & & \\
\hline $8 n+4=0140-32.3$ & $0.0221 \mathrm{mg} / 1$ & $0.0179 \mathrm{mg} / 1$ & $0.0221 \mathrm{mg} / 1$ & $0.0179 \mathrm{mg} / 1$ & 1 & \\
\hline 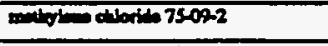 & $<0.010 \mathrm{mg} / \mathrm{l}$ & & $<0.010 \mathrm{mg} / 1$ & & 1 & \\
\hline 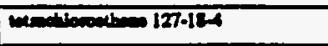 & $<0.010 \mathrm{mg} / 1$ & & $<0.010 \mathrm{mg} / 1$ & & 1 & \\
\hline 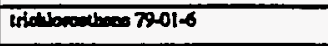 & $<0.010 \mathrm{mg} / 1$ & & $<0.010 \mathrm{mg} / 1$ & & 1 & \\
\hline Xyingen & $<0.010 \mathrm{mg} / \mathrm{l}$ & & $<0.010 \mathrm{mg} / 1$ & & 1 & \\
\hline 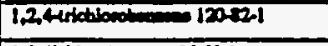 & $<0.010 \mathrm{mg} / 1$ & $<0.010 \mathrm{mg} / 1$ & $<0.010 \mathrm{mg} / 1$ & $<0.010 \mathrm{mg} / 1$ & 1 & \\
\hline 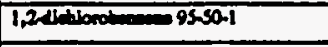 & $<0.010 \mathrm{mg} / \mathrm{l}$ & $<0.010 \mathrm{mg} / \mathrm{l}$ & $<0.010 \mathrm{mg} / \mathrm{l}$ & $<0.010 \mathrm{mg} / \mathrm{l}$ & 1 & \\
\hline 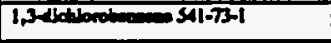 & $<0.010 \mathrm{mg} / \mathrm{l}$ & $<0.010 \mathrm{mg} / \mathrm{l}$ & $<0.010 \mathrm{mg} / \mathrm{l}$ & $<0.010 \mathrm{mg} / \mathrm{l}$ & 1 & \\
\hline 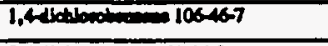 & $<0.010 \mathrm{mg} / \mathrm{l}$ & $<0.010 \mathrm{mg} / \mathrm{l}$ & $<0.010 \mathrm{mg} / 1$ & $<0.010 \mathrm{mg} / \mathrm{l}$ & 1 & \\
\hline 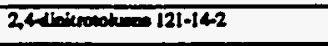 & $<0.010 \mathrm{mg} / 1$ & $<0.010 \mathrm{mg} / \mathrm{l}$ & $<0.010 \mathrm{mg} / \mathrm{l}$ & $<0.010 \mathrm{mg} / \mathrm{l}$ & 1 & \\
\hline 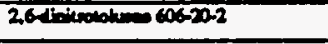 & $<0.010 \mathrm{mg} / 1$ & $<0.010 \mathrm{mg} / 1$ & $<0.010 \mathrm{mg} / \mathrm{l}$ & $<0.010 \mathrm{mg} / \mathrm{l}$ & 1 & \\
\hline 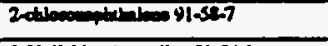 & $<0.010 \mathrm{mg} / \mathrm{l}$ & $<0.010 \mathrm{mg} / \mathrm{l}$ & $<0.010 \mathrm{mg} / \mathrm{l}$ & $<0.010 \mathrm{mg} / 1$ & 1 & \\
\hline 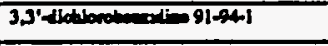 & $<0.010 \mathrm{mg} / \mathrm{l}$ & $<0.010 \mathrm{mg} / \mathrm{l}$ & $<0.010 \mathrm{mg} / \mathrm{l}$ & $<0.010 \mathrm{mg} / \mathrm{l}$ & 1 & \\
\hline 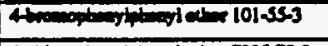 & $<0.010 \mathrm{mg} / \mathrm{l}$ & $<0.010 \mathrm{mg} / \mathrm{l}$ & $<0.010 \mathrm{mg} / \mathrm{L}$ & $<0.010 \mathrm{mg} / \mathrm{l}$ & 1 & \\
\hline 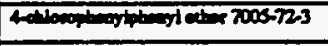 & $<0.010 \mathrm{mg} / \mathrm{l}$ & $<0.010 \mathrm{mg} / \mathrm{l}$ & $<0.010 \mathrm{mg} / 1$ & $<0.010 \mathrm{mg} / \mathrm{l}$ & 1 & \\
\hline $00=83.329$ & $<0.010 \mathrm{mg} / \mathrm{l}$ & $<0.010 \mathrm{mg} / \mathrm{l}$ & $<0.010 \mathrm{mg} / 1$ & $<0.010 \mathrm{mg} / 1$ & 1 & \\
\hline 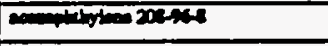 & $<0.010 \mathrm{mg} / \mathrm{h}$ & $<0.010 \mathrm{men} / \mathrm{l}$ & $<0.010 \mathrm{mg} / \mathrm{l}$ & $<0.010 \mathrm{mg} / \mathrm{l}$ & 1 & \\
\hline $120-12-7$ & $<0.010 \mathrm{mg} / \mathrm{l}$ & $<0.010 \mathrm{mg} / \mathrm{l}$ & $<0.010 \mathrm{mg} / 1$ & $<0.010 \mathrm{mg} / \mathrm{h}$ & 1 & : \\
\hline 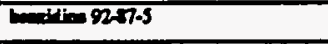 & $<0.050 \mathrm{mg} / \mathrm{h}$ & $<0.050 \mathrm{mg} / 1$ & $<0.050 \mathrm{mg} / 1$ & $<0.050 \mathrm{mg} / \mathrm{l}$ & 1 & \\
\hline S6-5353 & $<0.010 \mathrm{mg} / 1$ & $<0.010 \mathrm{mg} / 1$ & $<0.010 \mathrm{mg} / 1$ & $<0.010 \mathrm{mg} / 1$ & 1 & \\
\hline 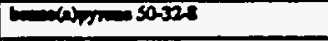 & $<0.010 \mathrm{mg} / \mathrm{l}$ & $<0.010 \mathrm{mg} / 1$ & $<0.010 \mathrm{mg} / 1$ & $<0.010 \mathrm{mg} / \mathrm{h}$ & 1 & \\
\hline 205992 & $<0.010 \mathrm{mg} / \mathrm{l}$ & $<0.010 \mathrm{mg} / 1$ & $<0.010 \mathrm{mg} /$ & $<0.010 \mathrm{mg} / \mathrm{l}$ & 1 & \\
\hline 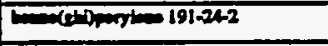 & $<0.010 \mathrm{mg} / \mathrm{l}$ & $<0.010 \mathrm{mg} / \mathrm{l}$ & $<0.010 \mathrm{mg} / 1$ & $<0.010 \mathrm{mg} / \mathrm{l}$ & 1 & \\
\hline 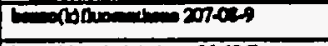 & $<0.010 \mathrm{mg} / \mathrm{l}$ & $<0.010 \mathrm{mg} / 1$ & $<0.010 \mathrm{mg} / \mathrm{s}$ & $<0.010 \mathrm{mg} / \mathrm{l}$ & 1 & \\
\hline 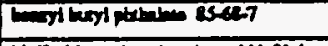 & $<0.010 \mathrm{mg} / 1$ & $<0.010 \mathrm{me} / \mathrm{l}$ & $<0.010 \mathrm{mgh}$ & $<0.010 \mathrm{mg} / \mathrm{l}$ & 1 & \\
\hline 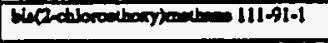 & $<0.010 \mathrm{mg} / 1$ & $<0.010 \mathrm{mg} / \mathrm{l}$ & $<0.010 \mathrm{mg} / 1$ & $<0.010 \mathrm{mg} / 1$ & 1 & \\
\hline 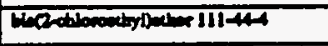 & $<0.010 \mathrm{mg} / \mathrm{l}$ & $<0.010 \mathrm{mg} / \mathrm{l}$ & $<0.010 \mathrm{mg} / \mathrm{l}$ & $<0.010 \mathrm{mg} /$ & 1 & \\
\hline 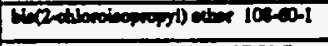 & $<0.010 \mathrm{mg} / \mathrm{l}$ & $<0.010 \mathrm{mgll}$ & $<0.010 \mathrm{mg} / \mathrm{l}$ & $<0.010 \mathrm{mg} / \mathrm{h}$ & 1 & \\
\hline 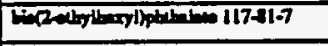 & $<0.010 \mathrm{mg} / \mathrm{h}$ & $<0.015 \mathrm{mg} / 1$ & $<0.010 \mathrm{mg} / \mathrm{l}$ & $<0.015 \mathrm{mg} / 1$ & 1 & \\
\hline 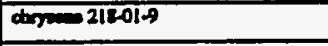 & $<0.010 \mathrm{mg} / \mathrm{l}$ & $<0.010 \mathrm{mg} / \mathrm{l}$ & $<0.010 \mathrm{mg} / \mathrm{l}$ & $<0.010 \mathrm{mg} / 1$ & 1 & \\
\hline 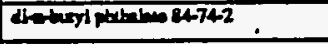 & $<0.010 \mathrm{mg} / \mathrm{l}$ & $<0.013 \mathrm{mg} / 1$ & $<0.010 \mathrm{mg} / \mathrm{l}$ & $<0.013 \mathrm{mg} / \mathrm{l}$ & 1 & \\
\hline 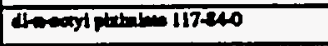 & $<0.010 \mathrm{mg} / 1$ & $<0.010 \mathrm{mg} / \mathrm{l}$ & $<0.010 \mathrm{mg} / \mathrm{l}$ & $<0.010 \mathrm{mg} / \mathrm{l}$ & 1 & \\
\hline 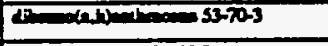 & $<0.010 \mathrm{mg} / 1$ & $<0.010 \mathrm{mg} / \mathrm{l}$ & $<0.010 \mathrm{me} / \mathrm{l}$ & $<0.010 \mathrm{mg} / 1$ & 1 & \\
\hline 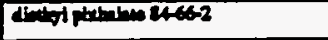 & $<0.010 \mathrm{mg} / 1$ & $<0.010 \mathrm{mg} / 1$ & $<0.010 \mathrm{mg} / \mathrm{l}$ & $<0.010 \mathrm{mg} / \mathrm{l}$ & 1 & \\
\hline 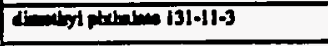 & $<0.010 \mathrm{mg} / \mathrm{l}$ & $<0.010 \mathrm{mg} / \mathrm{l}$ & $<0.010 \mathrm{mg} / \mathrm{l}$ & $<0.010 \mathrm{mg} / \mathrm{l}$ & 1 & \\
\hline $000=206140$ & $<0.010 \mathrm{mg} / \mathrm{l}$ & $<0.010 \mathrm{mg} / \mathrm{l}$ & $<0.010 \mathrm{mg} / \mathrm{l}$ & $<0.010 \mathrm{mg} / \mathrm{l}$ & 1 & \\
\hline $800000-73-7$ & $<0.010 \mathrm{mg} / \mathrm{l}$ & $<0.010 m g / 1$ & $<0.010 \mathrm{mg} / 1$ & $<0.010 \mathrm{mg} / \mathrm{h}$ & 1 & \\
\hline 118.741 & $<0.010 \mathrm{mg} / \mathrm{l}$ & $<0.010 \mathrm{mg} / \mathrm{l}$ & $<0.010 \mathrm{mg} / \mathrm{l}$ & $<0.010 \mathrm{mg} / 1$ & 1 & \\
\hline 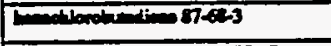 & $<0.010 \mathrm{mg} / \mathrm{l}$ & $<0.010 \mathrm{mg} / \mathrm{l}$ & $<0.010 \mathrm{mg} / \mathrm{l}$ & $<0.010 \mathrm{mg} / \mathrm{l}$ & 1 & \\
\hline 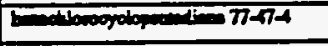 & $<0.010 \mathrm{mg} / \mathrm{h}$ & $<0.010 \mathrm{mg} / \mathrm{l}$ & $<0.010 \mathrm{mg} / \mathrm{l}$ & $<0.010 \mathrm{mg} / \mathrm{l}$ & 1 & \\
\hline $67.72-1$ & $<0.010 \mathrm{mg} / \mathrm{h}$ & $<0.010 \mathrm{mg} / \mathrm{l}$ & $<0.010 \mathrm{mg} / \mathrm{l}$ & $<0.010 \mathrm{mg} /$ & 1 & \\
\hline 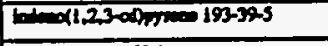 & $<0.010 \mathrm{mg} / 1$ & $<0.010 \mathrm{mg} / \mathrm{l}$ & $<0.010 \mathrm{mg} / \mathrm{l}$ & $<0.010 \mathrm{mg} / \mathrm{h}$ & 1 & \\
\hline 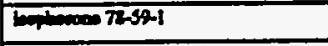 & $<0.010 \mathrm{mg} / \mathrm{i}$ & $<0.010 \mathrm{mg} / 1$ & $<0.010 \mathrm{mg} / \mathrm{l}$ & $<0.010 \mathrm{mg} / 1$ & 1 & \\
\hline 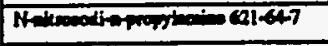 & $<0.010 \mathrm{mg} / 1$ & $<0.010 \mathrm{mg} / 1$ & $<0.010 \mathrm{mg} / \mathrm{l}$ & $<0.010 \mathrm{mg} / 1$ & 1 & \\
\hline 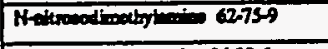 & $<0.010 \mathrm{mg} / \mathrm{l}$ & $<0.010 \mathrm{mg} / \mathrm{l}$ & $<0.010 \mathrm{mg} / \mathrm{l}$ & $<0.010 \mathrm{mg} / 1$ & 1 & \\
\hline 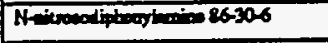 & $<0.010 \mathrm{mg} / 1$ & $<0.010 \mathrm{mg} / \mathrm{l}$ & $<0.010 \mathrm{mg} / \mathrm{l}$ & $<0.010 \mathrm{mg} / 1$ & 1 & \\
\hline 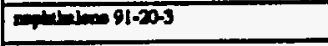 & $<0.010 \mathrm{mg} / \mathrm{l}$ & $<0.010 \mathrm{mg} / 1$ & $<0.010 \mathrm{mg} / \mathrm{l}$ & $<0.010 \mathrm{mg} / 1$ & 1 & \\
\hline 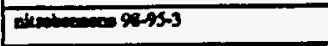 & $<0.010 \mathrm{mg} / \mathrm{l}$ & $<0.010 \mathrm{mg} / \mathrm{l}$ & $<0.010 \mathrm{mg} / \mathrm{l}$ & $<0.010 \mathrm{mg} / \mathrm{l}$ & 1 & \\
\hline 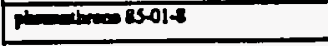 & $<0.010 \mathrm{mg} / 1$ & $<0.010 \mathrm{mg} / \mathrm{l}$ & $:<0.010 \mathrm{mg} / \mathrm{l}$ & $<0.010 \mathrm{mg} / 1$ & 1 & \\
\hline$m m-129000$ & $<0.010 \mathrm{mg} / \mathrm{l}$ & $<0.010 \mathrm{mg} / \mathrm{l}$ & $<0.010 \mathrm{mg} / \mathrm{l}$ & $<0.010 \mathrm{mg} / \mathrm{l}$ & 1 & \\
\hline 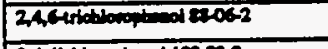 & $<0.030 \mathrm{mg} / \mathrm{l}$ & $<0.030 \mathrm{mg} / \mathrm{l}$ & $<0.030 \mathrm{mg} / \mathrm{I}$ & $<0.030 \mathrm{mg} / \mathrm{l}$ & 1 & \\
\hline 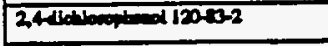 & $<0.030 \mathrm{mg} / \mathrm{l}$ & $<0.030 \mathrm{mg} / \mathrm{l}$ & $<0.030 \mathrm{mg} / 1$ & $<0.030 \mathrm{mg} / \mathrm{l}$ & 1 & \\
\hline 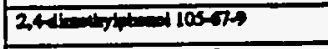 & $<0.030 \mathrm{mg} / \mathrm{l}$ & $<0.030 \mathrm{mg} / \mathrm{h}$ & $<0.030 \mathrm{mg} / \mathrm{l}$ & $<0.030 \mathrm{mg} / \mathrm{l}$ & 1 & \\
\hline 2,4dinieropteed 51-225s & $<0.050 \mathrm{mg} / \mathrm{l}$ & $<0.050 \mathrm{mg} / \mathrm{l}$ & $<0.050 \mathrm{mg} / \mathrm{l}$ & $<0.050 \mathrm{mg} / \mathrm{l}$ & 1 & \\
\hline
\end{tabular}


Outfoll 11

Part B and C Cootiveod

\begin{tabular}{|c|c|c|c|c|c|c|}
\hline \multirow{2}{*}{$\begin{array}{l}\text { Polbuant } \\
\text { CAs Number } \\
\text { (if evilible) }\end{array}$} & \multicolumn{2}{|c|}{$\begin{array}{l}\text { Maximem Vabes } \\
\text { (include nim) }\end{array}$} & \multicolumn{2}{|c|}{$\begin{array}{l}\text { Avernge Vabee } \\
\text { (ivelude mis) }\end{array}$} & \multirow{2}{*}{$\begin{array}{l}\text { Number } \\
\text { of } \\
\text { Shom } \\
\text { Everes } \\
\text { Sersipled }\end{array}$} & \multirow[b]{2}{*}{ Sources of Polktinte } \\
\hline & 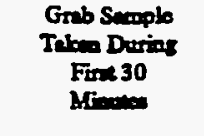 & $\begin{array}{l}\text { Foom-weighed } \\
\text { Compotive }\end{array}$ & 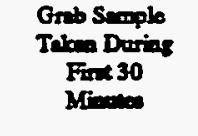 & $\begin{array}{l}\text { From-wrighed } \\
\text { Compotise }\end{array}$ & & \\
\hline 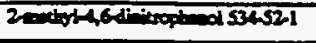 & $<0.050 \mathrm{mg} / 1$ & $<0.050 \mathrm{mg} / \mathrm{h}$ & $<0.050 \mathrm{mg} / 1$ & $<0.050 \mathrm{mg} / \mathrm{l}$ & 1 & \\
\hline 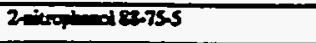 & $<0.030 \mathrm{mg} / 1$ & $<0.030 \mathrm{mg} / \mathrm{l}$ & $<0.030 \mathrm{mg} / 1$ & $<0.030 \mathrm{mg} / 1$ & 1 & \\
\hline 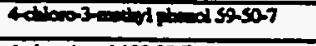 & $<0.030 \mathrm{mg} / \mathrm{l}$ & $<0.030 \mathrm{mg} / 1$ & $<0.030 \mathrm{mg} / 1$ & $<0.030 \mathrm{mg} / 1$ & 1 & \\
\hline 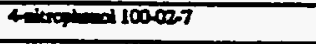 & $<0.050 \mathrm{mg} / \mathrm{h}$ & $<0.050 \mathrm{mg} / 1$ & $<0.050 \mathrm{mg} /$ & $<0.050 \mathrm{mg} / 1$ & 1 & \\
\hline 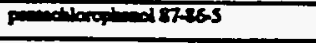 & $<0.050 \mathrm{mg} / 1$ & $<0.050 \mathrm{mg} / \mathrm{l}$ & $<0.050 \mathrm{mg} / 1$ & $<0.050 \mathrm{mg} / 1$ & 1 & \\
\hline 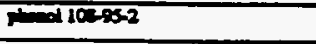 & $<0.030 \mathrm{mg} / 1$ & $<0.030 \mathrm{mg} / 1$ & $<0.030 \mathrm{mg} / 1$ & $<0.030 \mathrm{mg} / 1$ & 1 & \\
\hline $44-00072348$ & $<0.00001 \mathrm{mg} / 1$ & $<0.00001 \mathrm{mg} / \mathrm{h}$ & $<0.00001 \mathrm{mg} / \mathrm{l}$ & $<0.00001 \mathrm{mg} / \mathrm{l}$ & 1 & \\
\hline 4,4 DDE 72S5-9 & $<0.00001 \mathrm{mg} / \mathrm{l}$ & $<0.00001 \mathrm{mg} / \mathrm{h}$ & $<0.00001 \mathrm{mg} / \mathrm{l}$ & $<0.00001 \mathrm{mg} / \mathrm{l}$ & 1 & \\
\hline $4,4-000550-20-3$ & $<0.00001 \mathrm{mg} / 1$ & $<0.00001 \mathrm{mg} / \mathrm{l}$ & $<0.00001 \mathrm{mg} / \mathrm{l}$ & $<0.00001 \mathrm{mg} / 1$ & 1 & \\
\hline 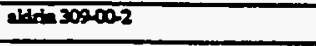 & $<0.00001 \mathrm{mg} / \mathrm{l}$ & $<0.00001 \mathrm{mg} / 1$ & $<0.00001 \mathrm{mg} / \mathrm{h}$ & $<0.00001 \mathrm{mg} / \mathrm{h}$ & 1 & \\
\hline 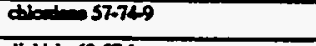 & $<0.00002 \mathrm{mg} / 1$ & $<0.00002 \mathrm{mg} / \mathrm{l}$ & $<0.00002 \mathrm{mg} / \mathrm{h}$ & $<0.00002 \mathrm{mg} / \mathrm{h}$ & 1 & \\
\hline dinciente-57-1 & $<0.00001 \mathrm{mg} / \mathrm{l}$ & $<0.00001 \mathrm{mg} / 1$ & $<0.00001 \mathrm{mg} / \mathrm{l}$ & $<0.00001 \mathrm{mg} / 1$ & 1 & \\
\hline 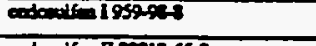 & $<0.00001 \mathrm{mg} / 1$ & $<0.00001 \mathrm{mg} / 1$ & $<0.00001 \mathrm{mg} / \mathrm{l}$ & $<0.00001 \mathrm{mg} / \mathrm{l}$ & 1 & \\
\hline 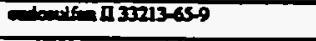 & $<0.00001 \mathrm{mg} / 1$ & $<0.00001 \mathrm{mg} / 1$ & $<0.00001 \mathrm{mg} / 1$ & $<0.00001 \mathrm{mg} / \mathrm{l}$ & 1 & \\
\hline 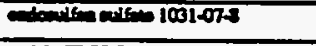 & $<0.00001 \mathrm{mg} / \mathrm{l}$ & $<0.00001 \mathrm{me} / 1$ & $<0.00001$ medl & $<0.00001 \mathrm{mg} / \mathrm{l}$ & 1 & \\
\hline tim 72208 & $<0.00005 \mathrm{mg} / \mathrm{l}$ & $<0.00005 \mathrm{mg} / \mathrm{l}$ & $<0.00005 \mathrm{mg} / \mathrm{l}$ & $<0.00005 \mathrm{mg} / \mathrm{l}$ & 1 & \\
\hline 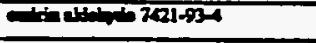 & $<0.00001 \mathrm{mg} / \mathrm{l}$ & $<0.00001 \mathrm{mg} / 1$ & $<0.00001 \mathrm{mg} / 1$ & $<0.00001 \mathrm{mg} / 1$ & 1 & \\
\hline bondion 76468 & $<0.00001 \mathrm{mg} / 1$ & $<0.00001 \mathrm{mg} / \mathrm{l}$ & $<0.00001$ medl & $<0.00001 \mathrm{mg} / \mathrm{l}$ & 1 & \\
\hline 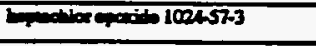 & $<0.00001 \mathrm{mg} / \mathrm{l}$ & $<0.00001 \mathrm{mg} / 1$ & $<0.00001 \mathrm{mg} / \mathrm{l}$ & $<0.00001 \mathrm{mg} / \mathrm{l}$ & 1 & \\
\hline cent-35-2 & $<0.0002 \mathrm{mg} / \mathrm{l}$ & $<0.0002 \mathrm{mo} / 1$ & $<0.0002 \mathrm{mg} / 1$ & $<0.0002 \mathrm{mg} / \mathrm{l}$ & 1 & \\
\hline 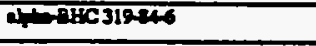 & $<0.00001 \mathrm{mg} / \mathrm{h}$ & $<0.00001 \mathrm{mg} / \mathrm{h}$ & $<0.00001 \mathrm{mg} / \mathrm{l}$ & $<0.00001 \mathrm{mg} / \mathrm{l}$ & 1 & \\
\hline DAHC 31985.7 & $<0.00001 \mathrm{men} / \mathrm{l}$ & $<0.00001 \mathrm{mg} / 1$ & $<0.00001 \mathrm{mg} / \mathrm{l}$ & $<0.00001 \mathrm{me} / \mathrm{l}$ & 1 & \\
\hline din-DHC 31965 & $0.000011 \mathrm{mg} / \mathrm{h}$ & $<0.00001 \mathrm{mg} / 1$ & $0.000011 \mathrm{men}$ & $<0.00001 \mathrm{mg} / \mathrm{l}$ & 1 & \\
\hline $8=2 \mathrm{atc} \operatorname{sen} 6$ & $<0.00001 \mathrm{mg} / \mathrm{s}$ & $<0.00001 \mathrm{mg} / \mathrm{l}$ & $<0.00001 \mathrm{med}$ & $<0.00001 \mathrm{mg} / \mathrm{l}$ & $\overline{1}$ & \\
\hline 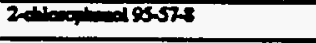 & $<0.030 \mathrm{med} / 1$ & $<0.030 \mathrm{mg} / \mathrm{l}$ & $<0.030 \mathrm{men} / 1$ & $<0.030 \mathrm{mg} / 1$ & 1 & \\
\hline Arodon $12225360221-9$ & $<0.0005 \mathrm{mg} / \mathrm{l}$ & $<0.0005 \mathrm{mg} / \mathrm{l}$ & $<0.0005 \mathrm{mg} / \mathrm{l}$ & $<0.0005 \mathrm{me} /$ & 1 & \\
\hline A & $<0.0005 \mathrm{mg} / \mathrm{l}$ & $<0.0005 \mathrm{mg} / \mathrm{l}$ & $<0.0005 \mathrm{me} / 1$ & $<0.0005 \mathrm{mg} / 1$ & 1 & \\
\hline Arodon $12211110+22-2$ & $<0.0005 \mathrm{mg} / 1$ & $<0.0005 \mathrm{me} / \mathrm{l}$ & $<0.0005$ minh & $<0.0005 \mathrm{mg} / \mathrm{l}$ & 1 & \\
\hline Arodoen $1222111 / 4-16-5$ & $<0.0005 \mathrm{mg} / 1$ & $<0.0005 \mathrm{mg} / \mathrm{l}$ & $<0.0005 \mathrm{mg} / \mathrm{l}$ & $<0.0005 \mathrm{mg} / \mathrm{l}$ & 1 & \\
\hline Arodon-12912512206 & $<0.0005 \mathrm{mg} / \mathrm{l}$ & $<0.0005 \mathrm{mg} / 1$ & $<0.0005 \mathrm{mg} / \mathrm{h}$ & $<0.0005 \mathrm{mg} / \mathrm{l}$ & 1 & \\
\hline Avodon 125011006625 & $<0.0005 \mathrm{mg} / \mathrm{l}$ & $<0.0005 \mathrm{mg} / \mathrm{l}$ & $<0.0005 \operatorname{mg} n$ & $<0.0005 \mathrm{mq} / \mathrm{h}$ & 1 & \\
\hline Aradon $10161257411-2$ & $<0.0005 \mathrm{mg} / 1$ & $<0.0005 \mathrm{mg} / \mathrm{l}$ & $<0.0005 \mathrm{me} / \mathrm{h}$ & $<0.0005 \mathrm{mg} / \Omega$ & 1 & \\
\hline Conots & $<0.010 \mathrm{mg} / \mathrm{l}$ & $<0.010 \mathrm{mg} / 1$ & $<0.010 \mathrm{me} / 1$ & $<0.010 \mathrm{mp} / 1$ & 1 & \\
\hline Anosinin & $<0.010 \mathrm{mg} / \mathrm{l}$ & $<0.010 \mathrm{mg} /$ & $<0.010 \mathrm{me} / \mathrm{l}$ & $<0.010 \mathrm{me} / 1$ & 1 & \\
\hline Acosbonitite & $<0.010 \mathrm{mg} / \mathrm{l}$ & $<0.010 \mathrm{mg} / 1$ & $<0.010 \mathrm{mg} / 1$ & $<0.010 \mathrm{mg} / \mathrm{H}$ & $I$ & \\
\hline 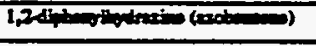 & Resulus pending & Reaulte pending & Realite pending & Reacults pending & 1 & \\
\hline & & & & & & \\
\hline & & & & & & \\
\hline & & & & & & \\
\hline & & & & & & \\
\hline & & & & & & \\
\hline & & & & & & \\
\hline & & & & & & \\
\hline & & & . & & & \\
\hline & & & & & & \\
\hline & & & & & & \\
\hline & & & & & & \\
\hline & & & & & & \\
\hline & & & & & & \\
\hline
\end{tabular}


NI. Discharge Informatuon (contenulad from page 3 of Form 2A)

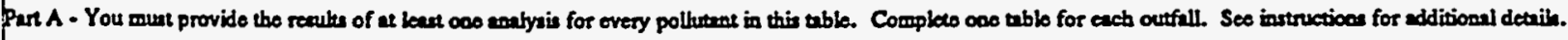

\begin{tabular}{|c|c|c|c|c|c|c|}
\hline $\begin{array}{l}\text { Pollutant } \\
\text { end } \\
\text { CAs Number } \\
\text { (if availible) }\end{array}$ & \multicolumn{2}{|c|}{$\begin{array}{l}\text { Maximum Valuce } \\
\text { (inchedo unita) }\end{array}$} & \multicolumn{2}{|c|}{$\begin{array}{l}\text { Avernge Values } \\
\text { (include unitu) }\end{array}$} & $\begin{array}{c}\text { Number } \\
\text { of } \\
\text { storm } \\
\text { Eveots } \\
\text { sempled }\end{array}$ & Sourceses of Pollutents \\
\hline pil and Gomen & $2 \mathrm{mg} / \mathrm{l}$ & & $2 \mathrm{mg} / \mathrm{l}$ & & 1 & . \\
\hline 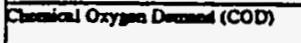 & $46 \mathrm{mg} / 1$ & $22 \mathrm{mg} / \mathrm{l}$ & $46 \mathrm{mg} / 1$ & $22 \mathrm{mg} / 1$ & 1 & \\
\hline 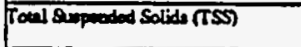 & $23 \mathrm{mg} / \mathrm{l}$ & $25 \mathrm{mg} / \mathrm{l}$ & $23 \mathrm{mg} / \mathrm{l}$ & $25 \mathrm{mg} / 1$ & 1 & \\
\hline 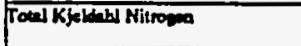 & $0.3 . \mathrm{mg} / \mathrm{l}$ & $0.5 \mathrm{mg} / \mathrm{l}$ & $0.3 \mathrm{mg} / \mathrm{l}$ & $0.5 \mathrm{mg} / \mathrm{h}$ & 1 & \\
\hline Fitne plue Nitrie Nitroger & $0.3 \mathrm{mg} / \mathrm{l}$ & $0.28 \mathrm{mg} / 1$ & $0.3 \mathrm{mg} / \mathrm{l}$ & $028 \mathrm{mg} / 1$ & 1 & \\
\hline Toeal Propionen & $0.16 \mathrm{mg} / \mathrm{l}$ & $<0.1 \mathrm{mg} / \mathrm{l}$ & $0.16 \mathrm{mg} / \mathrm{l}$ & $<0.1 \mathrm{mg} / \mathrm{l}$ & 1 & . \\
\hline
\end{tabular}

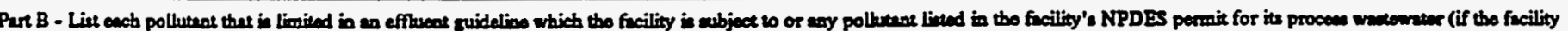

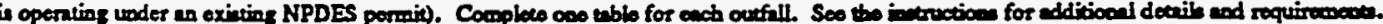

\begin{tabular}{|c|c|c|c|c|c|c|}
\hline \multirow{2}{*}{$\begin{array}{l}\text { Polluteal } \\
\text { and } \\
\text { CAs Number } \\
\text { (if muilibila) }\end{array}$} & \multicolumn{2}{|c|}{ 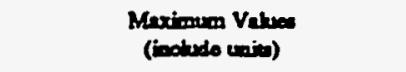 } & \multicolumn{2}{|c|}{$\begin{array}{l}\text { Avarese Values } \\
\text { (inoled enil) }\end{array}$} & \multirow{2}{*}{$\begin{array}{l}\text { Nurnber } \\
\text { of } \\
\text { storm } \\
\text { Eveced } \\
\text { Semplad }\end{array}$} & \multirow{2}{*}{$\begin{array}{c}: \\
\text { Souroes of Polkenent }\end{array}$} \\
\hline & 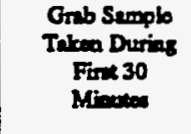 & $\begin{array}{l}\text { Flow-meithed } \\
\text { Compociter }\end{array}$ & 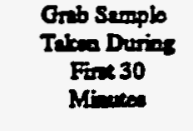 & $\begin{array}{l}\text { Flow-meiztrod } \\
\text { Compotine }\end{array}$ & & \\
\hline $70 \mathrm{~d}=000 \mathrm{y} 740.360$ & $<0.04 \mathrm{mg} / \mathrm{l}$ & $<0.04 \mathrm{mg} / \mathrm{l}$ & $<0.04 \mathrm{mg} / \mathrm{ll}$ & $<0.04 \mathrm{mg} / \mathrm{l}$ & 1 & \\
\hline Faming & $<0.002 \mathrm{mg} / \mathrm{l}$ & & $<0.002 \mathrm{mg} / \mathrm{l}$ & & 1 & \\
\hline Monow 7439076 & $0.0003 \mathrm{mg} / \mathrm{l}$ & $0.0004 \mathrm{mg} / 1$ & $0.0003 \mathrm{mg} / \mathrm{l}$ & $0.0004 \mathrm{mg} / 1$ & 1 & \\
\hline nom & $0.005 \mathrm{mg} / \mathrm{l}$ & & $0.005 \mathrm{mg} / \mathrm{l}$ & & 1 & \\
\hline $301-7 m 2-192$ & $<0.002 \mathrm{mg} / \mathrm{l}$ & $<0.002 \mathrm{mg} / \mathrm{l}$ & $<0.002 \mathrm{mg} / \mathrm{l}$ & $<0.002 \mathrm{mg} / \mathrm{l}$ & 1 & \\
\hline Silnen & $<10 \mathrm{mg} / \mathrm{l}$ & $<10 \mathrm{mg} / \mathrm{l}$ & $<10 \mathrm{mg} / \mathrm{l}$ & $<10 \mathrm{mg} / \mathrm{l}$ & 1 & \\
\hline Arthaneres & $0.091 \mathrm{mg} / \mathrm{l}$ & $<0.05 \mathrm{mg} / 1$ & $0.091 \mathrm{mg} / \mathrm{l}$ & $<0.05 \mathrm{mg} / 1$ & 1 & \\
\hline thenim 7429-90-5 & $1.42 \mathrm{mg} / \mathrm{l}$ & $0.47 \mathrm{mg} / \mathrm{l}$ & $1.42 \mathrm{mg} / 1$ & $0.47 \mathrm{mg} / \mathrm{l}$ & 1 & \\
\hline 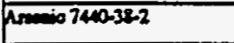 & $<0.04 \mathrm{mq} / 1$ & $<0.04 \mathrm{mg} / 1$ & $<0.04 \mathrm{mg} / \mathrm{l}$ & $<0.04 \mathrm{mg} / 1$ & 1 & \\
\hline 30007400128 & $0.024 \mathrm{mg} / \mathrm{l}$ & $0.022 \mathrm{mg} / \mathrm{l}$ & $0.024 \mathrm{mg} / \mathrm{l}$ & $0.022 \mathrm{mg} / \mathrm{l}$ & 1 & \\
\hline 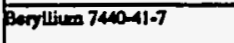 & $<0.0004 \mathrm{mg} / 1$ & $<0.0004 \mathrm{mg} / 1$ & $<0.0004 \mathrm{mg} / 1$ & $<0.0004 \mathrm{mg} / 1$ & 1 & \\
\hline $8 \sin 740-43-9$ & $<0.004 \mathrm{mg} / \mathrm{l}$ & $<0.004 \mathrm{mg} / \mathrm{l}$ & $<0.004 \mathrm{mg} / \mathrm{l}$ & $<0.004 \mathrm{mg} / 1$ & 1 & \\
\hline Fention $7400-\pi 0-2$ & $22.5 \mathrm{mg} / 1$ & $15.7 \mathrm{mg} / \mathrm{l}$ & $22.5 \mathrm{mg} / 1$ & $15.7 \mathrm{mg} / \mathrm{l}$ & 1 & \\
\hline $800=740-7.3$ & $<0.006 \mathrm{mg} / \mathrm{l}$ & $<0.006 \mathrm{mg} / \mathrm{l}$ & $<0.006 \mathrm{mg} / 1$ & $<0.006 \mathrm{mg} / 1$ & 1 & \\
\hline cotalit 740-84 & $<0.002 \mathrm{mg} / \mathrm{l}$ & $<0.002 \mathrm{mg} / 1$ & $<0.002 \mathrm{mg} / \mathrm{l}$ & $<0.002 \mathrm{mg} / 1$ & 1 & \\
\hline Conper 740-508 & $0.030 \mathrm{mg} / \mathrm{l}$ & $0.013 \mathrm{mg} / \mathrm{l}$ & $0.030 \mathrm{mg} / 1$ & $0.013 \mathrm{mg} / \mathrm{l}$ & 1 & \\
\hline 7000742696 & $1.95 \mathrm{mg} / 1$ & $0.63 \mathrm{mg} / \mathrm{l}$ & $1.95 \mathrm{mg} / \mathrm{l}$ & $0.63 \mathrm{mg} / \mathrm{l}$ & 1 & \\
\hline $201739-92-1$ & $<0.02 \mathrm{mg} / \mathrm{l}$ & $<0.02 \mathrm{mg} / \mathrm{l}$ & $<0.02 \mathrm{mg} / 1$ & $<0.02 \mathrm{mg} / 1$ & 1 & \\
\hline Albhem 7/39-93-2 & $<0.02 \mathrm{mg} / \mathrm{l}$ & $<0.02 \mathrm{mg} / 1$ & $<0.02 \mathrm{mg} / \mathrm{l}$ & $<0.02 \mathrm{mg} / 1$ & 1 & \\
\hline Mecomiven 7039954 & $2.94 \mathrm{mg} / 1$ & $1.92 \mathrm{mg} / 1$ & $2.94 \mathrm{mg} / 1$ & $1.92 \mathrm{mg} / 1$ & 1 & \\
\hline Meranom $7432 x+5$ & $0.102 \mathrm{mg} / \mathrm{l}$ & $0.036 \mathrm{mg} / \mathrm{l}$ & $0.102 \mathrm{mg} / \mathrm{l}$ & $0.036 \mathrm{mg} / \mathrm{l}$ & 1 & \\
\hline 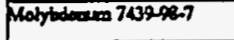 & $<0.006 \mathrm{mg} / \mathrm{l}$ & $<0.006 \mathrm{mg} / 1$ & $<0.006 \mathrm{mg} / 1$ & $<0.006 \mathrm{mg} / \mathrm{l}$ & 1 & \\
\hline$\sqrt{102017400020}$ & $<0.008 \mathrm{mg} / 1$ & $<0.008 \mathrm{mg} / 1$ & $<0.008 \mathrm{mg} / \mathrm{l}$ & $<0.008 \mathrm{mg} / \mathrm{l}$ & 1 & \\
\hline 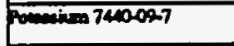 & $1.6 \mathrm{mg} / \mathrm{l}$ & $1.1 \mathrm{mg} / \mathrm{l}$ & $1.6 \mathrm{mg} / \mathrm{l}$ & $1.1 \mathrm{mg} / \mathrm{l}$ & 1 & \\
\hline simen 74a-224 & $<0.006 \mathrm{mg} / \mathrm{l}$ & $<0.006 \mathrm{mg} / 1$ & $<0.006 \mathrm{mg} / \mathrm{l}$ & $<0.006 \mathrm{mg} / \mathrm{l}$ & 1 & \\
\hline Paxm 740-20-5 & $1.07 \mathrm{mg} / 1$ & $0.77 \mathrm{mg} / 1$ & $1.07 \mathrm{mg} / 1$ & $0.77 \mathrm{mg} / 1$ & 1 & \\
\hline 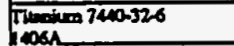 & $0.02 \mathrm{mg} / \mathrm{l}$ & $<0.01 \mathrm{mg} / \mathrm{l}$ & $0.02 \mathrm{mg} / 1$ & $<0.01 \mathrm{mg} / 1$ & 1 & \\
\hline $2000740-666$ & $0.22 \mathrm{mg} / 1$ & $0.09 \mathrm{mg} / \mathrm{h}$ & $0.22 \mathrm{mg} / \mathrm{l}$ & $0.09 \mathrm{mg} / 1$ & 1 & \\
\hline
\end{tabular}


Cosdianed from frowe.

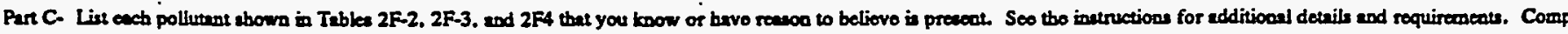
one trble for each outfall.

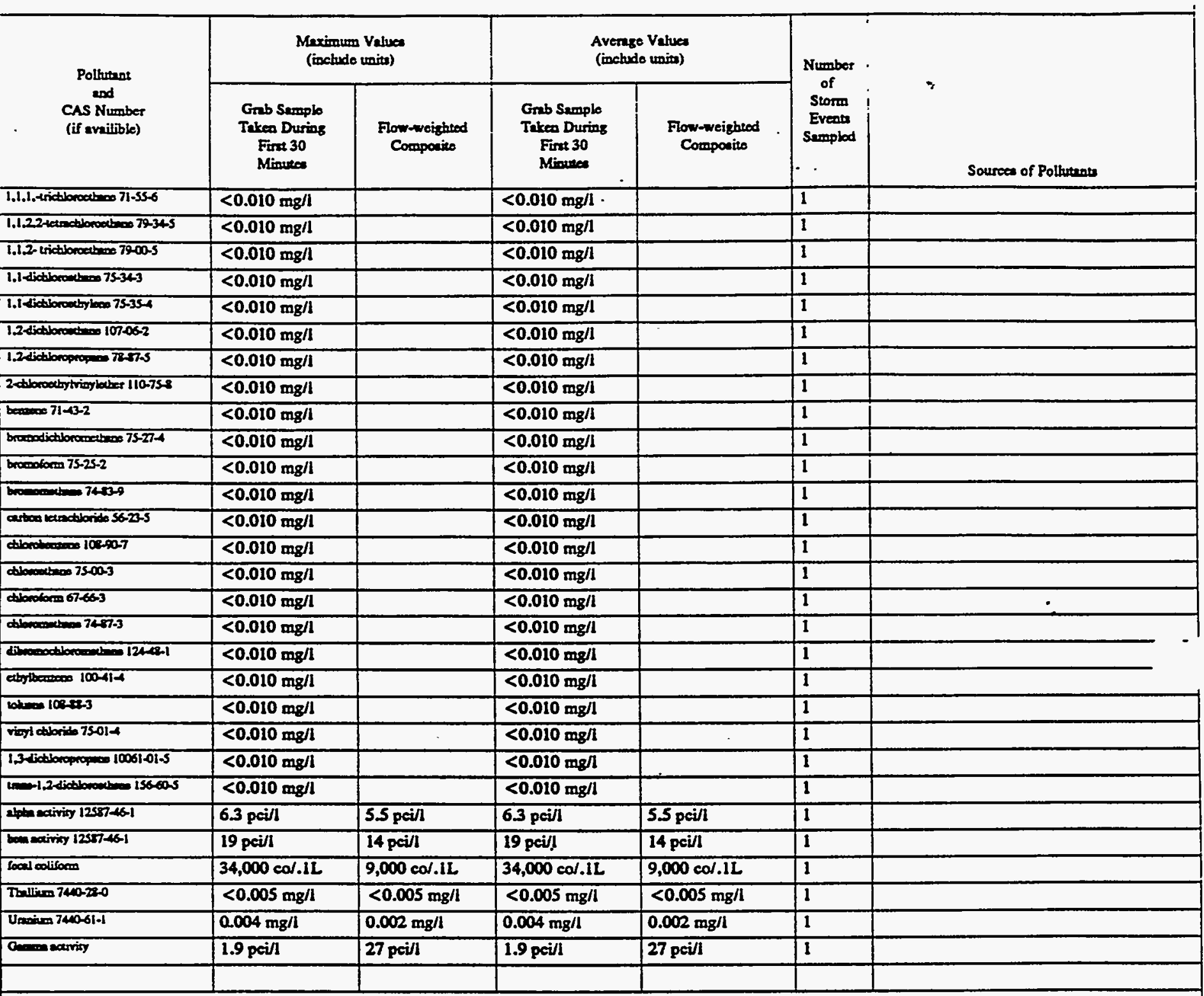

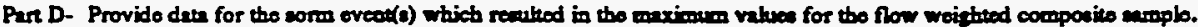

\begin{tabular}{|c|c|c|c|c|c|c|c|}
\hline $\begin{array}{l}1 . \\
\text { Dito of } \\
\text { Storm } \\
\text { Event }\end{array}$ & $\begin{array}{l}2 . \\
\text { Duration } \\
\text { of Storm } \\
\text { (in } \\
\text { minutos) }\end{array}$ & $\begin{array}{l}\text { Towil ninfill } \\
\text { during storm ovear } \\
\text { (in incteos) }\end{array}$ & 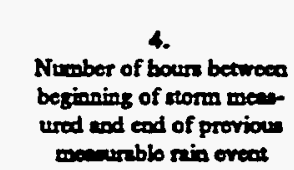 & 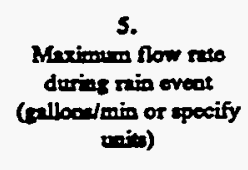 & $\begin{array}{l}6 . \\
\text { Tocil flow fros } \\
\text { nin eveat } \\
\text { (pallows or } \\
\text { spocify woin) }\end{array}$ & $\begin{array}{c}7 . \\
\text { Semon } \\
\text { aumple was } \\
\text { tuken }\end{array}$ & $\begin{array}{l}\text { Bo. } \\
\text { Procipiantion } \\
\text { (niafall. } \\
\text { esowmell) }\end{array}$ \\
\hline $8 / 26 / 91$ & 410 & 1.1 & 160.2 & 5,524 & $1,270,913$ & Summer & Reinfall \\
\hline
\end{tabular}

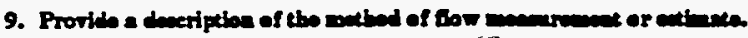

An ISCO 3230 Flow meter was used to obtain water level readings. This instrument was calibrated with the normal dry weather flow, if present, as level zero. The flow meter wes programmed with the characteristics of the conveyance so that flow rates and totel fows conld be calculated by the meter. 
Uutfall 14

Parra B and C Continused

\begin{tabular}{|c|c|c|c|c|c|c|}
\hline \multirow{2}{*}{$\begin{array}{l}\text { - Pollutuat } \\
\text { und } \\
\text { CAS Number } \\
\text { (if availible) }\end{array}$} & \multicolumn{2}{|c|}{$\begin{array}{l}\text { Merimum Valuea } \\
\text { (iveludo unin) }\end{array}$} & \multicolumn{2}{|c|}{$\begin{array}{l}\text { Averngs Veluce } \\
\text { (incluedo wnio) }\end{array}$} & \multirow{2}{*}{$\begin{array}{c}\text { Number } \\
\text { of } \\
\text { Stomen } \\
\text { Eveose } \\
\text { Semplad }\end{array}$} & \multirow[b]{2}{*}{ Sources of Polhutinas } \\
\hline & $\begin{array}{c}\text { Onbb Samplo } \\
\text { Tateon During } \\
\text { Fins } 30 \\
\text { Minote }\end{array}$ & $\begin{array}{l}\text { Flow-weightiod } \\
\text { Comporite }\end{array}$ & $\begin{array}{l}\text { Grab Semple } \\
\text { Tikea During } \\
\text { Firat } 30 \\
\text { Minues }\end{array}$ & $\begin{array}{l}\text { Flow-weighted } \\
\text { Compoticos }\end{array}$ & & \\
\hline Berien $740-32,3$ & $0.0414 \mathrm{mg} / 1$ & $0.0278 \mathrm{mg} / 1$ & $0.0414 \mathrm{mg} / \mathrm{l}$ & $0.0278 \mathrm{mg} / \mathrm{l}$ & 1 & \\
\hline 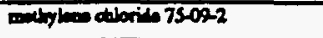 & $<0.010 \mathrm{mg} / \mathrm{l}$ & & $<0.010 \mathrm{mg} / 1$ & & 1 & \\
\hline 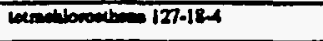 & $<0.010 \mathrm{mg} / 1$ & & $<0.010 \mathrm{mg} / \mathrm{l}$ & & 1 & \\
\hline 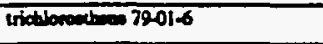 & $<0.010 \mathrm{mg} / \mathrm{l}$ & & $<0.010 \mathrm{mg} / \mathrm{l}$ & & 1 & \\
\hline Xyleosen & $<0.010 \mathrm{mg} / \mathrm{l}$ & & $<0.010 \mathrm{mg} / 1$ & & 1 & \\
\hline 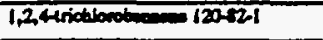 & $<0.010 \mathrm{mg} / \mathrm{h}$ & $<0.010 \mathrm{mg} / 1$ & $<0.010 \mathrm{mg} / 1$ & $<0.010 \mathrm{mg} / \mathrm{l}$ & 1 & \\
\hline 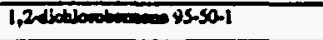 & $<0.010 \mathrm{mg} / \mathrm{l}$ & $<0.010 \mathrm{mg} / \mathrm{l}$ & $<0.010 \mathrm{mg} / 1$ & $<0.010 \mathrm{mg} / \mathrm{l}$ & 1 & \\
\hline 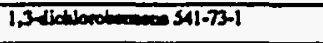 & $<0.010 \mathrm{mg} / 1$ & $<0.010 \mathrm{mg} / 1$ & $<0.010 \mathrm{mg} / \mathrm{l}$ & $<0.010 \mathrm{mg} / \mathrm{l}$ & 1 & \\
\hline 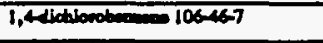 & $<0.010 \mathrm{mg} / \mathrm{l}$ & $<0.010 \mathrm{mg} / \mathrm{l}$ & $<0.010 \mathrm{mg} / \mathrm{l}$ & $<0.010 \mathrm{mg} / \mathrm{s}$ & 1 & \\
\hline 2,4 dishodoknes $121-142$ & $<0.010 \mathrm{mg} / \mathrm{l}$ & $<0.010 \mathrm{mg} / 1$ & $<0.010 \mathrm{mg} / \mathrm{l}$ & $<0.010 \mathrm{mg} / \mathrm{l}$ & 1 & \\
\hline 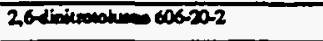 & $<0.010 \mathrm{mg} / \mathrm{l}$ & $<0.010 \mathrm{mg} / \mathrm{l}$ & $<0.010 \mathrm{mg} / \mathrm{l}$ & $<0.010 \mathrm{mg} / \mathrm{h}$ & 1 & - \\
\hline 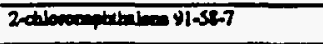 & $<0.010 \mathrm{mg} / \mathrm{l}$ & $<0.010 \mathrm{mg} / 1$ & $<0.010 \mathrm{mg} / \mathrm{I}$ & $<0.010 \mathrm{mg} / \mathrm{l}$ & 1 & \\
\hline 1,3'-dictionoticidive 91-241 & $<0.010 \mathrm{mg} / 1$ & $<0.010 \mathrm{mg} / \mathrm{l}$ & $<0.010 \mathrm{mg} / \mathrm{l}$ & $<0.010 \mathrm{mg} / \mathrm{ll}$ & 1 & \\
\hline 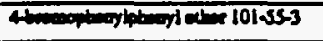 & $<0.010 \mathrm{mg} / \mathrm{l}$ & $<0.010 \mathrm{mg} / \mathrm{l}$ & $<0.010 \mathrm{mg} / \mathrm{l}$ & $<0.010 \mathrm{mg} / \mathrm{l}$ & 1 & \\
\hline 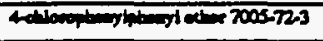 & $<0.010 \mathrm{mg} / \mathrm{l}$ & $<0.010 \mathrm{mg} / \mathrm{l}$ & $<0.010 \mathrm{mg} / 1$ & $<0.010 \mathrm{mg} / \mathrm{l}$ & 1 & \\
\hline complemes $83-329$ & $<0.010 \mathrm{mg} / \mathrm{l}$ & $<0.010 \mathrm{mg} / \mathrm{h}$ & $<0.010 \mathrm{mg} / \mathrm{l}$ & $<0.010 \mathrm{mg} / 1$ & 1 & \\
\hline 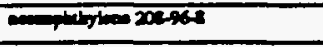 & $<0.010 \mathrm{mg} / 1$ & $<0.010 \mathrm{mg} / 1$ & $<0.010 \mathrm{mg} / \mathrm{l}$ & $<0.010 \mathrm{mg} / \mathrm{l}$ & 1 & \\
\hline $120-127$ & $<0.010 \mathrm{mg} / \mathrm{l}$ & $<0.010 \mathrm{mg} / \mathrm{l}$ & $<0.010 \mathrm{mg} / \mathrm{l}$ & $<0.010 \mathrm{mg} / \mathrm{l}$ & $I$ & \\
\hline idiens.5 & $<0.030 \mathrm{mg} / \mathrm{l}$ & $<0.030 \mathrm{mg} / \mathrm{l}$ & $<0.030 \mathrm{mg} / \mathrm{l}$ & $<0.030 \mathrm{mg} /$ & 1 & - \\
\hline 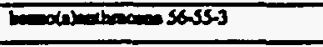 & $<0.010 \mathrm{mg} / \mathrm{l}$ & $<0.010 \mathrm{mg} / \mathrm{l}$ & $<0.010 \mathrm{mg} / \mathrm{l}$ & $<0.010 \mathrm{mg} / \mathrm{I}$ & 1 & \\
\hline 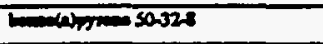 & $<0.010 \mathrm{mg} / \mathrm{l}$ & $<0.010 \mathrm{mg} / \mathrm{l}$ & $<0.010 \mathrm{mg} / \mathrm{l}$ & $<0.010 \mathrm{mg} / 1$ & 1 & \\
\hline $20592-2$ & $<0.010 \mathrm{mg} / \mathrm{l}$ & $<0.010 \mathrm{mg} / \mathrm{l}$ & $<0.010 \mathrm{mg} / 1$ & $<0.010 \mathrm{mg} / 1$ & 1 & \\
\hline 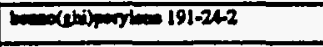 & $<0.010 \mathrm{mg} / \mathrm{h}$ & $<0.010 \mathrm{mg} / \mathrm{l}$ & $<0.010 \mathrm{mg} / \mathrm{l}$ & $<0.010 \mathrm{mg} / \mathrm{l}$ & 1 & \\
\hline 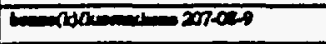 & $<0.010 \mathrm{mg} / 1$ & $<0.010 \mathrm{mg} / 1$ & $<0.010 \mathrm{mg} / \mathrm{h}$ & $<0.010 \mathrm{mg} / 1$ & 1 & \\
\hline 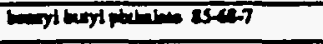 & $<0.010 \mathrm{mg} / 1$ & $<0.010 \mathrm{mg} / 1$ & $<0.010 \mathrm{mg} / \mathrm{l}$ & $<0.010 \mathrm{mg} / \mathrm{l}$ & 1 & \\
\hline 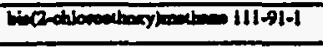 & $<0.010 \mathrm{mg} / \mathrm{h}$ & $<0.010 \mathrm{mg} / \mathrm{l}$ & $<0.010 \mathrm{mg} / \mathrm{l}$ & $<0.010 \mathrm{mg} / 1$ & 1 & \\
\hline 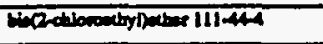 & $<0.010 \mathrm{mg} / \mathrm{l}$ & $<0.010 \mathrm{mg} / 1$ & $<0.010 \mathrm{mg} / \mathrm{l}$ & $<0.010 \mathrm{mg} / \mathrm{l}$ & 1 & \\
\hline 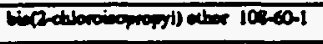 & $<0.010 \mathrm{mg} / \mathrm{l}$ & $<0.010 \mathrm{mg} / \mathrm{l}$ & $<0.010 \mathrm{mg} / \mathrm{l}$ & $<0.010 \mathrm{mg} / \mathrm{l}$ & 1 & \\
\hline 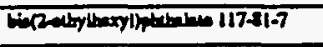 & $<0.010 \mathrm{mg} / 1$ & $0.012 \mathrm{mg} / 1$ & $<0.010 \mathrm{mg} / \mathrm{l}$ & $0.012 \mathrm{mg} / 1$ & 1 & \\
\hline dernes 218019 & $<0.010 \mathrm{mg} / 1$ & $<0.010 \mathrm{mg} / \mathrm{l}$ & $<0.010 \mathrm{mg} / 1$ & $<0.010 \mathrm{mg} / \mathrm{h}$ & 1 & \\
\hline 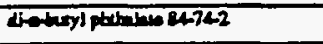 & $<0.010 \mathrm{mg} / 1$ & $<0.010 \mathrm{mg} / \mathrm{l}$ & $<0.010 \mathrm{mg} / \mathrm{l}$ & $<0.010 \mathrm{mg} / \mathrm{l}$ & 1 & \\
\hline 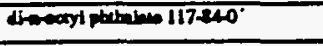 & $<0.010 \mathrm{mg} / 1$ & $0.030 \mathrm{mg} / \mathrm{l}$ & $<0.010 \mathrm{mg} / \mathrm{h}$ & $0.030 \mathrm{mg} / \mathrm{l}$ & 1 & \\
\hline 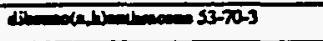 & $<0.010 \mathrm{mg} / 1$ & $<0.010 \mathrm{mg} / 1$ & $<0.010 \mathrm{mg} / \mathrm{l}$ & $<0.010 \mathrm{mg} / 1$ & 1 & \\
\hline 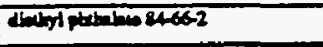 & $<0.010 \mathrm{mg} / \mathrm{l}$ & $<0.010 \mathrm{mg} / \mathrm{l}$ & $<0.010 \mathrm{mg} / \mathrm{l}$ & $<0.010 \mathrm{mg} / 1$ & 1 & \\
\hline 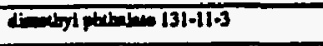 & $<0.010 \mathrm{mg} / \mathrm{I}$ & $<0.010 \mathrm{mg} / 1$ & $<0.010 \mathrm{mg} / \mathrm{l}$ & $<0.010 \mathrm{mg} / 1$ & 1 & \\
\hline $20000=200460$ & $<0.010 \mathrm{mg} / \mathrm{ll}$ & $<0.010 \mathrm{mg} / 1$ & $<0.010 \mathrm{mg} / \mathrm{l}$ & $<0.010 \mathrm{mg} / 1$ & 1 & \\
\hline $06=18-73-7$ & $<0.010 \mathrm{mg} / \mathrm{I}$ & $<0.010 \mathrm{mg} / \mathrm{l}$ & $<0.010 \mathrm{mg} / 1$ & $<0.010 \mathrm{mg} / 1$ & 1 & \\
\hline 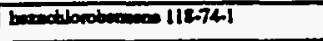 & $<0.010 \mathrm{mg} / 1$ & $<0.010 \mathrm{mg} / 1$ & $<0.010 \mathrm{mg} / \mathrm{l}$ & $<0.010 \mathrm{mg} / 1$ & 1 & \\
\hline 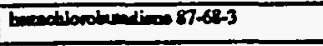 & $<0.010 \mathrm{mg} / 1$ & $<0.010 \mathrm{mg} / \mathrm{l}$ & $<0.010 \mathrm{mg} / \mathrm{l}$ & $<0.010 \mathrm{mg} / \mathrm{l}$ & 1 & \\
\hline 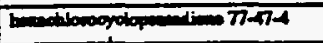 & $<0.010 \mathrm{mg} / \mathrm{l}$ & $<0.010 \mathrm{mg} /$ & $<0.010 \mathrm{mg} / \mathrm{l}$ & $<0.010 \mathrm{mg} / 1$ & 1 & \\
\hline 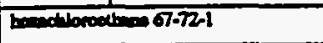 & $<0.010 \mathrm{mg} / \mathrm{l}$ & $<0.010 \mathrm{mg} / \mathrm{l}$ & $<0.010 \mathrm{mg} / \mathrm{l}$ & $<0.010 \mathrm{mg} / \mathrm{l}$ & 1 & \\
\hline 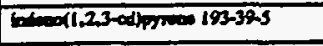 & $<0.010 \mathrm{mg} / \mathrm{l}$ & $<0.010 \mathrm{mg} / 1$ & $<0.010 \mathrm{mg} / 1$ & $<0,010 \mathrm{mg} / 1$ & 1 & \\
\hline 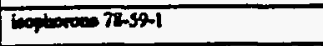 & $<0.010 \mathrm{mg} / \mathrm{l}$ & $<0.010 \mathrm{mg} / \mathrm{l}$ & $<0.010 \mathrm{mg} / \mathrm{h}$ & $<0.010 \mathrm{mg} / \mathrm{l}$ & 1 & \\
\hline 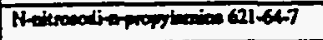 & $<0.010 \mathrm{mg} / \mathrm{t}$ & $<0.010 \mathrm{mg} / \mathrm{l}$ & $<0.010 \mathrm{mg} / \mathrm{l}$ & $<0.010 \mathrm{mg} / 1$ & 1 & \\
\hline 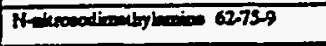 & $<0.010 \mathrm{mg} / \mathrm{l}$ & $<0.010 \mathrm{mg} / 1$ & $<0.010 \mathrm{mg} / 1$ & $<0.010 \mathrm{mg} / \mathrm{h}$ & 1 & \\
\hline 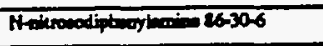 & $<0.010 \mathrm{mg} / \mathrm{l}$ & $<0.010 \mathrm{mg} / \mathrm{l}$ & $<0.010 \mathrm{mg} / \mathrm{l}$ & $<0.010 \mathrm{mg} / \mathrm{h}$ & 1 & \\
\hline 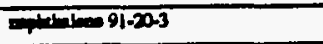 & $<0.010 \mathrm{mg} / 1$ & $<0.010 \mathrm{mg} / 1$ & $<0.010 \mathrm{mg} / 1$ & $<0.010 \mathrm{mg} / \mathrm{l}$ & 1 & \\
\hline menteseses-3 & $<0.010 \mathrm{mg} / \mathrm{l}$ & $<0.010 \mathrm{mg} / \mathrm{l}$ & $<0.010 \mathrm{mg} / \mathrm{l}$ & $<0.010 \mathrm{mg} / 1$ & 1 & \\
\hline 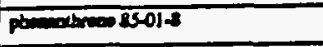 & $<0.010 \mathrm{mg} / \mathrm{l}$ & $<0.010 \mathrm{mg} / 1$ & $<0.010 \mathrm{mg} / \mathrm{I}$ & $<0.010 \mathrm{mg} / 1$ & 1 & \\
\hline mines 129000 & $<0.010 \mathrm{mg} / 1$ & $<0.010 \mathrm{mg} / 1$ & $<0.010 \mathrm{mg} / \mathrm{l}$ & $<0.010 \mathrm{mg} / 1$ & 1 & \\
\hline 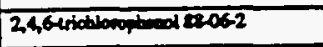 & $<0.030 \mathrm{mg} / 1$ & $<0.030 \mathrm{mg} / \mathrm{l}$ & $<0.030 \mathrm{mg} / \mathrm{l}$ & $<0.030 \mathrm{mg} / \mathrm{h}$ & 1 & \\
\hline 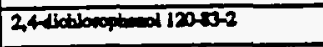 & $<0.030 \mathrm{mg} / \mathrm{l}$ & $<0.030 \mathrm{mg} / 1$ & $<0.030 \mathrm{mg} / \mathrm{l}$ & $<0.030 \mathrm{mg} / \mathrm{l}$ & 1 & \\
\hline 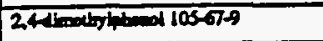 & $<0.030 \mathrm{mg} / \mathrm{l}$ & $<0.030 \mathrm{mg} / \mathrm{ll}$ & $<0.030 \mathrm{mg} / \mathrm{l}$ & $<0.030 \mathrm{mg} / \mathrm{l}$ & 1 & \\
\hline 2,4 dimitroped SI-220s & $<0.030 \mathrm{mg} / \mathrm{l}$ & $<0.030 \mathrm{mg} / \mathrm{l}$ & $<0.030 \mathrm{mg} / \mathrm{l}$ & $<0.030 \mathrm{mg} / 1$ & 1 & \\
\hline
\end{tabular}


Outfan 14

Pars B and C Costinuod

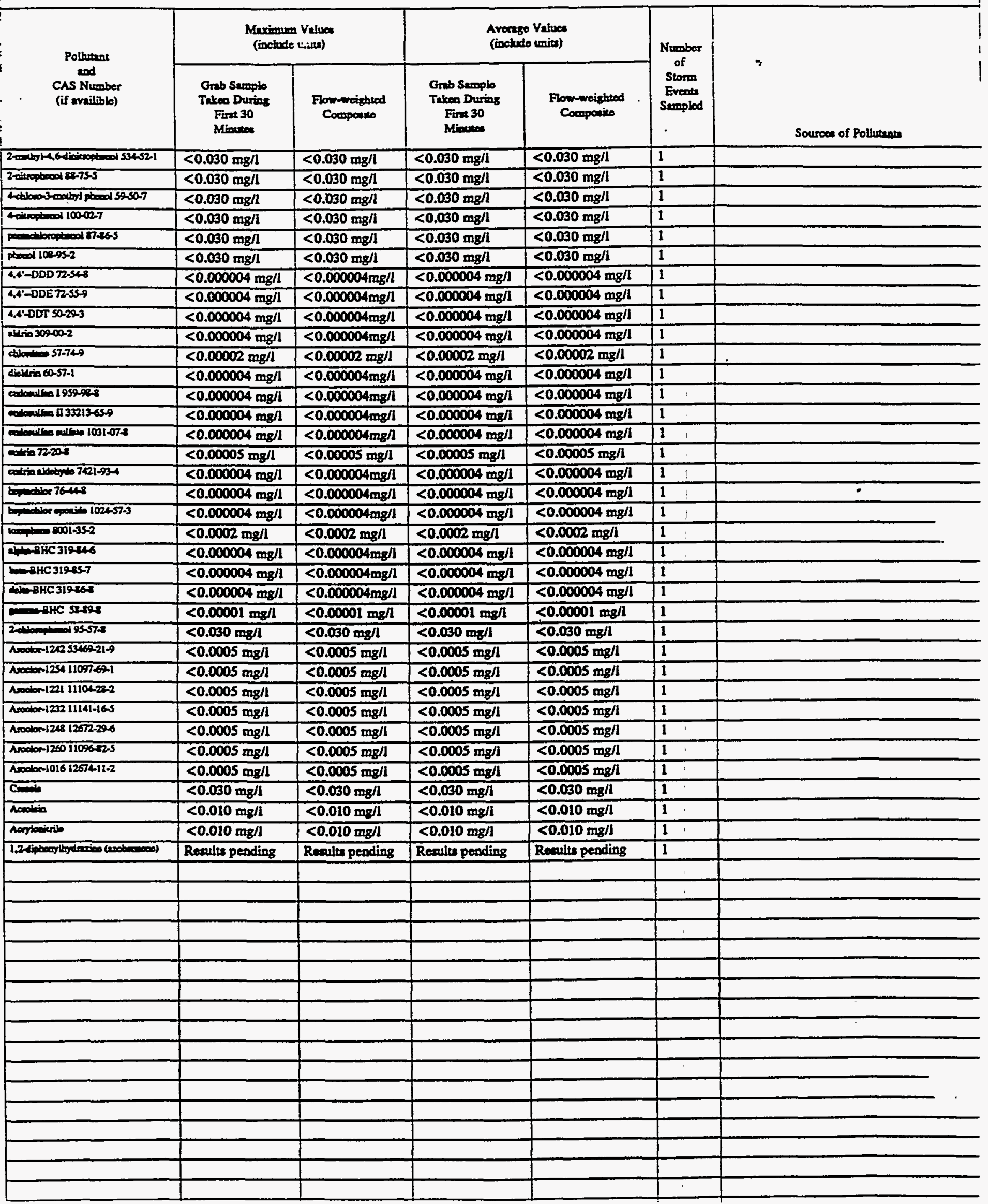




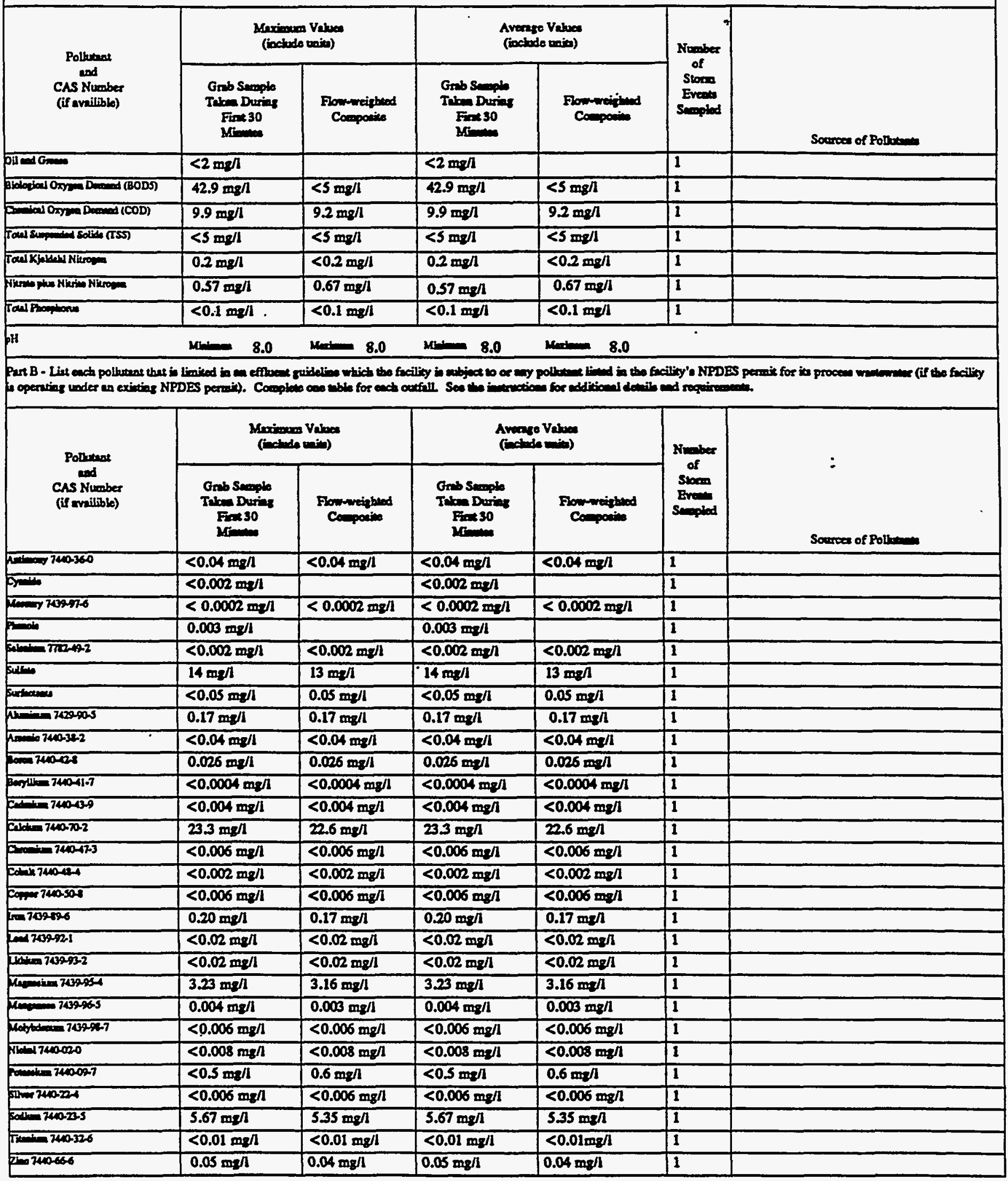


Cockinued from froot.

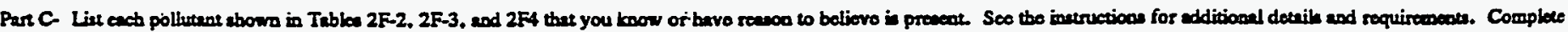
one table for cach outiall.

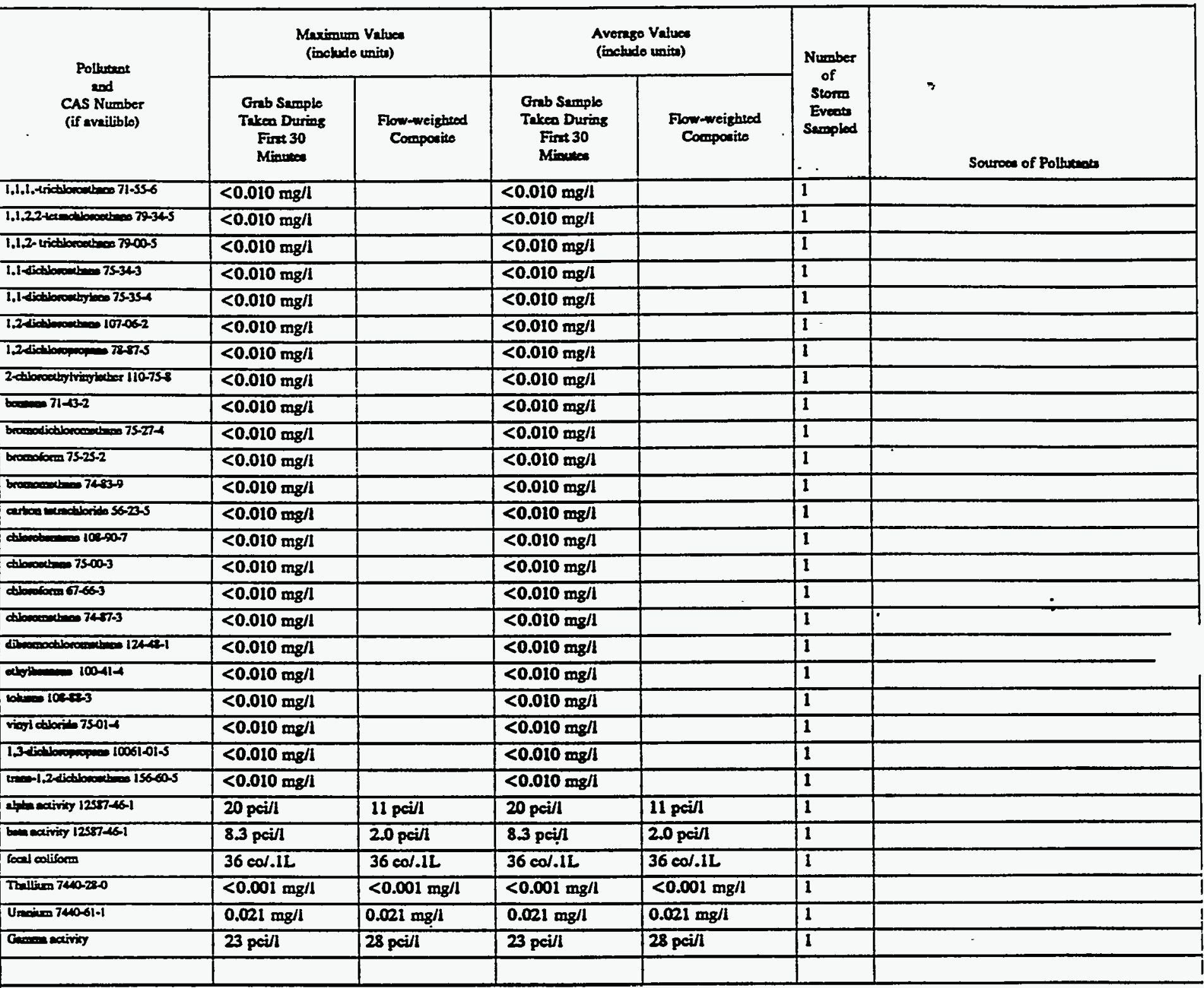

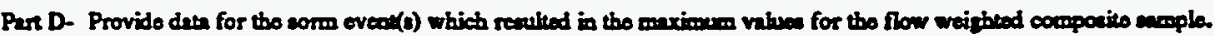

\begin{tabular}{|c|c|c|c|c|c|c|c|}
\hline $\begin{array}{l}1 . \\
\text { Detos of } \\
\text { Storm } \\
\text { Eveot }\end{array}$ & $\begin{array}{l}2 . \\
\text { Durition } \\
\text { of Storm } \\
\text { (in } \\
\text { mineses) }\end{array}$ & $\begin{array}{l}3 . \\
\text { Total minfill } \\
\text { during etomb ovent } \\
\text { (in inche) }\end{array}$ & 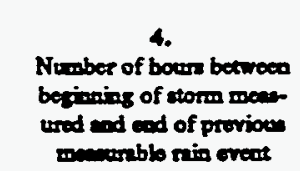 & 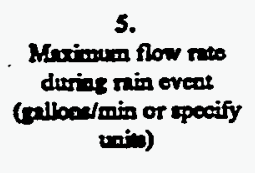 & $\begin{array}{l}6 . \\
\text { Total flow from } \\
\text { min eveot } \\
\text { (alloos or } \\
\text { pacify mita) }\end{array}$ & $\begin{array}{c}7 . \\
\text { Seacon } \\
\text { aemplo wes } \\
\text { tuken }\end{array}$ & $\begin{array}{l}8 . \\
\text { Form of } \\
\text { Precipiasion } \\
\text { (miafill, } \\
\text { ammels) }\end{array}$ \\
\hline $3 / 6 / 92$ & 420 & 0.76 & 138.9 & 15.6 & 3,204 & Wimet & Rninfall \\
\hline
\end{tabular}

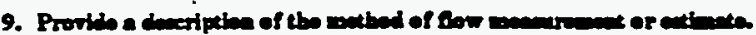

An ISCO 3230 Flow meter was used to obtain water level reading. This instrument was calibrated with the normal dry wenther flow, if present, as bere zero. The flow weter was progrnmmed with the chnracteristics of the convey ance so that flow rates and total flows could be calculated by the rneter. 
Qutrill 15

Parts B and C Consinenod

\begin{tabular}{|c|c|c|c|c|c|c|}
\hline \multirow{2}{*}{$\begin{array}{l}\text { Pollutaose } \\
\text { and } \\
\text { CAs Number } \\
\text { (if availiblo) }\end{array}$} & \multicolumn{2}{|c|}{$\begin{array}{l}\text { Meximum Valuea } \\
\text { (includo uning) }\end{array}$} & \multicolumn{2}{|c|}{$\begin{array}{l}\text { Avernge Values } \\
\text { (include unitu) }\end{array}$} & \multirow{2}{*}{$\begin{array}{c}\text { Number } \\
\text { of } \\
\text { Storm } \\
\text { Eveces } \\
\text { Sxropled }\end{array}$} & \multirow[b]{2}{*}{ Sourres of Polluenese } \\
\hline & $\begin{array}{l}\text { Oreb Semplo } \\
\text { Tukea During } \\
\text { First } 30 \\
\text { Minewed }\end{array}$ & $\begin{array}{l}\text { Frow-waighled } \\
\text { Comporice }\end{array}$ & 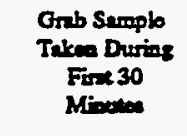 & $\begin{array}{l}\text { Flow-wrighted } \\
\text { Componins }\end{array}$ & & \\
\hline $\operatorname{Barkm} 740.32-3$ & $0.0252 \mathrm{mg} / 1$ & $0.0238 \mathrm{mg} / 1$ & $0.0252 \mathrm{mg} / 1$ & $0.0238 \mathrm{mg} / \mathrm{l}$ & 1 & \\
\hline 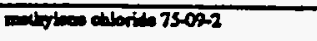 & $<0.010 \mathrm{mg} / 1$ & & $<0.010 \mathrm{mg} / 1$ & & $I$ & \\
\hline 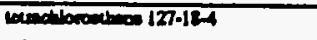 & $<0.010 \mathrm{mg} / \mathrm{l}$ & . & $<0.010 \mathrm{mg} / 1$ & & 1 & \\
\hline Irictlosedeon 79016 & $<0.010 \mathrm{mg} / \mathrm{l}$ & & $<0.010 \mathrm{mg} / \mathrm{l}$ & & $\overline{1}$ & \\
\hline xyleon & $<0.010 \mathrm{mg} / \mathrm{l}$ & & $<0.010 \mathrm{mg} / \mathrm{l}$ & & $I$ & \\
\hline 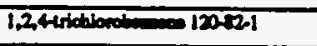 & $<0.010 \mathrm{mg} / \mathrm{l}$ & $<0.010 \mathrm{mg} / 1$ & $<0.010 \mathrm{mg} / 1$ & $<0.010 \mathrm{mg} / 1$ & 1 & \\
\hline 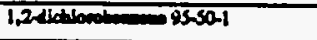 & $<0.010 \mathrm{mg} / \mathrm{l}$ & $<0.010 \mathrm{mg} / 1$ & $<0.010 \mathrm{mg} / 1$ & $<0.010 \mathrm{mg} / 1$ & 1 & $\bar{r}$ \\
\hline 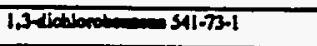 & $<0.010 \mathrm{mg} / \mathrm{l}$ & $<0.010 \mathrm{mg} / \mathrm{l}$ & $<0.010 \mathrm{mg} / \mathrm{l}$ & $<0.010 \mathrm{mg} / \mathrm{l}$ & 1 & \\
\hline 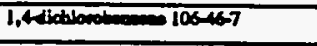 & $<0.010 \mathrm{mg} / \mathrm{l}$ & $<0.010 \mathrm{mg} / 1$ & $<0.010 \mathrm{mg} / \mathrm{l}$ & $<0.010 \mathrm{mg} / 1$ & 1 & \\
\hline 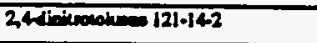 & $<0.010 \mathrm{mg} / \mathrm{l}$ & $<0.010 \mathrm{mg} / \mathrm{l}$ & $<0.010 \mathrm{mg} / 1$ & $<0.010 \mathrm{mg} / 1$ & 1 & \\
\hline 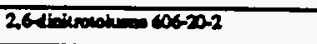 & $<0.010 \mathrm{mg} / \mathrm{l}$ & $<0.010 \mathrm{mg} / \mathrm{l}$ & $<0.010 \mathrm{mg} / 1$ & $<0.010 \mathrm{mg} / \mathrm{l}$ & 1 & \\
\hline 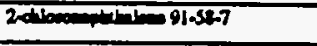 & $<0.010 \mathrm{mg} / \mathrm{l}$ & $<0.010 \mathrm{mg} / \mathrm{l}$ & $<0.010 \mathrm{mg} / \mathrm{l}$ & $<0.010 \mathrm{mg} / \mathrm{l}$ & 1 & \\
\hline 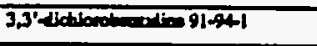 & $<0.020 \mathrm{mg} / \mathrm{l}$ & $<0.020 \mathrm{mg} / \mathrm{l}$ & $<0.020 \mathrm{mg} / 1$ & $<0.020 \mathrm{mg} / \mathrm{l}$ & $I$ & \\
\hline 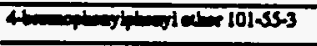 & $<0.010 \mathrm{mg} / \mathrm{l}$ & $<0.010 \mathrm{mg} / 1$ & $<0.010 \mathrm{mg} / 1$ & $<0.010 \mathrm{mg} / 1$ & 1 & \\
\hline 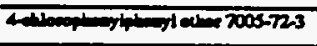 & $<0.010 \mathrm{mg} / \mathrm{l}$ & $<0.010 \mathrm{mg} / \mathrm{l}$ & $<0.010 \mathrm{mg} / \mathrm{l}$ & $<0.010 \mathrm{mg} / 1$ & 1 & \\
\hline 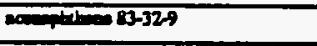 & $<0.010 \mathrm{mg} / \mathrm{l}$ & $<0.010 \mathrm{mg} / 1$ & $<0.010 \mathrm{mg} / \mathrm{l}$ & $<0.010 \mathrm{mg} / \mathrm{l}$ & 1 & \\
\hline 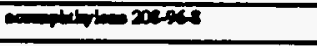 & $<0.010 \mathrm{mg} / \mathrm{l}$ & $<0.010 \mathrm{mg} / \mathrm{I}$ & $<0.010 \mathrm{mg} / \mathrm{l}$ & $<0.010 \mathrm{mg} / 1$ & 1 & \\
\hline $120-127$ & $<0.010 \mathrm{mg} / \mathrm{l}$ & $<0.010 \mathrm{mg} / \mathrm{l}$ & $<0.010 \mathrm{mg} / 1$ & $<0.010 \mathrm{mg} / 1$ & 1 & $\therefore$ \\
\hline inters 925 & $<0.050 \mathrm{mg} / \mathrm{l}$ & $<0.050 \mathrm{mg} / 1$ & $<0.050 \mathrm{mg} / \mathrm{l}$ & $<0.050 \mathrm{mg} / \mathrm{l}$ & 1 & \\
\hline 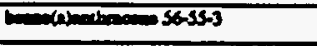 & $<0.010 \mathrm{mg} / \mathrm{l}$ & $<0.010 \mathrm{mg} / 1$ & $<0.010 \mathrm{mg} / \mathrm{l}$ & $<0.010 \mathrm{mg} / \mathrm{l}$ & 1 & \\
\hline 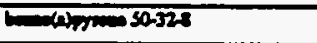 & $<0.010 \mathrm{mg} / 1$ & $<0.010 \mathrm{mg} / \mathrm{l}$ & $<0.010 \mathrm{mg} / \mathrm{l}$ & $<0.010 \mathrm{mg} / 1$ & 1 & \\
\hline 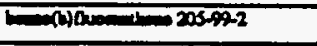 & $<0.010 \mathrm{mg} / \mathrm{l}$ & $<0.010 \mathrm{mg} / 1$ & $<0.010 \mathrm{mg} / \mathrm{l}$ & $<0.010 \mathrm{mg} / 1$ & 1 & \\
\hline 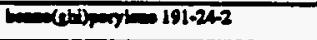 & $<0.010 \mathrm{mg} / \mathrm{l}$ & $<0.010 \mathrm{mg} / 1$ & $<0.010 \mathrm{mg} / 1$ & $<0.010 \mathrm{mg} / 1$ & 1 & \\
\hline 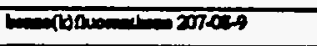 & $<0.010 \mathrm{mg} / \mathrm{l}$ & $<0.010 \mathrm{mg} / \mathrm{l}$ & $<0.010 \mathrm{mg} / 1$ & $<0.010 \mathrm{mg} / \mathrm{l}$ & 1 & \\
\hline 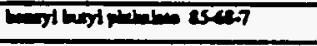 & $<0.010 \mathrm{mg} / \mathrm{i}$ & $<0.010 \mathrm{mg} / \mathrm{l}$ & $<0.010 \mathrm{mg} / \mathrm{h}$ & $<0.010 \mathrm{mg} / \mathrm{l}$ & 1 & \\
\hline 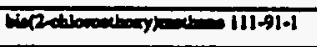 & $<0.010 \mathrm{mg} / \mathrm{l}$ & $<0.010 \mathrm{mg} / \mathrm{l}$ & $<0.010 \mathrm{mg} / \mathrm{l}$ & $<0.010 \mathrm{mg} / \mathrm{l}$ & 1 & \\
\hline 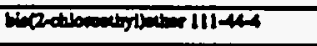 & $<0.010 \mathrm{mg} / \mathrm{l}$ & $<0.010 \mathrm{mg} / \mathrm{l}$ & $<0.010 \mathrm{mg} / \mathrm{l}$ & $<0.010 \mathrm{mg} / \mathrm{l}$ & 1 & \\
\hline 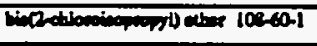 & $<0.010 \mathrm{mg} / \mathrm{l}$ & $<0.010 \mathrm{mg} / 1$ & $<0.010 \mathrm{mg} / \mathrm{l}$ & $<0.010 \mathrm{mg} / \mathrm{l}$ & 1 & \\
\hline 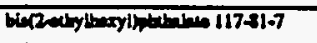 & $<0.010 \mathrm{mg} / \mathrm{l}$ & $<0.010 \mathrm{mg} / \mathrm{l}$ & $<0.010 \mathrm{mg} / \mathrm{l}$ & $<0.010 \mathrm{mg} / \mathrm{l}$ & 1 & \\
\hline chryou $31801 \mathrm{~A}$ & $<0.010 \mathrm{mg} / \mathrm{l}$ & $<0.010 \mathrm{mg} / \mathrm{l}$ & $<0.010 \mathrm{mg} / \mathrm{l}$ & $<0.010 \mathrm{mg} / \mathrm{l}$ & 1 & \\
\hline 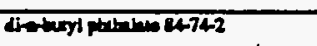 & $<0.010 \mathrm{mg} / 1$ & $<0.010 \mathrm{mg} / \mathrm{l}$ & $<0.010 \mathrm{mg} / \mathrm{h}$ & $<0.010 \mathrm{mg} / \mathrm{l}$ & 1 & \\
\hline 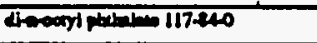 & $<0.010 \mathrm{mg} / \mathrm{l}$ & $<0.010 \mathrm{mg} / 1$ & $<0.010 \mathrm{mg} / \mathrm{l}$ & $<0.010 \mathrm{mg} / \mathrm{l}$ & 1 & \\
\hline 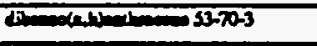 & $<0.010 \mathrm{mg} / \mathrm{l}$ & $<0.010 \mathrm{mg} / \mathrm{l}$ & $<0.010 \mathrm{mg} / \mathrm{l}$ & $<0.010 \mathrm{mg} / 1$ & 1 & \\
\hline 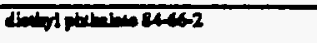 & $<0.010 \mathrm{mg} / !$ & $<0.010 \mathrm{mg} / \mathrm{l}$ & $<0.010 \mathrm{mg} / 1$ & $<0.010 \mathrm{mg} / \mathrm{l}$ & 1 & \\
\hline 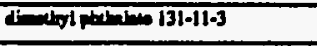 & $<0.010 \mathrm{mg} / 1$ & $<0.010 \mathrm{mg} / \mathrm{l}$ & $<0.010 \mathrm{mg} / \mathrm{I}$ & $<0.010 \mathrm{mg} / \mathrm{l}$ & 1 & \\
\hline 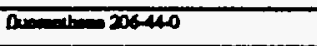 & $<0.010 \mathrm{mg} / \mathrm{l}$ & $<0.010 \mathrm{mg} / 1$ & $<0.010 \mathrm{mg} / \mathrm{l}$ & $<0.010 \mathrm{mg} h$ & 1 & \\
\hline Dences 16-73-7 & $<0.010 \mathrm{mg} / 1$ & $<0.010 \mathrm{mg} /$ & $<0.010 \mathrm{mg} / \mathrm{l}$ & $<0.010 \mathrm{mg} /$ & 1 & \\
\hline 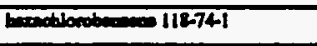 & $<0.010 \mathrm{mg} / \mathrm{l}$ & $<0.010 \mathrm{mg} / \mathrm{l}$ & $<0.010 \mathrm{mg} / \mathrm{l}$ & $<0.010 \mathrm{mg} / \mathrm{l}$ & 1 & \\
\hline 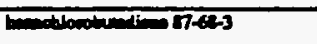 & $<0.010 \mathrm{mg} / \mathrm{l}$ & $<0.010 \mathrm{mg} / \mathrm{l}$ & $<0.010 \mathrm{mg} / 1$ & $<0.010 \mathrm{mg} / \mathrm{h}$ & 1 & \\
\hline 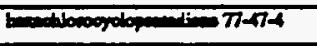 & $<0.010 \mathrm{mg} / 1$ & $<0.010 \mathrm{mg} / \mathrm{l}$ & $<0.010 \mathrm{mg} / 1$ & $<0.010 \mathrm{mg} / \mathrm{h}$ & 1 & \\
\hline -7.721 & $<0.010 \mathrm{mg} / \mathrm{l}$ & $<0.010 \mathrm{mg} / 1$ & $<0.010 \mathrm{mg} / 1$ & $<0.010 \mathrm{mg} /$ & 1 & \\
\hline 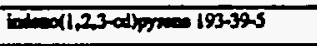 & $<0.010 \mathrm{mg} / \mathrm{l}$ & $<0.010 \mathrm{mg} / \mathrm{l}$ & $<0.010 \mathrm{mg} / \mathrm{l}$ & $<0.010 \mathrm{mg} / \mathrm{L}$ & 1 & \\
\hline 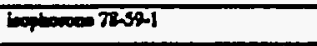 & $<0.010 \mathrm{mg} / 1$ & $<0.010 \mathrm{mg} / \mathrm{l}$ & $<0.010 \mathrm{mg} / \mathrm{l}$ & $<0.010 \mathrm{mg} / \mathrm{l}$ & 1 & \\
\hline 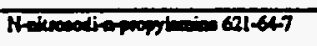 & $<0.010 \mathrm{mg} / \mathrm{l}$ & $<0.010 \mathrm{mg} / \mathrm{l}$ & $<0.010 \mathrm{mg} / 1$ & $<0.010 \mathrm{mg} / 1$ & 1 & \\
\hline 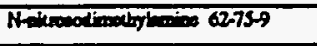 & $<0.010 \mathrm{mg} / \mathrm{l}$ & $<0.010 \mathrm{mg} / \mathrm{l}$ & $<0.010 \mathrm{mg} / \mathrm{i}$ & $<0.010 \mathrm{mg} / 1$ & 1 & \\
\hline 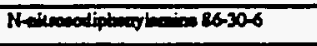 & $<0.010 \mathrm{mg} / \mathrm{l}$ & $<0.010 \mathrm{mg} / \mathrm{h}$ & $<0.010 \mathrm{mg} / \mathrm{l}$ & $<0.010 \mathrm{mg} / \mathrm{l}$ & 1 & \\
\hline atheos 91-20-3 & $<0.010 \mathrm{mg} / \mathrm{l}$ & $<0.010 \mathrm{mg} / 1$ & $<0.010 \mathrm{mg} / \mathrm{l}$ & $<0.010 \mathrm{mg} / 1$ & 1 & \\
\hline 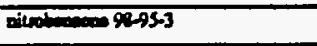 & $<0.010 \mathrm{mg} / \mathrm{l}$ & $<0.010 \mathrm{mg} / \mathrm{l}$ & $<0.010 \mathrm{mg} / \mathrm{l}$ & $<0.010 \mathrm{mg} / \mathrm{l}$ & 1 & \\
\hline 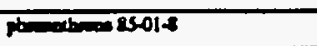 & $<0.010 \mathrm{mg} / \mathrm{l}$ & $<0.010 \mathrm{mg} / 1$ & $<0.010 \mathrm{mg} / \mathrm{l}$ & $<0.010 \mathrm{mg} / 1$ & 1 & \\
\hline $50=129000$ & $<0.010 \mathrm{mg} / \mathrm{l}$ & $<0.010 \mathrm{mg} / \mathrm{l}$ & $<0.010 \mathrm{mg} / \mathrm{l}$ & $<0.010 \mathrm{mg} / 1$ & 1 & \\
\hline 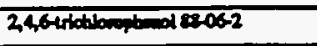 & $<0.030 \mathrm{mg} / 1$ & $<0.030 \mathrm{mg} / \mathrm{l}$ & $<0.030 \mathrm{mg} / \mathrm{l}$ & $<0.030 \mathrm{mg} / \mathrm{I}$ & 1 & \\
\hline 24tictionopheod 12003-2 & $<0.030 \mathrm{mg} / \mathrm{l}$ & $<0.030 \mathrm{mg} / \mathrm{l}$ & $<0.030 \mathrm{mg} / 1$ & $<0.030 \mathrm{mg} / \mathrm{l}$ & 1 & \\
\hline 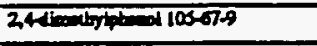 & $<0.030 \mathrm{mg} / \mathrm{l}$ & $<0.030 \mathrm{mg} / \mathrm{l}$ & $<0.030 \mathrm{mg} / 1$ & $<0.030 \mathrm{mg} / \mathrm{l}$ & 1 & \\
\hline 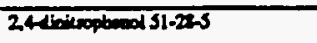 & $<0.050 \mathrm{mg} / \mathrm{l}$ & $<0.050 \mathrm{mg} / \mathrm{l}$ & $<0.050 \mathrm{mg} / \mathrm{l}$ & $<0.050 \mathrm{mg} / \mathrm{l}$ & 1 & \\
\hline
\end{tabular}


Outinll 15

Pare B and C Coationed

\begin{tabular}{|c|c|c|c|c|c|c|}
\hline \multirow{2}{*}{$\begin{array}{l}\text { Polbuens } \\
\text { and } \\
\text { CAS Number } \\
\text { (if mailible) }\end{array}$} & \multicolumn{2}{|c|}{ 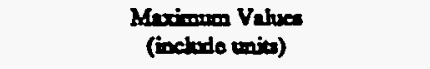 } & \multicolumn{2}{|c|}{$\begin{array}{l}\text { Avensere Valuen } \\
\text { (ibctudo uniede) }\end{array}$} & \multirow{2}{*}{$\begin{array}{c}\text { Number } \\
\text { of } \\
\text { Storm } \\
\text { Everentiod } \\
\text { Sermplod }\end{array}$} & \multirow[b]{2}{*}{ Sourdes of Polbetane } \\
\hline & $\begin{array}{c}\text { Grab Semple } \\
\text { Take Duries } \\
\text { Finc } 30 \\
\text { Aficties }\end{array}$ & $\begin{array}{l}\text { Flommerizuded } \\
\text { Compocine }\end{array}$ & 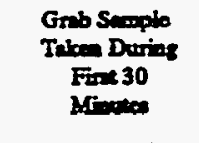 & $\begin{array}{l}\text { Flom-wrifbed } \\
\text { Comporite }\end{array}$ & & \\
\hline 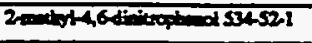 & $<0.050 \mathrm{mg} h$ & $<0.050 \mathrm{mg} / \mathrm{l}$ & $<0.050 \mathrm{mg} / \mathrm{l}$ & $<0.050 \mathrm{mg} / 1$ & 1 & \\
\hline 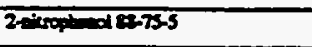 & $<0.030 \mathrm{mg} / \mathrm{l}$ & $<0.030 \mathrm{mg} / \mathrm{l}$ & $<0.030 \mathrm{mg} / 1$ & $<0.030 \mathrm{mg} / \mathrm{h}$ & 1 & \\
\hline 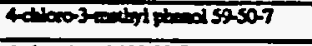 & $<0.030 \mathrm{mg} / 1$ & $<0.030 \mathrm{mg} / 1$ & $<0.030 \mathrm{mg} / 1$ & $<0.030 \mathrm{mg} / \mathrm{h}$ & 1 & \\
\hline Ankophead 100027 & $<0.050 \mathrm{mg} / \mathrm{h}$ & $<0.050 \mathrm{mg} / 1$ & $<0.050 \mathrm{mg} / 1$ & $<0.050 \mathrm{mg} /$ & 1 & \\
\hline 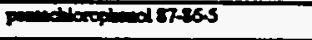 & $<0.050 \mathrm{mg} / 1$ & $<0.050 \mathrm{mg} / \mathrm{l}$ & $<0.050 \mathrm{mg} / 1$ & $<0.050 \mathrm{mg} / \mathrm{l}$ & 1 & \\
\hline 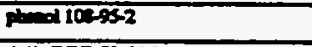 & $<0.030 \mathrm{mg} / 1$ & $<0.030 \mathrm{mg} / \mathrm{l}$ & $<0.030 \mathrm{mg} / \mathrm{h}$ & $<0.030 \mathrm{mg} / \mathrm{h}$ & 1 & \\
\hline 4, 4-DDD72548 & $<0.00001 \mathrm{mg} / 1$ & $<0.0000$ Img/h & $<0.00001 \mathrm{mg} / 1$ & $<0.00001 \mathrm{mg} / 1$ & 1 & \\
\hline 4,4-DDE72559 & $<0.00001 \mathrm{mg} / \mathrm{h}$ & $<0.00001 \mathrm{mg} / \mathrm{l}$ & $<0.00001 \mathrm{mg} / \mathrm{l}$ & $<0.00001 \mathrm{mg} / \mathrm{l}$ & 1 & \\
\hline 40.000 50-2023 & $0.000083 \mathrm{mg} / \mathrm{l}$ & $0.000082 \mathrm{mg} / \mathrm{h}$ & $0.000083 \mathrm{mg} / \mathrm{l}$ & $0.000082 \mathrm{mg} / \mathrm{l}$ & 1 & \\
\hline allate30902 & $<0.00001 \mathrm{mg} / \mathrm{l}$ & $<0.00001 \mathrm{mg} / \mathrm{h}$ & $<0.00001 \mathrm{mg} / \mathrm{l}$ & $<0.00001 \mathrm{mg} / \mathrm{l}$ & 1 & \\
\hline 572749 & $<0.00002 \mathrm{mg} / 1$ & $<0.00002 \mathrm{mg} / 1$ & $<0.00002 \mathrm{mg} / 1$ & $<0.00002 \mathrm{mg} / \mathrm{h}$ & 1 & \\
\hline deth 60.57.1 & $<0.00001 \mathrm{mg} / \mathrm{l}$ & $<0.00001 \mathrm{mg} / 1$ & $<0.00001 \mathrm{mg} / \mathrm{l}$ & $<0.00001 \mathrm{mg} / 1$ & 1 & \\
\hline 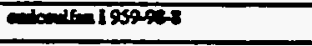 & $<0.00001 \mathrm{mg} n$ & $<0.00001 \mathrm{mg} / \mathrm{h}$ & $<0.00001 \mathrm{mg} / 1$ & $<0.00001 \mathrm{mg} / 1$ & 1 & \\
\hline 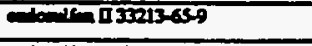 & $<0.00001 \mathrm{mg} / 1$ & $<0.00001 \mathrm{mg} / \mathrm{l}$ & $<0.00001 \mathrm{mg} / \mathrm{l}$ & $<0.00001 \mathrm{me} / \mathrm{n}$ & 1 & \\
\hline 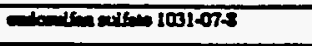 & $<0.00001 \mathrm{mg} / \mathrm{h}$ & $<0.00001 \mathrm{mg} / 1$ & $<0.00001 \mathrm{mg} / 1$ & $<0.00001 \mathrm{mg} / \mathrm{l}$ & 1 & \\
\hline $\cos 200$ & $<0.00005 \mathrm{mg} / \mathrm{l}$ & $<0.00005 \mathrm{mg} / 1$ & $<0.00005 \mathrm{mg} / 1$ & $<0.00005 \mathrm{mg} / \mathrm{h}$ & 1 & \\
\hline 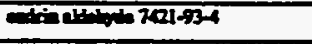 & $<0.00001 \mathrm{mg} / \mathrm{l}$ & $0.00001 \mathrm{mg} / 1$ & $<0.00001 \mathrm{mg} / \mathrm{l}$ & $0.00001 \mathrm{med}$ & 1 & . \\
\hline 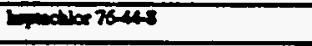 & $<0.00001 \mathrm{mg} / \mathrm{l}$ & $<0.00001 \mathrm{mg} / \mathrm{l}$ & $<0.00001 \mathrm{mg} / \mathrm{l}$ & $<0.00001 \mathrm{mg} /$ & 1 & \\
\hline 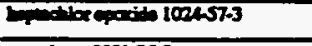 & $<0.00001 \mathrm{mg} / \mathrm{l}$ & $<0.00001 \mathrm{mg} / \mathrm{l}$ & $<0.00001 \mathrm{mg} / \mathrm{l}$ & $<0.00001 \mathrm{mg} / \mathrm{l}$ & 1 & \\
\hline $0001-001.35-2$ & $<0.0002 \mathrm{mg} / 1$ & $<0.0002 \mathrm{mg} / 1$ & $<0.0002 \mathrm{mg} / \mathrm{n}$ & $<0.0002 \mathrm{mg} / 1$ & 1 & \\
\hline 4h-315 31946 & $<0.00001 \mathrm{mg} / \mathrm{l}$ & $<0.00001 \mathrm{mg} / \mathrm{l}$ & $<0.00001 \mathrm{mg} / \mathrm{l}$ & $<0.00001 \mathrm{mg} / \mathrm{s}$ & 1 & \\
\hline -IHC 312557 & $<0.00001 \mathrm{mg} / \mathrm{l}$ & $<0.00001 \mathrm{mg} / \mathrm{l}$ & $<0.00001 \mathrm{mg} / \mathrm{l}$ & $<0.00001 \mathrm{mg} / \mathrm{l}$ & 1 & \\
\hline 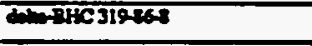 & $<0.00001 \mathrm{mg} / \mathrm{l}$ & $<0.00001 \mathrm{mg} / \mathrm{l}$ & $<0.00001 \mathrm{mg} / \mathrm{l}$ & $<0.00001 \mathrm{me} / 1$ & 1 & \\
\hline matc sasp & $<0.00001 \mathrm{mg} / \mathrm{l}$ & $<0.00001 \mathrm{mg} / \mathrm{l}$ & $<0.00001$ med & $<0.00001 \mathrm{me} / \mathrm{l}$ & 1 & \\
\hline 2 dhoritad 85578 & $<0.030 \mathrm{mg} / \mathrm{l}$ & $<0.030 \mathrm{mg} / \mathrm{l}$ & $<0.030 \mathrm{mg} / 1$ & $<0.030 \mathrm{mg} / \mathrm{l}$ & 1 & \\
\hline 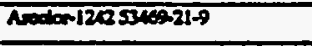 & $<0.0005 \mathrm{mg} / \mathrm{l}$ & $<0.0005 \mathrm{mg} / \mathrm{l}$ & $<0.0005 \mathrm{men}$ & $<0.0005 \mathrm{mg} h$ & 1 & \\
\hline Arodon-123611007-621 & $<0.0005 \mathrm{mg} / 1$ & $<0.0005 \mathrm{mg} / \mathrm{h}$ & $<0.0005 \mathrm{mg} / \mathrm{h}$ & $<0.0005 \mathrm{mg} / 1$ & 1 & \\
\hline Andor-121 11104-22-2 & $<0.0005 \mathrm{mg} / \mathrm{l}$ & $<0.0005 \mathrm{mg} / 1$ & $<0.0005 \mathrm{mg} / \mathrm{l}$ & $<0.0005 \mathrm{mg} / \mathrm{l}$ & 1 & \\
\hline Arodor-1202 IIIA1-16.5 & $<0.0005 \mathrm{mg} / \mathrm{h}$ & $<0.0005 \mathrm{mg} / \mathrm{l}$ & $<0.0005 \mathrm{mg} / \mathrm{l}$ & $<0.0005 \mathrm{mg} / 1$ & 1 & \\
\hline Anodor 12412512226 & $<0.0005 \mathrm{mg} / \mathrm{h}$ & $<0.0005 \mathrm{mg} / \mathrm{l}$ & $<0.0005 \mathrm{mg} / \mathrm{l}$ & $<0.0005 \mathrm{mg} / \mathrm{l}$ & 1 & \\
\hline Avoditor 126011006525 & $<0.0005 \mathrm{mg} / \mathrm{h}$ & $<0.0005 \mathrm{mg} / \mathrm{l}$ & $<0.0005 \mathrm{mg} / \mathrm{I}$ & $<0.0005 \mathrm{mg} / \mathrm{h}$ & 1 & \\
\hline Arodo-1016 1257411.2 & $<0.0005 \mathrm{mg} / \mathrm{h}$ & $<0.0005$ meg $/ 1$ & $<0.0005$ medl & $<0.0005 \mathrm{med}$ & 1 & \\
\hline Cande & $<0.010 \mathrm{mg} / 1$ & $<0.010 \mathrm{mg} / \mathrm{h}$ & $<0.010 \mathrm{mg} / 1$ & $<0.010 \mathrm{mg} / \mathrm{l}$ & 1 & \\
\hline Anowin & $<0.010 \mathrm{mg} / 1$ & $<0.010 \mathrm{mg} / \mathrm{l}$ & $<0.010 \mathrm{mg} / \mathrm{l}$ & $<0.010 \mathrm{mg} / \mathrm{h}$ & 1 & \\
\hline Aarkasitrib & $<0.010 \mathrm{mg} / \mathrm{L}$ & $<0.010 \mathrm{mg} / 1$ & $<0.010 \mathrm{mg} / 1$ & $<0.010 \mathrm{mg} / \mathrm{h}$ & 1 & \\
\hline 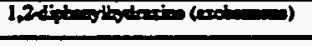 & Renults pending & Romults peoding & Reoults pendiog & Rezults pending & 1 & \\
\hline & & & & & & \\
\hline & & & & & & \\
\hline & & & & & & \\
\hline & & & & & & \\
\hline & & & & & & \\
\hline & & & & & & \\
\hline & & & & & & \\
\hline & & & & & & \\
\hline & & & & & & \\
\hline & & & & & & \\
\hline & & & & & & \\
\hline & & & & & & \\
\hline - & & & & & & \\
\hline
\end{tabular}


VII. Discharge Information (continuved from page 3 of Forre 2 )

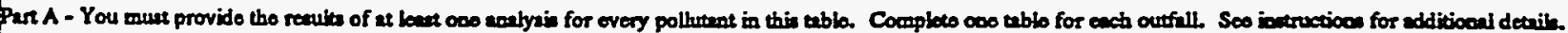

\begin{tabular}{|c|c|c|c|c|c|c|}
\hline \multirow{2}{*}{$\begin{array}{l}\text { Polturans } \\
\text { and } \\
\text { CAs Number } \\
\text { (if avilible) }\end{array}$} & \multicolumn{2}{|c|}{$\begin{array}{l}\text { Maximuser Values } \\
\text { (imolede unitu) }\end{array}$} & \multicolumn{2}{|c|}{$\begin{array}{l}\text { Avernso Yalues } \\
\text { (include units) }\end{array}$} & \multirow{2}{*}{$\begin{array}{l}\text { Number } \\
\text { of } \\
\text { Storm } \\
\text { Everes } \\
\text { Sempled }\end{array}$} & \multirow[b]{2}{*}{ Sources of Pollation } \\
\hline & $\begin{array}{l}\text { Grab Samplo } \\
\text { Tukeo During } \\
\text { Fins } 30 \\
\text { Minuece }\end{array}$ & $\begin{array}{l}\text { Forr-weightod } \\
\text { Compocite }\end{array}$ & $\begin{array}{l}\text { Grab Sumple } \\
\text { Taken Durring } \\
\text { First } 30 \\
\text { Minsed }\end{array}$ & $\begin{array}{c}\text { Fow-weizhiod } \\
\text { Compocito }\end{array}$ & & \\
\hline pil ead Groences & $3 \mathrm{mg} / \mathrm{l}$ & & $3 \mathrm{mg} / 1$ & & 1 & \\
\hline Diologial Oxypos Deaded (BODS) & $9.77 \mathrm{mg} / \mathrm{l}$ & $<5 \mathrm{mg} / \mathrm{l}$ & $9.77 \mathrm{mg} / 1$ & $<5 \mathrm{mg} / \mathrm{l}$ & 1 & \\
\hline Clewicel Oxypos Demend (COD) & $93 \mathrm{mg} / \mathrm{l}$ & $12 \mathrm{mg} / \mathrm{l}$ & $93 \mathrm{mg} / \mathrm{l}$ & $12 \mathrm{mg} / \mathrm{l}$ & 1 & \\
\hline Toul Seppoded Solted (TSO) & $96 \mathrm{mg} / \mathrm{l}$ & $5 \mathrm{mg} / \mathrm{l}$ & $96 \mathrm{mg} / \mathrm{ll}$ & $5 \mathrm{mg} / \mathrm{l}$ & 1 & \\
\hline 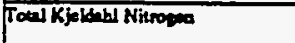 & $1.3 \mathrm{mg} / 1$ & $<0.2 \mathrm{mg} / \mathrm{l}$ & $1.3 \mathrm{mg} / \mathrm{l}$ & $<0.2 \mathrm{mg} / \mathrm{l}$ & 1 & \\
\hline Nirme phu Nitrits Nitrogese & $1.1 \mathrm{mg} / \mathrm{l}$ & $0.29 \mathrm{mg} / \mathrm{l}$ & $1.1 \mathrm{mg} / \mathrm{l}$ & $0.29 \mathrm{mg} / 1$ & 1 & \\
\hline Toual Phopoporm & $0.37 \mathrm{mg} / \mathrm{h}$ & $<0.1 \mathrm{mg} / \mathrm{l}$ & $0.37 \mathrm{mg} / 1$ & $<0.1 \mathrm{mg} / \mathrm{l}$, & 1 & \\
\hline pH & Mither 8.5 & Matum 8.5 & $M=1.5$ & Maximan 8.5 & & \\
\hline
\end{tabular}

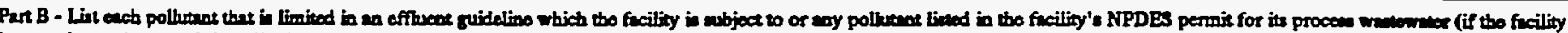

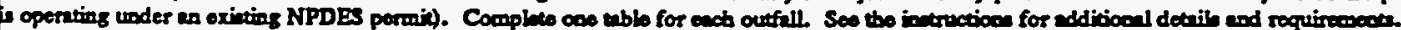

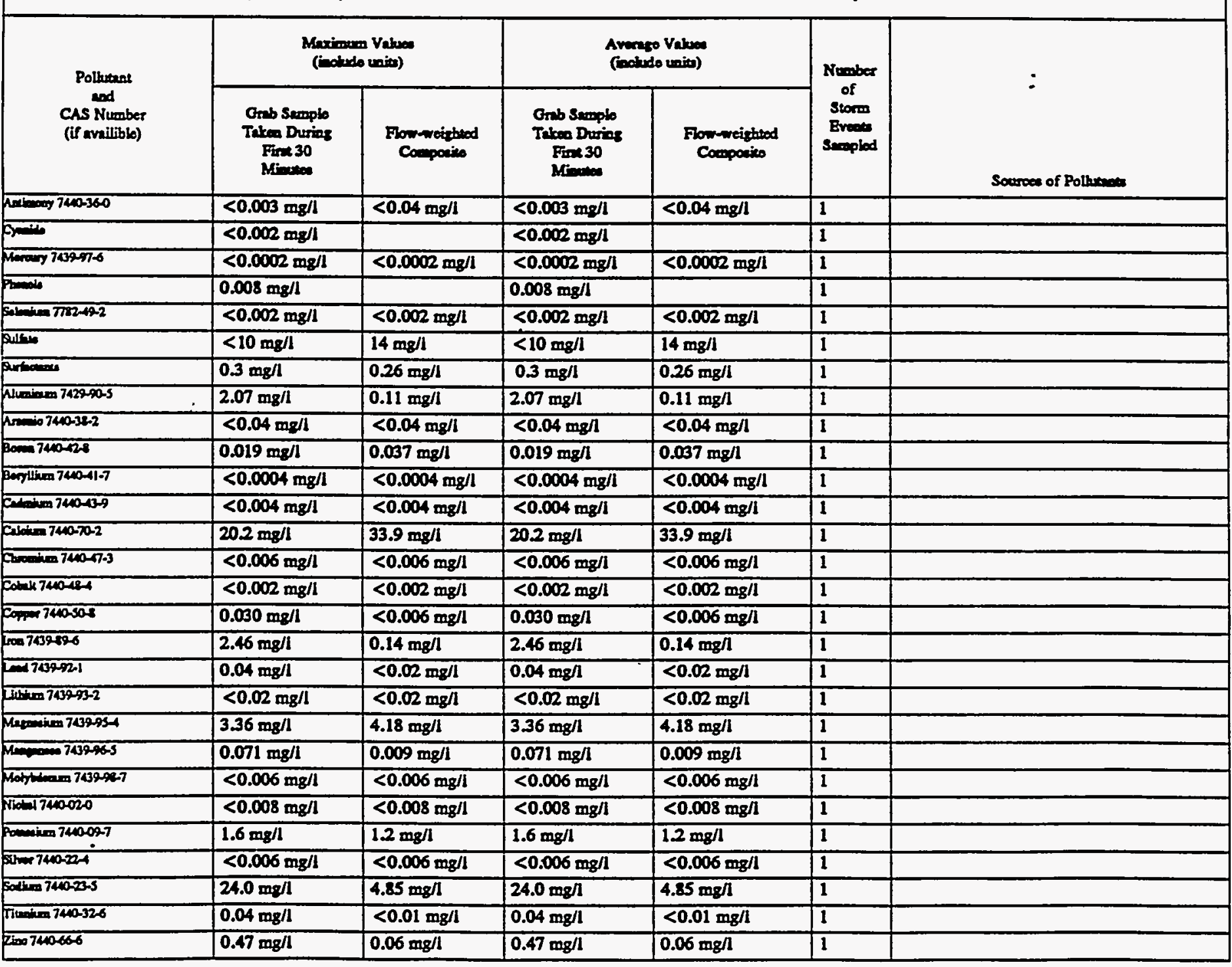


Cootinued from frose.

\begin{tabular}{|c|c|c|c|c|c|c|}
\hline Polhutant & Mexin & $\begin{array}{l}\text { Valuea } \\
\text { units) }\end{array}$ & & $\begin{array}{l}\text { Valuea } \\
\text { o unisu) }\end{array}$ & Number & \\
\hline $\begin{array}{l}\text { CAS Number } \\
\text { (if availible) }\end{array}$ & $\begin{array}{c}\text { Grab Sample } \\
\text { Taken During } \\
\text { Finst } 30 \\
\text { Mineweo }\end{array}$ & $\begin{array}{l}\text { Flow-meictiod } \\
\text { Comporite }\end{array}$ & $\begin{array}{c}\text { Greb Samplo } \\
\text { Takon During } \\
\text { Finst } 30 \\
\text { Minute }\end{array}$ & $\begin{array}{l}\text { Flow-weighted } \\
\text { Componito }\end{array}$ & $\begin{array}{l}\text { Storm } \\
\text { Event } \\
\text { Sxmpled }\end{array}$ & Sources of Pollutants \\
\hline 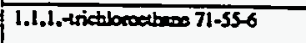 & $<0.010 \mathrm{mg} / 1$ & & $<0.010 \mathrm{mg} / 1$ & & 1 & \\
\hline 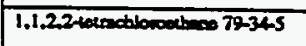 & $<0.010 \mathrm{mg} / \mathrm{l}$ & & $<0.010 \mathrm{mg} / \mathrm{l}$ & & 1 & \\
\hline 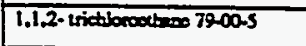 & $<0.010 \mathrm{mg} / \mathrm{l}$ & & $<0.010 \mathrm{mg} / \mathrm{l}$ & & 1 & . \\
\hline 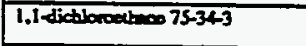 & $<0.010 \mathrm{mg} / \mathrm{l}$ & & $<0.010 \mathrm{mg} / \mathrm{l}$ & & 1 & \\
\hline 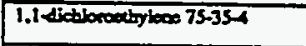 & $<0.010 \mathrm{mg} / \mathrm{l}$ & & $<0.010 \mathrm{mg} / \mathrm{l}$ & & 1 & \\
\hline 1.2-dictionectareso $107-06-2$ & $<0.010 \mathrm{mg} / \mathrm{l}$ & & $<0.010 \mathrm{mg} / \mathrm{l}$ & & 1 & \\
\hline 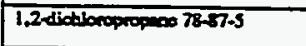 & $<0.010 \mathrm{mg} / \mathrm{l}$ & & $<0.010 \mathrm{mg} / \mathrm{l}$ & & 1 & \\
\hline 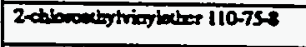 & $<0.010 \mathrm{mg} / \mathrm{l}$ & & $<0.010 \mathrm{mg} / \mathrm{l}$ & & 1 & \\
\hline $71-13-2$ & $<0.010 \mathrm{mg} / \mathrm{l}$ & & $<0.010 \mathrm{mg} / \mathrm{l}$ & & 1 & \\
\hline 75-27A & $<0.010 \mathrm{mg} / \mathrm{l}$ & & $<0.010 \mathrm{mg} / \mathrm{l}$ & & 1 & \\
\hline brow00600m 75-25-2 & $<0.010 \mathrm{mg} / \mathrm{l}$ & & $<0.010 \mathrm{mg} / \mathrm{l}$ & & 1 & \\
\hline 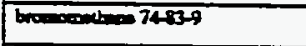 & $<0.010 \mathrm{mg} / \mathrm{l}$ & & $<0.010 \mathrm{mg} / \mathrm{l}$ & & 1 & \\
\hline 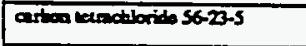 & $<0.010 \mathrm{mg} / 1$ & & $<0.010 \mathrm{mg} / \mathrm{h}$ & & 1 & \\
\hline $108-90-7$ & $<0.010 \mathrm{mg} / \mathrm{l}$ & & $<0.010 \mathrm{mg} / \mathrm{l}$ & & 1 & \\
\hline chlosecthen $75-00-3$ & $<0.010 \mathrm{mg} / \mathrm{l}$ & & $<0.010 \mathrm{mg} / \mathrm{l}$ & & 1 & \\
\hline chlomobocm 67-66-3 & $<0.010 \mathrm{mg} / \mathrm{l}$ & & $<0.010 \mathrm{mg} / \mathrm{l}$ & & 1 & $\because$ \\
\hline 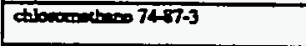 & $<0.010 \mathrm{mg} / 1$ & & $<0.010 \mathrm{mg} / 1$ & & 1 & \\
\hline 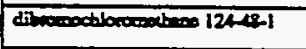 & $<0.010 \mathrm{mg} / 1$ & & $<0.010 \mathrm{mg} / \mathrm{l}$ & & 1 & \\
\hline 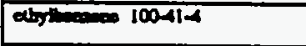 & $<0.010 \mathrm{mg} / \mathrm{l}$ & & $<0.010 \mathrm{mg} / \mathrm{l}$ & & 1 & \\
\hline 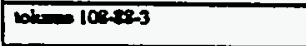 & $<0.010 \mathrm{mg} / \mathrm{l}$ & & $<0.010 \mathrm{mg} / \mathrm{l}$ & & 1 & \\
\hline vinglaboride 75-014 & $<0.010 \mathrm{mg} / \mathrm{l}$ & & $<0.010 \mathrm{mg} / \mathrm{h}$ & & 1 & \\
\hline 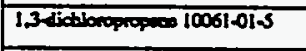 & $<0.010 \mathrm{mg} / \mathrm{l}$ & & $<0.010 \mathrm{mg} / \mathrm{l}$ & . & 1 & \\
\hline 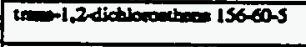 & $<0.010 \mathrm{mg} / \mathrm{l}$ & & $<0.010 \mathrm{mg} / \mathrm{l}$ & & 1 & \\
\hline athan exivity $12507-40-1$ & $8.6 \mathrm{pcill}$ & $4.5 \mathrm{pei} / \mathrm{l}$ & $8.6 \mathrm{pei} / 1$ & $4.5 \mathrm{peill}$ & 1 & \\
\hline omaceiving $12587-16-1$ & $17 \mathrm{pei} / \mathrm{l}$ & $3.6 \mathrm{pci} / 1$ & 17 pcill & $3.6 \mathrm{pei} / \mathrm{l}$ & 1 & \\
\hline froal oolibom & $560 \mathrm{col} .1 \mathrm{~L}$ & $60 \mathrm{co} / .1 \mathrm{~L}$ & $560 \mathrm{col} .1 \mathrm{~L}$ & $60 \mathrm{col} .1 \mathrm{~L}$ & 1 & \\
\hline Thallinem 7400-280 & $<0.001 \mathrm{mg} / \mathrm{l}$ & $<0.001 \mathrm{mg} / \mathrm{l}$ & $<0.001 \mathrm{mg} / 1$ & $<0.001 \mathrm{mg} / \mathrm{l}$ & 1 & \\
\hline Unaming 740-61-1 & $0.001 \mathrm{mg} / \mathrm{l}$ & $0.005 \mathrm{mg} / \mathrm{l}$ & $0.001 \mathrm{mg} / \mathrm{l}$ & $0.005 \mathrm{mg} / \mathrm{l}$ & 1 & \\
\hline Gonemearivg & 10 peill & $32 \mathrm{pci} / 1$ & $10 \mathrm{pec} / 1$ & $32 \mathrm{pei} / 1$ & 1 & \\
\hline
\end{tabular}

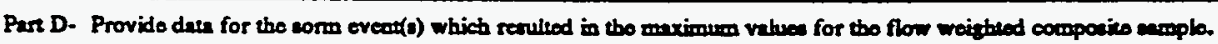

\begin{tabular}{|c|c|c|c|c|c|c|c|}
\hline $\begin{array}{l}1 . \\
\text { Date of } \\
\text { Storm } \\
\text { Eveat }\end{array}$ & $\begin{array}{l}2 . \\
\text { Duration } \\
\text { of Sionm } \\
\text { (in } \\
\text { miniten) }\end{array}$ & $\begin{array}{l}3 . \\
\text { Totel rinfull } \\
\text { during storm oveas } \\
\text { (in isebea) }\end{array}$ & 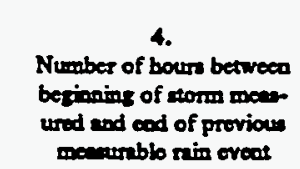 & $\begin{array}{l}\text { S. } \\
\text { Meximum flow mis } \\
\text { during rain ovent } \\
\text { (olloodmin or apecify } \\
\text { univ) }\end{array}$ & $\begin{array}{l}6 . \\
\text { Total flow from } \\
\text { min eveot } \\
\text { (pilloes or } \\
\text { peoify units) }\end{array}$ & $\begin{array}{c}7 . \\
\text { Season } \\
\text { semple was } \\
\text { viken }\end{array}$ & $\begin{array}{l}\text { Form of } \\
\text { Precipitution } \\
\text { (ninfill. } \\
\text { Eammalk) }\end{array}$ \\
\hline $\begin{array}{l}2 / 13 / 92 \\
3 / 6 / 92\end{array}$ & $\begin{array}{l}65 \\
420\end{array}$ & $\begin{array}{l}0.27 \\
0.76\end{array}$ & $\begin{array}{l}>395 \\
138.9\end{array}$ & $\begin{array}{l}\text { NA } \\
239\end{array}$ & $\begin{array}{l}\text { NA } \\
29,751\end{array}$ & $\begin{array}{l}\text { Winter (grab) } \\
\text { Winter } \\
\text { (comporito) }\end{array}$ & $\begin{array}{l}\text { Rainfall } \\
\text { Rainfall }\end{array}$ \\
\hline
\end{tabular}

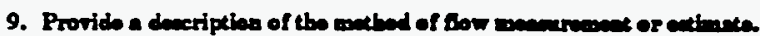

An ISCO 3230 Flow meter was wed to obthin water level readings. This instrument was cakibrated with the normal iry weather flow, if present, as level zero. The flow meter was programmed with the characteristics of the conveyance so that flor rates and total flows conld be calonlated by the meter. 
Outrall 16

Parta B and C Costianed

\begin{tabular}{|c|c|c|c|c|c|c|}
\hline \multirow{2}{*}{$\begin{array}{l}\text { Polluabe } \\
\text { and } \\
\text { CAS Number } \\
\text { (if evailibic) }\end{array}$} & \multicolumn{2}{|c|}{$\begin{array}{l}\text { Maximum Values } \\
\text { (inchude uniob) }\end{array}$} & \multicolumn{2}{|c|}{$\begin{array}{l}\text { Avernge Vahue } \\
\text { (inchude univ) }\end{array}$} & \multirow{2}{*}{$\begin{array}{l}\text { Nomber } \\
\text { of } \\
\text { Stomen } \\
\text { Evemen } \\
\text { Sempled }\end{array}$} & \multirow[b]{2}{*}{ Soerces of Pollewerese } \\
\hline & 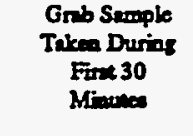 & $\begin{array}{l}\text { Flomweighed } \\
\text { Compoties }\end{array}$ & 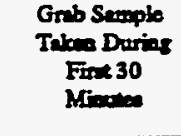 & $\begin{array}{c}\text { Fonmmigibed } \\
\text { Compolite } \\
.\end{array}$ & & \\
\hline 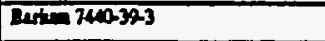 & $0.0343 \mathrm{mg} / \mathrm{l}$ & $0.0316 \mathrm{mg} / 1$ & $0.0343 \mathrm{mg} / \mathrm{l}$ & $0.0316 \mathrm{mg} / 1$ & 1 & \\
\hline 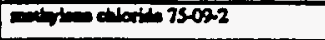 & $<0.010 \mathrm{mg} / \mathrm{l}$ & & $<0.010 \mathrm{mg} / \mathrm{l}$ & & 1 & \\
\hline 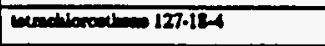 & $<0.010 \mathrm{mg} / \mathrm{l}$ & & $<0.010 \mathrm{mg} / \mathrm{I}$ & & I & \\
\hline Irideroved 7201.6 & $<0.010 \mathrm{mg} / \mathrm{l}$ & & $<0.010 \mathrm{mg} / 1$ & & 1 & \\
\hline$x y=$ & $<0.010 \mathrm{mg} / 1$ & & $<0.010 \mathrm{mg} / 1$ & & 1 & \\
\hline 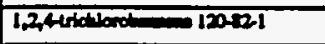 & $<0.010 \mathrm{mg} / 1$ & $<0.010 \mathrm{mg} / 1$ & $<0.010 \mathrm{mg} / \mathrm{l}$ & $<0.010 \mathrm{mg} / \mathrm{l}$ & 1 & \\
\hline 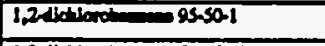 & $<0.010 \mathrm{mg} / 1$ & $<0.010 \mathrm{mg} / 1$ & $<0.010 \mathrm{mg} / 1$ & $<0.010 \mathrm{mg} / \mathrm{l}$ & 1 & \\
\hline 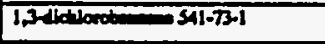 & $<0.010 \mathrm{mg} / \mathrm{l}$ & $<0.010 \mathrm{mg} / 1$ & $<0.010 \mathrm{mg} / \mathrm{l}$ & $<0.010 \mathrm{mg} / \mathrm{I}$ & 1 & \\
\hline 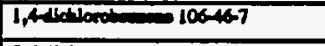 & $<0.010 \mathrm{mg} / \mathrm{l}$ & $<0.010 \mathrm{mg} / \mathrm{h}$ & $<0.010 \mathrm{mg} / \mathrm{l}$ & $<0.010 \mathrm{mg} / 1$ & 1 & \\
\hline 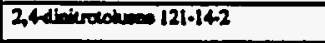 & $<0.010 \mathrm{mg} / \mathrm{l}$ & $<0.010 \mathrm{mg} / \mathrm{l}$ & $<0.010 \mathrm{mg} / \mathrm{l}$ & $<0.010 \mathrm{mg} / \mathrm{l}$ & 1 & \\
\hline 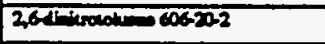 & $<0.010 \mathrm{mg} / 1$ & $<0.010 \mathrm{mg} / \mathrm{l}$ & $<0.010 \mathrm{mg} / \mathrm{h}$ & $<0.010 \mathrm{mg} / 1$ & 1 & \\
\hline 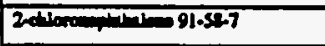 & $<0.010 \mathrm{mg} / \mathrm{l}$ & $<0.010 \mathrm{mg} / \mathrm{l}$ & $<0.010 \mathrm{mg} / \mathrm{l}$ & $<0.010 \mathrm{mg} / \mathrm{l}$ & 1 & \\
\hline 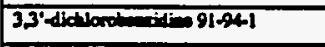 & $<0.020 \mathrm{mg} / \mathrm{l}$ & $<0.020 \mathrm{mg} / \mathrm{l}$ & $<0.020 \mathrm{mg} / \mathrm{l}$ & $<0.020 \mathrm{mg} / 1$ & 1 & \\
\hline 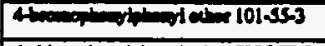 & $<0.010 \mathrm{mg} / \mathrm{l}$. & $<0.010 \mathrm{mg} / \mathrm{l}$ & $<0.010 \mathrm{me} / \mathrm{l}$ & $<0.010 \mathrm{mg} / \mathrm{l}$ & 1 & \\
\hline 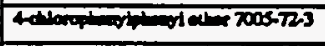 & $<0.010 \mathrm{mg} / \mathrm{l}$ & $<0.010 \mathrm{mg} / \mathrm{l}$ & $<0.010 m g h$ & $<0.010 \mathrm{mg} / \mathrm{I}$ & 1 & \\
\hline 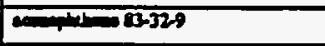 & $<0.010 \mathrm{mg} / \mathrm{l}$ & $<0.010 \mathrm{mg} / \mathrm{l}$ & $<0.010 \mathrm{mg} / \mathrm{h}$ & $<0.010 \mathrm{mg} / 1$ & 1 & \\
\hline 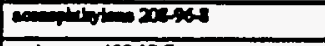 & $<0.010 \mathrm{mg} / \mathrm{l}$ & $<0.010 \mathrm{mg} / \mathrm{l}$ & $<0.010 \mathrm{mg} / \mathrm{L}$ & $<0.010 \mathrm{me} / 1$ & 1 & \\
\hline $120-127$ & $<0.010 \mathrm{mg} / \mathrm{I}$ & $<0.010 \mathrm{mg} / \mathrm{l}$ & $<0.010 \mathrm{mg} / \mathrm{l}$ & $<0.010 \mathrm{mg} / 1$ & 1 & $\therefore$ \\
\hline idibersat.5 & $<0.050 \mathrm{mg} / \mathrm{l}$ & $<0.050 \mathrm{mg} /$ & $<0.050 \mathrm{mg} / \mathrm{h}$ & $<0.050 \mathrm{mg} / 1$ & 1 & \\
\hline 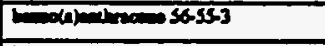 & $<0.010 \mathrm{mg} / \mathrm{l}$ & $<0.010 \mathrm{mg} /$ & $<0.010 \mathrm{mg} / 1$ & $<0.010 \mathrm{mg} / \mathrm{l}$ & 1 & \\
\hline 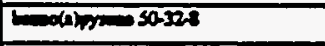 & $<0.010 \mathrm{mg} / \mathrm{l}$ & $<0.010 \mathrm{mg} / \mathrm{l}$ & $<0.010 \mathrm{mg} / 1$ & $<0.010 \mathrm{mg} / 1$ & 1 & \\
\hline 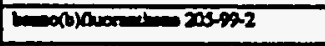 & $<0.010 \mathrm{mg} / \mathrm{l}$ & $<0.010 \mathrm{mg} / \mathrm{l}$ & $<0.010 \mathrm{mg} /$ & $<0.010 \mathrm{mg} /$ & 1 & \\
\hline 191.242 & $<0.010 \mathrm{mg} / \mathrm{l}$ & $<0.010 \mathrm{mg} / \mathrm{l}$ & $<0.010 \mathrm{mg} / \mathrm{h}$ & $<0.010 \mathrm{~m} / \mathrm{l}$ & 1 & \\
\hline 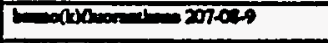 & $<0.010 \mathrm{mg} / 1$ & $<0.010 \mathrm{mg} / \mathrm{h}$ & $<0.010 \mathrm{mg} / \mathrm{h}$ & $<0.010 \mathrm{mg} / 1$ & 1 & \\
\hline 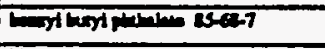 & $<0.010$ ug/1 & $<0.010 \mathrm{mg} / \mathrm{h}$ & $<0.010 \mathrm{mg} / \mathrm{I}$ & $<0.010 \mathrm{mg} / \mathrm{l}$ & 1 & \\
\hline 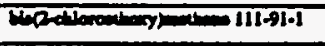 & $<0.010 \mathrm{mg} / \mathrm{l}$ & $<0.010 \mathrm{mg} / \mathrm{h}$ & $<0.010 \mathrm{mg} / 1$ & $<0.010 \mathrm{mg} / 1$ & 1 & \\
\hline 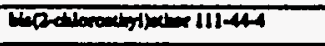 & $<0.010 \mathrm{mg} / \mathrm{l}$ & $<0.010 \mathrm{mg} / \mathrm{l}$ & $<0.010 \mathrm{mg} / \mathrm{l}$ & $<0.010 \mathrm{mg} / \mathrm{l}$ & 1 & \\
\hline 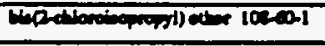 & $<0.010 \mathrm{mg} / \mathrm{l}$ & $<0.010 \mathrm{mg} / \mathrm{l}$ & $:<0.010 \mathrm{mg} / \mathrm{h}$ & $<0.010 \mathrm{mg} / \mathrm{l}$ & 1 & \\
\hline 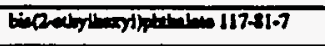 & $<0.010 \mathrm{mg} / \mathrm{l}$ & $<0.010 \mathrm{mg} / \mathrm{h}$ & $<0.010 \mathrm{mg} / \mathrm{h}$ & $<0.010 \mathrm{mg} / 1$ & 1 & \\
\hline chyom 21L-01.9 & $<0.010 \mathrm{mg} / \mathrm{l}$ & $<0.010 \mathrm{mg} / \mathrm{h}$ & $<0.010 \mathrm{mg} / \mathrm{h}$ & $<0.010 \mathrm{mg} / \mathrm{l}$ & 1 & \\
\hline 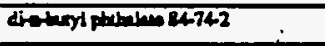 & $<0.010 \mathrm{mg} / \mathrm{l}$ & $<0.010 \mathrm{mg} / \mathrm{h}$ & $<0.010 \mathrm{mg} / \mathrm{h}$ & $<0.010 \mathrm{mg} / \mathrm{l}$ & 1 & \\
\hline 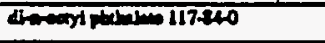 & $<0.010 \mathrm{mg} /$ & $<0.010 \mathrm{mg} / \mathrm{l}$ & $<0.010 \mathrm{mg} / \mathrm{h}$ & $<0.010 \mathrm{mg} / 1$ & 1 & \\
\hline 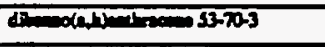 & $<0.010 \mathrm{mg} / \mathrm{l}$ & $<0.010 \mathrm{mg} / 1$ & $<0.010 \mathrm{mg} / 1$ & $<0.010 \mathrm{mg} / \mathrm{l}$ & 1 & \\
\hline 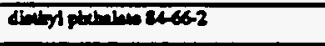 & $<0.010 \mathrm{mg} / \mathrm{l}$ & $<0.010 \mathrm{mg} / \mathrm{l}$ & $<0.010 \mathrm{mg} / \mathrm{h}$ & $<0.010 \mathrm{mg} / \mathrm{l}$ & 1 & \\
\hline 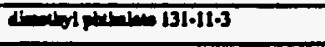 & $<0.010 \mathrm{mg} / \mathrm{l}$ & $<0.010 \mathrm{mg} / \mathrm{l}$ & $<0.010 \mathrm{mg} / 1$ & $<0.010 \mathrm{mg} / \mathrm{l}$ & 1 & \\
\hline 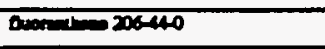 & $<0.010 \mathrm{mg} / \mathrm{l}$ & $<0.010 \mathrm{mg} / \mathrm{l}$ & $<0.010 \mathrm{mg} / \mathrm{l}$ & $<0.010 \mathrm{mg} / \mathrm{l}$ & 1 & \\
\hline 06000006737 & $<0.010 \mathrm{mg} / \mathrm{l}$ & $<0.010 \mathrm{mg} / \mathrm{h}$ & $<0.010 \mathrm{mg} / \mathrm{h}$ & $<0.010 \mathrm{mg} / \mathrm{l}$ & 1 & \\
\hline 11274 & $<0.010 \mathrm{mg} / \mathrm{l}$ & $<0.010 \mathrm{mg} / 1$ & $<0.010 \mathrm{mg} / 1$ & $<0.010 \mathrm{mg} / \mathrm{l}$ & 1 & \\
\hline 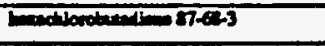 & $<0.010 \mathrm{mg} / \mathrm{l}$ & $<0.010 \mathrm{mg} / \mathrm{l}$ & $<0.010 \mathrm{mg} / 1$ & $<0.010 \mathrm{mg} / \mathrm{l}$ & 1 & \\
\hline 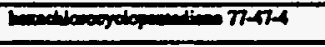 & $<0.010 \mathrm{mg} / \mathrm{l}$ & $<0.010 \mathrm{mg} / \mathrm{h}$ & $<0.010 \mathrm{mg} / 1$ & $<0.010 \mathrm{mg} / \mathrm{l}$ & 1 & \\
\hline $67-721$ & $<0.010 \mathrm{mg} / \mathrm{l}$ & $<0.010 \mathrm{mg} / \mathrm{h}$ & $<0.010 \mathrm{mg} / 1$ & $<0.010 \mathrm{mg} / \mathrm{l}$ & 1 & \\
\hline 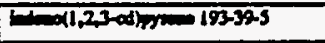 & $<0.010 \mathrm{mg} / 1$ & $<0.010 \mathrm{mg} / \mathrm{h}$ & $<0.010 \mathrm{mg} / 1$ & $<0.010 \mathrm{mg} / 1$ & 1 & \\
\hline 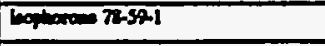 & $<0.010 \mathrm{mg} / \mathrm{l}$ & $<0.010 \mathrm{mg} / \mathrm{l}$ & $<0.010 \mathrm{mg} / \mathrm{l}$ & $<0.010 \mathrm{mg} / 1$ & 1 & \\
\hline 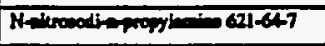 & $<0.010 \mathrm{mg} / \mathrm{l}$ & $<0.010 \mathrm{mg} / 1$ & $<0.010 \mathrm{mg} / 1$ & $<0.010 \mathrm{mg} / \mathrm{l}$ & 1 & \\
\hline 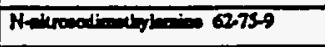 & $<0.010 \mathrm{mg} / \mathrm{l}$ & $<0.010 \mathrm{mg} / \mathrm{l}$ & $<0.010 \mathrm{mg} / \mathrm{l}$ & $<0.010 \mathrm{mg} / 1$ & 1 & \\
\hline 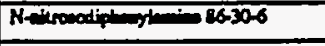 & $<0.010 \mathrm{mg} / \mathrm{l}$ & $<0.010 \mathrm{mg} / 1$ & $<0.010 \mathrm{mg} / 1$ & $<0.010 \mathrm{mg} / \mathrm{I}$ & 1 & \\
\hline 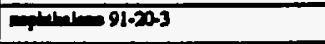 & $<0.010 \mathrm{mg} / \mathrm{l}$ & $<0.010 \mathrm{mg} / \mathrm{h}$ & $<0.010 \mathrm{mg} / 1$ & $<0.010 \mathrm{mg} / 1$ & 1 & \\
\hline 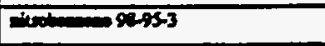 & $<0.010 \mathrm{mg} / \mathrm{l}$ & $<0.010 \mathrm{mg} / 1$ & $<0.010 \mathrm{mg} / 1$ & $<0.010 \mathrm{mg} / \mathrm{h}$ & 1 & \\
\hline 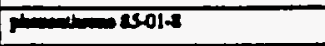 & $<0.010 \mathrm{mg} / \mathrm{l}$ & $<0.010 \mathrm{mg} / \mathrm{l}$ & $<0.010 \mathrm{mg} / 1$ & $<0.010$ men $h$ & 1 & \\
\hline 120000 & $<0.010 \mathrm{mg} / \mathrm{h}$ & $<0.010 \mathrm{mg} / \mathrm{l}$ & $<0.010 \mathrm{mq} / \mathrm{l}$ & $<0.010 \mathrm{mq} / 1$ & 1 & \\
\hline 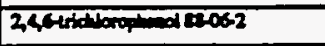 & $<0.030 \mathrm{mg} / \mathrm{l}$ & $<0.030 \mathrm{mg} / \mathrm{l}$ & $<0.030 \mathrm{mg} / 1$ & $<0.030 \mathrm{mg} / 1$ & 1 & \\
\hline 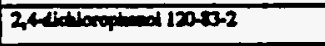 & $<0.030 \mathrm{mg} / \mathrm{l}$ & $<0.030 \mathrm{mg} / \mathrm{l}$ & $<0.030 \mathrm{mg} / \mathrm{l}$ & $<0.030 \mathrm{mg} / \mathrm{l}$ & 1 & \\
\hline $2,4 t^{2}$ dy & $<0.030 \mathrm{mg} / \mathrm{l}$ & $<0.030 \mathrm{mg} / \mathrm{l}$ & $<0.030 \mathrm{mg} / \mathrm{l}$ & $<0.030 \mathrm{mg} / \mathrm{I}$ & 1 & \\
\hline 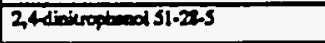 & $<0.030 \mathrm{mg} / \mathrm{l}$ & $<0.050 \mathrm{mg} / \mathrm{l}$ & $<0.030 \mathrm{mg} / \mathrm{l}$ & $<0.050 \mathrm{mg} / 1$ & $\longdiv { 1 }$ & \\
\hline
\end{tabular}


Outfall 16

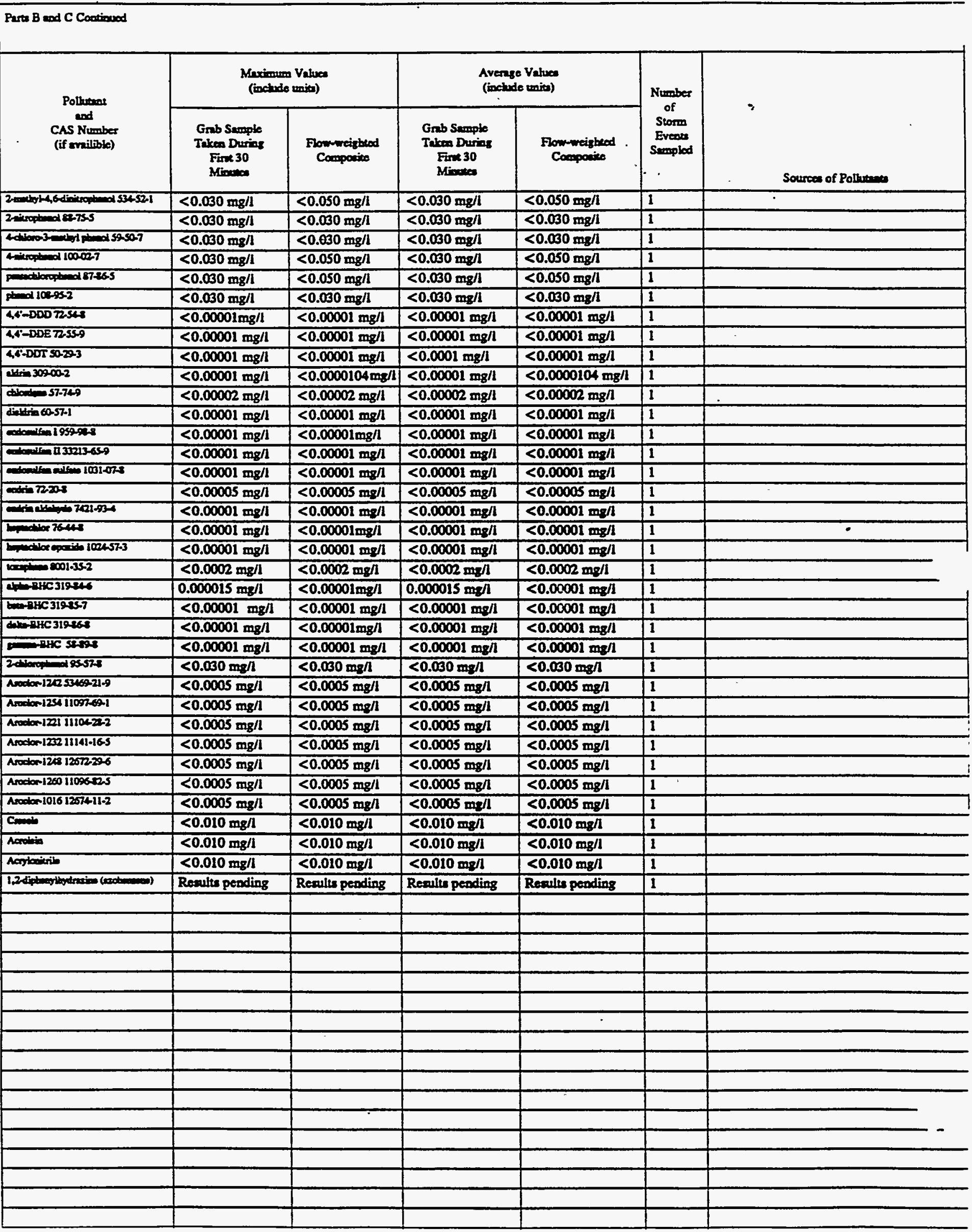


Va. Dicibure Informusion (continuad froon page 3 of Form 2F)

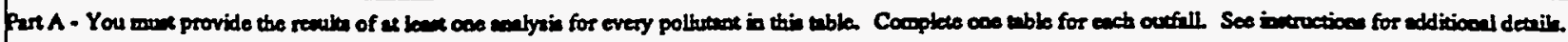

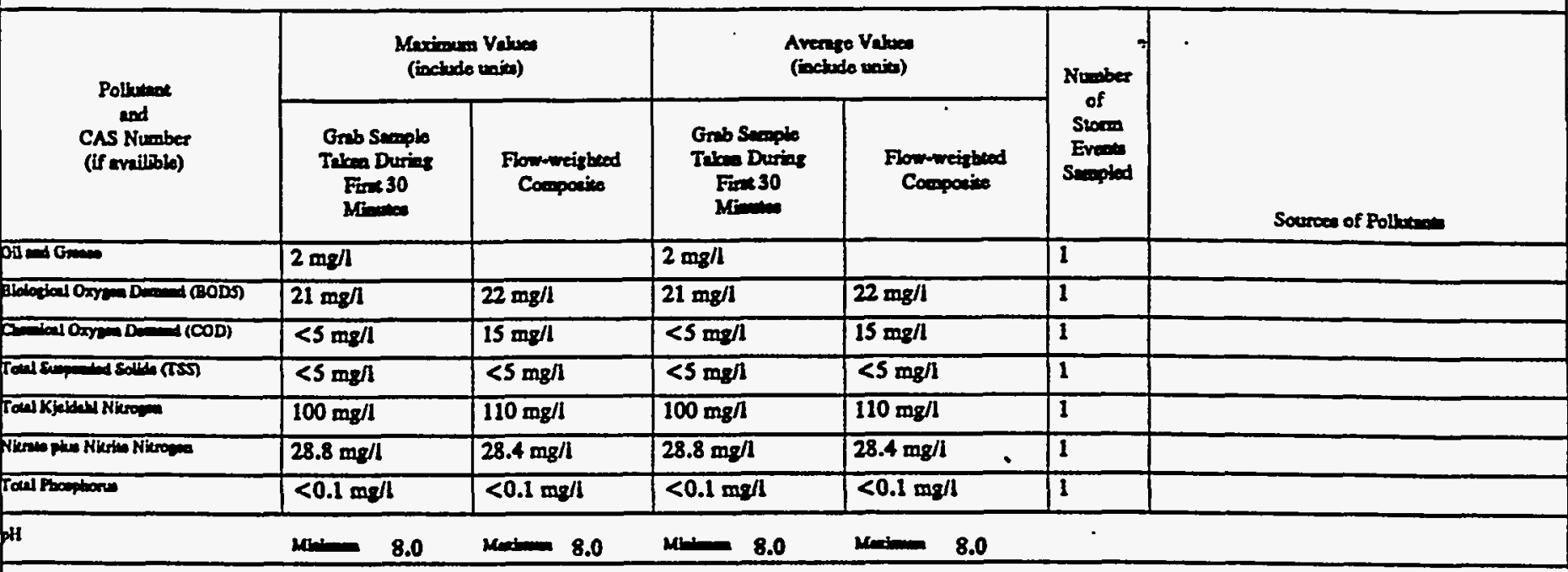

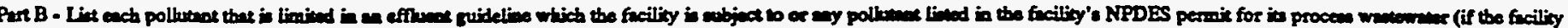

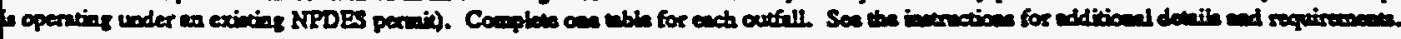

\begin{tabular}{|c|c|c|c|c|c|c|c|}
\hline \multirow{2}{*}{$\begin{array}{l}\text { Pollowe } \\
\text { CAs Number } \\
\text { (if avilbble) }\end{array}$} & & \multicolumn{2}{|c|}{ 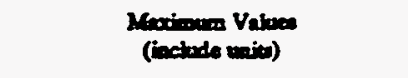 } & \multicolumn{2}{|c|}{ 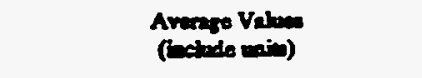 } & \multirow{2}{*}{$\begin{array}{l}\text { Nember } \\
\text { of } \\
\text { Stoon } \\
\text { Evemen } \\
\text { Semplod }\end{array}$} & \multirow{2}{*}{$\begin{array}{c}: \\
\text { Sources of Pollowion }\end{array}$} \\
\hline & & 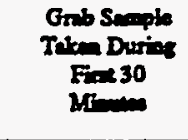 & $\begin{array}{l}\text { Fiom-weizbed } \\
\text { Compain }\end{array}$ & $\begin{array}{l}\text { Orbb Smpho } \\
\text { Trace Durins } \\
\text { Fis } 30 \\
\text { MI }\end{array}$ & $\begin{array}{l}\text { Flowmeigbed } \\
\text { Compotion }\end{array}$ & & \\
\hline $7800-360$ & & $<0.010 \mathrm{mg} / \mathrm{l}$ & $<0.010 \mathrm{mg} / \mathrm{l}$ & $<0.010 \mathrm{mg} / \mathrm{l}$ & $<0.010 \mathrm{mg} / \mathrm{l}$ & 1 & \\
\hline Frinth & & $<0.002 \mathrm{~m} / 1$ & & $<0.002 \mathrm{men} /$ & & 1 & \\
\hline 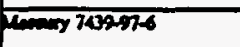 & & $<0.0002 \mathrm{mpll}$ & $<0.0002 \mathrm{mg} / \mathrm{l}$ & $<0.0002 \mathrm{mg} / \mathrm{l}$. & $<0.0002 \mathrm{mg} / \mathrm{h}$ & 1 & \\
\hline$x$ & & $0.002 \mathrm{mg} / \mathrm{h}$ & & $0.002 \mathrm{mg} / 1$ & & 1 & \\
\hline 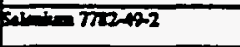 & & $<0.002 \mathrm{mg} / 1$ & $<0.002 \mathrm{mg} / 1$ & $<0.002 \mathrm{mg} / 1$ & $<0.002 \mathrm{mg} / \mathrm{l}$ & 1 & 7 \\
\hline sut/m & & $<10 \mathrm{mg} / \mathrm{l}$ & $<10 \mathrm{mg} / \mathrm{l}$ &.$<10 \mathrm{mg} / 1$ & $<10 \mathrm{mg} / \mathrm{l}$ & 1 & \\
\hline Perthocenes & & $<0.05 \mathrm{mg} / \mathrm{l}$ & $<0.05 \mathrm{mg} / \mathrm{l}$ & $<0.05 \mathrm{mg} / \mathrm{l}$ & $<0.05 \mathrm{mg} / \mathrm{L}$ & $I$ & \\
\hline 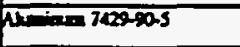 & & $<0.04 \mathrm{mg} / \mathrm{l}$ & $<0.04 \mathrm{mg} / 1$ & $<0.04 \mathrm{mg} / 1$ & $<0.04 \mathrm{mg} / 1$ & 1 & \\
\hline 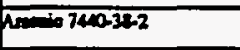 & $\bar{i}$ & $<0.04 \mathrm{mg} / \mathrm{l}$ & $<0.04 \mathrm{mg} / 1$ & $<0.04 \mathrm{mg} / 1$ & $<0.04 \mathrm{mg} / 1$ & 1 & \\
\hline 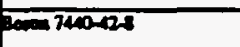 & & $0.009 \mathrm{me} / \mathrm{h}$ & $0.014 \mathrm{mg} / \mathrm{l}$ & $0.009 \mathrm{mg} / \mathrm{l}$ & $0.014 \mathrm{mg} / 1$ & 1 & \\
\hline Pentilien 740-1.7 & & $<0.0004 \mathrm{mg} / \mathrm{l}$ & $<0.0004 \mathrm{mg} / \mathrm{l}$ & $<0.0004 \mathrm{mg} / 1$ & $<0.0004 \mathrm{mg} / \mathrm{l}$ & 1 & \\
\hline Cons $740-139$ & & $<0.004 \mathrm{mg} / \mathrm{l}$ & $<0.004 \mathrm{mg} / \mathrm{l}$ & $<0.004 \mathrm{~mm} / \mathrm{l}$ & $<0.004 \mathrm{mg} / 1$ & 1 & \\
\hline Cascien $740-20-2$ & & $37.3 \mathrm{mg} / \mathrm{l}$ & $38.7 \mathrm{mg} / 1$ & $37.3 \mathrm{mgh}$ & $38.7 \mathrm{mg} / 1$ & 1 & \\
\hline $0=740-17.3$ & & $<0.006 \mathrm{mqd}$ & $<0.006 \mathrm{mg} / \mathrm{l}$ & $<0.006 \mathrm{~m} / 1$ & $<0.006 \mathrm{mg} / 1$ & 1 & \\
\hline 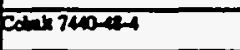 & & $0.005 \mathrm{mg} / \mathrm{h}$ & $0.005 \mathrm{mg} / \mathrm{h}$ & $0.005 \mathrm{mg} / \mathrm{l}$ & $0.005 \mathrm{mg} / \mathrm{l}$ & 1 & \\
\hline carm $74 a b a s$ & & $0.057 \mathrm{mg} / \mathrm{l}$ & $0.054 \mathrm{mg} / 1$ & $0.057 \mathrm{mq} / 1$ & $0.054 \mathrm{mg} / \mathrm{l}$ & 1 & \\
\hline $\operatorname{sen} 2439696$ & & $<0.06 \mathrm{mg} / \mathrm{h}$ & $<0.06 \mathrm{mg} / \mathrm{l}$ & $<0.06 \mathrm{mg} / 1$ & $<0.06 \mathrm{mg} / \mathrm{l}$ & 1 & \\
\hline med 7039-92.1 & & $<0.02 \mathrm{mg} / 1$ & $<0.02 \mathrm{mg} / 1$ & $<0.02 \mathrm{mg} / \mathrm{l}$ & $<0.02 \mathrm{mg} / \mathrm{l}$ & 1 & \\
\hline 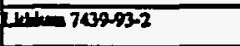 & & $<0.02 \mathrm{mg} / \mathrm{l}$ & $<0.02 \mathrm{mg} / 1$ & $<0.02 \mathrm{mg} / 1$ & $<0.02 \mathrm{mg} / \mathrm{l}$ & 1 & \\
\hline 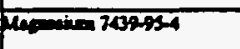 & & $32.9 \mathrm{mg} / \mathrm{l}$ & $34.4 \mathrm{mg} / 1$ & $32.9 \mathrm{mg} / 1$ & $34.4 \mathrm{mg} / \mathrm{h}$ & 1 & \\
\hline 700000005 & & $0.009 \mathrm{mg} / \mathrm{l}$ & $0.007 \mathrm{mg} / \mathrm{l}$ & $0.009 \mathrm{mg} / \mathrm{l}$ & $0.007 \mathrm{mg} / \mathrm{l}$ & 1 & \\
\hline 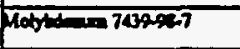 & & $<0.006 \mathrm{mg} / \mathrm{l}$ & $<0.006 \mathrm{mg} / \mathrm{l}$ & $<0.006 \mathrm{mg} / \mathrm{l}$ & $<0.006 \mathrm{mg} / \mathrm{l}$ & 1 & \\
\hline Nintal740000 & & $<0.008 \mathrm{mg} /$ & $<0.008 \mathrm{mg} /$ & $<0.008 \mathrm{mg} / 1$ & $<0.008 \mathrm{mg} / 1$ & 1 & \\
\hline Pommina 7400097 & & $1.5 \mathrm{mg} / \mathrm{l}$ & $1.7 \mathrm{mg} / \mathrm{l}$ & $1.5 \mathrm{mg} / \mathrm{l}$ & $1.7 \mathrm{mg} / 1$ & 1 & - \\
\hline 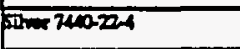 & & $<0.006 \mathrm{mg} / \mathrm{l}$ & $<0.006 \mathrm{mg} / \mathrm{l}$ & $<0.006 \mathrm{mg} / 1$ & $<0.006 \mathrm{mg} / \mathrm{l}$ & 1 & \\
\hline $740-225$ & & $2.82 \mathrm{mg} /$ & $2.86 \mathrm{mg} / \mathrm{h}$ & $2.82 \mathrm{mg} / 1$ & $2.86 \mathrm{mg} / 1$ & 1 & \\
\hline $2740-326$ & & $<0.02 \mathrm{mg} / 1$ & $<0.02 \mathrm{mg} / 1$ & $<0.02 \mathrm{mg} / 1$ & $<0.02 \mathrm{mg} / 1$ & 1 & \\
\hline 200740066 & & $<0.01 \mathrm{mg} / \mathrm{l}$ & $<0.01 \mathrm{mg} / \mathrm{l}$ & $<0.01 \mathrm{mg} / \mathrm{l}$ & $<0.01 \mathrm{mg} / 1$ & 1 & \\
\hline
\end{tabular}


Coctinued from front.

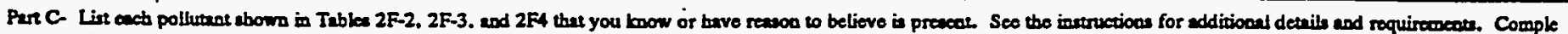
oce tublo for each outfall.

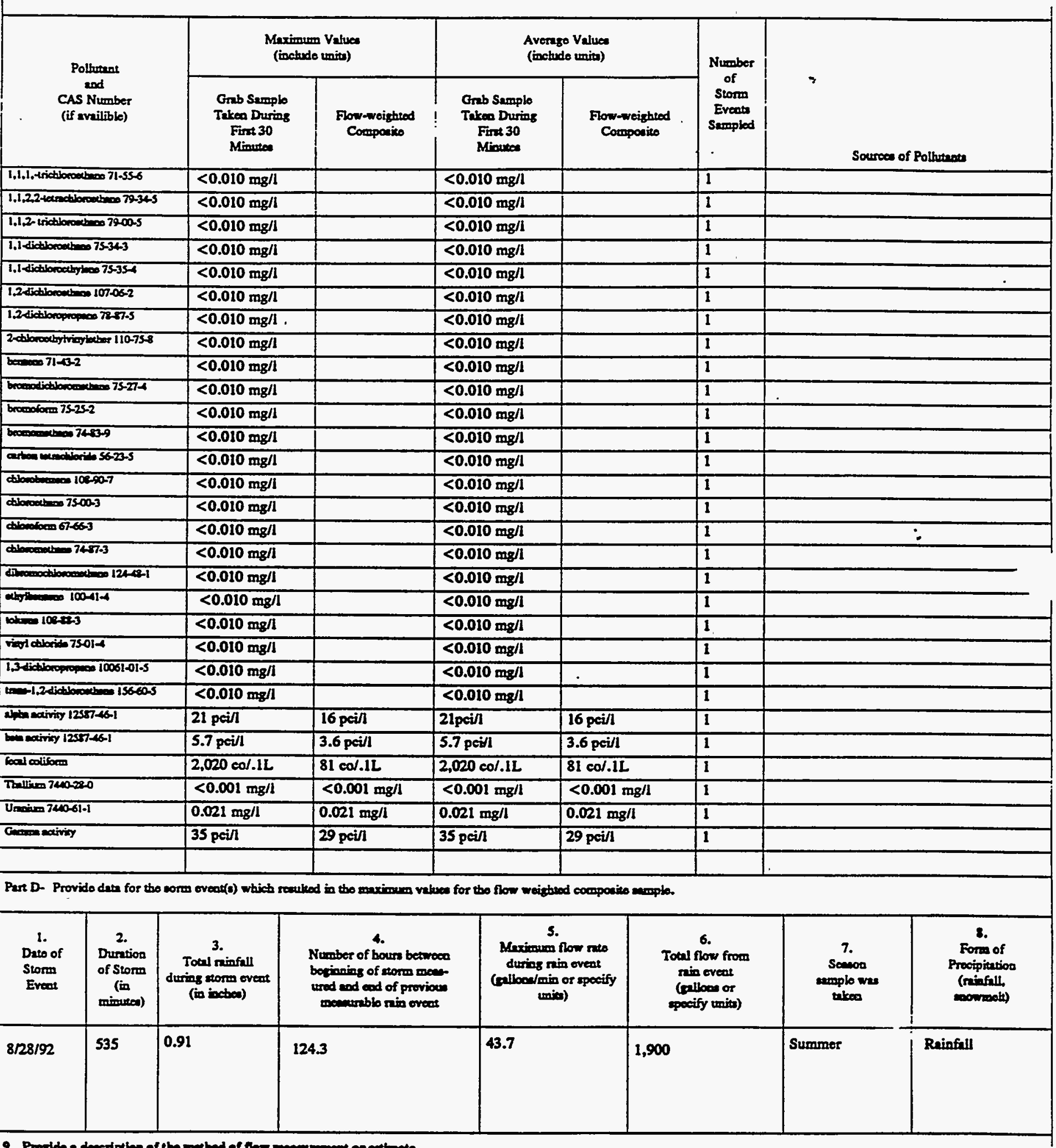

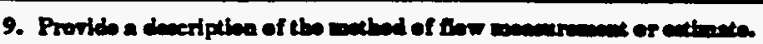

An ISCO 3230 Flow meter was used to obtain water level readinga. This inctrument was calibrated with the normal dry weather flow, if present, as level sero. The flow meter was programmed with the characteristics of the coeveyauce so that flow rates and total flow conid be calculated by the meter. 


\begin{tabular}{|c|c|c|c|c|c|c|}
\hline Parta B, and C Continuad & & : & & & & \\
\hline & Maxi & $\begin{array}{l}\text { Oa Values } \\
0 \text { unitu) }\end{array}$ & & $\begin{array}{l}\text { o Values } \\
\text { lo witas) }\end{array}$ & & \\
\hline $\begin{array}{l}\text { CAs Number } \\
\text { (if avilitible) }\end{array}$ & $\begin{array}{c}\text { Grab Semple } \\
\text { Taken Durizg } \\
\text { First 30 } \\
\text { Minutea }\end{array}$ & $\begin{array}{l}\text { Flow-weigheod } \\
\text { Compocico }\end{array}$ & $\begin{array}{l}\text { Grab Sample } \\
\text { Trkeo Durius } \\
\text { Fint } 30 \\
\text { Minureo }\end{array}$ & $\begin{array}{l}\text { Flow-weighted } \\
\text { Componito }\end{array}$ & $\begin{array}{l}\text { Storm = } \\
\text { Eveots } \\
\text { Seropled }\end{array}$ & Sources of Polhurate \\
\hline Burken 7400-3223 & $0.154 \mathrm{mg} / \mathrm{l}$ & $0.160 \mathrm{mg} / \mathrm{l}$ & $0.154 \mathrm{mg} / \mathrm{l}$ & $0.160 \mathrm{mg} / \mathrm{l}$ & 1 & \\
\hline 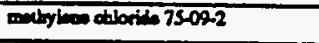 & $<0.010 \mathrm{mg} / \mathrm{l}$ & & $<0.010 \mathrm{mg} / \mathrm{h}$ & & 1 & \\
\hline 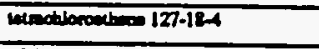 & $<0.010 \mathrm{mg} / \mathrm{l}$ & & $<0.010 \mathrm{mg} / \mathrm{l}$ & & 1 & \\
\hline trictlorembers 79016 & $<0.010 \mathrm{mg} / 1$ & & $<0.010 \mathrm{mg} / \mathrm{h}$ & & 1 & \\
\hline$x$ yleones & $<0.010 \mathrm{mg} / \mathrm{l}$ & & $<0.010 \mathrm{mg} / \mathrm{l}$ & & 1 & \\
\hline 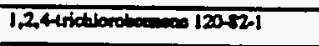 & $<0.010 \mathrm{mg} / \mathrm{l}$ & $<0.010 \mathrm{mg} / \mathrm{l}$ & $<0.010 \mathrm{mg} / 1$ & $<0.010 \mathrm{mg} / 1$ & 1 & \\
\hline 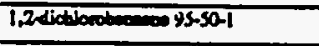 & $<0.010 \mathrm{mg} / 1$ & $<0.010 \mathrm{mg} / 1$ & $<0.010 \mathrm{mg} / \mathrm{l}$ & $<0.010 \mathrm{mg} / \mathrm{l}$ & 1 & \\
\hline 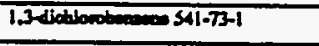 & $<0.010 \mathrm{mg} / \mathrm{l}$ & $<0.010 \mathrm{mg} / \mathrm{l}$ & $<0.010 \mathrm{mg} / \mathrm{l}$ & $<0.010 \mathrm{mg} / \mathrm{l}$ & 1 & \\
\hline 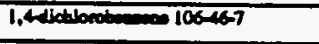 & $<0.010 \mathrm{mg} / \mathrm{h}$ & $<0.010 \mathrm{mg} / \mathrm{l}$ & $<0.010 \mathrm{mg} / \mathrm{l}$ & $<0.010 \mathrm{mg} / 1$ & 1 & \\
\hline 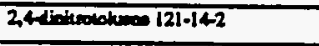 & $<0.010 \mathrm{mg} / \mathrm{l}$ & $<0.010 \mathrm{mg} / \mathrm{l}$ & $<0.010 \mathrm{mg} / \mathrm{l}$ & $<0.010 \mathrm{mg} / \mathrm{l}$ & 1 & \\
\hline 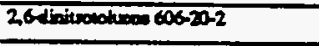 & $<0.010 \mathrm{mg} / \mathrm{l}$ & $<0.010 \mathrm{mg} / \mathrm{l}$ & $<0.010 \mathrm{mg} / \mathrm{l}$ & $<0.010 \mathrm{mg} / \mathrm{l}$ & 1 & \\
\hline 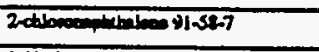 & $<0.010 \mathrm{mg} / \mathrm{l}$ & $<0.010 \mathrm{mg} / \mathrm{l}$ & $<0.010 \mathrm{mg} / \mathrm{l}$ & $<0.010 \mathrm{mg} / \mathrm{l}$ & 1 . & \\
\hline 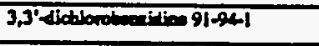 & $<0.010 \mathrm{mg} ! \mathrm{l}$ & $<0.010 \mathrm{mg} / 1$ & $<0.010 \mathrm{mg} / \mathrm{l}$ & $<0.010 \mathrm{mg} / 1$ & 1 & \\
\hline 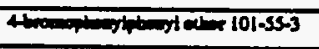 & $<0.010 \mathrm{mg} / \mathrm{l}$ & $<0.010 \mathrm{mg} / \mathrm{l}$ & $<0.010 \mathrm{mg} / \mathrm{l}$ & $<0.010 \mathrm{mg} / \mathrm{l}$ & 1 & \\
\hline 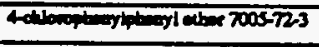 & $<0.010 \mathrm{mg} / \mathrm{l}$ & $<0.010 \mathrm{mg} / \mathrm{l}$ & $<0.010 \mathrm{mg} / 1$ & $<0.010 \mathrm{mg} / 1$ & 1 & \\
\hline 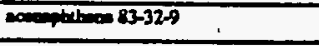 & $<0.010 \mathrm{mg} / \mathrm{h}$ & $<0.010 \mathrm{mg} / \mathrm{l}$ & $>0.010 \mathrm{mg} / \mathrm{l}$ & $<0.010 \mathrm{mg} / 1$ & 1 & \\
\hline maniminges $200 x-8$ & $<0.010 \mathrm{mg} / \mathrm{l}$ & $<0.010 \mathrm{mg} / \mathrm{l}$ & $<0.010 \mathrm{mg} / \mathrm{l}$ & $<0.010 \mathrm{mg} / \mathrm{l}$ & 1 & \\
\hline $120-127$ & $<0.010 \mathrm{mg} / \mathrm{l}$ & $<0.010 \mathrm{mg} / \mathrm{l}$ & $<0.010 \mathrm{mg} / \mathrm{l}$ & $<0.010 \mathrm{mg} / \mathrm{l}$ & 1 & \\
\hline niding 9275 & $<0.030 \mathrm{mg} / \mathrm{l}$ & $<0.030 \mathrm{mg} / \mathrm{l}$ & $<0.030 \mathrm{mg} / \mathrm{l}$ & $<0.030 \mathrm{mg} / \mathrm{l}$ & 1 & \\
\hline - & $<0.010 \mathrm{mg} / \mathrm{l}$ & $<0.010 \mathrm{mg} / \mathrm{l}$ & $<0.010 \mathrm{mg} / 1$ & $<0.010 \mathrm{mg} / 1$ & 1 & \\
\hline 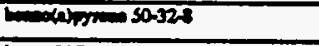 & $<0.010 \mathrm{mg} / \mathrm{l}$ & $<0.010 \mathrm{mg} / \mathrm{h}$ & $<0.010 \mathrm{mg} / 1$ & $<0.010 \mathrm{mg} / 1$ & 1 & \\
\hline 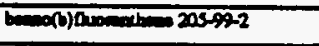 & $<0.010 \mathrm{mg} / \mathrm{l}$ & $<0.010 \mathrm{mg} / \mathrm{l}$ & $<0.010 \mathrm{mg} / \mathrm{l}$ & $<0.010 \mathrm{mg} / 1$ & 1 & \\
\hline - $x(1+1), 191-24-2$ & $<0.010 \mathrm{mg} / \mathrm{l}$ & $<0.010 \mathrm{mg} / \mathrm{l}$ & $<0.010 \mathrm{mg} / \mathrm{h}$ & $<0.010 \mathrm{mg} / \mathrm{l}$ & 1 & \\
\hline 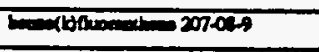 & $<0.010 \mathrm{mg} / \mathrm{l}$ & $<0.010 \mathrm{mg} / \mathrm{l}$ & $<0.010 \mathrm{mg} / \mathrm{l}$ & $<0.010 \mathrm{mf} / 1$ & 1 & \\
\hline 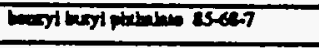 & $<0.010 \mathrm{mg} / \mathrm{l}$ & $<0.010 \mathrm{mg} / \mathrm{l}$ & $<0.010 \mathrm{mg} / \mathrm{h}$ & $<0.010 \mathrm{mg} / \mathrm{l}$ & 1 & \\
\hline 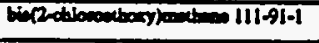 & $<0.010 \mathrm{mg} / \mathrm{l}$ & $<0.010 \mathrm{mg} / \mathrm{l}$ & $<0.010 \mathrm{mg} / \mathrm{l}$ & $<0.010 \mathrm{mg} / \mathrm{l}$ & 1 & \\
\hline 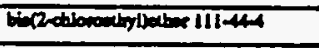 & $<0.010 \mathrm{mg} / \mathrm{l}$ & $<0.010 \mathrm{mg} / \mathrm{l}$ & $<0.010 \mathrm{mg} / 1$ & $<0.010 \mathrm{mg} / 1$ & 1 & \\
\hline 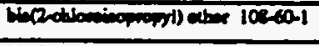 & $<0.010 \mathrm{mg} / \mathrm{l}$ & $<0.010 \mathrm{mg} / \mathrm{l}$ & $<0.010 \mathrm{mg} / \mathrm{l}$ & $<0.010 \mathrm{mg} / \mathrm{l}$ & 1 & \\
\hline 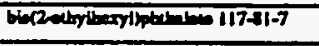 & $<0.010 \mathrm{mg} / \mathrm{l}$ & $<0.010 \mathrm{mg} / \mathrm{l}$ & $<0.010 \mathrm{mg} / \mathrm{l}$ & $<0.010 \mathrm{mg} / \mathrm{l}$ & 1 & \\
\hline ctrymen 21501-9 & $<0.010 \mathrm{mg} / \mathrm{l}$ & $<0.010 \mathrm{mg} / \mathrm{l}$ & $<0.010 \mathrm{mg} / \mathrm{l}$ & $<0.010 \mathrm{mg} / 1$ & 1 & \\
\hline 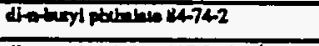 & $<0.010 \mathrm{mg} / \mathrm{l}$ & $<0.010 \mathrm{mg} / \mathrm{l}$ & $<0.010 \mathrm{mg} / \mathrm{l}$ & $<0.010 \mathrm{mg} / \mathrm{l}$ & 1 & \\
\hline 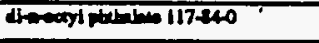 & $<0.010 \mathrm{mg} / 1$ & $<0.010 \mathrm{mg} / \mathrm{l}$ & $<0.010 \mathrm{mg} / 1$ & $<0.010 \mathrm{mg} / 1$ & 1 & \\
\hline 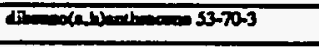 & $<0.010 \mathrm{mg} / \mathrm{l}$ & $<0.010 \mathrm{mg} / \mathrm{l}$ & $<0.010 \mathrm{mg} / \mathrm{l}$ & $<0.010 \mathrm{mg} / \mathrm{l}$ & 1 & \\
\hline 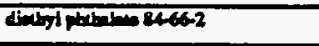 & $<0.010 \mathrm{mg} / \mathrm{l}$ & $<0.010 \mathrm{mg} / \mathrm{l}$ & $<0.010 \mathrm{mg} / 1$ & $<0.010 \mathrm{mg} / 1$ & 1 & \\
\hline 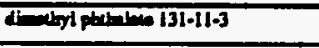 & $<0.010 \mathrm{mg} / \mathrm{l}$ & $<0.010 \mathrm{mg} / \mathrm{l}$ & $<0.010 \mathrm{mg} / \mathrm{l}$ & $<0.010 \mathrm{mg} / \mathrm{l}$ & 1 & \\
\hline 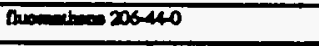 & $<0.010 \mathrm{mg} / \mathrm{l}$ & $<0.010 \mathrm{mg} / 1$ & $<0.010 \mathrm{mg} / \mathrm{l}$ & $<0.010 \mathrm{mg} / 1$ & 1 & \\
\hline 0 nomenes $26-73-7$ & $<0.010 \mathrm{mg} / \mathrm{l}$ & $<0.010 \mathrm{mg} / \mathrm{l}$ & $<0.010 \mathrm{mg} / 1$ & $<0.010 \mathrm{mg} / 1$ & 1 & \\
\hline 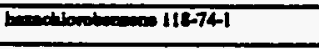 & $<0.010 \mathrm{mg} / \mathrm{l}$ & $<0.010 \mathrm{mg} / \mathrm{l}$ & $<0.010 \mathrm{mg} / 1$ & $<0.010 \mathrm{mg} / 1$ & 1 & \\
\hline 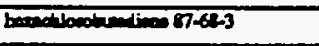 & $<0.010 \mathrm{mg} / \mathrm{l}$ & $<0.010 \mathrm{mg} / \mathrm{l}$ & $<0.010 \mathrm{mg} / \mathrm{l}$ & $<0.010 \mathrm{mg} / 1$ & 1 & \\
\hline 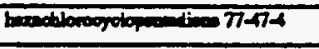 & $<0.010 \mathrm{mg} / \mathrm{l}$ & $<0.010 \mathrm{mg} / \mathrm{l}$ & $<0.010 \mathrm{mg} / \mathrm{l}$ & $<0.010 \mathrm{mg} / \mathrm{l}$ & 1 & \\
\hline 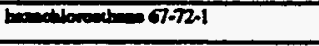 & $<0.010 \mathrm{mg} / 1$ & $<0.010 \mathrm{mg} / 1$ & $<0.010 \mathrm{mg} / \mathrm{l}$ & $<0.010 \mathrm{mg} / \mathrm{l}$ & 1 & \\
\hline 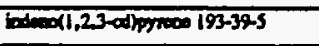 & $<0.010 \mathrm{mg} / \mathrm{l}$ & $<0.010 \mathrm{mg} / 1$ & $<0.010 \mathrm{mg} / \mathrm{l}$ & $<0.010 \mathrm{mg} / \mathrm{l}$ & 1 & \\
\hline isopheren 72-59-1 & $<0.010 \mathrm{mg} / \mathrm{l}$ & $<0.010 \mathrm{mg} / \mathrm{l}$ & $<0.010 \mathrm{mg} / \mathrm{l}$ & $<0.010 \mathrm{mg} / \mathrm{l}$ & 1 & \\
\hline 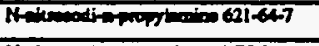 & $<0.010 \mathrm{mg} / \mathrm{l}$ & $<0.010 \mathrm{mg} / 1$ & $<0.010 \mathrm{mg} / 1$ & $<0.010 \mathrm{mg} / \mathrm{l}$ & 1 & \\
\hline 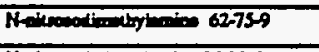 & $<0.010 \mathrm{mg} / \mathrm{l}$ & $<0.010 \mathrm{mg} / 1$ & $<0.010 \mathrm{mg} / \mathrm{l}$ & $<0.010 \mathrm{mg} / 1$ & 1 & \\
\hline 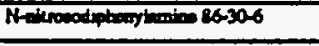 & $<0.010 \mathrm{mg} / \mathrm{l}$ & $<0.010 \mathrm{mg} / \mathrm{l}$ & $<0.010 \mathrm{mg} / \mathrm{l}$ & $<0.010 \mathrm{mg} / 1$ & 1 & \\
\hline 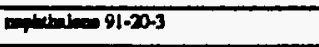 & $<0.010 \mathrm{mg} / \mathrm{l}$ & $<0.010 \mathrm{mg} / \mathrm{l}$ & $<0.010 \mathrm{mg} / \mathrm{l}$ & $<0.010 \mathrm{mg} / 1$ & 1 & \\
\hline 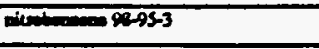 & $<0.010 \mathrm{mg} / \mathrm{l}$ & $<0.010 \mathrm{mg} / 1$ & $<0.010 \mathrm{mg} / \mathrm{l}$ & $<0.010 \mathrm{mg} / \mathrm{l}$ & 1 & \\
\hline 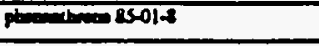 & $<0.010 \mathrm{mg} / 1$ & $<0.010 \mathrm{mg} / 1$ & $<0.010 \mathrm{mg} / \mathrm{l}$ & $<0.010 \mathrm{mg} / \mathrm{l}$ & 1 & \\
\hline $790=129000$ & $<0.010 \mathrm{mg} / \mathrm{l}$ & $<0.010 \mathrm{mg} / 1$ & $<0.010 \mathrm{mg} / 1$ & $<0.010 \mathrm{mg} / 1$ & 1 & \\
\hline 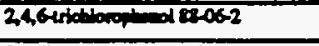 & $<0.030 \mathrm{mg} / \mathrm{l}$ & $<0.030 \mathrm{mg} / \mathrm{l}$ & $<0.030 \mathrm{mg} / \mathrm{l}$ & $<0.030 \mathrm{mg} / \mathrm{l}$ & 1 & \\
\hline 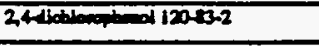 & $<0.030 \mathrm{mg} / \mathrm{l}$ & $<0.030 \mathrm{mg} / 1$ & $<0.030 \mathrm{mg} / 1$ & $<0.030 \mathrm{mg} / 1$ & 1 & \\
\hline 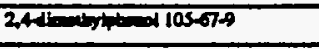 & $<0.030 \mathrm{mg} / \mathrm{h}$ & $<0.030 \mathrm{mg} / 1$ & $<0.030 \mathrm{mg} / 1$ & $<0.030 \mathrm{mg} / \mathrm{l}$ & 1 & \\
\hline 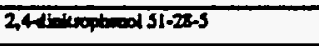 & $<0.030 \mathrm{mg} / \mathrm{I}$ & $<0.030 \mathrm{mg} / \mathrm{h}$ & $<0.030 \mathrm{mg} / 1$ & $<0.030 \mathrm{mg} / \mathrm{h}$ & 1 & \\
\hline
\end{tabular}


Outfall 17

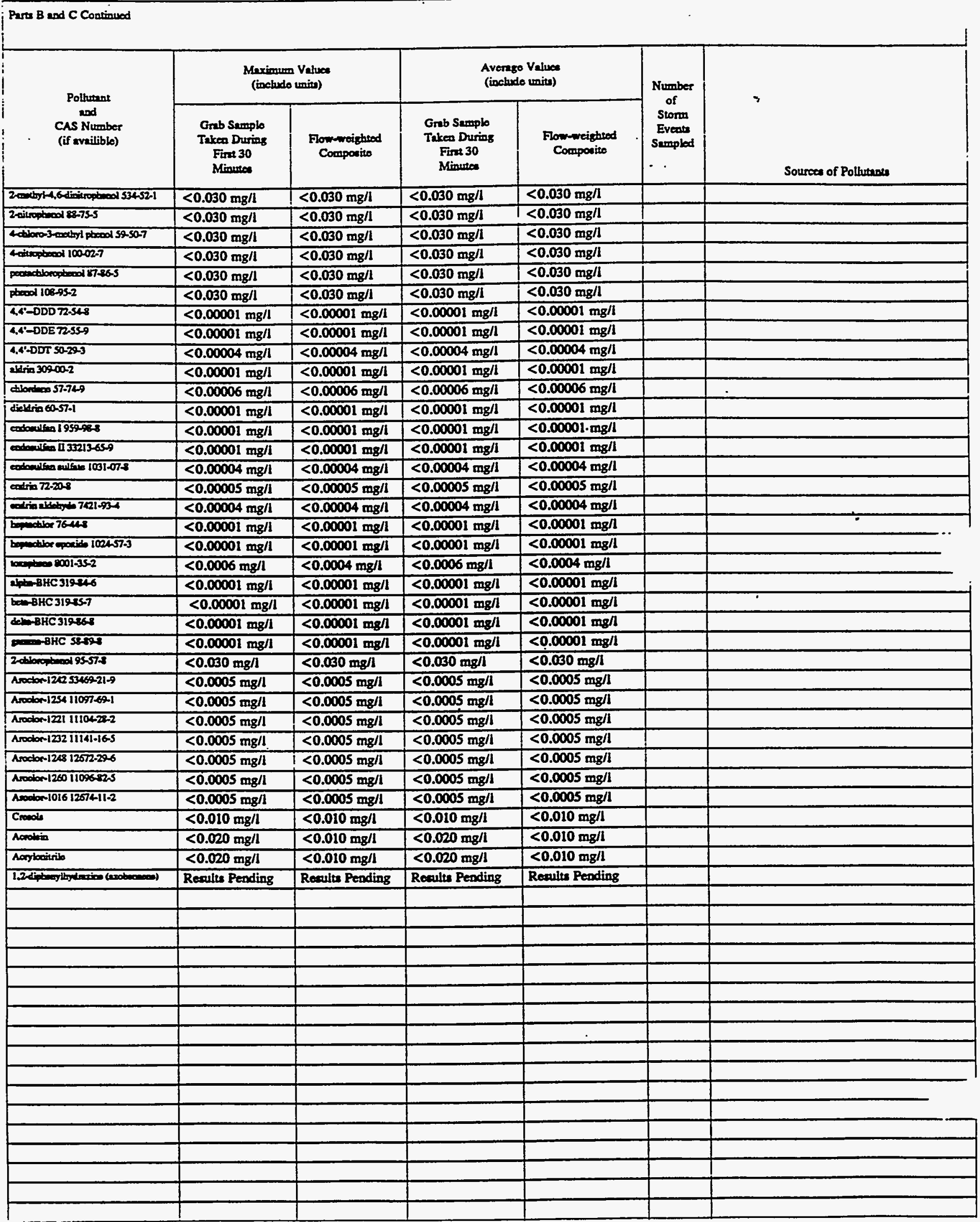


MII. Discharse Lnformanion (continued from page 3 of Form 2F)

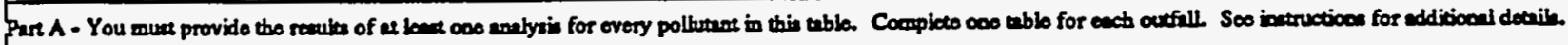

\begin{tabular}{|c|c|c|c|c|c|c|}
\hline \multirow{2}{*}{$\begin{array}{l}\text { Pollutent } \\
\text { and } \\
\text { CAs Number } \\
\text { (if maitiblo) }\end{array}$} & \multicolumn{2}{|c|}{$\begin{array}{l}\text { Maximum Valuex } \\
\text { (inchado unita) }\end{array}$} & \multicolumn{2}{|c|}{$\begin{array}{l}\text { Averase Vature } \\
\text { (inctudo uniti) }\end{array}$} & \multirow{2}{*}{ 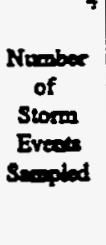 } & \multirow[b]{2}{*}{ Soursoes of Pollowines } \\
\hline & 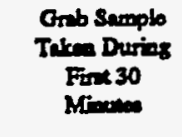 & $\begin{array}{l}\text { Fon-writived } \\
\text { Compocisos }\end{array}$ & 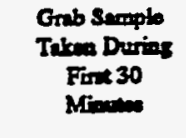 & Formmigtiod & & \\
\hline pil ad Genes & $<2 m g / l$ & & $<2 \mathrm{mg} / 1$ & & 1 & \\
\hline 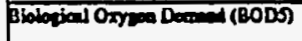 & $<5 \mathrm{mg} / \mathrm{l}$ & $56.4 \mathrm{mg} / \mathrm{h}$ & $<5 \mathrm{mg} / \mathrm{l}$ & $56.4 \mathrm{mg} / 1$ & 1 & \\
\hline (COD) & $32 \mathrm{mg} / \mathrm{l}$ & $9.7 \mathrm{mg} / \mathrm{l}$ & $32 \mathrm{mg} / \mathrm{l}$ & $9.7 \mathrm{mg} / \mathrm{l}$ & 1 & \\
\hline 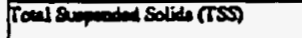 & $88 \mathrm{mg} / \mathrm{l}$ & $<5 \mathrm{mg} / \mathrm{l}$ & $88 \mathrm{mg} / 1$ & <5 mg/l & 1 & \\
\hline 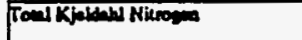 & $0.6 \mathrm{mg} / \mathrm{l}$ & $<0.2 \mathrm{mg} / \mathrm{l}$ & $0.6 \mathrm{mg} / 1$ & $<0.2 \mathrm{mg} / \mathrm{l}$ & 1 & \\
\hline 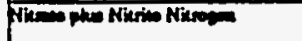 & $2.1 \mathrm{mgll}$ & $0.61 \mathrm{mg} / 1$ & $2.1 \mathrm{mg} / 1$ & $0.61 \mathrm{mg} / \mathrm{l}$ & 1 & \\
\hline Pand tromponse & $0.19 \mathrm{mg} / \mathrm{l}$ & $<0.1 \mathrm{mg} / \mathrm{l}$ & $0.19 \mathrm{mg} / 1$ & $<0.1 \mathrm{mg} / \mathrm{l}$ & 1 & \\
\hline H & $M i n=7.4$ & $\operatorname{Man} 7.4$ & Mame 7.4 & Madis 7.4 & & \\
\hline
\end{tabular}

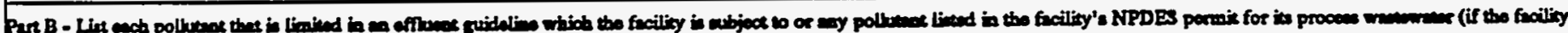

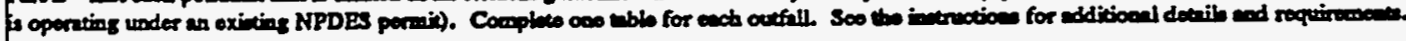

\begin{tabular}{|c|c|c|c|c|c|c|}
\hline \multirow{2}{*}{$\begin{array}{l}\text { Pollwenes } \\
\text { and } \\
\text { CAs Number } \\
\text { (if amiliblo) }\end{array}$} & \multicolumn{2}{|c|}{ 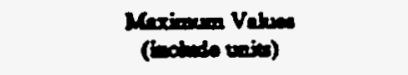 } & \multicolumn{2}{|c|}{ 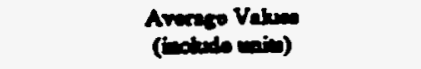 } & \multirow{2}{*}{$\begin{array}{l}\text { Nawber } \\
\text { of } \\
\text { Seorm } \\
\text { Evence } \\
\text { sempled }\end{array}$} & \multirow{2}{*}{$\begin{array}{l}\therefore \\
\text { Souroes of Polvenom }\end{array}$} \\
\hline & 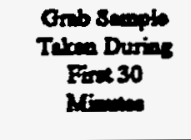 & $\begin{array}{l}\text { Flom-maighed } \\
\text { Compotive }\end{array}$ & 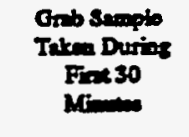 & $\begin{array}{l}\text { Flow-mointed } \\
\text { Componine }\end{array}$ & & \\
\hline $1=740-360$ & $<0.003 \mathrm{mg} / \mathrm{l}$ & $<0.04 \mathrm{mg} / \mathrm{l}$ & $<0.003 \mathrm{mg} / \mathrm{l}$ & $<0.04 \mathrm{mg} / \mathrm{l}$ & 1 & \\
\hline $20=10$ & $0.004 \mathrm{me} / 1$ & & $0.004 \mathrm{mp} / 1$ & & 1 & \\
\hline 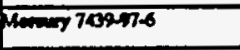 & $<0.0002 \mathrm{mg} / 1$ & $<0.0002 \mathrm{mg} / \mathrm{l}$ & $<0.0002 \mathrm{~min} / \mathrm{l}$ & $<0.0002 \mathrm{mg} / \mathrm{l}$ & 1 & \\
\hline$x$ & $0.002 \mathrm{mg} / \mathrm{l}$ & & 0.002 medl & & 1 & \\
\hline andem $772-422$ & $<0.002 \mathrm{mg} / 1$ & $<0.002 \mathrm{mg} / 1$ & $<0.002 \mathrm{mg} / \mathrm{l}$ & $<0.002 \mathrm{mg} / \mathrm{l}$ & 1 & \\
\hline (avinem & $36 \mathrm{mg} / 1$ & $17 \mathrm{mg} / \mathrm{h}$ & $36 \mathrm{mgll}$ & 17 mg/l & 1 & \\
\hline Purtionsen & $0.07 \mathrm{mg} / 1$ & $<0.05 \mathrm{mg} / \mathrm{l}$ & $0.07 \mathrm{mg} / 1$ & $<0.05 \mathrm{mg} / \mathrm{l}$ & 1 & \\
\hline 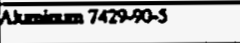 & $1.40 \mathrm{mg} / \mathrm{l}$ & $0.26 \mathrm{mg} / \mathrm{ll}$ & $1.40 \mathrm{mg} / 1$ & $0.26 \mathrm{mg} / \mathrm{h}$ & 1 & \\
\hline $100=740-3 x-2$ & $<0.04 \mathrm{mq} / \mathrm{l}$ & $<0.04 \mathrm{mg} / \mathrm{l}$ & $<0.04 \mathrm{mg} / 1$ & $<0.04 \mathrm{mg} / \mathrm{l}$ & 1 & \\
\hline 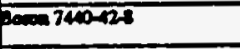 & $0.040 \mathrm{mg} / \mathrm{l}$ & $0.053 \mathrm{mg} / 1$ & $0.040 \mathrm{mg} / 1$ & $0.053 \mathrm{mg} / 1$ & 1 & \\
\hline 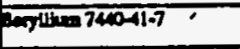 & $<0.0004 \mathrm{mg} / \mathrm{h}$ & $<0.0004 \mathrm{mg} / \mathrm{l}$ & $<0.0004 \mathrm{mg} / \mathrm{l}$ & $<0.0004 \mathrm{mg} / 1$ & 1 & \\
\hline $20 \sin 740-139$ & $<0.004 \mathrm{mg} / \mathrm{l}$ & $<0.004 \mathrm{mg} / 1$ & $<0.004 \mathrm{mg} / \mathrm{l}$ & $<0.004 \mathrm{mg} / \mathrm{l}$ & 1 & \\
\hline 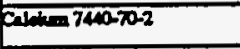 & $46.8 \mathrm{mg} / 1$ & $27.6 \mathrm{mg} / 1$ & $46.8 \mathrm{mg} / 1$ & $27.6 \mathrm{mg} / 1$ & 1 & \\
\hline $500.740-17.3$ & $<0.006 \mathrm{mg} / 1$ & $<0.006 \mathrm{mg} / 1$ & $<0.006 \mathrm{mg} / \mathrm{l}$ & $<0.006 \mathrm{mg} / 1$ & 1 & \\
\hline 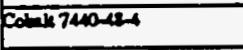 & $<0.002 \mathrm{mg} / \mathrm{l}$ & $<0.002 \mathrm{mg} / 1$ & $<0.002 \mathrm{mg} / \mathrm{h}$ & $<0.002 \mathrm{mg} / 1$ & 1 & \\
\hline 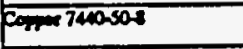 & $0.011 \mathrm{mg} / 1$ & $<0.006 \mathrm{mg} / \mathrm{l}$ & $0.011 \mathrm{mg} / 1$ & $<0.006 \mathrm{mg} / 1$ & 1 & \\
\hline 1007439526 & $1.51 \mathrm{mg} / 1$ & $0.25 \mathrm{mg} / 1$ & $1.51 \mathrm{mg} / \mathrm{l}$ & $0.25 \mathrm{mg} / \mathrm{l}$ & 1 & \\
\hline Fed 7439921 & $<0.02 \mathrm{mg} / 1$ & $<0.02 \mathrm{mg} / \mathrm{l}$ & $<0.02 \mathrm{mg} / \mathrm{l}$ & $<0.02 \mathrm{mg} / \mathrm{l}$ & 1 & \\
\hline 145kem 7/39-93-2 & $<0.02 \mathrm{mg} / \mathrm{l}$ & $<0.02 \mathrm{mg} / \mathrm{l}$ & $<0.02 \mathrm{mg} / \mathrm{l}$ & $<0.02 \mathrm{mg} / \mathrm{h}$ & 1 & \\
\hline Meposine 7039954- & $7.35 \mathrm{mg} / \mathrm{h}$ & $4.06 \mathrm{mg} / \mathrm{h}$ & $7.35 \mathrm{mg} / 1$ & $4.06 \mathrm{mg} / 1$ & 1 & \\
\hline 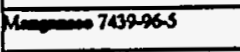 & $0.032 \mathrm{mg} / \mathrm{l}$ & $0.006 \mathrm{mg} / \mathrm{l}$ & $0.032 \mathrm{mgll}$ & $0.006 \mathrm{mg} / \mathrm{l}$ & 1 & \\
\hline 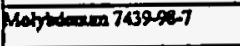 & $<0.006 \mathrm{mg} / \mathrm{l}$ & $<0.006 \mathrm{mg} / \mathrm{l}$ & $<0.006 \mathrm{mg} / \mathrm{l}$ & $<0.006 \mathrm{mg} / \mathrm{l}$ & 1 & \\
\hline$\sqrt{12017} 740020$ & $<0.008 \mathrm{mg} / \mathrm{h}$ & $<0.008 \mathrm{mg} / 1$ & $<0.008 \mathrm{mg} / \mathrm{l}$ & $<0.008 \mathrm{mg} / \mathrm{h}$ & 1 & \\
\hline 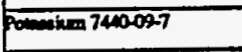 & $1.5 \mathrm{mg} / \mathrm{l}$ & $0.5 \mathrm{mg} / \mathrm{l}$ & $1.5 \mathrm{mg} / \mathrm{h}$ & $0.5 \mathrm{mg} / \mathrm{l}$ & 1 & \\
\hline $8 \lim 740025$ & $<0.006 \mathrm{mg} / \mathrm{h}$ & $<0.006 \mathrm{mg} / \mathrm{h}$ & $<0.006 \mathrm{mg} / \mathrm{l}$ & $<0.006 \mathrm{mg} / \mathrm{l}$ & 1 & \\
\hline 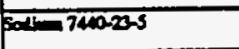 & $26.6 \mathrm{mg} / 1$ & $2.55 \mathrm{mg} / 1$ & $26.6 \mathrm{mg} / \mathrm{t}$ & $2.55 \mathrm{mg} / 1$ & 1 & \\
\hline riteriem 740-325 & $0.02 \mathrm{mg} / \mathrm{h}$ & $<0.01 \mathrm{mg} / \mathrm{l}$ & $0.02 \mathrm{mg} / 1$ & $<0.01 \mathrm{mg} / \mathrm{l}$ & 1 & \\
\hline 200740656 & $0.36 \mathrm{mg} / \mathrm{l}$ & $0.07 \mathrm{mg} / \mathrm{l}$ & $0.36 \mathrm{mg} / \mathrm{l}$ & $0.07 \mathrm{mg} / 1$ & 1 & \\
\hline
\end{tabular}


Continend from frons.

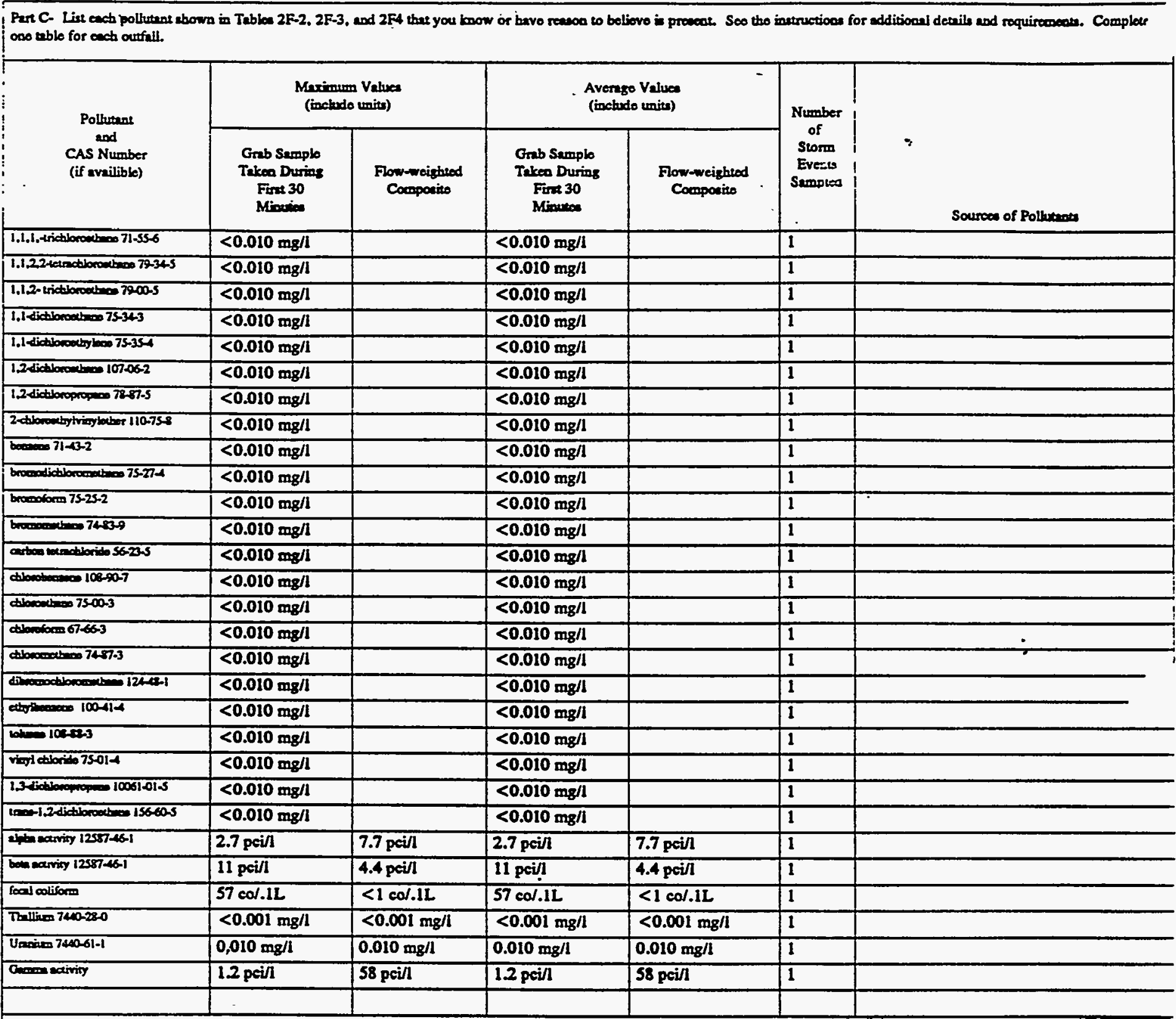

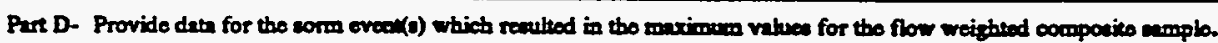

\begin{tabular}{|c|c|c|c|c|c|c|c|}
\hline $\begin{array}{l}1 . \\
\text { Date of } \\
\text { Storm } \\
\text { Event }\end{array}$ & $\begin{array}{l}2 . \\
\text { Duration } \\
\text { of Storm } \\
\text { (in }\end{array}$ & 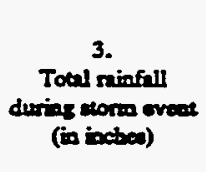 & 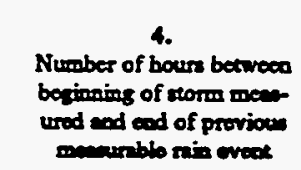 & 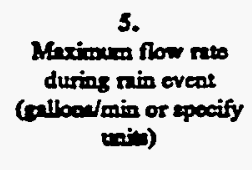 & $\begin{array}{l}6 . \\
\text { Total flow from } \\
\text { rain event } \\
\text { (allowe or } \\
\text { pacify union) }\end{array}$ & $\begin{array}{c}7 . \\
\text { Season } \\
\text { semplo we } \\
\text { uleon }\end{array}$ & $\begin{array}{l}8 . \\
\text { Form of } \\
\text { Precipitation } \\
\text { (rinfall. } \\
\text { andina) }\end{array}$ \\
\hline $3 / 6 / 92$ & 420 & 0.76 & 138.9 & 3.4 & 715 & Winter & Rainfall \\
\hline
\end{tabular}

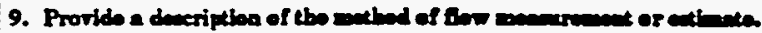

An ISCO 3230 Flow meter wes wed to obtain water lerd readings. This instrument was calibrated with the normal dry wenther flon, if present, as bere zero. The flow meter was programmed with the charecteritics of the coaveyence so that flow rates and total flows conld be calculated by the aneter. 
Outfall 18

Parte B and C Contioused

\begin{tabular}{|c|c|c|c|c|c|c|}
\hline \multirow{2}{*}{$\begin{array}{l}\text { Pollunant } \\
\text { end } \\
\text { CAs Numbor } \\
\text { (if aveilible) }\end{array}$} & \multicolumn{2}{|c|}{$\begin{array}{l}\text { Maximum Vahes } \\
\text { (iochedo units) }\end{array}$} & \multicolumn{2}{|c|}{$\begin{array}{l}\text { Averase Vahuea } \\
\text { (include unita) }\end{array}$} & \multirow{2}{*}{$\begin{array}{c}\text { Nomber } \\
\text { of } \\
\text { Stormi } \\
\text { Evocts } \\
\text { Sompled }\end{array}$} & \multirow[b]{2}{*}{ Sources of Pollweate } \\
\hline & $\begin{array}{l}\text { Orab Semplo } \\
\text { Teleen During } \\
\text { Firs } 30 \\
\text { Minece }\end{array}$ & $\begin{array}{l}\text { Flow-moigted } \\
\text { Compoites }\end{array}$ & $\begin{array}{l}\text { Greb Samplo } \\
\text { Takeo During } \\
\text { Firu } 30 \\
\text { Minotece }\end{array}$ & $\begin{array}{c}\text { Fow-woighed } \\
\text { Compocises } \\
\ldots\end{array}$ & & \\
\hline Berten 740-39-3 & $0.0592 \mathrm{mg} / \mathrm{h}$ & $0.0299 \mathrm{mg} / 1$ & $0.0592 \mathrm{mg} / 1$ & $0.0299 \mathrm{mg} / 1$ & 1 & \\
\hline 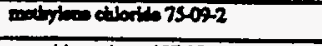 & $<0.010 \mathrm{mg} / \mathrm{l}$ & & $<0.010 \mathrm{mg} / 1$ & & 1 & \\
\hline 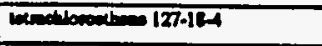 & $<0.010 \mathrm{mg} / \mathrm{l}$ & & $<0.010 \mathrm{mg} / 1$ & & 1 & \\
\hline Whatherouben 7901.6 & $<0.010 \mathrm{mg} / 1$ & & $<0.010 \mathrm{mg} / \mathrm{l}$ & & 1 & \\
\hline xylem & $<0.010 \mathrm{mg} / \mathrm{l}$ & & $<0.010 \mathrm{mg} / \mathrm{l}$ & & 1 & \\
\hline 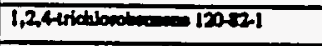 & $<0.010 \mathrm{mg} / 1$ & $<0.010 \mathrm{mg} / 1$ & $<0.010 \mathrm{mg} / \mathrm{l}$ & $<0.010 \mathrm{mg} / \mathrm{l}$ & $\overline{1}$ & \\
\hline 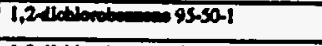 & $<0.010 \mathrm{mg} / \mathrm{l}$ & $<0.010 \mathrm{mg} / 1$ & $<0.010 \mathrm{mg} / \mathrm{l}$ & $<0.010 \mathrm{mg} / \mathrm{l}$ & 1 & \\
\hline 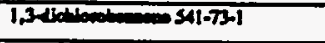 & $<0.010 \mathrm{mg} / \mathrm{l}$ & $<0.010 \mathrm{mg} / \mathrm{l}$ & $<0.010 \mathrm{mg} /$ & $<0.010 \mathrm{mg} / 1$ & 1 & \\
\hline 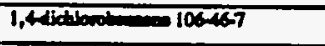 & $<0.010 \mathrm{mg} / \mathrm{l}$ & $<0.010 \mathrm{mg} / \mathrm{l}$ & $<0.010 \mathrm{mg} / \mathrm{l}$ & $<0.010 \mathrm{mg} / \mathrm{l}$ & 1 & \\
\hline 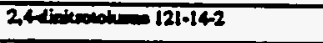 & $<0.010 \mathrm{mg} / \mathrm{h}$ & $<0.010 \mathrm{mg} / \mathrm{l}$ & $<0.010 \mathrm{mg} / 1$ & $<0.010 \mathrm{mg} / 1$ & 1 & \\
\hline 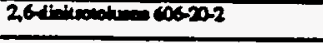 & $<0.010 \mathrm{mg} / \mathrm{l}$ & $<0.010 \mathrm{mg} / 1$ & $<0.010 \mathrm{mg} / 1$ & $<0.010 \mathrm{mg} / 1$ & 1 & \\
\hline 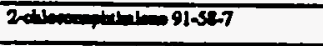 & $<0.010 \mathrm{mg} / \mathrm{l}$ & $<0.010 \mathrm{mg} / \mathrm{l}$ & $<0.010 \mathrm{mg} / \mathrm{l}$ & $<0.010 \mathrm{mg} / \mathrm{l}$ & I & \\
\hline 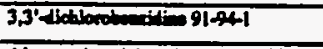 & $<0.010 \mathrm{mg} / \mathrm{l}$ & $<0.020 \mathrm{mg} / 1$ & $<0.010 \mathrm{mg} / 1$ & $<0.020 \mathrm{mg} / 1$ & 1 & \\
\hline 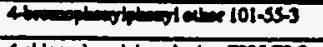 & $<0.010 \mathrm{mg} / 1$ & $<0.010 \mathrm{mg} / 1$ & $<0.010 \mathrm{mpl}$ & $<0.010 \mathrm{mg} / \mathrm{l}$ & 1 & \\
\hline 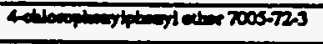 & $<0.010 \mathrm{mg} / \mathrm{l}$ & $<0.010 \mathrm{mg} / 1$ & $<0.010 \mathrm{mg} / \mathrm{t}$ & $<0.010 \mathrm{mg} / 1$ & 1 & \\
\hline $200000000003-329$ & $<0.010 \mathrm{mg} / \mathrm{l}$ & $<0.010 \mathrm{mg} / \mathrm{l}$ & $<0.010 \mathrm{mg} / \mathrm{l}$ & $<0.010 \mathrm{mg} / 1$ & 1 & \\
\hline 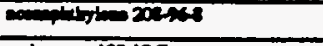 & $<0.010 \mathrm{mg} / \mathrm{l}$ & $<0.010 \mathrm{mg} / \mathrm{h}$ & $<0.010 \mathrm{mg} / \mathrm{l}$ & $<0.010 \mathrm{mg} / 1$ & 1 & \\
\hline $120-127$ & $<0.010 \mathrm{mg} / \mathrm{l}$ & $<0.010 \mathrm{mg} / \mathrm{l}$ & $<0.010 \mathrm{mg} / \mathrm{l}$ & $<0.010 \mathrm{mg} / 1$ & 1 & $\because$ \\
\hline 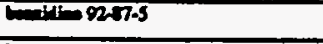 & $<0.030 \mathrm{mg} / 1$ & $<0.050 \mathrm{mg} / \mathrm{l}$ & $<0.030 \mathrm{mg} /$ & $<0.050 \mathrm{mg} / \mathrm{l}$ & 1 & \\
\hline 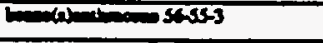 & $<0.010 \mathrm{mg} / 1$ & $<0.010 \mathrm{mg} / \mathrm{l}$ & $<0.010 \mathrm{mg} / \mathrm{l}$ & $<0.010 \mathrm{mg} / 1$ & 1 & \\
\hline 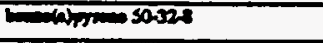 & $<0.010 \mathrm{mg} / \mathrm{l}$ & $<0.010 \mathrm{mg} / \mathrm{I}$ & $<0.010 \mathrm{mg} / \mathrm{l}$ & $<0.010 \mathrm{mg} / 1$ & 1 & \\
\hline 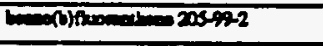 & $<0.010 \mathrm{mg} / 1$ & $<0.010 \mathrm{mg} / 1$ & $<0.010 \mathrm{mg} / 1$ & $<0.010 \mathrm{mg} / 1$ & 1 & \\
\hline $191-242$ & $<0.010 \mathrm{mg} / \mathrm{l}$ & $<0.010 \mathrm{mg} / \mathrm{h}$ & $<0.010 \mathrm{mg} / \mathrm{l}$ & $<0.010 \mathrm{mg} h$ & 1 & \\
\hline 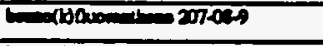 & $<0.010 \mathrm{mg} / \mathrm{L}$ & $<0.010 \mathrm{mq} / \mathrm{h}$ & $<0.010 \mathrm{mg} / \mathrm{I}$ & $<0.010 \mathrm{mg} / \mathrm{l}$ & 1 & \\
\hline 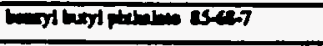 & $<0.010 \mathrm{mg} / \mathrm{l}$ & $<0.010 \mathrm{mg} / \mathrm{l}$ & $<0.010 \mathrm{mg} / \mathrm{l}$ & $<0.010 \mathrm{mg} / \mathrm{l}$ & 1 & \\
\hline 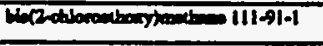 & $<0.010 \mathrm{mg} / \mathrm{l}$ & $<0.010 \mathrm{mg} / \mathrm{l}$ & $<0.010 \mathrm{mg} / \mathrm{l}$ & $<0.010 \mathrm{mg} / 1$ & 1 & \\
\hline 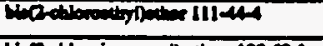 & $<0.010 \mathrm{mg} / 1$ & $<0.010 \mathrm{mg} / \mathrm{l}$ & $<0.010 \mathrm{mg} / \mathrm{l}$ & $<0.010 \mathrm{mg} / \mathrm{l}$ & 1 & \\
\hline 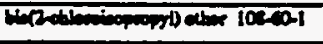 & $<0.010 \mathrm{mg} / \mathrm{l}$ & $<0.010 \mathrm{mgh}$. & $<0.010 \mathrm{mg} / \mathrm{l}$ & $<0.010 \mathrm{mg} / 1$ & 1 & \\
\hline 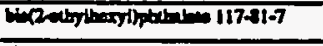 & $<0.010 \mathrm{mg} / \mathrm{l}$ & $<0.010 \mathrm{mg} / \mathrm{l}$ & $<0.010 \mathrm{mg} / \mathrm{l}$ & $<0.010 \mathrm{mg} / \mathrm{l}$ & 1 & \\
\hline tymoen 21L01-9 & $<0.010 \mathrm{mg} / \mathrm{l}$ & $<0.010 \mathrm{mg} / \mathrm{l}$ & $<0.010 \mathrm{mg} / \mathrm{l}$ & $<0.010 \mathrm{mg} / \mathrm{l}$ & 1 & \\
\hline 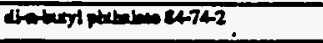 & $<0.010 \mathrm{mg} / \mathrm{l}$ & $<0.010 \mathrm{mg} / \mathrm{l}$ & $<0.010 \mathrm{mg} / \mathrm{l}$ & $<0.010 \mathrm{mg} / \mathrm{l}$ & 1 & \\
\hline 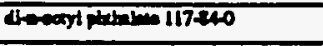 & $<0.010 \mathrm{mg} / \mathrm{l}$ & $<0.010 \mathrm{mg} / \mathrm{l}$ & $<0.010 \mathrm{mg} / \mathrm{l}$ & $<0.010 \mathrm{mg} / \mathrm{h}$ & 1 & \\
\hline $53270-3$ & $<0.010 \mathrm{mg} / \mathrm{l}$ & $<0.010 \mathrm{mg} / \mathrm{h}$ & $<0.010 \mathrm{mg} / 1$ & $<0.010 \mathrm{mg} / \mathrm{h}$ & 1 & \\
\hline 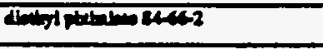 & $<0.010 \mathrm{mg} / 1$ & $<0.010 \mathrm{mg} / 1$ & $<0.010 \mathrm{mg} / 1$ & $<0.010 \mathrm{mg} / \mathrm{l}$ & 1 & \\
\hline 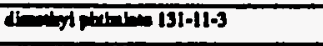 & $<0.010 \mathrm{mg} / \mathrm{l}$ & $<0.010 \mathrm{mg} / 1$ & $<0.010 \mathrm{mg} / \mathrm{l}$ & $<0.010 \mathrm{mg} / \mathrm{l}$ & 1 & \\
\hline 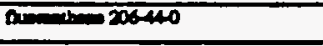 & $<0.010 \mathrm{mg} / \mathrm{l}$ & $<0.010 \mathrm{mg} / \mathrm{l}$ & $<0.010 \mathrm{mg} / \mathrm{I}$ & $<0.010 \mathrm{mg} / 1$ & 1 & \\
\hline $000006.72-7$ & $<0.010 \mathrm{mg} / \mathrm{l}$ & $<0.010 \mathrm{mg} / \mathrm{l}$ & $<0.010 \mathrm{mg} /$ & $<0.010 \mathrm{mg} / \mathrm{s}$ & 1 & \\
\hline 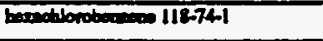 & $<0.010 \mathrm{mg} / \mathrm{l}$ & $<0.010 \mathrm{mg} / 1$ & $<0.010 \mathrm{mg} / \mathrm{l}$ & $<0.010 \mathrm{mg} / \mathrm{l}$ & 1 & \\
\hline 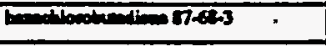 & $<0.010 \mathrm{mg} / \mathrm{l}$ & $<0.010 \mathrm{mg} / 1$ & $<0.010 \mathrm{mg} / \mathrm{l}$ & $<0.010 \mathrm{mg} / \mathrm{h}$ & 1 & \\
\hline 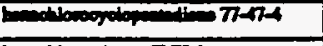 & $<0.010 \mathrm{mg} / \mathrm{l}$ & $<0.010 \mathrm{mg} / 1$ & $<0.010 \mathrm{mg} / \mathrm{l}$ & $<0.010 \mathrm{mg} / 1$ & 1 & \\
\hline 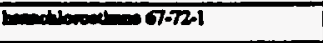 & $<0.010 \mathrm{mg} / \mathrm{l}$ & $<0.010 \mathrm{mg} / 1$ & $<0.010 \mathrm{mg} / \mathrm{l}$ & $<0.010 \mathrm{mg} / \mathrm{l}$ & 1 & \\
\hline 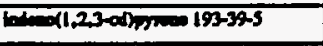 & $<0.010 \mathrm{mg} / \mathrm{l}$ & $<0.010 \mathrm{mg} / \mathrm{l}$ & $<0.010 \mathrm{mg} / \mathrm{l}$ & $<0.010 \mathrm{mg} / \mathrm{l}$ & 1 & \\
\hline 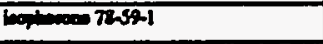 & $<0.010 \mathrm{mg} / \mathrm{l}$ & $<0.010 \mathrm{mg} / 1$ & $<0.010 \mathrm{mg} / 1$ & $<0.010 \mathrm{mg} / \mathrm{L}$ & 1 & \\
\hline 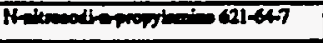 & $<0.010 \mathrm{mg} / \mathrm{l}$ & $<0.010 \mathrm{mg} / \mathrm{l}$ & $<0.010 \mathrm{mg} / 1$ & $<0.010 \mathrm{mg} / 1$ & 1 & \\
\hline 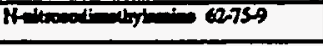 & $<0.010 \mathrm{mg} / \mathrm{l}$ & $<0.010 \mathrm{mg} / \mathrm{l}$ & $<0.010 \mathrm{mg} / \mathrm{l}$ & $<0.010 \mathrm{mg} / \mathrm{l}$ & 1 & \\
\hline 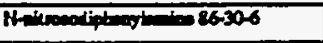 & $<0.010 \mathrm{mg} / \mathrm{l}$ & $<0.010 \mathrm{mg} / \mathrm{l}$ & $<0.010 \mathrm{mg} / 1$ & $<0.010 \mathrm{mg} / \mathrm{l}$ & 1 & \\
\hline $9+4=01-30-3$ & $<0.010 \mathrm{mg} / \mathrm{h}$ & $<0.010 \mathrm{mg} / 1$ & $<0.010 \mathrm{mg} / \mathrm{l}$ & $<0.010 \mathrm{mg} / \mathrm{l}$ & 1 & \\
\hline 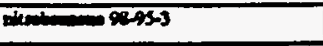 & $<0.010 \mathrm{mg} / \mathrm{l}$ & $<0.010 \mathrm{mg} / 1$ & $<0.010 \mathrm{mg} / \mathrm{l}$ & $<0.010 \mathrm{mg} / \mathrm{l}$ & 1 & \\
\hline 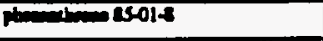 & $<0.010 \mathrm{mg} / \mathrm{h}$ & $<0.010 \mathrm{mg} / 1$ & $<0.010 \mathrm{mg} / 1$ & $<0.010 \mathrm{mg} / \mathrm{l}$ & 1 & \\
\hline 89000129000 & $<0.010 \mathrm{mg} / \mathrm{ll}$ & $<0.010 \mathrm{mg} / 1$ & $<0.010 \mathrm{mg} / 1$ & $<0.010 \mathrm{mg} /$ & 1 & \\
\hline 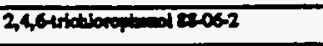 & $<0.030 \mathrm{mg} / \mathrm{l}$ & $<0.030 \mathrm{mg} / 1$ & $<0.030 \mathrm{mg} / \mathrm{l}$ & $<0.030 \mathrm{mg} / \mathrm{l}$ & 1 & \\
\hline 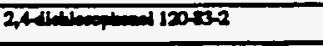 & $<0.030 \mathrm{mg} / 1$ & $<0.030 \mathrm{mg} / \mathrm{l}$ & $<0.030 \mathrm{mg} / 1$ & $<0.030 \mathrm{mg} / \mathrm{l}$ & 1 & \\
\hline 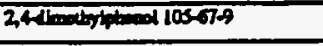 & $<0.030 \mathrm{mg} / \mathrm{h}$ & $<0.030 \mathrm{mg} / 1$ & $<0.030 \mathrm{mg} / 1$ & $<0.030 \mathrm{mg} / 1$ & 1 & \\
\hline 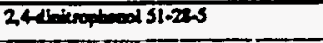 & $<0.030 \mathrm{mg} / \mathrm{l}$ & $<0.050 \mathrm{mg} / 1$ & $<0.030 \mathrm{mg} / \mathrm{l}$ & $<0.050 \mathrm{mg} / 1$ & 1 & \\
\hline
\end{tabular}


Outioll 18

Purt B ad C Continued

\begin{tabular}{|c|c|c|c|c|c|c|}
\hline \multirow{2}{*}{$\begin{array}{l}\text { Polbuent } \\
\text { and } \\
\text { Cas Number } \\
\text { (if avilible) }\end{array}$} & \multicolumn{2}{|c|}{$\begin{array}{l}\text { Mnximum Values } \\
\text { (inchele mia) }\end{array}$} & \multicolumn{2}{|c|}{ 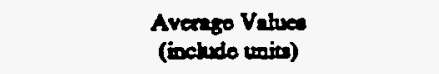 } & \multirow{2}{*}{$\begin{array}{l}\text { Number } \\
\text { of } \\
\text { Storm } \\
\text { Eveces } \\
\text { Stropled }\end{array}$} & \multirow[b]{2}{*}{ Sourcee of Pollutenes } \\
\hline & 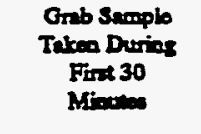 & $\begin{array}{l}\text { Flommoighters } \\
\text { Compocies }\end{array}$ & 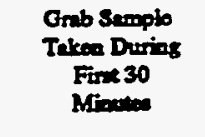 & $\begin{array}{l}\text { Flowmerighted } \\
\text { Cosposites }\end{array}$ & & \\
\hline 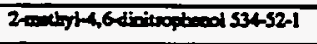 & $<0.030 \mathrm{mg} / 1$ & $<0.050 \mathrm{mg} / 1$ & $<0.030 \mathrm{mg} / 1$ & $<0.050 \mathrm{mg} / \mathrm{h}$ & 1 & \\
\hline 2-itroded ap-75-5 & $<0.030 \mathrm{mg} / \mathrm{l}$ & $<0.030 \mathrm{mg} / \mathrm{l}$ & $<0.030 \mathrm{mg} / \mathrm{h}$ & $<0.030 \mathrm{mg} / \mathrm{l}$ & 1 & \\
\hline 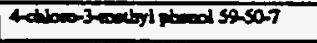 & $<0.030 \mathrm{mg} / 1$ & $<0.030 \mathrm{mg} / 1$ & $<0.030 \mathrm{mg} / 1$ & $<0.030 \mathrm{mg} / 1$ & 1 & \\
\hline 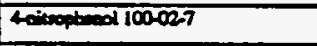 & $<0.030 \mathrm{mg} / \mathrm{l}$ & $<0.050 \mathrm{mg} / 1$ & $<0.030 \mathrm{mg} / \mathrm{l}$ & $<0.050 \mathrm{mg} / \mathrm{l}$ & 1 & \\
\hline 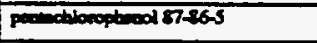 & $<0.030 \mathrm{mg} / \mathrm{l}$ & $<0.050 \mathrm{mg} / 1$ & $<0.030 \mathrm{mg} / \mathrm{l}$ & $<0.050 \mathrm{mg} / 1$ & 1 & \\
\hline Fiend loess-2 & $<0.030 \mathrm{mg} / \mathrm{l}$ & $<0.030 \mathrm{mg} / \mathrm{l}$ & $<0.030 \mathrm{mg} / \mathrm{l}$ & $<0.030 \mathrm{mg} / 1$ & 1 & \\
\hline $4,4-D D D 72-548$ & $<0.00001 \mathrm{mg} / 1$ & $<0.00001 \mathrm{mg} / 1$ & $<0.00001 \mathrm{mg} / 1$ & $<0.00001 \mathrm{mg} / \mathrm{l}$ & 1 & \\
\hline 4,4'-DDE 72-S5-9 & $<0.00001 \mathrm{mg} / \mathrm{h}$ & $<0.00001 \mathrm{mg} / 1$ & $<0.00001 \mathrm{mg} / \mathrm{l}$ & $<0.00001 \mathrm{mg} / \mathrm{l}$ & 1 & \\
\hline 4.4.-00T 50-22-3 & $<0.00001 \mathrm{mg} / \mathrm{l}$ & $<0.0000 \operatorname{lmg} / 1$ & $<0.00001 \mathrm{mg} / \mathrm{l}$ & $<0.00001 \mathrm{mg} / \mathrm{l}$ & 1 & \\
\hline $24 \mathrm{fin} 302-00-2$ & $<0.00001 \mathrm{mg} / \mathrm{h}$ & $<0.00001 \mathrm{mg} / l$ & $<0.00001 \mathrm{mg} / 1$ & $<0.00001 \mathrm{mg} / \mathrm{h}$ & 1 & \\
\hline कbont-57.74 & $<0.00002 \mathrm{mg} / \mathrm{l}$ & $<0.00002 \mathrm{mg} / \mathrm{l}$ & $<0.00002 \mathrm{mg} / \mathrm{l}$ & $<0.00002 \mathrm{mg} / \mathrm{h}$ & 1 & \\
\hline dentin 60-57-1 & $<0.00001 \mathrm{mg} / \mathrm{l}$ & $<0.00001 \mathrm{mg} / 1$ & $<0.00001 \mathrm{mg} / \mathrm{l}$ & $<0.00001 \mathrm{mg} / \mathrm{l}$ & 1 & \\
\hline 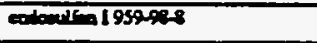 & $<0.00001 \mathrm{mg} / \mathrm{l}$ & $<0.00001 \mathrm{mg} / 1$ & $<0.00001 \mathrm{mg} / \mathrm{l}$ & $<0.00001 \mathrm{mg} / \mathrm{l}$ & 1 & \\
\hline 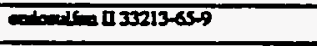 & $<0.00001 \mathrm{mg} / \mathrm{l}$ & $<0.00001 m g / 1$ & $<0.00001 \mathrm{mg} / \mathrm{l}$ & $<0.00001 \mathrm{mg} / \mathrm{l}$ & 1 & \\
\hline 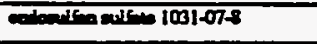 & $<0.00001 \mathrm{mg} / \mathrm{l}$ & $<0.00001 \mathrm{~m} / 1$ & $<0.00001 \mathrm{mg} / \mathrm{l}$ & $<0.00001 \mathrm{mg} / \mathrm{l}$ & 1 & \\
\hline $\operatorname{argin} 72-208$ & $<0.00005 \mathrm{mg} / \mathrm{l}$ & $<0.00005 \mathrm{mg} / 1$ & $<0.00005 \mathrm{mg} / \mathrm{h}$ & $<0.00005 \mathrm{mg} / 1$ & 1 & \\
\hline 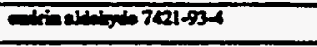 & $<0.00001 \mathrm{mg} / \mathrm{l}$ & $<0.00001 \mathrm{mg} / 1$ & $<0.00001 \mathrm{mg} / 1$ & $<0.00001 \mathrm{mg} h$ & 1 & \\
\hline 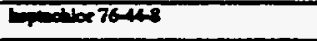 & $<0.00001 \mathrm{mg} / \mathrm{h}$ & $<0.00001 \mathrm{mg} / 1$ & $<0.00001 \mathrm{mg} / \mathrm{h}$ & $<0.00001 \mathrm{mg} / \mathrm{h}$ & 1 & $\because$ \\
\hline 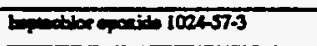 & $<0.00001 \mathrm{mg} / 1$ & $<0.00001 \mathrm{mg} / \mathrm{l}$ & $<0.00001 \mathrm{mg} / \mathrm{l}$ & $<0.00001 \mathrm{mg} / 1$ & 1 & \\
\hline 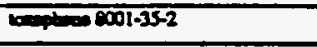 & $<0.0002 \mathrm{mg} / \mathrm{l}$ & $<0.0002 \mathrm{mg} / \mathrm{l}$ & $<0.0002 \mathrm{mg} / \mathrm{h}$ & $<0.0002$ mgh & 1 & 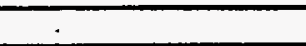 \\
\hline Ant-RHC 319446 & $<0.00001 \mathrm{mg} / \mathrm{h}$ & $<0.00001 \mathrm{mg} / \mathrm{h}$ & $<0.00001 \mathrm{mg} / 1$ & $<0.00001 \mathrm{mg} / \mathrm{l}$ & 1 & \\
\hline - & $<0.00001 \mathrm{mg} / 1$ & $<0.00001 \mathrm{mg} / 1$ & $<0.00001 \mathrm{mg} / \mathrm{l}$ & $<0.00001 \mathrm{mg} / \mathrm{h}$ & 1 & \\
\hline Wh-BHC 319868 & $<0.00001 \mathrm{mg} / \mathrm{h}$ & $<0.00001 \mathrm{mg} / 1$ & $<0.00001 \mathrm{mg} / \mathrm{l}$ & $<0.00001 \mathrm{mg} / \mathrm{l}$ & 1 & \\
\hline 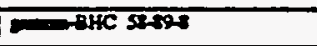 & $<0.00001 \mathrm{mg} / \mathrm{h}$ & $<0.00001 \mathrm{mg} / \mathrm{h}$ & $<0.00001 \mathrm{mg} / \mathrm{l}$ & $<0.00001 \mathrm{mg} / \mathrm{l}$ & 1 & \\
\hline 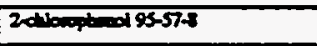 & $<0.030 \mathrm{mg} / \mathrm{l}$ & $<0.030 \mathrm{mg} / 1$ & $<0.030 \mathrm{mg} / \mathrm{l}$ & $<0.030 \mathrm{mg} / 1$ & 1 & \\
\hline Arodom $12 \times 25345921-9$ & $<0.0005 \mathrm{mg} / 1$ & $<0.0005 \mathrm{mg} / \mathrm{l}$ & $<0.0005 \mathrm{mg} /$ & $<0.0005 \mathrm{mg} / \mathrm{l}$ & 1 & \\
\hline Arodor $125411097-69-1$ & $<0.0005 \mathrm{mg} / \mathrm{l}$ & $<0.0005 \mathrm{mg} / \mathrm{l}$ & $<0.0005 \mathrm{mg} / \mathrm{h}$ & $<0.0005 \mathrm{mg} / \mathrm{l}$ & 1 & \\
\hline 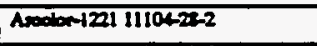 & $<0.0005 \mathrm{mg} / \mathrm{l}$ & $<0.0005 \mathrm{mg} / \mathrm{h}$ & $<0.0005 \mathrm{mg} / \mathrm{h}$ & $<0.0005 \mathrm{mg} / 1$ & 1 & \\
\hline Aroctom $122211141-16-5$ & $<0.0005 \mathrm{mg} / \mathrm{l}$ & $<0.0005 \mathrm{mg} / 1$ & $<0.0005 \mathrm{mg} / 1$ & $<0.0005 \mathrm{mg} / \mathrm{l}$ & 1 & \\
\hline A $1000100-124812572296$ & $<0.0005 \mathrm{mg} / 1$ & $<0.0005 \mathrm{mg} / \mathrm{l}$ & $<0.0005 \mathrm{mg} / \mathrm{l}$ & $<0.0005 \mathrm{mg} / 1$ & 1 & \\
\hline Arodoror 126011096525 & $<0.0005 \mathrm{mg} / 1$ & $<0.0005 \mathrm{mg} / 1$ & $<0.0005 \mathrm{mg} / \mathrm{h}$ & $<0.0005 \mathrm{mg} / \mathrm{l}$ & 1 & \\
\hline Alodon-1016 1267411.2 & $<0.0005 \mathrm{mg} / 1$ & $<0.0005 \mathrm{mg} / \mathrm{l}$ & $<0.0005 \mathrm{mg} / 1$ & $<0.0005 \mathrm{mg} / \mathrm{h}$ & 1 & \\
\hline Cromole & $<0.010 \mathrm{mp} / 1$ & $<0.010 \mathrm{mg} / \mathrm{h}$ & $<0.010 \mathrm{mg} / \mathrm{l}$ & $<0.010 \mathrm{mg} / \mathrm{l}$ & 1 & \\
\hline Acolowin & $<0.010 \mathrm{mg} / \mathrm{l}$ & $<0.010 \mathrm{mg} / \mathrm{l}$ & $<0.010 \mathrm{mg} / 1$ & $<0.010 \mathrm{mg} / 1$ & 1 & \\
\hline Anesboicrib & $<0.010 \mathrm{mg} / \mathrm{l}$ & $<0.010 \mathrm{mg} / 1$ & $<0.010 \mathrm{mg} / 1$ & $<0.010 \mathrm{mg} / \mathrm{l}$ & 1 & \\
\hline 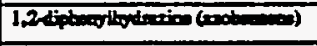 & Recults peoding & Renults peading & Recults peading & Reaulte pending & 1 & \\
\hline & & & & & & \\
\hline & & & & & & \\
\hline & & & & & & \\
\hline & & & & & & \\
\hline & & & & & & \\
\hline & & & & & & \\
\hline & & & & & & \\
\hline & & & & & & \\
\hline & & & & & & \\
\hline & & & & & & \\
\hline & & & & & & \\
\hline & & & & & & \\
\hline & & & & & & \\
\hline & & & & & & \\
\hline & & & & & & \\
\hline
\end{tabular}


VI. Discharge Informetion (continued from page 3 of Form $2 \mathrm{~F}$

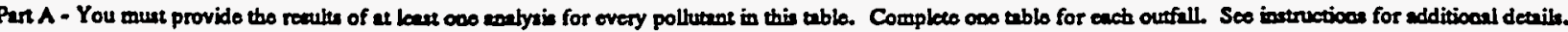

\begin{tabular}{|c|c|c|c|c|c|c|}
\hline \multirow{2}{*}{$\begin{array}{l}\text { Polturade } \\
\text { asd } \\
\text { CAS Number } \\
\text { (if availible) }\end{array}$} & \multicolumn{2}{|c|}{$\begin{array}{l}\text { Maximum Values } \\
\text { (includo tuiv) }\end{array}$} & \multicolumn{2}{|c|}{$\begin{array}{l}\text { Averngo Vabuea } \\
\text { (inchudo univs) }\end{array}$} & \multirow{2}{*}{$\begin{array}{l}\text { Number } \\
\text { of } \\
\text { Stomen } \\
\text { Eveoses } \\
\text { Sempled }\end{array}$} & \multirow[b]{2}{*}{ Sourece of Pollutants } \\
\hline & $\begin{array}{c}\text { Grab Sumple } \\
\text { Trkea During } \\
\text { Firut } 30 \\
\text { Minewa }\end{array}$ & $\begin{array}{l}\text { Flow-weighed } \\
\text { Composics }\end{array}$ & $\begin{array}{c}\text { Greb Sample } \\
\text { Takea During } \\
\text { Firu } 30 \\
\text { Minuteo }\end{array}$ & $\begin{array}{l}\text { Flow-wreightiod } \\
\text { Cocopponito }\end{array}$ & & \\
\hline pilmod Gomes & $3 \mathrm{mg} / \mathrm{l}$ & & $3 \mathrm{mg} / \mathrm{l}$ & & 1 & \\
\hline 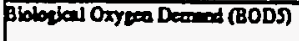 & $9.15 \mathrm{mg} / \mathrm{l}$ & $<5 \mathrm{mg} / 1$ & $9.15 \mathrm{mg} / 1$ & $<5 \mathrm{mg} / 1$ & 1 & \\
\hline Chomial Oxyous Deaned (COD) & $60 \mathrm{mg} / \mathrm{l}$ & $23 \mathrm{mg} / 1$ & $60 \mathrm{mg} / \mathrm{l}$ & $23 \mathrm{mg} / 1$ & 1 & \\
\hline Tond 2mped Solide (TSS) & $72 \mathrm{mg} / \mathrm{l}$ & $12 \mathrm{mg} / 1$ & $72 \mathrm{mg} / \mathrm{l}$ & $12 \mathrm{mg} / 1$ & 1 & \\
\hline 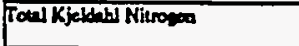 & $1.0 \mathrm{mg} / \mathrm{l}$ & $0.5 \mathrm{mg} / \mathrm{l}$ & $1.0 \mathrm{mg} / \mathrm{l}$ & $0.5 \mathrm{mg} / 1$ & 1 & \\
\hline 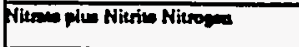 & $0.67 \mathrm{mg} / 1$ & $0.94 \mathrm{mg} / \mathrm{l}$ & $0.67 \mathrm{mg} / 1$ & $0.94 \mathrm{mg} / \mathrm{l}$ & 1 & \\
\hline Toal Prouphorum & $0.12 \mathrm{mg} / \mathrm{l}$ & $<0.1 \mathrm{mg} / \mathrm{l}$ & $0.12 \mathrm{mg} / 1$ & $<0.1 \mathrm{mg} / \mathrm{l}$ & 1 & \\
\hline
\end{tabular}

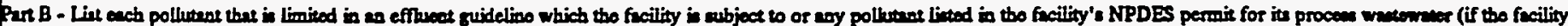

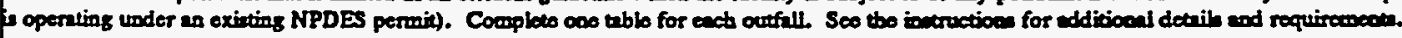

\begin{tabular}{|c|c|c|c|c|c|c|}
\hline \multirow{2}{*}{$\begin{array}{l}\text { Pollumant } \\
\text { end } \\
\text { CAs Number } \\
\text { (if availible) }\end{array}$} & \multicolumn{2}{|c|}{$\begin{array}{l}\text { Meximum Veluee } \\
\text { (inolude unital) }\end{array}$} & \multicolumn{2}{|c|}{$\begin{array}{l}\text { Averise Vahser } \\
\text { (include units) }\end{array}$} & \multirow{2}{*}{$\begin{array}{l}\text { Number } \\
\text { of } \\
\text { storm } \\
\text { Events } \\
\text { Semplod }\end{array}$} & \multirow[b]{2}{*}{ Sources of Pollwercen } \\
\hline & 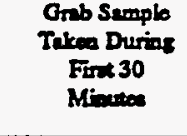 & $\begin{array}{l}\text { Fow-weighood } \\
\text { Componico }\end{array}$ & $\begin{array}{l}\text { Grab Samplo } \\
\text { Tabeo Diring } \\
\text { Find } 30 \\
\text { Minute }\end{array}$ & $\begin{array}{l}\text { Fow-weichted } \\
\text { Compocice }\end{array}$ & & \\
\hline Anilexp 7400-360 & $<0.04 \mathrm{mg} / \mathrm{l}$ & $<0.04 \mathrm{mg} / \mathrm{l}$ & $<0.04 \mathrm{mg} / \mathrm{l}$ & $<0.04 \mathrm{mg} / \mathrm{l}$ & 1 & \\
\hline orain & $<0.002 \mathrm{mg} / \mathrm{l}$ & & $<0.002 \mathrm{mg} / \mathrm{l}$ & & 1 & \\
\hline $\operatorname{monany} 7439076$ & $<0.0002 \mathrm{mg} / \mathrm{l}$ & $<0.0002 \mathrm{mg} / 1$ & $<0.0002 \mathrm{mg} / 1$ & $<0.0002 \mathrm{mg} / 1$ & 1 & \\
\hline מיס- & $0.004 \mathrm{mg} / \mathrm{l}$ & & $0.004 \mathrm{mg} / \mathrm{l}$ & & 1 & \\
\hline 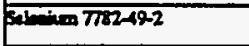 & $<0.002 \mathrm{mg} / \mathrm{l}$ & $<0.002 \mathrm{mg} / \mathrm{l}$ & $<0.002 \mathrm{mg} / \mathrm{l}$ & $<0.002 \mathrm{mg} / 1$ & 1 & \\
\hline Pullow & $14 \mathrm{mg} / \mathrm{l}$ & $<10 \mathrm{mg} / 1$ & $14 \mathrm{mg} / 1$ & $<10 \mathrm{mg} / 1$ & 1 & \\
\hline Purteneses & $0.16 \mathrm{mg} / 1$ & $0.10 \mathrm{mg} / \mathrm{l}$ & $0.16 \mathrm{mg} / 1$ & $0.10 \mathrm{mg} / \mathrm{l}$ & 1 & \\
\hline Then:ind 7429.90 .5 & $2.03 \mathrm{mg} / 1$ & $0.76 \mathrm{mg} / \mathrm{l}$ & $2.03 \mathrm{mg} / \mathrm{l}$ & $0.76 \mathrm{mg} / 1$ & 1 & \\
\hline $40=740-34-2$ & $<0.04 \mathrm{mg} / 1$ & $<0.04 \mathrm{mg} / 1$ & $<0.04 \mathrm{mg} / \mathrm{l}$ & $<0.04 \mathrm{mg} / 1$ & 1 & \\
\hline $300007400-128$ & $0.024 \mathrm{mg} / 1$ & $0.020 \mathrm{mg} / \mathrm{l}$ & $0.024 \mathrm{mg} / \mathrm{l}$ & $0.020 \mathrm{mg} / 1$ & 1 & \\
\hline Bengliting 7460-1.7 & $<0.0004 \mathrm{mg} / \mathrm{l}$ & $<0.0004 \mathrm{mg} / \mathrm{l}$ & $<0.0004 \mathrm{mg} / 1$ & $<0.0004 \mathrm{mg} / \mathrm{l}$ & 1 & \\
\hline 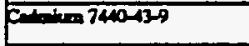 & $<0.004 \mathrm{mg} / \mathrm{l}$ & $<0.004 \mathrm{mg} / \mathrm{l}$ & $<0.004 \mathrm{mg} / \mathrm{l}$ & $<0.004 \mathrm{mg} / \mathrm{l}$ & 1 & \\
\hline Calohm $7400-x-2$ & $30.9 \mathrm{mg} / \mathrm{l}$ & $15.7 \mathrm{mg} / \mathrm{l}$ & $30.9 \mathrm{mg} / \mathrm{l}$ & $15.7 \mathrm{mg} / 1$ & 1 & \\
\hline 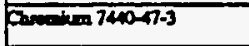 & $<0.006 \mathrm{mg} / \mathrm{l}$ & $<0.006 \mathrm{mg} / \mathrm{l}$ & $<0.006 \mathrm{mg} / 1$ & $<0.006 \mathrm{mg} / 1$ & 1 & \\
\hline Cotak 7440-18-4 & $<0.002 \mathrm{mg} / \mathrm{l}$ & $<0.002 \mathrm{mg} / 1$ & $<0.002 \mathrm{mg} / \mathrm{l}$ & $<0.002 \mathrm{mg} / \mathrm{l}$ & 1 & \\
\hline Formen 74050 sos & $0.012 \mathrm{mg} / \mathrm{h}$ & $<0.006 \mathrm{mg} / \mathrm{h}$ & $0.012 \mathrm{mg} / \mathrm{l}$ & $<0.006 \mathrm{mg} / \mathrm{l}$ & 1 & \\
\hline 5007039896 & $1.12 \mathrm{mg} / 1$ & $0.43 \mathrm{mg} / \mathrm{l}$ & $1.12 \mathrm{mg} / 1$ & $0.43 \mathrm{mg} / \mathrm{l}$ & 1 & \\
\hline $70403902-1$ & $<0.02 \mathrm{mg} / \mathrm{l}$ & $<0.02 \mathrm{mg} / \mathrm{l}$ & $<0.02 \mathrm{mg} / \mathrm{l}$ & $<0.02 \mathrm{mg} / \mathrm{h}$ & 1 & \\
\hline $7464003993-2$ & $<0.02 \mathrm{mg} / 1$ & $<0.02 \mathrm{mg} / \mathrm{l}$ & $<0.02 \mathrm{mg} / 1$ & $<0.02 \mathrm{mg} / 1$ & 1 & \\
\hline Mequativen 7439-954 & $3.28 \mathrm{mg} / \mathrm{l}$ & $1.31 \mathrm{mg} / \mathrm{l}$ & $3.28 \mathrm{mg} / \mathrm{l}$ & $1.31 \mathrm{mg} / 1$ & 1 & \\
\hline M. 7439065 & $0.033 \mathrm{mg} / \mathrm{l}$ & $0.012 \mathrm{mg} / \mathrm{l}$ & $0.033 \mathrm{mg} / \mathrm{h}$ & $0.012 \mathrm{mg} / 1$ & 1 & \\
\hline Mobtation 7439-96.7 & $<0.006 \mathrm{mg} / \mathrm{l}$ & $<0.006 \mathrm{mg} / 1$ & $<0.006 \mathrm{mg} / \mathrm{l}$ & $<0.006 \mathrm{mg} / 1$ & 1 & \\
\hline$\sqrt{1601740020}$ & $<0.008 \mathrm{mg} / \mathrm{l}$ & $<0.008 \mathrm{mg} / \mathrm{l}$ & $<0.008 \mathrm{mg} / \mathrm{l}$ & $<0.008 \mathrm{mg} / \mathrm{l}$ & 1 & \\
\hline Pomiven 740-027 & $5.5 \mathrm{mg} / 1$ & $1.9 \mathrm{mg} / \mathrm{l}$ & $5.5 \mathrm{mg} / 1$ & $1.9 \mathrm{mg} / 1$ & 1 & \\
\hline (4601740-204 & $<0.006 \mathrm{mg} / 1$ & $<0.006 \mathrm{mg} / 1$ & $<0.006 \mathrm{mg} / \mathrm{l}$ & $<0.006 \mathrm{mg} / 1$ & 1 & \\
\hline $501600740-225$ & $3.01 \mathrm{mg} / 1$ & $1.08 \mathrm{mg} / \mathrm{f}$ & $3.01 \mathrm{mg} / 1$ & $1.08 \mathrm{mg} / 1$ & 1 & \\
\hline Fitenicon 7400-32.6 & $<0.01 \mathrm{mg} / \mathrm{l}$ & $<0.01 \mathrm{mg} / \mathrm{l}$ & $<0.01 \mathrm{mg} / \mathrm{l}$ & $<0.01 \mathrm{mg} / 1$ & 1 & \\
\hline $2700740066-6$ & $0.21 \mathrm{mg} / \mathrm{l}$ & $0.10 \mathrm{mg} / \mathrm{l}$ & $0.21 \mathrm{mg} / \mathrm{l}$ & $0.10 \mathrm{mg} / \mathrm{l}$ & 1 & \\
\hline
\end{tabular}


Continued from fros.

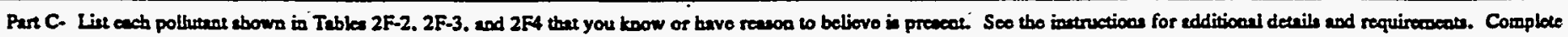
ose table for each outilil.

\begin{tabular}{|c|c|c|c|c|c|c|}
\hline \multirow{2}{*}{$\begin{array}{c}\text { Pollutang } \\
\text { and } \\
\text { CAS Number } \\
\text { (if avilible) }\end{array}$} & \multicolumn{2}{|c|}{$\begin{array}{l}\text { Meximum Values } \\
\text { (include units) }\end{array}$} & \multicolumn{2}{|c|}{$\begin{array}{l}\text { Averege Values } \\
\text { (includo unith) }\end{array}$} & \multirow{2}{*}{$\begin{array}{c}\text { Number } \\
\text { of } \\
\text { Storm } \\
\text { Events } \\
\text { Simpled }\end{array}$} & \multirow[b]{2}{*}{ Sources of Pollurunts } \\
\hline & $\begin{array}{c}\text { Grib Semplo } \\
\text { Taken During } \\
\text { Fint } 30 \\
\text { Minuter }\end{array}$ & $\begin{array}{l}\text { Flow-mreigtied } \\
\text { Compointo }\end{array}$ & $\begin{array}{l}\text { Grab Sumple } \\
\text { Taken During } \\
\text { Fint } 30 \\
\text { Minuece }\end{array}$ & $\begin{array}{l}\text { Flow meighted } \\
\text { Comporite }\end{array}$ & & \\
\hline $1.1,1$, trictionocten $71-55-6$ & $<0.010 \mathrm{mg} / 1$ & & $<0.010 \mathrm{mg} / \mathrm{l}$ & & 1 & \\
\hline 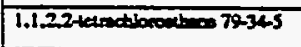 & $<0.010 \mathrm{mg} / \mathrm{l}$ & & $<0.010 \mathrm{mg} / 1$ & & 1 & \\
\hline 1.1.2- Lictibereol teo 7900-5 & $<0.010 \mathrm{mg} / \mathrm{l}$ & & $<0.010 \mathrm{mg} / \mathrm{l}$ & & 1 & \\
\hline 1.1 dictionoches 75-34-3 & $<0.010 \mathrm{mg} / 1$ & & $<0.010 \mathrm{mg} / \mathrm{l}$ & & 1 & \\
\hline I.1-dichlorouthyleses 75-35-4 & $<0.010 \mathrm{mg} / \mathrm{l}$ & & $<0.010 \mathrm{mg} / 1$ & & 1 & \\
\hline 1,2-dictioncuethes 107-06-2 & $<0.010 \mathrm{mg} / \mathrm{h}$ & & $<0.010 \mathrm{mg} / 1$ & & 1 & \\
\hline 1.2dichboropropes $72-57.5$ & $<0.010 \mathrm{mg} / \mathrm{l}$ & & $<0.010 \mathrm{mg} / 1$ & & 1 & \\
\hline 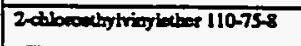 & $<0.010 \mathrm{mg} / \mathrm{l}$ & & $<0.010 \mathrm{mg} / 1$ & & 1 & \\
\hline becosos $71-43-2$ & $<0.010 \mathrm{mg} / \mathrm{l}$ & & $<0.010 \mathrm{mg} / 1$ & & 1 & \\
\hline 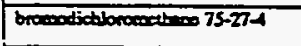 & $<0.010 \mathrm{mg} / 1$ & & $<0.010 \mathrm{mg} / 1$ & & 1 & . \\
\hline broweoform 75-25-2 & $<0.010 \mathrm{mg} / \mathrm{l}$ & & $<0.010 \mathrm{mg} / \mathrm{l}$ & & 1 & \\
\hline 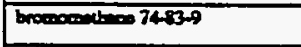 & $<0.010 \mathrm{mg} / \mathrm{h}$ & & $<0.010 \mathrm{mg} / \mathrm{l}$ & & 1 & \\
\hline artion menchlorite 56-22-5 & $<0.010 \mathrm{mg} / 1$ & & $<0.010 \mathrm{mg} / \mathrm{l}$ & & 1 & \\
\hline chibotaceos $108-90.7$ & $<0.010 \mathrm{mg} / 1$ & & $<0.010 \mathrm{mg} / \mathrm{l}$ & & 1 & \\
\hline $7500-3$ & $<0.010 \mathrm{mg} / 1$ & & $<0.010 \mathrm{mg} / l$ & & 1 & \\
\hline chiondoren 6768-3 & $<0.010 \mathrm{mg} / \mathrm{l}$ & & $<0.010 \mathrm{mg} /$ & & 1 & $:$ \\
\hline 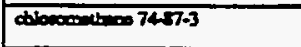 & $<0.010 \mathrm{mg} / \mathrm{l}$ & & $<0.010 \mathrm{mg} / 1$ & & 1 & \\
\hline 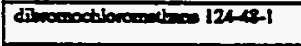 & $<0.010 \mathrm{mg} / 1$ & & $<0.010 \mathrm{mg} / 1$ & & 1 & \\
\hline 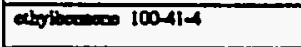 & $<0.010 \mathrm{mg} / 1$ & & $<0.010 \mathrm{mg} / \mathrm{l}$ & & 1 & \\
\hline botwes losen-3 & $<0.010 \mathrm{mg} / 1$ & & $<0.010 \mathrm{mg} / \mathrm{l}$ & & 1 & \\
\hline vigh chlotide 75-014 & $<0.010 \mathrm{mg} / 1$ & & $<0.010 \mathrm{mg} / \mathrm{l}$ & & 1 & \\
\hline 1.3-1idboropopees 10061-01-5 & $<0.010 \mathrm{mg} / 1$ & & $<0.010 \mathrm{mg} / \mathrm{l}$ & - & 1 & \\
\hline 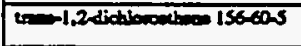 & $<0.010 \mathrm{mg} / 1$ & & $<0.010 \mathrm{mg} / 1$ & & 1 & \\
\hline 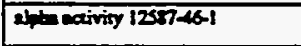 & $7.4 \mathrm{pcin}$ & 1.9 peill & $7.4 \mathrm{pci} / \mathrm{l}$ & $1.9 \mathrm{pci} / 1$ & 1 & \\
\hline tonexivisy $1258746-1$ & $18 \mathrm{pei} / 1$ & 5.2 peill & 18 peill & 5.2 peill & 1 & \\
\hline rocil colifocen & $20 \mathrm{co} / .1 \mathrm{~L}$ & $79 \mathrm{col} .1 \mathrm{~L}$ & $20 \mathrm{co} / .1 \mathrm{~L}$ & $79 \mathrm{col} .1 \mathrm{~L}$ & 1 & \\
\hline Thallinon 740-220 & $<0.003 \mathrm{mg} / \mathrm{l}$ & $<0.03 \mathrm{mg} / 1$ & $<0.003 \mathrm{mg} / \mathrm{l}$ & $<0.03 \mathrm{mg} / \mathrm{l}$ & 1 & \\
\hline Uaxim 740-61-1 & $0.001 \mathrm{mg} / 1$ & $0.003 \mathrm{mg} / \mathrm{l}$ & $0.001 \mathrm{mg} / 1$ & $0.003 \mathrm{mg} / \mathrm{l}$ & 1 & \\
\hline Gonneseciviny & $76 \mathrm{pci} / \mathrm{I}$ & 22 pei/l & 76 pcin & 22 peill & 1 & \\
\hline
\end{tabular}

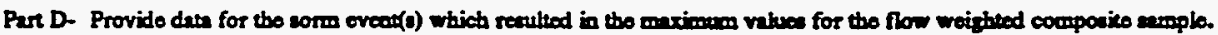

\begin{tabular}{|c|c|c|c|c|c|c|c|}
\hline $\begin{array}{l}1 . \\
\text { Detes of } \\
\text { Storm } \\
\text { Evecs }\end{array}$ & $\begin{array}{l}2 . \\
\text { Durition } \\
\text { of Storm } \\
\text { (in } \\
\text { miverea) }\end{array}$ & $\begin{array}{l}3 . \\
\text { Total ninfall } \\
\text { durias atorm oveat } \\
\text { (in iecbel) }\end{array}$ & 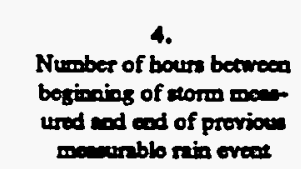 & $\begin{array}{l}\text { S. } \\
\text { Meximen flow rats } \\
\text { during nin eveat } \\
\text { (ollbadmin or specify } \\
\text { unin) }\end{array}$ & $\begin{array}{l}6 . \\
\text { Total fow from } \\
\text { nis event } \\
\text { (allows or } \\
\text { pecify tmita) }\end{array}$ & $\begin{array}{c}7 . \\
\text { Scaion } \\
\text { amplo whe } \\
\text { tiken }\end{array}$ & $\begin{array}{l}\text { 8. } \\
\text { Forma of } \\
\text { Procipiation } \\
\text { (ninfill. } \\
\text { aomman) }\end{array}$ \\
\hline $12 / 13 / 91$ & 250 & 0.25 & 88.2 & 78.6 & 5,600 & Fall & Rrinfull \\
\hline
\end{tabular}

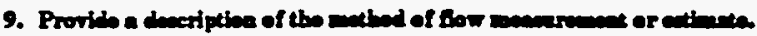

An ISCO 3230 Fow meter was used to obtain water level readings. This instrument was calibrated with the normal dry reather flow, if present, as level zero. The flow meter was programmed with the charncteristics of the conveyance so that flow rates and total flows condd be calculated by the meter. 
Ontfoll 19

Parte B and C Continuod

\begin{tabular}{|c|c|c|c|c|c|c|}
\hline \multirow{2}{*}{$\begin{array}{l}\text { Pollutuas } \\
\text { and } \\
\text { CAs Number } \\
\text { (if availibb) }\end{array}$} & \multicolumn{2}{|c|}{ 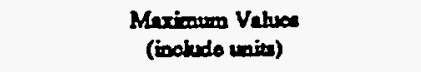 } & \multicolumn{2}{|c|}{$\begin{array}{l}\text { Average Values } \\
\text { (include unita) }\end{array}$} & \multirow{2}{*}{$\begin{array}{c}\text { Number } \\
\text { of } \\
\text { Storm } \\
\text { Eveced } \\
\text { Sempiod }\end{array}$} & \multirow[b]{2}{*}{ Sources of Pollumans } \\
\hline & $\begin{array}{l}\text { Grab Samplo } \\
\text { Taken Durias } \\
\text { Firas } 30 \\
\text { Mineses }\end{array}$ & $\begin{array}{l}\text { Flow-weighted } \\
\text { Comporice }\end{array}$ & $\begin{array}{l}\text { Grab Sumple } \\
\text { Treos Durias } \\
\text { Fins } 30 \\
\text { Mincece }\end{array}$ & $\begin{array}{c}\text { Flow-wreighted } \\
\text { Componivo } \\
. .\end{array}$ & & \\
\hline Beriven 7400-32.3 & $0.0359 \mathrm{mg} / \mathrm{l}$ & $0.0224 \mathrm{mg} / 1$ & $0.0359 \mathrm{mg} / \mathrm{l}$ & $0.0224 \mathrm{mg} / \mathrm{l}$ & 1 & \\
\hline 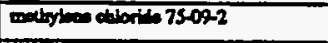 & $<0.010 \mathrm{mg} / \mathrm{l}$ & & $<0.010 \mathrm{mg} / 1$ & & 1 & \\
\hline 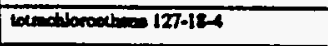 & $<0.010 \mathrm{mg} / \mathrm{l}$ & - & $<0.010 \mathrm{mg} / 1$ & & 1 & \\
\hline 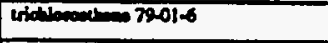 & $<0.010 \mathrm{mg} / \mathrm{l}$ & & $<0.010 \mathrm{mg} / \mathrm{l}$ & & 1 & \\
\hline Xyloos & $<0.010 \mathrm{mg} / \mathrm{l}$ & & $<0.010 \mathrm{mg} / \mathrm{h}$ & & 1 & \\
\hline 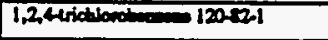 & $<0.010 \mathrm{mg} / \mathrm{l}$ & $<0.010 \mathrm{mg} / 1$ & $<0.010 \mathrm{mg} / 1$ & $<0.010 \mathrm{mg} / 1$ & 1 & \\
\hline 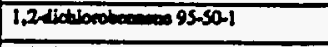 & $<0.010 \mathrm{mg} / 1$ & $<0.010 \mathrm{mg} / 1$ & $<0.010 \mathrm{mg} / \mathrm{l}$ & $<0.010 \mathrm{mg} / 1$ & 1 & \\
\hline 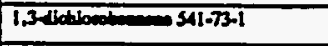 & $<0.010 \mathrm{mg} / \mathrm{l}$ & $<0.010 \mathrm{mg} / \mathrm{l}$ & $<0.010 \mathrm{mg} / 1$ & $<0.010 \mathrm{mg} / 1$ & 1 & \\
\hline 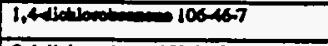 & $<0.010 \mathrm{mg} / 1$ & $<0.010 \mathrm{mg} / 1$ & $<0.010 \mathrm{mg} /$ & $<0.010 \mathrm{mg} / \mathrm{l}$ & 1 & \\
\hline 2,4 thitrondiven $121-14-2$ & $<0.010 \mathrm{mg} / 1$ & $<0.010 \mathrm{mg} / \mathrm{l}$ & $<0.010 \mathrm{mg} / 1$ & $<0.010 \mathrm{mg} / \mathrm{l}$ & 1 & \\
\hline 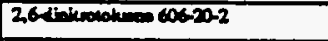 & $<0.010 \mathrm{mg} / 1$ & $<0.010 \mathrm{mg} / 1$ & $<0.010 \mathrm{mg} / 1$ & $<0.010 \mathrm{mg} / \mathrm{l}$ & 1 & \\
\hline 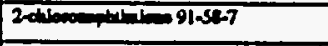 & $<0.010 \mathrm{mg} / 1$ & $<0.010 \mathrm{mg} / \mathrm{l}$ & $<0.010 \mathrm{mg} / 1$ & $<0.010 \mathrm{mg} / \mathrm{l}$ & 1 & $=$ \\
\hline 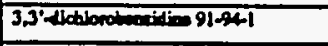 & $<0.020 \mathrm{mg} / \mathrm{l}$ & $<0.020 \mathrm{mg} / \mathrm{l}$ & $<0.020 \mathrm{mg} / \mathrm{l}$ & $<0.020 \mathrm{mg} / 1$ & 1 & \\
\hline 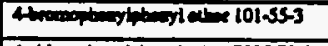 & $<0.010 \mathrm{mg} / \mathrm{l}$ & $<0.010 \mathrm{mg} / \mathrm{l}$ & $<0.010 \mathrm{mg} / \mathrm{l}$ & $<0.010 \mathrm{mg} / \mathrm{h}$ & 1 & \\
\hline 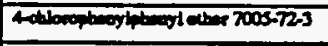 & $<0.010 \mathrm{mg} / 1$ & $<0.010 \mathrm{mg} / 1$ & $<0.010 \mathrm{mg} / \mathrm{l}$ & $<0.010 \mathrm{mg} / \mathrm{l}$ & 1 & \\
\hline 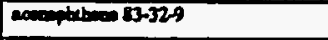 & $<0.010 \mathrm{mg} / \mathrm{l}$ & $<0.010 \mathrm{mg} / 1$ & $<0.010 \mathrm{mg} / \mathrm{l}$ & $<0.010 \mathrm{mg} / \mathrm{l}$ & 1 & \\
\hline 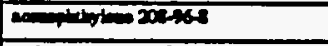 & $<0.010 \mathrm{mg} / 1$ & $<0.010 \mathrm{mg} / 1$ & $<0.010 \mathrm{mg} / \mathrm{l}$ & $<0.010 \mathrm{mg} / \mathrm{l}$ & 1 & \\
\hline-120.12 .7 & $<0.010 \mathrm{mg} / \mathrm{l}$ & $<0.010 \mathrm{mg} / \mathrm{l}$ & $<0.010 \mathrm{mg} / \mathrm{l}$ & $<0.010 \mathrm{mg} / 1$ & 1 & : \\
\hline 202575 & $<0.050 \mathrm{mg} / \mathrm{l}$ & $<0.050 \mathrm{mg} / \mathrm{l}$ & $<0.050 \mathrm{mg} / \mathrm{l}$ & $<0.050 \mathrm{mg} / \mathrm{l}$ & 1 & \\
\hline 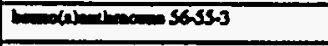 & $<0.010 \mathrm{mg} / \mathrm{l}$ & $<0.010 \mathrm{mg} / 1$ & $<0.010 \mathrm{mg} / \mathrm{l}$ & $<0.010 \mathrm{mg} / \mathrm{l}$ & 1 & \\
\hline 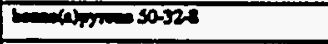 & $<0.010 \mathrm{mg} / \mathrm{l}$ & $<0.010 \mathrm{mg} / \mathrm{l}$ & $<0.010 \mathrm{mg} / \mathrm{l}$ & $<0.010 \mathrm{mg} / 1$ & 1 & \\
\hline 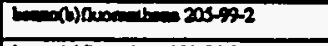 & $<0.010 \mathrm{mg} / \mathrm{l}$ & $<0.010 \mathrm{mg} / 1$ & $<0.010 \mathrm{mg} / \mathrm{l}$ & $<0.010 \mathrm{mg} / 1$ & 1 & \\
\hline 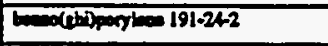 & $<0.010 \mathrm{mg} / 1$ & $<0.010 \mathrm{mg} / \mathrm{l}$ & $<0.010 \mathrm{mg} / \mathrm{l}$ & $<0.010 \mathrm{mg} / \mathrm{l}$ & 1 & \\
\hline 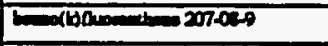 & $<0.010 \mathrm{mg} / 1$ & $<0.010 \mathrm{mg} / \mathrm{l}$ & $<0.010 \mathrm{mg} / \mathrm{l}$ & $<0.010 \mathrm{mg} / \mathrm{l}$ & 1 & \\
\hline 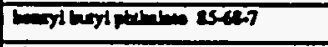 & $<0.010 \mathrm{mg} / \mathrm{l}$ & $<0.010 \mathrm{mg} / \mathrm{l}$ & $<0.010 \mathrm{mg} / \mathrm{l}$ & $<0.010 \mathrm{mg} / \mathrm{l}$ & 1 & \\
\hline 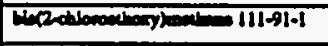 & $<0.010 \mathrm{mg} / \mathrm{l}$ & $<0.010 \mathrm{mg} / \mathrm{l}$ & $<0.010 \mathrm{mg} / 1$ & $<0.010 \mathrm{mg} / \mathrm{l}$ & 1 & \\
\hline 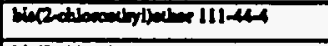 & $<0.010 \mathrm{mg} / \mathrm{l}$ & $<0.010 \mathrm{mg} / 1$ & $<0.010 \mathrm{mg} / 1$ & $<0.010 \mathrm{mg} / \mathrm{l}$ & 1 & \\
\hline 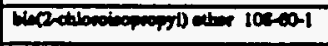 & $<0.010 \mathrm{mg} / \mathrm{l}$ & $<0.010 \mathrm{mg} / \mathrm{l}$ & $<0.010 \mathrm{mg} / \mathrm{l}$ & $<0.010 \mathrm{mg} / \mathrm{l}$ & 1 & \\
\hline 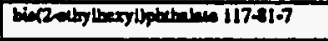 & $<0.010 \mathrm{mg} / 1$ & $<0.010 \mathrm{mg} / \mathrm{l}$ & $<0.010 \mathrm{mg} / 1$ & $<0.010 \mathrm{mg} / \mathrm{l}$ & 1 & \\
\hline 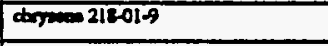 & $<0.010 \mathrm{mg} / \mathrm{l}$ & $<0.010 \mathrm{mg} / \mathrm{l}$ & $<0.010 \mathrm{mg} / 1$ & $<0.010 \mathrm{mg} / \mathrm{l}$ & 1 & \\
\hline 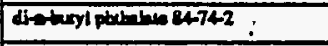 & $<0.010 \mathrm{mg} / 1$ & $<0.010 \mathrm{mg} / 1$ & $<0.010 \mathrm{mg} / \mathrm{l}$ & $<0.010 \mathrm{mg} / \mathrm{l}$ & 1 & \\
\hline 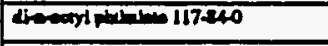 & $<0.010 \mathrm{mg} / \mathrm{l}$ & $<0.010 \mathrm{mg} / \mathrm{l}$ & $<0.010 \mathrm{mg} / 1$ & $<0.010 \mathrm{mg} / \mathrm{l}$ & 1 & \\
\hline 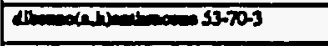 & $<0.010 \mathrm{mg} / \mathrm{l}$ & $<0.010 \mathrm{mg} / 1$ & $<0.010 \mathrm{mg} / \mathrm{l}$ & $<0.010 \mathrm{mg} / \mathrm{l}$ & 1 & \\
\hline 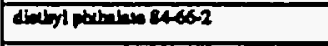 & $<0.010 \mathrm{mg} / \mathrm{l}$ & $<0.010 \mathrm{mg} / \mathrm{l}$ & $<0.010 \mathrm{mg} / \mathrm{l}$ & $<0.010 \mathrm{mg} / \mathrm{l}$ & 1 & \\
\hline 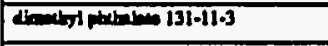 & $<0.010 \mathrm{mg} / \mathrm{l}$ & $<0.010 \mathrm{mg} / \mathrm{l}$ & $<0.010 \mathrm{mg} / \mathrm{l}$ & $<0.010$ mg/l & 1 & \\
\hline 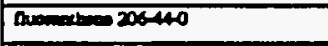 & $<0.010 \mathrm{mg} / 1$ & $<0.010 \mathrm{mg} / 1$ & $<0.010 \mathrm{mg} / 1$ & $<0.010 \mathrm{mg} / \mathrm{l}$ & 1 & \\
\hline Alowoses $26-75-7$ & $<0.010 \mathrm{mg} / \mathrm{l}$ & $<0.010 \mathrm{mg} / \mathrm{l}$ & $<0.010 \mathrm{mg} / 1$ & $<0.010 \mathrm{mg} / 1$ & 1 & \\
\hline 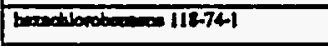 & $<0.010 \mathrm{mg} / \mathrm{l}$ & $<0.010 \mathrm{mg} / \mathrm{l}$ & $<0.010 \mathrm{mg} / \mathrm{l}$ & $<0.010 \mathrm{mg} / \mathrm{l}$ & 1 & \\
\hline 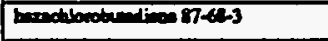 & $<0.010 \mathrm{mg} / \mathrm{l}$ & $<0.010 \mathrm{mg} / 1$ & $<0.010 \mathrm{mg} / 1$ & $<0.010 \mathrm{mg} / \mathrm{l}$ & 1 & \\
\hline 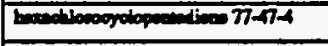 & $<0.010 \mathrm{mg} / \mathrm{l}$ & $<0.010 \mathrm{mg} / 1$ & $<0.010 \mathrm{mg} / 1$ & $<0.010 \mathrm{mg} / \mathrm{l}$ & 1 & \\
\hline 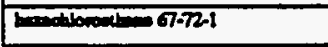 & $<0.010 \mathrm{mg} / \mathrm{l}$ & $<0.010 \mathrm{mg} / \mathrm{l}$ & $<0.010 \mathrm{mg} / \mathrm{l}$ & $<0.010 \mathrm{mg} / 1$ & 1 & \\
\hline 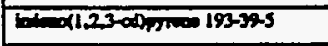 & $<0.010 \mathrm{mg} / \mathrm{l}$ & $<0.010 \mathrm{mg} / \mathrm{l}$ & $<0.010 \mathrm{mg} / \mathrm{l}$ & $<0.010 \mathrm{mg} / 1$ & 1 & \\
\hline mophoroen 72-59-1 & $<0.010 \mathrm{mq} / \mathrm{l}$ & $<0.010 \mathrm{mg} / 1$ & $<0.010 \mathrm{mg} / \mathrm{l}$ & $<0.010 \mathrm{mg} / \mathrm{l}$ & 1 & \\
\hline 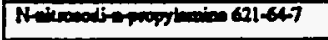 & $<0.010 \mathrm{mg} / 1$ & $<0.010 \mathrm{mg} / \mathrm{l}$ & $<0.010 \mathrm{mg} / \mathrm{l}$ & $<0.010 \mathrm{mg} / 1$ & 1 & \\
\hline 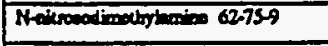 & $<0.010 \mathrm{mg} / \mathrm{l}$ & $<0.010 \mathrm{mg} / \mathrm{l}$ & $<0.010 \mathrm{mg} / \mathrm{l}$ & $<0.010 \mathrm{mg} / \mathrm{l}$ & 1 & \\
\hline 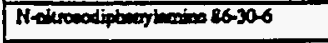 & $<0.010 \mathrm{mg} / \mathrm{l}$ & $<0.010 \mathrm{mg} / 1$ & $<0.010 \mathrm{mg} / 1$ & $<0.010 \mathrm{mg} / \mathrm{l}$ & 1 & \\
\hline Dpethaloos $91-20-3$ & $<0.010 \mathrm{mg} / 1$ & $<0.010 \mathrm{mg} / \mathrm{l}$ & $<0.010 \mathrm{mg} / \mathrm{l}$ & $<0.010 \mathrm{mg} / \mathrm{l}$ & 1 & \\
\hline nivolumenesings-3 & $<0.010 \mathrm{mg} / \mathrm{h}$ & $<0.010 \mathrm{mg} / 1$ & $<0.010 \mathrm{mg} / 1$ & $<0.010 \mathrm{mg} / 1$ & 1 & \\
\hline theras 85015 & $<0.010 \mathrm{mg} / \mathrm{l}$ & $<0.010 \mathrm{mg} / 1$ & $<0.010 \mathrm{mg} / \mathrm{l}$ & $<0.010 \mathrm{mg} / \mathrm{l}$ & 1 & \\
\hline $7 m 0=122000$ & $<0.010 \mathrm{mg} / \mathrm{l}$ & $<0.010 \mathrm{mg} / 1$ & $<0.010 \mathrm{mg} / 1$ & $<0.010 \mathrm{mg} / \mathrm{l}$ & 1 & \\
\hline 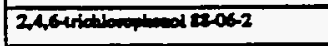 & $<0.030 \mathrm{mg} / 1$ & $<0.030 \mathrm{mg} / 1$ & $<0.030 \mathrm{mg} / \mathrm{l}$ & $<0.030 \mathrm{mg} / \mathrm{l}$ & 1 & \\
\hline 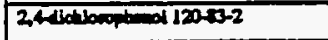 & $<0.030 \mathrm{mg} / \mathrm{l}$ & $<0.030 \mathrm{mg} / \mathrm{l}$ & $<0.030 \mathrm{mg} / \mathrm{l}$ & $<0.030 \mathrm{mg} / \mathrm{l}$ & 1 & \\
\hline 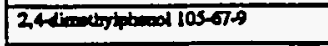 & $<0.030 \mathrm{mg} / \mathrm{l}$ & $<0.030 \mathrm{mg} / \mathrm{l}$ & $<0.030 \mathrm{mg} / \mathrm{l}$ & $<0.030 \mathrm{mg} / \mathrm{l}$ & 1 & \\
\hline 2,4diniteopheod SI-23-5 & $<0.050 \mathrm{mg} / \mathrm{l}$ & $<0.050 \mathrm{mg} / \mathrm{l}$ & $<0.050 \mathrm{mg} / \mathrm{l}$ & $<0.050 \mathrm{mg} / 1$ & $\sqrt{1}$ & \\
\hline
\end{tabular}


Outfall 19

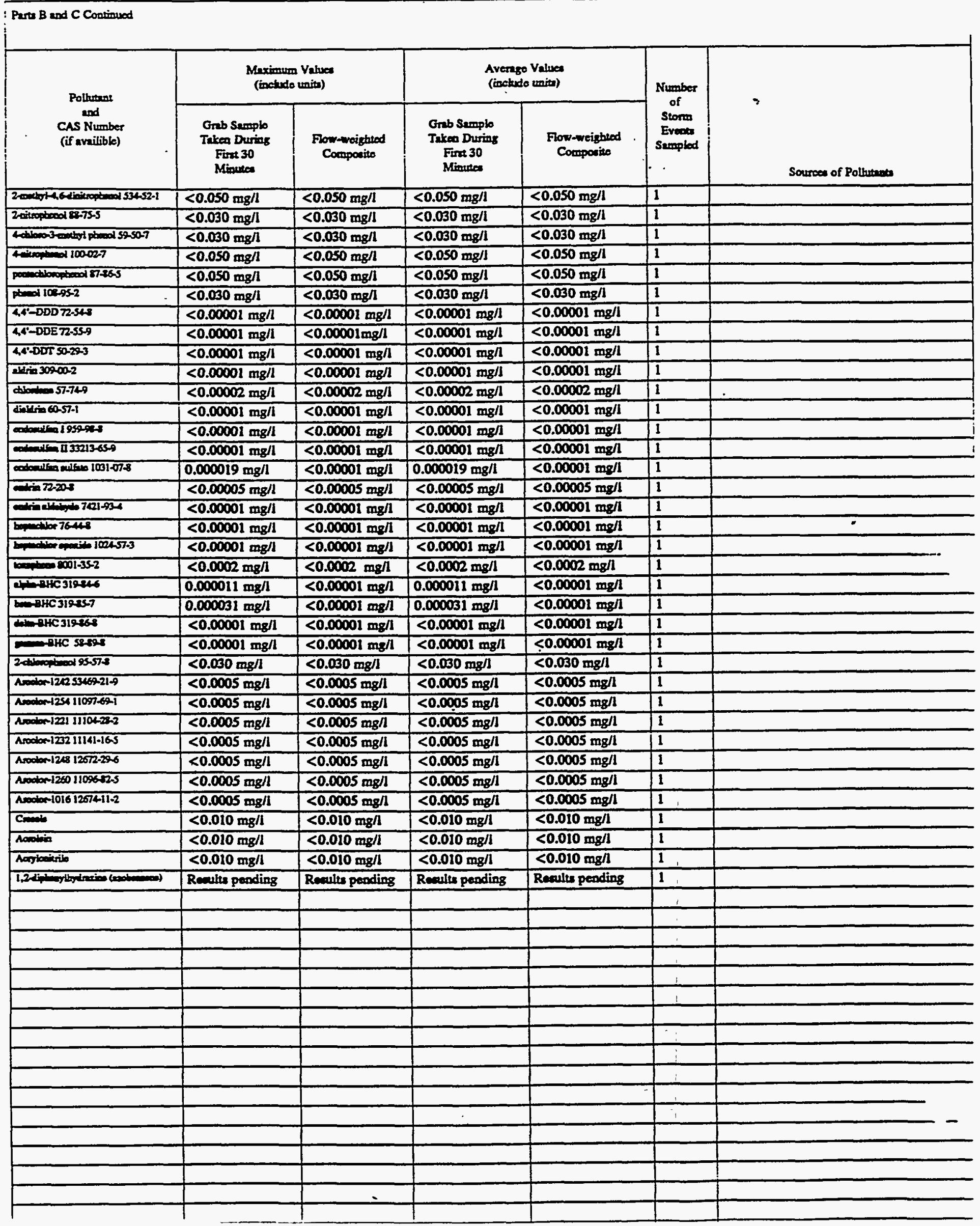




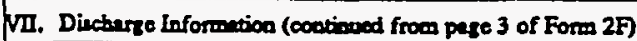

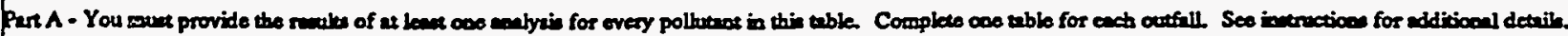

\begin{tabular}{|c|c|c|c|c|c|c|}
\hline \multirow{2}{*}{$\begin{array}{l}\text { Pollutene } \\
\text { and } \\
\text { CAs Number } \\
\text { (if availible) }\end{array}$} & \multicolumn{2}{|c|}{$\begin{array}{l}\text { Mrxicued Values } \\
\text { (ixclede unien) }\end{array}$} & \multicolumn{2}{|c|}{$\begin{array}{l}\text { Aversye Vabues } \\
\text { (ivelode unim) }\end{array}$} & \multirow{2}{*}{$\begin{array}{l}\text { Number } \\
\text { of } \\
\text { Storem } \\
\text { Eveots } \\
\text { Sempled }\end{array}$} & \multirow[b]{2}{*}{ Sources of Pollewer } \\
\hline & 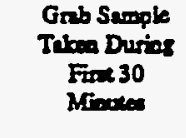 & $\begin{array}{c}\text { Flow-wrizbed } \\
\text { Compocits }\end{array}$ & 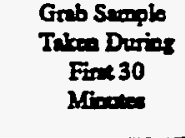 & $\begin{array}{l}\text { Fom-meighied } \\
\text { Compocies }\end{array}$ & & \\
\hline pil cod $\mathrm{G}=0$ & $<2 \mathrm{mg} / \mathrm{l}$ & & $<2 m g / 1$ & & 1 & \\
\hline Biolodial Oxyen Dened (BOD) & $<5 \mathrm{mg} / 1$ & $<5 \mathrm{mg} / \mathrm{l}$ & $<5 \mathrm{mg} / 1$ & $<5 \mathrm{mg} / \mathrm{l}$ & 1 & \\
\hline 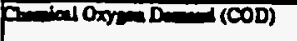 & $24 \mathrm{mg} / 1$ & $19 \mathrm{mg} / \mathrm{h}$ & $24 \mathrm{mg} / \mathrm{l}$ & $19 \mathrm{mg} / \mathrm{l}$ & 1 & \\
\hline Toul sapanded solith (ISS) & $9 \mathrm{men} / 1$ & $8.5 \mathrm{mg} / 1$ & $9 \mathrm{mg} / \mathrm{l}$ & $8.5 \mathrm{mg} / 1$ & 1 & \\
\hline 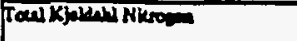 & $<0.2 \mathrm{mg} / \mathrm{t}$ & $0.6 \mathrm{mg} / 1$ & $<0.2 \mathrm{mg} / 1$ & $0.6 \mathrm{mg} / \mathrm{l}$ & 1 & \\
\hline 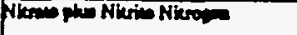 & $0.1 \mathrm{mg} / \mathrm{l}$ & $0.55 \mathrm{mg} / \mathrm{l}$ & $0.1 \mathrm{mg} / \mathrm{h}$ & $0.55 \mathrm{mg} / \mathrm{h}$ & 1 & \\
\hline Taul proptarin & $<0.1 \mathrm{mg} / \mathrm{l}$ & $0.12 \mathrm{mg} / 1$ & $<0.1 \mathrm{mg} / \mathrm{l}$ & $0.12 \mathrm{mg} / \mathrm{l}$ & 1 & \\
\hline PH & Minimen & Nodim 7.9 & Mining 7.9 & Mnimian 7.9 & & \\
\hline
\end{tabular}

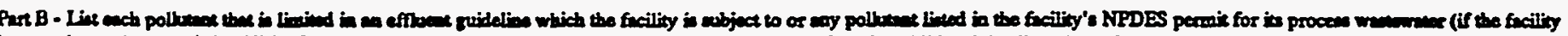

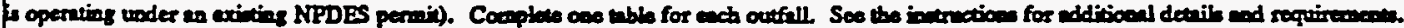

\begin{tabular}{|c|c|c|c|c|c|c|}
\hline \multirow{2}{*}{$\begin{array}{l}\text { Pollunese } \\
\text { and } \\
\text { CAs Numbur } \\
\text { (if maibibie) }\end{array}$} & \multicolumn{2}{|c|}{ 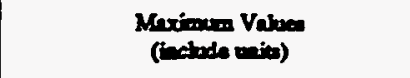 } & \multicolumn{2}{|c|}{$\begin{array}{l}\text { Averase Vikves } \\
\text { (iectede unim) }\end{array}$} & \multirow{2}{*}{$\begin{array}{c}\text { Number } \\
\text { of } \\
\text { Sionem } \\
\text { Evenes } \\
\text { Seropled }\end{array}$} & \multirow[b]{2}{*}{ Source of Pollew } \\
\hline & 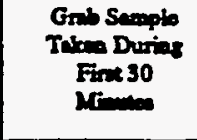 & $\begin{array}{l}\text { Fow-meighwod } \\
\text { Compociss }\end{array}$ & $\begin{array}{l}\text { Grab Semple } \\
\text { Tabu Dering } \\
\text { Fine } 30 \\
\text { Minos }\end{array}$ & $\begin{array}{l}\text { Flom-wrighted } \\
\text { Compotive }\end{array}$ & & \\
\hline tations, $740-360$ & $<0.04 \mathrm{mg} / 1$ & $<0.04 \mathrm{mg} / \mathrm{l}$ & $<0.04 \mathrm{mg} / \mathrm{l}$ & $<0.04 \mathrm{mg} / \mathrm{l}$ & 1 & \\
\hline $8 x$ & $<0.002 \mathrm{mg} / 1$ & & $<0.002 \mathrm{mgll}$ & & 1 & \\
\hline $702907-6$ & $<0.0002 \mathrm{mg} / 1$ & $<0.0002 \mathrm{mg} / \mathrm{h}$ & $<0.0002 \mathrm{mg} /{ }^{\circ}$ & $<0.0002 \mathrm{mg} / \mathrm{l}$ & 1 & \\
\hline Bnente & $0.002 \mathrm{mg} / \mathrm{l}$ & & $0.002 \mathrm{mg} / 1$ & & 1 & \\
\hline 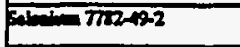 & $<0.002 \mathrm{mp} / \mathrm{l}$ & $<0.002 \mathrm{mg} / 1$ & $<0.002 \mathrm{mg} / 1$ & $<0.002 \mathrm{mg} / \mathrm{l}$ & 1 & \\
\hline solston & $<10 \mathrm{mg} / \mathrm{l}$ & $12 \mathrm{mg} / 1$ & $<10 \mathrm{mg} / \mathrm{l}$ & $12 \mathrm{mg} / 1$ & 1 & \\
\hline Surficaneses & $0.12 \mathrm{mg} / 1$ & $<0.05 \mathrm{~m} / 1$ & $0.12 \mathrm{mg} / \mathrm{h}$ & $<0.05 \mathrm{mg} / \mathrm{h}$ & 1 & \\
\hline 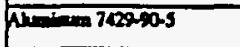 & $0.54 \mathrm{mg} / 1$ & $0.40 \mathrm{mg} / 1$ & $0.54 \mathrm{mg} / \mathrm{l}$ & $0.40 \mathrm{mg} / 1$ & 1 & \\
\hline $40-10740-36-2$ & $<0.04 \mathrm{mg} / \mathrm{l}$ & $<0.04 \mathrm{mg} / \mathrm{L}$ & $<0.04 \mathrm{mg} / 1$ & $<0.04 \mathrm{mg} / \mathrm{l}$ & 1 & \\
\hline 200000240008 & $0.010 \mathrm{mg} / 1$ & $0.049 \mathrm{mg} / \mathrm{l}$ & $0.010 \mathrm{mg} / 1$ & $0.049 \mathrm{mg} / 1$ & 1 & \\
\hline Forllien 740-1.-7 & $<0.0004 \mathrm{mg} / \mathrm{l}$ & $<0.0004 \mathrm{mg} / \mathrm{l}$ & $<0.0004 \mathrm{mg} / \mathrm{l}$ & $<0.0004 \mathrm{mg} / \mathrm{l}$ & 1 & \\
\hline $2 x=740-13-9$ & $<0.004 \mathrm{mg} / 1$ & $<0.004 \mathrm{mg} / \mathrm{l}$ & $<0.004 \mathrm{mg} / \mathrm{l}$ & $<0.004 \mathrm{mg} /$ & 1 & \\
\hline 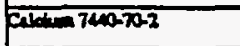 & $8.2 \mathrm{mg} / 1$ & $14.1 \mathrm{mg} /$ & $8.2 \mathrm{mg} / 1$ & $14.1 \mathrm{mg} / \mathrm{l}$ & 1 & \\
\hline 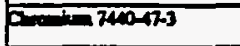 & $<0.006 \mathrm{mg} / 1$ & $<0.006 \mathrm{mg} / \mathrm{l}$ & $<0.006 \mathrm{mg} / \mathrm{l}$ & $<0.006 \mathrm{mg} h$ & $\sqrt{1}$ & \\
\hline Cotale 7410-424 & $<0.002 \mathrm{mg} / 1$ & $<0.002 \mathrm{mg} / \mathrm{l}$ & $<0.002 \mathrm{mg} / \mathrm{l}$ & $<0.002 \mathrm{mg} / \mathrm{l}$ & 1 & \\
\hline Conpor 7masos & $0.018 \mathrm{mg} / \mathrm{l}$ & $0.011 \mathrm{mg} / \mathrm{l}$ & $0.018 \mathrm{mg} / \mathrm{l}$ & $0.011 \mathrm{mg} / \mathrm{l}$ & 1 & \\
\hline 200143926 & $0.43 \mathrm{mg} / \mathrm{l}$ & $0.38 \mathrm{mg} / \mathrm{l}$ & $0.43 \mathrm{mg} / 1$ & $0.38 \mathrm{mg} / \mathrm{h}$ & 1 & \\
\hline mad 7092-21 & $<0.02 \mathrm{mg} / \mathrm{l}$ & $<0.02 \mathrm{mg} / \mathrm{l}$ & $<0.02 \mathrm{mg} / \mathrm{l}$ & $<0.02 \mathrm{mg} / \mathrm{l}$ & 1 & \\
\hline 145ing 7439-93-2 & $<0.02 \mathrm{mg} / 1$ & $<0.02 \mathrm{mg} / \mathrm{h}$ & $<0.02 \mathrm{mg} / \mathrm{l}$ & $<0.02 \mathrm{mg} / 1$ & 1 & \\
\hline Theming 709-9S4 & $0.61 \mathrm{mg} / 1$ & $1.47 \mathrm{mg} / \mathrm{I}$ & $0.61 \mathrm{mg} / \mathrm{l}$ & $1.47 \mathrm{mg} / \mathrm{l}$ & 1 & \\
\hline 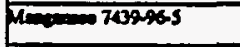 & $0.011 \mathrm{mg} / \mathrm{l}$ & $0.012 \mathrm{mg} / 1$ & $0.011 \mathrm{mg} / 1$ & $0.012 \mathrm{mg} / \mathrm{l}$ & 1 & \\
\hline 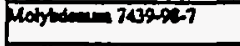 & $<0.006 \mathrm{mg} / \mathrm{l}$ & $<0.006 \mathrm{mg} / \mathrm{l}$ & $<0.006 \mathrm{mg} / \mathrm{l}$ & $<0.006 \mathrm{mg} / \mathrm{h}$ & 1 & \\
\hline 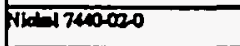 & $<0.008 \mathrm{mg} / 1$ & $<0.008 \mathrm{mg} / \mathrm{l}$ & $<0.008 \mathrm{mg} / 1$ & $<0.008 \mathrm{mg} / 1$ & 1 & \\
\hline 7400097 & $0.5 \mathrm{mg} / 1$ & $0.7 \mathrm{mg} / \mathrm{l}$ & $0.5 \mathrm{mg} / 1$ & $0.7 \mathrm{mg} / \mathrm{l}$ & 1 & \\
\hline (inden 740-20-4 & $<0.006 \mathrm{mg} / \mathrm{l}$ & $<0.006 \mathrm{mg} / \mathrm{l}$ & $<0.006 \mathrm{mg} / \mathrm{l}$ & $<0.006 \mathrm{mg} / \mathrm{l}$ & 1 & \\
\hline Podthen 7400225 & $0.49 \mathrm{mg} / \mathrm{l}$ & $0.93 \mathrm{mg} / 1$ & $0.49 \mathrm{mg} / 1$ & $0.93 \mathrm{mg} / \mathrm{h}$ & 1 & \\
\hline $75016-740-326$ & $<0.01 \mathrm{mg} / \mathrm{l}$ & $<0.01 \mathrm{mg} / 1$ & $<0.01 \mathrm{mg} / \mathrm{l}$ & $<0.01 \mathrm{mg} / 1$ & 1 & \\
\hline $20=240066$ & $0.05 \mathrm{mg} / 1$ & $0.05 \mathrm{mg} / \mathrm{l}$ & $0.05 \mathrm{mg} h$ & $0.05 \mathrm{mg} / \mathrm{l}$ & 1 & \\
\hline
\end{tabular}


Conduned from frote.

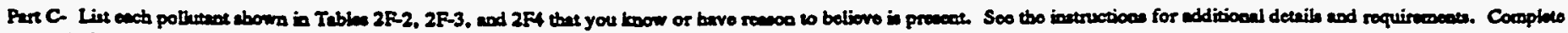
oso table for cach outfill.

\begin{tabular}{|c|c|c|c|c|c|c|}
\hline \multirow{2}{*}{$\begin{array}{l}\text { Polluwn } \\
\text { asd } \\
\text { CAS Niumber } \\
\text { (if aveusie) }\end{array}$} & \multicolumn{2}{|c|}{$\begin{array}{l}\text { Meximum Valuea } \\
\text { (inchedo unita) }\end{array}$} & \multicolumn{2}{|c|}{$\begin{array}{l}\text { Average Values } \\
\text { (inctudo units) }\end{array}$} & \multirow{2}{*}{$\begin{array}{c}\text { Number } \\
\text { of } \\
\text { Storm } \\
\text { Events } \\
\text { Semres }\end{array}$} & \multirow[b]{2}{*}{ Sources of Polbuting } \\
\hline & $\begin{array}{c}\text { Grab Siroplo } \\
\text { Taken Durias } \\
\text { Fink } 30 \\
\text { Mindues }\end{array}$ & $\begin{array}{l}\text { Flow-a eighed } \\
\text { Cunnocied }\end{array}$ & $\begin{array}{l}\text { Grab Sumple } \\
\text { Takea During } \\
\text { Find } 30 \\
\text { Minutes }\end{array}$ & $\begin{array}{l}\text { Flow-weightod } \\
\text { Componito }\end{array}$ & & \\
\hline 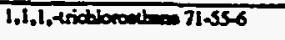 & $<0.010 \mathrm{mg} / 1$ & & $<0.010 \mathrm{mg} / 1$ & & 1 & \\
\hline 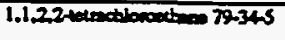 & $<0.010 \mathrm{mg} / \mathrm{l}$ & & $<0.010 \mathrm{mg} / 1$ & & 1 & \\
\hline 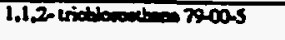 & $<0.010 \mathrm{mg} /$ & & $<0.010 \mathrm{mg} / 1$ & & 1 & \\
\hline 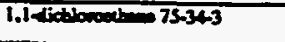 & $<0.010 \mathrm{mg} / 1$ & & $<0.010 \mathrm{mg} / 1$ & & 1 & \\
\hline 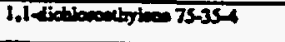 & $<0.010 \mathrm{mg} / 1$ & & $<0.010 \mathrm{mg} / 1$ & & 1 & \\
\hline 12 diablonoula os $10706-2$ & $<0.010 \mathrm{mg} / 1$ & & $<0.010 \mathrm{mg} / \mathrm{l}$ & & 1 & \\
\hline 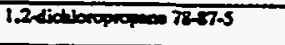 & $<0.010 \mathrm{mg} / 1$ & & $<0.010 \mathrm{mg} / 1$ & & 1 & \\
\hline 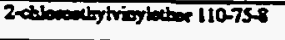 & $<0.010 \mathrm{mg} / 1$ & & $<0.010 \mathrm{mg} / \mathrm{l}$ & & 1 & . \\
\hline $71-13-2$ & $<0.010 \mathrm{mg} / 1$ & & $<0.010 \mathrm{mg} / 1$ & & 1 & \\
\hline bromolichborcenter 75-274 & $<0.010 \mathrm{mg} / 1$ & & $<0.010 \mathrm{mg} / 1$ & & 1 & \\
\hline becolocen $75-25-2$ & $<0.010 \mathrm{mg} / \mathrm{l}$ & & $<0.010 \mathrm{mg} / \mathrm{l}$ & & 1 & \\
\hline 7409 & $<0.010 \mathrm{mg} / \mathrm{l}$ & & $<0.010 \mathrm{mg} / 1$ & & 1 & \\
\hline 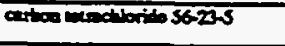 & $<0.010 \mathrm{mg} / \mathrm{l}$ & & $<0.010 \mathrm{mg} / 1$ & & 1 & \\
\hline 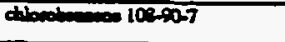 & $<0.010 \mathrm{mg} / \mathrm{l}$ & & $<0.010 \mathrm{mg} / 1$ & & 1 & \\
\hline chlowedberes $7500-3$ & $<0.010 \mathrm{mg} / \mathrm{l}$ & & $<0.010 \mathrm{mg} / \mathrm{l}$ & & 1 & \\
\hline dibosed $67-66-3$ & $<0.010 \mathrm{mg} / \mathrm{l}$ & & $<0.010 \mathrm{mg} / \mathrm{l}$ & & 1 & \\
\hline 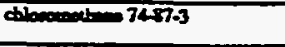 & $<0.010 \mathrm{mg} / 1$ & & $<0.010 \mathrm{~m} / \mathrm{l}$ & & 1 & - \\
\hline 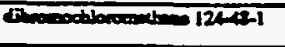 & $<0.010 \mathrm{mg} / \mathrm{l}$ & & $<0.010 \mathrm{mg} / \mathrm{l}$ & & 1 & \\
\hline 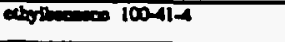 & $<0.010 \mathrm{mg} / 1$ & & $<0.010 \mathrm{mg} / \mathrm{l}$ & & 1 & \\
\hline $100-2 x-3$ & $<0.010 \mathrm{mg} / 1$ & & $<0.010 \mathrm{mg} / 1$ & & 1 & \\
\hline viegt ablarido 75014 & $<0.010 \mathrm{mg} / \mathrm{l}$ & & $<0.010 \mathrm{mg} / 1$ & & 1 & \\
\hline 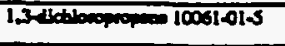 & $<0.910 \mathrm{mg} / \mathrm{l}$ & & $<0.010 \mathrm{mg} / \mathrm{l}$ & & 1 & \\
\hline 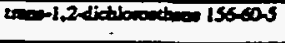 & $<0.010 \mathrm{mg} / 1$ & & $<0.010 \mathrm{mg} / 1$ & & 1 & \\
\hline ahte exivixy $126786-1$ & $13 \mathrm{pei} / \mathrm{l}$ & $5.0 \mathrm{pei} / \mathrm{l}$ & $13 \mathrm{pei} / \mathrm{l}$ & $5.0 \mathrm{gci} / \mathrm{l}$ & 1 & \\
\hline extiviny $12587-46-1$ & $5.7 \mathrm{pci} / 1$ & $9.8 \mathrm{poin}$ & $5.7 \mathrm{pcil}$ & $9.8 \mathrm{pei} / \mathrm{s}$ & 1 & \\
\hline Socal colifiom & $16 \mathrm{col} .1 \mathrm{~L}$ & $830 \mathrm{co} / .1 \mathrm{~L}$ & $16 \mathrm{co} / .1 \mathrm{~L}$ & $830 \mathrm{co} / .1 \mathrm{~L}$ & 1 & \\
\hline Thallians 740-220 & $<0.005 \mathrm{mg} / \mathrm{l}$ & $<0.005 \mathrm{mg} / 1$ & $<0.005 \mathrm{mg} / \mathrm{l}$ & $<0.005 \mathrm{mg} / \mathrm{l}$ & 1 & \\
\hline$U=-740-61-1$ & $<0,001 \mathrm{mg} / \mathrm{l}$ & $0.003 \mathrm{mg} / \mathrm{l}$ & $<0.001 \mathrm{mg} / 1$ & $0.003 \mathrm{mg} / \mathrm{l}$ & 1 & \\
\hline 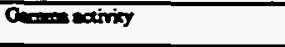 & 12 pein & 20 peill & $12 \mathrm{pei} / \mathrm{l}$ & 20 peill & 1 & \\
\hline & & & & & & - \\
\hline
\end{tabular}

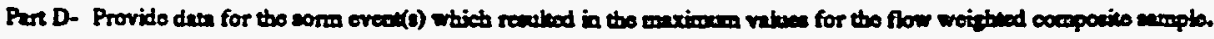

\begin{tabular}{|c|c|c|c|c|c|c|c|}
\hline $\begin{array}{l}1 . \\
\text { Date of } \\
\text { Storm } \\
\text { Ereas }\end{array}$ & $\begin{array}{l}2 . \\
\text { Durniog } \\
\text { of Storm } \\
\text { (in } \\
\text { misnees) }\end{array}$ & 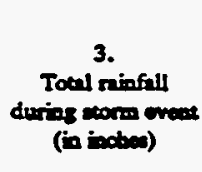 & 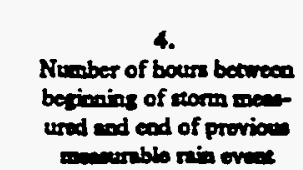 & 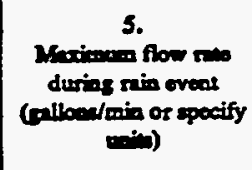 & $\begin{array}{l}6 . \\
\text { Total flow from } \\
\text { nix ovent } \\
\text { (ailoes or } \\
\text { pecify mix) }\end{array}$ & $\begin{array}{c}7 . \\
\text { Seacon } \\
\text { anplo was } \\
\text { taken }\end{array}$ & 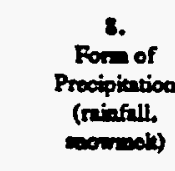 \\
\hline $8 / 26 / 91$ & 410 & 1.1 & 1602 & 213 & 42,376 & Summer & Rninfill \\
\hline
\end{tabular}

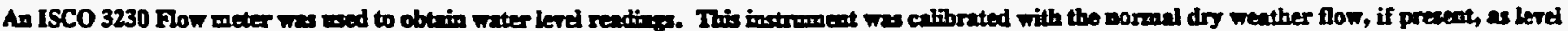

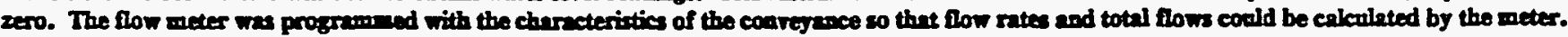


Outfall 20

Pars B ind C Continued

\begin{tabular}{|c|c|c|c|c|c|c|}
\hline \multirow{2}{*}{$\begin{array}{l}\text { Pollutual } \\
\text { ond } \\
\text { CAs Number } \\
\text { (if availiblo) }\end{array}$} & \multicolumn{2}{|c|}{ 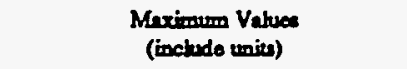 } & \multicolumn{2}{|c|}{$\begin{array}{l}\text { Avernge Valuea } \\
\text { (inabudo unitr) }\end{array}$} & \multirow{2}{*}{$\begin{array}{l}\text { Number } \\
\text { of } \\
\text { Storm } \\
\text { Evect } \\
\text { Sempled }\end{array}$} & \multirow[b]{2}{*}{ Sources of Polburases } \\
\hline & 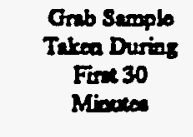 & $\begin{array}{l}\text { Flow-weighted } \\
\text { Componito }\end{array}$ & $\begin{array}{l}\text { Grab Samplo } \\
\text { Trean During } \\
\text { Fint } 30 \\
\text { Mincese }\end{array}$ & 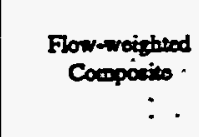 & & \\
\hline Bearion 7440-39-3 & $0.0079 \mathrm{mg} / \mathrm{l}$ & $0.0192 \mathrm{mg} / 1$ & $0.0079 \mathrm{mg} / 1$ & $0.0192 \mathrm{mg} / \mathrm{h}$ & 1 & \\
\hline 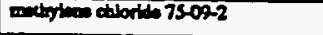 & $<0.010 \mathrm{mg} / \mathrm{l}$ & & $<0.010 \mathrm{mg} / \mathrm{l}$ & & 1 & \\
\hline mantibonowbere $127.18-4$ & $<0.010 \mathrm{mg} / \mathrm{l}$ & & $<0.010 \mathrm{mg} / \mathrm{l}$ & & 1 & \\
\hline 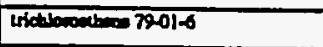 & $<0.010 \mathrm{mg} / \mathrm{l}$ & & $<0.010 \mathrm{mg} / \mathrm{l}$ & & 1 & \\
\hline xylem & $<0.010 \mathrm{mg} / 1$ & & $<0.010 \mathrm{mg} / \mathrm{l}$ & & 1 & \\
\hline 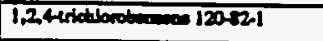 & $<0.010 \mathrm{mg} / \mathrm{l}$ & $<0.010 \mathrm{mg} / \mathrm{l}$ & $<0.010 \mathrm{mg} / \mathrm{l}$ & $<0.010 \mathrm{mg} / \mathrm{l}$ & 1 & \\
\hline 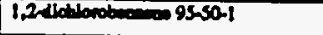 & $<0.010 \mathrm{mg} / \mathrm{l}$ & $<0.010 \mathrm{mg} / \mathrm{l}$ & $<0.010 \mathrm{mg} / \mathrm{l}$ & $<0.010 \mathrm{mg} / \mathrm{I}$ & 1 & \\
\hline 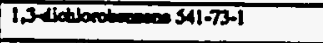 & $<0.010 \mathrm{mg} / 1$ & $<0.010 \mathrm{mg} / \mathrm{l}$ & $<0.010 \mathrm{mg} / \mathrm{l}$ & $<0.010 \mathrm{mg} / 1$ & 1 & \\
\hline 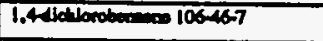 & $<0.010 \mathrm{mg} / \mathrm{l}$ & $<0.010 \mathrm{mg} / \mathrm{l}$ & $<0.010 \mathrm{mg} / \mathrm{l}$ & $<0.010 \mathrm{mg} / 1$ & 1 & \\
\hline 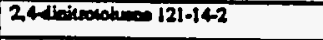 & $<0.010 \mathrm{mg} / 1$ & $<0.010 \mathrm{mg} / \mathrm{l}$ & $<0.010 \mathrm{mg} / \mathrm{l}$ & $<0.010 \mathrm{mg} / 1$ & 1 & \\
\hline 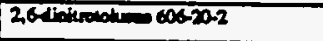 & $<0.010 \mathrm{mg} / \mathrm{l}$ & $<0.010 \mathrm{mg} / \mathrm{l}$ & $<0.010 \mathrm{mg} / \mathrm{l}$ & $<0.010 \mathrm{mg} / \mathrm{l}$ & 1 & \\
\hline 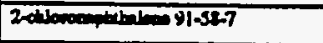 & $<0.010 \mathrm{mg} / \mathrm{l}$ & $<0.010 \mathrm{mg} / \mathrm{l}$ & $<0.010 \mathrm{mg} / \mathrm{l}$ & $<0.010 \mathrm{mg} / \mathrm{l}$ & 1 & \\
\hline 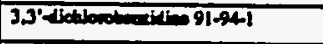 & $<0.010 \mathrm{mg} / \mathrm{l}$ & $<0.010 \mathrm{mg} / \mathrm{l}$ & $<0.010 \mathrm{mg} / \mathrm{l}$ & $<0.010 \mathrm{mg} / \mathrm{I}$ & 1 & \\
\hline 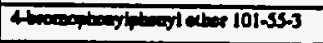 & $<0.010 \mathrm{mg} / \mathrm{l}$ & $<0.010 \mathrm{mg} / \mathrm{h}$ & $<0.010 \mathrm{mg} / 1$ & $<0.010 \mathrm{mg} / \mathrm{l}$ & 1 & \\
\hline 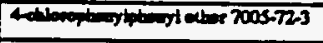 & $<0.010 \mathrm{mg} / \mathrm{l}$ & $<0.010 \mathrm{mg} / \mathrm{l}$ & $<0.010 \mathrm{mg} / 1$ & $<0.010 \mathrm{mg} / \mathrm{l}$ & 1 & \\
\hline 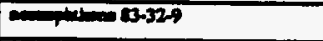 & $<0.010 \mathrm{mg} / \mathrm{l}$ & $<0.010 \mathrm{mg} / \mathrm{l}$ & $<0.010 \mathrm{mg} / \mathrm{l}$ & $<0.010 \mathrm{mg} /$ & 1 & \\
\hline 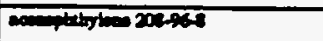 & $<0.010 \mathrm{mg} / \mathrm{l}$ & $<0.010 \mathrm{mg} / \mathrm{l}$ & $<0.010 \mathrm{mg} / \mathrm{l}$ & $<0.010 \mathrm{mg} / \mathrm{l}$ & 1 & \\
\hline$-120=12.7$ & $<0.010 \mathrm{mg} / \mathrm{l}$ & $<0.010 \mathrm{mg} / \mathrm{l}$ & $<0.010 \mathrm{mg} / 1$ & $<0.010 \mathrm{mg} / 1$ & 1 & $\because$ \\
\hline midises 925.5 & $<0.030 \mathrm{mg} / \mathrm{l}$ & $<0.030 \mathrm{mg} / \mathrm{l}$ & $<0.030 \mathrm{mg} / \mathrm{l}$ & $<0.030 \mathrm{mg} / \mathrm{l}$ & 1 & \\
\hline 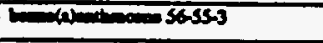 & $<0.010 \mathrm{mg} / \mathrm{h}$ & $<0.010 \mathrm{mg} / \mathrm{h}$ & $<0.010 \mathrm{mg} / 1$ & $<0.010 \mathrm{mg} / 1$ & 1 & \\
\hline 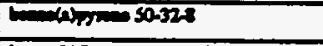 & $<0.010 \mathrm{mg} / \mathrm{l}$ & $<0.010 \mathrm{mg} / \mathrm{l}$ & $<0.010 \mathrm{mg} / 1$ & $<0.010 \mathrm{mg} / \mathrm{l}$ & 1 & \\
\hline $200-902$ & $<0.010 \mathrm{mg} / \mathrm{l}$ & $<0.010 \mathrm{mp} / 1$ & $<0.010 \mathrm{mg} / \mathrm{l}$ & $<0.010 \mathrm{mg} / 1$ & 1 & \\
\hline 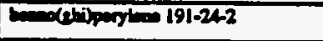 & $<0.010 \mathrm{mg} / \mathrm{l}$ & $<0.010 \mathrm{mg} / \mathrm{h}$ & $<0.010 \mathrm{mg} / \mathrm{h}$ & $<0.010 \mathrm{mg} / 1$ & 1 & \\
\hline 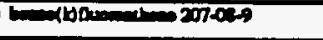 & $<0.010 \mathrm{mg} / \mathrm{l}$ & $<0.010 \mathrm{mg} / \mathrm{h}$ & $<0.010 \mathrm{mg} / \mathrm{l}$ & $<0.010 \mathrm{mg} / 1$ & 1 & \\
\hline 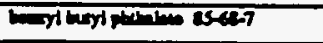 & $<0.010 \mathrm{mg} / \mathrm{l}$ & $<0.010 \mathrm{mg} / \mathrm{h}$ & $<0.010 \mathrm{mg} / \mathrm{l}^{\circ}$ & $<0.010 \mathrm{mg} / \mathrm{l}$ & 1 & \\
\hline 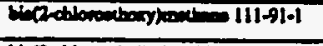 & $<0.010 \mathrm{mg} / \mathrm{l}$ & $<0.010 \mathrm{mg} / \mathrm{h}$ & $<0.010 \mathrm{mg} / 1$ & $<0.010 \mathrm{mg} / 1$ & 1 & \\
\hline 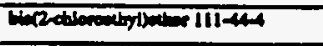 & $<0.010 \mathrm{mg} / \mathrm{l}$ & $<0.010 \mathrm{mg} / \mathrm{I}$ & $<0.010 \mathrm{mg} / \mathrm{l}$ & $<0.010 \mathrm{mg} / \mathrm{l}$ & 1 & \\
\hline 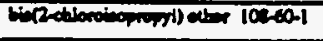 & $<0.010 \mathrm{mg} / \mathrm{l}$ & $<0.010 \mathrm{mg} / \mathrm{h}$ & $<0.010 \mathrm{mg} / 1$ & $<0.010 \mathrm{mg} / \mathrm{l}$ & 1 & \\
\hline 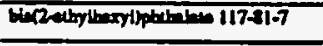 & $<0.010 \mathrm{mg} / \mathrm{l}$ & $0.010 \mathrm{mg} / \mathrm{l}$ & $<0.010 \mathrm{mg} / 1$ & $0.010 \mathrm{mg} / 1$ & 1 & \\
\hline 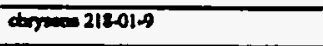 & $<0.010 \mathrm{mg} / \mathrm{l}$ & $<0.010 \mathrm{mg} / \mathrm{l}$ & $<0.010 \mathrm{mg} / \mathrm{l}$ & $<0.010 \mathrm{mg} / \mathrm{l}$ & 1 & \\
\hline 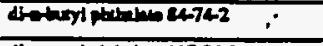 & $<0.010 \mathrm{mg} / \mathrm{l}$ & $<0.010 \mathrm{mg} / \mathrm{l}$ & $<0.010 \mathrm{mg} / 1$ & $<0.010 \mathrm{mg} / 1$ & 1 & \\
\hline 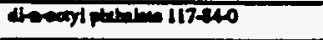 & $<0.010 \mathrm{mg} / \mathrm{l}$ & $0.025 \mathrm{mg} / 1$ & $<0.010 \mathrm{mg} / 1$ & $0.025 \mathrm{mg} / 1$ & 1 & \\
\hline 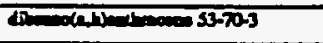 & $<0.010 \mathrm{mg} / \mathrm{l}$ & $<0.010 \mathrm{mg} / \mathrm{l}$ & $<0.010 \mathrm{mg} / \mathrm{l}$ & $<0.010 \mathrm{mg} / \mathrm{l}$ & 1 & \\
\hline 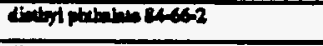 & $<0.010 \mathrm{mg} / \mathrm{l}$ & $<0.010 \mathrm{mg} / 1$ & $<0.010 \mathrm{mg} / 1$ & $<0.010 \mathrm{mg} / \mathrm{l}$ & 1 & \\
\hline 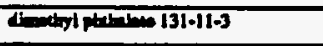 & $<0.010 \mathrm{mg} / \mathrm{l}$ & $<0.010 \mathrm{mg} / \mathrm{l}$ & $<0.010 \mathrm{mg} / 1$ & $<0.010 \mathrm{mg} / \mathrm{l}$ & 1 & \\
\hline$h=206-140$ & $<0.010 \mathrm{mg} / \mathrm{l}$ & $<0.010 \mathrm{mg} / \mathrm{l}$ & $<0.010 \mathrm{mg} / 1$ & $<0.010 \mathrm{mg} / \mathrm{l}$ & 1 & \\
\hline $00000-13-7$ & $<0.010 \mathrm{mg} / \mathrm{l}$ & $<0.010 \mathrm{mg} / 1$ & $<0.010 \mathrm{mg} / 1$ & $<0.010 \mathrm{mg} / \mathrm{l}$ & 1 & \\
\hline 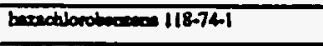 & $<0.010 \mathrm{mg} / \mathrm{l}$ & $<0.010 \mathrm{mg} / 1$ & $<0.010 \mathrm{mg} / \mathrm{l}$ & $<0.010 \mathrm{mg} / \mathrm{l}$ & 1 & \\
\hline 87.623 & $<0.010 \mathrm{mg} / \mathrm{l}$ & $<0.010 \mathrm{mg} / \mathrm{l}$ & $<0.010 \mathrm{mg} / 1$ & $<0.010 \mathrm{mg} / 1$ & 1 & \\
\hline 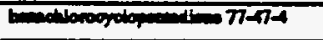 & $<0.010 \mathrm{mg} / 1$ & $<0.010 \mathrm{mg} / \mathrm{l}$ & $<0.010 \mathrm{mg} / \mathrm{l}$ & $<0.010 \mathrm{mg} / \mathrm{l}$ & 1 & \\
\hline 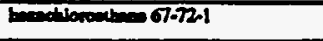 & $<0.010 \mathrm{mg} / 1$ & $<0.010 \mathrm{mg} / 1$ & $<0.010 \mathrm{mg} / 1$ & $<0.010 \mathrm{mg} / 1$ & 1 & \\
\hline 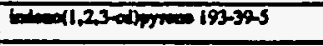 & $<0.010 \mathrm{mg} / \mathrm{l}$ & $<0.010 \mathrm{mg} / \mathrm{l}$ & $<0.010 \mathrm{mg} / \mathrm{l}$ & $<0.010 \mathrm{mg} / \mathrm{l}$ & 1 & \\
\hline 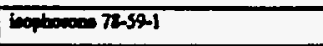 & $<0.010 \mathrm{mg} / \mathrm{l}$ & $<0.010 \mathrm{mg} / 1$ & $<0.010 \mathrm{mg} / 1$ & $<0.010 \mathrm{mg} / 1$ & 1 & \\
\hline 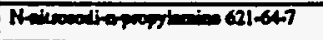 & $<0.010 \mathrm{mg} / \mathrm{l}$ & $<0.010 \mathrm{mg} / \mathrm{l}$ & $<0.010 \mathrm{mg} / \mathrm{l}$ & $<0.010 \mathrm{mg} / 1$ & 1 & \\
\hline 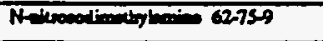 & $<0.010 \mathrm{mg} / \mathrm{l}$ & $<0.010 \mathrm{mg} / \mathrm{l}$ & $<0.010 \mathrm{mg} / \mathrm{l}$ & $<0.010 \mathrm{mg} / \mathrm{l}$ & 1 & \\
\hline 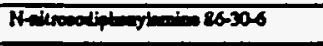 & $<0.010 \mathrm{mg} / \mathrm{l}$ & $<0.010 \mathrm{mg} / \mathrm{l}$ & $<0.010 \mathrm{mg} / 1$ & $<0.010 \mathrm{mg} / \mathrm{l}$ & 1 & \\
\hline minteren $91-20-3$ & $<0.010 \mathrm{mg} / \mathrm{l}$ & $<0.010 \mathrm{mg} / \mathrm{l}$ & $<0.010 \mathrm{mg} / \mathrm{l}$ & $<0.010 \mathrm{mg} / \mathrm{h}$ & 1 & \\
\hline 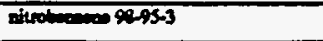 & $<0.010 \mathrm{mg} / \mathrm{l}$ & $<0.010 \mathrm{mg} / \mathrm{l}$ & $<0.010 \mathrm{mg} / \mathrm{l}$ & $<0.010 \mathrm{mg} / \mathrm{l}$ & 1 & \\
\hline N & $<0.010 \mathrm{mg} / \mathrm{l}$ & $<0.010 \mathrm{mg} / \mathrm{l}$ & $<0.010 \mathrm{mg} / 1$ & $<0.010 \mathrm{mg} / 1$ & 1 & \\
\hline 070001220000 & $<0.010 \mathrm{mg} / \mathrm{l}$ & $<0.010 \mathrm{mgll}$ & $<0.010 \mathrm{mg} / \mathrm{l}$ & $<0.010 \mathrm{mg} / \mathrm{l}$ & 1 & \\
\hline 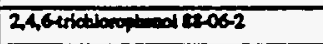 & $<0.030 \mathrm{mg} / 1$ & $<0.030 \mathrm{mg} / \mathrm{l}$ & $<0.030 \mathrm{mg} / \mathrm{l}$ & $<0.030 \mathrm{mg} / 1$ & 1 & \\
\hline 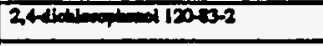 & $<0.030 \mathrm{mq} / \mathrm{l}$ & $<0.030 \mathrm{mg} / \mathrm{l}$ & $<0.030 \mathrm{mg} / 1$ & $<0.030 \mathrm{mg} / \mathrm{I}$ & 1 & \\
\hline 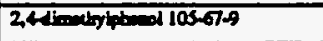 & $<0.030 \mathrm{mg} / \mathrm{l}$ & $<0.030 \mathrm{mg} / \mathrm{h}$ & $<0.030 \mathrm{mg} / \mathrm{l}$ & $<0.030 \mathrm{mg} / \mathrm{l}$ & 1 & \\
\hline 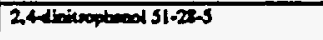 & $<0.030 \mathrm{mg} / \mathrm{l}$ & $<0.030 \mathrm{mg} / \mathrm{l}$ & $<0.030 \mathrm{mg} / \mathrm{l}$ & $<0.030 \mathrm{mg} / \mathrm{l}$ & $\sqrt{1}$ & \\
\hline
\end{tabular}


Oxtfin 20

Parta $B$ and C Cootinued

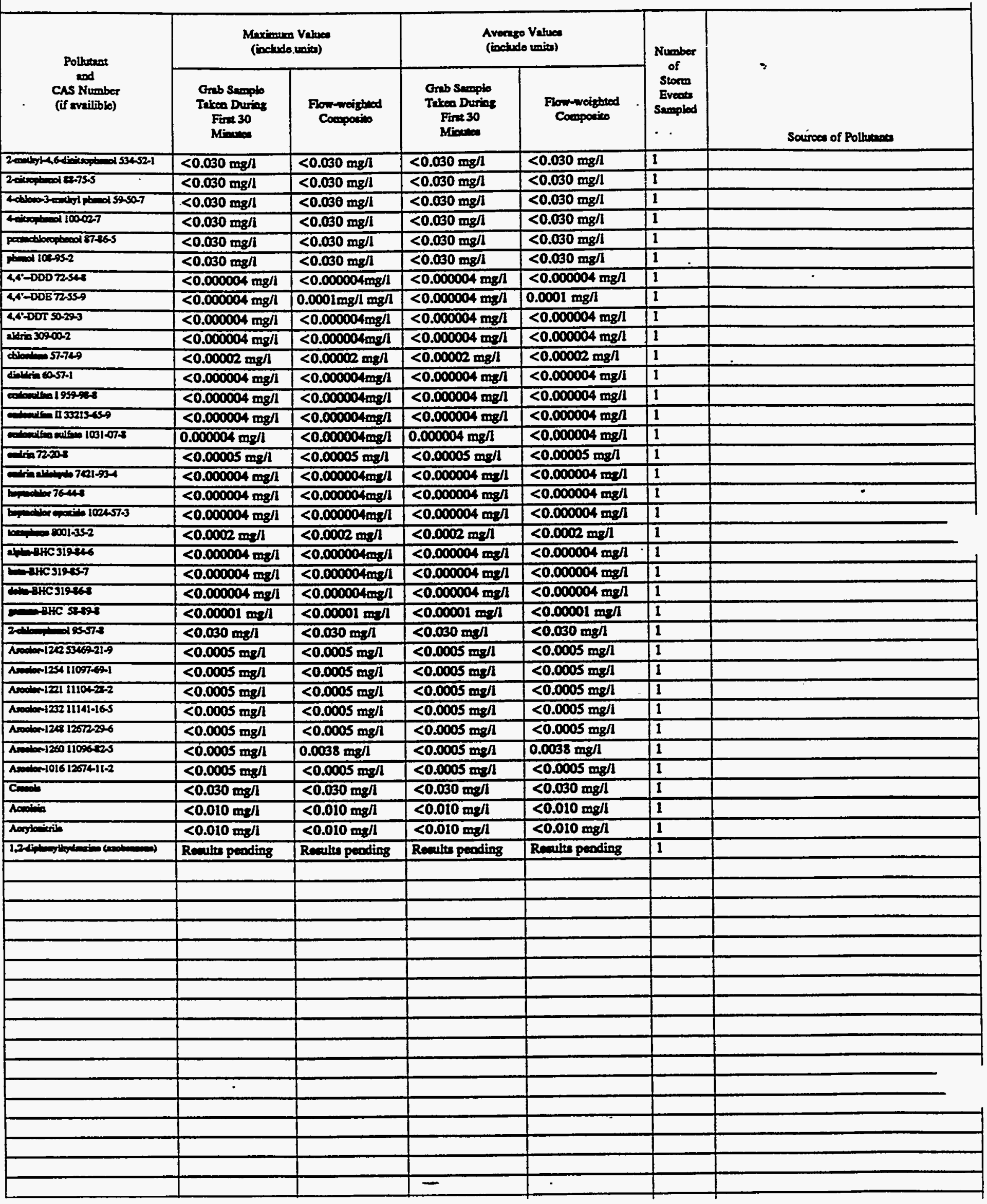


VII. Dixcharge Laformatioa (continued from page 3 of Form 2F)

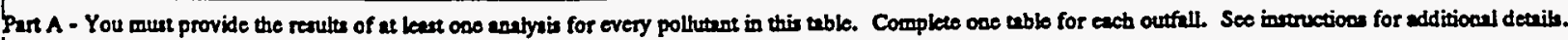

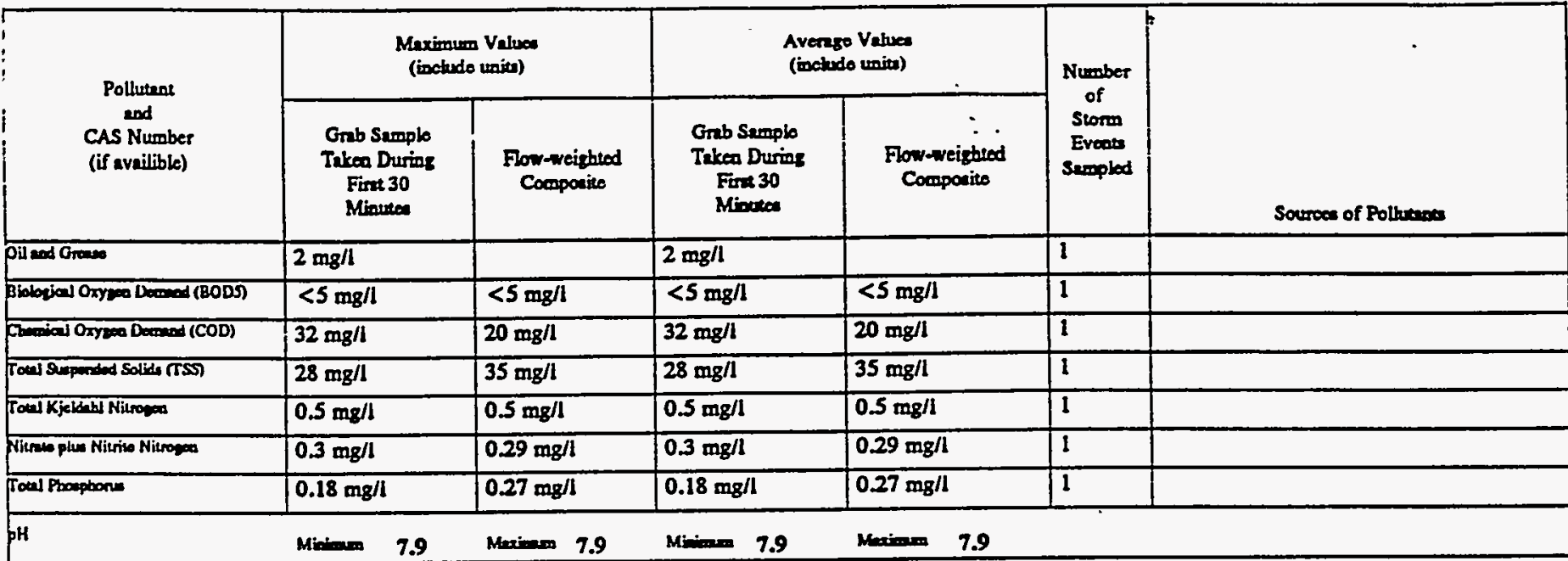

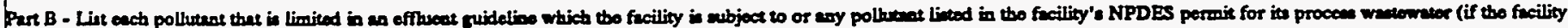

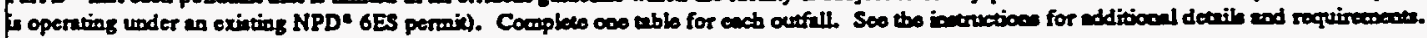

\begin{tabular}{|c|c|c|c|c|c|c|}
\hline \multirow{2}{*}{$\begin{array}{l}\text { Pollutant } \\
\text { and } \\
\text { CAs Number } \\
\text { (if aviliblo) }\end{array}$} & \multicolumn{2}{|c|}{$\begin{array}{l}\text { Maximem Valuce } \\
\text { (ivelude unitu) }\end{array}$} & \multicolumn{2}{|c|}{ 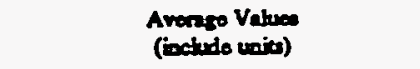 } & \multirow{2}{*}{$\begin{array}{l}\text { Number } \\
\text { of } \\
\text { Storm } \\
\text { Evecta } \\
\text { Sarepled }\end{array}$} & \multirow[b]{2}{*}{ Sources of Polluteres } \\
\hline & 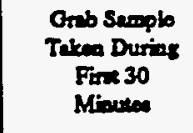 & $\begin{array}{l}\text { Flow-moipted } \\
\text { Compocico }\end{array}$ & $\begin{array}{l}\text { Onab Semplo } \\
\text { Trken Duries } \\
\text { Fin 30 } \\
\text { Minceses }\end{array}$ & $\begin{array}{c}\text { Flowneighed } \\
\text { Comporits }\end{array}$ & & \\
\hline trimosen 7400.360 & $<0.04 \mathrm{mg} / \mathrm{l}$ & $<0.04 \mathrm{mg} / \mathrm{l}$ & $<0.04 \mathrm{mg} / \mathrm{l}$ & $<0.04 \mathrm{mg} / \mathrm{l}$ & 1 & \\
\hline syonids & $<0.002 \mathrm{mg} / 1$ & & $<0.002 \mathrm{mg} / \mathrm{l}$ & & 1 & \\
\hline Mowany 7439-976 & $<0.0002 \mathrm{mg} / 1$ & $<0.0002 \mathrm{mg} / \mathrm{l}$ & $<0.0002 \mathrm{mg} / \mathrm{l}$ & $<0.0002 \mathrm{mg} / 1$ & 1 & \\
\hline pine & $0.004 \mathrm{mg} / \mathrm{l}$ & & $0.004 \mathrm{mg} / 1$ & & $I$ & \\
\hline saminom $7 \pi 2^{2}-122$ & $<0.002 \mathrm{mg} / \mathrm{l}$ & $<0.002 \mathrm{mg} / \mathrm{l}$ & $<0.002 \mathrm{mg} / \mathrm{l}$ & $<0.002 \mathrm{mg} / \mathrm{l}$ & 1 & \\
\hline puthen & $11 \mathrm{mg} / \mathrm{l}$ & $<10 \mathrm{mg} / \mathrm{l}$ & $11 \mathrm{mg} / 1$ & $<10 \mathrm{mg} / \mathrm{l}$ & 1 & \\
\hline Purtacteres & $0.15 \mathrm{mg} / \mathrm{l}$ & $0.08 \mathrm{mg} / \mathrm{h}$ & $0.15 \mathrm{mg} / \mathrm{I}$ & $0.08 \mathrm{mg} / \mathrm{l}$ & 1 & \\
\hline Thmines $742990-5$ & $0.87 \mathrm{mg} / \mathrm{l}$ & $0.83 \mathrm{mg} / \mathrm{l}$ & $0.87 \mathrm{mg} / \mathrm{l}$ & $0.83 \mathrm{mg} / \mathrm{l}$ & 1 & \\
\hline Tramo 740-32-2 & $<0.04 \mathrm{mg} / 1$ & $<0.04 \mathrm{mg} / \mathrm{l}$ & $<0.04 \mathrm{mg} / 1$ & $<0.04 \mathrm{mg} / \mathrm{l}$ & 1 & \\
\hline $3000074000-228$ & $0.022 \mathrm{mg} / \mathrm{l}$ & $0.020 \mathrm{mg} / 1$ & $0.022 \mathrm{mg} / \mathrm{l}$ & $0.020 \mathrm{mg} / \mathrm{l}$ & 1 & \\
\hline Boryllium 740a-1.7 & $<0.0004 \mathrm{mg} / 1$ & $<0.0004 \mathrm{mg} / 1$ & $<0.0004 \mathrm{mg} / \mathrm{l}$ & $<0.0004 \mathrm{mg} / \mathrm{h}$ & 1 & \\
\hline 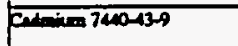 & $<0.004 \mathrm{mg} / \mathrm{l}$ & $<0.004 \mathrm{mg} / 1$ & $<0.004 \mathrm{mg} / \mathrm{l}$ & $<0.004 \mathrm{mg} / \mathrm{l}$ & 1 & \\
\hline Caloirm 7400-70-2 & $23.4 \mathrm{mg} / \mathrm{l}$ & $20.0 \mathrm{mg} / 1$ & $23.4 \mathrm{mg} / 1$ & $20.0 \mathrm{mg} / \mathrm{l}$ & 1 & \\
\hline Proming $740-17.3$ & $<0.006 \mathrm{mg} / \mathrm{l}$ & $<0.006 \mathrm{mg} / \mathrm{l}$ & $<0.006 \mathrm{mg} / 1$ & $<0.006 \mathrm{mg} / \mathrm{l}$ & 1 & \\
\hline Folate 740-184 & $<0.002 \mathrm{mg} / \mathrm{l}$ & $<0.002 \mathrm{mg} / 1$ & $<0.002 \mathrm{mg} / \mathrm{l}$ & $<0.002 \mathrm{mg} / \mathrm{h}$ & 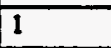 & \\
\hline carper 740-50s & $0.019 \mathrm{mg} / \mathrm{l}$ & $0.026 \mathrm{mg} / \mathrm{l}$ & $0.019 \mathrm{mg} / \mathrm{l}$ & $0.026 \mathrm{mg} / \mathrm{l}$ & 1 & \\
\hline 10007439696 & $1.07 \mathrm{mg} / \mathrm{l}$ & $2.10 \mathrm{mg} / 1$ & $1.07 \mathrm{mg} / 1$ & $2.10 \mathrm{mg} / 1$ & 1 & \\
\hline 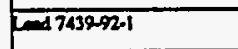 & $0.02 \mathrm{mg} / \mathrm{h}$ & $0.02 \mathrm{mg} / 1$ & $0.02 \mathrm{mg} / 1$ & $0.02 \mathrm{mg} / 1$ & 1 & \\
\hline 14timen 7439-93-2 & $<0.02 \mathrm{mg} / \mathrm{l}$ & $<0.02 \mathrm{mg} / \mathrm{l}$ & $<0.02 \mathrm{mg} / \mathrm{l}$ & $<0.02 \mathrm{mg} / 1$ & 1 & \\
\hline Megomakm 7039954 & $3.96 \mathrm{mg} / \mathrm{l}$ & $2.95 \mathrm{mg} / 1$ & $3.96 \mathrm{mg} / \mathrm{l}$ & $2.95 \mathrm{mg} / \mathrm{l}$ & 1 & \\
\hline Menasem $743296-5$ & $0.055 \mathrm{mg} / \mathrm{l}$ & $0.122 \mathrm{mg} / \mathrm{l}$ & $0.055 \mathrm{mg} / \mathrm{l}$ & $0.122 \mathrm{mg} / \mathrm{l}$ & 1 & \\
\hline 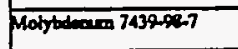 & $0.008 \mathrm{mg} / \mathrm{l}$ & $0.007 \mathrm{mg} / \mathrm{l}$ & $0.008 \mathrm{mg} / \mathrm{l}$ & $0.007 \mathrm{mg} / \mathrm{l}$ & 1 & \\
\hline$\sqrt{16401740020}$ & $<0.008 \mathrm{mg} / \mathrm{I}$ & $<0.008 \mathrm{mg} / \mathrm{l}$ & $<0.008 \mathrm{mg} / \mathrm{l}$ & $<0.008 \mathrm{mg} / \mathrm{l}$ & 1 & \\
\hline romiven 7400027 & $1.7 \mathrm{mg} / \mathrm{l}$ & $1.4 \mathrm{mg} / \mathrm{l}$ & $1.7 \mathrm{mg} / \mathrm{l}$ & $1.4 \mathrm{mg} / \mathrm{l}$ & 1 & \\
\hline simer 74ta-2a-4 & $<0.006 \mathrm{mg} / \mathrm{h}$ & $<0.006 \mathrm{mg} / 1$ & $<0.005 \mathrm{mg} / \mathrm{l}$ & $<0.006 \mathrm{mg} / \mathrm{l}$ & 1 & \\
\hline 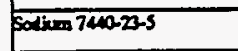 & $2.48 \mathrm{mg} / \mathrm{l}$ & $1.67 \mathrm{mg} / \mathrm{l}$ & $2.48 \mathrm{mg} / 1$ & $1.67 \mathrm{mg} / \mathrm{l}$ & 1 & \\
\hline 7 indm 740-32-6 & $0.01 \mathrm{mg} / \mathrm{l}$ & $0.02 \mathrm{mg} / \mathrm{l}$ & $0.01 \mathrm{mg} / 1$ & $0.02 \mathrm{mg} / \mathrm{l}$ & 1 & \\
\hline $2300740066-6$ & $0.12 \mathrm{mg} / 1$ & $0.19 \mathrm{mg} / \mathrm{l}$ & $0.12 \mathrm{mg} / \mathrm{I}$ & $0.19 \mathrm{mg} / 1$ & 1 & \\
\hline
\end{tabular}


Costinued froen froet.

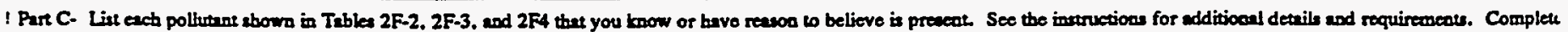
one table for act outfall.

\begin{tabular}{|c|c|c|c|c|c|c|}
\hline \multirow{2}{*}{$\begin{array}{l}\text { Polturans } \\
\text { and } \\
\text { CAs Number } \\
\text { (if availible) }\end{array}$} & \multicolumn{2}{|c|}{$\begin{array}{l}\text { Meximum Value } \\
\text { (inctude units) }\end{array}$} & \multicolumn{2}{|c|}{$\begin{array}{l}\text { Average Valuces } \\
\text { (inelude univ) }\end{array}$} & \multirow{2}{*}{$\begin{array}{l}\text { Number } \\
\text { of } \\
\text { Storm } \\
\text { Eveat } \\
\text { Sempled } \\
\text {. }\end{array}$} & \multirow{2}{*}{ Sources of Pollutintu } \\
\hline & $\begin{array}{c}\text { Grab Samplo } \\
\text { Takeo During } \\
\text { Fint } 30 \\
\text { Minutes }\end{array}$ & $\begin{array}{l}\text { Flow-weighted } \\
\text { Componite }\end{array}$ & $\begin{array}{c}\text { Grab Saroplo } \\
\text { Takeo Durios } \\
\text { First } 30 \\
\text { Minute }\end{array}$ & $\begin{array}{l}\text { Flow-weighted } \\
\text { Componite }\end{array}$ & & \\
\hline 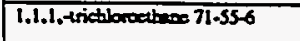 & $<0.010 \mathrm{mg} / 1$ & & $<0.010 \mathrm{mg} / \mathrm{l}$ & & 1 & \\
\hline 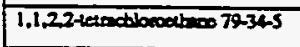 & $<0.010 \mathrm{mg} / 1$ & & $<0.010 \mathrm{mg} / 1$ & & 1 & \\
\hline 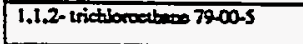 & $<0.010 \mathrm{mg} / \mathrm{l}$ & & $<0.010 \mathrm{mg} / 1$ & & 1 & \\
\hline 1.1 dichbrocthere 75-34-3 & $<0.010 \mathrm{mg} / \mathrm{l}$ & & $<0.010 \mathrm{mg} / \mathrm{l}$ & & 1 & \\
\hline 1.1-dictionoetbylace 75-35-4 & $<0.010 \mathrm{mg} / \mathrm{l}$ & & $<0.010 \mathrm{mg} / 1$ & & 1 & \\
\hline 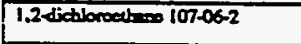 & $<0.010 \mathrm{mg} / \mathrm{l}$ & & $<0.010 \mathrm{mg} / 1$ & & 1 & \\
\hline 1.2-dictionopropacos 78-87.5 & $<0.010 \mathrm{mg} / 1$ & & $<0.010 \mathrm{mg} / \mathrm{l}$ & & 1 & \\
\hline 2-chlonoctbylvinglecter 110.75-8 & $<0.010 \mathrm{mg} / \mathrm{l}$ & & $<0.010 \mathrm{mg} / \mathrm{l}$ & & 1 & \\
\hline beoseces $71-43-2$ & $<0.010 \mathrm{mg} / \mathrm{l}$ & & $<0.010 \mathrm{mg} / \mathrm{l}$ & & 1 & \\
\hline broenodictibroceathere 75-274 & $<0.010 \mathrm{mg} / \mathrm{l}$ & & $<0.010 \mathrm{mg} / \mathrm{l}$ & & 1 & ; \\
\hline bremolom 75-25-2 & $<0.010 \mathrm{mg} / \mathrm{l}$ & & $<0.010 \mathrm{mg} / \mathrm{l}$ & & 1 & \\
\hline browomedtes 74-839 & $<0.010 \mathrm{mg} / \mathrm{l}$ & & $<0.010 \mathrm{mg} / 1$ & & 1 & \\
\hline cartion wetentibrith 56-23-5 & $<0.010 \mathrm{mg} / \mathrm{l}$ & & $<0.010 \mathrm{mg} / 1$ & & 1 & \\
\hline 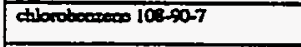 & $<0.010 \mathrm{mg} / !$ & & $<0.010 \mathrm{mg} / \mathrm{l}$ & & 1 & \\
\hline 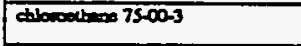 & $<0.010 \mathrm{mg} / \mathrm{l}$ & & $<0.010 \mathrm{mg} / 1$ & & 1 & \\
\hline ctibaform 67663 & $<0.010 \mathrm{mg} / \mathrm{l}$ & & $<0.010 \mathrm{mg} / 1$ & & 1 & $:$ \\
\hline chbocenthes 74-87-3 & $<0.010 \mathrm{mg} / \mathrm{l}$ & & $<0.010 \mathrm{mg} / \mathrm{l}$ & & 1 & \\
\hline 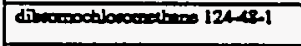 & $<0.010 \mathrm{mg} / 1$ & & $<0.010 \mathrm{mg} / \mathrm{l}$ & & 1 & \\
\hline etrylbecoses $100-414$ & $<0.010 \mathrm{mg} / 1$ & & $<0.010 \mathrm{mg} / \mathrm{l}$ & & 1 & \\
\hline whace 102-83 & $<0.010 \mathrm{mg} / \mathrm{l}$ & & $<0.010 \mathrm{mg} / \mathrm{l}$ & & 1 & \\
\hline visylaboride 75014 & $<0.010 \mathrm{mg} / 1$ & & $<0.010 \mathrm{mg} / \mathrm{l}$ & & 1 & \\
\hline 1.3-dictboropeopeces $10061-01-5$ & $<0.010 \mathrm{mg} / \mathrm{l}$ & & $<0.010 \mathrm{mg} / 1$ & - & 1 & \\
\hline 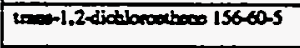 & $<0.010 \mathrm{mg} / \mathrm{l}$ & & $<0.010 \mathrm{mg} / 1$ & & 1 & \\
\hline aptemexivity $12557-16-1$ & 10 pcill & $4.5 \mathrm{pei} / \mathrm{l}$ & 10 pei/l & $4.5 \mathrm{pei} /$ & 1 & \\
\hline besenectivity 1258746.1 & 12 pcill & $5.6 \mathrm{pei} / 1$ & 12 peit & $5.6 \mathrm{pci} / \mathrm{l}$ & 1 & \\
\hline focel coliform & $8,000 \mathrm{col} .1 \mathrm{~L}$ & $<1 \mathrm{col} .1 \mathrm{~L}$ & $8,000 \mathrm{col} .1 \mathrm{~L}$ & $<1 \mathrm{co} / . \mathrm{LL}$ & 1 & \\
\hline Thalliem 740-220 & $<0.005 \mathrm{mg} / \mathrm{l}$ & $<0.005 \mathrm{mg} / \mathrm{l}$ & $<0.005 \mathrm{mg} / \mathrm{l}$ & $<0.005 \mathrm{mg} / \mathrm{l}$ & 1 & \\
\hline Uaxine 7440-61-1 & $0.002 \mathrm{mg} / \mathrm{l}$ & $0.002 \mathrm{mg} / 1$ & $0.002 \mathrm{mg} / \mathrm{l}$ & $0.002 \mathrm{mg} / 1$ & 1 & \\
\hline Genem saiving & -9.5 pei/l & 70 peill & -9.5 pei/l & $70 \mathrm{pci} / \mathrm{l}$ & 1 & \\
\hline
\end{tabular}

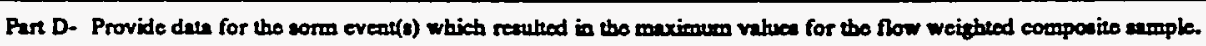

\begin{tabular}{|c|c|c|c|c|c|c|c|}
\hline $\begin{array}{c}1 . \\
\text { Dato of } \\
\text { Storm } \\
\text { Eveot }\end{array}$ & $\begin{array}{l}2 . \\
\text { Duration } \\
\text { of Storm } \\
\text { (in } \\
\text { minines) }\end{array}$ & $\begin{array}{l}3 . \\
\text { Tocal rimfall } \\
\text { during storm oveot } \\
\text { (in incten) }\end{array}$ & 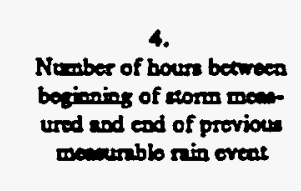 & 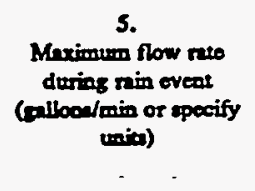 & $\begin{array}{l}6 . \\
\text { Tocal flow from } \\
\text { nin ovent } \\
\text { (gallow or } \\
\text { epeoify unio) }\end{array}$ & $\begin{array}{c}7 . \\
\text { Seasoan } \\
\text { acroplo was } \\
\text { taken }\end{array}$ & $\begin{array}{l}8 . \\
\text { Form of } \\
\text { Precipitation } \\
\text { (roinfall. } \\
\text { soommeln) }\end{array}$ \\
\hline $8 / 26 / 91$ & 410 & 1.2 & 1602 & 2,868 & 373,935 & Summer & Rainfall \\
\hline
\end{tabular}

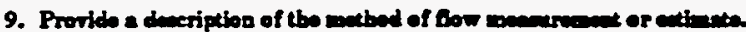

An ISCO 3230 Flow meter was used to obtain water level reading. This instrument was cabibrated with the normal dry weather flow, if present, as level zero. The flow meter was programed with the charecteritios of the codveyence so that flow rates and total flows conld be calcalated by the meter. 


\begin{tabular}{|c|c|c|c|c|c|c|}
\hline \multirow{2}{*}{$\begin{array}{l}\text { Pollurane } \\
\text { and } \\
\text { CAS Number } \\
\text { (if aveilible) }\end{array}$} & \multicolumn{2}{|c|}{$\begin{array}{l}\text { Maximum Valuce } \\
\text { (inchele units) }\end{array}$} & \multicolumn{2}{|c|}{$\begin{array}{l}\text { Avernge Values } \\
\text { (ibchodo unitu) }\end{array}$} & \multirow{2}{*}{$\begin{array}{c}\text { Number } \\
\text { of } \\
\text { Stoms } \\
\text { Everses } \\
\text { Semplad }\end{array}$} & \multirow[b]{2}{*}{ Sources of Pollutint } \\
\hline & $\begin{array}{c}\text { Grob Semplo } \\
\text { Taken During } \\
\text { First } 30 \\
\text { Minutes }\end{array}$ & $\begin{array}{l}\text { Fon-weighted } \\
\text { Componito }\end{array}$ & $\begin{array}{l}\text { Orab Samplo } \\
\text { Taken During } \\
\text { Fint } 30 \\
\text { Minuteas }\end{array}$ & $\begin{array}{l}\text { Flow-weighted } \\
\text { Coopposive }\end{array}$ & & \\
\hline Beringen 740-39-3 & $0.0300 \mathrm{mg} / \mathrm{l}$ & $0.0296 \mathrm{mg} / \mathrm{l}$ & $0.0300 \mathrm{mg} / 1$ & $0.0296 \mathrm{mg} / \mathrm{h}$ & 1 & \\
\hline 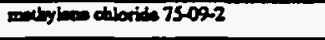 & $<0.010 \mathrm{mg} / \mathrm{l}$ & & $<0.010 \mathrm{mg} / 1$ & & 1 & \\
\hline 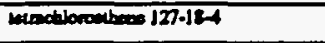 & $<0.010 \mathrm{mg} / \mathrm{l}$ & & $<0.010 \mathrm{mg} / \mathrm{l}$ & & 1 & \\
\hline 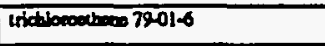 & $<0.010 \mathrm{mg} / 1$ & & $<0.010 \mathrm{mg} / \mathrm{l}$ & & 1 & \\
\hline Xylex & $<0.010 \mathrm{mg} / 1$ & & $<0.010 \mathrm{mg} / \mathrm{l}$ & & 1 & \\
\hline 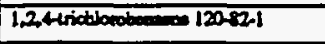 & $<0.010 \mathrm{~m} / \mathrm{d}$ & $<0.010 \mathrm{mg} / 1$ & $<0.010 \mathrm{mg} / \mathrm{l}$ & $<0.010 \mathrm{mg} / \mathrm{l}$ & 1 & \\
\hline 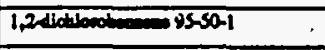 & $<0.010 \mathrm{mg} / \mathrm{l}$ & $<0.010 \mathrm{mg} / \mathrm{l}$ & $<0.010 \mathrm{mg} / \mathrm{l}$ & $<0.010 \mathrm{mg} / \mathrm{l}$ & 1 & \\
\hline 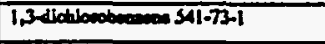 & $<0.010 \mathrm{mg} / 1$ & $<0.010 \mathrm{mg} / 1$ & $<0.010 \mathrm{mg} / \mathrm{l}$ & $<0.010 \mathrm{mg} / \mathrm{l}$ & 1 & \\
\hline 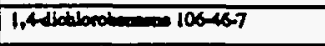 & $<0.010 \mathrm{mg} / 1$ & $<0.010 \mathrm{mg} / \mathrm{l}$ & $<0.010 \mathrm{mg} / 1$ & $<0.010 \mathrm{mg} / 1$ & 1 & \\
\hline 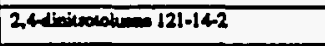 & $<0.010 \mathrm{mg} / \mathrm{l}$ & $<0.010 \mathrm{mg} / 1$ & $<0.010 \mathrm{mg} / \mathrm{l}$ & $<0.010 \mathrm{mg} / \mathrm{l}$ & 1 & \\
\hline 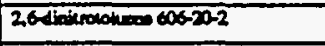 & $<0.010 \mathrm{mg} / 1$ & $<0.010 \mathrm{mg} / \mathrm{l}$ & $<0.010 \mathrm{mg} / \mathrm{l}$ & $<0.010 \mathrm{mg} / 1$ & 1 & \\
\hline 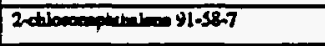 & $<0.010 \mathrm{mg} / 1$ & $<0.010 \mathrm{mg} / \mathrm{l}$ & $<0.010 \mathrm{mg} / \mathrm{l}$ & $<0.010 \mathrm{mg} / \mathrm{l}$ & 1 & \\
\hline 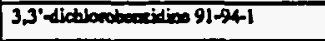 & $<0.010 \mathrm{mg} / \mathrm{l}$ & $<0.010 \mathrm{mg} / 1$ & $<0.010 \mathrm{mg} / \mathrm{l}$ & $<0.010 \mathrm{mg} / 1$ & 1 & \\
\hline 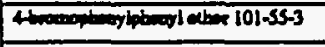 & $<0.010 \mathrm{mg} / \mathrm{l}$ & $<0.010 \mathrm{mg} / \mathrm{l}$ & $<0.010 \mathrm{mg} / \mathrm{l}$ & $<0.010 \mathrm{mg} / \mathrm{l}$ & 1 & \\
\hline 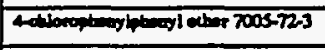 & $<0.010 \mathrm{mg} / \mathrm{l}$ & $<0.010 \mathrm{mg} / \mathrm{l}$ & $<0.010 \mathrm{mg} / \mathrm{l}$ & $<0.010 \mathrm{mg} / 1$ & 1 & \\
\hline 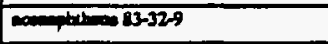 & $<0.010 \mathrm{mg} / 1$ & $<0.010 \mathrm{mg} / \mathrm{l}$ & $<0.010 \mathrm{mg} / \mathrm{l}$ & $<0.010 \mathrm{mg} / 1$ & 1 & \\
\hline 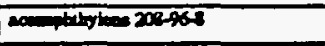 & $<0.010 \mathrm{mg} / \mathrm{l}$ & $<0.010 \mathrm{mg} / 1$ & $<0.010 \mathrm{mg} / \mathrm{l}$ & $<0.010 \mathrm{mg} / \mathrm{l}$ & 1 & \\
\hline $122-12.7$ & $<0.010 \mathrm{mg} / \mathrm{l}$ & $<0.010 \mathrm{mg} / \mathrm{l}$ & $<0.010 \mathrm{mg} /$ & $<0.010 \mathrm{mg} / \mathrm{l}$ & 1 & \\
\hline 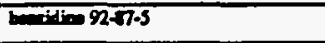 & $<0.030 \mathrm{mg} / 1$ & $<0.030 \mathrm{mg} / \mathrm{h}$ & $<0.030 \mathrm{mg} / \mathrm{l}$ & $<0.030 \mathrm{mg} / \mathrm{l}$ & 1 & $\vdots$ \\
\hline $56-55-3$ & $<0.010 \mathrm{mg} / 1$ & $<0.010 \mathrm{mg} / 1$ & $<0.010 \mathrm{mg} / \mathrm{l}$ & $<0.010 \mathrm{mg} / \mathrm{l}$ & 1 & \\
\hline 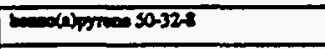 & $<0.010 \mathrm{mg} / \mathrm{l}$ & $<0.010 \mathrm{mg} / \mathrm{l}$ & $<0.010 \mathrm{mg} / \mathrm{l}$ & $<0.010 \mathrm{mg} / \mathrm{l}$ & 1 & \\
\hline 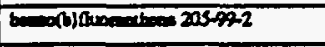 & $<0.010 \mathrm{mg} / \mathrm{l}$ & $<0.010 \mathrm{mg} n$ & $<0.010 \mathrm{mg} / \mathrm{l}$ & $<0.010 \mathrm{mg} / \mathrm{l}$ & 1 & \\
\hline 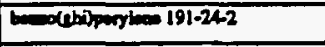 & $<0.010 \mathrm{mg} / \mathrm{l}$ & $<0.010 \mathrm{mg} / \mathrm{l}$ & $<0.010 \mathrm{mg} / \mathrm{l}$ & $<0.010 \mathrm{mg} / 1$ & 1 & \\
\hline 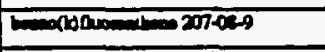 & $<0.010 \mathrm{mg} / \mathrm{l}$ & $<0.010 \mathrm{mg} / \mathrm{l}$ & $<0.010 \mathrm{mg} / 1$ & $<0.010 \mathrm{mg} / \mathrm{l}$ & 1 & \\
\hline 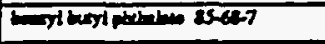 & $<0.010 \mathrm{mg} / 1$ & $<0.010 \mathrm{mg} / \mathrm{h}$ & $<0.010 \mathrm{mg} / 1$ & $<0.010 \mathrm{mg} / 1$ & 1 & \\
\hline 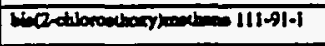 & $<0.010 \mathrm{mg} / \mathrm{l}$ & $<0.010 \mathrm{mg} / 1$ & $<0.010 \mathrm{mg} / 1$ & $<0.010 \mathrm{mg} / \mathrm{l}$ & 1 & \\
\hline 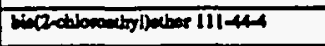 & $<0.010 \mathrm{mg} / \mathrm{l}$ & $<0.010 \mathrm{mg} / 1$ & $<0.010 \mathrm{mg} / \mathrm{l}$ & $<0.010 \mathrm{mg} / 1$ & 1 & \\
\hline 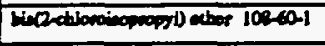 & $<0.010 \mathrm{mg} / \mathrm{l}$ & $<0.010 \mathrm{mg} / 1$ & $<0.010 \mathrm{mg} / 1$ & $<0.010 \mathrm{mg} / 1$ & 1 & \\
\hline 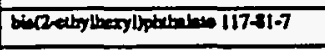 & $<0.010 \mathrm{mg} / \mathrm{l}$ & $0.015 \mathrm{mg} / \mathrm{l}$ & $<0.010 \mathrm{mg} /$ & $0.015 \mathrm{mg} / 1$ & 1 & \\
\hline drymed 21801A & $<0.010 \mathrm{mg} / \mathrm{l}$ & $<0.010 \mathrm{mg} / \mathrm{l}$ & $<0.010 \mathrm{mg} / \mathrm{l}$ & $<0.010 \mathrm{mg} / \mathrm{l}$ & 1 & \\
\hline 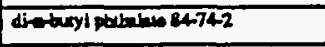 & $<0.010 \mathrm{mg} / \mathrm{l}$ & $<0.010 \mathrm{mg} / \mathrm{l}$ & $<0.010 \mathrm{mg} / \mathrm{l}$ & $<0.010 \mathrm{mg} / 1$ & 1 & \\
\hline 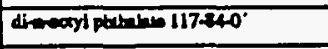 & $<0.010 \mathrm{mg} / \mathrm{l}$ & $0.033 \mathrm{mg} / 1$ & $<0.010 \mathrm{mg} / \mathrm{h}$ & $0.033 \mathrm{mg} / \mathrm{l}$ & 1 & \\
\hline 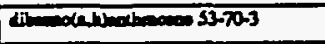 & $<0.010 \mathrm{mg} / 1$ & $<0.010 \mathrm{mg} / \mathrm{h}$ & $<0.010 \mathrm{mg} / 1$ & $<0.010 \mathrm{mg} / \mathrm{l}$ & 1 & \\
\hline 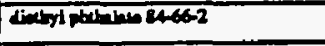 & $<0.010 \mathrm{mg} / \mathrm{l}$ & $<0.010 \mathrm{mg} / \mathrm{l}$ & $<0.010 \mathrm{mg} / \mathrm{l}$ & $<0.010 \mathrm{mg} / 1$ & 1 & \\
\hline 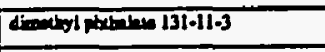 & $<0.010 \mathrm{mg} / 1$ & $<0.010 \mathrm{mg} / \mathrm{l}$ & $<0.010 \mathrm{mg} / \mathrm{l}$ & $<0.010 \mathrm{mg} / 1$ & 1 & \\
\hline 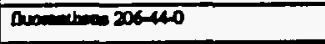 & $<0.010 \mathrm{mg} / \mathrm{l}$ & $<0.010 \mathrm{mg} / 1$ & $<0.010 \mathrm{mg} / \mathrm{l}$ & $<0.010 \mathrm{mg} / \mathrm{l}$ & 1 & \\
\hline 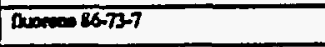 & $<0.010 \mathrm{mg} / \mathrm{l}$ & $<0.010 \mathrm{mg} / \mathrm{l}$ & $<0.010 \mathrm{mg} / 1$ & $<0.010 \mathrm{mg} / \mathrm{l}$ & 1 & \\
\hline 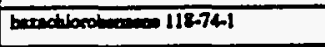 & $<0.010 \mathrm{mg} / \mathrm{l}$ & $<0.010 \mathrm{mg} / 1$ & $<0.010 \mathrm{mg} / 1$ & $<0.010 \mathrm{mg} / 1$ & 1 & \\
\hline 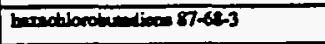 & $<0.010 \mathrm{mg} / \mathrm{l}$ & $<0.010 \mathrm{mg} / \mathrm{l}$ & $<0.010 \mathrm{mg} / \mathrm{l}$ & $<0.010 \mathrm{mg} / 1$ & 1 & \\
\hline 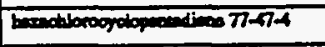 & $<0.010 \mathrm{mg} / \mathrm{l}$ & $<0.010 \mathrm{mg} / 1$ & $<0.010 \mathrm{mg} / 1$ & $<0.010 \mathrm{mg} / \mathrm{l}$ & 1 & \\
\hline 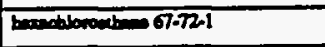 & $<0.010 \mathrm{mg} / 1$ & $<0.010 \mathrm{mg} / 1$ & $<0.010 \mathrm{mg} / \mathrm{l}$ & $<0.010 \mathrm{mg} / \mathrm{l}$ & 1 & \\
\hline 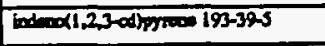 & $<0.010 \mathrm{mg} / 1$ & $<0.010 \mathrm{mg} / 1$ & $<0.010 \mathrm{mg} / \mathrm{l}$ & $<0.010 \mathrm{mg} / \mathrm{l}$ & 1 & \\
\hline insolos $\pi 2-59-1$ & $<0.010 \mathrm{mg} / 1$ & $<0.010 \mathrm{mg} / 1$ & $<0.010 \mathrm{mg} / 1$ & $<0.010 \mathrm{mg} / 1$ & 1 & \\
\hline 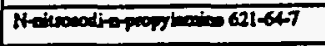 & $<0.010 \mathrm{mg} / 1$ & $<0.010 \mathrm{mg} / \mathrm{l}$ & $<0.010 \mathrm{mg} / 1$ & $<0.010 \mathrm{mg} / 1$ & 1 & \\
\hline 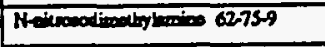 & $<0.010 \mathrm{mg} / \mathrm{l}$ & $<0.010 \mathrm{mg} / \mathrm{l}$ & $<0.010 \mathrm{mg} / \mathrm{l}$ & $<0.010 \mathrm{mg} / 1$ & 1 & \\
\hline 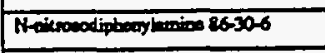 & $<0.010 \mathrm{mg} / 1$ & $<0.010 \mathrm{mg} / \mathrm{l}$ & $<0.010 \mathrm{mg} / \mathrm{l}$ & $<0.010 \mathrm{mg} / 1$ & 1 & \\
\hline 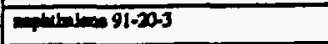 & $<0.010 \mathrm{mg} / \mathrm{l}$ & $<0.010 \mathrm{mg} / \mathrm{h}$ & $<0.010 \mathrm{mg} / 1$ & $<0.010 \mathrm{mg} / \mathrm{h}$ & 1 & \\
\hline 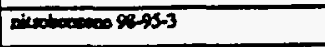 & $<0.010 \mathrm{mg} / 1$ & $<0.010 \mathrm{mg} / \mathrm{h}$ & $<0.010 \mathrm{mg} / \mathrm{h}$ & $<0.010 \mathrm{mg} / \mathrm{l}$ & 1 & \\
\hline 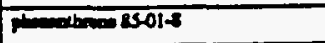 & $<0.010 \mathrm{mg} / \mathrm{l}$ & $<0.010 \mathrm{mg} / \mathrm{l}$ & $<0.010 \mathrm{mg} / 1$ & $<0.010 \mathrm{mg} / \mathrm{l}$ & 1 & \\
\hline Prone 120000 & $<0.010 \mathrm{mg} / \mathrm{l}$ & $<0.010 \mathrm{mg} / \mathrm{l}$ & $<0.010 \mathrm{mg} / \mathrm{l}$ & $<0.010 \mathrm{mg} / \mathrm{l}$ & 1 & \\
\hline 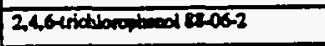 & $<0.030 \mathrm{mg} / 1$ & $<0.030 \mathrm{mg} / \mathrm{l}$ & $<0.030 \mathrm{mg} / \mathrm{l}$ & $<0.030 \mathrm{mg} / \mathrm{l}$ & 1 & \\
\hline 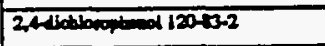 & $<0.030 \mathrm{mg} / \mathrm{l}$ & $<0.030 \mathrm{mg} / \mathrm{l}$ & $<0.030 \mathrm{mg} / 1$ & $<0.030 \mathrm{mg} / \mathrm{l}$ & 1 & \\
\hline 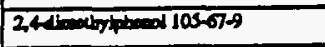 & $<0.030 \mathrm{mg} / 1$ & $<0.030 \mathrm{mg} / \mathrm{l}$ & $<0.030 \mathrm{mg} / \mathrm{l}$ & $<0.030 \mathrm{mg} / 1$ & 1 & \\
\hline 24 dritopotaed S1-225 & $<0.030 \mathrm{mg} / \mathrm{l}$ & $<0.030 \mathrm{mg} / \mathrm{l}$ & $<0.030 \mathrm{mg} / 1$ & $<0.030 \mathrm{mg} / \mathrm{l}$ & 1 & \\
\hline
\end{tabular}


Outfoll 21

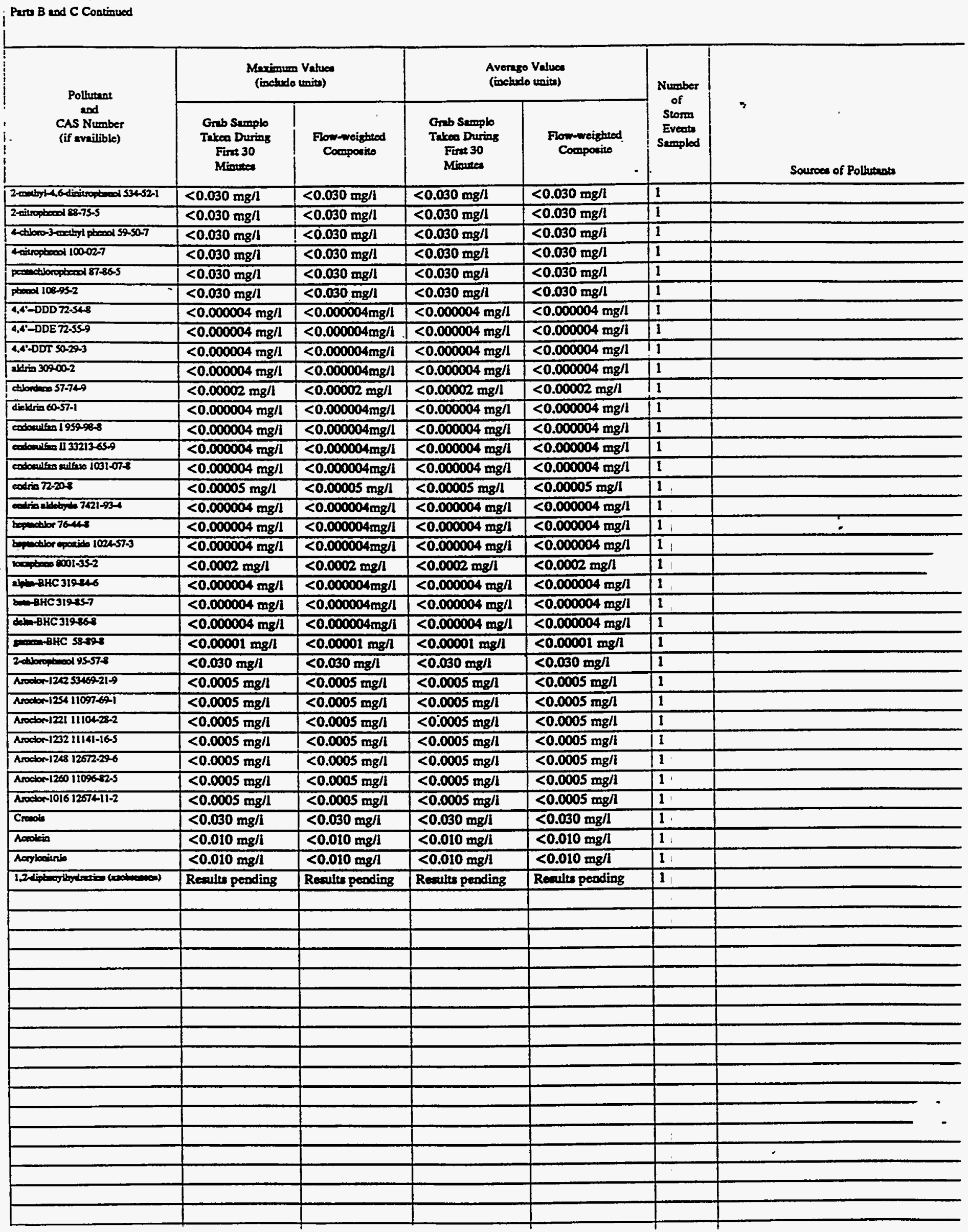


VII. Diacharge Lnformation (continued from page 3 of Form 2A

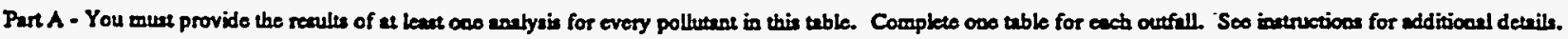

\begin{tabular}{|c|c|c|c|c|c|c|}
\hline \multirow{2}{*}{$\begin{array}{l}\text { Pollutent } \\
\text { and } \\
\text { CAs Number } \\
\text { (if eveilible) }\end{array}$} & \multicolumn{2}{|c|}{$\begin{array}{l}\text { Maximum Valuce } \\
\text { (ibebudo unius) }\end{array}$} & \multicolumn{2}{|c|}{$\begin{array}{l}\text { Average Vatuce } \\
\text { (inchude units) }\end{array}$} & \multirow{2}{*}{$\begin{array}{l}\text { Number } \\
\text { of } \\
\text { Storm } \\
\text { Eveous } \\
\text { Sempled }\end{array}$} & \multirow{2}{*}{ 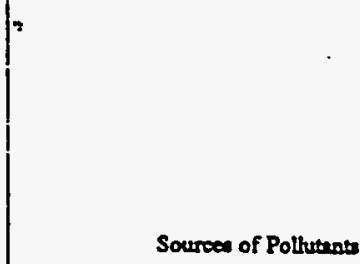 } \\
\hline & $\begin{array}{l}\text { Grb Semplo } \\
\text { Takeo During } \\
\text { Firut } 30 \\
\text { Minuteo }\end{array}$ & $\begin{array}{l}\text { Fow-woighed } \\
\text { Compotito }\end{array}$ & $\begin{array}{c}\text { Grbb Samplo } \\
\text { Takea During } \\
\text { Fird } 30 \\
\text { Minutee }\end{array}$ & $\begin{array}{l}\text { Flow-weighiod } \\
\text { Componits }\end{array}$ & & \\
\hline oil and Gremeces & $<2 \mathrm{mg} / \mathrm{l}$ & & $<2 \mathrm{mg} / 1$ & & 1 & \\
\hline Blopial Oxysen Drenead (BOD) & $5.67 \mathrm{mg} / 1$ & $<5 \mathrm{mg} / \mathrm{l}$ & $5.67 \mathrm{mg} / 1$ & $<5 \mathrm{mg} / \mathrm{l}$ & 1 & \\
\hline Cremial Oxy & $5.5 \mathrm{mg} / \mathrm{l}$ & $<5 \mathrm{mg} / \mathrm{l}$ & $5.5 \mathrm{mg} / \mathrm{l}$ & $<5 \mathrm{mg} / \mathrm{l}$ & 1 & \\
\hline Toet anpanded Solded (TSS) & $12 \mathrm{mg} / 1$ & $6 \mathrm{mg} / \mathrm{l}$ & $12 \mathrm{mg} / \mathrm{l}$ & $6 \mathrm{mg} / \mathrm{l}$ & 1 & \\
\hline 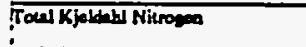 & $0.3 \mathrm{mg} / \mathrm{l}$ & $0.3 \mathrm{mg} / \mathrm{l}$ & $0.3 \mathrm{mg} / 1$ & $0.3 \mathrm{mg} / \mathrm{l}$ & 1 & \\
\hline Nitmen phen Nitrin Nitrosed & $0.94 \mathrm{mg} / \mathrm{l}$ & $2.021 \mathrm{mg} / 1$ & $0.94 \mathrm{mg} / \mathrm{l}$ & $2.021 \mathrm{mg} / 1$ & 1 & \\
\hline Toes Phouphorim & $0.11 \mathrm{mg} / \mathrm{l}$ & $<0.1 \mathrm{mg} / 1$ & $0.11 \mathrm{mg} / 1$ & $<0.1 \mathrm{mg} / 1$ & 1 & \\
\hline $\mathrm{pH}$ & Minimen 78 & Maviman 78 & Min:mum 78 & Mxims 7.8 & & \\
\hline
\end{tabular}

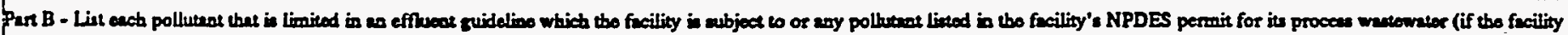

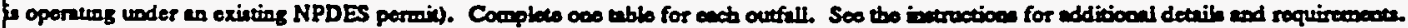

\begin{tabular}{|c|c|c|c|c|c|c|}
\hline \multirow{2}{*}{$\begin{array}{l}\text { Polthuane } \\
\text { aod } \\
\text { CAs Number } \\
\text { (if availible) }\end{array}$} & \multicolumn{2}{|c|}{$\begin{array}{l}\text { Marimerom Vahues } \\
\text { (ivatudo units) }\end{array}$} & \multicolumn{2}{|c|}{ 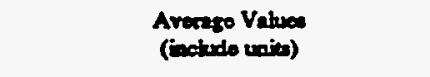 } & \multirow{2}{*}{$\begin{array}{l}\text { Number } \\
\text { of } \\
\text { Storm } \\
\text { Eveote } \\
\text { Sumpled }\end{array}$} & \multirow[b]{2}{*}{ Source of Polthrande } \\
\hline & 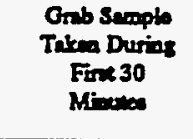 & $\begin{array}{l}\text { Flow-woigted } \\
\text { Componits }\end{array}$ & 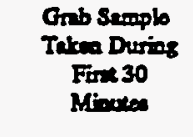 & $\begin{array}{l}\text { Flow-woighed } \\
\text { Compotite }\end{array}$ & & \\
\hline 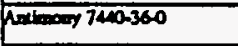 & $<0.04 \mathrm{mg} / 1$ & $<0.04 \mathrm{mg} / \mathrm{l}$ & $<0.04 \mathrm{mg} / \mathrm{l}$ & $<0.04 \mathrm{mg} / \mathrm{l}$ & 1 & \\
\hline$x=16$ & $<0.002 \mathrm{mg} / \mathrm{l}$ & & $<0.002 \mathrm{mg} / 1$ & & 1 & \\
\hline Meraey 7639-976 & $0.011 \mathrm{mg} / 1$ & $0.0013 \mathrm{mg} / 1$ & $0.011 \mathrm{mg} / 1$ & $0.0013 \mathrm{mg} / 1$ & 1 & \\
\hline proode & $<0.001 \mathrm{mg} / 1$ & & $<0.001 \mathrm{mg} / \mathrm{l}$ & & 1 & \\
\hline selming $7 \pi x^{2}-19-2$ & $<0.002 \mathrm{mg} / \mathrm{h}$ & $<0.002 \mathrm{mg} / \mathrm{l}$ & $<0.002 \mathrm{mg} / \mathrm{l}$ & $<0.002 \mathrm{mg} / \mathrm{l}$ & 1 & \\
\hline sulmen & $<10 \mathrm{mg} / 1$ & $<10 \mathrm{mg} / \mathrm{l}$ & $<10 \mathrm{mg} / \mathrm{l}$ & $<10 \mathrm{mg} / \mathrm{l}$ & 1 & \\
\hline Purfoconine & $<0.05 \mathrm{mg} / 1$ & $<0.05 \mathrm{mg} / \mathrm{l}$ & $<0.05 \mathrm{mg} / \mathrm{l}$ & $<0.05 \mathrm{mg} / \mathrm{l}$ & 1 & \\
\hline Aheminam 7622-00-5 & $1.01 \mathrm{mg} / 1$ & $0.91 \mathrm{mg} / \mathrm{l}$ & $1.01 \mathrm{mg} / \mathrm{l}$ & $0.91 \mathrm{mg} / 1$ & 1 & \\
\hline $4 s=0.6740-38-2$ & $<0.4 \mathrm{mg} / \mathrm{l}$ & $<0.4 \mathrm{mg} / \mathrm{l}$ & $<0.4 \mathrm{mg} / 1$ & $<0.4 \mathrm{mg} / \mathrm{l}$ & 1 & \\
\hline $30007400-28$ & $0.030 \mathrm{mg} / \mathrm{l}$ & $0.046 \mathrm{mg} / 1$ & $0.030 \mathrm{mg} / \mathrm{l}$ & $0.046 \mathrm{mg} / \mathrm{l}$ & 1 & \\
\hline Berillimen 740a-11.7 & $<0.0004 \mathrm{mg} / \mathrm{l}$ & $<0.0004 \mathrm{mg} / \mathrm{l}$ & $<0.0004 \mathrm{mg} / \mathrm{l}$ & $<0.0004 \mathrm{mg} / \mathrm{l}$ & 1 & \\
\hline Cancium 740-1399 & $<0.004 \mathrm{mg} / \mathrm{l}$ & $<0.004 \mathrm{mg} / \mathrm{l}$ & $<0.004 \mathrm{mg} /$ & $<0.004 \mathrm{mg} / 1$ & 1 & \\
\hline Clockm 740-70-2 & $16.8 \mathrm{mg} / \mathrm{l}$ & $24.0 \mathrm{mg} / 1$ & $16.8 \mathrm{mg} / \mathrm{l}$ & $24.0 \mathrm{mg} / \mathrm{h}$ & 1 & \\
\hline 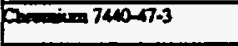 & $0.006 \mathrm{mg} / \mathrm{l}$ & $<0.006 \mathrm{mg} / \mathrm{l}$ & $0.006 \mathrm{mg} / \mathrm{l}$ & $<0.006 \mathrm{mg} / \mathrm{l}$ & 1 & \\
\hline Cobath 740-12-4 & $<0.002 \mathrm{mg} / \mathrm{l}$ & $<0.002 \mathrm{mg} / \mathrm{l}$ & $<0.002 \mathrm{mg} / \mathrm{l}$ & $<0.002 \mathrm{mg} / \mathrm{l}$ & 1 & \\
\hline 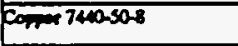 & $0.020 \mathrm{mg} / \mathrm{l}$ & $0.013 \mathrm{mg} / 1$ & $0.020 \mathrm{mg} / \mathrm{I}$ & $0.013 \mathrm{mg} / \mathrm{h}$ & 1 & \\
\hline$\sqrt{0007639-696}$ & $1.57 \mathrm{mg} / 1$ & $1.13 \mathrm{mg} / 1$ & $1.57 \mathrm{mg} / \mathrm{l}$ & $1.13 \mathrm{mg} / 1$ & 1 & \\
\hline fod 7439202-1 & $<0.02 \mathrm{mg} / \mathrm{l}$ & $<0.02 \mathrm{mg} / 1$ & $<0.02 \mathrm{mg} / \mathrm{l}$ & $<0.02 \mathrm{mg} / \mathrm{l}$ & 1 & \\
\hline 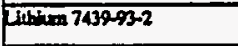 & $<0.02 \mathrm{mg} / \mathrm{l}$ & $<0.02 \mathrm{mg} / \mathrm{l}$ & $<0.02 \mathrm{mg} / 1$ & $<0.02 \mathrm{mg} / \mathrm{l}$ & 1 & \\
\hline Meposiken $7439-95-4$ & $3.82 \mathrm{mg} / 1$ & $6.34 \mathrm{mg} / \mathrm{l}$ & $3.82 \mathrm{mg} / 1$ & $6.34 \mathrm{mg} / 1$ & 1 & \\
\hline 743996.5 & $0.038 \mathrm{mg} / \mathrm{l}$ & $0.030 \mathrm{mg} / \mathrm{l}$ & $0.038 \mathrm{mg} / \mathrm{l}$ & $0.030 \mathrm{mg} / \mathrm{l}$ & 1 & \\
\hline 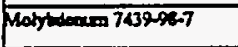 & $<0.006 \mathrm{mg} / \mathrm{l}$ & $<0.006 \mathrm{mg} / \mathrm{l}$ & $<0.006 \mathrm{mg} / \mathrm{l}$ & $<0.006 \mathrm{mg} / \mathrm{l}$ & 1 & \\
\hline$\sqrt{160177400000}$ & $<0.008 \mathrm{mg} / \mathrm{l}$ & $<0.008 \mathrm{mg} / \mathrm{l}$ & $<0.008 \mathrm{mg} / \mathrm{l}$ & $<0.008 \mathrm{mg} / \mathrm{l}$ & 1 & \\
\hline Polendikn 74009-7 & $1.2 \mathrm{mg} / \mathrm{l}$ & $1.4 \mathrm{mg} / \mathrm{l}$ & $1.2 \mathrm{mg} / \mathrm{l}$ & $1.4 \mathrm{mg} / \mathrm{l}$ & 1 & \\
\hline 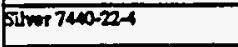 & $<0.006 \mathrm{mg} / \mathrm{l}$ & $<0.006 \mathrm{mg} / \mathrm{l}$ & $<0.006 \mathrm{mg} / \mathrm{l}$ & $<0.006 \mathrm{mg} / \mathrm{l}$ & 1 & \\
\hline sodingon 7400-22-5 & $2.91 \mathrm{mg} / 1$ & $4.66 \mathrm{mg} / 1$ & $2.91 \mathrm{mg} / \mathrm{l}$ & $4.66 \mathrm{mg} / \mathrm{l}$ & 1 & \\
\hline Thenden 740-32-6 & $0.02 \mathrm{mg} / \mathrm{l}$ & $0.01 \mathrm{mg} / \mathrm{l}$ & $0.02 \mathrm{mg} / \mathrm{l}$ & $0.01 \mathrm{mg} / 1$ & 1 & \\
\hline $2 i 007440-666$ & $0.02 \mathrm{mg} / \mathrm{l}$ & $0.02 \mathrm{mg} / \mathrm{l}$ & $0.02 \mathrm{mg} / 1$ & $0.02 \mathrm{mg} / \mathrm{l}$ & 1 & \\
\hline
\end{tabular}


Costinoed from tront.

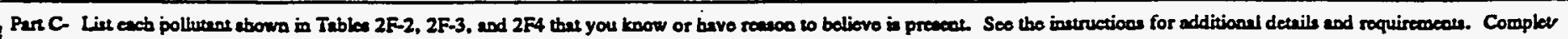
one uble for cacts outfall.

\begin{tabular}{|c|c|c|c|c|c|c|}
\hline \multirow{2}{*}{$\begin{array}{l}\text { Pollument } \\
\text { and } \\
\text { CAS Number } \\
\text { (if availible) }\end{array}$} & \multicolumn{2}{|c|}{$\begin{array}{l}\text { Maximum Values } \\
\text { (ixchudo unitu) }\end{array}$} & \multicolumn{2}{|c|}{$\begin{array}{l}\text { Averase Valuce } \\
\text { (inchude units) }\end{array}$} & \multirow{2}{*}{$\begin{array}{c}\text { Number } \\
\text { of } \\
\text { Storn } \\
\text { Evects } \\
\text { Sumpled }\end{array}$} & \multirow[b]{2}{*}{ Sources of Polbutanta } \\
\hline & $\begin{array}{l}\text { Grab Sumple } \\
\text { Teken During } \\
\text { Fint } 30 \\
\text { Minutee }\end{array}$ & $\begin{array}{l}\text { Flow-weighted } \\
\text { Componite }\end{array}$ & $\begin{array}{l}\text { Grab Sample } \\
\text { Taked During } \\
\text { Firat } 30 \\
\text { Minutes }\end{array}$ & $\begin{array}{l}\text { Flow-weighted } \\
\text { Compotite }\end{array}$ & & \\
\hline 1.1.1.trictibroetheos 71-55-6 & $<0.010 \mathrm{mg} / \mathrm{l}$ & & $<0.010 \mathrm{mg} / \mathrm{l}$ & & 1 & \\
\hline 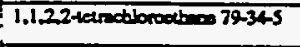 & $<0.010 \mathrm{mg} / 1$ & & $<0.010 \mathrm{mg} / \mathrm{l}$ & & 1 & \\
\hline 1.1.2-trichlorosthen 7900.5 & $<0.010 \mathrm{mg} / \mathrm{l}$ & & $<0.010 \mathrm{mg} / 1$ & & 1 & \\
\hline 1.1 dictborocthes 75-34-3 & $<0.010 \mathrm{mg} / \mathrm{l}$ & & $<0.010 \mathrm{mg} / \mathrm{l}$ & & 1 & \\
\hline 1.1-dictboredityleos 75-35-4 & $<0.010 \mathrm{mg} / \mathrm{l}$ & & $<0.010 \mathrm{mg} / 1$ & & 1 & \\
\hline 1.2 dichborombece $107.06-2$ & $<0.010 \mathrm{mg} / \mathrm{l}$ & & $<0.010 \mathrm{mg} / \mathrm{l}$ & & 1 & \\
\hline 1.2 dictionopioses $72-87.5$ & $<0.010 \mathrm{mg} / \mathrm{l}$ & & $<0.010 \mathrm{mg} / \mathrm{l}$ & & 1 & \\
\hline 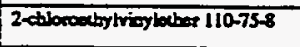 & $<0.010 \mathrm{mg} / \mathrm{l}$ & & $<0.010 \mathrm{mg} / \mathrm{l}$ & & 1 & \\
\hline $70000001-43-2$ & $<0.010 \mathrm{mg} / \mathrm{l}$ & & $<0.010 \mathrm{mg} / \mathrm{l}$ & & 1 & \\
\hline brocodictsoncuedates 75-274 & $<0.010 \mathrm{mg} / \mathrm{l}$ & - & $<0.010 \mathrm{mg} / \mathrm{l}$ & & 1 & \\
\hline brocosionm 75-25-2 & $<0.010 \mathrm{mg} / 1$ & & $<0.010 \mathrm{mg} / \mathrm{l}$ & & 1 & \\
\hline browocolateon 7483-9 & $<0.010 \mathrm{mg} / 1$ & & $<0.010 \mathrm{mg} / 1$ & & 1 & \\
\hline 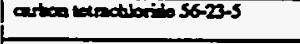 & $<0.010 \mathrm{mg} / 1$ & & $<0.010 \mathrm{mg} / 1$ & & 1 & \\
\hline 108907 & $<0.010 \mathrm{mg} / \mathrm{l}$ & & $<0.010 \mathrm{mg} / 1$ & & 1 & \\
\hline 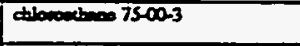 & $<0.010 \mathrm{mg} / \mathrm{l}$ & & $<0.010 \mathrm{mg} / 1$ & & 1 & \\
\hline chibocisoses 67-66-3 & $<0.010 \mathrm{mg} / 1$ & & $<0.010 \mathrm{mg} / 1$ & & 1 & $\dot{.}$ \\
\hline 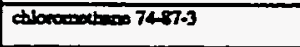 & $<0.010 \mathrm{mg} / \mathrm{l}$ & & $<0.010 \mathrm{mg} / \mathrm{l}$ & & 1 & \\
\hline 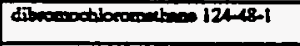 & $<0.010 \mathrm{mg} / 1$ & & $<0.010 \mathrm{mg} / 1$ & & 1 & \\
\hline 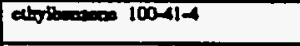 & $<0.010 \mathrm{mg} / \mathrm{l}$ & & $<0.010 \mathrm{mg} / \mathrm{l}$ & & 1 & \\
\hline loheses 108-2i-3 & $<0.010 \mathrm{mg} / \mathrm{l}$ & & $<0.010 \mathrm{mg} / 1$ & & 1 & - \\
\hline ringl charide 75014 & $<0.010 \mathrm{mg} / 1$ & & $<0.010 \mathrm{mg} / 1$ & & 1 & \\
\hline 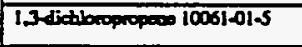 & $<0.010 \mathrm{mg} / \mathrm{l}$ & & $<0.010 \mathrm{mg} / \mathrm{l}$ & & 1 & \\
\hline 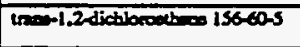 & $<0.010 \mathrm{mg} / \mathrm{l}$ & & $<0.010 \mathrm{mg} / \mathrm{l}$ & & 1 & \\
\hline atple exivity $12557-46-1$ & $1.7 \mathrm{pci} / \mathrm{l}$ & $2.1 \mathrm{pci} / 1$ & 1.7 pcill & $2.1 \mathrm{pci} / 1$ & 1 & \\
\hline betin saivity $12587-46-1$ & $1.2 \mathrm{pci} / \mathrm{l}$ & 5.9 peill & $12 \mathrm{pei} / 1$ & 5.9 poill & 1 & \\
\hline focal coliform & $15 \mathrm{co} / .1 \mathrm{~L}$ & $20 \mathrm{co} / .1 \mathrm{~L}$ & $15 \mathrm{col} .1 \mathrm{~L}$ & $20 \mathrm{co} / .1 \mathrm{~L}$ & 1 & \\
\hline Thallien 740-280 & $<0.4 \mathrm{mg} / 1$ & $<0.4 \mathrm{mg} / 1$ & $<0.4 \mathrm{mg} / \mathrm{l}$ & $<0.4 \mathrm{mg} / \mathrm{l}$ & 1 & \\
\hline Unsingo 740-61-1 & $0.004 \mathrm{mg} / \mathrm{l}$ & $0.005 \mathrm{mg} / \mathrm{l}$ & $0.004 \mathrm{mg} / \mathrm{l}$ & $0.005 \mathrm{mg} / \mathrm{l}$ & 1 & \\
\hline Genioneaivity & 100 peill & 9.3 peill & $100 \mathrm{pci} / \mathrm{l}$ & $9.3 \mathrm{pci} / 1$ & 1 & \\
\hline
\end{tabular}

Part D. Provido dete for the soim eveat(l) which rewhod in the maximum value for the flow weighed composite sample.

\begin{tabular}{|c|c|c|c|c|c|c|c|}
\hline $\begin{array}{l}1 . \\
\text { Dato of } \\
\text { Storm } \\
\text { Eveat }\end{array}$ & $\begin{array}{l}2 . \\
\text { Durtion } \\
\text { of Stom } \\
\text { (in } \\
\text { minutes) }\end{array}$ & $\begin{array}{l}3 . \\
\text { Total minfall } \\
\text { during sorm event } \\
\text { (in inchen) }\end{array}$ & 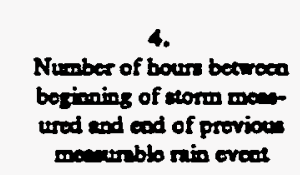 & $\begin{array}{l}\text { S. } \\
\text { Maximum flow rato } \\
\text { durias min oveat } \\
\text { (allow/min or eppecify } \\
\text { unis) }\end{array}$ & $\begin{array}{l}6 . \\
\text { Toul flow from } \\
\text { min oveat } \\
\text { (pllow or } \\
\text { pocify umit) }\end{array}$ & $\begin{array}{l}7 . \\
\text { Senion } \\
\text { amplo whs } \\
\text { tuken }\end{array}$ & 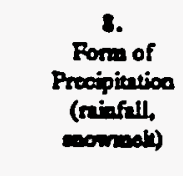 \\
\hline $1 / 3 / 92$ & 435 & 1.1 & 128.8 & 20.9 & 3,435 & Winter & Rninfall \\
\hline
\end{tabular}

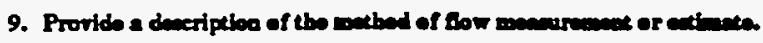

An ISCO 3230 Flow meter wes used to obtrin water leved readings. This instrument was calibrated with the normal dry weather flow, if present, as level zero. The flow meter was progrummed with the charecteristics of the conveyance so that flow rates and total flows conld be calcalated by the meter. 
Outfall 33

Parts B and C Continued

\begin{tabular}{|c|c|c|c|c|c|c|}
\hline \multirow{2}{*}{$\begin{array}{l}\text { Pollutant } \\
\text { and } \\
\text { CAS Numaber } \\
\text { (if avnilible) }\end{array}$} & \multicolumn{2}{|c|}{$\begin{array}{l}\text { Maximum Valuea } \\
\text { (inctude unita) }\end{array}$} & \multicolumn{2}{|c|}{$\begin{array}{l}\text { Avernge Valuea } \\
\text { (iacludo unina) }\end{array}$} & \multirow{2}{*}{$\begin{array}{c}\text { Number } \\
\text { of } \\
\text { Storm } \\
\text { Eveoses } \\
\text { Sempled }\end{array}$} & \multirow[b]{2}{*}{ Sources of Pollutants } \\
\hline & $\begin{array}{l}\text { Grab Sumple } \\
\text { Takeo Durias } \\
\text { Firz } 30 \\
\text { Minuteo }\end{array}$ & $\begin{array}{l}\text { Fow-weighted } \\
\text { Compounte }\end{array}$ & $\begin{array}{l}\text { Grab Semplo } \\
\text { Tuken Durims } \\
\text { Firnt } 30 \\
\text { Minueas }\end{array}$ & $\begin{array}{l}\text { Flow-weightiod } \\
\text { Comporine }\end{array}$ & & \\
\hline Beriken 740-39-3 & $0.0199 \mathrm{mg} / \mathrm{l}$ & $0.0255 \mathrm{mg} / \mathrm{l}$ & $0.0199 \mathrm{mg} / \mathrm{l}$ & $0.0255 \mathrm{mg} / 1$ & 1 & \\
\hline 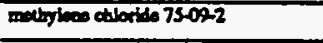 & $<0.010 \mathrm{mg} / \mathrm{l}$ & & $<0.010 \mathrm{mg} / \mathrm{I}$ & & 1 & \\
\hline letactionolbeos $127 \cdot 18-4$ & $<0.010 \mathrm{mg} / \mathrm{l}$ & & $<0.010 \mathrm{mg} / \mathrm{l}$ & & 1 & \\
\hline Irictsorouberes 79016 & $<0.010 \mathrm{mg} / \mathrm{l}$ & & $<0.010 \mathrm{mg} / \mathrm{l}$ & & 1 & $\cdot$ \\
\hline Xylaeder & $<0.010 \mathrm{mg} / \mathrm{l}$ & & $<0.010 \mathrm{mg} / 1$ & & 1 & \\
\hline 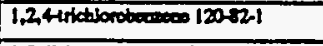 & $<0.010 \mathrm{mg} / \mathrm{l}$ & $<0.010 \mathrm{mg} / \mathrm{l}$ & $<0.010 \mathrm{mg} / \mathrm{I}$ & $<0.010 \mathrm{mg} / \mathrm{l}$ & 1 & \\
\hline 1.2-dictborobeseneos 95-50-1 & $<0.010 \mathrm{mg} / \mathrm{l}$ & $<0.010 \mathrm{mg} / \mathrm{l}$ & $<0.010 \mathrm{mg} / \mathrm{l}$ & $<0.010 \mathrm{mg} / 1$ & 1 & \\
\hline 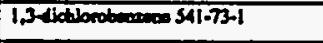 & $<0.010 \mathrm{mg} / 1$ & $<0.010 \mathrm{mg} / \mathrm{l}$ & $<0.010 \mathrm{mg} /$ & $<0.010 \mathrm{mg} / \mathrm{l}$ & 1 & \\
\hline 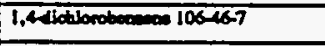 & $<0.010 \mathrm{mg} / \mathrm{l}$ & $<0.010 \mathrm{mg} / \mathrm{l}$ & $<0.010 \mathrm{mg} / \mathrm{l}$ & $<0.010 \mathrm{mg} / \mathrm{l}$ & 1 & \\
\hline 2,4 dreltrocolumes $121-14-2$ & $<0.010 \mathrm{mg} / \mathrm{l}$ & $<0.010 \mathrm{mg} / \mathrm{l}$ & $<0.010 \mathrm{mg} / \mathrm{l}$ & $<0.010 \mathrm{mg} / \mathrm{l}$ & 1 & \\
\hline 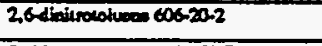 & $<0.010 \mathrm{mg} / \mathrm{l}$ & $<0.010 \mathrm{mg} / \mathrm{l}$ & $<0.010 \mathrm{mg} / \mathrm{l}$ & $<0.010 \mathrm{mg} / 1$ & 1 & \\
\hline 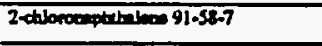 & $<0.010 \mathrm{mg} / \mathrm{l}$ & $<0.010 \mathrm{mg} / \mathrm{l}$ & $<0.010 \mathrm{mg} / \mathrm{l}$ & $<0.010 \mathrm{mg} / \mathrm{l}$ & 1 & \\
\hline 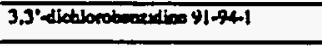 & $<0.010 \mathrm{mg} / \mathrm{l}$ & $<0.010 \mathrm{mg} / \mathrm{l}$ & $<0.010 \mathrm{mg} / \mathrm{l}$ & $<0.010 \mathrm{mg} / 1$ & 1 & \\
\hline 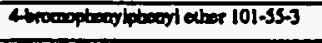 & $<0.010 \mathrm{mg} / \mathrm{l}$ & $<0.010 \mathrm{mg} / \mathrm{l}$ & $<0.010 \mathrm{mg} / \mathrm{l}$ & $<0.010 \mathrm{mg} / 1$ & 1 & \\
\hline 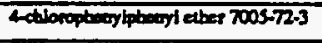 & $<0.010 \mathrm{mg} / \mathrm{I}$ & $<0.010 \mathrm{mg} / 1$ & $<0.010 \mathrm{mg} / \mathrm{l}$ & $<0.010 \mathrm{mg} / 1$ & 1 & \\
\hline 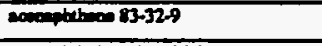 & $<0.010 \mathrm{mg} / \mathrm{l}$ & $<0.010 \mathrm{mg} / 1$ & $<0.010 \mathrm{mg} / \mathrm{l}$ & $<0.010 \mathrm{mg} / 1$ & 1 & \\
\hline 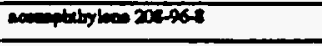 & $<0.010 \mathrm{mg} / \mathrm{I}$ & $<0.010 \mathrm{mg} / \mathrm{l}$ & $<0.010 \mathrm{mg} / \mathrm{l}$ & $<0.010 \mathrm{mg} / \mathrm{l}$ & 1 & \\
\hline 120.12 .7 & $<0.010 \mathrm{mg} / \mathrm{l}$ & $<0.010 \mathrm{mg} / \mathrm{l}$ & $<0.010 \mathrm{mg} / \mathrm{l}$ & $<0.010 \mathrm{mg} / 1$ & 1 & 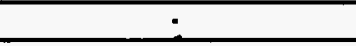 \\
\hline Datheng.5 & $<0.030 \mathrm{mg} / \mathrm{l}$ & $<0.030 \mathrm{mg} / 1$ & $<0.030 \mathrm{mg} / \mathrm{l}$ & $<0.030 \mathrm{mg} / \mathrm{l}$ & 1 & \\
\hline 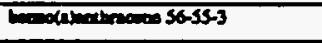 & $<0.010 \mathrm{mg} / 1$ & $<0.010 \mathrm{mg} / \mathrm{l}$ & $<0.010 \mathrm{mg} / 1$ & $<0.010 \mathrm{mg} / \mathrm{l}$ & 1 & \\
\hline 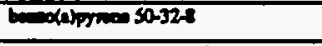 & $<0.010 \mathrm{mg} / \mathrm{l}$ & $<0.010 \mathrm{mg} / \mathrm{l}$ & $<0.010 \mathrm{mg} / \mathrm{l}$ & $<0.010 \mathrm{mg} / 1$ & 1 & \\
\hline 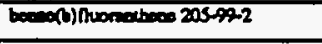 & $<0.010 \mathrm{mg} / \mathrm{l}$ & $<0.010 \mathrm{mg} / \mathrm{h}$ & $<0.010 \mathrm{mg} / 1$ & $<0.010 \mathrm{mg} / \mathrm{l}$ & 1 & \\
\hline 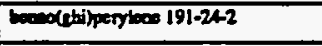 & $<0.010 \mathrm{mg} / \mathrm{l}$ & $<0.010 \mathrm{mg} / \mathrm{l}$ & $<0.010 \mathrm{mg} / 1$ & $<0.010 \mathrm{mg} / \mathrm{l}$ & 1 & \\
\hline 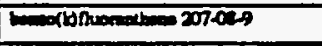 & $<0.010 \mathrm{mg} / \mathrm{l}$ & $<0.010 \mathrm{mg} / \mathrm{l}$ & $<0.010 \mathrm{mg} / 1$ & $<0.010 \mathrm{mg} / 1$ & 1 & \\
\hline 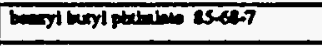 & $<0.010 \mathrm{mg} / \mathrm{l}$ & $<0.010 \mathrm{mg} / \mathrm{l}$ & $<0.010 \mathrm{mg} / \mathrm{l}$. & $<0.010 \mathrm{mg} / \mathrm{l}$ & 1 & \\
\hline 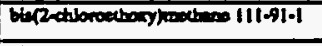 & $<0.010 \mathrm{mg} / \mathrm{l}$ & $<0.010 \mathrm{mg} / 1$ & $<0.010 \mathrm{mg} / \mathrm{l}$ & $<0.010 \mathrm{mg} / 1$ & 1 & \\
\hline 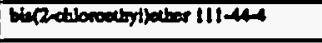 & $<0.010 \mathrm{mg} / \mathrm{l}$ & $<0.010 \mathrm{mg} / \mathrm{l}$ & $<0.010 \mathrm{mg} / \mathrm{l}$ & $<0.010 \mathrm{mg} / 1$ & 1 & \\
\hline 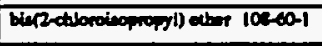 & $<0.010 \mathrm{mg} / \mathrm{l}$ & $<0.010 \mathrm{mg} / 1$ & $<0.010 \mathrm{mg} / \mathrm{l}$ & $<0.010 \mathrm{mg} / 1$ & 1 & \\
\hline 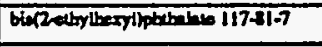 & $<0.010 \mathrm{mg} / \mathrm{l}$ & $<0.010 \mathrm{mg} / \mathrm{l}$ & $<0.010 \mathrm{mg} / \mathrm{l}$ & $<0.010 \mathrm{mg} / 1$ & 1 & \\
\hline doryeses 218.01A & $<0.010 \mathrm{mg} / 1$ & $<0.010 \mathrm{mg} / \mathrm{l}$ & $<0.010 \mathrm{mg} / 1$ & $<0.010 \mathrm{mg} / \mathrm{l}$ & 1 & \\
\hline 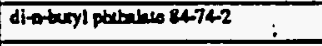 & $<0.010 \mathrm{mg} / \mathrm{l}$ & $<0.010 \mathrm{mg} / \mathrm{l}$ & $<0.010 \mathrm{mg} / \mathrm{l}$ & $<0.010 \mathrm{mg} / 1$ & 1 & \\
\hline 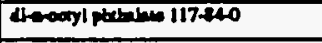 & $<0.010 \mathrm{mg} / \mathrm{l}$ & $<0.010 \mathrm{mg} / 1$ & $<0.010 \mathrm{mg} / \mathrm{l}$ & $<0.010 \mathrm{mg} / 1$ & 1 & \\
\hline 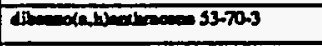 & $<0.010 \mathrm{mg} / 1$ & $<0.010 \mathrm{mg} / \mathrm{l}$ & $<0.010 \mathrm{mg} / \mathrm{l}$ & $<0.010 \mathrm{mg} / 1$ & 1 & \\
\hline 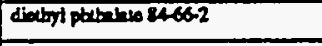 & $<0.010 \mathrm{mg} / \mathrm{l}$ & $<0.010 \mathrm{mg} / \mathrm{l}$ & $<0.010 \mathrm{mg} / \mathrm{l}$ & $<0.010 \mathrm{mg} / 1$ & 1 & \\
\hline 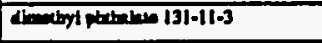 & $<0.010 \mathrm{mg} / \mathrm{l}$ & $<0.010 \mathrm{mg} / 1$ & $<0.010 \mathrm{mg} / \mathrm{l}$ & $<0.010 \mathrm{mg} / \mathrm{l}$ & 1 & \\
\hline 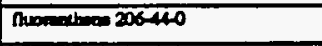 & $<0.010 \mathrm{mg} / \mathrm{l}$ & $<0.010 \mathrm{mg} / \mathrm{l}$ & $<0.010 \mathrm{mg} / \mathrm{l}$ & $<0.010 \mathrm{mg} / \mathrm{l}$ & 1 & \\
\hline 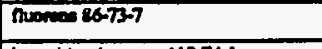 & $<0.010 \mathrm{mg} / \mathrm{l}$ & $<0.010 \mathrm{mg} / \mathrm{l}$ & $<0.010 \mathrm{mg} / 1$ & $<0.010 \mathrm{mg} / 1$ & 1 & \\
\hline 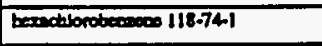 & $<0.010 \mathrm{mg} / \mathrm{l}$ & $<0.010 \mathrm{mg} / \mathrm{l}$ & $<0.010 \mathrm{mg} / 1$ & $<0.010 \mathrm{mg} / \mathrm{l}$ & 1 & \\
\hline 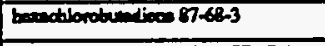 & $<0.010 \mathrm{mg} / \mathrm{l}$ & $<0.010 \mathrm{mg} / \mathrm{l}$ & $<0.010 \mathrm{mg} / \mathrm{l}$ & $<0.010 \mathrm{mg} / \mathrm{l}$ & 1 & \\
\hline 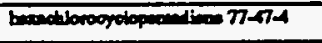 & $<0.010 \mathrm{mg} / \mathrm{l}$ & $<0.010 \mathrm{mg} / \mathrm{l}$ & $<0.010 \mathrm{mg} / 1$ & $<0.010 \mathrm{mg} / \mathrm{l}$ & 1 & \\
\hline berectionoubs $67.72-1$ & $<0.010 \mathrm{mg} / \mathrm{l}$ & $<0.010 \mathrm{mg} / \mathrm{l}$ & $<0.010 \mathrm{mg} / \mathrm{h}$ & $<0.010 \mathrm{mg} / \mathrm{l}$ & 1 & \\
\hline 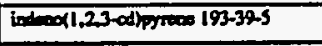 & $<0.010 \mathrm{mg} / \mathrm{l}$ & $<0.010 \mathrm{mg} / \mathrm{l}$ & $<0.010 \mathrm{mg} / \mathrm{l}$ & $<0.010 \mathrm{mg} / \mathrm{l}$ & 1 & \\
\hline boptonowe $72-59-1$ & $<0.010 \mathrm{mg} / \mathrm{l}$ & $<0.010 \mathrm{mg} / \mathrm{l}$ & $<0.010 \mathrm{mg} / 1$ & $<0.010 \mathrm{mg} / 1$ & 1 & \\
\hline 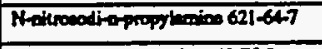 & $<0.010 \mathrm{mg} / 1$ & $<0.010 \mathrm{mg} / 1$ & $<0.010 \mathrm{mg} / \mathrm{l}$ & $<0.010 \mathrm{mg} / 1$ & 1 & \\
\hline 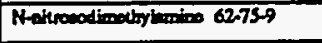 & $<0.010 \mathrm{mg} / \mathrm{l}$ & $<0.010 \mathrm{mg} / \mathrm{l}$ & $<0.010 \mathrm{mg} / \mathrm{l}$ & $<0.010 \mathrm{mg} / 1$ & 1 & \\
\hline 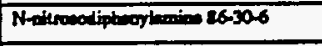 & $<0.010 \mathrm{mg} / 1$ & $<0.010 \mathrm{mg} / 1$ & $<0.010 \mathrm{mg} / 1$ & $<0.010 \mathrm{mg} / \mathrm{l}$ & 1 & \\
\hline onplochanaso 91-20-3 & $<0.010 \mathrm{mg} / \mathrm{l}$ & $<0.010 \mathrm{mg} / \mathrm{l}$ & $<0.010 \mathrm{mg} / 1$ & $<0.010 \mathrm{mg} / \mathrm{l}$ & 1 & \\
\hline 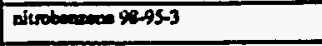 & $<0.010 \mathrm{mg} / \mathrm{l}$ & $<0.010 \mathrm{mg} / 1$ & $<0.010 \mathrm{mg} / \mathrm{l}$ & $<0.010 \mathrm{mg} / 1$ & 1 & \\
\hline 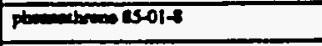 & $<0.010 \mathrm{mg} / \mathrm{l}$ & $<0.010 \mathrm{mg} / \mathrm{l}$ & $<0.010 \mathrm{mg} / \mathrm{l}$ & $<0.010 \mathrm{mg} / 1$ & 1 & \\
\hline $8970=129000$ & $<0.010 \mathrm{mg} / \mathrm{I}$ & $<0.010 \mathrm{mg} / \mathrm{l}$ & $<0.010 \mathrm{mg} / \mathrm{l}$ & $<0.010 \mathrm{mg} / 1$ & 1 & \\
\hline 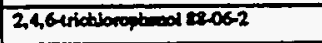 & $<0.030 \mathrm{mg} / \mathrm{l}$ & $<0.030 \mathrm{mg} / 1$ & $<0.030 \mathrm{mg} / \mathrm{l}$ & $<0.030 \mathrm{mg} / \mathrm{l}$ & 1 & \\
\hline 2,4tioblonopheod 12013.2 & $<0.030 \mathrm{mg} / \mathrm{l}$ & $<0.030 \mathrm{mg} / \mathrm{l}$ & $<0.030 \mathrm{mg} / \mathrm{l}$ & $<0.030 \mathrm{mg} / \mathrm{l}$ & 1 & \\
\hline 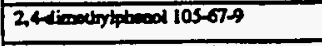 & $<0.030 \mathrm{mg} / 1$ & $<0.030 \mathrm{mg} / \mathrm{l}$ & $<0.030 \mathrm{mg} / \mathrm{l}$ & $<0.030 \mathrm{mg} / 1$ & 1 & \\
\hline$\longdiv { 2 , 4 \text { diviluopheod SI-25-S } }$ & $<0.050 \mathrm{mg} / 1$ & $<0.050 \mathrm{mg} / \mathrm{l}$ & $<0.050 \mathrm{mg} / \mathrm{l}$ & $<0.050 \mathrm{mg} / \mathrm{l}$ & 1 & \\
\hline
\end{tabular}


Outrall 33

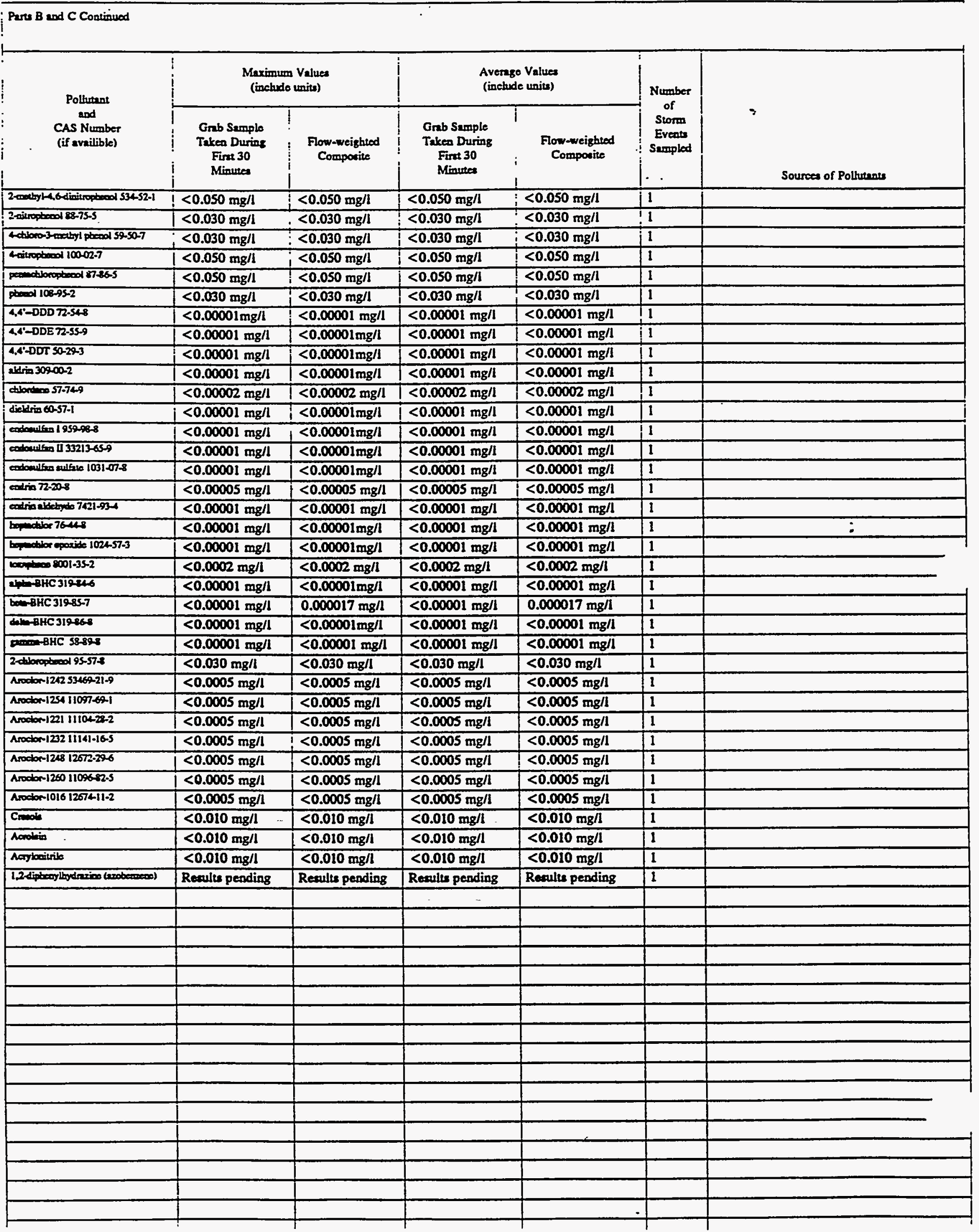


VII. Discterge Informetion (continuod from page 3 of Form 2A

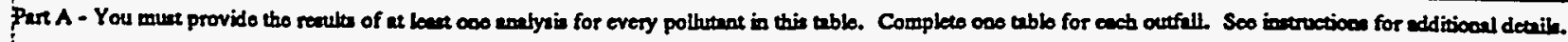

\begin{tabular}{|c|c|c|c|c|c|c|}
\hline \multirow{2}{*}{$\begin{array}{l}\text { Pollutual } \\
\text { and } \\
\text { CAS Number } \\
\text { (if availible) }\end{array}$} & \multicolumn{2}{|c|}{$\begin{array}{l}\text { Meximum Valuces } \\
\text { (include units) }\end{array}$} & \multicolumn{2}{|c|}{$\begin{array}{l}\text { Averngo Vahuea } \\
\text { (include unitr) }\end{array}$} & \multirow{2}{*}{$\begin{array}{l}\text { Nurober } \\
\text { of } \\
\text { Storm } \\
\text { Eveote } \\
\text { Sraspled }\end{array}$} & \multirow{2}{*}{ Souroen of Polthenese } \\
\hline & $\begin{array}{c}\text { Grab Semplo } \\
\text { Takea During } \\
\text { Fint } 30 \\
\text { Minowee }\end{array}$ & $\begin{array}{l}\text { Flow-weighted } \\
\text { Compocise }\end{array}$ & $\begin{array}{c}\text { Grab Samplo } \\
\text { Takeo During } \\
\text { Fina } 30 \\
\text { Mioume }\end{array}$ & $\begin{array}{l}\text { Flow-weigheded } \\
\text { Composito }\end{array}$ & & \\
\hline pil and Gronesos & $4 \mathrm{mg} / \mathrm{l}$ & & $4 \mathrm{mg} / \mathrm{l}$ & & 1 & \\
\hline 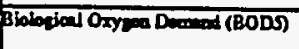 & $<5 \mathrm{mg} / \mathrm{l}$ & $<5 \mathrm{mg} / \mathrm{l}$ & $<5 \mathrm{mg} / \mathrm{l}$ & $<5 \mathrm{mg} / \mathrm{l}$ & 1 & \\
\hline Chenical Oxyper Deriend (COD) & $21 \mathrm{mg} / 1$ & $13 \mathrm{mg} / \mathrm{l}$ & $21 \mathrm{mg} / 1$ & $13 \mathrm{mg} / \mathrm{l}$ & 1 & \\
\hline 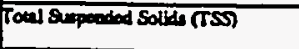 & $9.5 \mathrm{mg} / 1$ & $6.5 \mathrm{mg} / 1$ & $9.5 \mathrm{mg} / \mathrm{l}$ & $6.5 \mathrm{mg} / \mathrm{l}$ & 1 & \\
\hline Toul Kjoldent Nitrowes & $0.2 \mathrm{mg} / \mathrm{l}$ & $0.3 \mathrm{mg} / \mathrm{l}$ & $0.2 \mathrm{mg} / \mathrm{h}$ & $0.3 \mathrm{mg} / \mathrm{l}$ & 1 & \\
\hline 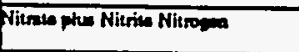 & $0.58 \mathrm{mg} / 1$ & $1.24 \mathrm{mg} / \mathrm{l}$ & $0.58 \mathrm{mg} / \mathrm{l}$ & $1.24 \mathrm{mg} / \mathrm{l}$ & 1 & \\
\hline 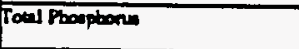 & $<0.1 \mathrm{mg} / \mathrm{l}$ & $<0.1 \mathrm{mg} / \mathrm{l}$ & $<0.1 \mathrm{mg} / 1$ & $<0.1 \mathrm{mg} / \mathrm{l}$ & 1 & \\
\hline & Minine 8.3 & Mriming 8.3 & Minim. 8.3 & Maximin 8.3 & & \\
\hline
\end{tabular}

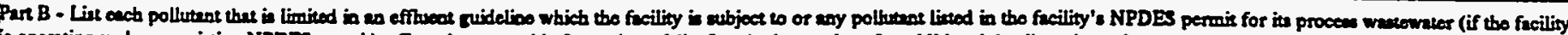

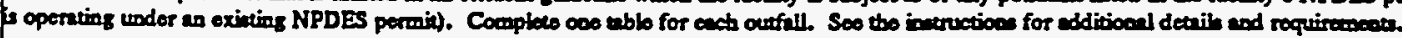

\begin{tabular}{|c|c|c|c|c|c|c|}
\hline \multirow{2}{*}{$\begin{array}{l}\text { Pollumeat } \\
\text { and } \\
\text { CAs Number } \\
\text { (if availiblo) }\end{array}$} & \multicolumn{2}{|c|}{ 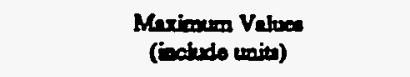 } & \multicolumn{2}{|c|}{$\begin{array}{l}\text { Averase Valuce } \\
\text { (iochedo unita) }\end{array}$} & \multirow{2}{*}{$\begin{array}{c}\text { Number } \\
\text { of } \\
\text { Storm } \\
\text { Eveot } \\
\text { Sumpled }\end{array}$} & \multirow[b]{2}{*}{ Sources of Pollurander } \\
\hline & $\begin{array}{c}\text { Greb Semplo } \\
\text { Takes During } \\
\text { First } 30 \\
\text { Misecteo }\end{array}$ & $\begin{array}{l}\text { Flow meighted } \\
\text { Compocite }\end{array}$ & 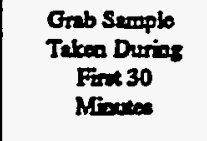 & $\begin{array}{l}\text { Flow-moizted } \\
\text { Comporite }\end{array}$ & & \\
\hline$\Rightarrow=7400-360$ & $<0.04 \mathrm{mg} / 1$ & $<0.04 \mathrm{mg} / 1$ & $<0.04 \mathrm{mg} / \mathrm{l}$ & $<0.04 \mathrm{mg} / \mathrm{l}$ & 1 & \\
\hline 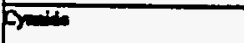 & $<0.002 \mathrm{~m} / / \mathrm{l}$ & & $<0.002 \mathrm{mg} / 1$ & & 1 & \\
\hline Menowy 7439-976 & $<0.0002 \mathrm{mg} / \mathrm{l}$ & $0.0003 \mathrm{mg} / \mathrm{l}$ & $<0.0002 \mathrm{mg} / \mathrm{l}$ & $0.0003 \mathrm{mg} / 1$ & 1 & \\
\hline Ren & $0.003 \mathrm{mg} / \mathrm{h}$ & & $0.003 \mathrm{mg} / \mathrm{l}$ & & 1 & \\
\hline 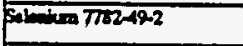 & $<0.002 \mathrm{mg} / \mathrm{l}$ & $<0.002 \mathrm{mg} / \mathrm{l}$ & $<0.002 \mathrm{mg} / \mathrm{l}$ & $<0.002 \mathrm{mg} / 1$ & 1 & \\
\hline pilnes & $10 \mathrm{mg} / \mathrm{l}$ & $33 \mathrm{mg} / \mathrm{h}$ & $10 \mathrm{mg} / \mathrm{l}$ & $33 \mathrm{mg} / 1$ & 1 & \\
\hline porthomens & $<0.05 \mathrm{mg} / \mathrm{h}$ & $<0.05 \mathrm{mg} / \mathrm{l}$ & $<0.05 \mathrm{mg} / \mathrm{I}$ & $<0.05 \mathrm{mg} / \mathrm{l}$ & 1 & \\
\hline Ahmingm 7429.90.5 & $0.56 \mathrm{mg} / \mathrm{l}$ & $0.43 \mathrm{mg} / \mathrm{l}$ & $0.56 \mathrm{mg} / \mathrm{l}$ & $0.43 \mathrm{mg} / \mathrm{l}$ & 1 & \\
\hline Aromio $7400-38-2$ & $<0.04 \mathrm{mg} / 1$ & $<0.04 \mathrm{mg} / \mathrm{l}$ & $<0.04 \mathrm{mg} / \mathrm{l}$ & $<0.04 \mathrm{mg} / \mathrm{l}$ & 1 & \\
\hline Bowoun 740-12.8 & $0.022 \mathrm{mg} / 1$ & $0.021 \mathrm{mg} / \mathrm{l}$ & $0.022 \mathrm{mg} / 1$ & $0.021 \mathrm{mg} / 1$ & 1 & \\
\hline Beryiliem $7400-1 / .7$ & $<0.0004 \mathrm{mg} / 1$ & $<0.0004 \mathrm{mg} / 1$ & $<0.0004 \mathrm{mg} / \mathrm{l}$ & $<0.0004 \mathrm{mg} / 1$ & 1 & \\
\hline Codixm 740409 & $<0.004 \mathrm{mg} / \mathrm{l}$ & $<0.004 \mathrm{mg} / \mathrm{l}$ & $<0.004 \mathrm{mg} / \mathrm{l}$ & $<0.004 \mathrm{mg} / \mathrm{l}$ & 1 & . \\
\hline asianon $7400-70-2$ & $13.5 \mathrm{mg} / 1$ & $24.3 \mathrm{mg} / \mathrm{l}$ & $13.5 \mathrm{mg} / \mathrm{l}$ & $24.3 \mathrm{mg} / \mathrm{l}$ & 1 & \\
\hline Crominan $740-67.3$ & $<0.006 \mathrm{mg} / 1$ & $<0.006 \mathrm{mg} / \mathrm{l}$ & $<0.006 \mathrm{mg} / 1$ & $<0.006 \mathrm{mg} / \mathrm{l}$ & 1 & \\
\hline Cotall 740a-4a-4 & $<0.002 \mathrm{mg} / \mathrm{l}$ & $<0.002 \mathrm{mg} / \mathrm{l}$ & $<0.002 \mathrm{mg} / 1$ & $<0.002 \mathrm{mg} / \mathrm{l}$ & 1 & \\
\hline 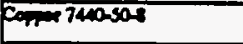 & $0.013 \mathrm{mg} / \mathrm{l}$ & $0.010 \mathrm{mg} / \mathrm{l}$ & $0.013 \mathrm{mg} / \mathrm{l}$ & $0.010 \mathrm{mg} / 1$ & 1 & \\
\hline 10007439896 & $0.64 \mathrm{mg} / 1$ & $0.46 \mathrm{mg} / \mathrm{l}$ & $0.64 \mathrm{mg} / \mathrm{l}$ & $0.46 \mathrm{mg} / \mathrm{l}$ & 1 & \\
\hline $70439-2.1$ & $<0.02 \mathrm{mg} / \mathrm{l}$ & $<0.02 \mathrm{mg} / \mathrm{h}$ & $<0.02 \mathrm{mg} / \mathrm{l}$ & $<0.02 \mathrm{mg} / 1$ & 1 & \\
\hline Tithem 7439-93-2 & $<0.02 \mathrm{mg} / \mathrm{l}$ & $<0.02 \mathrm{mg} / \mathrm{l}$ & $<0.02 \mathrm{mg} / \mathrm{l}$ & $<0.02 \mathrm{mg} / \mathrm{l}$ & 1 & \\
\hline Mromixum 7439-954 & $1.38 \mathrm{mg} / 1$ & $2.56 \mathrm{mg} / \mathrm{l}$ & $1.38 \mathrm{mg} / 1$ & $2.56 \mathrm{mg} / \mathrm{l}$ & 1 & \\
\hline 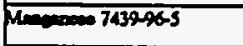 & $0.018 \mathrm{mg} / \mathrm{l}$ & $0.026 \mathrm{mg} / 1$ & $0.018 \mathrm{mg} / 1$ & $0.026 \mathrm{mg} / \mathrm{l}$ & 1 & \\
\hline 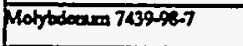 & $<0.006 \mathrm{mg} / \mathrm{l}$ & $<0.006 \mathrm{mg} / \mathrm{l}$ & $<0.006 \mathrm{mg} / \mathrm{l}$ & $<0.006 \mathrm{mg} / \mathrm{l}$ & 1 & \\
\hline Nothi 740020 & $<0.008 \mathrm{mg} / \mathrm{l}$ & $<0.008 \mathrm{mg} / \mathrm{l}$ & $<0.008 \mathrm{mg} / \mathrm{l}$ & $<0.008 \mathrm{mg} / \mathrm{l}$ & 1 & \\
\hline Promentiven 7400997 & $0.7 \mathrm{mg} / \mathrm{f}$ & $1.0 \mathrm{mg} / 1$ & $0.7 \mathrm{mg} / \mathrm{l}$ & $1.0 \mathrm{mg} / 1$ & 1 & \\
\hline $740140-224$ & $<0.006 \mathrm{mg} / \mathrm{l}$ & $<0.006 \mathrm{mg} / \mathrm{l}$ & $<0.006 \mathrm{mg} / 1$ & $<0.006 \mathrm{mg} / \mathrm{l}$ & 1 & \\
\hline sodken 740-20-5 & $0.35 \mathrm{mg} / \mathrm{l}$ & $3.08 \mathrm{mg} / \mathrm{l}$ & $0.35 \mathrm{mg} / \mathrm{l}$ & $3.08 \mathrm{mg} / \mathrm{l}$ & 1 & \\
\hline 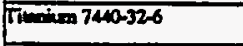 & $<0.01 \mathrm{mg} / \mathrm{I}$ & $<0.01 \mathrm{mg} / \mathrm{l}$ & $<0.01 \mathrm{mg} / \mathrm{l}$ & $<0.01 \mathrm{mg} / 1$ & 1 & \\
\hline $2 \ln 07400606$ & $0.20 \mathrm{mg} / \mathrm{l}$ & $0.15 \mathrm{mg} / \mathrm{l}$ & $0.20 \mathrm{mg} / \mathrm{l}$ & $0.15 \mathrm{mg} / 1$ & 1 & \\
\hline
\end{tabular}


Coothend from frox.

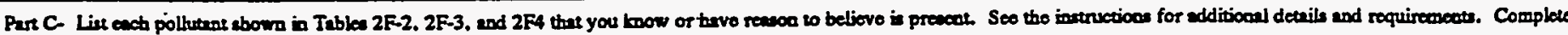
ono table for ench outfill

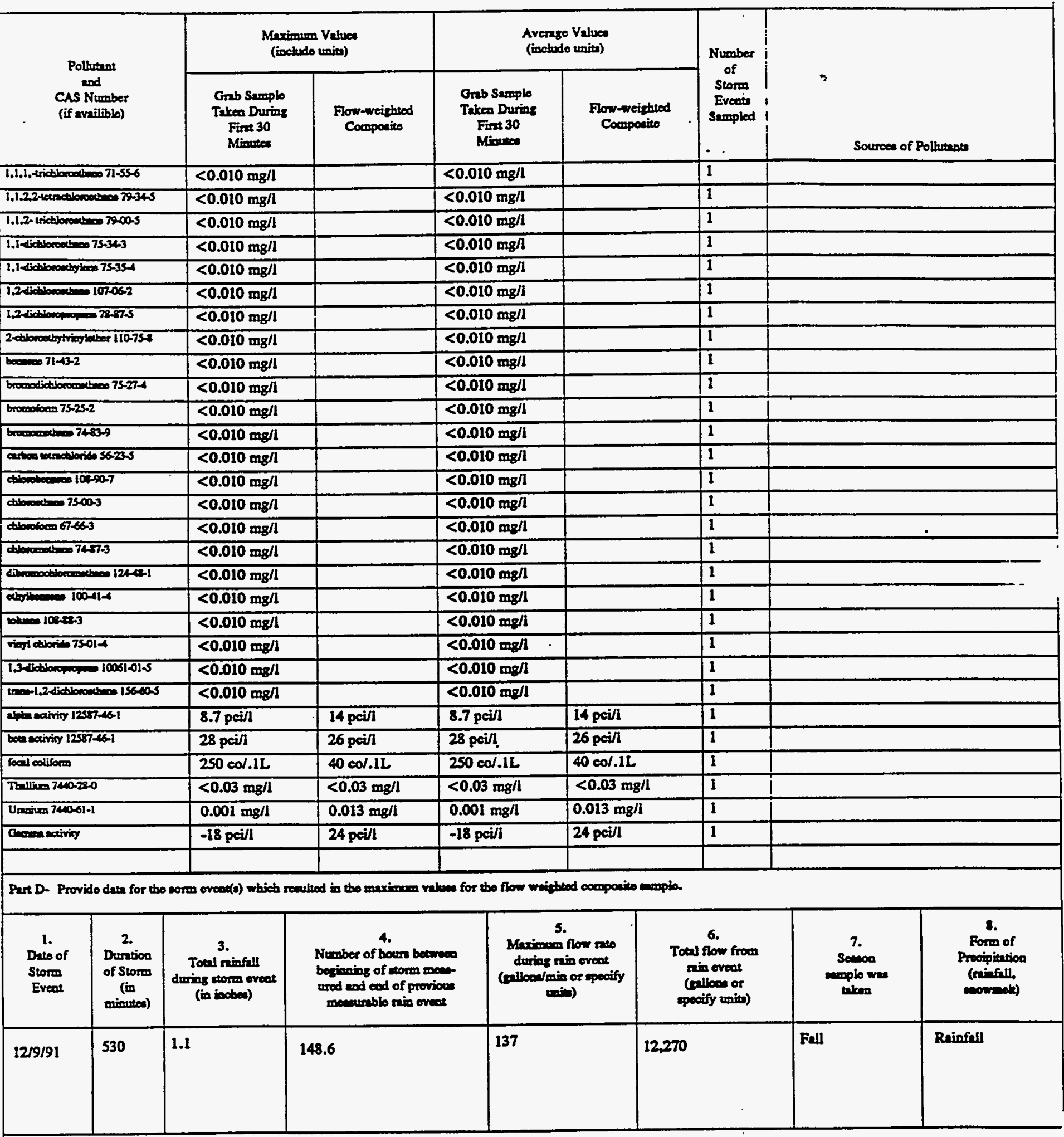

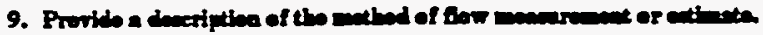

An ISCO 3230 Flow meter was wed to obthin water lerel reading. This intrment was calibrated with the sormal dry weather flow, if present, as lered

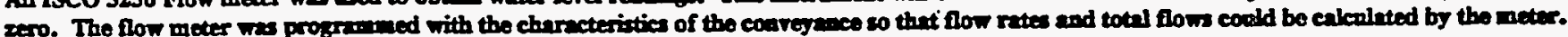


Outfall 41

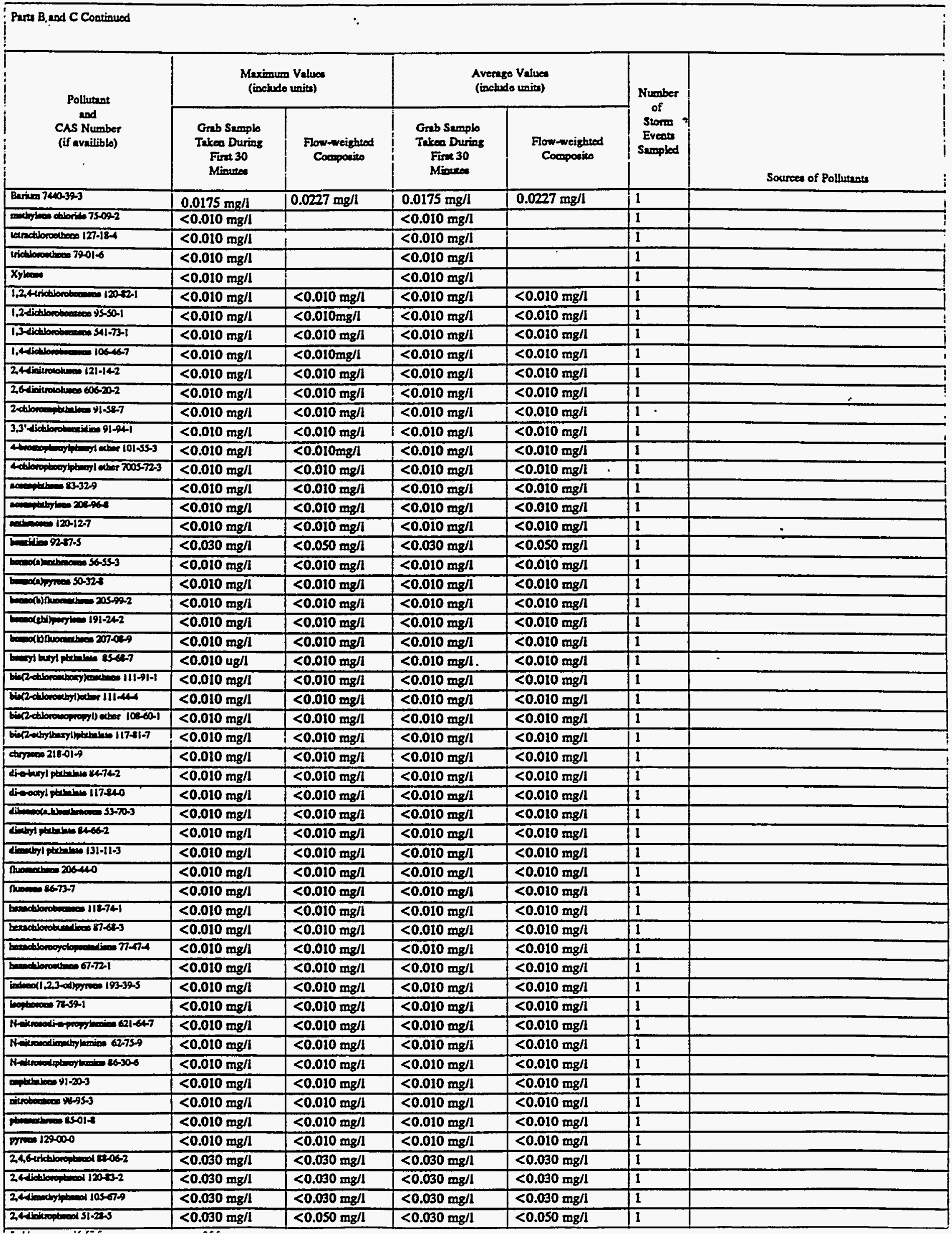


Outrall 41

\begin{tabular}{|c|c|c|c|c|c|c|}
\hline \multicolumn{7}{|l|}{ Parta B and C Continuod } \\
\hline \multirow{2}{*}{$\begin{array}{l}\text { Pollutunt } \\
\text { end } \\
\text { CAS Number } \\
\text { (if aveiliblo) }\end{array}$} & \multicolumn{2}{|c|}{$\begin{array}{l}\text { Maximingm Values } \\
\text { (inchude unitu) }\end{array}$} & \multicolumn{2}{|c|}{$\begin{array}{l}\text { Avernge Values } \\
\text { (inchude unin) }\end{array}$} & \multirow{2}{*}{$\begin{array}{l}\text { Number } \\
\text { of } \\
\text { Storm } \\
\text { Evenes } \\
\text { Sempled }\end{array}$} & \multirow[b]{2}{*}{ Sources of Pollumaner } \\
\hline & $\begin{array}{l}\text { Grab Semplo } \\
\text { Takeo During } \\
\text { Firn } 30 \\
\text { Minutea }\end{array}$ & $\begin{array}{l}\text { Flow-mointed } \\
\text { Cooponito }\end{array}$ & $\begin{array}{l}\text { Grab Semplo } \\
\text { Taken During } \\
\text { Firnt 30 } \\
\text { Minurea }\end{array}$ & $\begin{array}{l}\text { Flow-weighted } \\
\text { Comomponite }\end{array}$ & & \\
\hline 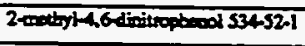 & $<0.030 \mathrm{mg} / \mathrm{l}$ & $<0.050 \mathrm{mg} / \mathrm{l}$ & $<0.030 \mathrm{mg} / \mathrm{l}$ & $<0.050 \mathrm{mg} / \mathrm{l}$ & 1 & \\
\hline 2 -incoptrosol $28-75-5$ & $<0.030 \mathrm{mg} / \mathrm{h}$ & $<0.030 \mathrm{mg} / \mathrm{l}$ & $<0.030 \mathrm{mg} / 1$ & $<0.030 \mathrm{mg} / \mathrm{l}$ & 1 & \\
\hline 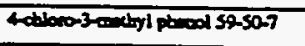 & $<0.030 \mathrm{mg} / \mathrm{l}$ & $<0.030 \mathrm{mg} / \mathrm{l}$ & $<0.030 \mathrm{mg} / \mathrm{l}$ & $<0.030 \mathrm{mg} / \mathrm{l}$ & 1 & \\
\hline 4 aiveopteod $100-02-7$ & $<0.030 \mathrm{mg} / \mathrm{l}$ & $<0.050 \mathrm{mg} / \mathrm{l}$ & $<0.030 \mathrm{mg} / \mathrm{l}$ & $<0.050 \mathrm{mg} / 1$ & 1 & \\
\hline 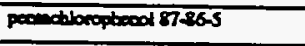 & $<0.030 \mathrm{mg} / \mathrm{l}$ & $<0.050 \mathrm{mg} / \mathrm{l}$ & $<0.030 \mathrm{mg} / \mathrm{l}$ & $<0.050 \mathrm{mg} / \mathrm{l}$ & 1 & \\
\hline 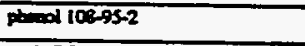 & $<0.030 \mathrm{mg} / \mathrm{l}$ & $<0.030 \mathrm{mg} / \mathrm{l}$ & $<0.030 \mathrm{mg} / 1$ & $<0.030 \mathrm{mg} / \mathrm{l}$ & 1 & \\
\hline $4,4-00072.548$ & $<0.00001 \mathrm{mg} / \mathrm{l}$ & $<0.00001 \mathrm{mg} / \mathrm{l}$ & $<0.00001 \mathrm{mg} / \mathrm{l}$ & $<0.00001 \mathrm{mg} / \mathrm{l}$ & 1 & \\
\hline 4.40DDE 72.5S-9 & $<0.00001 \mathrm{mg} / \mathrm{l}$ & $<0.00001 \mathrm{mg} / \mathrm{l}$ & $<0.00001 \mathrm{mg} / \mathrm{l}$ & $<0.00001 \mathrm{mg} / \mathrm{l}$ & 1 & \\
\hline 4,4-000T $50-22-3$ & $<0.00001 \mathrm{mg} / \mathrm{l}$ & $<0.00001 \mathrm{mg} / \mathrm{l}$ & $<0.00001 \mathrm{mg} / \mathrm{l}$ & $<0.00001 \mathrm{mg} / \mathrm{l}$ & 1 & \\
\hline atsin 302000-2 & $<0.00001 \mathrm{mg} / 1$ & $0.000057 \mathrm{mg} / \mathrm{l}$ & $<0.00001 \mathrm{mg} / \mathrm{l}$ & $0.000057 \mathrm{mg} / 1$ & 1 & \\
\hline oblotion S7.74-9 & $<0.00002 \mathrm{mg} / \mathrm{l}$ & $<0.00002 \mathrm{mg} / \mathrm{l}$ & $<0.00002 \mathrm{mg} / \mathrm{l}$ & $<0.00002 \mathrm{mg} / \mathrm{l}$ & 1 & . \\
\hline dientrie 60-57-1 & $<0.00001 \mathrm{mg} / \mathrm{l}$ & $<0.00001 \mathrm{mg} / \mathrm{l}$ & $<0.00001 \mathrm{mg} / \mathrm{l}$ & $<0.00001 \mathrm{mg} / \mathrm{l}$ & 1 & \\
\hline 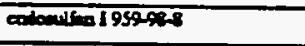 & $<0.00001 \mathrm{mg} / \mathrm{l}$ & $<0.00001 \mathrm{mg} / \mathrm{lt}$ & $<0.00001 \mathrm{mg} / \mathrm{l}$ & $<0.00001 \mathrm{mg} / \mathrm{l}$ & 1 & \\
\hline 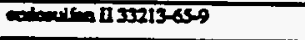 & $<0.00001 \mathrm{mg} / \mathrm{l}$ & $<0.00001 \mathrm{mg} / 1$ & $<0.00001 \mathrm{mg} / \mathrm{l}$ & $<0.00001 \mathrm{mg} / \mathrm{l}$ & 1 & \\
\hline 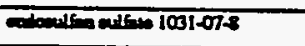 & $<0.00001 \mathrm{mg} / \mathrm{l}$ & $<0.00001 \mathrm{mg} / \mathrm{l}$ & $<0.00001 \mathrm{mg} / \mathrm{l}$ & $<0.00001 \mathrm{mg} / \mathrm{l}$ & 1 & \\
\hline cotris 72.200 & $<0.00005 \mathrm{mg} / \mathrm{l}$ & $<0.00005 \mathrm{mg} / \mathrm{l}$ & $<0.00005 \mathrm{mg} / \mathrm{h}$ & $<0.00005 \mathrm{mg} / \mathrm{l}$ & 1 & \\
\hline 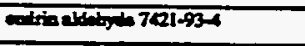 & $<0.00001 \mathrm{mg} / \mathrm{l}$ & $<0.00001 \mathrm{mg} / 1$ & $<0.00001 \mathrm{mg} / \mathrm{l}$ & $<0.00001 \mathrm{mg} / 1$ & 1 & \\
\hline bepection 76448 & $<0.00001 \mathrm{mg} / \mathrm{l}$ & $<0.00001 \mathrm{mg} / 1$ & $<0.00001 \mathrm{mg} / \mathrm{l}$ & $<0.00001 \mathrm{mg} / \mathrm{h}$ & 1 & \\
\hline 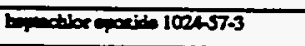 & $<0.00001 \mathrm{mg} / \mathrm{l}$ & $<0.00001 \mathrm{mg} / 1$ & $<0.00001 \mathrm{mg} / \mathrm{l}$ & $<0.00001 \mathrm{mg} / \mathrm{l}$ & 1 & \\
\hline $2001-35-2$ & $<0.0002 \mathrm{mg} / \mathrm{l}$ & $<0.0002 \mathrm{mg} / \mathrm{l}$ & $<0.0002 \mathrm{mg} / \mathrm{h}$ & $<0.0002 \mathrm{mg} / 1$ & 1 & - \\
\hline $2 \times-B H C 319-6$ & $<0.00001 \mathrm{mg} / \mathrm{l}$ & $<0.00001 \mathrm{mg} / 1$ & $<0.00001 \mathrm{mg} / \mathrm{l}$ & $<0.00001 \mathrm{mg} / \mathrm{l}$ & 1 & \\
\hline WHC 319859 & $<0.00001 \mathrm{mg} / \mathrm{l}$ & $<0.00001 \mathrm{mg} / \mathrm{l}$ & $<0.00001 \mathrm{mg} / \mathrm{l}$ & $<0.00001 \mathrm{mg} / \mathrm{l}$ & 1 & \\
\hline 45-8HC 312468 & $<0.00001 \mathrm{mg} / \mathrm{l}$ & $<0.00001 \mathrm{~m} / 1$ & $<0.00001 \mathrm{mg} / \mathrm{l}$ & $<0.00001 \mathrm{mg} / \mathrm{h}$ & 1 & \\
\hline $\sin 2 \mathrm{RHC} \sin 8$ & $<0.00001 \mathrm{mg} / 1$ & $<0.00001 \mathrm{mg} / \mathrm{l}$ & $<0.00001 \mathrm{mg} / \mathrm{l}$ & $<0.00001 \mathrm{mg} / \mathrm{l}$ & 1 & \\
\hline 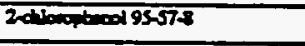 & $<0.030 \mathrm{mg} / 1$ & $<0.030 \mathrm{mg} / \mathrm{l}$ & $<0.030 \mathrm{mg} / \mathrm{l}$ & $<0.030 \mathrm{mg} / \mathrm{l}$ & 1 & \\
\hline Arodon-1212 53469-21-9 & $<0.0005 \mathrm{mg} / \mathrm{l}$ & $<0.0005 \mathrm{mg} / \mathrm{l}$ & $<0.0005 \mathrm{mg} / 1$ & $<0.0005 \mathrm{mg} / \mathrm{l}$ & 1 & \\
\hline Arodor-1254 1109769-1 & $<0.0005 \mathrm{mg} / 1$ & $<0.0005 \mathrm{mg} / 1$ & $<0.0005 \mathrm{mg} / 1$ & $<0.0005 \mathrm{mg} / \mathrm{l}$ & 1 & \\
\hline Arodon-1211110422-2 & $<0.0005 \mathrm{mg} / \mathrm{l}$ & $<0.0005 \mathrm{mg} / \mathrm{l}$ & $<0.0005 \mathrm{mg} / \mathrm{l}$ & $<0.0005 \mathrm{mg} / \mathrm{l}$ & 1 & \\
\hline Arocion-1202 IIIA1-16.5 & $<0.0005 \mathrm{mg} / \mathrm{l}$ & $<0.0005 \mathrm{mg} / \mathrm{l}$ & $<0.0005 \mathrm{mg} / \mathrm{l}$ & $<0.0005 \mathrm{mg} / \mathrm{l}$ & 1 & \\
\hline Arodion 1248 12672-296 & $<0.0005 \mathrm{mg} / \mathrm{l}$ & $<0.0005 \mathrm{mg} / \mathrm{l}$ & $<0.0005 \mathrm{mg} / \mathrm{l}$ & $<0.0005 \mathrm{mg} / \mathrm{l}$ & 1 & \\
\hline Anodion 126011096025 & $<0.0005 \mathrm{mg} / \mathrm{l}$ & $<0.0005 \mathrm{mg} / \mathrm{l}$ & $<0.0005 \mathrm{mg} / 1$ & $<0.0005 \mathrm{mg} / \mathrm{l}$ & 1 & \\
\hline Alooton-1016 126/4-11-2 & $<0.0005 \mathrm{mg} / \mathrm{l}$ & $<0.0005 \mathrm{mg} / \mathrm{l}$ & $<0.0005$ mg/l & $<0.0005 \mathrm{mg} / 1$ & 1 & \\
\hline Cronole & $<0.010 \mathrm{mg} / 1$ & $<0.010 \mathrm{mg} / \mathrm{l}$ & $<0.010 \mathrm{mg} / !$ & $<0.010 \mathrm{mg} / \mathrm{l}$ & 1 & \\
\hline Acoolnis & $<0.010 \mathrm{mg} / 1$ & $<0.010 \mathrm{mg} / \mathrm{l}$ & $<0.010 \mathrm{mg} / \mathrm{l}$ & $<0.010 \mathrm{mg} / \mathrm{l}$ & 1 & \\
\hline Angibcitith & $<0.010 \mathrm{mg} / \mathrm{l}$ & $<0.010 \mathrm{mg} / \mathrm{l}$ & $<0.010 \mathrm{mg} / 1$ & $<0.010 \mathrm{mg} / 1$ & 1 & \\
\hline 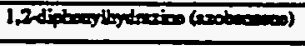 & Recults pending & Reaulls pending & Rowults peoding & Reaults pending & 1 & \\
\hline & & & & & & \\
\hline & & & & & & \\
\hline & & & & & & \\
\hline & & & & & & \\
\hline & & & & & & \\
\hline & & & & & & \\
\hline & & & & & & \\
\hline & & & & & & \\
\hline & & & & & & \\
\hline & & & & & & \\
\hline & & & & & & \\
\hline & & & & & & \\
\hline & & & & & & \\
\hline & & & & & & \\
\hline & & & & & & \\
\hline & & & & & & \\
\hline
\end{tabular}


VII. Discherge Information (continued from page 3 of Form 2F)

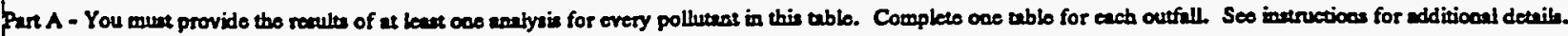

\begin{tabular}{|c|c|c|c|c|c|c|}
\hline \multirow{2}{*}{$\begin{array}{l}\text { Pollutuant } \\
\text { and } \\
\text { CAs Number } \\
\text { (if mailible) }\end{array}$} & \multicolumn{2}{|c|}{$\begin{array}{l}\text { Meximum Vabuea } \\
\text { (inelude unils) }\end{array}$} & \multicolumn{2}{|c|}{$\begin{array}{l}\text { Avengo Valuen } \\
\text { (inctude unitu) }\end{array}$} & \multirow{2}{*}{ 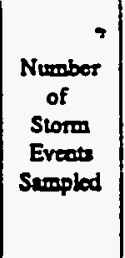 } & \multirow[b]{2}{*}{ Sources of Polbureses } \\
\hline & $\begin{array}{l}\text { Grab Sample } \\
\text { Takeo During } \\
\text { First } 30 \\
\text { Minesea }\end{array}$ & $\begin{array}{l}\text { Flow-weigtied } \\
\text { Composite }\end{array}$ & $\begin{array}{l}\text { Grab Samplo } \\
\text { Takea During } \\
\text { Firat } 30 \\
\text { Minutea }\end{array}$ & $\begin{array}{l}\text { Flow-weighted } \\
\text { Comporite }\end{array}$ & & \\
\hline 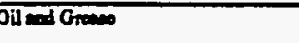 & $5 \mathrm{mg} / \mathrm{l}$ & & 5 mgil & & 1 & \\
\hline 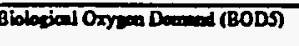 & $<5 \mathrm{mg} / 1$ & $<5 \mathrm{mg} / \mathrm{l}$ & $<5 m g / l$ & $<5 \mathrm{mg} / \mathrm{l}$ & 1 & \\
\hline 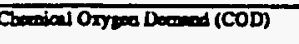 & $9.2 \mathrm{mg} / \mathrm{l}$ & $6.9 \mathrm{mg} / \mathrm{I}$ & $9.2 \mathrm{mg} / \mathrm{h}$ & $6.9 \mathrm{mg} / \mathrm{l}$ & 1 & \\
\hline Tow Serpond Solide (TSS) & $<5 \mathrm{mg} / \mathrm{l}$ & $<5 \mathrm{mg} / \mathrm{l}$ & $<5 \mathrm{mg} / \mathrm{l}$ & $<5 \mathrm{mg} / \mathrm{l}$ & 1 & \\
\hline 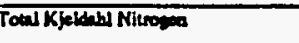 & $0.2 \mathrm{mg} / \mathrm{l}$ & $<0.2 \mathrm{mg} / \mathrm{l}$ & $0.2 \mathrm{mg} / \mathrm{l}$ & $<0.2 \mathrm{mg} / \mathrm{l}$ & 1 & \\
\hline 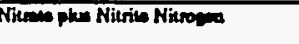 & $0.6 \mathrm{mg} / 1$ & $0.21 \mathrm{mg} / 1$ & $0.6 \mathrm{mg} / 1$ & $0.21 \mathrm{mg} / \mathrm{l}$ & 1 & \\
\hline 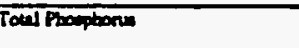 & $0.20 \mathrm{mg} / \mathrm{l}$ & $0.17 \mathrm{mg} / 1$ & $0.20 \mathrm{mg} / \mathrm{l}$ & $0.17 \mathrm{mg} / \mathrm{l}$ & 1 & \\
\hline H & \multicolumn{2}{|l|}{ Miniman 8.1} & Minimen 8.1 & Meximans 8.1 & $\dot{0}$ & - \\
\hline \multirow{2}{*}{$\begin{array}{l}\text { Polluenest } \\
\text { mod } \\
\text { CAs Number } \\
\text { (if aveiliblo) }\end{array}$} & \multicolumn{2}{|c|}{$\begin{array}{l}\text { Meximen Vaheon } \\
\text { (inchede units) }\end{array}$} & \multicolumn{2}{|c|}{$\begin{array}{l}\text { Arecese Vahuce } \\
\text { (includt toniv) }\end{array}$} & \multirow{2}{*}{$\begin{array}{l}\text { Number } \\
\text { of } \\
\text { Stom } \\
\text { Evesead } \\
\text { Sempled }\end{array}$} & \multirow[b]{2}{*}{ Source of Polhuresen } \\
\hline & $\begin{array}{l}\text { Orib Seropio } \\
\text { Tikeo Durins } \\
\text { Firse } 30 \\
\text { Minice }\end{array}$ & $\begin{array}{l}\text { Flow-weighted } \\
\text { Coupocito }\end{array}$ & $\begin{array}{l}\text { Grb Samplo } \\
\text { Takea Durieg } \\
\text { Fune } 30 \\
\text { Minutes }\end{array}$ & $\begin{array}{l}\text { Flow-wreighted } \\
\text { Cocoponito }\end{array}$ & & \\
\hline $1+10001740-360$ & $<0.04 \mathrm{mg} / 1$ & $<0.04 \mathrm{mg} / \mathrm{l}$ & $<0.04 \mathrm{mg} / \mathrm{l}$ & $<0.04 \mathrm{mg} / \mathrm{l}$ & 1 & \\
\hline 20 & $<0.002 \mathrm{mg} / \mathrm{l}$ & & $<0.002 \mathrm{mg} / 1$ & & 1 & \\
\hline 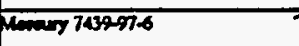 & $<0.0002 \mathrm{mg} / \mathrm{l}$ & $<0.0002 \mathrm{mg} / 1$ & $<0.0002 \mathrm{mg} / \mathrm{l}$ & $<0.0002 \mathrm{mg} / 1$ & 1 & \\
\hline 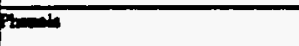 & $0.002 \mathrm{mg} / \mathrm{l}$ & & $0.002 \mathrm{mg} / \mathrm{l}$ & & 1 & \\
\hline $301=172+4-2$ & $<0.002 \mathrm{mg} / \mathrm{l}$ & $<0.002 \mathrm{mg} / \mathrm{l}$ & $<0.002 \mathrm{mg} / 1$ & $<0.002 \mathrm{mg} / \mathrm{l}$ & 1 & \\
\hline sinas & $15 \mathrm{mg} / \mathrm{h}$ & $14 \mathrm{mg} / 1$ & $15 \mathrm{mg} / 1$ & $14 \mathrm{mg} / \mathrm{l}$ & 1 & \\
\hline Purtinamenes & $<0.05 \mathrm{mg} / 1$ & $<0.05 \mathrm{mg} / \mathrm{l}$ & $<0.05 \mathrm{mg} / \mathrm{l}$ & $<0.05 \mathrm{mg} / \mathrm{l}$ & 1 & \\
\hline chenimom 7429-90-5 & $0.25 \mathrm{mg} / \mathrm{l}$ & $0.23 \mathrm{mg} / \mathrm{l}$ & $0.25 \mathrm{mg} / \mathrm{l}$ & $0.23 \mathrm{mg} / \mathrm{l}$ & 1 & \\
\hline Ansio $7400-34-2$ & $<0.04 \mathrm{mg} / 1$ & $<0.04 \mathrm{mg} / \mathrm{t}$ & $<0.04 \mathrm{mg} / 1$ & $<0.04 \mathrm{mg} / \mathrm{I}$ & 1 & \\
\hline 30007400128 & $0.020 \mathrm{mg} / \mathrm{l}$ & $0.015 \mathrm{mg} / \mathrm{l}$ & $0.020 \mathrm{mg} / \mathrm{l}$ & $0.015 \mathrm{mg} / \mathrm{l}$ & 1 & \\
\hline 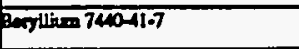 & $<0.0004 \mathrm{mg} / \mathrm{l}$ & $<0.0004 \mathrm{mg} / \mathrm{l}$ & $<0.0004 \mathrm{mg} / \mathrm{l}$ & $<0.0004 \mathrm{mg} / \mathrm{l}$ & 1 & \\
\hline Fandenes $740-139$ & $<0.004 \mathrm{mg} / \mathrm{l}$ & $<0.004 \mathrm{mg} / 1$ & $<0.004 \mathrm{mg} / \mathrm{l}$ & $<0.004 \mathrm{mg} / \mathrm{l}$ & 1 & \\
\hline calaten $7400-70-2$ & $20.6 \mathrm{mg} / \mathrm{l}$ & $19.5 \mathrm{mg} / 1$ & $20.6 \mathrm{mg} / \mathrm{l}$ & $19.5 \mathrm{mg} / \mathrm{l}$ & 1 & \\
\hline 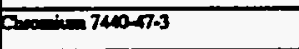 & $<0.006 \mathrm{mg} / \mathrm{h}$ & $<0.006 \mathrm{mg} / \mathrm{l}$ & $<0.006 \mathrm{mg} / \mathrm{l}$ & $<0.006 \mathrm{mg} / \mathrm{l}$ & 1 & \\
\hline Cotad 7440-4es & $<0.002 \mathrm{mg} / \mathrm{l}$ & $<0.002 \mathrm{mg} / 1$ & $<0.002 \mathrm{mg} / \mathrm{l}$ & $<0.002 \mathrm{mg} / \mathrm{l}$ & $I$ & \\
\hline 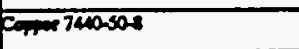 & $<0.006 \mathrm{mg} / \mathrm{l}$ & $<0.006 \mathrm{mg} / 1$ & $<0.006 \mathrm{mg} / 1$ & $<0.006 \mathrm{mg} / \mathrm{l}$ & 1 & \\
\hline 500763.026 & $0.21 \mathrm{mg} / \mathrm{n}$ & $0.19 \mathrm{mg} / \mathrm{L}$ & $0.21 \mathrm{mg} / 1$ & $0.19 \mathrm{mg} / \mathrm{l}$ & 1 & \\
\hline red 74392-1 & $<0.02 \mathrm{mg} / \mathrm{l}$ & $<0.02 \mathrm{mg} / \mathrm{l}$ & $<0.02 \mathrm{mg} / 1$ & $<0.02 \mathrm{mg} / \mathrm{l}$ & 1 & \\
\hline Whiser 7699-93-2 & $<0.02 \mathrm{mg} / \mathrm{l}$ & $<0.02 \mathrm{mg} / \mathrm{l}$ & $<0.02 \mathrm{mg} / \mathrm{l}$ & $<0.02 \mathrm{mg} / \mathrm{l}$ & 1 & \\
\hline 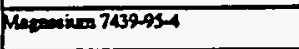 & $5.37 \mathrm{mg} / \mathrm{l}$ & $4.92 \mathrm{mg} / \mathrm{l}$ & $5.37 \mathrm{mg} / \mathrm{I}$ & $4.92 \mathrm{mg} / 1$ & 1 & \\
\hline 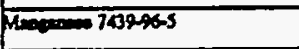 & $0.007 \mathrm{mg} / \mathrm{l}$ & $0.008 \mathrm{mg} / \mathrm{l}$ & $0.007 \mathrm{mg} / \mathrm{l}$ & $0.008 \mathrm{mg} / \mathrm{l}$ & 1 & \\
\hline Schpodacon 743998-7 & $<0.006 \mathrm{mg} / \mathrm{l}$ & $<0.006 \mathrm{mg} / \mathrm{l}$ & $<0.006 \mathrm{mg} / \mathrm{l}$ & $<0.006 \mathrm{mg} / \mathrm{l}$ & 1 & \\
\hline$\sqrt{162=1740020}$ & $<0.008 \mathrm{mg} / \mathrm{l}$ & $<0.008 \mathrm{mg} / \mathrm{l}$ & $<0.008 \mathrm{mg} / \mathrm{l}$ & $<0.008 \mathrm{mg} / \mathrm{l}$ & 1 & \\
\hline Pomixn 740027 & $1.0 \mathrm{mg} / \mathrm{l}$ & $1.0 \mathrm{mg} / \mathrm{l}$ & $1.0 \mathrm{mg} / 1$ & $1.0 \mathrm{mg} / \mathrm{l}$ & 1 & \\
\hline 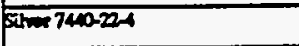 & $<0.006 \mathrm{mg} / \mathrm{l}$ & $<0.006 m g / 1$ & $<0.006 \mathrm{mg} / \mathrm{l}$ & $<0.006 \mathrm{mg} / \mathrm{l}$ & 1 & \\
\hline Pathon 740-23.5 & $3.15 \mathrm{mg} / \mathrm{l}$ & $2.92 \mathrm{mg} / \mathrm{l}$ & $3.15 \mathrm{mg} / 1$ & $2.92 \mathrm{mg} / \mathrm{l}$ & 1 & \\
\hline rimangon 740-326 & $<0.01 \mathrm{mg} / 1$ & $<0.01 \mathrm{mg} / 1$ & $<0.01 \mathrm{mg} / 1$ & $<0.01 \mathrm{mg} / \mathrm{l}$ & 1 & \\
\hline 200740666 & $0.09 \mathrm{mg} / \mathrm{l}$ & $0.08 \mathrm{mg} / \mathrm{l}$ & $0.09 \mathrm{mg} / \mathrm{l}$ & $0.08 \mathrm{mg} / \mathrm{l}$ & 1 & \\
\hline
\end{tabular}


Concined from from.

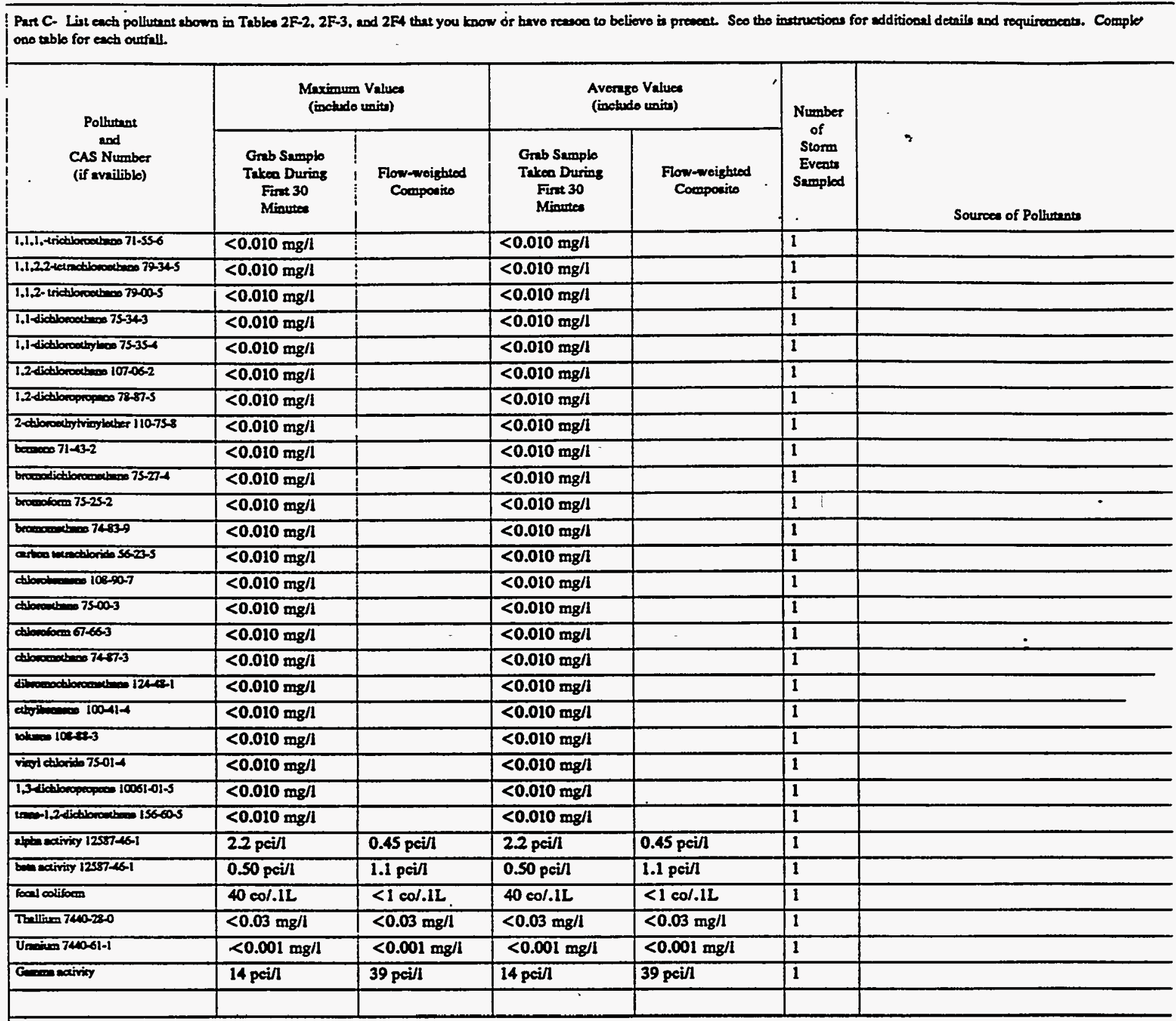

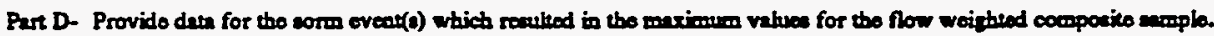

\begin{tabular}{|c|c|c|c|c|c|c|c|}
\hline $\begin{array}{l}1 . \\
\text { Dato of } \\
\text { Stores } \\
\text { Evees }\end{array}$ & $\begin{array}{l}2 . \\
\text { Durition } \\
\text { of Storm } \\
\text { (in } \\
\text { minima) }\end{array}$ & $\begin{array}{l}3 . \\
\text { Total ninfall } \\
\text { during rorm oveat } \\
\text { (in ixcben) }\end{array}$ & 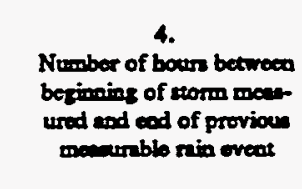 & 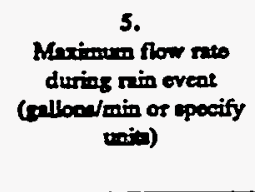 & $\begin{array}{l}6 . \\
\text { Tocal flow from } \\
\text { min ovent } \\
\text { (alloos or } \\
\text { specify unitu) }\end{array}$ & $\begin{array}{c}7 . \\
\text { Season } \\
\text { tamplo wes } \\
\text { tukeo }\end{array}$ & $\begin{array}{l}8 . \\
\text { Roria of } \\
\text { Procipitation } \\
\text { (nimfill. } \\
\text { neownela) }\end{array}$ \\
\hline $12 / 9 / 91$ & 530 & 1.1 & 148.6 & 19 & 7,290 & Fall & Rainfill \\
\hline
\end{tabular}

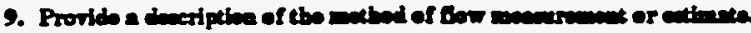

An ISCO 3230 Flow meter was used to obtain water level readings. This instrument was catibrated with the normel dry weather flow, if present, as ler. zero. The flow meter was programmed with the characteristics of the coavey ance so that flow rates and total nows conld be calculated by the meter. 
Outfall 42

\begin{tabular}{|c|c|c|c|c|c|c|}
\hline \multicolumn{7}{|l|}{ Purte B and C Continued } \\
\hline \multirow{2}{*}{$\begin{array}{l}\text { Pollutant } \\
\text { end } \\
\text { CAS Number } \\
\text { (if avniliblo) }\end{array}$} & \multicolumn{2}{|c|}{$\begin{array}{l}\text { Meximum Values } \\
\text { (inchude unita) }\end{array}$} & \multicolumn{2}{|c|}{$\begin{array}{l}\text { Avernge Valuea } \\
\text { (include unisu) }\end{array}$} & \multirow{2}{*}{\begin{tabular}{c|} 
Number \\
of \\
Stomm \\
Eventa \\
Srmopled
\end{tabular}} & \multirow[b]{2}{*}{ Sources of Polthrests } \\
\hline & $\begin{array}{c}\text { Grab Sample } \\
\text { Taken During } \\
\text { Fint } 30 \\
\text { Minurea }\end{array}$ & $\begin{array}{c}\text { Flowaweightod } \\
\text { Cocospotice }\end{array}$ & $\begin{array}{l}\text { Grab Sample } \\
\text { Takeo During } \\
\text { Firut } 30 \\
\text { Minutes }\end{array}$ & $\begin{array}{l}\text { Flow-weighted } \\
\text { Coomposice }\end{array}$ & & \\
\hline Bartum 740-32-3 & $0.0211 \mathrm{mg} / 1$ & $0.0185 \mathrm{mg} / 1$ & $0.0211 \mathrm{mg} / 1$ & $0.0185 \mathrm{mg} / \mathrm{l}$ & 1 & \\
\hline mothyloos dibride 7509-2 & $<0.010 \mathrm{mg} / \mathrm{l}$ & 7 & $<0.010 \mathrm{mg} / 1$ & & 1 & \\
\hline 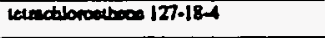 & $<0.010 \mathrm{mg} / \mathrm{l}$ & 1 & $<0.010 \mathrm{mg} / 1$ & & 1 & \\
\hline 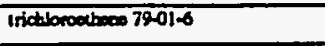 & $<0.010 \mathrm{mg} / \mathrm{l}$ & & $<0.010 \mathrm{mg} / \mathrm{l}$ & & 1 & \\
\hline Xyileose & $<0.010 \mathrm{mg} / \mathrm{l}$ & & $<0.010 \mathrm{mg} / \mathrm{l}$ & & 1 & \\
\hline 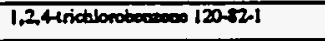 & $<0.010 \mathrm{mg} / \mathrm{l}$ & $<0.010 \mathrm{mg} / \mathrm{l}$ & $<0.010 \mathrm{mg} / \mathrm{l}$ & $<0.010 \mathrm{mg} / \mathrm{l}$ & 1 & \\
\hline 1.2-dictlonobonemen Y5-30-1 & $<0.010 \mathrm{mg} / \mathrm{l}$ & $<0.010 \mathrm{mg} / \mathrm{l}$ & $<0.010 \mathrm{mg} / \mathrm{l}$ & $<0.010 \mathrm{mg} / \mathrm{l}$ & 1 & \\
\hline 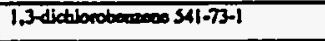 & $<0.010 \mathrm{mg} / \mathrm{l}$ & $<0.010 \mathrm{mg} / \mathrm{l}$ & $<0.010 \mathrm{mg} / \mathrm{l}$ & $<0.010 \mathrm{mg} / \mathrm{l}$ & 1 & \\
\hline 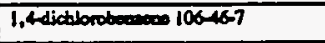 & $<0.010 \mathrm{mg} / \mathrm{l}$ & $<0.010 \mathrm{mg} / \mathrm{l}$ & $<0.010 \mathrm{mg} / \mathrm{l}$ & $<0.010 \mathrm{mg} / \mathrm{l}$ & 1 & \\
\hline 24dinitrolotives $121-14-2$ & $<0.010 \mathrm{mg} / \mathrm{l}$ & $<0.010 \mathrm{mg} / 1$ & $<0.010 \mathrm{mg} / \mathrm{l}$ & $<0.010 \mathrm{mg} / \mathrm{l}$ & 1 & \\
\hline 2,6-dinitrochoh $=00606-20-2$ & $<0.010 \mathrm{mg} / \mathrm{l}$ & $<0.010 \mathrm{mg} / \mathrm{l}$ & $<0.010 \mathrm{mg} / \mathrm{l}$ & $<0.010 \mathrm{mg} / 1$ & 1 & \\
\hline 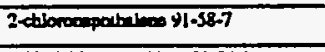 & $<0.010 \mathrm{mg} / \mathrm{l}$ & $<0.010 \mathrm{mg} / \mathrm{l}$ & $<0.010 \mathrm{mg} / \mathrm{l}$ & $<0.010 \mathrm{mg} / 1$ & 1. & \\
\hline 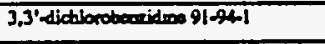 & $<0.010 \mathrm{mg} / \mathrm{l}$ & $<0.010 \mathrm{mg} / 1$ & $<0.010 \mathrm{mg} / 1$ & $<0.010 \mathrm{mg} / \mathrm{l}$ & 1 & \\
\hline 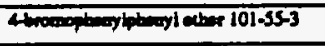 & $<0.010 \mathrm{mg} / \mathrm{l}$ & $<0.010 \mathrm{mg} / \mathrm{l}$ & $<0.010 \mathrm{mg} / \mathrm{l}$ & $<0.010 \mathrm{mg} / 1$ & 1 & \\
\hline 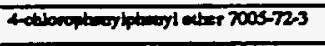 & $<0.010 \mathrm{mg} / \mathrm{l}$ & $<0.010 \mathrm{mg} / \mathrm{l}$ & $<0.010 \mathrm{mg} / \mathrm{l}$ & $<0.010 \mathrm{mg} / 1$ & 1 & \\
\hline 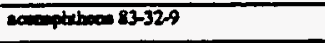 & $<0.010 \mathrm{mg} / 1$ & $<0.010 \mathrm{mg} / \mathrm{l}$ & $<0.010 \mathrm{mg} / \mathrm{l}$ & $<0.010 \mathrm{mg} / 1$ & 1 & \\
\hline 200 phetyloce $20096-8$ & $<0.010 \mathrm{mg} / \mathrm{l}$ & $<0.010 \mathrm{mg} / \mathrm{l}$ & $<0.010 \mathrm{mg} / 1$ & $<0.010 \mathrm{mg} / 1$ & 1 & \\
\hline anderome- $120-12-7$ & $<0.010 \mathrm{mg} / \mathrm{l}$ & $<0.010 \mathrm{mg} / \mathrm{l}$ & $<0.010 \mathrm{mg} / \mathrm{l}$ & $<0.010 \mathrm{mg} / \mathrm{l}$ & 1 & \\
\hline mative 925.5 & $<0.050 \mathrm{mg} / \mathrm{l}$ & $<0.050 \mathrm{mg} / \mathrm{l}$ & $<0.050 \mathrm{mg} / \mathrm{l}$ & $<0.050 \mathrm{mg} / 1$ & 1 & \\
\hline 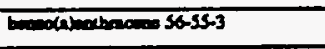 & $<0.010 \mathrm{mg} / \mathrm{l}$ & $<0.010 \mathrm{mg} / \mathrm{l}$ & $<0.010 \mathrm{mg} / \mathrm{l}$ & $<0.010 \mathrm{mg} / 1$ & 1 & \\
\hline 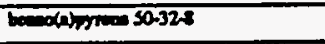 & $<0.010 \mathrm{mg} / \mathrm{l}$ & $<0.010 \mathrm{mg} / \mathrm{l}$ & $<0.010 \mathrm{mg} / \mathrm{l}$ & $<0.010 \mathrm{mg} / \mathrm{l}$ & 1 & \\
\hline 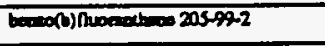 & $<0.010 \mathrm{mg} / \mathrm{l}$ & $<0.010 \mathrm{mg} / \mathrm{L}$ & $<0.010 \mathrm{mg} / 1$ & $<0.010 \mathrm{mg} / \mathrm{l}$ & 1 & \\
\hline 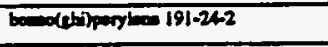 & $<0.010 \mathrm{mg} / \mathrm{l}$ & $<0.010 \mathrm{mg} / \mathrm{h}$ & $<0.010 \mathrm{mg} / \mathrm{h}$ & $<0.010 \mathrm{mg} / 1$ & 1 & \\
\hline 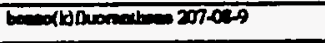 & $<0.010 \mathrm{mg} / \mathrm{l}$ & $<0.010 \mathrm{mg} / \mathrm{l}$ & $<0.010 \mathrm{mg} / \mathrm{l}$ & $<0.010 \mathrm{mg} / \mathrm{l}$ & 1 & \\
\hline 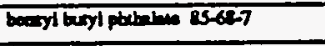 & $<0.010 \mathrm{mg} / \mathrm{l}$ & $<0.010 \mathrm{mg} / \mathrm{L}$ & $<0.010 \mathrm{mg} / 1$ & $<0.010 \mathrm{mg} / \mathrm{I}$ & 1 & \\
\hline 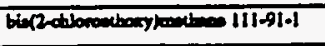 & $<0.010 \mathrm{mg} / 1$ & $<0.010 \mathrm{mg} / 1$ & $<0.010 \mathrm{mg} / \mathrm{l}$ & $<0.010 \mathrm{mg} / \mathrm{l}$ & 1 & \\
\hline bix(2) & $<0.010 \mathrm{mg} / \mathrm{l}$ & $<0.010 \mathrm{mg} / \mathrm{L}$ & $<0.010 \mathrm{mg} / \mathrm{l}$ & $<0.010 \mathrm{mg} / \mathrm{l}$ & 1 & \\
\hline biarizatloromomuppli) aber $10860-1$ & $<0.010 \mathrm{mg} / \mathrm{l}$ & $<0.010 \mathrm{mg} / \mathrm{l}$ & $<0.010 \mathrm{mg} / 1$ & $<0.010 \mathrm{mg} / \mathrm{l}$ & 1 & \\
\hline 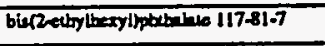 & $<0.010 \mathrm{mg} / \mathrm{l}$ & $<0.010 \mathrm{mg} / \mathrm{l}$ & $<0.010 \mathrm{mg} / \mathrm{l}$ & $<0.010 \mathrm{mg} / \mathrm{l}$ & 1 & \\
\hline chryoue $21501-9$ & $<0.010 \mathrm{mg} / \mathrm{l}$ & $<0.010 \mathrm{mg} / \mathrm{L}$ & $<0.010 \mathrm{mg} / \mathrm{l}$ & $<0.010 \mathrm{mg} / \mathrm{l}$ & 1 & \\
\hline ditoteryl pectalue 84-74-2 & $<0.010 \mathrm{mg} / \mathrm{l}$ & $<0.010 \mathrm{mg} / \mathrm{l}$ & $<0.010 \mathrm{mg} / 1$ & $<0.010 \mathrm{mg} / 1$ & 1 & \\
\hline ditwoxyl phethene 117840 & $0.011 \mathrm{mg} / \mathrm{l}$ & $<0.010 \mathrm{mg} / 1$ & $0.011 \mathrm{mg} / 1$ & $<0.010 \mathrm{mg} / \mathrm{l}$ & 1 & \\
\hline 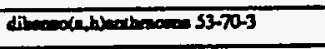 & $<0.010 \mathrm{mg} / \mathrm{l}$ & $<0.010 \mathrm{mg} / 1$ & $<0.010 \mathrm{mg} / 1$ & $<0.010 \mathrm{mg} / 1$ & 1 & \\
\hline 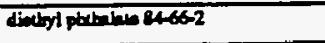 & $<0.010 \mathrm{mg} / 1$ & $<0.010 \mathrm{mg} / \mathrm{I}$ & $<0.010 \mathrm{mg} / \mathrm{l}$ & $<0.010 \mathrm{mg} / 1$ & 1 & \\
\hline 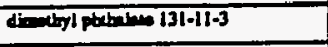 & $<0.010 \mathrm{mg} / \mathrm{l}$ & $<0.010 \mathrm{mg} / 1$ & $<0.010 \mathrm{mg} / \mathrm{l}$ & $<0.010 \mathrm{mg} / 1$ & 1 & \\
\hline 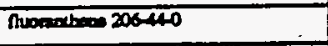 & $<0.010 \mathrm{mg} / \mathrm{l}$ & $<0.010 \mathrm{mg} / \mathrm{l}$ & $<0.010 \mathrm{mg} / \mathrm{l}$ & $<0.010 \mathrm{mg} / \mathrm{l}$ & 1 & \\
\hline Duomene $26.73-7$ & $<0.010 \mathrm{mg} / 1$ & $<0.010 \mathrm{mg} / \mathrm{l}$ & $<0.010 \mathrm{mg} / \mathrm{l}$ & $<0.010 \mathrm{mg} / \mathrm{l}$ & 1 & \\
\hline (18-74 & $<0.010 \mathrm{mg} / 1$ & $<0.010 \mathrm{mg} / 1$ & $<0.010 \mathrm{mg} / \mathrm{l}$ & $<0.010 \mathrm{mg} / \mathrm{l}$ & 1 & \\
\hline 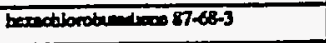 & $<0.010 \mathrm{mg} / 1$ & $<0.010 \mathrm{mg} / \mathrm{l}$ & $<0.010 \mathrm{mg} / \mathrm{l}$ & $<0.010 \mathrm{mg} / \mathrm{l}$ & 1 & \\
\hline 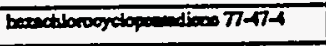 & $<0.010 \mathrm{mg} / \mathrm{l}$ & $<0.010 \mathrm{mg} / \mathrm{l}$ & $<0.010 \mathrm{mg} / \mathrm{l}$ & $<0.010 \mathrm{mg} / 1$ & 1 & \\
\hline $67.72-1$ & $<0.010 \mathrm{mg} / \mathrm{l}$ & $<0.010 \mathrm{mg} / 1$ & $<0.010 \mathrm{mg} / \mathrm{l}$ & $<0.010 \mathrm{mg} / \mathrm{l}$ & 1 & \\
\hline 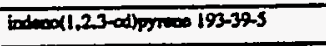 & $<0.010 \mathrm{mg} / 1$ & $<0.010 \mathrm{mg} / \mathrm{l}$ & $<0.010 \mathrm{mg} / \mathrm{l}$ & $<0.010 \mathrm{mg} / \mathrm{l}$ & 1 & \\
\hline $72-59-1$ & $<0.010 \mathrm{mg} / \mathrm{l}$ & $<0.010 \mathrm{mg} / \mathrm{l}$ & $<0.010 \mathrm{mg} / \mathrm{l}$ & $<0.010 \mathrm{mg} / \mathrm{l}$ & 1 & \\
\hline 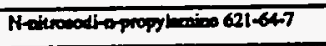 & $<0.010 \mathrm{mg} / \mathrm{l}$ & $<0.010 \mathrm{mg} / 1$ & $<0.010 \mathrm{mg} / \mathrm{l}$ & $<0.010 \mathrm{mg} / \mathrm{l}$ & 1 & \\
\hline 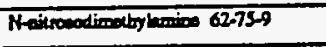 & $<0.010 \mathrm{mg} / \mathrm{l}$ & $<0.010 \mathrm{mg} / 1$ & $<0.010 \mathrm{mg} / \mathrm{l}$ & $<0.010 \mathrm{mg} / \mathrm{l}$ & 1 & \\
\hline 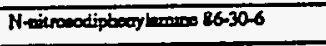 & $<0.010 \mathrm{mg} / \mathrm{l}$ & $<0.010 \mathrm{mg} / \mathrm{l}$ & $<0.010 \mathrm{mg} / \mathrm{l}$ & $<0.010 \mathrm{mg} / \mathrm{l}$ & 1 & \\
\hline $91-20-3$ & $<0.010 \mathrm{mg} / \mathrm{l}$ & $<0.010 \mathrm{mg} / \mathrm{l}$ & $<0.010 \mathrm{mg} / \mathrm{l}$ & $<0.010 \mathrm{mg} / \mathrm{l}$ & 1 & \\
\hline 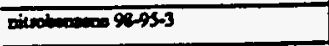 & $<0.010 \mathrm{mg} / \mathrm{l}$ & $<0.010 \mathrm{mg} / 1$ & $<0.010 \mathrm{mg} / 1$ & $<0.010 \mathrm{mg} / 1$ & 1 & \\
\hline 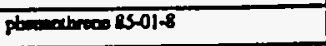 & $<0.010 \mathrm{mg} / \mathrm{l}$ & $<0.010 \mathrm{mg} / \mathrm{l}$ & $<0.010 \mathrm{mg} / \mathrm{l}$ & $<0.010 \mathrm{mg} / \mathrm{l}$ & 1 & \\
\hline prince 129000 & $<0.010 \mathrm{mg} / \mathrm{l}$ & $<0.010 \mathrm{mg} / 1$ & $<0.010 \mathrm{mg} / \mathrm{l}$ & $<0.010 \mathrm{mg} / \mathrm{l}$ & 1 & \\
\hline 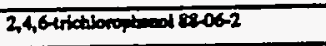 & $<0.030 \mathrm{mg} / \mathrm{l}$ & $<0.030 \mathrm{mg} / \mathrm{l}$ & $<0.030 \mathrm{mg} / \mathrm{l}$ & $<0.030 \mathrm{mg} / \mathrm{l}$ & 1 & \\
\hline 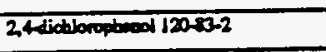 & $<0.030 \mathrm{mg} / \mathrm{l}$ & $<0.030 \mathrm{mg} / \mathrm{l}$ & $<0.030 \mathrm{mg} / 1$ & $<0.030 \mathrm{mg} / \mathrm{l}$ & 1 & \\
\hline 2, dim $105-679$ & $<0.030 \mathrm{mg} / \mathrm{l}$ & $<0.030 \mathrm{mg} / \mathrm{L}$ & $<0.030 \mathrm{mg} / \mathrm{l}$ & $<0.030 \mathrm{mg} / 1$ & 1 & \\
\hline 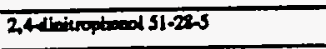 & $<0.050 \mathrm{mg} / \mathrm{l}$ & $<0.050 \mathrm{mg} / 1$ & $<0.050 \mathrm{mg} / \mathrm{l}$ & $<0.050 \mathrm{mg} / 1$ & 1 & \\
\hline
\end{tabular}


Outfall 42

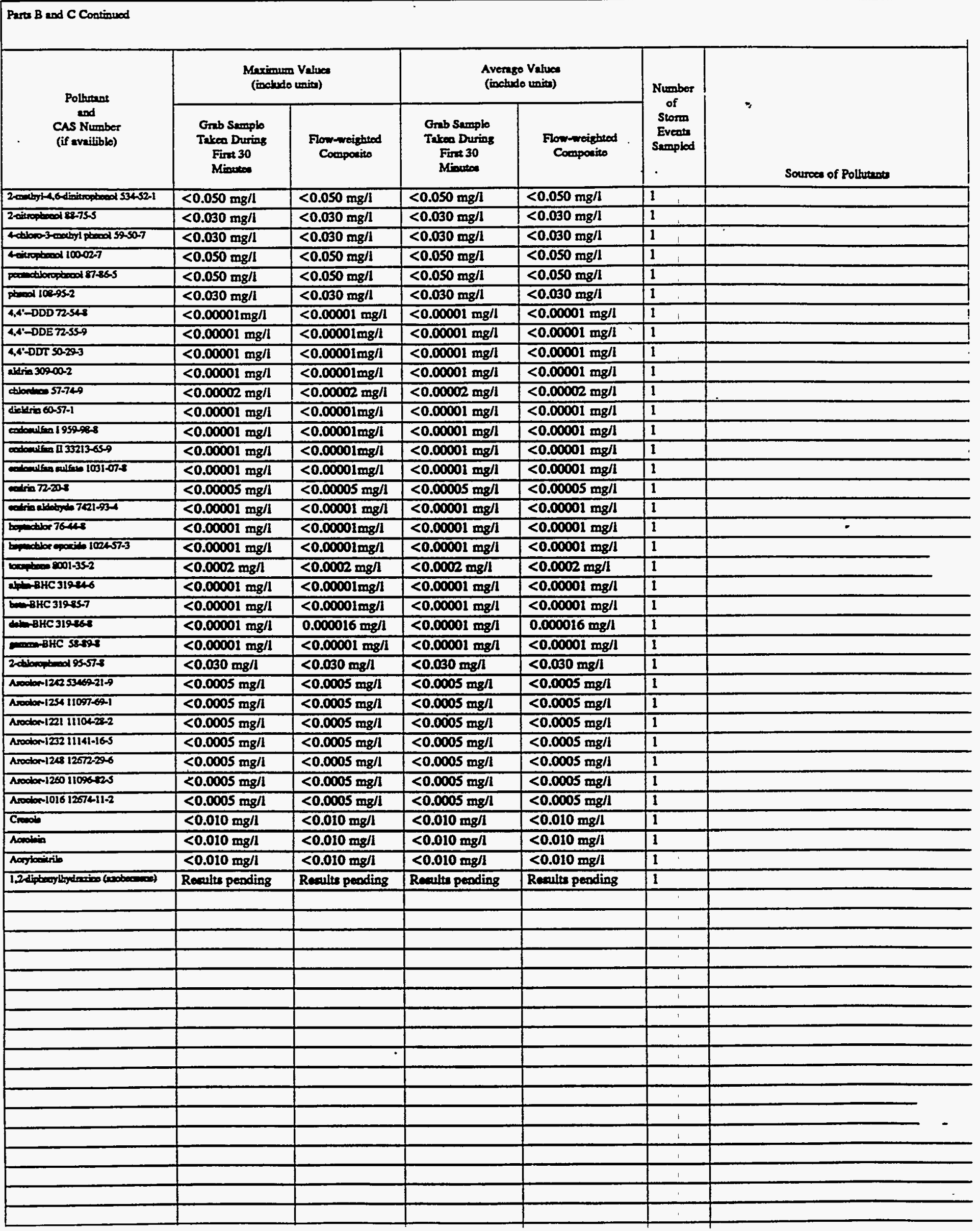


VII. Discharge Information (continued from page 3 of Form 27)

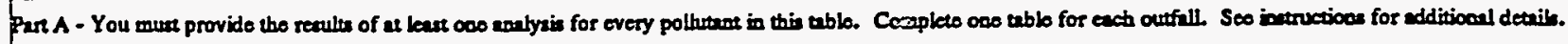

\begin{tabular}{|c|c|c|c|c|c|c|}
\hline \multirow{2}{*}{$\begin{array}{l}\text { Pollutine } \\
\text { and } \\
\text { CAS Number } \\
\text { (if availible) }\end{array}$} & \multicolumn{2}{|c|}{$\begin{array}{l}\text { Marimum Vahves } \\
\text { (inchudo univo) }\end{array}$} & \multicolumn{2}{|c|}{$\begin{array}{l}\text { Avoreso Values } \\
\text { (include units) }\end{array}$} & \multirow{2}{*}{$\begin{array}{l}\text { Number } \\
\text { of } \\
\text { Storm } \\
\text { Eveosen } \\
\text { Saropiled }\end{array}$} & \multirow[b]{2}{*}{ Sources of Polluenou } \\
\hline & $\begin{array}{c}\text { Grab Semple } \\
\text { Trkea During } \\
\text { Fine } 30 \\
\text { Minutes }\end{array}$ & $\begin{array}{l}\text { Fow-weightiod } \\
\text { Compotico }\end{array}$ & $\begin{array}{c}\text { Grab Sumple } \\
\text { Trken During } \\
\text { First } 30 \\
\text { Mioneses }\end{array}$ & $\begin{array}{l}\text { Frow-wrigted } \\
\text { Compotine }\end{array}$ & & \\
\hline pil ad Genes & $17 \mathrm{mg} / \mathrm{I}$ & & $17 \mathrm{mg} / \mathrm{t}$ & & 1 & \\
\hline Plobedeal Oxysea Deaned (BODS) & $<5 \mathrm{mg} / \mathrm{l}$ & $<5 \mathrm{mg} / \mathrm{l}$ & $<5 \mathrm{mg} / \mathrm{l}$ & $<5 \mathrm{mg} / 1$ & 1 & \\
\hline Chendal oxyra Dewed (COD) & $14 \mathrm{mg} / \mathrm{l}$ & $<5 \mathrm{mg} / \mathrm{l}$ & $14 \mathrm{mg} / \mathrm{l}$ & $<5 \mathrm{mg} / 1$ & 1 & \\
\hline 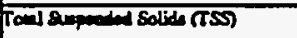 & $7.5 \mathrm{mg} / \mathrm{l}$ & $<5 \mathrm{mg} / \mathrm{l}$ & $7.5 \mathrm{mg} / 1$ & $<5 \mathrm{mg} / \mathrm{l}$ & 1 & \\
\hline 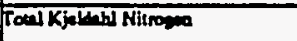 & $<0.2 \mathrm{mg} / \mathrm{l}$ & $<0.2 \mathrm{mg} / \mathrm{l}$ & $<0.2 \mathrm{mg} / 1$ & $<0.2 \mathrm{mg} / \mathrm{l}$ & 1 & \\
\hline Niture phene Nitrien Nikroger & $1.12 \mathrm{mg} / 1$ & $0.35 \mathrm{mg} / \mathrm{l}$ & $1.12 \mathrm{mg} / 1$ & $0.35 \mathrm{mg} / \mathrm{l}$ & 1 & \\
\hline Toul Propoporus & $<0.1 \mathrm{mg} / \mathrm{l}$ & $0.11 \mathrm{mg} / \mathrm{l}$ & $<0.1 \mathrm{mg} / \mathrm{l}$ & $0.11 \mathrm{mg} / \mathrm{l}$ & 1 & \\
\hline pH & 8.5 & 85 & Miming 8.5 & Mncinaln 8.5 & . & \\
\hline
\end{tabular}

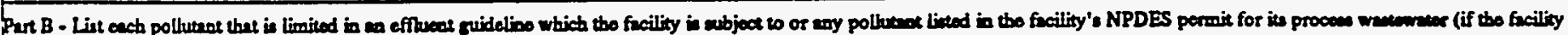

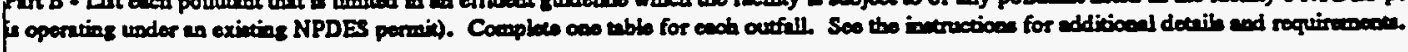

\begin{tabular}{|c|c|c|c|c|c|c|}
\hline \multirow{2}{*}{$\begin{array}{l}\text { Pollutant } \\
\text { and } \\
\text { CAs Number } \\
\text { (if aniliblo) }\end{array}$} & \multicolumn{2}{|c|}{$\begin{array}{l}\text { Maximum Vabues } \\
\text { (Foludo tmix) }\end{array}$} & \multicolumn{2}{|c|}{$\begin{array}{l}\text { Avecese Vives } \\
\text { (Fohdo units) }\end{array}$} & \multirow{2}{*}{$\begin{array}{l}\text { Number } \\
\text { of } \\
\text { storm } \\
\text { Everes } \\
\text { Suropied }\end{array}$} & \multirow[b]{2}{*}{ Souroces of Pollew } \\
\hline & 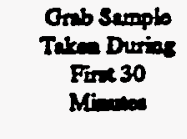 & $\begin{array}{l}\text { Flow-maighed } \\
\text { Compocito }\end{array}$ & 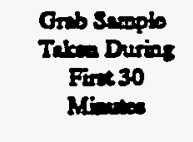 & $\begin{array}{l}\text { Flow-wriethrod } \\
\text { Compocied }\end{array}$ & & \\
\hline$p i=0007740-360$ & $<0.04 \mathrm{mg} / 1$ & $<0.04 \mathrm{mg} / \mathrm{ll}$ & $<0.04 \mathrm{mg} / \mathrm{l}$ & $<0.04 \mathrm{mg} / \mathrm{l}$ & 1 & \\
\hline ming & $<0.002 \mathrm{mgll}$ & & $<0.002 \mathrm{mg} / 1$ & & 1 & \\
\hline rewary 7439076 & $<0.0002 \mathrm{mg} / \mathrm{l}$ & $<0.0002 \mathrm{mg} / \mathrm{l}$ & $<0.0002 \mathrm{mg} / \mathrm{l}$. & $<0.0002 \mathrm{mg} / 1$ & 1 & \\
\hline himale & $0.002 \mathrm{mg} / \mathrm{l}$ & & $0.002 \mathrm{mg} / \mathrm{l}$ & & 1 & \\
\hline stanen $7 \pi 2-192$ & $<0.002 \mathrm{mg} / 1$ & $<0.002 \mathrm{mg} / \mathrm{l}$ & $<0.002 \mathrm{mg} / 1$ & $<0.002 \mathrm{mg} / \mathrm{l}$ & 1 & \\
\hline Pithe & $19 \mathrm{mg} / \mathrm{l}$ & $10 \mathrm{mg} / \mathrm{l}$ & $.19 \mathrm{mg} / \mathrm{l}$ & $10 \mathrm{mg} / \mathrm{l}$ & 1 & \\
\hline Putranons & $<0.05 \mathrm{mg} / \mathrm{l}$ & $<0.05 \mathrm{mg} / \mathrm{l}$ & $<0.05 \mathrm{mg} / \mathrm{l}$ & $<0.05 \mathrm{mg} / 1$ & 1 & \\
\hline thenion $7429900-5$ & $1.33 \mathrm{mg} / \mathrm{l}$ & $0.69 \mathrm{mg} / \mathrm{l}$ & $1.33 \mathrm{mg} / \mathrm{l}$ & $0.69 \mathrm{mg} / \mathrm{l}$ & 1 & \\
\hline $4=\lim ^{2} 740.38-2$ & $<0.04 \mathrm{mg} / \mathrm{l}$ & <0.04 mg/l & $<0.04 \mathrm{mg} / \mathrm{l}$ & $<0.04 \mathrm{mg} / \mathrm{l}$ & 1 & \\
\hline 20000740020 & $0.021 \mathrm{mg} / \mathrm{I}$ & $0.017 \mathrm{mg} / \mathrm{l}$ & $0.021 \mathrm{mg} / \mathrm{l}$ & $0.017 \mathrm{mg} / 1$ & 1 & \\
\hline 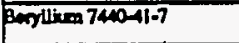 & $<0.0004 \mathrm{mg} / 1$ & $<0.0004 \mathrm{mg} / \mathrm{l}$ & $<0.0004 \mathrm{mg} / 1$ & $<0.0004 \mathrm{mg} / \mathrm{l}$ & 1 & \\
\hline Conatem 7440-43-9 & $<0.004 \mathrm{mg} / \mathrm{l}$ & $<0.004 \mathrm{mg} / \mathrm{l}$ & $<0.004 \mathrm{mg} / \mathrm{l}$ & $<0.004 \mathrm{mg} / 1$ & 1 & \\
\hline culoner $740-70-2$ & $16.6 \mathrm{mg} / \mathrm{l}$ & $13.8 \mathrm{mg} / 1$ & $16.6 \mathrm{mg} / 1$ & $13.8 \mathrm{mg} / \mathrm{l}$ & 1 & \\
\hline 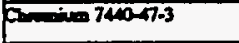 & $<0.006 \mathrm{mg} / \mathrm{l}$ & $<0.006 \mathrm{mg} / \mathrm{l}$ & $<0.006 \mathrm{mg} / \mathrm{l}$ & $<0.006 \mathrm{mg} / 1$ & 1 & \\
\hline Cobali 74ta- 8 -4 & $<0.002 \mathrm{mg} / \mathrm{l}$ & $<0.002 \mathrm{mg} / \mathrm{l}$ & $<0.002 \mathrm{mg} / \mathrm{l}$ & $<0.002 \mathrm{mg} / \mathrm{l}$ & 1 & \\
\hline 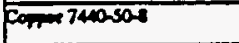 & $<0.006 \mathrm{mg} / \mathrm{l}$ & $<0.006 \mathrm{mg} / \mathrm{l}$ & $<0.006 \mathrm{mg} / \mathrm{l}$ & $<0.006 \mathrm{mg} / \mathrm{l}$ & 1 & \\
\hline 1000709096 & $1.58 \mathrm{mg} / \mathrm{l}$ & $0.84 \mathrm{mg} / \mathrm{l}$ & $1.58 \mathrm{mg} / 1$ & $0.84 \mathrm{mg} / \mathrm{l}$ & 1 & \\
\hline $\mid \operatorname{sed} 7639-29-1$ & $<0.02 \mathrm{mg} / \mathrm{l}$ & $<0.02 \mathrm{mg} / \mathrm{l}$ & $<0.02 \mathrm{mg} / \mathrm{l}$ & $<0.02 \mathrm{mg} / \mathrm{l}$ & 1 & \\
\hline Hakm 7439-93-2 & $<0.02 \mathrm{mg} / \mathrm{l}$ & $<0.02 \mathrm{mg} / \mathrm{l}$ & $<0.02 \mathrm{mg} / 1$ & $<0.02 \mathrm{mg} / 1$ & 1 & \\
\hline Magoniven 703995 & $2.03 \mathrm{mg} / \mathrm{l}$ & $1.80 \mathrm{mg} / 1$ & $2.03 \mathrm{mg} / \mathrm{l}$ & $1.80 \mathrm{mg} / 1$ & 1 & \\
\hline Mnomone $743996-5$ & $0.034 \mathrm{mg} / \mathrm{l}$ & $0.015 \mathrm{mg} / \mathrm{l}$ & $0.034 \mathrm{mg} / \mathrm{l}$ & $0.015 \mathrm{mg} / \mathrm{l}$ & 1 & \\
\hline Moptsanom 7439-96-7 & $<0.006 \mathrm{mg} / \mathrm{h}$ & $<0.006 \mathrm{mg} / \mathrm{l}$ & $<0.006 \mathrm{mg} / \mathrm{l}$ & $<0.006 \mathrm{mg} / \mathrm{l}$ & 1 & \\
\hline Nioled 7400020 & $<0.008 \mathrm{mg} / \mathrm{l}$ & $<0.008 \mathrm{mg} / 1$ & $<0.008 \mathrm{mg} / \mathrm{l}$ & $<0.008 \mathrm{mg} / 1$ & 1 & \\
\hline Prominom 7140027 & $0.7 \mathrm{mg} / \mathrm{l}$ & $0.7 \mathrm{mg} / 1$ & $0.7 \mathrm{mg} / \mathrm{l}$ & $0.7 \mathrm{mg} / \mathrm{h}$ & 1 & \\
\hline sinem 740-224 & $<0.006 \mathrm{mg} / 1$ & $<0.006 \mathrm{mg} / \mathrm{l}$ & $<0.006 \mathrm{mg} / \mathrm{l}$ & $<0.006 \mathrm{mg} / 1$ & 1 & \\
\hline $7400-23-5$ & $0.41 \mathrm{mg} / \mathrm{l}$ & $0.59 \mathrm{mg} / \mathrm{l}$ & $0.41 \mathrm{mg} / 1$ & $0.59 \mathrm{mg} / 1$ & 1 & \\
\hline 7 inden $740-32-6$ & $0.02 \mathrm{mg} / \mathrm{l}$ & $<0.01 \mathrm{mg} / 1$ & $0.02 \mathrm{mg} / \mathrm{l}$ & $<0.01 \mathrm{mg} / \mathrm{l}$ & 1 & \\
\hline 23007400666 & $0.05 \mathrm{mg} / \mathrm{l}$ & $0.02 \mathrm{mg} / \mathrm{l}$ & $0.05 \mathrm{mg} / \mathrm{l}$ & $0.02 \mathrm{mg} / 1$ & 1 & \\
\hline
\end{tabular}


Concinend from front.

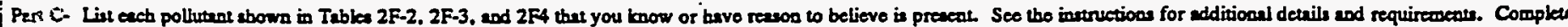
oos thisls for each ouffall.

\begin{tabular}{|c|c|c|c|c|c|c|}
\hline \multirow{2}{*}{$\begin{array}{l}\text { Polhume } \\
\text { and } \\
\text { CAS Number } \\
\text { (if avaitible) }\end{array}$} & \multicolumn{2}{|c|}{$\begin{array}{l}\text { Meximum Valuce } \\
\text { (inchido unito) }\end{array}$} & \multicolumn{2}{|c|}{$\begin{array}{l}\text { Averago Valuea } \\
\text { (inctudo unita) }\end{array}$} & \multirow{2}{*}{$\begin{array}{l}\text { Number } \\
\text { of } \\
\text { Storm } \\
\text { Evects } \\
\text { Saropled } \\
\text {. . }\end{array}$} & \multirow[b]{2}{*}{ Sourrees of Polhutast } \\
\hline & $\begin{array}{c}\text { Grab Sumplo } \\
\text { Takea During } \\
\text { Fint } 30 \\
\text { Mintec }\end{array}$ & $\begin{array}{l}\text { Flow-weighted } \\
\text { Componito }\end{array}$ & $\begin{array}{l}\text { Greb Samplo } \\
\text { Triken During } \\
\text { Firat } 30 \\
\text { Minute }\end{array}$ & $\begin{array}{l}\text { Flow-meighted } \\
\text { Comporito }\end{array}$ & & \\
\hline 1.1.1. trictibrocthesoc 71-55-6 & $<0.010 \mathrm{mg} / \mathrm{l}$ & & $<0.010 \mathrm{mg} / 1$ & & 1 & \\
\hline 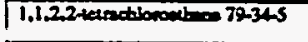 & $<0.010 \mathrm{mg} / \mathrm{l}$ & & $<0.010 \mathrm{mg} / 1$ & & 1 & \\
\hline 1.1.2- trictbocthen $7900-5$ & $<0.010 \mathrm{mg} / 1$ & & $<0.010 \mathrm{mg} / 1$ & & 1 & \\
\hline 1.1 dicblonoculanes 75-34-3 & $<0.010 \mathrm{mg} / 1$ & & $<0.010 \mathrm{mg} / 1$ & & 1 & \\
\hline 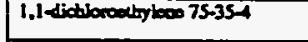 & $<0.010 \mathrm{mg} / \mathrm{l}$ & & $<0.010 \mathrm{mg} / 1$ & & 1 & \\
\hline 1,2-dictioncethes $10706-2$ & $<0.010 \mathrm{mg} / 1$ & & $<0.010 \mathrm{mg} / \mathrm{h}$ & & 1 & \\
\hline 1.2 dictloropiopines $72-87.5$ & $<0.010 \mathrm{mg} / 1$ & & $<0.010 \mathrm{mg} / 1$ & & 1 & \\
\hline 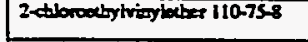 & $<0.010 \mathrm{mg} / 1$ & & $<0.010 \mathrm{mg} / \mathrm{l}$ & & 1 & \\
\hline $71-13-2$ & $<0.010 \mathrm{mg} / 1$ & & $<0.010 \mathrm{mg} / \mathrm{l}$ & & 1 & \\
\hline 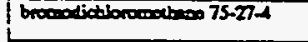 & $<0.010 \mathrm{mg} / 1$ & & $<0.010 \mathrm{mg} / 1$ & & 1 & \\
\hline browodocose 7s-2s-2 & $<0.010 \mathrm{mg} / 1$ & & $<0.010 \mathrm{mg} / 1$ & & $I$ & \\
\hline breomancthes 7483-9 & $<0.010 \mathrm{mg} / 1$ & & $<0.010 \mathrm{mg} / 1$ & & 1 & \\
\hline artan wandibride $56-23-5$ & $<0.010 \mathrm{mg} / \mathrm{l}$ & & $<0.010 \mathrm{mg} / 1$ & & 1 & \\
\hline 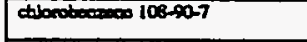 & $<0.010 \mathrm{mg} / 1$ & & $<0.010 \mathrm{mg} / 1$ & & 1 & \\
\hline chlowedhos $75-00-3$ & $<0.010 \mathrm{mg} / \mathrm{l}$ & & $<0.010 \mathrm{mg} / 1$ & & 1 & \\
\hline dibrofocion 67-66-3 & $<0.010 \mathrm{mg} / 1$ & & $<0.010 \mathrm{mg} / 1$ & & 1 & $\vdots$ \\
\hline chlovenethos 7477.3 & $<0.010 \mathrm{mg} / \mathrm{l}$ & & $<0.010 \mathrm{mg} / \mathrm{l}$ & & 1 & \\
\hline 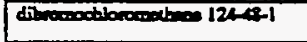 & $<0.010 \mathrm{mg} / 1$ & & $<0.010 \mathrm{mg} / \mathrm{l}$ & & 1 & \\
\hline abylinges 100414 & $<0.010 \mathrm{mg} / \mathrm{l}$ & & $<0.010 \mathrm{mg} / 1$ & & 1 & \\
\hline bothece $108-2=3$ & $<0.010 \mathrm{mg} / 1$ & & $<0.010 \mathrm{mg} / \mathrm{l}$ & & 1 & \\
\hline vingl chocide 75014 & $<0.010 \mathrm{mg} / 1$ & & $<0.010 \mathrm{mg} / \mathrm{l}$ & & 1 & \\
\hline 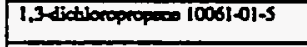 & $<0.010 \mathrm{mg} / 1$ & & $<0.010 \mathrm{mg} / \mathrm{h}$ & . & 1 & \\
\hline 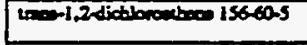 & $<0.010 \mathrm{mg} / 1$ & & $<0.010 \mathrm{mg} / 1$ & & 1 & \\
\hline apten exivity $12587-46-1$ & $3.8 \mathrm{pei} / 1$ & 0.48 peill & 3.8 peill & $0.48 \mathrm{pci} / \mathrm{l}$ & 1 & \\
\hline bete naivity $12587-46-1$ & 6.3 pcill & $7.2 \mathrm{pci} / 1$ & 6.3 pcill & $7.2 \mathrm{pci} / 1$ & 1 & \\
\hline focal coliform & $30 \mathrm{col} .1 \mathrm{~L}$ & $100 \mathrm{co} / .1 \mathrm{~L}$ & $30 \mathrm{co} / .1 \mathrm{~L}$ & $100 \mathrm{co} / .1 \mathrm{~L}$ & 1 & \\
\hline Thellinm 740-220 & $<0.03 \mathrm{mg} / 1$ & $<0.03 \mathrm{mg} / 1$ & $<0.03 \mathrm{mg} / 1$ & $<0.03 \mathrm{mg} / 1$ & 1 & \\
\hline Unasing 740-61-1 & $0: 005 \mathrm{mg} / 1$ & $0.003 \mathrm{mg} / \mathrm{l}$ & $0.005 \mathrm{mg} / \mathrm{l}$ & $0.003 \mathrm{mg} / 1$ & 1 & \\
\hline Gemolonging & 47 peill & 160 pсi/l & 47 pcill & $160 \mathrm{pci} / 1$ & 1 & \\
\hline
\end{tabular}

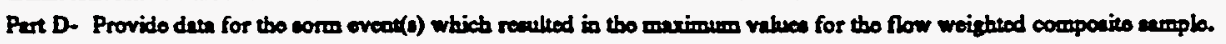

\begin{tabular}{|c|c|c|c|c|c|c|c|}
\hline $\begin{array}{l}1 . \\
\text { Dite of } \\
\text { Storm } \\
\text { Evens }\end{array}$ & $\begin{array}{l}2 . \\
\text { Duration } \\
\text { of Srorm } \\
\text { (in } \\
\text { minutes) }\end{array}$ & $\begin{array}{l}3 . \\
\text { Toul ninfill } \\
\text { during sorm oveot } \\
\text { (in inches) }\end{array}$ & 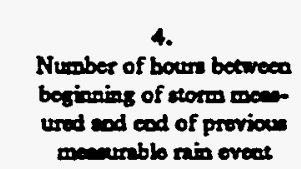 & $\begin{array}{l}\text { S. } \\
\text { Mariming flow nato } \\
\text { durins nin oveet } \\
\text { (Elloadmin or specify } \\
\text { unin) }\end{array}$ & $\begin{array}{l}6 . \\
\text { Total flow from } \\
\text { min evens } \\
\text { (alloos or } \\
\text { peoify unit) }\end{array}$ & $\begin{array}{c}7 . \\
\text { Season } \\
\text { sample wes } \\
\text { taken }\end{array}$ & 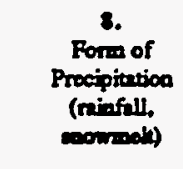 \\
\hline $12 / 9 / 91$ & 530 & 1.1 & 148.6 & 42.4 & 17,694 & Fall & Ruinfll \\
\hline
\end{tabular}

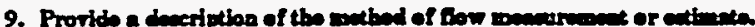

An ISCO 3230 For meter was used to obtain water level readings. This instrument was calibrated with the normal dry weather flow, if present, as level zero. The flow meter was progranmed with the characteristics of the conveyance so that flow rates and total flows conid be calculated by the meter. 
Outfoll 44

Parte B and C Continuad

\begin{tabular}{|c|c|c|c|c|c|c|}
\hline \multirow{2}{*}{$\begin{array}{l}\text { Polhutant } \\
\text { and } \\
\text { CAs Number } \\
\text { (if avaitible) }\end{array}$} & \multicolumn{2}{|c|}{$\begin{array}{l}\text { Meximum Vabuee } \\
\text { (includo unita) }\end{array}$} & \multicolumn{2}{|c|}{$\begin{array}{l}\text { Avenso Vahues } \\
\text { (inchude unita) }\end{array}$} & \multirow{2}{*}{$\begin{array}{l}\text { Number } \\
\text { of } \\
\text { Siond } \\
\text { Eveots } \\
\text { Sempled }\end{array}$} & \multirow[b]{2}{*}{ Sources of Pollutuats } \\
\hline & $\begin{array}{l}\text { Grab Semplo } \\
\text { Taken During } \\
\text { Fint } 30 \\
\text { Minuces }\end{array}$ & $\begin{array}{l}\text { Flow-weighted } \\
\text { Compocico }\end{array}$ & 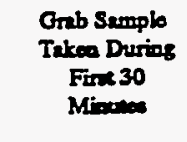 & $\begin{array}{l}\text { Flow-weighted } \\
\text { Componite }\end{array}$ & & \\
\hline Barken 7400-39-3 & $0.0254 \mathrm{mg} / \mathrm{l}$ & $0.0188 \mathrm{mg} / \mathrm{l}$ & $0.0254 \mathrm{mg} / 1$ & $0.0188 \mathrm{mg} / 1$ & 1 & \\
\hline 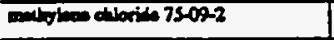 & $<0.010 \mathrm{mg} / 1$ & & $<0.010 \mathrm{mg} / \mathrm{l}$ & & 1 & \\
\hline Letactiboocheos $127-184$ & $<0.010 \mathrm{mg} / \mathrm{l}$ & & $<0.010 \mathrm{mg} / 1$ & & 1 & \\
\hline 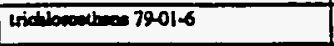 & $<0.010 \mathrm{mg} / \mathrm{l}$ & & $<0.010 \mathrm{mg} / \mathrm{h}$ & & 1 & \\
\hline$x y=$ & $<0.010 \mathrm{mg} / \mathrm{l}$ & & $<0.010 \mathrm{mg} / 1$ & & 1 & \\
\hline 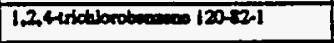 & $<0.010 \mathrm{mg} / \mathrm{l}$ & $<0.010 \mathrm{mg} / \mathrm{l}$ & $<0.010 \mathrm{mg} / \mathrm{l}$ & $<0.010 \mathrm{mg} / \mathrm{l}$ & 1 & \\
\hline 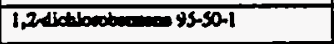 & $<0.010 \mathrm{mg} / \mathrm{l}$ & $<0.010 \mathrm{mg} / 1$ & $<0.010 \mathrm{mg} / \mathrm{l}$ & $<0.010 \mathrm{mg} / \mathrm{l}$ & 1 & \\
\hline 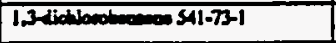 & $<0.010 \mathrm{mg} / \mathrm{l}$ & $<0.010 \mathrm{mg} / \mathrm{l}$ & $<0.010 \mathrm{mg} / 1$ & $<0.010 \mathrm{mg} / 1$ & 1 & \\
\hline 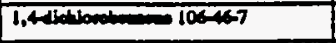 & $<0.010 \mathrm{mg} / \mathrm{l}$ & $<0.010 \mathrm{mg} / 1$ & $<0.010 \mathrm{mg} / \mathrm{l}$ & $<0.010 \mathrm{mg} / 1$ & 1 & \\
\hline 2,4 trictroduman $121-14-2$ & $<0.010 \mathrm{mq} / \mathrm{l}$ & $<0.010 \mathrm{mg} / 1$ & $<0.010 \mathrm{mg} / \mathrm{l}$ & $<0.010 \mathrm{mg} / 1$ & 1 & \\
\hline 2,6 divilocedheren $605-202-2$ & $<0.010 \mathrm{mg} / \mathrm{l}$ & $<0.010 \mathrm{mg} / \mathrm{l}$ & $<0.010 \mathrm{mg} / \mathrm{l}$ & $<0.010 \mathrm{mg} / \mathrm{l}$ & 1 & \\
\hline 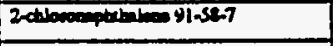 & $<0.010 \mathrm{mg} / 1$ & $<0.010 \mathrm{mg} / 1$ & $<0.010 \mathrm{mg} / \mathrm{l}$ & $<0.010 \mathrm{mg} / \mathrm{l}$ & 1 & \\
\hline 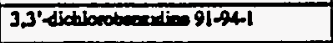 & $<0.010 \mathrm{mg} / 1$ & $<0.010 \mathrm{mg} / \mathrm{l}$ & $<0.010 \mathrm{mg} / \mathrm{l}$ & $<0.010 \mathrm{mg} / 1$ & 1 & \\
\hline 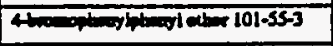 & $<0.010 \mathrm{mg} / \mathrm{l}$ & $<0.010 \mathrm{mg} / 1$ & $<0.010 \mathrm{mg} / \mathrm{l}$ & $<0.010 \mathrm{mg} / \mathrm{l}$ & 1 & \\
\hline 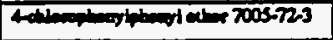 & $<0.010 \mathrm{mg} / \mathrm{I}$ & $<0.010 \mathrm{mg} / \mathrm{l}$ & $<0.010 \mathrm{mg} / 1$ & $<0.010 \mathrm{mg} / \mathrm{l}$ & 1 & \\
\hline 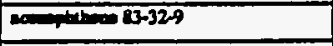 & $<0.010 \mathrm{mg} / 1$ & $<0.010 \mathrm{mg} / \mathrm{l}$ & $<0.010 \mathrm{mg} / \mathrm{l}$ & $<0.010 \mathrm{mg} / 1$ & 1 & \\
\hline 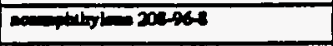 & $<0.010 \mathrm{mg} / 1$ & $<0.010 \mathrm{mg} / 1$ & $<0.010 \mathrm{mg} / 1$ & $<0.010 \mathrm{mg} / \mathrm{l}$ & 1 & \\
\hline$-120-12.7$ & $<0.010 \mathrm{mg} / 1$ & $<0.010 \mathrm{mg} / \mathrm{h}$ & $<0.010 \mathrm{mg} / 1$ & $<0.010 \mathrm{mg} / 1$ & 1 & \\
\hline nitim 927.5 & $<0.050 \mathrm{mg} / \mathrm{l}$ & $<0.050 \mathrm{mg} / \mathrm{l}$ & $<0.050 \mathrm{mg} / \mathrm{I}$ & $<0.050 \mathrm{mg} / \mathrm{l}$ & 1 & \\
\hline 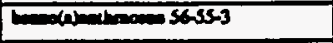 & $<0.010 \mathrm{mg} / \mathrm{l}$ & $<0.010 \mathrm{mg} / \mathrm{l}$ & $<0.010 \mathrm{mg} / 1$ & $<0.010 \mathrm{mg} / 1$ & 1 & \\
\hline 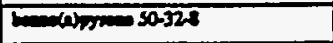 & $<0.010 \mathrm{mg} / \mathrm{l}$ & $<0.010 \mathrm{mg} / \mathrm{l}$ & $<0.010 \mathrm{mg} / 1$ & $<0.010 \mathrm{mg} / \mathrm{l}$ & 1 & \\
\hline 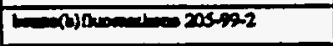 & $<0.010 \mathrm{mpg} / 1$ & $<0.010 \mathrm{mg} / \mathrm{l}$ & $<0.010 \mathrm{mg} / \mathrm{l}$ & $<0.010 \mathrm{mg} / 1$ & 1 & \\
\hline 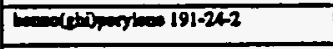 & $<0.010 \mathrm{mg} / \mathrm{l}$ & $<0.010 \mathrm{mg} / \mathrm{l}$ & $<0.010 \mathrm{mg} / \mathrm{l}$ & $<0.010 \mathrm{mg} / 1$ & 1 & \\
\hline 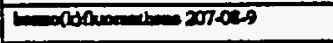 & $<0.010 \mathrm{mg} / 1$ & $<0.010 \mathrm{mg} / \mathrm{l}$ & $<0.010 \mathrm{mg} / \mathrm{l}$ & $<0.010 \mathrm{mg} / 1$ & 1 & \\
\hline 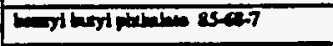 & $<0.010$ ug/l & $<0.010 \mathrm{mg} / \mathrm{l}$ & $<0.010 \mathrm{mg} / \mathrm{h}$ & $<0.010 \mathrm{mg} / 1$ & 1 & \\
\hline 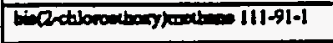 & $<0.010 \mathrm{mg} / 1$ & $<0.010 \mathrm{mg} / \mathrm{l}$ & $<0.010 \mathrm{mg} / 1$ & $<0.010 \mathrm{mg} / \mathrm{l}$ & 1 & \\
\hline 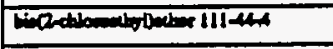 & $<0.010 \mathrm{mg} / 1$ & $<0.010 \mathrm{mg} / \mathrm{l}$ & $<0.010 \mathrm{mg} / \mathrm{l}$ & $<0.010 \mathrm{mg} / \mathrm{l}$ & 1 & \\
\hline 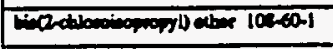 & $<0.010 \mathrm{mg} / \mathrm{l}$ & $<0.010 \mathrm{mg} / \mathrm{l}$ & $<0.010 \mathrm{mg} / 1$ & $<0.010 \mathrm{mg} / \mathrm{l}$ & 1 & \\
\hline 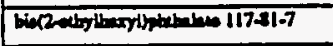 & $<0.010 \mathrm{mg} / 1$ & $<0.010 \mathrm{mg} / 1$ & $<0.010 \mathrm{mg} / \mathrm{l}$ & $<0.010 \mathrm{mg} / \mathrm{ll}$ & 1 & \\
\hline कyणn 215्णान & $<0.010 \mathrm{mg} / 1$ & $<0.010 \mathrm{mg} / \mathrm{l}$ & $<0.010 \mathrm{mg} / 1$ & $<0.010 \mathrm{mg} / 1$ & 1 & \\
\hline 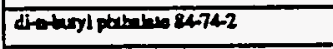 & $<0.010 \mathrm{mg} / \mathrm{l}$ & $<0.010 \mathrm{mg} / \mathrm{l}$ & $<0.010 \mathrm{mg} / 1$ & $<0.010 \mathrm{mg} / \mathrm{l}$ & 1 & \\
\hline 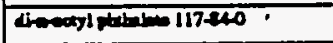 & $<0.010 \mathrm{mg} / 1$ & $<0.010 \mathrm{mg} / 1$ & $<0.010 \mathrm{mg} / 1$ & $<0.010 \mathrm{mg} / 1$ & 1 & \\
\hline 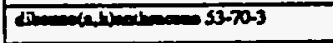 & $<0.010 \mathrm{mg} / \mathrm{l}$ & $<0.010 \mathrm{mg} / 1$ & $<0.010 \mathrm{mg} / \mathrm{l}$ & $<0.010 \mathrm{mg} / 1$ & 1 & \\
\hline 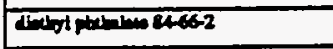 & $<0.010 \mathrm{mg} / \mathrm{l}$ & $<0.010 \mathrm{mg} / \mathrm{l}$ & $<0.010 \mathrm{mg} / \mathrm{l}$ & $<0.010 \mathrm{mg} / 1$ & 1 & \\
\hline 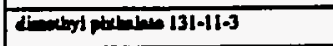 & $<0.010 \mathrm{mg} / 1$ & $<0.010 \mathrm{mg} / \mathrm{l}$ & $<0.010 \mathrm{mg} / \mathrm{l}$ & $<0.010 \mathrm{mg} / 1$ & 1 & \\
\hline $020=206440$ & $<0.010 \mathrm{mg} / \mathrm{l}$ & $<0.010 \mathrm{mg} / 1$ & $<0.010 \mathrm{mg} / \mathrm{l}$ & $<0.010 \mathrm{mg} / \mathrm{l}$ & $I$ & \\
\hline $040000-86-73-7$ & $<0.010 \mathrm{mg} / \mathrm{l}$ & $<0.010 \mathrm{mg} / 1$ & $<0.010 \mathrm{mg} / \mathrm{l}$ & $<0.010 \mathrm{mg} / \mathrm{l}$ & 1 & \\
\hline 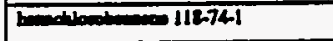 & $<0.010 \mathrm{mg} / 1$ & $<0.010 \mathrm{mg} / 1$ & $<0.010 \mathrm{mg} / \mathrm{l}$ & $<0.010 \mathrm{mg} / \mathrm{l}$ & 1 & \\
\hline 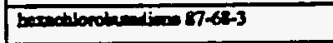 & $<0.010 \mathrm{mg} / \mathrm{l}$ & $<0.010 \mathrm{mg} / \mathrm{l}$ & $<0.010 \mathrm{mg} / 1$ & $<0.010 \mathrm{mg} / \mathrm{h}$ & 1 & \\
\hline 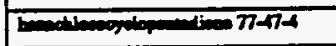 & $<0.010 \mathrm{mg} / \mathrm{l}$ & $<0.010 \mathrm{mg} / 1$ & $<0.010 \mathrm{mg} / \mathrm{l}$ & $<0.010 \mathrm{mg} / \mathrm{l}$ & 1 & \\
\hline 67.72 .1 & $<0.010 \mathrm{mg} / \mathrm{l}$ & $<0.010 \mathrm{mg} / \mathrm{l}$ & $<0.010 \mathrm{mg} / \mathrm{l}$ & $<0.010 \mathrm{mg} / 1$ & 1 & \\
\hline 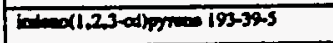 & $<0.010 \mathrm{mg} / \mathrm{l}$ & $<0.010 \mathrm{mg} / \mathrm{l}$ & $<0.010 \mathrm{mg} / 1$ & $<0.010 \mathrm{mg} / \mathrm{l}$ & 1 & \\
\hline 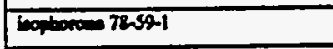 & $<0.010 \mathrm{mg} / \mathrm{l}$ & $<0.010 \mathrm{mg} / 1$ & $<0.010 \mathrm{mg} / 1$ & $<0.010 \mathrm{mg} / \mathrm{l}$ & 1 & \\
\hline 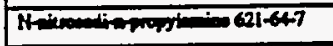 & $<0.010 \mathrm{mg} / \mathrm{l}$ & $<0.010 \mathrm{mg} / 1$ & $<0.010 \mathrm{mg} / 1$ & $<0.010 \mathrm{mg} / \mathrm{l}$ & 1 & \\
\hline 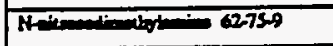 & $<0.010 \mathrm{mg} / \mathrm{l}$ & $<0.010 \mathrm{mg} / \mathrm{l}$ & $<0.010 \mathrm{mg} / \mathrm{l}$ & $<0.010 \mathrm{mg} / \mathrm{h}$ & 1 & \\
\hline 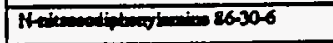 & $<0.010 \mathrm{mg} / \mathrm{l}$ & $<0.010 \mathrm{mg} / 1$ & $<0.010 \mathrm{mg} / \mathrm{l}$ & $<0.010 \mathrm{mg} / 1$ & 1 & \\
\hline antelaios 91-20-3 & $<0.010 \mathrm{mg} / \mathrm{l}$ & $<0.010 \mathrm{mg} / \mathrm{l}$ & $<0.010 \mathrm{mg} / \mathrm{l}$ & $<0.010 \mathrm{mg} / \mathrm{l}$ & 1 & \\
\hline 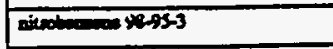 & $<0.010 \mathrm{mg} / \mathrm{l}$ & $<0.010 \mathrm{mg} / \mathrm{l}$ & $<0.010 \mathrm{mg} / \mathrm{h}$ & $<0.010 \mathrm{mg} / \mathrm{l}$ & 1 & \\
\hline $5=25014$ & $<0.010 \mathrm{mg} / \mathrm{l}$ & $<0.010 \mathrm{mg} / \mathrm{l}$ & $<0.010 \mathrm{mg} / \mathrm{l}$ & $<0.010 \mathrm{mg} / \mathrm{l}$ & 1 & \\
\hline prome- 1220000 & $<0.010 \mathrm{mg} / 1$ & $<0.010 \mathrm{mg} / 1$ & $<0.010 \mathrm{mg} / 1$ & $<0.010 \mathrm{mg} / \mathrm{l}$ & 1 & \\
\hline 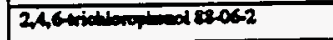 & $<0.030 \mathrm{mg} / \mathrm{l}$ & $<0.030 \mathrm{mg} / \mathrm{l}$ & $<0.030 \mathrm{mg} / \mathrm{h}$ & $<0.030 \mathrm{mg} / \mathrm{l}$ & 1 & \\
\hline 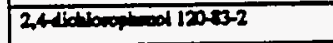 & $<0.030 \mathrm{mg} / 1$ & $<0.030 \mathrm{mg} / \mathrm{l}$ & $<0.030 \mathrm{mg} / \mathrm{l}$ & $<0.030 \mathrm{mg} / \mathrm{l}$ & 1 & \\
\hline 2.400105679 & $<0.030 \mathrm{mg} / \mathrm{l}$ & $<0.030 \mathrm{mg} / \mathrm{l}$ & $<0.030 \mathrm{mg} / \mathrm{l}$ & $<0.030 \mathrm{mg} / \mathrm{l}$ & 1 & \\
\hline 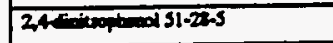 & $<0.050 \mathrm{mg} / !$ & $<0.050 \mathrm{mg} / \mathrm{l}$ & $<0.050 \mathrm{mg} / \mathrm{I}$ & $<0.050 \mathrm{mg} / \mathrm{l}$ & 1 & \\
\hline 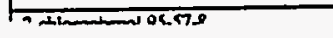 & $\operatorname{lnn} 2 n \operatorname{man}$ & $\operatorname{lnmanman}$ & $\operatorname{lnman} m a n$ & en man moll & 1 & \\
\hline
\end{tabular}


Outrall 44

Para B and C Coatinuod

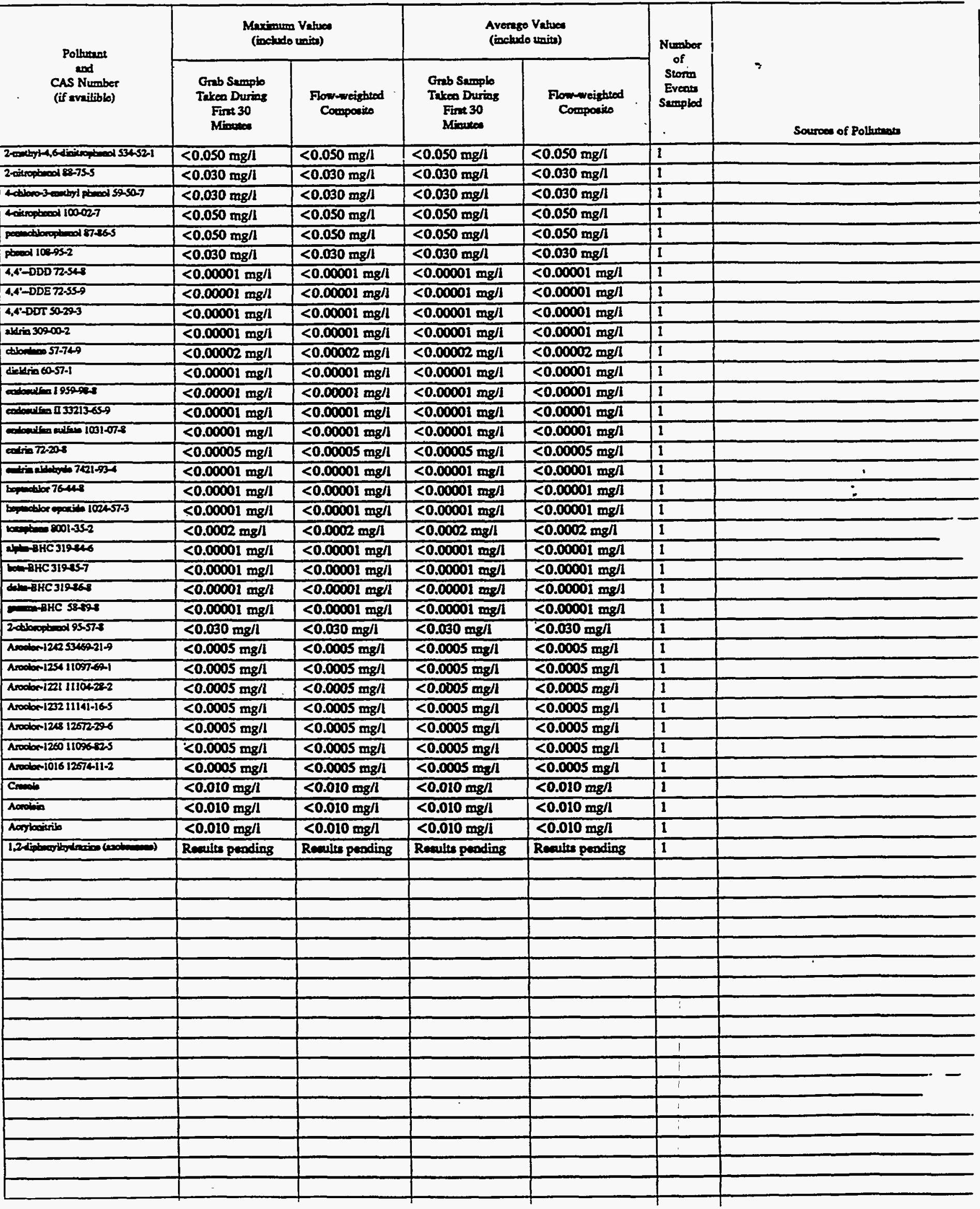




\begin{tabular}{|c|c|c|c|c|c|c|}
\hline \multirow{2}{*}{$\begin{array}{l}\text { Pollumant } \\
\text { and } \\
\text { CAS Number } \\
\text { (if availible) }\end{array}$} & \multicolumn{2}{|c|}{$\begin{array}{l}\text { Maximum Vabues } \\
\text { (ixchude waits) }\end{array}$} & \multicolumn{2}{|c|}{$\begin{array}{l}\text { Averse Valaes } \\
\text { (inchude uniey) }\end{array}$} & \multirow{2}{*}{$\begin{array}{l}\text { Norober } \\
\text { of } \\
\text { Sloom } \\
\text { Everom } \\
\text { Serplad }\end{array}$} & \multirow{2}{*}{$\begin{array}{l}7 . \\
\text { Soorese of Pollowents }\end{array}$} \\
\hline & 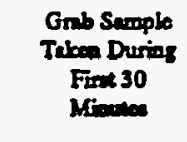 & $\begin{array}{l}\text { Flow-wrighted } \\
\text { Compotive }\end{array}$ & 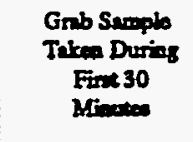 & $\begin{array}{c}\text { Fommeighed } \\
\text { Compoive }\end{array}$ & & \\
\hline Pil & $3 \mathrm{mg} /$ & & $3 \mathrm{mg} / 1$ & & 1 & \\
\hline Piolodical Oxyer Dened (BODS) & $<5 \mathrm{mg} / \mathrm{h}$ & $<5 \mathrm{mg} / 1$ & $<5 \mathrm{mg} / \mathrm{l}$ & $<5 \mathrm{mg} / \mathrm{l}$ & 1 & \\
\hline Chem $0 x y=D=d(C O D)$ & $5.9 \mathrm{mg} / \mathrm{l}$ & $11 \mathrm{mg} / 1$ & $5.9 \mathrm{mg} / 1$ & $11 \mathrm{mg} / 1$ & 1 & \\
\hline Poel supinder solsh (TSS) & $5.5 \mathrm{mg} / \mathrm{l}$ & $6.0 \mathrm{mg} / \mathrm{l}$ & $5.5 \mathrm{mg} / 1$ & $6.0 \mathrm{mg} / \mathrm{I}$ & 1 & \\
\hline 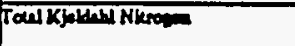 & $0.2 \mathrm{mg} / \mathrm{h}$ & $<0.2 \mathrm{mg} / \mathrm{l}$ & $0.2 \mathrm{mg} / 1$ & $<0.2 \mathrm{mg} / \mathrm{l}$ & 1 & \\
\hline 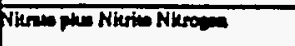 & $0.66 \mathrm{mg} / 1$ & $0.33 \mathrm{mg} / \mathrm{l}$ & $0.66 \mathrm{mg} / \mathrm{l}$ & $0.33 \mathrm{mg} / \mathrm{l}$ & 1 & \\
\hline Poell reopionem & $<0.1 \mathrm{mg} / 1$ & $<0.1 \mathrm{mg} / \mathrm{I}$ & $<0.1 \mathrm{mg} / \mathrm{l}$ & $<0.1 \mathrm{mg} / \mathrm{l}$ & 1 & \\
\hline br & Mintan 8.3 & Modimen 8.3 & Maimen 8.3 & $M a d=8.3$ & & \\
\hline
\end{tabular}

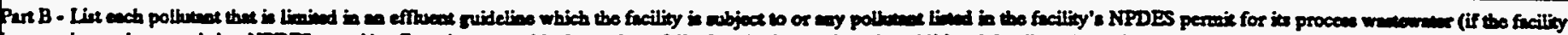

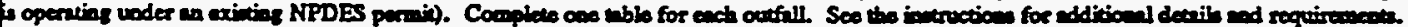

\begin{tabular}{|c|c|c|c|c|c|c|}
\hline \multirow{2}{*}{$\begin{array}{l}\text { Potluneme } \\
\text { and } \\
\text { CAs Nimber } \\
\text { (if milible) }\end{array}$} & \multicolumn{2}{|c|}{$\begin{array}{l}\text { Mexinam Vabee } \\
\text { (include unite) }\end{array}$} & \multicolumn{2}{|c|}{ 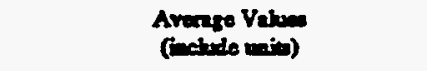 } & \multirow{2}{*}{ 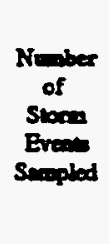 } & \multirow[b]{2}{*}{ 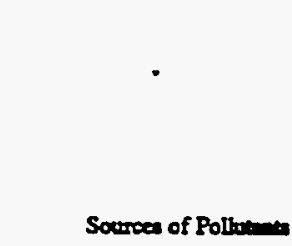 } \\
\hline & 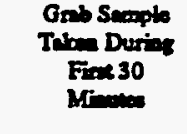 & $\begin{array}{l}\text { Flowneighed } \\
\text { Compocies }\end{array}$ & 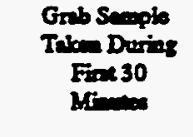 & $\begin{array}{l}\text { Flon-meighed } \\
\text { Conponise }\end{array}$ & & \\
\hline 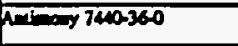 & $<0.04 \mathrm{mg} / 1$ & $<0.04 \mathrm{mg} /$ & $<0.04 \mathrm{mg} / 1$ & $<0.04 \mathrm{mg} / \mathrm{h}$ & 1 & \\
\hline Fins & $<0.002 \mathrm{mg} / 1$ & & $<0.002 \mathrm{mg} / 1$ & & 1 & \\
\hline 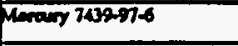 & $<0.0002 \mathrm{mg} / \mathrm{l}$ & $<0.0002 \mathrm{mg} / \mathrm{h}$ & $<0.0002 \mathrm{mg} / \mathrm{l}$ & $<0.0002 \mathrm{mg} / 1$ & 1 & \\
\hline thende & $0.003 \mathrm{mg} / \mathrm{l}$ & & $0.003 \mathrm{mg} / \mathrm{l}$ & & 1 & \\
\hline 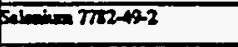 & $<0.002 \mathrm{mg} / \mathrm{l}$ & $<0.002 \mathrm{mg} / \mathrm{l}$ & $<0.002 \mathrm{mg} / 1$ & $<0.002 \mathrm{mg} / \mathrm{h}$ & 1 & \\
\hline sutame & $11 \mathrm{mg} / \mathrm{h}$ & $<10 \mathrm{mg} / \mathrm{h}$ & $11 \mathrm{mg} / 1$ & $<10 \mathrm{mg} / 1$ & 1 & \\
\hline Surteceneses & $<0.05 \mathrm{mg} / 1$ & $<0.05 \mathrm{mg} / 1$ & $<0.05 \mathrm{mg} / \mathrm{l}$ & $<0.05 \mathrm{mg} / \mathrm{h}$ & 1 & \\
\hline 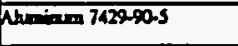 & $0.38 \mathrm{mg} / \mathrm{l}$ & $0.29 \mathrm{mg} / \mathrm{h}$ & $0.38 \mathrm{mg} / \mathrm{l}$ & $0.29 \mathrm{mg} / \mathrm{l}$ & 1 & \\
\hline $10 \sin 740-32-2$ & $<0.04 \mathrm{mg} / \mathrm{l}$ & $<0.04 \mathrm{mg} / 1$ & $<0.04 \mathrm{mg} / \mathrm{l}$ & $<0.04 \mathrm{mg} /$ & 1 & \\
\hline 300007400008 & $0.011 \mathrm{mg} / \mathrm{l}$ & $0.013 \mathrm{mg} / \mathrm{l}$ & $0.011 \mathrm{mg} / 1$ & 0.013 men & 1 & \\
\hline Berflliem 740-11.7 & $<0.0004 \mathrm{mg} / \mathrm{l}$ & $<0.0004 \mathrm{mg} / \mathrm{l}$ & $<0.0004 \mathrm{mg} / \mathrm{l}$ & $<0.0004 m g / h$ & 1 & \\
\hline Codaine 740-13-9 & $<0.004 \mathrm{mg} / \mathrm{l}$ & $<0.004 \mathrm{mg} / 1$ & $<0.004 \mathrm{mg} / 1$ & $<0.004 \mathrm{mg} / \mathrm{l}$ & 1 & \\
\hline 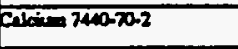 & $10.5 \mathrm{mg} / 1$ & $11.2 \mathrm{mg} / \mathrm{l}$ & $10.5 \mathrm{mg} / \mathrm{l}$ & $11.2 \mathrm{mg} / \mathrm{h}$ & 1 & \\
\hline 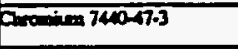 & $<0.006 \mathrm{mg} / \mathrm{l}$ & $<0.006 \mathrm{mg} / 1$ & $<0.006 \mathrm{mg} / 1$ & $<0.006 \mathrm{mg} /$ & 1 & \\
\hline Cotakik 740-424 & $<0.002 \mathrm{mg} / \mathrm{l}$ & $<0.002 \mathrm{mg} / \mathrm{ll}$ & $<0.002 \mathrm{mg} / 1$ & $<0.002 \mathrm{mg} / \mathrm{l}$ & 1 & \\
\hline Corpen 740-50s & $<0.006 \mathrm{mg} / \mathrm{l}$ & $<0.006 \mathrm{mg} / \mathrm{l}$ & $<0.006 \mathrm{mg} /$ & $<0.006 \mathrm{mg} / \mathrm{h}$ & 1 & \\
\hline 5007439256 & $0.47 \mathrm{mg} / \mathrm{l}$ & $0.36 \mathrm{mg} / \mathrm{l}$ & $0.47 \mathrm{mg} / \mathrm{l}$ & $0.36 \mathrm{mg} / \mathrm{f}$ & 1 & \\
\hline 7017392921 & $<0.02 \mathrm{mg} / \mathrm{l}$ & $<0.02 \mathrm{mg} / 1$ & $<0.02 \mathrm{mg} / 1$ & $<0.02 \mathrm{mg} / 1$ & 1 & . \\
\hline Inthe-7/39-93-2 & $<0.02 \mathrm{mg} / \mathrm{l}$ & $<0.02 \mathrm{mg} / 1$ & $<0.02 \mathrm{mg} h$ & $<0.02 \mathrm{mg} / \mathrm{l}$ & 1 & \\
\hline Meanowing 7439054 & $0.79 \mathrm{mg} / \mathrm{l}$ & $0.96 \mathrm{mg} / \mathrm{l}$ & $0.79 \mathrm{mg} / 1$ & $0.96 \mathrm{mq} / \mathrm{h}$ & 1 & \\
\hline 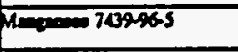 & $0.011 \mathrm{mg} / 1$ & $0.008 \mathrm{mg} / \mathrm{l}$ & $0.011 \mathrm{mg} / \mathrm{l}$ & $0.008 \mathrm{mg} / \mathrm{l}$ & 1 & \\
\hline 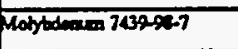 & $<0.006 \mathrm{mg} / \mathrm{l}$ & $<0.006 \mathrm{mg} / \mathrm{l}$ & $<0.006 \mathrm{mg} / \mathrm{l}$ & $<0.006 \mathrm{mg} / \mathrm{h}$ & 1 & \\
\hline Nikdel 740-020 & $<0.008 \mathrm{mg} / \mathrm{l}$ & $<0.008 \mathrm{mg} / \mathrm{h}$ & $<0.008 \mathrm{mg} / \mathrm{l}$ & $<0.008 \mathrm{mg} / \mathrm{l}$ & 1 & \\
\hline Pamenim 740027 & $<0.5 \mathrm{mg} / 1$ & $<0.5 \mathrm{mg} / \mathrm{l}$ & $<0.5 \mathrm{mg} / \mathrm{l}$ & $<0.5 \mathrm{mg} / \mathrm{h}$ & 1 & \\
\hline Pither 740ar2an & $<0.006 \mathrm{mg} / \mathrm{l}$ & $<0.006 \mathrm{mg} / \mathrm{l}$ & $<0.006 \mathrm{mg} / \mathrm{l}$ & $<0.006 \mathrm{mg} / \mathrm{h}$ & 1 & \\
\hline Poditem 740-20.5 & $0.25 \mathrm{mg} / 1$ & $0.28 \mathrm{mg} / \mathrm{h}$ & $0.25 \mathrm{mg} / \mathrm{h}$ & $0.28 \mathrm{mg} / \mathrm{h}$ & 1 & \\
\hline Fienden $7400-326$ & $<0.01 \mathrm{mg} / \mathrm{l}$ & $<0.01 \mathrm{mg} / \mathrm{l}$ & $<0.01 \mathrm{mg} / \mathrm{l}$ & $<0.01 \mathrm{mg} / \mathrm{l}$ & 1 & \\
\hline $2200740266-6$ & $0.17 \mathrm{mg} / \mathrm{I}$ & $0.13 \mathrm{mg} / \mathrm{l}$ & $0.17 \mathrm{mg} / \mathrm{l}$ & $0.13 \mathrm{mg} / 1$ & 1 & \\
\hline
\end{tabular}


Coothoud from frove.

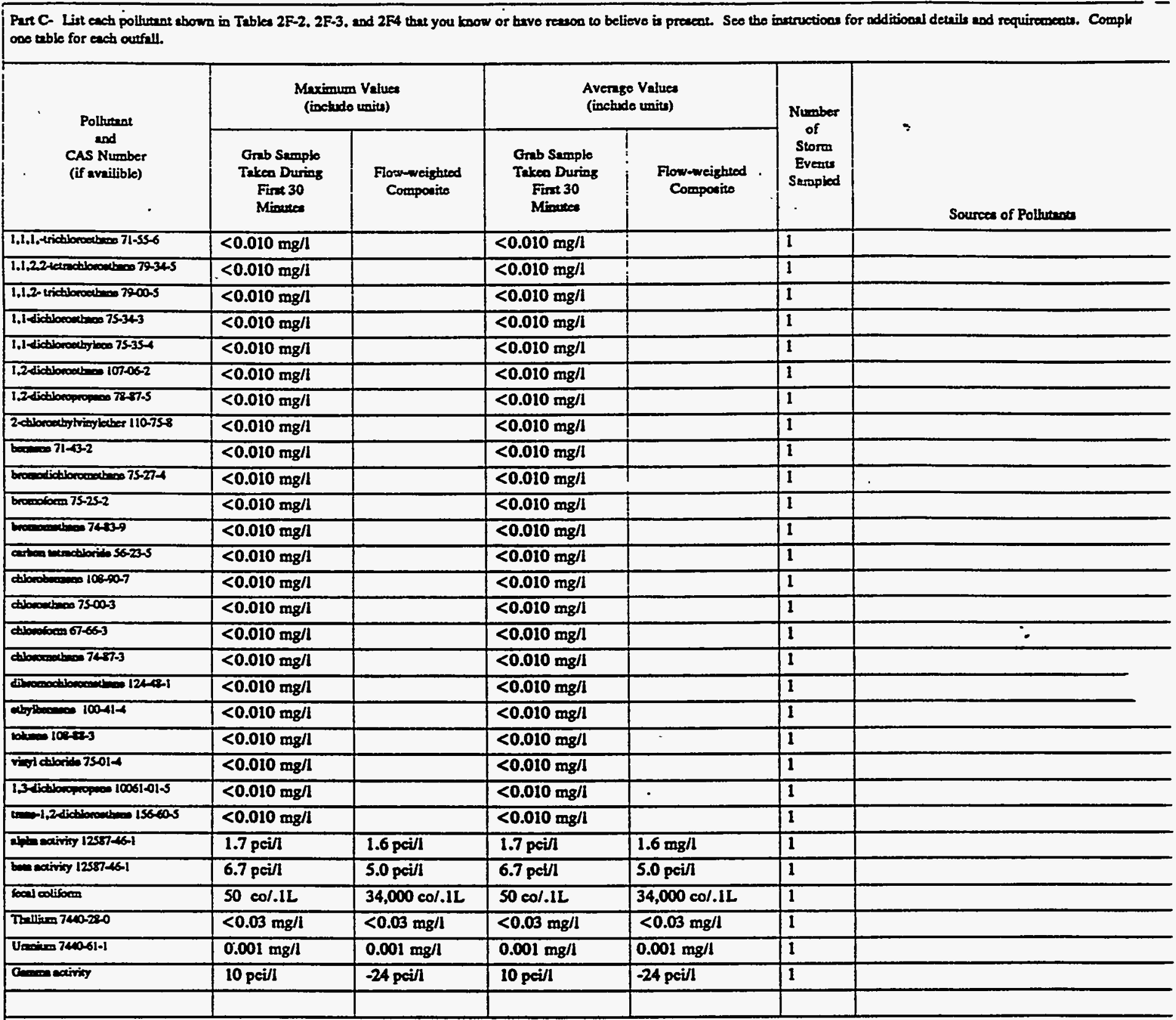

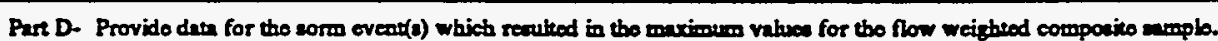

\begin{tabular}{|c|c|c|c|c|c|c|c|}
\hline $\begin{array}{l}1 . \\
\text { Dats of } \\
\text { Storm } \\
\text { Eveat }\end{array}$ & $\begin{array}{l}2 . \\
\text { Duntion } \\
\text { of Storm } \\
\text { (in } \\
\text { minten) }\end{array}$ & $\begin{array}{l}3 . \\
\text { Towil minfill } \\
\text { during stomen event } \\
\text { (in inchea) }\end{array}$ & 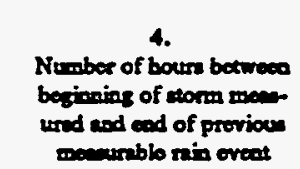 & 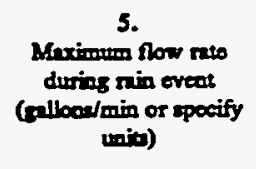 & $\begin{array}{l}6 . \\
\text { Total flow from } \\
\text { min evect } \\
\text { (gilloes or } \\
\text { epecify tenits) }\end{array}$ & $\begin{array}{l}7 . \\
\text { Semon } \\
\text { sample wes } \\
\text { ulbed }\end{array}$ & $\begin{array}{l}\text { Porm of } \\
\text { Preoipitution } \\
\text { (niafall, } \\
\text { commale) }\end{array}$ \\
\hline $12 / 9 / 91$ & 530 & 1.1 & 148.6 & 282 & 104,002 & Fall & Rrinfall \\
\hline
\end{tabular}

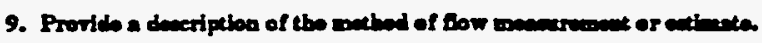

An ISCO 3230 Fow meter was med to obtain water level reatings. This instrumeat was catibrated with the normal dry weather flow, if present, as level zero. The flow meter was programmed with the charecteritios of the conveyance so that flow rates and total flow conld be calcalated by the renter. 
Outfoll 45

Parts B. and C Conciausd

\begin{tabular}{|c|c|c|c|c|c|c|}
\hline \multirow{2}{*}{$\begin{array}{l}\text { Pollutant } \\
\text { and } \\
\text { CAS Number } \\
\text { (if availible) }\end{array}$} & \multicolumn{2}{|c|}{$\begin{array}{l}\text { Meximum Values } \\
\text { (inctudo units) }\end{array}$} & \multicolumn{2}{|c|}{$\begin{array}{l}\text { Average Values } \\
\text { (include writs) }\end{array}$} & \multirow{2}{*}{$\begin{array}{c}\text { Number } \\
\text { of } \\
\text { Storm } \\
\text { Everst } \\
\text { Sempled }\end{array}$} & \multirow[b]{2}{*}{ Sources of Pollutunts } \\
\hline & $\begin{array}{l}\text { Grab Sample } \\
\text { Taken During } \\
\text { First } 30 \\
\text { Minute }\end{array}$ & $\begin{array}{l}\text { Flow-weighted } \\
\text { Componite }\end{array}$ & $\begin{array}{l}\text { Grab Samplo } \\
\text { Takea During } \\
\text { Firt } 30 \\
\text { Minutat }\end{array}$ & $\begin{array}{c}\text { Flow-weightod } \\
\text { Composito }\end{array}$ & & \\
\hline Beriven 7440-393 & $0.0102 \mathrm{mg} / \mathrm{l}$ & $0.0093 \mathrm{mg} / \mathrm{l}$ & $0.0102 \mathrm{mg} / 1$ & $0.0093 \mathrm{mg} / \mathrm{l}$ & 1 & \\
\hline molbyluos ctibride 75-02-2 & $<0.010 \mathrm{mg} / \mathrm{l}$ & & $<0.010 \mathrm{mg} / \mathrm{l}$ & & 1 & \\
\hline Leanctibreobecos 127.184 & $<0.010 \mathrm{mg} / \mathrm{l}$ & & $<0.010 \mathrm{mg} / \mathrm{l}$ & & 1 & \\
\hline 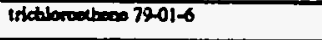 & $<0.010 \mathrm{mg} / \mathrm{l}$ & & $<0.010 \mathrm{mg} / 1$ & & 1 & \\
\hline xylomen & $<0.010 \mathrm{mg} / \mathrm{l}$ & & $<0.010 \mathrm{mg} / \mathrm{l}$ & & 1 & \\
\hline 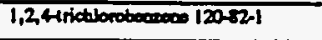 & $<0.010 \mathrm{mg} / \mathrm{l}$ & $<0.010 \mathrm{mg} / \mathrm{l}$ & $<0.010 \mathrm{mg} / \mathrm{l}$ & $<0.010 \mathrm{mg} / \mathrm{l}$ & 1 & \\
\hline 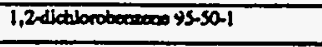 & $<0.010 \mathrm{mg} / \mathrm{l}$ & $<0.010 \mathrm{mg} / 1$ & $<0.010 \mathrm{mg} / \mathrm{l}$ & $<0.010 \mathrm{mg} / \mathrm{l}$ & 1 & \\
\hline 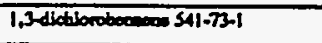 & $<0.010 \mathrm{mg} / \mathrm{l}$ & $<0.010 \mathrm{mg} / 1$ & $<0.010 \mathrm{mg} / \mathrm{l}$ & $<0.010 \mathrm{mg} / 1$ & 1 & \\
\hline 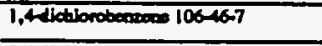 & $<0.010 \mathrm{mg} / \mathrm{l}$ & $<0.010 \mathrm{mg} / 1$ & $<0.010 \mathrm{mg} / \mathrm{l}$ & $<0.010 \mathrm{mg} / \mathrm{l}$ & 1 & \\
\hline 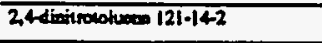 & $<0.010 \mathrm{mg} / \mathrm{l}$ & $<0.010 \mathrm{mg} / \mathrm{l}$ & $<0.010 \mathrm{mg} / 1$ & $<0.010 \mathrm{mg} / \mathrm{l}$ & 1 & \\
\hline 26-dinitrolohores $606-20.2$ & $<0.010 \mathrm{mg} / \mathrm{l}$ & $<0.010 \mathrm{mg} / 1$ & $<0.010 \mathrm{mg} / \mathrm{l}$ & $<0.010 \mathrm{mg} / \mathrm{l}$ & 1 & \\
\hline 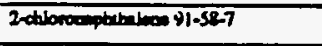 & $<0.010 \mathrm{mg} / 1$ & $<0.010 \mathrm{mg} / 1$ & $<0.010 \mathrm{mg} / \mathrm{l}$ & $<0.010 \mathrm{mg} / 1$ & 1 & \\
\hline 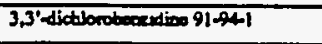 & $<0.010 \mathrm{mg} / \mathrm{l}$ & $<0.010 \mathrm{mg} / 1$ & $<0.010 \mathrm{mg} / 1$ & $<0.010 \mathrm{mg} / \mathrm{l}$ & 1 & \\
\hline 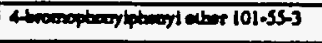 & $<0.010 \mathrm{mg} / \mathrm{l}$ & $<0.010 \mathrm{mg} / 1$ & $<0.010 \mathrm{mg} / 1$ & $<0.010 \mathrm{mg} / \mathrm{l}$ & 1 & \\
\hline 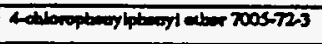 & $<0.010 \mathrm{mg} / \mathrm{l}$ & $<0.010 \mathrm{mg} / \mathrm{l}$ & $<0.010 \mathrm{mg} / 1$ & $<0.010 \mathrm{mg} / 1$ & 1 & \\
\hline 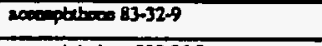 & $<0.010 \mathrm{mg} / 1$ & $<0.010 \mathrm{mg} / 1$ & $<0.010 \mathrm{mg} / \mathrm{l}$ & $<0.010 \mathrm{mg} / \mathrm{l}$ & 1 & \\
\hline 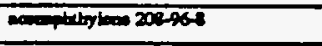 & $<0.010 \mathrm{mg} / 1$ & $<0.010 \mathrm{mg} / 1$ & $<0.010 \mathrm{mg} / \mathrm{l}$ & $<0.010 \mathrm{mg} / 1$ & 1 & \\
\hline $120-127$ & $<0.010 \mathrm{mg} / \mathrm{l}$ & $<0.010 \mathrm{mg} / \mathrm{l}$ & $<0.010 \mathrm{mg} / 1$ & $<0.010 \mathrm{mg} / 1$ & 1 & $\cdot$ \\
\hline idfon 927.5 & $<0.050 \mathrm{mg} / \mathrm{l}$ & $<0.050 \mathrm{mg} / \mathrm{l}$ & $<0.050 \mathrm{mg} / \mathrm{l}$ & $<0.050 \mathrm{mg} / \mathrm{l}$ & 1 & \\
\hline 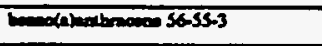 & $<0.010 \mathrm{mg} / 1$ & $<0.010 \mathrm{mg} / \mathrm{l}$ & $<0.010 \mathrm{mg} / 1$ & $<0.010 \mathrm{mg} / \mathrm{l}$ & 1 & \\
\hline 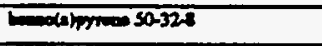 & $<0.010 \mathrm{mg} / \mathrm{t}$ & $<0.010 \mathrm{mg} / \mathrm{l}$ & $<0.010 \mathrm{mg} / \mathrm{l}$ & $<0.010 \mathrm{mg} / 1$ & 1 & \\
\hline 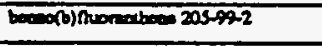 & $<0.010 \mathrm{mg} / \mathrm{l}$ & $<0.010 \mathrm{mg} / 1$ & $<0.010 \mathrm{mg} / \mathrm{l}$ & $<0.010 \mathrm{mg} / 1$ & 1 & \\
\hline 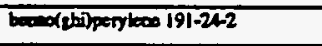 & $<0.010 \mathrm{mg} / 1$ & $<0.010 \mathrm{mg} / 1$ & $<0.010 \mathrm{mg} / \mathrm{I}$ & $<0.010 \mathrm{mg} / 1$ & 1 & \\
\hline 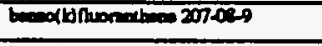 & $<0.010 \mathrm{mg} / \mathrm{l}$ & $<0.010 \mathrm{mg} / 1$ & $<0.010 \mathrm{mg} / \mathrm{l}$ & $<0.010 \mathrm{mg} / 1$ & 1 & \\
\hline 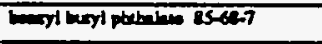 & $<0.010$ ug/l & $<0.010 \mathrm{mg} / \mathrm{l}$ & $<0.010 \mathrm{mg} / \mathrm{l}$ & $<0.010 \mathrm{mg} / 1$ & 1 & \\
\hline 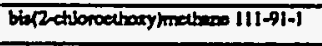 & $<0.010 \mathrm{mg} / \mathrm{l}$ & $<0.010 \mathrm{mg} / \mathrm{l}$ & $<0.010 \mathrm{mg} / \mathrm{l}$ & $<0.010 \mathrm{mg} / \mathrm{l}$ & 1 & $\dot{ }$ \\
\hline 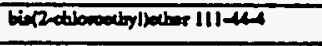 & $<0.010 \mathrm{mg} / 1$ & $<0.010 \mathrm{mg} / 1$ & $<0.010 \mathrm{mg} / \mathrm{l}$ & $<0.010 \mathrm{mg} / 1$ & 1 & \\
\hline 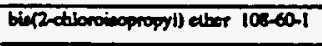 & $<0.010 \mathrm{mg} / \mathrm{l}$ & $<0.010 \mathrm{mg} / 1$ & $<0.010 \mathrm{mg} / \mathrm{l}$ & $<0.010 \mathrm{mg} / \mathrm{l}$ & 1 & \\
\hline 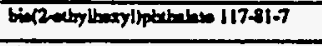 & $<0.010 \mathrm{mg} / 1$ & $<0.010 \mathrm{mg} / \mathrm{l}$ & $<0.010 \mathrm{mg} / \mathrm{l}$ & $<0.010 \mathrm{mg} / \mathrm{l}$ & 1 & \\
\hline 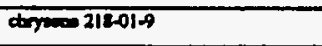 & $<0.010 \mathrm{mg} / \mathrm{l}$ & $<0.010 \mathrm{mg} / \mathrm{l}$ & $<0.010 \mathrm{mg} / \mathrm{l}$ & $<0.010 \mathrm{mg} / \mathrm{l}$ & 1 & \\
\hline diteberyl plabahes 24.742 & $<0.010 \mathrm{mg} / \mathrm{l}$ & $<0.010 \mathrm{mg} / \mathrm{l}$ & $<0.010 \mathrm{mg} / 1$ & $<0.010 \mathrm{mg} / \mathrm{l}$ & 1 & \\
\hline 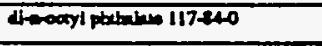 & $<0.010 \mathrm{mg} / \mathrm{l}$ & $<0.010 \mathrm{mg} / 1$ & $<0.010 \mathrm{mg} / \mathrm{l}$ & $<0.010 \mathrm{mg} / \mathrm{l}$ & 1 & \\
\hline 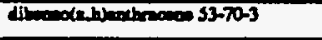 & $<0.010 \mathrm{mg} / \mathrm{l}$ & $<0.010 \mathrm{mg} / 1$ & $<0.010 \mathrm{mg} / \mathrm{l}$ & $<0.010 \mathrm{mg} / 1$ & 1 & \\
\hline 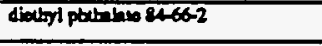 & $<0.010 \mathrm{mg} / 1$ & $<0.010 \mathrm{mg} / \mathrm{l}$ & $<0.010 \mathrm{mg} / \mathrm{l}$ & $<0.010 \mathrm{mg} / \mathrm{l}$ & 1 & \\
\hline 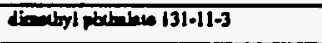 & $<0.010 \mathrm{mg} / \mathrm{l}$ & $<0.010 \mathrm{mg} / \mathrm{I}$ & $<0.010 \mathrm{mg} / \mathrm{l}$ & $<0.010 \mathrm{mg} / \mathrm{l}$ & 1 & \\
\hline Dromentheos $206-140$ & $<0.010 \mathrm{mg} / \mathrm{l}$ & $<0.010 \mathrm{mg} / \mathrm{l}$ & $<0.010 \mathrm{mg} / \mathrm{l}$ & $<0.010 \mathrm{mg} / \mathrm{l}$ & 1 & \\
\hline howence $26.73-7$ & $<0.010 \mathrm{mg} / 1$ & $<0.010 \mathrm{mg} / \mathrm{l}$ & $<0.010 \mathrm{mg} / 1$ & $<0.010 \mathrm{mg} / \mathrm{l}$ & 1 & \\
\hline 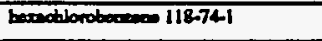 & $<0.010 \mathrm{mg} / 1$ & $<0.010 \mathrm{mg} / \mathrm{l}$ & $<0.010 \mathrm{mg} / 1$ & $<0.010 \mathrm{mg} / \mathrm{l}$ & 1 & \\
\hline 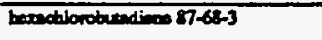 & $<0.010 \mathrm{mg} / \mathrm{l}$ & $<0.010 \mathrm{mg} / \mathrm{ll}$ & $<0.010 \mathrm{mg} / \mathrm{l}$ & $<0.010 \mathrm{mg} / \mathrm{l}$ & 1 & \\
\hline 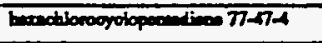 & $<0.010 \mathrm{mg} / \mathrm{I}$ & $<0.010 \mathrm{mg} / \mathrm{l}$ & $<0.010 \mathrm{mg} / 1$ & $<0.010 \mathrm{mg} / \mathrm{l}$ & 1 & \\
\hline 67.72 .1 & $<0.010 \mathrm{mg} / 1$ & $<0.010 \mathrm{mg} / 1$ & $<0.010 \mathrm{mg} / 1$ & $<0.010 \mathrm{mg} / 1$ & 1 & \\
\hline 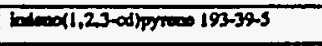 & $<0.010 \mathrm{mg} / \mathrm{l}$ & $<0.010 \mathrm{mg} / 1$ & $<0.010 \mathrm{mg} / 1$ & $<0.010 \mathrm{mg} / \mathrm{l}$ & 1 & \\
\hline inophoroen $72-52-1$ & $<0.010 \mathrm{mg} / 1$ & $<0.010 \mathrm{mg} / \mathrm{l}$ & $<0.010 \mathrm{mg} / 1$ & $<0.010 \mathrm{mg} / 1$ & 1 & \\
\hline 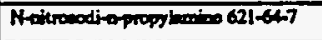 & $<0.010 \mathrm{mg} / \mathrm{h}$ & $<0.010 \mathrm{mg} / \mathrm{l}$ & $<0.010 \mathrm{mg} / 1$ & $<0.010 \mathrm{mg} / \mathrm{l}$ & 1 & \\
\hline 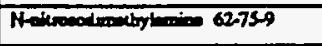 & $<0.010 \mathrm{mg} / 1$ & $<0.010 \mathrm{mg} / \mathrm{l}$ & $<0.010 \mathrm{mg} / \mathrm{l}$ & $<0.010 \mathrm{mg} / \mathrm{l}$ & 1 & \\
\hline 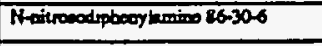 & $<0.010 \mathrm{mg} / \mathrm{l}$ & $<0.010 \mathrm{mg} / \mathrm{l}$ & $<0.010 \mathrm{mg} / \mathrm{l}$ & $<0.010 \mathrm{mg} / \mathrm{l}$ & 1 & \\
\hline Dopteraleos $91-20-3$ & $<0.010 \mathrm{mg} / \mathrm{l}$ & $<0.010 \mathrm{mg} / \mathrm{l}$ & $<0.010 \mathrm{mg} / 1$ & $<0.010 \mathrm{mg} / 1$ & 1 & \\
\hline 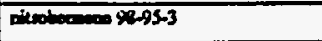 & $<0.010 \mathrm{mg} / \mathrm{l}$ & $<0.010 \mathrm{mg} / 1$ & $<0.010 \mathrm{mg} / 1$ & $<0.010 \mathrm{mg} / \mathrm{l}$ & 1 & \\
\hline 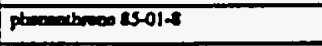 & $<0.010 \mathrm{mg} / \mathrm{l}$ & $<0.010 \mathrm{mg} / \mathrm{l}$ & $<0.010 \mathrm{mg} / \mathrm{l}$ & $<0.010 \mathrm{mg} / \mathrm{l}$ & 1 & \\
\hline monos 129000 & $<0.010 \mathrm{mg} / \mathrm{l}$ & $<0.010 \mathrm{mg} / \mathrm{l}$ & $<0.010 \mathrm{mg} / 1$ & $<0.010 \mathrm{mg} / 1$ & 1 & \\
\hline 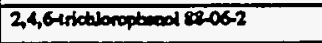 & $<0.030 \mathrm{mg} / \mathrm{I}$ & $<0.030 \mathrm{mg} / \mathrm{l}$ & $<0.030 \mathrm{mg} / \mathrm{l}$ & $<0.030 \mathrm{mg} / \mathrm{l}$ & 1 & \\
\hline 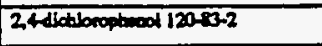 & $<0.030 \mathrm{mg} / 1$ & $<0.030 \mathrm{mg} / \mathrm{l}$ & $<0.030 \mathrm{mg} / 1$ & $<0.030 \mathrm{mg} / \mathrm{l}$ & 1 & \\
\hline 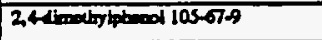 & $<0.030 \mathrm{mg} / \mathrm{l}$ & $<0.030 \mathrm{mg} / \mathrm{l}$ & $<0.030 \mathrm{mg} / \mathrm{l}$ & $<0.030 \mathrm{mg} / \mathrm{l}$ & 1 & \\
\hline 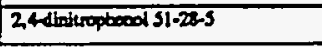 & $<0.050 \mathrm{mg} / \mathrm{l}$ & $<0.050 \mathrm{mg} / 1$ & $<0.050 \mathrm{mg} / 1$ & $<0.050 \mathrm{mg} / \mathrm{l}$ & 1 & \\
\hline
\end{tabular}


Outfall 45

Perte B axc C Costinaved

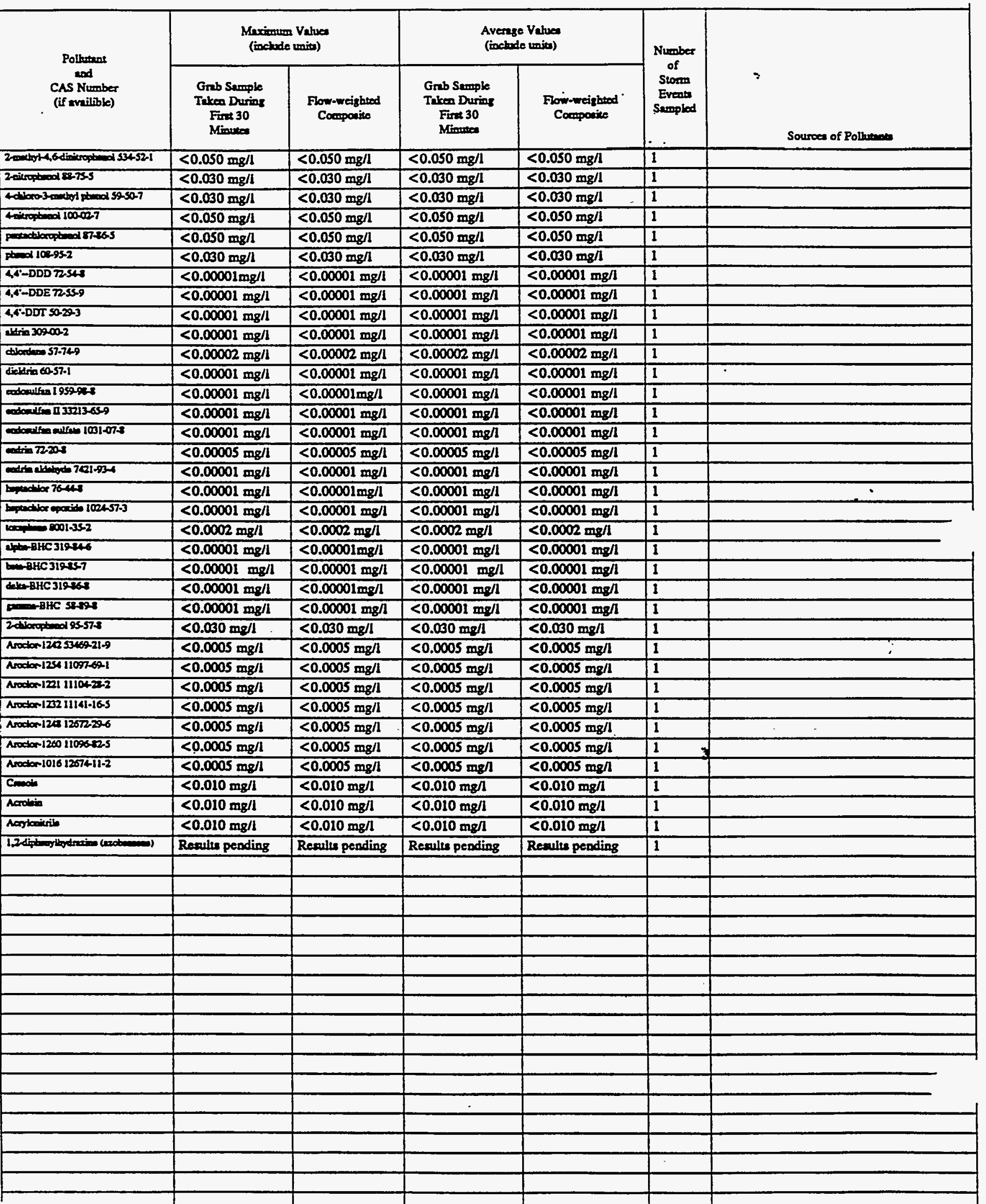


VII. Discharge Informetion (continuod from pago 3 of Form 2F)

Part A - You must provide the results of at least one anelyais for every pollutent in this table. Complete one table for each outfall. Sec instructiona for additional desaib.

\begin{tabular}{|c|c|c|c|c|c|c|}
\hline \multirow{2}{*}{$\begin{array}{l}\text { Pollutast } \\
\text { 2nd } \\
\text { CAs Number } \\
\text { (if availible) }\end{array}$} & \multicolumn{2}{|c|}{$\begin{array}{l}\text { Marimume Velues } \\
\text { (inctude unita) }\end{array}$} & \multicolumn{2}{|c|}{$\begin{array}{l}\text { Average Values } \\
\text { (inchude units). }\end{array}$} & \multirow{2}{*}{$\begin{array}{c}\text { Number } \\
\text { of } \\
\text { Storm } \\
\text { Evente } \\
\text { Sampled }\end{array}$} & \multirow{2}{*}{$=$} \\
\hline & $\begin{array}{l}\text { Grab Sampio } \\
\text { Taken During } \\
\text { Firnt } 30 \\
\text { Minute }\end{array}$ & $\begin{array}{l}\text { Flow-woightod } \\
\text { Comporive }\end{array}$ & $\begin{array}{c}\text { Grab Semplo } \\
\text { Takeo During } \\
\text { Fint } 30 \\
\text { Mintec }\end{array}$ & $\begin{array}{l}\text { Flow-weighted } \\
\text { Componite }\end{array}$ & & \\
\hline Oil and Grease & $3 \mathrm{mg} / \mathrm{l}$ & & $3 \mathrm{mg} / \mathrm{l}$ & & 1 & \\
\hline Biolofical Oxyoua Deomed (BOD) & $<5 \mathrm{mg} / \mathrm{l}$ & $<5 \mathrm{mg} / \mathrm{l}$ & $<5 \mathrm{mg} / \mathrm{l}$ & $<5 \mathrm{mg} / \mathrm{l}$ & 1 & \\
\hline Frearioal Oxy & $13 \mathrm{mg} / 1$ & $7.3 \mathrm{mg} / 1$ & $13 \mathrm{mg} / \mathrm{l}$ & $7.3 \mathrm{mg} / 1$ & 1 & \\
\hline Toul supposidod Solde (TSS) & $<5 \mathrm{mg} / \mathrm{l}$ & $6 \mathrm{mg} / \mathrm{l}$ & $<5 \mathrm{mg} / \mathrm{l}$ & $6 \mathrm{mg} / 1$ & 1 & \\
\hline Foel Kjedabi Nitroper & $0.5 \mathrm{mg} / \mathrm{l}$ & $<0.2 \mathrm{mg} / \mathrm{l}$ & $0.5 \mathrm{mg} / \mathrm{l}$ & $<0.2 \mathrm{mg} / \mathrm{l}$ & 1 & \\
\hline Nitane plus Nitrive Nitropes & $0.31 \mathrm{mg} / \mathrm{l}$ & $0.24 \mathrm{mg} / \mathrm{l}$ & $0.31 \mathrm{mg} / 1$ & $0.24 \mathrm{mg} / \mathrm{l}$ & 1 & \\
\hline Toul Proophorne & $<0.1 \mathrm{mg} / \mathrm{l}$ & $<0.1 \mathrm{mg} / \mathrm{l}$ & $<0.1 \mathrm{mg} / 1$ & $<0.1 \mathrm{mg} / \mathrm{l}$ & 1 & \\
\hline
\end{tabular}

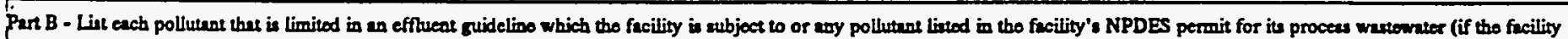

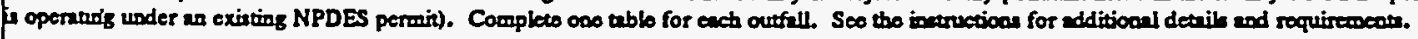

\begin{tabular}{|c|c|c|c|c|c|c|}
\hline \multirow{2}{*}{$\begin{array}{l}\text { Pollutant } \\
\text { end } \\
\text { CAs Number } \\
\text { (if availiblo) }\end{array}$} & \multicolumn{2}{|c|}{$\begin{array}{l}\text { Maximum Valucot } \\
\text { (inolude unita) }\end{array}$} & \multicolumn{2}{|c|}{$\begin{array}{l}\text { Averase Valuce } \\
\text { (inctedo units) }\end{array}$} & \multirow{2}{*}{$\begin{array}{l}\text { Nurober } \\
\text { of } \\
\text { Storm } \\
\text { Eveots } \\
\text { Semplod }\end{array}$} & \multirow[b]{2}{*}{ Sourees of Polthenesen } \\
\hline & $\begin{array}{c}\text { Grab Sumple } \\
\text { Takeo During } \\
\text { Firse } 30 \\
\text { Minuces }\end{array}$ & $\begin{array}{l}\text { Fow -weighted } \\
\text { Comporino }\end{array}$ & $\begin{array}{c}\text { Grab Sample } \\
\text { Takea During } \\
\text { Firse } 30 \\
\text { Minsuce }\end{array}$ & $\begin{array}{l}\text { Flow } \\
\text { Couspocightod }\end{array}$ & & \\
\hline $7400-360$ & $<0.04 \mathrm{mg} / \mathrm{l}$ & $<0.04 \mathrm{mg} / \mathrm{l}$ & $<0.04 \mathrm{mg} / \mathrm{l}$ & $<0.04 \mathrm{mg} / \mathrm{l}$ & 1 & \\
\hline Cyanith & $<0.002 \mathrm{mg} / 1$ & & $<0.002 \mathrm{mg} / \mathrm{l}$ & & 1 & \\
\hline Merainy 7439-976 & $<0.0002 \mathrm{mg} / \mathrm{l}$ & $<0.0002 \mathrm{mg} / 1$ & $<0.0002 \mathrm{mg} / \mathrm{l}$ & $<0.0002 \mathrm{mg} / \mathrm{l}$ & 1 & \\
\hline Prementes & $0.004 \mathrm{mg} / \mathrm{l}$ & & $0.004 \mathrm{mg} / \mathrm{l}$ & & 1 & $\because$ \\
\hline 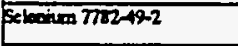 & $<0.002 \mathrm{mg} / \mathrm{l}$ & $<0.002 \mathrm{mg} / \mathrm{l}$ & $<0.002 \mathrm{mg} / \mathrm{l}$ & $<0.002 \mathrm{mg} / \mathrm{l}$ & 1 & \\
\hline pellowe & $8.6 \mathrm{mg} / \mathrm{l}$ & $9.2 \mathrm{mg} / \mathrm{l}$ & $8.6 \mathrm{mg} / \mathrm{h}$ & $9.2 \mathrm{mg} / 1$ & 1 & \\
\hline Surthosenses & $<0.05 \mathrm{mg} / \mathrm{l}$ & $0.09 \mathrm{mg} / \mathrm{l}$ & $<0.05 \mathrm{mg} / \mathrm{l}$ & $0.09 \mathrm{mg} / \mathrm{l}$ & 1 & \\
\hline Theminum 7 $422900-5$ & $0.22 \mathrm{mg} / 1$ & $0.34 \mathrm{mg} / \mathrm{l}$ & $0.22 \mathrm{mg} / \mathrm{l}$ & $0.34 \mathrm{mg} / 1$ & 1 & \\
\hline Armimos 740-38-2 & $<0.04 \mathrm{mg} / 1$ & $<0.04 \mathrm{mg} / \mathrm{l}$ & $<0.04 \mathrm{mg} / \mathrm{l}$ & $<0.04 \mathrm{mg} / \mathrm{l}$ & 1 & \\
\hline Bonden 7400428 & $0.019 \mathrm{mg} / \mathrm{l}$ & $0.015 \mathrm{mg} / \mathrm{l}$ & $0.019 \mathrm{mg} / 1$ & $0.015 \mathrm{mg} / 1$ & 1 & \\
\hline Boryllixm $7440-41.7$ & $<0.0004 \mathrm{mg} / \mathrm{l}$ & $<0.0004 \mathrm{mg} / \mathrm{l}$ & $<0.0004 \mathrm{mg} / \mathrm{l}$ & $<0.0004 \mathrm{mg} / 1$ & 1 & \\
\hline Fodming 740-139 & $<0.004 \mathrm{mg} / \mathrm{l}$ & $<0.004 \mathrm{mg} / \mathrm{l}$ & $<0.004 \mathrm{mg} / \mathrm{l}$ & $<0.004 \mathrm{mg} / \mathrm{l}$ & 1 & \\
\hline Calcher $740-70-2$ & $11.1 \mathrm{mg} / \mathrm{l}$ & $11.8 \mathrm{mg} / \mathrm{l}$ & $11.1 \mathrm{mg} / 1$ & $11.8 \mathrm{mg} / \mathrm{l}$ & 1 & \\
\hline Corestion 7410-17-3 & $<0.006 \mathrm{mg} / \mathrm{l}$ & $<0.006 \mathrm{mg} / 1$ & $<0.006 \mathrm{mg} / 1$ & $<0.006 \mathrm{mg} / 1$ & 1 & \\
\hline Cotalis 7440-48 & $<0.002 \mathrm{mg} / \mathrm{l}$ & $<0.002 \mathrm{mg} / \mathrm{l}$ & $<0.002 \mathrm{mg} / \mathrm{l}$ & $<0.002 \mathrm{mg} / \mathrm{l}$ & 1 & \\
\hline Coppor 7ma-sas & $0.007 \mathrm{mg} / \mathrm{l}$ & $<0.006 \mathrm{mg} / 1$ & $0.007 \mathrm{mg} / \mathrm{l}$ & $<0.006 \mathrm{mg} / 1$ & 1 & \\
\hline 50007432906 & $0.30 \mathrm{mg} / \mathrm{l}$ & $0.38 \mathrm{mg} / \mathrm{l}$ & $0.30 \mathrm{mg} / \mathrm{l}$ & $0.38 \mathrm{mg} / \mathrm{l}$ & 1 & \\
\hline ond 7/39-2.1 & $<0.02 \mathrm{mg} / 1$ & $<0.02 \mathrm{mg} / \mathrm{l}$ & $<0.02 \mathrm{mg} / \mathrm{l}$ & $<0.02 \mathrm{mg} / \mathrm{l}$ & 1 & \\
\hline Sthiven 7439-93-2 & $<0.02 \mathrm{mg} / 1$ & $<0.02 \mathrm{mg} / \mathrm{l}$ & $<0.02 \mathrm{mg} / \mathrm{l}$ & $<0.02 \mathrm{mg} / \mathrm{l}$ & 1 & \\
\hline Mepound 7439-95-4 & $1.17 \mathrm{mg} / \mathrm{l}$ & $1.25 \mathrm{mg} / 1$ & $1.17 \mathrm{mg} / \mathrm{l}$ & $1.25 \mathrm{mg} / \mathrm{l}$ & 1 & \\
\hline Menganowe 7439-96-5 & $0.010 \mathrm{mg} / \mathrm{l}$ & $0.014 \mathrm{mg} / \mathrm{l}$ & $0.010 \mathrm{mg} / 1$ & $0.014 \mathrm{mg} / \mathrm{l}$ & 1 & \\
\hline Molybsooum $7439996-7$ & $<0.006 \mathrm{mg} / \mathrm{l}$ & $<0.006 \mathrm{mg} / \mathrm{l}$ & $<0.006 \mathrm{mg} / \mathrm{l}$ & $<0.006 \mathrm{mg} / 1$ & 1 & \\
\hline$\sqrt{160617400020}$ & $<0.008 \mathrm{mg} / \mathrm{l}$ & $<0.008 \mathrm{mg} / \mathrm{l}$ & $<0.008 \mathrm{mg} / \mathrm{l}$ & $<0.008 \mathrm{mg} / \mathrm{l}$ & 1 & \\
\hline Promediven $740009-7$ & $0.6 \mathrm{mg} / \mathrm{l}$ & $0.7 \mathrm{mg} / \mathrm{l}$ & $0.6 \mathrm{mg} / \mathrm{l}$ & $0.7 \mathrm{mg} / \mathrm{l}$ & 1 & \\
\hline Sulvor 740.204 & $<0.006 \mathrm{mg} / \mathrm{l}$ & $<0.006 \mathrm{mg} / \mathrm{l}$ & $<0.006 \mathrm{mg} / \mathrm{l}$ & $<0.006 \mathrm{mg} / \mathrm{l}$ & 1 & \\
\hline 74ta-22-3 & $0.29 \mathrm{mg} / \mathrm{l}$ & $0.43 \mathrm{mg} / \mathrm{l}$ & $0.29 \mathrm{mg} / \mathrm{l}$ & $0.43 \mathrm{mg} / \mathrm{l}$ & 1 & \\
\hline Tiventan 7440-32-6 & $<0.01 \mathrm{mg} / \mathrm{l}$ & $<0.01 \mathrm{mg} / \mathrm{l}$ & $<0.01 \mathrm{mg} / \mathrm{l}$ & $<0.01 \mathrm{mg} / 1$ & 1 & \\
\hline $7000740-66-6$ & $0.02 \mathrm{mg} / \mathrm{l}$ & $0.03 \mathrm{mg} / \mathrm{l}$ & $0.02 \mathrm{mg} / \mathrm{l}$ & $0.03 \mathrm{mg} / \mathrm{l}$ & 1 & \\
\hline
\end{tabular}


Continued from tront.

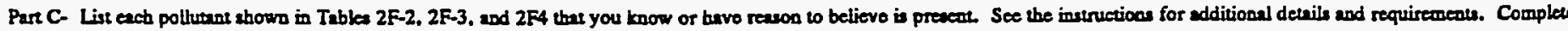
one table for each ourfall.

\begin{tabular}{|c|c|c|c|c|c|c|}
\hline \multirow{2}{*}{$\begin{array}{l}\text { Pollutant } \\
\text { and } \\
\text { CAS Number } \\
\text { (if availible) }\end{array}$} & \multicolumn{2}{|c|}{$\begin{array}{l}\text { Maximum Value } \\
\text { (inchude units) }\end{array}$} & \multicolumn{2}{|c|}{$\begin{array}{l}\text { Avernge Valuea } \\
\text { (inchudo units) }\end{array}$} & \multirow{2}{*}{$\begin{array}{c}\text { Number } \\
\text { of } \\
\text { Storm } \\
\text { Evente } \\
\text { Samplod }\end{array}$} & \multirow[b]{2}{*}{ S roces of Polturtenta } \\
\hline & $\begin{array}{c}\text { Grab Snmplo } \\
\text { Takeo During } \\
\text { Fint } 30 \\
\text { Minutea }\end{array}$ & $\begin{array}{l}\text { Flow-weighted } \\
\text { Comporite }\end{array}$ & $\begin{array}{l}\text { Grab Sample } \\
\text { Taken During } \\
\text { Firut } 30 \\
\text { Minutes }\end{array}$ & $\begin{array}{l}\text { Flowi-weighred } \\
\text { Compoite }\end{array}$ & & \\
\hline 1.1.1.-2rictioroctsens $71.55-6$ & $<0.010 \mathrm{mg} / 1$ & & $<0.010 \mathrm{mg} / \mathrm{l}$ & & 1 & \\
\hline 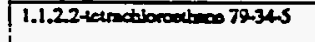 & $<0.010 \mathrm{mg} / \mathrm{l}$ & & $<0.010 \mathrm{mg} / 1$ & & 1 & \\
\hline 1.1.2-1rictibrootheres 79-00.5 & $<0.010 \mathrm{mg} / \mathrm{l}$ & & $<0.010 \mathrm{mg} / 1$ & & 1 & \\
\hline 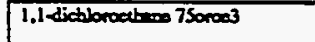 & $<0.010 \mathrm{mg} / 1$ & & $<0.010 \mathrm{mg} / 1$ & & 1 & \\
\hline 1.1-dictibroctby lewe 75-35-4 & $<0.010 \mathrm{mg} / 1$ & & $<0.010 \mathrm{mg} / \mathrm{l}$ & & 1 & \\
\hline 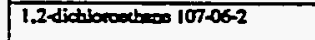 & $<0.010 \mathrm{mg} / \mathrm{l}$ & & $<0.010 \mathrm{mg} / \mathrm{l}$ & & 1 & \\
\hline 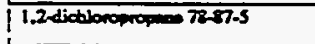 & $<0.010 \mathrm{mg} / \mathrm{l}$ & & $<0.010 \mathrm{mg} / 1$ & & 1 & \\
\hline 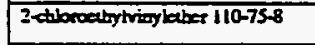 & $<0.010 \mathrm{mg} / \mathrm{l}$ & & $<0.010 \mathrm{mg} / \mathrm{l}$ & & 1 & \\
\hline $71-13-2$ & $<0.010 \mathrm{mg} / 1$ & & $<0.010 \mathrm{mg} / 1$ & & 1 & \\
\hline 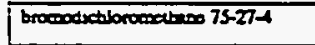 & $<0.010 \mathrm{mg} / 1$ & & $<0.010 \mathrm{mg} / \mathrm{l}$ & & 1 & \\
\hline brocoloem $75-25-2$ & $<0.010 \mathrm{mg} / \mathrm{l}$ & & $<0.010 \mathrm{mg} / \mathrm{l}$ & & 1 & $\cdot$ \\
\hline 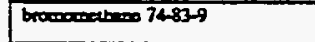 & $<0.010 \mathrm{mg} / 1$ & & $<0.010 \mathrm{mg} / 1$ & & 1 & \\
\hline artes vetnotbilats 56-23-5 & $<0.010 \mathrm{mg} / 1$ & & $<0.010 \mathrm{mg} / \mathrm{l}$ & & 1 & \\
\hline chlorobereseos $108-90.7$ & $<0.010 \mathrm{mg} / 1$ & & $<0.010 \mathrm{mg} / \mathrm{l}$ & & 1 & \\
\hline chlorcoubanos $7500-3$ & $<0.010 \mathrm{mg} / \mathrm{l}$ & & $<0.010 \mathrm{mg} / \mathrm{l}$ & & 1 & \\
\hline chboroform 67-66-3 & $<0.010 \mathrm{mg} / 1$ & & $<0.010 \mathrm{mg} / 1$ & & 1 & \\
\hline chloracoutanes 7487.3 & $<0.010 \mathrm{mg} / 1$ & & $<0.010 \mathrm{mg} / \mathrm{l}$ & & 1 & $\therefore$ \\
\hline 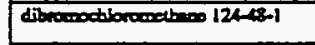 & $<0.010 \mathrm{mg} / \mathrm{l}$ & & $<0.010 \mathrm{mg} / 1$ & & 1 & \\
\hline 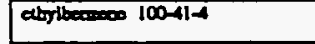 & $<0.010 \mathrm{mg} / \mathrm{l}$ & & $<0.010 \mathrm{mg} / 1$ & & 1 & \\
\hline tohen 108-88-3 & $<0.010 \mathrm{mg} / 1$ & & $<0.010 \mathrm{mg} / 1$ & & 1 & \\
\hline vingl dioride 75014 & $<0.010 \mathrm{mg} / \mathrm{l}$ & & $<0.010 \mathrm{mg} / \mathrm{l}$ & & 1 & \\
\hline 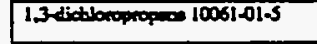 & $<0.010 \mathrm{mg} / 1$ & & $<0.010 \mathrm{mg} / 1$ & & 1 & \\
\hline 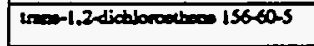 & $<0.010 \mathrm{mg} / 1$ & & $<0.010 \mathrm{mg} / 1$ & & 1 & - \\
\hline alptin exanty $12587-46-1$ & $3.9 \mathrm{pei} / \mathrm{l}$ & $1.7 \mathrm{pei} / 1$ & $3.9 \mathrm{pci} / 1$ & 1.7 poill & 1 & 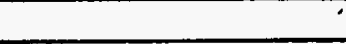 \\
\hline benamp $12587-16-1$ & $8.9 \mathrm{pci} / 1$ & $3.6 \mathrm{pci} / 1$ & $8.9 \mathrm{pci} / \mathrm{l}$ & $3.6 \mathrm{pci} / 1$ & 1 & \\
\hline loct odifocm & $<1 \mathrm{col} .1 \mathrm{~L}$ & $26,000 \mathrm{co} / .1 \mathrm{~L}$ & $<1 \mathrm{col} .1 \mathrm{~L}$ & $26,000 \mathrm{co} / .1 \mathrm{~L}$ & 1 & \\
\hline Thalliers 740-220 & $<0.03 \mathrm{mg} / \mathrm{l}$ & $<0.03 \mathrm{mg} / \mathrm{l}$ & $<0.03 \mathrm{mg} / \mathrm{l}$ & $<0.03 \mathrm{mg} / \mathrm{l}$ & 1 & \\
\hline Unaing $740-61-1$ & $0.001 \mathrm{mg} / \mathrm{l}$ & $0.002 \mathrm{mg} / \mathrm{l}$ & $0.001 \mathrm{mg} / 1$ & $0.002 \mathrm{mg} / 1$ & 1 & \\
\hline Consearivisy & 22 pcill & 16 pcill & 22 peill & $16 \mathrm{pci} / \mathrm{l}$ & 1 & \\
\hline
\end{tabular}

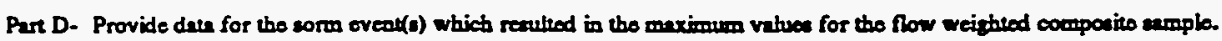

\begin{tabular}{|c|c|c|c|c|c|c|c|}
\hline $\begin{array}{l}1 . \\
\text { Date of } \\
\text { Storm } \\
\text { Eveat }\end{array}$ & $\begin{array}{l}2 . \\
\text { Durition } \\
\text { of Storm } \\
\text { (in } \\
\text { minuese) }\end{array}$ & $\begin{array}{l}3 . \\
\text { Totul ninfall } \\
\text { during storm ovens } \\
\text { (in ixoben) }\end{array}$ & $\begin{array}{l}\text { 4. } \\
\text { Number of bours betwoes } \\
\text { beginning of storm mone- } \\
\text { ured end cod of provious } \\
\text { meaminble min ovent }\end{array}$ & 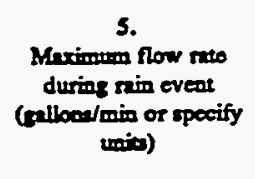 & $\begin{array}{l}6 . \\
\text { Total flow from } \\
\text { nin ovent } \\
\text { (ralloot or } \\
\text { ppecify twin) }\end{array}$ & $\begin{array}{c}7 . \\
\text { Secion } \\
\text { enmplo was } \\
\text { tiken }\end{array}$ & $\begin{array}{l}8 . \\
\text { Form of } \\
\text { Precipitusion } \\
\text { (ninfill, } \\
\text { enommaly) }\end{array}$ \\
\hline $12 / 9 / 91$ & 530 & 1.1 & 148.6 & 1.7 & 318 & Fall & Ruinfall \\
\hline
\end{tabular}

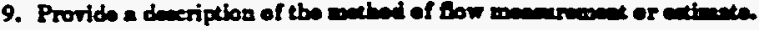

An ISCO 3230 Flow meter was used to obtain water leved readings. This instrument was calibrated with the normal dry weather flow, if present, as level zero. The flow meter was programmed with the characteristics of the convejance so that flow rates and total flows could be calcalated by the meter. 


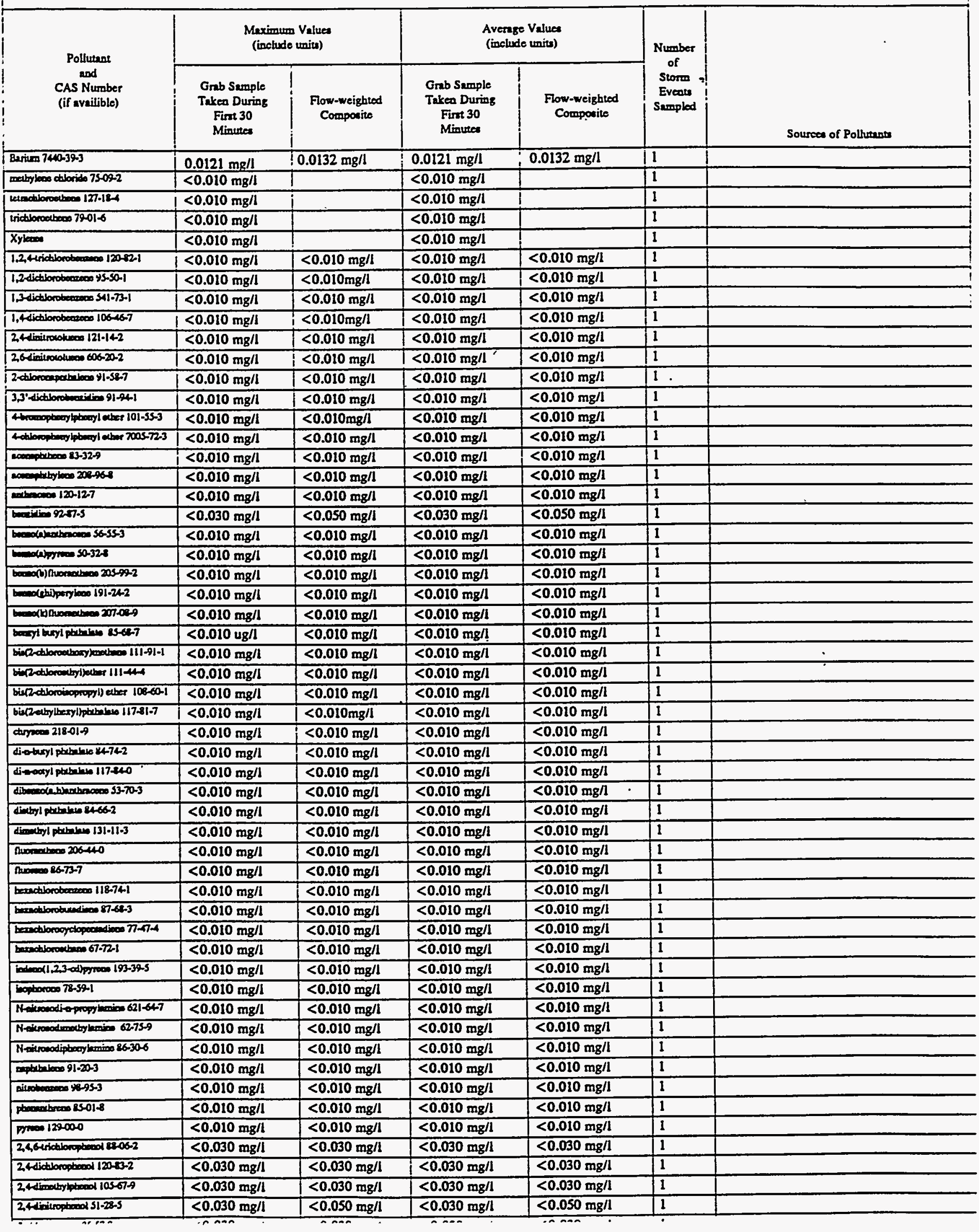


Outfall 46

\begin{tabular}{|c|c|c|c|c|c|c|}
\hline \multirow{2}{*}{$\begin{array}{l}\text { Pollutunt } \\
\text { and } \\
\text { CAS Number } \\
\text { (if availible) }\end{array}$} & \multicolumn{2}{|c|}{$\begin{array}{l}\text { Meximimm Value } \\
\text { (inchedo unitu) }\end{array}$} & \multicolumn{2}{|c|}{$\begin{array}{l}\text { Averege Values } \\
\text { (inchude unitu) }\end{array}$} & \multirow{2}{*}{$\begin{array}{c}\text { Number } \\
\text { of } \\
\text { Storm } \\
\text { Eveats } \\
\text { Sumpled }\end{array}$} & \multirow[b]{2}{*}{ Sourcen of Pollutans } \\
\hline & $\begin{array}{l}\text { Grab Sumplo } \\
\text { Taken During } \\
\text { Fint } 30 \\
\text { Minutes }\end{array}$ & $\begin{array}{l}\text { Fow-weighed } \\
\text { Componize }\end{array}$ & $\begin{array}{c}\text { Grab Sample } \\
\text { Taken During } \\
\text { Firnt } 30 \\
\text { Minutea }\end{array}$ & $\begin{array}{l}\text { Flow-weighted } \\
\text { Componte }\end{array}$ & & \\
\hline 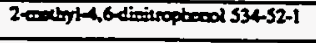 & $<0.030 \mathrm{mg} / 1$ & $<0.050 \mathrm{mg} / 1$ & $<0.030 \mathrm{mg} / \mathrm{l}$ & $<0.050 \mathrm{mg} / \mathrm{l}$ & 1 & \\
\hline 2 - eitropteod $88-75-5$ & $<0.030 \mathrm{mg} / \mathrm{l}$ & $<0.030 \mathrm{mg} / \mathrm{l}$ & $<0.030 \mathrm{mg} / 1$ & $<0.030 \mathrm{mg} / \mathrm{l}$ & 1 & \\
\hline 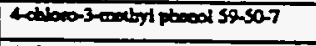 & $<0.030 \mathrm{mg} / \mathrm{l}$ & $<0.030 \mathrm{mg} / \mathrm{l}$ & $<0.030 \mathrm{mg} / 1$ & $<0.030 \mathrm{mg} / 1$ & 1 & \\
\hline 4 atropteod 100027 & $<0.030 \mathrm{mg} / 1$ & $<0.050 \mathrm{mg} / \mathrm{l}$ & $<0.030 \mathrm{mg} / \mathrm{l}$ & $<0.050 \mathrm{mg} / \mathrm{l}$ & 1 & \\
\hline 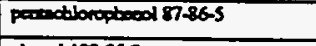 & $<0.030 \mathrm{mg} / 1$ & $<0.050 \mathrm{mg} / \mathrm{l}$ & $<0.030 \mathrm{mg} / \mathrm{l}$ & $<0.050 \mathrm{mg} / \mathrm{l}$ & 1 & \\
\hline$p h=d 108-93-2$ & $<0.030 \mathrm{mg} / \mathrm{l}$ & $<0.030 \mathrm{mg} / 1$ & $<0.030 \mathrm{mg} / \mathrm{l}$ & $<0.030 \mathrm{mg} / 1$ & 1 & \\
\hline $4.4^{2}-00072.548$ & $<0.00001 \mathrm{mg} / \mathrm{l}$ & $<0.00001 \mathrm{mg} / \mathrm{l}$ & $<0.00001 \mathrm{mg} / \mathrm{l}$ & $<0.00001 \mathrm{mg} / \mathrm{l}$ & 1 & \\
\hline $4,4^{\circ} \rightarrow$ DDE 72.55-9 & $<0.00001 \mathrm{mg} / 1$ & $<0.00001 \mathrm{mg} / \mathrm{l}$ & $<0.00001 \mathrm{mg} / \mathrm{l}$ & $<0.00001 \mathrm{mg} / \mathrm{l}$ & 1 & \\
\hline $4.4^{\circ}-0.00550-29.3$ & $<0.00001 \mathrm{mg} / \mathrm{l}$ & $<0.00001 \mathrm{mg} / 1$ & $<0.00001 \mathrm{mg} / \mathrm{l}$ & $<0.00001 \mathrm{mg} / \mathrm{l}$ & 1 & \\
\hline $2 \operatorname{tin} 309-00-2$ & $<0.00001 \mathrm{mg} / \mathrm{l}$ & $0.000022 \mathrm{mg} / \mathrm{l}$ & $<0.00001 \mathrm{mg} / \mathrm{l}$ & $0.000022 \mathrm{mg} / \mathrm{l}$ & 1 & \\
\hline ctionderes 57.749 & $<0.00002 \mathrm{mg} / 1$ & $<0.00002 \mathrm{mg} / \mathrm{l}$ & $<0.00002 \mathrm{mg} / \mathrm{l}$ & $<0.00002 \mathrm{mg} / \mathrm{l}$ & 1 & \\
\hline didating $60-57-1$ & $<0.00001 \mathrm{mg} / \mathrm{l}$ & $<0.00001 \mathrm{mg} / \mathrm{l}$ & $<0.00001 \mathrm{mg} / \mathrm{l}$ & $<0.00001 \mathrm{mg} / 1$ & 1 & \\
\hline ondonilen $1959+8$ & $<0.00001 \mathrm{mg} / \mathrm{l}$ & $<0.00001 \mathrm{mg} / \mathrm{l}$ & $<0.00001 \mathrm{mg} / \mathrm{l}$ & $<0.00001 \mathrm{mg} / \mathrm{l}$ & 1 & \\
\hline 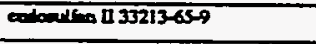 & $<0.00001 \mathrm{mg} / \mathrm{l}$ & $<0.00001 \mathrm{mg} / \mathrm{l}$ & $<0.00001 \mathrm{mg} / \mathrm{l}$ & $<0.00001 \mathrm{mg} / \mathrm{l}$ & 1 & \\
\hline 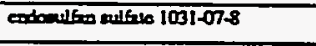 & $<0.00001 \mathrm{mg} / \mathrm{l}$ & $<0.00001 \mathrm{mg} / \mathrm{l}$ & $<0.00001 \mathrm{mg} / 1$ & $<0.00001 \mathrm{mg} / 1$ & 1 & \\
\hline cedin 72-20s & $<0.00005 \mathrm{mg} / \mathrm{l}$ & $<0.00005 \mathrm{mg} / \mathrm{l}$ & $<0.00005 \mathrm{mg} / \mathrm{l}$ & $<0.00005 \mathrm{mg} / 1$ & 1 & \\
\hline codria eldetydo 7421-934- & $<0.00001 \mathrm{mg} / \mathrm{l}$ & $<0.00001 \mathrm{mg} / \mathrm{l}$ & $<0.00001 \mathrm{mg} / \mathrm{l}$ & $<0.00001 \mathrm{mg} / 1$ & 1 & \\
\hline bepection 764 . & $<0.00001 \mathrm{mg} / \mathrm{l}$ & $<0.00001 \mathrm{mg} / \mathrm{l}$ & $<0.00001 \mathrm{mg} / \mathrm{l}$ & $<0.00001 \mathrm{mg} / \mathrm{l}$ & 1 &. \\
\hline 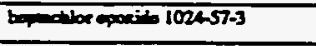 & $<0.00001 \mathrm{mg} / \mathrm{l}$ & $<0.00001 \mathrm{mg} / \mathrm{l}$ & $<0.00001 \mathrm{mg} / 1$ & $<0.00001 \mathrm{mg} / \mathrm{l}$ & 1 & \\
\hline $000 p$ becen $1001-35-2$ & $<0.0002 \mathrm{mg} / 1$ & $<0.0002 \mathrm{mg} / \mathrm{l}$ & $<0.0002 \mathrm{mg} / 1$ & $<0.0002 \mathrm{mg} / \mathrm{l}$ & 1 & \\
\hline $46-8+C$ 319246 & $<0.00001 \mathrm{mg} / \mathrm{l}$ & $<0.00001 \mathrm{mg} / 1$ & $<0.00001 \mathrm{mg} / \mathrm{l}$ & $<0.00001 \mathrm{mg} / \mathrm{l}$ & 1 & \\
\hline $500-81+31985-7$ & $<0.00001 \mathrm{mg} / \mathrm{l}$ & $<0.00001 \mathrm{mg} / \mathrm{l}$ & $<0.00001 \mathrm{mg} / \mathrm{l}$ & $<0.00001 \mathrm{mg} / \mathrm{l}$ & 1 & \\
\hline dab-BHC 312868 & $<0.00001 \mathrm{mg} / 1$ & $0.000014 \mathrm{mg} / \mathrm{l}$ & $<0.00001 \mathrm{mg} / 1$ & $0.000014 \mathrm{mg} / \mathrm{l}$ & 1 & \\
\hline 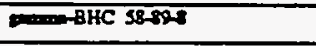 & $0.000011 \mathrm{mg} / 1$ & $<0.00001 \mathrm{mg} / \mathrm{l}$ & $0.000011 \mathrm{mg} / 1$ & $<0.00001 \mathrm{mg} / \mathrm{l}$ & 1 & \\
\hline 2ndtoroptioed 95-57- & $<0.030 \mathrm{mg} / \mathrm{l}$ & $<0.030 \mathrm{mg} / \mathrm{l}$ & $<0.030 \mathrm{mg} / \mathrm{l}$ & $<0.030 \mathrm{mg} / \mathrm{l}$ & 1 & \\
\hline Arodon-1212 S36G21-A & $<0.0005 \mathrm{mg} / \mathrm{l}$ & $<0.0005 \mathrm{mg} / \mathrm{l}$ & $<0.0005 \mathrm{mg} / \mathrm{l}$ & $<0.0005 \mathrm{mg} / \mathrm{l}$ & 1 & $\cdot$ \\
\hline Nocton-125 11097691 & $<0.0005 \mathrm{mg} / 1$ & $<0.0005 \mathrm{mg} / \mathrm{l}$ & $<0.0005 \mathrm{mg} / \mathrm{l}$ & $<0.0005 \mathrm{mg} / \mathrm{l}$ & 1 & \\
\hline Noodon $1211110425-2$ & $<0.0005 \mathrm{mg} / \mathrm{l}$ & $<0.0005 \mathrm{mg} / \mathrm{l}$ & $<0.0005 \mathrm{mg} / \mathrm{l}$ & $<0.0005 \mathrm{mg} / \mathrm{l}$ & 1 & \\
\hline Arodon-12211141-16-5 & $<0.0005 \mathrm{mg} / \mathrm{l}$ & $<0.0005 \mathrm{mg} / 1$ & $<0.0005 \mathrm{mg} / \mathrm{l}$ & $<0.0005 \mathrm{mg} / \mathrm{l}$ & 1 & \\
\hline Arodor-1263 12012-296 & $<0.0005 \mathrm{mg} / \mathrm{l}$ & $<0.0005 \mathrm{mg} / \mathrm{l}$ & $<0.0005 \mathrm{mg} / \mathrm{l}$ & $<0.0005 \mathrm{mg} / \mathrm{l}$ & 1 & \\
\hline Arodor $125011096-52-5$ & $<0.0005 \mathrm{mg} / 1$ & $<0.0005 \mathrm{mg} / \mathrm{l}$ & $<0.0005 \mathrm{mg} / \mathrm{l}$ & $<0.0005 \mathrm{mg} / \mathrm{l}$ & 1 & \\
\hline Acodon $10161207411-2$ & $<0.0005 \mathrm{mg} / \mathrm{l}$ & $<0.0005 \mathrm{mg} / 1$ & $<0.0005 \mathrm{mg} / \mathrm{l}$ & $<0.0005 \mathrm{mg} / 1$ & 1 & \\
\hline Cromolo & $<0.010 \mathrm{mg} / \mathrm{l}$ & $<0.010 \mathrm{mg} / \mathrm{l}$ & $<0.010 \mathrm{mg} / 1$ & $<0.010 \mathrm{mg} / 1$ & 1 & \\
\hline Anolose & $<0.010 \mathrm{mg} / \mathrm{l}$ & $<0.010 \mathrm{mg} / \mathrm{l}$ & $<0.010 \mathrm{mg} / \mathrm{l}$ & $<0.010 \mathrm{mg} / 1$ & 1 & \\
\hline Acyloserite & $<0.010 \mathrm{mg} / \mathrm{l}$ & $<0.010 \mathrm{mg} / \mathrm{l}$ & $<0.010 \mathrm{mg} / 1$ & $<0.010 \mathrm{mg} / 1$ & 1 & \\
\hline 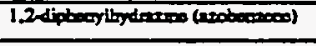 & Reaulte pending & Remulte pending & Reaults pending & Reavilu peoding & 1 & \\
\hline & & & & & & \\
\hline & & & & & & \\
\hline & & & & & & \\
\hline & & & & & & \\
\hline & & & & & & \\
\hline & & & & & & \\
\hline & & & & & & \\
\hline & & & & & & \\
\hline & & & & & & \\
\hline & & & & & & \\
\hline & & & & & & \\
\hline & & & & & & \\
\hline & & & & & & \\
\hline & & & & & & \\
\hline & & & & & & \\
\hline & & & & & & \\
\hline
\end{tabular}


VII. Dischergo laformetion (continued from page 3 of Form 2F)

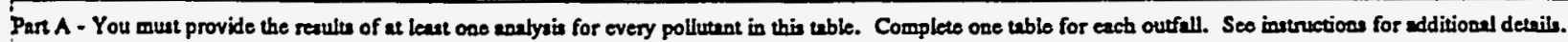

\begin{tabular}{|c|c|c|c|c|c|c|}
\hline $\begin{array}{l}\text { Polluent } \\
\text { ind } \\
\text { CAs Number } \\
\text { (if avilible) }\end{array}$ & \multicolumn{2}{|c|}{$\begin{array}{l}\text { Meximum Valuea } \\
\text { (inctudo unitu) }\end{array}$} & \multicolumn{2}{|c|}{$\begin{array}{l}\text { Avernge Values } \\
\text { (include unitu) }\end{array}$} & $\begin{array}{l}\text { Number } \\
\text { of } \\
\text { Storm } \\
\text { Events } \\
\text { Sempled }\end{array}$ & Souree of Pollureanes \\
\hline pil and Grase & $2 \mathrm{mg} / \mathrm{l}$ & & $2 \mathrm{mg} / 1$ & & 1 & \\
\hline Chectical Oxyoes Decoud (COD) & $24 \mathrm{mg} / \mathrm{h}$ & $14 \mathrm{mg} / \mathrm{l}$ & $24 \mathrm{mg} / \mathrm{l}$ & $14 \mathrm{mg} / \mathrm{l}$ & 1 & \\
\hline Tool Supended Solids (TSS) & $6 \mathrm{mg} / \mathrm{l}$ & $12 \mathrm{mg} / 1$ & $6 \mathrm{mg} / 1$ & $12 \mathrm{mg} / 1$ & 1 & \\
\hline Toul Kjeidehl Nilrower & $0.5 \mathrm{mg} / \mathrm{l}$ & $0.2 \mathrm{mg} / 1$ & $0.5 \mathrm{mg} / 1$ & $0.2 \mathrm{mg} / \mathrm{h}$ & 1 & \\
\hline Nitres ple Nitrive Nitrooses & $0.5 \mathrm{mg} / \mathrm{l}$ & $0.51 \mathrm{mg} / \mathrm{l}$ & $0.5 \mathrm{mg} / 1$ & $0.51 \mathrm{mg} / \mathrm{l}$ & 1 & \\
\hline Toul Propoptonem & $0.60 \mathrm{mg} / \mathrm{l}$ & $0.50 \mathrm{mg} / \mathrm{l}$ & $0.60 \mathrm{mg} / \mathrm{l}$ & $0.50 \mathrm{mg} / \mathrm{l}$ & 1 & \\
\hline
\end{tabular}

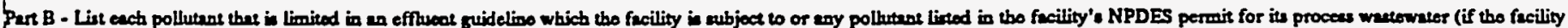

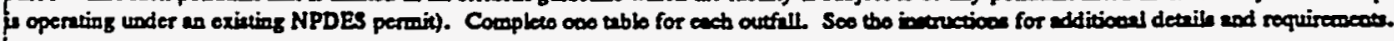

\begin{tabular}{|c|c|c|c|c|c|c|}
\hline \multirow{2}{*}{$\begin{array}{l}\text { Pollutent } \\
\text { and } \\
\text { CAs Number } \\
\text { (if eviliblo) }\end{array}$} & \multicolumn{2}{|c|}{$\begin{array}{l}\text { Maximum Vahoos } \\
\text { (inctudo unise) }\end{array}$} & \multicolumn{2}{|c|}{$\begin{array}{l}\text { Averese Vahuea } \\
\text { (ivoludo mise) }\end{array}$} & \multirow{2}{*}{$\begin{array}{l}\text { Number } \\
\text { of } \\
\text { Storm } \\
\text { Eveoti } \\
\text { Sumpled }\end{array}$} & \multirow[b]{2}{*}{ Sources of Polthromes } \\
\hline & $\begin{array}{c}\text { Grb Semple } \\
\text { Takeo Duriag } \\
\text { Find } 30 \\
\text { Misuree }\end{array}$ & $\begin{array}{l}\text { Flow-weighted } \\
\text { Comporits }\end{array}$ & $\begin{array}{l}\text { Greb Sarople } \\
\text { Tekeo Durrias } \\
\text { Fisu } 30 \\
\text { Minesese }\end{array}$ & $\begin{array}{l}\text { Forw-wreighted } \\
\text { Compoxito }\end{array}$ & & \\
\hline teimaty 7400-360 & $<0.04 \mathrm{mg} / 1$ & $<0.04 \mathrm{mg} / \mathrm{l}$ & $<0.04 \mathrm{mg} / \mathrm{l}$ & $<0.04 \mathrm{mg} / 1$ & 1 & \\
\hline Drander & $<0.002 \mathrm{mg} / \mathrm{l}$ & & $<0.002 \mathrm{mg} / \mathrm{l}$ & & 1 & \\
\hline Movery 7439976 & $0.0010 \mathrm{mg} / \mathrm{l}$ & $0.0010 \mathrm{mg} / \mathrm{l}$ & $0.0010 \mathrm{mg} / \mathrm{l}$ & $0.0010 \mathrm{mg} / \mathrm{l}$ & 1 & $\therefore$ \\
\hline phomes & $0.006 \mathrm{mg} / \mathrm{l}$ & & $0.006 \mathrm{mg} / \mathrm{l}$ & & 1 & \\
\hline 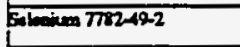 & $<0.002 \mathrm{mg} / \mathrm{l}$ & $<0.002 \mathrm{mg} / \mathrm{l}$ & $<0.002 \mathrm{mgll}$ & $<0.002 \mathrm{mg} / \mathrm{l}$ & 1 & \\
\hline Sulber & $36 \mathrm{mg} / 1$ & $30 \mathrm{mg} / \mathrm{l}$ & $36 \mathrm{mg} / 1$ & $30 \mathrm{mg} / \mathrm{l}$ & 1 & \\
\hline Surfectenes & $0.06 \mathrm{mg} / \mathrm{l}$ & $<0.05 \mathrm{mg} / \mathrm{l}$ & $0.06 \mathrm{mg} / \mathrm{l}$ & $<0.05 \mathrm{mg} / \mathrm{l}$ & 1 & \\
\hline Alumean 7429-90-5 & $0.24 \mathrm{mg} / \mathrm{l}$ & $0.21 \mathrm{mg} / \mathrm{l}$ & $0.24 \mathrm{mg} / \mathrm{l}$ & $0.21 \mathrm{mg} / \mathrm{l}$ & 1 & \\
\hline $1=000740-34-2$ & $<0.04 \mathrm{mg} / \mathrm{l}$ & $<0.04 \mathrm{mg} / \mathrm{l}$ & $<0.04 \mathrm{mg} / \mathrm{l}$ & $<0.04 \mathrm{mg} / \mathrm{l}$ & 1 & \\
\hline $300004740-128$ & $0.020 \mathrm{mg} / 1$ & $0.017 \mathrm{mg} / \mathrm{l}$ & $0.020 \mathrm{mg} / \mathrm{l}$ & $0.017 \mathrm{mg} / \mathrm{l}$ & 1 & \\
\hline Reryllium 74041.7 & $<0.0004 \mathrm{mg} / \mathrm{l}$ & $<0.0004 \mathrm{mg} / \mathrm{l}$ & $<0.0004 \mathrm{mg} / \mathrm{l}$ & $<0.0004 \mathrm{mg} / \mathrm{l}$ & 1 & \\
\hline Fadminos 7400-13-9 & $<0.004 \mathrm{mg} / \mathrm{l}$ & $<0.004 \mathrm{mg} / \mathrm{l}$ & $<0.004 \mathrm{mg} / 1$ & $<0.004 \mathrm{mg} / \mathrm{l}$ & 1 & \\
\hline pelaxion 740-70-2 & $27.3 \mathrm{mg} / 1$ & $22.7 \mathrm{mg} / \mathrm{l}$ & $27.3 \mathrm{mg} / \mathrm{l}$ & $22.7 \mathrm{mg} / \mathrm{l}$ & 1 & \\
\hline 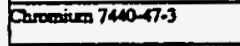 & $<0.006 \mathrm{mg} / 1$ & $<0.006 \mathrm{mg} / \mathrm{l}$ & $<0.006 \mathrm{mg} / 1$ & $<0.006 \mathrm{mg} / \mathrm{l}$ & 1 & \\
\hline Cotali 740aced & $<0.002 \mathrm{mg} / \mathrm{l}$ & $<0.002 \mathrm{mg} / \mathrm{h}$ & $<0.002 \mathrm{mg} / 1$ & $<0.002 \mathrm{mg} / \mathrm{l}$ & 1 & \\
\hline Fopper 7400-508 & $0.036 \mathrm{mg} / 1$ & $0.027 \mathrm{mg} / 1$ & $0.036 \mathrm{mg} / 1$ & $0.027 \mathrm{mg} / \mathrm{l}$ & 1 & \\
\hline 5000739896 & $0.22 \mathrm{mg} / 1$ & $0.24 \mathrm{mg} / \mathrm{l}$ & $0.22 \mathrm{mg} / 1$ & $0.24 \mathrm{mg} / \mathrm{l}$ & 1 & \\
\hline Fod 7ajon-1 & $<0.02 \mathrm{mg} / 1$ & $<0.02 \mathrm{mg} / \mathrm{l}$ & $<0.02 \mathrm{mg} / \mathrm{l}$ & $<0.02 \mathrm{mg} / 1$ & 1 & \\
\hline Folher 7439-93-2 & $<0.02 \mathrm{mg} / \mathrm{l}$ & $<0.02 \mathrm{mg} / \mathrm{l}$ & $<0.02 \mathrm{mg} / \mathrm{l}$ & $<0.02 \mathrm{mg} / 1$ & I & \\
\hline Meqpesure 7432954 & $5.44 \mathrm{mg} / 1$ & $4.37 \mathrm{mg} / \mathrm{l}$ & $5.44 \mathrm{mg} / \mathrm{l}$ & $4.37 \mathrm{mg} / 1$ & 1 & \\
\hline Menpenem 7439-96-5 & $0.015 \mathrm{mg} / \mathrm{l}$ & $0.016 \mathrm{mg} / 1$ & $0.015 \mathrm{mg} / 1$ & $0.016 \mathrm{mg} / 1$ & 1 & \\
\hline Motrodeam 743998-7 & $<0.006 \mathrm{mg} / \mathrm{l}$ & $<0.006 \mathrm{mg} / \mathrm{l}$ & $<0.006 \mathrm{mg} / \mathrm{l}$ & $<0.006 \mathrm{mg} / \mathrm{l}$ & 1 & \\
\hline Niscel 7400020 & $<0.008 \mathrm{mg} / \mathrm{l}$ & $<0.008 \mathrm{mg} / 1$ & $<0.008 \mathrm{mg} / \mathrm{l}$ & $<0.008 \mathrm{mg} / \mathrm{l}$ & 1 & \\
\hline Pomonom 7400097 & $1.7 \mathrm{mg} / \mathrm{l}$ & $1.5 \mathrm{mg} / 1$ & $1.7 \mathrm{mg} / 1$ & $1.5 \mathrm{mg} / 1$ & 1 & \\
\hline Sither 740-224 & $<0.006 \mathrm{mg} / \mathrm{l}$ & $<0.006 \mathrm{mg} / \mathrm{l}$ & $<0: 006 \mathrm{mg} / \mathrm{l}$ & $<0.006 \mathrm{mg} / \mathrm{l}$ & 1 & \\
\hline soding $7400.22-5$ & $3.27 \mathrm{mg} / \mathrm{l}$ & $2.69 \mathrm{mg} / \mathrm{l}$ & $3.27 \mathrm{mg} / 1$ & $2.69 \mathrm{mg} / \mathrm{l}$ & 1 & \\
\hline Tinesives 740-326-6 & $<0.01 \mathrm{mg} / \mathrm{l}$ & $<0.01 \mathrm{mg} / \mathrm{l}$ & $<0.01 \mathrm{mg} / \mathrm{l}$ & $<0.01 \mathrm{mg} / 1$ & 1 & \\
\hline $31007400-66-6$ & $0.17 \mathrm{mg} / 1$ & $0.15 \mathrm{mg} / \mathrm{l}$ & $0.17 \mathrm{mg} / \mathrm{l}$ & $0.15 \mathrm{mg} / \mathrm{l}$ & 1 & \\
\hline
\end{tabular}


Continow from front.

\begin{tabular}{|c|c|c|c|c|c|c|}
\hline \multirow{2}{*}{$\begin{array}{l}\text { Pollutuant } \\
\text { and } \\
\text { CAS Number } \\
\text { (if availible) }\end{array}$} & \multicolumn{2}{|c|}{$\begin{array}{l}\text { Meximumom Valuas } \\
\text { (ivelude units) }\end{array}$} & \multicolumn{2}{|c|}{$\begin{array}{l}\text { Average Values } \\
\text { (includo units) }\end{array}$} & \multirow{2}{*}{$\begin{array}{l}\text { Number } \\
\text { of } \\
\text { Storm } \\
\text { Events } \\
\text { Sempled } \\
\text { - }\end{array}$} & \multirow[b]{2}{*}{ Soures of Pollutents } \\
\hline & $\begin{array}{l}\text { Grab Semple } \\
\text { Taken During } \\
\text { Fint } 30 \\
\text { Minurea }\end{array}$ & $\begin{array}{l}\text { Fow-weighted } \\
\text { Composite }\end{array}$ & $\begin{array}{l}\text { Greb Sumple } \\
\text { Taken During } \\
\text { Firs } 30 \\
\text { Minuea }\end{array}$ & $\begin{array}{c}\text { Flow-weighrod } \\
\text { Componite }\end{array}$ & & \\
\hline 1.1.1.trictlored 71.556 & $<0.010 \mathrm{mg} / \mathrm{l}$ & & $<0.010 \mathrm{mg} / \mathrm{l}$ & & 1 & \\
\hline 1.1 .2 .2 tanchloroetbone $79-34-5$ & $<0.010 \mathrm{mg} / \mathrm{l}$ & & $<0.010 \mathrm{mg} / \mathrm{l}$ & & 1 & \\
\hline 1.1.2-trictborocheres 7900.5 & $<0.010 \mathrm{mg} / \mathrm{l}$ & & $<0.010 \mathrm{mg} / \mathrm{l}$ & & 1 & \\
\hline 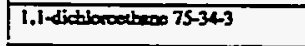 & $<0.010 \mathrm{mg} / \mathrm{l}$ & & $<0.010 \mathrm{mg} / \mathrm{l}$ & & I & \\
\hline 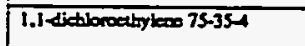 & $<0.010 \mathrm{mg} / \mathrm{l}$ & & $<0.010 \mathrm{mg} / \mathrm{l}$ & & 1 & \\
\hline 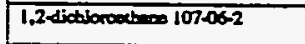 & $<0.010 \mathrm{mg} / 1$ & & $<0.010 \mathrm{mg} / \mathrm{l}$ & & 1 & \\
\hline 1.2-dictionopropares i2-87-5 & $<0.010 \mathrm{mg} / \mathrm{l}$ & & $<0.010 \mathrm{mg} / 1$ & & I & \\
\hline 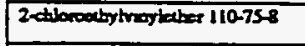 & $<0.010 \mathrm{mg} / \mathrm{l}$ & & $<0.010 \mathrm{mg} / \mathrm{l}$ & & 1 & \\
\hline $71-43-2$ & $<0.010 \mathrm{mg} / \mathrm{l}$ & & $<0.010 \mathrm{mg} / \mathrm{l}$ & & 1 & \\
\hline 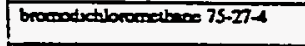 & $<0.010 \mathrm{mg} / \mathrm{l}$ & & $<0.010 \mathrm{mg} / \mathrm{l}$ & & I & \\
\hline browolorm $75-25-2$ & $<0.010 \mathrm{mg} / 1$ & & $<0.010 \mathrm{mg} / \mathrm{l}$ & & 1 & \\
\hline $7483-9$ & $<0.010 \mathrm{mg} / 1$ & & $<0.010 \mathrm{mg} / 1$ & & 1 & \\
\hline artos verantibides 56-23-5 & $<0.010 \mathrm{mg} / \mathrm{l}$ & & $<0.010 \mathrm{mg} / \mathrm{l}$ & & 1 & \\
\hline 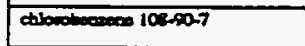 & $<0.010 \mathrm{mg} / \mathrm{l}$ & & $<0.010 \mathrm{mg} / \mathrm{l}$ & & 1 & \\
\hline 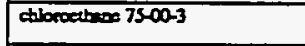 & $<0.010 \mathrm{mg} / \mathrm{l}$ & & $<0.010 \mathrm{mg} / 1$ & & 1 & \\
\hline 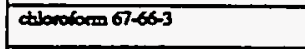 & $<0.010 \mathrm{mg} / \mathrm{l}$ & & $<0.010 \mathrm{mg} / \mathrm{l}$ & & 1 & ? \\
\hline 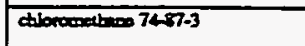 & $<0.010 \mathrm{mg} / \mathrm{l}$ & & $<0.010 \mathrm{mg} / \mathrm{l}$ & & 1 & \\
\hline 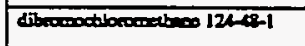 & $<0.010 \mathrm{mg} / \mathrm{h}$ & & $<0.010 \mathrm{mg} / 1$ & & 1 & \\
\hline 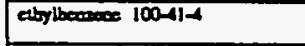 & $<0.010 \mathrm{mg} / \mathrm{l}$ & & $<0.010 \mathrm{mg} / \mathrm{l}$ & & 1 & \\
\hline Loheos $108-58-3$ & $<0.010 \mathrm{mg} / \mathrm{l}$ & & $<0.010 \mathrm{mg} / \mathrm{l}$ & & 1 & \\
\hline vivilabrido 75014 & $<0.010 \mathrm{mg} / 1$ & & $<0.010 \mathrm{mg} / \mathrm{l}$ & & 1 & \\
\hline 1.3-3ictlorepropace 10061-01-5 & $<0.010 \mathrm{mg} / \mathrm{l}$ & & $<0.010 \mathrm{mg} / \mathrm{l}$ & & 1 & \\
\hline 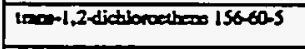 & $<0.010 \mathrm{mg} / \mathrm{l}$ & & $<0.010 \mathrm{mg} / \mathrm{l}$ & & 1 & ; \\
\hline apta satrivi $12587+46-1$ & $32 \mathrm{pci} / \mathrm{l}$ & $7.1 \mathrm{pci} / 1$ & $32 \mathrm{pci} / \mathrm{l}$ & $7.1 \mathrm{pci} / \mathrm{l}$ & 1 & \\
\hline 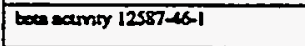 & $35 \mathrm{pei} / \mathrm{l}$ & 9.1 peill & 35 peill & $9.1 \mathrm{pci} / \mathrm{l}$ & 1 & \\
\hline soal wollibont & $1.500 \mathrm{col} .1 \mathrm{~L}$ & $180 \mathrm{co} / .1 \mathrm{~L}$ & $1,500 \mathrm{col} .1 \mathrm{~L}$ & $180 \mathrm{col} .1 \mathrm{~L}$ & 1 & \\
\hline Thellinen 7460-2300 & $<0.005 \mathrm{mg} / \mathrm{l}$ & $<0.005 \mathrm{mg} / \mathrm{l}$ & $<0.005 \mathrm{mg} / \mathrm{l}$ & $<0.005 \mathrm{mg} / \mathrm{l}$ & 1 & \\
\hline U & $0.006 \mathrm{mg} / \mathrm{l}$ & $0.006 \mathrm{mg} / \mathrm{l}$ & $0.006 \mathrm{mg} / \mathrm{t}$ & $0.006 \mathrm{mg} / \mathrm{l}$ & 1 & \\
\hline 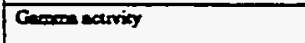 & 15 peill & 300 pcill & 15 peill & $300 \mathrm{pci} / 1$ & 1 & \\
\hline
\end{tabular}

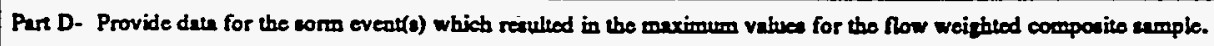

\begin{tabular}{|c|c|c|c|c|c|c|c|}
\hline $\begin{array}{l}1 . \\
\text { Date of } \\
\text { Storm } \\
\text { Eveat }\end{array}$ & $\begin{array}{l}2 . \\
\text { Durition } \\
\text { of Storm } \\
\text { (in } \\
\text { minutea) }\end{array}$ & $\begin{array}{l}3 . \\
\text { Toul ninfall } \\
\text { during roros eveat } \\
\text { (in inchea) }\end{array}$ & 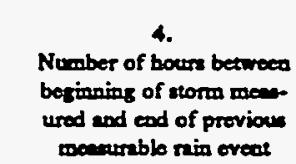 & $\begin{array}{l}\text { S. } \\
\text { Misximum flow nte } \\
\text { during nin eveat } \\
\text { (allonimin or specify } \\
\text { uniu) }\end{array}$ & $\begin{array}{c}6 . \\
\text { Toal how from } \\
\text { nin ovent } \\
\text { (ovllowe or } \\
\text { ppecify unitu) }\end{array}$ & $\begin{array}{c}7 . \\
\text { Seavon } \\
\text { semplo was } \\
\text { taken }\end{array}$ & $\begin{array}{l}8 . \\
\text { Form of } \\
\text { Procipitation } \\
\text { (nimfall. } \\
\text { noowmelt) }\end{array}$ \\
\hline $8 / 26 / 91$ & 410 & 1.4 & 160.2 & 3,652 & 599,755 & Summer & Rainfall \\
\hline
\end{tabular}

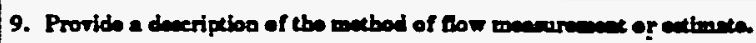

An ISCO 3230 Flow meter was used to obtain water level readings. This instrumeat was calibrated with the normal dry weather flow, if present, as level zero. The flow meter was programmed with the characteristics of the conveyance so that flow rates and total flows conld be calcalated by the meter. 
Outfoll 47

Parte B and C Continued

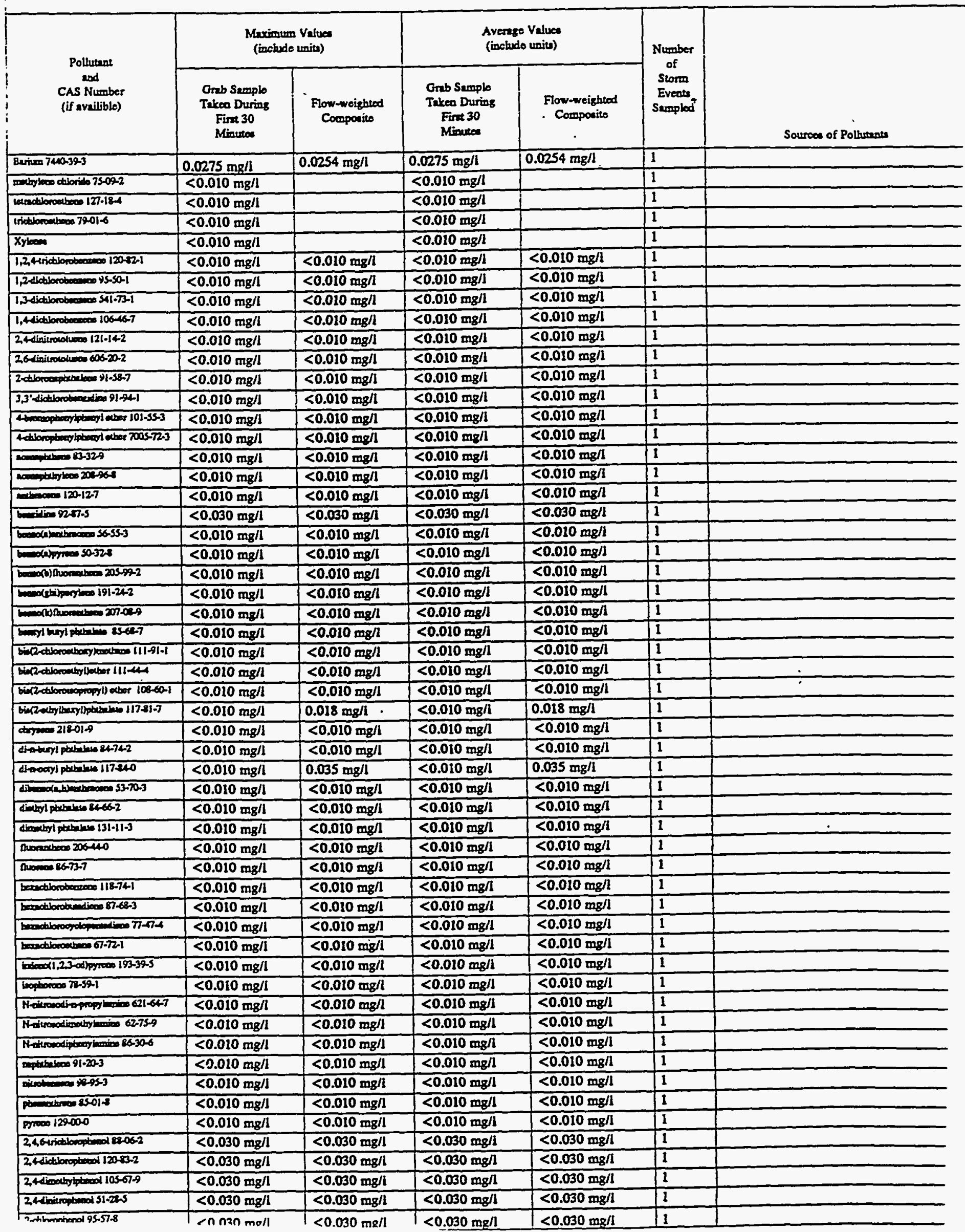


Outfail 47

Parta B end C Continuad

\begin{tabular}{|c|c|c|c|c|c|c|c|}
\hline \multirow{2}{*}{$\begin{array}{l}\text { Pollutunt } \\
\text { and } \\
\text { CAS Number } \\
\text { (if availible) }\end{array}$} & \multicolumn{2}{|c|}{$\begin{array}{l}\text { Maximum Valuea } \\
\text { (ioctude unios) }\end{array}$} & \multicolumn{2}{|c|}{$\begin{array}{l}\text { Avernge Values } \\
\text { (inebre unjit) }\end{array}$} & \multirow{2}{*}{$\begin{array}{c}\text { Number } \\
\text { of } \\
\text { Storm } \\
\text { Everts } \\
\text { Semplod }\end{array}$} & \multirow[b]{2}{*}{ Sourcen of Polhreats } & \\
\hline & $\begin{array}{l}\text { Grab Sumple } \\
\text { Takeo Durimg } \\
\text { Furst } 30 \\
\text { Minurea }\end{array}$ & $\begin{array}{l}\text { Flow-weightod } \\
\text { Compoutite }\end{array}$ & $\begin{array}{l}\text { Grab Sumple } \\
\text { Taken Durizg } \\
\text { First } 30 \\
\text { Mintea }\end{array}$ & $\begin{array}{l}\text { Flow-weighted } \\
\text { Comporise }\end{array}$ & & & \\
\hline 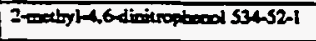 & $<0.030 \mathrm{mg} / 1$ & $<0.030 \mathrm{mg} / \mathrm{l}$ & $<0.030 \mathrm{mg} / \mathrm{l}$ & $<0.030 \mathrm{mg} / \mathrm{l}$ & 1 & & \\
\hline $2-$ ditroptroed $88.75-5$ & $<0.030 \mathrm{mg} / 1$ & $<0.030 \mathrm{mg} / \mathrm{l}$ & $<0.030 \mathrm{mg} / \mathrm{l}$ & $<0.030 \mathrm{mg} / \mathrm{l}$ & 1 & & \\
\hline 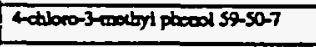 & $<0.030 \mathrm{mg} / 1$ & $<0.030 \mathrm{mg} / \mathrm{l}$ & $<0.030 \mathrm{mg} / \mathrm{l}$ & $<0.030 \mathrm{mg} / \mathrm{l}$ & 1 & & \\
\hline Laituoptood 100-02-7 & $<0.030 \mathrm{mg} / \mathrm{h}$ & $<0.030 \mathrm{mg} / \mathrm{l}$ & $<0.030 \mathrm{mg} / \mathrm{l}$ & $<0.030 \mathrm{mg} / \mathrm{l}$ & 1 & & \\
\hline peonodionopbool 8786-5 & $<0.030 \mathrm{mg} / \mathrm{l}$ & $<0.030 \mathrm{mg} / \mathrm{l}$ & $<0.030 \mathrm{mg} / \mathrm{l}$ & $<0.030 \mathrm{mg} / \mathrm{l}$ & 1 & & \\
\hline $1000-95-2$ & $<0.030 \mathrm{mg} / \mathrm{l}$ & $<0.030 \mathrm{mg} / \mathrm{l}$ & $<0.030 \mathrm{mg} / \mathrm{l}$ & $<0.030 \mathrm{mg} / \mathrm{l}$ & 1 & & \\
\hline $4.4^{\circ}-D D D 72-548$ & $<0.000004 \mathrm{mg} / \mathrm{l}$ & $<0.000004 \mathrm{mg} / 1$ & $<0.000004 \mathrm{mg} / \mathrm{l}$ & $<0.000004 \mathrm{mg} / 1$ & 1 & & \\
\hline $4,4^{4}-D D E$ 72-SS-9 & $<0.000004 \mathrm{mg} / \mathrm{l}$ & $<0.000004 \mathrm{mg} / \mathrm{l}$ & $<0.000004 \mathrm{mg} / \mathrm{l}$ & $<0.000004 \mathrm{mg} / \mathrm{l}$ & 1 & & \\
\hline 4,46-DDT SO-29-3 & $<0.000004 \mathrm{mg} / \mathrm{l}$ & $<0.000004 \mathrm{mg} / \mathrm{l}$ & $<0.000004 \mathrm{mg} / \mathrm{l}$ & $<0.000004 \mathrm{mg} / \mathrm{l}$ & 1 & & \\
\hline $2 \mathrm{ddrin} 30200-2$ & $<0.000004 \mathrm{mg} / \mathrm{l}$ & $<0.000004 \mathrm{mg} / \mathrm{l}$ & $<0.000004 \mathrm{mg} / \mathrm{l}$ & $<0.000004 \mathrm{mg} / \mathrm{l}$ & 1 & & \\
\hline chborden 57.74 & $<0.00002 \mathrm{mg} / 1$ & $<0.00002 \mathrm{mg} / \mathrm{l}$ & $<0.00002 \mathrm{mg} / \mathrm{l}$ & $<0.00002 \mathrm{mg} / \mathrm{l}$ & 1 & & \\
\hline dickidin $60-57-1$ & $<0.000004 \mathrm{mg} / \mathrm{l}$ & $<0.000004 \mathrm{mg} / \mathrm{l}$ & $<0.000004 \mathrm{mg} / \mathrm{l}$ & $<0.000004 \mathrm{mg} / \mathrm{l}$ & 1 & & \\
\hline odoulen 1959968 & $<0.000004 \mathrm{mg} / \mathrm{l}$ & $<0.000004 \mathrm{mg} / \mathrm{l}$ & $<0.000004 \mathrm{mg} / \mathrm{l}$ & $<0.000004 \mathrm{mg} / 1$ & 1 & & \\
\hline costoulfan 1133213659 & $<0.000004 \mathrm{mg} / \mathrm{l}$ & $<0.000004 \mathrm{mg} / \mathrm{l}$ & $<0.000004 \mathrm{mg} / \mathrm{h}$ & $<0.000004 \mathrm{mg} / \mathrm{l}$ & 1 & & \\
\hline 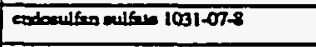 & $<0.000004 \mathrm{mg} / \mathrm{l}$ & $<0.000004 \mathrm{mg} / \mathrm{l}$ & $<0.000004 \mathrm{mg} / \mathrm{l}$ & $<0.000004 \mathrm{mg} / \mathrm{l}$ & 1 & & \\
\hline codris 72-2003 & $<0.00005 \mathrm{mg} / \mathrm{l}$ & $<0.00005 \mathrm{mg} / \mathrm{l}$ & $<0.00005 \mathrm{mg} / \mathrm{l}$ & $<0.00005 \mathrm{mg} / 1$ & 1 & & \\
\hline andrio aldetydo 7/21-93-1 & $<0.000004 \mathrm{mg} / \mathrm{l}$ & $<0.000004 \mathrm{mg} / \mathrm{l}$ & $<0.000004 \mathrm{mg} / \mathrm{l}$ & $<0.000004 \mathrm{mg} / \mathrm{l}$ & 1 & & \\
\hline tepondiber $76-46$ & $<0.000004 \mathrm{mg} / \mathrm{l}$ & $<0.000004 \mathrm{mg} / \mathrm{l}$ & $<0.000004 \mathrm{mg} / \mathrm{l}$ & $<0.000004 \mathrm{mg} / \mathrm{l}$ & 1 & - & \\
\hline bopectior epourdo $102457-3$ & $<0.000004 \mathrm{mg} / \mathrm{l}$ & $<0.000004 \mathrm{mg} / 1$ & $<0.000004 \mathrm{mg} / \mathrm{l}$ & $<0.000004 \mathrm{mg} / \mathrm{I}$ & 1 & & \\
\hline Woropheos C001-35-2 & $<0.0002 \mathrm{mg} / 1$ & $<0.0002 \mathrm{mg} / 1$ & $<0.0002 \mathrm{mg} / 1$ & $<0.0002 \mathrm{mg} / \mathrm{l}$ & 1 & & \\
\hline 4\$6-8HC 319016 & $<0.000004 \mathrm{mg} / \mathrm{l}$ & $<0.000004 \mathrm{mg} / 1$ & $<0.000004 \mathrm{mg} / 1$ & $<0.000004 \mathrm{mg} / \mathrm{l}$ & 1 & & \\
\hline bon-8HC 319-25-7 & $<0.000004 \mathrm{mg} / \mathrm{l}$ & $<0.000004 \mathrm{mg} / \mathrm{l}$ & $<0.000004 \mathrm{mg} / \mathrm{l}$ & $<0.000004 \mathrm{mg} / \mathrm{l}$ & 1 & & \\
\hline deb-BHC $319-868$ & $<0.000004 \mathrm{mg} / \mathrm{l}$ & $<0.000004 \mathrm{mg} / \mathrm{l}$ & $<0.000004 \mathrm{mg} / \mathrm{l}$ & $<0.000004 \mathrm{mg} / \mathrm{l}$ & 1 & & \\
\hline DEm-BHC $58-898$ & $<0.00001 \mathrm{mg} / \mathrm{l}$ & $<0.00001 \mathrm{mg} / \mathrm{l}$ & $<0.00001 \mathrm{mg} / 1$ & $<0.00001 \mathrm{mg} / \mathrm{l}$ & 1 & & \\
\hline 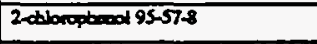 & $<0.030 \mathrm{mg} / 1$ & $<0.030 \mathrm{mg} / \mathrm{l}$ & $<0.030 \mathrm{mg} / 1$ & $<0.030 \mathrm{mg} / 1$ & 1 & & \\
\hline Arodor-1212 536021-9 & $<0.0005 \mathrm{mg} / 1$ & $<0.0005 \mathrm{mg} / \mathrm{l}$ & $<0.0005 \mathrm{mg} / \mathrm{l}$ & $<0.0005 \mathrm{mg} / \mathrm{l}$ & 1 & $\dot{5}$ & \\
\hline Aroctor $125411097-69$ & $<0.0005 \mathrm{mg} / \mathrm{l}$ & $<0.0005 \mathrm{mg} / 1$ & $<0.0005 \mathrm{mg} / \mathrm{l}$ & $<0.0005 \mathrm{mg} / 1$ & 1 & & \\
\hline Arodor-1201 11104-28-2 & $<0.0005 \mathrm{mg} / \mathrm{l}$ & $<0.0005 \mathrm{mg} / \mathrm{l}$ & $<0.0005 \mathrm{mg} / 1$ & $<0.0005 \mathrm{mg} / 1$ & 1 & & \\
\hline Aroctor 1232 III $11-16-5$ & $<0.0005 \mathrm{mg} / \mathrm{l}$ & $<0.0005 \mathrm{mg} / \mathrm{l}$ & $<0.0005 \mathrm{mg} / \mathrm{l}$ & $<0.0005 \mathrm{mg} / 1$ & 1 & & \\
\hline Anoctor-1248 12072-29-6 & $<0.0005 \mathrm{mg} / \mathrm{I}$ & $<0.0005 \mathrm{mg} / \mathrm{l}$ & $<0.0005 \mathrm{mg} / \mathrm{l}$ & $<0.0005 \mathrm{mg} / \mathrm{l}$ & 1 & & \\
\hline Aroctor $126011006-82-5$ & $<0.0005 \mathrm{mg} / \mathrm{l}$ & $<0.0005 \mathrm{mg} / \mathrm{l}$ & $<0.0005 \mathrm{mg} / 1$ & $<0.0005 \mathrm{mg} / 1$ & 1 & & \\
\hline Arodon-1016 12074-11-2 & $<0.0005 \mathrm{mg} / \mathrm{I}$ & $<0.0005 \mathrm{mg} / \mathrm{l}$ & $<0.0005 \mathrm{mg} / \mathrm{l}$ & $<0.0005 \mathrm{mg} / \mathrm{l}$ & 1 & & \\
\hline Croods & $<0.030 \mathrm{mg} / \mathrm{l}$ & $<0.030 \mathrm{mg} / \mathrm{l}$ & $<0.030 \mathrm{mg} / \mathrm{l}$ & $<0.030 \mathrm{mg} / \mathrm{l}$ & 1 & & \\
\hline Acrokin & $<0.010 \mathrm{mg} / \mathrm{l}$ & $<0.010 \mathrm{mg} / \mathrm{l}$ & $<0.010 \mathrm{mg} / 1$ & $<0.010 \mathrm{mg} / \mathrm{l}$ & 1 & & \\
\hline Acosplocitivito & $<0.010 \mathrm{mg} / \mathrm{l}$ & $<0.010 \mathrm{mg} / 1$ & $<0.010 \mathrm{mg} / \mathrm{l}$ & $<0.010 \mathrm{mg} / \mathrm{l}$ & 1 & & \\
\hline 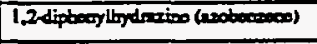 & Results pending & Results pending & Reaults pending & Recults pending & 1 & & \\
\hline & & & & & & & \\
\hline & & & & & & & \\
\hline & & & & & & & \\
\hline & & & & & & & \\
\hline & & & & & & & \\
\hline & & & & & & & \\
\hline & & & & & & & \\
\hline & & & & & & & \\
\hline & & & & & & & \\
\hline & & & & & & & \\
\hline & & & & & & & \\
\hline & & & & & & & \\
\hline & & & & & & & \\
\hline & & & & & & & \\
\hline
\end{tabular}


VII. Discharge Information (continuod from page 3 of Form 2F)

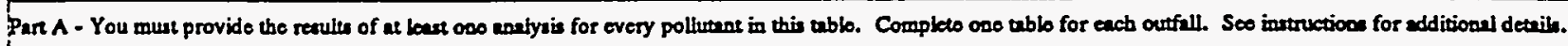

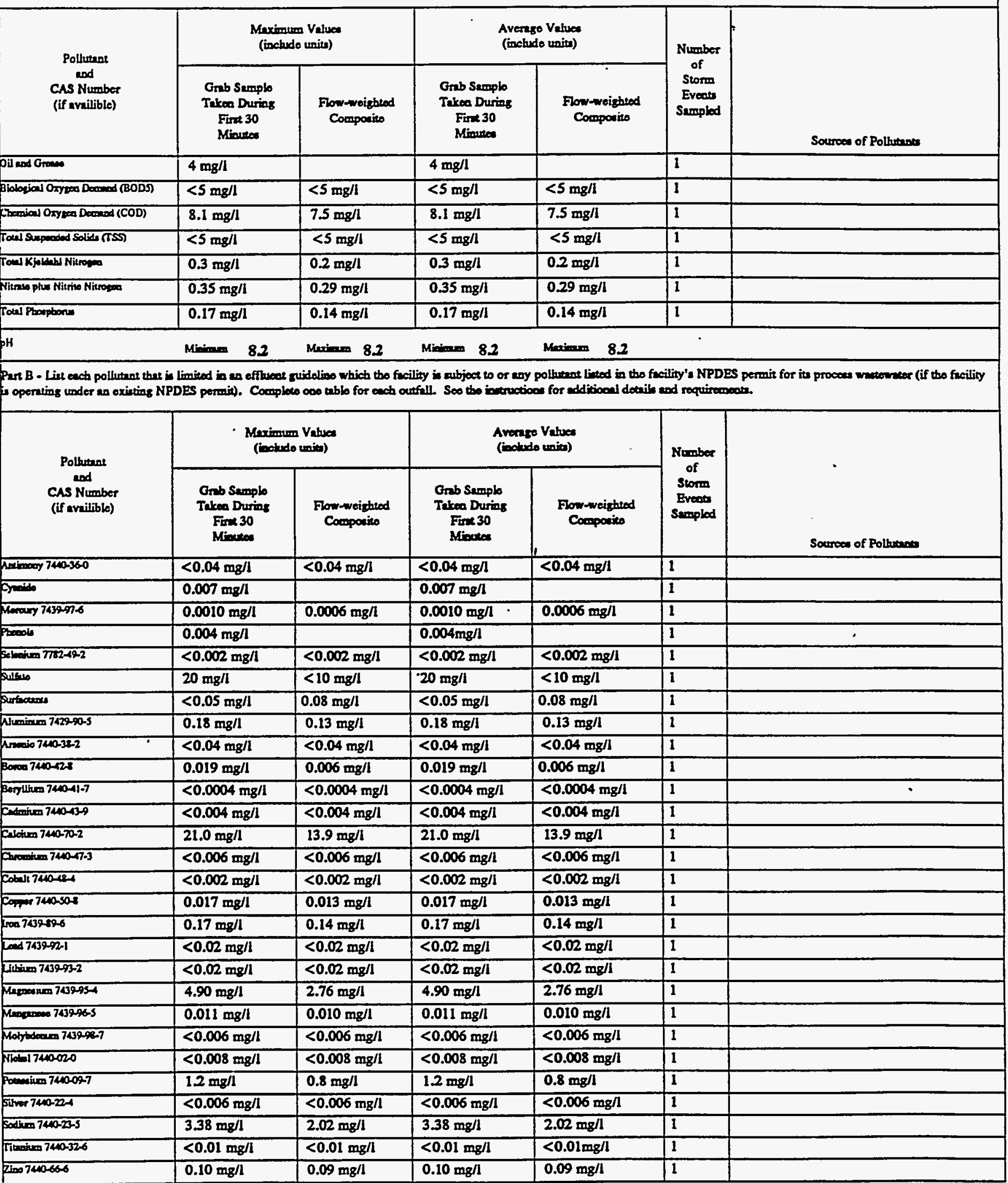




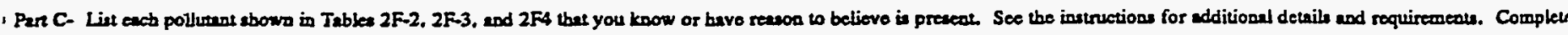
one tuble for each outull.

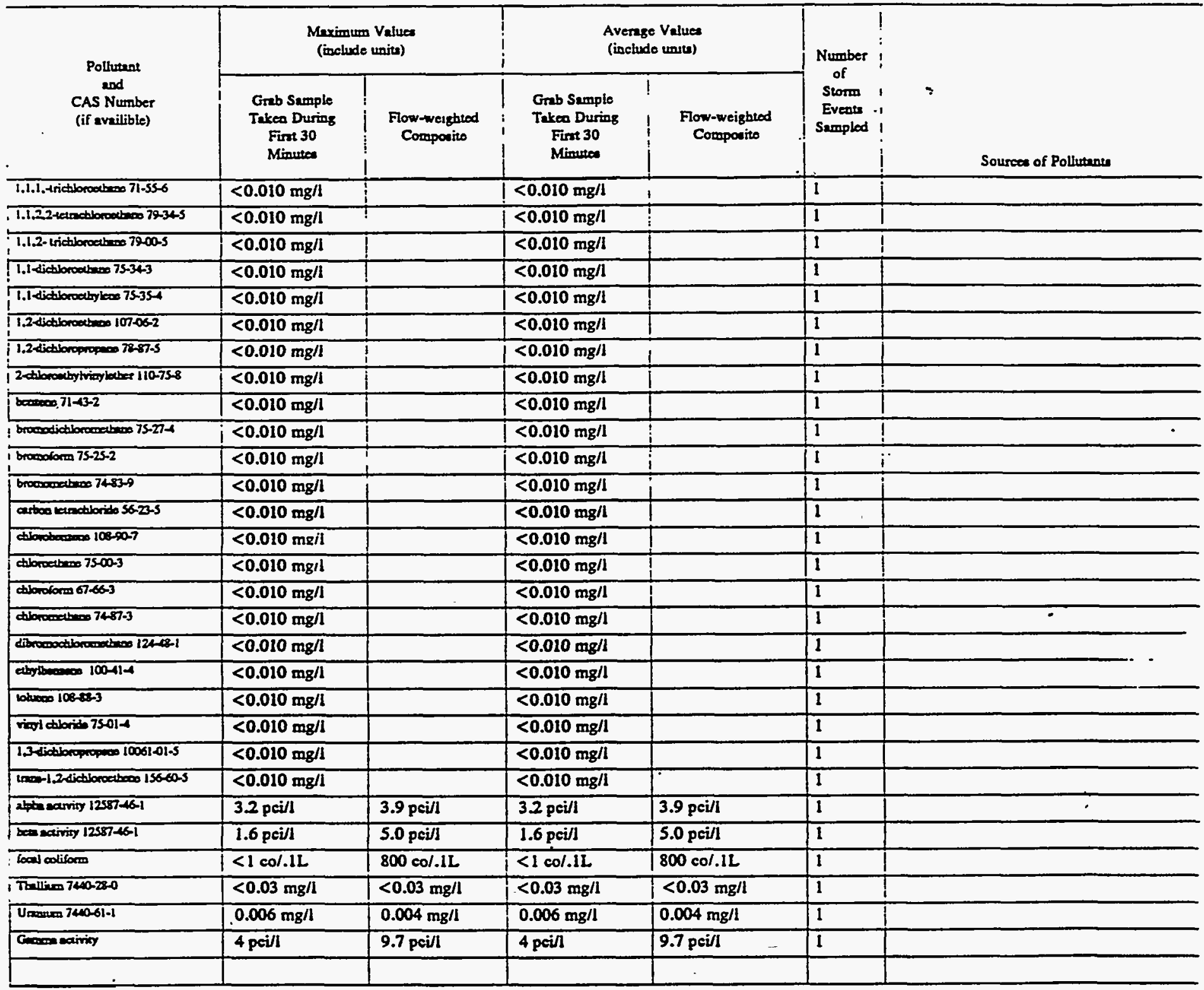

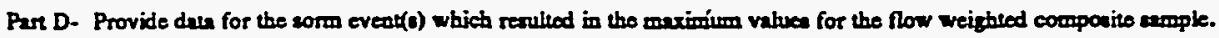

\begin{tabular}{|c|c|c|c|c|c|c|c|}
\hline $\begin{array}{l}1 . \\
\text { Deto of } \\
\text { Storm } \\
\text { Event }\end{array}$ & $\begin{array}{l}2 . \\
\text { Durniog } \\
\text { of Storm } \\
\text { (in } \\
\text { minutes) }\end{array}$ & $\begin{array}{l}3 . \\
\text { Total rinfall } \\
\text { during stomen event } \\
\text { (in inches) }\end{array}$ & $\begin{array}{l}\text { Number of bours between } \\
\text { beginning of atorm mean- } \\
\text { ured and eod of provious } \\
\text { menumblo rnin event }\end{array}$ & $\begin{array}{l}\text { S. } \\
\text { Meximum flow rato } \\
\text { during min eveol } \\
\text { (olloosimin or spocify } \\
\text { unim) }\end{array}$ & $\begin{array}{l}6 . \\
\text { Total flow from } \\
\text { nin oveas } \\
\text { (ralloos or } \\
\text { specify unita) }\end{array}$ & $\begin{array}{c}7 . \\
\text { Seavon } \\
\text { semple was } \\
\text { taked }\end{array}$ & $\begin{array}{l}8 . \\
\text { Form of } \\
\text { Procipitation } \\
\text { (rimfill. } \\
\text { soowmety) }\end{array}$ \\
\hline $12 / 9 / 91$ & 530 & 1.1 & 148.6 & 1,353 & 281,856 & Fall & Rainfall \\
\hline
\end{tabular}

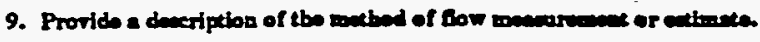

An ISCO 3230 Flow meter wes used to obtain water level readings. This instrument was calibrated with the normal dry wenther flow, if present, as le zero. The flow meter was programmed with the charcteristics of the conveyance so that flow rates and total flows could be calculated by the meter. 
Outfall 48

Parre B and C Continued

\begin{tabular}{|c|c|c|c|c|c|c|}
\hline \multirow{2}{*}{$\begin{array}{l}\text { Polluteot } \\
\text { and } \\
\text { CAS Number } \\
\text { (if availible) }\end{array}$} & \multicolumn{2}{|c|}{$\begin{array}{l}\text { Meximum Values } \\
\text { (inebude units) }\end{array}$} & \multicolumn{2}{|c|}{$\begin{array}{l}\text { Averase Valuea } \\
\text { (inebudo units) }\end{array}$} & \multirow{2}{*}{$\begin{array}{c}\text { Number } \\
\text { of } \\
\text { Storm: } \\
\text { Eveota } \\
\text { Sampled }\end{array}$} & \multirow[b]{2}{*}{ Sourcese of Polluturata } \\
\hline & $\begin{array}{c}\text { Grab Sample } \\
\text { Teken During } \\
\text { Firat } 30 \\
\text { Minutee }\end{array}$ & $\begin{array}{l}\text { Flow-wrighted } \\
\text { Composico }\end{array}$ & 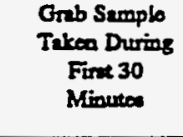 & $\begin{array}{l}\text { - Flow-woightod } \\
\text { Componito }\end{array}$ & & \\
\hline Barixom 7400-39-3 & $0.0222 \mathrm{mg} / \mathrm{l}$ & $0.0158 \mathrm{mg} / 1$ & $0.0222 \mathrm{mg} / \mathrm{l}$ & $0.0158 \mathrm{mg} / 1$ & 1 & \\
\hline mobyino otborite 7509-2 & $<0.010 \mathrm{mg} / \mathrm{l}$ & & $<0.010 \mathrm{mg} / \mathrm{l}$ & & 1 & \\
\hline 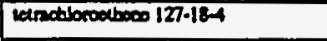 & $<0.010 \mathrm{mg} / \mathrm{l}$ & & $<0.010 \mathrm{mg} / 1$ & & 1 & \\
\hline Irichionoublowe 79016 & $<0.010 \mathrm{mg} / \mathrm{l}$ & & $<0.010 \mathrm{mg} / \mathrm{l}$ & & 1 & \\
\hline Xyloowen & $<0.010 \mathrm{mg} / 1$ & & $<0.0010 \mathrm{mg} / 1$ & & 1 & \\
\hline 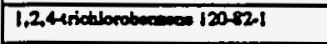 & $<0.010 \mathrm{mg} / 1$ & $<0.010 \mathrm{mg} / \mathrm{I}$ & $<0.010 \mathrm{mg} / \mathrm{l}$ & $<0.010 \mathrm{mg} / 1$ & 1 & \\
\hline 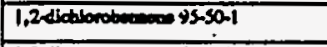 & $<0.010 \mathrm{mg} / \mathrm{l}$ & $<0.010 \mathrm{mg} / \mathrm{I}$ & $<0.010 \mathrm{mg} / \mathrm{l}$ & $<0.010 \mathrm{mg} / \mathrm{l}$ & 1 & \\
\hline 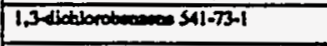 & $<0.010 \mathrm{mg} / \mathrm{l}$ & $<0.010 \mathrm{mg} / \mathrm{l}$ & $<0.010 \mathrm{mg} / \mathrm{l}$ & $<0.010 \mathrm{mg} / 1$ & 1 & \\
\hline 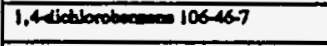 & $<0.010 \mathrm{mg} / \mathrm{l}$ & $<0.010 \mathrm{mg} / 1$ & $<0.010 \mathrm{mg} / 1$ & $<0.010 \mathrm{mg} / \mathrm{l}$ & 1 & \\
\hline 2.4dwitronohnere $121-142$ & $<0.010 \mathrm{mg} / \mathrm{l}$ & $<0.010 \mathrm{mg} / 1$ & $<0.010 \mathrm{mg} / \mathrm{l}$ & $<0.010 \mathrm{mg} / \mathrm{l}$ & 1 & \\
\hline 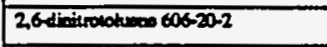 & $<0.010 \mathrm{mg} / \mathrm{l}$ & $<0.010 \mathrm{mg} / \mathrm{l}$ & $<0.010 \mathrm{mg} / \mathrm{l}$ & $<0.010 \mathrm{mg} / \mathrm{h}$ & 1 & \\
\hline 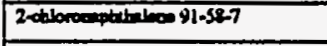 & $<0.010 \mathrm{mg} / \mathrm{l}$ & $<0.010 \mathrm{mg} / 1$ & $<0.010 \mathrm{mg} / 1$ & $<0.010 \mathrm{mg} / 1$ & 1. & \\
\hline 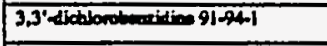 & $<0.010 \mathrm{mg} / \mathrm{l}$ & $<0.010 \mathrm{mg} / \mathrm{l}$ & $<0.010 \mathrm{mg} / 1$ & $<0.010 \mathrm{mg} / \mathrm{l}$ & 1 & \\
\hline 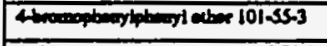 & $<0.010 \mathrm{mg} / \mathrm{l}$ & $<0.010 \mathrm{mg} / 1$ & $<0.010 \mathrm{mg} / \mathrm{l}$ & $<0.010 \mathrm{mg} / \mathrm{l}$ & 1 & \\
\hline 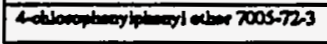 & $<0.010 \mathrm{mg} / 1$ & $<0.010 \mathrm{mg} / \mathrm{l}$ & $<0.010 \mathrm{mg} / \mathrm{I}$ & $<0.010 \mathrm{mg} / \mathrm{l}$ & 1 & \\
\hline 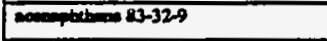 & $<0.010 \mathrm{mg} / \mathrm{h}$ & $<0.010 \mathrm{mg} / 1$ & $<0.010 \mathrm{mg} / \mathrm{l}$ & $<0.010 \mathrm{mg} / 1$ & 1 & \\
\hline 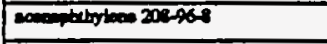 & $<0.010 \mathrm{mg} / \mathrm{l}$ & $<0.010 \mathrm{mg} / 1$ & $<0.010 \mathrm{mg} / \mathrm{l}$ & $<0.010 \mathrm{mg} / 1$ & 1 & \\
\hline $120-127$ & $<0.010 \mathrm{mg} / 1$ & $<0.010 \mathrm{mg} / \mathrm{l}$ & $<0.010 \mathrm{mg} / 1$ & $<0.010 \mathrm{mg} / \mathrm{l}$ & 1 & \\
\hline madien $92-7.5$ & $<0.050 \mathrm{mg} / \mathrm{l}$ & $<0.050 \mathrm{mg} / \mathrm{l}$ & $<0.050 \mathrm{mg} / \mathrm{l}$ & $<0.050 \mathrm{mg} / \mathrm{l}$ & 1 & \\
\hline 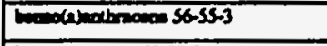 & $<0.010 \mathrm{mg} / 1$ & $<0.010 \mathrm{mg} / 1$ & $<0.010 \mathrm{mg} / 1$ & $<0.010 \mathrm{mg} / \mathrm{h}$ & 1 & \\
\hline 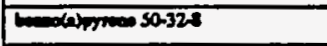 & $<0.010 \mathrm{mg} / 1$ & $<0.010 \mathrm{mg} / \mathrm{l}$ & $<0.010 \mathrm{mg} / 1$ & $<0.010 \mathrm{mg} / \mathrm{l}$ & 1 & \\
\hline 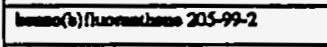 & $<0.010 \mathrm{mg} / \mathrm{l}$ & $<0.010 \mathrm{mg} / 1$ & $<0.010 \mathrm{mg} / 1$ & $<0.010 \mathrm{mg} / \mathrm{l}$ & 1 & \\
\hline 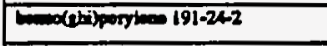 & $<0.010 \mathrm{mg} / \mathrm{l}$ & $<0.010 \mathrm{mg} / \mathrm{l}$ & $<0.010 \mathrm{mg} / \mathrm{l}$ & $<0.010 \mathrm{mg} / 1$ & 1 & \\
\hline 200.09 & $<0.010 \mathrm{mg} / \mathrm{l}$ & $<0.010 \mathrm{mg} / \mathrm{l}$ & $<0.010 \mathrm{mg} / 1$ & $<0.010 \mathrm{mg} / \mathrm{l}$ & 1 & \\
\hline 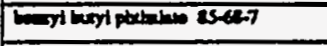 & $<0.010 \mathrm{mg} / 1$ & $<0.010 \mathrm{mg} / \mathrm{l}$ & $<0.010 \mathrm{mg} / 1$ & $<0.010 \mathrm{mg} / 1$ & 1 & \\
\hline 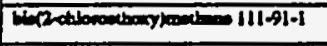 & $<0.010 \mathrm{mg} / \mathrm{l}$ & $<0.010 \mathrm{mg} / \mathrm{l}$ & $<0.010 \mathrm{mg} / 1$ & $<0.010 \mathrm{mg} / 1$ & 1 & $\therefore$ \\
\hline 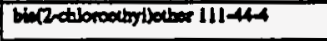 & $<0.010 \mathrm{mg} / 1$ & $<0.010 \mathrm{mg} / \mathrm{l}$ & $<0.010 \mathrm{mg} / 1$ & $<0.010 \mathrm{mg} / 1$ & 1 & \\
\hline 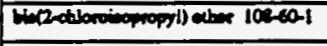 & $<0.010 \mathrm{mg} / \mathrm{l}$ & $<0.010 \mathrm{mg} / \mathrm{l}$ & $<0.010$ mg/l & $<0.010 \mathrm{mg} / \mathrm{l}$ & 1 & \\
\hline 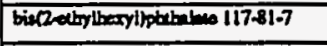 & $<0.010 \mathrm{mg} / \mathrm{l}$ & $<0.010 \mathrm{mg} / \mathrm{l}$ & $<0.010 \mathrm{mg} / \mathrm{l}$ & $<0.010 \mathrm{mg} / \mathrm{l}$ & 1 & \\
\hline कy=0 21801-9 & $<0.010 \mathrm{mg} / \mathrm{l}$ & $<0.010 \mathrm{mg} / 1$ & $<0.010 \mathrm{mg} / \mathrm{l}$ & $<0.010 \mathrm{mg} / \mathrm{l}$ & 1 & \\
\hline 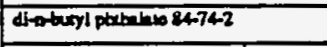 & $<0.010 \mathrm{mg} / \mathrm{l}$ & $<0.010 \mathrm{mg} / \mathrm{l}$ & $<0.010 \mathrm{mg} / \mathrm{l}$ & $<0.010 \mathrm{mg} / \mathrm{l}$ & 1 & \\
\hline 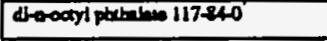 & $<0.010 \mathrm{mg} / 1$ & $<0.010 \mathrm{mg} / 1$ & $<0.010 \mathrm{mg} / 1$ & $<0.010 \mathrm{mg} / \mathrm{l}$ & 1 & \\
\hline 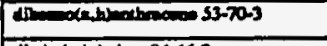 & $<0.010 \mathrm{mg} / 1$ & $<0.010 \mathrm{mg} / 1$ & $<0.010 \mathrm{mg} / \mathrm{h}$ & $<0.010 \mathrm{mg} / \mathrm{l}$ & 1 & \\
\hline dischil pathenes 84-66-2 & $<0.010 \mathrm{mg} / \mathrm{l}$ & $<0.010 \mathrm{mg} / \mathrm{l}$ & $<0.010 \mathrm{mg} / \mathrm{l}$ & $<0.010 \mathrm{mg} / 1$ & 1 & \\
\hline 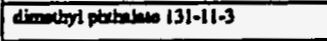 & $<0.010 \mathrm{mg} / 1$ & $<0.010 \mathrm{mg} / 1$ & $<0.010 \mathrm{mg} / \mathrm{l}$ & $<0.010 \mathrm{mg} / \mathrm{l}$ & 1 & \\
\hline 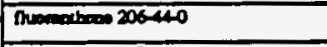 & $<0.010 \mathrm{mg} / \mathrm{l}$ & $<0.010 \mathrm{mg} / \mathrm{l}$ & $<0.010 \mathrm{mg} / \mathrm{l}$ & $<0.010 \mathrm{mg} / \mathrm{l}$ & 1 & \\
\hline $20000=86-73-7$ & $<0.010 \mathrm{mg} / 1$ & $<0.010 \mathrm{mg} / 1$ & $<0.010 \mathrm{mg} / \mathrm{l}$ & $<0.010 \mathrm{mg} / 1$ & 1 & \\
\hline 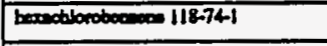 & $<0.010 \mathrm{mg} / 1$ & $<0.010 \mathrm{mg} / \mathrm{l}$ & $<0.010 \mathrm{mg} / \mathrm{l}$ & $<0.010 \mathrm{mg} / 1$ & 1 & \\
\hline 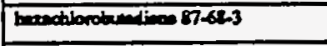 & $<0.010$ mg/i & $<0.010 \mathrm{mg} / \mathrm{l}$ & $<0.010 \mathrm{mg} / 1$ & $<0.010 \mathrm{mg} / 1$ & 1 & \\
\hline 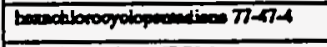 & $<0.010 \mathrm{mg} / \mathrm{l}$ & $<0.010 \mathrm{mg} / \mathrm{l}$ & $<0.010 \mathrm{mg} / \mathrm{l}$ & $<0.010 \mathrm{mg} / \mathrm{l}$ & 1 & \\
\hline 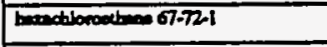 & $<0.010 \mathrm{mg} / \mathrm{I}$ & $<0.010 \mathrm{mg} / \mathrm{l}$ & $<0.010 \mathrm{mg} / 1$ & $<0.010 \mathrm{mg} / \mathrm{l}$ & 1 & \\
\hline 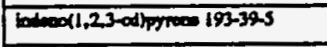 & $<0.010 \mathrm{mg} / \mathrm{l}$ & $<0.010 \mathrm{mg} / \mathrm{h}$ & $<0.010 \mathrm{mg} / \mathrm{I}$ & $<0.010 \mathrm{mg} / 1$ & 1 & \\
\hline Inophoracos $72-59-1$ & $<0.010 \mathrm{mg} / \mathrm{l}$ & $<0.010 \mathrm{mg} / 1$ & $<0.010 \mathrm{mg} / \mathrm{l}$ & $<0.010 \mathrm{mg} / \mathrm{l}$ & 1 & \\
\hline 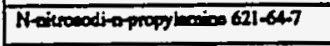 & $<0.010 \mathrm{mg} / \mathrm{l}$ & $<0.010 \mathrm{mg} / \mathrm{l}$ & $<0.010 \mathrm{mg} / 1$ & $<0.010 \mathrm{mg} / \mathrm{l}$ & 1 & \\
\hline 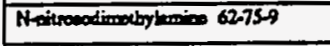 & $<0.010 \mathrm{mg} / \mathrm{l}$ & $<0.010 \mathrm{mg} / \mathrm{l}$ & $<0.010 \mathrm{mg} / \mathrm{l}$ & $<0.010 \mathrm{mg} / 1$ & 1 & \\
\hline 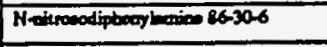 & $<0.010 \mathrm{mg} / \mathrm{l}$ & $<0.010 \mathrm{mg} / \mathrm{l}$ & $<0.010 \mathrm{mg} / 1$ & $<0.010 \mathrm{mg} / \mathrm{l}$ & 1 & \\
\hline 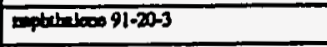 & $<0.010 \mathrm{mg} / \mathrm{l}$ & $<0.010 \mathrm{mg} / \mathrm{l}$ & $<0.010 \mathrm{mg} / \mathrm{l}$ & $<0.010 \mathrm{mg} / 1$ & 1 & \\
\hline 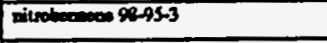 & $<0.010 \mathrm{mg} / \mathrm{l}$ & $<0.010 \mathrm{mg} / 1$ & $<0.010 \mathrm{mg} / 1$ & $<0.010 \mathrm{mg} / \mathrm{l}$ & 1 & \\
\hline 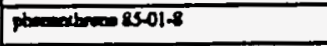 & $<0.010 \mathrm{mg} / 1$ & $<0.010 \mathrm{mg} / \mathrm{l}$ & $<0.010 \mathrm{mg} / \mathrm{l}$ & $<0.010 \mathrm{mg} / 1$ & 1 & \\
\hline prinos 1220000 & $<0.010 \mathrm{mg} / \mathrm{l}$ & $<0.010 \mathrm{mg} / \mathrm{l}$ & $<0.010 \mathrm{mg} / 1$ & $<0.010 \mathrm{mg} / \mathrm{l}$ & 1 & \\
\hline 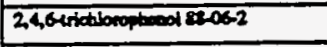 & $<0.030 \mathrm{mg} / \mathrm{l}$ & $<0.030 \mathrm{mg} / \mathrm{l}$ & $<0.030 \mathrm{mg} / \mathrm{H}$ & $<0.030 \mathrm{mg} / \mathrm{l}$ & 1 & \\
\hline 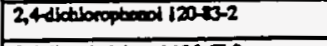 & $<0.030 \mathrm{mg} / \mathrm{l}$ & $<0.030 \mathrm{mg} / \mathrm{l}$ & $<0.030 \mathrm{mg} / 1$ & $<0.030 \mathrm{mg} / 1$ & 1 & \\
\hline 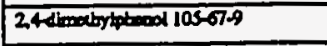 & $<0.030 \mathrm{mg} / \mathrm{h}$ & $<0.030 \mathrm{mg} / \mathrm{l}$ & $<0.030 \mathrm{mg} / 1$ & $<0.030 \mathrm{mg} / 1$ & 1 & \\
\hline 24 dinitroptroud 31.22 .5 & $<0.050 \mathrm{mg} / 1$ & $<0.050 \mathrm{mg} / \mathrm{l}$ & $<0.050 \mathrm{mg} / 1$ & $<0.050 \mathrm{mg} / 1$ & 1 & \\
\hline
\end{tabular}


Outfall 48

Part B and C Continived

\begin{tabular}{|c|c|c|c|c|c|c|}
\hline \multirow{2}{*}{$\begin{array}{l}\text { Polhutant } \\
\text { and } \\
\text { CAS Number } \\
\text { (if avmilible) }\end{array}$} & \multicolumn{2}{|c|}{$\begin{array}{l}\text { Meximam Values } \\
\text { (includo taits) }\end{array}$} & \multicolumn{2}{|c|}{$\begin{array}{l}\text { Averago Values } \\
\text { (inchudo uniua) }\end{array}$} & \multirow{2}{*}{$\begin{array}{l}\text { Number } \\
\text { of } \\
\text { Storm } \\
\text { Eveats } \\
\text { Sampled }\end{array}$} & \multirow[b]{2}{*}{ Sourcea of Polhuteats } \\
\hline & $\begin{array}{l}\text { Grab Sample } \\
\text { Takeo During } \\
\text { Firnt } 30 \\
\text { Minutea }\end{array}$ & $\begin{array}{l}\text { Flow-weightod } \\
\text { Composine }\end{array}$ & $\begin{array}{c}\text { Gnb Sample } \\
\text { Takea During } \\
\text { Fint } 30 \\
\text { Minutea }\end{array}$ & $\begin{array}{l}\text { Fiow-weightod } \\
\text { Composito }\end{array}$ & & \\
\hline 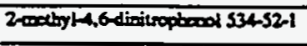 & $<0.050 \mathrm{mg} / \mathrm{l}$ & $<0.050 \mathrm{mg} / \mathrm{h}$ & $<0.050 \mathrm{mg} / 1$ & $<0.050 \mathrm{mg} / 1$ & 1 & \\
\hline 2 -iscopleod 82-75-5 & $<0.030 \mathrm{mg} / \mathrm{l}$ & $<0.030 \mathrm{mg} / \mathrm{l}$ & $<0.030 \mathrm{mg} / \mathrm{l}$ & $<0.030 \mathrm{mg} / \mathrm{l}$ & 1 & \\
\hline 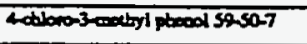 & $<0.030 \mathrm{mg} / \mathrm{l}$ & $<0.030 \mathrm{mg} / 1$ & $<0.030 \mathrm{mg} / \mathrm{l}$ & $<0.030 \mathrm{mg} / \mathrm{l}$ & 1 & \\
\hline Sinsopeod 10002.7 & $<0.050 \mathrm{mg} / \mathrm{l}$ & $<0.050 \mathrm{mg} / \mathrm{l}$ & $<0.050 \mathrm{mg} / \mathrm{l}$ & $<0.050 \mathrm{mg} / \mathrm{l}$ & 1 & \\
\hline 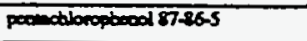 & $<0.050 \mathrm{mg} / \mathrm{l}$ & $<0.050 \mathrm{mg} / \mathrm{l}$ & $<0.050 \mathrm{mg} / \mathrm{l}$ & $<0.050 \mathrm{mg} / 1$ & 1 & \\
\hline phem ioc-95.2 & $<0.030 \mathrm{mg} / \mathrm{l}$ & $<0.030 \mathrm{mg} / \mathrm{l}$ & $<0.030 \mathrm{mg} / \mathrm{l}$ & $<0.030 \mathrm{mg} / \mathrm{l}$ & 1 & \\
\hline $4,4^{\circ}-000722-548$ & $<0.00001 \mathrm{mg} / \mathrm{l}$ & $<0.00001 \mathrm{mg} / \mathrm{l}$ & $<0.00001 \mathrm{mg} / \mathrm{l}$ & $<0.00001 \mathrm{mg} / \mathrm{l}$ & 1 & \\
\hline 4,4-00E 72-55-9 & $<0.00001 \mathrm{mg} / \mathrm{l}$ & $<0.00001 \mathrm{mg} / 1$ & $<0.00001 \mathrm{mg} / \mathrm{l}$ & $<0.00001 \mathrm{mg} / \mathrm{l}$ & 1 & \\
\hline 4.4.-DET S0-22.3 & $<0.00001 \mathrm{mg} / \mathrm{l}$ & $<0.00001 \mathrm{mg} / \mathrm{l}$ & $<0.00001 \mathrm{mg} / \mathrm{l}$ & $<0.00001 \mathrm{mg} / \mathrm{l}$ & 1 & \\
\hline 2hrin 30900-2 & $<0.00001 \mathrm{mg} / \mathrm{l}$ & $0.000020 \mathrm{mg} / \mathrm{l}$ & $<0.00001 \mathrm{mg} / \mathrm{l}$ & $0.000020 \mathrm{mg} / \mathrm{l}$ & 1 & \\
\hline dibories 57.749 & $<0.00002 \mathrm{mg} / \mathrm{l}$ & $<0.00002 \mathrm{mg} / \mathrm{l}$ & $<0.00002 \mathrm{mg} / 1$ & $<0.00002 \mathrm{mg} / 1$ & 1 & \\
\hline dielurin 60-57.1 & $<0.00001 \mathrm{mg} / \mathrm{l}$ & $<0.00001 \mathrm{mg} / \mathrm{l}$ & $<0.00001 \mathrm{mg} / \mathrm{l}$ & $<0.00001 \mathrm{mg} / \mathrm{l}$ & 1 & \\
\hline 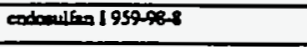 & $<0.00001 \mathrm{mg} / \mathrm{l}$ & $<0.00001 \mathrm{mg} / \mathrm{l}$ & $<0.00001 \mathrm{mg} / \mathrm{l}$ & $<0.00001 \mathrm{mg} / 1$ & 1 & \\
\hline 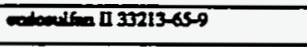 & $<0.00001 \mathrm{mg} / \mathrm{l}$ & $<0.00001 \mathrm{mg} / \mathrm{l}$ & $<0.00001 \mathrm{mg} / 1$ & $<0.00001 \mathrm{mg} / \mathrm{l}$ & 1 & \\
\hline 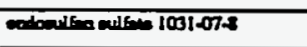 & $<0.00001 \mathrm{mg} / \mathrm{l}$ & $<0.00001 \mathrm{mg} / \mathrm{l}$ & $<0.00001 \mathrm{mg} / \mathrm{l}$ & $<0.00001 \mathrm{mg} / \mathrm{l}$ & 1 & \\
\hline castrie $72-205$ & $<0.00005 \mathrm{mg} / \mathrm{l}$ & $<0.00005 \mathrm{mg} / \mathrm{l}$ & $<0.00005 \mathrm{mg} / \mathrm{l}$ & $<0.00005 \mathrm{mg} / \mathrm{l}$ & 1 & \\
\hline 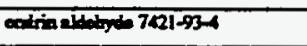 & $<0.00001 \mathrm{mg} / \mathrm{l}$ & $<0.00001 \mathrm{mg} / \mathrm{l}$ & $<0.00001 \mathrm{mg} / 1$ & $<0.00001 \mathrm{mg} / \mathrm{l}$ & 1 & \\
\hline topectiber 76 ans & $<0.00001 \mathrm{mg} / \mathrm{l}$ & $<0.00001 \mathrm{mg} / \mathrm{l}$ & $<0.00001 \mathrm{mg} / \mathrm{l}$ & $<0.00001 \mathrm{mg} / \mathrm{h}$ & 1 & \\
\hline 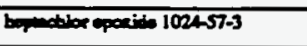 & $<0.00001 \mathrm{mg} / \mathrm{l}$ & $<0.00001 \mathrm{mg} / \mathrm{l}$ & $<0.00001 \mathrm{mg} / 1$ & $<0.00001 \mathrm{mg} / \mathrm{l}$ & 1 & \\
\hline 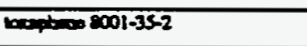 & $<0.0002 \mathrm{mg} / \mathrm{l}^{-}$ & $<0.0002 \mathrm{mg} / \mathrm{l}$ & $<0.0002 \mathrm{mg} / 1$ & $<0.0002 \mathrm{mg} / \mathrm{l}$ & 1 & \\
\hline 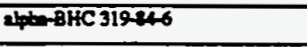 & $<0.00001 \mathrm{mg} / \mathrm{l}$ & $<0.00001 \mathrm{mg} / \mathrm{h}$ & $<0.00001 \mathrm{mg} / 1$ & $<0.00001 \mathrm{mg} / \mathrm{l}$ & 1 & \\
\hline BDOAHC 319-85-7 & $<0.00001 \mathrm{mg} / \mathrm{l}$ & $<0.00001 \mathrm{mg} / \mathrm{l}$ & $<0.00001 \mathrm{mg} / \mathrm{l}$ & $<0.00001 \mathrm{mg} / 1$ & $T$ & \\
\hline 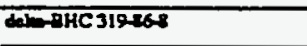 & $<0.00001 \mathrm{mg} / \mathrm{l}$ & $<0.000013 \mathrm{mg} / \mathrm{l}$ & $<0.00001 \mathrm{mg} / \mathrm{l}$ & $<0.000013 \mathrm{mg} / \mathrm{l}$ & 1 & \\
\hline $20-8 H C \operatorname{sen} 28$ & $<0.00001 \mathrm{mg} / \mathrm{l}$ & $<0.000012 \mathrm{mg} / \mathrm{l}$ & $<0.00001 \mathrm{mg} / \mathrm{l}$ & $<0.000012 \mathrm{mg} / 1$ & 1 & \\
\hline 2atlonopeod 95-57-8 & $<0.030 \mathrm{mg} / \mathrm{l}$ & $<0.030 \mathrm{mg} / \mathrm{l}$ & $<0.030 \mathrm{mg} / \mathrm{l}$ & $<0.030 \mathrm{mg} / \mathrm{l}$ & 1 & \\
\hline Anochon 1202 5316921-9 & $<0.0005 \mathrm{mg} / \mathrm{l}$ & $<0.0005 \mathrm{mg} / \mathrm{l}$ & $<0.0005 \mathrm{mg} / \mathrm{l}$ & $<0.0005 \mathrm{mg} / \mathrm{l}$ & 1 & . \\
\hline Arodor-12541100764 & $<0.0005 \mathrm{mg} / \mathrm{l}$ & $<0.0005 \mathrm{mg} / \mathrm{l}$ & $<0.0005 \mathrm{mg} / 1$ & $<0.0005 \mathrm{mg} / \mathrm{l}$ & 1 & \\
\hline Anoclon-12111104-23-2 & $<0.0005 \mathrm{mg} / \mathrm{l}$ & $<0.0005 \mathrm{mg} / \mathrm{l}$ & $<0.0005 \mathrm{mg} / \mathrm{l}$ & $<0.0005 \mathrm{mg} / \mathrm{l}$ & 1 & \\
\hline Arodor-1232 11141-16-5 & $<0.0005 \mathrm{mg} / \mathrm{l}$ & $<0.0005 \mathrm{mg} / \mathrm{l}$ & $<0.0005 \mathrm{mg} / \mathrm{h}$ & $<0.0005 \mathrm{mg} / \mathrm{l}$ & 1 & \\
\hline Aroobor-1248 12672-296 & $<0.0005 \mathrm{mg} / \mathrm{l}$ & $<0.0005 \mathrm{mg} / \mathrm{l}$ & $<0.0005 \mathrm{mg} / \mathrm{l}$ & $<0.0005 \mathrm{mg} / \mathrm{l}$ & 1 & \\
\hline Arodon-1250 11086525.5 & $<0.0005 \mathrm{mg} / \mathrm{l}$ & $<0.0005 \mathrm{mg} / \mathrm{l}$ & $<0.0005 \mathrm{mg} / \mathrm{l}$ & $<0.0005 \mathrm{mg} / \mathrm{l}$ & 1 & \\
\hline Aredon-1016 $1254-11.2$ & $<0.0005 \mathrm{mg} / \mathrm{l}$ & $<0.0005 \mathrm{mg} / 1$ & $<0.0005 \mathrm{mg} / \mathrm{l}$ & $<0.0005 \mathrm{mg} / \mathrm{l}$ & 1 & \\
\hline Creanole & $<0.010 \mathrm{mg} / \mathrm{l}$ & $<0.010 \mathrm{mg} / \mathrm{l}$ & $<0.010 \mathrm{mg} / \mathrm{h}$ & $<0.010 \mathrm{mg} / \mathrm{l}$ & 1 & \\
\hline Acroben & $<0.010 \mathrm{mg} / \mathrm{l}$ & $<0.010 \mathrm{mg} / \mathrm{l}$ & $<0.010 \mathrm{mg} / \mathrm{l}$ & $<0.010 \mathrm{mg} / \mathrm{l}$ & 1 & \\
\hline Aarylaciurito & $<0.010 \mathrm{mg} / 1$ & $<0.010 \mathrm{mg} / 1$ & $<0.010 \mathrm{mg} / \mathrm{l}$ & $<0.010 \mathrm{mg} / \mathrm{l}$ & 1 & \\
\hline 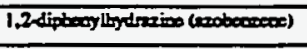 & Results peading & Rexults pending & Results pending & Recults pending & 1 & \\
\hline & & & & & & \\
\hline & & & & & & \\
\hline & & & & & & \\
\hline & & & & & & \\
\hline & & & & & & \\
\hline & & & & & & \\
\hline & & & & & & \\
\hline & & & & & & \\
\hline & & & & & & \\
\hline & & & & & & \\
\hline & & & & & & \\
\hline & & & & & & \\
\hline & & & & & & \\
\hline & & & & & & \\
\hline
\end{tabular}


VII. Discharge Information (continuad from page 3 of Form 2F

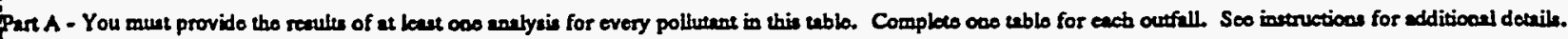

\begin{tabular}{|c|c|c|c|c|c|c|}
\hline \multirow{2}{*}{$\begin{array}{l}\text { Pollutant } \\
\text { and } \\
\text { CAS Number } \\
\text { (if availible) }\end{array}$} & \multicolumn{2}{|c|}{$\begin{array}{l}\text { Maximauran Valuces } \\
\text { (inebudo units) }\end{array}$} & \multicolumn{2}{|c|}{$\begin{array}{l}\text { Avengo Values } \\
\text { (inctude units) }\end{array}$} & \multirow{2}{*}{$\begin{array}{l}\text { Number } \\
\text { of } \\
\text { Storm } \\
\text { Evects } \\
\text { Sempled }\end{array}$} & \multirow[b]{2}{*}{ Sources of Pollutances } \\
\hline & $\begin{array}{l}\text { Greb Semple } \\
\text { Takea During } \\
\text { First } 30 \\
\text { Mioutes }\end{array}$ & $\begin{array}{l}\text { Flow-weighted } \\
\text { Componito }\end{array}$ & $\begin{array}{l}\text { Orab Sample } \\
\text { Taken During } \\
\text { Fint } 30 \\
\text { Minunes }\end{array}$ & $\begin{array}{l}\text { Fow-weightod } \\
\text { Componizo }\end{array}$ & & \\
\hline pil ead Granes & $<2 \mathrm{mg} / \mathrm{l}$ & & $<2 \mathrm{mg} / 1$ & & 1 & \\
\hline Plolopial Oxybeo Demend (BOD) & $<5 \mathrm{mg} / \mathrm{l}$ & $<5 \mathrm{mg} / \mathrm{l}$ & $<5 \mathrm{mg} / \mathrm{l}$ & $<5 \mathrm{mg} / \mathrm{l}$ & 1 & \\
\hline Chemial Oxypos Dewend (COD) & $5.5 \mathrm{mg} / 1$ & $9.2 \mathrm{mg} / \mathrm{l}$ & $5.5 \mathrm{mg} / 1$ & $9.2 \mathrm{mg} / 1$ & 1 & \\
\hline Toal suppodod Solide (TSS) & $<5 \mathrm{mg} / \mathrm{l}$ & $<5 \mathrm{mg} / 1$ & $<5 \mathrm{mg} / \mathrm{l}$ & $<5$ mg/l & 1 & \\
\hline Total Kjoblahl Nitroosos & $0.34 \mathrm{mg} / 1$ & $0.37 \mathrm{mg} / \mathrm{l}$ & $0.34 \mathrm{mg} / \mathrm{l}$ & $0.37 \mathrm{mg} / 1$ & 1 & \\
\hline Nitnes plew Nilrile Nitroses & $2.2 \mathrm{mg} / \mathrm{l}$ & $1.9 \mathrm{mg} / 1$ & $2.2 \mathrm{mg} / \mathrm{l}$ & $1.9 \mathrm{mg} / \mathrm{l}$ & 1 & \\
\hline 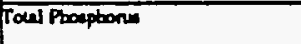 & $3.0 \mathrm{mg} / \mathrm{l}$ & $3.1 \mathrm{mg} / 1$ & $3.0 \mathrm{mg} / \mathrm{l}$ & $3.1 \mathrm{mg} / 1$ & 1 & \\
\hline $\mathrm{pH}$ & Mindmen 7.7 & Meximem 7.7 & Minimul 7.7 & Meximm 7.7 & & \\
\hline \multicolumn{7}{|c|}{ 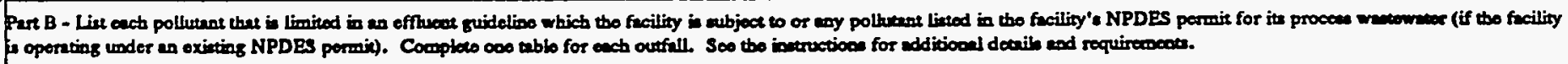 } \\
\hline \multirow{2}{*}{$\begin{array}{l}\text { Polbunt } \\
\text { and } \\
\text { CAS Number } \\
\text { (if avnilible) }\end{array}$} & \multicolumn{2}{|c|}{$\begin{array}{l}\text { Marionum Vahue } \\
\text { (include unitu) }\end{array}$} & \multicolumn{2}{|c|}{$\begin{array}{l}\text { Avecage Vahice } \\
\text { (bahdo unive) }\end{array}$} & \multirow{2}{*}{$\begin{array}{l}\text { Number } \\
\text { of } \\
\text { Storm } \\
\text { Evense } \\
\text { Samplod }\end{array}$} & \multirow[b]{2}{*}{ Sources of Pollutument } \\
\hline & 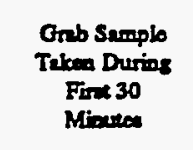 & $\begin{array}{l}\text { Flow-weighted } \\
\text { Compoisto }\end{array}$ & $\begin{array}{l}\text { Orab Seroplo } \\
\text { Takea During } \\
\text { Fins } 30 \\
\text { Minstes }\end{array}$ & $\begin{array}{l}\text { Fow-weighted } \\
\text { Compotito }\end{array}$ & & \\
\hline 19im 7400.360 & $<0.01 \mathrm{mg} / 1$ & $<0.01 \mathrm{mg} / \mathrm{l}$ & $<0.01 \mathrm{mg} / \mathrm{l}$ & $<0.01 \mathrm{mg} / \mathrm{l}$ & 1 & \\
\hline oyide & $<0.002 \mathrm{mg} / 1$ & & $<0.002 \mathrm{mg} / 1$ & & 1 & \\
\hline Merain 7439.97 .6 & $<0.0002 \mathrm{mg} / 1$ & $<0.0002 \mathrm{mg} / \mathrm{l}$ & $<0.0002 \mathrm{mg} / \mathrm{l}^{\circ}$ & $<0.0002 \mathrm{mg} / \mathrm{I}$ & 1 & \\
\hline Promole & $0.001 \mathrm{mg} / \mathrm{l}$ & & $0.001 \mathrm{mg} / \mathrm{l}$ & & 1 & $\bar{\vdots}$ \\
\hline Selvidm $7 \pi 2-49-2$ & $<0.002 \mathrm{mg} / \mathrm{I}$ & $<0.002 \mathrm{mg} / 1$ & $<0.002 \mathrm{mg} / \mathrm{l}$ & $<0.002 \mathrm{mg} / 1$ & 1 & \\
\hline Pellate & $400 \mathrm{mg} / \mathrm{l}$ & $400 \mathrm{mg} / 1$ & $400 \mathrm{mg} / 1$ & $400 \mathrm{mg} / \mathrm{l}$ & $\bar{I}$ & \\
\hline Purfoctered & $<0.05 \mathrm{mg} / \mathrm{l}$ & $<0.05 \mathrm{mg} / \mathrm{l}$ & $<0.05 \mathrm{mg} / \mathrm{l}$ & $<0.05 \mathrm{mg} / \mathrm{l}$ & 1 & \\
\hline 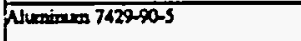 & $0.11 \mathrm{mg} / \mathrm{l}$ & $0.11 \mathrm{mg} / 1$ & $0.11 \mathrm{mg} / \mathrm{l}$ & $0.11 \mathrm{mg} / \mathrm{l}$ & 1 & \\
\hline Areadio 7460-38-2 & $<0.04 \mathrm{mg} / \mathrm{l}$ & $<0.04 \mathrm{mg} / \mathrm{l}$ & $<0.04 \mathrm{mg} / \mathrm{l}$ & $<0.04 \mathrm{mg} / 1$ & 1 & \\
\hline $800007440-124$ & $0.043 \mathrm{mg} / \mathrm{l}$ & $0.042 \mathrm{mg} / \mathrm{l}$ & $0.043 \mathrm{mg} / 1$ & $0.042 \mathrm{mg} / 1$ & 1 & \\
\hline Beryllium 7440-11-7 & $<0.0004 \mathrm{mg} / \mathrm{l}$ & $<0.0004 \mathrm{mg} / 1$ & $<0.0004 \mathrm{mg} / \mathrm{l}$ & $<0.0004 \mathrm{mg} / \mathrm{l}$ & 1 & \\
\hline Cudmiun 7400-63-9 & $<0.004 \mathrm{mg} / \mathrm{l}$ & $<0.004 \mathrm{mg} / 1$ & $<0.004 \mathrm{mg} / \mathrm{l}$ & $<0.004 \mathrm{mg} / 1$ & 1 & \\
\hline 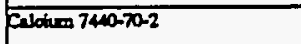 & $77.5 \mathrm{mg} / \mathrm{l}$ & $76.4 \mathrm{mg} / \mathrm{t}$ & $77.5 \mathrm{mg} / 1$ & $76.4 \mathrm{mg} / \mathrm{l}$ & 1 & \\
\hline Chroming $740-7.3$ & $<0.006 \mathrm{mg} / \mathrm{l}$ & $<0.006 \mathrm{mg} / \mathrm{l}$ & $<0.006 \mathrm{mg} / \mathrm{l}$ & $<0.006 \mathrm{mg} / \mathrm{l}$ & 1 & \\
\hline Fotal 7440-18-s & $<0.002 \mathrm{mg} / \mathrm{l}$ & $<0.002 \mathrm{mg} / \mathrm{l}$ & $<0.002 \mathrm{mg} / \mathrm{l}$ & $<0.002 \mathrm{mg} / \mathrm{l}$ & 1 & \\
\hline Coppen 740-50-8 & $0.008 \mathrm{mg} / \mathrm{l}$ & $0.008 \mathrm{mg} / \mathrm{l}$ & $0.008 \mathrm{mg} / \mathrm{l}$ & $0.008 \mathrm{mg} / \mathrm{l}$ & 1 & \\
\hline 1000 7439-896-6 & $0.22 \mathrm{mg} / \mathrm{l}$ & $0.16 \mathrm{mg} / \mathrm{l}$ & $0.22 \mathrm{mg} / \mathrm{l}$ & $0.16 \mathrm{mg} / \mathrm{l}$ & 1 & \\
\hline Fond 7439-92-1 & $<0.02 \mathrm{mg} / \mathrm{l}$ & $<0.02 \mathrm{mg} / 1$ & $<0.02 \mathrm{mg} / 1$ & $<0.02 \mathrm{mg} / \mathrm{l}$ & 1 & \\
\hline Intiven $743993-2$ & $<0.02 \mathrm{mg} / \mathrm{l}$ & $<0.02 \mathrm{mg} / \mathrm{l}$ & $<0.02 \mathrm{mg} / \mathrm{l}$ & $<0.02 \mathrm{mg} / 1$ & 1 & \\
\hline Meponiuno 7439995- & $23.0 \mathrm{mg} / \mathrm{l}$ & $22.7 \mathrm{mg} / \mathrm{l}$ & $23 \mathrm{mg} / \mathrm{l}$ & $22.7 \mathrm{mg} / 1$ & 1 & \\
\hline Mnow00000 743906-5 & $0.006 \mathrm{mg} / \mathrm{l}$ & $0.006 \mathrm{mg} / \mathrm{l}$ & $0.006 \mathrm{mg} / 1$ & $0.006 \mathrm{mg} / \mathrm{l}$ & 1 & \\
\hline Mahtodoum 7439-98-7 & $<0.006 \mathrm{mg} / \mathrm{l}$ & $<0.006 \mathrm{mg} / \mathrm{l}$ & $<0.006 \mathrm{mg} / \mathrm{l}$ & $<0.006 \mathrm{mg} / \mathrm{l}$ & 1 & \\
\hline Fiodel 7400020 & $<0.008 \mathrm{mg} / \mathrm{l}$ & $<0.008 \mathrm{mg} / 1$ & $<0.008 \mathrm{mg} / \mathrm{l}$ & $<0.008 \mathrm{mg} / \mathrm{l}$ & 1 & \\
\hline Potaniven 740-097 & $4.4 \mathrm{mg} / \mathrm{l}$ & $4.3 \mathrm{mg} / \mathrm{l}$ & $4.4 \mathrm{mg} / \mathrm{l}$ & $4.3 \mathrm{mg} / 1$ & 1 & \\
\hline Fither 740-22-4 & $<0.006 \mathrm{mg} / \mathrm{l}$ & $<0.006 \mathrm{mg} / \mathrm{l}$ & $<0.006 \mathrm{mg} / \mathrm{l}$ & $<0.006 \mathrm{mg} / \mathrm{ll}$ & 1 & \\
\hline Soliven $740-22-5$ & $59.7 \mathrm{mg} / 1$ & $57.9 \mathrm{mg} / \mathrm{l}$ & $59.7 \mathrm{mg} / \mathrm{l}$ & $57.9 \mathrm{mg} / \mathrm{l}$ & 1 & \\
\hline 71 moxian $7440-326$ & $<0.02 \mathrm{mg} / 1$ & $<0.02 \mathrm{mg} / 1$ & $<0.02 \mathrm{mg} / \mathrm{l}$ & $<0.02 \mathrm{mg} / \mathrm{l}$ & 1 & \\
\hline 21007400666 & $0.15 \mathrm{mg} / 1$ & $0.16 \mathrm{mg} / \mathrm{l}$ & $0.15 \mathrm{mg} / \mathrm{l}$ & $0.016 \mathrm{mg} / 1$ & 1 & \\
\hline
\end{tabular}


Continued trom froot.

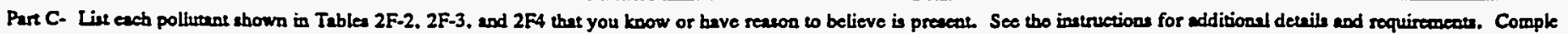
ine table for each ourfall.

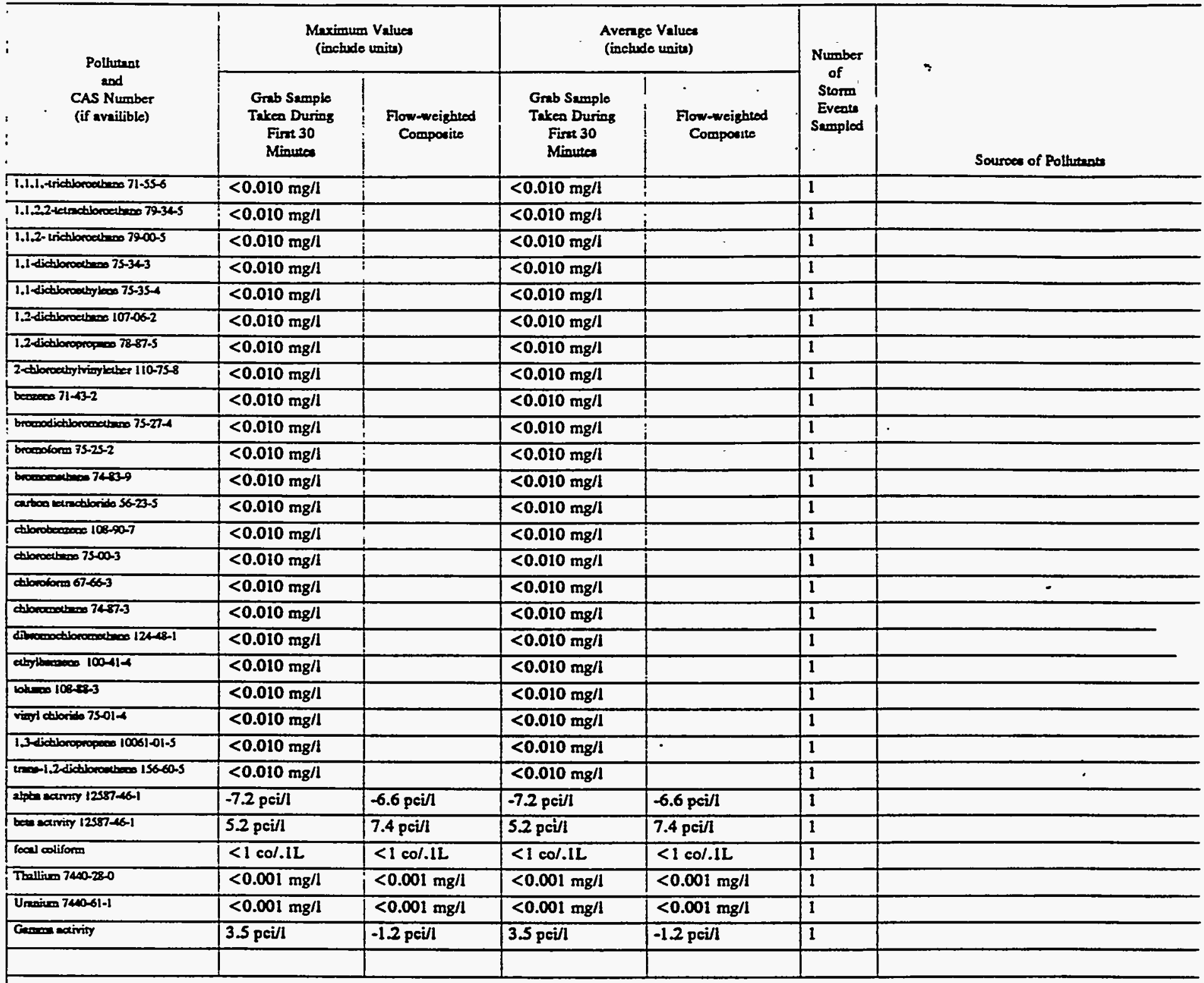

Purt D. Provide data for tho sorm eveat(s) which renuled in tho maximum valuel for the flow weighted componito amplo.

\begin{tabular}{|c|c|c|c|c|c|c|c|}
\hline $\begin{array}{l}1 . \\
\text { Deto of } \\
\text { Storm } \\
\text { Evcos }\end{array}$ & $\begin{array}{l}2 . \\
\text { Duration } \\
\text { of Storm } \\
\text { (in } \\
\text { minites) }\end{array}$ & $\begin{array}{l}3 . \\
\text { Toul rainfall } \\
\text { during storm eveat } \\
\text { (in incboe) }\end{array}$ & 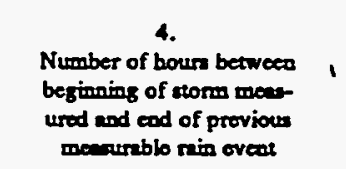 & $\begin{array}{l}\text { S. } \\
\text { Meximum flow rete } \\
\text { during nin event } \\
\text { (allone/min or specify } \\
\text { unitu) }\end{array}$ & $\begin{array}{l}6 . \\
\text { Total flow from } \\
\text { min event } \\
\text { (gallons or } \\
\text { spocify unitu) }\end{array}$ & $\begin{array}{c}7 . \\
\text { Season } \\
\text { sumple was } \\
\text { tuken }\end{array}$ & $\begin{array}{l}8 . \\
\text { Form of } \\
\text { Procipitation } \\
\text { (ninfall. } \\
\text { oween) }\end{array}$ \\
\hline $8 / 28 / 92$ & 535 & 0.91 & 124.3 & 15.5 & 2,550 & Summer & Rainfall \\
\hline
\end{tabular}

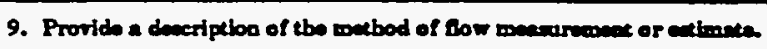

An ISCO 3230 Flow meter was used to obtain water leved readings. This instrment was calibrated with the normal dry weather flow, if present, as level zero. The fow meter was programmed with the characteristics of the conveyance so that flow rates and total flows could be calculated by the meter. 
Outfall 54

Parre B.end C Coatinued

\begin{tabular}{|c|c|c|c|c|c|c|}
\hline \multirow{2}{*}{$\begin{array}{l}\text { Pollutrant } \\
\text { and } \\
\text { CAS Numaber } \\
\text { (if availible) }\end{array}$} & \multicolumn{2}{|c|}{$\begin{array}{l}\text { Maximum Valuea } \\
\text { (inchude uritu) }\end{array}$} & \multicolumn{2}{|c|}{$\begin{array}{l}\text { Average Values } \\
\text { (inctudo unitu) }\end{array}$} & \multirow{2}{*}{$\begin{array}{c}\text { Number } \\
\text { of } \\
\text { Storm } \\
\text { Evects } \\
\text { Sempled }\end{array}$} & \multirow[b]{2}{*}{ Souroce of Pollutursta } \\
\hline & $\begin{array}{c}\text { Grab Semplo } \\
\text { Tikea During } \\
\text { Fint } 30 \\
\text { Minutea }\end{array}$ & $\begin{array}{l}\text { Fow-weightod } \\
\text { Compoito }\end{array}$ & $\begin{array}{l}\text { Grab Sumple } \\
\text { Taken During } \\
\text { Fint } 30 \\
\text { Minutes }\end{array}$ & $\begin{array}{c}\text { Flow-weightod } \\
\text { Composite }\end{array}$ & & \\
\hline Bensiden 7400-39-3 & $0.0342 \mathrm{mg} / 1$ & $0.0340 \mathrm{mg} / \mathrm{l}$ & $0.0342 \mathrm{mg} / \mathrm{l}$ & $0.0340 \mathrm{mg} / \mathrm{l}$ & 1 & \\
\hline meloymen atborido 75,092 & $<0.010 \mathrm{mg} / \mathrm{l}$ & & $<0.010 \mathrm{mg} / \mathrm{l}$ & & 1 & \\
\hline rearectionoolheos 127.184 & $<0.010 \mathrm{mg} / \mathrm{l}$ & & $<0.010 \mathrm{mg} / \mathrm{l}$ & & 1 & \\
\hline inichlorecthoos $7901-6$ & $<0.010 \mathrm{mg} / \mathrm{l}$ & & $<0.010 \mathrm{mg} / \mathrm{l}$ & & 1 & \\
\hline Xylomen & $<0.010 \mathrm{mg} / \mathrm{l}$ & & $<0.010 \mathrm{mg} / \mathrm{l}$ & & 1 & \\
\hline 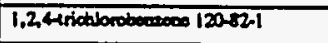 & $<0.010 \mathrm{mg} / \mathrm{l}$ & $<0.010 \mathrm{mg} / \mathrm{l}$ & $<0.010 \mathrm{mg} / \mathrm{l}$ & $<0.010 \mathrm{mg} / \mathrm{l}$ & 1 & \\
\hline 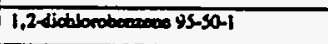 & $<0.010 \mathrm{mg} / \mathrm{l}$ & $<0.010 \mathrm{mg} / \mathrm{l}$ & $<0.010 \mathrm{mg} / \mathrm{l}$ & $<0.010 \mathrm{mg} / \mathrm{l}$ & 1 & \\
\hline 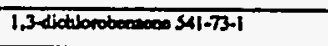 & $<0.010 \mathrm{mg} / \mathrm{l}$ & $<0.010 \mathrm{mg} / \mathrm{l}$ & $<0.010 \mathrm{mg} / \mathrm{l}$ & $<0.010 \mathrm{mg} / \mathrm{l}$ & 1 & \\
\hline 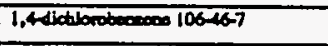 & $<0.010 \mathrm{mg} / \mathrm{l}$ & $<0.010 \mathrm{mg} / \mathrm{l}$ & $<0.010 \mathrm{mg} / \mathrm{l}$ & $<0.010 \mathrm{mg} / 1$ & 1 & \\
\hline 24, dinitrodutuen $121-142$ & $<0.010 \mathrm{mg} / \mathrm{l}$ & $<0.010 \mathrm{mg} / 1$ & $<0.010 \mathrm{mg} / \mathrm{l}$ & $<0.010 \mathrm{mg} / \mathrm{l}$ & 1 & \\
\hline 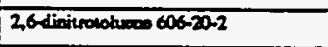 & $<0.010 \mathrm{mg} / \mathrm{l}$ & $<0.010 \mathrm{mg} / \mathrm{l}$ & $<0.010 \mathrm{mg} / \mathrm{l}$ & $<0.010 \mathrm{mg} / 1$ & 1 & \\
\hline 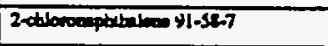 & $<0.010 \mathrm{mg} / \mathrm{l}$ & $<0.010 \mathrm{mg} / \mathrm{l}$ & $<0.010 \mathrm{mg} / \mathrm{l}$ & $<0.010 \mathrm{mg} / \mathrm{l}$ & 1 & \\
\hline 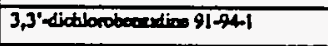 & $<0.010 \mathrm{mg} / \mathrm{l}$ & $<0.010 \mathrm{mg} / \mathrm{l}$ & $<0.010 \mathrm{mg} / \mathrm{l}$ & $<0.010 \mathrm{mg} / \mathrm{l}$ & 1 & \\
\hline 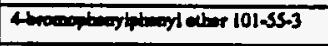 & $<0.010 \mathrm{mg} / \mathrm{l}$ & $<0.010 \mathrm{mg} / \mathrm{l}$ & $<0.010 \mathrm{mg} / \mathrm{l}$ & $<0.010 \mathrm{mg} / \mathrm{l}$ & 1 & \\
\hline 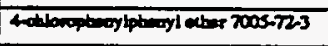 & $<0.010 \mathrm{mg} / \mathrm{l}$ & $<0.010 \mathrm{mg} / \mathrm{l}$ & $<0.010 \mathrm{mg} / 1$ & $<0.010 \mathrm{mg} / 1$ & 1 & \\
\hline 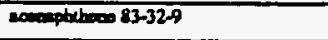 & $<0.010 \mathrm{mg} / \mathrm{l}$ & $<0.010 \mathrm{mg} / \mathrm{l}$ & $<0.010 \mathrm{mg} / \mathrm{l}$ & $<0.010 \mathrm{mg} / \mathrm{l}$ & 1 & \\
\hline comontaty & $<0.010 \mathrm{mg} / \mathrm{l}$ & $<0.010 \mathrm{mg} / \mathrm{l}$ & $<0.010 \mathrm{mg} / \mathrm{l}$ & $<0.010 \mathrm{mg} / \mathrm{l}$ & 1 & \\
\hline $120.12-7$ & $<0.010 \mathrm{mg} / 1$ & $<0.010 \mathrm{mg} / \mathrm{l}$ & $<0.010 \mathrm{mg} / \mathrm{l}$ & $<0.010 \mathrm{mg} / 1$ & 1 & \\
\hline 927.5 & $<0.030 \mathrm{mg} / \mathrm{l}$ & $<0.030 \mathrm{mg} / \mathrm{l}$ & $<0.030 \mathrm{mg} / \mathrm{l}$ & $<0.030 \mathrm{mg} / \mathrm{l}$ & 1 & \\
\hline 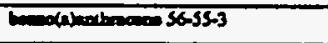 & $<0.010 \mathrm{mg} / \mathrm{l}$ & $<0.010 \mathrm{mg} / \mathrm{l}$ & $<0.010 \mathrm{mg} / \mathrm{l}$ & $<0.010 \mathrm{mg} / 1$ & 1 & \\
\hline 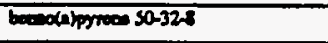 & $<0.010 \mathrm{mg} / \mathrm{l}$ & $<0.010 \mathrm{mg} / 1$ & $<0.010 \mathrm{mg} / \mathrm{l}$ & $<0.010 \mathrm{mg} / \mathrm{I}$ & 1 & \\
\hline 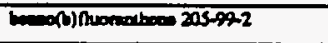 & $<0.010 \mathrm{mg} / \mathrm{l}$ & $<0.010 \mathrm{mg} / \mathrm{l}$ & $<0.010 \mathrm{mg} / \mathrm{l}$ & $<0.010 \mathrm{mg} / \mathrm{l}$ & 1 & \\
\hline 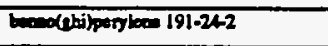 & $<0.010 \mathrm{mg} / \mathrm{l}$ & $<0.010 \mathrm{mg} / \mathrm{l}$ & $<0.010 \mathrm{mg} / \mathrm{l}$ & $<0.010 \mathrm{mg} / 1$ & 1 & \\
\hline 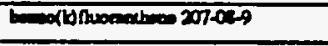 & $<0.010 \mathrm{mg} / \mathrm{l}$ & $<0.010 \mathrm{mg} / 1$ & $<0.010 \mathrm{mg} / \mathrm{h}$ & $<0.010 \mathrm{mg} / 1$ & 1 & \\
\hline 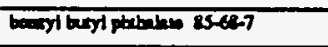 & $<0.010 \mathrm{mg} / \mathrm{l}$ & $<0.010 \mathrm{mg} / \mathrm{l}$ & $<0.010 \mathrm{mg} / \mathrm{l}$ & $<0.010 \mathrm{mg} / \mathrm{l}$ & 1 & \\
\hline 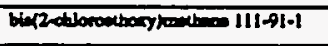 & $<0.010 \mathrm{mg} / \mathrm{l}$ & $<0.010 \mathrm{mg} / 1$ & $<0.010 \mathrm{mg} / \mathrm{l}$ & $<0.010 \mathrm{mg} / \mathrm{l}$ & 1 & \\
\hline 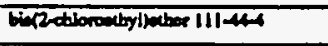 & $<0.010 \mathrm{mg} / \mathrm{l}$ & $<0.010 \mathrm{mg} / \mathrm{l}$ & $<0.010 \mathrm{mg} / 1$ & $<0.010 \mathrm{mg} / \mathrm{l}$ & 1 & \\
\hline 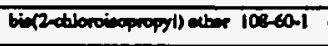 & $<0.010 \mathrm{mg} / \mathrm{l}$ & $<0.010 \mathrm{mg} / \mathrm{l}$ & $<0.010 \mathrm{mg} / \mathrm{l}$ & $<0.010 \mathrm{mg} / \mathrm{l}$ & 1 & \\
\hline 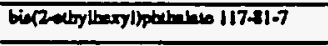 & $<0.010 \mathrm{mg} / \mathrm{l}$ & $<0.010 \mathrm{mg} / \mathrm{l}$ & $<0.010 \mathrm{mg} / 1$ & $<0.010 \mathrm{mg} / \mathrm{l}$ & 1 & \\
\hline chroweos $21801-9$ & $<0.010 \mathrm{mg} / \mathrm{l}$ & $<0.010 \mathrm{mg} / \mathrm{l}$ & $<0.010 \mathrm{mg} / 1$ & $<0.010 \mathrm{mg} / \mathrm{l}$ & 1 & \\
\hline 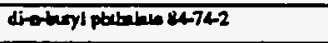 & $<0.010 \mathrm{mg} / \mathrm{l}$ & $<0.010 \mathrm{mg} / \mathrm{l}$ & $<0.010 \mathrm{mg} / 1$ & $<0.010 \mathrm{mg} / \mathrm{l}$ & 1 & \\
\hline 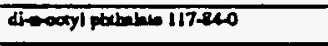 & $<0.010 \mathrm{mg} / \mathrm{l}$ & $<0.010 \mathrm{mg} / \mathrm{l}$ & $<0.010 \mathrm{mg} / \mathrm{l}$ & $<0.010 \mathrm{mg} / \mathrm{l}$ & 1 & \\
\hline 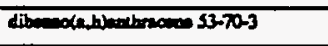 & $<0.010 \mathrm{mg} / \mathrm{l}$ & $<0.010 \mathrm{mg} / \mathrm{l}$ & $<0.010 \mathrm{mg} / \mathrm{l}$ & $<0.010 \mathrm{mg} / \mathrm{l}$ & 1 & \\
\hline 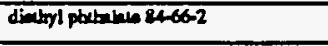 & $<0.010 \mathrm{mg} / \mathrm{l}$ & $<0.010 \mathrm{mg} / \mathrm{l}$ & $<0.010 \mathrm{mg} / \mathrm{l}$ & $<0.010 \mathrm{mg} / \mathrm{l}$ & 1 & \\
\hline 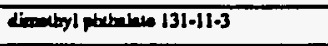 & $<0.010 \mathrm{mg} / \mathrm{l}$ & $<0.010 \mathrm{mg} / \mathrm{l}$ & $<0.010 \mathrm{mg} / 1$ & $<0.010 \mathrm{mg} / \mathrm{l}$ & 1 & \\
\hline Thoosedboses $206-140$ & $<0.010 \mathrm{mg} / \mathrm{l}$ & $<0.010 \mathrm{mg} / \mathrm{l}$ & $<0.010 \mathrm{mg} / 1$ & $<0.010 \mathrm{mg} / \mathrm{l}$ & 1 & \\
\hline Theosece $26-73-7$ & $<0.010 \mathrm{mg} / \mathrm{l}$ & $<0.010 \mathrm{mg} / \mathrm{l}$ & $<0.010 \mathrm{mg} / \mathrm{l}$ & $<0.010 \mathrm{mg} / \mathrm{l}$ & 1 & \\
\hline 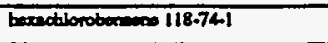 & $<0.010 \mathrm{mg} / 1$ & $<0.010 \mathrm{mg} / 1$ & $<0.010 \mathrm{mg} / \mathrm{l}$ & $<0.010 \mathrm{mg} / \mathrm{l}$ & 1 & \\
\hline 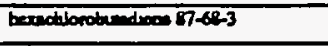 & $<0.010 \mathrm{mg} / \mathrm{l}$ & $<0.010 \mathrm{mg} / \mathrm{l}$ & $<0.010 \mathrm{mg} / 1$ & $<0.010 \mathrm{mg} / \mathrm{l}$ & 1 & \\
\hline 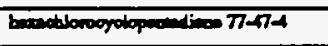 & $<0.010 \mathrm{mg} / \mathrm{l}$ & $<0.010 \mathrm{mg} / 1$ & $<0.010 \mathrm{mg} / 1$ & $<0.010 \mathrm{mg} / \mathrm{l}$ & 1 & \\
\hline 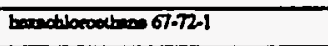 & $<0.010 \mathrm{mg} / 1$ & $<0.010 \mathrm{mg} / \mathrm{l}$ & $<0.010 \mathrm{mg} / 1$ & $<0.010 \mathrm{mg} / \mathrm{l}$ & 1 & \\
\hline 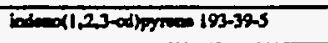 & $<0.010 \mathrm{mg} / 1$ & $<0.010 \mathrm{mg} / \mathrm{l}$ & $<0.010 \mathrm{mg} / \mathrm{l}$ & $<0.010 \mathrm{mg} / \mathrm{l}$ & 1 & \\
\hline inophoweres 72-59-1 & $<0.010 \mathrm{mg} / \mathrm{l}$ & $<0.010 \mathrm{mg} / \mathrm{l}$ & $<0.010 \mathrm{mg} / \mathrm{l}$ & $<0.010 \mathrm{mg} / \mathrm{l}$ & 1 & \\
\hline Nealsowodj-apropylamino $621-647$ & $<0.010 \mathrm{mg} / \mathrm{l}$ & $<0.010 \mathrm{mg} / \mathrm{l}$ & $<0.010 \mathrm{mg} / 1$ & $<0.010 \mathrm{mg} / \mathrm{l}$ & 1 & \\
\hline Naitrosodionothylanion 62.75-9 & $<0.010 \mathrm{mg} / \mathrm{l}$ & $<0.010 \mathrm{mg} / \mathrm{l}$ & $<0.010 \mathrm{mg} / \mathrm{l}$ & $<0.010 \mathrm{mg} / \mathrm{l}$ & 1 & \\
\hline 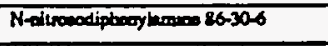 & $<0.010 \mathrm{mg} / \mathrm{l}$ & $<0.010 \mathrm{mg} / \mathrm{l}$ & $<0.010 \mathrm{mg} / 1$ & $<0.010 \mathrm{mg} / \mathrm{l}$ & 1 & \\
\hline $91-20-3$ & $<0.010 \mathrm{mg} / \mathrm{l}$ & $<0.010 \mathrm{mg} / \mathrm{l}$ & $<0.010 \mathrm{mg} / 1$ & $<0.010 \mathrm{mg} / 1$ & 1 & \\
\hline nitrobuesosese-95-3 & $<0.010 \mathrm{mg} / \mathrm{l}$ & $<0.010 \mathrm{mg} / \mathrm{l}$ & $<0.010 \mathrm{mg} / \mathrm{l}$ & $<0.010 \mathrm{mg} / \mathrm{l}$ & 1 & \\
\hline 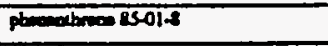 & $<0.010 \mathrm{mg} / \mathrm{l}$ & $<0.010 \mathrm{mg} / 1$ & $<0.010 \mathrm{mg} / 1$ & $<0.010 \mathrm{mg} / \mathrm{l}$ & 1 & \\
\hline Frines 129000 & $<0.010 \mathrm{mg} / \mathrm{l}$ & $<0.010 \mathrm{mg} / 1$ & $<0.010 \mathrm{mg} / \mathrm{l}$ & $<0.010 \mathrm{mg} / 1$ & 1 & \\
\hline 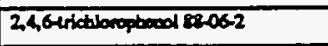 & $<0.030 \mathrm{mg} / 1$ & $<0.030 \mathrm{mg} / \mathrm{h}$ & $<0.030 \mathrm{mg} / \mathrm{l}$ & $<0.030 \mathrm{mg} / 1$ & 1 & \\
\hline 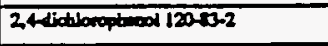 & $<0.030 \mathrm{mg} / 1$ & $<0.030 \mathrm{mg} / \mathrm{l}$ & $<0.030 \mathrm{mg} / \mathrm{l}$ & $<0.030 \mathrm{mg} / 1$ & 1 & \\
\hline 24dind 105-79 & $<0.030 \mathrm{mg} / \mathrm{l}$ & $<0.030 \mathrm{mg} / \mathrm{l}$ & $<0.030 \mathrm{mg} / \mathrm{l}$ & $<0.030 \mathrm{mg} / 1$ & 1 & \\
\hline 2.4diniropbeod S1.22.5 & $<0.030 \mathrm{mg} / \mathrm{l}$ & $<0.030 \mathrm{mg} / \mathrm{l}$ & $<0.030 \mathrm{mg} / \mathrm{l}$ & $<0.030 \mathrm{mg} / \mathrm{l}$ & 1 & \\
\hline
\end{tabular}


Outrall 54

Fars B and C Continued

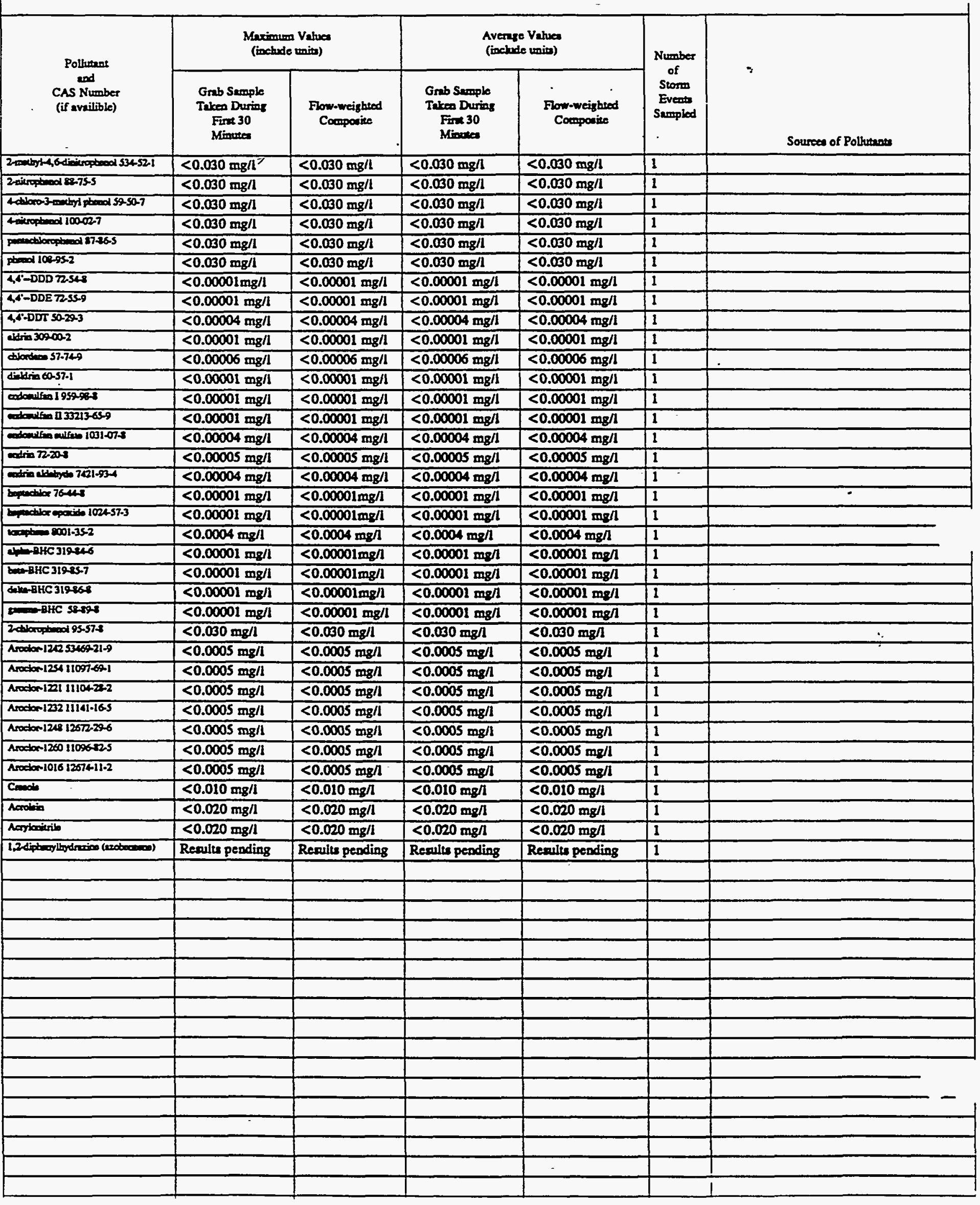




\section{Dixcharge Information (continued from page 3 of Form $2 \mathrm{~A}$}

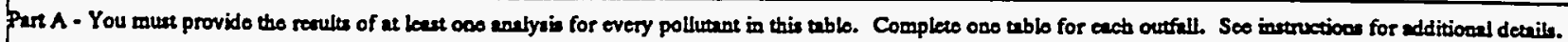

\begin{tabular}{|c|c|c|c|c|c|c|}
\hline \multirow{2}{*}{$\begin{array}{l}\text { Pollutane } \\
\text { and } \\
\text { CAs Number } \\
\text { (if availiblo) }\end{array}$} & \multicolumn{2}{|c|}{$\begin{array}{l}\text { Maximum Valuee } \\
\text { (ivelude units) }\end{array}$} & \multicolumn{2}{|c|}{$\begin{array}{l}\text { Average Valuce } \\
\text { (inelude unirs) }\end{array}$} & \multirow{2}{*}{$\begin{array}{l}\text { Number } \\
\text { of } \\
\text { Storm } \\
\text { Eveats } \\
\text { Sempled }\end{array}$} & \multirow[b]{2}{*}{ Sorm o D all } \\
\hline & $\begin{array}{l}\text { Orab Samplo } \\
\text { Takea During } \\
\text { Fint } 30 \\
\text { Mieurea }\end{array}$ & $\begin{array}{l}\text { Flow-weighted } \\
\text { Compouite }\end{array}$ & $\begin{array}{c}\text { Grab Samplo } \\
\text { Takeo Durivs } \\
\text { Finet } 30 \\
\text { Minutes }\end{array}$ & $\begin{array}{l}\text { Flow-weightod } \\
\text { Componito }\end{array}$ & & \\
\hline pil and 0 & $25 \mathrm{mg} / 1$ & & $25 \mathrm{mg} / \mathrm{l}$ & & 1 & \\
\hline Pidopical Oxy Dowed (BOD) & $<5 \mathrm{mg} / \mathrm{l}$ & $<5 \mathrm{mg} / \mathrm{l}$ & $<5 \mathrm{mg} / \mathrm{l}$ & $<5 \mathrm{mg} / \mathrm{l}$ & 1 & \\
\hline Cbenial Oxymes Deciond (COD) & $7.9 \mathrm{mg} / \mathrm{l}$ & $15 \mathrm{mg} / \mathrm{l}$ & $7.9 \mathrm{mg} / 1$ & $15 \mathrm{mg} / \mathrm{l}$ & 1 & \\
\hline Trael Sexpocided Solken (TSS) & $5.0 \mathrm{mg} / 1$ & $<5 \mathrm{mg} / 1$ & $5.0 \mathrm{mg} / \mathrm{l}$ & $<5 \mathrm{mg} / \mathrm{l}$ & 1 & \\
\hline Toal Kjeltabl Nitrosos & $0.3 \mathrm{mg} / \mathrm{l}$ & $<0.2 \mathrm{mg} / \mathrm{l}$ & $0.3 \mathrm{mg} / \mathrm{l}$ & $<0.2 \mathrm{mg} / \mathrm{l}$ & 1 & \\
\hline Nitrelo ptan Nitrito Nitrogese & $1.06 \mathrm{mg} / \mathrm{l}$ & $0.36 \mathrm{mg} / \mathrm{l}$ & $1.06 \mathrm{mg} / 1$ & $0.36 \mathrm{mg} / \mathrm{l}$ & 1 & \\
\hline Troal Propopere & $<0.1 \mathrm{mg} / \mathrm{l}$ & $<0.1 \mathrm{mg} / 1$ & $<0.1 \mathrm{mg} / \mathrm{l}$ & $<0.1 \mathrm{mg} / 1$ & 1 & \\
\hline $\mathrm{pH}$ & Minimen & Maximen 8.5 & Minimm 8.5 & Macimm 8.5 & & \\
\hline
\end{tabular}

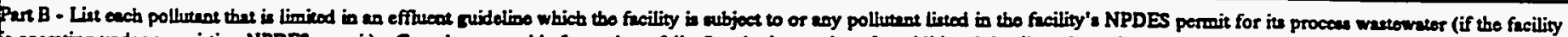

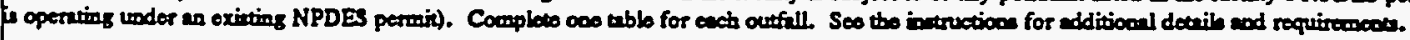

\begin{tabular}{|c|c|c|c|c|c|c|}
\hline \multirow{2}{*}{$\begin{array}{l}\text { Pollutung } \\
\text { Cand } \\
\text { CAs Number } \\
\text { (if mvilibb) }\end{array}$} & \multicolumn{2}{|c|}{$\begin{array}{l}\text { Maximumor Values } \\
\text { (inoludo unin) }\end{array}$} & \multicolumn{2}{|c|}{$\begin{array}{l}\text { Averngo Vahues } \\
\text { (ivelude units) }\end{array}$} & \multirow{2}{*}{$\begin{array}{c}\text { Number } \\
\text { of } \\
\text { Storm } \\
\text { Eveots } \\
\text { Sumpled } \\
\text {, }\end{array}$} & \multirow[b]{2}{*}{ Sources of Pollutanes } \\
\hline & $\begin{array}{l}\text { Grub Saroplo } \\
\text { Taken During } \\
\text { Find } 30 \\
\text { Mireted }\end{array}$ & $\begin{array}{l}\text { Flow-moighted } \\
\text { Comporino }\end{array}$ & $\begin{array}{l}\text { Greb Sumpbo } \\
\text { Tatoo During } \\
\text { Fint } 30 \\
\text { Minuteo }\end{array}$ & $\begin{array}{l}\text { Form-wreighted } \\
\text { Componito }\end{array}$ & & \\
\hline trimany $7400-360$ & $<0.04 \mathrm{mg} / \mathrm{l}$ & $<0.04 \mathrm{mg} / \mathrm{l}$ & $<0.04 \mathrm{mg} / \mathrm{l}$ & $<0.04 \mathrm{mg} / 1$ & 1 & \\
\hline 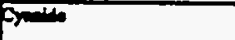 & $<0.002 \mathrm{mg} / 1$ & & $<0.002 \mathrm{mg} / \mathrm{l}$ & & 1 & \\
\hline mand 7009976 & $<0.0002 \mathrm{mg} / \mathrm{l}$ & $<0.0002 \mathrm{mg} / \mathrm{l}$ & $<0.0002 \mathrm{mg} / \mathrm{ll}$ & $<0.0002 \mathrm{mg} / \mathrm{l}$ & 1 & \\
\hline Promole & $0.002 \mathrm{mg} / \mathrm{l}$ & & $0.002 \mathrm{mg} / \mathrm{l}$ & & 1 & $\because$ \\
\hline salwanc $7 \pi 2+22$ & $<0.002 \mathrm{mg} / \mathrm{l}$ & $<0.002 \mathrm{mg} / \mathrm{h}$ & $<0.002 \mathrm{mg} / \mathrm{l}$ & $<0.002 \mathrm{mg} / \mathrm{l}$ & 1 & \\
\hline Pultem & $20 \mathrm{mg} / \mathrm{l}$ & $11 \mathrm{mg} / 1$ & $20 \mathrm{mg} / 1$ & $11 \mathrm{mg} / 1$ & $I$ & \\
\hline Perhoctenes & $<0.05 \mathrm{mg} / \mathrm{l}$ & $<0.05 \mathrm{mg} / \mathrm{l}$ & $<0.05 \mathrm{mg} / \mathrm{l}$ & $<0.05 \mathrm{mg} / 1$ & 1 & \\
\hline Ahming 7429-90-s & $0.61 \mathrm{mg} / \mathrm{l}$ & $0.42 \mathrm{mg} / \mathrm{l}$ & $0.61 \mathrm{mg} / \mathrm{l}$ & $0.42 \mathrm{mg} / \mathrm{l}$ & 1 & \\
\hline Arsodio 7400-32-2 & $<0.04 \mathrm{mg} / \mathrm{l}$ & $<0.04 \mathrm{mg} / \mathrm{l}$ & $<0.04 \mathrm{mg} / \mathrm{l}$ & $<0.04 \mathrm{mg} / \mathrm{l}$ & 1 & \\
\hline $3000097400-28$ & $0.018 \mathrm{mg} / \mathrm{l}$ & $0.014 \mathrm{mg} / \mathrm{l}$ & $0.018 \mathrm{mg} / 1$ & $0.014 \mathrm{mg} / \mathrm{l}$ & 1 & \\
\hline Berglthen 740-41-7 & $<0.0004 \mathrm{mg} / 1$ & $<0.0004 \mathrm{mg} / \mathrm{l}$ & $<0.0004 \mathrm{mg} / \mathrm{l}$ & $<0.0004 \mathrm{mg} / 1$ & 1 & \\
\hline Casmien 740-13-9 & $<0.004 \mathrm{mg} / \mathrm{l}$ & $<0.004 \mathrm{mg} / \mathrm{l}$ & $<0.004 \mathrm{mg} / \mathrm{l}$ & $<0.004 \mathrm{mg} / \mathrm{l}$ & 1 & \\
\hline Caloiem 740-70-2 & $15.4 \mathrm{mg} / \mathrm{l}$ & $12.4 \mathrm{mg} / \mathrm{l}$ & $15.4 \mathrm{mg} / \mathrm{l}$ & $12.4 \mathrm{mg} / 1$ & 1 & \\
\hline Doroming 7400-7.3 & $<0.006 \mathrm{mg} / \mathrm{l}$ & $<0.006 \mathrm{mg} / \mathrm{l}$ & $<0.006 \mathrm{mg} / 1$ & $<0.006 \mathrm{mg} / \mathrm{l}$ & 1 & \\
\hline Cobat 740-184 & $<0.002 \mathrm{mg} / \mathrm{l}$ & $<0.002 \mathrm{mg} / \mathrm{l}$ & $<0.002 \mathrm{mg} / \mathrm{l}$ & $<0.002 \mathrm{mg} / \mathrm{l}$ & 1 & \\
\hline Coppen 740-508 & $0.011 \mathrm{mg} / \mathrm{l}$ & $<0.006 \mathrm{mg} / \mathrm{l}$ & $0.011 \mathrm{mg} / 1$ & $<0.006 \mathrm{mg} / \mathrm{I}$ & 1 & \\
\hline 1007439296 & $0.63 \mathrm{mg} / \mathrm{l}$ & $0.48 \mathrm{mg} / \mathrm{l}$ & $0.63 \mathrm{mg} / \mathrm{l}$ & $0.48 \mathrm{mg} / \mathrm{l}$ & 1 & \\
\hline $\cos 743920-1$ & $<0.02 \mathrm{mg} / \mathrm{l}$ & $<0.02 \mathrm{mg} / \mathrm{l}$ & $<0.02 \mathrm{mg} / 1$ & $<0.02 \mathrm{mg} / \mathrm{l}$ & 1 & \\
\hline Fistom 7439-93-2 & $<0.02 \mathrm{mg} / \mathrm{l}$ & $<0.02 \mathrm{mg} / \mathrm{l}$ & $<0.02 \mathrm{mg} / 1$ & $<0.02 \mathrm{mg} / \mathrm{l}$ & 1 & \\
\hline 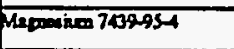 & $1.59 \mathrm{mg} / \mathrm{h}$ & $1.18 \mathrm{mg} / \mathrm{l}$ & $1.59 \mathrm{mg} / \mathrm{l}$ & $1.18 \mathrm{mg} / \mathrm{l}$ & 1 & \\
\hline $743996-5$ & $0.024 \mathrm{mg} / \mathrm{l}$ & $0.014 \mathrm{mg} / \mathrm{l}$ & $0.024 \mathrm{mg} / \mathrm{l}$ & $0.014 \mathrm{mg} / \mathrm{l}$ & 1 & \\
\hline 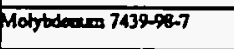 & $<0.006 \mathrm{mg} / \mathrm{l}$ & $<0.006 \mathrm{mg} / \mathrm{l}$ & $<0.006 \mathrm{mg} / \mathrm{l}$ & $<0.006 \mathrm{mg} / \mathrm{l}$ & 1 & \\
\hline Nioled 7400020 & $<0.008 \mathrm{mg} / \mathrm{l}$ & $<0.008 \mathrm{mg} / \mathrm{l}$ & $<0.008 \mathrm{mg} / \mathrm{l}$ & $<0.008 \mathrm{mg} / \mathrm{l}$ & 1 & \\
\hline 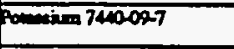 & $0.6 \mathrm{mg} / \mathrm{l}$ & $<0.5 \mathrm{mg} / \mathrm{l}$ & $0.6 \mathrm{mg} / \mathrm{l}$ & $<0.5 \mathrm{mg} / \mathrm{h}$ & 1 & \\
\hline (1) 7400-20-4 & $<0.006 \mathrm{mg} / \mathrm{l}$ & $<0.006 \mathrm{mg} / \mathrm{l}$ & $<0.006 \mathrm{mg} / \mathrm{l}$ & $<0.006 \mathrm{mg} / 1$ & 1 & \\
\hline sod4um 7440-22-5 & $0.34 \mathrm{mg} / \mathrm{l}$ & $0.26 \mathrm{mg} / 1$ & $0.34 \mathrm{mg} / \mathrm{l}$ & $0.26 \mathrm{mg} / \mathrm{l}$ & 1 & \\
\hline Fitadin $740-32-6$ & $<0.01 \mathrm{mg} / \mathrm{l}$ & $<0.01 \mathrm{mg} / \mathrm{l}$ & $<0.01 \mathrm{mg} / \mathrm{l}$ & $<0.01 \mathrm{mg} / \mathrm{l}$ & 1 & \\
\hline $2 i 00740-666$ & $0.28 \mathrm{mg} / \mathrm{l}$ & $0.15 \mathrm{mg} / 1$ & $0.28 \mathrm{mg} / \mathrm{l}$ & $0.15 \mathrm{mg} / \mathrm{l}$ & 1 & \\
\hline
\end{tabular}




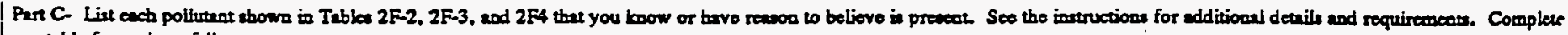
ose able for cacb outfill.

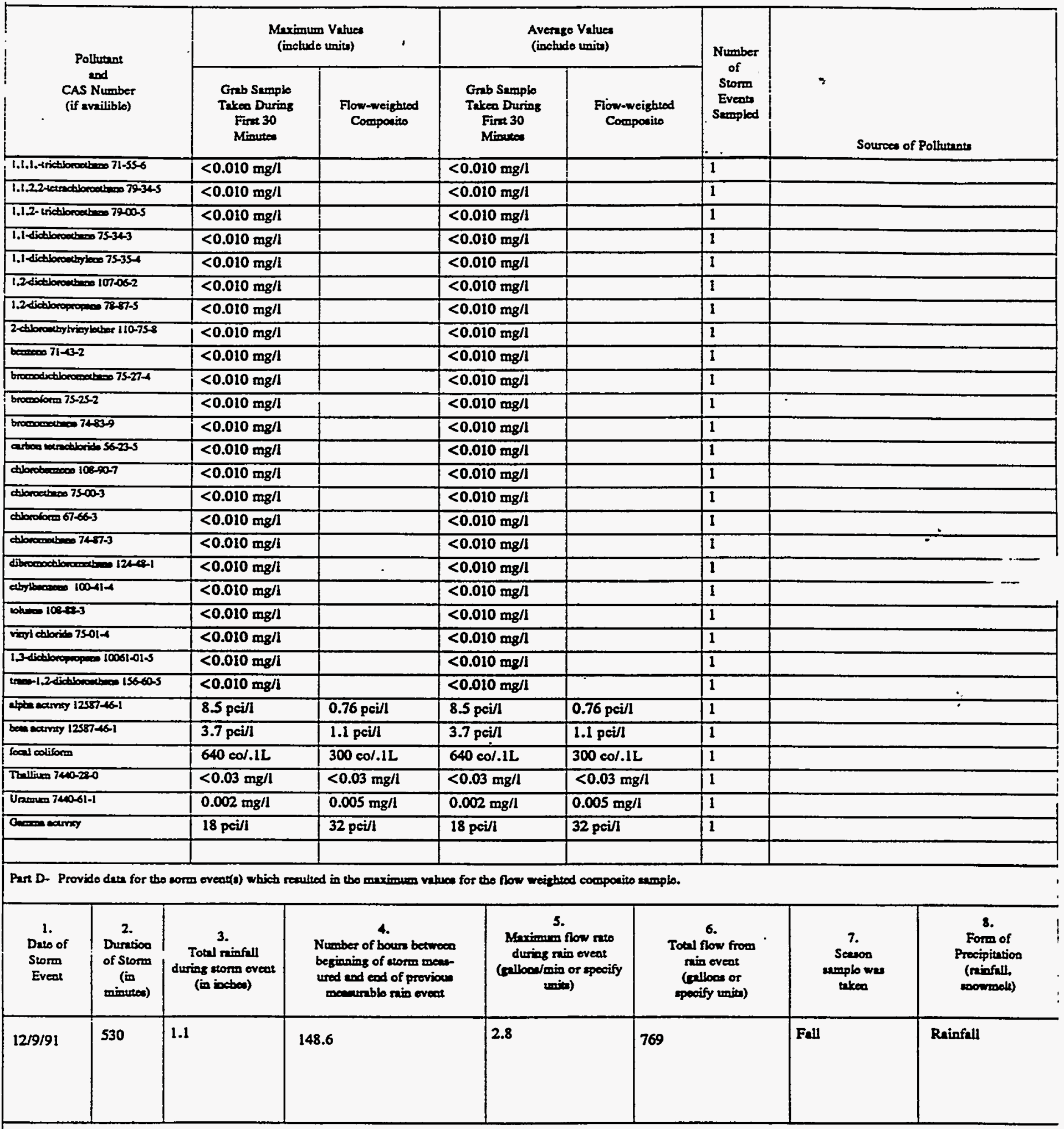

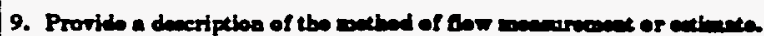

An ISCO 3230 Fon meter was med to obtain water level reading. This instrument was catibrated with the normal dry weather flow, if present, as leved zero. The flow meter was progrmmed with the chnreteristics of the convey ance so that flov rates and total Iows conld be calcolated by the meter. 
Outfall 57

Para B and C Continued

\begin{tabular}{|c|c|c|c|c|c|c|}
\hline \multirow{2}{*}{$\begin{array}{l}\text { Pollutent } \\
\text { ond } \\
\text { CAs Number } \\
\text { (if availiblo) }\end{array}$} & \multicolumn{2}{|c|}{$\begin{array}{l}\text { Maximum Values } \\
\text { (include taitu) }\end{array}$} & \multicolumn{2}{|c|}{$\begin{array}{l}\text { Aversse Vatuce } \\
\text { (incbude unitr) }\end{array}$} & \multirow{2}{*}{$\begin{array}{c}\text { Number } \\
\text { of } \\
\text { Stordif } \\
\text { Eveots } \\
\text { Samplod }\end{array}$} & \multirow[b]{2}{*}{ Sourcee of Pollhwangs } \\
\hline & $\begin{array}{l}\text { Greb Semplo } \\
\text { Takea Duriag } \\
\text { Firt } 30 \\
\text { Minutee }\end{array}$ & $\begin{array}{c}\text { Flow-weightod } \\
1 \\
1\end{array}$ & $\begin{array}{l}\text { Grab Samplo } \\
\text { Tikeo During } \\
\text { Firt } 30 \\
\text { Minurea }\end{array}$ & $\begin{array}{l}\text { Flow-weightod } \\
\text { Componite }\end{array}$ & & \\
\hline Barian 7400-39-3 & $0.0276 \mathrm{mg} / 1$ & $0.0199 \mathrm{mg} / \mathrm{l}$ & $0.0276 \mathrm{mg} / \mathrm{l}$ & $0.0199 \mathrm{mg} / \mathrm{l}$ & 1 & $\overline{-}$ \\
\hline 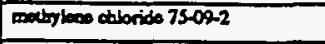 & $<0.010 \mathrm{mg} / \mathrm{l}$ & 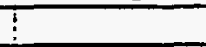 & $<0.010 \mathrm{mg} / \mathrm{l}$ & & 1 & \\
\hline 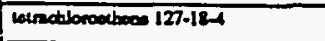 & $<0.010 \mathrm{mg} / \mathrm{l}$ & 7 & $<0.010 \mathrm{mg} / \mathrm{l}$ & & 1 & \\
\hline inidborodeon $79-01-6$ & $<0.010 \mathrm{mg} / \mathrm{l}$ & & $<0.010 \mathrm{mg} / \mathrm{l}$ & & 1 & \\
\hline xyloosen & $<0.010 \mathrm{mg} / \mathrm{I}$ & 1 & $<0.010 \mathrm{mg} / \mathrm{l}$ & & 1 & \\
\hline 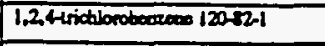 & $<0.010 \mathrm{mg} / \mathrm{l}$ & $<0.010 \mathrm{mg} / \mathrm{l}$ & $<0.010 \mathrm{mg} / \mathrm{l}$ & $<0.010 \mathrm{mg} / 1$ & 1 & \\
\hline 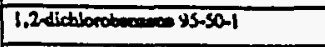 & $<0.010 \mathrm{mg} / \mathrm{l}$ & $<0.010 \mathrm{mg} / 1$ & $<0.010 \mathrm{mg} / \mathrm{l}$ & $<0.010 \mathrm{mg} / \mathrm{l}$ & 1 & \\
\hline 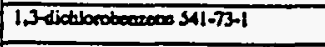 & $<0.010 \mathrm{mg} / \mathrm{l}$ & $:<0.010 \mathrm{mg} / \mathrm{l}$ & $<0.010 \mathrm{mg} / \mathrm{l}$ & $<0.010 \mathrm{mg} / \mathrm{l}$ & 1 & \\
\hline 1.4 dictlonobecoseos $106-16.7$ & $<0.010 \mathrm{mg} / \mathrm{l}$ & $<0.010 \mathrm{mg} / \mathrm{t}$ & $<0.010 \mathrm{mg} / \mathrm{l}$ & $<0.010 \mathrm{mg} / 1$ & 1 & \\
\hline 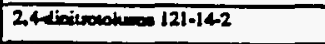 & $<0.010 \mathrm{mg} / \mathrm{l}$ & $<0.010 \mathrm{mg} / \mathrm{l}$ & $<0.010 \mathrm{mg} / \mathrm{l}$ & $<0.010 \mathrm{mg} / \mathrm{l}$ & 1 & \\
\hline 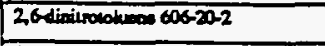 & $<0.010 \mathrm{mg} / \mathrm{l}$ & $<0.010 \mathrm{mg} / \mathrm{l}$ & $<0.010 \mathrm{mg} / \mathrm{l}$ & $<0.010 \mathrm{mg} / 1$ & 1 & \\
\hline 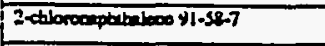 & $<0.010 \mathrm{mg} / \mathrm{l}$ & $<0.010 \mathrm{mg} / \mathrm{l}$ & $<0.010 \mathrm{mg} / \mathrm{l}$ & $<0.010 \mathrm{mg} / \mathrm{l}$ & 1. & \\
\hline 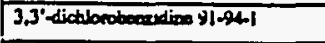 & $<0.010 \mathrm{mg} / \mathrm{l}$ & $<0.010 \mathrm{mg} / \mathrm{l}$ & $<0.010 \mathrm{mg} / \mathrm{l}$ & $<0.010 \mathrm{mg} / \mathrm{l}$ & 1 & \\
\hline 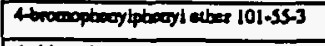 & $<0.010 \mathrm{mg} / \mathrm{l}$ & $<0.010 \mathrm{mg} / 1$ & $<0.010 \mathrm{mg} / 1$ & $<0.010 \mathrm{mg} / \mathrm{l}$ & 1 & \\
\hline 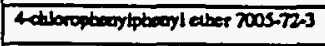 & $<0.010 \mathrm{mg} / \mathrm{l}$ & $<0.010 \mathrm{mg} / \mathrm{l}$ & $<0.010 \mathrm{mg} / \mathrm{l}$ & $<0.010 \mathrm{mg} / 1$ & 1 & \\
\hline 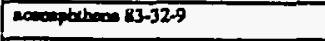 & $<0.010 \mathrm{mg} / \mathrm{l}$ & $<0.010 \mathrm{mg} / 1$ & $<0.010 \mathrm{mg} / \mathrm{l}$ & $<0.010 \mathrm{mg} / \mathrm{l}$ & 1 & \\
\hline 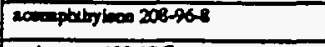 & $<0.010 \mathrm{mg} / \mathrm{l}$ & $<0.010 \mathrm{mg} / \mathrm{l}$ & $<0.010 \mathrm{mg} / \mathrm{l}$ & $<0.010 \mathrm{mg} / \mathrm{l}$ & 1 & \\
\hline wotheowoso $120-12.7$ & $<0.010 \mathrm{mg} / \mathrm{l}$ & $<0.010 \mathrm{mg} / \mathrm{l}$ & $<0.010 \mathrm{mg} / \mathrm{l}$ & $<0.010 \mathrm{mg} / \mathrm{ll}$ & 1 & \\
\hline Luidien 92.87.5 & $<0.050 \mathrm{mg} / \mathrm{l}$ & $<0.050 \mathrm{mg} / \mathrm{l}$ & $<0.050 \mathrm{mg} / \mathrm{l}$ & $<0.050 \mathrm{mg} / 1$ & 1 & \\
\hline 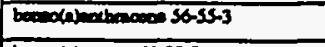 & $<0.010 \mathrm{mg} / \mathrm{l}$ & $<0.010 \mathrm{mg} / \mathrm{l}$ & $<0.010 \mathrm{mg} / \mathrm{l}$ & $<0.010 \mathrm{mg} / \mathrm{l}$ & 1 & \\
\hline 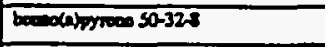 & $<0.010 \mathrm{mg} / \mathrm{l}$ & $<0.010 \mathrm{mg} / 1$ & $<0.010 \mathrm{mg} / \mathrm{l}$ & $<0.010 \mathrm{mg} / \mathrm{l}$ & 1 & \\
\hline 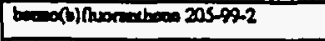 & $<0.010 \mathrm{mg} / \mathrm{I}$ & $<0.010 \mathrm{mg} / \mathrm{h}$ & $<0.010 \mathrm{mg} / \mathrm{l}$ & $<0.010 \mathrm{mg} / 1$ & 1 & \\
\hline 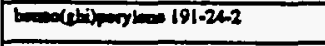 & $<0.010 \mathrm{mg} / \mathrm{l}$ & $<0.010 \mathrm{mg} / 1$ & $<0.010 \mathrm{mg} / \mathrm{l}$ & $<0.010 \mathrm{mg} / 1$ & 1 & \\
\hline 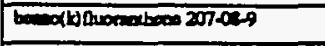 & $<0.010 \mathrm{mg} / \mathrm{l}$ & $<0.010 \mathrm{mg} / 1$ & $<0.010 \mathrm{mg} / \mathrm{l}$ & $<0.010 \mathrm{mg} / 1$ & 1 & \\
\hline 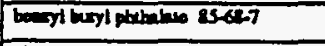 & $<0.010 \mathrm{ug} / 1$ & $<0.010 \mathrm{mg} / \mathrm{l}$ & $<0.010 \mathrm{mg} / \mathrm{l}$ & $<0.010 \mathrm{mg} / 1$ & 1 & \\
\hline 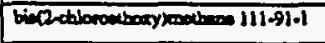 & $<0.010 \mathrm{mg} / \mathrm{l}$ & $<0.010 \mathrm{mg} / \mathrm{l}$ & $<0.010 \mathrm{mg} / 1$ & $<0.010 \mathrm{mg} / 1$ & 1 & $\because$ \\
\hline 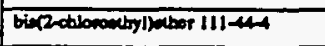 & $<0.010 \mathrm{mg} / \mathrm{l}$ & $<0.010 \mathrm{mg} / \mathrm{l}$ & $<0.010 \mathrm{mg} / 1$ & $<0.010 \mathrm{mg} / \mathrm{l}$ & 1 & \\
\hline 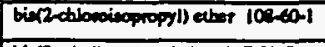 & $<0.010 \mathrm{mg} / \mathrm{l}$ & $<0.010 \mathrm{mg} / 1$ & $<0.010 \mathrm{mg} / 1$ & $<0.010 \mathrm{mg} / \mathrm{l}$ & 1 & \\
\hline bir(2-athyllexyl) & $<0.010 \mathrm{mg} / \mathrm{l}$ & $<0.010 \mathrm{mg} / \mathrm{l}$ & $<0.010 \mathrm{mg} / \mathrm{l}$ & $<0.010 \mathrm{mg} / \mathrm{l}$ & 1 & \\
\hline chryoues 218-01न & $<0.010 \mathrm{mg} / \mathrm{l}$ & $:<0.010 \mathrm{mg} / 1$ & $<0.010 \mathrm{mg} / \mathrm{l}$ & $<0.010 \mathrm{mg} / \mathrm{l}$ & 1 & \\
\hline 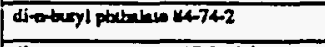 & $<0.010 \mathrm{mg} / \mathrm{l}$ & $1<0.010 \mathrm{mg} / \mathrm{l}$ & $<0.010 \mathrm{mg} / \mathrm{l}$ & $<0.010 \mathrm{mg} / \mathrm{l}$ & 1 & \\
\hline 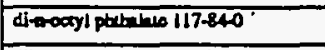 & $<0.010 \mathrm{mg} / 1$ & $<0.010 \mathrm{mg} / 1$ & $<0.010 \mathrm{mg} / \mathrm{l}$ & $<0.010 \mathrm{mg} / \mathrm{l}$ & 1 & \\
\hline 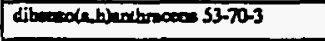 & $<0.010 \mathrm{mg} / 1$ & $<0.010 \mathrm{mg} / \mathrm{l}$ & $<0.010 \mathrm{mg} / 1$ & $<0.010 \mathrm{mg} / \mathrm{l}$ & 1 & \\
\hline 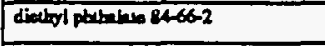 & $<0.010 \mathrm{mg} / \mathrm{l}$ & $<<0.010 \mathrm{mg} / 1$ & $<0.010 \mathrm{mg} / \mathrm{l}$ & $<0.010 \mathrm{mg} / 1$ & 1 & \\
\hline 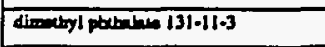 & $<0.010 \mathrm{mg} / \mathrm{l}$ & $<0.010 \mathrm{mg} / \mathrm{I}$ & $<0.010 \mathrm{mg} / \mathrm{l}$ & $<0.010 \mathrm{mg} / \mathrm{l}$ & 1 & \\
\hline 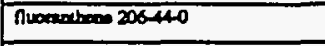 & $<0.010 \mathrm{mg} / \mathrm{l}$ & $<0.010 \mathrm{mg} / 1$ & $<0.010 \mathrm{mg} / 1$ & $<0.010 \mathrm{mg} / \mathrm{l}$ & 1 & \\
\hline Nowasom 26-73-7 & $<0.010 \mathrm{mg} / \mathrm{l}$ & $<0.010 \mathrm{mg} / \mathrm{l}$ & $<0.010 \mathrm{mg} / \mathrm{l}$ & $<0.010 \mathrm{mg} / \mathrm{l}$ & 1 & \\
\hline 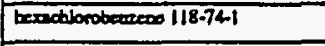 & $<0.010 \mathrm{mg} / \mathrm{l}$ & $<0.010 \mathrm{mg} / \mathrm{l}$ & $<0.010 \mathrm{mg} / \mathrm{l}$ & $<0.010 \mathrm{mg} / \mathrm{l}$ & 1 & \\
\hline 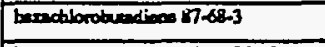 & $<0.010 \mathrm{mg} / \mathrm{l}$ & $<0.010 \mathrm{mg} / \mathrm{I}$ & $<0.010 \mathrm{mg} / 1$ & $<0.010 \mathrm{mg} / \mathrm{l}$ & 1 & \\
\hline 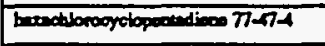 & $<0.010 \mathrm{mg} / 1$ & $<0.010 \mathrm{mg} / \mathrm{l}$ & $<0.010 \mathrm{mg} / \mathrm{l}$ & $<0.010 \mathrm{mg} / \mathrm{l}$ & 1 & \\
\hline beandtoroubano 67.72 .1 & $<0.010 \mathrm{mg} / \mathrm{l}$ & $<0.010 \mathrm{mg} / \mathrm{l}$ & $<0.010 \mathrm{mg} / \mathrm{l}$ & $<0.010 \mathrm{mg} / \mathrm{l}$ & 1 & \\
\hline 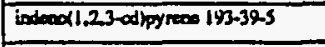 & $<0.010 \mathrm{mg} / 1$ & $<0.010 \mathrm{mg} / \mathrm{l}$ & $<0.010 \mathrm{mg} / \mathrm{l}$ & $<0.010 \mathrm{mg} / \mathrm{l}$ & 1 & \\
\hline 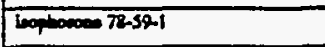 & $<0.010 \mathrm{mg} / \mathrm{l}$ & $<0.010 \mathrm{mg} / \mathrm{l}$ & $<0.010 \mathrm{mg} / \mathrm{l}$ & $<0.010 \mathrm{mg} / \mathrm{l}$ & 1 & \\
\hline 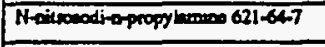 & $<0.010 \mathrm{mg} / \mathrm{l}$ & $<C .010 \mathrm{mg} / \mathrm{l}$ & $<0.010 \mathrm{mg} / 1$ & $<0.010 \mathrm{mg} / \mathrm{l}$ & 1 & \\
\hline 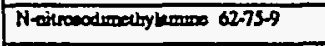 & $<0.010 \mathrm{mg} / \mathrm{l}$ & $<0.010 \mathrm{mg} / \mathrm{l}$ & $<0.010 \mathrm{mg} / \mathrm{l}$ & $<0.010 \mathrm{mg} / \mathrm{l}$ & 1 & \\
\hline 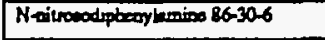 & $<0.010 \mathrm{mg} / \mathrm{l}$ & $<<0.010 \mathrm{mg} / \mathrm{l}$ & $<0.010 \mathrm{mg} / 1$ & $<0.010 \mathrm{mg} / \mathrm{l}$ & 1 & \\
\hline $91-20-3$ & $<0.010 \mathrm{mg} / \mathrm{l}$ & $<0.010 \mathrm{mg} / \mathrm{l}$ & $<0.010 \mathrm{mg} / 1$ & $<0.010 \mathrm{mg} / 1$ & 1 & \\
\hline 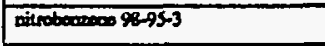 & $<0.010 \mathrm{mg} / 1$ & $<0.010 \mathrm{mg} / 1$ & $<0.010 \mathrm{mg} / 1$ & $<0.010 \mathrm{mg} / 1$ & 1 & \\
\hline pheonenthroos \&501.8 & $<0.010 \mathrm{mg} / \mathrm{l}$ & $<0.010 \mathrm{mg} / \mathrm{l}$ & $<0.010 \mathrm{mg} / \mathrm{l}$ & $<0.010 \mathrm{mg} / \mathrm{l}$ & 1 & \\
\hline mineo 129000 & $<0.010 \mathrm{mg} / 1$ & $<0.010 \mathrm{mg} / \mathrm{I}$ & $<0.010 \mathrm{mg} / \mathrm{l}$ & $<0.010 \mathrm{mg} / \mathrm{l}$ & 1 & \\
\hline 24,G-4rictionopenol 82,06-2 & $<0.030 \mathrm{mg} / \mathrm{l}$ & $<0.030 \mathrm{mg} / 1$ & $<0.030 \mathrm{mg} / 1$ & $<0.030 \mathrm{mg} / 1$ & 1 & \\
\hline 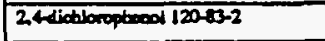 & $<0.030 \mathrm{mg} / 1$ & $<0.030 \mathrm{mg} / 1$ & $<0.030 \mathrm{mg} / \mathrm{l}$ & $<0.030 \mathrm{mg} / 1$ & 1 & \\
\hline 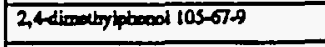 & $<0.030 \mathrm{mg} / \mathrm{l}$ & $<0.030 \mathrm{mg} / \mathrm{l}$ & $<0.030 \mathrm{mg} / \mathrm{l}$ & $<0.030 \mathrm{mg} / \mathrm{l}$ & 1 & \\
\hline 2.4dinitropheos S1-22-5 & $<0.050 \mathrm{mg} / \mathrm{l}$ & $<0.050 \mathrm{mg} / \mathrm{l}$ & $<0.050 \mathrm{mg} / \mathrm{l}$ & $<0.050 \mathrm{mg} / 1$ & 1 & \\
\hline
\end{tabular}


Outfall 57

Parts B and C Continied

\begin{tabular}{|c|c|c|c|c|c|c|}
\hline \multirow{2}{*}{$\begin{array}{l}\text { Poltument } \\
\text { abe } \\
\text { CAS Number } \\
\text { (if avnilibie) }\end{array}$} & \multicolumn{2}{|c|}{$\begin{array}{l}\text { Marimum Values } \\
\text { (inchude unitu) }\end{array}$} & \multicolumn{2}{|c|}{$\begin{array}{l}\text { Average Values } \\
\text { (inchude unisa) }\end{array}$} & \multirow{2}{*}{$\begin{array}{l}\text { Number } \\
\text { of } \\
\text { Storm } \\
\text { Everto } \\
\text { Sampled }\end{array}$} & \multirow[b]{2}{*}{ Sources of Polhtersen } \\
\hline & $\begin{array}{l}\text { Grab Samplo } \\
\text { Takeo Drurias } \\
\text { First } 30 \\
\text { Minutea }\end{array}$ & $\begin{array}{l}\text { Flow-wreighted } \\
\text { Componito }\end{array}$ & $\begin{array}{l}\text { Grab Semplo } \\
\text { Taken During } \\
\text { First } 30 \\
\text { Minines }\end{array}$ & $\begin{array}{l}\text { Flow-weightod } \\
\text { Componito }\end{array}$ & & \\
\hline 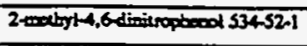 & $<0.050 \mathrm{mg} / 1$ & $<0.050 \mathrm{mg} / \mathrm{l}$ & $<0.050 \mathrm{mg} / 1$ & $<0.050 \mathrm{mg} / \mathrm{l}$ & 1 & \\
\hline 2 -ailopteod 88-75-5 & $<0.030 \mathrm{mg} / \mathrm{l}$ & $<0.030 \mathrm{mg} / \mathrm{l}$ & $<0.030 \mathrm{mg} / 1$ & $<0.030 \mathrm{mg} / \mathrm{l}$ & 1 & \\
\hline 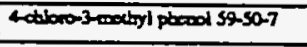 & $<0.030 \mathrm{mg} / \mathrm{l}$ & $<0.030 \mathrm{mg} / \mathrm{l}$ & $<0.030 \mathrm{mg} / \mathrm{l}$ & $<0.030 \mathrm{mg} / \mathrm{l}$ & 1 & \\
\hline 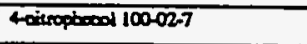 & $<0.050 \mathrm{mg} / 1$ & $<0.050 \mathrm{mg} / \mathrm{h}$ & $<0.050 \mathrm{mg} / \mathrm{l}$ & $<0.050 \mathrm{mg} / 1$ & 1 & \\
\hline pectectionopberol $87.86-5$ & $<0.050 \mathrm{mg} / \mathrm{l}$ & $<0.050 \mathrm{mg} / \mathrm{l}$ & $<0.050 \mathrm{mg} / \mathrm{l}$ & $<0.050 \mathrm{mg} / \mathrm{l}$ & 1 & \\
\hline pheod 108-9s-2 & $<0.030 \mathrm{mg} / \mathrm{l}$ & $<0.030 \mathrm{mg} / \mathrm{l}$ & $<0.030 \mathrm{mg} / 1$ & $<0.030 \mathrm{mg} / \mathrm{l}$ & 1 & \\
\hline 4.4-DDD 72-54-8 & $<0.00001 \mathrm{mg} / 1$ & $<0.00001 \mathrm{mg} / 1$ & $<0.00001 \mathrm{mg} / \mathrm{l}$ & $<0.00001 \mathrm{mg} / \mathrm{l}$ & 1 & \\
\hline 4.4'-DDE 72-55-9 & $<0.00001 \mathrm{mg} / \mathrm{l}$ & $<0.00001 \mathrm{mg} / 1$ & $<0.00001 \mathrm{mg} / 1$ & $<0.00001 \mathrm{mg} / 1$ & 1 & \\
\hline $4,4^{\circ}-00750-20-3$ & $<0.00001 \mathrm{mg} / \mathrm{l}$ & $<0.00001 \mathrm{mg} / 1$ & $<0.00001 \mathrm{mg} / \mathrm{l}$ & $<0.00001 \mathrm{mg} / \mathrm{l}$ & 1 & \\
\hline adisin $30900-2$ & $<0.00001 \mathrm{mg} / \mathrm{l}$ & $<0.00001 \mathrm{mg} / \mathrm{l}$ & $<0.00001 \mathrm{mg} / \mathrm{l}$ & $<0.00001 \mathrm{mg} / \mathrm{l}$ & 1 & \\
\hline cthordec0 57.749 & $<0.00002 \mathrm{mg} / \mathrm{l}$ & $<0.00002 \mathrm{mg} / 1$ & $<0.00002 \mathrm{mg} / 1$ & $<0.00002 \mathrm{mg} / \mathrm{l}$ & 1 & \\
\hline diekfion $60-57.1$ & $<0.00001 \mathrm{mg} / \mathrm{l}$ & $<0.00001 \mathrm{mg} / \mathrm{l}$ & $<0.00001 \mathrm{mg} / \mathrm{l}$ & $<0.00001 \mathrm{mg} / \mathrm{l}$ & 1 & \\
\hline 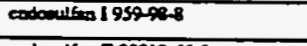 & $<0.00001 \mathrm{mg} / \mathrm{l}$ & $<0.00001 \mathrm{mg} / \mathrm{l}$ & $<0.00001 \mathrm{mg} / \mathrm{l}$ & $<0.00001 \mathrm{mg} / \mathrm{l}$ & 1 & \\
\hline 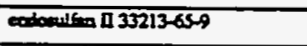 & $<0.00001 \mathrm{mg} / \mathrm{l}$ & $<0.00001 \mathrm{mg} / \mathrm{l}$ & $<0.00001 \mathrm{mg} / 1$ & $<0.00001 \mathrm{mg} / \mathrm{l}$ & 1 & \\
\hline 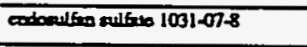 & $<0.00001 \mathrm{mg} / 1$ & $<0.00001 \mathrm{mg} / 1$ & $<0.00001 \mathrm{mg} / \mathrm{ll}$ & $<0.00001 \mathrm{mg} / 1$ & 1 & \\
\hline $\operatorname{cosin} 72-208$ & $<0.00005 \mathrm{mg} / \mathrm{l}$ & $<0.00005 \mathrm{mg} / \mathrm{l}$ & $<0.00005 \mathrm{mg} / \mathrm{l}$ & $<0.00005 \mathrm{mg} / \mathrm{l}$ & 1 & \\
\hline cadric abebodo $701-93-4$ & $<0.00001 \mathrm{mg} / \mathrm{l}$ & $<0.0001 \mathrm{mg} / \mathrm{l}$ & $<0.00001 \mathrm{mg} / \mathrm{l}$ & $<0.00001 \mathrm{mg} / \mathrm{l}$ & 1 & \\
\hline Bactlox 76448 & $<0.00001 \mathrm{mg} / \mathrm{l}$ & $<0.00001 \mathrm{mg} / 1$ & $<0.00001 \mathrm{mg} / \mathrm{h}$ & $<0.00001 \mathrm{mg} / \mathrm{l}$ & 1 & - \\
\hline 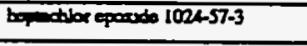 & $<0.00001 \mathrm{mg} / 1$ & $<0.00001 \mathrm{mg} / \mathrm{l}$ & $<0.00001 \mathrm{mg} / 1$ & $<0.00001 \mathrm{mg} / \mathrm{l}$ & 1 & \\
\hline 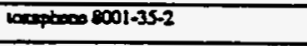 & $<0.0002 \mathrm{mg} / 1$ & $<0.0002 \mathrm{mg} / \mathrm{l}$ & $<0.0002 \mathrm{mg} / 1$ & $<0.0002 \mathrm{mg} / 1$ & 1 & \\
\hline $406-81+C 319846$ & $<0.00001 \mathrm{mg} / \mathrm{l}$ & $<0.00001 \mathrm{mg} / \mathrm{l}$ & $<0.00001 \mathrm{mg} / \mathrm{l}$ & $<0.00001 \mathrm{mg} / \mathrm{l}$ & 1 & \\
\hline BHC 31935.7 & $<0.00001 \mathrm{mg} / \mathrm{l}$ & $<0.00001 \mathrm{mg} / \mathrm{l}$ & $<0.00001 \mathrm{mg} / 1$ & $<0.00001 \mathrm{mg} / \mathrm{l}$ & 1 & \\
\hline dem-8HC 315868 & $<0.00001 \mathrm{mg} / 1$ & $<0.00001 \mathrm{mg} / 1$ & $<0.00001 \mathrm{mg} / 1$ & $<0.00001 \mathrm{mg} / 1$ & 1 & \\
\hline$=-8 H C 58-38$ & $0.000012 \mathrm{mg} / 1$ & $<0.00001 \mathrm{mg} / 1$ & $0.000012 \mathrm{mg} / 1$ & $<0.00001 \mathrm{mg} / \mathrm{l}$ & 1 & \\
\hline 2athoropteced $95-57.8$ & $<0.030 \mathrm{mg} / 1$ & $<0.030 \mathrm{mg} / 1$ & $<0.030 \mathrm{mg} / \mathrm{l}$ & $<0.030 \mathrm{mg} / 1$ & 1 & $\dot{5}$ \\
\hline Arodor-126253669-21-9 & $<0.0005 \mathrm{mg} / \mathrm{l}$ & $<0.0005 \mathrm{mg} / 1$ & $<0.0005 \mathrm{mg} / 1$ & $<0.0005 \mathrm{mg} / \mathrm{l}$ & 1 & 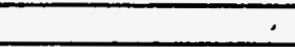 \\
\hline Arodoril234 11077-69-1 & $<0.0005 \mathrm{mg} / 1$ & $<0.0005 \mathrm{mg} / \mathrm{l}$ & $<0.0005 \mathrm{mg} / \mathrm{h}$ & $<0.0005 \mathrm{mg} / \mathrm{l}$ & 1 & \\
\hline Arodon-121 11104-28-2 & $<0.0005 \mathrm{mg} / \mathrm{l}$ & $<0.0005 \mathrm{mg} / \mathrm{l}$ & $<0.0005 \mathrm{mg} / 1$ & $<0.0005 \mathrm{mg} / 1$ & 1 & \\
\hline Aroctor-1232 :1141-16-5 & $<0.0005 \mathrm{mg} / \mathrm{l}$ & $<0.0005 \mathrm{mg} / \mathrm{l}$ & $<0.0005 \mathrm{mg} / \mathrm{l}$ & $<0.0005 \mathrm{mg} / \mathrm{l}$ & 1 & \\
\hline Arodon-1248 $12672-296$ & $<0.0005 \mathrm{mg} / 1$ & $<0.0005 \mathrm{mg} / \mathrm{l}$ & $<0.0005 \mathrm{mg} / 1$ & $<0.0005 \mathrm{mg} / \mathrm{l}$ & 1 & \\
\hline Arodon-1260 $1102682-5$ & $<0.0005 \mathrm{mg} / \mathrm{l}$ & $<0.0005 \mathrm{mg} / 1$ & $<0.0005 \mathrm{mg} / \mathrm{l}$ & $<0.0005 \mathrm{mg} / \mathrm{l}$ & 1 & \\
\hline Avodon-1016 12074-11-2 & $<0.0005 \mathrm{mg} / \mathrm{l}$ & $<0.0005 \mathrm{mg} / \mathrm{l}$ & $<0.0005 \mathrm{mg} / 1$ & $<0.0005 \mathrm{mg} / 1$ & 1 & \\
\hline Croons & $<0.010 \mathrm{mg} / 1$ & $<0.010 \mathrm{mg} / \mathrm{l}$ & $<0.010 \mathrm{mg} / 1$ & $<0.010 \mathrm{mg} / 1$ & 1 & \\
\hline Acrobin & $<0.010 \mathrm{mg} / \mathrm{l}$ & $<0.010 \mathrm{mg} / \mathrm{l}$ & $<0.010 \mathrm{mg} / \mathrm{l}$ & $<0.010 \mathrm{mg} / \mathrm{l}$ & 1 & \\
\hline Acrybociritis & $<0.010 \mathrm{mg} / \mathrm{l}$ & $<0.010 \mathrm{mg} / \mathrm{l}$ & $<0.010 \mathrm{mg} / \mathrm{l}$ & $<0.010 \mathrm{mg} / \mathrm{l}$ & 1 & \\
\hline 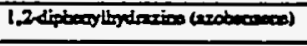 & Reoulte pending & Reaulte peoding & Reaules pending & Reaules pending & 1 & \\
\hline & & & & & & \\
\hline & & & & & & \\
\hline & & & & & & \\
\hline & & & & & & \\
\hline & & & & & & \\
\hline & & & & & & \\
\hline & & & & & & \\
\hline & & & & & & \\
\hline & & & & & & \\
\hline & & & & & & \\
\hline & & & & & & \\
\hline & & & & & & \\
\hline & & & & & & \\
\hline
\end{tabular}


VII. Discherge Lnformution (continued from pago 3 of Form 2P)

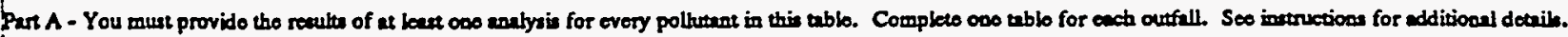

\begin{tabular}{|c|c|c|c|c|c|c|}
\hline \multirow{2}{*}{$\begin{array}{l}\text { Pollutaat } \\
\text { and } \\
\text { CAS Number } \\
\text { (if availible) }\end{array}$} & \multicolumn{2}{|c|}{$\begin{array}{l}\text { Maximuses Valuce } \\
\text { (iochude units) }\end{array}$} & \multicolumn{2}{|c|}{$\begin{array}{l}\text { Average Valuea } \\
\text { (iochude unity) }\end{array}$} & \multirow{2}{*}{$\begin{array}{l}\text { Number } \\
\text { of } \\
\text { Storm } \\
\text { Eveots } \\
\text { Sumpled }\end{array}$} & \multirow[b]{2}{*}{ Sources of Polluras } \\
\hline & $\begin{array}{c}\text { Gnb Semple } \\
\text { Takea During } \\
\text { Find } 30 \\
\text { Minose }\end{array}$ & $\begin{array}{l}\text { Flow-weightod } \\
\text { Comporite }\end{array}$ & $\begin{array}{l}\text { Grab Sumple } \\
\text { Takeo During } \\
\text { Fint } 30 \\
\text { Minuce }\end{array}$ & $\begin{array}{l}\text { Flow-weighted } \\
\text { Compotito }\end{array}$ & & \\
\hline pilm Gown & $3 \mathrm{mg} / \mathrm{h}$ & & $3 \mathrm{mg} / \mathrm{l}$ & & 1 & \\
\hline Biolopical Oxy wa Doun od (BODS) & $<5 \mathrm{mg} / \mathrm{l}$ & $<5 \mathrm{mg} / \mathrm{l}$ & $<5 \mathrm{mg} / \mathrm{l}$ & $<5 \mathrm{mg} / \mathrm{l}$ & 1 & \\
\hline 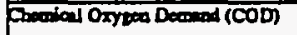 & $22 \mathrm{mg} / 1$ & $38 \mathrm{mg} / \mathrm{l}$ & $22 \mathrm{mg} / \mathrm{l}$ & $38 \mathrm{mg} / \mathrm{l}$ & 1 & \\
\hline Tom Stuppod solid (TSS) & $<5 \mathrm{mg} / \mathrm{l}$ & $6 \mathrm{mg} / \mathrm{h}$ & $<5 \mathrm{mg} / \mathrm{h}$ & $6 \mathrm{mg} / \mathrm{h}$ & 1 & \\
\hline ronal Kjoldehi Nitropose & $0.3 \mathrm{mg} / \mathrm{l}$ & $0.3 \mathrm{mg} / 1$ & $0.3 \mathrm{mg} / \mathrm{l}$ & $0.3 \mathrm{mg} / \mathrm{l}$ & T & \\
\hline 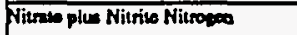 & $0.34 \mathrm{mg} / \mathrm{l}$ & $1.01 \mathrm{mg} / 1$ & $0.34 \mathrm{mg} / \mathrm{l}$ & $1.01 \mathrm{mg} / 1$ & 1 & \\
\hline Toul Proopbontus & $0.11 \mathrm{mg} / 1$ & $0.14 \mathrm{mg} / \mathrm{l}$ & $0.11 \mathrm{mg} / \mathrm{l}$ & $0.14 \mathrm{mg} / \mathrm{l}$ & 1 & \\
\hline
\end{tabular}

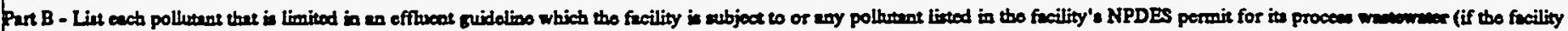

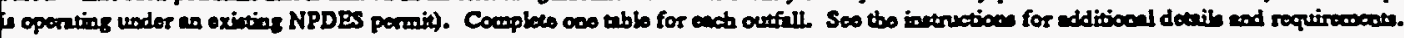

\begin{tabular}{|c|c|c|c|c|c|c|}
\hline \multirow{2}{*}{$\begin{array}{l}\text { Pollemant } \\
\text { and } \\
\text { CAs Number } \\
\text { (if availiblo) }\end{array}$} & \multicolumn{2}{|c|}{$\begin{array}{l}\text { Merimuson Valuece } \\
\text { (includo unin) }\end{array}$} & \multicolumn{2}{|c|}{$\begin{array}{l}\text { Averize Values } \\
\text { (includo unina) }\end{array}$} & \multirow{2}{*}{$\begin{array}{l}\text { Number } \\
\text { of } \\
\text { Storm } \\
\text { Eveous } \\
\text { Sempled }\end{array}$} & \multirow{2}{*}{ Sources of Polburenen } \\
\hline & 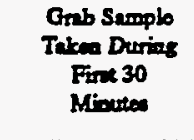 & $\begin{array}{l}\text { Frow-waighed } \\
\text { Cospotico }\end{array}$ & $\begin{array}{l}\text { Grab Semplo } \\
\text { Taken During } \\
\text { Fins } 30 \\
\text { Minated }\end{array}$ & $\begin{array}{c}\text { Flow-woighted } \\
\text { Compocies }\end{array}$ & & \\
\hline 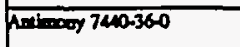 & $<0.04 \mathrm{mg} / \mathrm{l}$ & $<0.04 \mathrm{mg} / 1$ & $<0.04 \mathrm{mg} / \mathrm{l}$ & $<0.04 \mathrm{mg} / \mathrm{t}$ & 1 & \\
\hline sonits & $<0.002 \mathrm{mg} / 1$ & & $<0.002 \mathrm{mg} / \mathrm{l}$ & & 1 & \\
\hline Menary 7439-976 & $0.0005 \mathrm{mg} / \mathrm{l}$ & $0.0004 \mathrm{mg} / \mathrm{h}$ & $0.0005 \mathrm{mg} / \mathrm{l} \cdot$ & $0.0004 \mathrm{mg} / 1$ & 1 & \\
\hline ben & $<0.001 \mathrm{mg} / 1$ & & $<0.001 \mathrm{mg} / 1$ & & 1 & $\bar{\vdots}$ \\
\hline 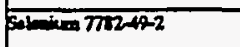 & $<0.002 \mathrm{mg} / \mathrm{l}$ & $<0.002 \mathrm{mg} / \mathrm{l}$ & $<0.002 \mathrm{mg} / 1$ & $<0.002 \mathrm{mg} / 1$ & 1 & \\
\hline Palab & $29 \mathrm{mg} / \mathrm{l}$ & $28 \mathrm{mg} / 1$ & $29 \mathrm{mg} / \mathrm{l}$ & $28 \mathrm{mg} / \mathrm{l}$ & 1 & \\
\hline Perteremes & $0.06 \mathrm{mg} / \mathrm{l}$ & $<0.05 \mathrm{mg} / 1$ & $0.06 \mathrm{mg} / \mathrm{l}$ & $<0.05 \mathrm{mg} / \mathrm{l}$ & 1 & \\
\hline Ahen $742290-5$ & $0.96 \mathrm{mg} / 1$ & $0.93 \mathrm{mg} / \mathrm{l}$ & $0.96 \mathrm{mg} / \mathrm{l}$ & $0.93 \mathrm{mg} / 1$ & 1 & \\
\hline 4 manio $7440-38-2$ & $<0.04 \mathrm{mg} / 1$ & $<0.04 \mathrm{mg} / \mathrm{l}$ & $<0.04 \mathrm{mg} / \mathrm{l}$ & $<0.04 \mathrm{mg} / 1$ & 1 & \\
\hline $300000740-128$ & $0.056 \mathrm{mg} / \mathrm{l}$ & $0.057 \mathrm{mg} / \mathrm{l}$ & $0.056 \mathrm{mg} / \mathrm{l}$ & $0.057 \mathrm{mg} / 1$ & 1 & \\
\hline Baryllivom 740-11.7 & $<0.0004 \mathrm{mg} / \mathrm{l}$ & $<0.0004 \mathrm{mg} / 1$ & $<0.0004 \mathrm{mg} / 1$ & $<0.0004 \mathrm{mg} / 1$ & 1 & \\
\hline 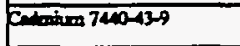 & $<0.004 \mathrm{mg} / \mathrm{l}$ & $<0.004 \mathrm{mg} / \mathrm{l}$ & $<0.004 \mathrm{mg} / \mathrm{l}$ & $<0.004 \mathrm{mg} / \mathrm{l}$ & 1 & \\
\hline Calower $7400-x 0-2$ & $42.4 \mathrm{mg} / \mathrm{l}$ & $39.7 \mathrm{mg} / \mathrm{l}$ & $42.4 \mathrm{mg} / 1$ & $39.7 \mathrm{mg} / \mathrm{l}$ & 1 & \\
\hline Furosiven 7400-17-3 & $<0.006 \mathrm{mg} / \mathrm{l}$ & $<0.006 \mathrm{mg} / \mathrm{l}$ & $<0.006 \mathrm{mg} / \mathrm{l}$ & $<0.006 \mathrm{mg} / \mathrm{l}$ & 1 & \\
\hline Cotall $740048-4$ & $<0.002 \mathrm{mg} / \mathrm{l}$ & $<0.002 \mathrm{mg} / \mathrm{l}$ & $<0.002 \mathrm{mg} / \mathrm{l}$ & $<0.002 \mathrm{mg} / \mathrm{l}$ & 1 & \\
\hline Compin 740asas & $<0.006 \mathrm{mg} / \mathrm{l}$ & $<0.006 \mathrm{mg} / \mathrm{l}$ & $<0.006 \mathrm{mg} / 1$ & $<0.006 \mathrm{mg} / 1$ & 1 & \\
\hline 5007439096 & $1.0 \mathrm{mg} / \mathrm{l}$ & $0.93 \mathrm{mg} / \mathrm{l}$ & $1.0 \mathrm{mg} / \mathrm{I}$ & $0.93 \mathrm{mg} / 1$ & 1 & \\
\hline 7043902.1 & $<0.02 \mathrm{mg} / \mathrm{l}$ & $<0.02 \mathrm{mg} / \mathrm{l}$ & $<0.02 \mathrm{mg} / \mathrm{l}$ & $<0.02 \mathrm{mg} / \mathrm{l}$ & 1 & \\
\hline Thenen 743993-2 & $<0.02 \mathrm{mg} / \mathrm{l}$ & $<0.02 \mathrm{mg} / \mathrm{l}$ & $<0.02 \mathrm{mg} / 1$ & $<0.02 \mathrm{mg} / 1$ & 1 & \\
\hline Maposiven 7439954 & $6.81 \mathrm{mg} / \mathrm{l}$ & $6.39 \mathrm{mg} / \mathrm{l}$ & $6.81 \mathrm{mg} / 1$ & $6.39 \mathrm{mg} / 1$ & 1 & \\
\hline 700000605 & $0.090 \mathrm{mg} / \mathrm{l}$ & $0.066 \mathrm{mg} / \mathrm{l}$ & $0.090 \mathrm{mg} / 1$ & $0.066 \mathrm{mg} / \mathrm{l}$ & 1 & \\
\hline 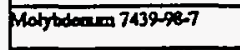 & $<0.006 \mathrm{mg} / \mathrm{l}$ & $<0.006 \mathrm{mg} / \mathrm{l}$ & $<0.006 \mathrm{mg} / \mathrm{l}$ & $<0.006 \mathrm{mg} / \mathrm{l}$ & 1 & \\
\hline$\sqrt{100 / 7440-020}$ & $<0.008 \mathrm{mg} / \mathrm{l}$ & $<0.008 \mathrm{mg} / \mathrm{l}$ & $<0.008 \mathrm{mg} / \mathrm{l}$ & $<0.008 \mathrm{mg} / \mathrm{l}$ & 1 & \\
\hline Prompixom 74009-7 & $2.4 \mathrm{mg} / \mathrm{l}$ & $2.5 \mathrm{mg} / 1$ & $2.4 \mathrm{mg} / \mathrm{l}$ & $2.5 \mathrm{mg} / \mathrm{l}$ & 1 & \\
\hline Sitwer 740025A & $<0.006 \mathrm{mg} / \mathrm{l}$ & $<0.006 \mathrm{mg} / \mathrm{I}$ & $<0.006 \mathrm{mg} / 1$ & $<0.006 \mathrm{mg} / \mathrm{l}$ & 1 & \\
\hline sodiven 740-23-5 & $2.53 \mathrm{mg} / \mathrm{l}$ & $2.33 \mathrm{mg} / \mathrm{l}$ & $2.53 \mathrm{mg} / \mathrm{l}$ & $2.33 \mathrm{mg} / 1$ & 1 & \\
\hline 75 mix 7460.326 & $<0.01 \mathrm{mg} / \mathrm{l}$ & $<0.01 \mathrm{mg} / \mathrm{l}$ & $<0.01 \mathrm{mg} / \mathrm{1}$ & $<0.01 \mathrm{mg} / 1$ & 1 & \\
\hline $2100740-66-6$ & $0.02 \mathrm{mg} / \mathrm{l}$ & $0.02 \mathrm{mg} / 1$ & $0.02 \mathrm{mg} / 1$ & $0.02 \mathrm{mg} / 1$ & 1 & \\
\hline
\end{tabular}


Continued from frone.

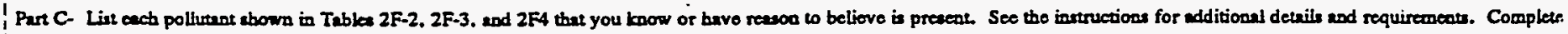
i ono tablo for cach ourfill.

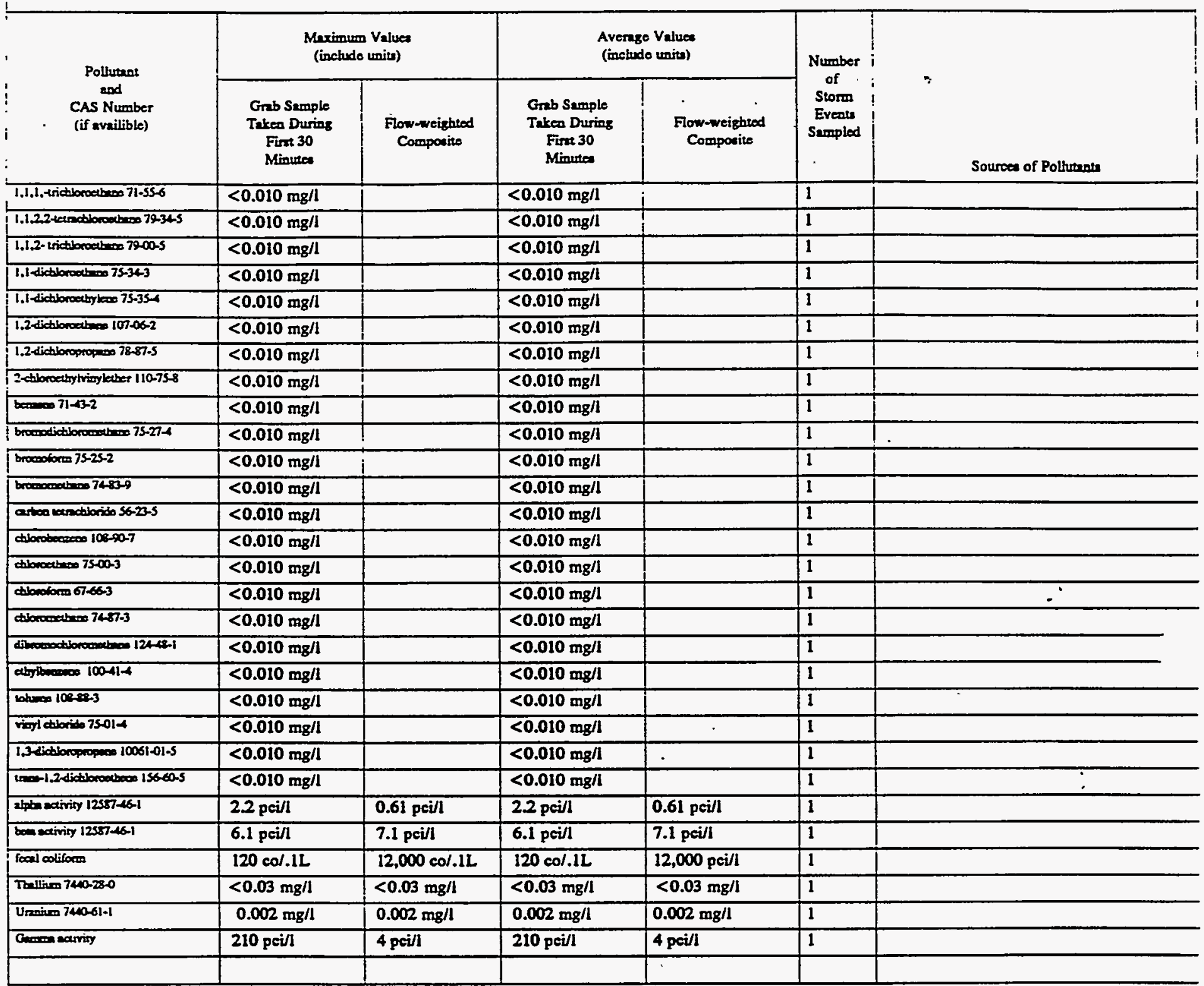

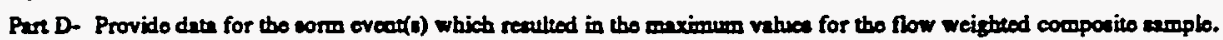

\begin{tabular}{|c|c|c|c|c|c|c|c|}
\hline $\begin{array}{l}1 . \\
\text { Date of } \\
\text { Storm } \\
\text { Eveat }\end{array}$ & $\begin{array}{l}2 . \\
\text { Duration } \\
\text { of Storm } \\
\text { (in } \\
\text { minuta) }\end{array}$ & 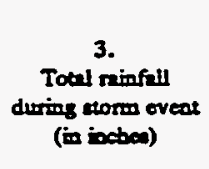 & 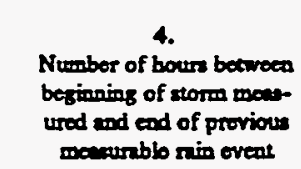 & $\begin{array}{l}\text { S. } \\
\text { Maximum flow nte } \\
\text { during min event } \\
\text { (alloos/min or specify } \\
\text { unin) }\end{array}$ & $\begin{array}{l}6 . \\
\text { Total flow from } \\
\text { nin evens } \\
\text { (rillows or } \\
\text { specify unitu) }\end{array}$ & $\begin{array}{c}7 . \\
\text { Scason } \\
\text { sample was } \\
\text { taken }\end{array}$ & $\begin{array}{l}\text { B. } \\
\text { Forms of } \\
\text { Procipitation } \\
\text { (nimfall. } \\
\text { socoment) }\end{array}$ \\
\hline $12 / 9 / 91$ & 530 & 1.1 & 148.6 & 57.9 & 1,480 & Fall & Rainfall \\
\hline
\end{tabular}

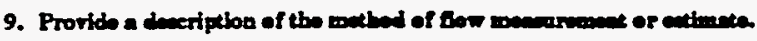

An ISCO 3230 Flow meter was wed to obtain water level readings. This instriment was colibrated with the normal dry weather flow, if preseat, as leve zero. The flow meter was programmed with the characteristics of the conveyance so that flow rates and total ilows conld be calcalated by the meter. 


\begin{tabular}{|c|c|c|c|c|c|c|}
\hline \multirow{2}{*}{$\begin{array}{l}\text { Polturant } \\
\text { and } \\
\text { CAs Number } \\
\text { (if availible) }\end{array}$} & \multicolumn{2}{|c|}{$\begin{array}{l}\text { Meximum Vahues } \\
\text { (ischudo units) }\end{array}$} & \multicolumn{2}{|c|}{$\begin{array}{l}\text { Average Valuea } \\
\text { (inchude units) }\end{array}$} & \multirow{2}{*}{$\begin{array}{l}\text { Number } \\
\text { of } \\
\text { Storm } \\
\text { Evente } \\
\text { Sroupled }\end{array}$} & \multirow[b]{2}{*}{ Sources of Polthread } \\
\hline & $\begin{array}{c}\text { Grab Sample } \\
\text { Takeo During } \\
\text { Fint } 30 \\
\text { Mininee }\end{array}$ & $\begin{array}{l}\text { Fow-woighted } \\
\text { Compocies }\end{array}$ & $\begin{array}{l}\text { Grab Semple } \\
\text { Takeo Dusing } \\
\text { Firt } 30 \\
\text { Minutea }\end{array}$ & $\begin{array}{l}\text { Flow-weighiced } \\
\text { Componito }\end{array}$ & & \\
\hline Betiunon 7440-32-3 & $0.0938 \mathrm{mg} / \mathrm{l}$ & $0.0824 \mathrm{mg} / \mathrm{l}$ & $0.0938 \mathrm{mg} / \mathrm{l}$ & $0.0824 \mathrm{mg} / \mathrm{l}$ & 1 & \\
\hline 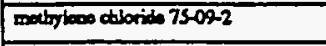 & $<0.010 \mathrm{mg} / \mathrm{I}$ & & $<0.010 \mathrm{mg} / \mathrm{l}$ & & 1 & \\
\hline 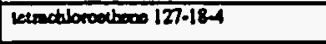 & $<0.010 \mathrm{mg} / \mathrm{l}$ & & $<0.010 \mathrm{mg} / 1$ & & 1 & \\
\hline 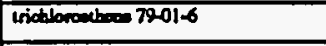 & $<0.010 \mathrm{mg} / \mathrm{I}$ & & $<0.010 \mathrm{mg} / \mathrm{l}$ & & 1 & \\
\hline Xyloosen & $<0.010 \mathrm{mg} / \mathrm{l}$ & & $<0.010 \mathrm{mg} / \mathrm{l}$ & & 1 & \\
\hline 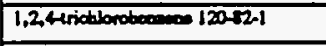 & $<0.010 \mathrm{mg} / \mathrm{l}$ & $<0.010 \mathrm{mg} / 1$ & $<0.010 \mathrm{mg} / \mathrm{l}$ & $<0.010 \mathrm{mg} / 1$ & 1 & \\
\hline 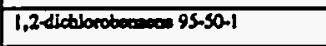 & $<0.010 \mathrm{mg} / \mathrm{l}$ & $<0.010 \mathrm{mg} / 1$ & $<0.010 \mathrm{mg} / \mathrm{l}$ & $<0.010 \mathrm{mg} / \mathrm{l}$ & 1 & \\
\hline 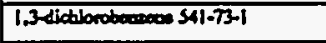 & $<0.010 \mathrm{mg} / \mathrm{l}$ & $<0.010 \mathrm{mg} / \mathrm{l}$ & $<0.010 \mathrm{mg} / \mathrm{l}$ & $<0.010 \mathrm{mg} / \mathrm{l}$ & 1 & \\
\hline 1,4 dicthoroboutooce $100-46-7$ & $<0.010 \mathrm{mg} / \mathrm{l}$ & $<0.010 \mathrm{mg} / \mathrm{l}$ & $<0.010 \mathrm{mg} / 1$ & $<0.010 \mathrm{mg} / \mathrm{l}$ & 1 & \\
\hline 24-dinitroubluose $121-142$ & $<0.010 \mathrm{mg} / \mathrm{l}$ & $<0.010 \mathrm{mg} / 1$ & $<0.010 \mathrm{mg} / \mathrm{l}$ & $<0.010 \mathrm{mg} / \mathrm{l}$ & 1 & \\
\hline 2,6-diniprosheroso $606-20-2$ & $<0.010 \mathrm{mg} / \mathrm{l}$ & $<0.010 \mathrm{mg} / 1$ & $<0.010 \mathrm{mg} / \mathrm{l}$ & $<0.010 \mathrm{mg} / \mathrm{l}$ & 1 & \\
\hline 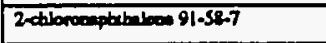 & $<0.010 \mathrm{mg} / \mathrm{l}$ & $<0.010 \mathrm{mg} / \mathrm{l}$ & $<0.010 \mathrm{mg} / 1$ & $<0.010 \mathrm{mg} / 1$ & 1 & \\
\hline 3.3.dictionobuatidine 91-94| & $<0.010 \mathrm{mg} / \mathrm{l}$ & $<0.010 \mathrm{mg} / \mathrm{l}$ & $<0.010 \mathrm{mg} / \mathrm{l}$ & $<0.010 \mathrm{mg} / \mathrm{l}$ & 1 & \\
\hline 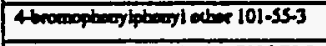 & $<0.010 \mathrm{mg} / \mathrm{l}$ & $<0.010 \mathrm{mg} / 1$ & $<0.010 \mathrm{mg} / \mathrm{l}$ & $<0.010 \mathrm{mg} / \mathrm{l}$ & 1 & \\
\hline 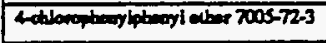 & $<0.010 \mathrm{mg} / \mathrm{l}$ & $<0.010 \mathrm{mg} / \mathrm{l}$ & $<0.010 \mathrm{mg} / \mathrm{l}$ & $<0.010 \mathrm{mg} / 1$ & 1 & \\
\hline 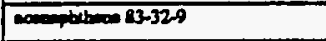 & $<0.010 \mathrm{mg} / 1$ & $<0.010 \mathrm{mg} / 1$ & $<0.010 \mathrm{mg} / 1$ & $<0.010 \mathrm{mg} / \mathrm{l}$ & 1 & \\
\hline 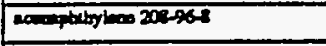 & $<0.010 \mathrm{mg} / \mathrm{l}$ & $<0.010 \mathrm{mg} / 1$ & $<0.010 \mathrm{mg} / 1$ & $<0.010 \mathrm{mg} / 1$ & 1 & \\
\hline $120-127$ & $<0.010 \mathrm{mg} / \mathrm{h}$ & $<0.010 \mathrm{mg} / \mathrm{l}$ & $<0.010 \mathrm{mg} / 1$ & $<0.010 \mathrm{mg} / \mathrm{l}$ & 1 & \\
\hline Doridion $92-77-5$ & $<0.050 \mathrm{mg} / \mathrm{l}$ & $<0.050 \mathrm{mg} / 1$ & $<0.050 \mathrm{mg} / \mathrm{l}$ & $<0.050 \mathrm{mg} / 1$ & 1 & \\
\hline 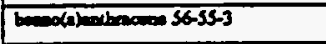 & $<0.010 \mathrm{mg} / \mathrm{l}$ & $<0.010 \mathrm{mg} / \mathrm{l}$. & $<0.010 \mathrm{mg} / 1$ & $<0.010 \mathrm{mg} / \mathrm{l}$ & 1 & \\
\hline $50-32 \pi$ & $<0.010 \mathrm{mg} / \mathrm{l}$ & $<0.010 \mathrm{mg} / 1$ & $<0.010 \mathrm{mg} / 1$ & $<0.010 \mathrm{mg} / \mathrm{l}$ & 1 & \\
\hline 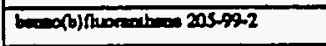 & $<0.010 \mathrm{mg} / \mathrm{l}$ & $<0.010 \mathrm{mg} / 1$ & $<0.010 \mathrm{mg} / \mathrm{l}$ & $<0.010 \mathrm{mg} / \mathrm{l}$ & 1 & \\
\hline 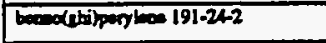 & $<0.010 \mathrm{mg} / \mathrm{l}$ & $<0.010 \mathrm{mg} / \mathrm{l}$ & $<0.010 \mathrm{mg} / \mathrm{I}$ & $<0.010 \mathrm{mg} / \mathrm{l}$ & 1 & \\
\hline 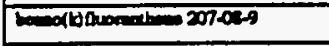 & $<0.010 \mathrm{mg} / \mathrm{l}$ & $<0.010 \mathrm{mg} / 1$ & $<0.010 \mathrm{mg} / \mathrm{l}$ & $<0.010 \mathrm{mg} / \mathrm{l}$ & 1 & \\
\hline 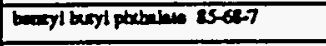 & $<0.010 \mathrm{mg} / 1$ & $<0.010 \mathrm{mg} / \mathrm{l}$ & $<0.010 \mathrm{mg} / 1$ & $<0.010 \mathrm{mg} / 1$ & 1 & \\
\hline 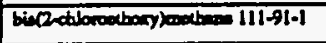 & $<0.010 \mathrm{mg} / 1$ & $<0.010 \mathrm{mg} / 1$ & $<0.010 \mathrm{mg} / \mathrm{l}$ & $<0.010 \mathrm{mg} / 1$ & 1 & $\because$ \\
\hline 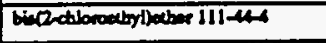 & $<0.010 \mathrm{mg} / 1$ & $<0.010 \mathrm{mg} / !$ & $<0.010 \mathrm{mg} / \mathrm{l}$ & $<0.010 \mathrm{mg} / \mathrm{l}$ & 1 & \\
\hline 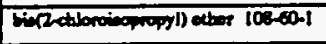 & $<0.010 \mathrm{mg} / \mathrm{l}$ & $<0.010 \mathrm{mg} / \mathrm{l}$ & $<0.010 \mathrm{mg} / \mathrm{l}$ & $<0.010 \mathrm{mg} / \mathrm{l}$ & 1 & \\
\hline 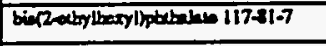 & $<0.010 \mathrm{mg} / \mathrm{l}$ & $<0.010 \mathrm{mg} / \mathrm{l}$ & $<0.010 \mathrm{mg} / \mathrm{l}$ & $<0.010 \mathrm{mg} / \mathrm{l}$ & 1 & \\
\hline ctryouen 218-01A & $<0.010 \mathrm{mg} / \mathrm{l}$ & $<0.010 \mathrm{mg} / \mathrm{l}$ & $<0.010 \mathrm{mg} / \mathrm{l}$ & $<0.010 \mathrm{mg} / \mathrm{l}$ & 1 & \\
\hline di-aturyi patalabo Q4-74-2 & $<0.010 \mathrm{mg} / \mathrm{l}$ & $<0.010 \mathrm{mg} / \mathrm{l}$ & $<0.010 \mathrm{mg} / \mathrm{l}$ & $<0.010 \mathrm{mg} / 1$ & 1 & \\
\hline 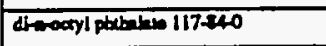 & $<0.010 \mathrm{mg} / \mathrm{l}$ & $<0.010 \mathrm{mg} / \mathrm{l}$ & $<0.010 \mathrm{mg} / \mathrm{l}$ & $<0.010 \mathrm{mg} / 1$ & 1 & \\
\hline 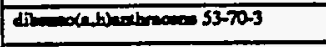 & $<0.010 \mathrm{mg} / \mathrm{l}$ & $<0.010 \mathrm{mg} / \mathrm{h}$ & $<0.010 \mathrm{mg} / \mathrm{l}$ & $<0.010 \mathrm{mg} / \mathrm{l}$ & 1 & \\
\hline 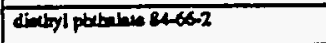 & $<0.010 \mathrm{mg} / 1$ & $<0.010 \mathrm{mg} / \mathrm{l}$ & $<0.010 \mathrm{mg} / \mathrm{l}$ & $<0.010 \mathrm{mg} / \mathrm{l}$ & 1 & \\
\hline dinechyl phethane $131-11-3$ & $<0.010 \mathrm{mg} / \mathrm{l}$ & $<0.010 \mathrm{mg} / \mathrm{l}$ & $<0.010 \mathrm{mg} / \mathrm{l}$ & $<0.010 \mathrm{mg} / 1$ & 1 & \\
\hline 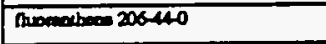 & $<0.010 \mathrm{mg} / \mathrm{l}$ & $<0.010 \mathrm{mg} / \mathrm{l}$ & $<0.010 \mathrm{mg} / \mathrm{l}$ & $<0.010 \mathrm{mg} / \mathrm{l}$ & 1 & \\
\hline thenence 86.73-7 & $<0.010 \mathrm{mg} / \mathrm{l}$ & $<0.010 \mathrm{mg} / \mathrm{l}$ & $<0.010 \mathrm{mg} / \mathrm{l}$ & $<0.010 \mathrm{mg} / \mathrm{l}$ & 1 & \\
\hline 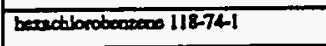 & $<0.010 \mathrm{mg} / \mathrm{l}$ & $<0.010 \mathrm{mg} / \mathrm{l}$ & $<0.010 \mathrm{mg} / 1$ & $<0.010 \mathrm{mg} / \mathrm{l}$ & $l$ & \\
\hline 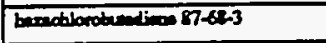 & $<0.010 \mathrm{mg} / \mathrm{l}$ & $<0.010 \mathrm{mg} / \mathrm{l}$ & $<0.010 \mathrm{mg} / \mathrm{l}$ & $<0.010 \mathrm{mg} / \mathrm{l}$ & 1 & \\
\hline 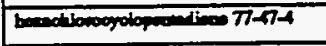 & $<0.010 \mathrm{mg} / \mathrm{l}$ & $<0.010 \mathrm{mg} / \mathrm{l}$ & $<0.010 \mathrm{mg} / \mathrm{l}$ & $<0.010 \mathrm{mg} / \mathrm{l}$ & 1 & \\
\hline 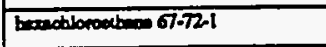 & $<0.010 \mathrm{mg} / 1$ & $<0.010 \mathrm{mg} / 1$ & $<0.010 \mathrm{mg} / \mathrm{l}$ & $<0.010 \mathrm{mg} / \mathrm{l}$ & $\mathfrak{I}$ & \\
\hline 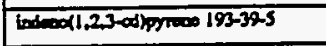 & $<0.010 \mathrm{mg} / \mathrm{l}$ & $<0.010 \mathrm{mg} / \mathrm{l}$ & $<0.010 \mathrm{mg} / \mathrm{l}$ & $<0.010 \mathrm{mg} / \mathrm{l}$ & 1 & \\
\hline Loplonocos 72-59-1 & $<0.010 \mathrm{mg} / 1$ & $<0.010 \mathrm{mg} / \mathrm{l}$ & $<0.010 \mathrm{mg} / 1$ & $<0.010 \mathrm{mg} / \mathrm{l}$ & 1 & \\
\hline 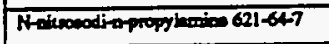 & $<0.010 \mathrm{mg} / 1$ & $<0.010 \mathrm{mg} / 1$ & $<0.010 \mathrm{mg} / 1$ & $<0.010 \mathrm{mg} / \mathrm{l}$ & 1 & \\
\hline 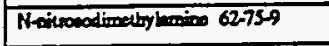 & $<0.010 \mathrm{mg} / \mathrm{l}$ & $<0.010 \mathrm{mg} / \mathrm{l}$ & $<0.010 \mathrm{mg} / \mathrm{l}$ & $<0.010 \mathrm{mg} / \mathrm{l}$ & 1 & \\
\hline 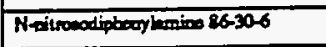 & $<0.010 \mathrm{mg} / \mathrm{l}$ & $<0.010 \mathrm{mg} / 1$ & $<0.010 \mathrm{mg} / \mathrm{l}$ & $<0.010 \mathrm{mg} / \mathrm{l}$ & 1 & \\
\hline $91-20-3$ & $<0.010 \mathrm{mg} / \mathrm{l}$ & $<0.010 \mathrm{mg} / \mathrm{l}$ & $<0.010 \mathrm{mg} / 1$ & $<0.010 \mathrm{mg} / \mathrm{l}$ & 1 & \\
\hline 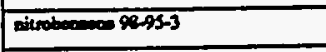 & $<0.010 \mathrm{mg} / \mathrm{l}$ & $<0.010 \mathrm{mg} / \mathrm{l}$ & $<0.010 \mathrm{mg} / \mathrm{l}$ & $<0.010 \mathrm{mg} / 1$ & 1 & \\
\hline 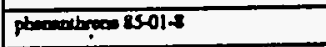 & $<0.010 \mathrm{mg} / \mathrm{l}$ & $<0.010 \mathrm{mg} / \mathrm{l}$ & $<0.010 \mathrm{mg} / \mathrm{l}$ & $<0.010 \mathrm{mg} / \mathrm{l}$ & 1 & \\
\hline promos 129000 & $<0.010 \mathrm{mg} / \mathrm{l}$ & $<0.010 \mathrm{mg} / \mathrm{l}$ & $<0.010 \mathrm{mg} / \mathrm{l}$ & $<0.010 \mathrm{mg} / 1$ & 1 & \\
\hline 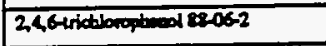 & $<0.030 \mathrm{mg} / \mathrm{l}$ & $<0.030 \mathrm{mg} / 1$ & $<0.030 \mathrm{mg} / \mathrm{l}$ & $<0.030 \mathrm{mg} / 1$ & 1 & \\
\hline 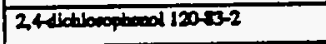 & $<0.030 \mathrm{mg} / \mathrm{l}$ & $<0.030 \mathrm{mg} / 1$ & $<0.030 \mathrm{mg} / \mathrm{l}$ & $<0.030 \mathrm{mg} / \mathrm{l}$ & 1 & \\
\hline 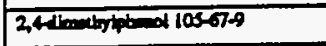 & $<0.030 \mathrm{mg} / \mathrm{l}$ & $<0.030 \mathrm{mg} /$ & $<0.030 \mathrm{mg} / \mathrm{l}$ & $<0.030 \mathrm{mg} / \mathrm{l}$ & 1 & \\
\hline 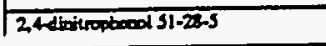 & $<0.050 \mathrm{mg} / \mathrm{l}$ & $<0.050 \mathrm{mg} / \mathrm{l}$ & $<0.050 \mathrm{mg} / \mathrm{l}$ & $<0.050 \mathrm{mg} / \mathrm{l}$ & 1 & \\
\hline
\end{tabular}


Outfall 58

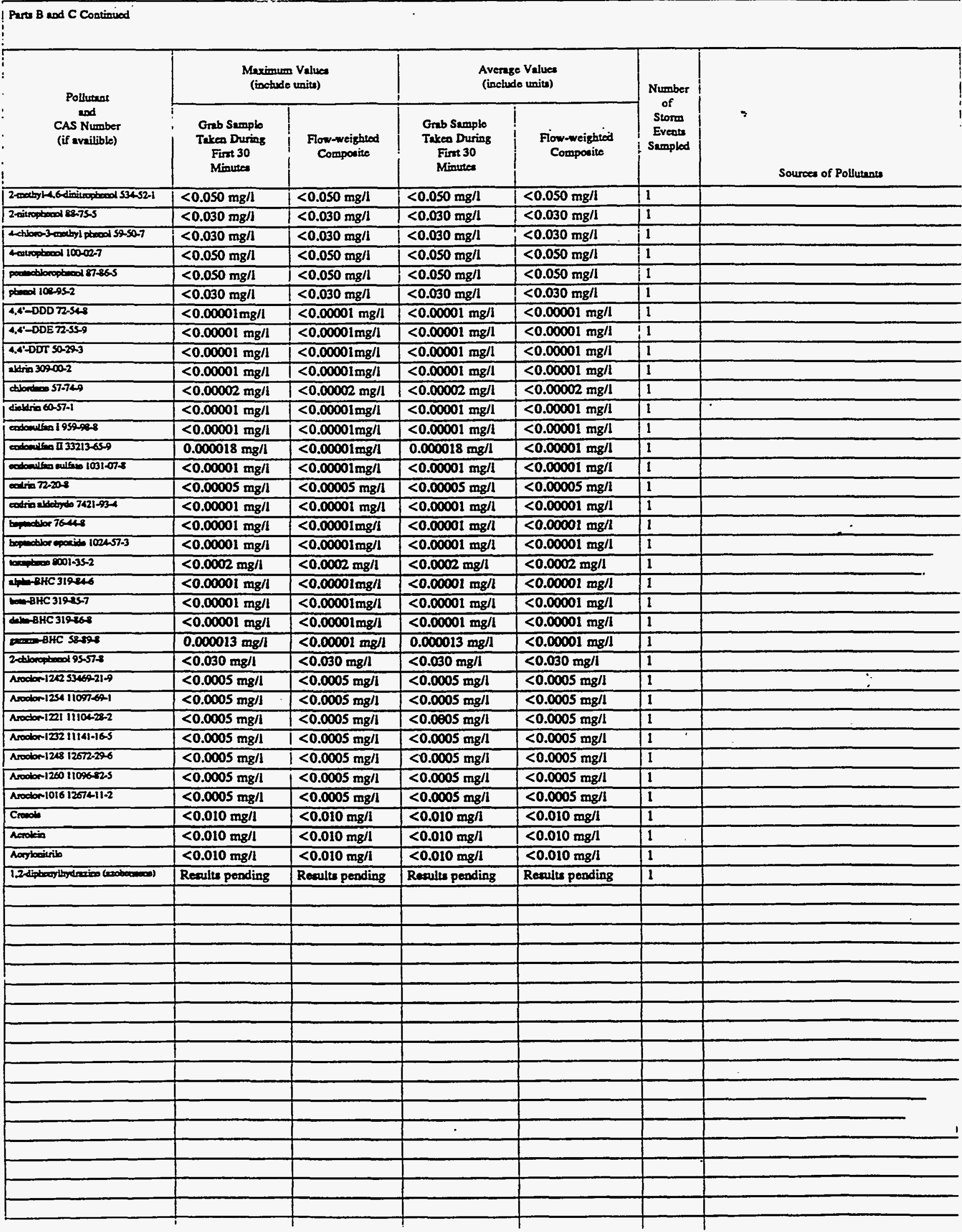


VII. Discharge Information (continuod from page 3 of Form 2F)

Pan A - You must provide the renulte of at leart one andysis for every polluant in this rable. Complete one table for eech outfall. Seo instructions for additional details.

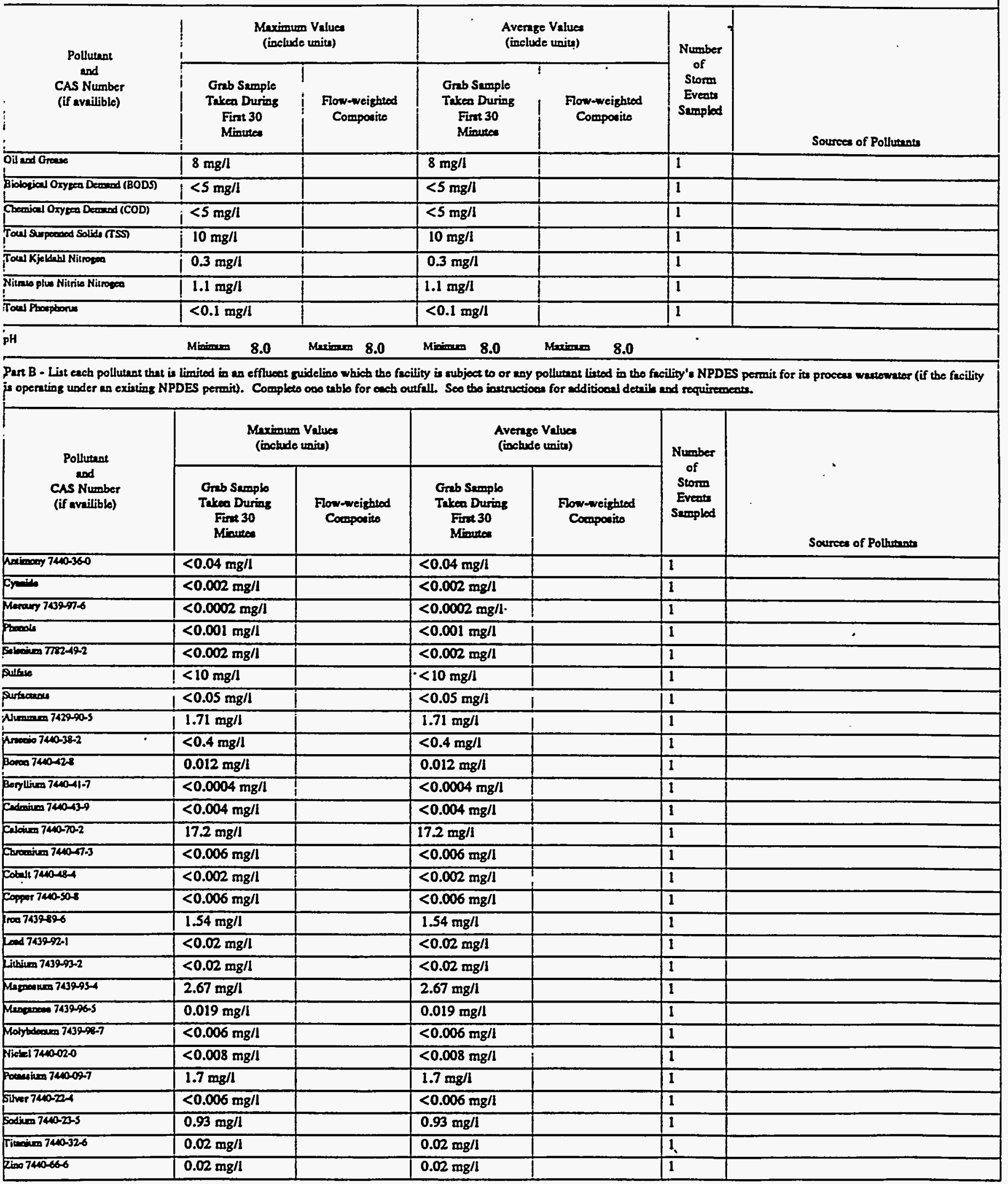


Continued from front.

Pant C- List each pollutant shown in Tables 2F-2. 2F-3, and 2F4 that you know or have reweo to believe is present. See the instruetsons for additional details and requirements. Cor ono reble for each outfall.

\begin{tabular}{|c|c|c|c|c|c|c|}
\hline $\begin{array}{l}\text { Polluteat } \\
\text { and } \\
\text { CAS Number } \\
\text { (if availible) }\end{array}$ & $\begin{array}{c}\text { Maxi } \\
\text { (ince } \\
\vdots \\
\text { Grab Sample } \\
\text { Taken During } \\
\text { Fint 30 } \\
\text { Minutet }\end{array}$ & $\begin{array}{c}\text { Flow-weightod } \\
\text { Comporite }\end{array}$ & $\begin{array}{l}\text { Grab Sxmple } \\
\text { Takea During } \\
\text { First } 30 \\
\text { Minutes }\end{array}$ & $\begin{array}{c}\text { Flow-weighiad } \\
\text { Composite }\end{array}$ & 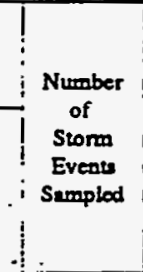 & Sources of Pollutents \\
\hline 1.1.1.-Anctioncetane 71-55-6 & $:<0.010 \mathrm{mg} / \mathrm{l}$ & & $<0.010 \mathrm{mg} / \mathrm{l}$ & $\overrightarrow{:}$ & 1 & i \\
\hline 1.1.22-ketractbocotedex 79-34-5 & $1<0.010 \mathrm{mg} / \mathrm{l}$ & & $<0.010 \mathrm{mg} / 1$ & $\div$ & 11 & 1 \\
\hline 1.1.2- trichboroctiono 7900.5 & i<0.010 mg/l & & $<0.010 \mathrm{mg} / \mathrm{l}$ & $i$ & $i 1$ & i \\
\hline 1.1-dichlonoetbex 75-34-3 & $<0.010 \mathrm{mg} / 1$ & & $<0.010 \mathrm{mg} / \mathrm{l}$ & $i$ & 1 & \\
\hline 1.1-dictsoroedsyleoe 75-35-4 & $<0.010 \mathrm{mg} / \mathrm{l}$ & . & $<0.010 \mathrm{mg} / \mathrm{l}$ & $i$ & 1 & \\
\hline 1.2 dictionoetheos $107.06-2$ & $<0.010 \mathrm{mg} / \mathrm{l}$ & & $<0.010 \mathrm{mg} / \mathrm{l}$ & $:$ & 1 & \\
\hline 1.2 dictlonopropeses $78-87.5$ & $<0.010 \mathrm{mg} / \mathrm{t}$ & - & $<0.010 \mathrm{mg} / \mathrm{l}$ & ! & 1 & \\
\hline 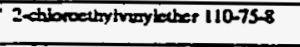 & $i<0.010 \mathrm{mg} / \mathrm{l}$ & $\div$ & $<0.010 \mathrm{mg} / \mathrm{l}$ & $i$ & 1 & \\
\hline beneses $71-42-2$ & $<0.010 \mathrm{mg} / \mathrm{l}$ & $\div$ & $<0.010 \mathrm{mg} / \mathrm{l}$ & $!$ & $1 !$ & \\
\hline 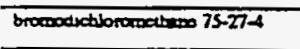 & $<0.010 \mathrm{mg} / \mathrm{l}$ & & $<0.010 \mathrm{mg} / 1$ & & 11 & $T$ \\
\hline bnemorom is.25.2 & i $<0.010 \mathrm{mg} / \mathrm{l}$ & & $1<0.010 \mathrm{mg} / 1$ & $\vdots$ & 1 & i" \\
\hline brocosacaries 7483-9 & $i<0.010 \mathrm{mg} / 1$ & & $<0.010 \mathrm{mg} / 1$ & & 1 & $i$ \\
\hline artoo ketractoride 56-23-5 & $<0.010 \mathrm{mg} / \mathrm{l}$ & & $<0.010 \mathrm{mg} / \mathrm{l}$ & & 1 & \\
\hline ctorobentores $108-90.7$ & $<0.010 \mathrm{mg} / 1$ & & $<0.010 \mathrm{mg} / 1$ & 1 & 1 & \\
\hline 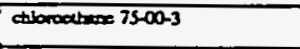 & $<0.010 \mathrm{mg} / \mathrm{l}$ & & $<0.010 \mathrm{mg} / \mathrm{l}$ & & 1 & \\
\hline chloroform 67-66-3 & $<0.010 \mathrm{mg} / \mathrm{l}$ & 7 & $<0.010 \mathrm{mg} / \mathrm{l}$ & & 1 & . \\
\hline thoncreateres 7487-3 & $<0.010 \mathrm{mg} / \mathrm{l}$ & & $<0.010 \mathrm{mg} / \mathrm{l}$ & & 1 & 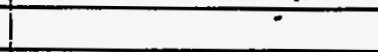 \\
\hline 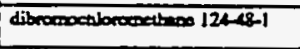 & $<0.010 \mathrm{mg} / \mathrm{l}$ & 1 & $<0.010 \mathrm{mg} / 1$ & & 1 & \\
\hline 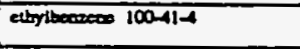 & $<0.010 \mathrm{mg} / \mathrm{l}$ & & $<0.010 \mathrm{mg} / \mathrm{l}$ & & 1 & \\
\hline wohuare 108-88-3 & $<0.010 \mathrm{mg} / \mathrm{l}$ & $\perp$ & $<0.010 \mathrm{mg} / \mathrm{l}$ & & 1 & \\
\hline vingl chloride 75-Ol-4 & $<0.010 \mathrm{mg} / \mathrm{l}$ & 7 & $<0.010 \mathrm{mg} / \mathrm{l}$ & & 1 & \\
\hline 1.3-dictionopropeces $10051-01-5$ & $<0.010 \mathrm{mg} / \mathrm{l}$ & 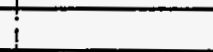 & $<0.010 \mathrm{mg} / \mathrm{l}$ & & 1 & \\
\hline 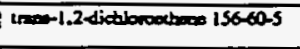 & $<0.010 \mathrm{mg} / \mathrm{l}$ & & $<0.010 \mathrm{mg} / 1$ & & 1 & $\dot{1}$ \\
\hline 1lphe nasurity $12587-46-1$ & $2.7 \mathrm{pci} / \mathrm{l}$ & & $2.7 \mathrm{pci} / \mathrm{l}$ & & 1 & \\
\hline earnay $12587-46-1$ & $9.5 \mathrm{pci} / \mathrm{l}$ & & 9.5 pcill & & 1 & \\
\hline rocal colliford & $630 \mathrm{co} / .1 \mathrm{~L}$ & $i$ & $630 \mathrm{co} / .1 \mathrm{~L}$ & $\vdots$ & 1 & $i$ \\
\hline Thallum 7440-2850 & $<0.4 \mathrm{mg} / 1$ & $!$ & $<0.4 \mathrm{mg} / \mathrm{l}$ & 1 & 1 & 1 \\
\hline Unsones $7440-61-1$ & $0: 002 \mathrm{mg} / \mathrm{l}$ & $i$ & $0.002 \mathrm{mg} / \mathrm{l}$ & & 1 & 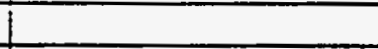 \\
\hline Gemenearnty & -6.3 peill & & $-6.3 \mathrm{pci} / \mathrm{l}$ & & 1 & \\
\hline & & & & & & \\
\hline
\end{tabular}

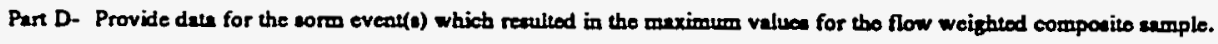

\begin{tabular}{|c|c|c|c|c|c|c|c|}
\hline $\begin{array}{l}1 . \\
\text { Date of } \\
\text { Storm } \\
\text { Evert }\end{array}$ & $\begin{array}{l}2 . \\
\text { Duntion } \\
\text { of Storm } \\
\text { (in } \\
\text { minutes) }\end{array}$ & $\begin{array}{l}3 . \\
\text { Total minfall } \\
\text { during storm event } \\
\text { (in isches) }\end{array}$ & $\begin{array}{l}4 \text {. } \\
\text { Number of hours betwoen } \\
\text { beginning of atom meas- } \\
\text { ured and ead of previous } \\
\text { meanublo nin eveat }\end{array}$ & $\begin{array}{l}\text { S. } \\
\text { Maximimow flow nite } \\
\text { during sain ovent } \\
\text { (rallona/min or specify } \\
\text { unim) }\end{array}$ & $\begin{array}{l}6 . \\
\text { Total flow from } \\
\text { rain event } \\
\text { (gillons or } \\
\text { specify unisu) }\end{array}$ & $\begin{array}{c}7 . \\
\text { Seteon } \\
\text { atemple whs } \\
\text { tikes }\end{array}$ & $\begin{array}{c}8 . \\
\text { Form of } \\
\text { Procipitation } \\
\text { (nimfill. } \\
\text { nommela) }\end{array}$ \\
\hline $1 / 3 / 92$ & 435 & 1.1 & 128.8 & 1.7 & 634 & Winter & Rninfall \\
\hline
\end{tabular}

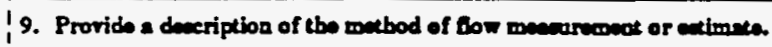

: An ISCO 3230 Flow meter was used to obtain water level reatings. This instrment was catibmted with the normal dry weather flow, if present, as lev. i zero. The flow meter was programmed with the characteristics of the conveyance so that flow rates and total flows could be calculated by the meter. 
Outfall 62

: Paru B and C Continued

\begin{tabular}{|c|c|c|c|c|c|c|}
\hline \multirow{2}{*}{$\begin{array}{l}\text { Pollutent } \\
\text { and } \\
\text { CAS Number } \\
\text { (if availible) }\end{array}$} & \multicolumn{2}{|c|}{$\begin{array}{l}\text { Maximum Valuces } \\
\text { (inchude units) }\end{array}$} & \multicolumn{2}{|c|}{$\begin{array}{l}\text { Avernge Valued } \\
\text { (isclude units) }\end{array}$} & \multirow{2}{*}{$\begin{array}{l}\text { Number } \\
\text { of } \\
\text { SLorm? } \\
\text { Events } \\
\text { Sumplod }\end{array}$} & \multirow[b]{2}{*}{ Sources of Pollutuats } \\
\hline & $\begin{array}{c}\text { Grab Sample } \\
\text { Taken During } \\
\text { Fint } 30 \\
\text { Minumes }\end{array}$ & $\begin{array}{l}\text { Flow-weightod } \\
\text { Composite }\end{array}$ & $\begin{array}{l}\text { Grab Sumple } \\
\text { Taken During } \\
\text { Firr 30 } \\
\text { Minule }\end{array}$ & $\begin{array}{l}\text { Flow-weightod } \\
\text { Comporine }\end{array}$ & & \\
\hline B2rium 740-39-3 & $0.0109 \mathrm{mg} / \mathrm{l}$ & & $0.0109 \mathrm{mg} / \mathrm{l}$ & & 1 & \\
\hline melbyleos chloride 75-092.2 & $<0.010 \mathrm{mg} / \mathrm{l}$ & & $<0.010 \mathrm{mg} / \mathrm{l}$ & & 1 & \\
\hline Letnotionocheos $127.18-4$ & $<0.010 \mathrm{mg} / \mathrm{l}$ & & $<0.010 \mathrm{mg} / \mathrm{t}$ & & 1 & \\
\hline 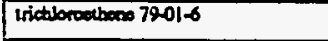 & $<0.010 \mathrm{mg} / \mathrm{l}$ & & $<0.010 \mathrm{mg} / 1$ & & 1 & \\
\hline Xyleoses & $<0.010 \mathrm{mg} / \mathrm{l}$ & & $<0.010 \mathrm{mg} / 1$ & & 1 & \\
\hline 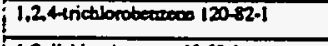 & $<0.010 \mathrm{mg} / \mathrm{l}$ & & $<0.010 \mathrm{mg} / \mathrm{l}$ & & 1 & \\
\hline 1.2 dictlorobecocose y5-50-1 & $<0.010 \mathrm{mg} / \mathrm{l}$ & & $<0.010 \mathrm{mg} / \mathrm{l}$ & & 1 & \\
\hline 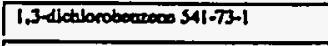 & $<0.010 \mathrm{mg} / \mathrm{l}$ & & $<0.010 \mathrm{mg} / \mathrm{l}$ & & 1 & \\
\hline 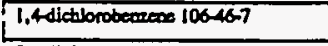 & $<0.010 \mathrm{mg} / \mathrm{l}$ & & $<0.010 \mathrm{mg} / 1$ & & 1 & \\
\hline 24dinitroudimes $121-14-2$ & $<0.010 \mathrm{mg} / 1$ & & $<0.010 \mathrm{mg} / \mathrm{l}$ & & 1 & \\
\hline 26 dinitrocheos $606.20-2$ & $<0.010 \mathrm{mg} / \mathrm{l}$ & & $<0.010 \mathrm{mg} / 1$ & & 1 & \\
\hline 2-ctsoronepteraleoes $41-58-7$ & $<0.010 \mathrm{mg} / \mathrm{l}$ & & $<0.010 \mathrm{mg} / \mathrm{l}$ & & 1. & \\
\hline 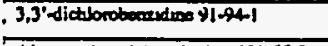 & $<0.010 \mathrm{mg} / \mathrm{l}$ & & $<0.010 \mathrm{mg} / \mathrm{l}$ & & 1 & \\
\hline 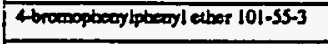 & $<0.010 \mathrm{mg} / \mathrm{l}$ & & $<0.010 \mathrm{mg} / \mathrm{l}$ & & 1 & \\
\hline 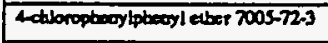 & $<0.010 \mathrm{mg} / \mathrm{l}$ & & $<0.010 \mathrm{mg} / 1$ & & 1 & \\
\hline 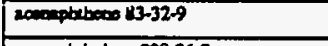 & $<0.010 \mathrm{mg} / \mathrm{l}$ & & $<0.010 \mathrm{mg} / \mathrm{l}$ & & 1 & \\
\hline 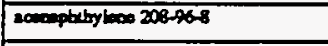 & $<0.010 \mathrm{mg} / \mathrm{l}$ & & $<0.010 \mathrm{mg} / 1$ & & 1 & \\
\hline wethesoses 120-12.7 & $<0.010 \mathrm{mg} / \mathrm{l}$ & & $<0.010 \mathrm{mg} / 1$ & & 1 & \\
\hline bencidine 92.87.5 & $<0.030 \mathrm{mg} / \mathrm{l}$ & & $<0.030 \mathrm{mg} / 1$ & & 1 & \\
\hline beoweda) & $<0.010 \mathrm{mg} / \mathrm{l}$ & & $<0.010 \mathrm{mg} / 1$ & & 1 & \\
\hline 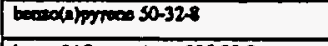 & $<0.010 \mathrm{mg} / \mathrm{l}$ & & $<0.010 \mathrm{mg} / 1$ & & 1 & \\
\hline 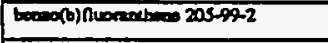 & $<0.010 \mathrm{mg} / \mathrm{l}$ & & $<0.010 \mathrm{mg} / \mathrm{l}$ & & 1 & \\
\hline 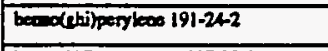 & $<0.010 \mathrm{mg} / \mathrm{l}$ & & $<0.010 \mathrm{mg} / \mathrm{l}$ & & 1 & \\
\hline 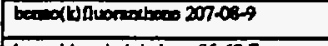 & $<0.010 \mathrm{mg} / \mathrm{l}$ & & $<0.010 \mathrm{mg} / 1$ & & 1 & \\
\hline 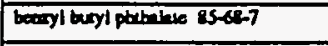 & $<0.010 \mathrm{mg} / \mathrm{l}$ & & $<0.010 \mathrm{mg} / \mathrm{l}$ & & 1 & \\
\hline 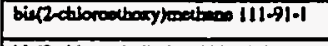 & $<0.010 \mathrm{mg} / \mathrm{l}$ & & $<0.010 \mathrm{mg} / \mathrm{t}$ & & 1 & $\because$ \\
\hline 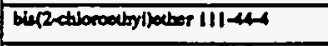 & $<0.010 \mathrm{mg} / \mathrm{l}$ & & $<0.010 \mathrm{mg} / \mathrm{l}$ & & 1 & \\
\hline 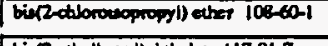 & $<0.010 \mathrm{mg} / \mathrm{l}$ & & $<0.010 \mathrm{mg} / \mathrm{l}$ & & 1 & \\
\hline 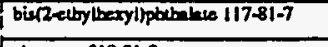 & $<0.010 \mathrm{mg} / \mathrm{l}$ & & $<0.010 \mathrm{mg} / \mathrm{l}$ & & 1 & \\
\hline ctaryeces 21801-9 & $<0.010 \mathrm{mg} / \mathrm{l}$ & & $<0.010 \mathrm{mg} / \mathrm{l}$ & & 1 & \\
\hline di-obaxyl phelainas 8474-2 & $<0.010 \mathrm{mg} / \mathrm{l}$ & & $<0.010 \mathrm{mg} / 1$ & & 1 & \\
\hline 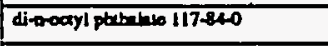 & $<0.010 \mathrm{mg} / \mathrm{l}$ & & $<0.010 \mathrm{mg} / \mathrm{l}$ & & 1 & \\
\hline 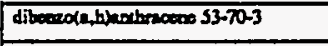 & $<0.010 \mathrm{mg} / \mathrm{l}$ & & $<0.010 \mathrm{mg} / \mathrm{t}$ & & 1 & \\
\hline 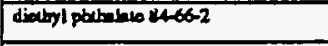 & $<0.010 \mathrm{mg} / \mathrm{l}$ & & $<0.010 \mathrm{mg} / \mathrm{h}$ & & 1 & \\
\hline dimolyl pathench $|3| \cdot||-3$ & $<0.010 \mathrm{mg} / \mathrm{l}$ & & $<0.010 \mathrm{mg} / \mathrm{l}$ & & 1 & \\
\hline Numentreos 20640 & $<0.010 \mathrm{mg} / \mathrm{l}$ & & $<0.010 \mathrm{mg} / \mathrm{l}$ & & 1 & \\
\hline Alowese 86-73-7 & $<0.010 \mathrm{mg} / \mathrm{l}$ & & $<0.010 \mathrm{mg} / \mathrm{l}$ & & 1 & \\
\hline berectlorobecreace $118-74.1$ & $<0.010 \mathrm{mg} / \mathrm{l}$ & & $<0.010 \mathrm{mg} / \mathrm{l}$ & & 1 & \\
\hline 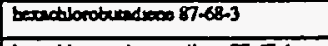 & $<0.010 \mathrm{mg} / \mathrm{l}$ & & $<0.010 \mathrm{mg} / \mathrm{l}$ & & 1 & \\
\hline 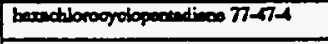 & $<0.010 \mathrm{mg} / \mathrm{l}$ & & $<0.010 \mathrm{mg} / \mathrm{l}$ & & 1 & \\
\hline 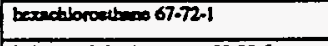 & $<0.010 \mathrm{mg} / \mathrm{I}$ & & $<0.010 \mathrm{mg} / \mathrm{l}$ & & 1 & \\
\hline indoed $(1,2,3-04)$ procos $193-39.5$ & $<0.010 \mathrm{mg} / \mathrm{l}$ & & $<0.010 \mathrm{mg} / 1$ & & 1 & \\
\hline isoptorenes 78-59-1 & $<0.010 \mathrm{mg} / 1$ & & $<0.010 \mathrm{mg} / \mathrm{l}$ & & 1 & \\
\hline N-tilrowodi-opropplamine $621-647$ & $<0.010 \mathrm{mg} / \mathrm{l}$ & & $<0.010 \mathrm{mg} / \mathrm{t}$ & & 1 & \\
\hline 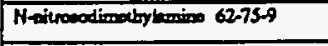 & $<0.010 \mathrm{mg} / \mathrm{l}$ & & $<0.010 \mathrm{mg} / \mathrm{l}$ & & 1 & \\
\hline 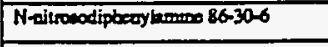 & $<0.010 \mathrm{mg} / \mathrm{l}$ & & $<0.010 \mathrm{mg} / \mathrm{l}$ & & 1 & \\
\hline 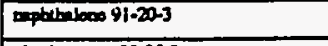 & $<0.010 \mathrm{mg} / \mathrm{l}$ & & $<0.010 \mathrm{mg} / \mathrm{l}$ & & 1 & \\
\hline ailuobroweeces 96-95-3 & $<0.010 \mathrm{mg} / \mathrm{l}$ & & $<0.010 \mathrm{mg} / \mathrm{l}$ & & 1 & \\
\hline phowothroos 85-01-8 & $<0.010 \mathrm{mg} / \mathrm{l}$ & & $<0.010 \mathrm{mg} / \mathrm{l}$ & & 1 & \\
\hline procose 1290000 & $<0.010 \mathrm{mg} / \mathrm{l}$ & & $<0.010 \mathrm{mg} / \mathrm{l}$ & & 1 & \\
\hline 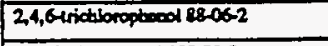 & $<0.030 \mathrm{mg} / 1$ & & $<0.030 \mathrm{mg} / \mathrm{l}$ & & 1 & - \\
\hline 2,4dichlorophood 120-83-2 & $<0.030 \mathrm{mg} / \mathrm{l}$ & & $<0.030 \mathrm{mg} / \mathrm{l}$ & & 1 & \\
\hline 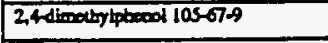 & $<0.030 \mathrm{mg} / \mathrm{l}$ & & $<0.030 \mathrm{mg} / \mathrm{l}$ & & 1 & \\
\hline 24-dinitropteond 51-28-5 & $<0.050 \mathrm{mg} / \mathrm{l}$ & & $<0.050 \mathrm{mg} / \mathrm{l}$ & & 1 & \\
\hline
\end{tabular}


Outfail 62

Pars B and C Cootinuod

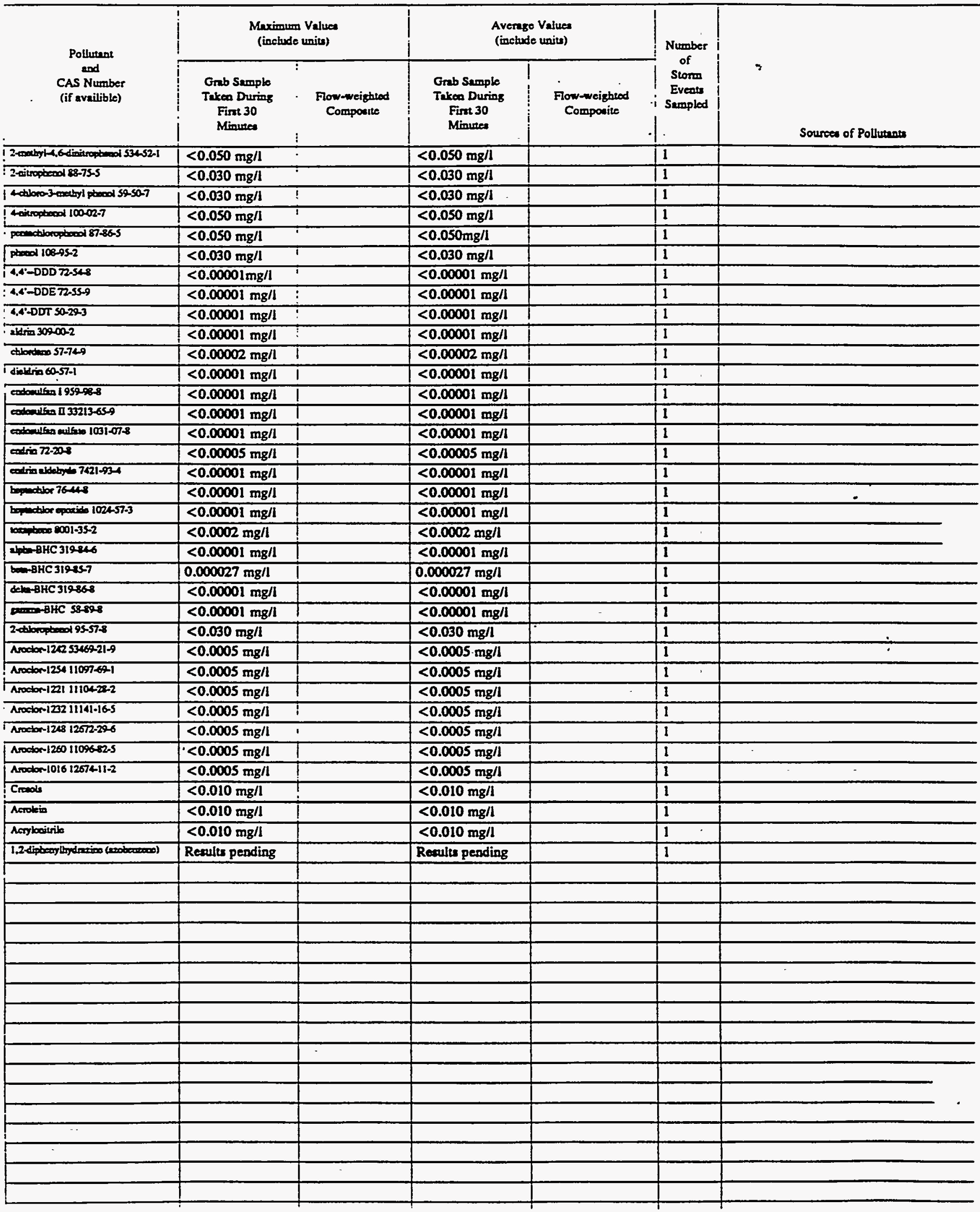


VI. Discharge Information (continuod from pege 3 of Form $2 \mathrm{~F}$

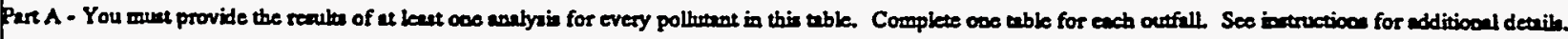

\begin{tabular}{|c|c|c|c|c|c|c|}
\hline \multirow{2}{*}{$\begin{array}{l}\text { Polbutant } \\
\text { end } \\
\text { CAS Number } \\
\text { (if availible) }\end{array}$} & \multicolumn{2}{|c|}{$\begin{array}{l}\text { Maximum Veluea } \\
\text { (include unitu) }\end{array}$} & \multicolumn{2}{|c|}{$\begin{array}{l}\text { Avernge Vahua } \\
\text { (include vaita). }\end{array}$} & \multirow{2}{*}{$\begin{array}{l}\text { Number } \\
\text { of } \\
\text { Siorm } \\
\text { Evenes } \\
\text { Sompled }\end{array}$} & \multirow[b]{2}{*}{ Soum of Polht } \\
\hline & $\begin{array}{l}\text { Grab Semple } \\
\text { Takea During } \\
\text { First } 30 \\
\text { Minowe }\end{array}$ & $\begin{array}{l}\text { Frow-weighted } \\
\text { Componite }\end{array}$ & $\begin{array}{l}\text { Grab Sumple } \\
\text { Taken During } \\
\text { Fint } 30 \\
\text { Mindo }\end{array}$ & $\begin{array}{l}\text { Frow-weightod } \\
\text { Compotice }\end{array}$ & & \\
\hline Dill and Graen & $<2 \mathrm{mg} / \mathrm{l}$ & & $<2 \mathrm{mg} / \mathrm{l}$ & & 1 & \\
\hline 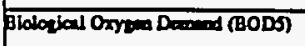 & $8.32 \mathrm{mg} / \mathrm{l}$ & $6.78 \mathrm{mg} / 1$ & $8.32 \mathrm{mg} / 1$ & $6.78 \mathrm{mg} / 1$ & 1 & \\
\hline Conerioal Oxypen Domed (COD) & $42 \mathrm{mg} / \mathrm{l}$ & $25 \mathrm{mg} / \mathrm{l}$ & $42 \mathrm{mg} / 1$ & $25 \mathrm{mg} / \mathrm{h}$ & 1 & \\
\hline Toul Sumpooded Solith (TSS) & $9.5 \mathrm{mg} / \mathrm{l}$ & $25 \mathrm{mg} / \mathrm{l}$ & $9.5 \mathrm{mg} / \mathrm{l}$ & $25 \mathrm{mg} / \mathrm{l}$ & 1 & \\
\hline Toul Kjektebl Nitrogen & $0.9 \mathrm{mg} / \mathrm{l}$ & $0.6 \mathrm{mg} / \mathrm{l}$ & $0.9 \mathrm{mg} / \mathrm{l}$ & $0.6 \mathrm{mg} / \mathrm{l}$ & 1 & \\
\hline Nitres phe Nitrie Nitropace & $2.0 \mathrm{mg} / \mathrm{l}$ & $1.4 \mathrm{mg} / \mathrm{l}$ & $2.0 \mathrm{mg} / \mathrm{l}$ & $1.4 \mathrm{mg} / \mathrm{l}$ & 1 & \\
\hline Taul Phopporis & $<0.1 \mathrm{mg} / \mathrm{l}$ & $0.11 \mathrm{mg} / 1$ & $<0.1 \mathrm{mg} / \mathrm{l}$ & $0.11 \mathrm{mg} / 1$ & 1 & \\
\hline
\end{tabular}

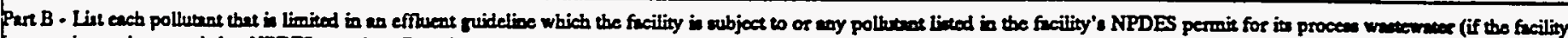

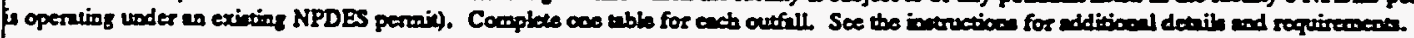

\begin{tabular}{|c|c|c|c|c|c|c|}
\hline \multirow{2}{*}{$\begin{array}{l}\text { Poltutent } \\
\text { end } \\
\text { CAs Number } \\
\text { (if nvaitible) }\end{array}$} & \multicolumn{2}{|c|}{$\begin{array}{l}\text { Meximin Vabuen } \\
\text { (inchude unita) }\end{array}$} & \multicolumn{2}{|c|}{$\begin{array}{l}\text { Avernge Vahues } \\
\text { (ipclude units) }\end{array}$} & \multirow{2}{*}{ 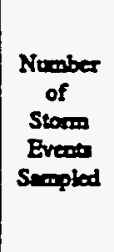 } & \multirow[b]{2}{*}{ Sources of Pollowinen } \\
\hline & $\begin{array}{l}\text { Grab Sample } \\
\text { Traem Durias } \\
\text { Fins } 30 \\
\text { Minecen }\end{array}$ & $\begin{array}{l}\text { Flow-wrightod } \\
\text { Componite }\end{array}$ & $\begin{array}{l}\text { Grab Semple } \\
\text { Tanon Durias } \\
\text { Find } 30 \\
\text { Minetes }\end{array}$ & $\begin{array}{l}\text { Flow-weicheod } \\
\text { Compocisco }\end{array}$ & & \\
\hline haimor 7401360 & $<0.04 \mathrm{mg} / \mathrm{h}$ & $<0.04 \mathrm{mg} / \mathrm{l}$ & $<0.04 \mathrm{mg} / \mathrm{l}$ & $<0.04 \mathrm{mg} / \mathrm{l}$ & 1 & \\
\hline Symide & $<0.002 \mathrm{mg} / \mathrm{l}$ & & $<0.002 \mathrm{mg} / 1$ & & 1 & \\
\hline Merainy 7439-97.6 & $0.0003 \mathrm{mg} / \mathrm{l}$ & $0.0004 \mathrm{mg} / \mathrm{l}$ & $0.0003 \mathrm{mg} / \mathrm{h}$ & $0.00004 \mathrm{mg} / \mathrm{l}$ & 1 & \\
\hline Pons & $0.004 \mathrm{mg} / 1$ & & $0.004 \mathrm{mg} / 1$ & & 1 & : \\
\hline seboinen $7 \pi 2-42-2$ & $<0.002 \mathrm{mg} / \mathrm{l}$ & $<0.002 \mathrm{mg} / \mathrm{l}$ & $<0.002 \mathrm{mg} / \mathrm{l}$ & $<0.002 \mathrm{mg} / \mathrm{l}$ & 1 & \\
\hline 50150 & $21 \mathrm{mg} / 1$ & $10 \mathrm{mg} / \mathrm{l}$ & $21 \mathrm{mg} / 1$ & $10 \mathrm{mg} / \mathrm{l}$ & 1 & \\
\hline Surtinaneses & $0.44 \mathrm{mg} / 1$ & $0.10 \mathrm{mg} / 1$ & $0.44 \mathrm{mg} / \mathrm{l}$ & $0.10 \mathrm{mg} / \mathrm{l}$ & 1 & \\
\hline Aromines 7129.90.5 & $0.36 \mathrm{mg} / \mathrm{l}$ & $0.90 \mathrm{mg} / \mathrm{l}$ & $0.36 \mathrm{mg} / \mathrm{l}$ & $0.90 \mathrm{mg} / \mathrm{l}$ & 1 & \\
\hline $4000000740-38-2$ & $<0.04 \mathrm{mg} / \mathrm{l}$ & $<0.04 \mathrm{mg} / \mathrm{l}$ & $<0.04 \mathrm{mg} / \mathrm{l}$ & $<0.04 \mathrm{mg} / \mathrm{l}$ & 1 & \\
\hline Borod 740-124 & $0.035 \mathrm{mg} / 1$ & $0.031 \mathrm{mg} / 1$ & $0.035 \mathrm{mg} / \mathrm{l}$ & $0.031 \mathrm{mg} / \mathrm{l}$ & 1 & \\
\hline Bergliven 740-1.-7 & $<0.0004 \mathrm{mg} / \mathrm{l}$ & $<0.0004 \mathrm{mg} / \mathrm{l}$ & $<0.0004 \mathrm{mg} / \mathrm{l}$ & $<0.0004 \mathrm{mg} / \mathrm{l}$ & 1 & \\
\hline Endaimm 740-13.9 & $<0.004 \mathrm{mg} / \mathrm{l}$ & $<0.004 \mathrm{mg} / \mathrm{l}$ & $<0.004 \mathrm{mg} / \mathrm{l}$ & $<0.004 \mathrm{mg} / \mathrm{l}$ & 1 & \\
\hline Eabaim 7400-200-2 & $18.7 \mathrm{mg} / 1$ & $18.8 \mathrm{mg} / \mathrm{l}$ & $18.7 \mathrm{mg} / \mathrm{l}$ & $18.8 \mathrm{mg} /$ & 1 & \\
\hline Fromidn 746-17.3 & $<0.006 \mathrm{mg} / \mathrm{l}$ & $<0.006 \mathrm{mg} / \mathrm{l}$ & $<0.006 \mathrm{mg} / \mathrm{l}$ & $<0.006 \mathrm{mg} / \mathrm{l}$ & 1 & \\
\hline Cotak 7400-124 & $<0.002 \mathrm{mg} / \mathrm{l}$ & $<0.002 \mathrm{mg} / \mathrm{l}$ & $<0.002 \mathrm{mg} / \mathrm{l}$ & $<0.002 \mathrm{mg} / \mathrm{l}$ & 1 & \\
\hline Foppoen 740a-sos & $0.009 \mathrm{mg} / \mathrm{l}$ & $0.012 \mathrm{mg} / \mathrm{h}$ & $0.009 \mathrm{mg} / \mathrm{l}$ & $0.012 \mathrm{mg} / \mathrm{l}$ & 1 & \\
\hline from $7039-196$ & $0.44 \mathrm{mg} / \mathrm{h}$ & $1.30 \mathrm{mg} / 1$ & $0.44 \mathrm{mg} / \mathrm{l}$ & $1.30 \mathrm{mg} / 1$ & 1 & \\
\hline med 7392-2.1 & $<0.02 \mathrm{mg} / 1$ & $<0.02 \mathrm{mg} / \mathrm{I}$ & $<0.02 \mathrm{mg} / \mathrm{l}$ & $<0.02 \mathrm{mg} / 1$ & 1 & \\
\hline 156hen 743993-2 & $<0.02 \mathrm{mg} / \mathrm{l}$ & $<0.02 \mathrm{mg} / \mathrm{l}$ & $<0.02 \mathrm{mg} / \mathrm{l}$ & $<0.02 \mathrm{mg} / \mathrm{h}$ & 1 & \\
\hline 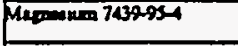 & $1.77 \mathrm{mg} / \mathrm{l}$ & $2.30 \mathrm{mg} / \mathrm{l}$ & $1.77 \mathrm{mg} / 1$ & $2.30 \mathrm{mg} / \mathrm{l}$ & 1 & \\
\hline Menpoen 7039-6.5 & $0.011 \mathrm{mg} / \mathrm{l}$ & $0.032 \mathrm{mg} / \mathrm{l}$ & $0.011 \mathrm{mg} / 1$ & $0.032 \mathrm{mg} / \mathrm{l}$ & 1 & \\
\hline Mobrocoun $7439.96-7$ & $<0.006 \mathrm{mg} / \mathrm{l}$ & $<0.006 \mathrm{mg} / \mathrm{l}$ & $<0.006 \mathrm{mg} / \mathrm{l}$ & $<0.006 \mathrm{mg} / \mathrm{l}$ & 1 & \\
\hline Nikd 7400020 & $<0.008 \mathrm{mg} / \mathrm{l}$ & $<0.008 \mathrm{mg} / \mathrm{h}$ & $<0.008 \mathrm{mg} / \mathrm{l}$ & $<0.008 \mathrm{mg} / \mathrm{l}$ & 1 & \\
\hline paraicm 740009-7 & $1.0 \mathrm{mg} / \mathrm{l}$ & $1.0 \mathrm{mg} / 1$ & $1.0 \mathrm{mg} / \mathrm{l}$ & $1.0 \mathrm{mg} / 1$ & 1 & \\
\hline Pitwe 7400-204 & $<0.006 \mathrm{mg} / \mathrm{l}$ & $<0.006 \mathrm{mg} / 1$ & $<0.006 \mathrm{mg} / 1$ & $<0.006 \mathrm{mg} / \mathrm{l}$ & 1 & \\
\hline sodkn= 74ta-2a-5 & $1.00 \mathrm{mg} / \mathrm{l}$ & $0.58 \mathrm{mg} / \mathrm{l}$ & $1.00 \mathrm{mg} / 1$ & $0.58 \mathrm{mg} / \mathrm{h}$ & 1 & \\
\hline Trieminen 740.326 & $<0.01 \mathrm{mg} / \mathrm{l}$ & $0.02 \mathrm{mg} / \mathrm{l}$ & $<0.01 \mathrm{mg} / \mathrm{l}$ & $0.02 \mathrm{mg} / \mathrm{l}$ & 1 & \\
\hline $\operatorname{lin} 07400-666$ & $0.15 \mathrm{mg} / \mathrm{l}$ & $0.19 \mathrm{mg} / 1$ & $0.15 \mathrm{mg} / \mathrm{l}$ & $0.19 \mathrm{mg} / \mathrm{l}$ & 1 & \\
\hline
\end{tabular}


Continued from front.

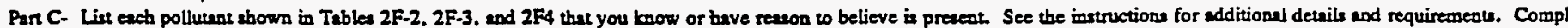
one tuble for each outfall.

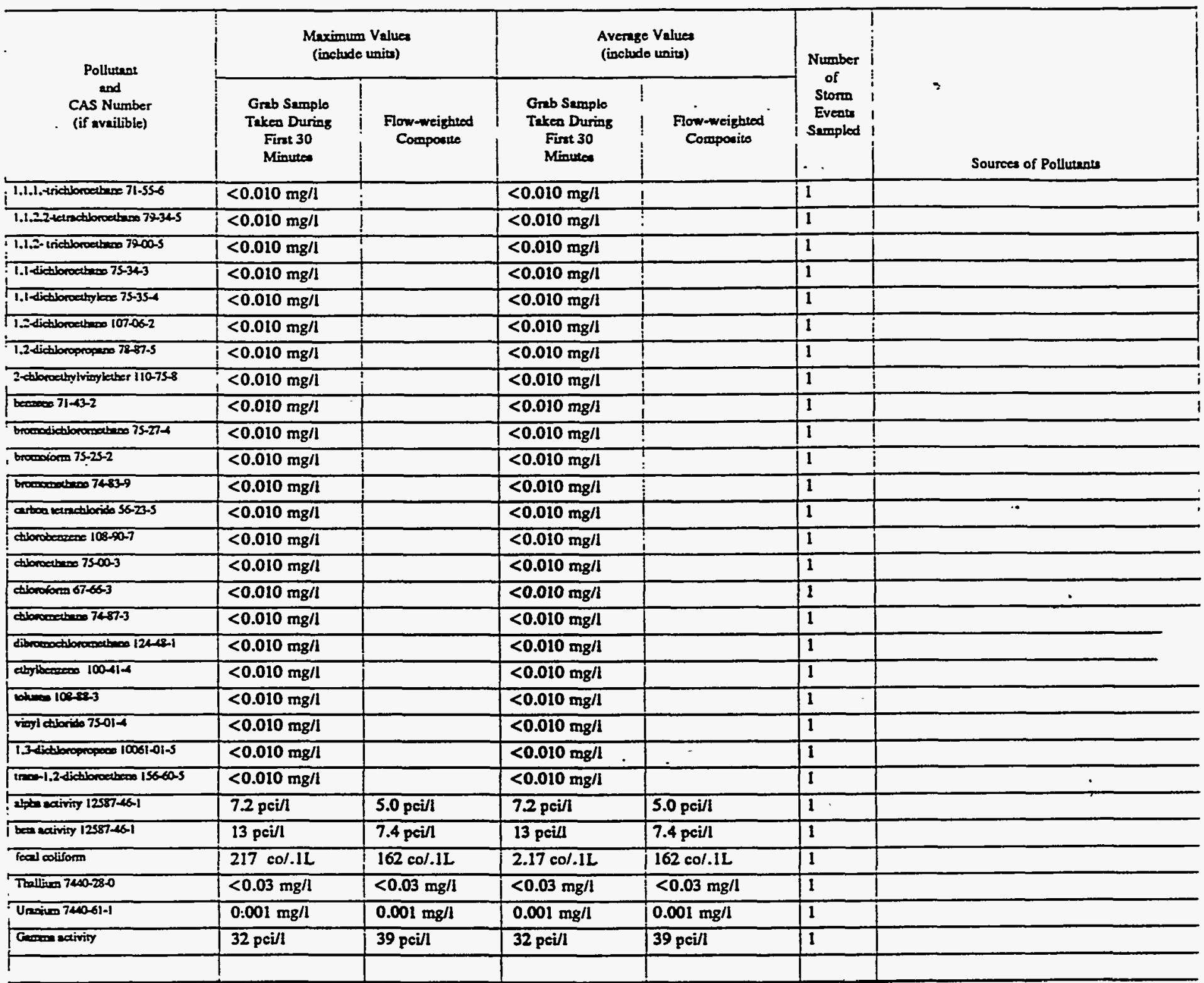

Part D- Provide dats for the sorm event(o) which reaulted in the maximum values for the flow weightod cocoposite sample.

\begin{tabular}{|c|c|c|c|c|c|c|c|}
\hline $\begin{array}{l}1 . \\
\text { Date of } \\
\text { Storm } \\
\text { Event }\end{array}$ & $\begin{array}{l}2 . \\
\text { Duntion } \\
\text { of Storm } \\
\text { (in } \\
\text { minirea) }\end{array}$ & $\begin{array}{l}3 . \\
\text { Total rinfill } \\
\text { durins storm ovent } \\
\text { (in inchea) }\end{array}$ & 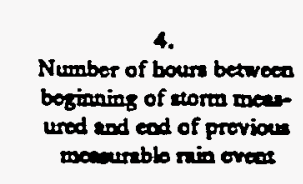 & $\begin{array}{l}\text { S. } \\
\text { Mrimum flow nuto } \\
\text { during min eveol } \\
\text { (olloci/min or epocify } \\
\text { unitu) }\end{array}$ & $\begin{array}{c}6 . \\
\text { Total flow from } \\
\text { min eveat } \\
\text { (alloos or } \\
\text { apeify unitu) }\end{array}$ & $\begin{array}{c}7 . \\
\text { Season } \\
\text { amplo wes } \\
\text { taken }\end{array}$ & $\begin{array}{l}\text { 8. } \\
\text { Form of } \\
\text { Precipintion } \\
\text { (ninfill, } \\
\text { enometh) }\end{array}$ \\
\hline $12 / 13 / 91$ & 250 & 025 & 88.2 & 90 & 8,100 & Fall & Rainfall \\
\hline
\end{tabular}

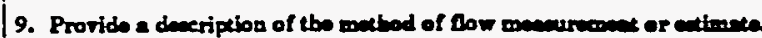

An ISCO 3230 Flow meter was msed to obtain water level readinge. This instrment was calibrated with the normal dry veather flow, if present, as level zero. The flow meter was programmed with the characteristics of the conveyance so that flow rates and total flows conid be calculated by the meter. 
Qutfall 64

Parta B and C Continused

\begin{tabular}{|c|c|c|c|c|c|c|}
\hline \multirow{2}{*}{$\begin{array}{l}\text { Pollutuat } \\
\text { and } \\
\text { CAS Number } \\
\text { (if availible) }\end{array}$} & \multicolumn{2}{|c|}{$\begin{array}{l}\text { Maximum Valuea } \\
\text { (includo units) }\end{array}$} & \multicolumn{2}{|c|}{$\begin{array}{l}\text { Averngo Valuea } \\
\text { (inchudo unitu) }\end{array}$} & \multirow{2}{*}{$\begin{array}{l}\text { Number } \\
\text { of } \\
\text { Storm } \\
\text { Everes } \\
\text { Sampled }\end{array}$} & \multirow[b]{2}{*}{ Sources of Polbutants } \\
\hline & $\begin{array}{c}\text { Onbb Semplo } \\
\text { Tuken Durimg } \\
\text { Firnt } 30 \\
\text { Minuece }\end{array}$ & $\begin{array}{l}\text { Flow-weighted } \\
\text { Compoeite }\end{array}$ & $\begin{array}{l}\text { Grzb Semplo } \\
\text { Takea During } \\
\text { Fint } 30 \\
\text { Minuteo }\end{array}$ & $\begin{array}{l}\text { Flow-weighred } \\
\text { Componito }\end{array}$ & & \\
\hline Barius 740-39-3 & $0.0325 \mathrm{mg} / 1$ & $0.0327 \mathrm{mg} / \mathrm{l}$ & $0.0325 \mathrm{mg} / \mathrm{l}$ & $0.0327 \mathrm{mg} / 1$ & 1 & \\
\hline 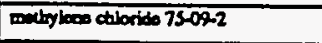 & $<0.010 \mathrm{mg} / \mathrm{l}$ & & $<0.010 \mathrm{mg} / \mathrm{l}$ & & 1 & \\
\hline 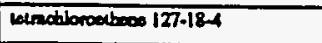 & $<0.010 \mathrm{mg} / 1$ & & $<0.010 \mathrm{mg} / 1$ & & 1 & \\
\hline 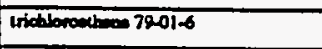 & $<0.010 \mathrm{mg} / \mathrm{l}$ & & $<0.010 \mathrm{mg} / 1$ & & 1 & \\
\hline$x y b=0$ & $<0.010 \mathrm{mg} / \mathrm{l}$ & & $<0.010 \mathrm{mg} / \mathrm{l}$ & & 1 & \\
\hline 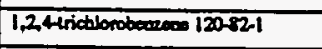 & $<0.010 \mathrm{mg} / \mathrm{l}$ & $<0.010 \mathrm{mg} / \mathrm{l}$ & $<0.010 \mathrm{mg} / \mathrm{l}$ & $<0.010 \mathrm{mg} / \mathrm{l}$ & 1 & \\
\hline 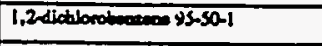 & $<0.010 \mathrm{mg} / \mathrm{l}$ & $<0.010 \mathrm{mg} / 1$ & $<0.010 \mathrm{mg} / \mathrm{l}$ & $<0.010 \mathrm{mg} / \mathrm{l}$ & 1 & \\
\hline 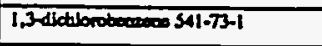 & $<0.010 \mathrm{mg} / \mathrm{l}$ & $<0.010 \mathrm{mg} / \mathrm{l}$ & $<0.010 \mathrm{mg} / \mathrm{l}$ & $<0.010 \mathrm{mg} / \mathrm{l}$ & 1 & \\
\hline 1.4-diationoboceono $106-16-7$ & $<0.010 \mathrm{mg} / \mathrm{l}$ & $<0.010 \mathrm{mg} / 1$ & $<0.010 \mathrm{mg} / \mathrm{l}$ & $<0.010 \mathrm{mg} / 1$ & 1 & \\
\hline 24dinitrochetuen $121-14-2$ & $<0.010 \mathrm{mg} / \mathrm{l}$ & $<0.010 \mathrm{mg} / \mathrm{l}$ & $<0.010 \mathrm{mg} / \mathrm{l}$ & $<0.010 \mathrm{mg} / \mathrm{l}$ & 1 & \\
\hline 26 dinitroutureses $600-20-2$ & $<0.010 \mathrm{mg} / \mathrm{l}$ & $<0.010 \mathrm{mg} / \mathrm{l}$ & $<0.010 \mathrm{mg} / \mathrm{l}$ & $<0.010 \mathrm{mg} / \mathrm{h}$ & 1 & \\
\hline 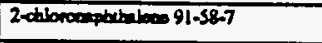 & $<0.010 \mathrm{mg} / \mathrm{l}$ & $<0.010 \mathrm{mg} / \mathrm{l}$ & $<0.010 \mathrm{mg} / \mathrm{l}$ & $<0.010 \mathrm{mg} / \mathrm{l}$ & 1 & \\
\hline 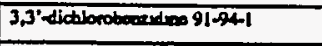 & $<0.020 \mathrm{mg} / \mathrm{l}$ & $<0.020 \mathrm{mg} / 1$ & $<0.020 \mathrm{mg} / \mathrm{l}$ & $<0.020 \mathrm{mg} / \mathrm{l}$ & 1 & \\
\hline 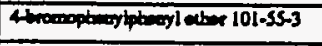 & $<0.010 \mathrm{mg} / \mathrm{l}$ & $<0.010 \mathrm{mg} / \mathrm{l}$ & $<0.010 \mathrm{mg} / \mathrm{h}$ & $<0.010 \mathrm{mg} / 1$ & 1 & \\
\hline 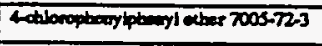 & $<0.010 \mathrm{mg} / \mathrm{l}$ & $<0.010 \mathrm{mg} / 1$ & $<0.010 \mathrm{mg} / \mathrm{l}$ & $<0.010 \mathrm{mg} / 1$ & 1 & \\
\hline 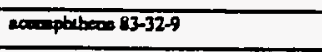 & $<0.010 \mathrm{mg} / \mathrm{l}$ & $<0.010 \mathrm{mg} / \mathrm{l}$ & $<0.010 \mathrm{mg} / \mathrm{l}$ & $<0.010 \mathrm{mg} / \mathrm{h}$ & 1 & \\
\hline 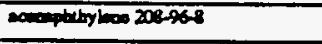 & $<0.010 \mathrm{mg} / 1$ & $<0.010 \mathrm{mg} / \mathrm{l}$ & $<0.010 \mathrm{mg} / \mathrm{l}$ & $<0.010 \mathrm{mg} / 1$ & 1 & \\
\hline $120-12.7$ & $<0.010 \mathrm{mg} / \mathrm{l}$ & $<0.010 \mathrm{mg} / \mathrm{h}$ & $<0.010 \mathrm{mg} / 1$ & $<0.010 \mathrm{mg} / \mathrm{l}$ & 1 & \\
\hline mation 9207.5 & $<0.050 \mathrm{mg} / \mathrm{l}$ & $<0.050 \mathrm{mg} / \mathrm{l}$ & $<0.050 \mathrm{mg} / 1$ & $<0.050 \mathrm{mg} / \mathrm{l}$ & 1 & \\
\hline 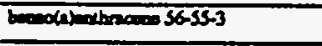 & $<0.010 \mathrm{mg} / 1$ & $<0.010 \mathrm{mg} / 1$ & $<0.010 \mathrm{mg} / 1$ & $<0.010 \mathrm{mg} / \mathrm{l}$ & 1 & \\
\hline 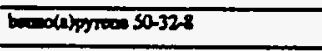 & $<0.010 \mathrm{mg} / \mathrm{l}$ & $<0.010 \mathrm{mg} / \mathrm{l}$ & $<0.010 \mathrm{mg} / \mathrm{l}$ & $<0.010 \mathrm{mg} / \mathrm{l}$ & 1 & \\
\hline 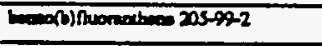 & $<0.010 \mathrm{mg} / \mathrm{l}$ & $<0.010 \mathrm{mg} / \mathrm{l}$ & $<0.010 \mathrm{mg} / \mathrm{l}$ & $<0.010 \mathrm{mg} / \mathrm{l}$ & 1 & \\
\hline 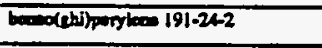 & $<0.010 \mathrm{mg} / \mathrm{l}$ & $<0.010 \mathrm{mg} / \mathrm{l}$ & $<0.010 \mathrm{mg} / 1$ & $<0.010 \mathrm{mg} / \mathrm{l}$ & 1 & \\
\hline 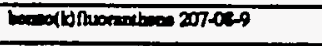 & $<0.010 \mathrm{mg} / \mathrm{I}$ & $<0.010 \mathrm{mg} / 1$ & $<0.010 \mathrm{mg} / \mathrm{l}$ & $<0.010 \mathrm{mg} / \mathrm{l}$ & 1 & \\
\hline 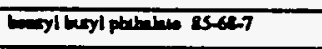 & $<0.010 u_{g} / l$ & $<0.010 \mathrm{mg} / \mathrm{h}$ & $<0.010 \mathrm{mg} / \mathrm{l}$ & $<0.010 \mathrm{mg} / \mathrm{l}$ & 1 & \\
\hline 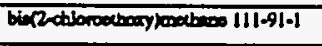 & $<0.010 \mathrm{mg} / \mathrm{l}$ & $<0.010 \mathrm{mg} / \mathrm{l}$ & $<0.010 \mathrm{mg} / \mathrm{l}$ & $<0.010 \mathrm{mg} / \mathrm{l}$ & 1 & . \\
\hline 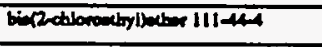 & $<0.010 \mathrm{mg} / 1$ & $<0.010 \mathrm{mg} / \mathrm{l}$ & $<0.010 \mathrm{mg} / \mathrm{l}$ & $<0.010 \mathrm{mg} / 1$ & 1 & \\
\hline 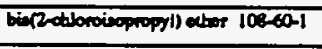 & $<0.010 \mathrm{mg} / 1$ & $<0.010 \mathrm{mg} / 1$ & $<0.010 \mathrm{mg} / \mathrm{l}$ & $<0.010 \mathrm{mg} / \mathrm{l}$ & 1 & \\
\hline 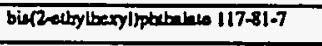 & $<0.010 \mathrm{mg} / \mathrm{l}$ & $<0.010 \mathrm{mg} / \mathrm{l}$ & $<0.010 \mathrm{mg} / \mathrm{l}$ & $<0.010 \mathrm{mg} / \mathrm{l}$ & 1 & \\
\hline ctryouses 218-01-9 & $<0.010 \mathrm{mg} / \mathrm{l}$ & $<0.010 \mathrm{mg} / \mathrm{l}$ & $<0.010 \mathrm{mg} / \mathrm{l}$ & $<0.010 \mathrm{mg} / \mathrm{l}$ & 1 & \\
\hline 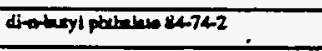 & $<0.010 \mathrm{mg} / \mathrm{l}$ & $<0.010 \mathrm{mg} / \mathrm{l}$ & $<0.010 \mathrm{mg} / 1$ & $<0.010 \mathrm{mg} / \mathrm{l}$ & 1 & \\
\hline 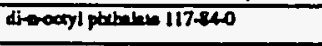 & $<0.010 \mathrm{mg} / \mathrm{l}$ & $<0.010 \mathrm{mg} / \mathrm{l}$ & $<0.010 \mathrm{mg} / \mathrm{l}$ & $<0.010 \mathrm{mg} / \mathrm{l}$ & 1 & \\
\hline 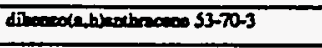 & $<0.010 \mathrm{mg} / \mathrm{l}$ & $<0.010 \mathrm{mg} / \mathrm{l}$ & $<0.010 \mathrm{mg} / \mathrm{l}$ & $<0.010 \mathrm{mg} / \mathrm{l}$ & 1 & \\
\hline 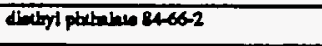 & $<0.010 \mathrm{mg} / 1$ & $<0.010 \mathrm{mg} / \mathrm{l}$ & $<0.010 \mathrm{mg} / \mathrm{l}$ & $<0.010 \mathrm{mg} / \mathrm{l}$ & 1 & \\
\hline 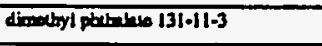 & $<0.010 \mathrm{mg} / \mathrm{l}$ & $<0.010 \mathrm{mg} / 1$ & $<0.010 \mathrm{mg} / \mathrm{l}$ & $<0.010 \mathrm{mg} / \mathrm{l}$ & $I$ & \\
\hline Avenonheres 20640 & $<0.010 \mathrm{mg} / \mathrm{l}$ & $<0.010 \mathrm{mg} / \mathrm{l}$ & $<0.010 \mathrm{mg} / \mathrm{l}$ & $<0.010 \mathrm{mg} / \mathrm{l}$ & 1 & \\
\hline Dhoveres 86-73-7 & $<0.010 \mathrm{mg} / \mathrm{l}$ & $<0.010 \mathrm{mg} / \mathrm{l}$ & $<0.010 \mathrm{mg} / \mathrm{h}$ & $<0.010 \mathrm{mg} / \mathrm{l}$ & 1 & \\
\hline 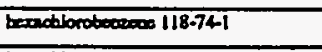 & $<0.010 \mathrm{mg} / \mathrm{l}$ & $<0.010 \mathrm{mg} / \mathrm{l}$ & $<0.010 \mathrm{mg} / 1$ & $<0.010 \mathrm{mg} / \mathrm{l}$ & 1 & \\
\hline 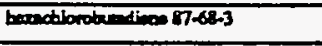 & $<0.010 \mathrm{mg} / \mathrm{l}$ & $<0.010 \mathrm{mg} / \mathrm{l}$ & $<0.010 \mathrm{mg} / \mathrm{l}$ & $<0.010 \mathrm{mg} / \mathrm{l}$ & 1 & \\
\hline 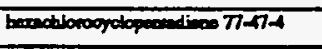 & $<0.010 \mathrm{mg} / 1$ & $<0.010 \mathrm{mg} / \mathrm{l}$ & $<0.010 \mathrm{mg} / \mathrm{l}$ & $<0.010 \mathrm{mg} / \mathrm{l}$ & 1 & \\
\hline 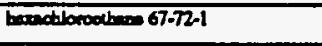 & $<0.010 \mathrm{mg} / \mathrm{l}$ & $<0.010 \mathrm{mg} / \mathrm{l}$ & $<0.010 \mathrm{mg} / \mathrm{l}$ & $<0.010 \mathrm{mg} / \mathrm{l}$ & 1 & \\
\hline 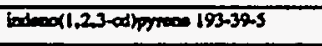 & $<0.010 \mathrm{mg} / \mathrm{l}$ & $<0.010 \mathrm{mg} / \mathrm{l}$ & $<0.010 \mathrm{mg} / \mathrm{l}$ & $<0.010 \mathrm{mg} / \mathrm{l}$ & 1 & \\
\hline noptopoos $72-59-1$ & $<0.010 \mathrm{mg} / \mathrm{l}$ & $<0.010 \mathrm{mg} / \mathrm{l}$ & $<0.010 \mathrm{mg} / \mathrm{l}$ & $<0.010 \mathrm{mg} / \mathrm{l}$ & 1 & \\
\hline 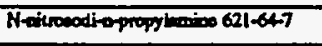 & $<0.010 \mathrm{mg} / \mathrm{l}$ & $<0.010 \mathrm{mg} / \mathrm{l}$ & $<0.010 \mathrm{mg} / \mathrm{l}$ & $<0.010 \mathrm{mg} / \mathrm{l}$ & 1 & \\
\hline 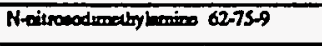 & $<0.010 \mathrm{mg} / \mathrm{t}$ & $<0.010 \mathrm{mg} / \mathrm{l}$ & $<0.010 \mathrm{mg} / 1$ & $<0.010 \mathrm{mg} / \mathrm{l}$ & 1 & \\
\hline 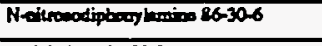 & $<0.010 \mathrm{mg} / 1$ & $<0.010 \mathrm{mg} / \mathrm{l}$ & $<0.010 \mathrm{mg} / 1$ & $<0.010 \mathrm{mg} / \mathrm{l}$ & 1 & \\
\hline 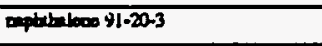 & $<0.010 \mathrm{mg} / \mathrm{t}$ & $<0.010 \mathrm{mg} / \mathrm{l}$ & $<0.010 \mathrm{mg} / \mathrm{l}$ & $<0.010 \mathrm{mg} / \mathrm{l}$ & $I$ & \\
\hline 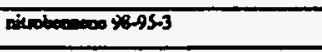 & $<0.010 \mathrm{mg} / \mathrm{l}$ & $<0.010 \mathrm{mg} / \mathrm{l}$ & $<0.010 \mathrm{mg} / 1$ & $<0.010 \mathrm{mg} / \mathrm{l}$ & 1 & \\
\hline 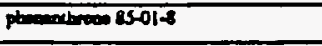 & $<0.010 \mathrm{mg} / \mathrm{t}$ & $<0.010 \mathrm{mg} / \mathrm{l}$ & $<0.010 \mathrm{mg} / 1$ & $<0.010 \mathrm{mg} / \mathrm{l}$ & 1 & \\
\hline prowos 122000 & $<0.010 \mathrm{mg} / 1$ & $<0.010 \mathrm{mg} / \mathrm{l}$ & $<0.010 \mathrm{mg} / \mathrm{l}$ & $<0.010 \mathrm{mg} / \mathrm{l}$ & 1 & \\
\hline 2,4,64rictlonopheod a2-06-2 & $<0.030 \mathrm{mg} / \mathrm{l}$ & $<0.030 \mathrm{mg} / \mathrm{l}$ & $<0.030 \mathrm{mg} / \mathrm{l}$ & $<0.030 \mathrm{mg} / \mathrm{l}$ & 1 & \\
\hline 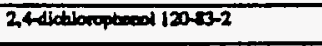 & $<0.030 \mathrm{mg} / \mathrm{l}$ & $<0.030 \mathrm{mg} / \mathrm{l}$ & $<0.030 \mathrm{mg} / \mathrm{l}$ & $<0.030 \mathrm{mg} / \mathrm{l}$ & 1 & \\
\hline 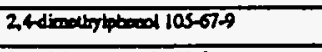 & $<0.030 \mathrm{mg} / \mathrm{l}$ & $<0.030 \mathrm{mg} / \mathrm{l}$ & $<0.030 \mathrm{mg} / \mathrm{l}$ & $<0.030 \mathrm{mg} / \mathrm{l}$ & 1 & \\
\hline 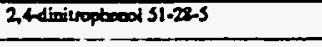 & $<0.050 \mathrm{mg} / \mathrm{l}$ & $<0.050 \mathrm{mg} / \mathrm{l}$ & $<0.050 \mathrm{mg} / \mathrm{l}$ & $<0.050 \mathrm{mg} / \mathrm{l}$ & 1 & \\
\hline
\end{tabular}


Outfall 64

, Parts B and C Continuod

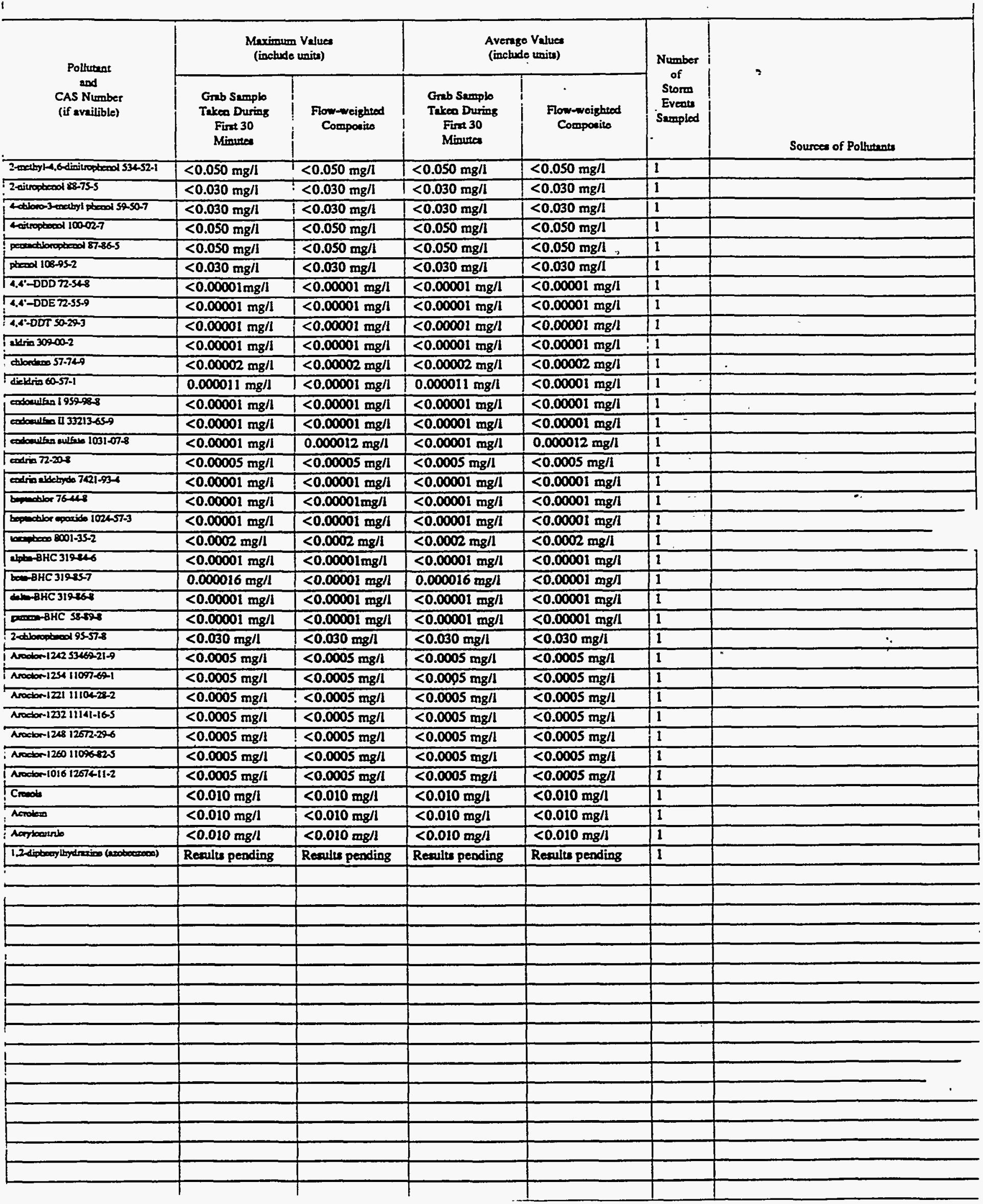


EPAID Number (copy from hrem 1 of Eomll

Form Approved OMB No. 2040-0086 Ointfall 67

Approval expires 5-31-92

VII. Dischargo laformation (continued from page 3 of Form 2A)

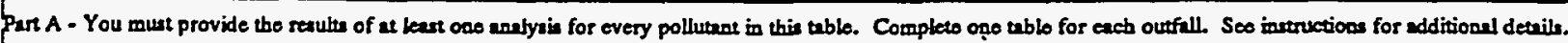

\begin{tabular}{|c|c|c|c|c|c|c|}
\hline \multirow{2}{*}{$\begin{array}{l}\text { Pollutant } \\
\text { end } \\
\text { CAs Number } \\
\text { (if availible) }\end{array}$} & \multicolumn{2}{|c|}{$\begin{array}{l}\text { Maximum Valuea } \\
\text { (include unitr) }\end{array}$} & \multicolumn{2}{|c|}{$\begin{array}{l}\text { Avernge Valuea } \\
\text { (iveludo units) }\end{array}$} & \multirow{2}{*}{$\begin{array}{l}\text { Number } \\
\text { of } \\
\text { Stomen } \\
\text { Events } \\
\text { Semplod }\end{array}$} & \multirow[b]{2}{*}{ Sources of Polhutante } \\
\hline & $\begin{array}{l}\text { Grab Sumple } \\
\text { Tuken During } \\
\text { Fint } 30 \\
\text { Minutes }\end{array}$ & $\begin{array}{l}\text { Flow-weighted } \\
\text { Componite }\end{array}$ & $\begin{array}{l}\text { Grab Sumple } \\
\text { Takeo During } \\
\text { Find } 30 \\
\text { Minutec }\end{array}$ & $\begin{array}{c}\text { Flow-weithted } \\
\text { Comporito }\end{array}$ & & \\
\hline 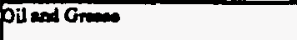 & $2 \mathrm{mg} / \mathrm{l}$ & & $2 \mathrm{mg} / \mathrm{l}$ & & 1 & \\
\hline 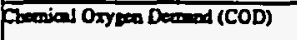 & $25 \mathrm{mg} / 1$ & $22 \mathrm{mg} / \mathrm{l}$ & $25 \mathrm{mg} / \mathrm{l}$ & $22 \mathrm{mg} / \mathrm{l}$ & 1 & \\
\hline Toul Sapposted Sollde (TSS) & $20 \mathrm{mg} / \mathrm{l}$ & $35 \mathrm{mg} / 1$ & $20 \mathrm{mg} / \mathrm{l}$ & $35 \mathrm{mg} / 1$ & 1 & \\
\hline Toul Kjelthel Nitroson & $0.8 \mathrm{mg} / 1$ & $0.3 \mathrm{mg} / \mathrm{I}$ & $0.8 \mathrm{mg} / \mathrm{l}$ & $0.3 \mathrm{mg} / \mathrm{l}$ & 1 & \\
\hline 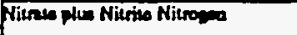 & $0.2 \mathrm{mg} / \mathrm{l}$ & $0.28 \mathrm{mg} / \mathrm{l}$ & $0.2 \mathrm{mg} / \mathrm{l}$ & $0.28 \mathrm{mg} / 1$ & 1 & \\
\hline Toul Prouphonse & $0.11 \mathrm{mg} / \mathrm{l}$ & $0.14 \mathrm{mg} / \mathrm{l}$ & $0.11 \mathrm{mg} / 1$ & $0.14 \mathrm{mg} / \mathrm{I}$ & 1 & \\
\hline
\end{tabular}

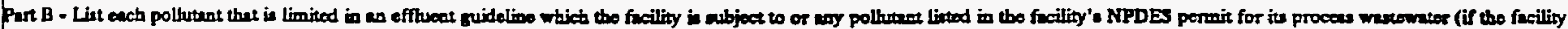

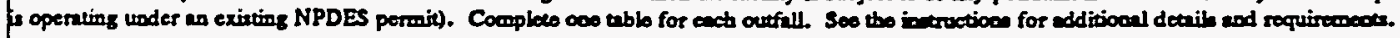

\begin{tabular}{|c|c|c|c|c|c|c|}
\hline \multirow{2}{*}{$\begin{array}{l}\text { Pollutane } \\
\text { and } \\
\text { CAs Number } \\
\text { (if availible) }\end{array}$} & \multicolumn{2}{|c|}{$\begin{array}{l}\text { Maximum Valuea } \\
\text { (Bochedo unies) }\end{array}$} & \multicolumn{2}{|c|}{$\begin{array}{l}\text { Average Valuese } \\
\text { (incledo unis) }\end{array}$} & \multirow{2}{*}{$\begin{array}{l}\text { Number } \\
\text { of } \\
\text { storm } \\
\text { Eveode } \\
\text { Semplod }\end{array}$} & \multirow[b]{2}{*}{ Sources of Polbutents } \\
\hline & 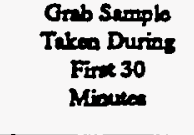 & $\begin{array}{l}\text { Flow-weizhted } \\
\text { Compotits }\end{array}$ & 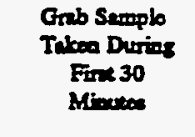 & $\begin{array}{l}\text { Flow-weightod } \\
\text { Couporite }\end{array}$ & & \\
\hline 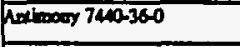 & $<0.04 \mathrm{mg} / \mathrm{l}$ & $<0.04 \mathrm{mg} / \mathrm{l}$ & $<0.04 \mathrm{mg} / \mathrm{l}$ & $<0.04 \mathrm{mg} / \mathrm{l}$ & 1 & \\
\hline $\operatorname{son}^{2} x^{2}$ & $<0.002 \mathrm{mg} / \mathrm{l}$ & & $<0.002 \mathrm{mg} / 1$ & & 1 & \\
\hline Menowy 7039976 & $<0.0002 \mathrm{mg} / \mathrm{l}$ & $<0.0002 \mathrm{mg} / \mathrm{l}$ & $<0.0002 \mathrm{mg} / \mathrm{l}$ & $<0.0002 \mathrm{mg} / 1$ & $T$ & $\because$ \\
\hline meools & $0.005 \mathrm{mg} / \mathrm{l}$ & & $0.005 \mathrm{mg} / \mathrm{l}$ & & 1 & \\
\hline 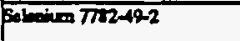 & $<0.002 \mathrm{mg} / \mathrm{l}$ & $<0.002 \mathrm{mg} / \mathrm{l}$ & $<0.002 \mathrm{mg} / \mathrm{l}$ & $<0.002 \mathrm{mg} / \mathrm{l}$ & 1 & \\
\hline sulenso & $<10 \mathrm{mg} / \mathrm{l}$ & $<10 \mathrm{mg} / \mathrm{l}$ & $<10 \mathrm{mg} / \mathrm{l}$ & $<10 \mathrm{mg} / \mathrm{l}$ & 1 & \\
\hline Purtecoens & $0.05 \mathrm{mg} / \mathrm{l}$ & $<0.05 \mathrm{mg} / \mathrm{l}$ & $0.05 \mathrm{mg} / \mathrm{l}$ & $<0.05 \mathrm{mg} / \mathrm{l}$ & 1 & \\
\hline Ahmingor $742990-5$ & $0.50 \mathrm{mg} / 1$ & $0.90 \mathrm{mg} / \mathrm{l}$ & $0.50 \mathrm{mg} / 1$ & $0.90 \mathrm{mg} / \mathrm{l}$ & 1 & \\
\hline 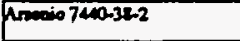 & $<0.04 \mathrm{mg} / \mathrm{I}$ & $<0.04 \mathrm{mg} / \mathrm{l}$ & $<0.04 \mathrm{mg} / 1$ & $<0.04 \mathrm{mg} / \mathrm{h}$ & 1 & \\
\hline $3000007400-128$ & $0.015 \mathrm{mg} / \mathrm{l}$ & $0.030 \mathrm{mg} / \mathrm{l}$ & $0.015 \mathrm{mg} / 1$ & $0.030 \mathrm{mg} / 1$ & 1 & \\
\hline Benplicus 740-11-7 & $<0.0004 \mathrm{mg} / 1$ & $<0.0004 \mathrm{mg} / \mathrm{l}$ & $<0.0004 \mathrm{mg} / \mathrm{l}$ & $<0.0004 \mathrm{mg} / \mathrm{l}$ & 1 & \\
\hline and $740-13-9$ & $<0.004 \mathrm{mg} / \mathrm{l}$ & $<0.004 \mathrm{mg} / \mathrm{l}$ & $<0.004 \mathrm{mg} / \mathrm{l}$ & $<0.004 \mathrm{mg} / \mathrm{l}$ & 1 & \\
\hline Caloivm 7400.702 & $17.4 \mathrm{mg} / 1$ & $18.7 \mathrm{mg} / \mathrm{h}$ & $17.4 \mathrm{mg} / 1$ & $18.7 \mathrm{mg} / \mathrm{l}$ & 1 & \\
\hline Coranim $740-10.3$ & $<0.006 \mathrm{mg} / \mathrm{l}$ & $<0.006 \mathrm{mg} / \mathrm{l}$ & $<0.006 \mathrm{mg} / \mathrm{l}$ & $<0.006 \mathrm{mg} / \mathrm{l}$ & 1 & \\
\hline Cotall 74040424 & $<0.002 \mathrm{mg} / \mathrm{l}$ & $<0.002 \mathrm{mg} / \mathrm{l}$ & $<0.002 \mathrm{mg} / \mathrm{l}$ & $<0.002 \mathrm{mg} / \mathrm{l}$ & 1 & \\
\hline capper 740.5as & $0.018 \mathrm{mg} / \mathrm{l}$ & $0.017 \mathrm{mg} / \mathrm{l}$ & $0.018 \mathrm{mg} / \mathrm{l}$ & $0.017 \mathrm{mg} / 1$ & 1 & \\
\hline 10007439896 & $0.64 \mathrm{mg} / \mathrm{l}$ & $1.16 \mathrm{mg} / \mathrm{l}$ & $0.64 \mathrm{mg} / 1$ & $1.16 \mathrm{mg} / \mathrm{l}$ & 1 & \\
\hline $\cos 7439-2.1$ & $<0.02 \mathrm{mg} / \mathrm{l}$ & $<0.02 \mathrm{mg} / 1$ & $<0.02 \mathrm{mg} / \mathrm{l}$ & $<0.02 \mathrm{mg} / 1$ & 1 & \\
\hline fusing 7439-93-2 & $<0.02 \mathrm{mg} / \mathrm{l}$ & $<0.02 \mathrm{mg} / 1$ & $<0.02 \mathrm{mg} / \mathrm{l}$ & $<0.02 \mathrm{mg} / 1$ & 1 & \\
\hline Megoundon 7439-95-4 & $2.26 \mathrm{mg} / \mathrm{I}$ & $2.72 \mathrm{mg} / \mathrm{l}$ & $2.26 \mathrm{mg} / 1$ & $2.72 \mathrm{mg} / 1$ & 1 & \\
\hline Moponem $72390 x-3$ & $0.041 \mathrm{mg} / 1$ & $0.065 \mathrm{mg} / \mathrm{l}$ & $0.041 \mathrm{mg} / \mathrm{l}$ & $0.065 \mathrm{mg} / \mathrm{h}$ & 1 & \\
\hline Motbdacom 743\%98-7 & $<0.006 \mathrm{mg} / \mathrm{l}$ & $<0.006 \mathrm{mg} / 1$ & $<0.006 \mathrm{mg} / \mathrm{l}$ & $<0.006 \mathrm{mg} / \mathrm{I}$ & 1 & \\
\hline Niolel 740020 & $<0.008 \mathrm{mg} / \mathrm{l}$ & $<0.008 \mathrm{mg} / 1$ & $<0.008 \mathrm{mg} / \mathrm{l}$ & $<0.008 \mathrm{mg} / \mathrm{l}$ & 1 & \\
\hline Promeniven 740009-7 & $1.4 \mathrm{mg} / 1$ & $1.6 \mathrm{mg} / \mathrm{l}$ & $1.4 \mathrm{mg} / \mathrm{l}$ & $1.6 \mathrm{mg} / \mathrm{l}$ & 1 & \\
\hline sither 7400-204 & $<0.006$ tng/l & $<0.006 \mathrm{mg} / \mathrm{l}$ & $<0.006 \mathrm{mg} / \mathrm{l}$ & $<0.006 \mathrm{mg} / 1$ & 1 & \\
\hline sodikes 740-20-5 & $1.11 \mathrm{mg} / 1$ & $1.45 \mathrm{mg} / \mathrm{l}$ & $1.11 \mathrm{mg} / 1$ & $1.45 \mathrm{mg} / 1$ & 1 & \\
\hline Tivascon 740-32.6 & $<0.01 \mathrm{mg} / 1$ & $0.01 \mathrm{mg} / 1$ & $<0.01 \mathrm{mg} / 1$ & $0.01 \mathrm{mg} / 1$ & 1 & \\
\hline 2000740.066 & $0.14 \mathrm{mg} / \mathrm{I}$ & $0.23 \mathrm{mg} / \mathrm{l}$ & $0.14 \mathrm{mg} / \mathrm{l}$ & $0.23 \mathrm{mg} / 1$ & 1 & \\
\hline
\end{tabular}


Cootioned from front.

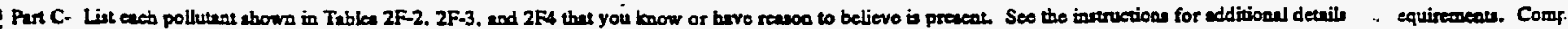
one tuble for each outfall.

\begin{tabular}{|c|c|c|c|c|c|c|}
\hline \multirow{2}{*}{$\begin{array}{l}\text { Poltutant } \\
\text { and } \\
\text { CAS Number } \\
\text { (if availible) }\end{array}$} & \multicolumn{2}{|c|}{$\begin{array}{l}\text { Maximum Values } \\
\text { (include univa) }\end{array}$} & \multicolumn{2}{|c|}{$\begin{array}{l}\text { Average Values } \\
\text { (inchude unita) }\end{array}$} & \multirow{2}{*}{$\begin{array}{l}\text { Nuraber } \\
\text { of } \\
\text { Storm } \\
\text { Eveat } \\
\text { Semplod }\end{array}$} & \\
\hline & $\begin{array}{c}\text { Greb Semple } \\
\text { Takea During } \\
\text { Fint } 30 \\
\text { Minives }\end{array}$ & $\begin{array}{l}\text { Fow-weighted } \\
\text { Composite }\end{array}$ & $\begin{array}{c}\text { Grab Sumplo } \\
\text { Taken During } \\
\text { Firnt } 30 \\
\text { Minence }\end{array}$ & $\begin{array}{l}\text { Flow-weighted } \\
\text { Componite }\end{array}$ & & srees of Pollutoness \\
\hline 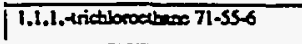 & $<0.010 \mathrm{mg} / \mathrm{l}$ & & $<0.010 \mathrm{mg} / 1$ & & 1 & \\
\hline 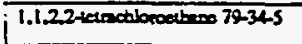 & $<0.010 \mathrm{mg} / \mathrm{l}$ & & $<0.010 \mathrm{mg} / \mathrm{l}$ & & 1 & \\
\hline 1.1.2- trictloroctiber $7900-5$ & $<0.010 \mathrm{mg} / \mathrm{l}$ & & $<0.010 \mathrm{mg} / \mathrm{l}$ & & 1 & \\
\hline 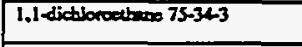 & $<0.010 \mathrm{mg} / \mathrm{l}$ & & $<0.010 \mathrm{mg} / \mathrm{l}$ & & 1 & \\
\hline 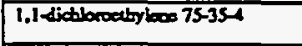 & $<0.010 \mathrm{mg} / \mathrm{l}$ & & $<0.010 \mathrm{mg} / \mathrm{l}$ & & 1 & \\
\hline 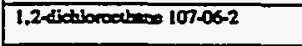 & $<0.010 \mathrm{mg} / \mathrm{l}$ & & $<0.010 \mathrm{mg} / \mathrm{l}$ & & 1 & \\
\hline 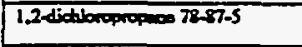 & $<0.010 \mathrm{mg} / 1$ & & $<0.010 \mathrm{mg} / \mathrm{l}$ & & 1 & \\
\hline 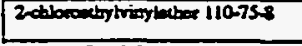 & $<0.010 \mathrm{mg} / \mathrm{l}$ & & $<0.010 \mathrm{mg} / \mathrm{l}$ & & 1 & \\
\hline $71+3-2$ & $<0.010 \mathrm{mg} / \mathrm{l}$ & & $<0.010 \mathrm{mg} / \mathrm{l}$ & & 1 & \\
\hline 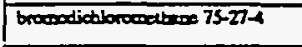 & $<0.010 \mathrm{mg} / \mathrm{l}$ & & $<0.010 \mathrm{mg} / 1$ & & 1 & \\
\hline broporm 7S-25-2 & $<0.010 \mathrm{mg} / \mathrm{l}$ & & $<0.010 \mathrm{mg} / \mathrm{l}$ & & 1 & \\
\hline $7489-9$ & $<0.010 \mathrm{mg} / \mathrm{l}$ & & $<0.010 \mathrm{mg} / \mathrm{l}$ & & 1 & \\
\hline arteos netracthride S6-22-5 & $<0.010 \mathrm{mg} / \mathrm{l}$ & & $<0.010 \mathrm{mg} / \mathrm{l}$ & & 1 & \\
\hline chloroberen $108-90.7$ & $<0.010 \mathrm{mg} / \mathrm{l}$ & & $<0.010 \mathrm{mg} / \mathrm{l}$ & & 1 & \\
\hline absontanos 75-00-3 & $<0.010 \mathrm{mg} / \mathrm{l}$ & & $<0.010 \mathrm{mg} / \mathrm{l}$ & & 1 & \\
\hline chlorobonem 67-66-3 & $<0.010 \mathrm{mg} / \mathrm{l}$ & & $<0.010 \mathrm{mg} / 1$ & & 1 & $\div$ \\
\hline chorocotateme $74.87-3$ & $<0.010 \mathrm{mg} / \mathrm{l}$ & & $<0.010 \mathrm{mg} / \mathrm{l}$ & & 1 & \\
\hline 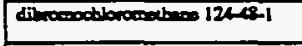 & $<0.010 \mathrm{mg} / 1$ & & $<0.010 \mathrm{mg} / 1$ & & 1 & \\
\hline 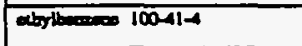 & $<0.010 \mathrm{mg} / \mathrm{l}$ & & $<0.010 \mathrm{mg} / \mathrm{l}$ & & 1 & \\
\hline 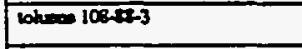 & $<0.010 \mathrm{mg} / \mathrm{l}$ & & $<0.010 \mathrm{mg} / \mathrm{l}$ & & 1 & \\
\hline 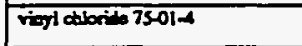 & $<0.010 \mathrm{mg} / \mathrm{l}$ & & $<0.010 \mathrm{mg} / \mathrm{l}$ & & 1 & \\
\hline 1.3-diationopropecos $10061-01-5$ & $<0.010 \mathrm{mg} / \mathrm{l}$ & & $<0.010 \mathrm{mg} / \mathrm{l}$ & . & 1 & \\
\hline Lno-1.2didtbrodbeos $156-60.5$ & $<0.010 \mathrm{mg} / 1$ & & $<0.010 \mathrm{mg} / 1$ & & $I$ & ; \\
\hline 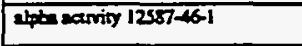 & $1.9 \mathrm{pci} / 1$ & $4.1 \mathrm{pci} / 1$ & $1.9 \mathrm{pci} / 1$ & $4.1 \mathrm{peill}$ & 1 & \\
\hline bou ecarvity $12587.46-1$ & 15 pcill & $6.5 \mathrm{pci} / \mathrm{l}$ & $15 \mathrm{pcin}$ & $6.5 \mathrm{pci} / \mathrm{l}$ & 1 & \\
\hline fool ooliform & $4,500 \mathrm{col} .1 \mathrm{~L}$ & $320 \mathrm{co} / .1 \mathrm{~L}$ & $4,500 \mathrm{col} .1 \mathrm{~L}$ & $320 \mathrm{col} .1 \mathrm{~L}$ & 1 & \\
\hline Thellivor 74a-2850 & $<0.005 \mathrm{mg} / \mathrm{l}$ & $<0.005 \mathrm{mg} / \mathrm{l}$ & $<0.005 \mathrm{mg} / \mathrm{l}$ & $<0.005 \mathrm{mg} / \mathrm{l}$ & 1 & \\
\hline Unanges 74061-1 & $0.001 \mathrm{mg} / 1$ & $0.001 \mathrm{mg} / \mathrm{l}$ & $0.001 \mathrm{mg} / \mathrm{I}$ & $0.001 \mathrm{mg} / \mathrm{l}$ & 1 & \\
\hline Cecrome exprity & $7.4 \mathrm{pei} / \mathrm{l}$ & $3.5 \mathrm{pei} / 1$ & 7.4 peill & $3.5 \mathrm{pci} / \mathrm{l}$ & 1 & \\
\hline
\end{tabular}

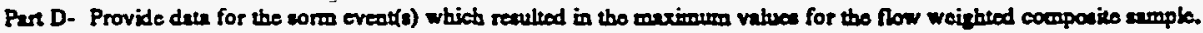

\begin{tabular}{|c|c|c|c|c|c|c|c|}
\hline $\begin{array}{l}1 . \\
\text { Deto of } \\
\text { Storm } \\
\text { Eveot }\end{array}$ & $\begin{array}{l}2 . \\
\text { Duntion } \\
\text { of Storm } \\
\text { (in } \\
\text { minutes) }\end{array}$ & $\begin{array}{c}3 . \\
\text { Total ninfall } \\
\text { during stosm eveat } \\
\text { (in incos) }\end{array}$ & 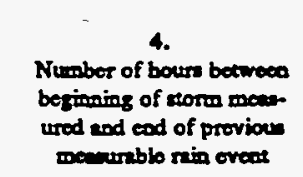 & 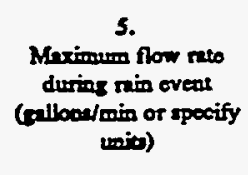 & $\begin{array}{l}6 . \\
\text { Toul flow from } \\
\text { nim event } \\
\text { (gallons or } \\
\text { spooify unita) }\end{array}$ & $\begin{array}{c}7 . \\
\text { Seeson } \\
\text { sample was } \\
\text { tuken }\end{array}$ & $\begin{array}{l}8 . \\
\text { Form of } \\
\text { Procipitation } \\
\text { (nimfull. } \\
\text { soommelt) }\end{array}$ \\
\hline $8 / 26 / 91$ & 410 & 1.2 & 1602 & 5,309 & 888,663 & Summer & Rainfall \\
\hline
\end{tabular}

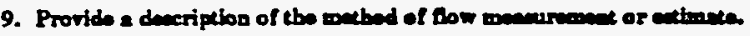

An ISCO 3230 Flow meter was med to obtnin water level readings. This instument was calibrated with the normal dry weather flow, if present, as leve zero. The fow meter was programmed with the characteristics of the convegance so that flow rates and total flows conld be calculated by the meter. 
Outfall 67

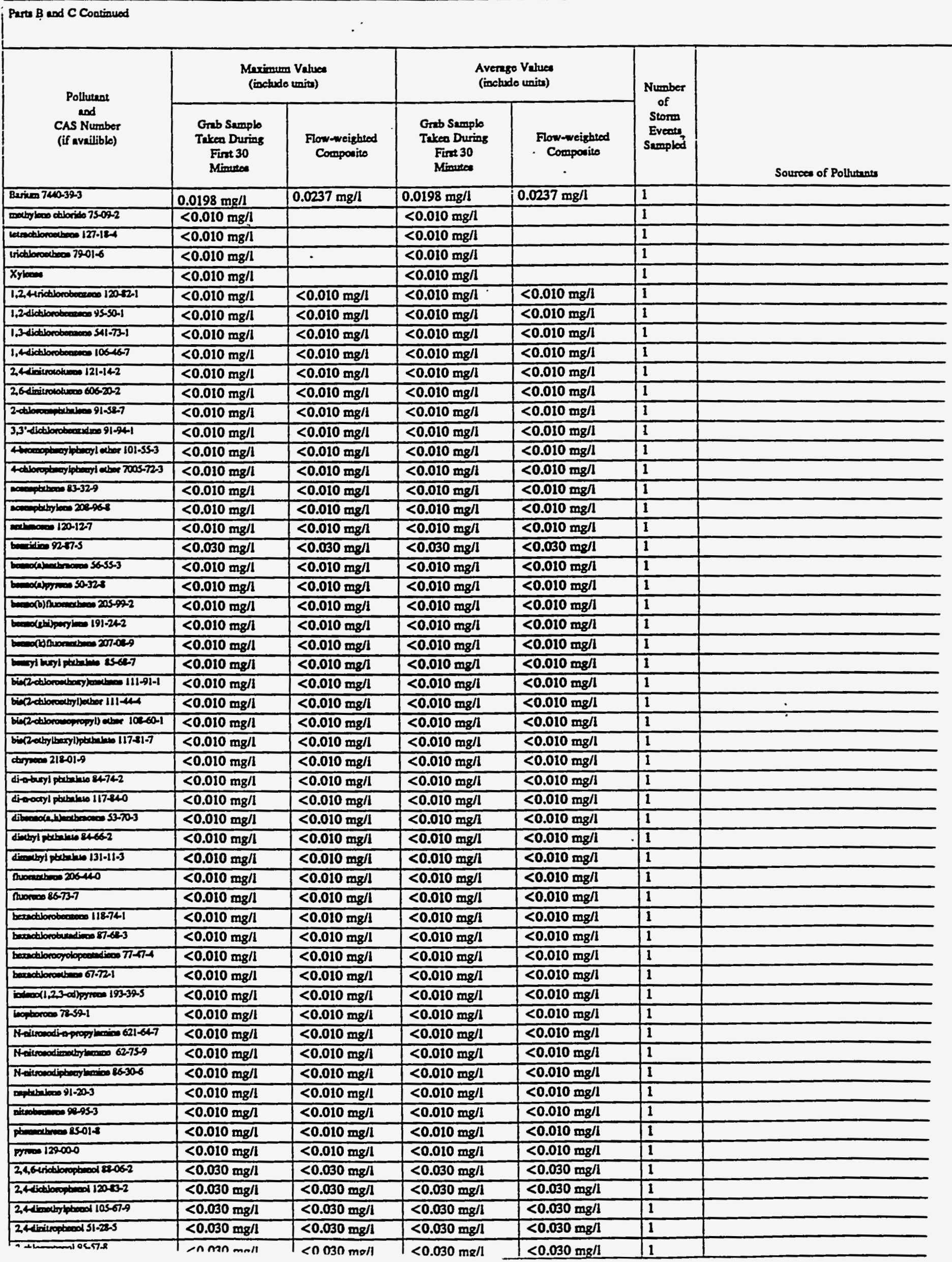


$\frac{\text { Outfall } 67}{\text { Para } B \text { and } C C}$

Para B and C Continued

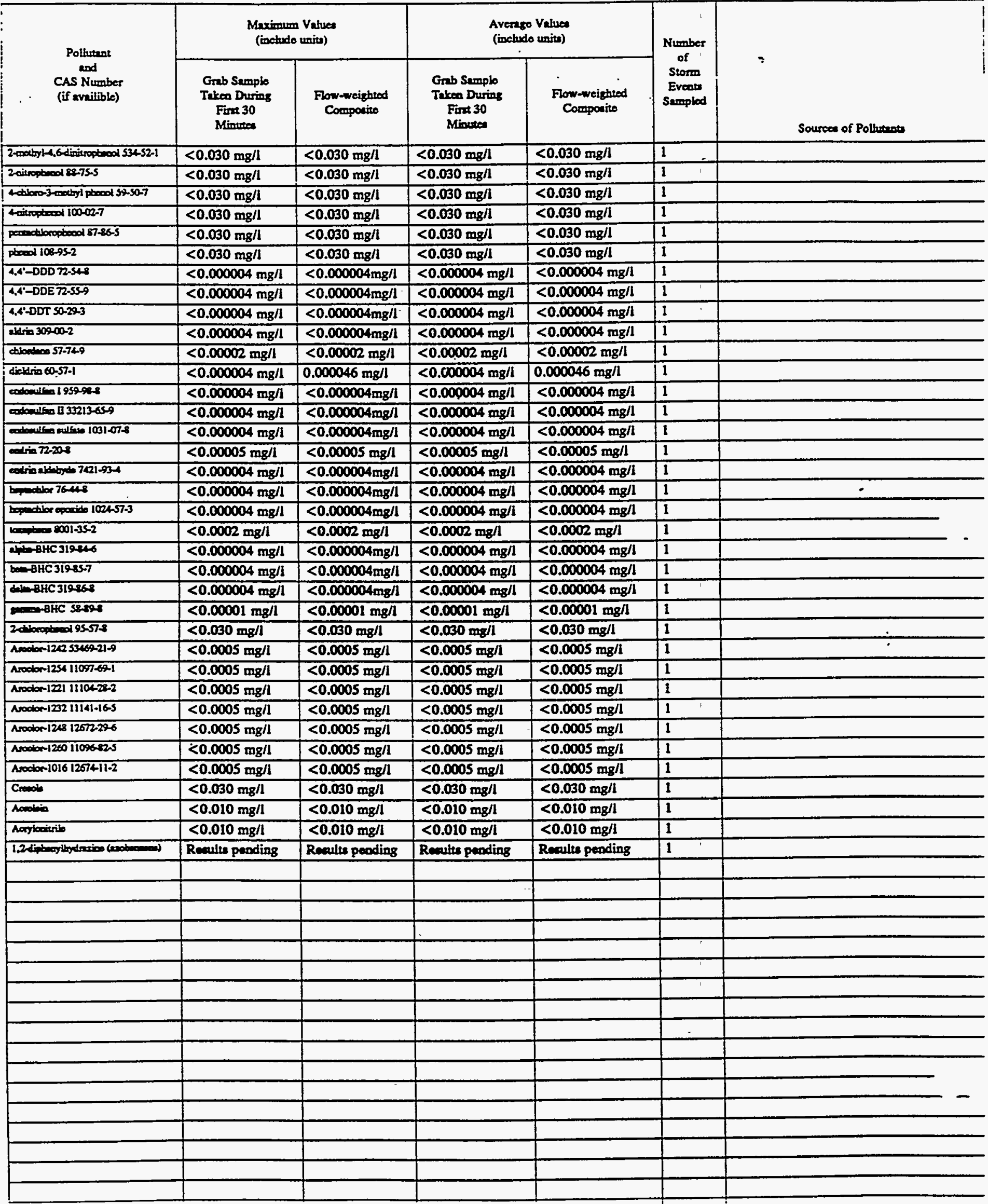


VI. Diecharge Informetion (contioned froon pege 3 of Form 2F)

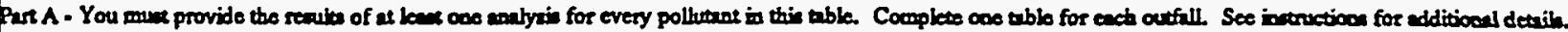

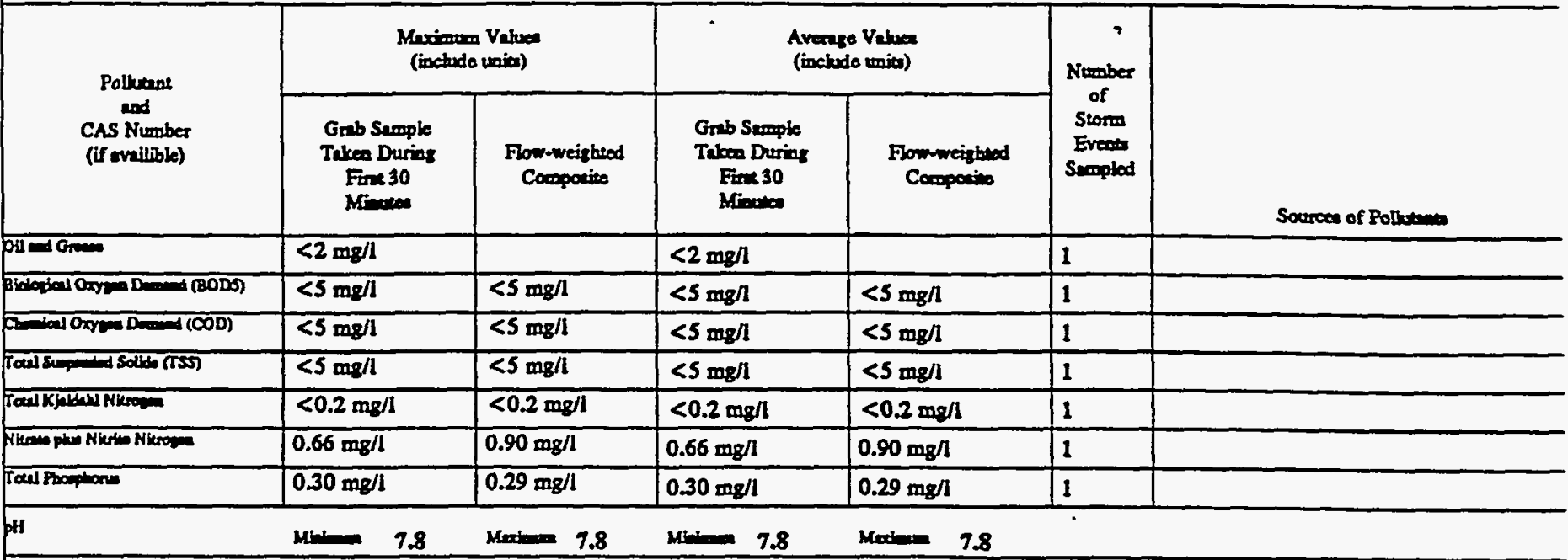

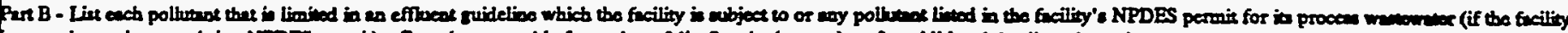

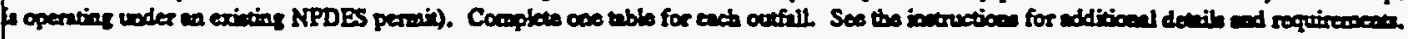

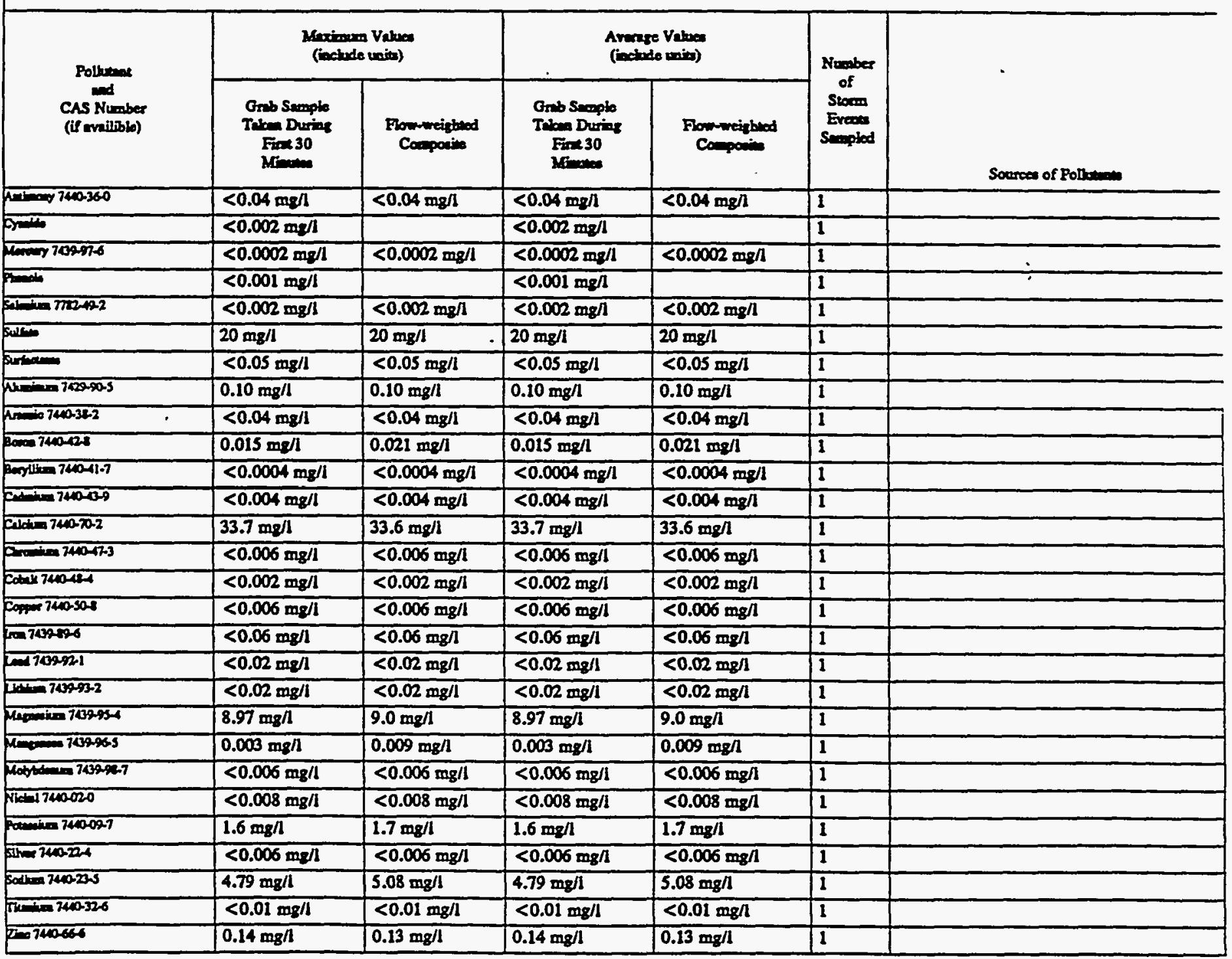


Continued from front.

; Par C- List each pollutant abown in Tables 2F-2, 2F-3, and 2F4 the you know or have reason to believe is preacot. See the instructions for additional details and requirementh. C one table for each outfall.

\begin{tabular}{|c|c|c|c|c|c|c|}
\hline \multirow{2}{*}{$\begin{array}{l}\text { Polbunt } \\
\text { and } \\
\text { CAS Number } \\
\text { (if arrilible) }\end{array}$} & \multicolumn{2}{|c|}{$\begin{array}{l}\text { Meximum Values } \\
\text { (inctude units) }\end{array}$} & \multicolumn{2}{|c|}{$\begin{array}{l}\text { Average Values } \\
\text { (inchudo units) }\end{array}$} & \multirow{2}{*}{$\begin{array}{l}\text { Number } \\
\text { of } \\
\text { Storm } \\
\text { Events } \\
\text { Sampled }\end{array}$} & \multirow[b]{2}{*}{ Sources of Pollutants } \\
\hline & $\begin{array}{l}\text { Grab Semplo } \\
\text { Taken During } \\
\text { Firnt } 30 \\
\text { Minutes }\end{array}$ & $\begin{array}{l}\text { Fow-weightod } \\
\text { Componito }\end{array}$ & $\begin{array}{l}\text { Grab Sample } \\
\text { Trken During } \\
\text { Finst } 30 \\
\text { Minutea }\end{array}$ & $\begin{array}{c}\text { Flow-weighted } \\
\text { Composite }\end{array}$ & & \\
\hline 1.1.1.trictbroctione $71-55-6$ & $<0.010 \mathrm{mg} / \mathrm{l}$ & & $<0.010 \mathrm{mg} / 1$ & & 1 & \\
\hline 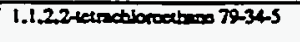 & $<0.010 \mathrm{mg} / \mathrm{l}$ & & $<0.010 \mathrm{mg} / \mathrm{l}$ & & 1 & \\
\hline 1.1.2- Lrictborodtares 79-00-5 & $<0.010 \mathrm{mg} / \mathrm{l}$ & & $<0.010 \mathrm{mg} / \mathrm{l}$ & & 1 & \\
\hline 1,1 dictionodien $75-34-3$ & $<0.010 \mathrm{mg} / \mathrm{l}$ & & $<0.010 \mathrm{mg} / \mathrm{l}$ & & 1 & \\
\hline I.1-dictionoubylooe 75-35-4 & $<0.010 \mathrm{mg} / 1$ & & $<0.010 \mathrm{mg} / 1$ & & 1 & \\
\hline 1.2 didibococtenes $107.06-2$ & $<0.010 \mathrm{mg} / 1$ & & $<0.010 \mathrm{mg} / 1$ & & 1 & \\
\hline 1.2didboroppoperes $78-87.5$ & $<0.010 \mathrm{mg} / 1$ & & $<0.010 \mathrm{mg} / 1$ & & 1 & \\
\hline 2 -ctionochytriaylecter $110-75-8$ & $<0.010 \mathrm{mg} / \mathrm{l}$ & & $<0.010 \mathrm{mg} / 1$ & & 1 & \\
\hline $71-13-2$ & $<0.010 \mathrm{mg} / \mathrm{l}$ & & $<0.010 \mathrm{mg} / 1$ & & 1 & \\
\hline broundictibrouthere $75-27-4$ & $<0.010 \mathrm{mg} / 1$ & & $<0.010 \mathrm{mg} / 1$ & & 1 & \\
\hline broconfoxm 75-25-2 & $<0.010 \mathrm{mg} / \mathrm{l}$ & & $<0.010 \mathrm{mg} / \mathrm{l}$ & & 1 & \\
\hline 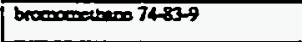 & $<0.010 \mathrm{mg} / \mathrm{l}$ & & $<0.010 \mathrm{mg} / \mathrm{l}$ & & 1 & \\
\hline cartion menctbride 56.22.5 & $<0.010 \mathrm{mg} / \mathrm{l}$ & & $<0.010 \mathrm{mg} / 1$ & & 1 & \\
\hline dibanabeneos 102907 & $<0.010 \mathrm{mg} / \mathrm{l}$ & & $<0.010 \mathrm{mg} / \mathrm{l}$ & & 1 & \\
\hline $75-0023$ & $<0.010 \mathrm{mg} / 1$ & & $<0.010 \mathrm{mg} / \mathrm{l}$ & & 1 & \\
\hline chboroborm $67-66-3$ & $0.012 \mathrm{mg} / \mathrm{l}$ & & $0.012 \mathrm{mg} / \mathrm{l}$ & & 1 & $\cdot$ \\
\hline 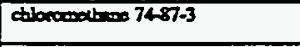 & $<0.010 \mathrm{mg} / 1$ & & $<0.010 \mathrm{mg} / \mathrm{l}$ & & 1 & \\
\hline 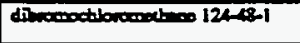 & $<0.010 \mathrm{mg} / 1$ & & $<0.010 \mathrm{mg} / \mathrm{l}$ & & 1 & \\
\hline 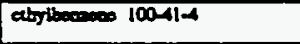 & $<0.010 \mathrm{mg} / \mathrm{l}$ & & $<0.010 \mathrm{mg} / 1$ & & 1 & \\
\hline lokmes 100882.3 & $<0.010 \mathrm{mg} / 1$ & & $<0.010 \mathrm{mg} / \mathrm{l}$ & & 1 & \\
\hline vingl ablondo 75014 & $<0.010 \mathrm{mg} / \mathrm{l}$ & & $<0.010 \mathrm{mg} / 1$ & & 1 & \\
\hline 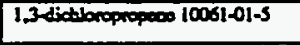 & $<0.010 \mathrm{mg} / \mathrm{l}$ & & $<0.010 \mathrm{mg} / 1$ & & 1 & \\
\hline 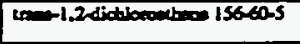 & $<0.010 \mathrm{mg} / 1$ & & $<0.010 \mathrm{mg} / \mathrm{l}$ & & 1 & $\cdot$ \\
\hline alpte exinty $12587-16-1$ & 12 pcill & $6.3 \mathrm{pci} / \mathrm{l}$ & $1.2 \mathrm{pci} / \mathrm{l}$ & $6.3 \mathrm{pei} / \mathrm{l}$ & 1 & \\
\hline $12537-16-1$ & $4.3 \mathrm{pci} / 1$ & 1.2 pcill & 4.3 pcill & $1.2 \mathrm{pci} / 1$ & 1 & \\
\hline fecol coliforo & $<1 \mathrm{co} / .1 \mathrm{~L}$ & $<1 \mathrm{col} .1 \mathrm{~L}$ & $<1 \mathrm{co} / . \mathrm{lL}$ & $<1 \mathrm{col} .1 \mathrm{~L}$ & 1 & \\
\hline Thalling 740-280 & $<0.03 \mathrm{mg} / \mathrm{l}$ & $<0.03 \mathrm{mg} / 1$ & $<0.03 \mathrm{mg} / 1$ & $<0.03 \mathrm{mg} / \mathrm{l}$ & 1 & \\
\hline Uraium 7406-61-1 & $<0.001 \mathrm{mg} / \mathrm{l}$ & $<0.001 \mathrm{mg} / \mathrm{l}$ & $<0.001 \mathrm{mg} / 1$ & $<0.001 \mathrm{mg} / 1$ & 1 & \\
\hline Ce...m savity & $87 \mathrm{pcill}$ & -11 pei/l & 87 pcill & -11 pcinl & 1 & \\
\hline
\end{tabular}

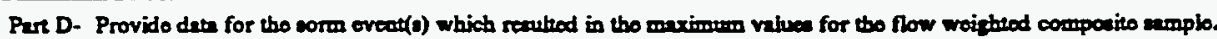

\begin{tabular}{|c|c|c|c|c|c|c|c|}
\hline $\begin{array}{l}1 . \\
\text { Dato of } \\
\text { Storm } \\
\text { Eveat }\end{array}$ & $\begin{array}{l}2 . \\
\text { Durition } \\
\text { of Storm } \\
\text { (in } \\
\text { minuten) }\end{array}$ & $\begin{array}{l}3 . \\
\text { Total ninfill } \\
\text { duries storm eveas } \\
\text { (in incbea) }\end{array}$ & $\begin{array}{l}\text { 4. } \\
\text { Number of hours between } \\
\text { beginning of utorm meas- } \\
\text { ured and ead of provious } \\
\text { meanrable nin oveat }\end{array}$ & $\begin{array}{l}\text { S. } \\
\text { Maximum flow nate } \\
\text { during nin event } \\
\text { (alloovimin or specify } \\
\text { unity) }\end{array}$ & $\begin{array}{c}6 . \\
\text { Toul flow from } \\
\text { nin event } \\
\text { (eallow or } \\
\text { specify units) }\end{array}$ & $\begin{array}{c}7 . \\
\text { Seanin } \\
\text { umple was } \\
\text { viten }\end{array}$ & $\begin{array}{l}\text { 8. } \\
\text { Form of } \\
\text { Procipitution } \\
\text { (rinfall. } \\
\text { enowmels) }\end{array}$ \\
\hline $5 / 8 / 92$ & 695 & 0.66 & $>134.3$ & 3.4 & 391 & Spring & Rainfall \\
\hline
\end{tabular}

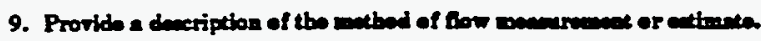

An ISCO 3230 Flow meter was nsed to obtain water level readings. This instrument was catihrated with the normal dry weather flow, if present, as levzero. The fow meter was programmed with the characteristics of the conveyance so that flow rates and total flows could be calcainted by the meter. 
Outfall 71

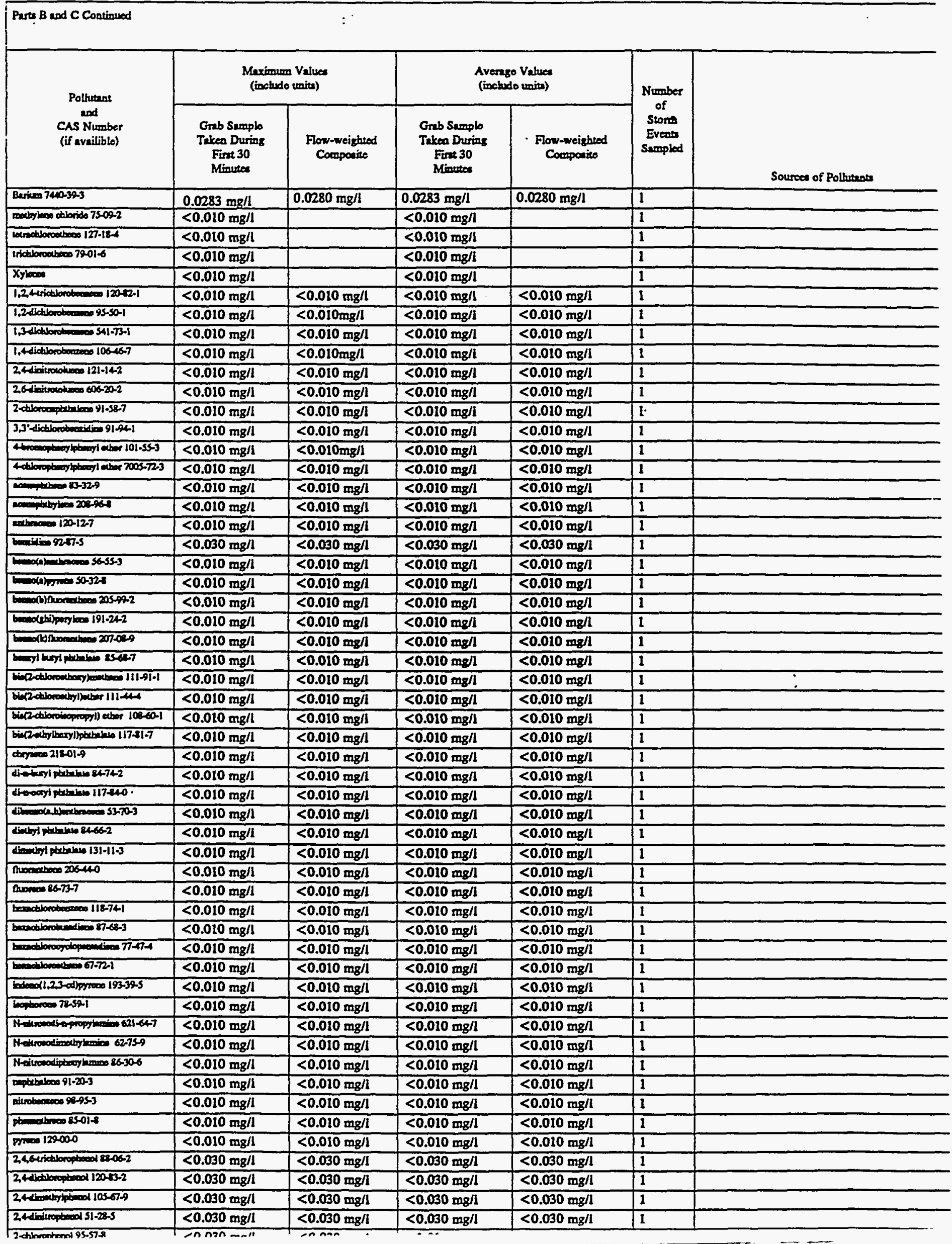


Outfall 71

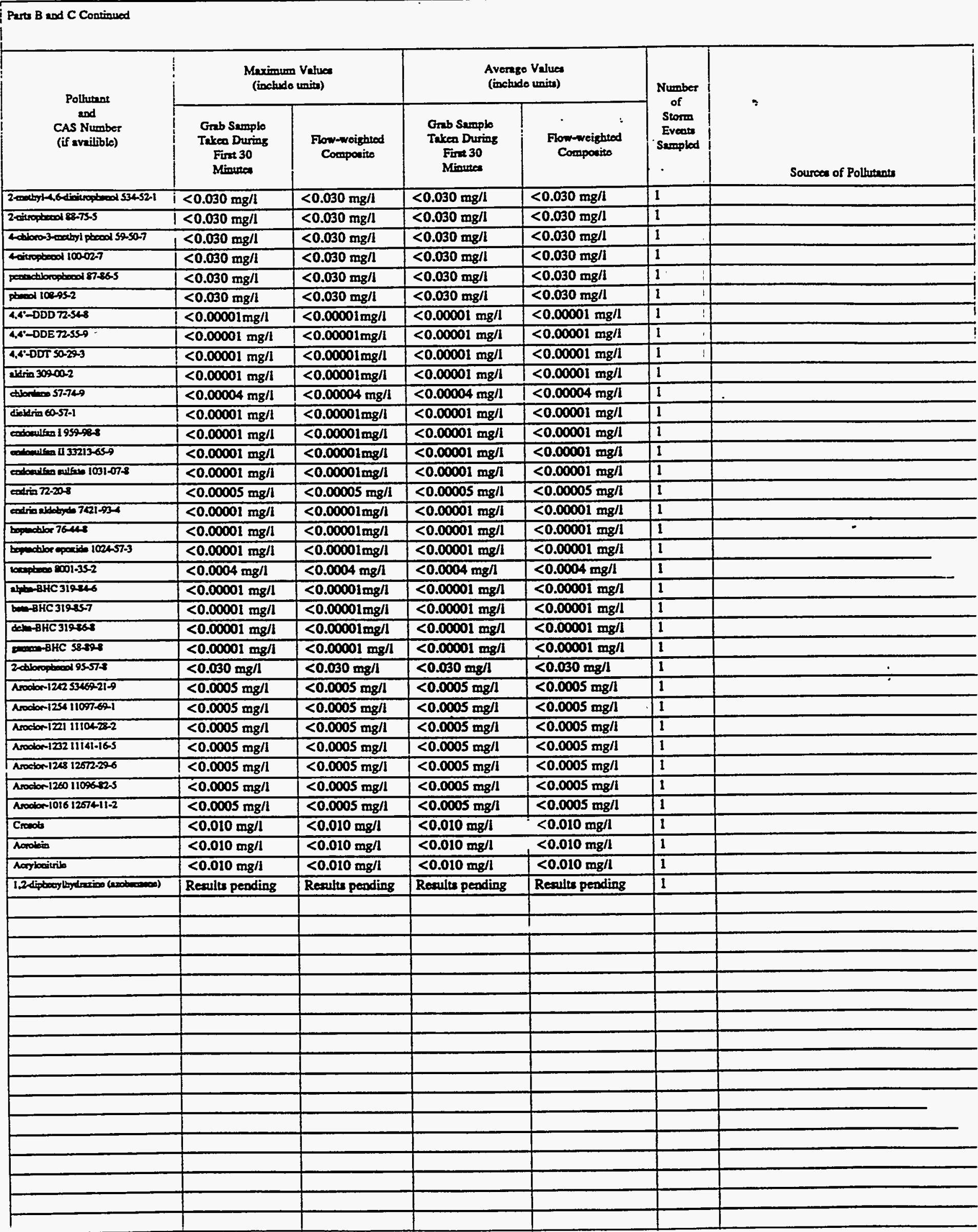


VI. Diecturge Informution (continued from page 3 of Form 2F)

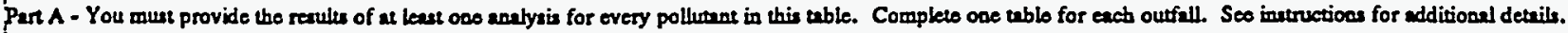

\begin{tabular}{|c|c|c|c|c|c|c|}
\hline \multirow{2}{*}{$\begin{array}{l}\text { Pollutune } \\
\text { and } \\
\text { CAS Number } \\
\text { (if nvailiblo) }\end{array}$} & \multicolumn{2}{|c|}{$\begin{array}{l}\text { Meximum Values } \\
\text { (includo unita) }\end{array}$} & \multicolumn{2}{|c|}{$\begin{array}{l}\text { Aversge Values } \\
\text { (includo units) }\end{array}$} & \multirow{2}{*}{$\begin{array}{l}\text { Number } \\
\text { of } \\
\text { Storm } \\
\text { Eveoti } \\
\text { Suroplod }\end{array}$} & \multirow[b]{2}{*}{ Sources of Pollumenses } \\
\hline & $\begin{array}{c}\text { Grab Sumplo } \\
\text { Takeo During } \\
\text { Firat } 30 \\
\text { Minutso }\end{array}$ & $\begin{array}{l}\text { Frow-weighted } \\
\text { Comporite }\end{array}$ & $\begin{array}{l}\text { Grab Semplo } \\
\text { Takeo During } \\
\text { Fint } 30 \\
\text { Mineweon }\end{array}$ & $\begin{array}{l}\text { Fow-woightod } \\
\text { Compouito }\end{array}$ & & \\
\hline pil and Grance & $2 \mathrm{mg} / \mathrm{l}$ & & $2 \mathrm{mg} / \mathrm{l}$ & & I & \\
\hline Bioberial Oxy & $8.96 \mathrm{mg} / \mathrm{l}$ & $<5 \mathrm{mg} / \mathrm{l}$ & $8.96 \mathrm{mg} / \mathrm{l}$ & $<5 \mathrm{mg} / \mathrm{l}$ & 1 & \\
\hline Chemical Oxypos Deraed (COD) & $53 \mathrm{mg} / \mathrm{l}$ & $26 \mathrm{mg} / \mathrm{I}$ & $53 \mathrm{mg} / 1$ & $26 \mathrm{mg} / \mathrm{l}$ & 1 & \\
\hline Toul Supocided Solide (TSS) & $50 \mathrm{mg} / \mathrm{l}$ & $35 \mathrm{mg} / \mathrm{l}$ & $50 \mathrm{mg} / 1$ & $35 \mathrm{mg} / \mathrm{l}$ & 1 & \\
\hline Toal Kjeldabl Nitroosen & $1.2 \mathrm{mg} / \mathrm{l}$ & $0.2 \mathrm{mg} / \mathrm{l}$ & $1.2 \mathrm{mg} / \mathrm{l}$ & $0.2 \mathrm{mg} / 1$ & 1 & \\
\hline Nirnes plue Nitrite Nitroes & $0.75 \mathrm{mg} / \mathrm{l}$ & $0.21 \mathrm{mg} / \mathrm{l}$ & $0.75 \mathrm{mg} / \mathrm{l}$ & $0.21 \mathrm{mg} / \mathrm{l}$ & 1 & \\
\hline Troeal Prouptorene & $0.22 \mathrm{mg} / \mathrm{l}$ & $<0.1 \mathrm{mg} / \mathrm{l}$ & $0.22 \mathrm{mg} / \mathrm{l}$ & $<0.1 \mathrm{mg} / 1$ & 1 & \\
\hline
\end{tabular}

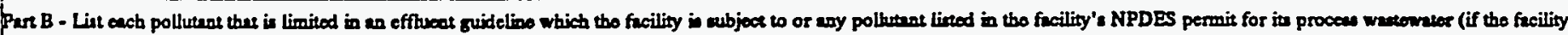

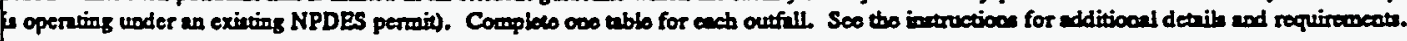

\begin{tabular}{|c|c|c|c|c|c|c|}
\hline \multirow{2}{*}{$\begin{array}{l}\text { Pollutant } \\
\text { and } \\
\text { CAs Number } \\
\text { (if avnilibio) }\end{array}$} & \multicolumn{2}{|c|}{$\begin{array}{l}\text { Meximum Valuce } \\
\text { (inctedo units) }\end{array}$} & \multicolumn{2}{|c|}{$\begin{array}{l}\text { Avernge Vahue } \\
\text { (includo unito) }\end{array}$} & \multirow{2}{*}{$\begin{array}{l}\text { Number } \\
\text { of } \\
\text { Seorm } \\
\text { Evert } \\
\text { Sempled }\end{array}$} & \multirow{2}{*}{ Sources of Pollumente } \\
\hline & $\begin{array}{l}\text { Grab Samplo } \\
\text { Taken During } \\
\text { Firx } 30 \\
\text { Mirexea }\end{array}$ & $\begin{array}{l}\text { Flom-wrighted } \\
\text { Comporine }\end{array}$ & $\begin{array}{l}\text { Greb Semplo } \\
\text { Triben During } \\
\text { Firs } 30 \\
\text { Minde }\end{array}$ & $\begin{array}{l}\text { Frow-weighted } \\
\text { Componito }\end{array}$ & & \\
\hline primeory $7400-360$ & $<0.003 \mathrm{mg} / \mathrm{l}$ & $<0.04 \mathrm{mg} / \mathrm{l}$ & $<0.003 \mathrm{mg} / \mathrm{l}$ & $<0.04 \mathrm{mg} / \mathrm{l}$ & 1 & \\
\hline $9 \sin x$ & $<0.002 \mathrm{mg} / \mathrm{l}$ & & $<0.002 \mathrm{mg} / \mathrm{l}$ & & 1 & \\
\hline Merary 7439976 & $0.0002 \mathrm{mg} / \mathrm{l}$ & $<0.0002 \mathrm{mg} / \mathrm{l}$ & $0.0002 \mathrm{mg} / \mathrm{l}$ & $<0.0002 \mathrm{mg} / \mathrm{l}$ & 1 & \\
\hline Phoods & $0.015 \mathrm{mg} / \mathrm{l}$ & & $0.015 \mathrm{mg} / \mathrm{l}$ & & 1 & $\vdots$ \\
\hline Selaino $7782-19-2$ & $<0.002 \mathrm{mg} / \mathrm{l}$ & $<0.002 \mathrm{mg} / \mathrm{l}$ & $<0.002 \mathrm{mg} / \mathrm{l}$ & $<0.002 \mathrm{mg} / \mathrm{l}$ & 1 & \\
\hline pelfere & $<10 \mathrm{mg} / \mathrm{l}$ & $<10 \mathrm{mg} / \mathrm{l}$ & $<10 \mathrm{mg} / \mathrm{l}$ & $<10 \mathrm{mg} / \mathrm{l}$ & 1 & \\
\hline Surteduanes & $0.09 \mathrm{mg} / \mathrm{l}$ & $0.19 \mathrm{mg} / \mathrm{l}$ & $0.09 \mathrm{mg} / \mathrm{l}$ & $0.19 \mathrm{mg} / \mathrm{l}$ & 1 & \\
\hline Mumanom 7429-90-5 & $1.38 \mathrm{mg} / \mathrm{l}$ & $0.51 \mathrm{mg} / \mathrm{l}$ & $1.38 \mathrm{mg} / \mathrm{h}$ & $0.51 \mathrm{mg} / 1$ & 1 & \\
\hline Arsocis 7440-38-2 & $<0.04 \mathrm{mg} / \mathrm{I}$ & $<0.04 \mathrm{mg} / \mathrm{h}$ & $<0.04 \mathrm{mg} / \mathrm{l}$ & $<0.04 \mathrm{mg} / \mathrm{l}$ & 1 & \\
\hline $800000740-124$ & $0.019 \mathrm{mg} / \mathrm{l}$ & $<0.006 \mathrm{mg} / \mathrm{l}$ & $0.019 \mathrm{mg} / \mathrm{l}$ & $<0.006 \mathrm{mg} / \mathrm{ll}$ & 1 & \\
\hline Boryllium 7410-41-7 & $<0.0004 \mathrm{mg} / \mathrm{l}$ & $<0.0004 \mathrm{mg} / \mathrm{l}$ & $<0.0004 \mathrm{mg} / \mathrm{l}$ & $<0.0004 \mathrm{mg} / \mathrm{l}$ & 1 & \\
\hline Cadmiven 7440-13-9 & $<0.004 \mathrm{mg} / \mathrm{l}$ & $<0.004 \mathrm{mg} / \mathrm{l}$ & $<0.004 \mathrm{mg} / 1$ & $<0.004 \mathrm{mg} / 1$ & 1 & \\
\hline Feloim 7460-70-2 & $18.4 \mathrm{mg} / \mathrm{l}$ & $13.2 \mathrm{mg} / 1$ & $18.4 \mathrm{mg} / 1$ & $13.2 \mathrm{mg} / \mathrm{l}$ & 1 & \\
\hline Chrouneros 7410-17.3 & $<0.006 \mathrm{mg} / \mathrm{l}$ & $<0.006 \mathrm{mg} / \mathrm{l}$ & $<0.006 \mathrm{mg} / \mathrm{l}$ & $<0.006 \mathrm{mg} / \mathrm{l}$ & 1 & \\
\hline Coball 7400-18-4 & $<0.002 \mathrm{mg} / \mathrm{l}$ & $<0.002 \mathrm{mg} / \mathrm{h}$ & $<0.002 \mathrm{mg} / \mathrm{l}$ & $<0.002 \mathrm{mg} / \mathrm{l}$ & 1 & \\
\hline Co,pon 740-508 & $0.094 \mathrm{mg} / \mathrm{l}$ & $0.025 \mathrm{mg} / \mathrm{l}$ & $0.094 \mathrm{mg} / 1$ & $0.025 \mathrm{mg} / \mathrm{l}$ & 1 & \\
\hline 50007039896 & $1.85 \mathrm{mg} / \mathrm{l}$ & $0.61 \mathrm{mg} / \mathrm{l}$ & $1.85 \mathrm{mg} / 1$ & $0.61 \mathrm{mg} / 1$ & 1 & \\
\hline 10ad 7439-21-1 & $0.02 \mathrm{mg} / \mathrm{l}$ & $<0.02 \mathrm{mg} / 1$ & $0.02 \mathrm{mg} / \mathrm{l}$ & $<0.02 \mathrm{mg} / \mathrm{l}$ & 1 & \\
\hline Lubiven 7099-93-2 & $<0.02 \mathrm{mg} / \mathrm{l}$ & $<0.02 \mathrm{mg} / \mathrm{l}$ & $<0.02 \mathrm{mg} / \mathrm{l}$ & $<0.02 \mathrm{mg} / \mathrm{l}$ & 1 & \\
\hline Mepowiken 7439-95-4 & $3.29 \mathrm{mg} / \mathrm{l}$ & $1.54 \mathrm{mg} / \mathrm{l}$ & $3.29 \mathrm{mg} / \mathrm{l}$ & $1.54 \mathrm{mg} / \mathrm{l}$ & 1 & \\
\hline$M=0000007439065$ & $0.062 \mathrm{mg} / \mathrm{l}$ & $0.018 \mathrm{mg} / \mathrm{h}$ & $0.062 \mathrm{mg} / \mathrm{l}$ & $0.018 \mathrm{mg} / \mathrm{l}$ & 1 & \\
\hline Mobfodoon 7439-98-7 & $<0.006 \mathrm{mg} / \mathrm{l}$ & $<0.006 \mathrm{mg} / \mathrm{l}$ & $<0.006 \mathrm{mg} / \mathrm{l}$ & $<0.006 \mathrm{mg} / \mathrm{l}$ & 1 & \\
\hline Niodal 17400020 & $<0.008 \mathrm{mg} / \mathrm{l}$ & $<0.008 \mathrm{mg} / \mathrm{l}$ & $<0.008 \mathrm{mg} / \mathrm{l}$ & $<0.008 \mathrm{mg} / \mathrm{l}$ & 1 & \\
\hline 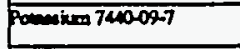 & $0.8 \mathrm{mg} / \mathrm{l}$ & $<0.5 \mathrm{mg} / \mathrm{l}$ & $0.8 \mathrm{mg} / \mathrm{l}$ & $<0.5 \mathrm{mg} / 1$ & 1 & \\
\hline sitwor 7400-204 & $<0.006 \mathrm{mg} / 1$ & $<0.006 \mathrm{mg} / \mathrm{l}$ & $<0.006 \mathrm{mg} / \mathrm{l}$ & $<0.006 \mathrm{mg} / \mathrm{l}$ & 1 & \\
\hline Sodiven 740-23-5 & $1.59 \mathrm{mg} / \mathrm{l}$ & $0.29 \mathrm{mg} / 1$ & $1.59 \mathrm{mg} / 1$ & $0.29 \mathrm{mg} / 1$ & 1 & \\
\hline Fitenium 7440-326 & $0.03 \mathrm{mg} / \mathrm{l}$ & $<0.01 \mathrm{mg} / \mathrm{l}$ & $0.03 \mathrm{mg} / 1$ & $<0.01 \mathrm{mg} / 1$ & 1 & \\
\hline $2700740066-6$ & $0.15 \mathrm{mg} / \mathrm{l}$ & $0.05 \mathrm{mg} / \mathrm{l}$ & $0.15 \mathrm{mg} / \mathrm{l}$ & $0.05 \mathrm{mg} / 1$ & 1 & \\
\hline
\end{tabular}




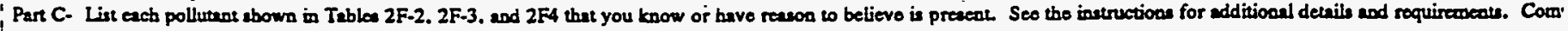
one tublo for each outrall.

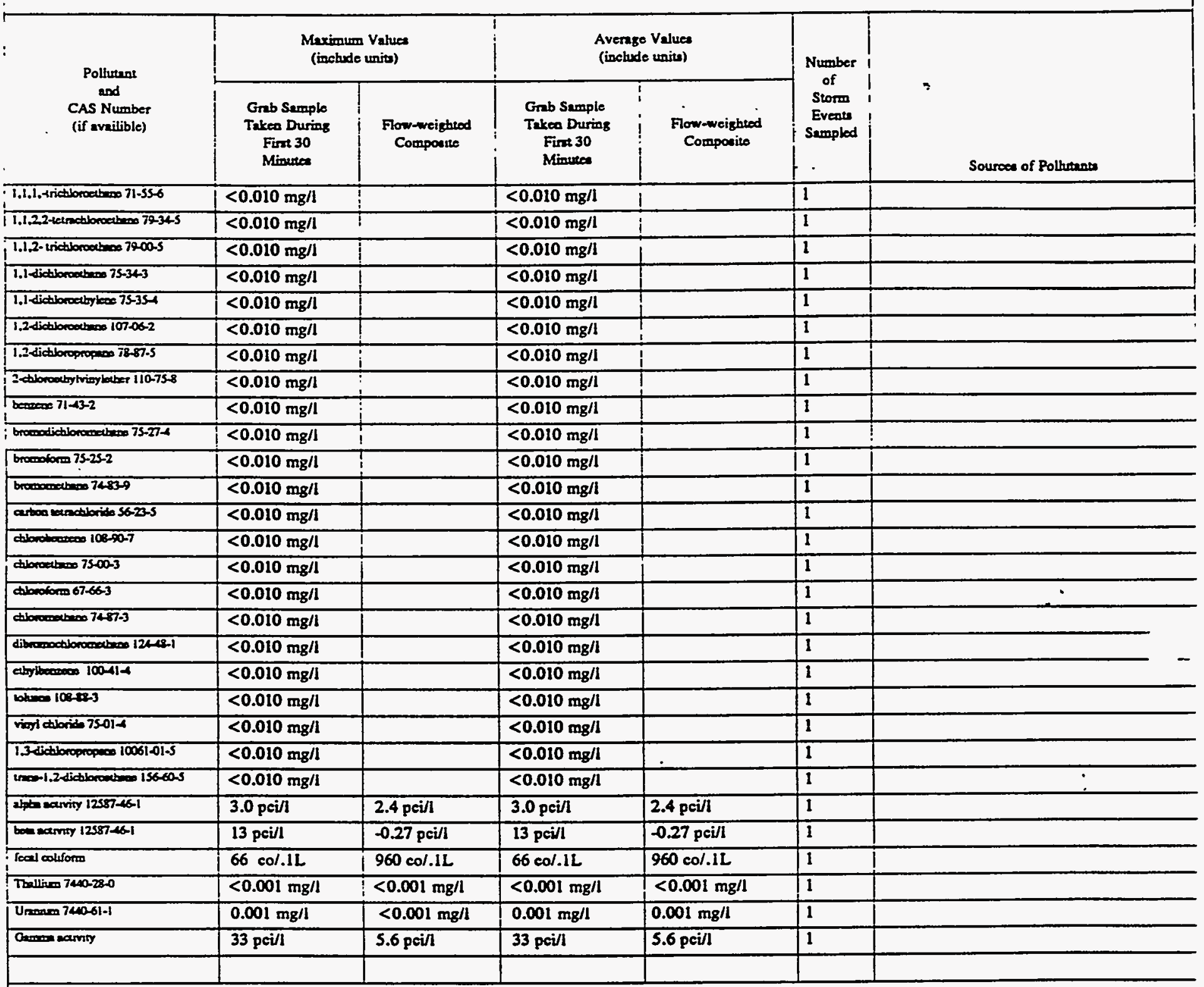

Par D. Provide des for the sorm event(y) which resulted in the maximim valuce for tho flow weightod composite ample.

\begin{tabular}{|c|c|c|c|c|c|c|c|}
\hline $\begin{array}{l}\text { I. } \\
\text { Date of } \\
\text { Storm } \\
\text { Eveat }\end{array}$ & $\begin{array}{l}2 . \\
\text { Duration } \\
\text { of Storm } \\
\text { (in } \\
\text { minutea) }\end{array}$ & $\begin{array}{l}3 . \\
\text { Total rinfill } \\
\text { during storm oveas } \\
\text { (in inches) }\end{array}$ & 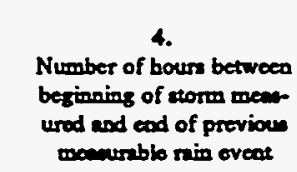 & $\begin{array}{l}\text { S. } \\
\text { Meximum flow rats } \\
\text { during min oveat } \\
\text { (alloos/min or specify } \\
\text { unitu) }\end{array}$ & $\begin{array}{c}6 . \\
\text { Total flow from } \\
\text { min event } \\
\text { (oalloow or } \\
\text { ppecify uniti) }\end{array}$ & $\begin{array}{c}7 . \\
\text { Seacon } \\
\text { eacople wes } \\
\text { tuken }\end{array}$ & $\begin{array}{l}\text { 8. } \\
\text { Form of } \\
\text { Procipitation } \\
\text { (niafill, } \\
\text { cownelt) }\end{array}$ \\
\hline $\begin{array}{l}2 / 13 / 92 \\
3 / 6 / 92\end{array}$ & $\begin{array}{l}65 \\
420\end{array}$ & $\begin{array}{l}0.27 \\
0.76\end{array}$ & $\begin{array}{l}>395 \\
138.9\end{array}$ & $\begin{array}{l}\text { NA } \\
5.6\end{array}$ & $\begin{array}{l}\text { NA } \\
779\end{array}$ & $\begin{array}{l}\text { Winter (grab) } \\
\text { Winter } \\
\text { (composite) }\end{array}$ & $\begin{array}{l}\text { Ruinfall } \\
\text { Rninfall }\end{array}$ \\
\hline
\end{tabular}

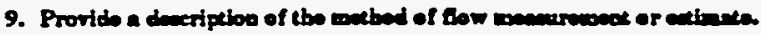

An ISCO 3230 Flow meter was used to obtain water letel readings. This instrument was calibrated with the normal dry weather flow, if present, as leved zero. The flow meter was programmed with the characteristics of the conveyance so that flow rates and total flows conid be calculated by the meter. 
Outiall 86

Parts B and C Costinued

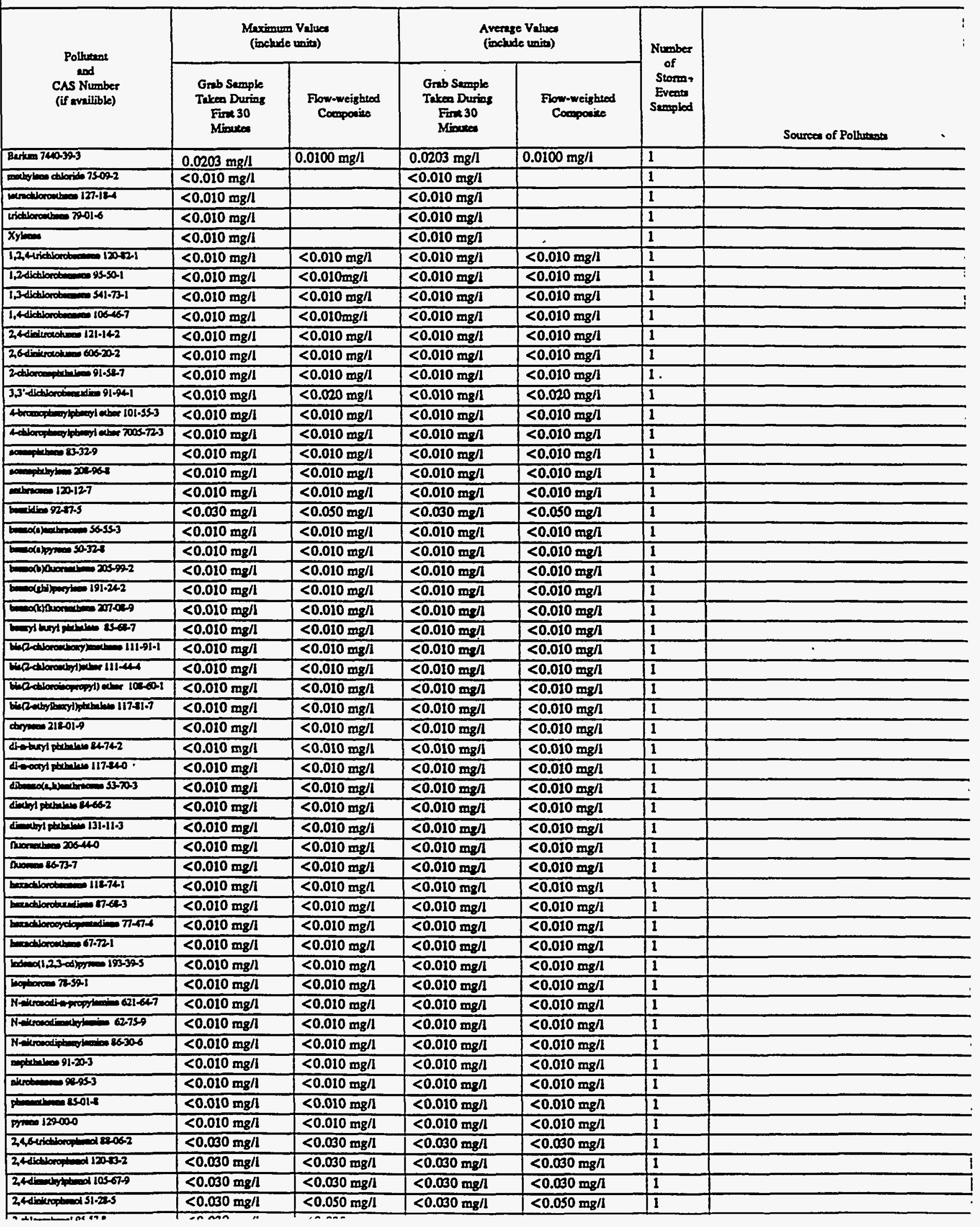


Outfoll 86

Pars B and C Contenued

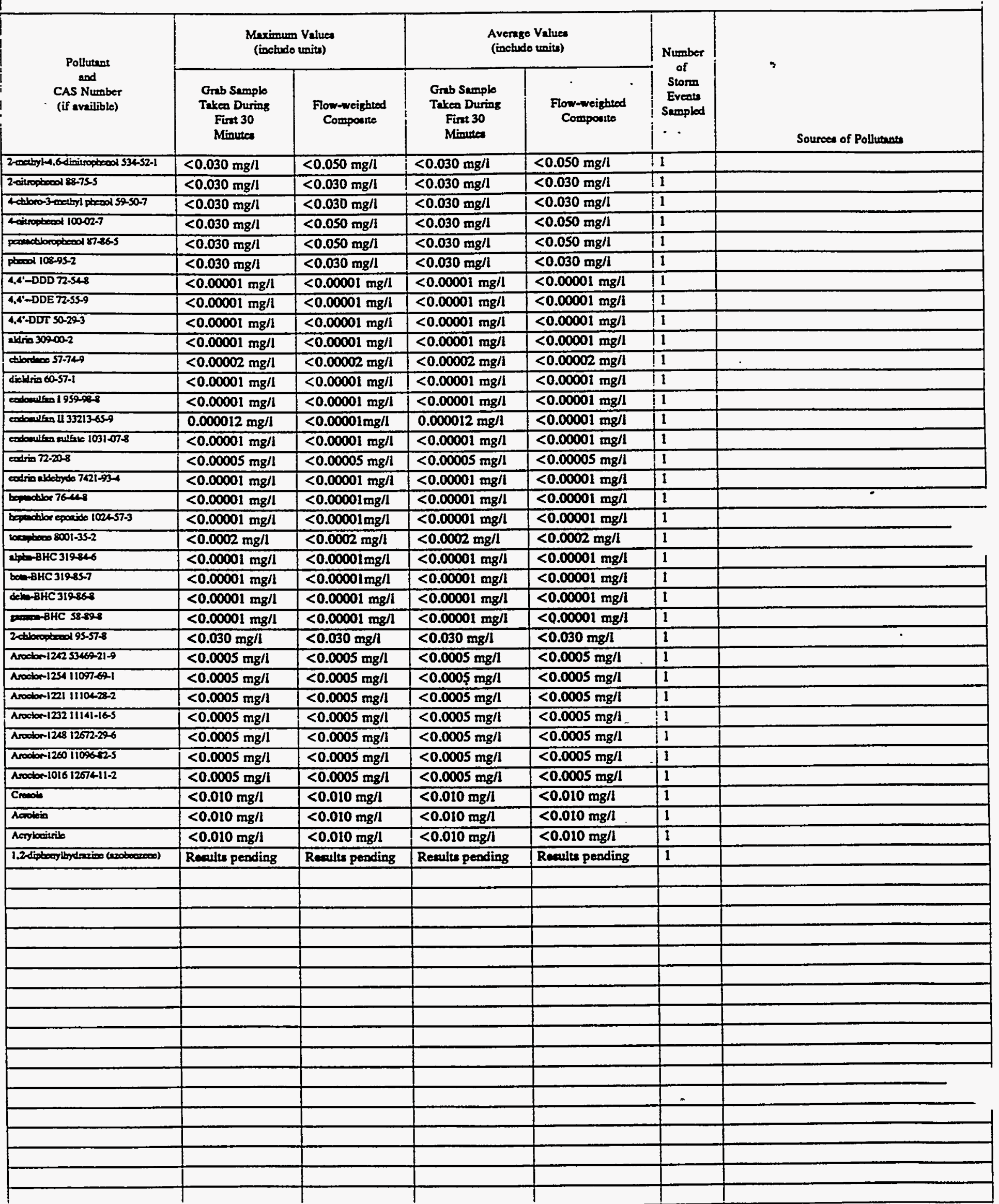


iva. Discharge Information (continued from page 3 of Form $2 \mathrm{~F}$ )

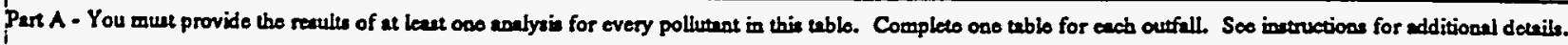

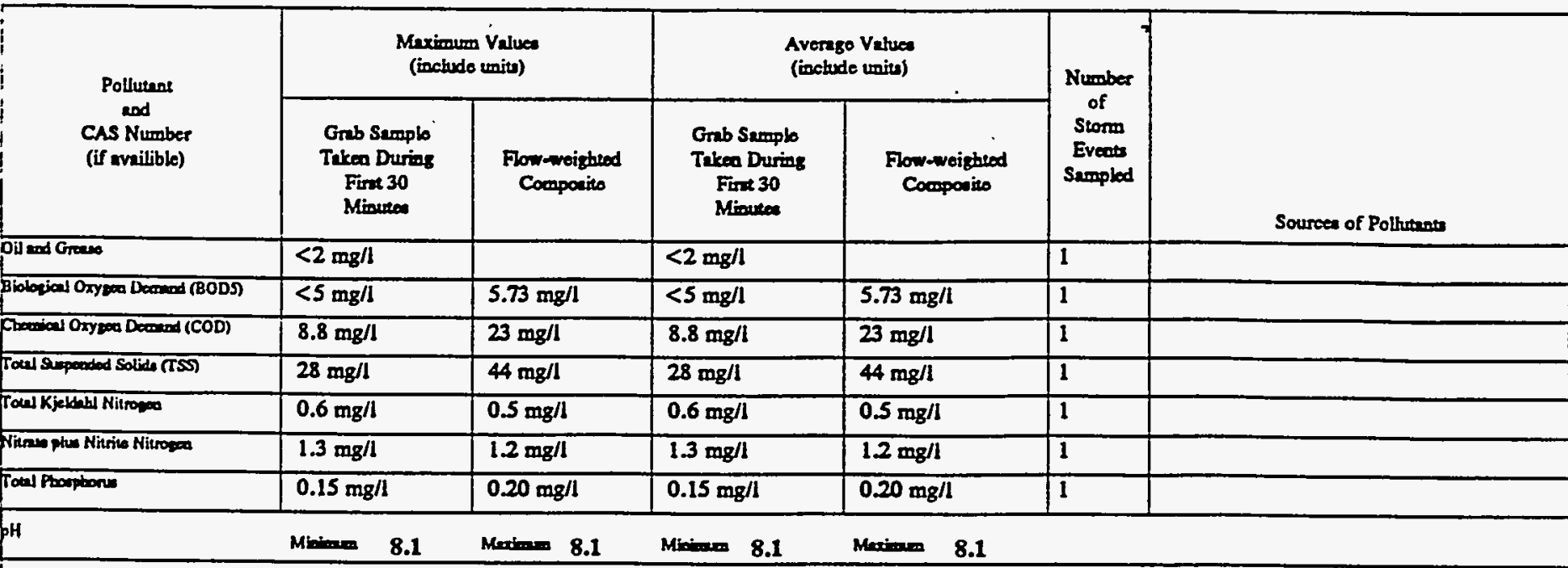

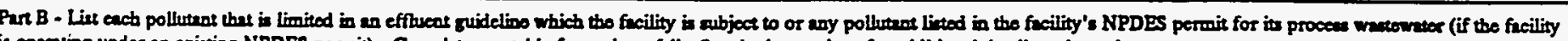

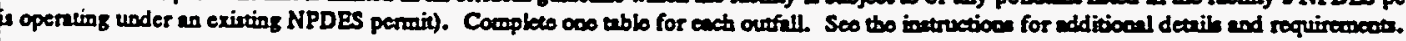

\begin{tabular}{|c|c|c|c|c|c|c|}
\hline \multirow{2}{*}{$\begin{array}{l}\text { Pollutant } \\
\text { end } \\
\text { CAs Number } \\
\text { (if availible) }\end{array}$} & \multicolumn{2}{|c|}{ 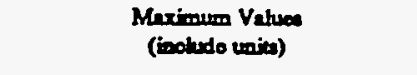 } & \multicolumn{2}{|c|}{$\begin{array}{l}\text { Average Vahue } \\
\text { (inchudo unio) }\end{array}$} & \multirow{2}{*}{$\begin{array}{l}\text { Number } \\
\text { of } \\
\text { storm } \\
\text { Eveoter } \\
\text { Semplad }\end{array}$} & \multirow{2}{*}{ Sources of Polberose } \\
\hline & 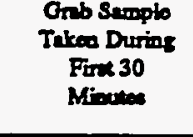 & $\begin{array}{l}\text { Fow-wrighted } \\
\text { Compocine }\end{array}$ & 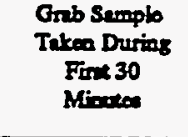 & $\begin{array}{l}\text { Flow-weighted } \\
\text { Compocites }\end{array}$ & & \\
\hline Artimasy 7400-36-0 & $<0.04 \mathrm{mg} / \mathrm{l}$ & $<0.04 \mathrm{mg} / \mathrm{l}$ & $<0.04 \mathrm{mg} / \mathrm{l}$ & $<0.04 \mathrm{mg} / 1$ & 1 & \\
\hline 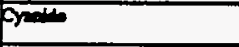 & $<0.002 \mathrm{mg} / \mathrm{l}$ & & $<0.002 \mathrm{mg} / \mathrm{l}$ & & 1 & \\
\hline Movoury 7439-97-6 & $<0.0002 \mathrm{mg} / \mathrm{l}$ & $<0.0002 \mathrm{mg} / \mathrm{l}$ & $<0.0002 \mathrm{mg} / 1$ & $<0.0002 \mathrm{mg} / 1$ & 1 & $\because$ \\
\hline Promele & $0.004 \mathrm{mg} / \mathrm{l}$ & & $0.004 \mathrm{mg} / \mathrm{l}$ & 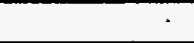 & 1 & \\
\hline setecongm $7782-12-2$ & $<0.002 \mathrm{mg} / \mathrm{l}$ & $<0.002 \mathrm{mg} / \mathrm{l}$ & $<0.002 \mathrm{mg} / \mathrm{l}$ & $<0.002 \mathrm{mg} / \mathrm{l}$ & 1 & \\
\hline Pultose & $26 \mathrm{mg} / \mathrm{l}$ & $19 \mathrm{mg} / \mathrm{l}$ & $26 \mathrm{mg} / 1$ & $19 \mathrm{mg} / \mathrm{l}$ & 1 & \\
\hline Purfocienes & $0.12 \mathrm{mg} / \mathrm{l}$ & $0.05 \mathrm{mg} / \mathrm{l}$ & $0.12 \mathrm{mg} / 1$ & $0.05 \mathrm{mg} / \mathrm{l}$ & 1 & \\
\hline Avenam 7429 ands & $1.35 \mathrm{mg} / 1$ & $1.83 \mathrm{mg} / 1$ & $1.35 \mathrm{mg} / \mathrm{l}$ & $1.83 \mathrm{mg} / 1$ & 1 & \\
\hline Armomion 74ta-38-2 & $<0.04 \mathrm{mg} / \mathrm{l}$ & $<0.04 \mathrm{mg} / \mathrm{l}$ & $<0.04 \mathrm{mg} / \mathrm{l}$ & $<0.04 \mathrm{mg} / \mathrm{l}$ & 1 & \\
\hline $300007400-128$ & $0.016 \mathrm{mg} / \mathrm{l}$ & $0.019 \mathrm{mg} / \mathrm{l}$ & $0.016 \mathrm{mg} / \mathrm{l}$ & $0.019 \mathrm{mg} / 1$ & 1 & \\
\hline 30 rilem 740-41-7 & $<0.0004 \mathrm{mg} / 1$ & $<0.0004 \mathrm{mg} / \mathrm{l}$ & $<0.0004 \mathrm{mg} / \mathrm{l}$ & $<0.0004 \mathrm{mg} / \mathrm{l}$ & 1 & \\
\hline $740-139$ & $<0.004 \mathrm{mg} / \mathrm{l}$ & $<0.004 \mathrm{mg} / \mathrm{l}$ & $<0.004 \mathrm{mg} / 1$ & $<0.004 \mathrm{mg} / \mathrm{l}$ & 1 & \\
\hline cenciom 74a-xo-2 & $30.9 \mathrm{mg} / \mathrm{l}$ & $32.5 \mathrm{mg} / 1$ & $30.9 \mathrm{mg} / \mathrm{l}$ & $32.5 \mathrm{mg} / 1$ & 1 & \\
\hline Chromanom $740-107.3$ & $<0.006 \mathrm{mg} / \mathrm{l}$ & $<0.006 \mathrm{mg} / \mathrm{l}$ & $<0.006 \mathrm{mg} / \mathrm{l}$ & $<0.006 \mathrm{mg} / \mathrm{l}$ & 1 & \\
\hline Cobale 740-18-4 & $<0.002 \mathrm{mg} / \mathrm{l}$ & $<0.002 \mathrm{mg} / 1$ & $<0.002 \mathrm{mg} / 1$ & $<0.002 \mathrm{mg} / \mathrm{l}$ & 1 & \\
\hline 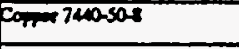 & $0.008 \mathrm{mg} / \mathrm{l}$ & $0.010 \mathrm{mg} / \mathrm{l}$ & $0.008 \mathrm{mg} / 1$ & $0.010 \mathrm{mg} / \mathrm{l}$ & 1 & \\
\hline 10007439096 & $1.33 \mathrm{mg} / \mathrm{l}$ & $1.71 \mathrm{mg} / 1$ & $1.33 \mathrm{mg} / \mathrm{l}$ & $1.71 \mathrm{mg} / 1$ & 1 & \\
\hline 7.m439201 & $<0.02 \mathrm{mg} / \mathrm{l}$ & $<0.02 \mathrm{mg} / \mathrm{h}$ & $<0.02 \mathrm{mg} / \mathrm{l}$ & $<0.02 \mathrm{mg} / 1$ & 1 & \\
\hline Hiblem 7439-93-2 & $<0.02 \mathrm{mg} / \mathrm{l}$ & $<0.02 \mathrm{mg} / 1$ & $<0.02 \mathrm{mg} / \mathrm{l}$ & $<0.02 \mathrm{mg} / \mathrm{l}$ & 1 & \\
\hline Meanixen 7439-95-4 & $3.36 \mathrm{mg} / \mathrm{l}$ & $4.14 \mathrm{mg} / 1$ & $3.36 \mathrm{mg} / \mathrm{l}$ & $4.14 \mathrm{mg} / \mathrm{l}$ & 1 & \\
\hline M. $7439060-5$ & $0.039 \mathrm{mg} / \mathrm{l}$ & $0.044 \mathrm{mg} / \mathrm{l}$ & $0.039 \mathrm{mg} / \mathrm{l}$ & $0.044 \mathrm{mg} / \mathrm{l}$ & 1 & \\
\hline Motydoun 7439-96-7 & $<0.006 \mathrm{mg} / \mathrm{l}$ & $<0.006 \mathrm{mg} / \mathrm{l}$ & $<0.006 \mathrm{mg} / \mathrm{l}$ & $<0.006 \mathrm{mg} / \mathrm{l}$ & 1 & \\
\hline Nitotal 7400200 & $<0.008 \mathrm{mg} / \mathrm{l}$ & $<0.008 \mathrm{mg} / \mathrm{l}$ & $<0.008 \mathrm{mg} / \mathrm{l}$ & $<0.008 \mathrm{mg} / \mathrm{l}$ & 1 & \\
\hline Pom 7400997 & $0.9 \mathrm{mg} / \mathrm{l}$ & $12 \mathrm{mg} / \mathrm{h}$ & $0.9 \mathrm{mg} / \mathrm{l}$ & $1.2 \mathrm{mg} / 1$ & 1 & \\
\hline Fither 74a0-2at & $<0.006 \mathrm{mg} / \mathrm{l}$ & $<0.006 \mathrm{mg} / \mathrm{l}$ & $<0.006 \mathrm{mg} / \mathrm{l}$ & $<0.006 \mathrm{mg} / \mathrm{l}$ & 1 & \\
\hline sosing 7400-20-5 & $1.96 \mathrm{mg} / \mathrm{l}$ & $2.11 \mathrm{mg} / 1$ & $1.96 \mathrm{mg} / \mathrm{l}$ & $2.11 \mathrm{mg} / 1$ & 1 & \\
\hline Titand $740-32.6$ & $0.02 \mathrm{mg} / \mathrm{l}$ & $0.02 \mathrm{mg} / \mathrm{I}$ & $0.02 \mathrm{mg} / 1$ & $0.02 \mathrm{mg} / 1$ & 1 & \\
\hline iino 740-66-6 & $0.03 \mathrm{mg} / \mathrm{l}$ & $0.04 \mathrm{mg} / \mathrm{l}$ & $0.03 \mathrm{mg} / 1$ & $0.04 \mathrm{mg} / \mathrm{l}$ & 1 & \\
\hline
\end{tabular}




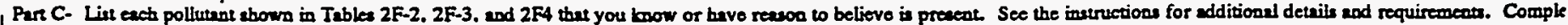
one table for each outfall.

\begin{tabular}{|c|c|c|c|c|c|c|}
\hline \multirow{2}{*}{$\begin{array}{l}\text { Pollutant } \\
\text { and } \\
\text { CAS Number } \\
\text { (if avnilible) }\end{array}$} & \multicolumn{2}{|c|}{$\begin{array}{l}\text { Maxionum Valuet } \\
\text { (inciude unisa) }\end{array}$} & \multicolumn{2}{|c|}{$\begin{array}{l}\text { Avernge Valueas } \\
\text { (includo unrus) }\end{array}$} & \multirow{2}{*}{$\begin{array}{c}\text { Number } \\
\text { of } \\
\text { Storm } \\
\text { Everass } \\
\text { Sumpled }\end{array}$} & \multirow[b]{2}{*}{ Sourese ss Polturabet } \\
\hline & $\begin{array}{l}\text { Grab Sumple } \\
\text { Taken Durizs } \\
\text { Fint } \\
\text { Minuce }\end{array}$ & $\begin{array}{l}\text { Flow-wreighted } \\
\text { Componito }\end{array}$ & $\begin{array}{l}\text { Grab Sxmplo } \\
\text { Taken During } \\
\text { Firnt } 30 \\
\text { Minutea }\end{array}$ & $\begin{array}{l}\text { Flow-weighted } \\
\text { Componite }\end{array}$ & & \\
\hline 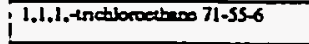 & $<0.010 \mathrm{mg} / \mathrm{l}$ & & $<0.010 \mathrm{mg} / 1$ & & 1 & \\
\hline 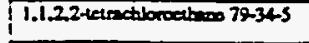 & $<0.010 \mathrm{mg} / 1$ & & $<0.010 \mathrm{mg} / \mathrm{l}$ & & 1 & \\
\hline 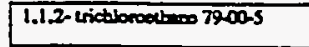 & $<0.010 \mathrm{mg} / \mathrm{l}$ & & $<0.010 \mathrm{mg} / \mathrm{l}$ & & 1 & \\
\hline 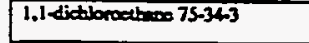 & $<0.010 \mathrm{mg} / \mathrm{l}$ & & $<0.010 \mathrm{mg} / \mathrm{l}$ & & 1 & \\
\hline 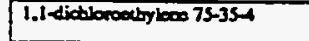 & $<0.010 \mathrm{mg} / 1$ & & $<0.010 \mathrm{mg} / 1$ & & 1 & \\
\hline 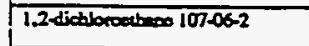 & $<0.010 \mathrm{mg} / \mathrm{l}$ & & $<0.010 \mathrm{mg} / 1$ & & 1 & \\
\hline 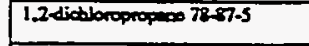 & $<0.010 \mathrm{mg} / \mathrm{l}$ & & $<0.010 \mathrm{mg} / \mathrm{l}$ & & 1 & \\
\hline 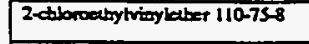 & $<0.010 \mathrm{mg} / \mathrm{l}$ & & $<0.010 \mathrm{mg} / \mathrm{l}$ & & 1 & \\
\hline bosecose $71-13.2$ & $<0.010 \mathrm{mg} / \mathrm{l}$ & & $<0.010 \mathrm{mg} / \mathrm{l}$ & & 1 & \\
\hline 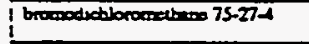 & $<0.010 \mathrm{mg} / \mathrm{l}$ & & $<0.010 \mathrm{mg} / \mathrm{l}$ & & 1 & \\
\hline broconoform 75-25-2 & $<0.010 \mathrm{mg} / \mathrm{l}$ & & $<0.010 \mathrm{mg} / \mathrm{l}$ & & 1 & \\
\hline bromonaten $7483-9$ & $<0.010 \mathrm{mg} / \mathrm{l}$ & & $<0.010 \mathrm{mg} / \mathrm{l}$ & & 1 & \\
\hline 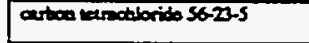 & $<0.010 \mathrm{mg} / 1$ & & $<0.010 \mathrm{mg} / \mathrm{l}$ & & 1 & \\
\hline theobec0000 $108-90-7$ & $<0.010 \mathrm{mg} / \mathrm{l}$ & & $<0.010 \mathrm{mg} / \mathrm{l}$ & & 1 & \\
\hline 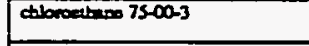 & $<0.010 \mathrm{mg} / 1$ & & $<0.010 \mathrm{mg} / \mathrm{l}$ & & 1 & \\
\hline thorotomen 67-66-3 & $<0.010 \mathrm{mg} / \mathrm{l}$ & & $<0.010 \mathrm{mg} / 1$ & & 1 & $\therefore$ \\
\hline 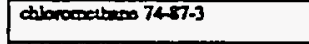 & $<0.010 \mathrm{mg} / 1$ & & $<0.010 \mathrm{mg} / \mathrm{l}$ & & 1 & \\
\hline 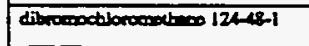 & $<0.010 \mathrm{mg} / \mathrm{l}$ & & $<0.010 \mathrm{mg} / \mathrm{l}$ & & 1 & \\
\hline 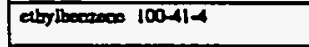 & $<0.010 \mathrm{mg} / \mathrm{l}$ & & $<0.010 \mathrm{mg} / \mathrm{l}$ & & 1 & \\
\hline whence $100-82-3$ & $<0.010 \mathrm{mg} / \mathrm{l}$ & & $<0.010 \mathrm{mg} / \mathrm{l}$ & & 1 & \\
\hline vidil atorido 75014 & $<0.010 \mathrm{mg} / \mathrm{l}$ & & $<0.010 \mathrm{mg} / \mathrm{l}$ & & 1 & \\
\hline 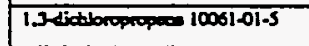 & $<0.010 \mathrm{mg} / 1$ & & $<0.010 \mathrm{mg} / 1$ & & 1 & \\
\hline 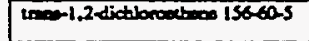 & $<0.010 \mathrm{mg} / 1$ & & $<0.010 \mathrm{mg} / \mathrm{ll}$ & & 1 & $\because$ \\
\hline 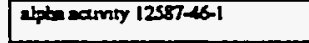 & $7.8 \mathrm{pcill}$ & $8.3 \mathrm{pci} / \mathrm{l}$ & $7.8 \mathrm{pcil}$ & $8.3 \mathrm{pci} / \mathrm{l}$ & 1 & \\
\hline beos sarnity $12587 \pm 6-1$ & $11 \mathrm{pei} / \mathrm{l}$ & $7.4 \mathrm{pei} / 1$ & Il peill. & 7.4 peill & 1 & \\
\hline Pocal ooleform & $446 \mathrm{co} / .1 \mathrm{~L}$ & $87 \mathrm{col} .1 \mathrm{~L}$ & $446 \mathrm{co} / .1 \mathrm{~L}$ & $87 \mathrm{co} / . \mathrm{lL}$ & 1 & \\
\hline Thellines 7400-2850 & $<0.03 \mathrm{mg} / \mathrm{l}$ & $<0.03 \mathrm{mg} / \mathrm{l}$ & $<0.03 \mathrm{mg} / \mathrm{l}$ & $<0.03 \mathrm{mg} / \mathrm{l}$ & 1 & \\
\hline Urasing 7400-61-1 & $0.006 \mathrm{mg} / \mathrm{l}$ & $0.010 \mathrm{mg} / 1$ & $0.006 \mathrm{mg} / \mathrm{l}$ & $0.010 \mathrm{mg} / \mathrm{l}$ & 1 & \\
\hline 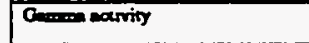 & $36 \mathrm{pei} / \mathrm{l}$ & $-25 \mathrm{pei} / 1$ & $36 \mathrm{pci} / \mathrm{l}$ & $-25 \mathrm{pci} / 1$ & 1 & \\
\hline
\end{tabular}

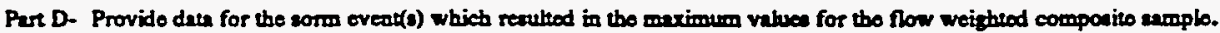

\begin{tabular}{|c|c|c|c|c|c|c|c|}
\hline $\begin{array}{l}1 . \\
\text { Detso of } \\
\text { Storm } \\
\text { Eveal }\end{array}$ & $\begin{array}{l}2 . \\
\text { Durntion } \\
\text { of Storm } \\
\text { (in } \\
\text { miniten) }\end{array}$ & $\begin{array}{l}3 . \\
\text { Total ninfill } \\
\text { durios storm eveat } \\
\text { (in iven) }\end{array}$ & 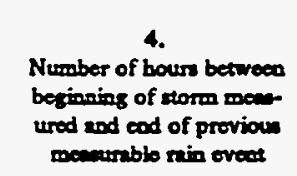 & $\begin{array}{c}\text { S. } \\
\text { Meximin flow nue } \\
\text { during min event } \\
\text { (alloce/min or specify } \\
\text { umin) }\end{array}$ & $\begin{array}{l}6 . \\
\text { Total flow from } \\
\text { nin oveat } \\
\text { (oullow or } \\
\text { peoify unito) }\end{array}$ & $\begin{array}{c}7 . \\
\text { Senson } \\
\text { camplo was } \\
\text { taked }\end{array}$ & $\begin{array}{c}8 . \\
\text { Form of } \\
\text { Procipination } \\
\text { (rinfall. } \\
\text { exomalus) }\end{array}$ \\
\hline $5 / 8 / 92$ & 695 & 0.66 & $>134.3$ & 314 & 15,905 & Spring & Rainfall \\
\hline
\end{tabular}

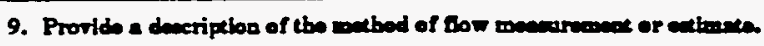

An ISCO 3230 Fow meter was med to obtain water level readings: This instrumem was calibrated with the normal iry weather flow, if present, as level zero. The flow meter wes programmed with the characteristics of the conveyance so that flow rates and total llows conld be calculated by the meter. 


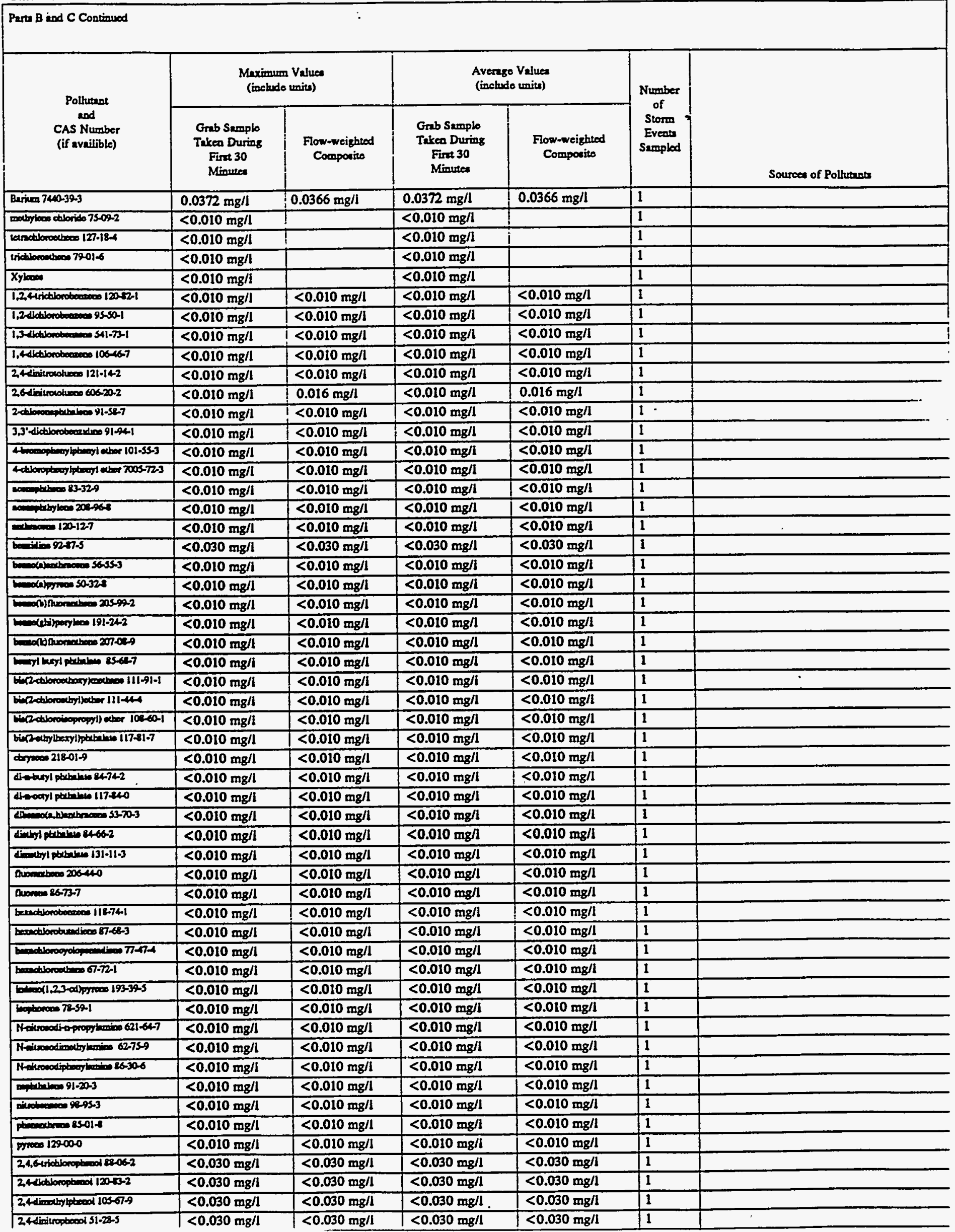


Outfall 98

Pars B and C Continued

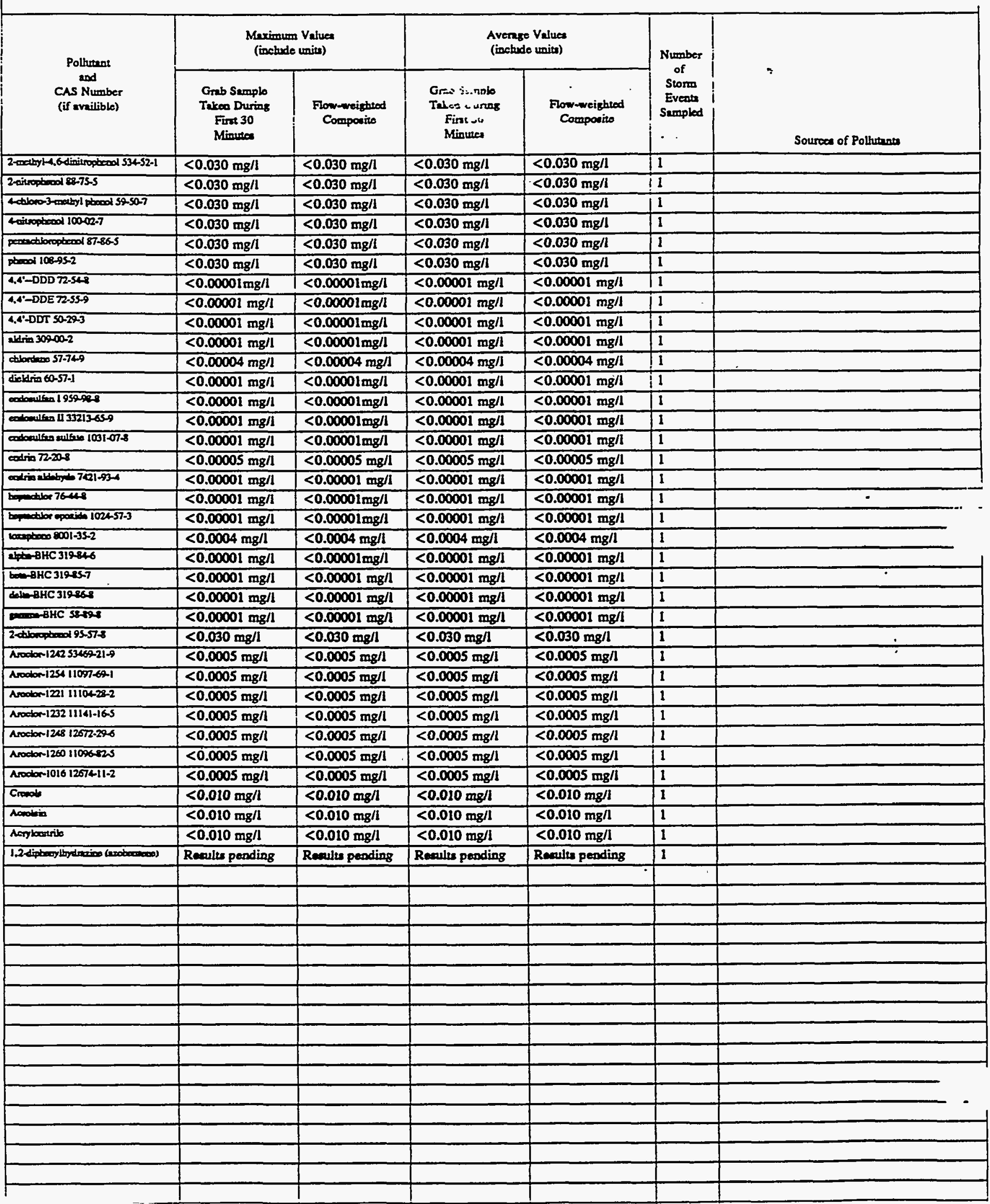


VIT. Discharge Lnformation (contioued from pago 3 of Form 2F)

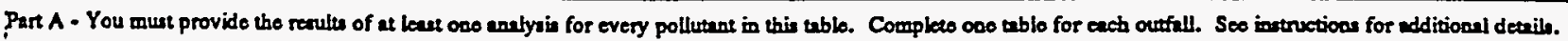

\begin{tabular}{|c|c|c|c|c|c|c|}
\hline \multirow{2}{*}{$\begin{array}{l}\text { Pollutent } \\
\text { nod } \\
\text { CAs Number } \\
\text { (if avilible) }\end{array}$} & \multicolumn{2}{|c|}{$\begin{array}{l}\text { Maximinn Vatuca } \\
\text { (include units) }\end{array}$} & \multicolumn{2}{|c|}{$\begin{array}{l}\text { Avernge Valued } \\
\text { (inctude units) }\end{array}$} & \multirow{2}{*}{$\begin{array}{l}\text { Number } \\
\text { of } \\
\text { Storm } \\
\text { Eveots } \\
\text { Sxemplod }\end{array}$} & \multirow[b]{2}{*}{ Sources of Pollutuats } \\
\hline & $\begin{array}{l}\text { Grab Sumple } \\
\text { Trkec During } \\
\text { Firut } 30 \\
\text { Minures }\end{array}$ & $\begin{array}{l}\text { Flow-weightod } \\
\text { Comporito }\end{array}$ & 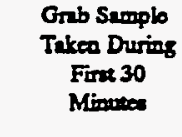 & $\begin{array}{l}\text { Flow-weightod } \\
\text { Componito }\end{array}$ & & \\
\hline pil and Graes & $<2 \mathrm{mg} / \mathrm{l}$ & & $<2 \mathrm{mg} / \mathrm{l}$ & & 1 & \\
\hline Biolofical Oxytea Deocoud (BOD) & $<5 \mathrm{mg} / \mathrm{l}$ & $<5 \mathrm{mg} / \mathrm{l}$ & $<5 \mathrm{mg} / \mathrm{l}$ & $<5 \mathrm{mg} / \mathrm{l}$ & I & \\
\hline Frenical Oxy Deaned (COD) & $5.8 \mathrm{mg} / \mathrm{l}$ & $12 \mathrm{mg} / \mathrm{l}$ & $5.8 \mathrm{mg} / 1$ & $12 \mathrm{mg} / 1$ & I & \\
\hline Toenl Suppordod Salid (TSS) & $<5 \mathrm{mg} / \mathrm{l}$ & $<5 \mathrm{mg} / 1$ & $<5 \mathrm{mg} / \mathrm{l}$ & $<5 \mathrm{mg} / \mathrm{l}$ & 1 & \\
\hline Toed Kjeldebl Nitron & $0.4 \mathrm{mg} / 1$ & $0.5 \mathrm{mg} / 1$ & $0.4 \mathrm{mg} / 1$ & $0.5 \mathrm{mg} / \mathrm{l}$ & 1 & \\
\hline Nirnes plus Nitrite Nitroeses & $0.38 \mathrm{mg} / \mathrm{l}$ & $0.38 \mathrm{mg} / \mathrm{l}$ & $0.38 \mathrm{mg} / \mathrm{l}$ & $0.38 \mathrm{mg} / 1$ & l & \\
\hline Toul Prowptonse & $<0.1 \mathrm{mg} / \mathrm{l}$ & $<0.1 \mathrm{mg} / \mathrm{l}$ & $<0.1 \mathrm{mg} / \mathrm{l}$ & $<0.1 \mathrm{mg} / 1$ & 1 & \\
\hline $\mathrm{pH}$ & Miniment & Meximm 8.0 & Minim 8.0 & Maximan & & \\
\hline
\end{tabular}

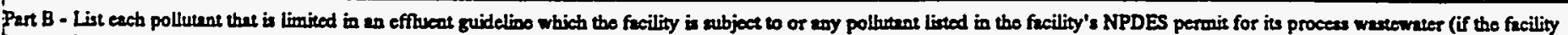

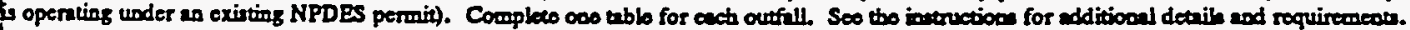

\begin{tabular}{|c|c|c|c|c|c|c|}
\hline \multirow{2}{*}{$\begin{array}{l}\text { Pollutuat } \\
\text { end } \\
\text { CAs Number } \\
\text { (if availible) }\end{array}$} & \multicolumn{2}{|c|}{ 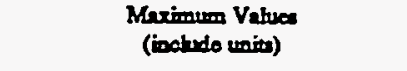 } & \multicolumn{2}{|c|}{$\begin{array}{l}\text { Avernge Vabue } \\
\text { (inchido unitr) }\end{array}$} & \multirow{2}{*}{$\begin{array}{l}\text { Nurobor } \\
\text { of } \\
\text { Storm } \\
\text { Eveots } \\
\text { Sampled }\end{array}$} & \multirow[b]{2}{*}{ Sources of Pollementes } \\
\hline & $\begin{array}{l}\text { Grab Samplo } \\
\text { Takea During } \\
\text { Firs } 30 \\
\text { Mineree }\end{array}$ & $\begin{array}{l}\text { Flow-weighted } \\
\text { Compotico }\end{array}$ & $\begin{array}{l}\text { Grab Semplo } \\
\text { Treec During } \\
\text { Fine } 30 \\
\text { Mineteo }\end{array}$ & $\begin{array}{l}\text { Frow-wrighted } \\
\text { Compocite }\end{array}$ & & \\
\hline tximoon $7400-360$ & $<0.04 \mathrm{mg} / \mathrm{l}$ & $<0.04 \mathrm{mg} / \mathrm{l}$ & $<0.04 \mathrm{mg} / \mathrm{l}$ & $<0.04 \mathrm{mg} / 1$ & 1 & \\
\hline Brasich & $<0.002 \mathrm{mg} / \mathrm{l}$ & & $<0.002 \mathrm{mg} / \mathrm{l}$ & & 1 & \\
\hline Menery 7439-976 & $<0.0002 \mathrm{mg} / 1$ & $<0.0002 \mathrm{mg} / \mathrm{l}$ & $<0.0002 \mathrm{mg} / \mathrm{l}$ & $<0.0002 \mathrm{mg} / \mathrm{l}$ & 1 & \\
\hline ponote & $0.004 \mathrm{mg} / \mathrm{l}$ & & $0.004 \mathrm{mg} / 1$ & & 1 & ? \\
\hline Feboinm $7782-19-2$ & $<0.002 \mathrm{mg} / \mathrm{l}$ & $<0.002 \mathrm{mg} / \mathrm{l}$ & $<0.002 \mathrm{mg} / \mathrm{l}$ & $<0.002 \mathrm{mg} / \mathrm{l}$ & 1 & \\
\hline Sulare & $<10 \mathrm{mg} / \mathrm{l}$ & $<10 \mathrm{mg} / \mathrm{l}$ & $<10 \mathrm{mg} / 1$ & $<10 \mathrm{mg} / \mathrm{l}$ & 1 & \\
\hline Perfectenes & $<0.05 \mathrm{mg} / \mathrm{l}$ & $<0.05 \mathrm{mg} / 1$ & $<0.05 \mathrm{mg} / \mathrm{l}$ & $<0.05 \mathrm{mg} / 1$ & 1 & \\
\hline Alumber $7429-90-5$ & $<0.04 \mathrm{mg} / \mathrm{l}$ & $0.07 \mathrm{mg} / \mathrm{I}$ & $<0.04 \mathrm{mg} / \mathrm{l}$ & $0.07 \mathrm{mg} / \mathrm{l}$ & 1 & \\
\hline Arsodio $740.38-2$ & $<0.04 \mathrm{mg} / \mathrm{l}$ & $<0.04 \mathrm{mg} / \mathrm{l}$ & $<0.04 \mathrm{mg} / \mathrm{l}$ & $<0.04 \mathrm{mg} / 1$ & 1 & \\
\hline $80000740-258$ & $0.006 \mathrm{mg} / \mathrm{l}$ & $0.007 \mathrm{mg} / 1$ & $0.006 \mathrm{mg} / \mathrm{l}$ & $0.007 \mathrm{mg} / 1$ & 1 & \\
\hline Berglifine 744041.7 & $<0.0004 \mathrm{mg} / \mathrm{l}$ & $<0.0004 \mathrm{mg} / \mathrm{l}$ & $<0.0004 \mathrm{mg} / \mathrm{l}$ & $<0.0004 \mathrm{mg} / 1$ & I & \\
\hline 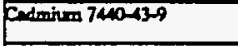 & $<0.004 \mathrm{mg} / \mathrm{l}$ & $<0.004 \mathrm{mg} / \mathrm{l}$ & $<0.004 \mathrm{mg} / 1$ & $<0.004 \mathrm{mg} / 1$ & 1 & \\
\hline Caloium 7440-70-2 & $8.5 \mathrm{mg} / 1$ & $10.0 \mathrm{mg} / \mathrm{l}$ & $8.5 \mathrm{mg} / \mathrm{l}$ & $10.0 \mathrm{mg} / 1$ & 1 & \\
\hline Chromium $7400-7.3$ & $<0.006 \mathrm{mg} / \mathrm{l}$ & $<0.006 \mathrm{mg} / \mathrm{l}$ & $<0.006 \mathrm{mg} / 1$ & $<0.006 \mathrm{mg} / \mathrm{l}$ & 1 & \\
\hline Cobalit 7440-184 & $<0.002 \mathrm{mg} / 1$ & $<0.002 \mathrm{mg} / 1$ & $<0.002 \mathrm{mg} / 1$ & $<0.002 \mathrm{mg} / \mathrm{l}$ & 1 & \\
\hline Coppon 740-50-8 & $<0.006 \mathrm{mg} / 1$ & $0.008 \mathrm{mg} / \mathrm{l}$ & $<0.006 \mathrm{mg} / \mathrm{l}$ & $0.008 \mathrm{mg} / \mathrm{l}$ & 1 & \\
\hline 1007439896 & $<0.06 \mathrm{mg} / 1$ & $0.18 \mathrm{mg} / \mathrm{l}$ & $<0.06 \mathrm{mg} / \mathrm{l}$ & $0.18 \mathrm{mg} / 1$ & 1 & \\
\hline Fond 7439-92-1 & $<0.02 \mathrm{mg} / \mathrm{l}$ & $<0.02 \mathrm{mg} / 1$ & $<0.02 \mathrm{mg} / 1$ & $<0.02 \mathrm{mg} / 1$ & 1 & \\
\hline Sithilum $7139993-2$ & $<0.02 \mathrm{mg} / \mathrm{l}$ & $<0.02 \mathrm{mg} / \mathrm{l}$ & $<0.02 \mathrm{mg} / \mathrm{l}$ & $<0.02 \mathrm{mg} / 1$ & 1 & \\
\hline Meposiun 7639954 & $2.07 \mathrm{mg} / \mathrm{l}$ & $227 \mathrm{mg} / \mathrm{l}$ & $2.07 \mathrm{mg} / \mathrm{l}$ & $227 \mathrm{mg} / 1$ & 1 & \\
\hline Menteoson $743996-5$ & $0.009 \mathrm{mg} / \mathrm{l}$ & $0.035 \mathrm{mg} / \mathrm{l}$ & $0.009 \mathrm{mg} / \mathrm{l}$ & $0.035 \mathrm{mg} / 1$ & 1 & \\
\hline Molytdeanom 743998-7 & $0.008 \mathrm{mg} / \mathrm{l}$ & $0.011 \mathrm{mg} / \mathrm{l}$ & $0.008 \mathrm{mg} / \mathrm{l}$ & $0.011 \mathrm{mg} / 1$ & 1 & \\
\hline Nictel 7440000 & $<0.008 \mathrm{mg} / \mathrm{l}$ & $<0.008 \mathrm{mg} / 1$ & $<0.008 \mathrm{mg} / \mathrm{l}$ & $<0.008 \mathrm{mg} / \mathrm{l}$ & 1 & \\
\hline Potersian 74009-7 & $<0.5 \mathrm{mg} / \mathrm{l}$ & $<0.5 \mathrm{mg} / \mathrm{l}$ & $<0.5 \mathrm{mg} / \mathrm{l}$ & $<0.5 \mathrm{mg} / 1$ & 1 & \\
\hline fither 740-204 & $<0.006 \mathrm{mg} / \mathrm{l}$ & $<0.006 \mathrm{mg} / \mathrm{l}$ & $<0.006 \mathrm{mg} / \mathrm{l}$ & $<0.006 \mathrm{mg} / 1$ & 1 & \\
\hline Sodhen 74ta-2a-5 & $0.42 \mathrm{mg} / \mathrm{l}$ & $0.61 \mathrm{mg} / \mathrm{l}$ & $0.42 \mathrm{mg} / \mathrm{l}$ & $0.61 \mathrm{mg} / 1$ & 1 & \\
\hline Titeringor 7440-32-6 & $<0.01 \mathrm{mg} / \mathrm{l}$ & $<0.01 \mathrm{mg} / 1$ & $<0.01 \mathrm{mg} / \mathrm{l}$ & $<0.01 \mathrm{mg} / 1$ & 1 & \\
\hline $21007440-66-6$ & $0.13 \mathrm{mg} / \mathrm{l}$ & $0.14 \mathrm{mg} / 1$ & $0.13 \mathrm{mg} / \mathrm{l}$ & $0.14 \mathrm{mg} / 1$ & 1 & \\
\hline
\end{tabular}


Coptinued from trook.

Pert C- List each poltutan shown in Table 2F-2, 2F-3, and 2F4 that you know or beve reason wo believe in present See the instructions for additional details and requiremente. Coon one tuble for cach ourfall.

\begin{tabular}{|c|c|c|c|c|c|c|}
\hline \multirow{2}{*}{$\begin{array}{l}\text { Polbutans } \\
\text { and } \\
\text { CAS Number } \\
\text { (if aveilible) }\end{array}$} & \multicolumn{2}{|c|}{$\begin{array}{l}\text { Marimum Values } \\
\text { (include unim) }\end{array}$} & \multicolumn{2}{|c|}{$\begin{array}{l}\text { Average Values } \\
\text { (imclude inita) }\end{array}$} & \multirow{2}{*}{$\begin{array}{l}\text { Nimber } \\
\text { of } \\
\text { Storm } \\
\text { Events } \\
\text { Srompled }\end{array}$} & \multirow[b]{2}{*}{ Source of Polhutan: } \\
\hline & $\begin{array}{c}\text { Greb Semple } \\
\text { Takea Durias } \\
\text { Find } 30 \\
\text { Minuces }\end{array}$ & $\begin{array}{l}\text { Flow-weighted } \\
\text { Compouite }\end{array}$ & $\begin{array}{c}\text { Grab Surople } \\
\text { Taken During } \\
\text { Fint } 30 \\
\text { Mforear }\end{array}$ & $\begin{array}{l}\text { Flow-weighted } \\
\text { Componite }\end{array}$ & & \\
\hline $1,1,1,-6$ richlaraction $71 \cdot 55-6$ & $<0.010 \mathrm{mg} / \mathrm{l}$ & & $<0.010 \mathrm{mg} / 1$ & & 1 & \\
\hline 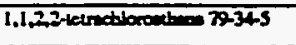 & $<0.010 \mathrm{mg} / 1$ & & $<0.010 \mathrm{mg} / 1$ & & 1 & \\
\hline 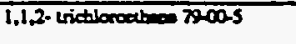 & $<0.010 \mathrm{mg} / 1$ & & $<0.010 \mathrm{mg} / 1$ & & 1 & \\
\hline 1.1-dichloroethenes $75-34-3$ & $<0.010 \mathrm{mg} / \mathrm{l}$ & & $<0.010 \mathrm{mg} / \mathrm{l}$ & & 1 & \\
\hline 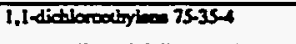 & $<0.010 \mathrm{mg} / \mathrm{l}$ & & $<0.010 \mathrm{mg} / 1$ & & 1 & \\
\hline 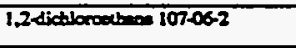 & $<0.010 \mathrm{mg} / \mathrm{t}$ & & $<0.010 \mathrm{mg} / \mathrm{l}$ & & 1 & \\
\hline 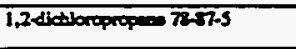 & $<0.010 \mathrm{mg} / \mathrm{l}$ & & $<0.010 \mathrm{mg} / \mathrm{l}$ & & 1 & \\
\hline 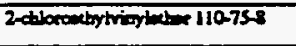 & $<0.010 \mathrm{mg} / 1$ & & $<0.010 \mathrm{mg} / \mathrm{l}$ & & 1 & \\
\hline $71-1322$ & $<0.010 \mathrm{mg} / 1$ & & $<0.010 \mathrm{mg} / \mathrm{l}$ & & 1 & \\
\hline $75-274$ & $<0.010 \mathrm{mg} / \mathrm{l}$ & & $<0.010 \mathrm{mg} / \mathrm{l}$ & & 1 & \\
\hline bromotarm 75-25-2 & $<0.010 \mathrm{mg} / \mathrm{l}$ & & $<0.010 \mathrm{mg} / 1$ & & 1 & \\
\hline 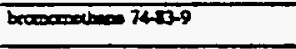 & $<0.010 \mathrm{mg} / 1$ & & $<0.010 \mathrm{mg} / 1$ & & 1 & \\
\hline arten wasctiarides6-23-5 & $<0.010 \mathrm{mg} / \mathrm{l}$ & & $<0.010 \mathrm{mg} / \mathrm{l}$ & & 1 & \\
\hline chlorobecosol $102-90.7$ & $<0.010 \mathrm{mg} / \mathrm{l}$ & & $<0.010 \mathrm{mg} / 1$ & & 1 & \\
\hline cloredbetes $75-00-3$ & $<0.010 \mathrm{mg} / \mathrm{l}$ & & $<0.010 \mathrm{mg} / \mathrm{l}$ & & 1 & \\
\hline chlardang 67-66-3 & $<0.010 \mathrm{mg} / \mathrm{l}$ & & $<0.010 \mathrm{mg} / \mathrm{l}$ & & 1 & $\dot{-}$ \\
\hline 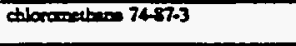 & $<0.010 \mathrm{mg} / 1$ & & $<0.010 \mathrm{mg} / \mathrm{l}$ & & 1 & \\
\hline 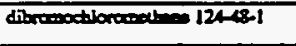 & $<0.010 \mathrm{mg} / 1$ & & $<0.010 \mathrm{mg} / 1$ & & 1 & \\
\hline 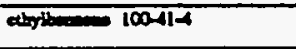 & $<0.010 \mathrm{mg} / 1$ & & $<0.010 \mathrm{mg} / \mathrm{l}$ & & 1 & \\
\hline loh $=100-62-3$ & $<0.010 \mathrm{mg} / 1$ & & $<0.010 \mathrm{mg} / \mathrm{l}$ & & 1 & \\
\hline vistationide 75014 & $<0.010 \mathrm{mg} / 1$ & & $<0.010 \mathrm{mg} / \mathrm{l}$ & & 1 & \\
\hline 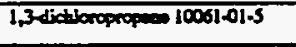 & $<0.010 \mathrm{mg} / \mathrm{l}$ & & $<0.010 \mathrm{mg} / \mathrm{h}$ & & 1 & \\
\hline 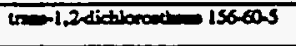 & $<0.010 \mathrm{mg} / 1$ & & $<0.010 \mathrm{mg} / \mathrm{l}$ & & 1 & $\because$ \\
\hline athe ectiviny $1257-16-1$ & $3.9 \mathrm{psi} / 1$ & 11 poill & $3.9 \mathrm{pci} / 1$ & $11 \mathrm{pci} / 1$ & 1 & \\
\hline boxes sainty $12577-46-1$ & 3.7 peill & $14 \mathrm{pci} / \mathrm{l}$ & $3.7 \mathrm{pci} / \mathrm{l}$ & $14 \mathrm{pci} / 1$ & 1 & \\
\hline Soal odifocm & $13 \mathrm{co} .1 \mathrm{~L}$ & $12 \mathrm{col} .1 \mathrm{~L}$ & $13 \mathrm{col} .1 \mathrm{~L}$ & $12 \mathrm{co} / .1 \mathrm{~L}$ & 1 & \\
\hline Thallim 2440-200 & $<0.001 \mathrm{mg} / \mathrm{l}$ & $<0.001 \mathrm{mg} / \mathrm{l}$ & $<0.001 \mathrm{mg} / \mathrm{l}$ & $<0.001 \mathrm{mg} / 1$ & 1 & \\
\hline Unimen 74061.1 & $0.001 \mathrm{mg} / 1$ & $0.003 \mathrm{mg} / 1$ & $0.001 \mathrm{mg} / 1$ & $0.003 \mathrm{mg} / 1$ & 1 & \\
\hline Gowedivixy & $26 \mathrm{pci} / \mathrm{l}$ & 25 pcill & 26 peil & 25 peill & 1 & \\
\hline
\end{tabular}

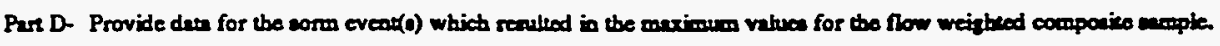

\begin{tabular}{|c|c|c|c|c|c|c|c|}
\hline $\begin{array}{l}1 . \\
\text { Date of } \\
\text { Storm } \\
\text { Eveal }\end{array}$ & $\begin{array}{l}2 . \\
\text { Durition } \\
\text { of Storm } \\
\text { (ix } \\
\text { minites) }\end{array}$ & $\begin{array}{l}3 . \\
\text { Towi ninfill } \\
\text { durias atorm event } \\
\text { (in inches) }\end{array}$ & 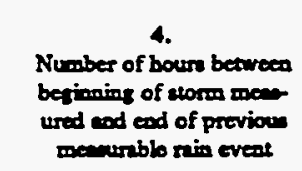 & 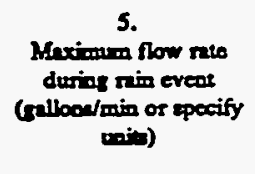 & $\begin{array}{c}6 . \\
\text { Totil flow from } \\
\text { nin event } \\
\text { (salioos or } \\
\text { epecify usit) }\end{array}$ & $\begin{array}{c}-. \\
\text { Seacos } \\
\text { andis wen } \\
\text { taken }\end{array}$ & $\begin{array}{l}8 . \\
\text { Form of } \\
\text { Precipination } \\
\text { (ninfill, } \\
\text { anmela) }\end{array}$ \\
\hline $3 / 6 / 92$ & 420 & 0.76 & 138.9 & 158 & 9,517 & Wimter & Rninfll \\
\hline
\end{tabular}

9. Provide a description of the mabod of flow mencurewent or catimate.

An ISCO 3230 Flow meter was used to obtain water level readings. This instrument was colibrated with the normal dry weather flow, if pretent, as leved zero. The flow meter wes programmed with the characterintics of the conreyance so that flow rates and totel flows conld be calculated by the meter. 
Outfnll 99

Pars B and C Continuad

\begin{tabular}{|c|c|c|c|c|c|c|}
\hline \multirow{2}{*}{$\begin{array}{l}\text { Pollumat } \\
\text { and } \\
\text { CAS Number } \\
\text { (if availible) }\end{array}$} & \multicolumn{2}{|c|}{$\begin{array}{l}\text { Maximum Valuea } \\
\text { (include unit) }\end{array}$} & \multicolumn{2}{|c|}{$\begin{array}{l}\text { Avernge Values } \\
\text { (inchude unitu) }\end{array}$} & \multirow{2}{*}{$\begin{array}{l}\text { Number } \\
\text { of } \\
\text { Storm" } \\
\text { Evoots } \\
\text { Semplod }\end{array}$} & \multirow[b]{2}{*}{ Soureces of Pollutuats } \\
\hline & $\begin{array}{c}\text { Grob Sample } \\
\text { Takea During } \\
\text { Firts } 30 \\
\text { Minurea }\end{array}$ & $\begin{array}{l}\text { Fow-weighrod } \\
\text { Componits }\end{array}$ & $\begin{array}{c}\text { Grab Semple } \\
\text { Taken During } \\
\text { First } 30 \\
\text { Minurea }\end{array}$ & $\begin{array}{l}\text { Flow-weighred } \\
\text { Cocoposite }\end{array}$ & & \\
\hline Bariven 7400-39-3 & $0.0056 \mathrm{mg} / \mathrm{l}$ & $0.0082 \mathrm{mg} / 1$ & $0.0056 \mathrm{mg} / \mathrm{l}$ & $0.0082 \mathrm{mg} / \mathrm{l}$ & 1 & \\
\hline methyleos chloride $7509-2$ & $<0.010 \mathrm{mg} / \mathrm{l}$ & & $<0.010 \mathrm{mg} / \mathrm{l}$ & & 1 & \\
\hline 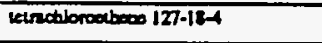 & $<0.010 \mathrm{mg} / \mathrm{l}$ & & $<0.010 \mathrm{mg} / \mathrm{l}$ & & 1 & \\
\hline 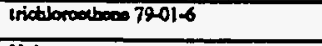 & $<0.010 \mathrm{mg} / \mathrm{l}$ & & $<0.010 \mathrm{mg} / \mathrm{l}$ & & 1 & \\
\hline Xylemen & $<0.010 \mathrm{mg} / \mathrm{l}$ & & $<0.010 \mathrm{mg} / \mathrm{l}$ & & 1 & \\
\hline 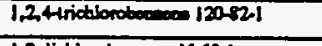 & $<0.010 \mathrm{mg} / \mathrm{l}$ & $<0.010 \mathrm{mg} / \mathrm{l}$ & $<0.010 \mathrm{mg} / \mathrm{l}$ & $<0.010 \mathrm{mg} / 1$ & 1 & \\
\hline 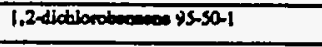 & $<0.010 \mathrm{mg} / \mathrm{l}$ & $<0.010 \mathrm{mg} / 1$ & $<0.010 \mathrm{mg} / \mathrm{l}$ & $<0.010 \mathrm{mg} / \mathrm{l}$ & 1 & \\
\hline 1,3 -dictionobocosos $341-73-1$ & $<0.010 \mathrm{mg} / \mathrm{l}$ & $<0.010 \mathrm{mg} / \mathrm{l}$ & $<0.010 \mathrm{mg} / \mathrm{l}$ & $<0.010 \mathrm{mg} / \mathrm{l}$ & 1 & \\
\hline 1.4 dictionobecenes $106-46-7$ & $<0.010 \mathrm{mg} / \mathrm{l}$ & $<0.010 \mathrm{mg} / \mathrm{h}$ & $<0.010 \mathrm{mg} / \mathrm{l}$ & $<0.010 \mathrm{mg} / \mathrm{l}$ & 1 & \\
\hline 2,4 dinitroloturese $121-142$ & $<0.010 \mathrm{mg} / \mathrm{l}$ & $<0.010 \mathrm{mg} / \mathrm{l}$ & $<0.010 \mathrm{mg} / 1$ & $<0.010 \mathrm{mg} / \mathrm{l}$ & 1 & \\
\hline 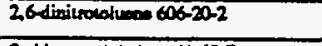 & $<0.010 \mathrm{mg} / \mathrm{l}$ & $<0.010 \mathrm{mg} / 1$ & $<0.010 \mathrm{mg} / \mathrm{l}$ & $<0.010 \mathrm{mg} / 1$ & 1 & \\
\hline 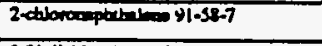 & $<0.010 \mathrm{mg} / \mathrm{l}$ & $<0.010 \mathrm{mg} / \mathrm{l}$ & $<0.010 \mathrm{mg} / \mathrm{l}$ & $<0.010 \mathrm{mg} / \mathrm{l}$ & 1 & \\
\hline 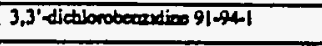 & $<0.010 \mathrm{mg} / \mathrm{l}$ & $<0.020 \mathrm{mg} / \mathrm{l}$ & $<0.010 \mathrm{mg} / 1$ & $<0.020 \mathrm{mg} / \mathrm{l}$ & 1 & \\
\hline 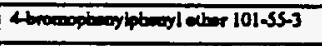 & $<0.010 \mathrm{mg} / 1$ & $<0.010 \mathrm{mg} / 1$ & $<0.010 \mathrm{mg} / \mathrm{l}$ & $<0.010 \mathrm{mg} / \mathrm{l}$ & 1 & \\
\hline 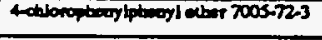 & $<0.010 \mathrm{mg} / \mathrm{l}$ & $<0.010 \mathrm{mg} / 1$ & $<0.010 \mathrm{mg} / 1$ & $<0.010 \mathrm{mg} / 1$ & 1 & \\
\hline 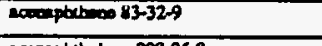 & $<0.010 \mathrm{mg} / 1$ & $<0.010 \mathrm{mg} / \mathrm{l}$ & $<0.010 \mathrm{mg} / \mathrm{l}$ & $<0.010 \mathrm{mg} / \mathrm{l}$ & 1 & \\
\hline 20000 pesty $16000208 \% 8$ & $<0.010 \mathrm{mg} / \mathrm{l}$ & $<0.010 \mathrm{mg} / \mathrm{l}$ & $<0.010 \mathrm{mg} / \mathrm{l}$ & $<0.010 \mathrm{mg} / \mathrm{l}$ & 1 & \\
\hline $120-12.7$ & $<0.010 \mathrm{mg} / \mathrm{l}$ & $<0.010 \mathrm{mg} / 1$ & $<0.010 \mathrm{mg} / \mathrm{l}$ & $<0.010 \mathrm{mg} / \mathrm{l}$ & 1 & \\
\hline 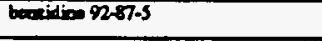 & $<0.050 \mathrm{mg} / \mathrm{l}$ & $<0.050 \mathrm{mg} / \mathrm{l}$ & $<0.050 \mathrm{mg} / \mathrm{l}$ & $<0.050 \mathrm{mg} / 1$ & 1 & \\
\hline 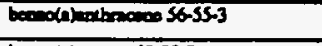 & $<0.010 \mathrm{mg} / \mathrm{l}$ & $<0.010 \mathrm{mg} / \mathrm{l}$ & $<0.010 \mathrm{mg} / \mathrm{l}$ & $<0.010 \mathrm{mg} / 1$ & 1 & \\
\hline 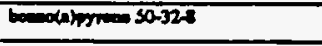 & $<0.010 \mathrm{mg} / \mathrm{l}$ & $<0.010 \mathrm{mg} / \mathrm{l}$ & $<0.010 \mathrm{mg} / \mathrm{l}$ & $<0.010 \mathrm{mg} / 1$ & 1 & \\
\hline 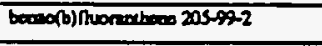 & $<0.010 \mathrm{mg} / 1$ & $<0.010 \mathrm{mg} / \mathrm{l}$ & $<0.010 \mathrm{mg} / \mathrm{l}$ & $<0.010 \mathrm{mg} / 1$ & 1 & \\
\hline 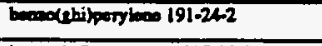 & $<0.010 \mathrm{mg} / 1$ & $<0.010 \mathrm{mg} / 1$ & $<0.010 \mathrm{mg} / \mathrm{l}$ & $<0.010 \mathrm{mg} / 1$ & 1 & \\
\hline 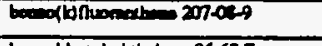 & $<0.010 \mathrm{mg} / 1$ & $<0.010 \mathrm{mg} / 1$ & $<0.010 \mathrm{mg} / \mathrm{l}$ & $<0.010 \mathrm{mg} / 1$ & 1 & \\
\hline 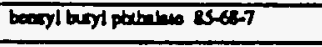 & $<0.010 \mathrm{mg} / \mathrm{I}$ & $<0.010 \mathrm{mg} / \mathrm{l}$ & $<0.010 \mathrm{mghl}$ & $<0.010 \mathrm{mg} / 1$ & 1 & \\
\hline 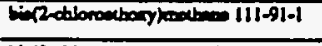 & $<0.010 \mathrm{mg} / \mathrm{l}$ & $<0.010 \mathrm{mg} / \mathrm{l}$ & $<0.010 \mathrm{mg} / 1$ & $<0.010 \mathrm{mg} / 1$ & 1 & \\
\hline 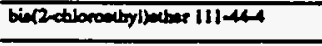 & $<0.010 \mathrm{mg} / \mathrm{l}$ & $<0.010 \mathrm{mg} / 1$ & $<0.010 \mathrm{mg} / \mathrm{l}$ & $<0.010 \mathrm{mg} / 1$ & 1 & \\
\hline 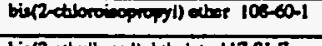 & $<0.010 \mathrm{mg} / \mathrm{l}$ & $<0.010 \mathrm{mg} / \mathrm{l}$ & $<0.010 \mathrm{mg} / 1$ & $<0.010 \mathrm{mg} / \mathrm{l}$ & 1 & \\
\hline 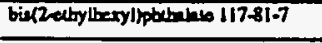 & $<0.010 \mathrm{mg} / \mathrm{l}$ & $<0.010 \mathrm{mg} / 1$ & $<0.010 \mathrm{mg} / \mathrm{l}$ & $<0.010 \mathrm{mg} / \mathrm{l}$ & 1 & \\
\hline atrpeon 21ह0|A & $<0.010 \mathrm{mg} / \mathrm{l}$ & $<0.010 \mathrm{mg} / 1$ & $<0.010 \mathrm{mg} / 1$ & $<0.010 \mathrm{mg} / \mathrm{l}$ & 1 & \\
\hline 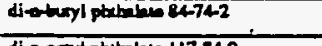 & $<0.010 \mathrm{mg} / \mathrm{l}$ & $<0.010 \mathrm{mg} / \mathrm{l}$ & $<0.010 \mathrm{mg} / \mathrm{l}$ & $<0.010 \mathrm{mg} / \mathrm{l}$ & 1 & \\
\hline 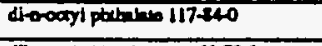 & $<0.010 \mathrm{mg} / 1$ & $<0.010 \mathrm{mg} / \mathrm{l}$ & $<0.010 \mathrm{mg} / 1$ & $<0.010 \mathrm{mg} / 1$ & 1 & \\
\hline 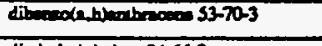 & $<0.010 \mathrm{mg} / 1$ & $<0.010 \mathrm{mg} / \mathrm{l}$ & $<0.010 \mathrm{mg} / \mathrm{l}$ & $<0.010 \mathrm{mg} / 1$ & 1 & \\
\hline 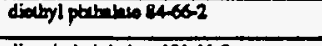 & $<0.010 \mathrm{mg} / \mathrm{l}$ & $<0.010 \mathrm{mg} / \mathrm{l}$ & $<0.010 \mathrm{mg} / \mathrm{l}$ & $<0.010 \mathrm{mg} / \mathrm{l}$ & 1 & \\
\hline 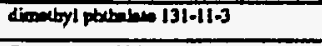 & $<0.010 \mathrm{mg} / \mathrm{I}$ & $<0.010 \mathrm{mg} / 1$ & $<0.010 \mathrm{mg} / \mathrm{l}$ & $<0.010 \mathrm{mg} / 1$ & 1 & \\
\hline 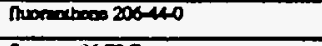 & $<0.010 \mathrm{mg} / \mathrm{l}$ & $<0.010 \mathrm{mg} / \mathrm{l}$ & $<0.010 \mathrm{mg} / \mathrm{l}$ & $<0.010 \mathrm{mg} / 1$ & 1 & \\
\hline Shovocos 86-73-7 & $<0.010 \mathrm{mg} / \mathrm{l}$ & $<0.010 \mathrm{mg} / \mathrm{l}$ & $<0.010 \mathrm{mg} / \mathrm{l}$ & $<0.010 \mathrm{mg} / 1$ & 1 & \\
\hline 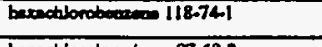 & $<0.010 \mathrm{mg} / \mathrm{L}$ & $<0.010 \mathrm{mg} / \mathrm{l}$ & $<0.010 \mathrm{mg} / \mathrm{l}$ & $<0.010 \mathrm{mg} / 1$ & 1 & \\
\hline 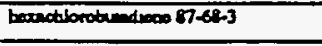 & $<0.010 \mathrm{mg} / \mathrm{I}$ & $<0.010 \mathrm{mg} / \mathrm{l}$ & $<0.010 \mathrm{mg} / 1$ & $<0.010 \mathrm{mg} / 1$ & 1 & \\
\hline 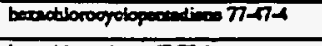 & $<0.010 \mathrm{mg} / 1$ & $<0.010 \mathrm{mg} / \mathrm{l}$ & $<0.010 \mathrm{mg} / \mathrm{l}$ & $<0.010 \mathrm{mg} / \mathrm{l}$ & 1 & \\
\hline hereactorowhere 67.72 .1 & $<0.010 \mathrm{mg} / \mathrm{l}$ & $<0.010 \mathrm{mg} / 1$ & $<0.010 \mathrm{mg} / \mathrm{l}$ & $<0.010 \mathrm{mg} / \mathrm{t}$ & 1 & \\
\hline 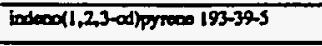 & $<0.010 \mathrm{mg} / \mathrm{l}$ & $<0.010 \mathrm{mg} / \mathrm{l}$ & $<0.010 \mathrm{mg} / \mathrm{l}$ & $<0.010 \mathrm{mg} / \mathrm{l}$ & 1 & \\
\hline boptorowe 72-59-1 & $<0.010 \mathrm{mg} / \mathrm{l}$ & $<0.010 \mathrm{mg} / \mathrm{l}$ & $<0.010 \mathrm{mg} / \mathrm{l}$ & $<0.010 \mathrm{mg} / 1$ & 1 & \\
\hline 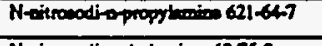 & $<0.010 \mathrm{mg} / \mathrm{I}$ & $<0.010 \mathrm{mg} / \mathrm{l}$ & $<0.010 \mathrm{mg} / \mathrm{l}$ & $<0.010 \mathrm{mg} / \mathrm{l}$ & 1 & \\
\hline 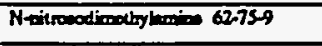 & $<0.010 \mathrm{mg} / \mathrm{l}$ & $<0.010 \mathrm{mg} / \mathrm{l}$ & $<0.010 \mathrm{mg} / \mathrm{l}$ & $<0.010 \mathrm{mg} / \mathrm{l}$ & 1 & \\
\hline 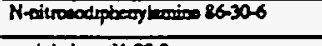 & $<0.010 \mathrm{mg} / \mathrm{l}$ & $<0.010 \mathrm{mg} / 1$ & $<0.010 \mathrm{mg} / \mathrm{l}$ & $<0.010 \mathrm{mg} / 1$ & 1 & \\
\hline 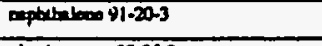 & $<0.010 \mathrm{mg} / \mathrm{l}$ & $<0.010 \mathrm{mg} / 1$ & $<0.010 \mathrm{mg} / \mathrm{l}$ & $<0.010 \mathrm{mg} / 1$ & 1 & \\
\hline 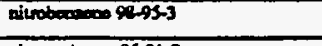 & $<0.010 \mathrm{mg} / \mathrm{l}$ & $<0.010 \mathrm{mg} / 1$ & $<0.010 \mathrm{mg} / \mathrm{l}$ & $<0.010 \mathrm{mg} / 1$ & 1 & \\
\hline 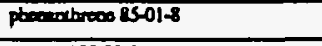 & $<0.010 \mathrm{mg} / \mathrm{l}$ & $<0.010 \mathrm{mg} / \mathrm{l}$ & $<0.010 \mathrm{mg} / \mathrm{I}$ & $<0.010 \mathrm{mg} / 1$ & 1 & \\
\hline pyroos 129000 & $<0.010 \mathrm{mg} / \mathrm{l}$ & $<0.010 \mathrm{mg} / \mathrm{l}$ & $<0.010 \mathrm{mg} / \mathrm{l}$ & $<0.010 \mathrm{mg} / 1$ & 1 & \\
\hline 2,4,64rictionophenol 28062 & $<0.030 \mathrm{mg} / \mathrm{l}$ & $<0.030 \mathrm{mg} / \mathrm{l}$ & $<0.030 \mathrm{mg} / \mathrm{l}$ & $<0.030 \mathrm{mg} / 1$ & 1 & \\
\hline 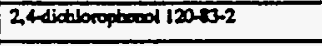 & $<0.030 \mathrm{mg} / \mathrm{l}$ & $<0.030 \mathrm{mg} / \mathrm{l}$ & $<0.030 \mathrm{mg} / \mathrm{l}$ & $<0.030 \mathrm{mg} / \mathrm{l}$ & 1 & \\
\hline 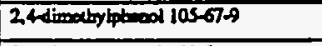 & $<0.030 \mathrm{mg} / \mathrm{l}$ & $<0.030 \mathrm{mg} / \mathrm{l}$ & $<0.030 \mathrm{mg} / \mathrm{l}$ & $<0.030 \mathrm{mg} / \mathrm{l}$ & 1 & \\
\hline 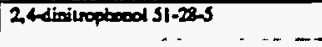 & $<0.050 \mathrm{mg} / \mathrm{l}$ & $<0.050 \mathrm{mg} / \mathrm{l}$ & $<0.050 \mathrm{mg} / \mathrm{l}$ & $<0.050 \mathrm{mg} / \mathrm{l}$ & 1 & \\
\hline
\end{tabular}


Outfall 99

; Parr B and C Concinued

\begin{tabular}{|c|c|c|c|c|c|c|}
\hline \multirow{2}{*}{$\begin{array}{l}\text { Pollutuns } \\
\text { aod } \\
\text { CAS Number } \\
\text { (if availible) }\end{array}$} & \multicolumn{2}{|c|}{$\begin{array}{l}\text { Maxinumom Valuea } \\
\text { (inchude units) }\end{array}$} & \multicolumn{2}{|c|}{$\begin{array}{l}\text { Averngo Values } \\
\text { (include unitu) }\end{array}$} & \multirow{2}{*}{$\begin{array}{l}\text { Number } \\
\text { of } \\
\text { Storm } \\
\text { Eveatu } \\
\text { Srompiod }\end{array}$} & \multirow[b]{2}{*}{ Sourstes of Pollutents } \\
\hline & $\begin{array}{c}\text { Grab Semple } \\
\text { Teken During } \\
\text { Firr } 30 \\
\text { Minutes }\end{array}$ & $\begin{array}{l}\text { Flow-weighted } \\
\text { Compocite }\end{array}$ & $\begin{array}{l}\text { Grab Sumple } \\
\text { Takeo During } \\
\text { Firt } 30 \\
\text { Minutea }\end{array}$ & $\begin{array}{c}\text { Fow-weighted } \\
\text { Composite }\end{array}$ & & \\
\hline 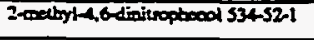 & $<0.050 \mathrm{mg} / \mathrm{l}$ & $<0.050 \mathrm{mg} / \mathrm{l}$ & $<0.050 \mathrm{mg} / \mathrm{l}$ & $<0.050 \mathrm{mg} / \mathrm{l}$ & 1 & \\
\hline 2 -iilophecol $48-75-5$ & $<0.030 \mathrm{mg} / \mathrm{l}$ & $<0.030 \mathrm{mg} / \mathrm{l}$ & $<0.030 \mathrm{mg} / \mathrm{l}$ & $<0.030 \mathrm{mg} / \mathrm{l}$ & 1 & \\
\hline 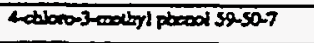 & $<0.030 \mathrm{mg} / 1$ & $<0.030 \mathrm{mg} / 1$ & $<0.030 \mathrm{mg} / \mathrm{l}$ & $<0.030 \mathrm{mg} / \mathrm{l}$ & 1 & \\
\hline 10000000027 & $<0.050 \mathrm{mg} / \mathrm{l}$ & $<0.050 \mathrm{mg} / \mathrm{l}$ & $<0.050 \mathrm{mg} / \mathrm{l}$ & $<0.050 \mathrm{mg} / \mathrm{l}$ & 1 & \\
\hline 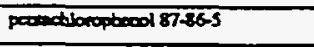 & $<0.050 \mathrm{mg} / \mathrm{l}$ & $<0.050 \mathrm{mg} / \mathrm{l}$ & $<0.050 \mathrm{mg} / \mathrm{l}$ & $<0.050 \mathrm{mg} / 1$ & 1 & \\
\hline pheood 108-95-2 & $<0.030 \mathrm{mg} / \mathrm{l}$ & $<0.030 \mathrm{mg} / \mathrm{l}$ & $<0.030 \mathrm{mg} / \mathrm{l}$ & $<0.030 \mathrm{mg} / \mathrm{l}$ & 1 & \\
\hline $4.4^{\circ}-000072-548$ & $<0.00001 \mathrm{mg} / \mathrm{l}$ & $<0.00001 \mathrm{mg} / \mathrm{l}$ & $<0.00001 \mathrm{mg} / \mathrm{l}$ & $<0.00001 \mathrm{mg} / \mathrm{l}$ & 1 & \\
\hline Q.4'DDEE 72.55-9 & $<0.00001 \mathrm{mg} / \mathrm{l}$ & $<0.00001 \mathrm{mg} / \mathrm{l}$ & $<0.00001 \mathrm{mg} / \mathrm{l}$ & $<0.00001 \mathrm{mg} / \mathrm{l}$ & 1 & \\
\hline 4.4.000T 50-22-3 & $0.000064 \mathrm{mg} / \mathrm{l}$ & $<0.00001 \mathrm{mg} / \mathrm{l}$ & $0.000064 \mathrm{mg} / \mathrm{l}$ & $<0.00001 \mathrm{mg} / \mathrm{l}$ & 1 & \\
\hline adria $302-00-2$ & $<0.00001 \mathrm{mg} / \mathrm{I}$ & $<0.00001 \mathrm{mg} / \mathrm{l}$ & $<0.00001 \mathrm{mg} / \mathrm{l}$ & $<0.00001 \mathrm{mg} / \mathrm{l}$ & 1 & \\
\hline chlonderes $57-749$ & $<0.00002 \mathrm{mg} / \mathrm{l}$ & $<0.00002 \mathrm{mg} / \mathrm{l}$ & $<0.00002 \mathrm{mg} / \mathrm{l}$ & $<0.00002 \mathrm{mg} / \mathrm{l}$ & 1 & \\
\hline diebsin $60.57-1$ & $0.000012 \mathrm{mg} / \mathrm{l}$ & $<0.00001 \mathrm{mg} / \mathrm{l}$ & $0.000012 \mathrm{mg} / \mathrm{l}$ & $<0.00001 \mathrm{mg} / \mathrm{l}$ & 1 & \\
\hline adoulen $195968-8$ & $<0.00001 \mathrm{mg} / \mathrm{l}$ & $<0.00001 \mathrm{mg} / 1$ & $<0.00001 \mathrm{mg} / \mathrm{l}$ & $<0.00001 \mathrm{mg} / \mathrm{l}$ & 1 & \\
\hline $33213-65-9$ & $0.000012 \mathrm{mg} / 1$ & $<0.00001 \mathrm{mg} / \mathrm{l}$ & $0.000012 \mathrm{mg} / 1$ & $<0.00001 \mathrm{mg} / \mathrm{l}$ & 1 & \\
\hline acteollenen eulase 1031.078 & $<0.00001 \mathrm{mg} / \mathrm{l}$ & $<0.00001 \mathrm{mg} / \mathrm{l}$ & $<0.00001 \mathrm{mg} / \mathrm{l}$ & $<0.00001 \mathrm{mg} / \mathrm{l}$ & 1 & \\
\hline odrin $72-20-8$ & $<0.00005 \mathrm{mg} / \mathrm{l}$ & $<0.00005 \mathrm{mg} / \mathrm{l}$ & $<0.00005 \mathrm{mg} / \mathrm{l}$ & $<0.00005 \mathrm{mg} / 1$ & 1 & \\
\hline 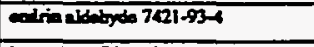 & $<0.00001 \mathrm{mg} / \mathrm{l}$ & $<0.00001 \mathrm{mg} / \mathrm{l}$ & $<0.00001 \mathrm{mg} / \mathrm{l}$ & $<0.00001 \mathrm{mg} / \mathrm{l}$ & 1 & \\
\hline bependiber $76-44$ & $<0.00001 \mathrm{mg} / \mathrm{l}$ & $<0.00001 \mathrm{mg} / \mathrm{l}$ & $<0.00001 \mathrm{mg} / \mathrm{l}$ & $<0.00001 \mathrm{mg} / 1$ & 1 & $=$ \\
\hline 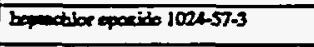 & $<0.00001 \mathrm{mg} / \mathrm{l}$ & $<0.00001 \mathrm{mg} / \mathrm{l}$ & $<0.00001 \mathrm{mg} / \mathrm{l}$ & $<0.00001 \mathrm{mg} / \mathrm{l}$ & 1 & \\
\hline $000+0000001-35-2$ & $<0.0002 \mathrm{mg} / \mathrm{l}$ & $<0.0002 \mathrm{mg} / \mathrm{l}$ & $<0.0002 \mathrm{mg} / 1$ & $<0.0002 \mathrm{mg} / 1$ & 1 & \\
\hline ato-8HC 319846 & $<0.00001 \mathrm{mg} / \mathrm{l}$ & $<0.00001 \mathrm{mg} / \mathrm{l}$ & $<0.00001 \mathrm{mg} / \mathrm{l}$ & $<0.00001 \mathrm{mg} / 1$ & 1 & \\
\hline $5=-8 H C$ 319-85-7 & $<0.00001 \mathrm{mg} / 1$ & $<0.00001 \mathrm{mg} / \mathrm{l}$ & $<0.00001 \mathrm{mg} / \mathrm{l}$ & $<0.00001 \mathrm{mg} / \mathrm{l}$ & 1 & \\
\hline delofic 319868 & $<0.00001 \mathrm{mg} / \mathrm{l}$ & $<0.00001 \mathrm{mg} / 1$ & $<0.00001 \mathrm{mg} / \mathrm{l}$ & $<0.00001 \mathrm{mg} / \mathrm{l}$ & 1 & \\
\hline $00-8$ HC 58.898 & $<0.00001 \mathrm{mg} / \mathrm{l}$ & $<0.00001 \mathrm{mg} / \mathrm{l}$ & $<0.00001 \mathrm{mg} / \mathrm{l}$ & $<0.00001 \mathrm{mg} / \mathrm{I}$ & 1 & \\
\hline 2 adboropheod 95-57. & $<0.030 \mathrm{mg} / \mathrm{l}$ & $<0.030 \mathrm{mg} / \mathrm{l}$ & $<0.030 \mathrm{mg} / \mathrm{l}$ & $<0.030 \mathrm{mg} / \mathrm{l}$ & 1 & $\therefore$ \\
\hline Arodor $120253460-219$ & $<0.0005 \mathrm{mg} / \mathrm{l}$ & $<0.0005 \mathrm{mg} / \mathrm{ll}$ & $<0.0005 \mathrm{mg} / \mathrm{l}$ & $<0.0005 \mathrm{mg} / \mathrm{l}$ & 1 & \\
\hline Anoctor-125111097-691 & $<0.0005 \mathrm{mg} / \mathrm{l}$ & $<0.0005 \mathrm{mg} / 1$ & $<0.0005 \mathrm{mg} / \mathrm{l}$ & $<0.0005 \mathrm{mg} / \mathrm{l}$ & 1 & \\
\hline Arodion-1201 11104-22-2 & $<0.0005 \mathrm{mg} / \mathrm{l}$ & $<0.0005 \mathrm{mg} / \mathrm{l}$ & $<0.0005 \mathrm{mg} / \mathrm{l}$ & $<0.0005 \mathrm{mg} / \mathrm{l}$ & 1 & \\
\hline Arodor-1232 III11-16-3 & $<0.0005 \mathrm{mg} / \mathrm{l}$ & $<0.0005 \mathrm{mg} / \mathrm{l}$ & $<0.0005 \mathrm{mg} / 1$ & $<0.0005 \mathrm{mg} / \mathrm{l}$ & 1 & \\
\hline Aroctor-1248 12572-29-6 & $<0.0005 \mathrm{mg} / \mathrm{l}$ & $<0.0005 \mathrm{mg} / \mathrm{l}$ & $<0.0005 \mathrm{mg} / \mathrm{l}$ & $<0.0005 \mathrm{mg} / \mathrm{l}$ & 1 & \\
\hline Aroctor-1250 I1100682-5 & $<0.0005 \mathrm{mg} / \mathrm{l}$ & $<0.0005 \mathrm{mg} / \mathrm{l}$ & $<0.0005 \mathrm{mg} / \mathrm{l}$ & $<0.0005 \mathrm{mg} / \mathrm{l}$ & 1 & \\
\hline Arodon $101612574-11-2$ & $<0.0005 \mathrm{mg} / 1$ & $<0.0005 \mathrm{mg} / \mathrm{l}$ & $<0.0005 \mathrm{mg} / \mathrm{l}$ & $<0.0005 \mathrm{mg} / 1$ & 1 & \\
\hline Creande & $<0.010 \mathrm{mg} / \mathrm{l}$ & $<0.010 \mathrm{mg} / \mathrm{l}$ & $<0.010 \mathrm{mg} / 1$ & $<0.010 \mathrm{mg} / 1$ & 1 & \\
\hline \begin{tabular}{|l|} 
Aordinin \\
\end{tabular} & $<0.010 \mathrm{mg} / 1$ & $<0.010 \mathrm{mg} / \mathrm{l}$ & $<0.010 \mathrm{mg} / \mathrm{l}$ & $<0.010 \mathrm{mg} / \mathrm{l}$ & 1 & \\
\hline Acylocounte & $<0.010 \mathrm{mg} / 1$ & $<0.010 \mathrm{mg} / \mathrm{l}$ & $<0.010 \mathrm{mg} / 1$ & $<0.010 \mathrm{mg} / 1$ & 1 & \\
\hline 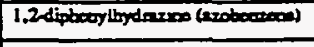 & Reaulls peoding & Resules pending & Results pending & Rasules pending & 1 & \\
\hline & & & & & & \\
\hline & & & & & & \\
\hline & & & & & & \\
\hline & & & & & & \\
\hline & & & & & & \\
\hline & & & & & & \\
\hline & & & & & & \\
\hline & & & & & & \\
\hline & & & & & & \\
\hline & & & & & & \\
\hline & & & & & & \\
\hline & & & & & & \\
\hline & & & & & & \\
\hline & & & & & & \\
\hline
\end{tabular}


VI. Dichergo Lnformation (continued from pago 3 of Form 2F)

Part A - You must provido the results of as least one enalysia for every pollutent in this cuble. Complese one lable for ench ouffall. See instructions for additional delailt.

\begin{tabular}{|c|c|c|c|c|c|c|}
\hline \multirow{2}{*}{$\begin{array}{l}\text { Pollutant } \\
\text { and } \\
\text { CAs Number } \\
\text { (if avnilible) }\end{array}$} & \multicolumn{2}{|c|}{$\begin{array}{l}\text { Maximum Valuea } \\
\text { (inchude units) }\end{array}$} & \multicolumn{2}{|c|}{$\begin{array}{l}\text { Avernge Values } \\
\text { (inchude unita) }\end{array}$} & \multirow{2}{*}{$\begin{array}{l}\text { Number } \\
\text { of } \\
\text { Storm } \\
\text { Eveos } \\
\text { Saropled }\end{array}$} & \multirow[b]{2}{*}{ Souree of Poithmests } \\
\hline & $\begin{array}{l}\text { Greb Sumple } \\
\text { Taken During } \\
\text { Firm } 30 \\
\text { Minutece }\end{array}$ & $\begin{array}{l}\text { Fow-weighted } \\
\text { Componite }\end{array}$ & $\begin{array}{l}\text { Gasb Sumple } \\
\text { Tiken Duriog } \\
\text { Fint } 30 \\
\text { Minurea }\end{array}$ & $\begin{array}{c}\text { Floweweightiod } \\
\text { Comporite }\end{array}$ & & \\
\hline pil and Groese & $<2 \mathrm{mg} / 1$ & & $<2 \mathrm{mg} / \mathrm{l}$ & & 1 & \\
\hline 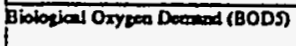 & $<5 \mathrm{mg} / \mathrm{l}$ & $5.9 \mathrm{mg} / 1$ & $<5 \mathrm{mg} / 1$ & $5.9 \mathrm{mg} / \mathrm{l}$ & 1 & \\
\hline Chearial Oxyper Deaned (COD) & $34 \mathrm{mg} / \mathrm{l}$ & $31 \mathrm{mg} / \mathrm{l}$ & $34 \mathrm{mg} / \mathrm{l}$ & $31 \mathrm{mg} / \mathrm{l}$ & 1 & \\
\hline Toul Sappoded Solde (TSS) & $16 \mathrm{mg} / \mathrm{l}$ & $61 \mathrm{mg} / \mathrm{l}$ & $16 \mathrm{mg} / \mathrm{l}$ & $61 \mathrm{mg} / 1$ & 1 & \\
\hline Toual Kjeldabl Nitroger & $1.2 \mathrm{mg} / \mathrm{l}$ & $1.0 \mathrm{mg} / \mathrm{l}$ & $1.2 \mathrm{mg} / \mathrm{l}$ & $1.0 \mathrm{mg} / 1$ & 1 & \\
\hline 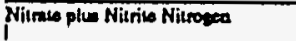 & $1.10 \mathrm{mg} / \mathrm{l}$ & $0.9 \mathrm{mg} / \mathrm{l}$ & $1.0 \mathrm{mg} / \mathrm{l}$ & $0.9 \mathrm{mg} / \mathrm{l}$ & 1 & \\
\hline Toud Plowptonen & $0.20 \mathrm{mg} / \mathrm{l}$ & $0.17 \mathrm{mg} / 1$ & $0.20 \mathrm{mg} / \mathrm{l}$ & $0.17 \mathrm{mg} / \mathrm{l}$ & 1 & \\
\hline pH & Minimus $\quad 7.7$ & Mariond 7.7 & Minimen 7.7 & Meximan 7.7 & & \\
\hline \multicolumn{7}{|c|}{ 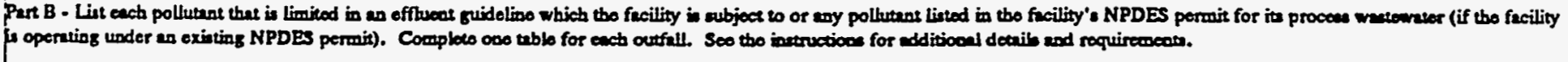 } \\
\hline \multirow{2}{*}{$\begin{array}{l}\text { Pollutant } \\
\text { and } \\
\text { CAS Number } \\
\text { (if aveiliblo) }\end{array}$} & \multicolumn{2}{|c|}{$\begin{array}{l}\text { Merionum Values } \\
\text { (includo units) }\end{array}$} & \multicolumn{2}{|c|}{$\begin{array}{l}\text { Avernse Values } \\
\text { (ioctudo unito) }\end{array}$} & \multirow{2}{*}{$\begin{array}{c}\text { Number } \\
\text { of } \\
\text { Stomm } \\
\text { Eveots } \\
\text { Sampled }\end{array}$} & \multirow[b]{2}{*}{ Sources of Polthertans } \\
\hline & $\begin{array}{l}\text { Onab Sumplo } \\
\text { Takeo During } \\
\text { Fisse } 30 \\
\text { Minute }\end{array}$ & $\begin{array}{l}\text { Flow-weightod } \\
\text { Componive }\end{array}$ & $\begin{array}{l}\text { Onb Semplo } \\
\text { Tekea Durius } \\
\text { Fink } 30 \\
\text { Minued }\end{array}$ & $\begin{array}{l}\text { Frow-wreighted } \\
\text { Comperite }\end{array}$ & & \\
\hline Tximony 740-36-0 & $<0.04 \mathrm{mg} / \mathrm{l}$ & $<0.04 \mathrm{mg} / \mathrm{l}$ & $<0.04 \mathrm{mg} / \mathrm{l}$ & $<0.04 \mathrm{mg} / \mathrm{l}$ & 1 & \\
\hline fyaside & $<0.002 \mathrm{mg} / 1$ & & $<0.002 \mathrm{mg} / \mathrm{l}$ & & 1 & \\
\hline Mmany 7439-976 & $0.0004 \mathrm{mg} / \mathrm{l}$ & $0.0003 \mathrm{mg} / 1$ & $0.0004 \mathrm{mg} / \mathrm{l}$ & $0.0003 \mathrm{mg} / \mathrm{l}$ & I & \\
\hline procole & $0.002 \mathrm{mg} / 1$ & & $0.002 \mathrm{mg} / \mathrm{l}$ & & 1 & \\
\hline etraiven $7782-992$ & $<0.002 \mathrm{mg} / \mathrm{l}$ & $<0.002 \mathrm{mg} / \mathrm{l}$ & $<0.002 \mathrm{mg} / \mathrm{l}$ & $<0.002 \mathrm{mg} / \mathrm{l}$ & 1 & \\
\hline Pulfas & $25 \mathrm{mg} / 1$ & $14 \mathrm{mg} / \mathrm{h}$ & $25 \mathrm{mg} / \mathrm{l}$ & $14 \mathrm{mg} / \mathrm{l}$ & 1 & \\
\hline Purthotenses & $<0.05 \mathrm{mg} / \mathrm{l}$ & $0.13 \mathrm{mg} / \mathrm{l}$ & $<0.05 \mathrm{mg} / \mathrm{l}$ & $0.13 \mathrm{mg} / \mathrm{l}$ & 1 & \\
\hline 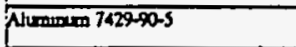 & $0.50 \mathrm{mg} / \mathrm{l}$ & $1.15 \mathrm{mg} / 1$ & $0.50 \mathrm{mg} / \mathrm{l}$ & $1.15 \mathrm{mg} / \mathrm{l}$ & 1 & \\
\hline Arowio 7440-38-2 & $<0.04 \mathrm{mg} / 1$ & $<0.04 \mathrm{mg} / \mathrm{l}$ & $<0.04 \mathrm{mg} / \mathrm{l}$ & $<0.04 \mathrm{mg} / \mathrm{l}$ & 1 & \\
\hline $301000740-128$ & $0.052 \mathrm{mg} / \mathrm{l}$ & $0.037 \mathrm{mg} / \mathrm{l}$ & $0.052 \mathrm{mg} / \mathrm{l}$ & $0.037 \mathrm{mg} / \mathrm{l}$ & 1 & \\
\hline Boplingm 7440-1-7 & $<0.0004 \mathrm{mg} / 1$ & $<0.0004 \mathrm{mg} / 1$ & $<0.0004 \mathrm{mg} / 1$ & $<0.0004 m g / l$ & 1 & \\
\hline Cestriven $740-13-9$ & $<0.004 \mathrm{mg} / 1$ & $<0.004 \mathrm{mg} / \mathrm{l}$ & $<0.004 \mathrm{mg} / \mathrm{l}$ & $<0.004 \mathrm{mg} / \mathrm{l}$ & 1 & \\
\hline artion $7400-70-2$ & $15.2 \mathrm{mg} / \mathrm{l}$ & $12.3 \mathrm{mg} / \mathrm{l}$ & $15.2 \mathrm{mg} / 1$ & $12.3 \mathrm{mg} / \mathrm{l}$ & 1 & \\
\hline Croctium $740-67-3$ & $<0.006 \mathrm{mg} / \mathrm{l}$ & $<0.006 \mathrm{mg} / \mathrm{l}$ & $<0.006 \mathrm{mg} / 1$ & $<0.006 \mathrm{mg} / \mathrm{l}$ & 1 & \\
\hline Cotali $7400-28-4$ & $<0.002 \mathrm{mg} / 1$ & $<0.002 \mathrm{mg} / \mathrm{l}$ & $<0.002 \mathrm{mg} / \mathrm{l}$ & $<0.002 \mathrm{mg} / 1$ & 1 & \\
\hline Coppor 740-502 & $0.010 \mathrm{mg} / \mathrm{l}$ & $0.009 \mathrm{mg} / \mathrm{l}$ & $0.010 \mathrm{mg} / \mathrm{l}$ & $0.009 \mathrm{mg} / \mathrm{l}$ & 1 & \\
\hline 10007439896 & $0.87 \mathrm{mg} / \mathrm{l}$ & $1.59 \mathrm{mg} / 1$ & $0.87 \mathrm{mg} / \mathrm{l}$ & $1.59 \mathrm{mg} / \mathrm{l}$ & 1 & \\
\hline Fod 7439on-1 & $<0.02 \mathrm{mg} / \mathrm{l}$ & $<0.02 \mathrm{mg} / \mathrm{l}$ & $<0.02 \mathrm{mg} / 1$ & $<0.02 \mathrm{mg} / \mathrm{l}$ & 1 & \\
\hline Tubiven 7039-93-2 & $<0.02 \mathrm{mg} / \mathrm{l}$ & $<0.02 \mathrm{mg} / 1$ & $<0.02 \mathrm{mg} / 1$ & $<0.02 \mathrm{mg} / \mathrm{l}$ & 1 & \\
\hline Mapoesm 7/39-9SS & $2.42 \mathrm{mg} / \mathrm{l}$ & $2.15 \mathrm{mg} / \mathrm{l}$ & $2.42 \mathrm{mg} / \mathrm{l}$ & $2.15 \mathrm{mg} / \mathrm{l}$ & 1 & \\
\hline 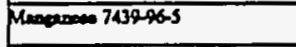 & $0.122 \mathrm{mg} / \mathrm{l}$ & $0.121 \mathrm{mg} / \mathrm{l}$ & $0.122 \mathrm{mg} / 1$ & $0.121 \mathrm{mg} / \mathrm{l}$ & 1 & \\
\hline Matpdocoum 763998.7 & $<0.006 \mathrm{mg} / \mathrm{l}$ & $<0.006 \mathrm{mg} / \mathrm{ll}$ & $<0.006 \mathrm{mg} / \mathrm{l}$ & $<0.006 \mathrm{mg} / \mathrm{l}$ & 1 & \\
\hline Nicted 740002-0 & $<0.008 \mathrm{mg} / \mathrm{l}$ & $<0.008 \mathrm{mg} / \mathrm{l}$ & $<0.008 \mathrm{mg} / \mathrm{l}$ & $<0.008 \mathrm{mg} / \mathrm{l}$ & I & \\
\hline 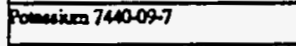 & $3.5 \mathrm{mg} / \mathrm{l}$ & $2.1 \mathrm{mg} / \mathrm{l}$ & $3.5 \mathrm{mg} / 1$ & $2.1 \mathrm{mg} / 1$ & 1 & \\
\hline situr 740-20- & $<0.006 \mathrm{mg} / \mathrm{l}$ & $<0.006 \mathrm{mg} / \mathrm{l}$ & $<0.006 \mathrm{mg} / \mathrm{l}$ & $<0.006 \mathrm{mg} / 1$ & 1 & \\
\hline sodium 7400-20-5 & $1.82 \mathrm{mg} / \mathrm{l}$ & $1.25 \mathrm{mg} / \mathrm{l}$ & $1.82 \mathrm{mg} / 1$ & $1.25 \mathrm{mg} / \mathrm{l}$ & 1 & \\
\hline Fitesion 740-32.6 & $<0.01 \mathrm{mg} / \mathrm{l}$ & $0.02 \mathrm{mg} / \mathrm{l}$ & $<0.01 \mathrm{mg} / \mathrm{l}$ & $0.02 \mathrm{mg} / 1$ & 1 & \\
\hline $2000740-606$ & $0.12 \mathrm{mg} / \mathrm{l}$ & $0.09 \mathrm{mg} / \mathrm{l}$ & $0.12 \mathrm{mg} / \mathrm{l}$ & $0.09 \mathrm{mg} / \mathrm{l}$ & 1 & \\
\hline
\end{tabular}


Contiond from frope.

Pant C. List each poltutant showa on Tablea 2F-2. 2F-3. and 2F4 that you know or bave reason to believe is preacot. See the instruetions for additional details end requireacents. Compl. oxe tuble for each ourfall.

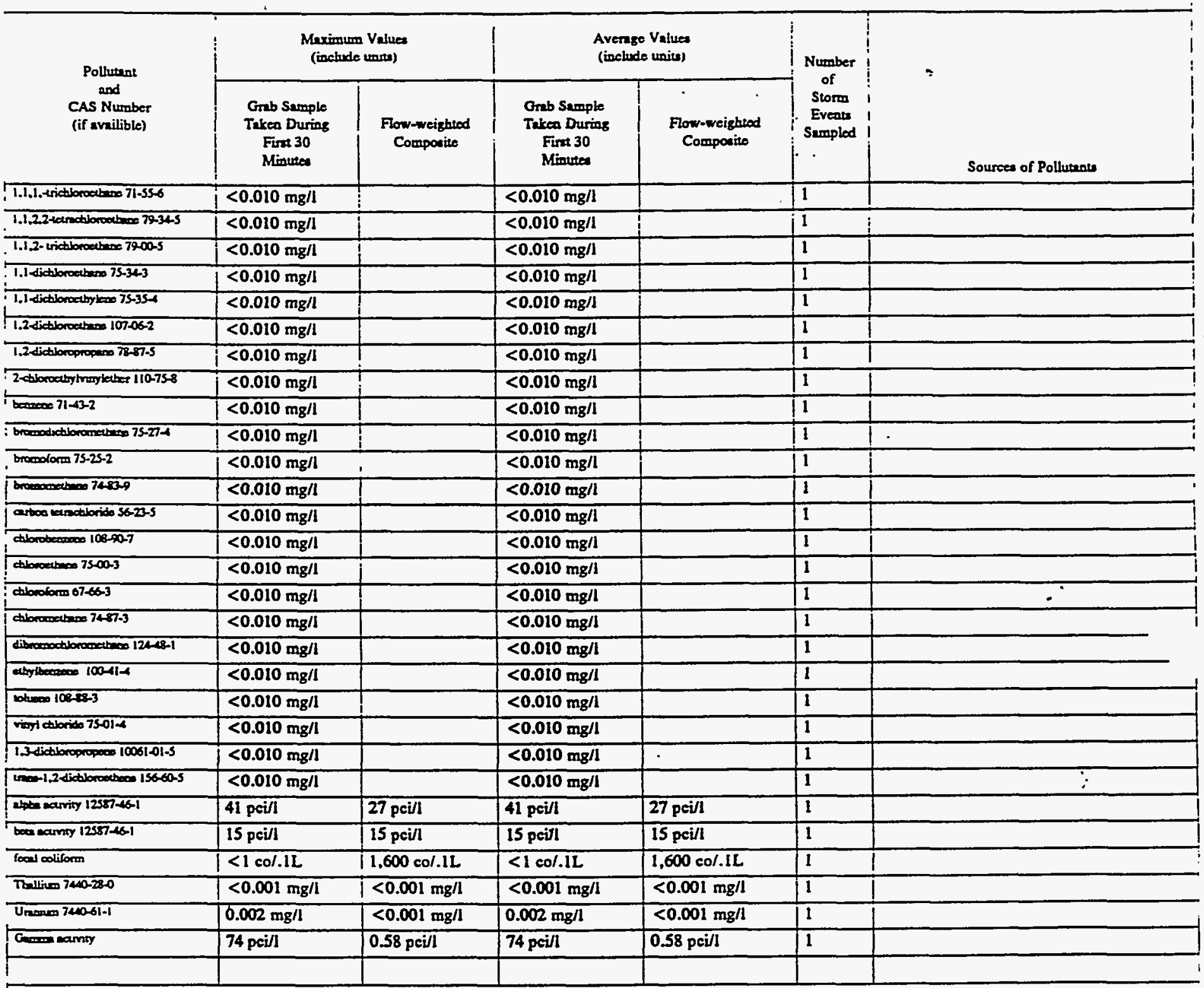

Part D. Provide date for the sorm event(s) which renulted in the maximum values for the flow weighted componite armple.

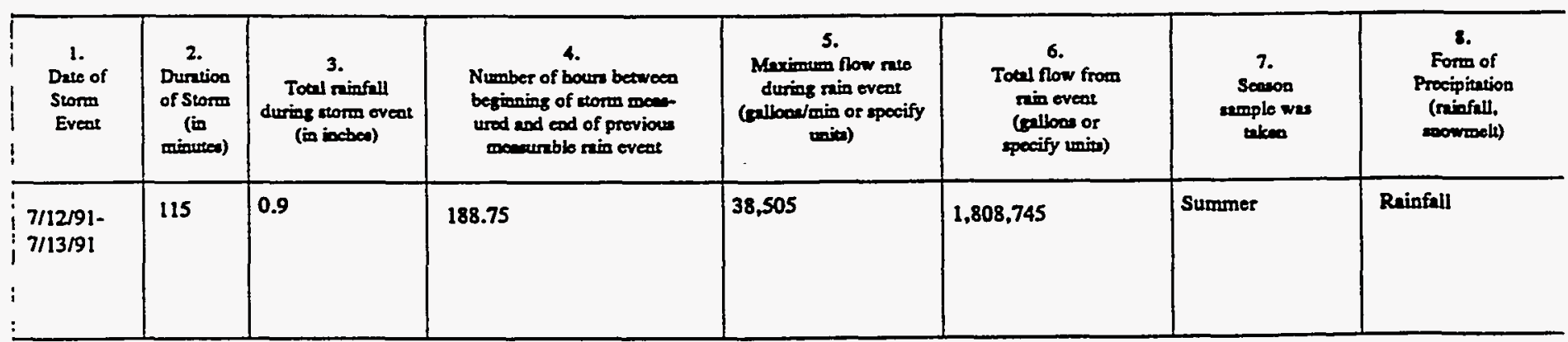

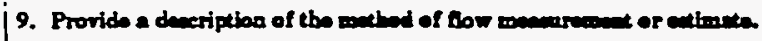

An ISCO 3230 Flow meter was osed to obtain water level readings. This instrument wes calibrated with the normal iry weather flow, if preseat, as letel zero. The flow meter was programmed with the characteristics of the conrejance so that flow rates and total flows conld be calculated by the meter. 
i)utfall 109

Pars B and C Cootinuod

\begin{tabular}{|c|c|c|c|c|c|c|}
\hline \multirow{2}{*}{$\begin{array}{l}\text { Pollutant } \\
\text { and } \\
\text { CAS Number } \\
\text { (if availible) }\end{array}$} & \multicolumn{2}{|c|}{$\begin{array}{l}\text { Maximum Valuee } \\
\text { (includo unita) }\end{array}$} & \multicolumn{2}{|c|}{$\begin{array}{l}\text { Avernge Valuea } \\
\text { (include vaits) }\end{array}$} & \multirow{2}{*}{$\begin{array}{c}\text { Number } \\
\text { of } \\
\text { Sloma } \\
\text { Eveats } \\
\text { Simpled }\end{array}$} & \multirow[b]{2}{*}{ Sources of Pollutants } \\
\hline & $\begin{array}{c}\text { Grab Sromplo } \\
\text { Takec During } \\
\text { Fint } 30 \\
\text { Minute }\end{array}$ & $\begin{array}{l}\text { Fow-weighted } \\
\text { Comporive }\end{array}$ & $\begin{array}{l}\text { Grab Sumplo } \\
\text { Teken Duriog } \\
\text { Fint } 30 \\
\text { Minutes }\end{array}$ & $\begin{array}{l}\text { Flow-weighted } \\
\text { Compoive }\end{array}$ & & \\
\hline Beriven 740-39-3 & $0.0273 \mathrm{mg} / \mathrm{l}$ & $0.282 \mathrm{mg} / \mathrm{l}$ & $0.0273 \mathrm{mg} / 1$ & $0.282 \mathrm{mg} / \mathrm{l}$ & 1 & \\
\hline 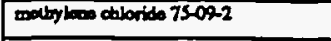 & $<0.010 \mathrm{mg} / \mathrm{l}$ & & $<0.010 \mathrm{mg} / \mathrm{l}$ & & 1 & \\
\hline Letractionentoon $127.18-4$ & $<0.010 \mathrm{mg} / \mathrm{l}$ & & $<0.010 \mathrm{mg} / \mathrm{l}$ & & 1 & \\
\hline iniction eathen $7901-6$ & $<0.010 \mathrm{mg} / \mathrm{l}$ & & $<0.010 \mathrm{mg} / \mathrm{l}$ & & 1 & \\
\hline xyilowe & $<0.010 \mathrm{mg} / \mathrm{l}$ & & $<0.010 \mathrm{mg} / \mathrm{l}$ & & 1 & \\
\hline 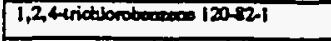 & $<0.010 \mathrm{mg} / \mathrm{l}$ & $<0.010 \mathrm{mg} / \mathrm{l}$ & $<0.010 \mathrm{mg} / 1$ & $<0.010 \mathrm{mg} / \mathrm{l}$ & 1 & \\
\hline 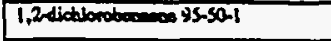 & $<0.010 \mathrm{mg} / \mathrm{l}$ & $<0.010 \mathrm{mg} / \mathrm{l}$ & $<0.010 \mathrm{mg} / \mathrm{l}$ & $<0.010 \mathrm{mg} / \mathrm{l}$ & 1 & \\
\hline 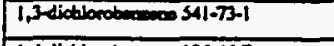 & $<0.010 \mathrm{mg} / \mathrm{l}$ & $<0.010 \mathrm{mg} / \mathrm{l}$ & $<0.010 \mathrm{mg} / \mathrm{l}$ & $<0.010 \mathrm{mg} / \mathrm{l}$ & 1 & \\
\hline 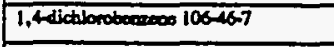 & $<0.010 \mathrm{mg} / \mathrm{l}$ & $<0.010 \mathrm{mg} / \mathrm{l}$ & $<0.010 \mathrm{mg} / \mathrm{l}$ & $<0.010 \mathrm{mg} / \mathrm{l}$ & 1 & \\
\hline 2.4dinuroudienes 121.142 & $<0.010 \mathrm{mg} / \mathrm{l}$ & $<0.010 \mathrm{mg} / \mathrm{l}$ & $<0.010 \mathrm{mg} / \mathrm{l}$ & $<0.010 \mathrm{mg} / 1$ & 1 & \\
\hline 20-dinitroloheros $606-20-2$ & $<0.010 \mathrm{mg} / \mathrm{l}$ & $<0.010 \mathrm{mg} / 1$ & $<0.010 \mathrm{mg} / \mathrm{l}$ & $<0.010 \mathrm{mg} / \mathrm{l}$ & 1 & \\
\hline 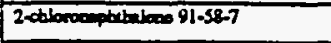 & $<0.010 \mathrm{mg} / \mathrm{l}$ & $<0.010 \mathrm{mg} / \mathrm{l}$ & $<0.010 \mathrm{mg} / \mathrm{l}$ & $<0.010 \mathrm{mg} / \mathrm{l}$ & 1. & \\
\hline 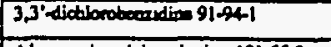 & $<0.010 \mathrm{mg} / \mathrm{l}$ & $<0.010 \mathrm{mg} / \mathrm{l}$ & $<0.010 \mathrm{mg} / \mathrm{l}$ & $<0.010 \mathrm{mg} / \mathrm{l}$ & 1 & \\
\hline 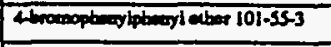 & $<0.010 \mathrm{mg} / \mathrm{l}$ & $<0.010 \mathrm{mg} / \mathrm{l}$ & $<0.010 \mathrm{mg} / \mathrm{l}$ & $<0.010 \mathrm{mg} / \mathrm{l}$ & 1 & \\
\hline 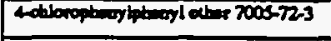 & $<0.010 \mathrm{mg} / \mathrm{l}$ & $<0.010 \mathrm{mg} / \mathrm{l}$ & $<0.010 \mathrm{mg} / 1$ & $<0.010 \mathrm{mg} / \mathrm{l}$ & 1 & \\
\hline 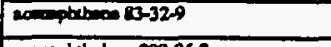 & $<0.010 \mathrm{mg} / \mathrm{l}$ & $<0.010 \mathrm{mg} / \mathrm{l}$ & $<0.010 \mathrm{mg} / \mathrm{l}$ & $<0.010 \mathrm{mg} / \mathrm{l}$ & 1 & \\
\hline 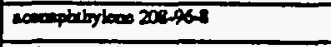 & $<0.010 \mathrm{mg} / \mathrm{l}$ & $<0.010 \mathrm{mg} / 1$ & $<0.010 \mathrm{mg} / \mathrm{l}$ & $<0.010 \mathrm{mg} / \mathrm{l}$ & 1 & \\
\hline $120-127$ & $<0.010 \mathrm{mg} / 1$ & $<0.010 \mathrm{mg} / \mathrm{t}$ & $<0.010 \mathrm{mg} / \mathrm{l}$ & $<0.010 \mathrm{mg} / \mathrm{l}$ & 1 & \\
\hline Didisen 9207.5 & $<0.030 \mathrm{mg} / \mathrm{h}$ & $<0.030 \mathrm{mg} / \mathrm{I}$ & $<0.030 \mathrm{mg} / \mathrm{l}$ & $<0.030 \mathrm{mg} / \mathrm{h}$ & 1 & \\
\hline 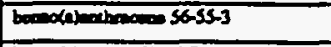 & $<0.010 \mathrm{mg} / 1$ & $<0.010 \mathrm{mg} / 1$ & $<0.010 \mathrm{mg} / 1$ & $<0.010 \mathrm{mg} / \mathrm{l}$ & 1 & \\
\hline 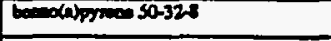 & $<0.010 \mathrm{mg} / \mathrm{l}$ & $<0.010 \mathrm{mg} / \mathrm{l}$ & $<0.010 \mathrm{mg} / \mathrm{l}$ & $<0.010 \mathrm{mg} / \mathrm{l}$ & 1 & \\
\hline 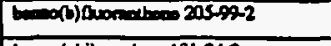 & $<0.010 \mathrm{mg} / \mathrm{l}$ & $<0.010 \mathrm{mg} / \mathrm{l}$ & $<0.010 \mathrm{mg} / \mathrm{l}$ & $<0.010 \mathrm{mg} / 1$ & 1 & \\
\hline beodedi) $191-24-2$ & $<0.010 \mathrm{mg} / \mathrm{l}$ & $<0.010 \mathrm{mg} / 1$ & $<0.010 \mathrm{mg} / \mathrm{l}$ & $<0.010 \mathrm{mg} / \mathrm{l}$ & 1 & \\
\hline 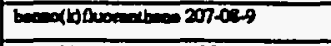 & $<0.010 \mathrm{mg} / 1$ & $<0.010 \mathrm{mg} / 1$ & $<0.010 \mathrm{mg} / \mathrm{l}$ & $<0.010 \mathrm{mg} / \mathrm{l}$ & 1 & \\
\hline 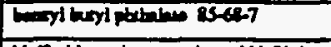 & $<0.010 \mathrm{mg} / \mathrm{l}$ & $<0.010 \mathrm{mg} / \mathrm{l}$ & $<0.010 \mathrm{mg} / 1$ & $<0.010 \mathrm{mg} / \mathrm{l}$ & 1 & \\
\hline 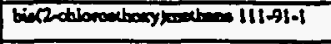 & $<0.010 \mathrm{mg} / \mathrm{l}$ & $<0.010 \mathrm{mg} / \mathrm{l}$ & $<0.010 \mathrm{mg} / \mathrm{l}$ & $<0.010 \mathrm{mg} / \mathrm{l}$ & 1 & $\because$ \\
\hline 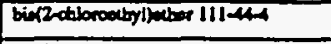 & $<0.010 \mathrm{mg} / 1$ & $<0.010 \mathrm{mg} / \mathrm{l}$ & $<0.010 \mathrm{mg} / 1$ & $<0.010 \mathrm{mg} / \mathrm{l}$ & 1 & \\
\hline 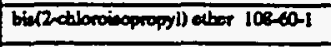 & $<0.010 \mathrm{mg} / \mathrm{l}$ & $<0.010 \mathrm{mg} / \mathrm{I}$ & $<0.010 \mathrm{mg} / 1$ & $<0.010 \mathrm{mg} / \mathrm{l}$ & 1 & \\
\hline 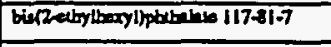 & $<0.010 \mathrm{mg} / \mathrm{l}$ & $<0.010 \mathrm{mg} / 1$ & $<0.010 \mathrm{mg} / \mathrm{l}$ & $<0.010 \mathrm{mg} / \mathrm{l}$ & 1 & \\
\hline armoses 21801-9 & $<0.010 \mathrm{mg} / \mathrm{l}$ & $<0.010 \mathrm{mg} / \mathrm{l}$ & $<0.010 \mathrm{mg} / \mathrm{l}$ & $<0.010 \mathrm{mg} / \mathrm{l}$ & 1 & \\
\hline difoteryl potalates 2474-2 & $0.046 \mathrm{mg} / \mathrm{l}$ & $0.061 \mathrm{mg} / \mathrm{l}$ & $0.046 \mathrm{mg} / \mathrm{l}$ & $0.061 \mathrm{mg} / \mathrm{l}$ & 1 & \\
\hline 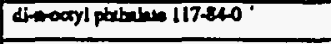 & $<0.010 \mathrm{mg} / \mathrm{l}$ & $<0.010 \mathrm{mg} / 1$ & $<0.010 \mathrm{mg} / 1$ & $<0.010 \mathrm{mg} / \mathrm{l}$ & 1 & \\
\hline 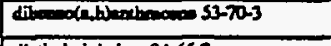 & $<0.010 \mathrm{mg} / \mathrm{l}$ & $<0.010 \mathrm{mg} / \mathrm{l}$ & $<0.010 \mathrm{mg} / 1$ & $<0.010 \mathrm{mg} / 1$ & 1 & \\
\hline 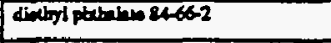 & $<0.010 \mathrm{mg} / \mathrm{l}$ & $<0.010 \mathrm{mg} / \mathrm{l}$ & $<0.010 \mathrm{mg} / \mathrm{l}$ & $<0.010 \mathrm{mg} / \mathrm{l}$ & 1 & \\
\hline 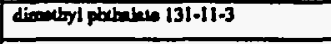 & $<0.010 \mathrm{mg} / 1$ & $<0.010 \mathrm{mg} / \mathrm{l}$ & $<0.010 \mathrm{mg} / \mathrm{l}$ & $<0.010 \mathrm{mg} / \mathrm{l}$ & 1 & \\
\hline shomentacose $206-140$ & $<0.010 \mathrm{mg} / \mathrm{l}$ & $<0.010 \mathrm{mg} / \mathrm{l}$ & $<0.010 \mathrm{mg} / 1$ & $<0.010 \mathrm{mg} / \mathrm{h}$ & 1 & \\
\hline Thooveos 85-73-7 & $<0.010 \mathrm{mg} / \mathrm{l}$ & $<0.010 \mathrm{mg} / \mathrm{s}$ & $<0.010 \mathrm{mg} / 1$ & $<0.010 \mathrm{mg} / 1$ & 1 & \\
\hline $112-741$ & $<0.010 \mathrm{mg} / \mathrm{l}$ & $<0.010 \mathrm{mg} / \mathrm{l}$ & $<0.010 \mathrm{mg} / \mathrm{l}$ & $<0.010 \mathrm{mg} / \mathrm{l}$ & 1 & \\
\hline 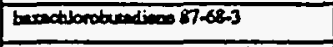 & $<0.010 \mathrm{mg} / \mathrm{l}$ & $<0.010 \mathrm{mg} / \mathrm{l}$ & $<0.010 \mathrm{mg} / \mathrm{l}$ & $<0.010 \mathrm{mg} / \mathrm{l}$ & 1 & \\
\hline 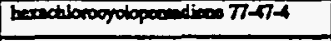 & $<0.010 \mathrm{mg} / \mathrm{l}$ & $<0.010 \mathrm{mg} / \mathrm{l}$ & $<0.010 \mathrm{mg} / \mathrm{l}$ & $<0.010 \mathrm{mg} / 1$ & 1 & \\
\hline 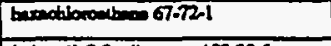 & $<0.010 \mathrm{mg} / 1$ & $<0.010 \mathrm{mg} / 1$ & $<0.010 \mathrm{mg} / 1$ & $<0.010 \mathrm{mg} / \mathrm{l}$ & 1 & \\
\hline 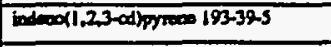 & $<0.010 \mathrm{mg} / \mathrm{l}$ & $<0.010 \mathrm{mg} / \mathrm{l}$ & $<0.010 \mathrm{mg} / \mathrm{l}$ & $<0.010 \mathrm{mg} / \mathrm{l}$ & 1 & \\
\hline incoponoced 72.59-1 & $<0.010 \mathrm{mg} / \mathrm{l}$ & $<0.010 \mathrm{mg} / \mathrm{l}$ & $<0.010 \mathrm{mg} / \mathrm{l}$ & $<0.010 \mathrm{mg} / \mathrm{l}$ & 1 & \\
\hline 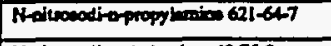 & $<0.010 \mathrm{mg} / \mathrm{l}$ & $<0.010 \mathrm{mg} / 1$ & $<0.010 \mathrm{mg} / 1$ & $<0.010 \mathrm{mg} / 1$ & 1 & \\
\hline 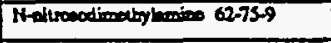 & $<0.010 \mathrm{mg} / \mathrm{l}$ & $<0.010 \mathrm{mg} / \mathrm{l}$ & $<0.010 \mathrm{mg} / \mathrm{l}$ & $<0.010 \mathrm{mg} / 1$ & 1 & \\
\hline 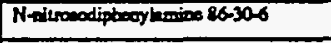 & $<0.010 \mathrm{mg} / \mathrm{I}$ & $<0.010 \mathrm{mg} / \mathrm{l}$ & $<0.010 \mathrm{mg} / \mathrm{l}$ & $<0.010 \mathrm{mg} / 1$ & 1 & \\
\hline 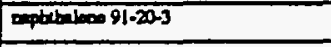 & $<0.010 \mathrm{mg} / \mathrm{l}$ & $<0.010 \mathrm{mg} / \mathrm{l}$ & $<0.010 \mathrm{mg} / \mathrm{l}$ & $<0.010 \mathrm{mg} / \mathrm{l}$ & 1 & \\
\hline 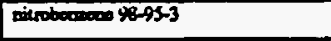 & $<0.010 \mathrm{mg} / \mathrm{l}$ & $<0.010 \mathrm{mg} / \mathrm{l}$ & $<0.010 \mathrm{mg} / \mathrm{l}$ & $<0.010 \mathrm{mg} / 1$ & 1 & \\
\hline 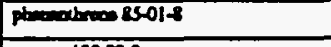 & $<0.010 \mathrm{mg} / \mathrm{l}$ & $<0.010 \mathrm{mg} / \mathrm{l}$ & $<0.010 \mathrm{mg} / \mathrm{l}$ & $<0.010 \mathrm{mg} / 1$ & 1 & \\
\hline promos 122000 & $<0.010 \mathrm{mg} / \mathrm{l}$ & $<0.010 \mathrm{mg} / \mathrm{l}$ & $<0.010 \mathrm{mg} / \mathrm{l}$ & $<0.010 \mathrm{mg} / \mathrm{l}$ & 1 & \\
\hline 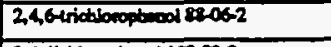 & $<0.030 \mathrm{mg} / \mathrm{I}$ & $<0.030 \mathrm{mg} / 1$ & $<0.030 \mathrm{mg} / \mathrm{l}$ & $<0.030 \mathrm{mg} / 1$ & 1 & \\
\hline 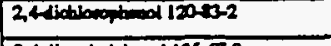 & $<0.030 \mathrm{mg} / \mathrm{I}$ & $<0.030 \mathrm{mg} / \mathrm{l}$ & $<0.030 \mathrm{mg} / \mathrm{l}$ & $<0.030 \mathrm{mg} / 1$ & 1 & \\
\hline 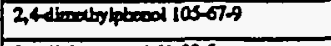 & $<0.030 \mathrm{mg} / \mathrm{l}$ & $<0.030 \mathrm{mg} / \mathrm{l}$ & $<0.030 \mathrm{mg} / \mathrm{l}$ & $<0.030 \mathrm{mg} / \mathrm{l}$ & 1 & \\
\hline 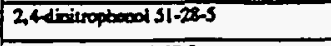 & $<0.030 \mathrm{mg} / \mathrm{l}$ & $<0.030 \mathrm{mg} / \mathrm{l}$ & $<0.030 \mathrm{mg} / 1$ & $<0.030 \mathrm{mg} / \mathrm{l}$ & 1 & \\
\hline
\end{tabular}


Outrall 109

\begin{tabular}{|c|c|c|c|c|c|c|}
\hline \multirow{2}{*}{$\begin{array}{l}\text { Pollumant } \\
\text { and } \\
\text { CAS Number } \\
\text { (if availible) }\end{array}$} & \multicolumn{2}{|c|}{$\begin{array}{l}\text { Maximum Valuea } \\
\text { (inchedo uniss) }\end{array}$} & \multicolumn{2}{|c|}{$\begin{array}{l}\text { Aversese Values } \\
\text { (ixctudo unia) }\end{array}$} & \multirow{2}{*}{$\begin{array}{c}\text { Nuraber } \\
\text { of } \\
\text { Storm } \\
\text { Eveas } \\
\text { Semplod }\end{array}$} & \multirow[b]{2}{*}{ Sources of Pollutents } \\
\hline & $\begin{array}{l}\text { Grab Sumple } \\
\text { Tukea During } \\
\text { Firz } 30 \\
\text { Minute }\end{array}$ & $\begin{array}{l}\text { Flow-weighted } \\
\text { Comporite }\end{array}$ & $\begin{array}{l}\text { Greb Sumple } \\
\text { Tuken Duriag } \\
\text { First } 30 \\
\text { Minutex }\end{array}$ & $\begin{array}{l}\text { Flow-weighted } \\
\text { Componite }\end{array}$ & & \\
\hline 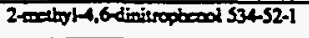 & $<0.030 \mathrm{mg} / \mathrm{l}$ & $<0.030 \mathrm{mg} / \mathrm{h}$ & $<0.030 \mathrm{mg} / \mathrm{l}$ & $<0.030 \mathrm{mg} / 1$ & 1 & \\
\hline 2 -fitropbrood 88.75-5 & $<0.030 \mathrm{mg} / \mathrm{l}$ & $<0.030 \mathrm{mg} / \mathrm{l}$ & $<0.030 \mathrm{mg} / \mathrm{l}$ & $<0.030 \mathrm{mg} / \mathrm{l}$ & 1 & \\
\hline 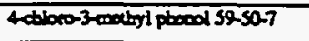 & $<0.030 \mathrm{mg} / 1$ & $<0.030 \mathrm{mg} / \mathrm{l}$ & $<0.030 \mathrm{mg} / \mathrm{l}$ & $<0.030 \mathrm{mg} / \mathrm{l}$ & 1 & \\
\hline 4 sitophood $100-027$ & $<0.030 \mathrm{mg} / \mathrm{l}$ & $<0.030 \mathrm{mg} / \mathrm{l}$ & $<0.030 \mathrm{mg} / \mathrm{l}$ & $<0.030 \mathrm{mg} / 1$ & 1 & \\
\hline 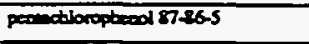 & $<0.030 \mathrm{mg} / \mathrm{l}-$ & $<0.030 \mathrm{mg} / \mathrm{l}$ & $<0.030 \mathrm{mg} / \mathrm{l}$ & $<0.030 \mathrm{mg} / \mathrm{l}$ & 1 & \\
\hline pheod 108-95-2 & $<0.030 \mathrm{mg} / \mathrm{l}$ & $<0.030 \mathrm{mg} / \mathrm{l}$ & $<0.030 \mathrm{mg} / \mathrm{l}$ & $<0.030 \mathrm{mg} / 1$ & 1 & \\
\hline $4,4^{\circ}-D D D 72-548$ & $<0.000004 \mathrm{mg} / \mathrm{l}$ & $<0.000004 \mathrm{mg} / \mathrm{l}$ & $<0.000004 \mathrm{mg} / \mathrm{l}$ & $<0.000004 \mathrm{mg} / \mathrm{l}$ & 1 & \\
\hline 4.4-DDE 72-55-9 & $<0.000004 \mathrm{mg} / \mathrm{l}$ & $<0.000004 \mathrm{mg} / \mathrm{l}$ & $<0.000004 \mathrm{mg} / \mathrm{l}$ & $<0.000004 \mathrm{mg} / 1$ & 1 & \\
\hline $4,4^{2}-00$ Tr $50-29-3$ & $<0.000004 \mathrm{mg} / \mathrm{l}$ & $<0.000004 \mathrm{mg} / \mathrm{l}$ & $<0.000004 \mathrm{mg} / \mathrm{l}$ & $<0.000004 \mathrm{mg} / 1$ & 1 & \\
\hline aldrien $30900-2$ & $<0.000004 \mathrm{mg} / \mathrm{l}$ & $<0.000004 \mathrm{mg} / \mathrm{l}$ & $<0.000004 \mathrm{mg} / \mathrm{l}$ & $<0.000004 \mathrm{mg} / \mathrm{l}$ & 1 & \\
\hline 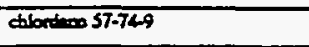 & $<0.00002 \mathrm{mg} / \mathrm{l}$ & $<0.00002 \mathrm{mg} / \mathrm{l}$ & $<0.00002 \mathrm{mg} / 1$ & $<0.00002 \mathrm{mg} / 1$ & 1 & \\
\hline dieturia 60-57-1 & $<0.000004 \mathrm{mg} / \mathrm{l}$ & $<0.000004 \mathrm{mg} / 1$ & $<0.000004 \mathrm{mg} / \mathrm{l}$ & $<0,000004 \mathrm{mg} / \mathrm{l}$ & 1 & \\
\hline condonulfisan $1959-98$ & $<0.000004 \mathrm{mg} / \mathrm{l}$ & $<0.000004 \mathrm{mg} / 1$ & $<0.000004 \mathrm{mg} / \mathrm{l}$ & $<0.000004 \mathrm{mg} / \mathrm{l}$ & 1 & \\
\hline odowalfon II 33213-65-9 & $<0.000004 \mathrm{mg} / \mathrm{l}$ & $<0.000004 \mathrm{mg} / 1$ & $<0.000004 \mathrm{mg} / 1$ & $<0.000004 \mathrm{mg} / \mathrm{l}$ & 1 & \\
\hline 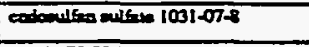 & $<0.000004 \mathrm{mg} / 1$ & $<0.000004 \mathrm{mg} / \mathrm{h}$ & $<0.000004 \mathrm{mg} / \mathrm{l}$ & $<0.000004 \mathrm{mg} / \mathrm{l}$ & 1 & \\
\hline adrin $72-20-8$ & $<0.00005 \mathrm{mg} / 1$ & $<0.00005 \mathrm{mg} / 1$ & $<0.00005 \mathrm{mg} / 1$ & $<0.00005 \mathrm{mg} / \mathrm{l}$ & 1 & \\
\hline 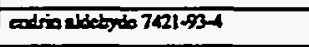 & $<0.000004 \mathrm{mg} / \mathrm{l}$ & $<0.000004 \mathrm{mg} / 1$ & $<0.000004 \mathrm{mg} / \mathrm{l}$ & $<0.000004 \mathrm{mg} / \mathrm{l}$ & 1 & \\
\hline topeaction 76-648 & $<0.000004 \mathrm{mg} / \mathrm{l}$ & $<0.000004 \mathrm{mg} / \mathrm{l}$ & $<0.000004 \mathrm{mg} / \mathrm{l}$ & $<0.000004 \mathrm{mg} / \mathrm{l}$ & 1 & \\
\hline byocther apocith 102457.3 & $<0.000004 \mathrm{mg} / \mathrm{l}$ & $<0.000004 \mathrm{mg} / 1$ & $<0.000004 \mathrm{mg} / 1$ & $<0.000004 \mathrm{mg} / \mathrm{l}$ & 1 & \\
\hline 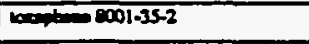 & $<0.0002 \mathrm{mg} / 1$ & $<0.0002 \mathrm{mg} / \mathrm{l}$ & $<0.0002 \mathrm{mg} / 1$ & $<0.0002 \mathrm{mg} / \mathrm{l}$ & 1 & \\
\hline $246-8$ HC 319246 & $<0.000004 \mathrm{mg} / \mathrm{l}$ & $<0.000004 \mathrm{mg} / \mathrm{l}$ & $<0.000004 \mathrm{mg} / \mathrm{l}$ & $<0.000004 \mathrm{mg} / \mathrm{l}$ & 1 & \\
\hline ben-BHC $319-85-7$ & $<0.000004 \mathrm{mg} / \mathrm{l}$ & $<0.000004 \mathrm{mg} / 1$ & $<0.000004 \mathrm{mg} / 1$ & $<0.000004 \mathrm{mg} / \mathrm{l}$ & 1 & \\
\hline delm-8HC 319.868 & $<0.000004 \mathrm{mg} / \mathrm{l}$ & $<0.000004 \mathrm{mg} / 1$ & $<0.000004 \mathrm{mg} / \mathrm{h}$ & $<0.000004 \mathrm{mg} / 1$ & 1 & \\
\hline 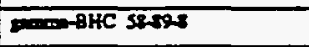 & $<0.00001 \mathrm{mg} / \mathrm{l}$ & $<0.00001 \mathrm{mg} / \mathrm{l}$ & $<0.0001 \mathrm{mg} / \mathrm{l}$ & $<0.0001 \mathrm{mg} / \mathrm{l}$ & 1 & \\
\hline 2-dborophool 93-57-8 & $<0.030 \mathrm{mg} / \mathrm{l}$ & $<0.030 \mathrm{mg} / \mathrm{l}$ & $<0.030 \mathrm{mg} / \mathrm{h}$ & $<0.030 \mathrm{mg} / \mathrm{l}$ & 1 & $\dot{5}$ \\
\hline Arodor-121235346921-9 & $<0.0005 \mathrm{mg} / 1$ & $<0.0005 \mathrm{mg} / \mathrm{l}$ & $<0.0005 \mathrm{mg} / 1$ & $<0.0005 \mathrm{mg} / \mathrm{l}$ & 1 & \\
\hline Aroctor-1234 11097-69-1 & $<0.0005 \mathrm{mg} / \mathrm{l}$ & $<0.0005 \mathrm{mg} / \mathrm{l}$ & $<0.0005 \mathrm{mg} / \mathrm{l}$ & $<0.0005 \mathrm{mg} / \mathrm{l}$ & 1 & \\
\hline Arodor 1221 1110+22-2 & $<0.0005 \mathrm{mg} / \mathrm{l}$ & $<0.0005 \mathrm{mg} / \mathrm{l}$ & $<0.0005 \mathrm{mg} / \mathrm{l}$ & $<0.0005 \mathrm{mg} / \mathrm{l}$ & 1 & \\
\hline Arodon $123211141-16-5$ & $<0.0005 \mathrm{mg} / \mathrm{l}$ & $<0.0005 \mathrm{mg} / \mathrm{l}$ & $<0.0005 \mathrm{mg} / \mathrm{l}$ & $<0.0005 \mathrm{mg} / \mathrm{l}$ & 1 & \\
\hline Arodor $121212072-296$ & $<0.0005 \mathrm{mg} / \mathrm{l}$ & $<0.0005 \mathrm{mg} / \mathrm{l}$ & $<0.0005 \mathrm{mg} / \mathrm{l}$ & $<0.0005 \mathrm{mg} / \mathrm{l}$ & 1 & \\
\hline Arodbar 125011006525 & $<0.0005 \mathrm{mg} / \mathrm{l}$ & $<0.0005 \mathrm{mg} / \mathrm{l}$ & $<0.0005 \mathrm{mg} / \mathrm{l}$ & $<0.0005 \mathrm{mg} / 1$ & 1 & \\
\hline Arodion-1016 12574-11-2 & $<0.0005 \mathrm{mg} / \mathrm{l}$ & $<0.0005 \mathrm{mg} / 1$ & $<0.0005 \mathrm{mg} / \mathrm{h}$ & $<0.0005 \mathrm{mg} / \mathrm{l}$ & 1 & \\
\hline Creonots & $<0.030 \mathrm{mg} / \mathrm{l}$ & $<0.030 \mathrm{mg} / \mathrm{l}$ & $<0.030 \mathrm{mg} / \mathrm{l}$ & $<0.030 \mathrm{mg} / \mathrm{l}$ & 1 & \\
\hline Acolders & $<0.010 \mathrm{mg} / \mathrm{l}$ & $<0.010 \mathrm{mg} / \mathrm{l}$ & $<0.010 \mathrm{mg} / \mathrm{l}$ & $<0.010 \mathrm{mg} / 1$ & 1 & \\
\hline Aospocirib & $<0.010 \mathrm{mg} / 1$ & $<0.010 \mathrm{mg} / \mathrm{l}$ & $<0.010 \mathrm{mg} / \mathrm{l}$ & $<0.010 \mathrm{mg} / \mathrm{l}$ & 1 & \\
\hline 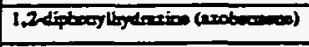 & Recults pending & Resulus pending & Results pending & Rosultu pending & 1 & \\
\hline & & & & & & \\
\hline & & & & & & \\
\hline & & & & & & \\
\hline & & & & & & \\
\hline & & & & & & \\
\hline & & & & & & \\
\hline & & & & & & \\
\hline & & & & & & \\
\hline & & & & & & \\
\hline & & & . & & & \\
\hline & & & & & & \\
\hline & & & & & & \\
\hline & & & & & & \\
\hline & & & & & & \\
\hline
\end{tabular}


VII. Discherge Information (continuod from page 3 of Form 2F

Part A - You must provide the results of at leart one anclysis for every pollutent in this tuble. Complete one table for cach outflll. See instructions for additional details.

\begin{tabular}{|c|c|c|c|c|c|c|}
\hline \multirow{2}{*}{$\begin{array}{l}\text { Pollutant } \\
\text { and } \\
\text { CAs Number } \\
\text { (if availible) }\end{array}$} & \multicolumn{2}{|c|}{$\begin{array}{l}\text { Marimum Valuea } \\
\text { (inchude units) }\end{array}$} & \multicolumn{2}{|c|}{$\begin{array}{l}\text { Avenge Values } \\
\text { (inelude uniu) }\end{array}$} & \multirow{2}{*}{$\begin{array}{l}\text { T } \\
\text { Number } \\
\text { of } \\
\text { Storm } \\
\text { Everts } \\
\text { Sempled }\end{array}$} & \multirow[b]{2}{*}{ Sources of Pollutums } \\
\hline & $\begin{array}{c}\text { Gab Semple } \\
\text { Takeo During } \\
\text { Fint } 30 \\
\text { Minute }\end{array}$ & $\begin{array}{l}\text { Flow-weighted } \\
\text { Comporite }\end{array}$ & $\begin{array}{l}\text { Grab Sample } \\
\text { Takeo During } \\
\text { Fins } 30 \\
\text { Minuteo }\end{array}$ & $\begin{array}{l}\text { Flow-weighted } \\
\text { Compotite }\end{array}$ & & \\
\hline Pil and Granes & $2 \mathrm{mg} / \mathrm{l}$ & & $2 \mathrm{mg} / \mathrm{l}$ & & $\overline{1}$ & \\
\hline Cherical Oxybeo Demend (COD) & $19 \mathrm{mg} / \mathrm{l}$ & $9.4 \mathrm{mg} / \mathrm{l}$ & $19 \mathrm{mg} / \mathrm{l}$ & $9.4 \mathrm{mg} / 1$ & 1 & \\
\hline Toal Sampented Solide (TSS) & $6 \mathrm{mg} / \mathrm{l}$ & $<5 \mathrm{mg} / \mathrm{l}$ & $6 \mathrm{mg} / \mathrm{l}$ & $<5 \mathrm{mg} / \mathrm{l}$ & 1 & \\
\hline Tocal Kjeldabl Nitrosen & $1.8 \mathrm{mg} / \mathrm{l}$ & $<0.2 \mathrm{mg} / \mathrm{l}$ & $1.8 \mathrm{mg} / \mathrm{l}$ & $<0.2 \mathrm{mg} / \mathrm{l}$ & 1 & \\
\hline Nitrate ples Nitrice Nitropea & $0.5 \mathrm{mg} / \mathrm{l}$ & $2.2 \mathrm{mg} / \mathrm{l}$ & $0.5 \mathrm{mg} / \mathrm{l}$ & $2.2 \mathrm{mg} / \mathrm{l}$ & $\overline{1}$ & \\
\hline Toxal Phooptorns & $<0.1 \mathrm{mg} / \mathrm{l}$ & $<0.1 \mathrm{mg} / \mathrm{l}$ & $<0.1 \mathrm{mg} / \mathrm{l}$ & $<0.1 \mathrm{mg} / \mathrm{l}$ & $\bar{I}$ & \\
\hline
\end{tabular}

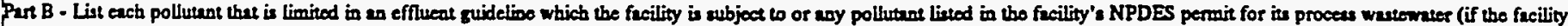

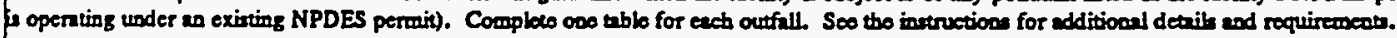

\begin{tabular}{|c|c|c|c|c|c|c|}
\hline \multirow{2}{*}{$\begin{array}{l}\text { Polluteal } \\
\text { eod } \\
\text { CAs Number } \\
\text { (if availible) }\end{array}$} & \multicolumn{2}{|c|}{$\begin{array}{l}\text { Meximusos Valuese } \\
\text { (inchude unitu) }\end{array}$} & \multicolumn{2}{|c|}{$\begin{array}{l}\text { Avorges Vabues } \\
\text { (inchudo units) }\end{array}$} & \multirow{2}{*}{$\begin{array}{l}\text { Nurober } \\
\text { of } \\
\text { Storm } \\
\text { Eveatir } \\
\text { Suropied }\end{array}$} & \multirow{2}{*}{ Sourcee of Pollutente } \\
\hline & 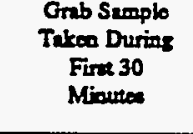 & $\begin{array}{l}\text { From-weighed } \\
\text { Componits }\end{array}$ & $\begin{array}{l}\text { Grab Semple } \\
\text { Takeo During } \\
\text { Firs } 30 \\
\text { Minutee }\end{array}$ & $\begin{array}{l}\text { Fow-weizbred } \\
\text { Composite }\end{array}$ & & \\
\hline Tolimory 740-3650 & $<0.04 \mathrm{mg} / \mathrm{l}$ & $<0.04 \mathrm{mg} / \mathrm{l}$ & $<0.04 \mathrm{mg} / \mathrm{l}$ & $<0.04 \mathrm{mg} / \mathrm{l}$ & 1 & \\
\hline Fyaide & $<0.002 \mathrm{mg} / \mathrm{l}$ & & $<0.002 \mathrm{mg} / \mathrm{l}$ & & 1 & \\
\hline remany 7439-97-6 & $0.0003 \mathrm{mg} / 1$ & $0.0003 \mathrm{mg} / \mathrm{l}$ & $0.0003 \mathrm{mg} /{ }^{*}$ & $0.0003 \mathrm{mg} / 1$ & $\sqrt{1}$ & \\
\hline Promente & $0.003 \mathrm{mg} / \mathrm{l}$ & & $0.003 \mathrm{mg} / 1$ & & 1 & $\therefore$ \\
\hline seloginan $772-192$ & $<0.002 \mathrm{mg} / \mathrm{l}$ & $<0.002 \mathrm{mg} / \mathrm{l}$ & $<0.002 \mathrm{mg} / \mathrm{l}$ & $<0.002 \mathrm{mg} / \mathrm{l}$ & 1 & \\
\hline Sulsero & $34 \mathrm{mg} / \mathrm{l}$ & $24 \mathrm{mg} / \mathrm{l}$ & $34 \mathrm{mg} / \mathrm{l}$ & $24 \mathrm{mg} / \mathrm{l}$ & 1 & \\
\hline surbetensis & $<0.05 \mathrm{mg} / \mathrm{l}$ & $0.06 \mathrm{mg} / \mathrm{l}$ & $<0.05 \mathrm{mg} / \mathrm{l}$ & $0.06 \mathrm{mg} / \mathrm{l}$ & 1 & \\
\hline Ahminm 7429.90 .5 & $0.22 \mathrm{mg} / \mathrm{l}$ & $0.36 \mathrm{mg} / 1$ & $0.22 \mathrm{mg} / 1$ & $0.36 \mathrm{mg} / \mathrm{l}$ & 1 & \\
\hline $1000007440-38-2$ & $<0.04 \mathrm{mg} / \mathrm{l}$ & $<0.04 \mathrm{mg} / \mathrm{l}$ & $<0.04 \mathrm{mg} / \mathrm{l}$ & $<0.04 \mathrm{mg} / 1$ & 1 & \\
\hline $3010007440-128$ & $0.017 \mathrm{mg} / \mathrm{l}$ & $0.037 \mathrm{mg} / \mathrm{l}$ & $0.017 \mathrm{mg} / \mathrm{l}$ & $0.037 \mathrm{mg} / \mathrm{l}$ & 1 & \\
\hline Beryllium 740-41.7 & $<0.0004 \mathrm{mg}^{\prime} / \mathrm{l}$ & $<0.0004 \mathrm{mg} / 1$ & $<0.0004 \mathrm{mg} / \mathrm{l}$ & $<0.0004 \mathrm{mg} / \mathrm{l}$ & 1 & \\
\hline Codmingos 740-43-9 & $<0.004 \mathrm{mg} / \mathrm{l}$ & $0.006 \mathrm{mg} / \mathrm{l}$ & $<0.004 \mathrm{mg} / \mathrm{l}$ & $0.006 \mathrm{mg} / \mathrm{I}$ & 1 & \\
\hline Celoiven 7440-70-2 & $11.6 \mathrm{mg} / 1$ & $17.8 \mathrm{mg} / \mathrm{l}$ & $11.6 \mathrm{mg} / 1$ & $17.8 \mathrm{mg} / \mathrm{l}$ & 1 & \\
\hline Cbramium 740-77.3 & $<0.006 \mathrm{mg} / \mathrm{l}$ & $<0.006 \mathrm{mg} / \mathrm{l}$ & $<0.006 \mathrm{mg} / \mathrm{l}$ & $<0.006 \mathrm{mg} / \mathrm{l}$ & 1 & \\
\hline Cotall 7400-18-4 & $<0.002 \mathrm{mg} / \mathrm{l}$ & $<0.002 \mathrm{mg} / \mathrm{l}$ & $<0.002 \mathrm{mg} / \mathrm{l}$ & $<0.002 \mathrm{mg} / 1$ & 1 & \\
\hline Copper 7440-50-8 & $<0.006 \mathrm{mg} / \mathrm{l}$ & $<0.006 \mathrm{mg} / \mathrm{l}$ & $<0.006 \mathrm{mg} / \mathrm{l}$ & $<0.006 \mathrm{mg} / \mathrm{l}$ & 1 & \\
\hline 7000439896 & $0.45 \mathrm{mg} / \mathrm{l}$ & $0.33 \mathrm{mg} / \mathrm{l}$ & $0.45 \mathrm{mg} / \mathrm{l}$ & $0.33 \mathrm{mg} / 1$ & 1 & \\
\hline mad 7439-92-1 & $<0.02 \mathrm{mg} / \mathrm{l}$ & $<0.02 \mathrm{mg} / \mathrm{l}$ & $<0.02 \mathrm{mg} / 1$ & $<0.02 \mathrm{mg} / 1$ & 1 & \\
\hline Jibiven 7439-93-2 & $<0.02 \mathrm{mg} / 1$ & $<0.02 \mathrm{mg} / 1$ & $<0.02 \mathrm{mg} / \mathrm{l}$ & $<0.02 \mathrm{mg} / 1$ & 1 & \\
\hline Mequecivon 7439-95-4 & $1.55 \mathrm{mg} / \mathrm{l}$ & $3.90 \mathrm{mg} / \mathrm{l}$ & $1.55 \mathrm{mg} / 1$ & $3.90 \mathrm{mg} / \mathrm{l}$ & 1 & \\
\hline Masponece 7439-96-5 & $0.011 \mathrm{mg} / \mathrm{l}$ & $0.011 \mathrm{mg} / 1$ & $0.011 \mathrm{mg} / \mathrm{l}$ & $0.011 \mathrm{mg} / \mathrm{l}$ & $I$ & \\
\hline Molybdoour 743996.7 & $<0.006 \mathrm{mg} / \mathrm{l}$ & $<0.006 \mathrm{mg} / \mathrm{l}$ & $<0.006 \mathrm{mg} / \mathrm{l}$ & $<0.006 \mathrm{mg} / \mathrm{l}$ & 1 & \\
\hline Niotel 1740020 & $<0.008 \mathrm{mg} / \mathrm{l}$ & $<0.008 \mathrm{mg} / \mathrm{l}$ & $<0.008 \mathrm{mg} / \mathrm{l}$ & $<0.008 \mathrm{mg} / \mathrm{l}$ & 1 & \\
\hline Ponming 740009-7 & $1.2 \mathrm{mg} / 1$ & $2.9 \mathrm{mg} / \mathrm{l}$ & $1.2 \mathrm{mg} / \mathrm{l}$ & $2.9 \mathrm{mg} / 1$ & 1 & \\
\hline setwen 740-204 & $<0.006 \mathrm{mg} / \mathrm{l}$ & $<0.006 \mathrm{mg} / \mathrm{l}$ & $<0.006 \mathrm{mg} / \mathrm{l}$ & $<0.006 \mathrm{mg} / \mathrm{h}$ & 1 & \\
\hline Sodiun 7410-20-5 & $0.67 \mathrm{mg} / \mathrm{h}$ & $1.70 \mathrm{mg} / \mathrm{l}$ & $0.67 \mathrm{mg} / 1$ & $1.70 \mathrm{mg} / 1$ & 1 & \\
\hline Titasinom 7460-32-6 & $<0.01 \mathrm{mg} / \mathrm{l}$ & $<0.01 \mathrm{mg} / \mathrm{l}$ & $<0.01 \mathrm{mg} / 1$ & $<0.01 \mathrm{mg} / \mathrm{l}$ & 1 & \\
\hline $26007440-66-6$ & $0.02 \mathrm{mg} / 1$ & $0.02 \mathrm{mg} / \mathrm{l}$ & $0.02 \mathrm{mg} / 1$ & $0.02 \mathrm{mg} / 1$ & 1 & \\
\hline
\end{tabular}


Contloued from front.

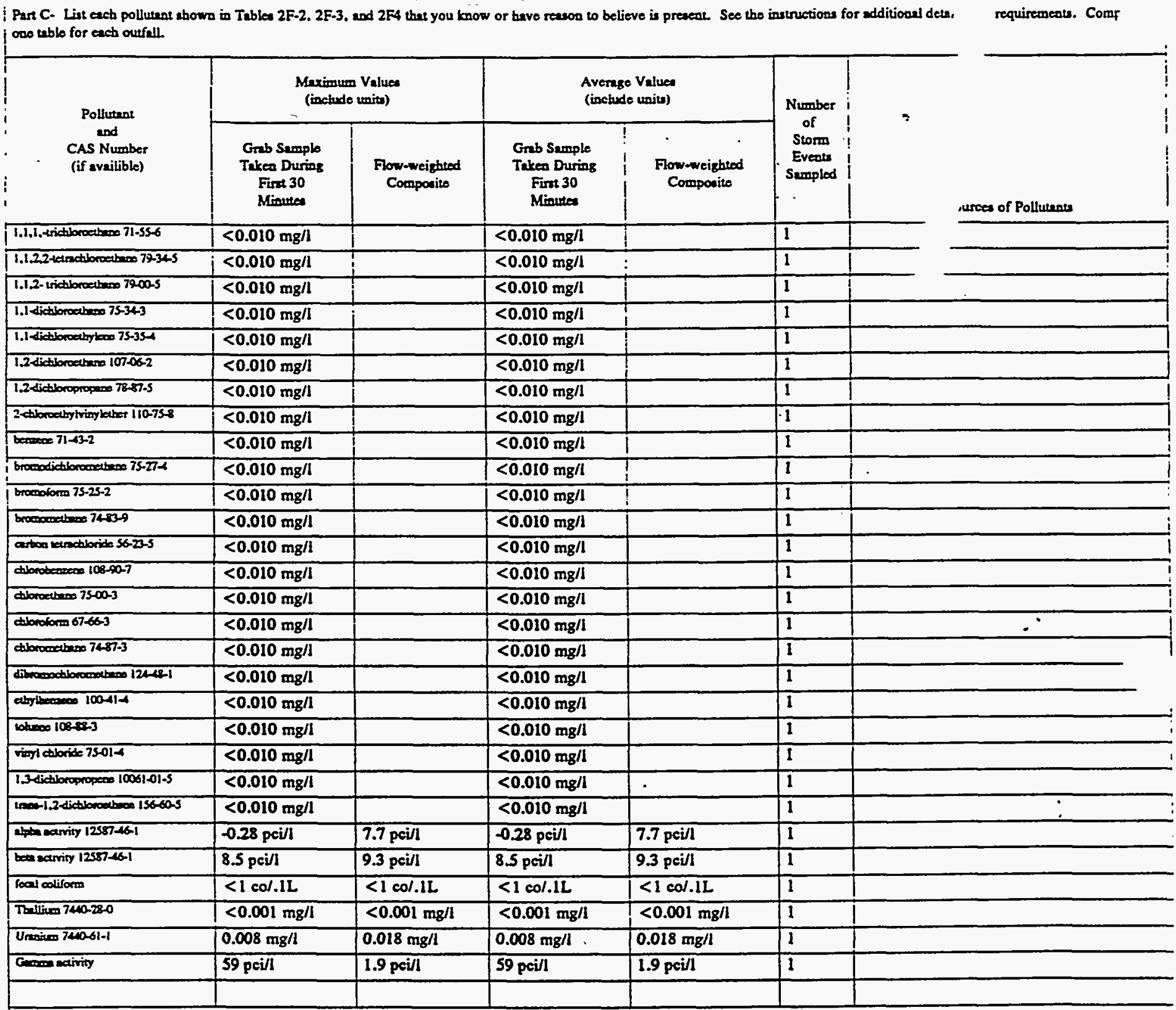

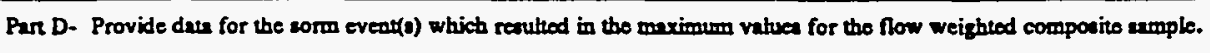

\begin{tabular}{|c|c|c|c|c|c|c|c|}
\hline $\begin{array}{l}1 . \\
\text { Date of } \\
\text { Stom } \\
\text { Event }\end{array}$ & $\begin{array}{l}2 . \\
\text { Durntion } \\
\text { of Storm } \\
\text { (in } \\
\text { minutea) }\end{array}$ & $\begin{array}{l}3 . \\
\text { Totol ninfall } \\
\text { during storm eveot } \\
\text { (in incbes) }\end{array}$ & $\begin{array}{l}4 \text {. } \\
\text { Number of bous between } \\
\text { bezinning of storm meat. } \\
\text { uned end ead of previous } \\
\text { monamblo min eveat }\end{array}$ & $\begin{array}{l}\text { S. } \\
\text { Meximum flow rate } \\
\text { during nin oveat } \\
\text { (sallomimin or spocify } \\
\text { unita) }\end{array}$ & $\begin{array}{c}6 . \\
\text { Total flow from } \\
\text { nim ovent } \\
\text { (aillons or } \\
\text { spocify unitu) }\end{array}$ & $\begin{array}{c}7 . \\
\text { Season } \\
\text { semple was } \\
\text { taked }\end{array}$ & $\begin{array}{l}8 . \\
\text { Form of } \\
\text { Procipitation } \\
\text { (rninfull. } \\
\text { soowmets) }\end{array}$ \\
\hline $\begin{array}{l}7 / 12 / 91- \\
7 / 13 / 91\end{array}$ & 120 & 1.1 & 188.75 & 655 & 12,373 & Summer & Ruinfall \\
\hline
\end{tabular}

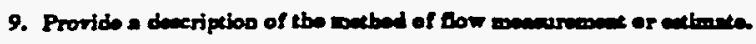

An ISCO 3230 Flow meter was used to obtain water level reatings. This instrument was calibrated with the normal dry weather flow, if present, as level zero. The fow meter was programmed with the characteristics of the conveyance so that flow rates and total flows conld be calculated by the meter. 
Outfall 110

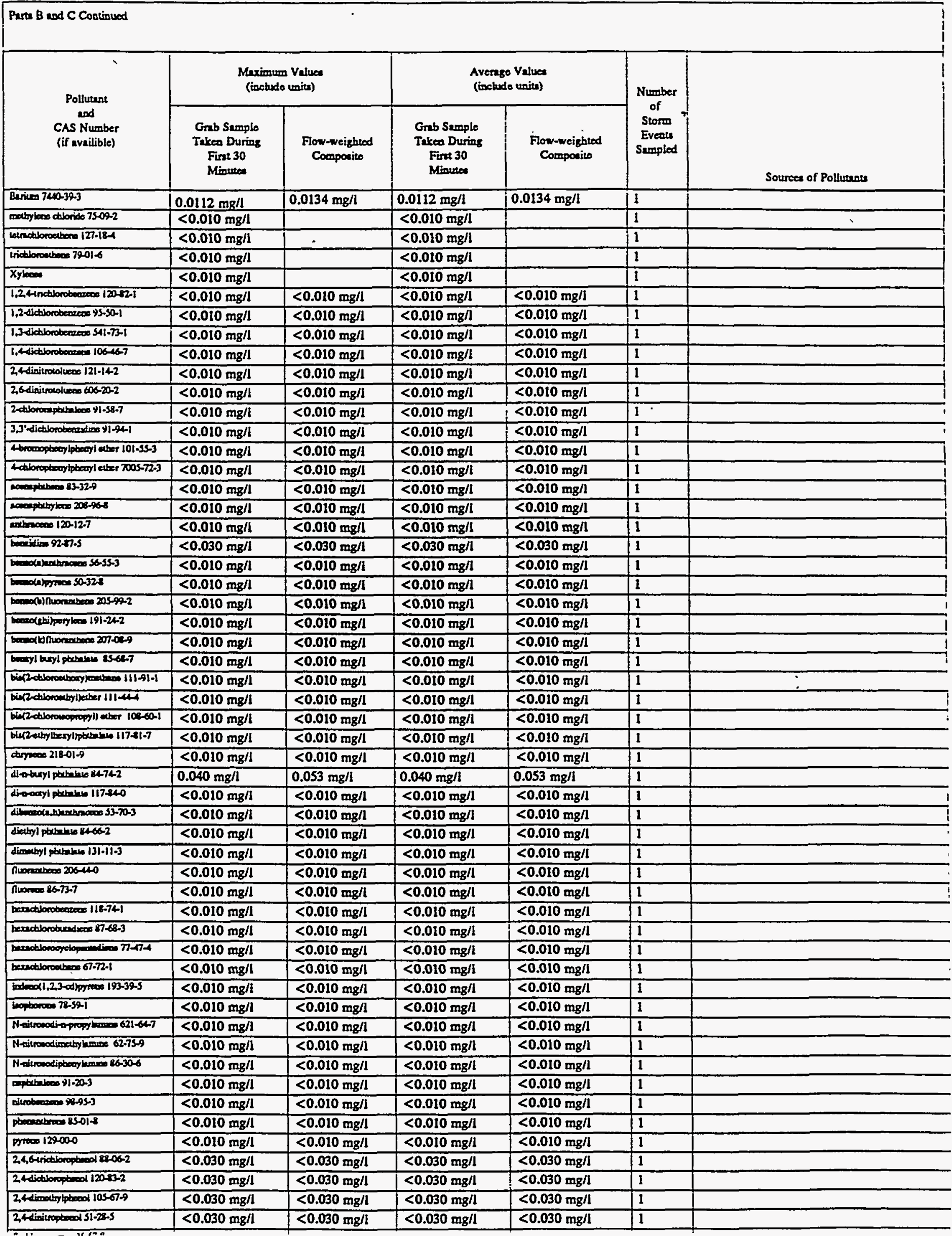


Outfall 110

Paru B and C Continuod

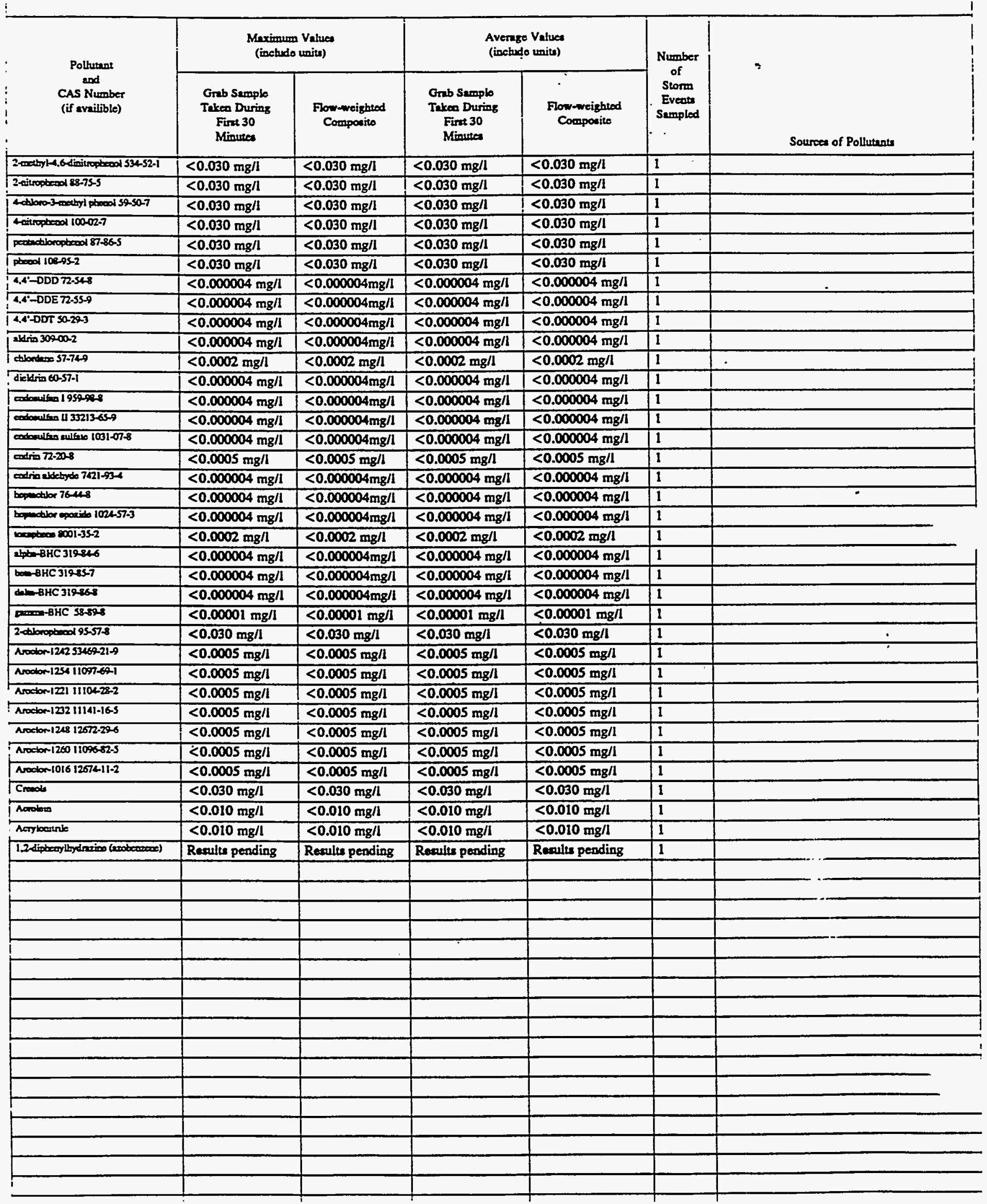


VII. Discharge Information (continuod from page 3 of Form 2F)

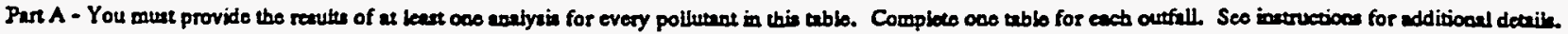

\begin{tabular}{|c|c|c|c|c|c|c|}
\hline \multirow{2}{*}{$\begin{array}{l}\text { Pollutant } \\
\text { and } \\
\text { CAS Number } \\
\text { (if avuiliblo) }\end{array}$} & \multicolumn{2}{|c|}{$\begin{array}{l}\text { Maximum Vahuen } \\
\text { (iseludo waits) }\end{array}$} & \multicolumn{2}{|c|}{$\begin{array}{l}\text { Averngo Valuee } \\
\text { (inchedo, units) }\end{array}$} & \multirow{2}{*}{$\begin{array}{c}\text { T } \\
\text { Nuraber } \\
\text { of } \\
\text { Siorm } \\
\text { Eveose } \\
\text { Sempled }\end{array}$} & \multirow[b]{2}{*}{ Sourcen of Poltencer } \\
\hline & $\begin{array}{l}\text { Grab Semplo } \\
\text { Takea Duriag } \\
\text { Firt } 30 \\
\text { Minuta }\end{array}$ & $\begin{array}{l}\text { Fow-weighted } \\
\text { Componite }\end{array}$ & 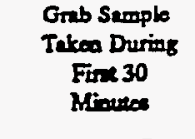 & $\begin{array}{l}\text { Fow-weighiod } \\
\text { Cocopories }\end{array}$ & & \\
\hline oiled Goneso & $<2 \mathrm{mg} / \mathrm{l}$ & & $<2 \mathrm{mg} / \mathrm{l}$ & & 1 & \\
\hline Crecrical Oxy & $14 \mathrm{mg} / \mathrm{l}$ & $12 \mathrm{mg} / 1$ & $14 \mathrm{mg} / 1$ & $12 \mathrm{mg} / 1$ & 1 & \\
\hline 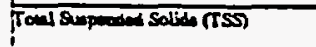 & $<5 \mathrm{mg} / \mathrm{l}$ & $16 \mathrm{mg} / \mathrm{l}$ & $<5 \mathrm{mg} / 1$ & $16 \mathrm{mg} / \mathrm{l}$ & 1 & \\
\hline Toul Kjetentil Nitropen & $1.0 \mathrm{mg} / \mathrm{l}$ & $0.4 \mathrm{mg} / \mathrm{l}$ & $1.0 \mathrm{mg} / \mathrm{h}$ & $0.4 \mathrm{mg} / \mathrm{l}$ & 1 & \\
\hline 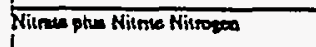 & $1.1 \mathrm{mg} / \mathrm{l}$ & $0.53 \mathrm{mg} / \mathrm{ll}$ & $1.1 \mathrm{mg} / \mathrm{l}$ & $0.53 \mathrm{mg} / \mathrm{l}$ & 1 & \\
\hline Toal Propoptons & $0.12 \mathrm{mg} / 1$ & $<0.1 \mathrm{mg} / \mathrm{l}$ & $0.12 \mathrm{mg} / 1$ & $<0.1 \mathrm{mg} / \mathrm{l}$ & 1 & \\
\hline
\end{tabular}

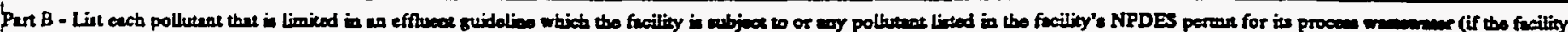

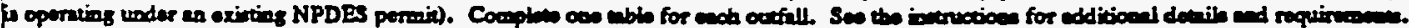

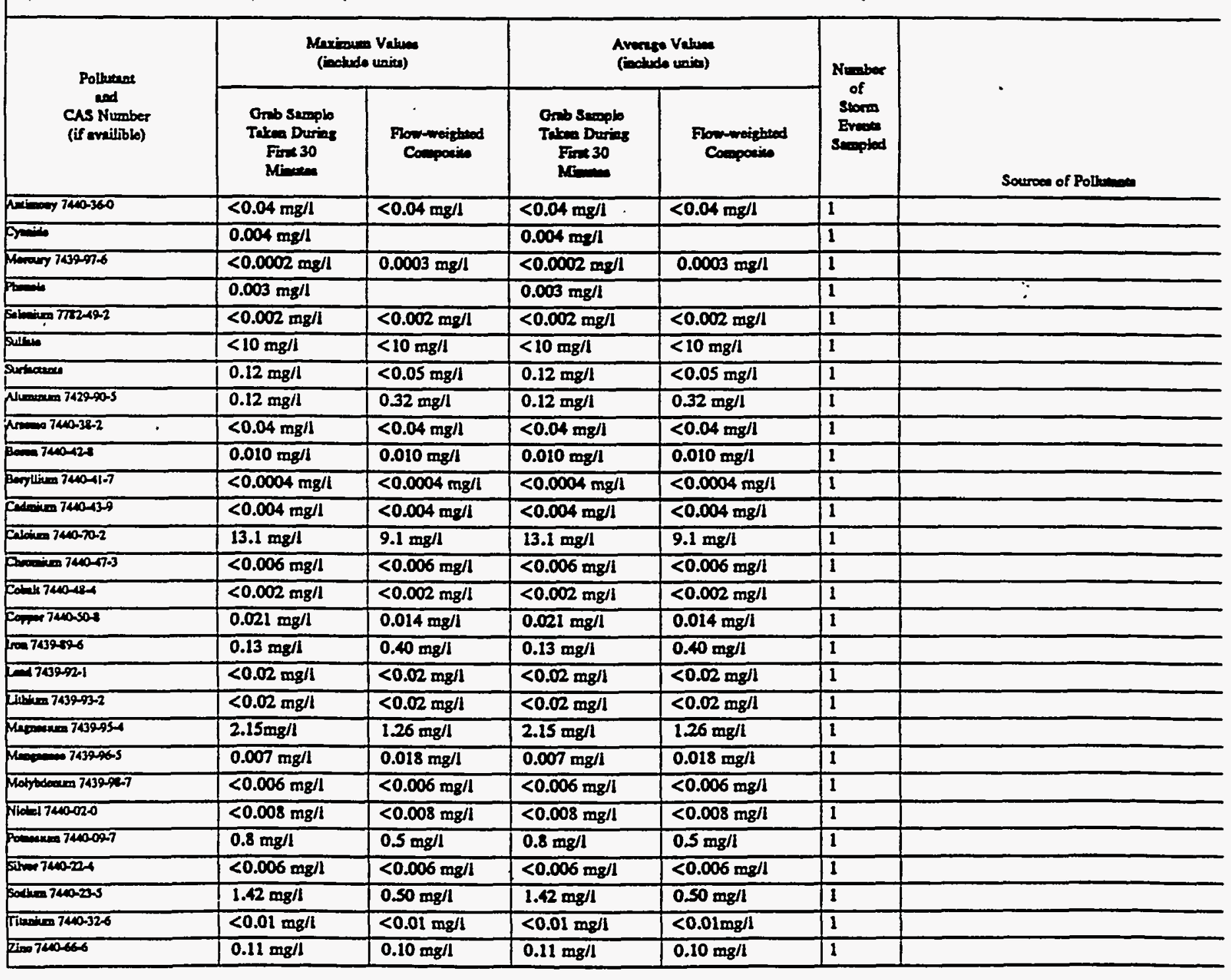




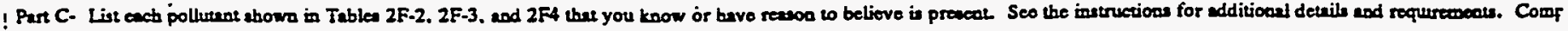
one uble for cach outill.

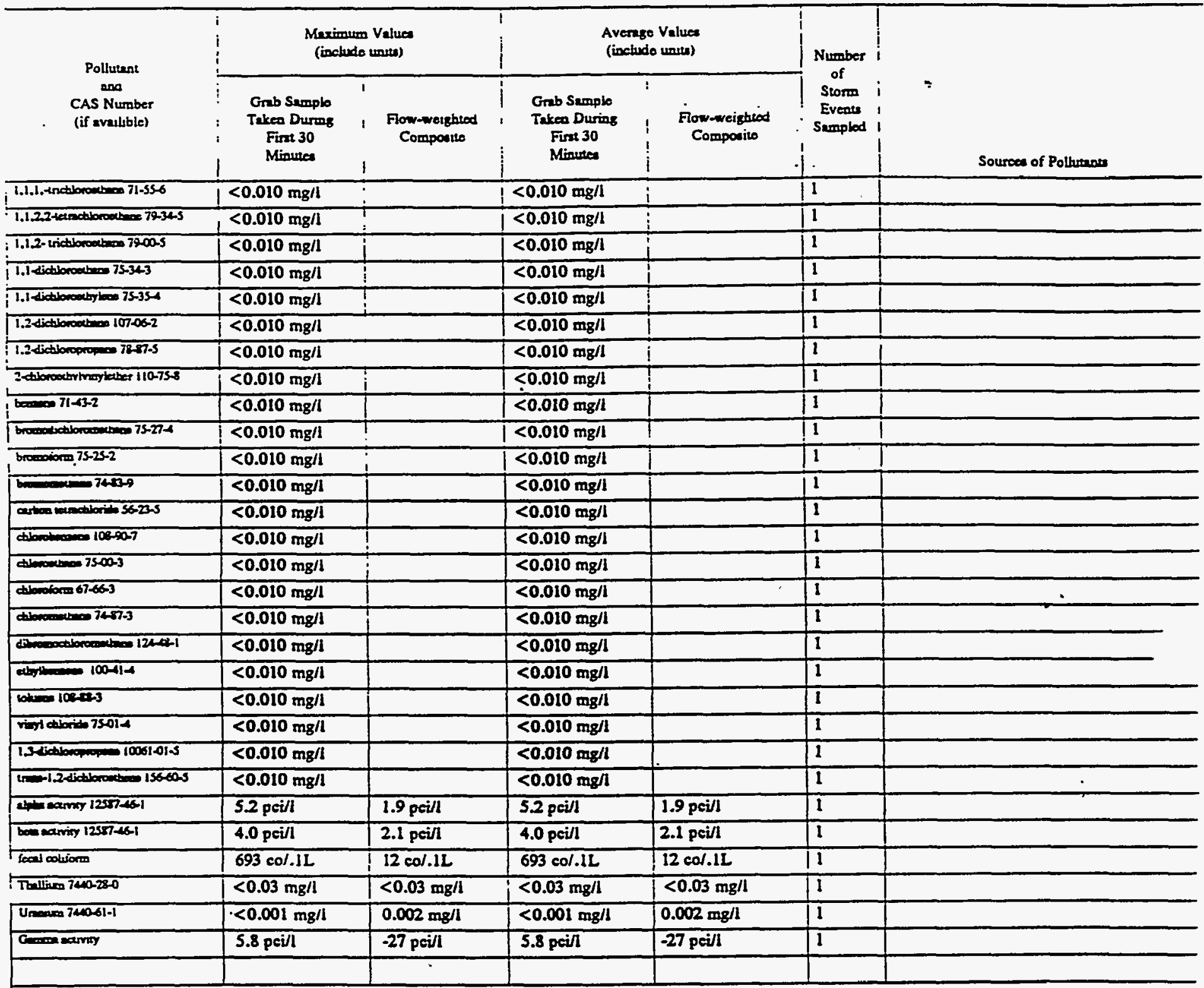

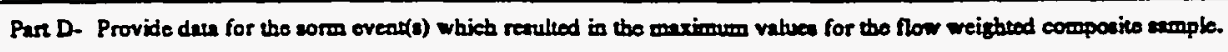

\begin{tabular}{|c|c|c|c|c|c|c|c|}
\hline $\begin{array}{l}1 . \\
\text { Date of } \\
\text { Storm } \\
\text { Evect }\end{array}$ & $\begin{array}{l}2 . \\
\text { Durition } \\
\text { of Storm } \\
\text { (in } \\
\text { minitea) }\end{array}$ & 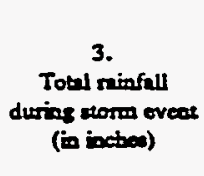 & 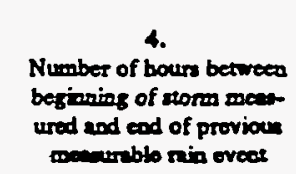 & 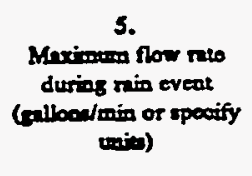 & $\begin{array}{l}6 . \\
\text { Total flow from } \\
\text { nin eveal } \\
\text { (gallows or } \\
\text { specify unitu) }\end{array}$ & $\begin{array}{c}7 . \\
\text { Scaion } \\
\text { uncole wase } \\
\text { unceo }\end{array}$ & $\begin{array}{l}\text { Eorim of } \\
\text { Precipitation } \\
\text { (ninfall. } \\
\text { nommati) }\end{array}$ \\
\hline $5 / 8 / 92$ & 695 & 0.66 & $>134.3$ & 19.5 & 1,333 & Spring & Rninfall \\
\hline
\end{tabular}

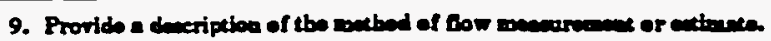

An ISCO 3230 Flow meter was nsed to obtain water level readings. This insurwent was calibrated with the normal dry wenther flow, if preate, as leve zero. The flow meter wes programmed with the chareteristics of the conveyence so that flow rates and total flowe conld be calculated by the rueter. 
Qutfall 113

Barta B and C Coatinued

\begin{tabular}{|c|c|c|c|c|c|c|}
\hline \multirow{2}{*}{$\begin{array}{l}\text { Polbutuas } \\
\text { and } \\
\text { CAs Number } \\
\text { (if availible) }\end{array}$} & \multicolumn{2}{|c|}{$\begin{array}{l}\text { Meximim Vahues } \\
\text { (inctude unita) }\end{array}$} & \multicolumn{2}{|c|}{$\begin{array}{l}\text { Avernge Valuce } \\
\text { (inctudo unita) }\end{array}$} & \multirow{2}{*}{$\begin{array}{l}\text { Number } \\
\text { of } \\
\text { Storm? } \\
\text { Eveosed } \\
\text { Sumpled }\end{array}$} & \multirow[b]{2}{*}{ Sourcese of Polturesons } \\
\hline & $\begin{array}{l}\text { Greb Semple } \\
\text { Taken During } \\
\text { Firs } 30 \\
\text { Minerea }\end{array}$ & $\begin{array}{l}\text { Flow-weighed } \\
\text { Compoixos }\end{array}$ & $\begin{array}{l}\text { Grab Sumpio } \\
\text { Tekea During } \\
\text { Firze } 30 \\
\text { Minutea }\end{array}$ & $\begin{array}{l}\text { Fow-weighiod } \\
\text { Coospousto }\end{array}$ & & \\
\hline Beriken 740a-30-3 & $0.0098 \mathrm{mg} / \mathrm{t}$ & $0.0083 \mathrm{mg} / \mathrm{l}$ & $0.0098 \mathrm{mg} / \mathrm{I}$ & $0.0083 \mathrm{mg} / \mathrm{l}$ & 1 & \\
\hline 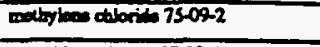 & $<0.010 \mathrm{mg} / \mathrm{l}$ & & $<0.010 \mathrm{mg} / 1$ & & 1 & \\
\hline 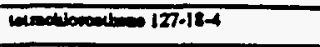 & $<0.010 \mathrm{mg} / 1$ & & $<0.010 \mathrm{mg} / 1$ & & 1 & \\
\hline 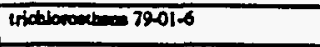 & $<0.010 \mathrm{mg} / 1$ & & $<0.010 \mathrm{mg} / \mathrm{l}$ & & 1 & $x$ \\
\hline xylan & $<0.010 \mathrm{mg} / \mathrm{l}$ & & $<0.010 \mathrm{mg} / \mathrm{l}$ & & 1 & \\
\hline 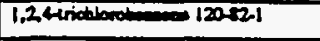 & $<0.010 \mathrm{mg} / \mathrm{l}$ & $<0.010 \mathrm{mg} / \mathrm{l}$ & $<0.010 \mathrm{mg} / \mathrm{l}$ & $<0.010 \mathrm{mg} / 1$ & 1 & \\
\hline 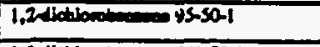 & $<0.010 \mathrm{mg} / \mathrm{l}$ & $<0.010 \mathrm{mg} / \mathrm{h}$ & $<0.010 \mathrm{mg} / \mathrm{I}$ & $<0.010 \mathrm{mg} / \mathrm{l}$ & 1 & \\
\hline 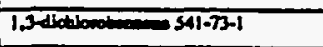 & $<0.010 \mathrm{mg} / 1$ & $<0.010 \mathrm{mg} / 1$ & $<0.010 \mathrm{mg} / \mathrm{l}$ & $<0.010 \mathrm{mg} / \mathrm{l}$ & 1 & \\
\hline 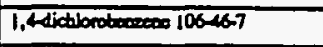 & $<0.010 \mathrm{mg} / 1$ & $<0.010 \mathrm{mg} / \mathrm{l}$ & $<0.010 \mathrm{mg} / 1$ & $<0.010 \mathrm{mg} / \mathrm{l}$ & 1 & \\
\hline 2.46initreatemen $121-142$ & $<0.010 \mathrm{mg} / \mathrm{I}$ & $<0.010 \mathrm{mg} / \mathrm{l}$ & $<0.010 \mathrm{mg} / \mathrm{l}$ & $<0.010 \mathrm{mg} / \mathrm{l}$ & 1 & \\
\hline 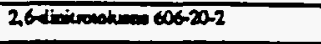 & $<0.010 \mathrm{mg} / \mathrm{l}$ & $<0.010 \mathrm{mg} / 1$ & $<0.010 \mathrm{mg} / \mathrm{l}$ & $<0.010 \mathrm{mg} / 1$ & 1 & \\
\hline 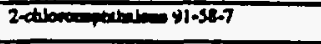 & $<0.010 \mathrm{mg} / \mathrm{l}$ & $<0.010 \mathrm{mg} / \mathrm{l}$ & $<0.010 \mathrm{mg} / \mathrm{l}$ & $<0.010 \mathrm{mg} / \mathrm{l}$ & $1 \cdot$ & \\
\hline 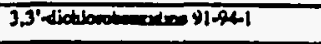 & $<0.010 \mathrm{mg} / 1$ & $<0.010 \mathrm{mg} / \mathrm{l}$ & $<0.010 \mathrm{mg} / \mathrm{l}$ & $<0.010 \mathrm{mg} / \mathrm{l}$ & 1 & \\
\hline 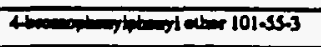 & $<0.010 \mathrm{mg} / 1$ & $<0.010 \mathrm{mg} / 1$ & $<0.010 \mathrm{mg} / 1$ & $<0.010 \mathrm{mg} / 1$ & 1 & \\
\hline 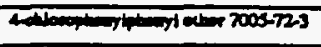 & $<0.010 \mathrm{mg} / 1$ & $<0.010 \mathrm{mg} / \mathrm{l}$ & $<0.010 \mathrm{mg} / \mathrm{l}$ & $<0.010 \mathrm{mg} / 1$ & 1 & \\
\hline 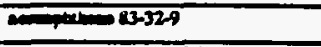 & $<0.010 \mathrm{mg} / 1$ & $<0.010 \mathrm{mg} / \mathrm{l}$ & $<0.010 \mathrm{mg} / \mathrm{l}$ & $<0.010 \mathrm{mg} / \mathrm{l}$ & 1 & \\
\hline 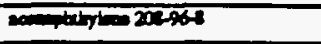 & $<0.010 \mathrm{mg} / 1$ & $<0.010 \mathrm{mg} / \mathrm{l}$ & $<0.010 \mathrm{mg} / \mathrm{h}$ & $<0.010 \mathrm{mg} / \mathrm{l}$ & 1 & \\
\hline 120.127 & $<0.010 \mathrm{mg} / \mathrm{l}$ & $<0.010 \mathrm{mg} / \mathrm{l}$ & $<0.010 \mathrm{mg} / \mathrm{l}$ & $<0.010 \mathrm{mg} / \mathrm{l}$ & 1 & \\
\hline midims 927s & $<0.030 \mathrm{mg} / 1$ & $<0.030 \mathrm{mg} / \mathrm{l}$ & $<0.030 \mathrm{mg} / \mathrm{L}$ & $<0.030 \mathrm{mg} / \mathrm{l}$ & 1 & \\
\hline 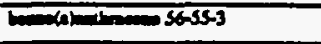 & $<0.010 \mathrm{mg} / \mathrm{l}$ & $<0.010 \mathrm{mg} / 1$ & $<0.010 \mathrm{mg} / \mathrm{L}$ & $<0.010 \mathrm{mg} / \mathrm{l}$ & 1 & \\
\hline 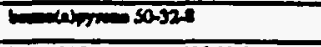 & $<0.010 \mathrm{mg} / \mathrm{l}$ & $<0.010 \mathrm{mg} / \mathrm{l}$ & $<0.010 \mathrm{mg} / \mathrm{l}$ & $<0.010 \mathrm{mg} / \mathrm{l}$ & 1 & \\
\hline$=0$ & $<0.010 \mathrm{mg} / 1$ & $<0.010 \mathrm{mg} / \mathrm{l}$ & $<0.010 \mathrm{mg} / \mathrm{L}$ & $<0.010 \mathrm{mg} / 1$ & $I$ & \\
\hline 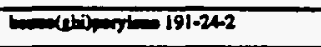 & $<0.010 \mathrm{mg} / 1$ & $<0.010 \mathrm{mg} / \mathrm{l}$ & $<0.010 \mathrm{mg} / 1$ & $<0.010 \mathrm{mg} / \mathrm{l}$ & 1 & \\
\hline - (1) & $<0.010 \mathrm{mg} / \mathrm{l}$ & $<0.010 \mathrm{mg} / \mathrm{l}$ & $<0.010 \mathrm{mg} / \mathrm{l}$ & $<0.010 \mathrm{mgll}$ & 1 & \\
\hline 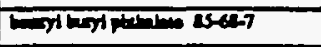 & $<0.010 \mathrm{mg} / 1$ & $<0.010 \mathrm{med} / 1$ & $<0.010 \mathrm{mg} / \mathrm{n}$ & $<0.010 \mathrm{mg} / 1$ & $I$ & \\
\hline His & $<0.010 \mathrm{mg} / \mathrm{l}$ & $<0.010 \mathrm{mg} / 1$ & $<0.010 \mathrm{mg} / 1$ & $<0.010 \mathrm{mg} / 1$ & 1 & \\
\hline 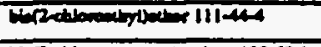 & $<0.010 \mathrm{mg} / \mathrm{l}$ & $<0.010 \mathrm{mg} / \mathrm{l}$ & $<0.010 \mathrm{mg} / \mathrm{l}$ & $<0.010 \mathrm{mg} / \mathrm{l}$ & 1 & \\
\hline 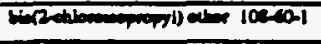 & $<0.010 \mathrm{mg} / \mathrm{l}$ & $<0.010 \mathrm{mg} / \mathrm{l}$ & $<0.010 \mathrm{mg} / \mathrm{h}$ & $<0.010 \mathrm{mg} / 1$ & 1 & \\
\hline 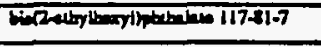 & $<0.010 \mathrm{mg} / \mathrm{l}$ & $<0.010 \mathrm{mg} / \mathrm{l}$ & $<0.010 \mathrm{mg} / \mathrm{L}$ & $<0.010 \mathrm{mg} / \mathrm{l}$ & 1 & \\
\hline ctrmen-212019 & $<0.010 \mathrm{mg} / \mathrm{l}$ & $<0.010 \mathrm{mg} / \mathrm{l}$ & $<0.010 \mathrm{mg} / \mathrm{L}$ & $<0.010 \mathrm{mg} / \mathrm{l}$ & 1 & \\
\hline 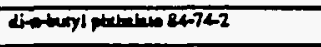 & $<0.010 \mathrm{mg} / 1$ & $<0.010 \mathrm{mg} / \mathrm{l}$ & $<0.010 \mathrm{mg} / \mathrm{h}$ & $<0.010 \mathrm{mg} / \mathrm{l}$ & 1 & \\
\hline 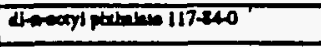 & $<0.010 \mathrm{mg} / 1$ & $<0.010 \mathrm{mg} / \mathrm{l}$ & $<0.010 \mathrm{mg} / 1$ & $<0.010 \mathrm{mg} / \mathrm{l}$ & 1 & \\
\hline 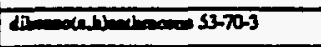 & $<0.010 \mathrm{mg} / 1$ & $<0.010 \mathrm{mg} / \mathrm{l}$ & $<0.010 \mathrm{mg} / 1$ & $<0.010 \mathrm{mg} / \mathrm{l}$ & 1 & \\
\hline 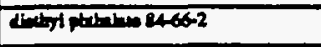 & $<0.010 \mathrm{mg} / 1$ & $<0.010 \mathrm{mg} / \mathrm{l}$ & $<0.010 \mathrm{mg} / 1$ & $<0.010 \mathrm{mg} / \mathrm{l}$ & 1 & \\
\hline 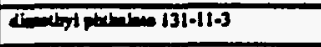 & $<0.010 \mathrm{mg} / \mathrm{l}$ & $<0.010 \mathrm{mg} / \mathrm{l}$ & $<0.010 \mathrm{mg} / 1$ & $<0.010 \mathrm{mg} / \mathrm{l}$ & 1 & \\
\hline 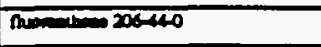 & $<0.010 \mathrm{mg} / \mathrm{l}$ & $<0.010 \mathrm{mg} / \mathrm{h}$ & $<0.010 \mathrm{mg} / \mathrm{l}$ & $<0.010 \mathrm{mg} / \mathrm{l}$ & 1 & \\
\hline onem-26-73-7 & $<0.010 \mathrm{mg} / 1$ & <0.010 mg/l & $<0.010 \mathrm{mg} / 1$ & $<0.010 \mathrm{mg} / \mathrm{l}$ & 1 & \\
\hline 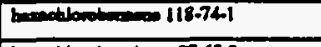 & $<0.010 \mathrm{mg} / \mathrm{l}$ & $<0.010 \mathrm{mg} / \mathrm{l}$ & $<0.010 \mathrm{mg} / \mathrm{l}$ & $<0.010 \mathrm{mg} / \mathrm{l}$ & 1 & \\
\hline 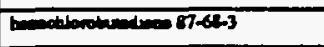 & $<0.010 \mathrm{mg} / \mathrm{l}$ & $<0.010 \mathrm{mg} / \mathrm{l}$ & $<0.010 \mathrm{mg} / \mathrm{l}$ & $<0.010 \mathrm{mg} / \mathrm{l}$ & 1 & \\
\hline 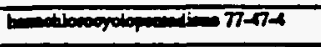 & $<0.010 \mathrm{mg} / \mathrm{l}$ & $<0.010 \mathrm{mg} / \mathrm{l}$ & $<0.010 \mathrm{mg} / \mathrm{l}$ & $<0.010 \mathrm{mg} / \mathrm{l}$ & 1 & \\
\hline $0.7 .72-1$ & $<0.010 \mathrm{mg} / \mathrm{l}$ & $<0.010 \mathrm{mg} / \mathrm{l}$ & $<0.010 \mathrm{mg} / 1$ & $<0.010 \mathrm{mg} / \mathrm{l}$ & 1 & \\
\hline 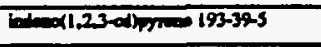 & $<0.010 \mathrm{mg} / \mathrm{l}$ & $<0.010 \mathrm{mg} / \mathrm{l}$ & $<0.010 \mathrm{mg} / 1$ & $<0.010 \mathrm{mg} / \mathrm{s}$ & 1 & \\
\hline inoporoes $x-59-1$ & $<0.010 \mathrm{mg} / 1$ & $<0.010 \mathrm{mg} / 1$ & $<0.010 \mathrm{mg} / 1$ & $<0.010 \mathrm{mg} / 1$ & 1 & \\
\hline 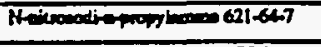 & $<0.010 \mathrm{mg} / 1$ & $<0.010 \mathrm{mg} / \mathrm{t}$ & $<0.010 \mathrm{mg} / 1$ & $<0.010 \mathrm{mg} / \mathrm{l}$ & 1 & \\
\hline 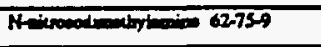 & $<0.010 \mathrm{mg} / \mathrm{l}$ & $<0.010 \mathrm{mg} / 1$ & $<0.010 \mathrm{mg} / \mathrm{l}$ & $<0.010 \mathrm{mg} / 1$ & 1 & \\
\hline 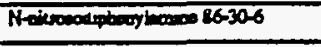 & $<0.010 \mathrm{mg} / \mathrm{I}$ & $<0.010 \mathrm{mg} / 1$ & $<0.010 \mathrm{mg} / 1$ & $<0.010 \mathrm{mg} / \mathrm{l}$ & 1 & \\
\hline athelos $91.20-3$ & $<0.010 \mathrm{mg} / \mathrm{l}$ & $<0.010 \mathrm{mg} / 1$ & $<0.010 \mathrm{mg} / \mathrm{l}$ & $<0.010 \mathrm{mg} / 1$ & 1 & \\
\hline 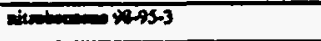 & $<0.010 \mathrm{mg} / 1$ & $<0.010 \mathrm{mg} / \mathrm{l}$ & $<0.010 \mathrm{mg} / \mathrm{l}$ & $<0.010 \mathrm{mg} / \mathrm{l}$ & 1 & \\
\hline Nementines 25015 & $<0.010 \mathrm{mg} / \mathrm{l}$ & $<0.010 \mathrm{mg} / \mathrm{l}$ & $<0.010 \mathrm{mg} / \mathrm{l}$ & $<0.010 \mathrm{mg} / 1$ & 1 & \\
\hline $570=122000$ & $<0.010 \mathrm{mg} / \mathrm{l}$ & $<0.010 \mathrm{mg} / \mathrm{l}$ & $<0.010 \mathrm{mg} / \mathrm{l}$ & $<0.010 \mathrm{mg} / \mathrm{l}$ & 1 & \\
\hline 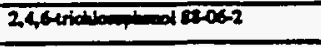 & $<0.030 \mathrm{mg} / \mathrm{l}$ & $<0.030 \mathrm{mg} / \mathrm{l}$ & $<0.030 \mathrm{mg} / 1$ & $<0.030 \mathrm{mg} / \mathrm{l}$ & 1 & \\
\hline 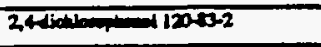 & $<0.030 \mathrm{mg} / \mathrm{I}$ & $<0.030 \mathrm{mg} / \mathrm{l}$ & $<0.030 \mathrm{mg} / \mathrm{l}$ & $<0.030 \mathrm{mg} / \mathrm{l}$ & 1 & \\
\hline 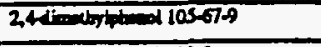 & $<0.030 \mathrm{mg} / \mathrm{l}$ & $<0.030 \mathrm{mg} / \mathrm{l}$ & $<0.030 \mathrm{mg} / \mathrm{I}$ & $<0.030 \mathrm{mg} / \mathrm{l}$ & 1 & \\
\hline 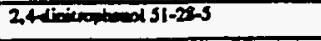 & $<0.030 \mathrm{mg} / 1$ & $<0.030 \mathrm{mg} / \mathrm{l}$ & $<0.030 \mathrm{mg} / 1$ & $<0.030 \mathrm{mg} / \mathrm{l}$ & 1 & \\
\hline
\end{tabular}


Outrail 113

Parre B and C Concinuod

\begin{tabular}{|c|c|c|c|c|c|c|}
\hline \multirow{2}{*}{$\begin{array}{l}\text { Polluteast } \\
\text { and } \\
\text { CAS Number } \\
\text { (if avaibible) }\end{array}$} & \multicolumn{2}{|c|}{$\begin{array}{l}\text { Maximum Values } \\
\text { (inctude units) }\end{array}$} & \multicolumn{2}{|c|}{$\begin{array}{l}\text { Average Valuea } \\
\text { (inclade uartu) }\end{array}$} & \multirow{2}{*}{$\begin{array}{c}\text { Number } \\
\text { of } \\
\text { Storm } \\
\text { Eventu } \\
\text { Simplod }\end{array}$} & \multirow[b]{2}{*}{ Sources of Polluteats } \\
\hline & $\begin{array}{l}\text { Grab Ssuoplo } \\
\text { Taken During } \\
\text { First } 30 \\
\text { Minutes }\end{array}$ & $\begin{array}{l}\text { Fow-weighted } \\
\text { Compoure }\end{array}$ & $\begin{array}{l}\text { Grab Sampic } \\
\text { Takeo During } \\
\text { Fint 30 } \\
\text { Minuteo }\end{array}$ & $\begin{array}{c}\text { Fow weighted } \\
\text { Compocite }\end{array}$ & & \\
\hline 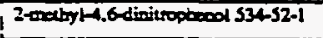 & $<0.030 \mathrm{mg} / \mathrm{l}$ & $<0.030 \mathrm{mg} / \mathrm{l}$ & $<0.030 \mathrm{mg} / 1$ & $<0.030 \mathrm{mg} / \mathrm{h}$ & 1 & \\
\hline 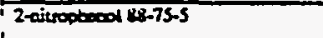 & $<0.030 \mathrm{mg} / \mathrm{l}$ & $<0.030 \mathrm{mg} / \mathrm{l}$ & $<<0.030 \mathrm{mg} / \mathrm{l}$ & $<0.030 \mathrm{mg} / \mathrm{l}$ & I & \\
\hline 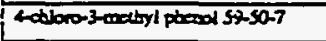 & $1-<0.030 \mathrm{mg} / \mathrm{l}$ & $<0.030 \mathrm{mg} / \mathrm{l}$ & $<0.030 \mathrm{mg} / \mathrm{l}$ & $<0.030 \mathrm{mg} / \mathrm{l}$ & 1 & \\
\hline 4 onsopbool $10002-7$ & $<0.030 \mathrm{mg} / \mathrm{l}$ & $<0.030 \mathrm{mg} / 1$ & $<0.030 \mathrm{mg} / \mathrm{l}$ & $<0.030 \mathrm{mg} / 1$ & 1 & \\
\hline peonationodecol 87-86-5 & $<0.030 \mathrm{mg} / \mathrm{l}$ & $<0.030 \mathrm{mg} / 1$ & $<0.030 \mathrm{mg} / \mathrm{l}$ & $<0.030 \mathrm{mg} / 1$ & 1 & \\
\hline Denod 108-95-2 & $<0.030 \mathrm{mg} / \mathrm{l}$ & $<0.030 \mathrm{mg} / \mathrm{l}$ & $<0.030 \mathrm{mg} / 1$ & $!<0.030 \mathrm{mg} / \mathrm{l}$ & 1 & \\
\hline $4,4-00072-548$ & $<0.00001 \mathrm{mg} / \mathrm{l}$ & $<0.00001 \mathrm{mg} / \mathrm{l}$ & $<0.00001 \mathrm{mg} / \mathrm{l}$ & $T<0.00001 \mathrm{mg} / \mathrm{l}$ & 1 & \\
\hline $4.4-D D E 72.55-9$ & $<0.00001 \mathrm{mg} / 1$ & $<0.00001 \mathrm{mg} / \mathrm{l}$ & $<0.00001 \mathrm{mg} / \mathrm{l}$ & $<0.00001 \mathrm{mg} / \mathrm{l}$ & 1 & \\
\hline 4.4-Dor $50-29-3$ & $<0.00001 \mathrm{mg} / \mathrm{l}$ & $<0.00001 \mathrm{mg} / \mathrm{l}$ & $<0.00001 \mathrm{mg} / \mathrm{l}$ & $<0.00001 \mathrm{mg} / \mathrm{l}$ & 1 & \\
\hline abrie $30900-2$ & $<0.00001 \mathrm{mg} / \mathrm{l}$ & $<0.00001 \mathrm{mg} / \mathrm{l}$ & $<0.00001 \mathrm{mg} / \mathrm{h}$ & $<0.00001 \mathrm{mg} / \mathrm{l}$ & 1 & \\
\hline ctlonteos 57.720 & $<0.00004 \mathrm{mg}$ II & $<0.00004 \mathrm{mg} / \mathrm{l}$ & $<0.00004 \mathrm{mg} / \mathrm{l}$ & $<0.00004 \mathrm{mg} / \mathrm{l}$ & 1 & \\
\hline diethen 60.57.1 & $<0.00001 \mathrm{mg} / \mathrm{l}$ & $<0.0000$ lmg/l & $<0.00001 \mathrm{mg} / \mathrm{l}$ & $<0.00001 \mathrm{mg} / \mathrm{l}$ & 1 & \\
\hline 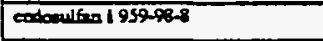 & $<0.00001 \mathrm{mg} / \mathrm{l}$ & $<0.00001 \mathrm{mg} / 1$ & $<0.00001 \mathrm{mg} / \mathrm{l}$ & $<0.00001 \mathrm{mg} / \mathrm{h}$ & 1 & \\
\hline collond 60 ol $33213-650$ & $<0.00001 \mathrm{mg} / \mathrm{l}$ & $<0.00001 \mathrm{mg} / \mathrm{l}$ & $<0.00001 \mathrm{mg} / \mathrm{l}$ & $<0.00001 \mathrm{mg} / \mathrm{l}$ & 1 & \\
\hline 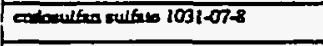 & $<0.00001 \mathrm{mg} / \mathrm{l}$ & $<0.0000 \operatorname{lmg} / \mathrm{l}$ & $<0.00001 \mathrm{mg} / \mathrm{l}$ & $<0.00001 \mathrm{mg} / \mathrm{l}$ & 1 & \\
\hline $\cos 1 \mathrm{sin} 72-20-8$ & $<0.00005 \mathrm{mg} / \mathrm{l}$ & $<0.00005 \mathrm{mg} / \mathrm{l}$ & $<0.00005 \mathrm{mg} / 1$ & $<0.00005 \mathrm{mg} / \mathrm{l}$ & 1 & \\
\hline adrie aldebydo 7421-93-4 & $<0.00001 \mathrm{mg} / \mathrm{l}$ & $<0.00001 \mathrm{mg} / \mathrm{l}$ & $<0.00001 \mathrm{mg} / \mathrm{l}$ & $<0.00001 \mathrm{mg} / \mathrm{l}$ & 1 & \\
\hline Emectior $76-148$ & $<0.00001 \mathrm{mg} / \mathrm{l}$ & $<0.00001 \mathrm{mg} / 1$ & $<0.00001 \mathrm{mg} / \mathrm{l}$ & $<0.00001 \mathrm{mg} / \mathrm{l}$ & 1 & \\
\hline 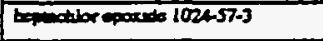 & $<0.00001 \mathrm{mg} / \mathrm{l}$ & $<0.00001 \mathrm{mg} / \mathrm{l}$ & $<0.00001 \mathrm{mg} / \mathrm{l}$ & $<0.00001 \mathrm{mg} / \mathrm{l}$ & 1 & \\
\hline $00=001-35-2$ & $<0.0004 \mathrm{mg} / \mathrm{l}$ & $<0.0004 \mathrm{mg} / \mathrm{l}$ & $<0.0004 \mathrm{mg} / \mathrm{l}$ & $<0.0004 \mathrm{mg} / \mathrm{l}$ & 1 & \\
\hline $246-\mathrm{BHC} 319846$ & $<0.00001 \mathrm{mg} / \mathrm{l}$ & $<0.00001 \mathrm{mg} / 1$ & $<0.00001 \mathrm{mg} / \mathrm{l}$ & $<0.00001 \mathrm{mg} / \mathrm{l}$ & 1 & \\
\hline-8 HC 31985-7 & $<0.00001 \mathrm{mg} / \mathrm{l}$ & $<0.00001 \mathrm{mg} / \mathrm{h}$ & $<0.00001 \mathrm{mg} / \mathrm{l}$ & $<0.00001 \mathrm{mg} / \mathrm{l}$ & 1 & \\
\hline din-2HC 319668 & $<0.00001 \mathrm{mg} / \mathrm{l}$ & $<0.00001 \mathrm{mg} / 1$ & $<0.00001 \mathrm{mg} / \mathrm{l}$ & $<0.00001 \mathrm{mg} / \mathrm{l}$ & 1 & \\
\hline 8 - BHC 58-598 & $<0.00001 \mathrm{mg} / \mathrm{l}$ & $<0.00001 \mathrm{mg} / \mathrm{l}$ & $<0.00001 \mathrm{mg} / 1$ & $<0.00001 \mathrm{mg} / \mathrm{l}$ & 1 & \\
\hline 2 -atibuptand ys-575 & $<0.030 \mathrm{mg} / 1$ & $<0.030 \mathrm{mg} / \mathrm{l}$ & $<0.030 \mathrm{mg} / \mathrm{h}$ & $<0.030 \mathrm{mg} / 1$ & 1 & $\therefore$ \\
\hline Aredor $1212533642.21-9$ & $<0.0005 \mathrm{mg} / \mathrm{l}$ & $<0.0005 \mathrm{mg} / \mathrm{l}$ & $<0.0005 \mathrm{mg} / 1$ & $<0.0005 \mathrm{mg} / 1$ & 1 & \\
\hline Arodom 1254 11097691 & $<0.0005 \mathrm{mg} / \mathrm{l}$ & $<0.0005 \mathrm{mg} / \mathrm{l}$ & $<0.0005 \mathrm{mg} / \mathrm{l}$ & $<0.0005 \mathrm{mg} / 1$ & 1 & \\
\hline Arodor-1221 $11106-282-2$ & $<0.0005 \mathrm{mg} / \mathrm{l}$ & $<0.0005 \mathrm{mg} / \mathrm{l}$ & $<0.0005 \mathrm{mg} / \mathrm{l}$ & $<0.0005 \mathrm{mg} / \mathrm{l}$ & 1 & \\
\hline Aroctor $123211141-16-5$ & $<0.0005 \mathrm{mg} / \mathrm{l}$ & $<0.0005 \mathrm{mg} / 1$ & $<0.0005 \mathrm{mg} / \mathrm{l}$ & $<0.0005 \mathrm{mg} / \mathrm{l}$ & 11 & \\
\hline Anocbor-1248 12672.296 & $<0.0005 \mathrm{mg} / \mathrm{l}$ & $<0.0005 \mathrm{mg} / \mathrm{l}$ & $<0.0005 \mathrm{mg} / \mathrm{l}$ & $<0.0005 \mathrm{mg} / \mathrm{I}$ & 1 & \\
\hline Aloodor $126011006-825$ & $<0.0005 \mathrm{mg} / \mathrm{l}$ & $<0.0005 \mathrm{mg} /$ & $<0.0005 \mathrm{mg} / \mathrm{h}$ & $<0.0005 \mathrm{mg} / \mathrm{l}$ & 1 & \\
\hline Aroctor-1016 12074-11-2 & $<0.0005 \mathrm{mg} / \mathrm{l}$ & $<0.0005 \mathrm{mg} / \mathrm{l}$ & $<0.0005 \mathrm{mg} / \mathrm{l}$ & $<0.0005 \mathrm{mg} / \mathrm{l}$ & 1 & \\
\hline Croode & $<0.010 \mathrm{mg} / \mathrm{l}$ & $<0.010 \mathrm{mg} / \mathrm{l}$ & $<0.010 \mathrm{mg} / \mathrm{l}$ & $<0.010 \mathrm{mg} / \mathrm{l}$ & 1 & \\
\hline Anoteon & $<0.010 \mathrm{mg} / \mathrm{I}$ & $<0.010 \mathrm{mg} / \mathrm{l}$ & $<0.010 \mathrm{mg} / \mathrm{l}$ & $<0.010 \mathrm{mg} / 1$ & 1 & \\
\hline Aarykorinte & $<0.010 \mathrm{mg} / \mathrm{l}$ & $<0.010 \mathrm{mg} / \mathrm{l}$ & $<0.010 \mathrm{mg} / \mathrm{l}$ & $<0.010 \mathrm{mg} / \mathrm{l}$ & $i$ & \\
\hline 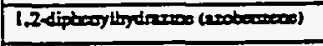 & Recults pending & Reaulus peoding & Reaults pending & Renules pending & 1 & \\
\hline & & & & & & \\
\hline & & & & & & \\
\hline & & & & & & \\
\hline & & & & & & \\
\hline & & & & & & \\
\hline & & & & & & \\
\hline & & & & & & \\
\hline & & & & & & \\
\hline & & & & & & \\
\hline & & & & & & \\
\hline & & & & & & \\
\hline & & & & & & \\
\hline & & & & & & \\
\hline & & & & & & \\
\hline
\end{tabular}


VII. Dischurge Information (continued from page 3 of Form 2F

Part A - You must provide the reautu of at leant one analysis for every pollutant in this table. Complete one able for each ounfill. See instruetions for additional devilts.

\begin{tabular}{|c|c|c|c|c|c|}
\hline \multirow{2}{*}{$\begin{array}{l}\text { Pollutent } \\
\text { and } \\
\text { CAS Number } \\
\text { (if avvilible) }\end{array}$} & \multicolumn{2}{|c|}{$\begin{array}{l}\text { Meximum Values } \\
\text { (include units) }\end{array}$} & \multicolumn{2}{|c|}{$\begin{array}{l}\text { Avernge Values } \\
\text { (inchude units) }\end{array}$} & \multirow{2}{*}{$\begin{array}{l}\text { Number } \\
\text { of } \\
\text { Stomm } \\
\text { Evects } \\
\text { Semplod }\end{array}$} \\
\hline & $\begin{array}{l}\text { Grab Sumpie } \\
\text { Taken During } \\
- \text { First } 30 \\
\text { Minutea }\end{array}$ & $\begin{array}{l}\text { Fow -weightod } \\
\text { Composics }\end{array}$ & $\begin{array}{c}\text { Greb Semple } \\
\text { Taken During } \\
\text { Fist } 30 \\
\text { Minerea }\end{array}$ & $\begin{array}{l}\text { Flow-weightod } \\
\text { Componito }\end{array}$ & \\
\hline Dil and Greas & $3 \mathrm{mg} / \mathrm{l}$ & & $3 \mathrm{mg} / 1$ & & 1 \\
\hline Biblopical Oxyeer Dexped (BOD) & $18.4 \mathrm{mg} / \mathrm{l}$ & $7.50 \mathrm{mg} / \mathrm{l}$ & $18.4 \mathrm{mg} / \mathrm{l}$ & $7.50 \mathrm{mg} / \mathrm{l}$ & 1 \\
\hline Chanial Oxygea Deanend (COD) & $24 \mathrm{mg} / 1$ & $34 \mathrm{mg} / \mathrm{l}$ & $24 \mathrm{mg} / 1$ & $34 \mathrm{mg} / \mathrm{l}$ & 1 \\
\hline Toul Suppentod Solide (TSS) & $32 \mathrm{mg} / 1$ & $30 \mathrm{mg} / 1$ & $32 \mathrm{mg} / \mathrm{l}$ & $30 \mathrm{mg} / \mathrm{l}$ & 1 \\
\hline Toeal Kjektand Nitrogon & $0.7 \mathrm{mg} / \mathrm{l}$ & $1.2 \mathrm{mg} / \mathrm{l}$ & $0.7 \mathrm{mg} / 1$ & $1.2 \mathrm{mg} / \mathrm{l}$ & 1 \\
\hline Nitrele ple Nitrite Nitroyea & $0.82 \mathrm{mg} / \mathrm{l}$ & $0.87 \mathrm{mg} / \mathrm{l}$ & $0.82 \mathrm{mg} / 1$ & $0.87 \mathrm{mg} / \mathrm{i}$ & 1 \\
\hline Toeal Pboptortu & $0.2 \mathrm{mg} / \mathrm{l}$ & $0.1 \mathrm{mg} / \mathrm{l}$ & $0.2 \mathrm{mg} / \mathrm{l}$ & $0.1 \mathrm{mg} / \mathrm{l}$ & 1 \\
\hline
\end{tabular}

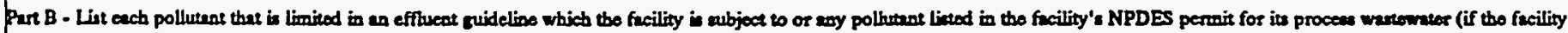

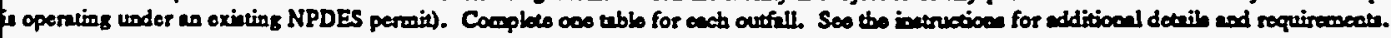

\begin{tabular}{|c|c|c|c|c|c|c|}
\hline \multirow{2}{*}{$\begin{array}{l}\text { Pollumane } \\
\text { and } \\
\text { CAs Number } \\
\text { (if availiblo) }\end{array}$} & \multicolumn{2}{|c|}{$\begin{array}{l}\text { Maximusn Valuces } \\
\text { (inchudo units) }\end{array}$} & \multicolumn{2}{|c|}{$\begin{array}{l}\text { Arenger Valuce } \\
\text { (include tritu) }\end{array}$} & \multirow{2}{*}{$\begin{array}{l}\text { Number } \\
\text { of } \\
\text { Storm } \\
\text { Eveose } \\
\text { Semplod }\end{array}$} & \multirow[b]{2}{*}{ 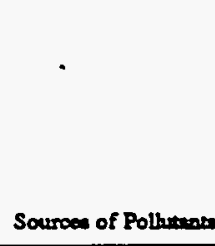 } \\
\hline & $\begin{array}{c}\text { Grab Semple } \\
\text { Taken During } \\
\text { Fint 30 } \\
\text { Minute }\end{array}$ & $\begin{array}{c}\text { Flow-weighed } \\
\text { Composito }\end{array}$ & $\begin{array}{l}\text { Gabb Semple } \\
\text { Taken During } \\
\text { Fira } 30 \\
\text { Mrouteo }\end{array}$ & $\begin{array}{l}\text { Frow-weighted } \\
\text { Comporito }\end{array}$ & & \\
\hline polowoy $7400-360$ & $<0.04 \mathrm{mg} / \mathrm{l}$ & $<0.04 \mathrm{mg} / \mathrm{l}$ & $<0.04 \mathrm{mg} / \mathrm{I}$ & $<0.04 \mathrm{mg} / \mathrm{l}$ & 1 & \\
\hline 8 manis & $<0.002 \mathrm{mg} / 1$ & & $<0.002 \mathrm{mg} / \mathrm{l}$ & & 1 & \\
\hline Merany 7639-976 & $<0.0002 \mathrm{mg} / \mathrm{I}$ & $<0.0002 \mathrm{mg} / \mathrm{l}$ & $<0.0002 \mathrm{mg} / \mathrm{i}$ & $<0.0002 \mathrm{mg} / \mathrm{l}$ & 1 & \\
\hline phen & $0.003 \mathrm{mg} / \mathrm{l}$ & & $0.003 \mathrm{mg} / 1$ & & 1 & $\cdot$ \\
\hline Salaim $7702-49-2$ & $<0.002 \mathrm{mg} / \mathrm{l}$ & $<0.002 \mathrm{mg} / \mathrm{l}$ & $<0.002 \mathrm{mg} / \mathrm{l}$ & $<0.002 \mathrm{mg} / \mathrm{l}$ & 1 & \\
\hline pulfue & $18 \mathrm{mg} / \mathrm{l}$ & $15 \mathrm{mg} / 1$ & $18 \mathrm{mg} / 1$ & $15 \mathrm{mg} / \mathrm{l}$ & 1 & \\
\hline Purthaneses & $0.06 \mathrm{mg} / \mathrm{l}$ & $0.09 \mathrm{mg} / \mathrm{l}$ & $0.06 \mathrm{mg} / \mathrm{l}$ & $0.09 \mathrm{mg} / \mathrm{l}$ & 1 & \\
\hline Alumanom $7429-90.5$ & $2.34 \mathrm{mg} / \mathrm{l}$ & $0.72 \mathrm{mg} / 1$ & $2.34 \mathrm{mg} / \mathrm{l}$ & $0.72 \mathrm{mg} / 1$ & 1 & \\
\hline tracosic 7440-38-2 & $<0.04 \mathrm{mg} / \mathrm{l}$ & $<0.04 \mathrm{mg} / \mathrm{l}$ & $<0.04 \mathrm{mg} / \mathrm{l}$ & $<0.04 \mathrm{mg} / 1$ & 1 & \\
\hline $8000007410-128$ & $0.044 \mathrm{mg} / 1$ & $0.041 \mathrm{mg} / 1$ & $0.044 \mathrm{mg} / 1$ & $0.041 \mathrm{mg} / 1$ & 1 & \\
\hline Bonglitum 740-11.7 & $<0.0004 \mathrm{mg} / \mathrm{l}$ & $<0.0004 \mathrm{mg} / \mathrm{l}$ & $<0.0004 \mathrm{mg} / \mathrm{l}$ & $<0.0004 \mathrm{mg} / \mathrm{l}$ & 1 & \\
\hline Cadmium 740-30-9 & $<0.004 \mathrm{mg} / \mathrm{l}$ & $<0.004 \mathrm{mg} / \mathrm{l}$ & $<0.004 \mathrm{mg} / \mathrm{l}$ & $<0.004 \mathrm{mg} / \mathrm{l}$ & 1 & \\
\hline celotim 7440-xo-2 & $16.1 \mathrm{mg} / \mathrm{l}$ & $15.7 \mathrm{mg} / \mathrm{l}$ & $16.1 \mathrm{mg} / \mathrm{l}$ & $15.7 \mathrm{mg} / \mathrm{l}$ & 1 & \\
\hline Daremian $7460-17.3$ & $<0.006 \mathrm{mg} / \mathrm{l}$ & $<0.006 \mathrm{mg} / \mathrm{l}$ & $<0.006 \mathrm{mg} / \mathrm{l}$ & $<0.006 \mathrm{mg} / \mathrm{l}$ & 1 & \\
\hline Soball 7440-18-4 & $<0.002 \mathrm{mg} / \mathrm{l}$ & $<0.002 \mathrm{mg} / 1$ & $<0.002 \mathrm{mg} / 1$ & $<0.002 \mathrm{mg} / \mathrm{h}$ & 1 & \\
\hline Coprom 74taso-sor & $0.021 \mathrm{mg} / \mathrm{l}$ & $0.021 \mathrm{mg} / 1$ & $0.021 \mathrm{mg} / 1$ & $0.021 \mathrm{mg} / \mathrm{l}$ & 1 & \\
\hline 50047099696 & $3.03 \mathrm{mg} / \mathrm{l}$ & $1.10 \mathrm{mg} / \mathrm{l}$ & $3.03 \mathrm{mg} / \mathrm{l}$ & $1.10 \mathrm{mg} / \mathrm{l}$ & 1 & 1 \\
\hline$[-47630-2-1$ & $<0.02 \mathrm{mg} / \mathrm{l}$ & $<0.02 \mathrm{mg} / \mathrm{l}$ & $<0.02 \mathrm{mg} / \mathrm{l}$ & $<0.02 \mathrm{mg} / \mathrm{l}$ & 1 & \\
\hline Whiven 7439-93-2 & $<0.02 \mathrm{mg} / \mathrm{l}$ & $<0.02 \mathrm{mg} / \mathrm{l}$ & $<0.02 \mathrm{mg} / 1$ & $<0.02 \mathrm{mg} / \mathrm{l}$ & 1 & \\
\hline Meposiue 7439-954-4 & $2.62 \mathrm{mg} / \mathrm{l}$ & $1.86 \mathrm{mg} / 1$ & $2.62 \mathrm{mg} / 1$ & $1.86 \mathrm{mg} / \mathrm{l}$ & 1 & \\
\hline Manances 7432-x6-5 & $0.093 \mathrm{mg} / \mathrm{l}$ & $0.036 \mathrm{mg} / \mathrm{l}$ & $0.093 \mathrm{mg} / \mathrm{l}$ & $0.036 \mathrm{mg} / 1$ & 1 & \\
\hline Motrodooum 743998-7 & $<0.006 \mathrm{mg} / \mathrm{l}$ & $<0.006 \mathrm{mg} / \mathrm{l}$ & $<0.006 \mathrm{mg} / 1$ & $<0.006 \mathrm{mg} / \mathrm{l}$ & 1 & \\
\hline Niobel 7400-02-0 & $<0.008 \mathrm{mg} / 1$ & $<0.008 \mathrm{mg} / \mathrm{l}$ & $<0.008 \mathrm{mg} / 1$ & $<0.008 \mathrm{mg} / 1$ & 1 & \\
\hline Pomenon 74009-7 & $2.0 \mathrm{mg} / 1$ & $1.9 \mathrm{mg} / \mathrm{l}$ & $2.0 \mathrm{mg} / \mathrm{l}$ & $1.9 \mathrm{mg} / 1$ & 1 & \\
\hline Bituor 7402-22-1 & $<0.006 \mathrm{mg} / \mathrm{l}$ & $<0.006 \mathrm{mg} / \mathrm{l}$ & $<0.006 \mathrm{mg} / 1$ & $<0.006 \mathrm{mg} / 1$ & 1 & \\
\hline sodson 7ma-23.5 & $0.51 \mathrm{mg} / \mathrm{l}$ & $0.95 \mathrm{mg} / 1$ & $0.51 \mathrm{mg} / 1$ & $0.95 \mathrm{mg} / 1$ & 1 & \\
\hline 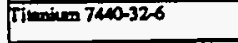 & $0.04 \mathrm{mg} / \mathrm{l}$ & $0.01 \mathrm{mg} / \mathrm{l}$ & $0.04 \mathrm{mg} / 1$ & $0.01 \mathrm{mg} / \mathrm{l}$ & 1 & \\
\hline $24007460-606$ & $0.28 \mathrm{mg} / \mathrm{l}$ & $0.18 \mathrm{mg} / \mathrm{l}$ & $0.28 \mathrm{mg} / \mathrm{l}$ & $0.18 \mathrm{mg} / \mathrm{l}$ & 1 & \\
\hline
\end{tabular}


Continued from front.

Part C. List each pollutane shown in Tables 2F-2. 2F-3. and 2F4 that you know or bave reason to believe is preaent. See the instructions for additional detrils and requirements. Com. one table for cact outfall.

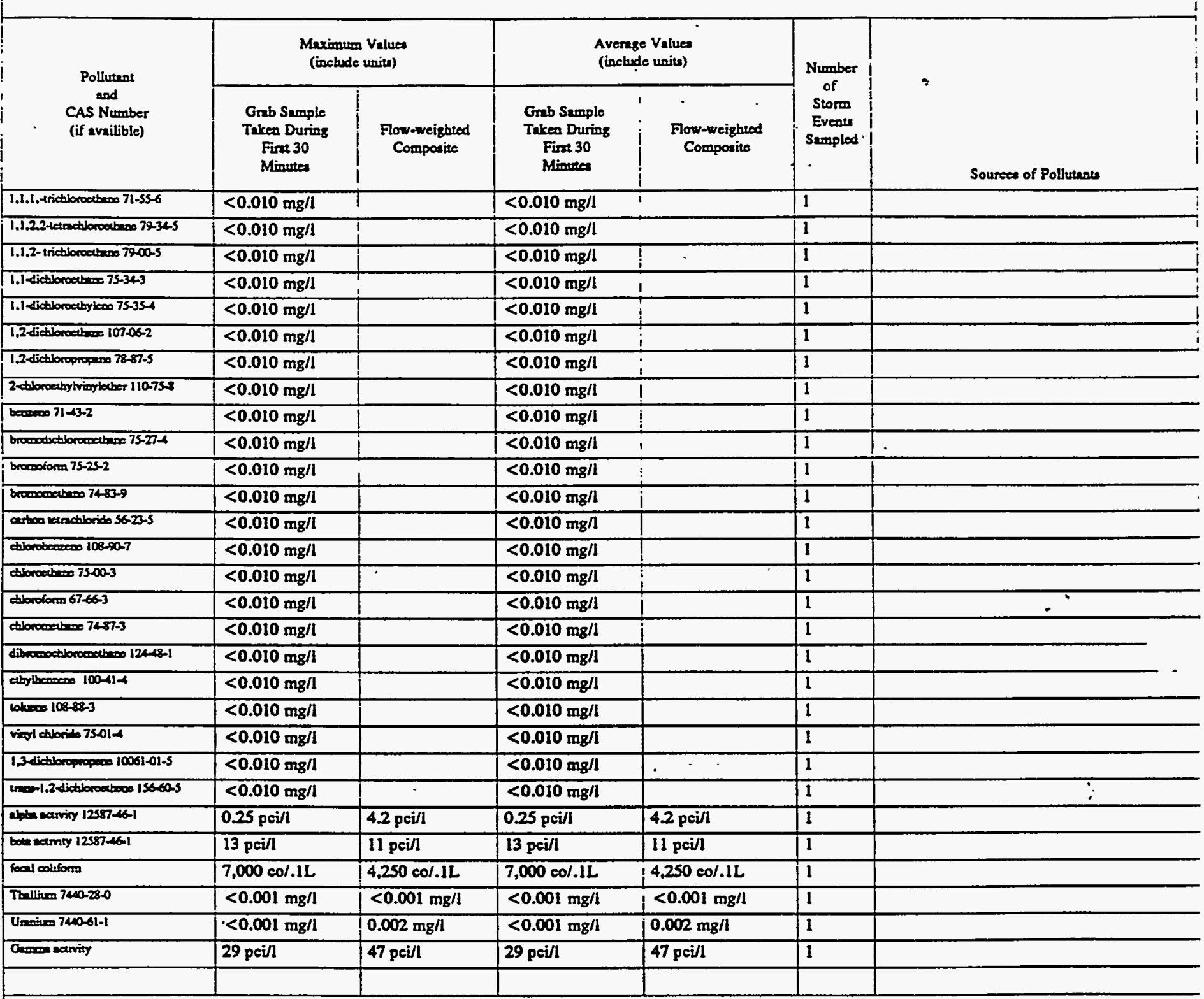

Part D. Provide data for the sorm eveat(s) which reaulted in the maximum values for the flow weighed componito maple.

\begin{tabular}{|c|c|c|c|c|c|c|c|}
\hline $\begin{array}{l}1 . \\
\text { Date of } \\
\text { Storm } \\
\text { Eveat }\end{array}$ & $\begin{array}{l}2 . \\
\text { Durntion } \\
\text { of Stores } \\
\text { (in } \\
\text { minites) }\end{array}$ & $\begin{array}{l}3 . \\
\text { Toul rinfill } \\
\text { during storm eveat } \\
\text { (in iscbes) }\end{array}$ & $\begin{array}{l}\text { 4. } \\
\text { Number of boun berween } \\
\text { beginning of atorm mean. } \\
\text { ured and end of previoun } \\
\text { menumblo sin ovent }\end{array}$ & $\begin{array}{l}\text { S. } \\
\text { Meximum flow nte } \\
\text { during min event } \\
\text { (allosidmin or specify } \\
\text { univ) }\end{array}$ & $\begin{array}{l}6 . \\
\text { Total flow from } \\
\text { nim eveat } \\
\text { (oallow or } \\
\text { specify units) }\end{array}$ & $\begin{array}{c}7 . \\
\text { Seavon } \\
\text { exmple was } \\
\text { cuken }\end{array}$ & $\begin{array}{l}\text { s. } \\
\text { Form of } \\
\text { Precipitation } \\
\text { (rainfall. } \\
\text { coowmell) }\end{array}$ \\
\hline $7 / 24 / 91$ & 185 & 0.7 & 154.2 & 1,414 & 108,024 & Summer & Rainfall \\
\hline
\end{tabular}

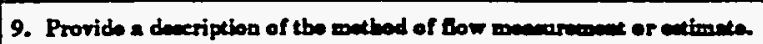

An ISCO 3230 Fow meter was used to obtain water level readings. This instrument was calibrated with the normal dry weather flow, if present, as leve zero. The flow meter was programmed with the characteristics of the conveyance so that flow rates and total flows conid be calcalated by the meter. 
Outfall 114

Pars B and C Continued

\begin{tabular}{|c|c|c|c|c|c|c|}
\hline \multirow{2}{*}{$\begin{array}{l}\text { Pollurant } \\
\text { and } \\
\text { CAS Number } \\
\text { (if availible) }\end{array}$} & \multicolumn{2}{|c|}{$\begin{array}{l}\text { Meximum Values } \\
\text { (inctudo unirs) }\end{array}$} & \multicolumn{2}{|c|}{$\begin{array}{l}\text { Averngo Velues } \\
\text { (inctudo unine) }\end{array}$} & \multirow{2}{*}{$\begin{array}{l}\text { Number } \\
\text { of } \\
\text { Storm } \\
\text { Evects } \\
\text { Sumpled }\end{array}$} & \multirow[b]{2}{*}{ Source of Pollutento } \\
\hline & $\begin{array}{l}\text { Greb Sumple } \\
\text { Takeo During } \\
\text { Fint } 30 \\
\text { Minutes }\end{array}$ & $\begin{array}{l}\text { Fow-weighted } \\
\text { Compocito }\end{array}$ & $\begin{array}{l}\text { Greb Sumplo } \\
\text { Takea During } \\
\text { Furt } 30 \\
\text { Minurea }\end{array}$ & $\begin{array}{l}\text { Flow-weighted } \\
\text { Composite }\end{array}$ & & \\
\hline Bariven 740-39-3 & $0.0342 \mathrm{mg} / \mathrm{l}$ & $0.0251 \mathrm{mg} / 1$ & $0.0342 \mathrm{mg} / \mathrm{l}$ & $0.0251 \mathrm{mg} / \mathrm{l}$ & 1 & \\
\hline modyleon ctioride 75-022-2 & $<0.010 \mathrm{mg} / \mathrm{l}$ & & $<0.010 \mathrm{mg} / \mathrm{l}$ & & 1 & \\
\hline Letnotbonocheos 127.184 & $<0.010 \mathrm{mg} / \mathrm{l}$ & & $<0.010 \mathrm{mg} / \mathrm{l}$ & & 1 & \\
\hline 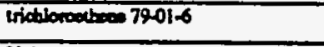 & $<0.010 \mathrm{mg} / \mathrm{l}$ & & $<0.010 \mathrm{mg} / \mathrm{l}$ & & 1 & \\
\hline Xyleowe & $<0.010 \mathrm{mg} / \mathrm{l}$ & & $<0.010 \mathrm{mg} / \mathrm{l}$ & & 1 & \\
\hline 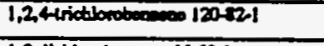 & $<0.010 \mathrm{mg} / \mathrm{l}$ & $<0.010 \mathrm{mg} / \mathrm{l}$ & $<0.010 \mathrm{mg} / \mathrm{l}$ & $<0.010 \mathrm{mg} / \mathrm{l}$ & 1 & \\
\hline 1.2-dictionotarecoss-50-1 & $<0.010 \mathrm{mg} / \mathrm{l}$ & $<0.010 \mathrm{mg} / \mathrm{l}$ & $<0.010 \mathrm{mg} / 1$ & $<0.010 \mathrm{mg} / 1$ & 1 & \\
\hline 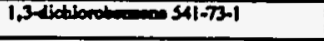 & $<0.010 \mathrm{mg} / \mathrm{l}$ & $<0.010 \mathrm{mg} / \mathrm{L}$ & $<0.010 \mathrm{mg} / \mathrm{l}$ & $<0.010 \mathrm{mg} / 1$ & 1 & \\
\hline 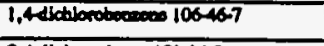 & $<0.010 \mathrm{mg} / \mathrm{l}$ & $<0.010 \mathrm{mg} / 1$ & $<0.010 \mathrm{mg} / \mathrm{l}$ & $<0.010 \mathrm{mg} / 1$ & 1 & \\
\hline 24-dinitrolokenos $121-142$ & $<0.010 \mathrm{mg} / \mathrm{l}$ & $<0.010 \mathrm{mg} / \mathrm{l}$ & $<0.010 \mathrm{mg} / \mathrm{l}$ & $<0.010 \mathrm{mg} / \mathrm{l}$ & 1 & \\
\hline 2.0-dinitrocotucose $606-30-2$ & $<0.010 \mathrm{mg} / \mathrm{l}$ & $<0.010 \mathrm{mg} / 1$ & $<0.010 \mathrm{mg} / \mathrm{l}$ & $<0.010 \mathrm{mg} / \mathrm{l}$ & 1 & \\
\hline 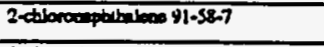 & $<0.010 \mathrm{mg} / \mathrm{l}$ & $<0.010 \mathrm{mg} / \mathrm{l}$ & $<0.010 \mathrm{mg} / \mathrm{l}$ & $<0.010 \mathrm{mg} / \mathrm{l}$ & 1 & \\
\hline 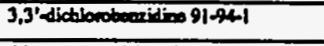 & $<0.010 \mathrm{mg} / \mathrm{l}$ & $<0.010 \mathrm{mg} / \mathrm{l}$ & $<0.010 \mathrm{mg} / \mathrm{l}$ & $<0.010 \mathrm{mg} / \mathrm{l}$ & 1 & \\
\hline 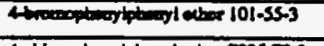 & $<0.010 \mathrm{mg} / \mathrm{l}$ & $<0.010 \mathrm{mg} / \mathrm{i}$ & $<0.010 \mathrm{mg} / \mathrm{l}$ & $<0.010 \mathrm{mg} / \mathrm{l}$ & 1 & \\
\hline 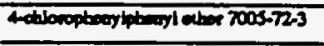 & $<0.010 \mathrm{mg} / \mathrm{l}$ & $<0.010 \mathrm{mg} / 1$ & $<0.010 \mathrm{mg} / \mathrm{l}$ & $<0.010 \mathrm{mg} / 1$ & 1 & \\
\hline 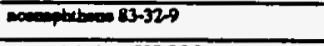 & $<0.010 \mathrm{mg} / 1$ & $<0.010 \mathrm{mg} / \mathrm{l}$ & $<0.010 \mathrm{mg} / \mathrm{l}$ & $<0.010 \mathrm{mg} / \mathrm{l}$ & 1 & \\
\hline mamplestybor 208964 & $<0.010 \mathrm{mg} / \mathrm{l}$ & $<0.010 \mathrm{mg} / 1$ & $<0.010 \mathrm{mg} / \mathrm{l}$ & $<0.010 \mathrm{mg} / \mathrm{l}$ & 1 & \\
\hline 120.127 & $<0.010 \mathrm{mg} / 1$ & $<0.010 \mathrm{mg} / 1$ & $<0.010 \mathrm{mg} / \mathrm{ll}$ & $<0.010 \mathrm{mg} / 1$ & 1 & \\
\hline 600 dition 9287.5 & $<0.030 \mathrm{mg} / \mathrm{l}$ & $<0.030 \mathrm{mg} / \mathrm{l}$ & $<0.030 \mathrm{mg} / \mathrm{l}$ & $<0.030 \mathrm{mg} / \mathrm{l}$ & 1 & \\
\hline 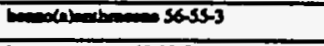 & $<0.010 \mathrm{mg} / \mathrm{l}$ & $<0.010 \mathrm{mg} / \mathrm{l}$ & $<0.010 \mathrm{mg} / \mathrm{l}$ & $<0.010 \mathrm{mg} / 1$ & 1 & \\
\hline$=0(a)+700=50-324$ & $<0.010 \mathrm{mg} / \mathrm{l}$ & $<0.010 \mathrm{mg} / 1$ & $<0.010 \mathrm{mg} / \mathrm{l}$ & $<0.010 \mathrm{mg} / 1$ & 1 & \\
\hline 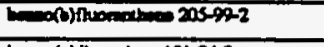 & $<0.010 \mathrm{mg} / \mathrm{l}$ & $<0.010 \mathrm{mg} / 1$ & $<0.010 \mathrm{mg} / 1$ & $<0.010 \mathrm{mg} / 1$ & 1 & \\
\hline 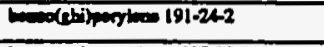 & $<0.010 \mathrm{mg} / \mathrm{l}$ & $<0.010 \mathrm{mg} / 1$ & $<0.010 \mathrm{mg} / \mathrm{l}$ & $<0.010 \mathrm{mg} / 1$ & 1 & \\
\hline 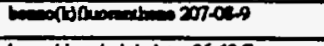 & $<0.010 \mathrm{mg} / \mathrm{I}$ & $<0.010 \mathrm{mg} / \mathrm{l}$ & $<0.010 \mathrm{mg} / \mathrm{l}$ & $<0.010 \mathrm{mg} / \mathrm{l}$ & 1 & \\
\hline 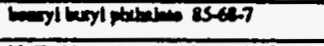 & $<0.010 \mathrm{mg} / 1$ & $<0.010 \mathrm{mg} / \mathrm{l}$ & $<0.010 \mathrm{mg} / \mathrm{l}$ & $<0.010 \mathrm{mg} / 1$ & 1 & \\
\hline 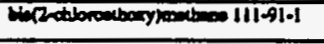 & $<0.010 \mathrm{mg} / \mathrm{l}$ & $<0.010 \mathrm{mg} / 1$ & $<0.010 \mathrm{mg} / \mathrm{h}$ & $<0.010 \mathrm{mg} / 1$ & 1 & ; \\
\hline 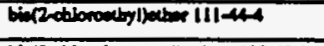 & $<0.010 \mathrm{mg} / 1$ & $<0.010 \mathrm{mg} / 1$ & $<0.010 \mathrm{mg} / \mathrm{h}$ & $<0.010 \mathrm{mg} / \mathrm{l}$ & 1 & \\
\hline 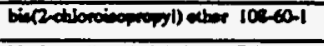 & $<0.010 \mathrm{mg} / \mathrm{l}$ & $<0.010 \mathrm{mg} / \mathrm{l}$ & $<0.010 \mathrm{mg} / \mathrm{l}$ & $<0.010 \mathrm{mg} / 1$ & 1 & \\
\hline 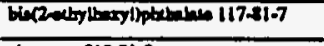 & $<0.010 \mathrm{mg} / \mathrm{l}$ & $<0.010 \mathrm{mg} / 1$ & $<0.010 \mathrm{mg} / \mathrm{l}$ & $<0.010 \mathrm{mg} / \mathrm{l}$ & 1 & \\
\hline dryene 21201 A & $<0.010 \mathrm{mg} / 1$ & $<0.010 \mathrm{mg} / 1$ & $<0.010 \mathrm{mg} / \mathrm{l}$ & $<0.010 \mathrm{mg} / \mathrm{l}$ & 1 & \\
\hline disteryl ptathabio $84-74-2$ & $0.029 \mathrm{mg} / \mathrm{l}$ & $0.012 \mathrm{mg} / 1$ & $0.029 \mathrm{mg} / \mathrm{l}$ & $0.012 \mathrm{mg} / 1$ & 1 & \\
\hline 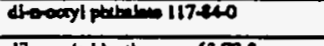 & $<0.010 \mathrm{mg} / 1$ & $<0.010 \mathrm{mg} / 1$ & $<0.010 \mathrm{mg} / \mathrm{l}$ & $<0.010 \mathrm{mg} / \mathrm{l}$ & 1 & \\
\hline 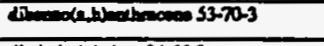 & $<0.010 \mathrm{mg} / \mathrm{l}$ & $<0.010 \mathrm{mg} / 1$ & $<0.010 \mathrm{mg} / \mathrm{l}$ & $<0.010 \mathrm{mg} / 1$ & 1 & \\
\hline 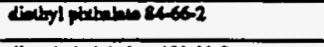 & $<0.010 \mathrm{mg} / 1$ & $<0.010 \mathrm{mg} / \mathrm{l}$ & $<0.010 \mathrm{mg} / 1$ & $<0.010 \mathrm{mg} / \mathrm{l}$ & 1 & \\
\hline 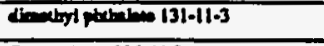 & $<0.010 \mathrm{mg} / \mathrm{l}$ & $<0.010 \mathrm{mg} / \mathrm{l}$ & $<0.010 \mathrm{mg} / 1$ & $<0.010 \mathrm{mg} / \mathrm{l}$ & 1 & \\
\hline 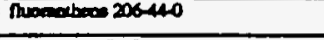 & $<0.010 \mathrm{mg} / \mathrm{l}$ & $<0.010 \mathrm{mg} / \mathrm{l}$ & $<0.010 \mathrm{mg} / \mathrm{l}$ & $<0.010 \mathrm{mg} / 1$ & 1 & \\
\hline 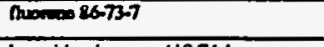 & $<0.010 \mathrm{mg} / \mathrm{l}$ & $<0.010 \mathrm{mg} / \mathrm{l}$ & $<0.010 \mathrm{mg} / \mathrm{l}$ & $<0.010 \mathrm{mg} / 1$ & 1 & \\
\hline 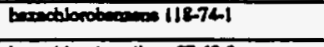 & $<0.010 \mathrm{mg} / \mathrm{l}$ & $<0.010 \mathrm{mg} / 1$ & $<0.010 \mathrm{mg} / \mathrm{l}$ & $<0.010 \mathrm{mg} / 1$ & 1 & \\
\hline 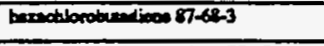 & $<0.010 \mathrm{mg} / 1$ & $<0.010 \mathrm{mg} / \mathrm{L}$ & $<0.010 \mathrm{mg} / \mathrm{l}$ & $<0.010 \mathrm{mg} / \mathrm{l}$ & 1 & \\
\hline 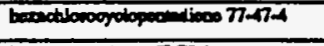 & $<0.010 \mathrm{mg} / 1$ & $<0.010 \mathrm{mg} / \mathrm{h}$ & $<0.010 \mathrm{mg} / \mathrm{h}$ & $<0.010 \mathrm{mg} / \mathrm{ll}$ & 1 & \\
\hline 67.72 .1 & $<0.010 \mathrm{mg} / \mathrm{l}$ & $<0.010 \mathrm{mg} / 1$ & $<0.010 \mathrm{mg} / \mathrm{l}$ & $<0.010 \mathrm{mg} / \mathrm{l}$ & 1 & \\
\hline 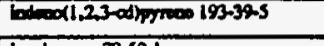 & $<0.010 \mathrm{mg} / \mathrm{l}$ & $<0.010 \mathrm{mg} / 1$ & $<0.010 \mathrm{mg} / \mathrm{l}$ & $<0.010 \mathrm{mg} / \mathrm{l}$ & 1 & \\
\hline Woptencosos 72.591 & $<0.010 \mathrm{mg} / \mathrm{l}$ & $<0.010 \mathrm{mg} / \mathrm{l}$ & $<0.010 \mathrm{mg} / \mathrm{l}$ & $<0.010 \mathrm{mg} / 1$ & 1 & \\
\hline 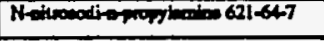 & $<0.010 \mathrm{mg} / 1$ & $<0.010 \mathrm{mg} / \mathrm{l}$ & $<0.010 \mathrm{mg} / \mathrm{l}$ & $<0.010 \mathrm{mg} / \mathrm{l}$ & 1 & \\
\hline 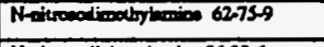 & $<0.010 \mathrm{mg} / \mathrm{l}$ & $<0.010 \mathrm{mg} / \mathrm{l}$ & $<0.010 \mathrm{mg} / \mathrm{l}$ & $<0.010 \mathrm{mg} / \mathrm{l}$ & 1 & \\
\hline 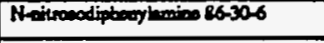 & $<0.010 \mathrm{mg} / 1$ & $<0.010 \mathrm{mg} / \mathrm{L}$ & $<0.010 \mathrm{mg} / 1$ & $<0.010 \mathrm{mg} / 1$ & 1 & \\
\hline mptednesen 91-20-3 & $<0.010 \mathrm{mg} / \mathrm{l}$ & $<0.010 \mathrm{mg} / 1$ & $<0.010 \mathrm{mg} / \mathrm{h}$ & $<0.010 \mathrm{mg} / \mathrm{l}$ & 1 & \\
\hline 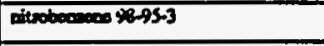 & $<0.010 \mathrm{mg} / \mathrm{l}$ & $<0.010 \mathrm{mg} / 1$ & $<0.010 \mathrm{mg} / 1$ & $<0.010 \mathrm{mg} / 1$ & 1 & \\
\hline 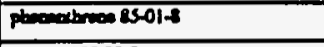 & $<0.010 \mathrm{mg} / \mathrm{l}$ & $<0.010 \mathrm{mg} / \mathrm{l}$ & $<0.010 \mathrm{mg} / \mathrm{h}$ & $<0.010 \mathrm{mg} / 1$ & 1 & \\
\hline proce 129000 & $<0: 010 \mathrm{mg} / \mathrm{l}$ & $<0.010 \mathrm{mg} / \mathrm{I}$ & $<0.010 \mathrm{mg} / \mathrm{l}$ & $<0.010 \mathrm{mg} / \mathrm{l}$ & 1 & \\
\hline 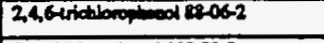 & $<0.030 \mathrm{mg} / \mathrm{I}$ & $<0.030 \mathrm{mg} / 1$ & $<0.030 \mathrm{mg} / 1$ & $<0.030 \mathrm{mg} / \mathrm{l}$ & 1 & \\
\hline 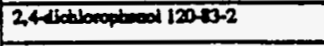 & $<0.030 \mathrm{mg} / \mathrm{l}$ & $<0.030 \mathrm{mg} / \mathrm{I}$ & $<0.030 \mathrm{mg} / 1$ & $<0.030 \mathrm{mg} / 1$ & 1 & \\
\hline 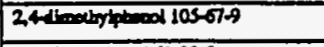 & $<0.030 \mathrm{mg} / 1$ & $<0.030 \mathrm{mg} / \mathrm{l}$ & $<0.030 \mathrm{mg} / \mathrm{h}$ & $<0.030 \mathrm{mg} / 1$ & 1 & \\
\hline 2,4diainophenot SI-22-5 & $<0.030 \mathrm{mg} / \mathrm{l}$ & $<0.030 \mathrm{mg} / \mathrm{l}$ & $<0.030 \mathrm{mg} / \mathrm{l}$ & $<0.030 \mathrm{mg} / 1$ & 1 & \\
\hline
\end{tabular}




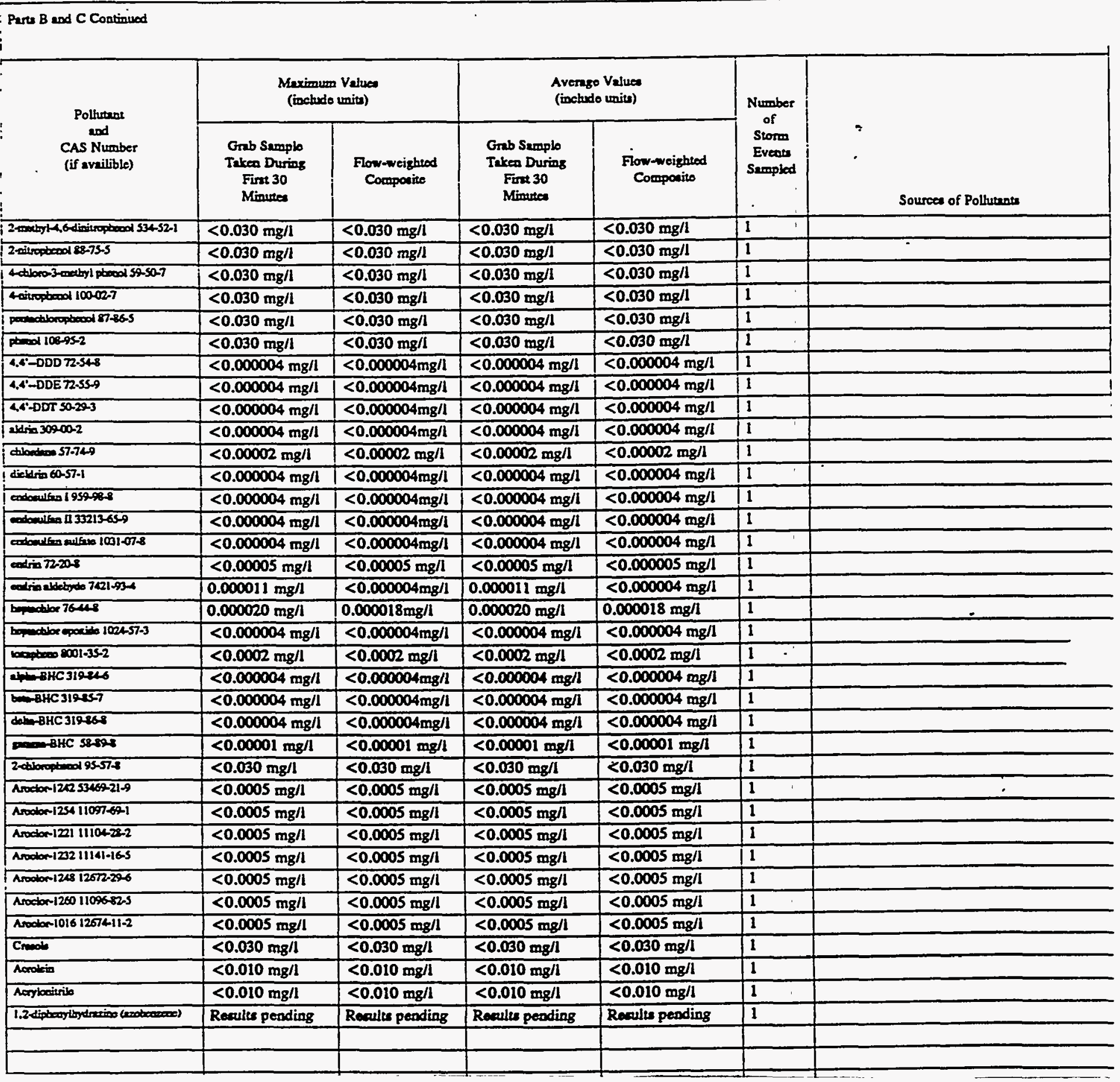

Continued from front.

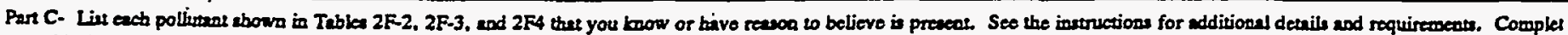
one table for eact outfall.

\begin{tabular}{|c|c|c|c|c|c|}
\hline \multirow{2}{*}{$\begin{array}{l}\text { Polhutant } \\
\text { and } \\
\text { CAS Nurnber } \\
\text { (if availible) }\end{array}$} & \multicolumn{2}{|c|}{$\begin{array}{l}\text { Merimum Values } \\
\text { (inchude unres) }\end{array}$} & \multicolumn{2}{|c|}{$\begin{array}{l}\text { Avernge Value: } \\
\text { (inebude uniss! }\end{array}$} & \multirow{2}{*}{$\begin{array}{c}\text { Number } \\
\text { of } \\
\text { Storm } \\
\text { Even'v } \\
\text { Sumpiea }\end{array}$} \\
\hline & $\begin{array}{c}\text { Grab Sample } \\
\text { Takeo During } \\
\text { First } 30\end{array}$ & $\begin{array}{l}\text { Flow-weighted } \\
\text { Cosoposite }\end{array}$ & $\begin{array}{c}\text { Grab Sample } \\
\text { Taken Durios } \\
\text { Fint } 30\end{array}$ & $\begin{array}{l}\text {. } \\
\text { Com-weighted }\end{array}$ & \\
\hline
\end{tabular}


Outfall 135

\begin{tabular}{|c|c|c|c|c|c|c|}
\hline \multicolumn{7}{|l|}{ Parte B and C Continued } \\
\hline \multirow{2}{*}{$\begin{array}{l}\text { Pollutenes } \\
\text { and } \\
\text { CAS Number } \\
\text { (if avnilible) }\end{array}$} & \multicolumn{2}{|c|}{$\begin{array}{l}\text { Maximum Vahuea } \\
\text { (includo unita) }\end{array}$} & \multicolumn{2}{|c|}{$\begin{array}{l}\text { Average Vehues } \\
\text { (ischedo unitu) }\end{array}$} & \multirow{2}{*}{$\begin{array}{l}\text { Nimber } \\
\text { of } \\
\text { Siorm } \\
\text { Evente } \\
\text { Samplad }\end{array}$} & \multirow[b]{2}{*}{ Sources of Pollutents } \\
\hline & $\begin{array}{c}\text { Onab Seoplo } \\
\text { Taken Durimg } \\
\text { Fint } 30 \\
\text { Minuce }\end{array}$ & $\begin{array}{l}\text { Flow-weighted } \\
\text { Compocito }\end{array}$ & $\begin{array}{l}\text { Grab Sample } \\
\text { Trkes Durring } \\
\text { Fint } 30 \\
\text { Minurea }\end{array}$ & $\begin{array}{l}\text { Flow-weighted } \\
\text { Componito }\end{array}$ & & \\
\hline Berieco 740-39-3 & $0.0207 \mathrm{mg} / \mathrm{l}$ & $0.0250 \mathrm{mg} / 1$ & $0.0207 \mathrm{mg} / 1$ & $0.0250 \mathrm{mg} / 1$ & 1 & \\
\hline montyleos abloribo $75-092-2$ & $<0.010 \mathrm{mg} / \mathrm{l}$ & & $<0.010 \mathrm{mg} / \mathrm{l}$ & & 1 & \\
\hline 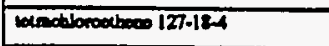 & $<0.010 \mathrm{mg} / \mathrm{l}$ & & $<0.010 \mathrm{mg} / 1$ & & 1 & \\
\hline 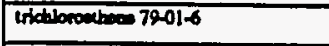 & $<0.010 \mathrm{mg} / \mathrm{l}$ & & $<0.010 \mathrm{mg} / \mathrm{l}$ & & 1 & \\
\hline Xyloenes & $<0.010 \mathrm{mg} / \mathrm{l}$ & & $<0.010 \mathrm{mg} / \mathrm{l}$ & & 1 & \\
\hline 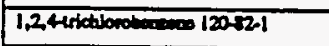 & $<0.010 \mathrm{mg} / \mathrm{l}$ & $<0.010 \mathrm{mg} / \mathrm{l}$ & $<0.010 \mathrm{mg} / \mathrm{l}$ & $<0.010 \mathrm{mg} / \mathrm{l}$ & 1 & \\
\hline 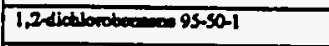 & $<0.010 \mathrm{mg} / \mathrm{l}$ & $<0.010 \mathrm{mg} / \mathrm{l}$ & $<0.010 \mathrm{mg} / \mathrm{l}$ & $<0.010 \mathrm{mg} / \mathrm{l}$ & 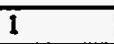 & \\
\hline 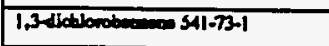 & $<0.010 \mathrm{mg} / \mathrm{l}$ & $<0.010 \mathrm{mg} / \mathrm{l}$ & $<0.010 \mathrm{mg} / \mathrm{l}$ & $<0.010 \mathrm{mg} / \mathrm{l}$ & 1 & \\
\hline 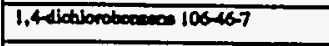 & $<0.010 \mathrm{mg} / 1$ & $<0.010 \mathrm{mg} / \mathrm{l}$ & $<0.010 \mathrm{mg} / \mathrm{l}$ & $<0.010 \mathrm{mg} / \mathrm{l}$ & 1 & \\
\hline 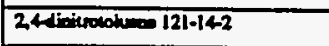 & $<0.010 \mathrm{mg} / \mathrm{l}$ & $<0.010 \mathrm{mg} / \mathrm{l}$ & $<0.010 \mathrm{mg} / \mathrm{l}$ & $<0.010 \mathrm{mg} / 1$ & 1 & \\
\hline 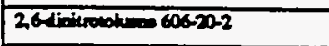 & $<0.010 \mathrm{mg} / \mathrm{l}$ & $<0.010 \mathrm{mg} / \mathrm{l}$ & $<0.010 \mathrm{mg} / \mathrm{l}$ & $<0.010 \mathrm{mg} / \mathrm{l}$ & 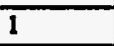 & \\
\hline 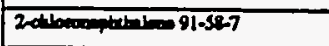 & $<0.010 \mathrm{mg} / \mathrm{l}$ & $<0.010 \mathrm{mg} / \mathrm{l}$ & $<0.010 \mathrm{mg} / 1$ & $<0.010 \mathrm{mg} / \mathrm{l}$ & 1 & \\
\hline 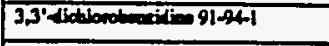 & $<0.010 \mathrm{mg} / 1$ & $<0.010 \mathrm{mg} / \mathrm{l}$ & $<0.010 \mathrm{mg} / \mathrm{l}$ & $<0.010 \mathrm{mg} / 1$ & 1 & \\
\hline 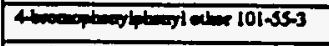 & $<0.010 \mathrm{mg} / \mathrm{l}$ & $<0.010 \mathrm{mg} / \mathrm{l}$ & $<0.010 \mathrm{mg} / \mathrm{l}$ & $<0.010 \mathrm{mg} / \mathrm{l}$ & 1 & \\
\hline 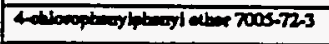 & $<0.010 \mathrm{mg} / \mathrm{l}$ & $<0.010 \mathrm{mg} / 1$ & $<0.010 \mathrm{mg} / 1$ & $<0.010 \mathrm{mg} / \mathrm{l}$ & 1 & \\
\hline 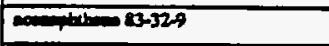 & $<0.010 \mathrm{mg} / \mathrm{l}$ & $<0.010 \mathrm{mg} / \mathrm{l}$ & $<0.010 \mathrm{mg} / 1$ & $<0.010 \mathrm{mg} / 1$ & 1 & \\
\hline 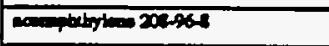 & $<0.010 \mathrm{mg} / \mathrm{l}$ & $<0.010 \mathrm{mg} / \mathrm{l}$ & $<0.010 \mathrm{mg} / \mathrm{l}$ & $<0.010 \mathrm{mg} / \mathrm{l}$ & 1 & \\
\hline$-120-127$ & $<0.010 \mathrm{mg} / \mathrm{l}$ & $<0.010 \mathrm{mg} / 1$ & $<0.010 \mathrm{mg} / \mathrm{l}$ & $<0.010 \mathrm{mg} / \mathrm{l}$ & 1 & \\
\hline Didiv 9257.5 & $<0.030 \mathrm{mg} / \mathrm{l}$ & $<0.030 \mathrm{mg} / \mathrm{l}$ & $<0.030 \mathrm{mg} / \mathrm{l}$ & $<0.030 \mathrm{mg} / \mathrm{l}$ & 1 & \\
\hline $56555-3$ & $<0.010 \mathrm{mg} / \mathrm{l}$ & $<0.010 \mathrm{mg} / \mathrm{l}$ & $<0.010 \mathrm{mg} / \mathrm{l}$ & $<0.010 \mathrm{mg} / \mathrm{l}$ & 1 & \\
\hline $50-20128$ & $<0.010 \mathrm{mg} / \mathrm{l}$ & $<0.010 \mathrm{mg} / \mathrm{l}$ & $<0.010 \mathrm{mg} / \mathrm{l}$ & $<0.010 \mathrm{mg} / 1$ & 1 & \\
\hline 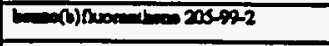 & $<0.010 \mathrm{mg} / \mathrm{l}$ & $<0.010 \mathrm{mg} / 1$ & $<0.010 \mathrm{mg} / 1$ & $<0.010 \mathrm{mg} / 1$ & 1 & \\
\hline 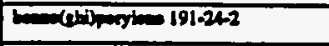 & $<0.010 \mathrm{mg} / \mathrm{l}$ & $<0.010 \mathrm{mg} / 1$ & $<0.010 \mathrm{mg} / \mathrm{l}$ & $<0.010 \mathrm{mg} / \mathrm{h}$ & 1 & \\
\hline 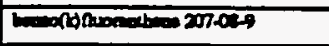 & $<0.010 \mathrm{mg} / \mathrm{l}$ & $<0.010 \mathrm{mg} / \mathrm{l}$ & $<0.010 \mathrm{mg} / 1$ & $<0.010 \mathrm{mg} / 1$ & 1 & \\
\hline 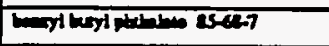 & $<0.010 \mathrm{mg} / \mathrm{l}$ & $<0.010 \mathrm{mg} / \mathrm{l}$ & $<0.010 \mathrm{mql} \mathrm{i}^{\circ}$ & $<0.010 \mathrm{mg} / \mathrm{l}$ & 1 & \\
\hline 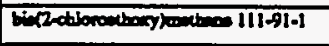 & $<0.010 \mathrm{mg} / \mathrm{l}$ & $<0.010 \mathrm{mg} / \mathrm{l}$ & $<0.010 \mathrm{mg} / \mathrm{l}$ & $<0.010 \mathrm{mg} / \mathrm{l}$ & 1 & - \\
\hline 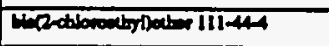 & $<0.010 \mathrm{mg} / \mathrm{l}$ & $<0.010 \mathrm{mg} / \mathrm{l}$ & $<0.010 \mathrm{mg} / 1$ & $<0.010 \mathrm{mg} / 1$ & $T$ & \\
\hline 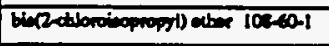 & $<0.010 \mathrm{mg} / \mathrm{l}$ & $<0.010 \mathrm{mg} / 1$ & $<0.010 \mathrm{mg} / \mathrm{l}$ & $<0.010 \mathrm{mg} / 1$ & 1 & \\
\hline 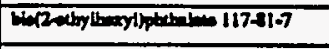 & $<0.010 \mathrm{mg} / 1$ & $<0.010 \mathrm{mg} / \mathrm{l}$ & $<0.010 \mathrm{mg} / 1$ & $<0.010 \mathrm{mg} / \mathrm{l}$ & 1 & \\
\hline 由ryooco 218-01A & $<0.010 \mathrm{mg} / \mathrm{l}$ & $<0.010 \mathrm{mg} / \mathrm{l}$ & $<0.010 \mathrm{mg} / \mathrm{l}$ & $<0.010 \mathrm{mg} / 1$ & 1 & \\
\hline 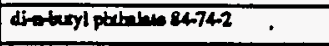 & $0.040 \mathrm{mg} / \mathrm{l}$ & $0.053 \mathrm{mg} / \mathrm{l}$ & $0.040 \mathrm{mg} / \mathrm{h}$ & $0.053 \mathrm{mg} / \mathrm{l}$ & 1 & \\
\hline 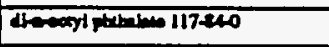 & $<0.010 \mathrm{mg} / 1$ & $<0.010 \mathrm{mg} / \mathrm{l}$ & $<0.010 \mathrm{mg} / \mathrm{l}$ & $<0.010 \mathrm{mg} / 1$ & 1 & \\
\hline 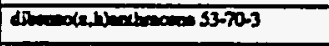 & $<0.010 \mathrm{mg} / \mathrm{l}$ & $<0.010 \mathrm{mg} / \mathrm{l}$ & $<0.010 \mathrm{mgl}$ & $<0.010 \mathrm{mg} / \mathrm{l}$ & 1 & \\
\hline 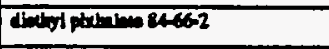 & $<0.010 \mathrm{mg} / \mathrm{l}$ & $<0.010 \mathrm{mg} / \mathrm{l}$ & $<0.010 \mathrm{mg} / 1$ & $<0.010 \mathrm{mg} / \mathrm{l}$ & 1 & \\
\hline 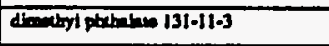 & $<0.010 \mathrm{mg} / \mathrm{l}$ & $<0.010 \mathrm{mg} / \mathrm{l}$ & $<0.010 \mathrm{mg} / 1$ & $<0.010 \mathrm{mg} / 1$ & 1 & \\
\hline Devandhere 200440 & $<0.010 \mathrm{mg} / 1$ & $<0.010 \mathrm{mg} / \mathrm{l}$ & $<0.010 \mathrm{mg} / \mathrm{l}$ & $<0.010 \mathrm{mg} / \mathrm{l}$ & 1 & \\
\hline Devomen $26-73-7$ & $<0.010 \mathrm{mg} / \mathrm{l}$ & $<0.010 \mathrm{mg} / \mathrm{l}$ & $<0.010 \mathrm{mg} / \mathrm{l}$ & $<0.010 \mathrm{mg} / 1$ & 1 & \\
\hline beractionoberesen IIR-74-1 & $<0.010 \mathrm{mg} / \mathrm{l}$ & $<0.010 \mathrm{mg} / 1$ & $<0.010 \mathrm{mg} / \mathrm{l}$ & $<0.010 \mathrm{mg} / \mathrm{l}$ & 1 & \\
\hline 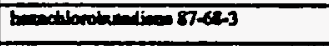 & $<0.010 \mathrm{mg} / 1$ & $<0.010 \mathrm{mg} / \mathrm{l}$ & $<0.010 \mathrm{mg} / \mathrm{l}$ & $<0.010 \mathrm{mg} / \mathrm{l}$ & $I$ & \\
\hline 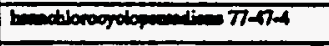 & $<0.010 \mathrm{mg} / \mathrm{l}$ & $<0.010 \mathrm{mg} / \mathrm{l}$ & $<0.010 \mathrm{mg} / \mathrm{l}$ & $<0.010 \mathrm{mg} / 1$ & 1 & \\
\hline 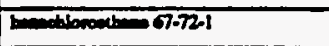 & $<0.010 \mathrm{mg} / \mathrm{l}$ & $<0.010 \mathrm{mg} / 1$ & $<0.010 \mathrm{mg} / 1$ & $<0.010 \mathrm{mg} / \mathrm{l}$ & 1 & \\
\hline 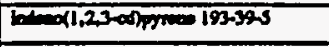 & $<0.010 \mathrm{mg} / \mathrm{l}$ & $<0.010 \mathrm{mg} / \mathrm{l}$ & $<0.010 \mathrm{mg} / 1$ & $<0.010 \mathrm{mg} / \mathrm{l}$ & 1 & \\
\hline 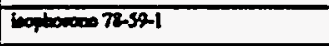 & $<0.010 \mathrm{mg} / \mathrm{l}$ & $<0.010 \mathrm{mg} / \mathrm{l}$ & $<0.010 \mathrm{mg} / \mathrm{l}$ & $<0.010 \mathrm{mg} / \mathrm{l}$ & 1 & \\
\hline 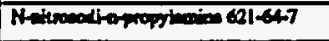 & $<0.010 \mathrm{mg} / \mathrm{l}$ & $<0.010 \mathrm{mg} / \mathrm{l}$ & $<0.010 \mathrm{mg} / 1$ & $<0.010 \mathrm{mg} / \mathrm{l}$ & 1 & \\
\hline 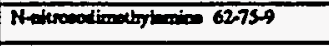 & $<0.010 \mathrm{mg} / \mathrm{l}$ & $<0.010 \mathrm{mg} / \mathrm{l}$ & $<0.010 \mathrm{mg} / \mathrm{l}$ & $<0.010 \mathrm{mg} / 1$ & 1 & \\
\hline 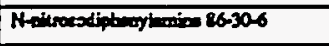 & $<0.010 \mathrm{mg} / \mathrm{l}$ & $<0.010 \mathrm{mg} / 1$ & $<0.010 \mathrm{mg} / 1$ & $<0.010 \mathrm{mg} / \mathrm{l}$ & 1 & \\
\hline 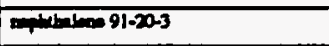 & $<0.010 \mathrm{mg} / \mathrm{l}$ & $<0.010 \mathrm{mg} / 1$ & $<0.010 \mathrm{mg} / \mathrm{l}$ & $<0.010 \mathrm{mg} / \mathrm{l}$ & 1 & \\
\hline minotios-3 & $<0.010 \mathrm{mg} / \mathrm{l}$ & $<0.010 \mathrm{mg} / \mathrm{l}$ & $<0.010 \mathrm{mg} / \mathrm{l}$ & $<0.010 \mathrm{mg} / 1$ & 1 & \\
\hline 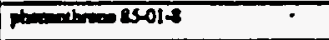 & $<0.010 \mathrm{mg} / \mathrm{l}$ & $<0.010 \mathrm{mg} / \mathrm{l}$ & $<0.010 \mathrm{mg} / \mathrm{l}$ & $<0.010 \mathrm{mg} / 1$ & 1 & \\
\hline momos 129000 & $<0.010 \mathrm{mg} / 1$ & $<0.010 \mathrm{mg} / \mathrm{l}$ & $<0.010 \mathrm{mg} / 1$ & $<0.010 \mathrm{mg} / \mathrm{l}$ & $I$ & \\
\hline 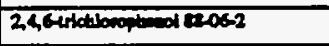 & $<0.030 \mathrm{mg} / 1$ & $<0.030 \mathrm{mg} / \mathrm{l}$ & $<0.030 \mathrm{mg} / 1$ & $<0.030 \mathrm{mg} / \mathrm{l}$ & 1 & \\
\hline 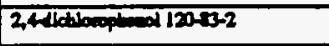 & $<0.030 \mathrm{mg} / \mathrm{l}$ & $<0.030 \mathrm{mg} / \mathrm{ll}$ & $<0.030 \mathrm{mg} / \mathrm{l}$ & $<0.030 \mathrm{mg} / \mathrm{l}$ & 1 & \\
\hline 2,4-dingedy $105-79$ & $<0.030 \mathrm{mg} / \mathrm{l}$ & $<0.030 \mathrm{mg} / \mathrm{l}$ & $<0.030 \mathrm{mg} / \mathrm{l}$ & $<0.030 \mathrm{mg} / \mathrm{l}$ & 1 & \\
\hline 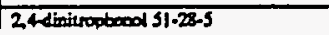 & $<0.030 \mathrm{mg} / \mathrm{l}$ & Come & & & & \\
\hline
\end{tabular}


Outfall 135

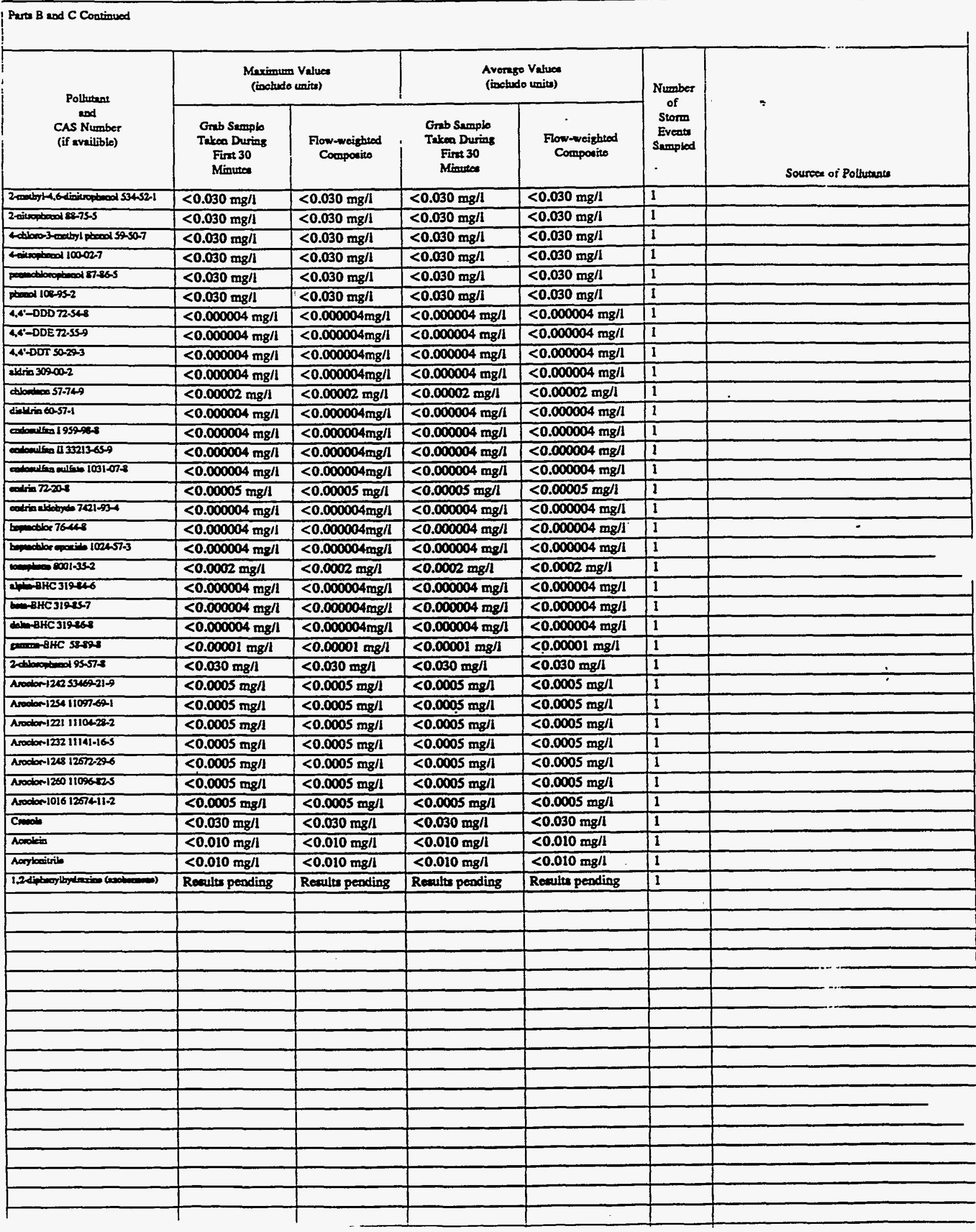


ivi. Discherge laformation (continued from page 3 of Form 2B)

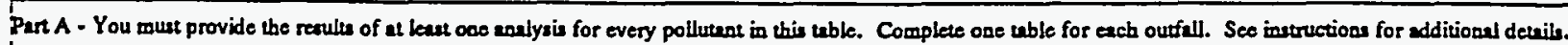

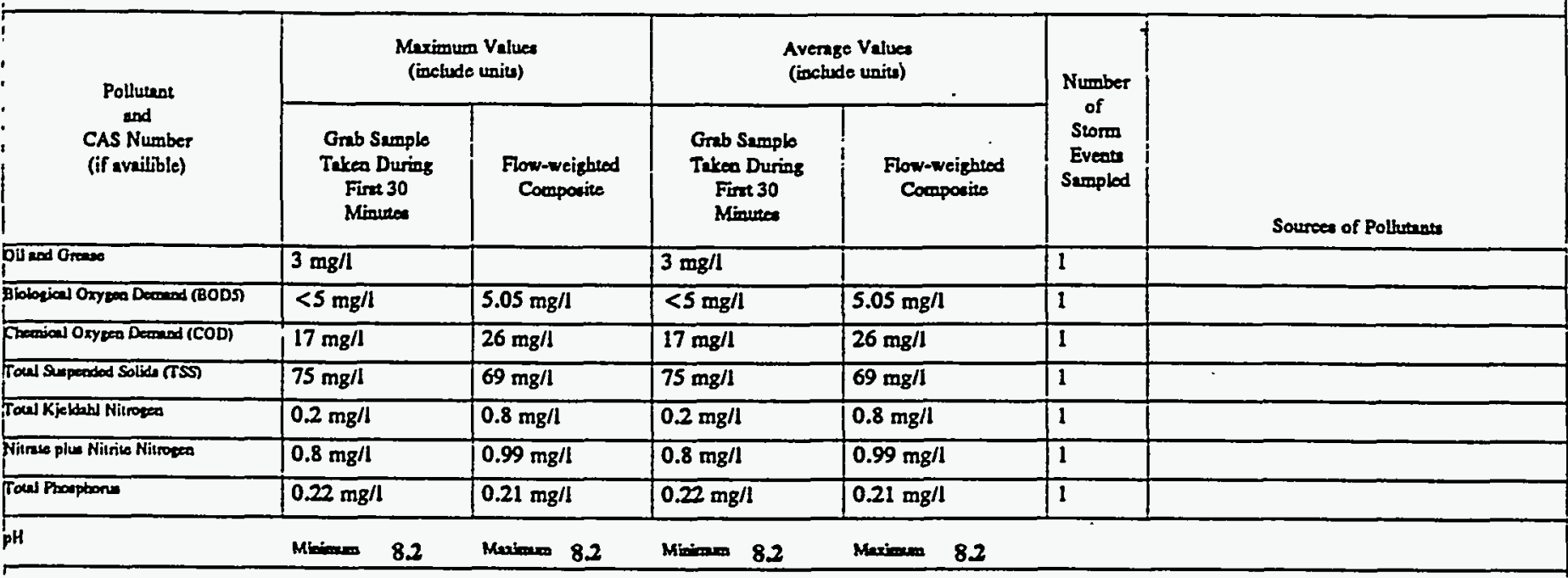

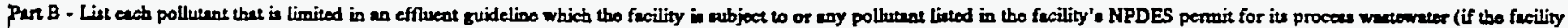

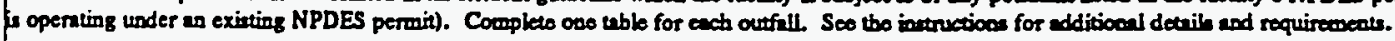

\begin{tabular}{|c|c|c|c|c|c|c|}
\hline trimoay $740-360$ & $<0.04 \mathrm{mg} / \mathrm{l}$ & $<0.04 \mathrm{mg} / \mathrm{l}$ & $<0.04 \mathrm{mg} / \mathrm{l}$ & $<0.04 \mathrm{mg} / \mathrm{l}$ & 1 & \\
\hline Movoury 7439-97.6 & $0.0004 \mathrm{mg} / \mathrm{l}$ & $0.0005 \mathrm{mg} / 1$ & $0.0004 \mathrm{mg} / 1$ & $0.0005 \mathrm{mg} / \mathrm{h}$ & 1 & \\
\hline preods & $0.003 \mathrm{mg} / \mathrm{l}$ & & $0.003 \mathrm{mg} / \mathrm{l}$ & & 1 & 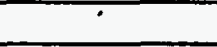 \\
\hline Peteorum $778249-2$ & $<0.002 \mathrm{mg} / \mathrm{l}$ & $<0.002 \mathrm{mg} / \mathrm{l}$ & $<0.002 \mathrm{mg} / \mathrm{l}$ & $<0.002 \mathrm{mg} / \mathrm{l}$ & 1 & \\
\hline Pulfate & $14 \mathrm{mg} / \mathrm{l}$ & $22 \mathrm{mg} / \mathrm{l}$ & $14 \mathrm{mg} / \mathrm{l}$ & $22 \mathrm{mg} / \mathrm{l}$ & 1 & \\
\hline purtacens & $<0.05 \mathrm{mg} / \mathrm{l}$ & $<0.05 \mathrm{mg} / \mathrm{l}$ & $<0.05 \mathrm{mg} / \mathrm{l}$ & $<0.05 \mathrm{mg} / \mathrm{l}$ & 1 & \\
\hline Alumanom 7422-90-3 & $2.89 \mathrm{mg} / 1$ & $2.61 \mathrm{mg} / \mathrm{l}$ & $2.89 \mathrm{mg} / \mathrm{l}$ & $2.61 \mathrm{mg} / \mathrm{l}$ & 1 & \\
\hline Cedmilum 744a-43-9 & $<0.004 \mathrm{mg} / \mathrm{l}$ & $<0.004 \mathrm{mg} / \mathrm{l}$ & $<0.004 \mathrm{mg} / \mathrm{l}$ & $<0.004 \mathrm{mg} / \mathrm{l}$ & 1 & \\
\hline Calcium 7440-20-2 & $27.0 \mathrm{mg} / \mathrm{I}$ & $31.1 \mathrm{mg} / 1$ & $27.0 \mathrm{mg} / 1$ & $31.1 \mathrm{mg} / \mathrm{l}$ & 1 & \\
\hline 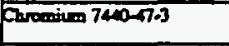 & $<0.006 \mathrm{mg} / \mathrm{l}$ & $<0.006 \mathrm{mg} / \mathrm{l}$ & $<0.006 \mathrm{mg} / \mathrm{l}$ & $<0.006 \mathrm{mg} / \mathrm{l}$ & 1 & \\
\hline Cotali 7440-184- & $<0.002 \mathrm{mg} / \mathrm{l}$ & $0.002 \mathrm{mg} / \mathrm{l}$ & $<0.002 \mathrm{mg} / \mathrm{l}$ & $0.002 \mathrm{mg} / \mathrm{l}$ & 1 & \\
\hline Copper 7400-sos & $0.019 \mathrm{mg} / \mathrm{l}$ & $0.015 \mathrm{mg} / \mathrm{l}$ & $0.019 \mathrm{mg} / \mathrm{l}$ & $0.015 \mathrm{mg} / \mathrm{l}$ & 1 & \\
\hline 10007439826 & $3.32 \mathrm{mg} / \mathrm{l}$ & $2.58 \mathrm{mg} / \mathrm{l}$ & $3.32 \mathrm{mg} / 1$ & $2.58 \mathrm{mg} / \mathrm{l}$ & 1 & \\
\hline 70ed 749-2.1 & $<0.02 \mathrm{mg} / \mathrm{l}$ & $<0.02 \mathrm{mg} / 1$ & $<0.02 \mathrm{mg} / 1$ & $<0.02 \mathrm{mg} / 1$ & 1 & \\
\hline Aubinom 7439-93-2 & $<0.02 \mathrm{mg} / \mathrm{l}$ & $<0.02 \mathrm{mg} / \mathrm{l}$ & $<0.02 \mathrm{mg} / \mathrm{l}$ & $<0.02 \mathrm{mg} / \mathrm{l}$ & 1 & \\
\hline Magoounum 7439-95-4 & $3.00 \mathrm{mg} / \mathrm{l}$ & $3.51 \mathrm{mg} / 1$ & $3.00 \mathrm{mg} / 1$ & $3.51 \mathrm{mg} / 1$ & 1 & \\
\hline 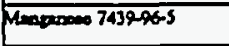 & $0.082 \mathrm{mg} / \mathrm{l}$ & $0.091 \mathrm{mg} / \mathrm{l}$ & $0.082 \mathrm{mg} / \mathrm{l}$ & $0.091 \mathrm{mg} / \mathrm{l}$ & 1 & \\
\hline Motybdoon 7439-98-7 & $<0.006 \mathrm{mg} / \mathrm{l}$ & $<0.006 \mathrm{mg} / \mathrm{l}$ & $<0.006 \mathrm{mg} / \mathrm{l}$ & $<0.006 \mathrm{mg} / 1$ & 1 & \\
\hline
\end{tabular}


Cookinued from frons.

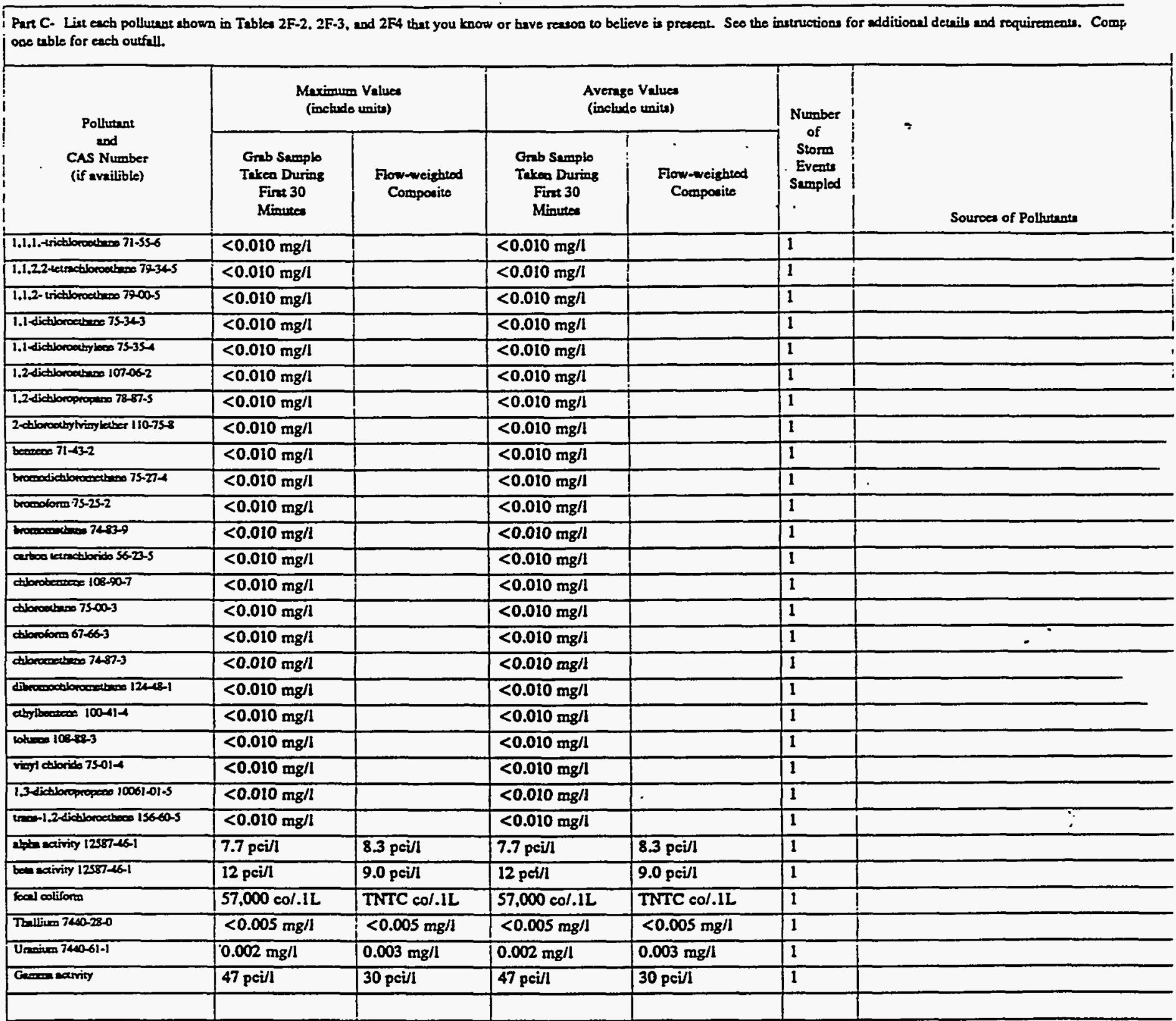

Part D. Provide dala for the sorm evear(0) which reauled in the maximum valued for the flow weightod componito aemplo.

\begin{tabular}{|c|c|c|c|c|c|c|c|}
\hline $\begin{array}{l}1 . \\
\text { Date of } \\
\text { Storm } \\
\text { Eveat }\end{array}$ & $\begin{array}{l}2 . \\
\text { Duratron } \\
\text { of Sione } \\
\text { (in } \\
\text { minutea) }\end{array}$ & $\begin{array}{l}3 . \\
\text { Tooul ninfell } \\
\text { during storm event } \\
\text { (in inchen) }\end{array}$ & 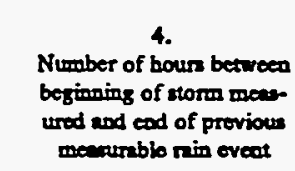 & $\begin{array}{c}\text { S. } \\
\text { Maximum flow nato } \\
\text { duriag min eveat } \\
\text { (alloce/min or spocify } \\
\text { unit) }\end{array}$ & $\begin{array}{c}6 . \\
\text { Toul flow from } \\
\text { nil oveat } \\
\text { (allons or } \\
\text { specify units) }\end{array}$ & $\begin{array}{c}7 . \\
\text { Seavon } \\
\text { earoplo was } \\
\text { tukeo }\end{array}$ & $\begin{array}{l}8 . \\
\text { Form of } \\
\text { Precipitation } \\
\text { (ninfill, } \\
\text { seowmely) }\end{array}$ \\
\hline $8 / 26 / 91$ & 410 & 12 & 160.2 & 27 & 8,426 & Summer & Rainfall \\
\hline
\end{tabular}

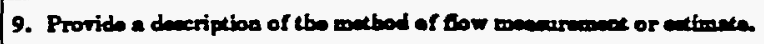

An ISCO 3230 Flow meter wos nsed to obtain water level readings. This instrument was calibrated with the normal dry veather flow, if present, as level zero. The flow meter was programmed with the characteristics of the conveyance so that flow rates and total nows conld be calculated by the meter. 


\begin{tabular}{|c|c|c|c|c|c|c|}
\hline \multirow{2}{*}{$\begin{array}{l}\text { Pollutuant } \\
\text { and } \\
\text { CAS Number } \\
\text { (if availible) }\end{array}$} & \multicolumn{2}{|c|}{$\begin{array}{l}\text { Maximaum Veluea } \\
\text { (include unitu) }\end{array}$} & \multicolumn{2}{|c|}{$\begin{array}{l}\text { Avernge Values } \\
\text { (includo unius) }\end{array}$} & \multirow{2}{*}{$\begin{array}{l}\text { Number } \\
\text { of } \\
\text { Storm" } \\
\text { Events } \\
\text { Seropled }\end{array}$} & \multirow[b]{2}{*}{ Sources of Polluments } \\
\hline & $\begin{array}{l}\text { Grab Sxmplo } \\
\text { Taken During } \\
\text { Fint } 30 \\
\text { Minutea }\end{array}$ & $\begin{array}{l}\text { Flow-weizhted } \\
\text { Comporine }\end{array}$ & $\begin{array}{l}\text { Greb Sample } \\
\text { Takea During } \\
\text { Fint } 30 \\
\text { Minutea }\end{array}$ & $\begin{array}{l}\text { Flow-weighrod } \\
\text { Componite }\end{array}$ & & \\
\hline Aarium 7400.39-3 & $0.0376 \mathrm{mg} / \mathrm{l}$ & $0.0328 \mathrm{mg} / \mathrm{l}$ & $0.0376 \mathrm{mg} / \mathrm{l}$ & $0.0328 \mathrm{mg} / \mathrm{l}$ & 1 & \\
\hline 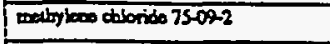 & $<0.010 \mathrm{mg} / 1$ & & $<0.010 \mathrm{mg} / \mathrm{l}$ & & 1 & \\
\hline Letrectloroocheos $127.18-4$ & $<0.010 \mathrm{mg} / \mathrm{l}$ & & $<0.010 \mathrm{mg} / 1$ & & 1 & \\
\hline irictionomberso $7901-5$ & $<0.010 \mathrm{mg} / 1$ & & $<0.010 \mathrm{mg} / \mathrm{l}$ & & 1 & \\
\hline Xyleacen & $<0.010 \mathrm{mg} / 1$ & & $<0.010 \mathrm{mg} / \mathrm{l}$ & & 1 & \\
\hline 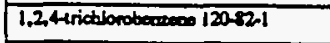 & $<0.010 \mathrm{mg} / \mathrm{l}$ & $<0.010 \mathrm{mg} / \mathrm{t}$ & $<0.010 \mathrm{mg} / \mathrm{l}$ & $<0.010 \mathrm{mg} / \mathrm{l}$ & 1 & \\
\hline 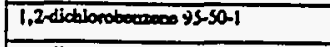 & $<0.010 \mathrm{mg} / \mathrm{l}$ & $<0.010 \mathrm{mg} / 1$ & $<0.010 \mathrm{mg} / \mathrm{l}$ & $<0.010 \mathrm{mg} / \mathrm{l}$ & 1 & \\
\hline 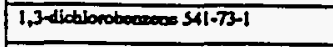 & $<0.010 \mathrm{mg} / \mathrm{l}$ & $<0.010 \mathrm{mg} / \mathrm{l}$ & $<0.010 \mathrm{mg} / \mathrm{l}$ & $<0.010 \mathrm{mg} / \mathrm{l}$ & 1 & \\
\hline 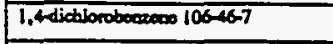 & $<0.010 \mathrm{mg} / \mathrm{l}$ & $<0.010 \mathrm{mg} / \mathrm{l}$ & $<0.010 \mathrm{mg} / \mathrm{l}$ & $<0.010 \mathrm{mg} / \mathrm{l}$ & 1 & \\
\hline 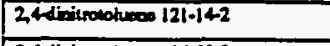 & $<0.010 \mathrm{mg} / \mathrm{l}$ & $<0.010 \mathrm{mg} / \mathrm{l}$ & $<0.010 \mathrm{mg} / \mathrm{l}$ & $<0.010 \mathrm{mg} / \mathrm{l}$ & 1 & \\
\hline 2,6-dinitrololiveos 606-200-2 & $<0.010 \mathrm{mg} / \mathrm{l}$ & $<0.010 \mathrm{mg} / \mathrm{l}$ & $<0.010 \mathrm{mg} / 1$ & $<0.010 \mathrm{mg} / \mathrm{l}$ & 1 & \\
\hline 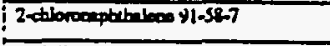 & $<0.010 \mathrm{mg} / \mathrm{l}$ & $<0.010 \mathrm{mg} / \mathrm{l}$ & $<0.010 \mathrm{mg} / \mathrm{l}$ & $<0.010 \mathrm{mg} / \mathrm{l}$ & $1^{\circ}$ & \\
\hline 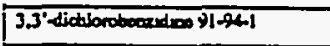 & $<0.010 \mathrm{mg} / \mathrm{l}$ & $<0.010 \mathrm{mg} / \mathrm{l}$ & $<0.010 \mathrm{mg} / 1$ & $<0.010 \mathrm{mg} / \mathrm{l}$ & 1 & \\
\hline 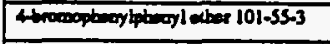 & $<0.010 \mathrm{mg} / 1$ & $<0.010 \mathrm{mg} / \mathrm{ll}$ & $<0.010 \mathrm{mg} / \mathrm{l}$ & $<0.010 \mathrm{mg} / 1$ & 1 & \\
\hline 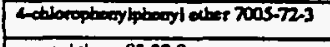 & $<0.010 \mathrm{mg} / \mathrm{l}$ & $<0.010 \mathrm{mg} / \mathrm{t}$ & $<0.010 \mathrm{mg} / \mathrm{l}$ & $<0.010 \mathrm{mg} / \mathrm{l}$ & 1 & \\
\hline $83-32-9$ & $<0.010 \mathrm{mg} / \mathrm{l}$ & $<0.010 \mathrm{mg} / \mathrm{l}$ & $<0.010 \mathrm{mg} / \mathrm{l}$ & $<0.010 \mathrm{mg} / \mathrm{l}$ & 1 & \\
\hline 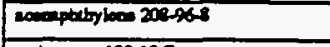 & $<0.010 \mathrm{mg} / \mathrm{l}$ & $<0.010 \mathrm{mg} / 1$ & $<0.010 \mathrm{mg} / \mathrm{l}$ & $<0.010 \mathrm{mg} / \mathrm{l}$ & 1 & \\
\hline 120.12 .7 & $<0.010 \mathrm{mg} / \mathrm{l}$ & $<0.010 \mathrm{mg} / \mathrm{l}$ & $<0.010 \mathrm{mg} / \mathrm{l}$ & $<0.010 \mathrm{mg} / \mathrm{l}$ & 1 & 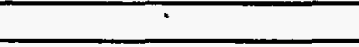 \\
\hline becosidino 9287.5 & $<0.030 \mathrm{mg} / \mathrm{l}$ & $<0.030 \mathrm{mg} / 1$ & $<0.030 \mathrm{mg} / \mathrm{l}$ & $<0.030 \mathrm{mg} / \mathrm{l}$ & 1 & \\
\hline 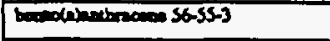 & $<0.010 \mathrm{mg} / \mathrm{l}$ & $<0.010 \mathrm{mg} / 1$ & $<0.010 \mathrm{mg} / \mathrm{l}$ & $<0.010 \mathrm{mg} / \mathrm{l}$ & 1 & \\
\hline 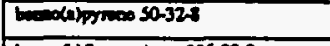 & $<0.010 \mathrm{mg} / \mathrm{l}$ & $<0.010 \mathrm{mg} / \mathrm{l}$ & $<0.010 \mathrm{mg} / \mathrm{l}$ & $<0.010 \mathrm{mg} / \mathrm{l}$ & 1 & \\
\hline 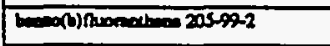 & $<0.010 \mathrm{mg} / \mathrm{l}$ & $<0.010 \mathrm{mg} / \mathrm{l}$ & $<0.010 \mathrm{mg} / \mathrm{l}$ & $<0.010 \mathrm{mg} / \mathrm{l}$ & 1 & \\
\hline 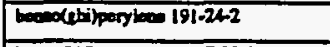 & $<0.010 \mathrm{mg} / \mathrm{l}$ & $<0.010 \mathrm{mg} / \mathrm{l}$ & $<0.010 \mathrm{mg} / \mathrm{l}$ & $<0.010 \mathrm{mg} / \mathrm{l}$ & 1 & \\
\hline 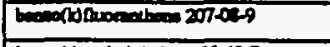 & $<0.010 \mathrm{mg} / \mathrm{l}$ & $<0.010 \mathrm{mg} / \mathrm{l}$ & $<0.010 \mathrm{mg} / \mathrm{l}$ & $<0.010 \mathrm{mg} / \mathrm{l}$ & 1 & \\
\hline 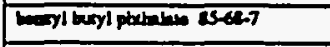 & $<0.010 \mathrm{mg} / \mathrm{l}$ & $<0.010 \mathrm{mg} / 1$ & $<0.010 \mathrm{mg} / 1$ & $<0.010 \mathrm{mg} / \mathrm{l}$ & 1 & \\
\hline 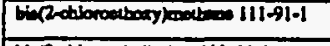 & $<0.010 \mathrm{mg} / 1$ & $<0.010 \mathrm{mg} / \mathrm{l}$ & $<0.010 \mathrm{mg} / \mathrm{l}$ & $<0.010 \mathrm{mg} / 1$ & 1 & 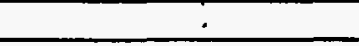 \\
\hline 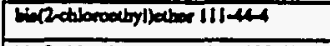 & $<0.010 \mathrm{mg} / \mathrm{l}$ & $<0.010 \mathrm{mg} / \mathrm{l}$ & $<0.010 \mathrm{mg} / \mathrm{l}$ & $<0.010 \mathrm{mg} / \mathrm{l}$ & 1 & \\
\hline 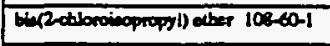 & $<0.010 \mathrm{mg} / \mathrm{l}$ & $<0.010 \mathrm{mg} / \mathrm{l}$ & $<0.010 \mathrm{mg} / \mathrm{L}$ & $<0.010 \mathrm{mg} / \mathrm{h}$ & 1 & \\
\hline 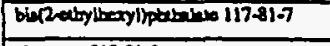 & $<0.010 \mathrm{mg} / \mathrm{l}$ & $<0.010 \mathrm{mg} / \mathrm{l}$ & $<0.010 \mathrm{mg} / 1$ & $<0.010 \mathrm{mg} / \mathrm{l}$ & 1 & \\
\hline drpocen 21801-9 & $<0.010 \mathrm{mg} / \mathrm{l}$ & $<0.010 \mathrm{mg} / \mathrm{l}$ & $<0.010 \mathrm{mg} / \mathrm{l}$ & $<0.010 \mathrm{mg} / 1$ & 1 & \\
\hline dioteryl ptethene 84-74-2, & $<0.010 \mathrm{mg} / \mathrm{l}$ & $<0.010 \mathrm{mg} / \mathrm{I}$ & $<0.010 \mathrm{mg} / \mathrm{l}$ & $<0.010 \mathrm{mg} / \mathrm{l}$ & 1 & \\
\hline 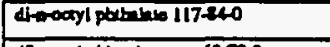 & $<0.010 \mathrm{mg} / \mathrm{l}$ & $<0.010 \mathrm{mg} / \mathrm{I}$ & $<0.010 \mathrm{mg} / \mathrm{l}$ & $<0.010 \mathrm{mg} / \mathrm{l}$ & 1 & \\
\hline 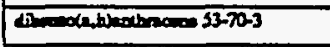 & $<0.010 \mathrm{mg} / \mathrm{l}$ & $<0.010 \mathrm{mg} / \mathrm{l}$ & $<0.010 \mathrm{mg} / \mathrm{l}$ & $<0.010 \mathrm{mg} / \mathrm{h}$ & 1 & \\
\hline 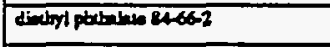 & $<0.010 \mathrm{mg} / \mathrm{l}$ & $<0.010 \mathrm{mg} / \mathrm{l}$ & $<0.010 \mathrm{mg} / \mathrm{l}$ & $<0.010 \mathrm{mg} / 1$ & 1 & \\
\hline 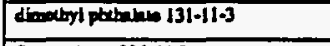 & $<0.010 \mathrm{mg} / \mathrm{l}$ & $<0.010 \mathrm{mg} / \mathrm{l}$ & $<0.010 \mathrm{mg} / \mathrm{l}$ & $<0.010 \mathrm{mg} / 1$ & 1 & \\
\hline 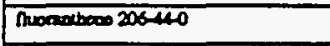 & $<0.010 \mathrm{mg} / \mathrm{l}$ & $<0.010 \mathrm{mg} / \mathrm{l}$ & $<0.010 \mathrm{mg} / 1$ & $<0.010 \mathrm{mg} / \mathrm{h}$ & 1 & \\
\hline nooneon 86-73-7 & $<0.010 \mathrm{mg} / 1$ & $<0.010 \mathrm{mg} / \mathrm{l}$ & $<0.010 \mathrm{mg} / \mathrm{l}$ & $<0.010 \mathrm{mg} / \mathrm{l}$ & 1 & \\
\hline 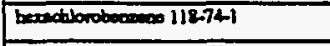 & $<0.010 \mathrm{mg} / \mathrm{l}$ & $<0.010 \mathrm{mg} / \mathrm{l}$ & $<0.010 \mathrm{mg} / \mathrm{l}$ & $<0.010 \mathrm{mg} / \mathrm{l}$ & 1 & \\
\hline 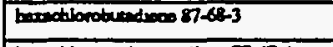 & $<0.010 \mathrm{mg} / \mathrm{l}$ & $<0.010 \mathrm{mg} / \mathrm{l}$ & $<0.010 \mathrm{mg} / \mathrm{l}$ & $<0.010 \mathrm{mg} / \mathrm{h}$ & 1 & \\
\hline 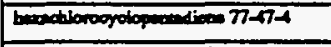 & $<0.010 \mathrm{mg} / \mathrm{l}$ & $<0.010 \mathrm{mg} / \mathrm{l}$ & $<0.010 \mathrm{mg} / \mathrm{l}$ & $<0.010 \mathrm{mg} / \mathrm{l}$ & 1 & \\
\hline 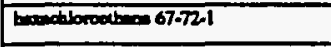 & $<0.010 \mathrm{mg} / \mathrm{l}$ & $<0.010 \mathrm{mg} / \mathrm{l}$ & $<0.010 \mathrm{mg} / \mathrm{l}$ & $<0.010 \mathrm{mg} / \mathrm{l}$ & 1 & \\
\hline 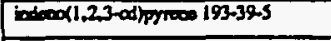 & $<0.010 \mathrm{mg} / 1$ & $<0.010$ mg/l & $<0.010 \mathrm{mg} / \mathrm{h}$ & $<0.010 \mathrm{mg} / \mathrm{l}$ & 1 & \\
\hline inopbovoses 78-59-1 & $<0.010 \mathrm{mg} / \mathrm{l}$ & $<0.010 \mathrm{mg} / 1$ & $<0.010 \mathrm{mg} / 1$ & $<0.010 \mathrm{mg} / \mathrm{l}$ & 1 & \\
\hline 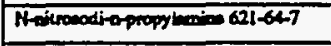 & $<0.010 \mathrm{mg} / \mathrm{l}$ & $<0.010 \mathrm{mg} / \mathrm{l}$ & $<0.010 \mathrm{mg} / \mathrm{s}$ & $<0.010 \mathrm{mg} / \mathrm{l}$ & 1 & \\
\hline 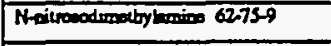 & $<0.010 \mathrm{mg} / 1$ & $<0.010 \mathrm{mg} / 1$ & $<0.010 \mathrm{mg} / \mathrm{l}$ & $<0.010 \mathrm{mg} / \mathrm{l}$ & 1 & \\
\hline 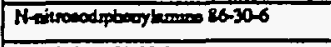 & $<0.010 \mathrm{mg} / \mathrm{l}$ & $<0.010 \mathrm{mg} / \mathrm{l}$ & $<0.010 \mathrm{mg} / 1$ & $<0.010 \mathrm{mg} / \mathrm{l}$ & 1 & \\
\hline anptiniber 91.20 .3 & $<0.010 \mathrm{mg} / \mathrm{l}$ & $<0.010 \mathrm{mg} / \mathrm{l}$ & $<0.010 \mathrm{mg} / 1$ & $<0.010 \mathrm{mg} / 1$ & 1 & \\
\hline 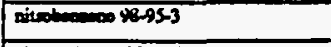 & $<0.010 \mathrm{mg} / \mathrm{l}$ & $<0.010 \mathrm{mg} / 1$ & $<0.010 \mathrm{mg} / 1$ & $<0.010 \mathrm{mg} / \mathrm{l}$ & 1 & \\
\hline 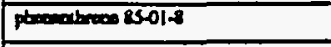 & $<0.010 \mathrm{mg} / \mathrm{l}$ & $<0.010 \mathrm{mg} / \mathrm{l}$ & $<0.010 \mathrm{mg} / 1$ & $<0.010 \mathrm{mg} / \mathrm{l}$ & 1 & \\
\hline 897000129000 & $<0.010 \mathrm{mg} / \mathrm{l}$ & $<0.010 \mathrm{mg} / \mathrm{l}$ & $<0.010 \mathrm{mg} / \mathrm{l}$ & $<0.010 \mathrm{mg} / \mathrm{l}$ & 1 & \\
\hline 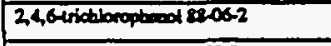 & $<0.030 \mathrm{mg} / \mathrm{l}$ & $<0.030 \mathrm{mg} / \mathrm{l}$ & $<0.030 \mathrm{mg} / \mathrm{l}$ & $<0.030 \mathrm{mg} / \mathrm{l}$ & 1 & \\
\hline 2,4dictionophesed 120-503-2 & $<0.030 \mathrm{mg} / \mathrm{l}$ & $<0.030 \mathrm{mg} / \mathrm{l}$ & $<0.030 \mathrm{mg} / 1$ & $<0.030 \mathrm{mg} / \mathrm{l}$ & 1 & \\
\hline 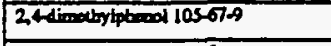 & $<0.030 \mathrm{mg} / \mathrm{l}$ & $<0.030 \mathrm{mg} / \mathrm{l}$ & $<0.030 \mathrm{mg} / \mathrm{l}$ & $<0.030 \mathrm{mg} / \mathrm{l}$ & 1 & \\
\hline 24tinitropteond 51-22-5 & $<0.030 \mathrm{mg} / \mathrm{l}$ & $<0.030 \mathrm{mg} / \mathrm{l}$ & $<0.030 \mathrm{mg} / 1$ & $<0.030 \mathrm{mg} / \mathrm{l}$ & 1 & \\
\hline
\end{tabular}


Outfall 212

Pars B and C Continuod

\begin{tabular}{|c|c|c|c|c|c|c|}
\hline \multirow{2}{*}{$\begin{array}{l}\text { Pollutant } \\
\text { and } \\
\text { CAS Number } \\
\text { (if availible) }\end{array}$} & \multicolumn{2}{|c|}{$\begin{array}{l}\text { Maximum Vatuea } \\
\text { (include units) }\end{array}$} & \multicolumn{2}{|c|}{$\begin{array}{l}\text { Average Valuea } \\
\text { (ibciude units) }\end{array}$} & \multirow{2}{*}{$\begin{array}{l}\text { Number } \\
\text { of } \\
\text { Stom } \\
\text { Events } \\
\text { Sampiod }\end{array}$} & \multirow[b]{2}{*}{ Sources of Pollumants } \\
\hline & $\begin{array}{l}\text { Grab Sample } \\
\text { Taken During } \\
\text { Fint } 30 \\
\text { Minuteo }\end{array}$ & $\begin{array}{l}\text { Frow-weighted } \\
\text { Composite }\end{array}$ & $\begin{array}{l}\text { Grab Semple } \\
\text { Takes During } \\
\text { Firnt } 30 \\
\text { Minite }\end{array}$ & $\begin{array}{c}\text { Fow-weigheod } \\
\text { i }\end{array}$ & & \\
\hline 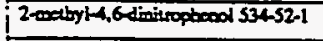 & $<0.030 \mathrm{mg} / \mathrm{l}$ & $<0.030 \mathrm{mg} / \mathrm{l}$ & $<0.030 \mathrm{mg} / \mathrm{l}$ & $<0.030 \mathrm{mg} / \mathrm{l}$ & 1 & \\
\hline 2-sitropheosol 828-75-5 & $<0.030 \mathrm{mg} / \mathrm{l}$ & $<0.030 \mathrm{mg} / \mathrm{l}$ & $<0.030 \mathrm{mg} / \mathrm{l}$ & $<0.030 \mathrm{mg} / 1$ & 1 & \\
\hline 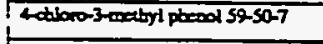 & $<0.030 \mathrm{mg} / \mathrm{l}$ & $<0.030 \mathrm{mg} / \mathrm{i}$ & $<0.030 \mathrm{mg} / \mathrm{l}$ & $<0.030 \mathrm{mg} / \mathrm{l}$ & 1 & \\
\hline Anitropteod 10002.7 & $<0.030 \mathrm{mg} / \mathrm{l}$ & $<0.030 \mathrm{mg} / \mathrm{l}$ & $<0.030 \mathrm{mg} / \mathrm{l}$ & $<0.030 \mathrm{mg} / \mathrm{l}$ & 1 & \\
\hline peractionopheod $87-86-5$ & $<0.030 \mathrm{mg} / \mathrm{l}$ & $<0.030 \mathrm{mg} / \mathrm{l}$ & $<0.030 \mathrm{mg} / \mathrm{l}$ & $<0.030 \mathrm{mg} / \mathrm{l}$ & 1 & \\
\hline ptoend 108-95-2 & $<0.030 \mathrm{mg} / \mathrm{l}$ & $<0.030 \mathrm{mg} / 1$ & $<0.030 \mathrm{mg} / \mathrm{l}$ & $<0.030 \mathrm{mg} / \mathrm{l}$ & 1 & \\
\hline $4,4-0 D D 72-548$ & $<0.000004 \mathrm{mg} / \mathrm{l}$ & $<0.000004 \mathrm{mg} / \mathrm{l}$ & $<0.000004 \mathrm{mg} / \mathrm{l}$ & $1<0.000004 \mathrm{mg} / \mathrm{l}$ & 1 & \\
\hline 4.4.-DDE 72-55-9 & $<0.000004 \mathrm{mg} / \mathrm{l}$ & $<0.000004 \mathrm{mg} / \mathrm{l}$ & $<0.000004 \mathrm{mg} / \mathrm{l}$ & $<0.000004 \mathrm{mg} / \mathrm{l}$ & I & \\
\hline 4.4-DDT SO-29-3 & $<0.000004 \mathrm{mg} / \mathrm{l}$ & $<0.000004 \mathrm{mg} / \mathrm{l}$ & $<0.000004 \mathrm{mg} / \mathrm{l}$ & I $<0.000004 \mathrm{mg} / \mathrm{l}$ & 1 & \\
\hline aldria $309-00-2$ & $<0.000004 \mathrm{mg} / \mathrm{l}$ & $<0.000004 \mathrm{mg} / \mathrm{l}$ & $<0.000004 \mathrm{mg} / \mathrm{l}$ & $<0.000004 \mathrm{mg} / \mathrm{l}$ & 1 & \\
\hline chlonderes 57.74-9 & $<0.00002 \mathrm{mg} / \mathrm{l}$ & $<0.00002 \mathrm{mg} / \mathrm{l}$ & $<0.00002 \mathrm{mg} / 1$ & $<0.00002 \mathrm{mg} / \mathrm{l}$ & 1 & \\
\hline dieldria 60-57-1 & $<0.000004 \mathrm{mg} / \mathrm{l}$ & $<0.000004 \mathrm{mg} / \mathrm{l}$ & $<0.000004 \mathrm{mg} / \mathrm{l}$ & $<0.000004 \mathrm{mg} / \mathrm{l}$ & 1 & \\
\hline endovulfan I 959-98-8 & $<0.000004 \mathrm{mg} / \mathrm{l}$ & $<0.000004 \mathrm{mg} / \mathrm{l}$ & $<0.000004 \mathrm{mg} / \mathrm{l}$ & $<0.000004 \mathrm{mg} / 1$ & 1 & \\
\hline Crodocaltsen II 33213-65-9 & $<0.000004 \mathrm{mg} / \mathrm{l}$ & $<0.000004 \mathrm{mg} / \mathrm{l}$ & $<0.000004 \mathrm{mg} / \mathrm{l}$ & $<0.000004 \mathrm{mg} / \mathrm{l}$ & 1 & \\
\hline contandengulfow 1031.078 & $<0.000004 \mathrm{mg} / \mathrm{l}$ & $<0.000004 \mathrm{mg} / \mathrm{l}$ & $<0.000004 \mathrm{mg} / 1$ & $<0.000004 \mathrm{mg} / 1$ & 1 & \\
\hline codric 72.208 & $<0.00005 \mathrm{mg} / \mathrm{l}$ & $<0.00005 \mathrm{mg} / 1$ & $<0.00005 \mathrm{mg} / \mathrm{l}$ & $<0.00005 \mathrm{mg} / \mathrm{l}$ & 1 & \\
\hline codrin aldedy $7421-934$ & $<0.000004 \mathrm{mg} / \mathrm{l}$ & $<0.000004 \mathrm{mg} / \mathrm{l}$ & $<0.000004 \mathrm{mg} / \mathrm{l}$ & $<0.000004 \mathrm{mg} / \mathrm{l}$ & 1 & 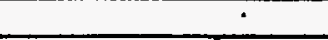 \\
\hline bepmotion $76-148$ & $<0.000004 \mathrm{mg} / \mathrm{l}$ & $<0.000004 \mathrm{mg} / \mathrm{l}$ & $<0.000004 \mathrm{mg} / \mathrm{l}$ & $<0.000004 \mathrm{mg} / \mathrm{l}$ & 1 & \\
\hline 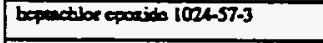 & $<0.000004 \mathrm{mg} / \mathrm{l}$ & $<0.000004 \mathrm{mg} / \mathrm{l}$ & $<0.000004 \mathrm{mg} / \mathrm{l}$ & $<0.000004 \mathrm{mg} / \mathrm{l}$ & 1 & \\
\hline 1arphoos $0001-35-2$ & $<0.0002 \mathrm{mg} / 1$ & $<0.0002 \mathrm{mg} / \mathrm{I}$ & $<0.0002 \mathrm{mg} / \mathrm{l}$ & $<0.0002 \mathrm{mg} / \mathrm{l}$ & 1 & \\
\hline apbo-8HC 319846 & $<0.000004 \mathrm{mg} / \mathrm{l}$ & $<0.000004 \mathrm{mg} / \mathrm{l}$ & $<0.000004 \mathrm{mg} / \mathrm{l}$ & $<0.000004 \mathrm{mg} / \mathrm{l}$ & 1 & \\
\hline $80-B H C$ ISISE-7 & $<0.000004 \mathrm{mg} / \mathrm{l}$ & $<0.000004 \mathrm{mg} / \mathrm{I}$ & $<0.000004 \mathrm{mg} / 1$ & $<0.000004 \mathrm{mg} / \mathrm{l}$ & 1 & \\
\hline dwo-8HC 319868 & $<0.000004 \mathrm{mg} / \mathrm{h}$ & $<0.000004 \mathrm{mg} / \mathrm{l}$ & $<0.000004 \mathrm{mg} / \mathrm{l}$ & $<0.000004 \mathrm{mg} / \mathrm{l}$ & 1 & \\
\hline Den-8HC $58-598$ & $<0.00001 \mathrm{mg} / \mathrm{l}$ & $<0.00001 \mathrm{mg} / \mathrm{l}$ & $<0.00001 \mathrm{mg} / 1$ & $<0.00001 \mathrm{mg} / \mathrm{l}$ & 1 & \\
\hline 2-chlonopocol 95-57.8 & $<0.030 \mathrm{mg} / \mathrm{l}$ & $<0.030 \mathrm{mg} / \mathrm{l}$ & $<0.030 \mathrm{mg} / 1$ & $<0.030 \mathrm{mg} / 1$ & 1 & 1 \\
\hline Arodor-120253467-21-9 & $<0.0005 \mathrm{mg} / \mathrm{l}$ & $<0.0005 \mathrm{mg} / 1$ & $<0.0005 \mathrm{mg} / \mathrm{l}$ & $<0.0005 \mathrm{mg} / 1$ & 1 & \\
\hline Anocton-1254 $11097-69-1$ & $<0.0005 \mathrm{mg} / \mathrm{l}$ & $<0.0005 \mathrm{mg} / \mathrm{l}$ & $<0.0005 \mathrm{mg} / \mathrm{ll}$ & $<0.0005 \mathrm{mg} / \mathrm{l}$ & 1 & \\
\hline Arocion $122111104-28-2$ & $<0.0005 \mathrm{mg} / \mathrm{l}$ & $<0.0005 \mathrm{mg} / \mathrm{l}$ & $<0.0005 \mathrm{mg} / \mathrm{l}$ & $<0.0005 \mathrm{mg} / \mathrm{I}$ & 1 & \\
\hline Aroctor-1232 II141-16-5 & $<0.0005 \mathrm{mg} / \mathrm{l}$ & $<0.0005 \mathrm{mg} / \mathrm{l}$ & $<0.0005 \mathrm{mg} / \mathrm{l}$ & $<0.0005 \mathrm{mg} / \mathrm{l}$ & 1 & \\
\hline Arocton $124812572-296$ & $<0.0005 \mathrm{mg} / \mathrm{l}$ & $<0.0005 \mathrm{mg} / 1$ & $<0.0005 \mathrm{mg} / 1$ & $<0.0005 \mathrm{mg} / \mathrm{l}$ & 1 & \\
\hline Arodon $126011006822-5$ & $<0.0005 \mathrm{mg} / \mathrm{l}$ & $<0.0005 \mathrm{mg} / \mathrm{l}$ & $<0.0005 \mathrm{mg} / \mathrm{l}$ & $<0.0005 \mathrm{mg} / \mathrm{l}$ & 1 & \\
\hline Arodor-1016 $1267411-2$ & $<0.0005 \mathrm{mg} / \mathrm{l}$ & $<0.0005 \mathrm{mg} / \mathrm{l}$ & $<0.0005 \mathrm{mg} / 1$ & $<0.0005 \mathrm{mg} / \mathrm{l}$ & 1 & \\
\hline Creade & $<0.030 \mathrm{mg} / 1$ & $<0.030 \mathrm{mg} / \mathrm{l}$ & $<0.030 \mathrm{mg} / \mathrm{l}$ & $<0.030 \mathrm{mg} / \mathrm{l}$ & 1 & \\
\hline Aaroling & $<0.010 \mathrm{mg} / \mathrm{l}$ & $<0.010 \mathrm{mg} / \mathrm{l}$ & $<0.010 \mathrm{mg} / 1$ & $<0.010 \mathrm{mg} / \mathrm{l}$ & 1 & \\
\hline Acrybaitrib & $<0.010 \mathrm{mg} / \mathrm{l}$ & $<0.010 \mathrm{mg} / \mathrm{l}$ & $<0.010 \mathrm{mg} / 1$ & $<0.010 \mathrm{mg} / \mathrm{l}$ & 1 & \\
\hline 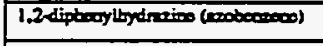 & Results pending & Reaults pending & Results pending & Reaults pending & 1 & \\
\hline & & & & & & \\
\hline & & & & & & \\
\hline & & & & & & \\
\hline & & & & & & \\
\hline & & & & & & \\
\hline & & & & & & \\
\hline & & & & & & \\
\hline & & & & & & \\
\hline & & & & & & \\
\hline & & & & & & \\
\hline & & & & & & \\
\hline & & & & & & \\
\hline & & & & & & \\
\hline & & & & & & \\
\hline & & & & & & \\
\hline
\end{tabular}


VII. Discharge Information (continuad from page 3 of Form 2F)

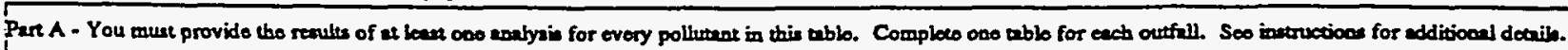

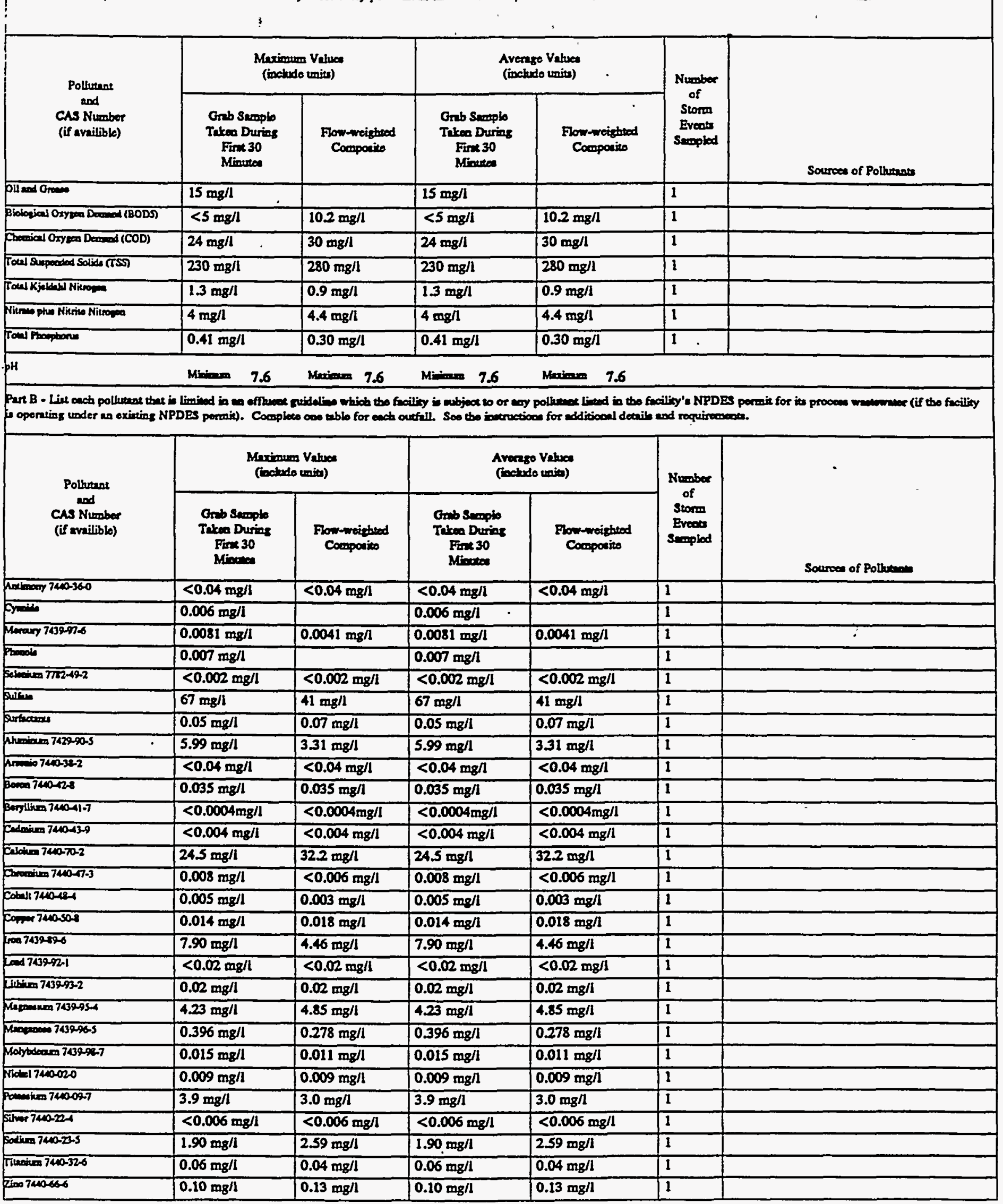


Continued from frons.

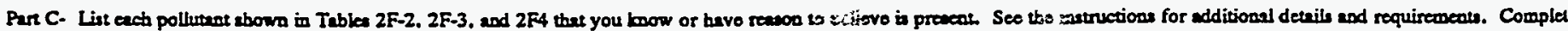
one table for each outfall.

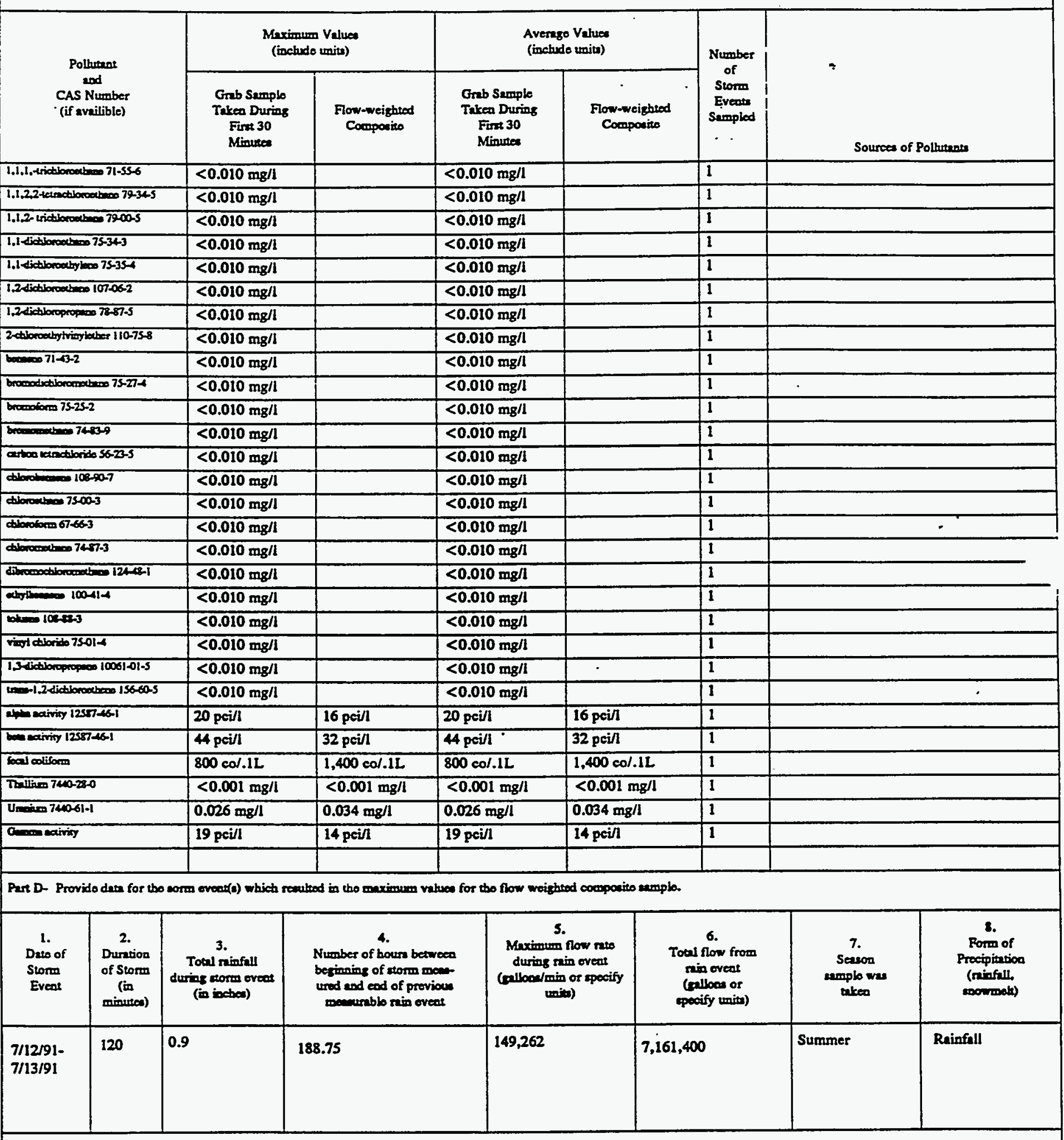

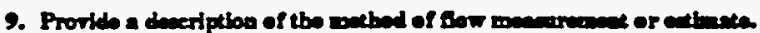

An ISCO 3230 Flow meter was med to obtain water level readings. This instroment was catibrated with the normel dry weather flow, if present, as level zero. The flow meter was progrimmed with the characteristics of the converance so that flow rates and total flows could be calculated by the meter. 
North-South Pipe

Parta B and C Continuad

\begin{tabular}{|c|c|c|c|c|c|c|}
\hline \multirow{2}{*}{$\begin{array}{l}\text { Polbunat } \\
\text { and } \\
\text { CAs Number } \\
\text { (if availiblo) }\end{array}$} & \multicolumn{2}{|c|}{$\begin{array}{l}\text { Marimum Values } \\
\text { (include units) }\end{array}$} & \multicolumn{2}{|c|}{$\begin{array}{l}\text { Avernge Values } \\
\text { (ibchodo unitr) }\end{array}$} & \multirow{2}{*}{$\begin{array}{c}\text { Number, } \\
\text { of } \\
\text { Storrec } \\
\text { Eveoce } \\
\text { Semplod }\end{array}$} & \multirow[b]{2}{*}{ Sourcos of Polbrandes } \\
\hline & $\begin{array}{c}\text { Grab Samplo } \\
\text { Taken Durias } \\
\text { Fint } 30 \\
\text { Minuteos }\end{array}$ & $\begin{array}{l}\text { Flow-woighed } \\
\text { Compories }\end{array}$ & $\begin{array}{l}\text { Greb Sumplo } \\
\text { Trken During } \\
\text { Firt 30 } \\
\text { Minuteos }\end{array}$ & $\begin{array}{l}\text { Flow wreightod } \\
\text { Componito }\end{array}$ & & \\
\hline Barimom 7400-39-3 & $0.0792 \mathrm{mg} / \mathrm{l}$ & $0.0609 \mathrm{mg} / \mathrm{l}$ & $0.0792 \mathrm{mg} / \mathrm{l}$ & $0.0609 \mathrm{mg} / 1$ & 1 & \\
\hline 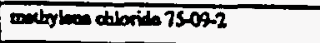 & $<0.010 \mathrm{mg} / \mathrm{h}$ & & $<0.010 \mathrm{mg} / \mathrm{l}$ & & 1 & \\
\hline 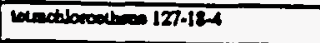 & $<0.010 \mathrm{mg} / 1$ & & $<0.010 \mathrm{mg} / 1$ & & 1 & \\
\hline 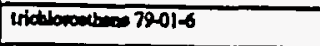 & $<0.010 \mathrm{mg} / \mathrm{l}$ & & $<0.010 \mathrm{mg} / 1$ & & 1 & \\
\hline xylanes & $<0.010 \mathrm{mg} / \mathrm{l}$ & & $<0.010 \mathrm{mgll}$ & & 1 & \\
\hline 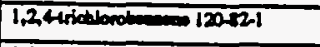 & $<0.010 \mathrm{mg} / 1$ & $<0.010 \mathrm{mg} / 1$ & $<0.010 \mathrm{mg} / \mathrm{l}$ & $<0.010 \mathrm{mg} / \mathrm{l}$ & 1 & \\
\hline 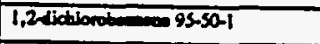 & $<0.010 \mathrm{mg} / \mathrm{l}$ & $<0.010 \mathrm{mg} / \mathrm{l}$ & $<0.010 \mathrm{mg} / \mathrm{l}$ & $<0.010 \mathrm{mg} / 1$ & 1 & \\
\hline 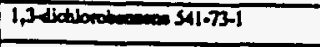 & $<0.010 \mathrm{mg} / \mathrm{l}$ & $<0.010 \mathrm{mg} / \mathrm{l}$ & $<0.010 \mathrm{mg} / \mathrm{l}$ & $<0.010 \mathrm{mg} / \mathrm{l}$ & 1 & \\
\hline 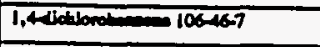 & $<0.010 \mathrm{mg} / \mathrm{l}$ & $<0.010 \mathrm{mg} / \mathrm{l}$ & $<0.010 \mathrm{mg} / 1$ & $<0.010 \mathrm{mg} / \mathrm{l}$ & 1 & \\
\hline 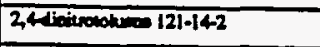 & $<0.010 \mathrm{mg} / \mathrm{l}$ & $<0.010 \mathrm{mg} / \mathrm{l}$ & $<0.010 \mathrm{mg} / 1$ & $<0.010 \mathrm{mg} / 1$ & 1 & \\
\hline 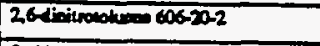 & $<0.010 \mathrm{mg} / \mathrm{l}$ & $<0.010 \mathrm{mg} / 1$ & $<0.010 \mathrm{mg} / 1$ & $<0.010 \mathrm{mg} / \mathrm{l}$ & 1 & \\
\hline 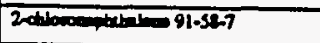 & $<0.010 \mathrm{mg} / 1$ & $<0.010 \mathrm{mg} / \mathrm{I}$ & $<0.010 \mathrm{mg} / \mathrm{l}$ & $<0.010 \mathrm{mg} / \mathrm{l}$ & 1 & \\
\hline 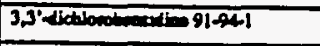 & $<0.010 \mathrm{mg} / \mathrm{l}$ & $<0.010 \mathrm{mg} / \mathrm{h}$ & $<0.010 \mathrm{mg} / 1$ & $<0.010 \mathrm{mg} / \mathrm{l}$ & 1 & \\
\hline 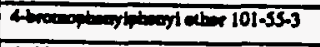 & $<0.010 \mathrm{mq} / 1$ & $<0.010 \mathrm{mg} / \mathrm{h}$ & $<0.010 \mathrm{mg} / \mathrm{l}$ & $<0.010 \mathrm{mg} / \mathrm{l}$ & 1 & \\
\hline 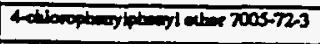 & $<0.010 \mathrm{mg} / \mathrm{l}$ & $<0.010 \mathrm{mg} /$ & $<0.010 \mathrm{mg} / 1$ & $<0.010 \mathrm{mg} / \mathrm{l}$ & 1 & \\
\hline $83-329$ & $<0.010 \mathrm{mg} / \mathrm{l}$ & $<0.010 \mathrm{mg} / 1$ & $<0.010 \mathrm{mg} / \mathrm{l}$ & $<0.010 \mathrm{mg} / \mathrm{l}$ & 1 & \\
\hline 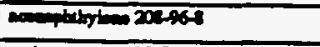 & $<0.010 \mathrm{mg} / \mathrm{l}$ & $<0.010 \mathrm{mg} / 1$ & $<0.010 \mathrm{mg} / \mathrm{l}$ & $<0.010 \mathrm{mg} / \mathrm{l}$ & 1 & $\therefore$ \\
\hline 120.127 & $<0.010 \mathrm{mg} / 1$ & $<0.010 \mathrm{mg} / \mathrm{h}$ & $<0.010 \mathrm{mg} / 1$ & $<0.010 \mathrm{mg} / \mathrm{l}$ & 1 & \\
\hline ition 92075 & $<0.030 \mathrm{mg} / \mathrm{l}$ & $<0.030 \mathrm{mg} / \mathrm{l}$ & $<0.030 \mathrm{mg} / 1$ & $<0.030 \mathrm{mg} / \mathrm{l}$ & 1 & \\
\hline 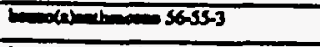 & $<0.010 \mathrm{mg} / \mathrm{h}$ & $<0.010 \mathrm{mg} / 1$ & $<0.010 \mathrm{mg} / \mathrm{l}$ & $<0.010 \mathrm{mg} / \mathrm{l}$ & 1 & \\
\hline 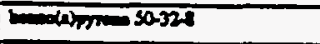 & $<0.010 \mathrm{mg} / \mathrm{l}$ & $<0.010 \mathrm{mg} / 1$ & $<0.010 \mathrm{mg} / 1$ & $<0.010 \mathrm{mg} / 1$ & 1 & \\
\hline 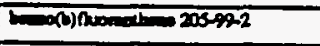 & $<0.010 \mathrm{mg} / \mathrm{l}$ & $<0.010 \mathrm{mg} / \mathrm{1}$ & $<0.010 \mathrm{mg} / 1$ & $<0.010 \mathrm{mg} / \mathrm{l}$ & 1 & \\
\hline 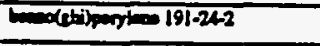 & $<0.010 \mathrm{mg} / \mathrm{l}$ & $<0.010 \mathrm{mq} / 1$ & $<0.010 \mathrm{mg} / 1$ & $<0.010 \mathrm{mg} / 1$ & 1 & \\
\hline 207029 & $<0.010 \mathrm{mg} / \mathrm{l}$ & $<0.010 \mathrm{mg} / 1$ & $<0.010 \mathrm{mg} / \mathrm{l}$ & $<0.010 \mathrm{mg} / 1$ & $\mathbf{I}$ & \\
\hline 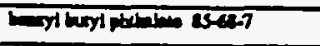 & $<0.010 \mathrm{mg} / \mathrm{l}$ & $<0.010 \mathrm{mq} / 1$ & $<0.010 \mathrm{mg} / \mathrm{l}$ & $<0.010 \mathrm{mg} / \mathrm{h}$ & 1 & $\therefore$ \\
\hline 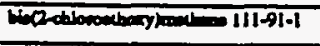 & $<0.010 \mathrm{mg} / \mathrm{l}$ & $<0.010 \mathrm{mg} / 1$ & $<0.010 \mathrm{mg} / \mathrm{l}$ & $<0.010 \mathrm{mg} / \mathrm{l}$ & 1 & \\
\hline 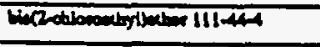 & $<0.010 \mathrm{mg} / \mathrm{l}$ & $<0.010 \mathrm{mg} / \mathrm{h}$ & $<0.010 \mathrm{mg} / 1$ & $<0.010 \mathrm{mg} / 1$ & 1 & \\
\hline 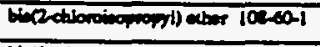 & $<0.010 \mathrm{mg} / \mathrm{l}$ & $<0.010 \mathrm{mg} / 1$ & $<0.010 \mathrm{mg} / \mathrm{I}$ & $<0.010 \mathrm{mg} / \mathrm{l}$ & 1 & \\
\hline 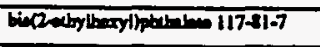 & $<0.010 \mathrm{mg} / \mathrm{l}$ & $<0.010 \mathrm{mg} / \mathrm{l}$ & $<0.010 \mathrm{mg} / \mathrm{l}$ & $<0.010 \mathrm{mg} / \mathrm{l}$ & 1 & \\
\hline 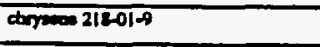 & $<0.010 \mathrm{mg} / \mathrm{l}$ & $<0.010 \mathrm{mg} / \mathrm{I}$ & $<0.010 \mathrm{mg} / \mathrm{l}$ & $<0.010 \mathrm{mg} / \mathrm{l}$ & 1 & \\
\hline 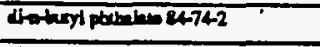 & $0.037 \mathrm{mg} / \mathrm{l}$ & $<0.010 \mathrm{mg} / 1$ & $0.037 \mathrm{mg} / \mathrm{l}$ & $<0.010 \mathrm{mg} / \mathrm{l}$ & 1 & \\
\hline 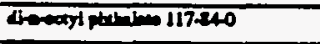 & $<0.010 \mathrm{mg} / 1$ & $<0.010 \mathrm{mg} / \mathrm{l}$ & $<0.010 \mathrm{mg} / 1$ & $<0.010 \mathrm{mg} / \mathrm{l}$ & 1 & \\
\hline 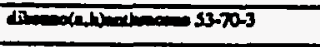 & $<0.010 \mathrm{mg} / \mathrm{l}$ & $<0.010 \mathrm{mg} / 1$ & $<0.010 \mathrm{mg} / 1$ & $<0.010 \mathrm{mg} / 1$ & 1 & \\
\hline 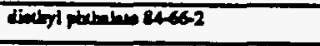 & $<0.010 \mathrm{mg} / \mathrm{l}$ & $<0.010 \mathrm{mg} / 1$ & $<0.010 \mathrm{mg} / \mathrm{l}$ & $<0.010 \mathrm{mg} / \mathrm{l}$ & 1 & \\
\hline 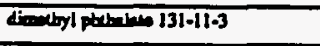 & $<0.010 \mathrm{mg} / \mathrm{l}$ & $<0.010 \mathrm{mg} / \mathrm{h}$ & $<0.010 \mathrm{mg} / \mathrm{l}$ & $<0.010 \mathrm{mg} / 1$ & 1 & \\
\hline 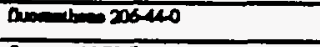 & $<0.010 \mathrm{mg} / \mathrm{l}$ & $<0.010 \mathrm{me} / 1$ & $<0.010 \mathrm{mg} / \mathrm{l}$ & $<0.010 \mathrm{mg} / \mathrm{l}$ & 1 & \\
\hline neovene $46-73-7$ & $<0.010 \mathrm{mg} / \mathrm{l}$ & $<0.010 \mathrm{mg} / \mathrm{l}$ & $<0.010 \mathrm{mg} / 1$ & $<0.010 \mathrm{mg} / \mathrm{l}$ & 1 & \\
\hline 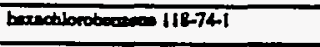 & $<0.010 \mathrm{mg} / 1$ & $<0.010 \mathrm{mg} / \mathrm{l}$ & $<0.010 \mathrm{mg} / \mathrm{h}$ & $<0.010 \mathrm{mg} / 1$ & 1 & \\
\hline 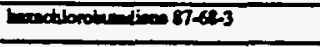 & $<0.010 \mathrm{mg} / \mathrm{l}$ & $<0.010 \mathrm{mg} / \mathrm{l}$ & $<0.010 \mathrm{mg} / \mathrm{l}$ & $<0.010 \mathrm{mg} / \mathrm{l}$ & 1 & \\
\hline 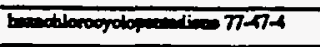 & $<0.010 \mathrm{mg} / \mathrm{l}$ & $<0.010 \mathrm{mg} / \mathrm{l}$ & $<0.010 \mathrm{mg} / \mathrm{l}$ & $<0.010 \mathrm{mg} / \mathrm{l}$ & 1 & \\
\hline 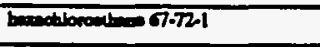 & $<0.010 \mathrm{mg} / \mathrm{l}$ & $<0.010 \mathrm{mg} / 1$ & $<0.010 \mathrm{mg} / 1$ & $<0.010 \mathrm{mg} / \mathrm{l}$ & 1 & \\
\hline 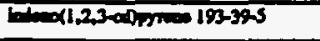 & $<0.010 \mathrm{mg} / \mathrm{l}$ & $<0.010 \mathrm{mg} / 1$ & $<0.010 \mathrm{mg} / 1$ & $<0.010 \mathrm{mg} / \mathrm{l}$ & 1 & \\
\hline 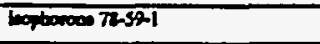 & $<0.010 \mathrm{mg} / 1$ & $<0.010 \mathrm{mg} / 1$ & $<0.010 \mathrm{mg} / \mathrm{l}$ & $<0.010 \mathrm{mg} / \mathrm{l}$ & 1 & \\
\hline 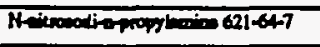 & $<0.010 \mathrm{mg} / \mathrm{l}$ & $<0.010 \mathrm{mg} / 1$ & $<0.010 \mathrm{mg} / \mathrm{l}$ & $<0.010 \mathrm{mg} / \mathrm{ll}$ & 1 & \\
\hline 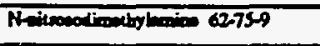 & $<0.010 \mathrm{mg} / \mathrm{l}$ & $<0.010 \mathrm{mg} / \mathrm{l}$ & $<0.010 \mathrm{mg} / 1$ & $<0.010 \mathrm{mg} / \mathrm{l}$ & 1 & \\
\hline 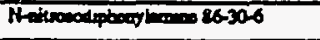 & $<0.010 \mathrm{mg} / 1$ & $<0.010 \mathrm{mg} / \mathrm{l}$ & $<0.010 \mathrm{mg} / \mathrm{l}$ & $<0.010 \mathrm{mg} / 1$ & 1 & \\
\hline ontheros 91-20-3 & $<0.010 \mathrm{mg} / \mathrm{l}$ & $<0.010 \mathrm{mg} / \mathrm{l}$ & $<0.010 \mathrm{mg} / 1$ & $<0.010 \mathrm{mg} / \mathrm{l}$ & 1 & \\
\hline 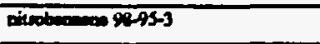 & $<0.010 \mathrm{mg} / \mathrm{l}$ & $<0.010 \mathrm{mg} / \mathrm{l}$ & $<0.010 \mathrm{mg} / \mathrm{l}$ & $<0.010 \mathrm{mg} / \mathrm{l}$ & 1 & \\
\hline 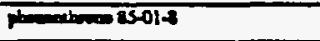 & $<0.010 \mathrm{mg} / \mathrm{l}$ & $<0.010 \mathrm{mg} / \mathrm{l}$ & $<0.010 \mathrm{mg} / \mathrm{l}$ & $<0.010 \mathrm{mg} / \mathrm{l}$ & 1 & \\
\hline $\min 122000$ & $<0.010 \mathrm{mg} / \mathrm{l}$ & $<0.010 \mathrm{mg} / 1$ & $<0.010 \mathrm{mg} / \mathrm{l}$ & $<0.010 \mathrm{mg} / \mathrm{l}$ & 1 & \\
\hline 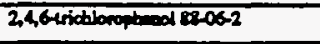 & $<0.030 \mathrm{mg} / \mathrm{l}$ & $<0.030 \mathrm{mg} / \mathrm{l}$ & $<0.030 \mathrm{mg} / \mathrm{l}$ & $<0.030 \mathrm{mg} / \mathrm{l}$ & 1 & \\
\hline 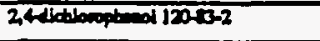 & $<0.030 \mathrm{mg} / \mathrm{l}$ & $<0.030 \mathrm{mg} / 1$ & $<0.030 \mathrm{mg} / \mathrm{l}$ & $<0.030 \mathrm{mg} / \mathrm{l}$ & 1 & \\
\hline 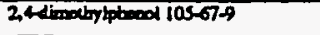 & $<0.030 \mathrm{mg} / \mathrm{l}$ & $<0.030 \mathrm{mg} / \mathrm{l}$ & $<0.030 \mathrm{mg} / \mathrm{l}$ & $<0.030 \mathrm{mg} / \mathrm{l}$ & 1 & \\
\hline
\end{tabular}


North-South Pipe

Para B and C Continued

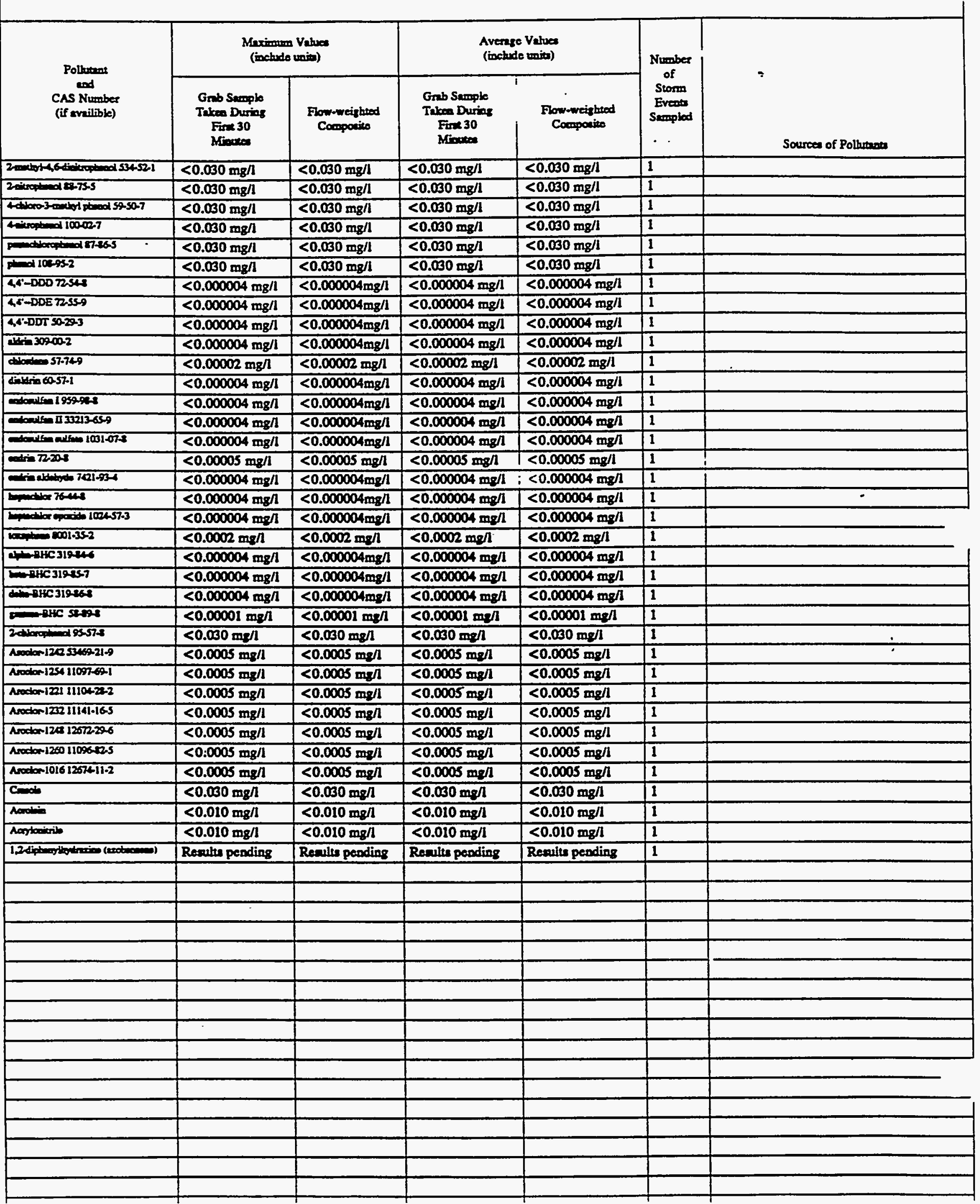


VII. Discharge Information (continuod from page 3 of Form 2F)

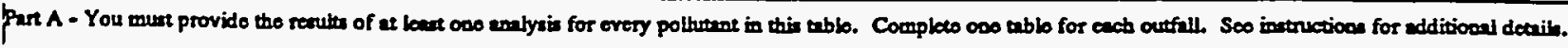

\begin{tabular}{|c|c|c|c|c|c|c|}
\hline \multirow{2}{*}{$\begin{array}{l}\text { Pollutane } \\
\text { and } \\
\text { CAs Number } \\
\text { (if avnilible) }\end{array}$} & \multicolumn{2}{|c|}{$\begin{array}{l}\text { Meximusm Vatues } \\
\text { (includo unitr) }\end{array}$} & \multicolumn{2}{|c|}{$\begin{array}{l}\text { Averags Vatuces } \\
\text { (inchudo units) }\end{array}$} & \multirow{2}{*}{$\begin{array}{l}\text { Nurober } \\
\text { of } \\
\text { Storm } \\
\text { Evertar } \\
\text { Sempled }\end{array}$} & \multirow[b]{2}{*}{ Source of Pollinimes } \\
\hline & 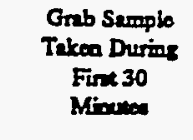 & $\begin{array}{l}\text { Frow-weighted } \\
\text { Compocitos }\end{array}$ & $\begin{array}{c}\text { Grab Semplo } \\
\text { Takec Durius } \\
\text { Fins } 30 \\
\text { Minewee }\end{array}$ & $\begin{array}{l}\text { Fow-weizhtod } \\
\text { Compocico }\end{array}$ & & \\
\hline fil and Granes & $<2 m g / l$ & & $<2 \mathrm{mg} / \mathrm{l}$ & & 1 & \\
\hline 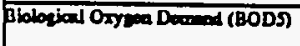 & $8.60 \mathrm{mg} / \mathrm{l}$ & $7.84 \mathrm{mg} / \mathrm{l}$ & $8.60 \mathrm{mg} / \mathrm{l}$ & $7.84 \mathrm{mg} / \mathrm{l}$ & 1 & \\
\hline Cherial Oxy Dowed (COD) & $68 \mathrm{mg} / \mathrm{h}$ & $27 \mathrm{mg} / \mathrm{l}$ & $68 \mathrm{mg} / \mathrm{l}$ & $27 \mathrm{mg} / 1$ & 1 & \\
\hline Toual Surpended Solden (TSS) & $14 \mathrm{mg} / 1$ & $<5 \mathrm{mg} / \mathrm{l}$ & $14 \mathrm{mg} / \mathrm{l}$ & $<5 \mathrm{mg} / \mathrm{l}$ & 1 & \\
\hline 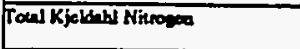 & $1 \mathrm{mg} / \mathrm{l}$ & $0.5 \mathrm{mg} / \mathrm{l}$ & $1 \mathrm{mg} / \mathrm{l}$ & $0.5 \mathrm{mg} / \mathrm{l}$ & 1 & \\
\hline Nitrie ptum Nitrites Nitropen & $1.3 \mathrm{mg} / \mathrm{l}$ & $0.88 \mathrm{mg} / \mathrm{l}$ & $1.3 \mathrm{mg} / \mathrm{l}$ & $0.88 \mathrm{mg} / \mathrm{l}$ & 1 & \\
\hline Toul Phoophosum & $<0.1 \mathrm{mg} / 1$ & $<0.1 \mathrm{mg} / \mathrm{l}$ & $<0.1 \mathrm{mg} / \mathrm{l}$ & $<0.1 \mathrm{mg} / 1$ & 1 & \\
\hline pH & Miniener 8.2 & Mexi-am 8.2 & Minimen 8.2 & Matimin 8.2 & & \\
\hline
\end{tabular}

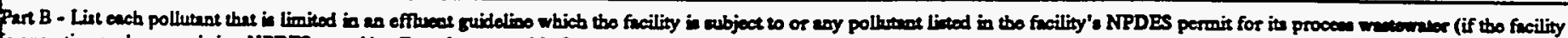

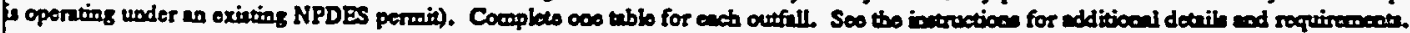

\begin{tabular}{|c|c|c|c|c|c|c|}
\hline \multirow{2}{*}{$\begin{array}{l}\text { Polluranar } \\
\text { ood } \\
\text { CAs Number } \\
\text { (if avilible) }\end{array}$} & \multicolumn{2}{|c|}{ 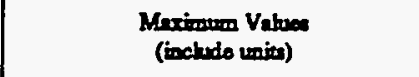 } & \multicolumn{2}{|c|}{$\begin{array}{l}\text { Averase Vabree } \\
\text { (iveludo unies) }\end{array}$} & \multirow{2}{*}{$\begin{array}{l}\text { Number } \\
\text { of } \\
\text { Storm } \\
\text { Eveote } \\
\text { Stopplad }\end{array}$} & \multirow{2}{*}{ 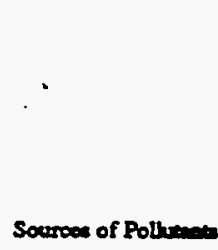 } \\
\hline & 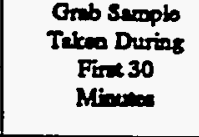 & $\begin{array}{l}\text { Flom-wrighed } \\
\text { Compotice }\end{array}$ & 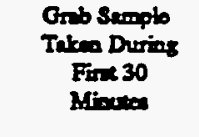 & $\begin{array}{l}\text { Flom-meightod } \\
\text { Compotice }\end{array}$ & & \\
\hline Animody 74a-36-0 & $<0.04 \mathrm{mg} / \mathrm{l}$ & $<0.04 \mathrm{mg} / 1$ & $<0.04 \mathrm{mg} / \mathrm{l}$ & $<0.04 \mathrm{mg} / 1$ & 1 & \\
\hline 5 & $<0.002 \mathrm{mg} / \mathrm{l}$ & & $<0.002 \mathrm{mg} / \mathrm{l}$ & & 1 & \\
\hline 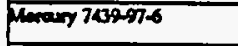 & $0.0002 \mathrm{mg} / \mathrm{t}$ & $<0.0002 \mathrm{mg} / \mathrm{l}$ & $0.0002 \mathrm{mg} / \mathrm{l}$ & $<0.0002 \mathrm{mg} / \mathrm{l}$ & 1 & \\
\hline phench & $0.006 \mathrm{mg} / \mathrm{l}$ & & $0.006 \mathrm{mg} / 1$ & & 1 & 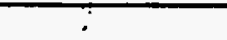 \\
\hline 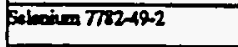 & $<0.002 \mathrm{mg} / \mathrm{l}$ & $<0.002 \mathrm{mg} / 1$ & $<0.002 \mathrm{mg} / \mathrm{l}$ & $<0.002 \mathrm{mg} / \mathrm{l}$ & 1 & \\
\hline Aution & $16 \mathrm{mg} / \mathrm{l}$ & $12 \mathrm{mg} / \mathrm{l}$ & $16 \mathrm{mg} / 1$ & $12 \mathrm{mg} / 1$ & 1 & \\
\hline surfocteres & $0.46 \mathrm{mg} / \mathrm{l}$ & $0.29 \mathrm{mg} / 1$ & $0.46 \mathrm{mg} / 1$ & $0.29 \mathrm{mg} / \mathrm{l}$ & 1 & \\
\hline Mhomen 7429-90-5 & $0.66 \mathrm{mg} / \mathrm{l}$ & $0.30 \mathrm{mg} / \mathrm{l}$ & $0.66 \mathrm{mg} / \mathrm{l}$ & $0.30 \mathrm{mg} / \mathrm{l}$ & 1 & \\
\hline 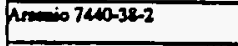 & $<0.04 \mathrm{mg} / \mathrm{l}$ & $<0.04 \mathrm{mg} / \mathrm{l}$ & $<0.04 \mathrm{mg} / \mathrm{l}$ & $<0.04 \mathrm{mg} / \mathrm{l}$ & 1 & \\
\hline 80000 740-24 & $0.035 \mathrm{mg} / \mathrm{l}$ & $0.070 \mathrm{mg} / \mathrm{l}$ & $0.035 \mathrm{mg} / \mathrm{l}$ & $0.070 \mathrm{mg} / \mathrm{l}$ & 1 & \\
\hline Berfllinom 740-41.7 & $<0.0004 \mathrm{mg} / \mathrm{l}$ & $<0.0004 \mathrm{mg} / 1$ & $<0.0004 \mathrm{mg} / \mathrm{l}$ & $<0.0004 \mathrm{mg} / \mathrm{ll}$ & 1 & \\
\hline 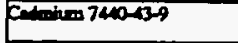 & $<0.004 \mathrm{mg} / \mathrm{l}$ & $<0.004 \mathrm{mg} / \mathrm{l}$ & $<0.004 \mathrm{mg} / \mathrm{ll}$ & $<0.004 \mathrm{mg} / \mathrm{l}$ & 1 & \\
\hline 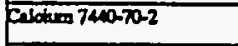 & $30.4 \mathrm{mg} / \mathrm{l}$ & $17.7 \mathrm{mg} / \mathrm{l}$ & $30.4 \mathrm{mg} / 1$ & $17.7 \mathrm{mg} / 1$ & 1 & \\
\hline 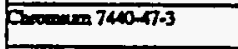 & $<0.006 \mathrm{mg} / \mathrm{l}$ & $<0.006 \mathrm{mg} / \mathrm{l}$ & $<0.006 \mathrm{mg} / \mathrm{l}$ & $<0.006 \mathrm{mg} / \mathrm{l}$ & 1 & \\
\hline Comall 740-49- & $<0.002 \mathrm{mg} / \mathrm{l}$ & $<0.002 \mathrm{mg} / \mathrm{l}$ & $<0.002 \mathrm{mg} / \mathrm{l}$ & $<0.002 \mathrm{mg} / \mathrm{l}$ & 1 & \\
\hline Compen 74ta-sos & $<0.006 \mathrm{mg} / \mathrm{l}$ & $<0.006 \mathrm{mg} / \mathrm{l}$ & $<0.006 \mathrm{mg} / 1$ & $<0.006 \mathrm{mg} / \mathrm{l}$ & 1 & \\
\hline 10007432826 & $0.67 \mathrm{mg} / 1$ & $0.31 \mathrm{mg} / \mathrm{l}$ & $0.67 \mathrm{mg} / 1$ & $0.31 \mathrm{mg} / 1$ & 1 & \\
\hline 1001739-201 & $<0.02 \mathrm{mg} / \mathrm{l}$ & $<0.02 \mathrm{mg} / 1$ & $<0.02 \mathrm{mg} / 1$ & $<0.02 \mathrm{mg} / 1$ & 1 & \\
\hline Filtiden 743999-2 & $<0.02 \mathrm{mg} / \mathrm{l}$ & $<0.02 \mathrm{mg} / 1$ & $<0.02 \mathrm{mg} / \mathrm{l}$ & $<0.02 \mathrm{mg} / 1$ & 1 & \\
\hline 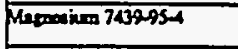 & $3.56 \mathrm{mg} / 1$ & $1.92 \mathrm{mg} / 1$ & $3.56 \mathrm{mg} / \mathrm{l}$ & $1.92 \mathrm{mg} / 1$ & 1 & \\
\hline 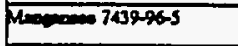 & $0.041 \mathrm{mg} / \mathrm{l}$ & $0.009 \mathrm{mg} / \mathrm{l}$ & $0.041 \mathrm{mg} / 1$ & $0.009 \mathrm{mg} / \mathrm{l}$ & 1 & \\
\hline 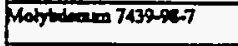 & $<0.006 \mathrm{mg} / \mathrm{l}$ & $<0.006 \mathrm{mg} / \mathrm{l}$ & $<0.006 \mathrm{mg} / \mathrm{l}$ & $<0.006 \mathrm{mg} / \mathrm{l}$ & 1 & \\
\hline Vichel 7400000 & $<0.008 \mathrm{mg} / \mathrm{l}$ & $<0.008 \mathrm{mg} / \mathrm{l}$ & $<0.008 \mathrm{mg} / 1$ & $<0.008 \mathrm{mg} / \mathrm{l}$ & 1 & \\
\hline 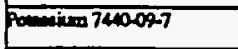 & $1.4 \mathrm{mg} / \mathrm{l}$ & $1.4 \mathrm{mg} / \mathrm{l}$ & $1.4 \mathrm{mg} / \mathrm{l}$ & $1.4 \mathrm{mg} / \mathrm{l}$ & 1 & \\
\hline sithe 740-204 & $<0.006 \mathrm{mg} / 1$ & $<0.006 \mathrm{mg} / \mathrm{l}$ & $<0.006 \mathrm{mg} / 1$ & $<0.006 \mathrm{mg} / \mathrm{l}$ & 1 & \\
\hline sodiven 7400235 & $1.40 \mathrm{mg} / 1$ & $1.12 \mathrm{mg} / \mathrm{l}$ & $1.40 \mathrm{mg} / 1$ & $1.12 \mathrm{mg} / 1$ & 1 & \\
\hline Tivenimen 7400-326-6 & $<0.01 \mathrm{mg} / \mathrm{l}$ & $<0.01 \mathrm{mg} / \mathrm{l}$ & $<0.01 \mathrm{mg} / 1$ & $<0.01 \mathrm{mg} / \mathrm{l}$ & 1 & \\
\hline 21007410666 & $0.02 \mathrm{mg} / 1$ & $0.01 \mathrm{mg} / 1$ & $0.02 \mathrm{mg} / \mathrm{l}$ & $0.01 \mathrm{mg} / \mathrm{l}$ & 1 & \\
\hline
\end{tabular}


Contiand from froes.

\begin{tabular}{|c|c|c|c|c|c|c|}
\hline \multirow{2}{*}{$\begin{array}{l}\text { Pollunase } \\
\text { and } \\
\text { CAS Number } \\
\text { (if aveilible) }\end{array}$} & \multicolumn{2}{|c|}{$\begin{array}{l}\text { Meximum Vatuea } \\
\text { (inetudo units) }\end{array}$} & \multicolumn{2}{|c|}{$\begin{array}{l}\text { Avernge Values } \\
\text { (inctudo units) }\end{array}$} & \multirow{2}{*}{$\begin{array}{l}\text { Number } \\
\text { of } \\
\text { Storm } \\
\text { Eveatix } \\
\text { Sempled }\end{array}$} & \multirow[b]{2}{*}{ Sources of Polbutant } \\
\hline & $\begin{array}{l}\text { Grab Samplo } \\
\text { Taken During } \\
\text { Fint } 30 \\
\text { Minoteo }\end{array}$ & $\begin{array}{l}\text { Flon-woighted } \\
\text { Compocito }\end{array}$ & $\begin{array}{l}\text { Grab Sumplo } \\
\text { Taken During } \\
\text { Finn } 30 \\
\text { Minuteo }\end{array}$ & $\begin{array}{l}\text { Flow-weighted } \\
\text { Componite }\end{array}$ & & \\
\hline 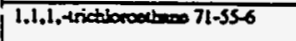 & $<0.010 \mathrm{mg} / \mathrm{l}$ & & $<0.010 \mathrm{mg} / \mathrm{h}$ & & 1 & \\
\hline 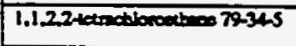 & $<0.010 \mathrm{mg} / \mathrm{l}$ & & $<0.010 \mathrm{mg} / 1$ & & 1 & \\
\hline 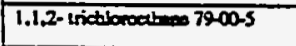 & $<0.010 \mathrm{mg} / \mathrm{l}$ & & $<0.010 \mathrm{mg} / 1$ & & 1 & \\
\hline 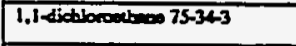 & $<0.010 \mathrm{mg} / 1$ & & $<0.010 \mathrm{mg} / 1$ & & 1 & \\
\hline 1,1 dictibrocelbyloos 75-35-4 & $<0.010 \mathrm{mg} / 1$ & & $<0.010 \mathrm{mg} / 1$ & & 1 & \\
\hline 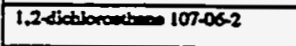 & $<0.010 \mathrm{mg} / \mathrm{l}$ & & $<0.010 \mathrm{mg} / 1$ & & 1 & \\
\hline 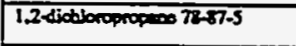 & $<0.010 \mathrm{mg} / 1$ & & $<0.010 \mathrm{mg} / \mathrm{l}$ & & 1 & \\
\hline 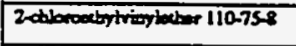 & $<0.010 \mathrm{mg} / 1$ & & $<0.010 \mathrm{mg} / \mathrm{l}$ & & 1 & \\
\hline $5000000071-13-2$ & $<0.010 \mathrm{mg} / \mathrm{l}$ & & $<0.010 \mathrm{mg} / \mathrm{l}$ & & 1 & \\
\hline 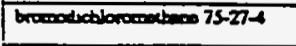 & $<0.010 \mathrm{mg} / 1$ & & $<0.010 \mathrm{mg} / 1$ & & 1 & \\
\hline $75.25-2$ & $<0.010 \mathrm{mg} / 1$ & & $<0.010 \mathrm{mg} / \mathrm{l}$ & & 1 & \\
\hline 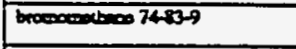 & $<0.010 \mathrm{mg} / \mathrm{h}$ & & $<0.010 \mathrm{mg} / 1$ & & 1 & \\
\hline 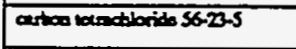 & $<0.010 \mathrm{mg} / \mathrm{l}$ & & $<0.010 \mathrm{mg} / \mathrm{l}$ & & 1 & \\
\hline 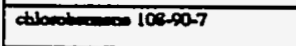 & $<0.010 \mathrm{mg} / \mathrm{l}$ & & $<0.010 \mathrm{mg} / \mathrm{l}$ & & 1 & \\
\hline $75,-00-3$ & $<0.010 \mathrm{mg} / \mathrm{l}$ & & $<0.010 \mathrm{mg} / \mathrm{l}$ & & 1 & \\
\hline 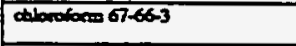 & $<0.010 \mathrm{mg} / 1$ & & $<0.010 \mathrm{mg} / \mathrm{l}$ & & 1 & - \\
\hline 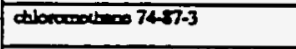 & $<0.010 \mathrm{mg} / 1$ & & $<0.010 \mathrm{mg} / \mathrm{l}$ & & 1 & \\
\hline 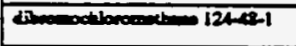 & $<0.010 \mathrm{mg} / 1$ & & $<0.010 \mathrm{mg} / \mathrm{l}$ & & 1 & \\
\hline 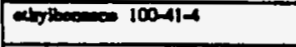 & $<0.010 \mathrm{mg} / \mathrm{l}$ & & $<0.010 \mathrm{mg} / \mathrm{h}$ & & 1 & \\
\hline 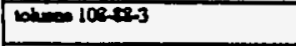 & $<0.010 \mathrm{mg} / 1$ & & $<0.010 \mathrm{mg} / 1$ & & 1 & \\
\hline ring darit 75-014 & $<0.010 \mathrm{mg} / \mathrm{l}$ & & $<0.010 \mathrm{mg} / 1$ & & 1 & \\
\hline 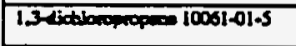 & $<0.010 \mathrm{mg} / 1$ & & $<0.010 \mathrm{mg} / \mathrm{l}$ & & 1 & \\
\hline 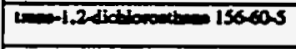 & $<0.010 \mathrm{mg} / \mathrm{l}$ & & $<0.010 \mathrm{mg} / \mathrm{I}$ & & 1 & . \\
\hline 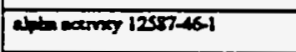 & 13 peill & $3.4 \mathrm{peill}$ & 13 paill & 3.4 peill & 1 & \\
\hline mearving $1255746-1$ & $17 \mathrm{pei} / \mathrm{s}$ & $7.6 \mathrm{peil}$ & 17pei/l & $7.6 \mathrm{pci} / \mathrm{s}$ & 1 & \\
\hline thal coliberen & $40,000 \mathrm{col} .1 \mathrm{~L}$ & $18,000 \mathrm{col} .1 \mathrm{~L}$ & $40,000 \mathrm{co} / .1 \mathrm{~L}$ & $18,000 \mathrm{col} .1 \mathrm{~L}$ & 1 & \\
\hline Thallien $7400-2000$ & $<0.001 \mathrm{mg} / 1$ & $<0.001 \mathrm{mg} / \mathrm{l}$ & $<0.001 \mathrm{mg} / 1$ & $<0.001 \mathrm{mg} / 1$ & 1 & \\
\hline Uasing 7400-61-1 & $0.008 \mathrm{mg} / 1$ & $0.004 \mathrm{mg} / \mathrm{l}$ & $0.008 \mathrm{mg} / 1$ & $0.004 \mathrm{mg} / \mathrm{l}$ & 1 & \\
\hline Omoneseniving & 48 peill & $26 \mathrm{pei} / \mathrm{s}$ & $48 \mathrm{pei} / \mathrm{l}$ & $26 \mathrm{pcill}$ & 1 & \\
\hline
\end{tabular}

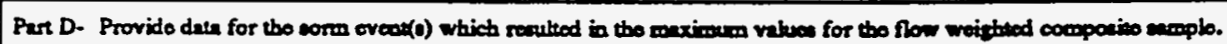

\begin{tabular}{|c|c|c|c|c|c|c|c|}
\hline $\begin{array}{l}1 . \\
\text { Dato of } \\
\text { Stom } \\
\text { Eveat }\end{array}$ & $\begin{array}{l}2 . \\
\text { Dirstion } \\
\text { of Stonns } \\
\text { (in } \\
\text { minuman) }\end{array}$ & $\begin{array}{l}3 . \\
\text { Toul ninfill } \\
\text { during inom ovent } \\
\text { (io incon) }\end{array}$ & 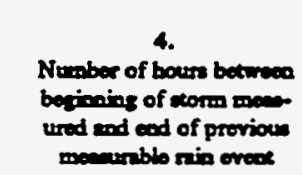 & 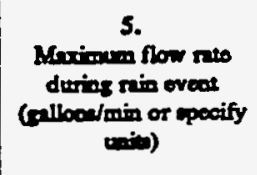 & 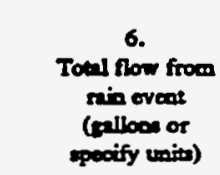 & $\begin{array}{c}7 . \\
\text { Semoco } \\
\text { tireo }\end{array}$ & 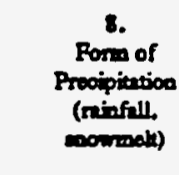 \\
\hline $9 / 24 / 91$ & 365 & 2.0 & 133.5 & 717 & 105,790 & Fall & Rainfall \\
\hline
\end{tabular}

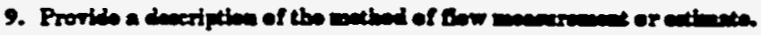

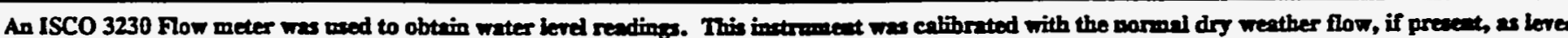
zero. The flow meter was programmed with the characterictics of the conveyance so that flow rates and total flows conid be calenlated by the meter. 


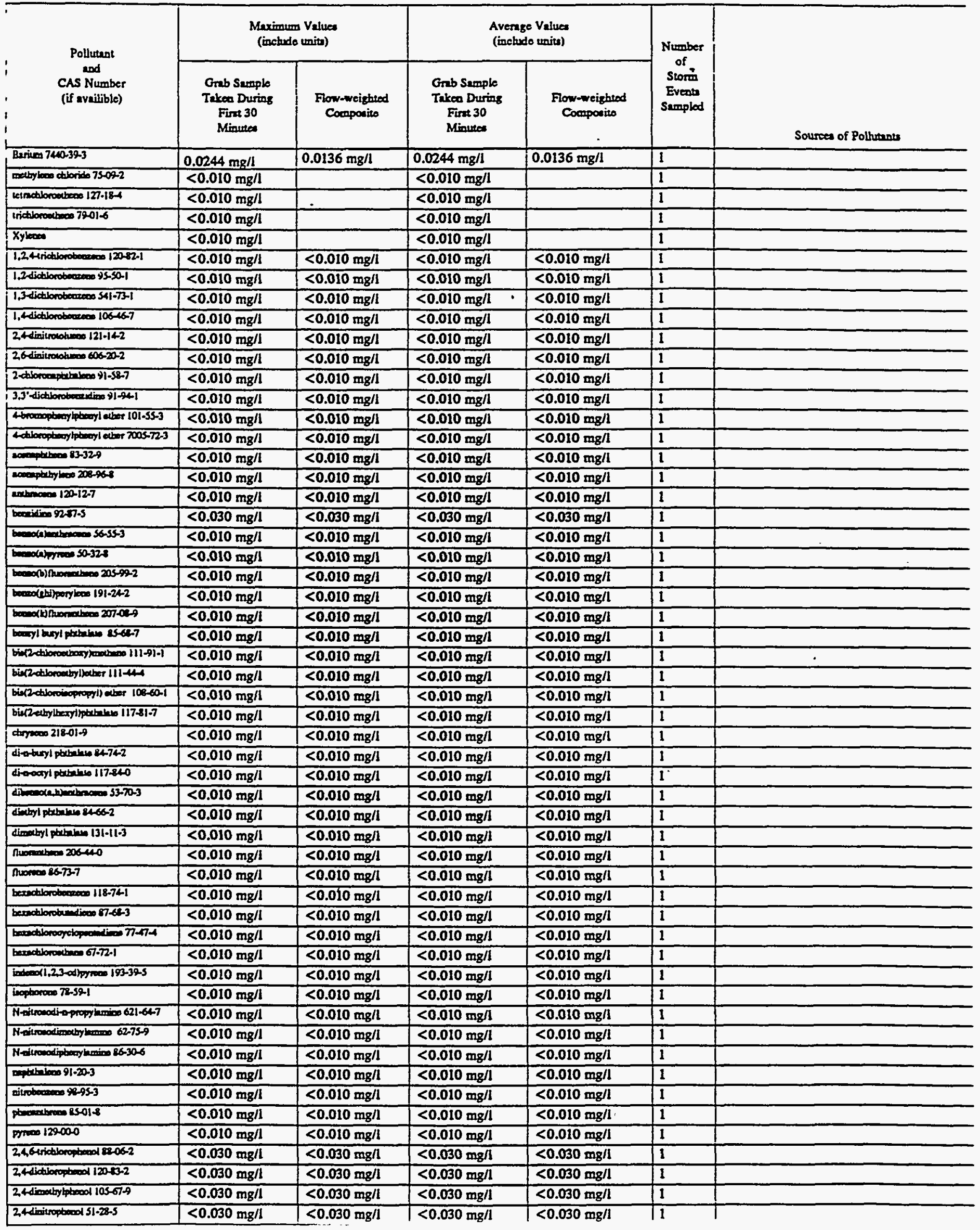




\begin{tabular}{|c|c|c|c|c|c|c|}
\hline \multicolumn{7}{|l|}{ Parts B and C Continued } \\
\hline \multirow{2}{*}{$\begin{array}{l}\text { Pollutent } \\
\text { and } \\
\text { CAS Number } \\
\text { (if availible) }\end{array}$} & \multicolumn{2}{|c|}{$\begin{array}{l}\text { Maximumon Valuea } \\
\text { (ivetude unitu) }\end{array}$} & \multicolumn{2}{|c|}{$\begin{array}{l}\text { Avernge Valuee } \\
\text { (inehudo units) }\end{array}$} & \multirow{2}{*}{$\begin{array}{l}\text { Number } \\
\text { of } \\
\text { Storm } \\
\text { Eveats } \\
\text { Seroplod }\end{array}$} & \multirow[b]{2}{*}{ Sources of Poltureast } \\
\hline & $\begin{array}{l}\text { Grab Samplo } \\
\text { Teken Durims } \\
\text { Fint } 30 \\
\text { Minuted }\end{array}$ & $\begin{array}{l}\text { Flow-weightiod } \\
\text { Componite }\end{array}$ & $\begin{array}{l}\text { Greb Sumple } \\
\text { Tekeo Durias } \\
\text { First } 30 \\
\text { Minures }\end{array}$ & $\begin{array}{l}\text { Flow-weighted } \\
\text { Cocompouite }\end{array}$ & & \\
\hline 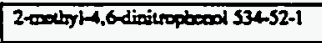 & $<0.030 \mathrm{mg} / \mathrm{l}$ & $<0.030 \mathrm{mg} / \mathrm{l}$ & $<0.030 \mathrm{mg} / \mathrm{l}$ & $<0.030 \mathrm{mg} / \mathrm{l}$ & 1 & \\
\hline 2 - riveophood 88-73-5 & $<0.030 \mathrm{mg} / 1$ & $<0.030 \mathrm{mg} / \mathrm{l}$ & $<0.030 \mathrm{mg} / \mathrm{l}$ & $<0.030 \mathrm{mg} / \mathrm{l}$ & 1 & \\
\hline 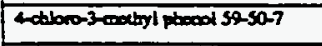 & $<0.030 \mathrm{mg} / 1$ & $<0.030 \mathrm{mg} / \mathrm{l}$ & $<0.030 \mathrm{mg} / \mathrm{l}$ & $<0.030 \mathrm{mg} / \mathrm{l}$ & 1 & \\
\hline 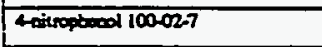 & $<0.030 \mathrm{mg} / \mathrm{l}$ & $<0.030 \mathrm{mg} / \mathrm{l}$ & $<0.030 \mathrm{mg} / \mathrm{l}$ & $<0.030 \mathrm{mg} / \mathrm{l}$ & 1 & \\
\hline periectibroptecol 87-86-5 & $<0.030 \mathrm{mg} / \mathrm{I}$ & $<0.030 \mathrm{mg} / 1$ & $<0.030 \mathrm{mg} / \mathrm{l}$ & $<0.030 \mathrm{mg} / \mathrm{l}$ & 1 & \\
\hline pheol locess-2 & $<0.030 \mathrm{mg} / \mathrm{l}$ & $<0.030 \mathrm{mg} / \mathrm{h}$ & $<0.030 \mathrm{mg} / \mathrm{l}$ & $<0.030 \mathrm{mg} / \mathrm{l}$ & 1 & \\
\hline $4.4^{\prime}-000072-548$ & $<0.00001 \mathrm{mg} / \mathrm{h}$ & $<0.00001 \mathrm{mg} / 1$ & $<0.00001 \mathrm{mg} / \mathrm{l}$ & $<0.00001 \mathrm{mg} / 1$ & 1 & \\
\hline 4.4'-ODE 72-55-9 & $<0.00001 \mathrm{mg} / \mathrm{l}$ & $<0.00001 \mathrm{mg} / 1$ & $<0.00001 \mathrm{mg} / \mathrm{l}$ & $<0.00001 \mathrm{mg} / 1$ & 1 & \\
\hline $4,4^{4}-0 D T 50-22-3$ & $<0.00001 \mathrm{mg} / 1$ & $<0.00001 \mathrm{mg} / \mathrm{l}$ & $<0.00001 \mathrm{mg} / \mathrm{l}$ & $<0.00001 \mathrm{mg} / \mathrm{l}$ & 1 & \\
\hline $24-30900-2$ & $<0.00001 \mathrm{mg} / \mathrm{l}$ & $<0.00001 \mathrm{mg} / \mathrm{l}$ & $<0.00001 \mathrm{mg} / \mathrm{l}$ & $<0.00001 \mathrm{mg} / \mathrm{l}$ & 1 & \\
\hline chlondere 57.749 & $<0.00002 \mathrm{mg} / 1$ & $<0.00002 \mathrm{mg} / \mathrm{l}$ & $<0.00002 \mathrm{mg} / 1$ & $<0.00002 \mathrm{mg} / \mathrm{l}$ & 1 & \\
\hline diekaria 60-57-1 & $<0.00001 \mathrm{mg} / \mathrm{l}$ & $<0.00001 \mathrm{mg} / \mathrm{l}$ & $<0.00001 \mathrm{mg} / \mathrm{I}$ & $<0.00001 \mathrm{mg} / \mathrm{l}$ & 1 & \\
\hline codoenilean $1939-90-8$ & $<0.00001 \mathrm{mg} / \mathrm{l}$ & $<0.0000$ lmg $/ 1$ & $<0.00001 \mathrm{mg} / \mathrm{l}$ & $<0.00001 \mathrm{mg} / \mathrm{l}$ & 1 & \\
\hline costonulfon II 33213-65-9 & $<0.00001 \mathrm{mg} / \mathrm{l}$ & $<0.00001 \mathrm{mg} / 1$ & $<0.00001 \mathrm{mg} / \mathrm{l}$ & $<0.00001 \mathrm{mg} / \mathrm{l}$ & 1 & \\
\hline 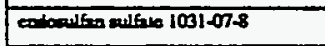 & $<0.00001 \mathrm{mg} / \mathrm{l}$ & $<0.00001 \mathrm{mg} / 1$ & $<0.00001 \mathrm{mg} / \mathrm{l}$ & $<0.00001 \mathrm{mg} / \mathrm{l}$ & 1 & \\
\hline actrin 72028 & $<0.00005 \mathrm{mg} / \mathrm{l}$ & $<0.00005 \mathrm{mg} / \mathrm{l}$ & $<0.00005 \mathrm{mg} / \mathrm{l}$ & $<0.00005 \mathrm{mg} / 1$ & 1 & \\
\hline codrin eldety to $7421-93-4$ & $<0.00001 \mathrm{mg} / \mathrm{l}$ & $<0.00001 \mathrm{mg} / \mathrm{l}$ & $<0.00001 \mathrm{mg} / \mathrm{l}$ & $<0.00001 \mathrm{mg} / \mathrm{l}$ & 1 & \\
\hline topection 76448 & $<0.00001 \mathrm{mg} / \mathrm{l}$ & $<0.00001 \mathrm{mg} / \mathrm{h}$ & $<0.00001 \mathrm{mg} / \mathrm{l}$ & $<0.00001 \mathrm{mg} / \mathrm{l}$ & 1 & \\
\hline 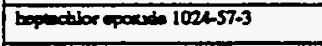 & $<0.00001 \mathrm{mg} / \mathrm{l}$ & $<0.00001 \mathrm{mg} / \mathrm{l}$ & $<0.00001 \mathrm{mg} / \mathrm{h}$ & $<0.00001 \mathrm{mg} / \mathrm{l}$ & 1 & \\
\hline 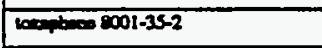 & $<0.0002 \mathrm{mg} / \mathrm{l}$ & $<0.0002 \mathrm{mg} / \mathrm{h}$ & $<0.0002 \mathrm{mg} / \mathrm{l}$ & $<0.0002 \mathrm{mg} / \mathrm{l}$ & 1 & \\
\hline 446-8HC 319856 & $<0.00001 \mathrm{mg} / \mathrm{l}$ & $<0.00001 \mathrm{mg} / 1$ & $<0.00001 \mathrm{mg} / \mathrm{l}$ & $<0.00001 \mathrm{mg} / 1$ & 1 & \\
\hline BOHC 319-85-7 & $<0.00001 \mathrm{mg} / \mathrm{l}$ & $<0.00001 \mathrm{mg} / 1$ & $<0.00001 \mathrm{mg} / \mathrm{l}$ & $<0.00001 \mathrm{mg} / \mathrm{l}$ & 1 & \\
\hline detwo-BHC 319365 & $<0.00001 \mathrm{mg} / 1$ & $<0.00001 \mathrm{mg} / 1$ & $<0.00001 \mathrm{mg} / \mathrm{l}$ & $<0.00001 \mathrm{mg} / \mathrm{I}$ & 1 & \\
\hline 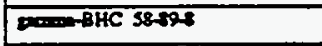 & $<0.00001 \mathrm{mg} / 1$ & $<0.00001 \mathrm{mg} / 1$ & $<0.00001 \mathrm{mg} / \mathrm{l}$ & $<0.00001 \mathrm{mg} / \mathrm{I}$ & 1 & \\
\hline 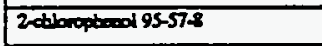 & $<0.030 \mathrm{mg} / 1$ & $<0.030 \mathrm{mg} / \mathrm{l}$ & $<0.030 \mathrm{mg} / 1$ & $<0.030 \mathrm{mg} / \mathrm{l}$ & 1 & \\
\hline Arodor 1202 5366-21-9 & $<0.0005 \mathrm{mg} / 1$ & $<0.0005 \mathrm{mg} / 1$ & $<0.0005 \mathrm{mg} / 1$ & $<0.0005 \mathrm{mg} / \mathrm{l}$ & 1 & $\because$ \\
\hline Arodion-1254 1100769-1 & $<0.0005 \mathrm{mg} / \mathrm{l}$ & $<0.0005 \mathrm{mg} / \mathrm{l}$ & $<0.0005 \mathrm{mg} / 1$ & $<0.0005 \mathrm{mg} / 1$ & 1 & \\
\hline Aroctor-1221 1110428-2 & $<0.0005 \mathrm{mg} / \mathrm{l}$ & $<0.0005 \mathrm{mg} / \mathrm{l}$ & $<0.0005 \mathrm{mg} / \mathrm{l}$ & $<0.0005 \mathrm{mg} / \mathrm{l}$ & 1 & \\
\hline Anodon-123211141-16-5 & $<0.0005 \mathrm{mg} / \mathrm{l}$ & $<0.0005 \mathrm{mg} / 1$ & $<0.0005 \mathrm{mg} / 1$ & $<0.0005 \mathrm{mg} / \mathrm{l}$ & 1 & \\
\hline Arodon-1248 12672-29-6 & $<0.0005 \mathrm{mg} / \mathrm{l}$ & $<0.0005 \mathrm{mg} / \mathrm{l}$ & $<0.0005 \mathrm{mg} / \mathrm{h}$ & $<0.0005 \mathrm{mg} / 1$ & 1 & \\
\hline Aroctor-1260 $11096-82.5$ & $<0.0005 \mathrm{mg} / \mathrm{l}$ & $<0.0005 \mathrm{mg} / \mathrm{l}$ & $<0.0005 \mathrm{mg} / \mathrm{l}$ & $<0.0005 \mathrm{mg} / \mathrm{l}$ & 1 & \\
\hline Arodosor1016 1207411-2 & $<0.0005 \mathrm{mg} / 1$ & $<0.0005 \mathrm{mg} / \mathrm{l}$ & $<0.0005 \mathrm{mg} / 1$ & $<0.0005 \mathrm{mg} / 1$ & 1 & \\
\hline Crmale & $<0.030 \mathrm{mg} / \mathrm{l}$ & $<0.030 \mathrm{mg} / 1$ & $<0.030 \mathrm{mg} / \mathrm{l}$ & $<0.030 \mathrm{mg} / \mathrm{l}$ & 1 & \\
\hline Aonoluis & $<0.010 \mathrm{mg} / \mathrm{l}$ & $<0.010 \mathrm{mg} / \mathrm{l}$ & $<0.010 \mathrm{mg} / \mathrm{l}$ & $<0.010 \mathrm{mg} / \mathrm{l}$ & 1 & \\
\hline Aoryboitrito & $<0.010 \mathrm{mg} / \mathrm{l}$ & $<0.010 \mathrm{mg} / 1$ & $<0.010 \mathrm{mg} / \mathrm{l}$ & $<0.010 \mathrm{mg} / \mathrm{l}$ & 1 & \\
\hline 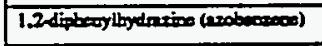 & Rosults pending & Reoults pending & Resulle pending & Results pending & 1 & \\
\hline & & & & & & \\
\hline & & & & & & \\
\hline & & & & & & \\
\hline & & & & & & \\
\hline & & & & & & \\
\hline & & & & & & \\
\hline & & & & & & \\
\hline & & & & & & \\
\hline & & & & & & \\
\hline & & & & & & \\
\hline & & & & & & \\
\hline & & & - & & & \\
\hline & & & & & & \\
\hline & & & & & & \\
\hline & & & & & & \\
\hline
\end{tabular}


NI. Discherge Information (continued from page 3 of Form 2P)

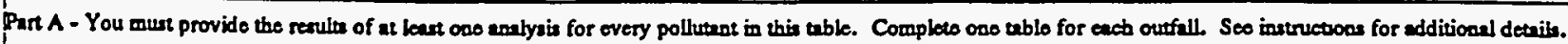

\begin{tabular}{|c|c|c|c|c|c|c|}
\hline \multirow{2}{*}{$\begin{array}{l}\text { Pollutans } \\
\text { and } \\
\text { CAS Number } \\
\text { (if availiblo) }\end{array}$} & \multicolumn{2}{|c|}{$\begin{array}{l}\text { Maximum Values } \\
\text { (ibchede unitu) }\end{array}$} & \multicolumn{2}{|c|}{$\begin{array}{l}\text { Average Velues } \\
\text { (ibebude uniss) }\end{array}$} & \multirow{2}{*}{\begin{tabular}{c|} 
\\
Number \\
of \\
Siom \\
Events \\
Semplod
\end{tabular}} & \multirow[b]{2}{*}{ Sourcen of Polluthest } \\
\hline & $\begin{array}{l}\text { Grab Semple } \\
\text { Taken During } \\
\text { Firat } 30 \\
\text { Minutea }\end{array}$ & $\begin{array}{l}\text { Flow-weightod } \\
\text { Compouixo }\end{array}$ & $\begin{array}{l}\text { Grab Semplo } \\
\text { Takea During } \\
\text { Firt } 30 \\
\text { Minotion }\end{array}$ & $\begin{array}{l}\text { Flow-wrighiod } \\
\text { Componte }\end{array}$ & & \\
\hline 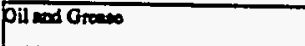 & $<2 \mathrm{mg} / \mathrm{l}$ & & $<2 \mathrm{mg} / \mathrm{l}$ & & 1 & \\
\hline Bbotoxial Oxyou Deanced (BODY) & $<5 \mathrm{mg} / \mathrm{l}$ & $5.40 \mathrm{mg} / \mathrm{l}$ & $<5 \mathrm{mg} / 1$ & $5.40 \mathrm{mg} / \mathrm{l}$ & 1 & \\
\hline Clowial Oxypea Deand (COD) & $11 \mathrm{mg} / \mathrm{l}$ & $20 \mathrm{mg} / \mathrm{l}$ & $11 \mathrm{mg} / \mathrm{l}$ & $20 \mathrm{mg} / 1$ & 1 & \\
\hline Toul Surpoded salide (TSS) & $84 \mathrm{mg} / \mathrm{l}$ & $300 \mathrm{mg} / \mathrm{l}$ & $84 \mathrm{mg} / \mathrm{l}$ & $300 \mathrm{mg} / \mathrm{l}$ & 1 & \\
\hline Toul Kjebabl Nitroned & $0.6 \mathrm{mg} / \mathrm{l}$ & $1 \mathrm{mg} / \mathrm{l}$ & $0.6 \mathrm{mg} / \mathrm{l}$ & $1 \mathrm{mg} / \mathrm{l}$ & 1 & \\
\hline 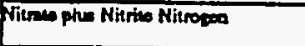 & $55 \mathrm{mg} / \mathrm{l}$ & $11 \mathrm{mg} / \mathrm{l}$ & $55 \mathrm{mg} / 1$ & $11 \mathrm{mg} / 1$ & 1 & \\
\hline Toul Phoophon: & $0.20 \mathrm{mg} / \mathrm{l}$ & $0.18 \mathrm{mg} / \mathrm{l}$ & $0.20 \mathrm{mg} / \mathrm{l}$ & $0.18 \mathrm{mg} / 1$ & 1 & \\
\hline $\mathrm{pH}$ & Minins 8.0 & Maring- 8.0 & Minimum 8.0 & $\begin{array}{ll}\text { Mrim } & 8.0 \\
\end{array}$ & & \\
\hline
\end{tabular}

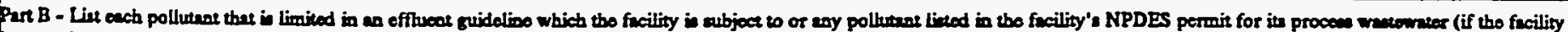

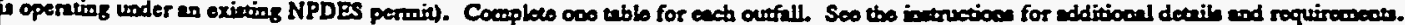

\begin{tabular}{|c|c|c|c|c|c|c|}
\hline \multirow{2}{*}{$\begin{array}{l}\text { Pollutand } \\
\text { end } \\
\text { CAs Nuraber } \\
\text { (if muiliblo) }\end{array}$} & \multicolumn{2}{|c|}{$\begin{array}{l}\text { Meximum Vahear } \\
\text { (inoludo unita) }\end{array}$} & \multicolumn{2}{|c|}{ 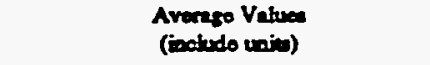 } & \multirow{2}{*}{$\begin{array}{l}\text { Number } \\
\text { of } \\
\text { Storm } \\
\text { Eveous } \\
\text { Seropled }\end{array}$} & \multirow{2}{*}{ Soureces of Polthenn } \\
\hline & 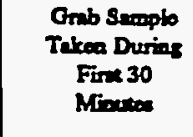 & $\begin{array}{l}\text { Frow-weigheod } \\
\text { Compotico }\end{array}$ & 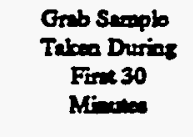 & $\begin{array}{l}\text { Fow-weightod } \\
\text { Compotico }\end{array}$ & & \\
\hline Axtimoor $7400-360$ & $<0.04 \mathrm{mg} / \mathrm{l}$ & $<0.04 \mathrm{mg} / \mathrm{l}$ & $<0.04 \mathrm{mg} / 1$ & $<0.04 \mathrm{mg} / \mathrm{l}$ & 1 & \\
\hline aringen & $<0.002 \mathrm{mg} / \mathrm{l}$ & & $<0.002 \mathrm{mg} / 1$ & & 1 & \\
\hline Memang 7439-976 & $0.0003 \mathrm{mg} / \mathrm{ll}$ & $0.0005 \mathrm{mg} / \mathrm{l}$ & $0.0003 \mathrm{mg} / \mathrm{l}$ & $0.0005 \mathrm{mg} / \mathrm{h}$ & 1 & \\
\hline sis & $0.005 \mathrm{mg} / \mathrm{l}$ & & $0.005 \mathrm{mg} / \mathrm{l}$ & & 1 & $\because$ \\
\hline Selocion $772-492$ & $<0.002 \mathrm{mg} / \mathrm{l}$ & $<0.002 \mathrm{mg} / \mathrm{l}$ & $<0.002 \mathrm{mg} / 1$ & $<0.002 \mathrm{mg} / 1$ & 1 & \\
\hline sulferes & $110 \mathrm{mg} / \mathrm{l}$ & $62 \mathrm{mg} / 1$ & $110 \mathrm{mg} / \mathrm{l}$ & $62 \mathrm{mg} / 1$ & 1 & \\
\hline purticaenes & $<0.05 \mathrm{mg} / \mathrm{l}$ & $<0.05 \mathrm{mg} / 1$ & $<0.05 \mathrm{mg} / \mathrm{l}$ & $<0.05 \mathrm{mg} / \mathrm{l}$ & 1 & \\
\hline 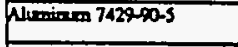 & $9.90 \mathrm{mg} / \mathrm{l}$ & $9.61 \mathrm{mg} / \mathrm{l}$ & $9.90 \mathrm{mg} / 1$ & $9.61 \mathrm{mg} / \mathrm{l}$ & 1 & \\
\hline Anomion $740.32-2$ & $<0.04 \mathrm{mg} / \mathrm{l}$ & $<0.04 \mathrm{mg} / \mathrm{l}$ & $<0.04 \mathrm{mg} / 1$ & $<0.04 \mathrm{mg} / 1$ & 1 & \\
\hline 30000 7400-20s & $0.077 \mathrm{mg} / \mathrm{l}$ & $0.073 \mathrm{mg} / \mathrm{l}$ & $0.077 \mathrm{mg} / \mathrm{l}$ & $0.073 \mathrm{mg} / \mathrm{l}$ & 1 & \\
\hline Bonflium 740-41.7 & $<0.0004 \mathrm{mg} / \mathrm{l}$ & $<0.0004 \mathrm{mg} / \mathrm{l}$ & $<0.0004 \mathrm{mg} / l$ & $<0.0004 \mathrm{mg} / \mathrm{l}$ & 1 & \\
\hline 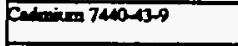 & $<0.004 \mathrm{mg} / \mathrm{l}$ & $<0.004 \mathrm{mg} / \mathrm{l}$ & $<0.004 \mathrm{mg} / \mathrm{l}$ & $<0.004 \mathrm{mg} / \mathrm{l}$ & 1 & \\
\hline Cabions 740-70-2 & $134 \mathrm{mg} / \mathrm{l}$ & $69.3 \mathrm{mg} / \mathrm{l}$ & $134 \mathrm{mg} / \mathrm{l}$ & $69.3 \mathrm{mg} / \mathrm{l}$ & 1 & \\
\hline Dorowien 740-7.3 & $0.011 \mathrm{mg} / 1$ & $0.011 \mathrm{mg} / \mathrm{l}$ & $0.011 \mathrm{mg} / 1$ & $0.011 \mathrm{mg} / 1$ & 1 & \\
\hline Cobali $740-18-4$ & $0.004 \mathrm{mg} / \mathrm{l}$ & $0.004 \mathrm{mg} / \mathrm{l}$ & $0.004 \mathrm{mg} / \mathrm{l}$ & $0.004 \mathrm{mg} / \mathrm{l}$ & $I$ & \\
\hline Coppon 740a-sos & $0.023 \mathrm{mg} / \mathrm{l}$ & $0.019 \mathrm{mg} / \mathrm{l}$ & $0.023 \mathrm{mg} / \mathrm{l}$ & $0.019 \mathrm{mg} / 1$ & 1 & \\
\hline $\operatorname{son} 7039+296$ & $15.1 \mathrm{mg} / 1$ & $14.0 \mathrm{mg} / \mathrm{l}$ & $15.1 \mathrm{mg} / 1$ & $14.0 \mathrm{mg} / \mathrm{l}$ & 1 & \\
\hline End 7439-92-1 & $0.02 \mathrm{mg} / 1$ & $<0.02 \mathrm{mg} / 1$ & $0.02 \mathrm{mg} / \mathrm{l}$ & $<0.02 \mathrm{mg} / 1$ & 1 & \\
\hline Ithinem 7439-93-2 & $0.02 \mathrm{mg} / 1$ & $<0.02 \mathrm{mg} / 1$ & $0.02 \mathrm{mg} / 1$ & $<0.02 \mathrm{mg} / 1$ & 1 & \\
\hline Mesosing 7632-93-4 & $21.3 \mathrm{mg} / 1$ & $10.7 \mathrm{mg} / 1$ & $21.3 \mathrm{mg} / \mathrm{l}$ & $10.7 \mathrm{mg} / \mathrm{l}$ & 1 & \\
\hline 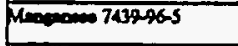 & $0.229 \mathrm{mg} / \mathrm{l}$ & $0.263 \mathrm{mg} / \mathrm{l}$ & $0.229 \mathrm{mg} / \mathrm{l}$ & $0.263 \mathrm{mg} / \mathrm{l}$ & 1 & \\
\hline 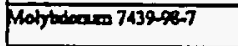 & $<0.006 \mathrm{mg} / \mathrm{I}$ & $0.007 \mathrm{mg} / \mathrm{l}$ & $<0.006 \mathrm{mg} / \mathrm{l}$ & $0.007 \mathrm{mg} / \mathrm{l}$ & 1 & \\
\hline$\sqrt{\text { richal } 740020}$ & $0.010 \mathrm{mg} / 1$ & $0.009 \mathrm{mg} / \mathrm{l}$ & $0.010 \mathrm{mg} / 1$ & $0.009 \mathrm{mg} / \mathrm{l}$ & 1 & \\
\hline 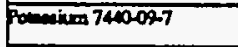 & $5.7 \mathrm{mg} / \mathrm{l}$ & $6.0 \mathrm{mg} / \mathrm{l}$ & $5.7 \mathrm{mg} / \mathrm{l}$ & $6.0 \mathrm{mg} / \mathrm{l}$ & 1 & \\
\hline sither 740204 & $<0.006 \mathrm{mg} / \mathrm{l}$ & $<0.006 \mathrm{mg} / \mathrm{l}$ & $<0.006 \mathrm{mg} / 1$ & $<0.006 \mathrm{mg} / \mathrm{l}$ & 1 & \\
\hline adisum 740-23-5 & $32.4 \mathrm{mg} / \mathrm{l}$ & $12.1 \mathrm{mg} / \mathrm{l}$ & $32.4 \mathrm{mg} / \mathrm{l}$ & $12.1 \mathrm{mg} / \mathrm{l}$ & 1 & \\
\hline Tincinan 7440-32-6 & $0.08 \mathrm{mg} / \mathrm{l}$ & $0.07 \mathrm{mg} / \mathrm{l}$ & $0.08 \mathrm{mg} / \mathrm{l}$ & $0.07 \mathrm{mg} / \mathrm{l}$ & 1 & \\
\hline 2707440666 & $0.09 \mathrm{mg} / \mathrm{l}$ & $0.07 \mathrm{mg} / \mathrm{l}$ & $0.09 \mathrm{mg} / 1$ & $0.07 \mathrm{mg} / \mathrm{l}$ & 1 & \\
\hline
\end{tabular}


Cootiound from front.

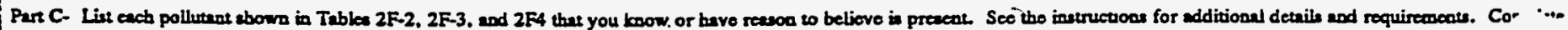
oso ublo for cach oufull.

\begin{tabular}{|c|c|c|c|c|c|c|}
\hline \multirow{2}{*}{$\begin{array}{l}\text { Polhutunt } \\
\text { and } \\
\text { CAS Number } \\
\text { (if availible) }\end{array}$} & \multicolumn{2}{|c|}{$\begin{array}{l}\text { Maximum Valuea } \\
\text { (inchude units) }\end{array}$} & \multicolumn{2}{|c|}{$\begin{array}{l}\text { Average Values } \\
\text { (ixclude urits) }\end{array}$} & \multirow{2}{*}{$\begin{array}{c}\text { Number } \\
\text { of } \\
\text { Storm } \\
\text { Events } \\
\text { Srmpied }\end{array}$} & \multirow[b]{2}{*}{ Sources of Polhurants } \\
\hline & $\begin{array}{l}\text { Grib Sample } \\
\text { Takea During } \\
\text { First } 30 \\
\text { Minues }\end{array}$ & $\begin{array}{l}\text { Flow-weighted } \\
\text { Composito }\end{array}$ & 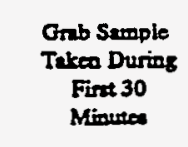 & $\begin{array}{l}\text { Flow-werghted } \\
\text { Componts }\end{array}$ & & \\
\hline 1.1.1. trictioreatene 71-55-6 & $<0.010 \mathrm{mg} / \mathrm{l}$ & & $<0.010 \mathrm{mg} / 1$ & & 1 & \\
\hline 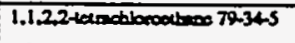 & $<0.010 \mathrm{mg} / 1$ & & $<0.010 \mathrm{mg} / \mathrm{l}$ & & I & \\
\hline 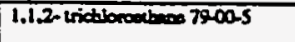 & $<0.010 \mathrm{mg} / \mathrm{l}$ & & $<0.010 \mathrm{mg} / \mathrm{l}$ & & 1 & \\
\hline 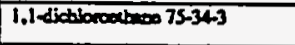 & $<0.010 \mathrm{mg} / \mathrm{l}$ & & $<0.010 \mathrm{mg} / \mathrm{l}$ & & 1 & \\
\hline 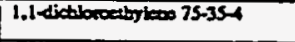 & $<0.010 \mathrm{mg} / \mathrm{l}$ & & $<0.010 \mathrm{mg} / \mathrm{l}$ & & 1 & \\
\hline 1.2 dictbonodten $107.06-2$ & $<0.010 \mathrm{mg} / 1$ & & $<0.010 \mathrm{mg} / \mathrm{l}$ & & 1 & \\
\hline 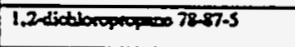 & $<0.010 \mathrm{mg} / \mathrm{l}$ & & $<0.010 \mathrm{mg} / \mathrm{l}$ & & 1 & \\
\hline 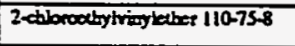 & $<0.010 \mathrm{mg} / 1$ & & $<0.010 \mathrm{mg} / \mathrm{l}$ & & 1 & \\
\hline beomed 71-13-2 & $<0.010 \mathrm{mg} / 1$ & & $<0.010 \mathrm{mg} / 1$ & & 1 & \\
\hline brocodictionatand $75-274$ & $<0.010 \mathrm{mg} / 1$ & & $<0.010 \mathrm{mg} / \mathrm{l}$ & & 1 & \\
\hline breatolorm 75-2S-2 & $<0.010 \mathrm{mg} / \mathrm{l}$ & & $<0.010 \mathrm{mg} / \mathrm{l}$ & & 1 & \\
\hline brecousations 7483-9 & $<0.010 \mathrm{mg} / \mathrm{l}$ & & $<0.010 \mathrm{mg} / \mathrm{l}$ & & 1 & \\
\hline artaon betenctioride 56-22-5 & $<0.010 \mathrm{mg} / \mathrm{l}$ & & $<0.010 \mathrm{mg} / 1$ & & 1 & \\
\hline chlorobereoces $108-90.7$ & $<0.010 \mathrm{mg} / \mathrm{l}$ & & $<0.010 \mathrm{mg} / 1$ & & 1 & \\
\hline $75-00-3$ & $<0.010 \mathrm{mg} / \mathrm{l}$ & & $<0.010 \mathrm{mg} / \mathrm{l}$ & & 1 & \\
\hline Dboroform 6-66-3 & $<0.010 \mathrm{mg} / 1$ & & $<0.010 \mathrm{mg} / \mathrm{l}$ & & 1 & \\
\hline 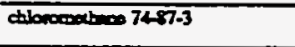 & $<0.010 \mathrm{mg} / 1$ & & $<0.010 \mathrm{mg} / \mathrm{l}$ & & 1 & 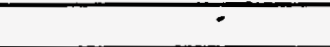 \\
\hline 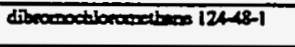 & $<0.010 \mathrm{mg} / 1$ & & $<0.010 \mathrm{mg} / \mathrm{l}$ & & 1 & \\
\hline 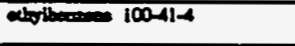 & $<0.010 \mathrm{mg} / \mathrm{l}$ & & $<0.010 \mathrm{mg} / \mathrm{l}$ & & 1 & \\
\hline whes $102-2-3$ & $<0.010 \mathrm{mg} / 1$ & & $<0.010 \mathrm{mg} / 1$ & & 1 & \\
\hline rivgl chboidt 75-014 & $<0.010 \mathrm{mg} / \mathrm{l}$ & & $<0.010 \mathrm{mg} / \mathrm{l}$ & & 1 & \\
\hline 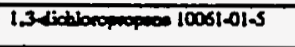 & $<0.010 \mathrm{mg} / \mathrm{l}$ & & $<0.010 \mathrm{mg} / 1$ & & 1 & \\
\hline 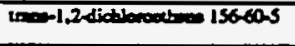 & $<0.010 \mathrm{mg} / 1$ & & $<0.010 \mathrm{mg} / 1$ & $\bar{\sigma}$ & 1 & \\
\hline apteativity $12587-46-1$ & $230 \mathrm{pci} / \mathrm{l}$ & 76 peill & $230 \mathrm{pci} / 1$ & 76 peill & 1 & 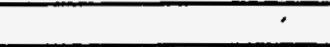 \\
\hline bedeaividy $1258746-1$ & 200 pcill & 77 pcill & 200 pein & 77 peill & 1 & \\
\hline focel oolifion & $8,700 \mathrm{co} / .1 \mathrm{~L}$ & $9,000 \mathrm{co} / .1 \mathrm{~L}$ & $8,700 \mathrm{co} / .1 \mathrm{~L}$ & $9,000 \mathrm{co} / .1 \mathrm{~L}$ & 1 & \\
\hline Thallim 7440-28-0 & $<0.001 \mathrm{mg} / \mathrm{l}$ & $<0.001 \mathrm{mg} / \mathrm{l}$ & $<0.001 \mathrm{mg} / \mathrm{l}$ & $<0.001 \mathrm{mg} / !$ & 1 & \\
\hline Unenting 746061-1 & $0.451 \mathrm{mg} / \mathrm{l}$ & $0.182 \mathrm{mg} / 1$ & $0.451 \mathrm{mg} / \mathrm{l}$ & $0.182 \mathrm{mg} / \mathrm{l}$ & 1 & \\
\hline Germeneativity & 35 peill & 22 pcill & 35 peill & 22 pcill & 1 & \\
\hline & & & & & & \\
\hline
\end{tabular}

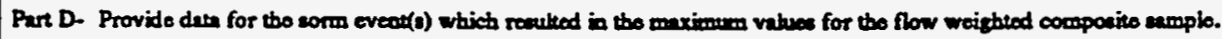

\begin{tabular}{|c|c|c|c|c|c|c|c|}
\hline $\begin{array}{l}1 . \\
\text { Date of } \\
\text { Stom } \\
\text { Event }\end{array}$ & $\begin{array}{l}2 . \\
\text { Durion } \\
\text { of Storm } \\
\text { (in } \\
\text { minutea) }\end{array}$ & $\begin{array}{l}3 . \\
\text { Totel ninfill } \\
\text { durias torm eveot } \\
\text { (in ivebeo) }\end{array}$ & 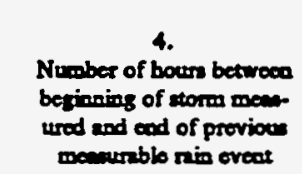 & 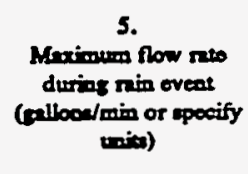 & $\begin{array}{l}6 . \\
\text { Tocal flow from } \\
\text { min ovent } \\
\text { (oullows or } \\
\text { spocify uniw) }\end{array}$ & 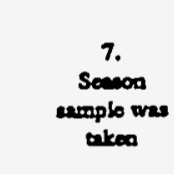 & $\begin{array}{l}\text { 8. } \\
\text { Form of } \\
\text { Precipitusion } \\
\text { (ninfill, } \\
\text { nowmolt) }\end{array}$ \\
\hline $9 / 24 / 91$ & 365 & 2.0 & 133.5 & 1,011 & 301,018 & Fall & Rainfull \\
\hline
\end{tabular}

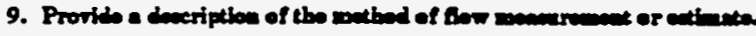

An ISCO 3230 Flow meter was wed to obtain water leved reading. This indromeat was calibrated with the normal dry weather flow, if preant, as le zero. The flow meter was programmed with the characteriatics of the conveyance so that flow rates and total flows conld be calculated by the meter. 


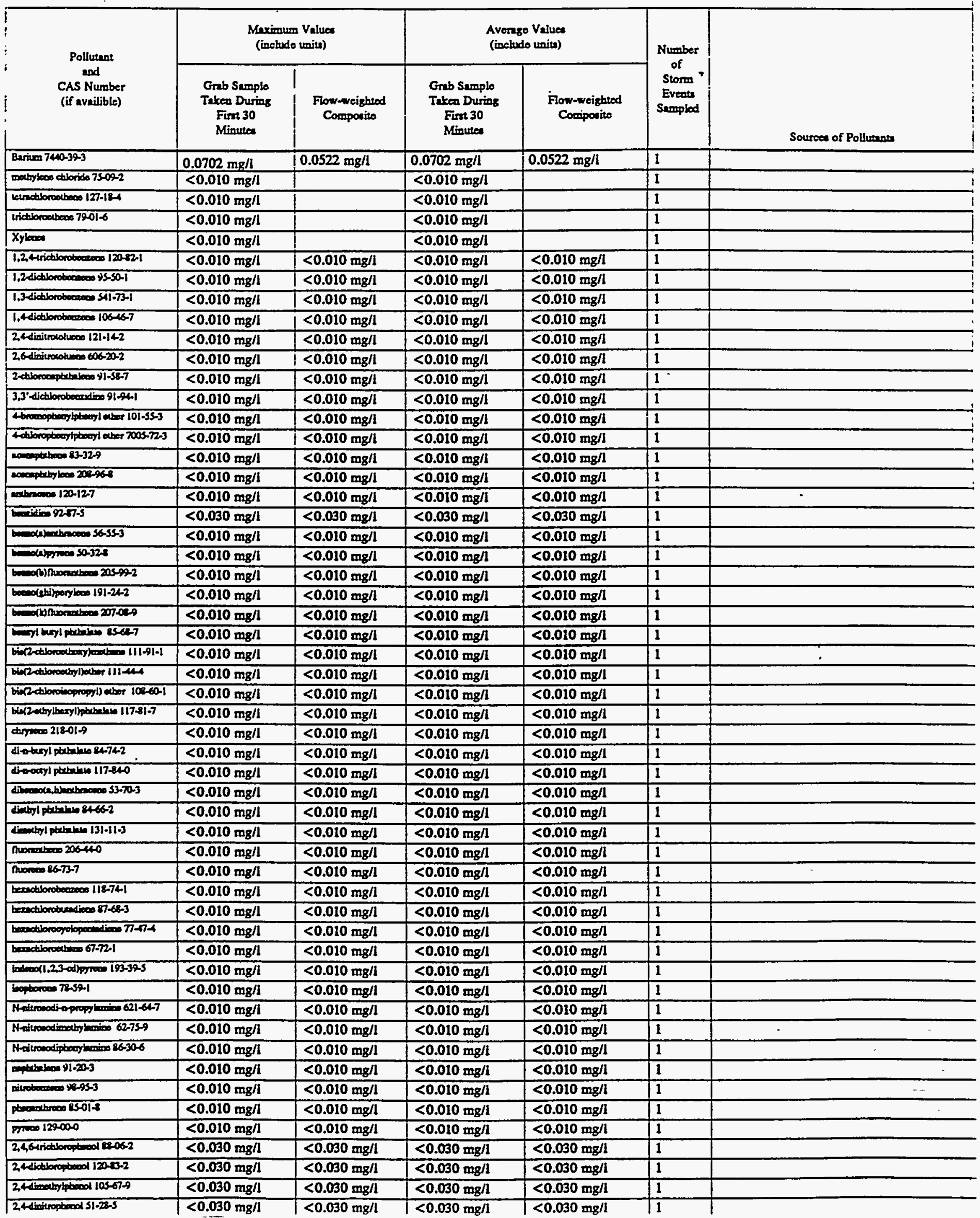


Outfall S2

Para B and C Contioied

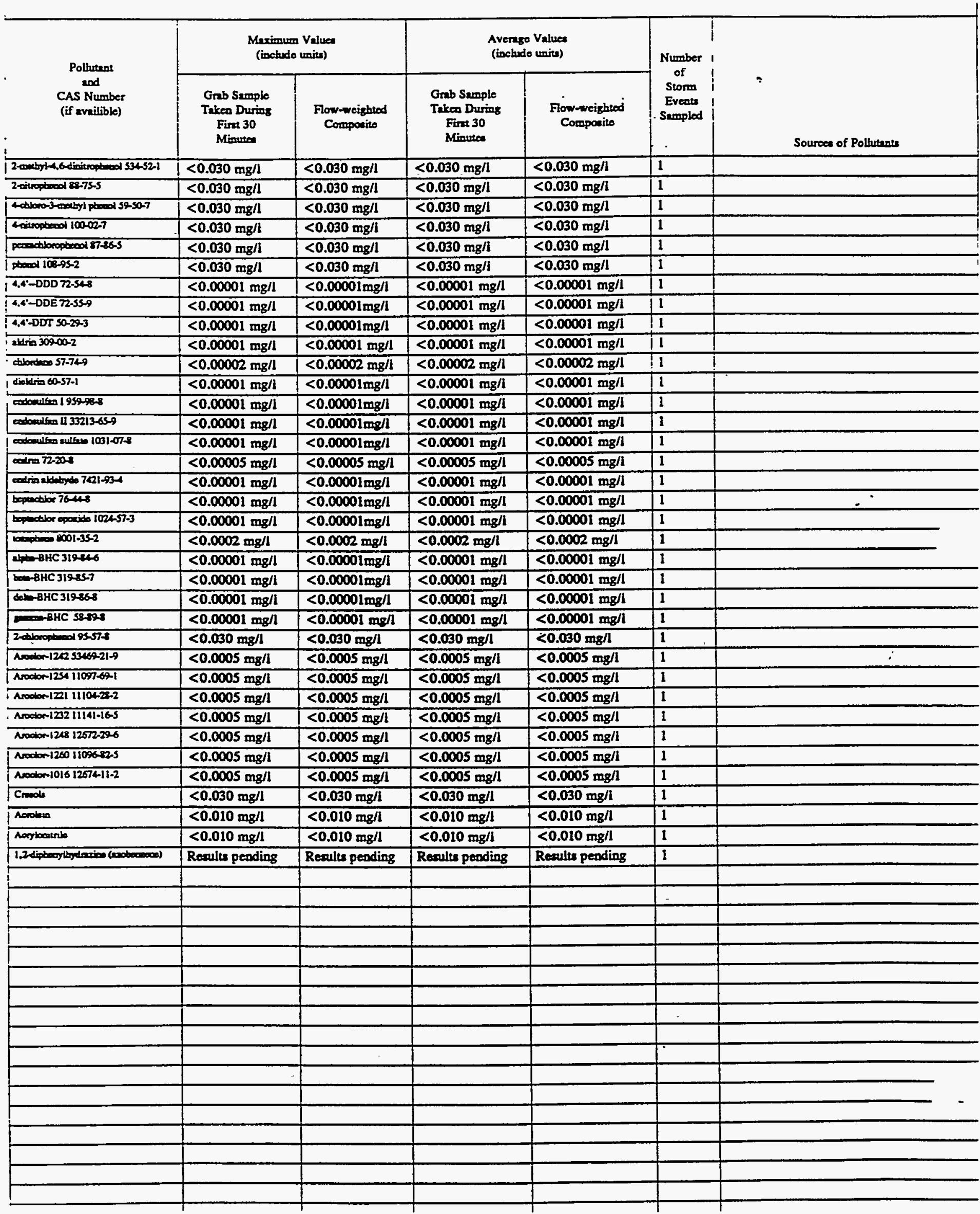


VII, Discharge Information (concinuod from page 3 of Form 2A)

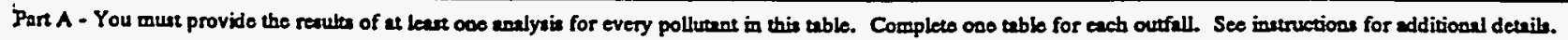

\begin{tabular}{|c|c|c|c|c|c|c|}
\hline \multirow{2}{*}{$\begin{array}{c}\text { Poltumens } \\
\text { and } \\
\text { CAS Number } \\
\text { (if avialible) }\end{array}$} & \multicolumn{2}{|c|}{$\begin{array}{l}\text { Maximum Valuee } \\
\text { (inctude units) }\end{array}$} & \multicolumn{2}{|c|}{$\begin{array}{l}\text { Averngo Values } \\
\text { (inetudo unisa) }\end{array}$} & \multirow{2}{*}{$\begin{array}{c}\text { Number } \\
\text { of } \\
\text { Storm } \\
\text { Eveate } \\
\text { Saroplod }\end{array}$} & \multirow[b]{2}{*}{ Sources of Pollwate } \\
\hline & $\begin{array}{c}\text { Grab Sumple } \\
\text { Takes During } \\
\text { Firn 30 } \\
\text { Mineres }\end{array}$ & $\begin{array}{l}\text { Flow-meizhted } \\
\text { Composito }\end{array}$ & $\begin{array}{l}\text { Grab Samplo } \\
\text { Takoo During } \\
\text { Fint } 30 \\
\text { Minuteo }\end{array}$ & $\begin{array}{l}\text { Flow-moigtited } \\
\text { Cocoporito }\end{array}$ & & \\
\hline pulead Groan & $<2 \mathrm{mg} / \mathrm{l}$ & & $<2 \mathrm{mg} / \mathrm{l}$ & & 1 & \\
\hline Blologial Oxyea Deaned (BOD) & $<5 \mathrm{mg} / 1$ & $5.23 \mathrm{mg} / 1$ & $<5 \mathrm{mg} / \mathrm{l}$ & $5.23 \mathrm{mg} / 1$ & 1 & \\
\hline Chermial Oxyera Dearean (COD) & $26 \mathrm{mg} / \mathrm{l}$ & $23 \mathrm{mg} / \mathrm{l}$ & $26 \mathrm{mg} / 1$ & $23 \mathrm{mg} / \mathrm{l}$ & 1 & \\
\hline Fotal Supendad Solide (TSS) & $650 \mathrm{mg} / \mathrm{l}$ & $370 \mathrm{mg} / \mathrm{l}$ & $650 \mathrm{mg} / \mathrm{l}$ & $370 \mathrm{mg} / 1$ & 1 & \\
\hline 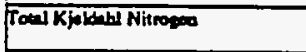 & $0.6 \mathrm{mg} / \mathrm{l}$ & $0.5 \mathrm{mg} / \mathrm{l}$ & $0.6 \mathrm{mg} / \mathrm{l}$ & $0.5 \mathrm{mg} / \mathrm{l}$ & 1 & \\
\hline Mitrese phe Nitrive Nitrosen & $0.78 \mathrm{mg} / \mathrm{l}$ & $10.64 \mathrm{mg} / 1$ & $0.78 \mathrm{mg} / \mathrm{l}$ & $10.64 \mathrm{mg} / \mathrm{l}$ & 1 & \\
\hline Tool Prophorese & $0.41 \mathrm{mg} / \mathrm{l}$ & $0.6 \mathrm{mg} / \mathrm{l}$ & $0.41 \mathrm{mg} / \mathrm{l}$ & $0.6 \mathrm{mg} / \mathrm{l}$ & 1 & \\
\hline pH & Minm 82 & Mximm 8.2 & Minimen 82 & Mecion 82 & & \\
\hline
\end{tabular}

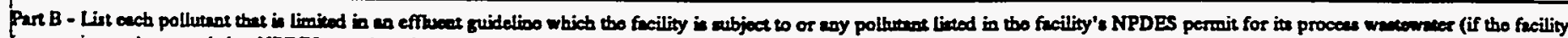

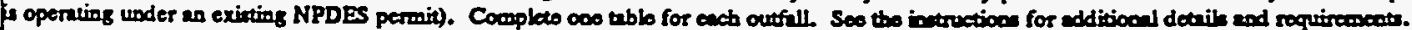

\begin{tabular}{|c|c|c|c|c|c|c|}
\hline \multirow{2}{*}{$\begin{array}{l}\text { Pollutant } \\
\text { and } \\
\text { CAs Number } \\
\text { (if aveiliblo) }\end{array}$} & \multicolumn{2}{|c|}{$\begin{array}{l}\text { Maximum Values } \\
\text { (inchudo unita) }\end{array}$} & \multicolumn{2}{|c|}{ 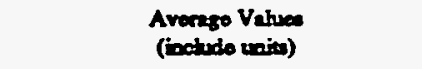 } & \multirow{2}{*}{$\begin{array}{c}\text { Nomber } \\
\text { of } \\
\text { Storm } \\
\text { Eveosed } \\
\text { Sempled }\end{array}$} & \multirow{2}{*}{ 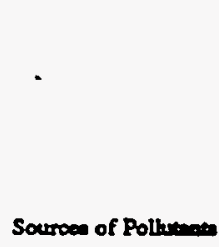 } \\
\hline & 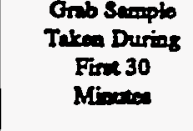 & $\begin{array}{l}\text { Fom-wrigted } \\
\text { Compocies }\end{array}$ & $\begin{array}{l}\text { Orab Semplo } \\
\text { Tebon During } \\
\text { Fint } 30 \\
\text { Miourece }\end{array}$ & $\begin{array}{l}\text { Flow-moizheod } \\
\text { Cocopocito }\end{array}$ & & \\
\hline Animang $740-360$ & $<0.04 \mathrm{mg} / \mathrm{l}$ & $<0.04 \mathrm{mg} / 1$ & $<0.04 \mathrm{mg} / \mathrm{l}$ & $<0.04 \mathrm{mg} / \mathrm{l}$ & 1 & - \\
\hline Frailo & $<0.002 \mathrm{mg} / \mathrm{h}$ & & $<0.002 \mathrm{mg} / \mathrm{l}$ & & 1 & \\
\hline Monowy $7439+76$ & $0.0002 \mathrm{mg} / \mathrm{l}$ & $0.0004 \mathrm{mg} / \mathrm{l}$ & $0.0002 \mathrm{mg} / 1^{\circ}$ & $0.0004 \mathrm{mg} / 1$ & 1 & \\
\hline roods & $0.009 \mathrm{mg} / \mathrm{I}$ & & $0.009 \mathrm{mg} / \mathrm{l}$ & & 1 & $\cdot$ \\
\hline Sonimon $778269-2$ & $<0.002 \mathrm{mg} / \mathrm{l}$ & $<0.002 \mathrm{mg} / \mathrm{l}$ & $<0.002 \mathrm{mg} / \mathrm{l}$ & $<0.002 \mathrm{mg} / 1$ & 1 & \\
\hline pulan & $21 \mathrm{mg} / \mathrm{l}$ & $<10 \mathrm{mg} / \mathrm{l}$ & $21 \mathrm{mg} / 1$ & $<10 \mathrm{mg} / \mathrm{l}$ & $I$ & \\
\hline Perthoveres & $<0.05 \mathrm{mg} / \mathrm{l}$ & $<0.05 \mathrm{mg} / \mathrm{l}$ & $<0.05 \mathrm{mg} / \mathrm{l}$ & $<0.05 \mathrm{mg} / \mathrm{l}$ & 1 & \\
\hline Muminam 7429-90.5 & $17.4 \mathrm{mg} / \mathrm{l}$ & $14.2 \mathrm{mg} / 1$ & $17.4 \mathrm{mg} / \mathrm{l}$ & $14.2 \mathrm{mg} / \mathrm{l}$ & 1 & \\
\hline trowic 74a-38-2 & $0.07 \mathrm{mg} / \mathrm{l}$ & $0.06 \mathrm{mg} / \mathrm{l}$ & $0.07 \mathrm{mg} / 1$ & $0.06 \mathrm{mg} / \mathrm{l}$ & 1 & \\
\hline Bovod 7410-228 & $0.016 \mathrm{mg} / \mathrm{l}$ & $0.012 \mathrm{mg} / \mathrm{l}$ & $0.016 \mathrm{mg} / 1$ & $0.012 \mathrm{mg} / \mathrm{l}$ & 1 & \\
\hline Benflition 740-41.7 & $0.0007 \mathrm{mg} / \mathrm{l}$ & $0.0006 \mathrm{mg} / \mathrm{l}$ & $0.0007 \mathrm{mg} / \mathrm{l}$ & $0.0006 \mathrm{mg} / 1$ & 1 & \\
\hline Fadming 740-13-9 & $<0.004 \mathrm{mg} / 1$ & $0.005 \mathrm{mg} / \mathrm{l}$ & $<0.004 \mathrm{mg} / \mathrm{l}$ & $0.005 \mathrm{mg} / 1$ & 1 & \\
\hline Felicien $7440-70-2$ & $8.9 \mathrm{mg} / \mathrm{l}$ & $9.5 \mathrm{mg} / 1$ & $8.9 \mathrm{mg} / \mathrm{l}$ & $9.5 \mathrm{mg} / 1$ & 1 & \\
\hline Prowive 740-7.3 & $0.026 \mathrm{mg} / \mathrm{l}$ & $0.022 \mathrm{mg} / \mathrm{l}$ & $0.026 \mathrm{mg} / 1$ & $0.022 \mathrm{mg} / \mathrm{l}$ & 1 & \\
\hline Cotall 7440-48-4 & $0.011 \mathrm{mg} / 1$ & $0.009 \mathrm{mg} / \mathrm{l}$ & $0.011 \mathrm{mg} / 1$ & $0.009 \mathrm{mg} / 1$ & 1 & \\
\hline Coppor 7440-50-8 & $0.039 \mathrm{mg} / \mathrm{l}$ & $0.031 \mathrm{mg} / 1$ & $0.039 \mathrm{mg} / \mathrm{l}$ & $0.031 \mathrm{mg} / 1$ & 1 & \\
\hline roo 7439-896-6 & $33.8 \mathrm{mg} / \mathrm{l}$ & $28.5 \mathrm{mg} / 1$ & $33.8 \mathrm{mg} / \mathrm{l}$ & $28.5 \mathrm{mg} / \mathrm{l}$ & 1 & \\
\hline Ind 7439-92-1 & $0.05 \mathrm{mg} / \mathrm{l}$ & $0.04 \mathrm{mg} / \mathrm{l}$ & $0.05 \mathrm{mg} / 1$ & $0.04 \mathrm{mg} / \mathrm{l}$ & 1 & \\
\hline 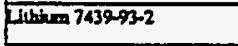 & $<0.02 \mathrm{mg} / \mathrm{l}$ & $<0.02 \mathrm{mg} / 1$ & $<0.02 \mathrm{mg} / 1$ & $<0.02 \mathrm{mg} / 1$ & 1 & \\
\hline Meponaum 7439-954 & $1.66 \mathrm{mg} / \mathrm{l}$ & $1.56 \mathrm{mg} / 1$ & $1.66 \mathrm{mg} / \mathrm{l}$ & $1.56 \mathrm{mg} / \mathrm{l}$ & 1 & \\
\hline 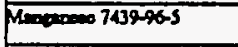 & $0.365 \mathrm{mg} / \mathrm{l}$ & $0.295 \mathrm{mg} / \mathrm{l}$ & $0.365 \mathrm{mg} / 1$ & $0.295 \mathrm{mg} / 1$ & 1 & \\
\hline Mobtdionm 7439-92-7 & $<0.006 \mathrm{mg} / \mathrm{l}$ & $<0.006 \mathrm{mg} / 1$ & $<0.006 \mathrm{mg} / 1$ & $<0.006 \mathrm{mg} / 1$ & 1 & \\
\hline$\sqrt{10017400020}$ & $0.019 \mathrm{mg} / \mathrm{l}$ & $0.017 \mathrm{mg} / \mathrm{l}$ & $0.019 \mathrm{mg} / \mathrm{l}$ & $0.017 \mathrm{mg} / \mathrm{l}$ & 1 & \\
\hline Pomenixas 7400027 & $5.2 \mathrm{mg} / \mathrm{l}$ & $5.3 \mathrm{mg} / \mathrm{l}$ & $5.2 \mathrm{mg} / 1$ & $5.3 \mathrm{mg} / \mathrm{l}$ & 1 & \\
\hline ither 740-20-4 & $<0.006 \mathrm{mg} / \mathrm{l}$ & $<0.006 \mathrm{mg} / 1$ & $<0.006 \mathrm{mg} / 1$ & $<0.006 \mathrm{mg} / 1$ & 1 & \\
\hline Sodthen 740-2a-s & $0.50 \mathrm{mg} / 1$ & $0.52 \mathrm{mg} / 1$ & $0.50 \mathrm{mg} / 1$ & $0.52 \mathrm{mg} / \mathrm{l}$ & 1 & \\
\hline 740.326 & $0.14 \mathrm{mg} / \mathrm{l}$ & $0.12 \mathrm{mg} / 1$ & $0.14 \mathrm{mg} / 1$ & $0.12 \mathrm{mg} / 1$ & 1 & \\
\hline $\sin 0740666$ & $0.13 \mathrm{mg} / \mathrm{l}$ & $0.11 \mathrm{mg} / \mathrm{l}$ & $0.13 \mathrm{mg} / \mathrm{l}$ & $0.11 \mathrm{mg} / \mathrm{l}$ & 1 & \\
\hline
\end{tabular}


Contioned from trove.

\begin{tabular}{|c|c|c|c|c|c|c|}
\hline \multirow{2}{*}{$\begin{array}{l}\text { Pollutuant } \\
\text { end } \\
\text { CAS Number } \\
\text { (if aveilible) }\end{array}$} & \multicolumn{2}{|c|}{$\begin{array}{l}\text { Meximumom Vahues } \\
\text { (iochude units) }\end{array}$} & \multicolumn{2}{|c|}{$\begin{array}{l}\text { Avernge Vatuea } \\
\text { (inchude units) }\end{array}$} & \multirow{2}{*}{ 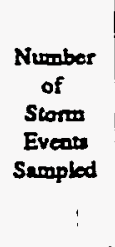 } & \multirow[b]{2}{*}{ - surces of Poltutanas } \\
\hline & $\begin{array}{l}\text { Grab Sumple } \\
\text { Tukea Duriag } \\
\text { Firn } 30 \\
\text { Minuex }\end{array}$ & $\begin{array}{l}\text { Frow-weighted } \\
\text { Compoxire }\end{array}$ & $\begin{array}{c}\text { Grab Sumple } \\
\text { Taken During } \\
\text { Firt } 30 \\
\text { Minutese }\end{array}$ & $\begin{array}{c}\text { Flow-weigited } \\
\text { Compocito }\end{array}$ & & \\
\hline 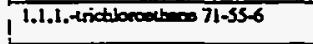 & $<0.010 \mathrm{mg} / \mathrm{l}$ & & $<0.010 \mathrm{mg} / 1$ & & 1 & \\
\hline 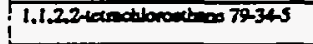 & $<0.010 \mathrm{mg} / \mathrm{l}$ & & $<0.010 \mathrm{mg} / \mathrm{l}$ & & 1 & \\
\hline 1.1.2- trictionocolmen $79000-5$ & $<0,010 \mathrm{mg} / \mathrm{l}$ & & $<0.010 \mathrm{mg} / 1$ & & 1 & \\
\hline 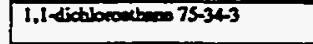 & $<0.010 \mathrm{mg} / \mathrm{l}$ & & $<0.010 \mathrm{mg} / \mathrm{h}$ & & 1 & \\
\hline 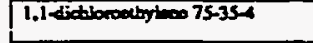 & $<0.010 \mathrm{mg} / \mathrm{l}$ & & $<0.010 \mathrm{mg} / 1$ & & 1 & \\
\hline 1.2 didtorouthece 107.062 & $<0.010 \mathrm{mg} / \mathrm{l}$ & & $<0.010 \mathrm{mg} / \mathrm{l}$ & & 1 & - \\
\hline 1.3 didtibropropene $72-57.5$ & $<0.010 \mathrm{mg} / \mathrm{l}$ & & $<0.010 \mathrm{mg} / \mathrm{L}$ & & 1 & \\
\hline 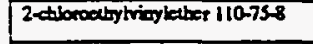 & $<0.010 \mathrm{mg} / \mathrm{l}$ & & $<0.010 \mathrm{mg} / \mathrm{l}$ & & 1 & \\
\hline $71-13-2$ & $<0.010 \mathrm{mg} / 1$ & & $<0.010 \mathrm{mg} / \mathrm{l}$ & & 1 & \\
\hline 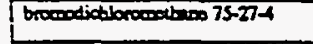 & $<0.010 \mathrm{mg} / \mathrm{l}$ & & $<0.010 \mathrm{mg} / \mathrm{l}$ & & 1 & \\
\hline browodions 75-25-2 & $<0.010 \mathrm{mg} / 1$ & & $<0.010 \mathrm{mg} / \mathrm{l}$ & & 1 & \\
\hline bromanomber 74839 & $<0.010 \mathrm{mg} / 1$ & & $<0.010 \mathrm{mg} / \mathrm{l}$ & & 1 & \\
\hline artace manchloride $56-23-5$ & $<0.010 \mathrm{mg} / \mathrm{l}$ & & $<0.010 \mathrm{mg} / \mathrm{l}$ & & 1 & \\
\hline 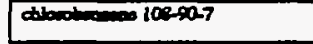 & $<0.010 \mathrm{mg} / 1$ & & $<0.010 \mathrm{mg} / 1$ & & 1 & \\
\hline atbonowhene $75,-00-3$ & $<0.010 \mathrm{mg} / 1$ & & $<0.010 \mathrm{mg} / \mathrm{l}$ & & 1 & \\
\hline dibofocin $67-66-3$ & $<0.010 \mathrm{mg} / \mathrm{l}$ & & $<0.010 \mathrm{mg} / \mathrm{l}$ & & 1 & \\
\hline 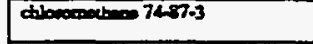 & $<0.010 \mathrm{mg} / \mathrm{l}$ & & $<0.010 \mathrm{mg} / 1$ & & 1 & - \\
\hline 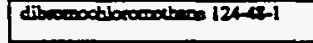 & $<0.010 \mathrm{mg} / 1$ & & $<0.010 \mathrm{mg} / \mathrm{l}$ & & 1 & \\
\hline atringeses 100014 & $<0.010 \mathrm{mg} / 1$ & & $<0.010 \mathrm{mg} / \mathrm{l}$ & & 1 & \\
\hline 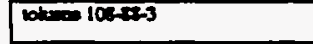 & $<0.010 \mathrm{mg} / \mathrm{l}$ & & $<0.010 \mathrm{mg} / \mathrm{l}$ & & 1 & \\
\hline vingl dibribo 75014 & $<0.010 \mathrm{mg} / 1$ & & $<0.010 \mathrm{mg} / \mathrm{l}$ & & 1 & \\
\hline 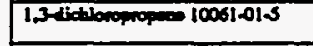 & $<0.010 \mathrm{mg} / \mathrm{l}$ & & $<0.010 \mathrm{mg} / \mathrm{l}$ & & 1 & \\
\hline 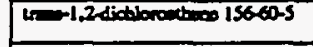 & $<0.010 \mathrm{mg} / \mathrm{l}$ & & $<0.010 \mathrm{mg} / \mathrm{l}$ & & 1 & $\therefore$ \\
\hline atseaciving $1258746-1$ & $12 \mathrm{pci} / 1$ & $1.0 \mathrm{pei} / \mathrm{l}$ & 12 psill & $1.0 \mathrm{pei} / \mathrm{l}$ & 1 & \\
\hline bearixy $125578-1$ & $30 \mathrm{pci} / \mathrm{l}$ & $4.6 \mathrm{poi} / \mathrm{l}$ & $30 \mathrm{pcill}$ & $4.6 \mathrm{pcill}$ & 1 & \\
\hline 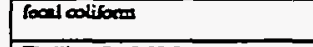 & $27,000 \mathrm{col} .1 \mathrm{~L}$ & $<1 \mathrm{col} .1 \mathrm{~L}$ & $27,000 \mathrm{col} .1 \mathrm{~L}$ & $<1 \mathrm{col} .1 \mathrm{~L}$ & 1 & \\
\hline Thallien 740-220 & $<0.001 \mathrm{mg} / \mathrm{l}$ & $<0.001 \mathrm{mg} / \mathrm{l}$ & $<0.001 \mathrm{mg} / 1$ & $<0.001 \mathrm{mg} / 1$ & 1 & \\
\hline Unsing 74061-1 & $0.003 \mathrm{mg} / \mathrm{l}$ & $0.003 \mathrm{mg} / \mathrm{l}$ & $0.003 \mathrm{mg} / \mathrm{l}$ & $0.003 \mathrm{mg} / \mathrm{l}$ & 1 & \\
\hline Gesomenecivity & $41 \mathrm{pei} / \mathrm{p}$ & 5 peill & $41 \mathrm{pei} / 1$ & 5 poin & 1 & \\
\hline
\end{tabular}

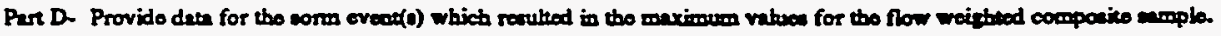

\begin{tabular}{|c|c|c|c|c|c|c|c|}
\hline $\begin{array}{l}1 . \\
\text { Dete of } \\
\text { Storm } \\
\text { Eveast }\end{array}$ & $\begin{array}{l}2 . \\
\text { Dinstion } \\
\text { of Storm } \\
\text { (in }\end{array}$ & $\begin{array}{l}3 . \\
\text { Total minfill } \\
\text { derixe ntorm oveer } \\
\text { (in inction) }\end{array}$ & 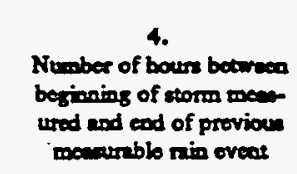 & $\begin{array}{l}\text { S. } \\
\text { Maximain flow rato } \\
\text { duries nin oveat } \\
\text { (ollowdmin or epocify } \\
\text { unies) }\end{array}$ & $\begin{array}{l}6 . \\
\text { Tosil flow from } \\
\text { nin ovent } \\
\text { (Eallows or } \\
\text { specify unitu) }\end{array}$ & $\begin{array}{c}7 . \\
\text { Semiog } \\
\text { axmole was } \\
\text { alkeo }\end{array}$ & $\begin{array}{l}8 . \\
\text { Form of } \\
\text { Procipitation } \\
\text { (ninfall. } \\
\text { nommeth) }\end{array}$ \\
\hline $9 / 24 / 91$ & 365 & 1.3 & 133.5 & 20 & 1,547 & Fall & Ruinfall \\
\hline
\end{tabular}

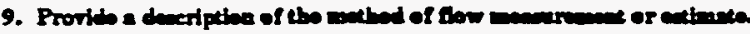

An ISCO 3230 Flow meter was ned to obthin water leved readings. This intrument was calibrated with the sormal dry weather tion, if present, as 4 zero. The flow meter was progermmed with the characteristics of the conveyence so that flow rates and total flows conid be calcolated by the meter. 
Outfall S3

Parte B and C Continuod

\begin{tabular}{|c|c|c|c|c|c|c|}
\hline \multirow{2}{*}{$\begin{array}{l}\text { Pollutens } \\
\text { and } \\
\text { CAS Number } \\
\text { (if availiblo) }\end{array}$} & \multicolumn{2}{|c|}{$\begin{array}{l}\text { Maximumon Valuea } \\
\text { (inchade unitu) }\end{array}$} & \multicolumn{2}{|c|}{$\begin{array}{l}\text { Average Values } \\
\text { (inchude units) }\end{array}$} & \multirow{2}{*}{$\begin{array}{c}\text { Number } \\
\text { of } \\
\text { Stom } \\
\text { Eveats } \\
\text { Sempled }\end{array}$} & \multirow[b]{2}{*}{ Sources of Pollutunts } \\
\hline & $\begin{array}{l}\text { Grab Sumplo } \\
\text { Takea Duriog } \\
\text { Firat } 30 \\
\text { Minutea }\end{array}$ & $\begin{array}{l}\text { Fow weightod } \\
\text { Componise }\end{array}$ & $\begin{array}{l}\text { Grab Sumple } \\
\text { Takea During } \\
\text { Firat } 30 \\
\text { Minute }\end{array}$ & $\begin{array}{c}\text { Fow-weightod } \\
\text { Componito } \\
.\end{array}$ & & \\
\hline Barium 7400-39-3 & $0.0231 \mathrm{mg} / \mathrm{l}$ & $0.0192 \mathrm{mg} / 1$ & $0.0231 \mathrm{mg} / 1$ & $0.0192 \mathrm{mg} / 1$ & 1 & \\
\hline meatyloce chloride 75-09-2 & $<0.010 \mathrm{mg} / \mathrm{l}$ & & $<0.010 \mathrm{mg} / \mathrm{l}$ & & 1 & \\
\hline 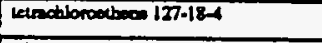 & $<0.010 \mathrm{mg} / 1$ & & $<0.010 \mathrm{mg} / \mathrm{l}$ & & 1 & \\
\hline Inictsonedbeos 7901.6 & $<0.010 \mathrm{mg} / \mathrm{l}$ & & $<0.010 \mathrm{mg} / \mathrm{l}$ & & 1 & \\
\hline Xylowed & $<0.010 \mathrm{mg} / 1$ & & $<0.010 \mathrm{mg} / \mathrm{l}$ & & 1 & \\
\hline 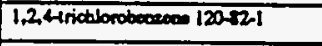 & $<0.010 \mathrm{mg} / \mathrm{l}$ & $<0.010 \mathrm{mg} / \mathrm{l}$ & $<0.010 \mathrm{mg} / 1$ & $<0.010 \mathrm{mg} / \mathrm{l}$ & 1 & \\
\hline 1.2-dichlorobomecos 45.30-1 & $<0.010 \mathrm{mg} / \mathrm{l}$ & $<0.010 \mathrm{mg} / \mathrm{l}$ & $<0.010 \mathrm{mg} / 1$ & $<0.010 \mathrm{mg} / \mathrm{l}$ & 1 & \\
\hline 1,3-dictionoborecose 541-73-1 & $<0.010 \mathrm{mg} / \mathrm{l}$ & $<0.010 \mathrm{mg} / \mathrm{l}$ & $<0.010 \mathrm{mg} / 1$ & $<0.010 \mathrm{mg} / 1$ & 1 & \\
\hline 1.4dictionoberenes $100-16-7$ & $<0.010 \mathrm{mg} / \mathrm{l}$ & $<0.010 \mathrm{mg} / \mathrm{l}$ & $<0.010 \mathrm{mg} / 1$ & $<0.010 \mathrm{mg} / \mathrm{l}$ & 1 & \\
\hline 24 dinitrocoturees $|2| \cdot 14-2$ & $<0.010 \mathrm{mg} / \mathrm{l}$ & $<0.010 \mathrm{mg} / \mathrm{l}$ & $<0.010 \mathrm{mg} / \mathrm{l}$ & $<0.010 \mathrm{mg} / \mathrm{l}$ & 1 & \\
\hline 2.6dinitrodoluseos $606-20-2$ & $<0.010 \mathrm{mg} / \mathrm{l}$ & $<0.010 \mathrm{mg} / 1$ & $<0.010 \mathrm{mg} / \mathrm{l}$ & $<0.010 \mathrm{mg} / \mathrm{l}$ & 1 & \\
\hline 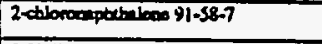 & $<0.010 \mathrm{mg} / \mathrm{l}$ & $<0.010 \mathrm{mg} / \mathrm{l}$ & $<0.010 \mathrm{mg} / 1$ & $<0.010 \mathrm{mg} / \mathrm{l}$ & $1^{\circ}$ & \\
\hline 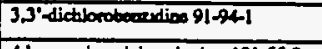 & $<0.010 \mathrm{mg} / \mathrm{l}$ & $<0.010 \mathrm{mg} / \mathrm{l}$ & $<0.010 \mathrm{mg} / \mathrm{l}$ & $<0.010 \mathrm{mg} / \mathrm{l}$ & 1 & \\
\hline 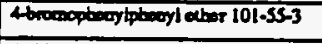 & $<0.010 \mathrm{mg} / 1$ & $<0.010 \mathrm{mg} / 1$ & $<0.010 \mathrm{mg} / 1$ & $<0.010 \mathrm{mg} / 1$ & 1 & \\
\hline 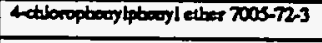 & $<0.010 \mathrm{mg} / 1$ & $<0.010 \mathrm{mg} / \mathrm{l}$ & $<0.010 \mathrm{mg} / 1$ & $<0.010 \mathrm{mg} / \mathrm{l}$ & 1 & \\
\hline 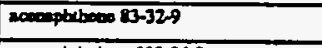 & $<0.010 \mathrm{mg} / \mathrm{l}$ & $<0.010 \mathrm{mg} / \mathrm{l}$ & $<0.010 \mathrm{mg} / \mathrm{l}$ & $<0.010 \mathrm{mg} / \mathrm{l}$ & 1 & \\
\hline 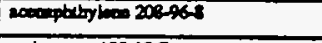 & $<0.010 \mathrm{mg} / \mathrm{l}$ & $<0.010 \mathrm{mg} / \mathrm{l}$ & $<0.010 \mathrm{mg} / 1$ & $<0.010 \mathrm{mg} / \mathrm{l}$ & 1 & \\
\hline antrocose $120-12.7$ & $<0.010 \mathrm{mg} / 1$ & $<0.010 \mathrm{mg} / 1$ & $<0.010 \mathrm{mg} / \mathrm{l}$ & $<0.010 \mathrm{mg} / \mathrm{l}$ & 1 & \\
\hline 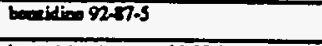 & $<0.030 \mathrm{mg} / \mathrm{l}$ & $<0.030 \mathrm{mg} / \mathrm{l}$ & $<0.030 \mathrm{mg} / \mathrm{l}$ & $<0.030 \mathrm{mg} / \mathrm{l}$ & 1 & \\
\hline 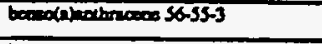 & $<0.010 \mathrm{mg} / \mathrm{l}$ & $<0.010 \mathrm{mg} / \mathrm{l}$ & $<0.010 \mathrm{mg} / \mathrm{l}$ & $<0.010 \mathrm{mg} / \mathrm{l}$ & 1 & \\
\hline 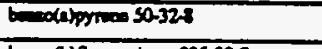 & $<0.010 \mathrm{mg} / \mathrm{l}$ & $<0.010 \mathrm{mg} / \mathrm{l}$ & $<0.010 \mathrm{mg} / \mathrm{l}$ & $<0.010 \mathrm{mg} / 1$ & 1 & \\
\hline 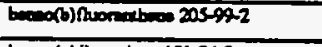 & $<0.010 \mathrm{mg} / \mathrm{l}$ & $<0.010 \mathrm{mg} / \mathrm{l}$ & $<0.010 \mathrm{mg} / 1$ & $<0.010 \mathrm{mg} / \mathrm{l}$ & 1 & \\
\hline beopodbi)peryloce $191-242$ & $<0.010 \mathrm{mg} / \mathrm{l}$ & $<0.010 \mathrm{mg} / \mathrm{l}$ & $<0.010 \mathrm{mg} / \mathrm{l}$ & $<0.010 \mathrm{mg} / \mathrm{l}$ & 1 & \\
\hline 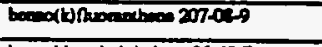 & $<0.010 \mathrm{mg} / \mathrm{l}$ & $<0.010 \mathrm{mg} / \mathrm{l}$ & $<0.010 \mathrm{mg} / 1$ & $<0.010 \mathrm{mg} / \mathrm{I}$ & 1 & \\
\hline 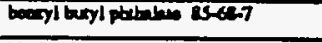 & $<0.010 \mathrm{mg} / 1$ & $<0.010 \mathrm{mg} / \mathrm{l}$ & $<0.010 \mathrm{mg} / \mathrm{l}$ & $<0.010 \mathrm{mg} / \mathrm{l}$ & 1 & \\
\hline 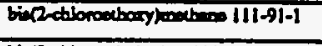 & $<0.010 \mathrm{mg} / \mathrm{l}$ & $<0.010 \mathrm{mg} / \mathrm{l}$ & $<0.010 \mathrm{mg} / \mathrm{l}$ & $<0.010 \mathrm{mg} / \mathrm{l}$ & 1 & $\because$ \\
\hline 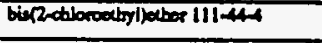 & $<0.010 \mathrm{mg} / \mathrm{ll}$ & $<0.010 \mathrm{mg} / \mathrm{l}$ & $<0.010 \mathrm{mg} / 1$ & $<0.010 \mathrm{mg} / \mathrm{I}$ & 1 & \\
\hline 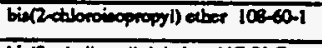 & $<0.010 \mathrm{mg} / \mathrm{I}$ & $<0.010 \mathrm{mg} / \mathrm{l}$ & $<0.010 \mathrm{mg} / \mathrm{l}$ & $<0.010 \mathrm{mg} / \mathrm{I}$ & 1 & \\
\hline 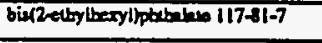 & $<0.010 \mathrm{mg} / \mathrm{l}$ & $<0.010 \mathrm{mg} / 1$ & $<0.010 \mathrm{mg} / \mathrm{l}$ & $<0.010 \mathrm{mg} / \mathrm{l}$ & 1 & \\
\hline chorouces $218-01-9$ & $<0.010 \mathrm{mg} / \mathrm{l}$ & $<0.010 \mathrm{mg} / \mathrm{l}$ & $<0.010 \mathrm{mg} / \mathrm{l}$ & $<0.010 \mathrm{mg} / \mathrm{l}$ & 1 & \\
\hline ditoturyl pablestes 84-74-2 & $<0.010 \mathrm{mg} / \mathrm{l}$ & $<0.010 \mathrm{mg} / \mathrm{l}$ & $<0.010 \mathrm{mg} / \mathrm{l}$ & $<0.010 \mathrm{mg} / \mathrm{l}$ & 1 & \\
\hline 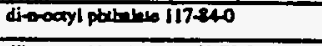 & $<0.010 \mathrm{mg} / \mathrm{l}$ & $<0.010 \mathrm{mg} / \mathrm{l}$ & $<0.010 \mathrm{mg} / \mathrm{l}$ & $<0.010 \mathrm{mg} / \mathrm{l}$ & 1 & \\
\hline 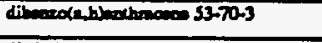 & $<0.010 \mathrm{mg} / \mathrm{l}$ & $<0.010 \mathrm{mg} / \mathrm{l}$ & $<0.010 \mathrm{mg} / \mathrm{l}$ & $<0.010 \mathrm{mg} / 1$ & 1 & \\
\hline dieatyl pathelato 84-66-2 & $<0.010 \mathrm{mg} / \mathrm{l}$ & $<0.010 \mathrm{mg} / \mathrm{l}$ & $<0.010 \mathrm{mg} / 1$ & $<0.010 \mathrm{mg} / \mathrm{l}$ & 1 & \\
\hline 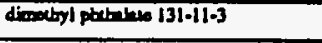 & $<0.010 \mathrm{mg} / \mathrm{l}$ & $<0.010 \mathrm{mg} / \mathrm{l}$ & $<0.010 \mathrm{mg} / \mathrm{l}$ & $<0.010 \mathrm{mg} / 1$ & 1 & \\
\hline Theosentheos $206-40$ & $<0.010 \mathrm{mg} / 1$ & $<0.010 \mathrm{mg} / \mathrm{l}$ & $<0.010 \mathrm{mg} / \mathrm{l}$ & $<0.010 \mathrm{mg} / 1$ & 1 & \\
\hline Nuonowe 26-73-7 & $<0.010 \mathrm{mg} / \mathrm{l}$ & $<0.010 \mathrm{mg} / \mathrm{l}$ & $<0.010 \mathrm{mg} / 1$ & $<0.010 \mathrm{mg} / \mathrm{l}$ & 1 & \\
\hline 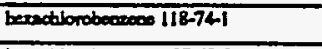 & $<0.010 \mathrm{mg} / \mathrm{l}$ & $<0.010 \mathrm{mg} / \mathrm{l}$ & $<0.010 \mathrm{mg} / 1$ & $<0.010 \mathrm{mg} / \mathrm{l}$ & 1 & \\
\hline 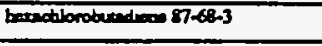 & $<0.010 \mathrm{mg} / \mathrm{l}$ & $<0.010 \mathrm{mg} / \mathrm{l}$ & $<0.010 \mathrm{mg} / \mathrm{l}$ & $<0.010 \mathrm{mg} / \mathrm{l}$ & 1 & \\
\hline 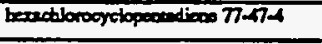 & $<0.010 \mathrm{mg} / \mathrm{l}$ & $<0.010 \mathrm{mg} / \mathrm{l}$ & $<0.010 \mathrm{mg} / \mathrm{l}$ & $<0.010 \mathrm{mg} / 1$ & 1 & \\
\hline 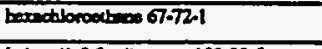 & $<0.010 \mathrm{mg} / 1$ & $<0.010 \mathrm{mg} / \mathrm{l}$ & $<0.010 \mathrm{mg} / \mathrm{l}$ & $<0.010 \mathrm{mg} / \mathrm{l}$ & 1 & \\
\hline 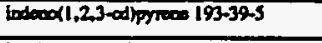 & $<0.010 \mathrm{mg} / \mathrm{I}$ & $<0.010 \mathrm{mg} / \mathrm{l}$ & $<0.010 \mathrm{mg} / \mathrm{l}$ & $<0.010 \mathrm{mg} / \mathrm{l}$ & 1 & \\
\hline inopteroos 72-59-1 & $<0.010 \mathrm{mg} / 1$ & $<0.010 \mathrm{mg} / \mathrm{I}$ & $<0.010 \mathrm{mg} / \mathrm{l}$ & $<0.010 \mathrm{mg} / 1$ & 1 & \\
\hline Nedilrowodj-e-propylemino $621-647$ & $<0.010 \mathrm{mg} / \mathrm{l}$ & $<0.010 \mathrm{mg} / \mathrm{l}$ & $<0.010 \mathrm{mg} / \mathrm{l}$ & $<0.010 \mathrm{mg} / \mathrm{l}$ & 1 & \\
\hline$N$ - throbodimaty bemino 62.759 & $<0.010 \mathrm{mg} / \mathrm{l}$ & $<0.010 \mathrm{mg} / \mathrm{l}$ & $<0.010 \mathrm{mg} / \mathrm{l}$ & $<0.010 \mathrm{mg} / \mathrm{l}$ & 1 & \\
\hline Nalitroodipteogleming 26-30-6 & $<0.010 \mathrm{mg} / \mathrm{l}$ & $<0.010 \mathrm{mg} / 1$ & $<0.010 \mathrm{mg} / \mathrm{l}$ & $<0.010 \mathrm{mg} / 1$ & 1 & \\
\hline noptetadoon $91-20-3$ & $<0.010 \mathrm{mg} / \mathrm{l}$ & $<0.010 \mathrm{mg} / \mathrm{l}$ & $<0.010 \mathrm{mg} / \mathrm{L}$ & $<0.010 \mathrm{mg} / 1$ & 1 & \\
\hline Dinobanemen $96-95.3$ & $<0.010 \mathrm{mg} / 1$ & $<0.010 \mathrm{mg} / \mathrm{l}$ & $<0.010 \mathrm{mg} / \mathrm{l}$ & $<0.010 \mathrm{mg} / 1$ & 1 & \\
\hline 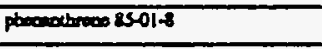 & $<0.010 \mathrm{mg} / \mathrm{l}$ & $<0.010 \mathrm{mg} / 1$ & $<0.010 \mathrm{mg} / \mathrm{l}$ & $<0.010 \mathrm{mg} / 1$ & 1 & \\
\hline primos 1220000 & $<0.010 \mathrm{mg} / \mathrm{l}$ & $<0.010 \mathrm{mg} / \mathrm{l}$ & $<0.010 \mathrm{mg} / \mathrm{l}$ & $<0.010 \mathrm{mg} / \mathrm{l}$ & 1 & \\
\hline 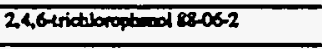 & $<0.030 \mathrm{mg} / \mathrm{l}$ & $<0.030 \mathrm{mg} / 1$ & $<0.030 \mathrm{mg} / \mathrm{l}$ & $<0.030 \mathrm{mg} / \mathrm{l}$ & 1 & \\
\hline 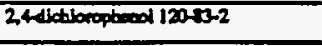 & $<0.030 \mathrm{mg} / \mathrm{l}$ & $<0.030 \mathrm{mg} / \mathrm{l}$ & $<0.030 \mathrm{mg} / \mathrm{l}$ & $<0.030 \mathrm{mg} / \mathrm{l}$ & 1 & \\
\hline 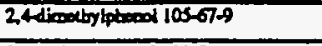 & $<0.030 \mathrm{mg} / \mathrm{l}$ & $<0.030 \mathrm{mg} / 1$ & $<0.030 \mathrm{mg} / \mathrm{l}$ & $<0.030 \mathrm{mg} / \mathrm{l}$ & 1 & \\
\hline 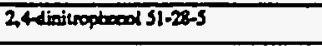 & $<0.030 \mathrm{mg} / \mathrm{l}$ & $<0.030 \mathrm{mg} / \mathrm{l}$ & $<0.030 \mathrm{mg} / \mathrm{l}$ & $<0.030 \mathrm{mg} / \mathrm{I}$ & 1 & \\
\hline
\end{tabular}


Outfall 53

Parts B and C Continued

\begin{tabular}{|c|c|c|c|c|c|c|}
\hline \multirow{2}{*}{$\begin{array}{l}\text { Polluteant } \\
\text { and } \\
\text { CAS Number } \\
\text { (if availible) }\end{array}$} & \multicolumn{2}{|c|}{$\begin{array}{l}\text { Meximouro Valuea } \\
\text { (include units) }\end{array}$} & \multicolumn{2}{|c|}{$\begin{array}{l}\text { Avernge Values } \\
\text { (inctudo unirs) }\end{array}$} & \multirow{2}{*}{$\begin{array}{c}\text { Number } \\
\text { of } \\
\text { Storm } \\
\text { Eveats } \\
\text { Seropled }\end{array}$} & \multirow[b]{2}{*}{ Sources of Pollutents } \\
\hline & $\begin{array}{l}\text { Grab Sumple } \\
\text { Taken Duriog } \\
\text { Firnt } 30 \\
\text { Minure }\end{array}$ & $\begin{array}{l}\text { Flow woighted } \\
\text { Compotite }\end{array}$ & $\begin{array}{c}\text { Grab Semple } \\
\text { Taien During } \\
\text { "intr } 30 \\
\text { Minutes }\end{array}$ & $\begin{array}{l}\text { Fiow-weightrod } \\
\text { Composine }\end{array}$ & & \\
\hline 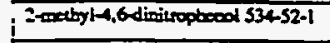 & $<0.030 \mathrm{mg} / \mathrm{l}$ & $<0.030 \mathrm{mg} / \mathrm{l}$ & $<0.030 \mathrm{mg} / \mathrm{l}$ & $<0.030 \mathrm{mg} / \mathrm{l}$ & 1 & \\
\hline 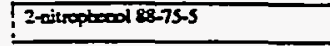 & $<0.030 \mathrm{mg} / \mathrm{l}$ & $<0.030 \mathrm{mg} / 1$ & $<0.030 \mathrm{mg} / \mathrm{l}$ & $<0.030 \mathrm{mg} / \mathrm{l}$ & 1 & \\
\hline 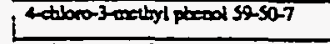 & $<0.030 \mathrm{mg} / \mathrm{l}$ & $<0.030 \mathrm{mg} / 1$ & $<0.030 \mathrm{mg} / \mathrm{l}$ & $<0.030 \mathrm{mg} / \mathrm{l}$ & 1 & \\
\hline 4 nitroptecol $100-02-7$ & $<0.030 \mathrm{mg} / \mathrm{l}$ & $<0.030 \mathrm{mg} / \mathrm{l}$ & $<0.030 \mathrm{mg} / \mathrm{l}$ & $<0.030 \mathrm{mg} / \mathrm{l}$ & 1 & \\
\hline 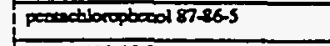 & $<0.030 \mathrm{mg} / \mathrm{l}$ & $<0.030 \mathrm{mg} / 1$ & $<0.030 \mathrm{mg} / \mathrm{l}$ & $<0.030 \mathrm{mg} / \mathrm{l}$ & 1 & \\
\hline pteen $108-95-2$ & $<0.030 \mathrm{mg} / \mathrm{l}$ & $<0.030 \mathrm{mg} / 1$ & $<0.030 \mathrm{mg} / \mathrm{l}$ & $<0.030 \mathrm{mg} / 1$ & 1 & \\
\hline $4.4^{\circ}-0 D D 72.548$ & $<0.00001 \mathrm{mg} / \mathrm{l}$ & $1<0.00001 \mathrm{mg} / \mathrm{l}$ & $<0.00001 \mathrm{mg} / 1$ & $<0.00001 \mathrm{mg} / \mathrm{l}$ & 1 & \\
\hline 4.4-DDE 72-55-9 & $<0.00001 \mathrm{mg} / \mathrm{l}$ & $<0.00001 \mathrm{mg} / \mathrm{l}$ & $<0.00001 \mathrm{mg} / \mathrm{l}$ & $<0.00001 \mathrm{mg} / \mathrm{l}$ & 1 & \\
\hline $4.4^{\prime}-\mathrm{DDT} 50-29-3$ & $<0.00001 \mathrm{mg} / \mathrm{l}$ & $<0.00001 \mathrm{mg} / 1$ & $<0.00001 \mathrm{mg} / \mathrm{l}$ & $<0.00001 \mathrm{mg} / \mathrm{l}$ & 1 & \\
\hline atdrin 302-00-2 & $<0.00001 \mathrm{mg} / \mathrm{l}$ & $<0.00001 \mathrm{mg} / \mathrm{l}$ & $<0.00001 \mathrm{mg} / 1$ & $<0.00001 \mathrm{mg} / \mathrm{l}$ & 1 & \\
\hline 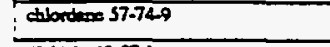 & $<0.00002 \mathrm{mg} / \mathrm{l}$ & $<0.00002 \mathrm{mg} / \mathrm{l}$ & $<0.00002 \mathrm{mg} / \mathrm{l}$ & $<0.00002 \mathrm{mg} / \mathrm{l}$ & 1 & \\
\hline dixldrin 60-57.1 & $<0.00001 \mathrm{mg} / \mathrm{l}$ & $<0.00001 \mathrm{mg} / 1$ & $<0.00001 \mathrm{mg} / \mathrm{l}$ & $<0.00001 \mathrm{mg} / \mathrm{l}$ & 1 & $\cdot$ \\
\hline 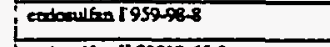 & $<0.00001 \mathrm{mg} / \mathrm{l}$ & $<0.00001 \mathrm{mg} / \mathrm{l}$ & $<0.00001 \mathrm{mg} / \mathrm{l}$ & $<0.00001 \mathrm{mg} / \mathrm{l}$ & 1 & \\
\hline 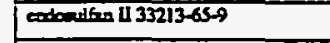 & $<0.00001 \mathrm{mg} / \mathrm{l}$ & $<0.00001 \mathrm{mg} / \mathrm{l}$ & $<0.00001 \mathrm{mg} / 1$ & $<0.00001 \mathrm{mg} / \mathrm{l}$ & 1 & \\
\hline 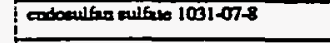 & $<0.00001 \mathrm{mg} / \mathrm{l}$ & $<0.00001 \mathrm{mg} / 1$ & $<0.00001 \mathrm{mg} / \mathrm{l}$ & $<0.00001 \mathrm{mg} / \mathrm{l}$ & 1 & \\
\hline $\cos 2 \pi 2.20-8$ & $<0.00005 \mathrm{mg} / \mathrm{l}$ & $<0.00005 \mathrm{mg} / \mathrm{l}$ & $<0.00005 \mathrm{mg} / \mathrm{l}$ & $<0.00005 \mathrm{mg} / \mathrm{l}$ & 1 & \\
\hline edrinabetyde 7421-934-4 & $<0.00001 \mathrm{mg} / \mathrm{l}$ & $<0.00001 \mathrm{mg} / \mathrm{l}$ & $<0.00001 \mathrm{mg} / \mathrm{l}$ & $<0.00001 \mathrm{mg} / \mathrm{l}$ & 1 & \\
\hline topaction $76-4-8$ & $<0.00001 \mathrm{mg} / 1$ & $<0.00001 \mathrm{mg} / !$ & $<0.00001 \mathrm{mg} / \mathrm{l}$ & $<0.00001 \mathrm{mg} / \mathrm{l}$ & 1 & \\
\hline 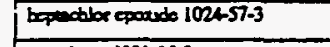 & $<0.00001 \mathrm{mg} / \mathrm{l}$ & $<0.00001 \mathrm{mg} / \mathrm{l}$ & $<0.00001 \mathrm{mg} / \mathrm{l}$ & $<0.00001 \mathrm{mg} / \mathrm{l}$ & 1 & \\
\hline $1000+600001-35-2$ & $<0.0002 \mathrm{mg} / \mathrm{l}$ & $<0.0002 \mathrm{mg} / 1$ & $<0.0002 \mathrm{mg} / \mathrm{l}$ & $<0.0002 \mathrm{mg} / \mathrm{l}$ & 1 & \\
\hline $246-8$ मC 319246 & $<0.00001 \mathrm{mg} / 1$ & $<0.00001 \mathrm{mg} / \mathrm{l}$ & $<0.00001 \mathrm{mg} / \mathrm{l}$ & $<0.00001 \mathrm{mg} / \mathrm{l}$ & 1 & \\
\hline - 8 HC $319-25-7$ & $<0.00001 \mathrm{mg} / 1$ & $<0.00001 \mathrm{mg} / \mathrm{l}$ & $<0.00001 \mathrm{mg} / \mathrm{l}$ & $<0.00001 \mathrm{mg} / \mathrm{l}$ & 1 & \\
\hline dele-BHC 319868 & $<0.00001 \mathrm{mg} / \mathrm{l}$ & $<0.00001 \mathrm{mg} / \mathrm{l}$ & $<0.00001 \mathrm{mg} / \mathrm{l}$ & $<0.00001 \mathrm{mg} / 1$ & 1 & \\
\hline $0-8 H C 58.898$ & $<0.00001 \mathrm{mg} / \mathrm{l}$ & $<0.00001 \mathrm{mg} / \mathrm{l}$ & $<0.00001 \mathrm{mg} / \mathrm{l}$ & $<0.00001 \mathrm{mg} / \mathrm{l}$ & 1 & \\
\hline 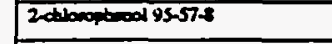 & $<0.030 \mathrm{mg} / \mathrm{l}$ & $<0.030 \mathrm{mg} / \mathrm{h}$ & $<0.030 \mathrm{mg} / \mathrm{l}$ & $<0.030 \mathrm{mg} / \mathrm{l}$ & 1 & \\
\hline Arodtar-1212 S366-21-9 & $<0.0005 \mathrm{mg} / \mathrm{l}$ & $<0.0005 \mathrm{mg} / \mathrm{l}$ & $<0.0005 \mathrm{mg} / 1$ & $<0.0005 \mathrm{mg} / \mathrm{l}$ & 1 & $\because$ \\
\hline Arodor-1254 $11097-69-1$ & $<0.0005 \mathrm{mg} / 1$ & $<0.0005 \mathrm{mg} / \mathrm{l}$ & $<0.0005 \mathrm{mg} / 1$ & $<0.0005 \mathrm{mg} / \mathrm{l}$ & 1 & \\
\hline Aroctor-1221 11104-28-2 & $<0.0005 \mathrm{mg} / \mathrm{l}$ & $<0.0005 \mathrm{mg} / \mathrm{l}$ & $<0.0005 \mathrm{mg} / \mathrm{l}$ & $<0.0005 \mathrm{mg} / \mathrm{l}$ & 1 & \\
\hline Aroctor $123211141 \cdot 16-5$ & $<0.0005 \mathrm{mg} / \mathrm{l}$ & $<0.0005 \mathrm{mg} / \mathrm{l}$ & $<0.0005 \mathrm{mg} / \mathrm{l}$ & $<0.0005 \mathrm{mg} / \mathrm{l}$ & 1 & \\
\hline Aroctor-1248 12672-29-6 & $<0.0005 \mathrm{mg} / \mathrm{l}$ & $<0.0005 \mathrm{mg} / \mathrm{l}$ & $<0.0005 \mathrm{mg} / \mathrm{l}$ & $<0.0005 \mathrm{mg} / \mathrm{l}$ & 1 & \\
\hline Aroctor-1260 $11096682-5$ & $<0.0005 \mathrm{mg} / \mathrm{l}$ & $<0.0005 \mathrm{mg} / 1$ & $<0.0005 \mathrm{mg} / \mathrm{l}$ & $<0.0005 \mathrm{mg} / \mathrm{l}$ & 1 & \\
\hline Arodor-1016 1264-11-2 & $<0.0005 \mathrm{mg} / \mathrm{l}$ & $<0.0005 \mathrm{mg} / \mathrm{l}$ & $<0.0005 \mathrm{mg} / \mathrm{l}$ & $<0.0005 \mathrm{mg} / \mathrm{l}$ & 1 & \\
\hline Croote & $<0.030 \mathrm{mg} / \mathrm{l}$ & $<0.030 \mathrm{mg} / \mathrm{l}$ & $<0.030 \mathrm{mg} / \mathrm{l}$ & $<0.030 \mathrm{mg} / \mathrm{l}$ & 1 & \\
\hline Acolvia & $<0.010 \mathrm{mg} / \mathrm{l}$ & $<0.010 \mathrm{mg} / 1$ & $<0.010 \mathrm{mg} / \mathrm{l}$ & $<0.010 \mathrm{mg} / \mathrm{l}$ & 1 & \\
\hline Acryboitrite & $<0.010 \mathrm{mg} / 1$ & $<0.010 \mathrm{mg} / \mathrm{I}$ & $<0.010 \mathrm{mg} / \mathrm{l}$ & $<0.010 \mathrm{mg} / \mathrm{l}$ & 1 & \\
\hline 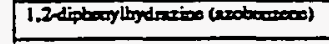 & Results pending & Results pending & Results pending & Results pending & 1 & \\
\hline & & & & & & \\
\hline & & & & & & \\
\hline & & & & & & \\
\hline & & & & & & \\
\hline & & & & & & \\
\hline & & & & & & \\
\hline & & & & & & \\
\hline & & & & & & \\
\hline & & & & & 7 & \\
\hline & & & & & & \\
\hline & & & & & & \\
\hline & & & & & 1 & \\
\hline & & & & & & \\
\hline & & & & & & \\
\hline & & - & & & & . \\
\hline
\end{tabular}


VI. Discharge Loformation (continuad from page 3 of Form 2A

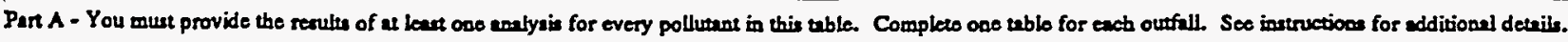

\begin{tabular}{|c|c|c|c|c|c|c|}
\hline \multirow{2}{*}{$\begin{array}{l}\text { Pollutent } \\
\text { and } \\
\text { CAs Number } \\
\text { (if aveilible) }\end{array}$} & \multicolumn{2}{|c|}{$\begin{array}{l}\text { Maximum Values } \\
\text { (include unita) }\end{array}$} & \multicolumn{2}{|c|}{$\begin{array}{l}\text { Avernge Valuce } \\
\text { (inchede unitu) }\end{array}$} & \multirow{2}{*}{$\begin{array}{c}\text { Numbor } \\
\text { of } \\
\text { Storm } \\
\text { Eveat } \\
\text { Semoled }\end{array}$} & \multirow[b]{2}{*}{ Sources of Pollhutens } \\
\hline & $\begin{array}{l}\text { Grab Stomple } \\
\text { Takea During } \\
\text { Firse } 30 \\
\text { Minues }\end{array}$ & $\begin{array}{l}\text { Flow-weightod } \\
\text { Componice }\end{array}$ & $\begin{array}{l}\text { Grab Sample } \\
\text { Taken Duriag } \\
\text { Fura } 30 \\
\text { Minutea }\end{array}$ & $\begin{array}{l}\text { Flow-weightiod } \\
\text { Compocito }\end{array}$ & & \\
\hline Dil end Grans & $<2 \mathrm{mg} / 1$ & & $<2 \mathrm{mg} / \mathrm{l}$ & & 1 & \\
\hline Biolopial Oxyecon Deconed (BODS) & $8.48 \mathrm{mg} / \mathrm{l}$ & $5.56 \mathrm{mg} / \mathrm{l}$ & $8.48 \mathrm{mg} / \mathrm{l}$ & $5.56 \mathrm{mg} / 1$ & 1 & \\
\hline Chemial Oxyena Derread (COD) & $45 \mathrm{mg} / \mathrm{l}$ & $34 \mathrm{mg} / \mathrm{l}$ & $45 \mathrm{mg} / 1$ & $34 \mathrm{mg} / 1$ & 1 & \\
\hline Toeal Serpeoded Solide (TSS) & $120 \mathrm{mg} / \mathrm{l}$ & $72 \mathrm{mg} / \mathrm{l}$ & $120 \mathrm{mg} / \mathrm{l}$ & $72 \mathrm{mg} / \mathrm{l}$ & 1 & \\
\hline Toal Kjelatal Niluoper & $0.6 \mathrm{mg} / \mathrm{l}$ & $0.4 \mathrm{mg} / \mathrm{l}$ & $0.6 \mathrm{mg} / \mathrm{l}$ & $0.4 \mathrm{mg} / 1$ & 1 & \\
\hline Nirnes plue Nitrise Nitrogen & $0.51 \mathrm{mg} / \mathrm{I}$ & $\longdiv { 0 . 3 5 \mathrm { mg } / \mathrm { l } }$ & $0.51 \mathrm{mg} / 1$ & $0.35 \mathrm{mg} / \mathrm{h}$ & 1 & \\
\hline Toul Phooptonse & $1.4 \mathrm{mg} / \mathrm{l}$ & $0.4 \mathrm{mg} / \mathrm{l}$ & $1.4 \mathrm{mg} / \mathrm{l}$ & $0.4 \mathrm{mg} / \mathrm{l}$ & 1 & \\
\hline BH & Minimom 8.1 & Merimem 8.1 & Minimere 8.1 & Mexims 8.1 & & \\
\hline \multicolumn{7}{|c|}{ 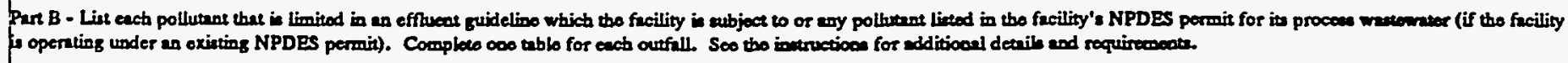 } \\
\hline \multirow{2}{*}{$\begin{array}{l}\text { Pollutent } \\
\text { and } \\
\text { CAs Number } \\
\text { (if avilibie) }\end{array}$} & \multicolumn{2}{|c|}{$\begin{array}{l}\text { Merimum Vather } \\
\text { (inoludo unitr) }\end{array}$} & \multicolumn{2}{|c|}{$\begin{array}{l}\text { Averngo Veluee } \\
\text { (inchedo emits) }\end{array}$} & \multirow{2}{*}{$\begin{array}{c}\text { Number } \\
\text { of } \\
\text { Storm } \\
\text { Evoota } \\
\text { Sempled }\end{array}$} & \multirow[b]{2}{*}{ Sources of Pollowen } \\
\hline & $\begin{array}{l}\text { Grab Semplo } \\
\text { Tuken During } \\
\text { Fint } 30 \\
\text { Misused }\end{array}$ & $\begin{array}{l}\text { Flow weighed } \\
\text { Coomporito }\end{array}$ & $\begin{array}{l}\text { Grab Semple } \\
\text { Taken During } \\
\text { Fint } 30 \\
\text { Minumes }\end{array}$ & $\begin{array}{l}\text { Flow-meighted } \\
\text { Compocies }\end{array}$ & & \\
\hline Axtimany $740-360$ & $<0.04 \mathrm{mg} / 1$ & $<0.04 \mathrm{mg} / \mathrm{l}$ & $<0.04 \mathrm{mg} / 1$ & $<0.04 \mathrm{mg} / 1$ & 1 & \\
\hline 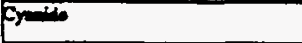 & $<0.002 \mathrm{mg} / \mathrm{l}$ & & $<0.002 \mathrm{mg} / \mathrm{l}$ & & 1 & \\
\hline Manany 7439-916 & $<0.0002 \mathrm{mg} / 1$ & $0.0003 \mathrm{mg} / \mathrm{l}$ & $<0.0002 \mathrm{mg} / \mathrm{t}$ & $0.0003 \mathrm{mg} / 1$ & 1 & \\
\hline proods & $0.002 \mathrm{mg} / \mathrm{l}$ & & $0.002 \mathrm{mg} / 1$ & & 1 & $\bar{\vdots}$ \\
\hline Soloden $778249-2$ & $<0.002 \mathrm{mg} / \mathrm{l}$ & $<0.002 \mathrm{mg} / \mathrm{h}$ & $<0.002 \mathrm{mg} / 1$ & $<0.002 \mathrm{mg} / \mathrm{l}$ & 1 & \\
\hline pulan & $<10 \mathrm{mg} / \mathrm{l}$ & $63 \mathrm{mg} / \mathrm{l}$ & $<10 \mathrm{mg} / \mathrm{l}$ & $63 \mathrm{mg} / \mathrm{l}$ & 1 & \\
\hline Purficansese & $2.5 \mathrm{mg} / \mathrm{l}$ & $<0.05 \mathrm{mg} / \mathrm{l}$ & $2.5 \mathrm{mg} / \mathrm{l}$ & $<0.05 \mathrm{mg} / \mathrm{l}$ & 1 & \\
\hline Aluminan 7429-90.3 & $4.10 \mathrm{mg} / 1$ & $4.80 \mathrm{mg} / 1$ & $4.10 \mathrm{mg} / \mathrm{l}$ & $4.80 \mathrm{mg} / \mathrm{l}$ & 1 & \\
\hline $4 x-30740-38-2$ & $<0.04 \mathrm{mg} / \mathrm{l}$ & $<0.04 \mathrm{mg} / \mathrm{l}$ & $<0.04 \mathrm{mg} / \mathrm{l}$ & $<0.04 \mathrm{mg} / 1$ & 1 & \\
\hline $300001740-128$ & $0.023 \mathrm{mg} / \mathrm{l}$ & $0.021 \mathrm{mg} / 1$ & $0.023 \mathrm{mg} / \mathrm{l}$ & $0.021 \mathrm{mg} / 1$ & 1 & \\
\hline Beryllines 740-11-7 & $<0.0004 \mathrm{mg} / \mathrm{l}$ & $<0,0004 \mathrm{mg} / \mathrm{l}$ & $<0.0004 \mathrm{mg} / \mathrm{l}$ & $<0.0004 \mathrm{mg} / \mathrm{l}$ & 1 & \\
\hline Cosming 74a-13-9 & $<0.004 \mathrm{mg} / \mathrm{l}$ & $<0.004 \mathrm{mg} / \mathrm{l}$ & $<0.004 \mathrm{mg} / 1$ & $<0.004 \mathrm{mg} / \mathrm{l}$ & 1 & \\
\hline 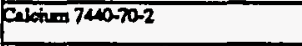 & $18.9 \mathrm{mg} / \mathrm{l}$ & $16.8 \mathrm{mg} / \mathrm{l}$ & $18.9 \mathrm{mg} / \mathrm{l}$ & $16.8 \mathrm{mg} / \mathrm{l}$ & 1 & \\
\hline Chromives 7460-7.3.3 & $<0.006 \mathrm{mg} / \mathrm{l}$ & $<0.006 \mathrm{mg} / \mathrm{l}$ & $<0.006 \mathrm{mg} / \mathrm{l}$ & $<0.006 \mathrm{mg} / \mathrm{l}$ & 1 & \\
\hline 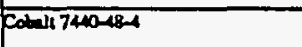 & $0.002 \mathrm{mg} / \mathrm{l}$ & $0.002 \mathrm{mg} / \mathrm{l}$ & $0.002 \mathrm{mg} / 1$ & $0.002 \mathrm{mg} / \mathrm{l}$ & 1 & \\
\hline 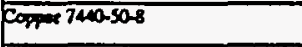 & $0.009 \mathrm{mg} / \mathrm{l}$ & $0.011 \mathrm{mg} / 1$ & $0.009 \mathrm{mg} / 1$ & $0.011 \mathrm{mg} / 1$ & 1 & \\
\hline 20067439896 & $6.62 \mathrm{mg} / \mathrm{l}$ & $7.35 \mathrm{mg} / \mathrm{l}$ & $6.62 \mathrm{mg} / 1$ & $7.35 \mathrm{mg} / \mathrm{l}$ & 1 & \\
\hline Fead $72392-1$ & $<0.02 \mathrm{mg} / \mathrm{l}$ & $<0.02 \mathrm{mg} / \mathrm{l}$ & $<0.02 \mathrm{mg} / \mathrm{l}$ & $<0.02 \mathrm{mg} / \mathrm{l}$ & 1 & \\
\hline 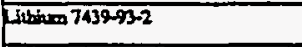 & $<0.02 \mathrm{mg} / \mathrm{l}$ & $<0.02 \mathrm{mg} / 1$ & $<0.02 \mathrm{mg} / \mathrm{l}$ & $<0.02 \mathrm{mg} / 1$ & 1 & \\
\hline Mesosion 7439-95-4 & $1.90 \mathrm{mg} / \mathrm{l}$ & $1.75 \mathrm{mg} / \mathrm{l}$ & $1.90 \mathrm{mg} / \mathrm{l}$ & $1.75 \mathrm{mg} / \mathrm{l}$ & 1 & \\
\hline Meanow0 743996-5 & $0.085 \mathrm{mg} / \mathrm{l}$ & $0.083 \mathrm{mg} / 1$ & $0.085 \mathrm{mg} / \mathrm{l}$ & $0.083 \mathrm{mg} / \mathrm{l}$ & 1 & \\
\hline roftroam 743992-7 & $<0.006 \mathrm{mg} / \mathrm{l}$ & $<0.006 \mathrm{mg} / \mathrm{l}$ & $<0.006 \mathrm{mg} / \mathrm{l}$ & $<0.006 \mathrm{mg} / 1$ & 1 & \\
\hline Nictel 1740020 & $<0.008 \mathrm{mg} / \mathrm{l}$ & $<0.008 \mathrm{mg} / 1$ & $<0.008 \mathrm{mg} / \mathrm{l}$ & $<0.008 \mathrm{mg} / \mathrm{l}$ & 1 & \\
\hline Pomaiver 7440027 & $8.4 \mathrm{mg} / \mathrm{l}$ & $6.4 \mathrm{mg} / \mathrm{l}$ & $8.4 \mathrm{mg} / \mathrm{l}$ & $6.4 \mathrm{mg} / 1$ & 1 & \\
\hline Pitwor 7440-22-4 & $<0.006 \mathrm{mg} / \mathrm{l}$ & $<0.006 \mathrm{mg} / \mathrm{l}$ & $<0.006 \mathrm{mg} / 1$ & $<0.006 \mathrm{mg} / \mathrm{l}$ & 1 & \\
\hline Sodives 740-22-3 & $1.71 \mathrm{mg} / 1$ & $0.91 \mathrm{mg} / \mathrm{l}$ & $1.71 \mathrm{mg} / 1$ & $0.91 \mathrm{mg} / \mathrm{l}$ & 1 & \\
\hline Finesiden $7440-326$ & $0.05 \mathrm{mg} / \mathrm{l}$ & $0.05 \mathrm{mg} / \mathrm{l}$ & $0.05 \mathrm{mg} / \mathrm{l}$ & $0.05 \mathrm{mg} / 1$ & 1 & \\
\hline 210074406666 & $0.15 \mathrm{mg} / \mathrm{l}$ & $0.11 \mathrm{mg} / \mathrm{l}$ & $0.15 \mathrm{mg} / \mathrm{l}$ & $0.11 \mathrm{mg} / \mathrm{l}$ & 1 & \\
\hline
\end{tabular}


Cocinoed from frow.

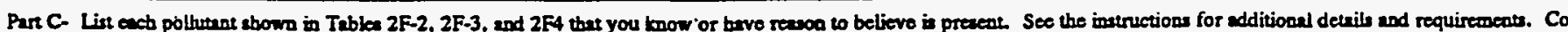
one uable for each outfall.

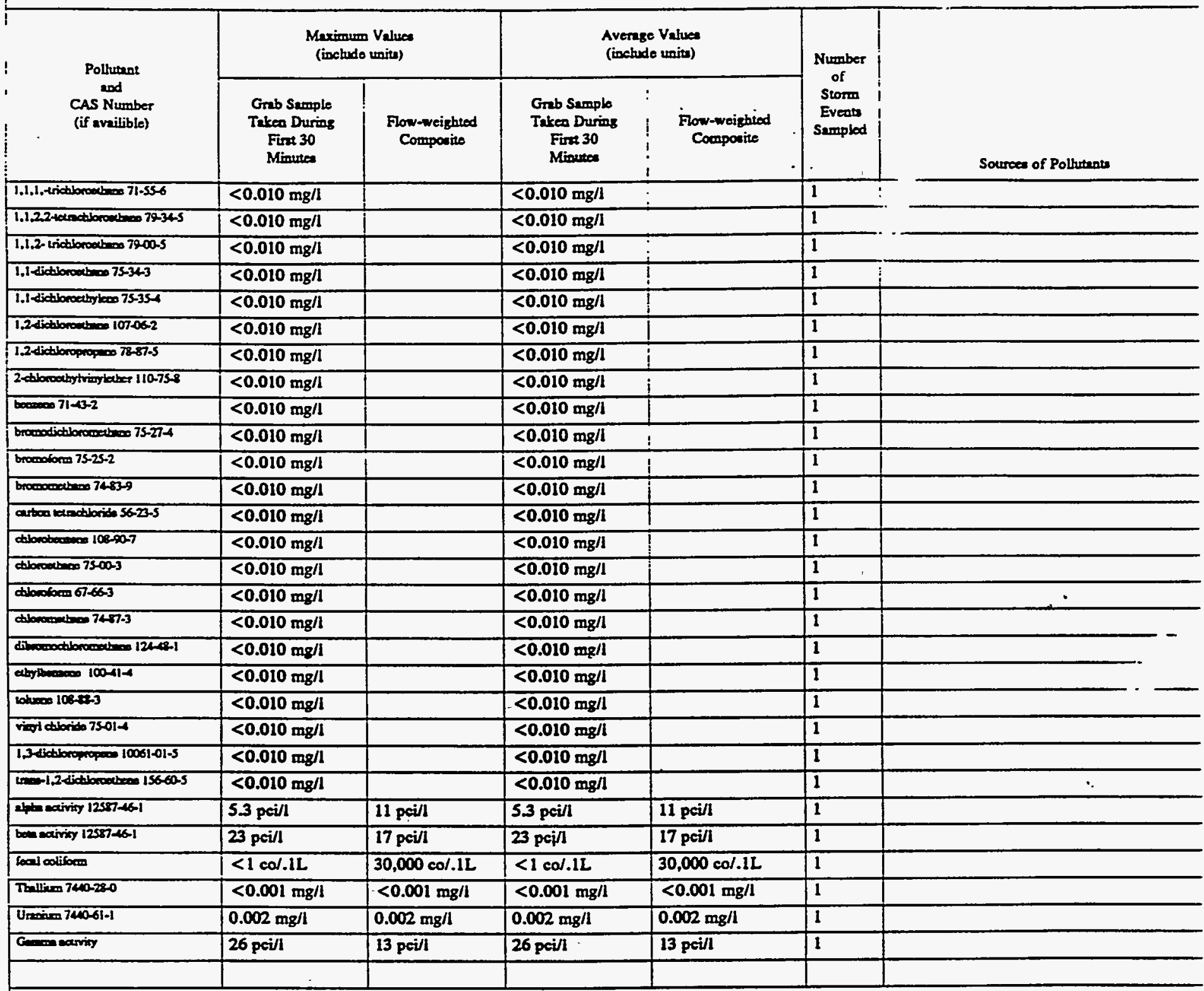

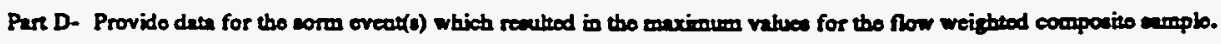

\begin{tabular}{|c|c|c|c|c|c|c|c|}
\hline $\begin{array}{l}1 . \\
\text { Dets of } \\
\text { Storm } \\
\text { Eveos }\end{array}$ & $\begin{array}{l}2 . \\
\text { Durition } \\
\text { of Storm } \\
\text { (in } \\
\text { minites) }\end{array}$ & 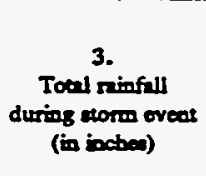 & 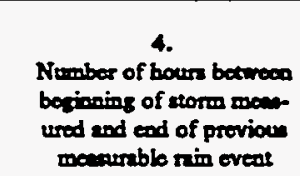 & $\begin{array}{l}\text { S. } \\
\text { Maximus flow rate } \\
\text { during nim event } \\
\text { (allowimin or epocify } \\
\text { unis) }\end{array}$ & $\begin{array}{l}6 . \\
\text { Total flow from } \\
\text { rin event } \\
\text { (enllows or } \\
\text { epecify unitr) }\end{array}$ & $\begin{array}{c}7 . \\
\text { Seasoo } \\
\text { samplo wa } \\
\text { tuken }\end{array}$ & $\begin{array}{l}8 . \\
\text { Porm of } \\
\text { Procipination } \\
\text { (niafill, } \\
\text { comenelt) }\end{array}$ \\
\hline $9 / 24 / 91$ & 365 & 1.2 & 133.5 & 15 & 5,777 & Fall & Ruinfall \\
\hline
\end{tabular}

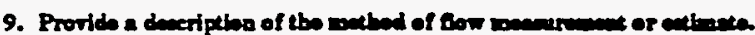

An ISCO 3230 Flow meter was nsed to obtain water level readings. This instrement was calibrated with the normal dry weather flow, if present, as leT, zero. The flow meter was programmed with the characteristics of the conveyance so that flow rates and total flows corid be calculated by the meter. 
Dutfall S4

\begin{tabular}{|c|c|c|c|c|c|c|}
\hline \multirow{2}{*}{$\begin{array}{l}\text { Pollutant } \\
\text { and } \\
\text { CAS Number } \\
\text { (if aviulible) }\end{array}$} & \multicolumn{2}{|c|}{$\begin{array}{l}\text { Maximum Valuee } \\
\text { (include units) }\end{array}$} & \multicolumn{2}{|c|}{$\begin{array}{l}\text { Avernge Vatuea } \\
\text { (imelude unitr) }\end{array}$} & \multirow{2}{*}{$\begin{array}{c}\text { Number } \\
\text { of } \\
\text { Storm } \\
\text { Evert } \\
\text { Semplod } \\
.\end{array}$} & \multirow[b]{2}{*}{ Soureces of Pollutunts } \\
\hline & $\begin{array}{c}\text { Gab Samplo } \\
\text { Takeo Durias } \\
\text { Fint } 30 \\
\text { Minurea }\end{array}$ & $\begin{array}{l}\text { Flow-weighed } \\
\text { Cormpoices }\end{array}$ & $\begin{array}{l}\text { Grab Sumple } \\
\text { Taken During } \\
\text { Firnt 30 } \\
\text { Minuet }\end{array}$ & $\begin{array}{l}\text { Flow-weightod } \\
\text { Compocits }\end{array}$ & & \\
\hline Bentiven 7440-39-3 & $0.0163 \mathrm{mg} / \mathrm{l}$ & $0.0164 \mathrm{mg} / \mathrm{l}$ & $0.0163 \mathrm{mg} / 1$ & $0.0164 \mathrm{mg} / \mathrm{l}$ & 1 & \\
\hline 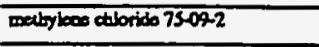 & $<0.010 \mathrm{mg} / \mathrm{l}$ & & $<0.010 \mathrm{mg} / \mathrm{l}$ & & 1 & \\
\hline Vetractionoodecos 127.184 & $<0.010 \mathrm{mg} / \mathrm{l}$ & & $<0.010 \mathrm{mg} / \mathrm{l}$ & & 1 & \\
\hline 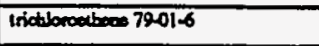 & $<0.010 \mathrm{mg} / 1$ & & $<0.010 \mathrm{mg} / 1$ & & 1 & \\
\hline Xyloosen & $<0.010 \mathrm{mg} / \mathrm{l}$ & & $<0.010 \mathrm{mg} / \mathrm{l}$ & & 1 & \\
\hline 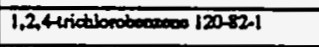 & $<0.010 \mathrm{mg} / \mathrm{l}$ & $<0.010 \mathrm{mg} / 1$ & $<0.010 \mathrm{mg} / \mathrm{l}$ & $<0.010 \mathrm{mg} / 1$ & 1 & \\
\hline 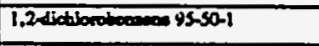 & $<0.010 \mathrm{mg} / \mathrm{l}$ & $<0.010 \mathrm{mg} / \mathrm{l}$ & $<0.010 \mathrm{mg} / \mathrm{l}$ & $<0.010 \mathrm{mg} / \mathrm{l}$ & 1 & \\
\hline 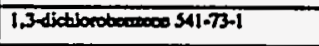 & $<0.010 \mathrm{mg} / \mathrm{l}$ & $<0.010 \mathrm{mg} / 1$ & $<0.010 \mathrm{mg} / \mathrm{l}$ & $<0.010 \mathrm{mg} / 1$ & 1 & \\
\hline 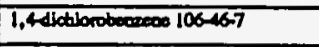 & $<0.010 \mathrm{mg} / \mathrm{l}$ & $<0.010 \mathrm{mg} / \mathrm{l}$ & $<0.010 \mathrm{mg} / \mathrm{l}$ & $<0.010 \mathrm{mg} / \mathrm{l}$ & 1 & \\
\hline 24dinivoloturese $121 \cdot 14-2$ & $<0.010 \mathrm{mg} / \mathrm{l}$ & $<0.010 \mathrm{mg} / \mathrm{l}$ & $<0.010 \mathrm{mg} / \mathrm{l}$ & $<0.010 \mathrm{mg} / 1$ & 1 & \\
\hline 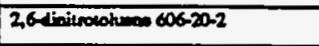 & $<0.010 \mathrm{mg} / \mathrm{l}$ & $<0.010 \mathrm{mg} / \mathrm{l}$ & $<0.010 \mathrm{mg} / \mathrm{l}$ & $<0.010 \mathrm{mg} / \mathrm{l}$ & 1 & \\
\hline 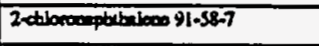 & $<0.010 \mathrm{mg} / \mathrm{l}$ & $<0.010 \mathrm{mg} / 1$ & $<0.010 \mathrm{mg} / \mathrm{l}$ & $<0.010 \mathrm{mg} / \mathrm{l}$ & 1 . & \\
\hline 3.3'dichlordonidion $91-941$ & $<0.010 \mathrm{mg} / \mathrm{l}$ & $<0.010 \mathrm{mg} / \mathrm{l}$ & $<0.010 \mathrm{mg} / \mathrm{l}$ & $<0.010 \mathrm{mg} / 1$ & 1 & \\
\hline 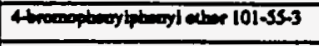 & $<0.010 \mathrm{mg} / \mathrm{l}$ & $<0.010 \mathrm{mg} / 1$ & $<0.010 \mathrm{mg} / \mathrm{l}$ & $<0.010 \mathrm{mg} / 1$ & 1 & \\
\hline 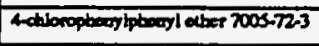 & $<0.010 \mathrm{mg} / 1$ & $<0.010 \mathrm{mg} / \mathrm{l}$ & $<0.010 \mathrm{mg} / 1$ & $<0.010 \mathrm{mg} / \mathrm{l}$ & 1 & \\
\hline 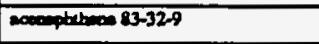 & $<0.010 \mathrm{mg} / \mathrm{l}$ & $<0.010 \mathrm{mg} / 1$ & $<0.010 \mathrm{mg} / \mathrm{l}$ & $<0.010 \mathrm{mg} / 1$ & 1 & \\
\hline 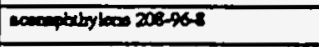 & $<0.010 \mathrm{mg} / \mathrm{l}$ & $<0.010 \mathrm{mg} / \mathrm{l}$ & $<0.010 \mathrm{mg}^{\prime / 1}$ & $<0.010 \mathrm{mg} / 1$ & 1 & \\
\hline whenowe $122-12.7$ & $<0.010 \mathrm{mg} / \mathrm{l}$ & $<0.010 \mathrm{mg} / \mathrm{l}$ & $<0.010 \mathrm{mg} / 1$ & $<0.010 \mathrm{mg} / 1$ & 1 & \\
\hline iditoro 927.5 & $<0.030 \mathrm{mg} / \mathrm{l}$ & $<0.030 \mathrm{mg} / 1$ & $<0.030 \mathrm{mg} / 1$ & $<0.030 \mathrm{mg} / \mathrm{l}$ & 1 & \\
\hline 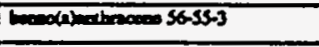 & $<0.010 \mathrm{mg} / \mathrm{l}$ & $<0.010 \mathrm{mg} / \mathrm{l}$ & $<0.010 \mathrm{mg} / \mathrm{l}$ & $<0.010 \mathrm{mg} / 1$ & 1 & \\
\hline 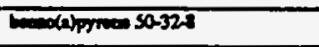 & $<0.010 \mathrm{mg} / 1$ & $<0.010 \mathrm{mg} / \mathrm{l}$ & $<0.010 \mathrm{mg} / \mathrm{l}$ & $<0.010 \mathrm{mg} / 1$ & 1 & \\
\hline 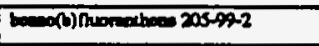 & $<0.010 \mathrm{mg} / \mathrm{l}$ & $<0.010 \mathrm{mg} / 1$ & $<0.010 \mathrm{mg} / \mathrm{l}$ & $<0.010 \mathrm{mg} / \mathrm{l}$ & 1 & \\
\hline 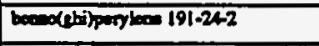 & $<0.010 \mathrm{mg} / \mathrm{l}$ & $<0.010 \mathrm{mg} / \mathrm{l}$ & $<0.010 \mathrm{mg} / \mathrm{l}$ & $<0.010 \mathrm{mg} / \mathrm{l}$ & 1 & \\
\hline 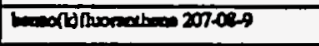 & $<0.010 \mathrm{mg} / 1$ & $<0.010 \mathrm{mg} / \mathrm{l}$ & $<0.010 \mathrm{mg} / \mathrm{l}$ & $<0.010 \mathrm{mg} / 1$ & 1 & \\
\hline 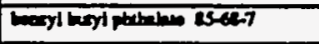 & $<0.010 \mathrm{mg} / 1$ & $<0.010 \mathrm{mg} / \mathrm{l}$ & $<0.010 \mathrm{mg} / \mathrm{l}$ & $<0.010 \mathrm{mg} / \mathrm{l}$ & 1 & \\
\hline 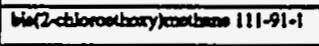 & $<0.010 \mathrm{mg} / \mathrm{l}$ & $<0.010 \mathrm{mg} / 1$ & $<0.010 \mathrm{mg} / \mathrm{l}$ & $<0.010 \mathrm{mg} / \mathrm{H}$ & 1 & $\therefore$ \\
\hline 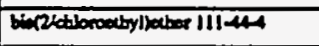 & $<0.010 \mathrm{mg} / \mathrm{l}$ & $<0.010 \mathrm{mg} / 1$ & $<0.010 \mathrm{mg} / \mathrm{l}$ & $<0.010 \mathrm{mg} / 1$ & 1 & \\
\hline 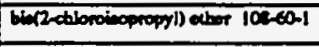 & $<0.010 \mathrm{mg} / \mathrm{l}$ & $<0.010 \mathrm{mg} / \mathrm{l}$ & $<0.010 \mathrm{mg} / \mathrm{l}$ & $<0.010 \mathrm{mg} / \mathrm{l}$ & 1 & \\
\hline 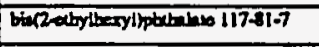 & $<0.010$ mg $/ 1$ & $<0.010 \mathrm{mg} / \mathrm{l}$ & $<0.010 \mathrm{mg} / \mathrm{l}$ & $<0.010 \mathrm{mg} / \mathrm{l}$ & 1 & \\
\hline dormas 21E0|- & $<0.010 \mathrm{mg} / \mathrm{l}$ & $<0.010 \mathrm{mg} / \mathrm{l}$ & $<0.010 \mathrm{mg} / \mathrm{l}$ & $<0.010 \mathrm{mg} / \mathrm{l}$ & 1 & \\
\hline 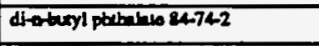 & $<0.010 \mathrm{mg} / \mathrm{l}$ & $<0.010 \mathrm{mg} / \mathrm{l}$ & $<0.010 \mathrm{mg} / \mathrm{l}$ & $<0.010 \mathrm{mg} / 1$ & 1 & \\
\hline 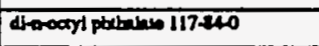 & $<0.010 \mathrm{mg} / \mathrm{l}$ & $<0.010 \mathrm{mg} / 1$ & $<0.010 \mathrm{mg} / 1$ & $<0.010 \mathrm{mg} / 1$ & 1 & \\
\hline 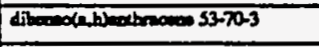 & $<0.010 \mathrm{mg} / \mathrm{l}$ & $<0.010 \mathrm{mg} / \mathrm{l}$ & $<0.010 \mathrm{mg} / \mathrm{I}$ & $<0.010 \mathrm{mg} / \mathrm{l}$ & 1 & \\
\hline 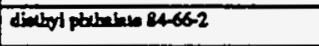 & $<0.010 \mathrm{mg} / \mathrm{l}$ & $<0.010 \mathrm{mg} / \mathrm{l}$ & $<0.010 \mathrm{mg} / \mathrm{I}$ & $<0.010 \mathrm{mg} / \mathrm{l}$ & 1 & \\
\hline 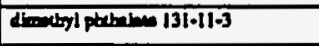 & $<0.010 \mathrm{mg} / 1$ & $<0.010 \mathrm{mg} / 1$ & $<0.010 \mathrm{mg} / 1$ & $<0.010 \mathrm{mg} / \mathrm{l}$ & 1 & \\
\hline Anowathoos 20040 & $<0.010 \mathrm{mg} / \mathrm{l}$ & $<0.010 \mathrm{mg} / \mathrm{l}$ & $<0.010 \mathrm{mg} / \mathrm{l}$ & $<0.010 \mathrm{mg} / \mathrm{l}$ & 1 & \\
\hline Theoren $26-73-7$ & $<0.010 \mathrm{mg} / 1$ & $<0.010 \mathrm{mg} / 1$ & $<0.010 \mathrm{mg} / \mathrm{l}$ & $<0.010 \mathrm{mg} / 1$ & 1 & \\
\hline 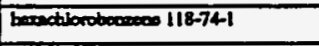 & $<0.010 \mathrm{mg} / \mathrm{l}$ & $<0.010 \mathrm{mg} / \mathrm{l}$ & $<0.010 \mathrm{mg} / \mathrm{l}$ & $<0.010 \mathrm{mg} / \mathrm{l}$ & 1 & \\
\hline 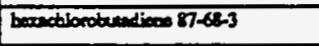 & $<0.010 \mathrm{mg} / \mathrm{l}$ & $<0.010 \mathrm{mg} / \mathrm{l}$ & $<0.010 \mathrm{mg} / \mathrm{I}$ & $<0.010 \mathrm{mg} / \mathrm{l}$ & 1 & \\
\hline 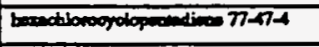 & $<0.010 \mathrm{mg} / \mathrm{l}$ & $<0.010 \mathrm{mg} / \mathrm{l}$ & $<0.010 \mathrm{mg} / 1$ & $<0.010 \mathrm{mg} / \mathrm{l}$ & 1 & \\
\hline 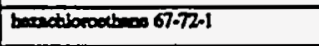 & $<0.010 \mathrm{mg} / \mathrm{l}$ & $<0.010 \mathrm{mg} / 1$ & $<0.010 \mathrm{mg} / 1$ & $<0.010 \mathrm{mg} / 1$ & 1 & \\
\hline 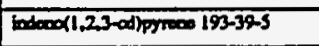 & $<0.010 \mathrm{mg} / 1$ & $<0.010 \mathrm{mg} / \mathrm{l}$ & $<0.010 \mathrm{mg} / \mathrm{l}$ & $<0.010 \mathrm{mg} / \mathrm{l}$ & 1 & \\
\hline Mophoroce 72-59-1 & $<0.010 \mathrm{mg} / \mathrm{l}$ & $<0.010 \mathrm{mg} / \mathrm{l}$ & $<0.010 \mathrm{mg} / \mathrm{l}$ & $<0.010 \mathrm{mg} / 1$ & 1 & \\
\hline 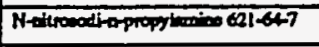 & $<0.010 \mathrm{mg} / 1$ & $<0.010 \mathrm{mg} / 1$ & $<0.010 \mathrm{mg} / \mathrm{l}$ & $<0.010 \mathrm{mg} / \mathrm{l}$ & 1 & \\
\hline 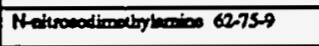 & $<0.010 \mathrm{mg} / 1$ & $<0.010 \mathrm{mg} / \mathrm{l}$ & $<0.010 \mathrm{mg} / 1$ & $<0.010 \mathrm{mg} / 1$ & 1 & \\
\hline 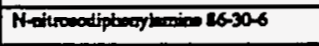 & $<0.010 \mathrm{mg} / 1$ & $<0.010 \mathrm{mg} / \mathrm{l}$ & $<0.010 \mathrm{mg} / \mathrm{l}$ & $<0.010 \mathrm{mg} / \mathrm{l}$ & 1 & \\
\hline $5+1+100091-20-3$ & $<0.010 \mathrm{mg} / 1$ & $<0.010 \mathrm{mg} / \mathrm{l}$ & $<0.010 \mathrm{mg} / 1$ & $<0.010 \mathrm{mg} / 1$ & 1 & \\
\hline 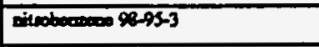 & $<0.010 \mathrm{mg} / \mathrm{l}$ & $<0.010 \mathrm{mg} / \mathrm{l}$ & $<0.010 \mathrm{mg} / 1$ & $<0.010 \mathrm{mg} / \mathrm{l}$ & 1 & \\
\hline 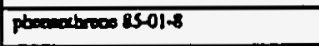 & $<0.010 \mathrm{mg} / \mathrm{l}$ & $<0.010 \mathrm{mg} / \mathrm{l}$ & $<0.010 \mathrm{mg} / \mathrm{l}$ & $<0.010 \mathrm{mg} / 1$ & 1 & \\
\hline procos 129000 & $<0.010 \mathrm{mg} / 1$ & $<0.010 \mathrm{mg} / \mathrm{l}$ & $<0.010 \mathrm{mg} / \mathrm{l}$ & $<0.010 \mathrm{mg} / \mathrm{l}$ & 1 & \\
\hline 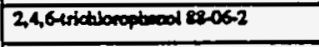 & $<0.030 \mathrm{mg} / \mathrm{l}$ & $<0.030 \mathrm{mg} / \mathrm{l}$ & $<0.030 \mathrm{mg} / \mathrm{l}$ & $<0.030 \mathrm{mg} / 1$ & 1 & \\
\hline 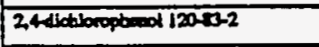 & $<0.030 \mathrm{mg} / 1$ & $<0.030 \mathrm{mg} / \mathrm{l}$ & $<0.030 \mathrm{mg} / \mathrm{l}$ & $<0.030 \mathrm{mg} / \mathrm{l}$ & 1 & \\
\hline 24dinotylyoed 105-7A & $<0.030 \mathrm{mg} / 1$ & $<0.030 \mathrm{mg} / \mathrm{h}$ & $<0.030 \mathrm{mg} / \mathrm{l}$ & $<0.030 \mathrm{mg} / \mathrm{l}$ & 1 & \\
\hline 24dinitropteond 51.226-5 & $<0.030 \mathrm{mg} / 1$ & $<0.030 \mathrm{mg} / \mathrm{l}$ & $<0.030 \mathrm{mg} / \mathrm{l}$ & $<0.030 \mathrm{mg} / \mathrm{l}$ & 1 & \\
\hline
\end{tabular}


Outfall 54

Part B and C Contrined

\begin{tabular}{|c|c|c|c|c|c|c|}
\hline \multirow{2}{*}{$\begin{array}{l}\text { Pollutant } \\
\text { iod } \\
\text { CAS Number } \\
\text { (if availible) }\end{array}$} & \multicolumn{2}{|c|}{$\begin{array}{l}\text { Maximum Values } \\
\text { (include units) }\end{array}$} & \multicolumn{2}{|c|}{$\begin{array}{l}\text { Averngo Values } \\
\text { (include units) }\end{array}$} & \multirow{2}{*}{$\begin{array}{l}\text { Number } \\
\text { of } \\
\text { Slorm } \\
\text { Eveate } \\
\text { Sempled }\end{array}$} & \multirow[b]{2}{*}{ Sources of Polluanats } \\
\hline & $\begin{array}{l}\text { Grab Srmplo } \\
\text { Traken During } \\
\text { First } 30 \\
\text { Minurea }\end{array}$ & $\begin{array}{l}\text { Flow-weighted } \\
\text { Composite }\end{array}$ & $\begin{array}{l}\text { Greb Sumple } \\
\text { Tukes During } \\
\text { First } 30 \\
\text { Minutes }\end{array}$ & $\begin{array}{l}\text { Fiow-weightiod } \\
\text { Componite }\end{array}$ & & \\
\hline 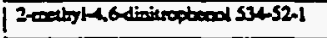 & $<0.030 \mathrm{mg} / \mathrm{l}$ & $<0.030 \mathrm{mg} / \mathrm{l}$ & $<0.030 \mathrm{mg} / \mathrm{ll}$ & $<0.030 \mathrm{mg} / \mathrm{l}$ & 1 & \\
\hline 2-aitropheod 288-75-5 & $<0.030 \mathrm{mg} / \mathrm{l}$ & $<0.030 \mathrm{mg} / 1$ & $<0.030 \mathrm{mg} / \mathrm{l}$ & $<0.030 \mathrm{mg} / \mathrm{l}$ & 1 & \\
\hline 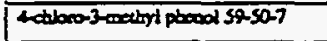 & $<0.030 \mathrm{mg} / \mathrm{l}$ & $<0.030 \mathrm{mg} / \mathrm{l}$ & $<0.030 \mathrm{mg} / \mathrm{I}$ & $<0.030 \mathrm{mg} / \mathrm{l}$ & 1 & \\
\hline Fisopted $10002-7$ & $<0.030 \mathrm{mg} / \mathrm{l}$ & $<0.030 \mathrm{mg} / \mathrm{l}$ & $<0.030 \mathrm{mg} / \mathrm{l}$ & $<0.030 \mathrm{mg} / \mathrm{l}$ & 1 & \\
\hline pecoctionopheod $8786-5$ & $<0.030 \mathrm{mg} / \mathrm{l}$ & $<0.030 \mathrm{mg} / \mathrm{l}$ & $<0.030 \mathrm{mg} / \mathrm{l}$ & $<0.030 \mathrm{mg} / \mathrm{h}$ & 1 & \\
\hline pheod $102095-2$ & $<0.030 \mathrm{mg} / \mathrm{l}$ & $<0.030 \mathrm{mg} / \mathrm{I}$ & $<0.030 \mathrm{mg} / \mathrm{l}$ & $<0.030 \mathrm{mg} / \mathrm{h}$ & 1 & \\
\hline $4.4-D D D 72-548$ & $<0.00001 \mathrm{mg} / \mathrm{l}$ & $<0.00001 \mathrm{mg} / \mathrm{l}$ & $<0.00001 \mathrm{mg} / \mathrm{l}$ & $<0.00001 \mathrm{mg} / \mathrm{l}$ & 1 & \\
\hline 4.4-DDE 72-5S-9 & $<0.00001 \mathrm{mg} / \mathrm{l}$ & $<0.00001 \mathrm{mg} / \mathrm{l}$ & $<0.00001 \mathrm{mg} / \mathrm{l}$ & $<0.00001 \mathrm{mg} / \mathrm{l}$ & 1 & \\
\hline $4,46000150-20-3$ & $<0.00001 \mathrm{mg} / \mathrm{l}$ & $<0.00001 \mathrm{mg} / \mathrm{l}$ & $<0.00001 \mathrm{mg} / \mathrm{l}$ & $<0.00001 \mathrm{mg} / \mathrm{l}$ & 1 & \\
\hline $24 \sin 30900-2$ & $<0.00001 \mathrm{mg} / \mathrm{l}$ & $<0.00001 \mathrm{mg} / 1$ & $<0.00001 \mathrm{mg} / \mathrm{l}$ & $<0.00001 \mathrm{mg} / \mathrm{l}$ & 1 & \\
\hline chlordece 57-74 & $<0.00002 \mathrm{mg} / 1$ & $<0.00002 \mathrm{mg} / \mathrm{l}$ & $<0.00002 \mathrm{mg} / 1$ & $<0.00002 \mathrm{mg} / 1$ & 1 & \\
\hline dinatria 60-57-1 & $<0.00001 \mathrm{mg} / \mathrm{l}$ & $<0.00001 \mathrm{mg} / \mathrm{l}$ & $<0.00001 \mathrm{mg} / 1$ & $<0.00001 \mathrm{mg} / \mathrm{l}$ & 1 & \\
\hline 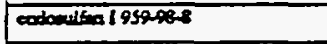 & $<0.00001 \mathrm{mg} / \mathrm{l}$ & $<0.00001 \mathrm{mg} / 1$ & $<0.00001 \mathrm{mg} / \mathrm{l}$ & $<0.00001 \mathrm{mg} / \mathrm{l}$ & 1 & \\
\hline 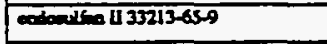 & $<0.00001 \mathrm{mg} / \mathrm{l}$ & $<0.00001 \mathrm{mg} / \mathrm{l}$ & $<0.00001 \mathrm{mg} / \mathrm{l}$ & $<0.00001 \mathrm{mg} / \mathrm{l}$ & 1 & \\
\hline 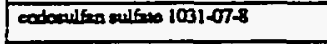 & $<0.00001 \mathrm{mg} / \mathrm{l}$ & $<0.00001 \mathrm{mg} / 1$ & $<0.00001 \mathrm{mg} / \mathrm{l}$ & $<0.00001 \mathrm{mg} / \mathrm{l}$ & 1 & \\
\hline $\cos 27^{2}-202$ & $<0.00005 \mathrm{mg} / 1$ & $<0.00005 \mathrm{mg} / \mathrm{l}$ & $<0.00005 \mathrm{mg} / \mathrm{l}$ & $<0.00005 \mathrm{mg} / \mathrm{l}$ & 1 & \\
\hline anfrie aldabyde 7/21-93-4 & $<0.00001 \mathrm{mg} / \mathrm{l}$ & $<0.00001 \mathrm{mg} / \mathrm{l}$ & $<0.00001 \mathrm{mg} / \mathrm{l}$ & $<0.00001 \mathrm{mg} / \mathrm{l}$ & 1 & \\
\hline bormotelox $76-148$ & $<0.00001 \mathrm{mg} / \mathrm{l}$ & $<0.00001 \mathrm{mg} / 1$ & $<0.00001 \mathrm{mg} / \mathrm{l}$ & $<0.00001 \mathrm{mg} / 1$ & 1 & \\
\hline 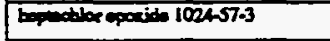 & $<0.00001 \mathrm{mg} / \mathrm{l}$ & $<0.00001 \mathrm{mg} / 1$ & $<0.00001 \mathrm{mg} / \mathrm{l}$ & $<0.00001 \mathrm{mg} / 1$ & 1 & \\
\hline 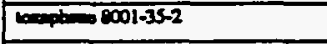 & $<0.0002 \mathrm{mg} / \mathrm{l}$ & $<0.0002 \mathrm{mg} / 1$ & $<0.0002 \mathrm{mg} / \mathrm{l}$ & $<0.0002 \mathrm{mg} / \mathrm{l}$ & 1 & \\
\hline $4+28+10319266$ & $<0.00001 \mathrm{mg} / \mathrm{l}$ & $<0.00001 \mathrm{mg} / \mathrm{l}$ & $<0.00001 \mathrm{mg} / \mathrm{l}$ & $<0.00001 \mathrm{mg} / \mathrm{I}$ & 1 & \\
\hline BD-DHC $319.95-7$ & $<0.00001 \mathrm{mg} / \mathrm{l}$ & $<0.00001 \mathrm{mg} / 1$ & $<0.00001 \mathrm{mg} / 1$ & $<0.00001 \mathrm{mg} / \mathrm{l}$ & 1 & \\
\hline de-AKC 31986 & $<0.00001 \mathrm{mg} / \mathrm{l}$ & $<0.00001 \mathrm{mg} / \mathrm{l}$ & $<0.00001 \mathrm{mg} / \mathrm{l}$ & $<0.00001 \mathrm{mg} / \mathrm{l}$ & 1 & \\
\hline 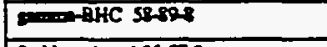 & $<0.00001 \mathrm{mg} / \mathrm{l}$ & $<0.00001 \mathrm{mg} / 1$ & $<0.00001 \mathrm{mg} / \mathrm{l}$ & $<0.00001 \mathrm{mg} / \mathrm{l}$ & 1 & \\
\hline 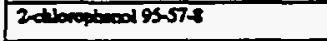 & $<0.030 \mathrm{mg} / 1$ & $<0.030 \mathrm{mg} / \mathrm{l}$ & $<0.030 \mathrm{mg} / \mathrm{l}$ & $<0.030 \mathrm{mg} / 1$ & 1 & \\
\hline Arodion-12R2 S3669-21-9 & $<0.0005 \mathrm{mg} / \mathrm{l}$ & $<0.0005 \mathrm{mg} / 1$ & $<0.0005 \mathrm{mg} / \mathrm{h}$ & $<0.0005 \mathrm{mg} / \mathrm{l}$ & 1 & \\
\hline Arodon-1234 1100769-1 & $<0.0005 \mathrm{mg} / \mathrm{l}$ & $<0.0005 \mathrm{mg} / \mathrm{l}$ & $<0.0005 \mathrm{mg} / \mathrm{h}$ & $<0.0005 \mathrm{mg} / \mathrm{l}$ & 1 & \\
\hline Aroctoriz21 1110422-2 & $<0.0005 \mathrm{mg} / \mathrm{l}$ & $<0.0005 \mathrm{mg} / \mathrm{I}$ & $<0.0005 \mathrm{mg} / \mathrm{l}$ & $<0.0005 \mathrm{mg} / 1$ & 1 & \\
\hline Aractan-123211141-16.5 & $<0.0005 \mathrm{mg} / 1$ & $<0.0005 \mathrm{mg} / \mathrm{l}$ & $<0.0005 \mathrm{mg} / \mathrm{l}$ & $<0.0005 \mathrm{mg} / \mathrm{l}$ & 1 & \\
\hline Arodor-1268 12672.296 & $<0.0005 \mathrm{mg} / \mathrm{l}$ & $<0.0005 \mathrm{mg} / \mathrm{l}$ & $<0.0005 \mathrm{mg} / \mathrm{i}$ & $<0.0005 \mathrm{mg} / 1$ & 1 & \\
\hline Arodor $126011096-125$ & $<0.0005 \mathrm{mg} / \mathrm{l}$ & $<0.0005 \mathrm{mg} / \mathrm{l}$ & $<0.0005 \mathrm{mg} / 1$ & $<0.0005 \mathrm{mg} / \mathrm{l}$ & 1 & \\
\hline Arodion $10161267411-2$ & $<0.0005 \mathrm{mg} / \mathrm{l}$ & $<0.0005 \mathrm{mg} / \mathrm{l}$ & $<0.0005 \mathrm{mg} / \mathrm{l}$ & $<0.0005 \mathrm{mg} / \mathrm{l}$ & 1 & \\
\hline Crosole & $<0.030 \mathrm{mg} / \mathrm{l}$ & $<0.030 \mathrm{mg} / \mathrm{l}$ & $<0.030 \mathrm{mg} / \mathrm{l}$ & $<0.030 \mathrm{mg} / \mathrm{l}$ & 1 & \\
\hline Aorolein & $<0.010 \mathrm{mg} / \mathrm{l}$ & $<0.010 \mathrm{mg} / \mathrm{l}$ & $<0.010 \mathrm{mg} / \mathrm{l}$ & $<0.010 \mathrm{mg} / \mathrm{l}$ & 1 & \\
\hline Aopylosinite & $<0.010 \mathrm{mg} / \mathrm{l}$ & $<0.010 \mathrm{mg} / 1$ & $<0.010 \mathrm{mg} / \mathrm{l}$ & $<0.010 \mathrm{mg} / \mathrm{l}$ & 1 & \\
\hline 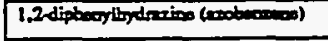 & Resulte pending & Reaults pending & Recultu pending & Recults pending & 1 & \\
\hline & & & & & & \\
\hline & & & & & & \\
\hline & & & & & & \\
\hline & & & & & & \\
\hline & & & & & & \\
\hline & & & & & & \\
\hline & & & & & & \\
\hline & & & & & & \\
\hline & & & & & & \\
\hline & & & & & & \\
\hline & & & & & & \\
\hline & & & & & & \\
\hline & & & & & & \\
\hline & & & & & & \\
\hline & & & & & & \\
\hline
\end{tabular}


Va. Discherge Informetion (continuad from paye 3 of Form 2F)

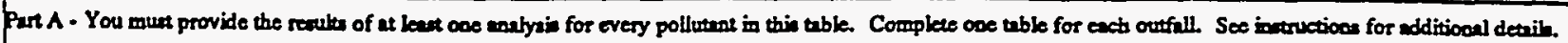

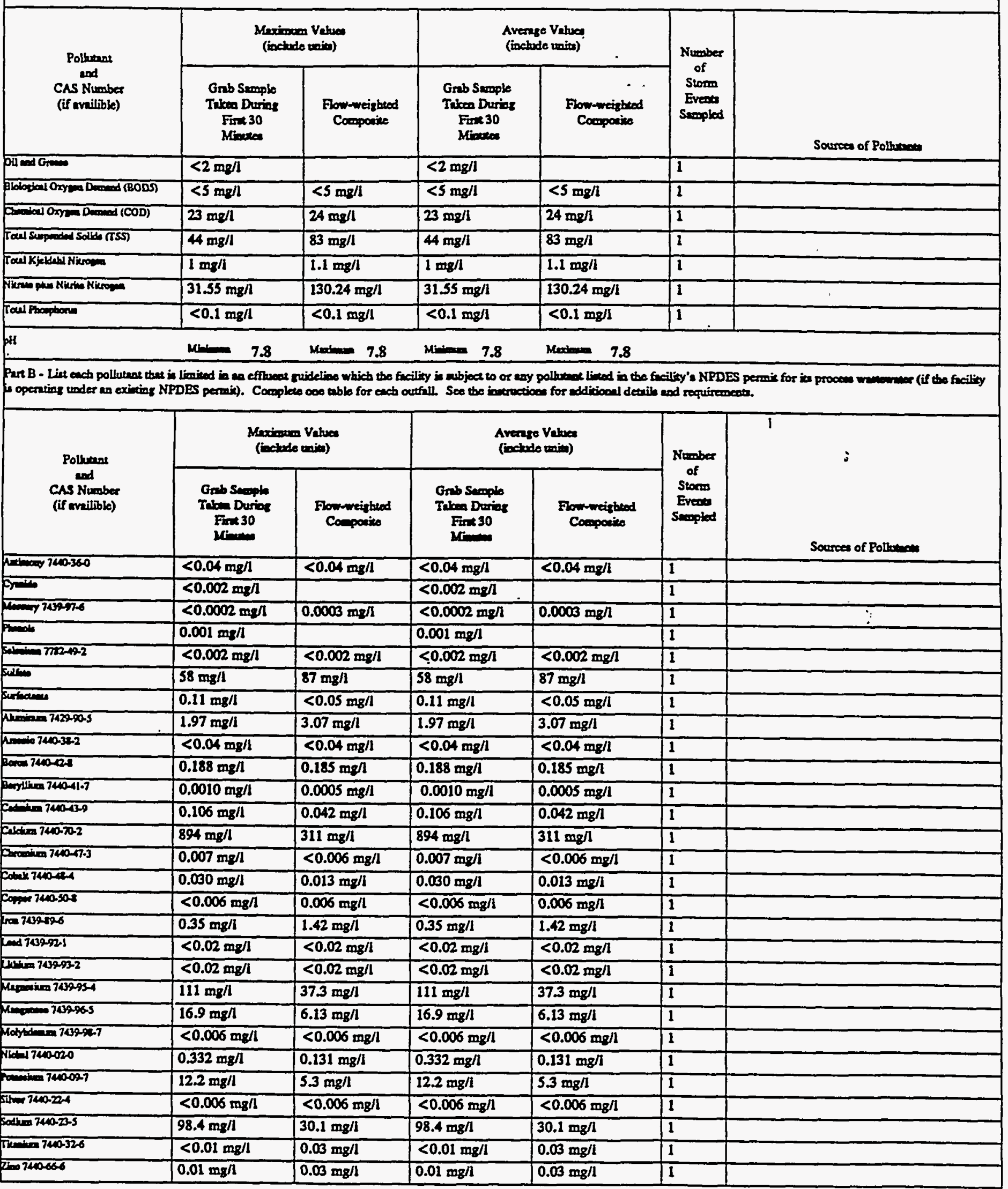


Conkinued from frost.

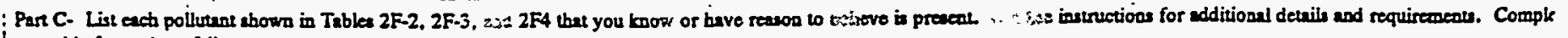
I one tuble for each outfall.

\begin{tabular}{|c|c|c|c|c|c|c|}
\hline \multirow{2}{*}{$\begin{array}{c}\text { Pollutent } \\
\text { Cod } \\
\text { CAS Number } \\
\text { (if availible) }\end{array}$} & \multicolumn{2}{|c|}{$\begin{array}{l}\text { Merimum Values } \\
\text { (inctude unitu) }\end{array}$} & \multicolumn{2}{|c|}{$\begin{array}{c}\text { Average Values } \\
\text { (includo units) }\end{array}$} & \multirow{2}{*}{$\begin{array}{l}\text { Number } \\
\text { of } \\
\text { Storm } \\
\text { Eveats } \\
\text { Sumpled } \\
\text {. . }\end{array}$} & \multirow[b]{2}{*}{ Sources of Pollhtant } \\
\hline & $\begin{array}{c}\text { Grab Samnie } \\
\text { Takeo Duans } \\
\text { Fint 3ú } \\
\text { Minuted }\end{array}$ & $\begin{array}{l}\text { Flow-weighted } \\
\text { Compotito }\end{array}$ & $\begin{array}{l}\text { Grab Sample } \\
\text { Taken During } \\
\text { Fint } 30 \\
\text { Minutes }\end{array}$ & $\begin{array}{l}\text { Flow-weighted } \\
\text { Componito }\end{array}$ & & \\
\hline $1,1,1$, trichloroctionoso $71-55-6$ & $<0.010 \mathrm{mg} / 1$ & & $<0.010 \mathrm{mg} / 1$ & & 1 & 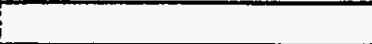 \\
\hline 1.1 .2 .2 tetrachloroet $79-34-5$ & $<0.010 \mathrm{mg} / \mathrm{l}$ & & $<0.010 \mathrm{mg} / 1$ & & 1 & 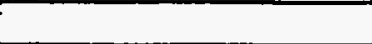 \\
\hline 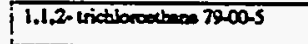 & $<0.010 \mathrm{mg} / \mathrm{l}$ & & $<0.010 \mathrm{mg} / 1$ & & 1 & 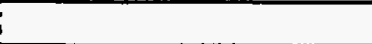 \\
\hline 1.1 didibocabeos $75-34-3$ & $<0.010 \mathrm{mg} / \mathrm{l}$ & & $<0.010 \mathrm{mg} / \mathrm{l}$ & & 1 & \\
\hline 1.1-dictibonethyleos 75-35-4 & $<0.010 \mathrm{mg} / 1$ & & $<0.010 \mathrm{mg} / 1$ & & 1 & \\
\hline 1.2 dictibrodber $10706-2$ & $<0.010 \mathrm{mg} / \mathrm{l}$ & & $<0.010 \mathrm{mg} / \mathrm{l}$ & & 1 & \\
\hline 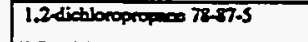 & $<0.010 \mathrm{mg} / \mathrm{l}$ & & $<0.010 \mathrm{mg} / 1$ & & 1 & \\
\hline 2-oblonombylvery bectuer 110-75-8 & $<0.010 \mathrm{mg} / 1$ & & $<0.010 \mathrm{mg} / \mathrm{l}$ & & 1 & \\
\hline $71-43-2$ & $<0.010 \mathrm{mg} / \mathrm{l}$ & & $<0.010 \mathrm{mg} / \mathrm{l}$ & & 1 & \\
\hline bromodicherathere 75-274 & $<0.010 \mathrm{mg} / 1$ & & $<0.010 \mathrm{mg} / \mathrm{l}$ & & 1 & \\
\hline brocoloros 75-25-2 & $<0.010 \mathrm{mg} / \mathrm{l}$ & & $<0.010 \mathrm{mg} / \mathrm{l}$ & & 1 & \\
\hline $6 r 040000000074839$ & $<0.010 \mathrm{mg} / \mathrm{l}$ & & $<0.010 \mathrm{mg} / !$ & & 1 & \\
\hline artece vexactibrith S6-23-5 & $<0.010 \mathrm{mg} / \mathrm{l}$ & & $<0.010 \mathrm{mg} / 1$ & & 1 & \\
\hline 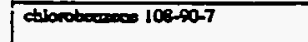 & $<0.010 \mathrm{mg} / 1$ & & $<0.010 \mathrm{mg}^{\prime l}$ & & 1 & \\
\hline chborcectine $75,00-3$ & $<0.010 \mathrm{mg} / 1$ & & $<0.010 \mathrm{mg} / 1$ & & 1 & \\
\hline ctionoform 67-66-3 & $<0.010 \mathrm{mg} / \mathrm{l}$ & & $<0.010 \mathrm{mg} / \mathrm{l}$ & & 1 & $\dot{*}$ \\
\hline 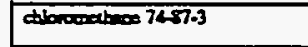 & $<0.010 \mathrm{mg} / \mathrm{l}$ & & $<0.010 \mathrm{mg} / \mathrm{l}$ & & 1 & \\
\hline 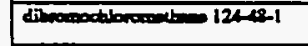 & $<0.010 \mathrm{mg} / \mathrm{l}$ & & $<0.010 \mathrm{mg} / 1$ & & 1 & \\
\hline 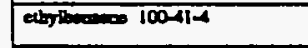 & $<0.010 \mathrm{mg} / 1$ & & $<0.010 \mathrm{mg} / \mathrm{l}$ & & 1 & \\
\hline bhen $102-3$ & $<0.010 \mathrm{mg} / \mathrm{l}$ & & $<0.010 \mathrm{mg} / 1$ & & 1 & \\
\hline vingl ablonile 75-014 & $<0.010 \mathrm{mg} / 1$ & & $<0.010 \mathrm{mg} / \mathrm{l}$ & & 1 & \\
\hline 1.3 dictionanopere $10061.01-5$ & $<0.010 \mathrm{mg} / 1$ & & $<0.010 \mathrm{mg} / \mathrm{l}$ & . & 1 & \\
\hline 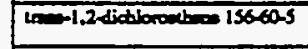 & $<0.010 \mathrm{mg} / \mathrm{l}$ & & $<0.010 \mathrm{mg} / 1$ & & 1 & $\because$ \\
\hline abbe eavity $12587-46.1$ & $250 \mathrm{pci} / 1$ & 47 pcill & $250 \mathrm{pci} / 1$ & 47 peill & 1 & \\
\hline been sarnty $12587-46-1$ & $2600 \mathrm{pci} / 1$ & $760 \mathrm{pci} / 1$ & 2,600pedl & 760 peill & 1 & \\
\hline rocal odisons & $3,300 \mathrm{co} / . \mathrm{IL}$ & $6,700 \mathrm{co} / .1 \mathrm{~L}$ & $3,300 \mathrm{co} / .1 \mathrm{~L}$ & $6,700 \mathrm{co} / .1 \mathrm{~L}$ & 1 & \\
\hline Tralliver 746-28-0 & $<0.001 \mathrm{mg} / \mathrm{l}$ & $<0.001 \mathrm{mg} / \mathrm{l}$ & $<0.001 \mathrm{mg} / \mathrm{l}$ & $<0.001 \mathrm{mg} / \mathrm{l}$ & 1 & \\
\hline Urasion 740-61-1 & $0.113 \mathrm{mg} / 1$ & $0.031 \mathrm{mg} / \mathrm{l}$ & $0.113 \mathrm{mg} / \mathrm{l}$ & $0.031 \mathrm{mg} / 1$ & 1 & \\
\hline Gen.in earingy & 14 peill & 0.78 peill & $14 \mathrm{pci} / 1$ & $0.78 \mathrm{pci} / 1$ & 1 & \\
\hline
\end{tabular}

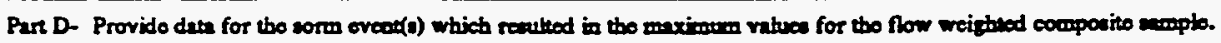

\begin{tabular}{|c|c|c|c|c|c|c|c|}
\hline $\begin{array}{l}1 . \\
\text { Dato of } \\
\text { Stores } \\
\text { Eveat }\end{array}$ & $\begin{array}{l}2 . \\
\text { Durntion } \\
\text { of Storm } \\
\text { (in } \\
\text { mininta) }\end{array}$ & $\begin{array}{l}3 . \\
\text { Toed ninfill } \\
\text { durios storm ovene } \\
\text { (in incha) }\end{array}$ & 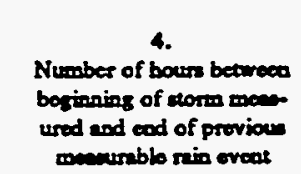 & $\begin{array}{l}\text { S. } \\
\text { Maximum flow nto } \\
\text { during nin eveat } \\
\text { (gillock/min or specify } \\
\text { unity) }\end{array}$ & $\begin{array}{l}6 . \\
\text { Total flow from } \\
\text { nin eveot } \\
\text { (alliow or } \\
\text { epeoify unito) }\end{array}$ & $\begin{array}{c}7 . \\
\text { Seavon } \\
\text { anplo was } \\
\text { takes }\end{array}$ & $\begin{array}{l}\text { 8. } \\
\text { Form of } \\
\text { Procipintion } \\
\text { (riafill, } \\
\text { mominely) }\end{array}$ \\
\hline $9 / 24 / 91$ & 365 & 1.6 & 133.5 & 1,142 & 566,066 & Fall & Ruinfall \\
\hline
\end{tabular}

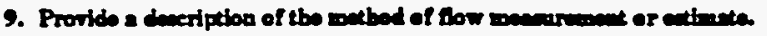

An ISCO 3230 Flow meter wes nsed to obtain water level readimg. This instrument was calibrated with the normul dry weather flow, if present, es leved zero. The fow meter was programmed with the characterities of the conveynce so that flow rattes and total flows conld be calcelated by the meter. 
Outfall S5

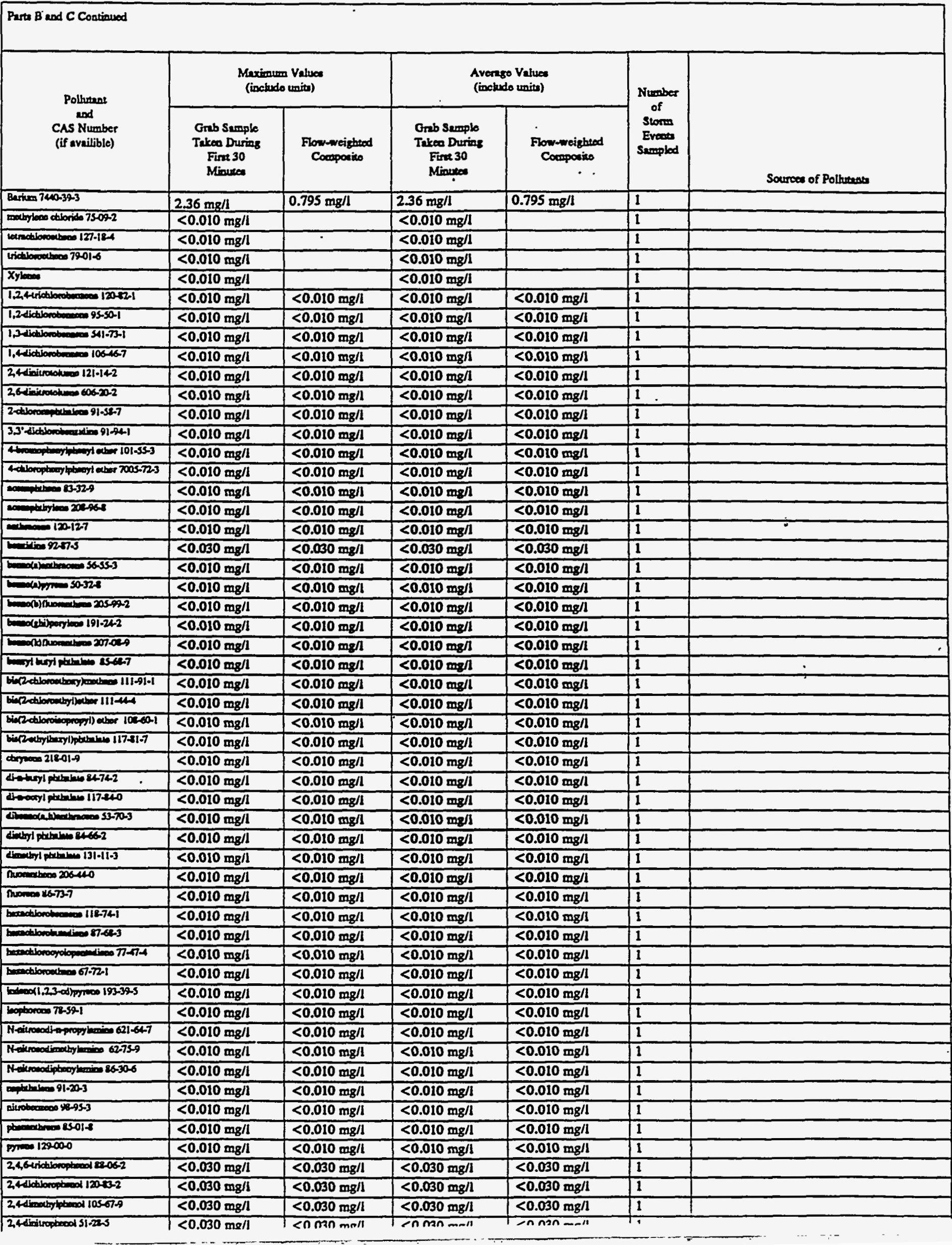




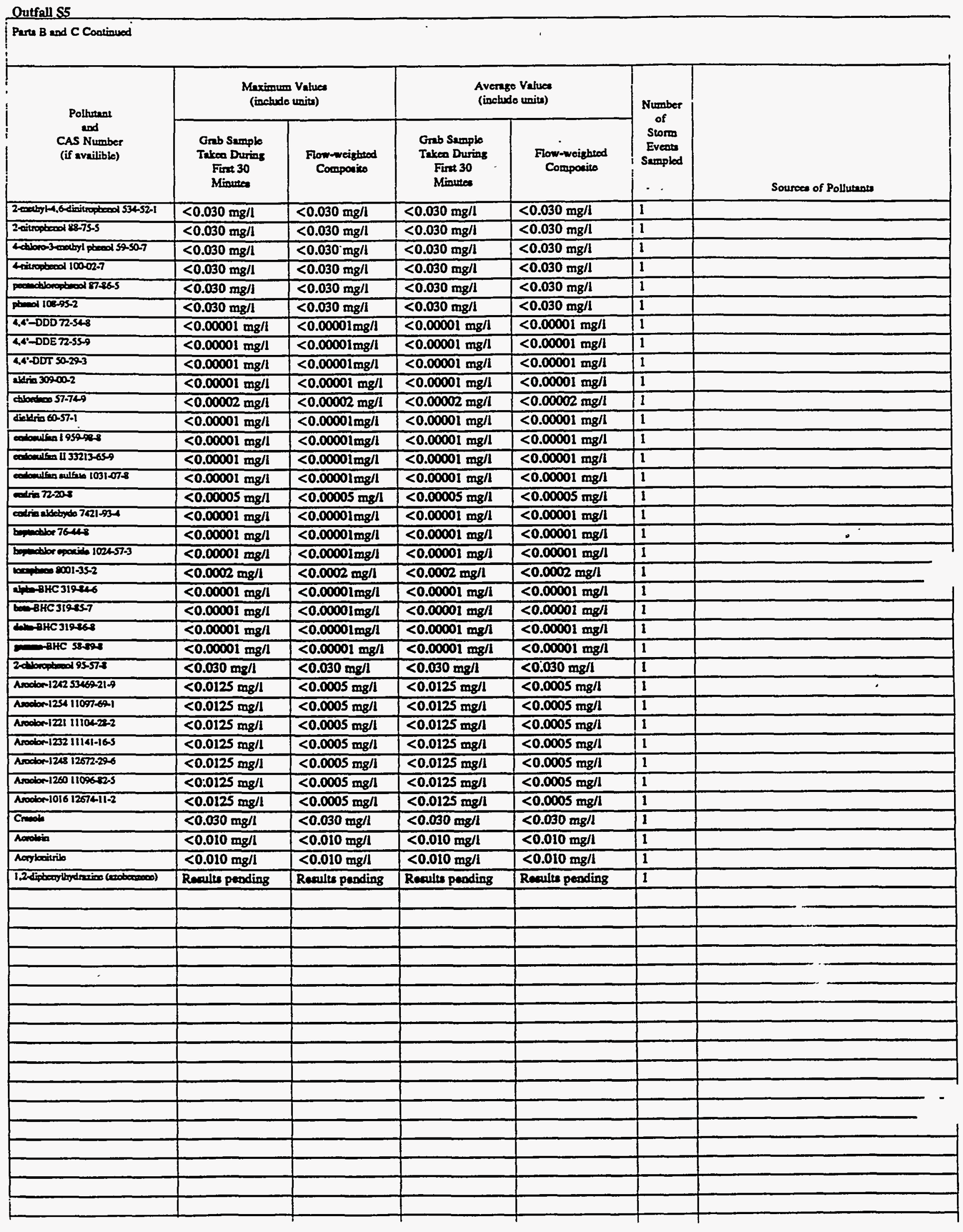


VII. Dircharge Information (continued from pege 3 of Form 2F)

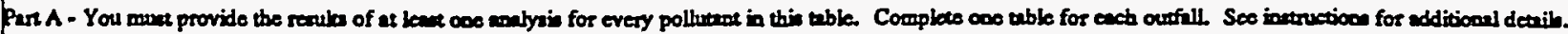

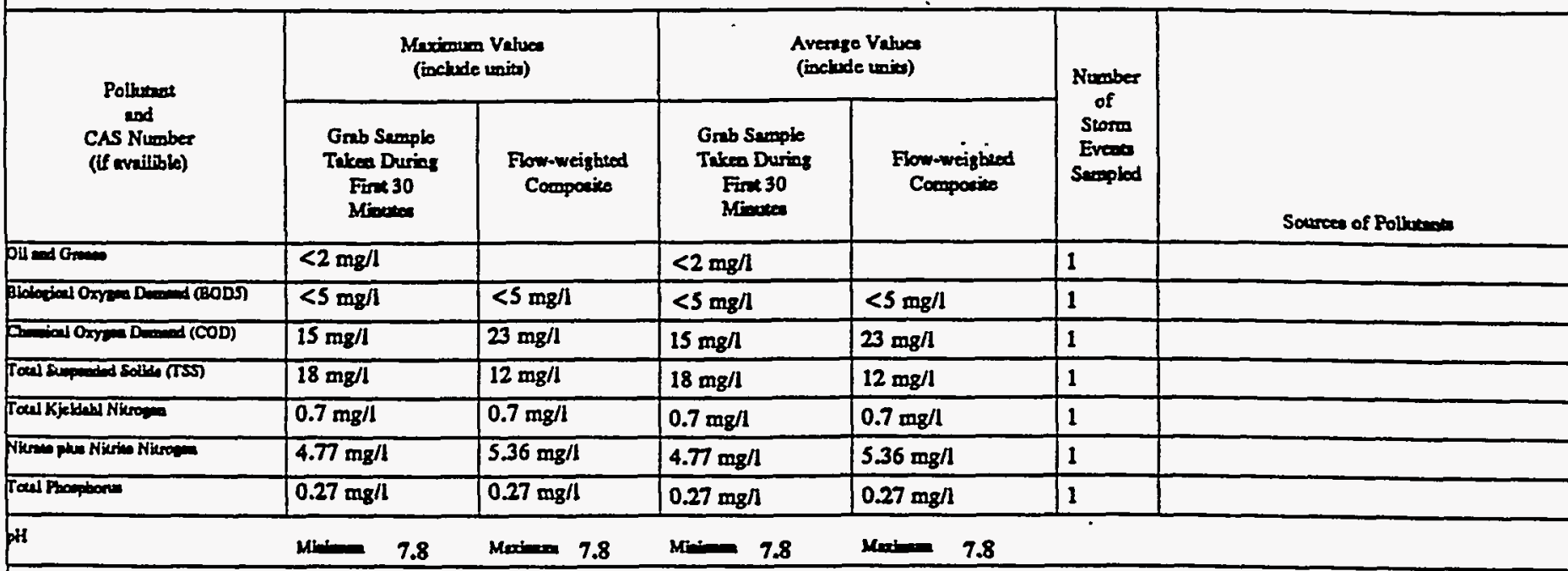

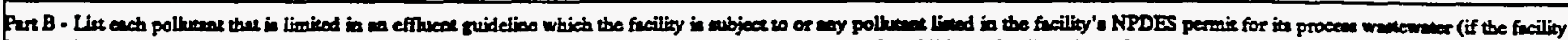

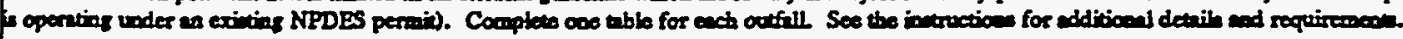

\begin{tabular}{|c|c|c|c|c|c|c|}
\hline \multirow{2}{*}{$\begin{array}{l}\text { Pollowax } \\
\text { ned } \\
\text { CAs Number } \\
\text { (if milbile) }\end{array}$} & \multicolumn{2}{|c|}{$\begin{array}{l}\text { Meximoum Valua } \\
\text { (inclusdo uniol) }\end{array}$} & \multicolumn{2}{|c|}{$\begin{array}{l}\text { Average Vabous } \\
\text { (ivelude vaida) }\end{array}$} & \multirow{2}{*}{$\begin{array}{c}\text { Nimbers } \\
\text { of } \\
\text { Stores } \\
\text { Evese } \\
\text { Sempled }\end{array}$} & \multirow{2}{*}{ Souree of Pollowenes } \\
\hline & 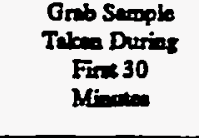 & $\begin{array}{l}\text { Form-weighed } \\
\text { Compotice }\end{array}$ & 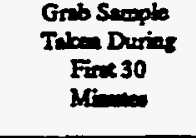 & $\begin{array}{l}\text { From-weighed } \\
\text { Cospocive }\end{array}$ & & \\
\hline 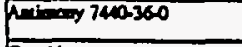 & $<0.04 \mathrm{mg} / \mathrm{l}$ & $<0.04 \mathrm{mg} / \mathrm{l}$ & $<0.04 \mathrm{mg} / \mathrm{l}$ & $<0.04 \mathrm{mg} / 1$ & 1 & \\
\hline Pyide & $<0.002 \mathrm{mg} / \mathrm{l}$ & & $<0.002 \mathrm{mg} / \mathrm{l}$ & & 1 & \\
\hline 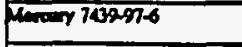 & $<0.0002 \mathrm{mg} / 1$ & $<0.0002 \mathrm{mg} / 1$ & $<0.0002 \mathrm{mg} h$ & $<0.0002 \mathrm{mg} / 1$ & 1 & \\
\hline Pat & $0.003 \mathrm{mq} / \mathrm{l}$ & & $0.003 \mathrm{mg} / \mathrm{l}$ & & 1 & \\
\hline 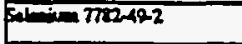 & $<0.002 \mathrm{mg} / \mathrm{l}$ & $<0.002 \mathrm{mg} / \mathrm{l}$ & $<0.002 \mathrm{mg} /$ & $<0.002 \mathrm{mg} / 1$ & 1 & \\
\hline sulaten & $18 \mathrm{mg} / \mathrm{l}$ & $41 \mathrm{mg} / 1$ & $18 \mathrm{mg} / 1$ & $41 \mathrm{mg} / \mathrm{l}$ & 1 & \\
\hline Surtaces & $<0.05 \mathrm{mg} / \mathrm{l}$ & $<0.05 \mathrm{mg} / \mathrm{l}$ & $<0.05 \mathrm{mg} / \mathrm{l}$ & $<0.05 \mathrm{mg} / \mathrm{l}$ & 1 & \\
\hline Thmonom 7429-90.5 & $2.22 \mathrm{mg} / \mathrm{l}$ & $2.41 \mathrm{mg} / \mathrm{l}$ & $2.22 \mathrm{mg} / 1$ & $2.41 \mathrm{mg} / \mathrm{I}$ & 1 & \\
\hline $5=-740.32-2$ & $<0.04 \mathrm{mg} / \mathrm{l}$ & $<0.04 \mathrm{mg} / \mathrm{l}$ & $<0.04 \mathrm{mg} / \mathrm{l}$ & $<0.04 \mathrm{mg} / \mathrm{l}$ & 1 & \\
\hline $80000740-108$ & $0.016 \mathrm{mg} / \mathrm{l}$ & $0.018 \mathrm{mg} / 1$ & $0.016 \mathrm{mg} / \mathrm{l}$ & $0.018 \mathrm{mg} / \mathrm{l}$ & 1 & \\
\hline Beryllinom 740-1-7 & $<0.0004 \mathrm{mg} / \mathrm{l}$ & $<0.0004 \mathrm{mg} / \mathrm{l}$ & $<0.0004 \mathrm{mg} / \mathrm{l}$ & $<0.0004 \mathrm{mg} / \mathrm{l}$ & 1 & \\
\hline Feding $740-13-9$ & $<0.004 \mathrm{mg} / \mathrm{l}$ & $<0.004 \mathrm{mg} / \mathrm{l}$ & $<0.004 \mathrm{mg} / \mathrm{l}$ & $<0.004 \mathrm{mg} / \mathrm{l}$ & 1 & \\
\hline Cabien 7400-xo-2 & $42.3 \mathrm{mg} / \mathrm{l}$ & $45.7 \mathrm{mg} / 1$ & $42.3 \mathrm{mg} / 1$ & $45.7 \mathrm{mg} / 1$ & 1 & \\
\hline Drenen $740-17.3$ & $<0.006 \mathrm{mg} / \mathrm{l}$ & $<0.006 \mathrm{mg} / \mathrm{l}$ & $<0.006 \mathrm{mg} / \mathrm{l}$ & $<0.006 \mathrm{mg} / \mathrm{l}$ & 1 & \\
\hline Coban 740-42. & $<0.002 \mathrm{mg} / \mathrm{l}$ & $<0.002 \mathrm{mg} / 1$ & $<0.002 \mathrm{mg} / \mathrm{l}$ & $<0.002 \mathrm{mg} / 1$ & 1 & \\
\hline Carm 24a-sas & $<0.006 \mathrm{mg} / \mathrm{l}$ & $<0.006 \mathrm{mg} / 1$ & $<0.006 \operatorname{mog} / 1$ & $<0.006 \mathrm{mg} / \mathrm{l}$ & 1 & \\
\hline 50702006 & $3.32 \mathrm{mg} / \mathrm{I}$ & $3.70 \mathrm{mg} / \mathrm{h}$ & $3.32 \mathrm{mg} / \mathrm{l}$ & $3.70 \mathrm{mg} / 1$ & 1 & \\
\hline 2017639821 & $<0.02 \mathrm{mg} / 1$ & $<0.02 \mathrm{mg} / \mathrm{l}$ & $<0.02 \mathrm{mg} / \mathrm{l}$ & $<0.02 \mathrm{mg} / \mathrm{l}$ & 1 & \\
\hline 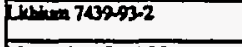 & $<0.02 \mathrm{mg} / \mathrm{l}$ & $<0.02 \mathrm{mg} / \mathrm{l}$ & $<0.02 \mathrm{mg} / 1$ & $<0.02 \mathrm{mg} / \mathrm{l}$ & 1 & \\
\hline Meomine 7239994 & $4.54 \mathrm{mg} / 1$ & $4.94 \mathrm{mg} / \mathrm{l}$ & $4.54 \mathrm{mg} / \mathrm{h}$ & $4.94 \mathrm{mg} / \mathrm{l}$ & 1 & \\
\hline 7000065 & $0.046 \mathrm{mg} / \mathrm{l}$ & $0.050 \mathrm{mg} / \mathrm{l}$ & $0.046 \mathrm{mg} / \mathrm{l}$ & $0.050 \mathrm{mg} / \mathrm{l}$ & 1 & \\
\hline 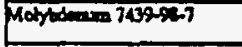 & $<0.006 \mathrm{mg} / \mathrm{I}$ & $<0.006 \mathrm{mg} / \mathrm{l}$ & $<0.006 \mathrm{mg} / \mathrm{l}$ & $<0.006 \mathrm{mg} / \mathrm{l}$ & 1 & \\
\hline Notal 740000 & $<0.008 \mathrm{mg} / 1$ & $<0.008 \mathrm{mg} / \mathrm{l}$ & $<0.008 \mathrm{mg} / \mathrm{l}$ & $<0.008 \mathrm{mg} / \mathrm{l}$ & 1 & 1 \\
\hline promina 740027 & $4.0 \mathrm{mg} / \mathrm{l}$ & $3.9 \mathrm{mg} / \mathrm{l}$ & $4.0 \mathrm{mg} / \mathrm{I}$ & $3.9 \mathrm{mg} / \mathrm{l}$ & 1 & \\
\hline ithen 740-20A & $<0.006 \mathrm{mg} / \mathrm{I}$ & $<0.006 \mathrm{mg} / \mathrm{l}$ & $<0.006 \mathrm{mg} / 1$ & $<0.006 \mathrm{mg} / \mathrm{l}$ & 1 & \\
\hline Sodiken 7400-22-5 & $3.24 \mathrm{mg} / \mathrm{l}$ & $3.67 \mathrm{mg} / 1$ & $3.24 \mathrm{mg} / 1$ & $3.67 \mathrm{mg} / 1$ & 1 & \\
\hline 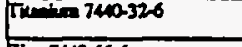 & $0.03 \mathrm{mg} / \mathrm{l}$ & $0.03 \mathrm{mg} / 1$ & $0.03 \mathrm{mg} / \mathrm{l}$ & $0.03 \mathrm{mg} / 1$ & 1 & \\
\hline 200740666 & $0.02 \mathrm{mg} / 1$ & $0.03 \mathrm{mg} / 1$ & $0.02 \mathrm{mg} / 1$ & $0.03 \mathrm{mg} / \mathrm{l}$ & 1 & \\
\hline
\end{tabular}


Combend from frove.

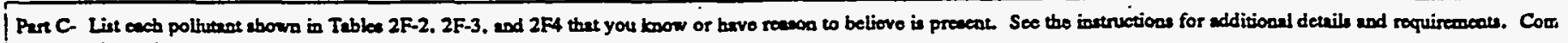
one table for each outfall.

\begin{tabular}{|c|c|c|c|c|c|c|}
\hline \multirow{2}{*}{$\begin{array}{l}\text { Polburat } \\
\text { and } \\
\text { CAS Number } \\
\text { (if availible) }\end{array}$} & \multicolumn{2}{|c|}{$\begin{array}{l}\text { Merimum Values } \\
\text { (ioclude units) }\end{array}$} & \multicolumn{2}{|c|}{$\begin{array}{l}\text { Avernge Values } \\
\text { (inchudo unita) }\end{array}$} & \multirow{2}{*}{$\begin{array}{l}\text { Number } \\
\text { of } \\
\text { Storm } \\
\text { Events } \\
\text { Samplad }\end{array}$} & \multirow[b]{2}{*}{ Sources of Pollutente } \\
\hline & $\begin{array}{l}\text { Gnb Sumplo } \\
\text { Takeo During } \\
\text { Fint } 30 \\
\text { Minutee }\end{array}$ & $\begin{array}{l}\text { Fow-weighted } \\
\text { Componite }\end{array}$ & $\begin{array}{l}\text { Grab Sumple } \\
\text { Triven During } \\
\text { First } 30 \\
\text { Minte }\end{array}$ & $\begin{array}{l}\text { Flow-weighted } \\
\text { Componite }\end{array}$ & & \\
\hline 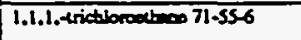 & $<0.010 \mathrm{mg} / \mathrm{l}$ & & $<0.010 \mathrm{mg} / 1$ & & 1 & \\
\hline 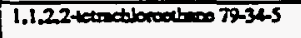 & $<0.010 \mathrm{mg} / 1$ & & $<0.010 \mathrm{mg} / 1$ & & 1 & \\
\hline 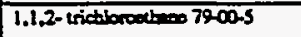 & $<0.010 \mathrm{mg} / 1$ & & $<0.010 \mathrm{mg} / 1$ & & 1 & \\
\hline 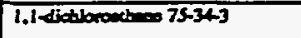 & $<0.010 \mathrm{mg} / 1$ & & $<0.010 \mathrm{mg} / 1$ & & 1 & \\
\hline 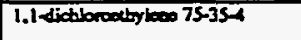 & $<0.010 \mathrm{mg} / 1$ & & $<0.010 \mathrm{mg} / 1$ & & 1 & \\
\hline 1.2 dictlorocthacos $107.06-2$ & $<0.010 \mathrm{mg} / \mathrm{l}$ & & $<0.010 \mathrm{mg} / \mathrm{l}$ & & 1 & \\
\hline 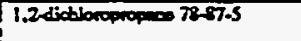 & $<0.010 \mathrm{mg} / \mathrm{l}$ & & $<0.010 \mathrm{mg} / \mathrm{l}$ & & 1 & \\
\hline 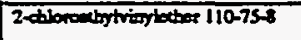 & $<0.010 \mathrm{mg} / \mathrm{l}$ & & $<0.010 \mathrm{mg} / 1$ & & 1 & \\
\hline $7=0-13-2$ & $<0.010 \mathrm{mg} / \mathrm{l}$ & & $<0.010 \mathrm{mg} / 1$ & & 1 & \\
\hline 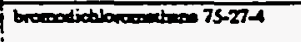 & $<0.010 \mathrm{mg} / 1$ & & $<0.010 \mathrm{mg} / 1$ & & 1 & \\
\hline $6700000000.75-25-2$ & $<0.010 \mathrm{mg} / \mathrm{l}$ & & $<0.010 \mathrm{mg} / 1$ & & 1 & \\
\hline $7483-9$ & $<0.010 \mathrm{mg} / 1$ & & $<0.010 \mathrm{mg} / 1$ & & 1 & \\
\hline artoo locinotibrido $\$ 6-23-5$ & $<0.010 \mathrm{mg} / \mathrm{l}$ & & $<0.010 \mathrm{mg} / \mathrm{l}$ & & 1 & \\
\hline 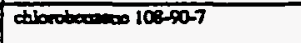 & $<0.010 \mathrm{mg} / \mathrm{l}$ & & $<0.010 \mathrm{mg} / 1$ & & 1 & \\
\hline chlocothes 75-00-3 & $<0.010 \mathrm{mg} / \mathrm{l}$ & & $<0.010 \mathrm{mg} / 1$ & & 1 & \\
\hline dibrolote 67-66-3 & $<0.010 \mathrm{mg} / 1$ & & $<0.010 \mathrm{mg} / 1$ & & 1 &. \\
\hline 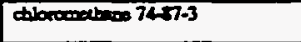 & $<0.010 \mathrm{mg} / 1$ & & $<0.010 \mathrm{mg} / \mathrm{l}$ & & 1 & \\
\hline 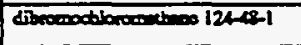 & $<0.010 \mathrm{mg} / \mathrm{l}$ & & $<0.010 \mathrm{mg} / \mathrm{l}$ & & 1 & \\
\hline ditylbareson 100414 & $<0.010 \mathrm{mg} / \mathrm{l}$ & & $<0.010 \mathrm{mg} / 1$ & & 1 & \\
\hline lohnes $102-3$ & $<0.010 \mathrm{mg} / \mathrm{l}$ & & $<0.010 \mathrm{mg} / \mathrm{l}$ & & 1 & \\
\hline viegl chiorice 75014 & $<0.010 \mathrm{mg} / \mathrm{l}$ & & $<0.010 \mathrm{mg} / \mathrm{l}$ & & 1 & \\
\hline 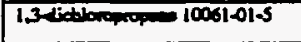 & $<0.010 \mathrm{mg} / 1$ & & $<0.010 \mathrm{mg} / \mathrm{l}$ & . & 1 & \\
\hline 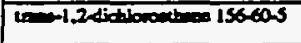 & $<0.010 \mathrm{mg} / \mathrm{l}$ & & $<0.010 \mathrm{mg} / 1$ & & 1 & $\because$ \\
\hline alpta ectivixy $12587+46-1$ & $8.7 \mathrm{pci} / 1$ & $160 \mathrm{pci} / \mathrm{l}$ & 8.7 peill & 160 pcill & 1 & \\
\hline bosediving $12557-46-1$ & 19 peill & 83 poill & 19 poill & $83 \mathrm{pci} / \mathrm{l}$ & 1 & \\
\hline focel coliforem & $1,800 \mathrm{co} .1 \mathrm{~L}$ & $1,400 \mathrm{col} .1 \mathrm{~L}$ & $1,800 \mathrm{col} .1 \mathrm{~L}$ & $1,400 \mathrm{co} / .1 \mathrm{~L}$ & 1 & \\
\hline Thallinen 740-28-0 & $<0.03 \mathrm{mg} / 1$ & $<0.03 \mathrm{mg} / \mathrm{l}$ & $<0.03 \mathrm{mg} / \mathrm{l}$ & $<0.03 \mathrm{mg} / \mathrm{l}$ & 1 & \\
\hline Uasian 740061-1 & $0.009 \mathrm{mg} / \mathrm{l}$ & $0.010 \mathrm{mg} / \mathrm{l}$ & $0.009 \mathrm{mg} / \mathrm{l}$ & $0.010 \mathrm{mg} / \mathrm{l}$ & 1 & \\
\hline Genen activisy & -25 peill & -23 peill & -25 peinl & -23 pcill & 1 & \\
\hline
\end{tabular}

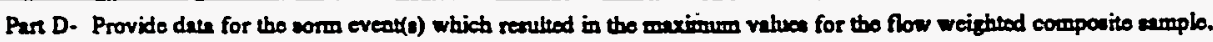

\begin{tabular}{|c|c|c|c|c|c|c|c|}
\hline $\begin{array}{c}1 . \\
\text { Date of } \\
\text { Storm } \\
\text { Event }\end{array}$ & $\begin{array}{l}2 . \\
\text { Duration } \\
\text { of Storn } \\
\text { (in } \\
\text { minutes) }\end{array}$ & $\begin{array}{l}3 . \\
\text { Total minfall } \\
\text { during morm ovent } \\
\text { (in incen) }\end{array}$ & 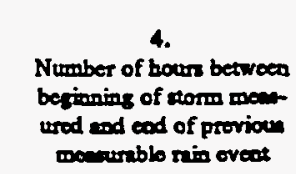 & 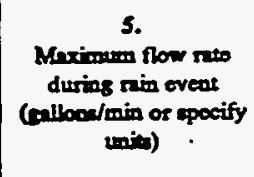 & $\begin{array}{l}6 . \\
\text { Total flow from } \\
\text { rim oveos } \\
\text { (alloow or } \\
\text { specify unim) }\end{array}$ & $\begin{array}{c}7 . \\
\text { Seanos } \\
\text { enimple was } \\
\text { unkeo }\end{array}$ & $\begin{array}{l}\text { 8. } \\
\text { Form of } \\
\text { Precipitation } \\
\text { (ninfull. } \\
\text { nommelt) }\end{array}$ \\
\hline $12 / 9 / 91$ & 530 & 1.1 & 148.6 & 54.9 & 11,019 & Fall & Rainfall \\
\hline
\end{tabular}

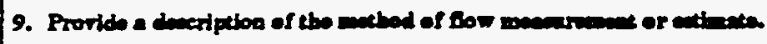

An ISCO 3230 Flow meter was used to obtain water level readiogs. This instrament was catibrated with the normal dry weather flow, if present, as level zero. The flow meter was programmed with the charecteristics of the conveyance so that flow rates and totol flows conld be calculated by the meter. 
Outrall S6

Purts B and C Coatinuod

\begin{tabular}{|c|c|c|c|c|c|c|}
\hline \multirow{2}{*}{$\begin{array}{l}\text { Polbutane } \\
\text { and } \\
\text { CAS Number } \\
\text { (if avnilible) }\end{array}$} & \multicolumn{2}{|c|}{$\begin{array}{l}\text { Meximung Vahuea } \\
\text { (inchude units) }\end{array}$} & \multicolumn{2}{|c|}{$\begin{array}{l}\text { Aversege Vahues } \\
\text { (ioclude unitu) }\end{array}$} & \multirow{2}{*}{$\begin{array}{l}\text { Number } \\
\text { of } \\
\text { Storn } \\
\text { Eventa } \\
\text { Sempled }\end{array}$} & \multirow[b]{2}{*}{ Sounces of Pollumans } \\
\hline & $\begin{array}{c}\text { Grab Semple } \\
\text { Taken Durieg } \\
\text { Firk } 30 \\
\text { Minulece }\end{array}$ & $\begin{array}{l}\text { Flom-weigbled } \\
\text { Compoticos }\end{array}$ & $\begin{array}{c}\text { Greb Semple } \\
\text { Taken Duriag } \\
\text { Firk } 30 \\
\text { Misued }\end{array}$ & $\begin{array}{l}\text { Fow-weizhedd } \\
\text { Componies }\end{array}$ & & \\
\hline Bertem 7400.32-3 & $0.0315 \mathrm{mg} / 1$ & $0.0330 \mathrm{mg} / 1$ & $0.0315 \mathrm{mg} / \mathrm{h}$ & $0.0330 \mathrm{mg} / \mathrm{l}$ & 1 & \\
\hline 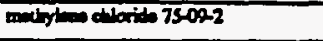 & $<0.010 \mathrm{mg} / \mathrm{l}$ & & $<0.010 \mathrm{mg} / \mathrm{l}$ & & 1 & \\
\hline 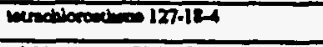 & $<0.010 \mathrm{mg} / \mathrm{l}$ & & $<0.010 \mathrm{mg} / \mathrm{l}$ & & 1 & \\
\hline 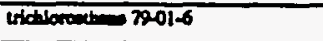 & $<0.010 \mathrm{mg} / 1$ & & $<0.010 \mathrm{mg} / 1$ & & 1 & \\
\hline xylemen & $<0.010 \mathrm{mg} / \mathrm{l}$ & & $<0.010 \mathrm{mg} / \mathrm{l}$ & & 1 & \\
\hline 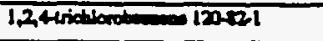 & $<0.010 \mathrm{mg} / \mathrm{l}$ & $<0.010 \mathrm{mg} / 1$ & $<0.010 \mathrm{mg} /$ & $<0.010 \mathrm{mg} / \mathrm{h}$ & 1 & \\
\hline 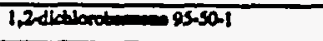 & $<0.010 \mathrm{mg} / \mathrm{l}$ & $<0.010 \mathrm{mg} / 1$ & $<0.010 \mathrm{mg} / \mathrm{l}$ & $<0.010 \mathrm{mg} / 1$ & 1 & \\
\hline 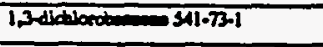 & $<0.010 \mathrm{mg} / \mathrm{l}$ & $<0.010 \mathrm{mg} / 1$ & $<0.010 \mathrm{mg} / \mathrm{l}$ & $<0.010 \mathrm{mg} / \mathrm{l}$ & 1 & \\
\hline 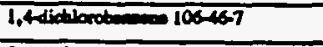 & $<0.010 \mathrm{mg} / \mathrm{l}$ & $<0.010 \mathrm{mg} / \mathrm{l}$ & $<0.010 \mathrm{mg} / 1$ & $<0.010 \mathrm{mg} / \mathrm{l}$ & 1 & \\
\hline 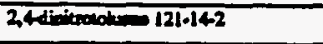 & $<0.010 \mathrm{mg} / 1$ & $<0.010 \mathrm{mg} / \mathrm{l}$ & $<0.010 \mathrm{mg} / \mathrm{l}$ & $<0.010 \mathrm{mg} / \mathrm{l}$ & 1 & \\
\hline 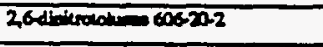 & $<0.010 \mathrm{mg} / \mathrm{I}$ & $<0.010 \mathrm{mg} / 1$ & $<0.010 \mathrm{mg} / \mathrm{l}$ & $<0.010 \mathrm{mg} / \mathrm{h}$ & 1 & \\
\hline 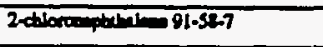 & $<0.010 \mathrm{mg} / \mathrm{l}$ & $<0.010 \mathrm{mg} / \mathrm{l}$ & $<0.010 \mathrm{mg} / \mathrm{l}$ & $<0.010 \mathrm{mg} / 1$ & 1 & \\
\hline 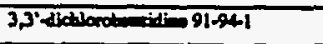 & $<0.010 \mathrm{mg} / \mathrm{l}$ & $<0.010 \mathrm{mg} / \mathrm{l}$ & $<0.010 \mathrm{mg} / \mathrm{l}$ & $<0.010 \mathrm{mg} / \mathrm{l}$ & 1 & \\
\hline 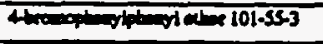 & $<0.010 \mathrm{mg} / \mathrm{l}$ & $<0.010 \mathrm{mg} / 1$ & $<0.010 \mathrm{mg} / \mathrm{l}$ & $<0.010 \mathrm{mg} / 1$ & 1 & \\
\hline 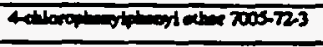 & $<0.010 \mathrm{mg} / \mathrm{l}$ & $<0.010 \mathrm{mg} / 1$ & $<0.010 \mathrm{mg} / \mathrm{l}$ & $<0.010 \mathrm{mg} / \mathrm{l}$ & 1 & \\
\hline conopithos 13325 & $<0.010 \mathrm{mg} / \mathrm{h}$ & $<0.010 \mathrm{mg} / 1$ & $<0.010 \mathrm{mg} / \mathrm{l}$ & $<0.010 \mathrm{mg} / 1$ & 1 & \\
\hline 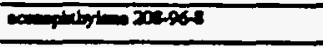 & $<0.010 \mathrm{mg} / \mathrm{l}$ & $<0.010 \mathrm{mg} / 1$ & $<0.010 \mathrm{mg} / 1$ & $<0.010 \mathrm{mg} / \mathrm{l}$ & 1 & \\
\hline $120-127$ & $<0.010 \mathrm{mg} / \mathrm{l}$ & $<0.010 \mathrm{mg} / 1$ & $<0.010 \mathrm{mg} / 1$ & $<0.010 \mathrm{mg} / \mathrm{l}$ & 1 & \\
\hline$=2 \sqrt{1-9} 927.5$ & $<0.050 \mathrm{mg} / \mathrm{l}$ & $<0.050 \mathrm{mg} / \mathrm{l}$ & $<0.050 \mathrm{mg} / \mathrm{l}$ & $<0.050 \mathrm{mg} / \mathrm{h}$ & 1 & \\
\hline 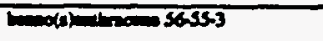 & $<0.010 \mathrm{mg} / \mathrm{l}$ & $<0.010 \mathrm{mg} / \mathrm{l}$ & $<0.010 \mathrm{mg} / 1$ & $<0.010 \mathrm{mg} / 1$ & 1 & \\
\hline 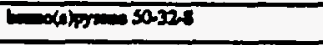 & $<0.010 \mathrm{mg} / 1$ & $<0.010 \mathrm{mg} / \mathrm{l}$ & $<0.010 \mathrm{mg} / 1$ & $<0.010 \mathrm{mg} / \mathrm{l}$ & 1 & \\
\hline 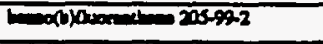 & $<0.010 \mathrm{mg} / \mathrm{l}$ & $<0.010 \mathrm{mg} / \mathrm{L}$ & $<0.010 \mathrm{mg} / \mathrm{l}$ & $<0.010 \mathrm{mg} / \mathrm{L}$ & 1 & \\
\hline 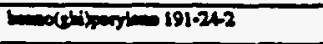 & $<0.010 \mathrm{mg} / \mathrm{l}$ & $<0.010 \mathrm{mg} / \mathrm{l}$ & $<0.010 \mathrm{mg} / \mathrm{l}$ & $<0.010 \mathrm{mg} / 1$ & 1 & \\
\hline 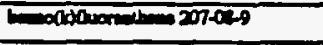 & $<0.010 \mathrm{mg} / \mathrm{l}$ & $<0.010 \mathrm{mg} / 1$ & $<0.010 \mathrm{mg} / \mathrm{l}$ & $<0.010 \mathrm{mg} / \mathrm{l}$ & 1 & \\
\hline 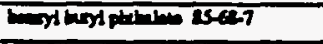 & $<0.010 \mathrm{mg} / 1$ & $<0.010 \mathrm{mg} / \mathrm{l}$ & $<0.010 \mathrm{mg} / \mathrm{s}$ & $<0.010 \mathrm{mg} / \mathrm{l}$ & 1 & \\
\hline 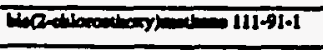 & $<0.010 \mathrm{mg} / \mathrm{l}$ & $<0.010 \mathrm{mg} / 1$ & $<0.010 \mathrm{me} / \mathrm{l}$ & $<0.010 \mathrm{mg} / 1$ & 1 & \\
\hline 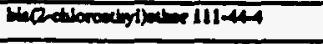 & $<0.010 \mathrm{mg} / 1$ & $<0.010 \mathrm{mg} / 1$ & $<0.010 \mathrm{mg} / \mathrm{l}$ & $<0.010 \mathrm{mg} / \mathrm{h}$ & 1 & \\
\hline 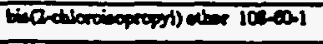 & $<0.010 \mathrm{mg} / 1$ & $<0.010 \mathrm{mg} / \mathrm{l}$ & $<0.010 \mathrm{mg} / 1$ & $<0.010 \mathrm{mg} / \mathrm{l}$ & 1 & \\
\hline 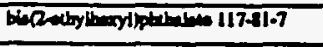 & $<0.010 \mathrm{mg} / \mathrm{l}$ & $<0.010 \mathrm{mg} / \mathrm{l}$ & $<0.010 \mathrm{mg} / 1$ & $<0.010 \mathrm{mg} / \mathrm{l}$ & 1 & \\
\hline 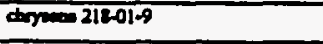 & $<0.010 \mathrm{mg} / \mathrm{l}$ & $<0.010 \mathrm{mg} / \mathrm{l}$ & $<0.010 \mathrm{mg} / \mathrm{l}$ & $<0.010 \mathrm{mg} / \mathrm{l}$ & 1 & \\
\hline 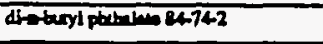 & $<0.010 \mathrm{mg} / \mathrm{l}$ & $12 \mathrm{mg} / \mathrm{l}$ & $<0.010 \mathrm{mg} / 1$ & $12 \mathrm{mg} / \mathrm{l}$ & 1 & \\
\hline 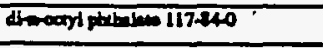 & $<0.010 \mathrm{mg} / 1$ & $<0.010 \mathrm{mg} / \mathrm{l}$ & $<0.010 \mathrm{mg} / 1$ & $<0.010 \mathrm{mg} / \mathrm{h}$ & 1 & \\
\hline 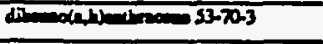 & $<0.010 \mathrm{mg} / \mathrm{l}$ & $<0.010 \mathrm{mg} / 1$ & $<0.010 \mathrm{mg} / \mathrm{l}$ & $<0.010 \mathrm{mg} /$ & 1 & \\
\hline 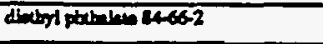 & $<0.010 \mathrm{mg} / \mathrm{l}$ & $<0.010 \mathrm{mg} / \mathrm{l}$ & $<0.010 \mathrm{mg} / 1$ & $<0.010 \mathrm{mg} / \mathrm{h}$ & 1 & \\
\hline 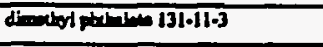 & $<0.010 \mathrm{mg} / 1$ & $<0.010 \mathrm{mg} / \mathrm{l}$ & $<0.010 \mathrm{mg} / \mathrm{l}$ & $<0.010 \mathrm{mg} / \mathrm{h}$ & 1 & \\
\hline 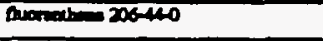 & $<0.010 \mathrm{mg} / \mathrm{l}$ & $<0.010 \mathrm{mg} /$ & $<0.010 \mathrm{mg} / \mathrm{l}$ & $<0.010 \mathrm{mg} / 1$ & 1 & \\
\hline acomene.73-7 & $<0.010 \mathrm{mg} / 1$ & $<0.010 \mathrm{mg} / \mathrm{l}$ & $<0.010 \mathrm{mg} / \mathrm{l}$ & $<0.010 \mathrm{mg} / \mathrm{l}$ & 1 & \\
\hline 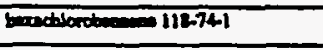 & $<0.010 \mathrm{mg} / \mathrm{l}$ & $<0.010 \mathrm{mg} / \mathrm{l}$ & $<0.010 \mathrm{mg} / \mathrm{l}$ & $<0.010 \mathrm{mg} / \mathrm{l}$ & 1 & \\
\hline 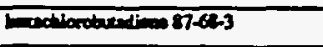 & $<0.010 \mathrm{mg} / 1$ & $<0.010 \mathrm{mg} / 1$ & $<0.010 \mathrm{mg} / \mathrm{l}$ & $<0.010 \mathrm{mg} / \mathrm{l}$ & 1 & \\
\hline 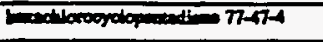 & $<0.010 \mathrm{mg} / \mathrm{l}$ & $<0.010 \mathrm{mg} / \mathrm{l}$ & $<0.010 \mathrm{mg} / \mathrm{l}$ & $<0.010 \mathrm{mg} / \mathrm{l}$ & 1 & \\
\hline 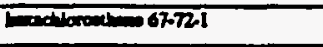 & $<0.010 \mathrm{mg} / 1$ & $<0.010 \mathrm{mg} / 1$ & $<0.010 \mathrm{mg} / 1$ & $<0.010 \mathrm{mg} / \mathrm{l}$ & 1 & \\
\hline 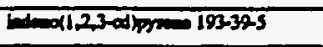 & $<0.010 \mathrm{mg} / \mathrm{l}$ & $<0.010 \mathrm{mg} / \mathrm{l}$ & $<0.010 \mathrm{mg} / \mathrm{l}$ & $<0.010 \mathrm{mg} / \mathrm{l}$ & 1 & \\
\hline t259-1 & $<0.010 \mathrm{mg} / \mathrm{l}$ & $<0.010 \mathrm{mg} / \mathrm{l}$ & $<0.010 \mathrm{mg} / 1$ & $<0.010 \mathrm{mg} / \mathrm{l}$ & 1 & \\
\hline 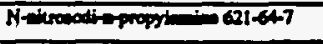 & $<0.010 \mathrm{mg} / \mathrm{l}$ & $<0.010 \mathrm{mg} / \mathrm{l}$ & $<0.010 \mathrm{mg} / 1$ & $<0.010 \mathrm{mg} / \mathrm{h}$ & 1 & \\
\hline 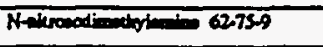 & $<0.010 \mathrm{mg} / \mathrm{l}$ & $<0.010 \mathrm{mg} / \mathrm{l}$ & $<0.010 \mathrm{mg} / \mathrm{l}$ & $<0.010 \mathrm{mg} / \mathrm{l}$ & 1 & \\
\hline 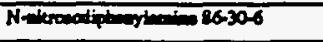 & $<0.010 \mathrm{mg} / \mathrm{I}$ & $<0.010 \mathrm{mg} / \mathrm{l}$ & $<0.010 \mathrm{mg} / 1$ & $<0.010 \mathrm{mg} / \mathrm{h}$ & 1 & \\
\hline anding $91-20-3$ & $<0.010 \mathrm{mg} / \mathrm{l}$ & $<0.010 \mathrm{mg} / 1$ & $<0.010 \mathrm{mg} / 1$ & $<0.010 \mathrm{mg} / \mathrm{h}$ & 1 & \\
\hline 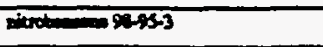 & $<0.010 \mathrm{mg} / \mathrm{l}$ & $<0.010 \mathrm{mg} / \mathrm{l}$ & $<0.010 \mathrm{mg} / 1$ & $<0.010 \mathrm{mg} /$ & 1 & \\
\hline 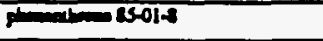 & $<0.010 \mathrm{mg} / 1$ & $<0.010 \mathrm{mg} / \mathrm{l}$ & $<0.010 \mathrm{mg} / \mathrm{l}$ & $<0.010 \mathrm{mg} / \mathrm{l}$ & 1 & \\
\hline 8700120000 & $<0.010 \mathrm{mg} / 1$ & $<0.010 \mathrm{mg} / 1$ & $<0.010 \mathrm{mg} / 1$ & $<0.010 \mathrm{mg} / \mathrm{l}$ & 1 & \\
\hline 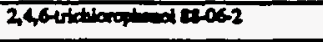 & $<0.030 \mathrm{mg} / \mathrm{l}$ & $<0.030 \mathrm{mg} / \mathrm{l}$ & $<0.030 \mathrm{mg} / 1$ & $<0.000 \mathrm{mg} / \mathrm{h}$ & 1 & \\
\hline 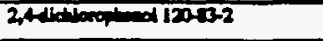 & $<0.030 \mathrm{mg} / \mathrm{I}$ & $<0.030 \mathrm{mg} / \mathrm{H}$ & $<0.030 \mathrm{mg} / \mathrm{l}$ & $<0.030 \mathrm{mg} / \mathrm{l}$ & 1 & \\
\hline 24 twithonot 105679 & $<0.030 \mathrm{mg} / \mathrm{l}$ & $<0.030 \mathrm{mg} / \mathrm{l}$ & $<0.030 \mathrm{mg} / \mathrm{l}$ & $<0.030 \mathrm{mg} / \mathrm{h}$ & 1 & \\
\hline 24dminophend S1-22.5 & $<0.050 \mathrm{mg} / \mathrm{l}$ & $<0.050 \mathrm{mg} / \mathrm{L}$ & $<0.050 \mathrm{mg} / \mathrm{l}$ & $<0.050 \mathrm{mg} \Omega$ & 1 & \\
\hline
\end{tabular}


Qutfall $\$ 6$

Pars B and C Continued

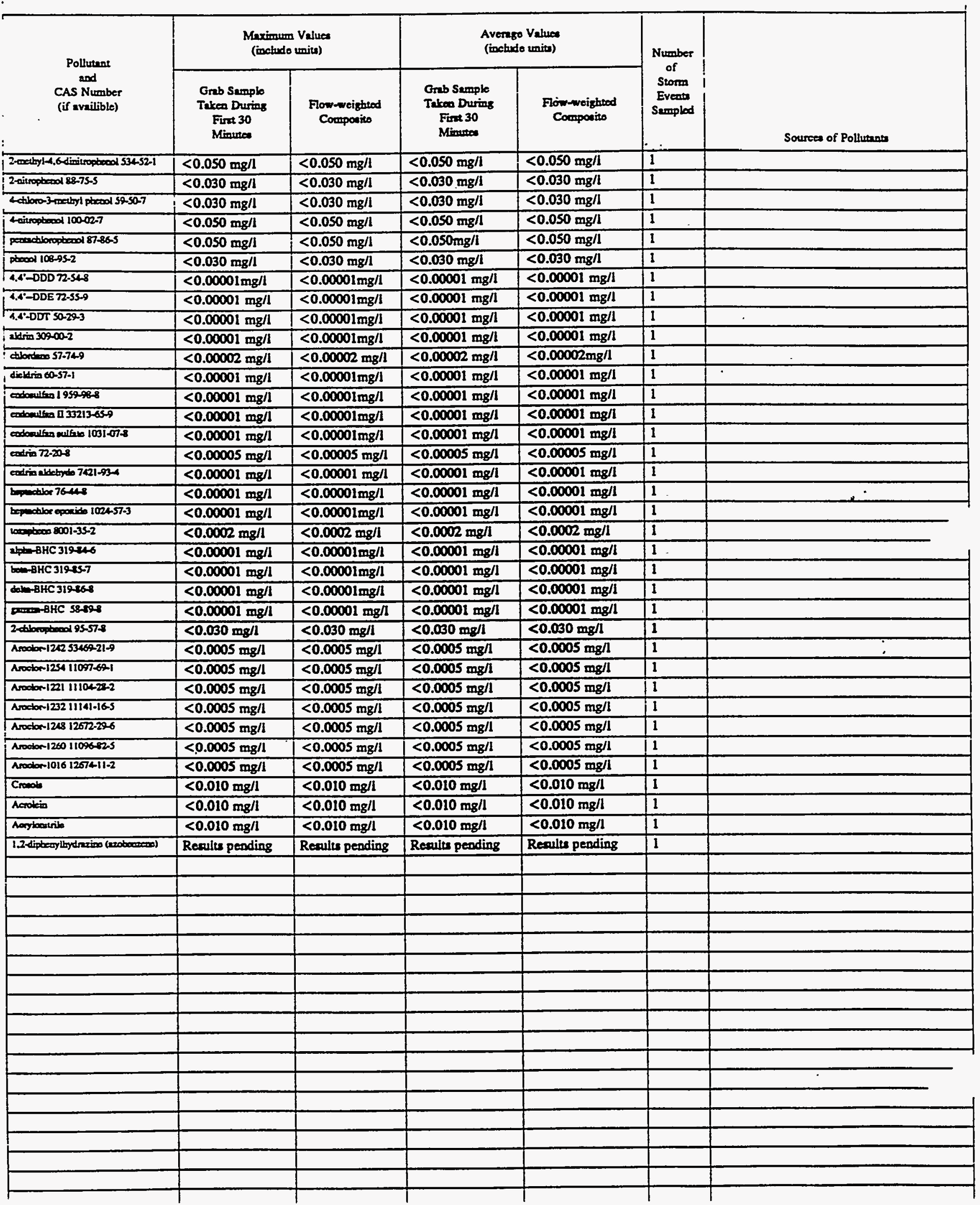


VII. Discharge Information (contialuod from page 3 of Form 2F)

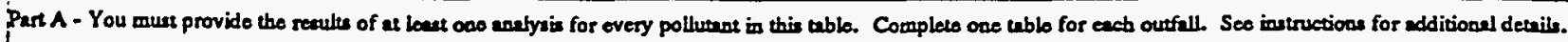

\begin{tabular}{|c|c|c|c|c|c|c|}
\hline \multirow{2}{*}{$\begin{array}{l}\text { Pollutuat } \\
\text { and } \\
\text { CAS Number } \\
\text { (if availiblo) }\end{array}$} & \multicolumn{2}{|c|}{$\begin{array}{l}\text { Marimuen Values } \\
\text { (irchude unita) }\end{array}$} & \multicolumn{2}{|c|}{$\begin{array}{l}\text { Average Vatuea } \\
\text { (inchude units) }\end{array}$} & \multirow{2}{*}{$\begin{array}{l}\text { Number } \\
\text { of } \\
\text { Storm } \\
\text { Eveats } \\
\text { Semplod }\end{array}$} & \multirow[b]{2}{*}{ Sourcece of Pollutantas } \\
\hline & $\begin{array}{c}\text { Grab Sampis } \\
\text { Taken During } \\
\text { Firz } 30 \\
\text { Mioutea }\end{array}$ & $\begin{array}{l}\text { Flow-weighted } \\
\text { Compotice }\end{array}$ & $\begin{array}{c}\text { Grab Sample } \\
\text { Takea During } \\
\text { Fint } 30 \\
\text { Minuteo }\end{array}$ & $\begin{array}{l}\text { Frow weighiod } \\
\text { Componito }\end{array}$ & & \\
\hline pid end Greese & $<2 \mathrm{mg} / \mathrm{l}$ & & $<2 \mathrm{mg} / \mathrm{l}$ & & 1 & \\
\hline 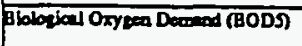 & $<5 \mathrm{mg} / \mathrm{l}$ & $<5 \mathrm{mg} / \mathrm{l}$ & $<5 \mathrm{mg} / \mathrm{l}$ & $<5 \mathrm{mg} / \mathrm{l}$ & $\bar{I}$ & \\
\hline 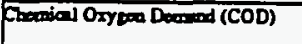 & $14 \mathrm{mg} / \mathrm{l}$ & $16 \mathrm{mg} / 1$ & $14 \mathrm{mg} / \mathrm{l}$ & $16 \mathrm{mg} / 1$ & 1 & \\
\hline Pot Supundod Solide (TSS) & $110 \mathrm{mg} / 1$ & $55 \mathrm{mg} / \mathrm{l}$ & $110 \mathrm{mg} / \mathrm{l}$ & $55 \mathrm{mg} / \mathrm{l}$ & 1 & \\
\hline Toul Xjelhebl Nilrosea & $0.4 \mathrm{mg} / \mathrm{l}$ & $0.5 \mathrm{mg} / 1$ & $0.4 \mathrm{mg} / 1$ & $0.5 \mathrm{mg} / 1$ & 1 & \\
\hline Nitrion plum Nilrice Nitroges & $1.4 \mathrm{mg} / \mathrm{l}$ & $1.3 \mathrm{mg} / \mathrm{l}$ & $1.4 \mathrm{mg} / \mathrm{l}$ & $1.3 \mathrm{mg} / 1$ & 1 & \\
\hline Toal Prosportus & $0.22 \mathrm{mg} / \mathrm{l}$ & $0.15 \mathrm{mg} / \mathrm{l}$ & $0.22 \mathrm{mg} / \mathrm{l}$ & $0.15 \mathrm{mg} / \mathrm{l}$ & 1 & \\
\hline
\end{tabular}

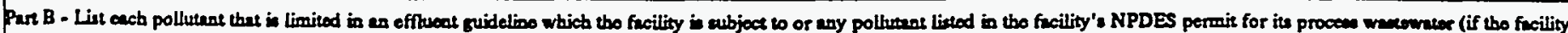

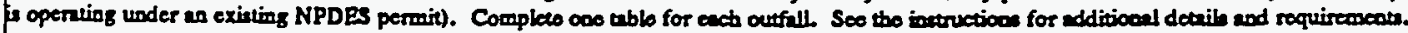

\begin{tabular}{|c|c|c|c|c|c|c|}
\hline \multirow{2}{*}{$\begin{array}{l}\text { Pollutase } \\
\text { and } \\
\text { CAs Number } \\
\text { (if avilible) }\end{array}$} & \multicolumn{2}{|c|}{ 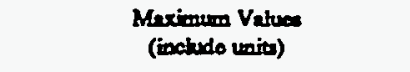 } & \multicolumn{2}{|c|}{$\begin{array}{l}\text { Aversese Vahues } \\
\text { (inctude unitu) }\end{array}$} & \multirow{2}{*}{$\begin{array}{l}\text { Number } \\
\text { of } \\
\text { Storm } \\
\text { Eveosta } \\
\text { Sampled }\end{array}$} & \multirow{2}{*}{$\begin{array}{c}: \\
\text { Source of Pollutuen }\end{array}$} \\
\hline & 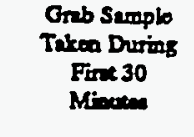 & $\begin{array}{l}\text { Flow-wreighted } \\
\text { Componito }\end{array}$ & $\begin{array}{c}\text { Orab Sumple } \\
\text { Takea Duriog } \\
\text { Fine } 30 \\
\text { Mineme }\end{array}$ & $\begin{array}{l}\text { Fow-weighiod } \\
\text { Compoito }\end{array}$ & & \\
\hline Aximeon $7400-360$ & $<0.003 \mathrm{mg} / \mathrm{l}$ & $<0.003 \mathrm{mg} / \mathrm{l}$ & $<0.003 \mathrm{mg} / \mathrm{l}$ & $<0.003 \mathrm{mg} / \mathrm{l}$ & 1 & \\
\hline symiso & $<0.002 \mathrm{mg} / \mathrm{l}$ & & $<0.002 \mathrm{mg} / \mathrm{l}$ & & 1 & \\
\hline Mewary 7439-976 & $<0.0002 \mathrm{mg} / 1$ & $<0.0002 \mathrm{mg} / \mathrm{l}$ & $<0.0002 \mathrm{mg} / \mathrm{l}$ & $<0.0002 \mathrm{mg} / 1$ & 1 & \\
\hline Pande & $0.003 \mathrm{mg} / \mathrm{l}$ & & $0.003 \mathrm{mg} / \mathrm{l}$ & & 1 & $:$ \\
\hline etraing $782-492$ & $<0.002 \mathrm{mg} / 1$ & $<0.002 \mathrm{mg} / \mathrm{l}$ & $<0.002 \mathrm{mg} / \mathrm{l}$ & $<0.002 \mathrm{mg} / \mathrm{l}$ & 1 & \\
\hline Pulfen & $22 \mathrm{mg} / \mathrm{l}$ & $<10 \mathrm{mg} / \mathrm{l}$ & $22 \mathrm{mg} / 1$ & $<10 \mathrm{mg} / \mathrm{l}$ & 1 & \\
\hline Purteceneses & $<0.05 \mathrm{mg} / \mathrm{l}$ & $<0.05 \mathrm{mg} / \mathrm{l}$ & $<0.05 \mathrm{mg} / \mathrm{l}$ & $<0.05 \mathrm{mg} / \mathrm{l}$ & 1 & \\
\hline Ahrmingon $7429090-5$ & $2.71 \mathrm{mg} / \mathrm{l}$ & $1.71 \mathrm{mg} / \mathrm{l}$ & $2.71 \mathrm{mg} / 1$ & $1.71 \mathrm{mg} / 1$ & 1 & \\
\hline $450.00740-38.2$ & $<0.04 \mathrm{mg} / \mathrm{l}$ & $<0.04 \mathrm{mg} / \mathrm{l}$ & $<0.04 \mathrm{mg} / \mathrm{l}$ & $<0.04 \mathrm{mg} / \mathrm{l}$ & 1 & \\
\hline Boron 740-128 & $0.337 \mathrm{mg} / \mathrm{l}$ & $0.324 \mathrm{mg} / \mathrm{l}$ & $0.337 \mathrm{mg} / \mathrm{l}$ & $0.324 \mathrm{mg} / 1$ & 1 & \\
\hline Berylling 740-1.7 & $<0.0004 \mathrm{mg} / \mathrm{l}$ & $<0.0004 \mathrm{mg} / \mathrm{I}$ & $<0.0004 \mathrm{mg} / 1$ & $<0.0004 \mathrm{mg} / \mathrm{l}$ & 1 & \\
\hline Canderion 7440-43-9 & $<0.004 \mathrm{mg} / \mathrm{l}$ & $<0.004 \mathrm{mg} / 1$ & $<0.004 \mathrm{mg} / \mathrm{l}$ & $<0.004 \mathrm{mg} / \mathrm{l}$ & 1 & \\
\hline 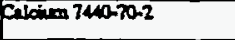 & $61.8 \mathrm{mg} / 1$ & $48.3 \mathrm{mg} / \mathrm{l}$ & $61.8 \mathrm{mg} / 1$ & $48.3 \mathrm{mg} / \mathrm{l}$ & 1 & \\
\hline cocosing 7400-77-3 & $<0.006 \mathrm{mg} / \mathrm{l}$ & $<0.006 \mathrm{mg} / \mathrm{l}$ & $<0.006 \mathrm{mg} / \mathrm{l}$ & $<0.006 \mathrm{mg} / \mathrm{l}$ & 1 & \\
\hline Cotalik 740-484 & $0.003 \mathrm{mg} / \mathrm{l}$ & $<0.002 \mathrm{mg} / 1$ & $0.003 \mathrm{mg} / 1$ & $<0.002 \mathrm{mg} / 1$ & 1 & \\
\hline Copwer 7410-50-8 & $<0.006 \mathrm{mg} / 1$ & $<0.006 \mathrm{mg} / \mathrm{l}$ & $<0.006 \mathrm{mg} / \mathrm{l}$ & $<0.006 \mathrm{mg} / 1$ & 1 & \\
\hline $50067039-596$ & $2.30 \mathrm{mg} / 1$ & $1.48 \mathrm{mg} / \mathrm{l}$ & $2.30 \mathrm{mg} / \mathrm{l}$ & $1.48 \mathrm{mg} / 1$ & 1 & \\
\hline 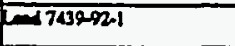 & $<0.02 \mathrm{mg} / 1$ & $<0.02 \mathrm{mg} / \mathrm{l}$ & $<0.02 \mathrm{mg} / \mathrm{l}$ & $<0.02 \mathrm{mg} / 1$ & 1 & \\
\hline Whituren 7439-93-2 & $<0.02 \mathrm{mg} / \mathrm{l}$ & $<0.02 \mathrm{mg} / \mathrm{l}$ & $<0.02 \mathrm{mg} / \mathrm{l}$ & $<0.02 \mathrm{mg} / \mathrm{l}$ & 1 & \\
\hline Maposingen 7439-95-4 & $9.12 \mathrm{mg} / \mathrm{l}$ & $7.60 \mathrm{mg} / 1$ & $9.12 \mathrm{mg} / \mathrm{l}$ & $7.60 \mathrm{mg} / \mathrm{l}$ & 1 & \\
\hline $743996-5$ & $0.112 \mathrm{mg} / \mathrm{l}$ & $0.080 \mathrm{mg} / \mathrm{l}$ & $0.112 \mathrm{mg} / 1$ & $0.080 \mathrm{mg} / 1$ & 1 & \\
\hline 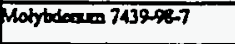 & $<0.006 \mathrm{mg} / 1$ & $<0.006 \mathrm{mg} / \mathrm{l}$ & $<0.006 \mathrm{mg} / \mathrm{l}$ & $<0.006 \mathrm{mg} / \mathrm{l}$ & 1 & \\
\hline 56017400020 & $<0.008 \mathrm{mg} / \mathrm{l}$ & $<0.008 \mathrm{mg} / \mathrm{l}$ & $<0.008 \mathrm{mg} / \mathrm{l}$ & $<0.008 \mathrm{mg} / \mathrm{l}$ & 1 & \\
\hline 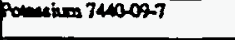 & $3.6 \mathrm{mg} / 1$ & $3.7 \mathrm{mg} / \mathrm{h}$ & $3.6 \mathrm{mg} / \mathrm{l}$ & $3.7 \mathrm{mg} / \mathrm{l}$ & 1 & \\
\hline Sibuen 7440-20-4 & $<0.006 \mathrm{mg} / \mathrm{l}$ & $<0.006 \mathrm{mg} / \mathrm{l}$ & $<0.006 \mathrm{mg} / 1$ & $<0.006 \mathrm{mg} / \mathrm{l}$ & 1 & \\
\hline sodiven 740-20-3 & $2.25 \mathrm{mg} / 1$ & $2.34 \mathrm{mg} / 1$ & $2.25 \mathrm{mg} / 1$ & $2.34 \mathrm{mg} / 1$ & 1 & $\dot{-}$ \\
\hline Tiveniug 740-32-6 & $0.03 \mathrm{mg} / \mathrm{h}$ & $0.02 \mathrm{mg} / 1$ & $0.03 \mathrm{mg} / \mathrm{l}$ & $0.02 \mathrm{mg} / \mathrm{l}$ & 1 & \\
\hline 25000740666 & $0.06 \mathrm{mg} / \mathrm{l}$ & $0.04 \mathrm{mg} / \mathrm{l}$ & $0.06 \mathrm{mg} / 1$ & $0.04 \mathrm{mg} / \mathrm{l}$ & 1 & \\
\hline
\end{tabular}


Coskinoed from front.

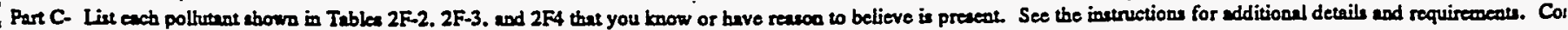
one tablo for each outfall.

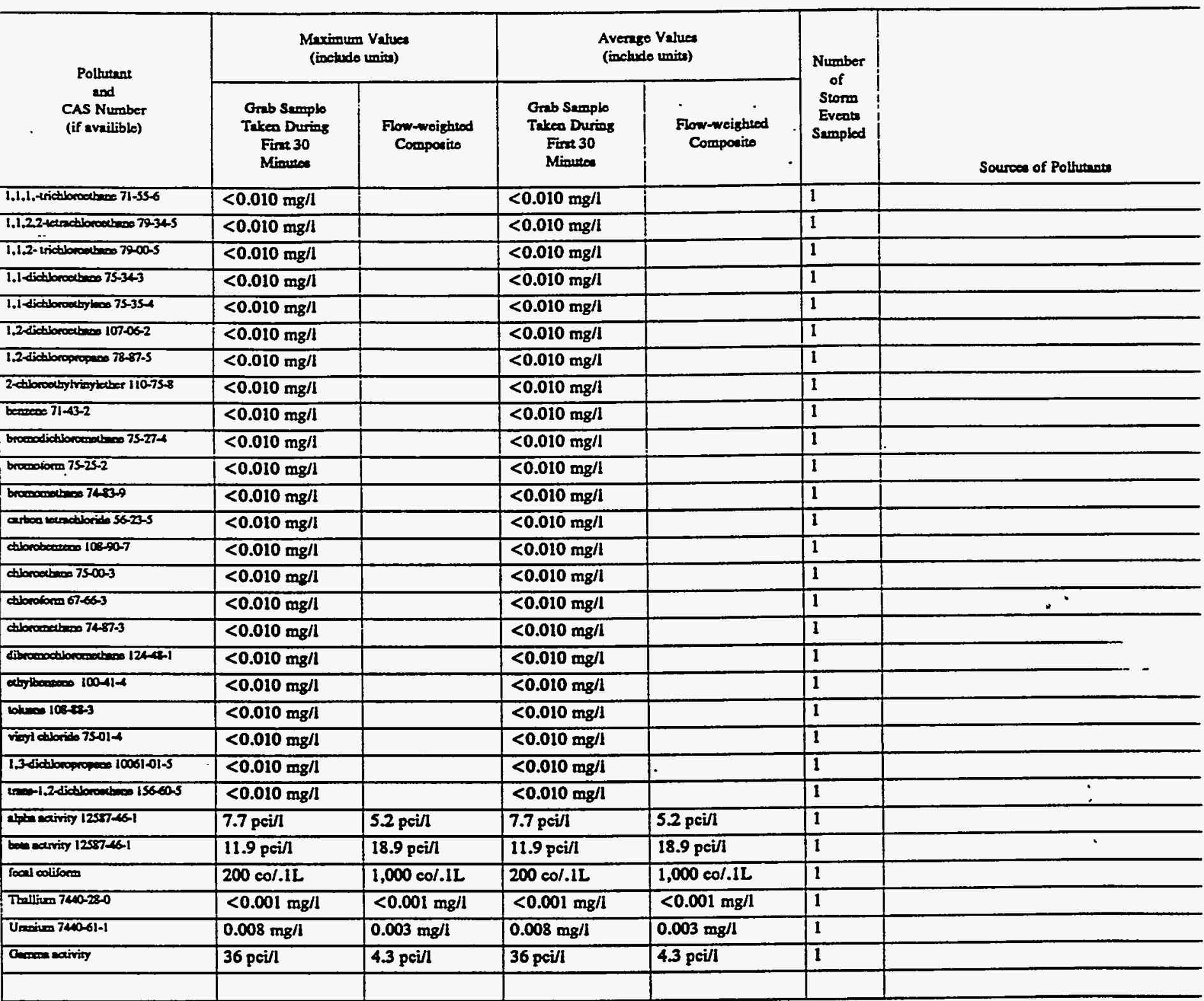

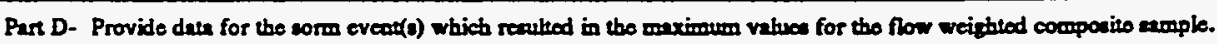

\begin{tabular}{|c|c|c|c|c|c|c|c|}
\hline $\begin{array}{l}1 . \\
\text { Date of } \\
\text { Stomn } \\
\text { Evese }\end{array}$ & $\begin{array}{l}2 . \\
\text { Duntion } \\
\text { of Storm } \\
\text { (in } \\
\text { minures) }\end{array}$ & 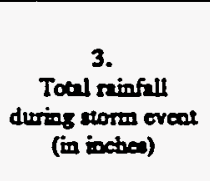 & $\begin{array}{l}4 . \\
\text { Nurber of bour between } \\
\text { beginning of storm men- } \\
\text { urod end acd of provious } \\
\text { momarnble min evere }\end{array}$ & $\begin{array}{l}\text { S. } \\
\text { Maximum flow mis } \\
\text { during nin eveot } \\
\text { (gilloodmin or epecify } \\
\text { tris) }\end{array}$ & $\begin{array}{l}6 . \\
\text { Total flow from } \\
\text { nin oveos } \\
\text { (oallowe or } \\
\text { specify unito) }\end{array}$ & $\begin{array}{c}7 . \\
\text { Season } \\
\text { samplo was } \\
\text { taken }\end{array}$ & $\begin{array}{l}8 . \\
\text { Fom of } \\
\text { Procipitution } \\
\text { (ninfull. } \\
\text { momelt) }\end{array}$ \\
\hline $\begin{array}{l}10 / 14 / 91- \\
10 / 15 / 91\end{array}$ & 650 & 1.5 & 206.25 & 521 & 117,505 & Fall & Rainfall \\
\hline
\end{tabular}

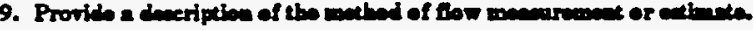

An ISCO 3230 Flow meter was used to obtain water level readings. This instrument was catibrated with the normal dry weather flow, if present, as leve zero. The flow meter was programmed with the chnrecteristics of the coaveyance so that flow rates and total flows could be calculated by the meter. 
Outfall 57

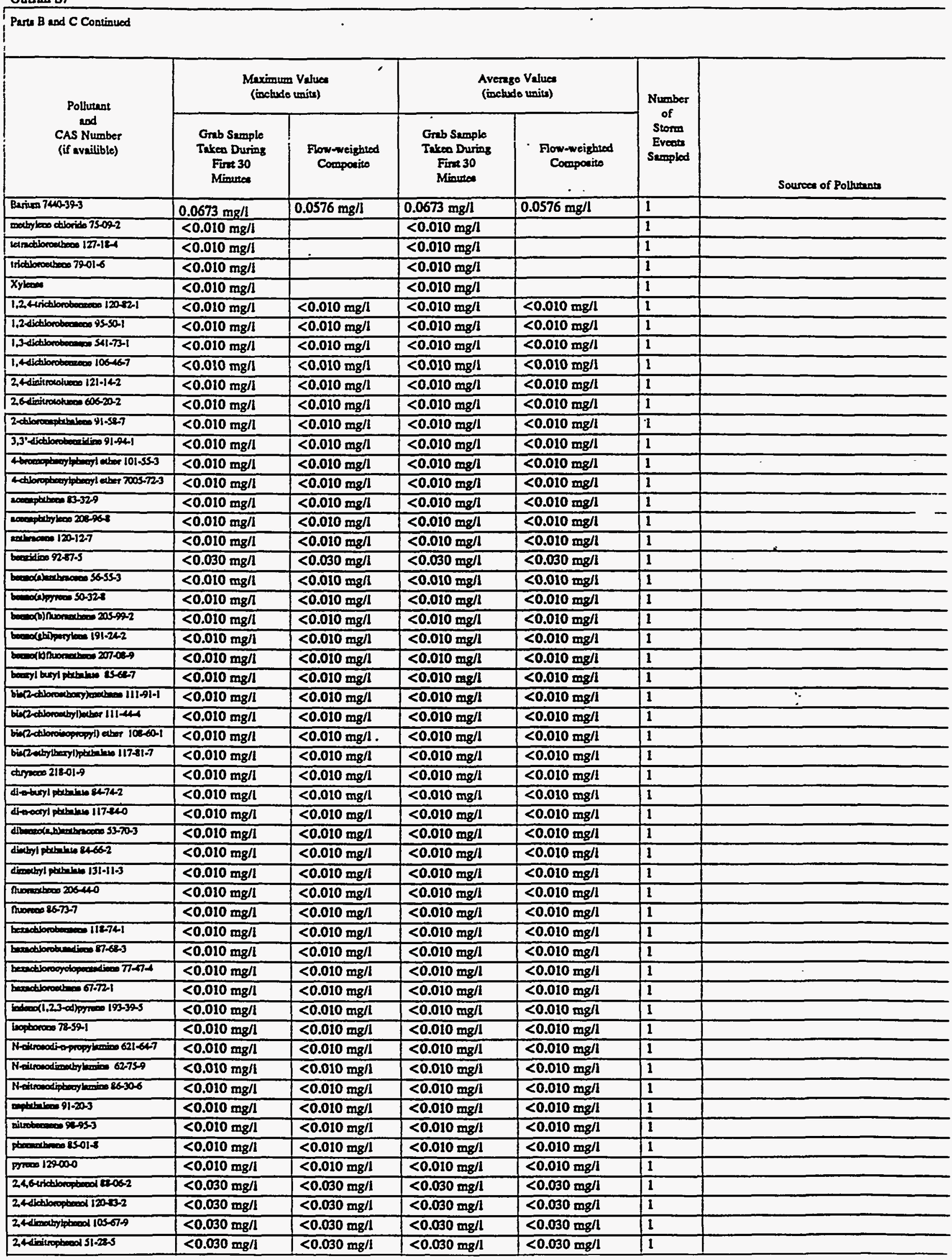


Outfall 57

Pars $B$ and $C$ Costinived

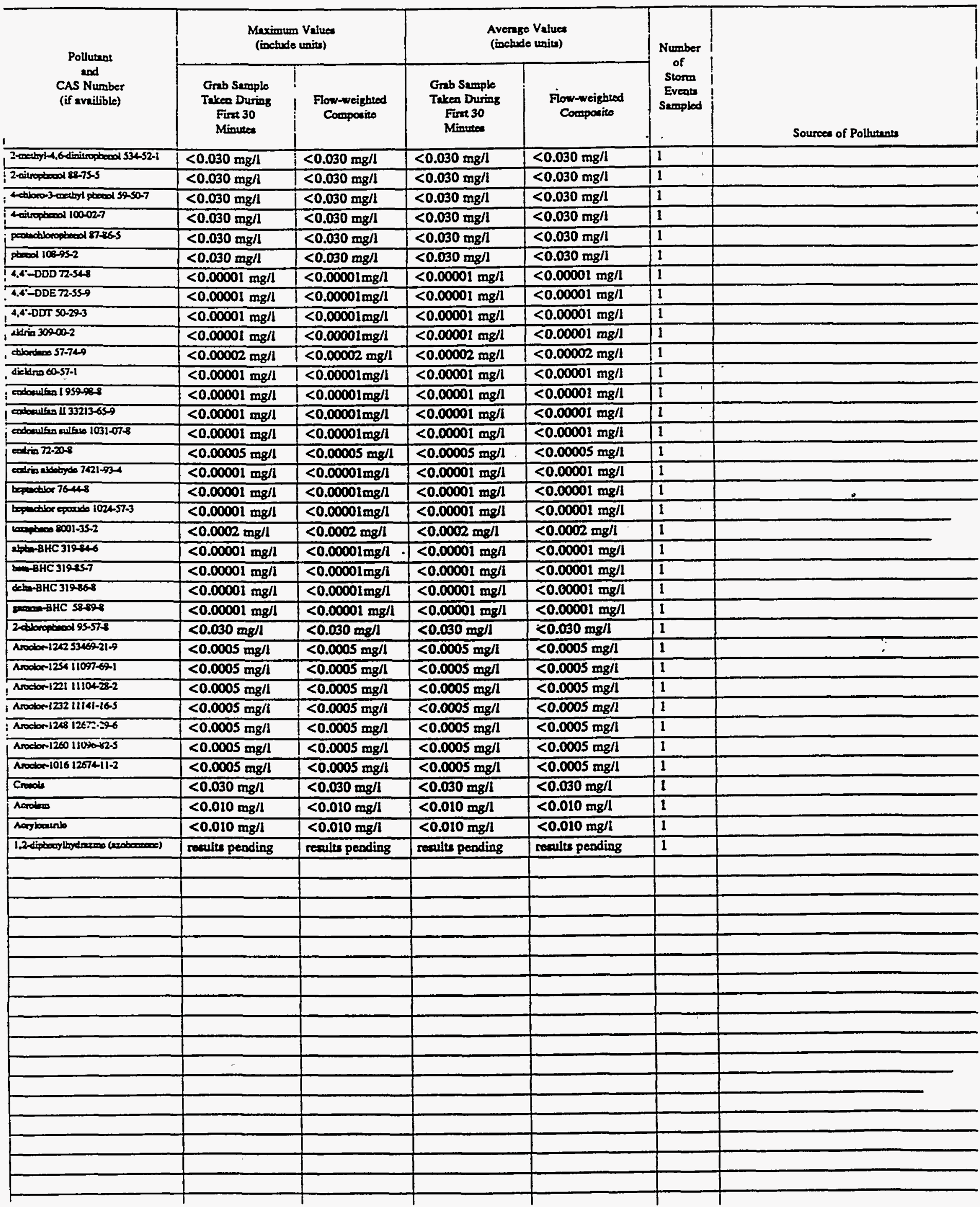


VII. Discharge Informetion (continuad from page 3 of Form 2F)

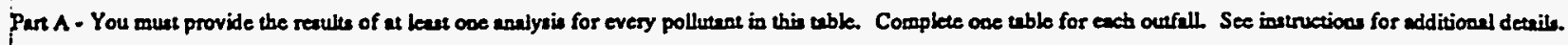

\begin{tabular}{|c|c|c|c|c|c|c|}
\hline \multirow{2}{*}{$\begin{array}{l}\text { Pollutunt } \\
\text { and } \\
\text { CAS Number } \\
\text { (if aveilible) }\end{array}$} & \multicolumn{2}{|c|}{$\begin{array}{l}\text { Mnximum Values } \\
\text { (inctude tnits) }\end{array}$} & \multicolumn{2}{|c|}{$\begin{array}{l}\text { Aversge Valuea } \\
\text { (inchude units) }\end{array}$} & \multirow{2}{*}{$\begin{array}{l}\text { Number } \\
\text { of } \\
\text { Storm } \\
\text { Events } \\
\text { Semplod }\end{array}$} & \multirow[b]{2}{*}{ Souree of Polthrants } \\
\hline & $\begin{array}{l}\text { Grab Sample } \\
\text { Taken During } \\
\text { Firnt } 30 \\
\text { Minunea }\end{array}$ & $\begin{array}{l}\text { Flow-weigbted } \\
\text { Componite }\end{array}$ & $\begin{array}{c}\text { Grab Semple } \\
\text { Taken During } \\
\text { Firn } 30 \\
\text { Minuea }\end{array}$ & $\begin{array}{l}\text { Flow-weighted } \\
\text { Comporive }\end{array}$ & & \\
\hline bil and Groues & $7 \mathrm{mg} / \mathrm{l}$ & & $7 \mathrm{mg} / \mathrm{l}$ & & 1 & \\
\hline Chemial Oxyper Desend (COD) & $26 \mathrm{mg} / 1$ & $31 \mathrm{mg} / \mathrm{l}$ & $26 \mathrm{mg} / \mathrm{l}$ & $31 \mathrm{mg} / 1$ & 1 & \\
\hline 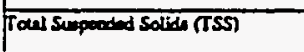 & $16 \mathrm{mg} / \mathrm{l}$ & $20 \mathrm{mg} / \mathrm{l}$ & $16 \mathrm{mg} / \mathrm{l}$ & $20 \mathrm{mg} / 1$ & 1 & \\
\hline Toed Kje thebl Nitrogero & $0.8 \mathrm{mg} / \mathrm{l}$ & $0.9 \mathrm{mg} / \mathrm{l}$ & $0.8 \mathrm{mg} / \mathrm{l}$ & $0.9 \mathrm{mg} / \mathrm{l}$ & 1 & \\
\hline Nitue plue Nitrie Nitrogen & $0.27 \mathrm{mg} / \mathrm{l}$ & $0.37 \mathrm{mg} / 1$ & $0.27 \mathrm{mg} / 1$ & $0.37 \mathrm{mg}$ & 1 & \\
\hline 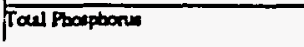 & $0.19 \mathrm{mg} / \mathrm{l}$ & $0.20 \mathrm{mg} / \mathrm{l}$ & $0.19 \mathrm{mg} / 1$ & $0.20 \mathrm{mg} / \mathrm{l}$ & 1 & \\
\hline
\end{tabular}

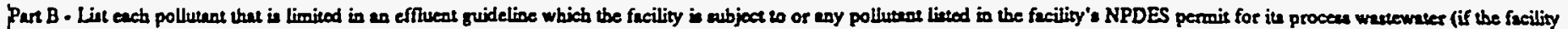
is operuting under en existin. NPDES permil). Complete coe tuble for each outfall. See the inetruction for additioal detrilh and requirementa.

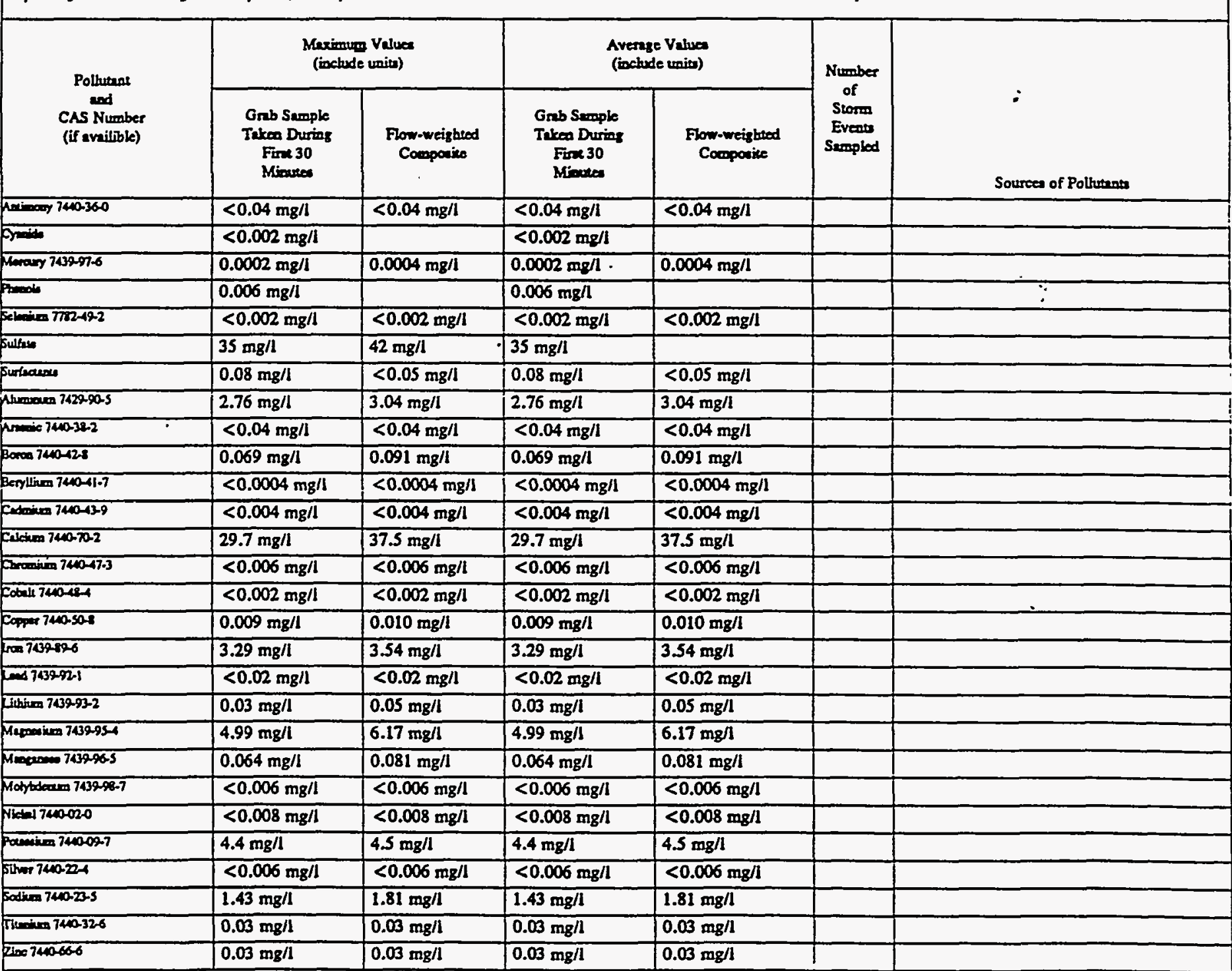


Part C- Liat each pollutunt stown in Tables 2F-2, 2F-3, and 2F4 that you know or tave reason to believe is present. See the instruetions for additional details and requiremeots. Con one uble for each ourfall.

\begin{tabular}{|c|c|c|c|c|c|c|}
\hline & $\begin{array}{l}\text { Mexi } \\
\text { (ine }\end{array}$ & $\begin{array}{l}\text { in Values } \\
\text { je units) }\end{array}$ & $\begin{array}{l}\text { Ave } \\
\text { (in }\end{array}$ & $\begin{array}{l}\text { ge Values } \\
\text { de unita) }\end{array}$ & $\begin{array}{l}\text { ! } \\
\text { - Number }\end{array}$ & ' \\
\hline $\begin{array}{l}\text { CAS Number } \\
\text { (if availible) }\end{array}$ & $\begin{array}{l}\text { Grab Sample } \\
\text { Taked During } \\
\text { Fint } 30 \\
\text { Minutes }\end{array}$ & $\begin{array}{c}\text { Flow-weightod } \\
\text { Componite }\end{array}$ & $\begin{array}{c}\text { Grab Sample } \\
\text { Taken During } \\
\text { Fint } 30 \\
\text { Minurea }\end{array}$ & $\begin{array}{l}\text { Flow-weighied } \\
\text { Composite }\end{array}$ & $\begin{array}{l}\text { Storm } \\
\text { Events } \\
\text { Sempled } \\
\text {. }\end{array}$ & Source of Pollumana \\
\hline 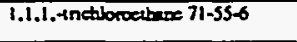 & $<0.010 \mathrm{mg} / 1$ & & $i<0.010 \mathrm{mg} / \mathrm{l}$ & & 1 & 1 \\
\hline 1.1.22-tetenchloretherse 79-345 & $:<0.010 \mathrm{mg} / 1$ & . & $:<0.010 \mathrm{mg} / \mathrm{l}$ & & 1 & $!$ \\
\hline 1.1.2- trichbordectere 7900-5 & $:<0.010 \mathrm{mg} / \mathrm{l}$ & & $<0.010 \mathrm{mg} / 1$ & & 1 & ! \\
\hline 1.1 dictionecthen 75-34-3 & $i<0.010 \mathrm{mg} / \mathrm{l}$ & 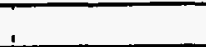 & $<0.010 \mathrm{mg} / \mathrm{l}$ & : & 1 & $T$ \\
\hline 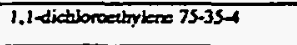 & $<0.010 \mathrm{mg} / 1$ & & $<0.010 \mathrm{mg} / 1$ & & 1 & $!$ \\
\hline 1.I-dictbroethere 107-06-2 & $<0.010 \mathrm{mg} / 1$ & & $<0.010 \mathrm{mg} / \mathrm{l}$ & - & 1 & 1 \\
\hline 1.2-dichlorepropenos $78-87.5$ & $<0.010 \mathrm{mg} / \mathrm{l}$ & & $1<0.010 \mathrm{mg} / 1$ & & 1 & $\mathrm{i}$ \\
\hline I-ctionathylviongleter $110-75-8$ & $<0.010 \mathrm{mg} / \mathrm{l}$ & $\overline{3}$ & $<0.010 \mathrm{mg} / 1$ & $\bullet$ & 1 & 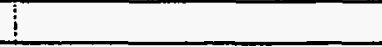 \\
\hline $71-43.2$ & $<0.010 \mathrm{mg} / \mathrm{l}$ & & $1<0.010 \mathrm{mg} / \mathrm{l}$ & $\cdot$ & il & 1 \\
\hline 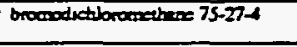 & , <0.010 mg/l & & $1<0.010 \mathrm{mg} / \mathrm{l}$ & $\cdot$ & 1 & \\
\hline broconotion $75.25-2$ & $i<0.010 \mathrm{mg} / \mathrm{l}$ & & $i<0.010 \mathrm{mg} / \mathrm{l}$ & $i$ & $i$ & 1 \\
\hline 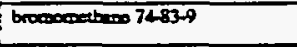 & $1<0.010 \mathrm{mg} / \mathrm{l}$ & $\therefore$ & $\mathrm{i}<0.010 \mathrm{mg} / \mathrm{l}$ & ; & 1 & \\
\hline 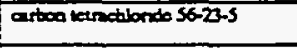 & $1<0.010 \mathrm{mg} / \mathrm{l}$ & $i$ & $<0.010 \mathrm{mg} / \mathrm{t}$ & 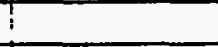 & 1 & \\
\hline chlorobesecos $108-90-7$ & i $<0.010 \mathrm{mg} / 1$ & ; & $<0.010 \mathrm{mg} / \mathrm{l}$ & . & 1 & \\
\hline 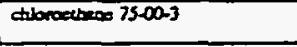 & $1<0.010 \mathrm{mg} / \mathrm{l}$ & $\therefore$ & $<0.010 \mathrm{mg} / 1$ & $i$ & 1 & \\
\hline atoonotord $67-66-3$ & $<0.010 \mathrm{mg} / \mathrm{l}$ & 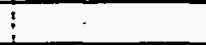 & $<0.010 \mathrm{mg} / \mathrm{l}$ & 1 & 1 & 1 \\
\hline ctoreanethene 74-87-3 & $<0.010 \mathrm{mg} / 1$ & $\vdots$ & $<0.010 \mathrm{mg} / \mathrm{l}$ & 7 & 1 & \\
\hline dibromotibrecothere $124-48-1$ & $i^{<0.010 \mathrm{mg} / \mathrm{l}}$ & $!$ & $<0.010 \mathrm{mg} / 1$ & ! & 1 & \\
\hline 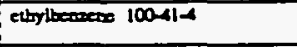 & $i<0.010 \mathrm{mg} / \mathrm{i}$ & $\therefore$ & $<0.010 \mathrm{mg} / \mathrm{l}$ & $!$ & 1 & \\
\hline cotheres $108-83-3$ & $<0.010 \mathrm{mg} / \mathrm{l}$ & i & $<0.010 \mathrm{mg} / \mathrm{l}$ & 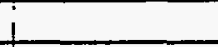 & 1 & \\
\hline vityl ctlonide 75.01A & $<0.010 \mathrm{mg} / 1$ & & $<0.010 \mathrm{mg} / \mathrm{l}$ & & 1 & \\
\hline IJ-Jictioropropene 1006l-01-5 & $<0.010 \mathrm{mg} / \mathrm{l}$ & & $<0.010 \mathrm{mg} / \mathrm{l}$ & 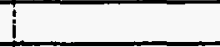 & 1 & \\
\hline 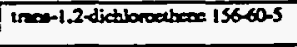 & 1 $<0.010 \mathrm{mg} / 1$ & & $<0.010 \mathrm{mg} / 1$ & & 1 & $\therefore$ \\
\hline slphe earvity $12587-66-1$ & $250 \mathrm{pci} / \mathrm{l}$ & $6.2 \mathrm{pci} / \mathrm{l}$ & $250 \mathrm{pci} / 1$ & $6.2 \mathrm{pci} / \mathrm{l}$ & 1 & \\
\hline betarimty $12587-16-1$ & 120 pcill & $8.3 \mathrm{pci} / 1$ & $120 p c i / 1$ & $8.3 \mathrm{pci} / 1$ & 1 & 1 \\
\hline Tecal oolifoms & $1.300 \mathrm{co} / .1 \mathrm{~L}$ & $30 \mathrm{col} .1 \mathrm{~L}$ & $1.300 \mathrm{co} / .1 \mathrm{~L}$ & $30 \mathrm{co} / .1 \mathrm{~L}$ & 11 & $!$ \\
\hline Thallimen 7440-28-0 & $<0.03 \mathrm{mg} / \mathrm{l}$ & $<0.03 \mathrm{mg} / \mathrm{l}$ & $<0.03 \mathrm{mg} / \mathrm{l}$ & $<0.03 \mathrm{mg} / \mathrm{l}$ & 11 & i \\
\hline Unarem 7440-61-1 & $1^{0.347 \mathrm{mg} / 1}$ & $0.560 \mathrm{mg} / \mathrm{l}$ & $0.347 \mathrm{mg} / 1$ & $0.560 \mathrm{mg} / \mathrm{l}$ & $i$ & 1 \\
\hline Garrice eavity & -2 pci/l & $24 \mathrm{pci} / \mathrm{l}$ & -2 pcill & $24 \mathrm{pci} / \mathrm{l}$ & 1 & \\
\hline I & & & & & i & \\
\hline
\end{tabular}

Pan D- Provide dak for the som eveat(s) which ranulied in the maximum values for tho flow weighted componte anmple.

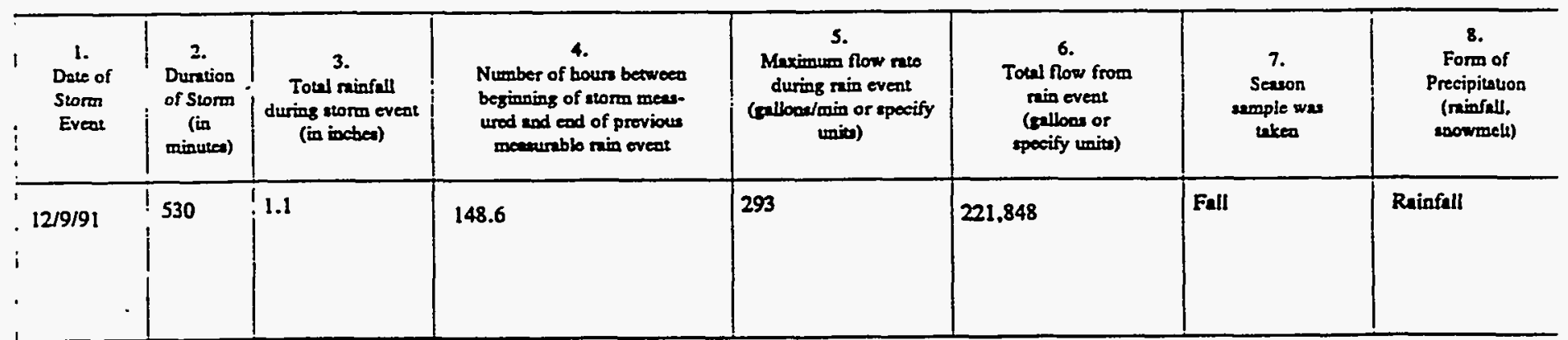

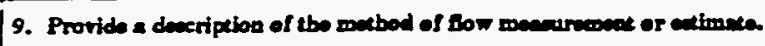

An ISCO 3230 Flow meter was used to obtain water level readings. This instrument was calibrated with the normal dry weather flow, if preseat, as leve zero. The flow meter was programmed with the characteristics of the conveyance so that flow mates and total flows could be calculated by the meter. 
Outfall 58

Parts B and C Continued

\begin{tabular}{|c|c|c|c|c|c|c|}
\hline \multirow{2}{*}{$\begin{array}{l}\text { Pollutane } \\
\text { and } \\
\text { CAS Number } \\
\text { (if availible) }\end{array}$} & \multicolumn{2}{|c|}{$\begin{array}{l}\text { Meximum Valuea } \\
\text { (inchude units) }\end{array}$} & \multicolumn{2}{|c|}{$\begin{array}{l}\text { Avenge Values } \\
\text { (inchude unius) }\end{array}$} & \multirow{2}{*}{$\begin{array}{c}\text { Number } \\
\text { of } \\
\text { Storm } \\
\text { Events } \\
\text { Stropled }\end{array}$} & \multirow[b]{2}{*}{ Sources of Polluzeses } \\
\hline & $\begin{array}{l}\text { Grab Sample } \\
\text { Taken Duriog } \\
\text { First } 30 \\
\text { Minutea }\end{array}$ & $\begin{array}{l}\text { Fow-weighrod } \\
\text { Composite }\end{array}$ & $\begin{array}{l}\text { Grab Sample } \\
\text { Taken Duriag } \\
\text { First } 30 \\
\text { Minutea }\end{array}$ & $\begin{array}{l}\text { Flow-weighted } \\
\text { Composite }\end{array}$ & & \\
\hline Beriem 740-39-3 & $0.0377 \mathrm{mg} / \mathrm{l}$ & $0.0438 \mathrm{mg} / \mathrm{h}$ & $0.0377 \mathrm{mg} / \mathrm{l}$ & $0.0438 \mathrm{mg} / \mathrm{l}$ & 1 & \\
\hline 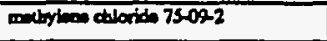 & $<0.010 \mathrm{mg} / 1$ & & $<0.010 \mathrm{mg} / \mathrm{l}$ & & 1 & \\
\hline 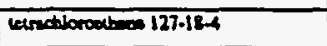 & $<0.010 \mathrm{mg} / \mathrm{l}$ & & $<0.010 \mathrm{mg} /$ & & 1 & \\
\hline 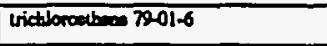 & $<0.010 \mathrm{mg} / \mathrm{l}$ & & $<0.010 \mathrm{mg} / \mathrm{l}$ & & 1 & \\
\hline Xyleowe & $<0.010 \mathrm{mg} / 1$ & & $<0.010 \mathrm{mg} / 1$ & & 1 & \\
\hline 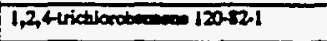 & $<0.010 \mathrm{mg} / \mathrm{l}$ & $<0.010 \mathrm{mg} / \mathrm{l}$ & $<0.010 \mathrm{mg} / \mathrm{l}$ & $<0.010 \mathrm{mg} / \mathrm{l}$ & 1 & \\
\hline 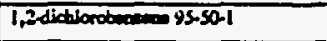 & $<0.010 \mathrm{mg} / \mathrm{l}$ & $<0.010 \mathrm{mg} / \mathrm{l}$ & $<0.010 \mathrm{mg} / \mathrm{l}$ & $<0.010 \mathrm{mg} / 1$ & 1 & \\
\hline 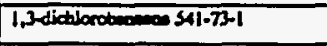 & $<0.010 \mathrm{mg} / \mathrm{l}$ & $<0.010 \mathrm{mg} / \mathrm{l}$ & $<0.010 \mathrm{mg} / \mathrm{l}$ & $<0.010 \mathrm{mg} / \mathrm{l}$ & 1 & \\
\hline 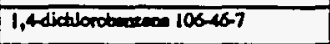 & $<0.010 \mathrm{mg} / \mathrm{l}$ & $<0.010 \mathrm{mg} / \mathrm{l}$ & $<0.010 \mathrm{mg} / \mathrm{l}$ & $<0.010 \mathrm{mg} / \mathrm{I}$ & 1 & \\
\hline 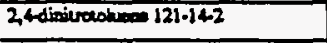 & $<0.010 \mathrm{mg} / \mathrm{l}$ & $<0.010 \mathrm{mg} / \mathrm{l}$ & $<0.010 \mathrm{mg} / \mathrm{h}$ & $<0.010 \mathrm{mg} / 1$ & 1 & \\
\hline 2,6-dinitrocotam $606-20,2$ & $<0.010 \mathrm{mg} / 1$ & $<0.010 \mathrm{mg} / 1$ & $<0.010 \mathrm{mg} / \mathrm{L}$ & $<0.010 \mathrm{mg} / \mathrm{l}$ & 1 & \\
\hline 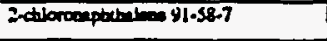 & $<0.010 \mathrm{mg} / \mathrm{l}$ & $<0.010 \mathrm{mg} / 1$ & $<0.010 \mathrm{mg} /$ & $<0.010 \mathrm{mg} / 1$ & 1. & \\
\hline 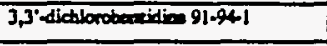 & $<0.010 \mathrm{mg} / 1$ & $<0.020 \mathrm{mg} / \mathrm{l}$ & $<0.010 \mathrm{mg} / \mathrm{l}$ & $<0.020 \mathrm{mg} / \mathrm{l}$ & 1 & \\
\hline 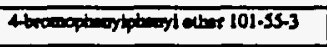 & $<0.010 \mathrm{mg} / \mathrm{l}$ & $<0.010 \mathrm{mg} / 1$ & $<0.010 \mathrm{mg} / \mathrm{l}$ & $<0.010 \mathrm{mg} / \mathrm{l}$ & 1 & \\
\hline 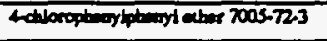 & $<0.010 \mathrm{mg} / 1$ & $<0.010 \mathrm{mg} / \mathrm{l}$ & $<0.010 \mathrm{mg} / \mathrm{l}$ & $<0.010 \mathrm{mg} / \mathrm{l}$ & 1 & \\
\hline $20000+16-0.23-32-9$ & $<0.010 \mathrm{mg} / \mathrm{l}$ & $<0.010 \mathrm{mg} / \mathrm{l}$ & $<0.010 \mathrm{mg} / 1$ & $<0.010 \mathrm{mg} / \mathrm{l}$ & 1 & \\
\hline 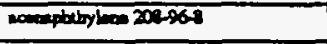 & $<0.010 \mathrm{mg} / \mathrm{l}$ & $<0.010 \mathrm{mg} / \mathrm{l}$ & $<0.010 \mathrm{mg} / \mathrm{l}$ & $<0.010 \mathrm{mg} / \mathrm{l}$ & 1 & \\
\hline entrocosed $120-12.7$ & $<0.010 \mathrm{mg} / \mathrm{l}$ & $<0.010 \mathrm{mg} / 1$ & $<0.010 \mathrm{mg} / \mathrm{l}$ & $<0.010 \mathrm{mg} / \mathrm{l}$ & 1 & \\
\hline batidion 92.57 .5 & $<0.050 \mathrm{mg} / \mathrm{l}$ & $<0.050 \mathrm{mg} / 1$ & $<0.050 \mathrm{mg} / 1$ & $<0.050 \mathrm{mg} / \mathrm{l}$ & 1 & \\
\hline 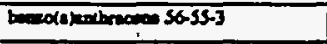 & $<0.010 \mathrm{mg} / \mathrm{l}$ & $<0.010 \mathrm{mg} / \mathrm{l}$ & $<0.010 \mathrm{mg} / \mathrm{l}$ & $<0.010 \mathrm{mg} / \mathrm{l}$ & 1 & \\
\hline 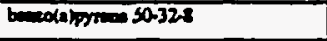 & $<0.010 \mathrm{mg} / \mathrm{l}$ & $<0.010 \mathrm{mg} / 1$ & $<0.010 \mathrm{mg} / \mathrm{l}$ & $<0.010 \mathrm{mg} / \mathrm{l}$ & 1 & \\
\hline 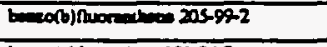 & $<0.010 \mathrm{mg} / \mathrm{l}$ & $<0.010 \mathrm{mg} / \mathrm{l}$ & $<0.010 \mathrm{mg} / \mathrm{l}$ & $<0.010 \mathrm{mg} / \mathrm{l}$ & 1 & \\
\hline 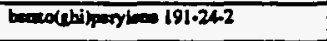 & $<0.010 \mathrm{mg} / \mathrm{l}$ & $<0.010 \mathrm{mg} / 1$ & $<0.010 \mathrm{mg} / 1$ & $<0.010 \mathrm{mg} / \mathrm{l}$ & 1 & \\
\hline 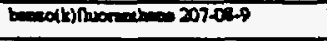 & $<0.010 \mathrm{mg} / \mathrm{l}$ & $<0.010 \mathrm{mg} / \mathrm{l}$ & $<0.010 \mathrm{mg} / \mathrm{h}$ & $<0.010 \mathrm{mg} / \mathrm{l}$ & 1 & \\
\hline 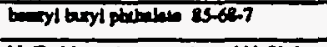 & $35 \mathrm{mg} / \mathrm{l}$ & $19 \mathrm{mg} / \mathrm{l}$ & $35 \mathrm{mg} / \mathrm{l}$ & $19 \mathrm{mg} / 1$ & 1 & \\
\hline 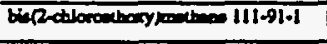 & $<0.010 \mathrm{mg} / !$ & $<0.010 \mathrm{mg} / 1$ & $<0.010 \mathrm{mg} / \mathrm{l}$ & $<0.010 \mathrm{mg} / \mathrm{h}$ & 1 & $\because$ \\
\hline 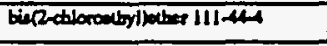 & $<0.010 \mathrm{mg} / \mathrm{l}$ & $<0.010 \mathrm{mg} / \mathrm{l}$ & $<0.010 \mathrm{mg} / \mathrm{l}$ & $<0.010 \mathrm{mg} / \mathrm{l}$ & 1 & \\
\hline 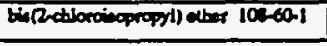 & $<0.010 \mathrm{mg} / \mathrm{l}$ & $<0.010 \mathrm{mg} / 1$ & $<0.010 \mathrm{mg} / \mathrm{l}$ & $<0.010 \mathrm{mg} / \mathrm{l}$ & 1 & \\
\hline 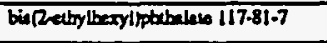 & $100 \mathrm{mg} / \mathrm{l}$ & $78 \mathrm{mg} / \mathrm{l}$ & $100 \mathrm{mg} / \mathrm{l}$ & $78 \mathrm{mg} / \mathrm{l}$ & 1 & \\
\hline doryoue $218-01.9$ & $<0.010 \mathrm{mg} / \mathrm{I}$ & $<0.010 \mathrm{mg} / \mathrm{l}$ & $<0.010 \mathrm{mg} / \mathrm{l}$ & $<0.010 \mathrm{mg} / \mathrm{l}$ & 1 & \\
\hline 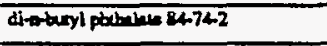 & $77 \mathrm{mg} / \mathrm{l}$ & $140 \mathrm{mg} / \mathrm{l}$ & $77 \mathrm{mg} / \mathrm{h}$ & $140 \mathrm{mg} / \mathrm{l}$ & 1 & \\
\hline 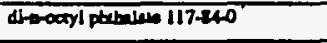 & $<0.010 \mathrm{mg} / \mathrm{l}$ & $<0.010 \mathrm{mg} / \mathrm{l}$ & $<0.010 \mathrm{mg} / \mathrm{l}$ & $<0.010 \mathrm{mg} / \mathrm{l}$ & 1 & \\
\hline 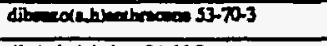 & $<0.010 \mathrm{mg} / \mathrm{l}$ & $<0.010 \mathrm{mg} / \mathrm{l}$ & $<0.010 \mathrm{mg} / \mathrm{l}$ & $<0.010 \mathrm{mg} / 1$ & 1 & \\
\hline 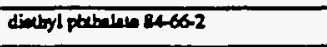 & $<0.010 \mathrm{mg} / \mathrm{l}$ & $<0.010 \mathrm{mg} / \mathrm{l}$ & $<0.010 \mathrm{mg} / \mathrm{l}$ & $<0.010 \mathrm{mg} / \mathrm{I}$ & 1 & \\
\hline 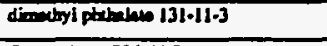 & $<0.010 \mathrm{mg} / \mathrm{l}$ & $<0.010 \mathrm{mg} / \mathrm{l}$ & $<0.010 \mathrm{mg} / 1$ & $<0.010 \mathrm{mg} / \mathrm{h}$ & 1 & \\
\hline 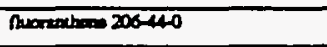 & $<0.010 \mathrm{mg} / \mathrm{l}$ & $<0.010 \mathrm{mg} / \mathrm{l}$ & $<0.010 \mathrm{mg} / \mathrm{l}$ & $<0.010 \mathrm{mg} / 1$ & 1 & \\
\hline nemene 26.73-7 & $<0.010 \mathrm{mg} / \mathrm{l}$ & $<0.010 \mathrm{mg} / \mathrm{l}$ & $<0.010 \mathrm{mg} / \mathrm{l}$ & $<0.010 \mathrm{mg} / 1$ & 1 & \\
\hline 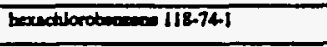 & $<0.010 \mathrm{mg} / \mathrm{l}$ & $<0.010 \mathrm{mg} / \mathrm{l}$ & $<0.010 \mathrm{mg} / \mathrm{l}$ & $<0.010 \mathrm{mg} / 1$ & 1 & \\
\hline 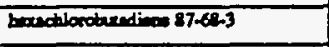 & $<0.010 \mathrm{mg} / \mathrm{l}$ & $<0.010 \mathrm{mg} / \mathrm{l}$ & $<0.010 \mathrm{mg} / \mathrm{l}$ & $<0.010 \mathrm{mg} / \mathrm{l}$ & 1 & \\
\hline 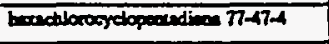 & $<0.010 \mathrm{mg} / \mathrm{l}$ & $<0.010 \mathrm{mg} / \mathrm{l}$ & $<0.010 \mathrm{mg} / \mathrm{l}$ & $<0.010 \mathrm{mg} / 1$ & 1 & \\
\hline 67.72 .1 & $<0.010 \mathrm{mg} / \mathrm{l}$ & $<0.010 \mathrm{mg} / \mathrm{l}$ & $<0.010 \mathrm{mg} / \mathrm{l}$ & $<0.010 \mathrm{mg} / \mathrm{l}$ & 1 & \\
\hline 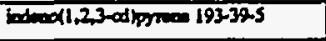 & $<0.010 \mathrm{mg} / \mathrm{l}$ & $<0.010 \mathrm{mg} / \mathrm{l}$ & $<0.010 \mathrm{mg} / \mathrm{l}$ & $<0.010 \mathrm{mg} / 1$ & 1 & \\
\hline incphoroen 72-591 & $<0.010 \mathrm{mg} / 1$ & $<0.010 \mathrm{mg} / 1$ & $<0.010 \mathrm{mg} / \mathrm{l}$ & $<0.010 \mathrm{mg} / \mathrm{l}$ & 1 & \\
\hline 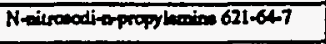 & $<0.010 \mathrm{mg} / \mathrm{I}$ & $<0.010 \mathrm{mg} / \mathrm{l}$ & $<0.010 \mathrm{mg} / \mathrm{l}$ & $<0.010 \mathrm{mg} / \mathrm{l}$ & 1 & \\
\hline 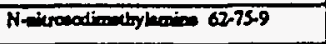 & $<0.010 \mathrm{mg} / \mathrm{l}$ & $<0.010 \mathrm{mg} / \mathrm{l}$ & $<0.010 \mathrm{mg} / \mathrm{l}$ & $<0.010 \mathrm{mg} / 1$ & 1 & \\
\hline 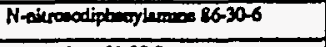 & $<0.010 \mathrm{mg} / \mathrm{I}$ & $<0.010 \mathrm{mg} / \mathrm{l}$ & $<0.010 \mathrm{mg} / \mathrm{l}$ & $<0.010 \mathrm{mg} / 1$ & 1 & \\
\hline mpletheloon $91.20-3$ & $<0.010 \mathrm{mg} / \mathrm{l}$ & $<0.010 \mathrm{mg} / \mathrm{l}$ & $<0.010 \mathrm{mg} / \mathrm{l}$ & $<0.010 \mathrm{mg} / \mathrm{I}$ & 1 & \\
\hline 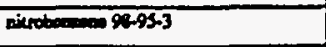 & $<0.010 \mathrm{mg} / \mathrm{l}$ & $<0.010 \mathrm{mg} / \mathrm{l}$ & $<0.010 \mathrm{mg} / \mathrm{l}$ & $<0.010 \mathrm{mg} / \mathrm{l}$ & 1 & \\
\hline ptomation 2501.8 & $<0.010 \mathrm{mg} / \mathrm{l}$ & $<0.010 \mathrm{mg} / \mathrm{l}$ & $<0.010 \mathrm{mg} / 1$ & $<0.010 \mathrm{mg} / 1$ & 1 & \\
\hline 070000129000 & $<0.010 \mathrm{mg} / \mathrm{l}$ & $<0.010 \mathrm{mg} / \mathrm{l}$ & $<0.010 \mathrm{mg} / \mathrm{l}$ & $<0.010 \mathrm{mg} / \mathrm{l}$ & 1 & \\
\hline 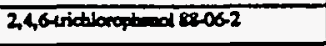 & $<0.030 \mathrm{mg} / \mathrm{l}$ & $<0.030 \mathrm{mg} / \mathrm{l}$ & $<0.030 \mathrm{mg} / \mathrm{l}$ & $<0.030 \mathrm{mg} / 1$ & 1 & \\
\hline 24-dictibeopheod 120-13-2 & $<0.030 \mathrm{mg} / \mathrm{l}$ & $<0.030 \mathrm{mg} / \mathrm{l}$ & $<0.030 \mathrm{mg} / \mathrm{l}$ & $<0.030 \mathrm{mg} / \mathrm{l}$ & 1 & \\
\hline 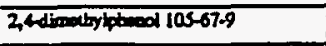 & $<0.030 \mathrm{mg} / \mathrm{l}$ & $<0.030 \mathrm{mg} / \mathrm{h}$ & $<0.030 \mathrm{mg} / \mathrm{l}$ & $<0.030 \mathrm{mg} / \mathrm{l}$ & 1 & \\
\hline 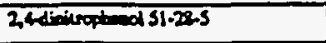 & $<0.050 \mathrm{mg} / \mathrm{l}$ & $<0.050 \mathrm{mg} / \mathrm{l}$ & $<0.050 \mathrm{mg} / 1$ & $<0.050 \mathrm{mg} / \mathrm{l}$ & 1 & \\
\hline
\end{tabular}


Outfall 58

Pars B and C Continiod

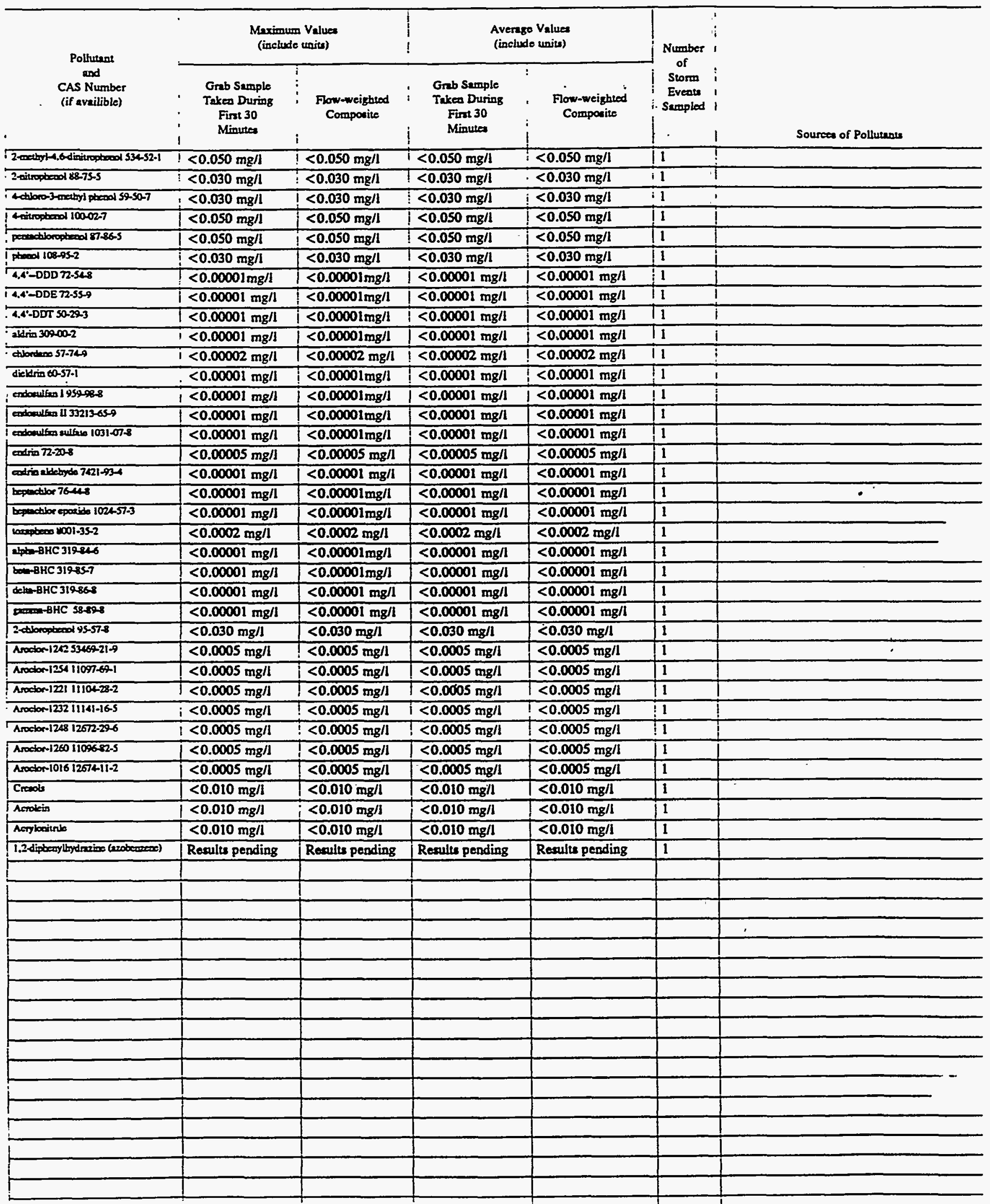


VII. Discharge Information (continuod frocos page 3 of Form 2A)

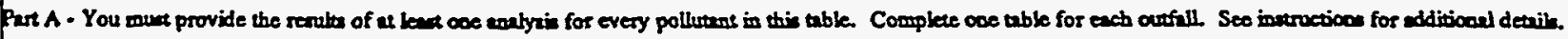

\begin{tabular}{|c|c|c|c|c|c|c|}
\hline \multirow{2}{*}{$\begin{array}{l}\text { Poltumat } \\
\text { end } \\
\text { CAs Number } \\
\text { (if milible) }\end{array}$} & \multicolumn{2}{|c|}{$\begin{array}{l}\text { Maximpon Valuear } \\
\text { (ivectude waib) }\end{array}$} & \multicolumn{2}{|c|}{$\begin{array}{l}\text { Average Valuea } \\
\text { (inebude unial) }\end{array}$} & \multirow{2}{*}{$\begin{array}{l}\text { Nomber } \\
\text { of } \\
\text { Storm } \\
\text { Evente } \\
\text { Sumplad }\end{array}$} & \multirow[b]{2}{*}{ Sourese of Polluranes } \\
\hline & 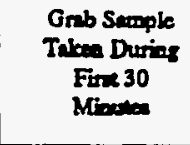 & $\begin{array}{l}\text { Fon-weighed } \\
\text { Compocice }\end{array}$ & 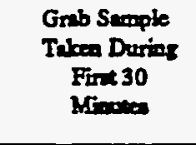 & $\begin{array}{c}\text { From-weigheded } \\
\text { Comporice }\end{array}$ & & \\
\hline Pil & $4 \mathrm{mg} / \mathrm{l}$ & & $4 \mathrm{mg} / 1$ & & 1 & \\
\hline 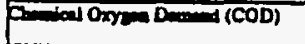 & $<5 \mathrm{mg} / 1$ & $37 \mathrm{mg} / 1$ & $<5 \mathrm{mg} / \mathrm{l}$ & $37 \mathrm{mg} / 1$ & 1 & \\
\hline Toul Suppod solith (TSS) & $80 \mathrm{mg} / \mathrm{l}$ & $64 \mathrm{mg} / 1$ & $80 \mathrm{mg} / 1$ & $64 \mathrm{mg} / \mathrm{l}$ & 1 & \\
\hline Toul Kjolleb Niturom & $0.8 \mathrm{mg} / \mathrm{l}$ & $0.7 \mathrm{mg} / \mathrm{l}$ & $0.8 \mathrm{mg} / 1$ & $0.7 \mathrm{mg} / \mathrm{l}$ & 1 & \\
\hline Nhen phe Nikrie Nikrogen & $0.4 \mathrm{mg} / 1$ & $0.7 \mathrm{mg} / \mathrm{l}$ & $0.4 \mathrm{mp} / \mathrm{l}$ & $0.7 \mathrm{mg} / \mathrm{l}$ & 1 & \\
\hline 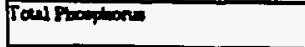 & $0.30 \mathrm{mg} / \mathrm{I}$ & $0.21 \mathrm{mg} / \mathrm{l}$ & $0.30 \mathrm{mg} / 1$ & $0.21 \mathrm{mg} / 1$ & 1 & \\
\hline
\end{tabular}

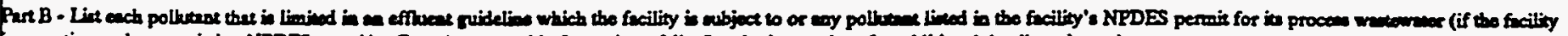

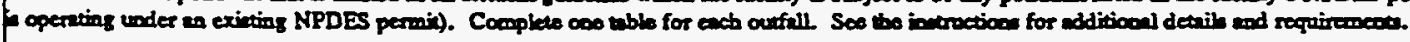

\begin{tabular}{|c|c|c|c|c|c|c|}
\hline \multirow{2}{*}{$\begin{array}{l}\text { Pollutuase } \\
\text { cod } \\
\text { CAs Number } \\
\text { (if availible) }\end{array}$} & \multicolumn{2}{|c|}{ 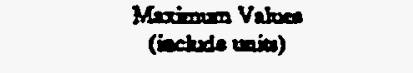 } & \multicolumn{2}{|c|}{$\begin{array}{l}\text { Avange Valuea } \\
\text { (Eelude trien) }\end{array}$} & \multirow{2}{*}{$\begin{array}{l}\text { Number } \\
\text { of } \\
\text { Storm } \\
\text { Eveoce } \\
\text { Sempled }\end{array}$} & \multirow[b]{2}{*}{ Sourcen of Pollhen } \\
\hline & 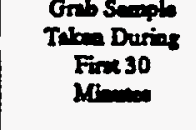 & $\begin{array}{l}\text { Flow-wrighted } \\
\text { Compotide }\end{array}$ & $\begin{array}{c}\text { Grib Semple } \\
\text { Tabo During } \\
\text { Fine } 30 \\
\text { Mfouce }\end{array}$ & $\begin{array}{l}\text { Flow-weizbrod } \\
\text { Compotite }\end{array}$ & & \\
\hline 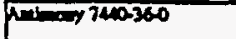 & $<0.003 \mathrm{mg} / \mathrm{l}$ & $<0.003 \mathrm{mg} / \mathrm{t}$ & $<0.003 \mathrm{mg} / 1$ & $<0.003 \mathrm{mg} / \mathrm{l}$ & 1 & \\
\hline Prans & $<0.002 \mathrm{mg} / \mathrm{l}$ & & $<0.002 \mathrm{mg} / 1$ & & 1 & \\
\hline Mond $709097-6$ & $<0.0002 \mathrm{mg} / \mathrm{h}$ & $<0.0002 \mathrm{mg} / \mathrm{l}$ & $<0.0002 \mathrm{mg} / 1$ & $<0.0002 \mathrm{mg} / \mathrm{h}$ & 1 & $\because$ \\
\hline then & $0.003 \mathrm{mg} / 1$ & & $0.003 \mathrm{mg} / 1$ & & 1 & \\
\hline 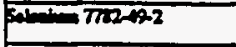 & $<0.002 \mathrm{mg} / \mathrm{l}$ & $<0.002 \mathrm{mg} / \mathrm{l}$ & $\leq 0.002 \mathrm{mg} / \mathrm{l}$ & $<0.002 \mathrm{mg} / \mathrm{l}$ & 1 & \\
\hline Sorthen & $44 \mathrm{mg} / \mathrm{h}$ & $67 \mathrm{mg} / \mathrm{l}$ & $44 \mathrm{mg} / 1$ & $67 \mathrm{mg} / \mathrm{l}$ & 1 & \\
\hline Furtwateses & $<0.05 \mathrm{mg} / 1$ & $<0.05 \mathrm{mg} / \mathrm{l}$ & $<0.05 \mathrm{mg} / \mathrm{h}$ & $<0.05 \mathrm{mg} / 1$ & 1 & \\
\hline Murian $742890-5$ & $3.54 \mathrm{mg} / 1$ & $3.34 \mathrm{mg} / \mathrm{l}$ & $3.54 \mathrm{mg} / \mathrm{l}$ & $3.34 \mathrm{mg} / 1$ & 1 & \\
\hline $4=140.322$ & $<0.04 \mathrm{mg} / \mathrm{l}$ & $<0.04 \mathrm{mg} / \mathrm{l}$ & $<0.04 \mathrm{mg} / 1$ & $<0.04 \mathrm{mg} / \mathrm{l}$ & 1 & \\
\hline 80000140008 & $0.022 \mathrm{mg} / \mathrm{n}$ & $0.020 \mathrm{mg} / \mathrm{l}$ & $0.022 \mathrm{mg} / 1$ & $0.020 \mathrm{mg} / 1$ & 1 & \\
\hline Beryliben 740-11.7 & $<0.0004 \mathrm{mg} / 1$ & $<0.0004 \mathrm{mg} / \mathrm{l}$ & $<0.0004 \mathrm{mg} / \mathrm{l}$ & $<0.0004 \mathrm{mg} / \mathrm{l}$ & .1 & \\
\hline Ed $740-139$ & $<0.004 \mathrm{mg} / \mathrm{l}$ & $<0.004 \mathrm{mg} / \mathrm{l}$ & $<0.004 \mathrm{mg} / \mathrm{l}$ & $<0.004 \mathrm{mg} / \mathrm{l}$ & 1 & \\
\hline Falan $740-70-2$ & $44 \mathrm{mg} / \mathrm{h}$ & $42.3 \mathrm{mg} / 1$ & $44 \mathrm{mg} / \mathrm{l}$ & $42.3 \mathrm{mg} / 1$ & 1 & \\
\hline Dreming 740-17.3 & $<0.006 \mathrm{mg} / 1$ & $<0.006 \mathrm{mg} / \mathrm{l}$ & $<0.006 \mathrm{mg} / \mathrm{l}$ & $<0.006 \mathrm{mg} / \mathrm{l}$ & 1 & \\
\hline $\operatorname{cosec} 740-24$ & $0.002 \mathrm{mg} / 1$ & $<0.002 \mathrm{mg} / \mathrm{l}$ & $0.002 \mathrm{mg} / 1$ & $<0.002 \mathrm{mg} / 1$ & 1 & \\
\hline Cormon 7400-50-4 & $<0.006 \mathrm{mg} / 1$ & $<0.006 \mathrm{mg} / \mathrm{l}$ & $<0.006 \mathrm{mg} / \mathrm{l}$ & $<0.006 \mathrm{mg} / \mathrm{i}$ & 1 & \\
\hline $50=743256$ & $4.61 \mathrm{mg} / \mathrm{l}$ & $4.18 \mathrm{mg} / 1$ & $4.61 \mathrm{mg} / 1$ & $4.18 \mathrm{mg} / \mathrm{l}$ & 1 & \\
\hline Loed 7439-921 & $<0.02 \mathrm{mg} / 1$ & $<0.02 \mathrm{mg} / 1$ & $<0.02 \mathrm{mg} / \mathrm{h}$ & $<0.02 \mathrm{mg} / \mathrm{l}$ & 1 & \\
\hline 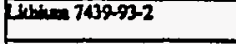 & $<0.02 \mathrm{mg} / 1$ & $<0.02 \mathrm{mg} / 1$ & $<0.02 \mathrm{mg} / 1$ & $<0.02 \mathrm{mg} / \mathrm{l}$ & 1 & \\
\hline M400mive 7439-95-4 & $6.90 \mathrm{mg} / \mathrm{l}$ & $6.68 \mathrm{mg} / \mathrm{l}$ & $6.90 \mathrm{mg} / 1$ & $6.68 \mathrm{mg} / 1$ & 1 & \\
\hline 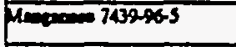 & $0.161 \mathrm{mg} / \mathrm{h}$ & $0.132 \mathrm{mg} / \mathrm{l}$ & $0.161 \mathrm{mg} / 1$ & $0.132 \mathrm{mg} / \mathrm{l}$ & 1 & \\
\hline 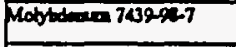 & $<0.006 \mathrm{mg} / \mathrm{l}$ & $<0.006 \mathrm{mg} / \mathrm{l}$ & $<0.006 \mathrm{mg} / \mathrm{l}$ & $<0.006 \mathrm{mg} / 1$ & 1 & \\
\hline 1601740000 & $<0.008 \mathrm{mg} / \mathrm{l}$ & $<0.008 \mathrm{mg} / \mathrm{l}$ & $<0.008 \mathrm{mg} / \mathrm{l}$ & $<0.008 \mathrm{mg} /$ & 1 & \\
\hline Porminas 740.027 & $8.0 \mathrm{mg} / \mathrm{l}$ & $7.8 \mathrm{mg} / \mathrm{l}$ & $8.0 \mathrm{mg} / \mathrm{l}$ & $7.8 \mathrm{mg} / \mathrm{l}$ & 1 & \\
\hline inner 740.24 & $<0.006 \mathrm{mg} / \mathrm{h}$ & $<0.006 \mathrm{mg} / \mathrm{l}$ & $<0.006 \mathrm{mg} / \mathrm{l}$ & $<0.006 \mathrm{mg} / 1$ & 1 & \\
\hline Sodsina $740-223-5$ & $3.81 \mathrm{mg} / \mathrm{l}$ & $3.97 \mathrm{mg} / 1$ & $3.81 \mathrm{mg} / \mathrm{h}$ & $3.97 \mathrm{mg} / \mathrm{I}$ & 1 & \\
\hline Timand 740-326 & $0.04 \mathrm{mg} / 1$ & $0.04 \mathrm{mg} / 1$ & $0.04 \mathrm{mg} /$ & $0.04 \mathrm{mg} / 1$ & 1 & \\
\hline 2007400606 & $0.03 \mathrm{mg} / 1$ & $0.03 \mathrm{mg} / 1$ & $0.03 \mathrm{mg} / \mathrm{l}$ & $0.03 \mathrm{mg} / 1$ & 1 & \\
\hline
\end{tabular}


Continued from fronk.

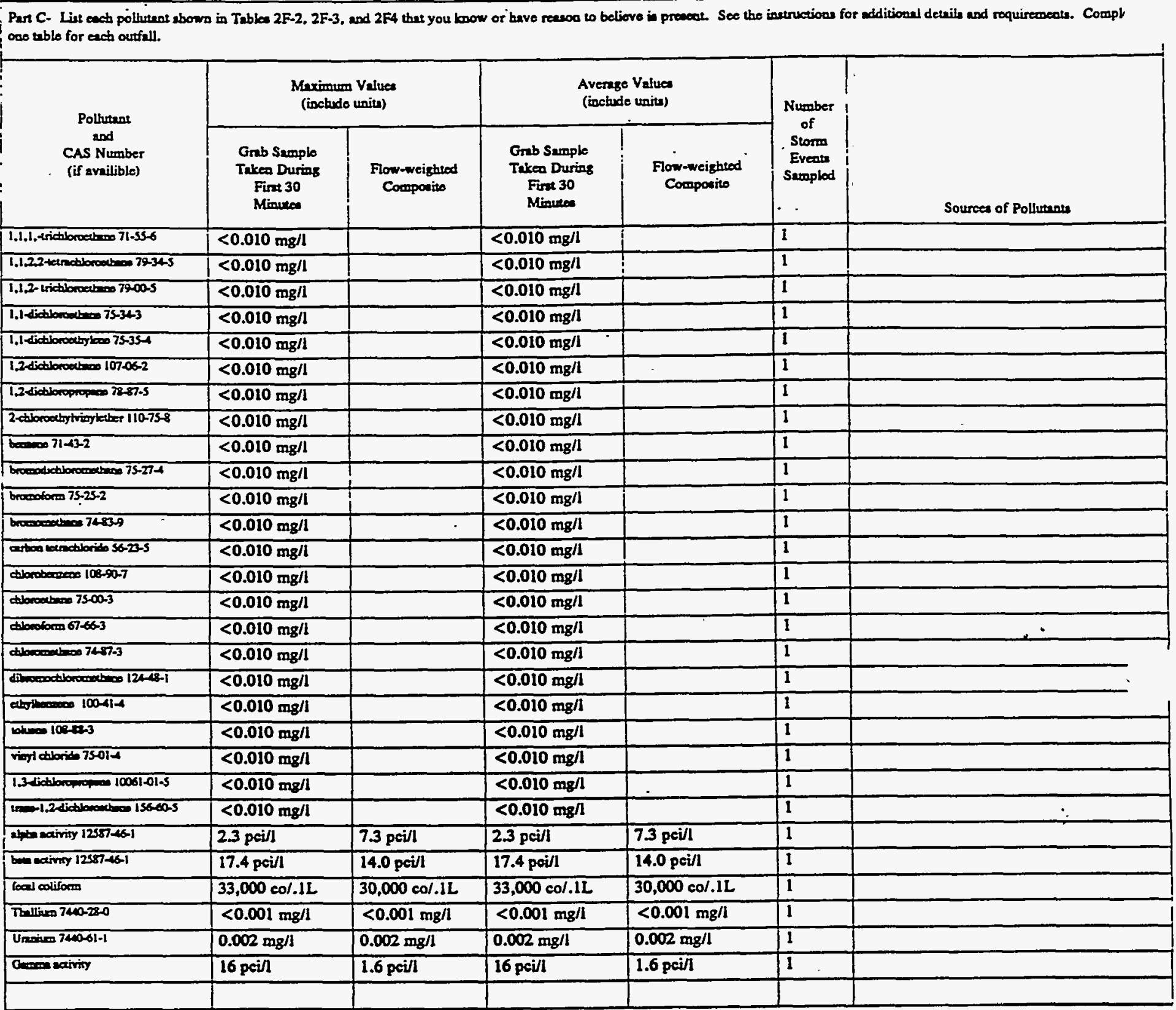

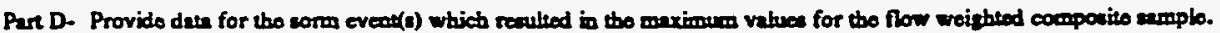

\begin{tabular}{|c|c|c|c|c|c|c|c|}
\hline $\begin{array}{l}1 . \\
\text { Date of } \\
\text { Storms } \\
\text { Evect }\end{array}$ & $\begin{array}{l}2 . \\
\text { Duntion } \\
\text { of Sion } \\
\text { (in } \\
\text { minuten) }\end{array}$ & $\begin{array}{l}3 . \\
\text { Total ninf all } \\
\text { during norm oveat } \\
\text { (in inces) }\end{array}$ & 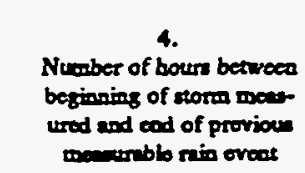 & $\begin{array}{l}\text { S. } \\
\text { Meximun flow mes } \\
\text { during rain eveat } \\
\text { (riloce/onin or spocify } \\
\text { unis) }\end{array}$ & $\begin{array}{l}6 . \\
\text { Total flow from } \\
\text { rin eveat } \\
\text { (alloos or } \\
\text { specify unitu) }\end{array}$ & $\begin{array}{c}7 . \\
\text { Scaico } \\
\text { exmplo was } \\
\text { unteo }\end{array}$ & $\begin{array}{l}8 . \\
\text { Form of } \\
\text { Precipitation } \\
\text { (rainfull. } \\
\text { soowmell) }\end{array}$ \\
\hline $\begin{array}{l}10 / 14 / 91- \\
10 / 15 / 91\end{array}$ & 650 & 1.6 & 226.25 & 204 & 133,065 & Fall & Rainfall \\
\hline
\end{tabular}

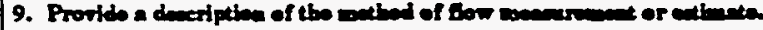

An ISCO 3230 Flow meter was need to obtein water leved readings. This instrunent was calibrated with the normal dry weather flow, if present, as level zero. The flow meter was programmed with the charecteristics of the conreyance so that now rates and total flows conld be calculated by the meter. 
Outrall S9

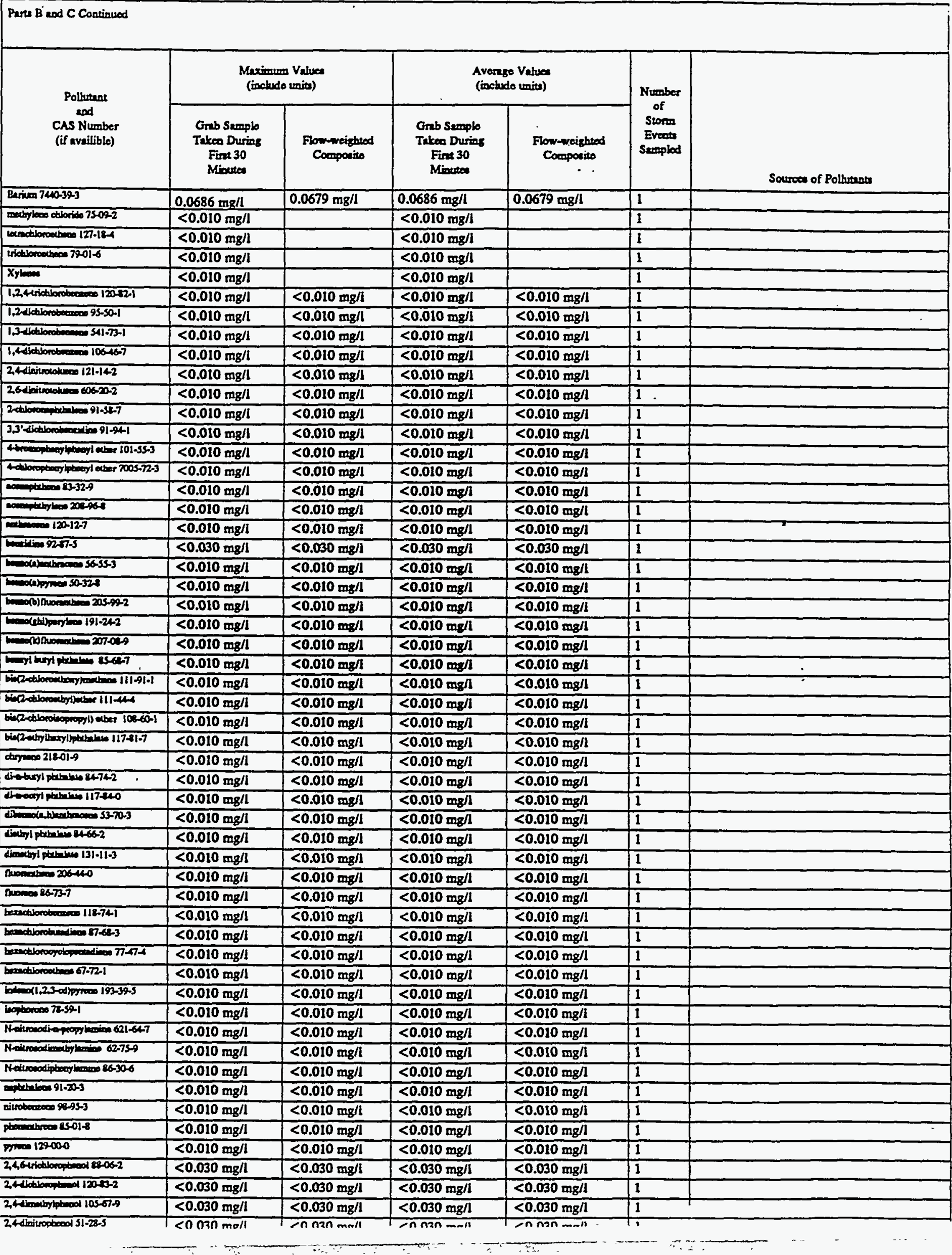


Outfall 59

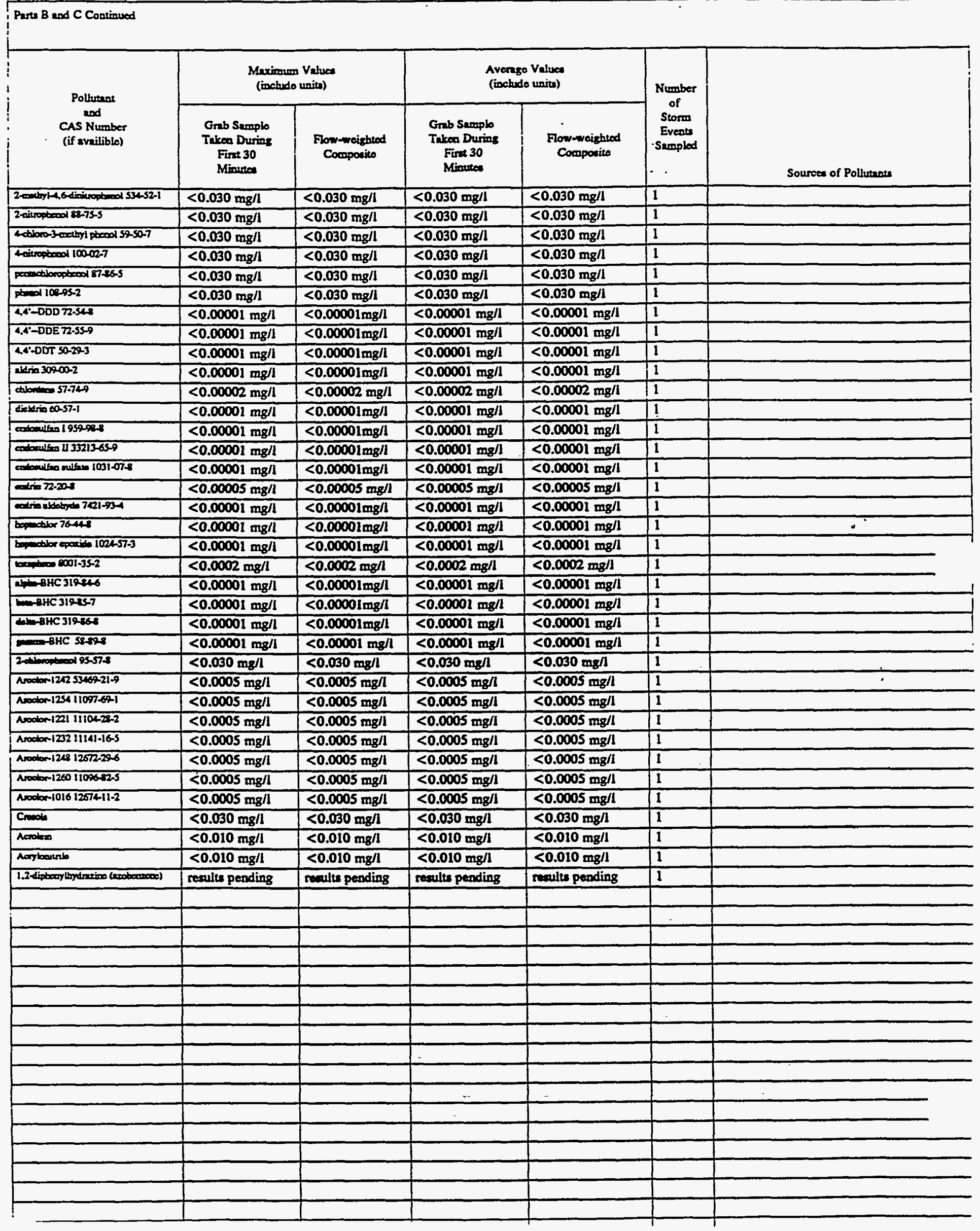


VI. Discherge Loformation (continwed from page 3 of Form 2P)

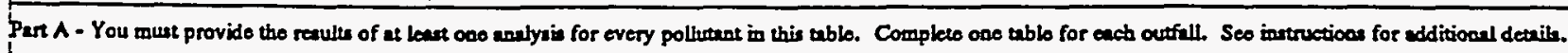

\begin{tabular}{|c|c|c|c|c|c|c|}
\hline \multirow{2}{*}{$\begin{array}{l}\text { Pollutant } \\
\text { end } \\
\text { CAs Number } \\
\text { (if availible) }\end{array}$} & \multicolumn{2}{|c|}{$\begin{array}{l}\text { Marimurn Values } \\
\text { (incluxde unitr) }\end{array}$} & \multicolumn{2}{|c|}{$\begin{array}{l}\text { Avernge Values } \\
\text { (inchude units) }\end{array}$} & \multirow{2}{*}{$\begin{array}{l}\text { Number } \\
\text { of } \\
\text { Slome } \\
\text { Evense } \\
\text { Sampled }\end{array}$} & \multirow[b]{2}{*}{ Sources of Pollhutents } \\
\hline & $\begin{array}{c}\text { Grab Somple } \\
\text { Taken During } \\
\text { Fint 30 } \\
\text { Minuse }\end{array}$ & $\begin{array}{l}\text { Flow-woighted } \\
\text { Cocopoutito }\end{array}$ & $\begin{array}{l}\text { Grib Sumplo } \\
\text { Takes During } \\
\text { Firt } 30 \\
\text { Minuteo }\end{array}$ & $\begin{array}{l}\text { Flow-iveighted } \\
\text { Componito }\end{array}$ & & \\
\hline pil mod Groences & $3 \mathrm{mg} / 1$ & & $3 \mathrm{mg} / \mathrm{l}$ & & 1 & \\
\hline Piolopial Oxyen Demend (BODS) & $10.32 \mathrm{mg} / 1$ & $<5 \mathrm{mg} / \mathrm{l}$ & $10.32 \mathrm{mg} / 1$ & $<5 \mathrm{mg} / 1$ & 1 & \\
\hline Cremial Oxyea Denand (COD) & $39 \mathrm{mg} / \mathrm{l}$ & $51 \mathrm{mg} / 1$ & $39 \mathrm{mg} / \mathrm{I}$ & $51 \mathrm{mg} / 1$ & 1 & \\
\hline Toul Supponded Solide (TSS) & $52 \mathrm{mg} / \mathrm{l}$ & $7.0 \mathrm{mg} / \mathrm{l}$ & $52 \mathrm{mg} / \mathrm{l}$ & $7.0 \mathrm{mg} / 1$ & 1 & \\
\hline Toal Kjethebl Nitrosen & $1.5 \mathrm{mg} / \mathrm{l}$ & $0.8 \mathrm{mg} / \mathrm{l}$ & $1.5 \mathrm{mg} / 1$ & $0.8 \mathrm{mg} / 1$ & 1 & \\
\hline Fikan phue Nierine Nieroges & $0.2 \mathrm{mg} / \mathrm{l}$ & $0.7 \mathrm{mg} / \mathrm{l}$ & $0.2 \mathrm{mg} / \mathrm{ll}$ & $0.7 \mathrm{mg} / \mathrm{l}$ & 1 & \\
\hline 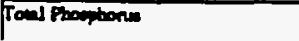 & $0.17 \mathrm{mg} / \mathrm{l}$ & $0.12 \mathrm{mg} / \mathrm{l}$ & $0.17 \mathrm{mg} / \mathrm{l}$ & $0.12 \mathrm{mg} / \mathrm{ll}$ & 1 & \\
\hline
\end{tabular}

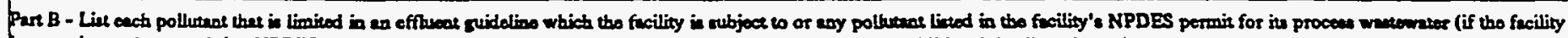

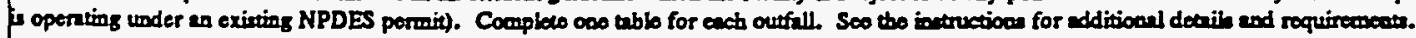

\begin{tabular}{|c|c|c|c|c|c|c|}
\hline \multirow{2}{*}{$\begin{array}{l}\text { Poltutuant } \\
\text { and } \\
\text { CAS Number } \\
\text { (if nviliblo) }\end{array}$} & \multicolumn{2}{|c|}{ 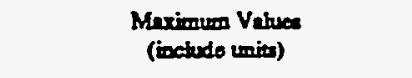 } & \multicolumn{2}{|c|}{$\begin{array}{l}\text { Avenge Values } \\
\text { (ineckede urita) }\end{array}$} & \multirow{2}{*}{$\begin{array}{l}\text { Number } \\
\text { of } \\
\text { Storm } \\
\text { Eveose } \\
\text { Sempled }\end{array}$} & \multirow{2}{*}{ Sourcen of Polbuesese } \\
\hline & 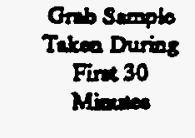 & $\begin{array}{l}\text { Flow-wroizhtod } \\
\text { Compocixs }\end{array}$ & $\begin{array}{l}\text { Orab Semplo } \\
\text { Tabeo During } \\
\text { Fura } 30 \\
\text { Mineces }\end{array}$ & $\begin{array}{l}\text { Flow-wreightrod } \\
\text { Cosposito }\end{array}$ & & \\
\hline hatimed $7460-360$ & $<0.003 \mathrm{mg} / 1$ & $<0.003 \mathrm{mg} / \mathrm{l}$ & $<0.003 \mathrm{mg}^{\prime l}$ & $<0.003 \mathrm{mg} / \mathrm{l}$ & 1 & \\
\hline$y=2$ & $<0.002 \mathrm{mg} / 1$ & & $<0.002 \mathrm{mg} / 1$ & & 1 & \\
\hline 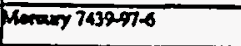 & $<0.0002 \mathrm{mg} / \mathrm{l}$ & $<0.0002 \mathrm{mg} / \mathrm{l}$ & $<0.0002 \mathrm{~m} / \mathrm{l}$ & $<0.0002 \mathrm{mg} / \mathrm{l}$ & 1 & \\
\hline proods & $0.003 \mathrm{mg} / \mathrm{l}$ & & $0.003 \mathrm{mg} / \mathrm{h}$ & & 1 & ! \\
\hline 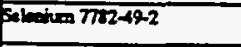 & $<0.002 \mathrm{mg} / 1$ & $<0.002 \mathrm{mg} / \mathrm{l}$ & $<0.002 \mathrm{mg} / 1$ & $<0.002 \mathrm{mg} / \mathrm{l}$ & 1 & \\
\hline suling & $75 \mathrm{mg} / 1$ & $82 \mathrm{mg} / 1$ & $75 \mathrm{mg} / 1$ & $82 \mathrm{mg} / \mathrm{l}$ & 1 & \\
\hline Purteones & $<0.05 \mathrm{mg} / \mathrm{l}$ & $<0.05 \mathrm{mg} / \mathrm{l}$ & $<0.05 \mathrm{mg} / \mathrm{l}$ & $<0.05 \mathrm{mg} / \mathrm{l}$ & $I$ & \\
\hline Aheningm 7429.90.5 & $1.97 \mathrm{mg} / \mathrm{l}$ & $0.32 \mathrm{mg} / \mathrm{l}$ & $1.97 \mathrm{mg} / \mathrm{l}$ & $0.32 \mathrm{mg} / 1$ & 1 & \\
\hline Ansodio 740-36-2 & $<0.04 \mathrm{mg} / \mathrm{l}$ & $<0.04 \mathrm{mg} / \mathrm{l}$ & $<0.04 \mathrm{mg} / \mathrm{l}$ & $<0.04 \mathrm{mg} / \mathrm{l}$ & 1 & \\
\hline $300000740-28$ & $0.021 \mathrm{mg} / \mathrm{l}$ & $0.016 \mathrm{mg} / \mathrm{l}$ & $0.021 \mathrm{mg} / \mathrm{l}$ & $0.016 \mathrm{mg} / \mathrm{l}$ & 1 & \\
\hline Bergliven 740-11.7 & $<0.0004 \mathrm{mg} / \mathrm{l}$ & $<0.0004 \mathrm{mg} / \mathrm{l}$ & $<0.0004 \mathrm{mg} / \mathrm{l}$ & $<0.0004 \mathrm{mg} / \mathrm{l}$ & 1 & \\
\hline $740-43-9$ & $<0.004 \mathrm{mg} / \mathrm{l}$ & $<0.004 \mathrm{mg} / \mathrm{l}$ & $<0.004 \mathrm{mg} / \mathrm{l}$ & $<0.004 \mathrm{mg} / 1$ & 1 & \\
\hline Eevoinen 7440.70-2 & $39.9 \mathrm{mg} / \mathrm{l}$ & $43.2 \mathrm{mg} / 1$ & $39.9 \mathrm{mg} / \mathrm{l}$ & $43.2 \mathrm{mg} / \mathrm{l}$ & 1 & \\
\hline Broming 7460-7.3 & $<0.006 \mathrm{mg} / \mathrm{l}$ & $<0.006 \mathrm{mg} / \mathrm{l}$ & $<0.006 \mathrm{mg} / \mathrm{l}$ & $<0.006 \mathrm{mg} / \mathrm{h}$ & 1 & \\
\hline Cobeck 740048-4 & $0.002 \mathrm{mg} / \mathrm{l}$ & $<0.002 \mathrm{mg} / \mathrm{l}$ & $0.002 \mathrm{mg} / \mathrm{l}$ & $<0.002 \mathrm{mg} / 1$ & 1 & \\
\hline Coppor 740-50-8 & $<0.006 \mathrm{mg} / \mathrm{l}$ & $<0.006 \mathrm{mg} / \mathrm{l}$ & $<0.006 \mathrm{mg} / \mathrm{l}$ & $<0.006 \mathrm{mg} / \mathrm{l}$ & 1 & \\
\hline 10007439696 & $2.12 \mathrm{mg} / 1$ & $0.30 \mathrm{mg} / 1$ & $2.12 \mathrm{mg} / 1$ & $0.30 \mathrm{mg} / 1$ & 1 & \\
\hline $\operatorname{lod} 7439-2-1$ & $<0.02 \mathrm{mg} / 1$ & $<0.02 \mathrm{mg} / 1$ & $<0.02 \mathrm{mg} / \mathrm{l}$ & $<0.02 \mathrm{mg} / \mathrm{l}$ & 1 & \\
\hline Iubiven 7139-93-2 & $<0.02 \mathrm{mg} / \mathrm{l}$ & $<0.02 \mathrm{mg} / \mathrm{l}$ & $<0.02 \mathrm{mg} / \mathrm{l}$ & $<0.02 \mathrm{mg} / 1$ & 1 & \\
\hline Megomanom 7/39-954 & $6.12 \mathrm{mg} / 1$ & $5.59 \mathrm{mg} / 1$ & $6.12 \mathrm{mg} / 1$ & $5.59 \mathrm{mg} / \mathrm{h}$ & 1 & \\
\hline 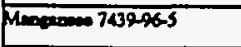 & $0.213 \mathrm{mg} / \mathrm{l}$ & $0.027 \mathrm{mg} / \mathrm{l}$ & $0.213 \mathrm{mg} / 1$ & $0.027 \mathrm{mg} / \mathrm{l}$ & 1 & \\
\hline Mathoan 7439-98-7 & $<0.006 \mathrm{mg} / \mathrm{l}$ & $<0.006 \mathrm{mg} / \mathrm{l}$ & $<0.006 \mathrm{mg} / \mathrm{ll}$ & $<0.006 \mathrm{mg} / 1$ & 1 & \\
\hline Nitel 740020 & $<0.008 \mathrm{mg} / \mathrm{l}$ & $<0.008 \mathrm{mg} / \mathrm{l}$ & $<0.008 \mathrm{mg} / \mathrm{l}$ & $<0.008 \mathrm{mg} / \mathrm{l}$ & 1 & \\
\hline Premeixen $74009-9$ & $6.3 \mathrm{mg} / 1$ & $3.6 \mathrm{mg} / \mathrm{l}$ & $6.3 \mathrm{mg} / \mathrm{l}$ & $3.6 \mathrm{mg} / \mathrm{l}$ & 1 & \\
\hline sinem 740.20-1 & $<0.006 \mathrm{mg} / 1$ & $<0.006 \mathrm{mg} / \mathrm{l}$ & $<0.006 \mathrm{mg} / \mathrm{l}$ & $<0.006 \mathrm{mg} / 1$ & 1 & \\
\hline Sadien 740-20-5 & $0.99 \mathrm{mg} / \mathrm{l}$ & $1.04 \mathrm{mg} / 1$ & $0.99 \mathrm{mg} / \mathrm{l}$ & $1.04 \mathrm{mg} / 1$ & 1 & \\
\hline 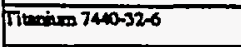 & $0.03 \mathrm{mg} / \mathrm{l}$ & $<0.01 \mathrm{mg} / 1$ & $0.03 \mathrm{mg} / \mathrm{l}$ & $<0.01 \mathrm{mg} / \mathrm{l}$ & 1 & \\
\hline $200740-606$ & $0.06 \mathrm{mg} / 1$ & $0.02 \mathrm{mg} / \mathrm{l}$ & $0.06 \mathrm{mg} / \mathrm{l}$ & $0.02 \mathrm{mg} / 1$ & 1 & \\
\hline
\end{tabular}


Continued from front.

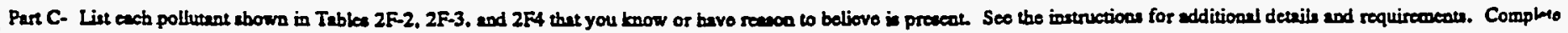
ono table for cach ouffall.

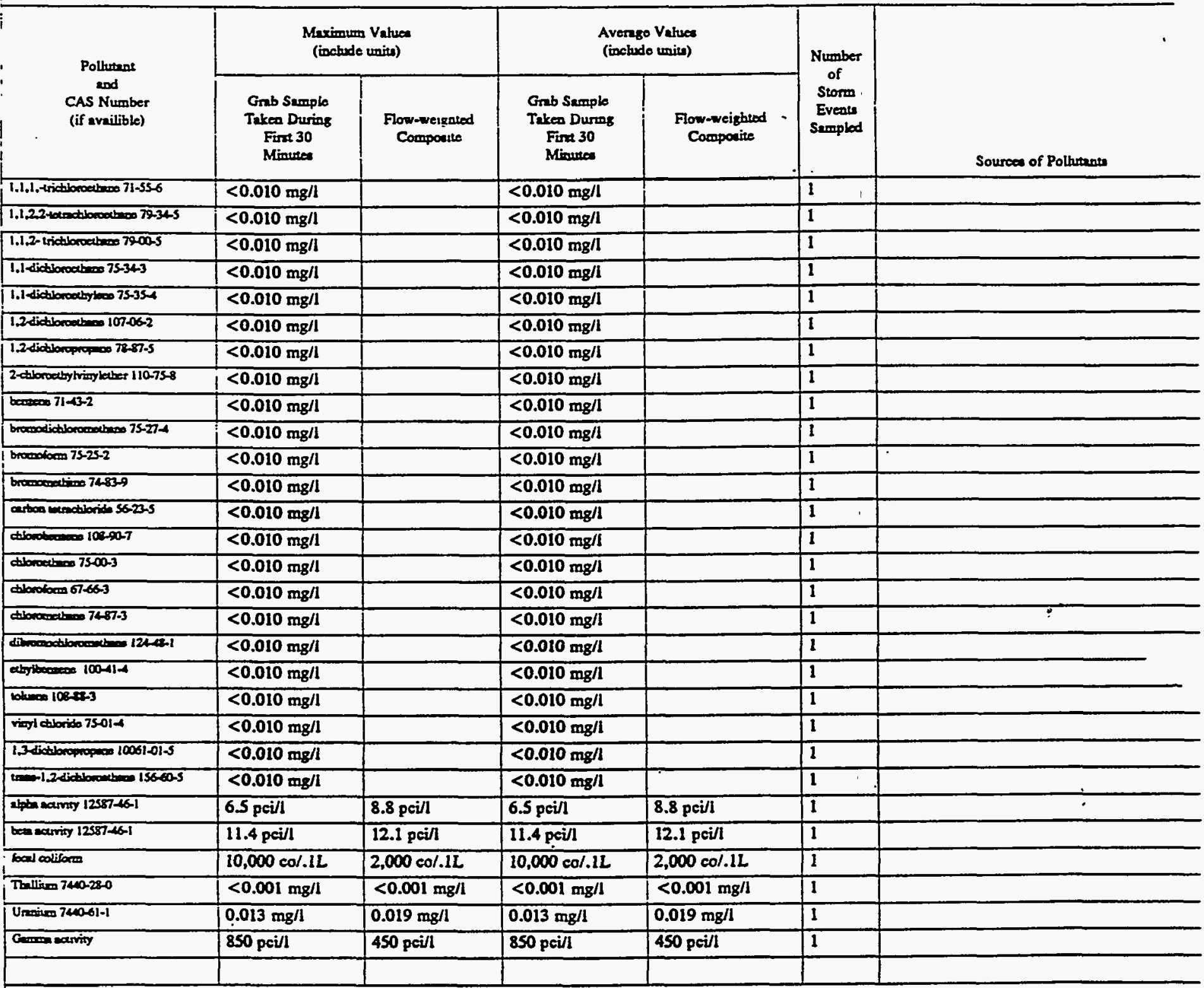

Part D- Provide date for the sorm ovent(l) which reatited in the weximum vatues for the flow weightod composite sample.

\begin{tabular}{|c|c|c|c|c|c|c|c|}
\hline $\begin{array}{l}1 . \\
\text { Dawo of } \\
\text { Storm } \\
\text { Eveal }\end{array}$ & $\begin{array}{l}2 . \\
\text { Durition } \\
\text { of Storm } \\
\text { (in } \\
\text { minutea) }\end{array}$ & $\begin{array}{l}3 . \\
\text { Total ninfill } \\
\text { during storm event } \\
\text { (in inchea) }\end{array}$ & 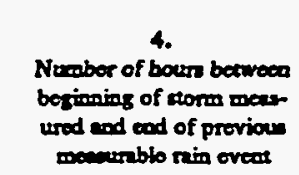 & $\begin{array}{l}\text { S. } \\
\text { Mrrimum flow nat } \\
\text { during nin oveat } \\
\text { (ollood/min or specify } \\
\text { unio) }\end{array}$ & $\begin{array}{l}6 . \\
\text { Toeal flow from } \\
\text { rim eveot } \\
\text { (ealloos or } \\
\text { epocify unitu) }\end{array}$ & $\begin{array}{c}7 . \\
\text { Semon } \\
\text { enomple was } \\
\text { taken }\end{array}$ & $\begin{array}{l}\text { 8. } \\
\text { Form of } \\
\text { Procipioncion } \\
\text { (rinfall, } \\
\text { soowmets) }\end{array}$ \\
\hline $\begin{array}{l}10 / 14 / 91-1 \\
0 / 15 / 91\end{array}$ & 650 & 1.5 & 226.25 & 141 & 105,106 & Fall & Rainfall \\
\hline
\end{tabular}

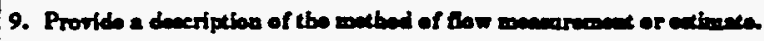

An ISCO 3230 Flow meter was used to obtsin water level readings. This instrument was catibrated with the normal dry weather flow, if present, as ler zero. The flow meter was programmed with the characteristics of the conveyance so that flow rates and total nows conld be calculated by the meter. 
Outfall S10

Parte B and C Continued

\begin{tabular}{|c|c|c|c|c|c|c|}
\hline \multirow{2}{*}{$\begin{array}{l}\text { Pollutunt } \\
\text { and } \\
\text { CAS Number } \\
\text { (if avnilible) }\end{array}$} & \multicolumn{2}{|c|}{$\begin{array}{l}\text { Meximusm Valuea } \\
\text { (inchude units) }\end{array}$} & \multicolumn{2}{|c|}{$\begin{array}{l}\text { Avengo Values } \\
\text { (iochudo units) }\end{array}$} & \multirow{2}{*}{$\begin{array}{l}\text { Number } \\
\text { of } \\
\text { Storm } \\
\text { Evecss } \\
\text { Sempled }\end{array}$} & \multirow[b]{2}{*}{ Sources of Polturants } \\
\hline & $\begin{array}{c}\text { Grab Samplo } \\
\text { Taken Duriog } \\
\text { First } 30 \\
\text { Minutea }\end{array}$ & $\begin{array}{l}\text { Fow-weightod } \\
\text { Compocite }\end{array}$ & $\begin{array}{c}\text { Grab Semplo } \\
\text { Takeo Durias } \\
\text { First } 30 \\
\text { Minuece }\end{array}$ & $\begin{array}{c}\text { Fow-weightod } \\
\text { Componito } \\
.\end{array}$ & & \\
\hline Reriven 740-32-3 & $0.0536 \mathrm{mg} / \mathrm{l}$ & $0.0541 \mathrm{mg} / 1$ & $0.0536 \mathrm{mg} / 1$ & $0.0541 \mathrm{mg} / \mathrm{l}$ & 1 & \\
\hline 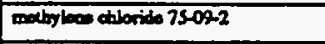 & $<0.010 \mathrm{mg} / \mathrm{l}$ & & $<0.010 \mathrm{mg} / 1$ & & 1 & \\
\hline 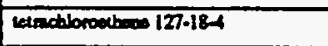 & $<0.010 \mathrm{mg} / \mathrm{l}$ & & $<0.010 \mathrm{mg} / 1$ & & 1 & \\
\hline 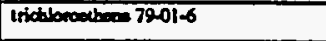 & $<0.010 \mathrm{mg} / \mathrm{l}$ & & $<0.010 \mathrm{mg} / 1$ & & 1 & \\
\hline xyloosen & $<0.010 \mathrm{mg} / \mathrm{l}$ & & $<0.010 \mathrm{mg} / 1$ & & 1 & \\
\hline 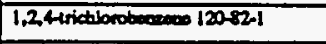 & $<0.010 \mathrm{mg} / \mathrm{l}$ & $<0.010 \mathrm{mg} / 1$ & $<0.010 \mathrm{mg} / \mathrm{l}$ & $<0.010 \mathrm{mg} / \mathrm{l}$ & 1 & \\
\hline 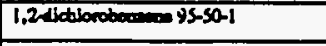 & $<0.010 \mathrm{mg} / \mathrm{l}$ & $<0.010 \mathrm{mg} / \mathrm{l}$ & $<0.010 \mathrm{mg} / \mathrm{l}$ & $<0.010 \mathrm{mg} / \mathrm{l}$ & 1 & \\
\hline 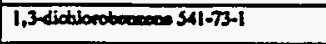 & $<0.010 \mathrm{mg} / \mathrm{l}$ & $<0.010 \mathrm{mg} / 1$ & $<0.010 \mathrm{mg} / 1$ & $<0.010 \mathrm{mg} / \mathrm{l}$ & 1 & \\
\hline 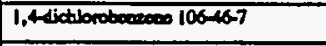 & $<0.010 \mathrm{mg} / \mathrm{l}$ & $<0.010 \mathrm{mg} / 1$ & $<0.010 \mathrm{mg} / 1$ & $<0.010 \mathrm{mg} / \mathrm{l}$ & 1 & \\
\hline 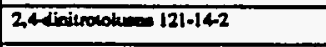 & $<0.010 \mathrm{mg} / \mathrm{l}$ & $<0.010 \mathrm{mg} / 1$ & $<0.010 \mathrm{mg} / \mathrm{l}$ & $<0.010 \mathrm{mg} / 1$ & 1 & \\
\hline 26-dinitrocolveoce 606-20-2 & $<0.010 \mathrm{mg} / \mathrm{l}$ & $<0.010 \mathrm{mg} / \mathrm{l}$ & $<0.010 \mathrm{mg} / \mathrm{l}$ & $<0.010 \mathrm{mg} / \mathrm{l}$ & 1 & \\
\hline 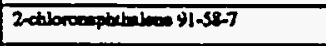 & $<0.010 \mathrm{mg} / \mathrm{l}$ & $<0.010 \mathrm{mg} / 1$ & $<0.010 \mathrm{mg} / \mathrm{l}$ & $<0.010 \mathrm{mg} / \mathrm{l}$ & I. & \\
\hline 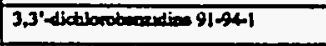 & $<0.010 \mathrm{mg} / \mathrm{l}$ & $<0.010 \mathrm{mg} / \mathrm{l}$ & $<0.010 \mathrm{mg} / \mathrm{l}$ & $<0.010 \mathrm{mg} / \mathrm{l}$ & 1 & \\
\hline 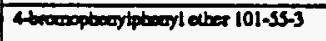 & $<0.010 \mathrm{mg} / 1$ & $<0.010 \mathrm{mg} / \mathrm{l}$ & $<0.010 \mathrm{mg} / \mathrm{l}$ & $<0.010 \mathrm{mg} / 1$ & 1 & \\
\hline 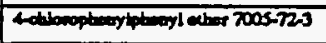 & $<0.010 \mathrm{mg} / \mathrm{l}$ & $<0.010 \mathrm{mg} / 1$ & $<0.010 \mathrm{mg} / 1$ & $<0.010 \mathrm{mg} / 1$ & 1 & \\
\hline 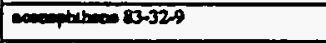 & $<0.010 \mathrm{mg} / \mathrm{l}$ & $<0.010 \mathrm{mg} / \mathrm{l}$ & $<0.010 \mathrm{mg} / \mathrm{l}$ & $<0.010 \mathrm{mg} / \mathrm{l}$ & 1 & \\
\hline 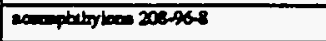 & $<0.010 \mathrm{mg} / \mathrm{l}$ & $<0.010 \mathrm{mg} / \mathrm{l}$ & $<0.010 \mathrm{mg} / 1$ & $<0.010 \mathrm{mg} / 1$ & 1 & \\
\hline wothanos $120-12.7$ & $<0.010 \mathrm{mg} / 1$ & $<0.010 \mathrm{mg} / \mathrm{l}$ & $<0.010 \mathrm{mg} / 1$ & $<0.010 \mathrm{mg} / 1$ & 1 & \\
\hline maves 927, & $<0.030 \mathrm{mg} / \mathrm{l}$ & $<0.030 \mathrm{mg} / \mathrm{l}$ & $<0.030 \mathrm{mg} / \mathrm{l}$ & $<0.030 \mathrm{mg} / \mathrm{l}$ & 1 & \\
\hline 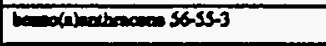 & $<0.010 \mathrm{mg} / \mathrm{l}$ & $<0.010 \mathrm{mg} / 1$ & $<0.010 \mathrm{mg} / \mathrm{l}$ & $<0.010 \mathrm{mg} / \mathrm{l}$ & 1 & \\
\hline 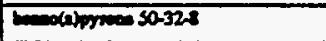 & $<0.010 \mathrm{mg} / \mathrm{l}$ & $<0.010 \mathrm{mg} / \mathrm{l}$ & $<0.010 \mathrm{mg} / 1$ & $<0.010 \mathrm{mg} / 1$ & 1 & \\
\hline 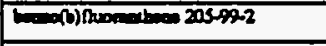 & $<0.010 \mathrm{mg} / \mathrm{l}$ & $<0.010 \mathrm{mg} / \mathrm{l}$ & $<0.010 \mathrm{mg} / 1$ & $<0.010 \mathrm{mg} / 1$ & 1 & \\
\hline 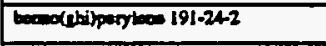 & $<0.010 \mathrm{mg} / \mathrm{l}$ & $<0.010 \mathrm{mg} / \mathrm{l}$ & $<0.010 \mathrm{mg} / \mathrm{l}$ & $<0.010 \mathrm{mg} / \mathrm{l}$ & 1 & \\
\hline 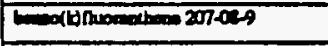 & $<0.010 \mathrm{mg} / \mathrm{l}$ & $<0.010 \mathrm{mg} / \mathrm{l}$ & $<0.010 \mathrm{mg} / \mathrm{l}$ & $<0.010 \mathrm{mg} / \mathrm{l}$ & 1 & \\
\hline 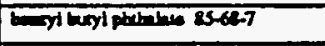 & $<0.010 \mathrm{mg} / \mathrm{l}$ & $<0.010 \mathrm{mg} / \mathrm{l}$ & $<0.010 \mathrm{mg} / 1$ & $<0.010 \mathrm{mg} / \mathrm{l}$ & 1 & \\
\hline 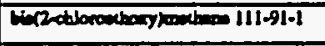 & $<0.010 \mathrm{mg} / \mathrm{l}$ & $<0.010 \mathrm{mg} / \mathrm{l}$ & $<0.010 \mathrm{mg} / \mathrm{l}$ & $<0.010 \mathrm{mg} / \mathrm{l}$ & 1 & $\div$ \\
\hline 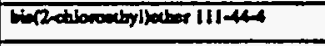 & $<0.010 \mathrm{mg} / \mathrm{l}$ & $<0.010 \mathrm{mg} / \mathrm{l}$ & $<0.010 \mathrm{mg} / \mathrm{l}$ & $<0.010 \mathrm{mg} / \mathrm{l}$ & 1 & \\
\hline 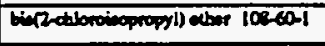 & $<0.010 \mathrm{mg} / \mathrm{l}$ & $<0.010 \mathrm{mg} / \mathrm{l}$ & $<0.010 \mathrm{mg} / 1$ & $<0.010 \mathrm{mg} / \mathrm{l}$ & 1 & \\
\hline 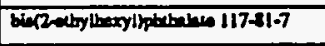 & $<0.010 \mathrm{mg} / \mathrm{l}$ & $<0.010 \mathrm{mg} / \mathrm{l}$ & $<0.010 \mathrm{mg} / 1$ & $<0.010 \mathrm{mg} / \mathrm{l}$ & 1 & \\
\hline dxymen 21201A & $<0.010 \mathrm{mg} / \mathrm{l}$ & $<0.010 \mathrm{mg} / \mathrm{l}$ & $<0.010 \mathrm{mg} / 1$ & $<0.010 \mathrm{mg} / \mathrm{l}$ & 1 & \\
\hline 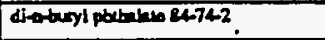 & $<0.010 \mathrm{mg} / \mathrm{l}$ & $<0.010 \mathrm{mg} / 1$ & $<0.010 \mathrm{mg} / \mathrm{l}$ & $<0.010 \mathrm{mg} / \mathrm{l}$ & 1 & \\
\hline 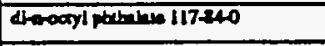 & $<0.010 \mathrm{mg} / 1$ & $<0.010 \mathrm{mg} / 1$ & $<0.010 \mathrm{mg} / 1$ & $<0.010 \mathrm{mg} / \mathrm{l}$ & 1 & \\
\hline 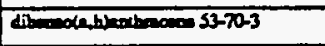 & $<0.010 \mathrm{mg} / 1$ & $<0.010 \mathrm{mg} / 1$ & $<0.010 \mathrm{mg} / 1$ & $<0.010 \mathrm{mg} / \mathrm{L}$ & 1 & \\
\hline 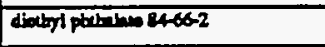 & $<0.010 \mathrm{mg} / \mathrm{l}$ & $<0.010 \mathrm{mg} / 1$ & $<0.010 \mathrm{mg} / \mathrm{l}$ & $<0.010 \mathrm{mg} / 1$ & 1 & \\
\hline 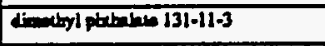 & $<0.010 \mathrm{mg} / \mathrm{l}$ & $<0.010 \mathrm{mg} / \mathrm{l}$ & $<0.010 \mathrm{mg} / 1$ & $<0.010 \mathrm{mg} / \mathrm{l}$ & 1 & \\
\hline Theornethene 206-140 & $<0.010 \mathrm{mg} / \mathrm{l}$ & $<0.010 \mathrm{mg} / 1$ & $<0.010 \mathrm{mg} / \mathrm{l}$ & $<0.010 \mathrm{mg} / \mathrm{l}$ & 1 & \\
\hline Romosese 26-73-7 & $<0.010 \mathrm{mg} / \mathrm{l}$ & $<0.010 \mathrm{mg} / \mathrm{l}$ & $<0.010 \mathrm{mg} / \mathrm{l}$ & $<0.010 \mathrm{mg} / \mathrm{l}$ & 1 & \\
\hline 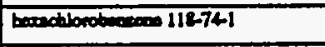 & $<0.010 \mathrm{mg} / \mathrm{l}$ & $<0.010 \mathrm{mg} / 1$ & $<0.010 \mathrm{mg} / \mathrm{l}$ & $<0.010 \mathrm{mg} / \mathrm{l}$ & 1 & \\
\hline $\begin{array}{ll} & \\
\end{array}$ & $<0.010 \mathrm{mg} / \mathrm{l}$ & $<0.010 \mathrm{mg} / 1$ & $<0.010 \mathrm{mg} / 1$ & $<0.010 \mathrm{mg} / \mathrm{l}$ & 1 & \\
\hline 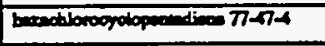 & $<0.010 \mathrm{mg} / \mathrm{l}$ & $<0.010 \mathrm{mg} / \mathrm{l}$ & $<0.010 \mathrm{mg} / \mathrm{l}$ & $<0.010 \mathrm{mg} / \mathrm{l}$ & 1 & \\
\hline 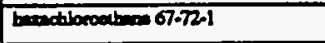 & $<0.010 \mathrm{mg} / 1$ & $<0.010 \mathrm{mg} / 1$ & $<0.010 \mathrm{mg} / 1$ & $<0.010 \mathrm{mg} / \mathrm{l}$ & 1 & \\
\hline 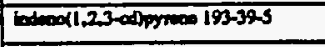 & $<0.010 \mathrm{mg} / \mathrm{l}$ & $<0.010 \mathrm{mg} / \mathrm{l}$ & $<0.010 \mathrm{mg} / 1$ & $<0.010 \mathrm{mg}_{\mathrm{g}} / \mathrm{l}$ & 1 & \\
\hline 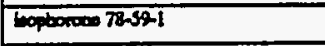 & $<0.010 \mathrm{mg} / \mathrm{l}$ & $<0.010 \mathrm{mg} / \mathrm{l}$ & $<0.010 \mathrm{mg} / \mathrm{l}$ & $<0.010 \mathrm{mg} / \mathrm{l}$ & 1 & \\
\hline 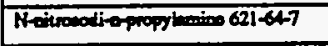 & $<0.010 \mathrm{mg} / \mathrm{l}$ & $<0.010 \mathrm{mg} / \mathrm{l}$ & $<0.010 \mathrm{mg} / \mathrm{l}$ & $<0.010 \mathrm{mg} / \mathrm{l}$ & 1 & \\
\hline 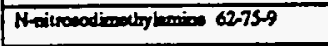 & $<0.010 \mathrm{mg} / \mathrm{l}$ & $<0.010 \mathrm{mg} / 1$ & $<0.010 \mathrm{mg} / \mathrm{l}$ & $<0.010 \mathrm{mg} / \mathrm{l}$ & 1 & \\
\hline 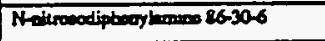 & $<0.010 \mathrm{mg} / \mathrm{l}$ & $<0.010 \mathrm{mg} / \mathrm{l}$ & $<0.010 \mathrm{mg} / \mathrm{l}$ & $<0.010 \mathrm{mg} / 1$ & 1 & \\
\hline $91-20-3$ & $<0.010 \mathrm{mg} / \mathrm{l}$ & $<0.010 \mathrm{mg} / \mathrm{l}$ & $<0.010 \mathrm{mg} / \mathrm{l}$ & $<0.010 \mathrm{mg} / \mathrm{l}$ & 1 & \\
\hline 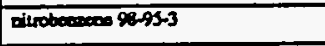 & $<0.010 \mathrm{mg} / 1$ & $<0.010 \mathrm{mg} / \mathrm{l}$ & $<0.010 \mathrm{mg} / 1$ & $<0.010 \mathrm{mg} / 1$ & 1 & \\
\hline 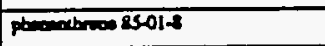 & $<0.010 \mathrm{mg} / \mathrm{l}$ & $<0.010 \mathrm{mg} / \mathrm{l}$ & $<0.010 \mathrm{mg} / 1$ & $<0.010 \mathrm{mg} / 1$ & 1 & \\
\hline prinos 1290000 & $<0.010 \mathrm{mg} / \mathrm{l}$ & $<0.010 \mathrm{mg} / \mathrm{l}$ & $<0.010 \mathrm{mg} / 1$ & $<0.010 \mathrm{mg} / \mathrm{l}$ & 1 & \\
\hline 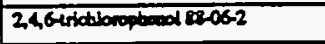 & $<0.030 \mathrm{mg} / 1$ & $<0.030 \mathrm{mg} / \mathrm{l}$ & $<0.030 \mathrm{mg} / \mathrm{l}$ & $<0.030 \mathrm{mg} / \mathrm{l}$ & 1 & \\
\hline 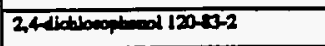 & $<0.030 \mathrm{mg} / \mathrm{l}$ & $<0.030 \mathrm{mg} / \mathrm{l}$ & $<0.030 \mathrm{mg} / \mathrm{l}$ & $<0.030 \mathrm{mg} / \mathrm{l}$ & 1 & \\
\hline 2,4imatyol 105-67-9 & $<0.030 \mathrm{mg} / \mathrm{l}$ & $<0.030 \mathrm{mg} / 1$ & $<0.030 \mathrm{mg} / 1$ & $<0.030 \mathrm{mg} / \mathrm{l}$ & 1 & \\
\hline 24dimitroptreed 51-225 & $<0.030 \mathrm{mg} / \mathrm{l}$ & $<0.030 \mathrm{mg} / 1$ & $<0.030 \mathrm{mg} / \mathrm{l}$ & $<0.030 \mathrm{mg} / 1$ & 1 & \\
\hline
\end{tabular}


Outfall S10

Pares B and C Continued

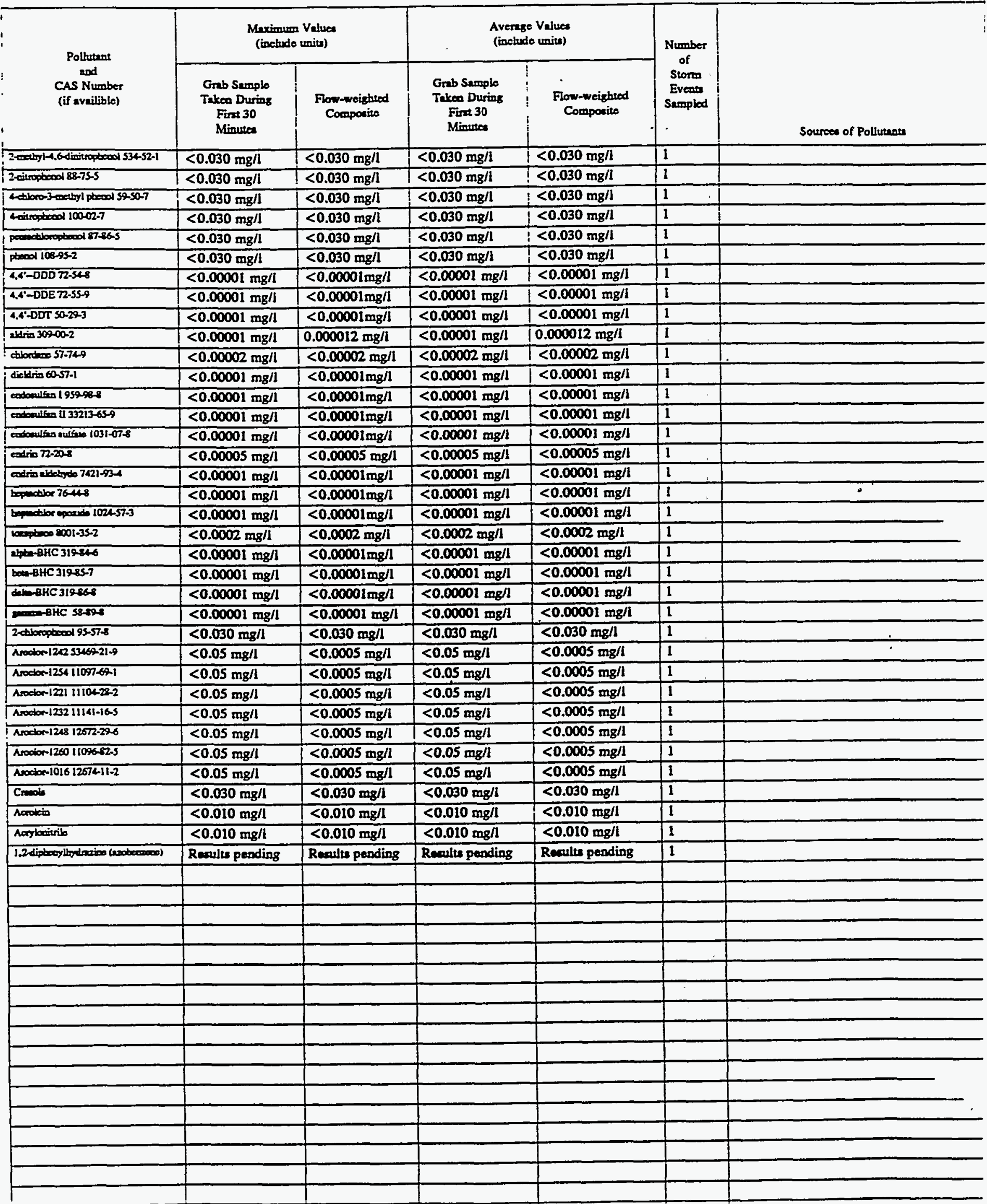


VII. Discherge Informetion (continued from page 3 of Form 2F

Pan A - You must provide the reaula of a leart one anelysis for every pollunent in this table. Complete one uble for cech ouffall. See instructions for additional details.

\begin{tabular}{|c|c|c|c|c|c|c|}
\hline \multirow{2}{*}{$\begin{array}{l}\text { Polluteant } \\
\text { and } \\
\text { CAS Number } \\
\text { (if availible) }\end{array}$} & \multicolumn{2}{|c|}{$\begin{array}{l}\text { Meximuno Valuea } \\
\text { (inchude unisu) }\end{array}$} & \multicolumn{2}{|c|}{$\begin{array}{l}\text { Averege Valuea } \\
\text { (inchude univo) }\end{array}$} & \multirow{2}{*}{$\begin{array}{l}\text { Number } \\
\text { of } \\
\text { Swotm } \\
\text { Event } \\
\text { Sumpled }\end{array}$} & \multirow[b]{2}{*}{ Sources of Polthrans } \\
\hline & $\begin{array}{c}\text { Greb Semple } \\
\text { Takeo During } \\
\text { Finet } 30 \\
\text { Minewex }\end{array}$ & $\begin{array}{l}\text { Foow-weighted } \\
\text { Componito }\end{array}$ & $\begin{array}{c}\text { Grab Semplo } \\
\text { Takea During } \\
\text { Fint } 30 \\
\text { Minutes }\end{array}$ & $\begin{array}{l}\text { Frow-wöightod } \\
\text { Composite }\end{array}$ & & \\
\hline pil and Groens & $<2 \mathrm{mg} / \mathrm{l}$ & & $<2 \mathrm{mg} / 1$ & & 1 & \\
\hline 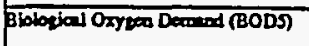 & $20.99 \mathrm{mg} / \mathrm{l}$ & $27.62 \mathrm{mg} / \mathrm{l}$ & $20.99 \mathrm{mg} / \mathrm{l}$ & $27.62 \mathrm{mg} / 1$ & I & \\
\hline 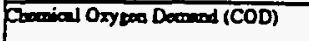 & $69 \mathrm{mg} / \mathrm{l}$ & $62 \mathrm{mg} / 1$ & $69 \mathrm{mg} / 1$ & $62 \mathrm{mg} / 1$ & 1 & \\
\hline Toal Suppored Salide (TSO) & $13 \mathrm{mg} / \mathrm{I}$ & $18 \mathrm{mg} / \mathrm{h}$ & $13 \mathrm{mg} / \mathrm{h}$ & $18 \mathrm{mg} / \mathrm{l}$ & 1 & \\
\hline Toeal Kjelatel Nilloosen & $0.7 \mathrm{mg} / 1$ & $0.6 \mathrm{mg} / \mathrm{l}$ & $0.7 \mathrm{mg} / 1$ & $0.6 \mathrm{mg} / \mathrm{l}$ & 1 & \\
\hline Nitne plum Nitrice Nilroses & $<0.1 \mathrm{mg} / \mathrm{l}$ & $0.2 \mathrm{mg} / \mathrm{l}$ & $<0.1 \mathrm{mg} / 1$ & $0.2 \mathrm{mg} / \mathrm{l}$ & 1 & \\
\hline Toul Phooptorse & $0.15 \mathrm{mg} / 1$ & $0.12 \mathrm{mg} / 1$ & $0.15 \mathrm{mg} / \mathrm{l}$ & $0.12 \mathrm{mg} / 1$ & 1 & \\
\hline $\mathrm{pH}$ & Mein 82 & Marimen 8.2 & Minim 8.2 & Maxims 8.2 & & \\
\hline
\end{tabular}

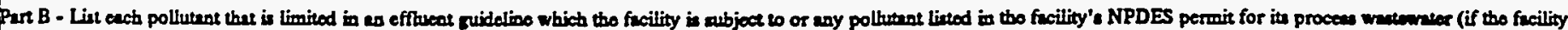

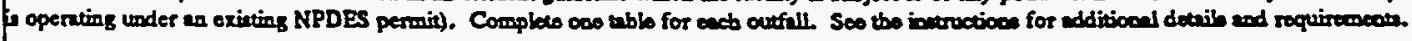

\begin{tabular}{|c|c|c|c|c|c|c|}
\hline \multirow{2}{*}{$\begin{array}{l}\text { Polbuant } \\
\text { eod } \\
\text { CAs Number } \\
\text { (if avnilible) }\end{array}$} & \multicolumn{2}{|c|}{$\begin{array}{l}\text { Mexiondon Vilan } \\
\text { (ivolude units) }\end{array}$} & \multicolumn{2}{|c|}{$\begin{array}{l}\text { Averaso Values } \\
\text { (ivectude units) }\end{array}$} & \multirow{2}{*}{$\begin{array}{c}\text { Numbor } \\
\text { of } \\
\text { storm } \\
\text { Evente } \\
\text { Serupiod }\end{array}$} & \multirow{2}{*}{$\begin{array}{l}\therefore \\
\text { Sources of Polverome }\end{array}$} \\
\hline & 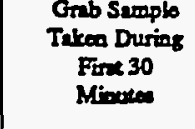 & $\begin{array}{l}\text { Foow-weithitod } \\
\text { Componito }\end{array}$ & $\begin{array}{l}\text { Grab Sumpto } \\
\text { Tarea During } \\
\text { Firse } 30 \\
\text { Minocece }\end{array}$ & $\begin{array}{l}\text { Flow-weighted } \\
\text { Cocosposits }\end{array}$ & & \\
\hline 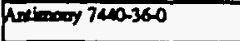 & $<0.003 \mathrm{mg} / \mathrm{l}$ & $<0.003 \mathrm{mg} / 1$ & $<0.003 \mathrm{mg} / \mathrm{l}$ & $<0.003 \mathrm{mg} / 1$ & 1 & \\
\hline Symide & $<0.002 \mathrm{mg} / \mathrm{l}$ & & $<0.002 \mathrm{mg} / \mathrm{l}$ & & 1 & \\
\hline Manary 703997.6 & $<0.0002 \mathrm{mg} / \mathrm{l}$ & $<0.0002 \mathrm{mg} / 1$ & $<0.0002 \mathrm{mg} / 1$ & $<0.0002 \mathrm{mg} / \mathrm{l}$ & 1 & \\
\hline pronde & $0.004 \mathrm{mg} / \mathrm{l}$ & & $0.004 \mathrm{mg} / \mathrm{l}$ & & 1 & $\vdots$ \\
\hline Fenoum $7782-49-2$ & $<0.002 \mathrm{mg} / \mathrm{l}$ & $<0.002 \mathrm{mg} / \mathrm{l}$ & $<0.002 m g / h$ & $<0.002 \mathrm{mg} / \mathrm{l}$ & 1 & \\
\hline Sulthe & $92 \mathrm{mg} / 1$ & $92 \mathrm{mg} / 1$ & $92 \mathrm{mg} / 1$ & $92 \mathrm{mg} / \mathrm{l}$ & 1 & \\
\hline Purtacteres & $<0.05 \mathrm{mg} / \mathrm{l}$ & $<0.05 \mathrm{mg} / 1$ & $<0.05 \mathrm{mg} / \mathrm{l}$ & $<0.05 \mathrm{mg} / \mathrm{l}$ & 1 & \\
\hline Numianom 742090-5 & $0.72 \mathrm{mg} / \mathrm{l}$ & $0.65 \mathrm{mg} / \mathrm{l}$ & $0.72 \mathrm{mg} / 1$ & $0.65 \mathrm{mg} / \mathrm{l}$ & 1 & \\
\hline$n=0000740-38-2$ & $<0.04 \mathrm{mg} / \mathrm{l}$ & $<0.04 \mathrm{mg} / 1$ & $<0.04 \mathrm{mg} / \mathrm{l}$ & $<0.04 \mathrm{mg} / \mathrm{l}$ & 1 & \\
\hline Borou 7400124 & $0.094 \mathrm{mg} / \mathrm{l}$ & $0.105 \mathrm{mg} / 1$ & $0.094 \mathrm{mg} / \mathrm{l}$ & $0.105 \mathrm{mg} / \mathrm{l}$ & 1 & \\
\hline Berylliven 74to-11.7 & $<0.0004 \mathrm{mg} / \mathrm{l}$ & $<0.0004 \mathrm{mg} / \mathrm{l}$ & $<0.0004 \mathrm{mg} / 1$ & $<0.0004 \mathrm{mg} / 1$ & 1 & \\
\hline Cadmingen 740-13-9 & $<0.004 \mathrm{mg} / \mathrm{l}$ & $<0.004 \mathrm{mg} / \mathrm{l}$ & $<0.004 \mathrm{mg} / \mathrm{l}$ & $<0.004 \mathrm{mg} / \mathrm{l}$ & 1 & \\
\hline celoingor $740-70-2$ & $40.2 \mathrm{mg} / \mathrm{l}$ & $40.6 \mathrm{mg} / 1$ & $40.2 \mathrm{mg} / 1$ & $40.6 \mathrm{mg} / \mathrm{l}$ & 1 & \\
\hline 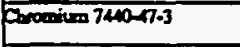 & $<0.006 \mathrm{mg} / \mathrm{l}$ & $<0.006 \mathrm{mg} / \mathrm{l}$ & $<0.006 \mathrm{mg} / \mathrm{l}$ & $<0.006 \mathrm{mg} / \mathrm{l}$ & 1 & \\
\hline Cotalis 7400-48s & $<0.002 \mathrm{mg} / \mathrm{l}$ & $<0.002 \mathrm{mg} / \mathrm{l}$ & $<0.002 \mathrm{mg} / \mathrm{l}$ & $<0.002 \mathrm{mg} / \mathrm{l}$ & 1 & \\
\hline Copper 7400-50s & $<0.006 \mathrm{mg} / \mathrm{l}$ & $<0.006 \mathrm{mg} / \mathrm{l}$ & $<0.006 \mathrm{mg} / \mathrm{l}$ & $<0.006 \mathrm{mg} / \mathrm{l}$ & 1 & \\
\hline 10007639896 & $1.21 \mathrm{mg} / \mathrm{I}$ & $1.19 \mathrm{mg} / \mathrm{l}$ & $1.21 \mathrm{mg} / 1$ & $1.19 \mathrm{mg} / 1$ & 1 & \\
\hline Fend 743902-1 & $<0.02 \mathrm{mg} / \mathrm{l}$ & $<0.02 \mathrm{mg} / 1$ & $<0.02 \mathrm{mg} / \mathrm{l}$ & $<0.02 \mathrm{mg} / \mathrm{l}$ & 1 & \\
\hline Fithive $7439993-2$ & $<0.02 \mathrm{mg} / 1$ & $0.02 \mathrm{mg} / 1$ & $<0.02 \mathrm{mg} / 1$ & $0.02 \mathrm{mg} / \mathrm{l}$ & 1 & \\
\hline Meposium 7439-9S-4 & $7.35 \mathrm{mg} / \mathrm{l}$ & $7.32 \mathrm{mg} / 1$ & $7.35 \mathrm{mg} / 1$ & $7.32 \mathrm{mg} / \mathrm{l}$ & 1 & \\
\hline Mmanowos $743996-5$ & $0.360 \mathrm{mg} / \mathrm{l}$ & $0.393 \mathrm{mg} / 1$ & $0.360 \mathrm{mg} / \mathrm{l}$ & $0.393 \mathrm{mg} / \mathrm{l}$ & 1 & \\
\hline 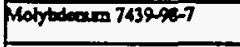 & $<0.006 \mathrm{mg} / \mathrm{l}$ & $<0.006 \mathrm{mg} / \mathrm{l}$ & $<0.006 \mathrm{mg} / \mathrm{l}$ & $<0.006 \mathrm{mg} / \mathrm{l}$ & 1 & \\
\hline Nitoln 1740020 & $<0.008 \mathrm{mg} / \mathrm{l}$ & $<0.008 \mathrm{mg} / 1$ & $<0.008 \mathrm{mg} / \mathrm{l}$ & $<0.008 \mathrm{mg} / \mathrm{l}$ & 1 & \\
\hline Promiven 74009-7 & $7.8 \mathrm{mg} / \mathrm{l}$ & $7.4 \mathrm{mg} / 1$ & $7.8 \mathrm{mg} / \mathrm{l}$ & $7.4 \mathrm{mg} / 1$ & 1 & \\
\hline $4 \mathrm{~s}=\mathrm{7400-2-4}$ & $<0.006 \mathrm{mg} / \mathrm{l}$ & $<0.006 \mathrm{mg} / \mathrm{l}$ & $<0.006 \mathrm{mg} / \mathrm{l}$ & $<0.006 \mathrm{mg} / 1$ & 1 & \\
\hline sodhem 740-20-3 & $4.29 \mathrm{mg} / \mathrm{l}$ & $4.32 \mathrm{mg} / 1$ & $4.29 \mathrm{mg} / 1$ & $4.32 \mathrm{mg} / 1$ & 1 & \\
\hline Fiveninga 7400-32.6 & $0.01 \mathrm{mg} / \mathrm{l}$ & $0.01 \mathrm{mg} / 1$ & $0.01 \mathrm{mg} / 1$ & $0.01 \mathrm{mg} / \mathrm{l}$ & 1 & \\
\hline 20007400656 & $<0.01 \mathrm{mg} / 1$ & $0.05 \mathrm{mg} / 1$ & $<0.01 \mathrm{mg} / 1$ & $0.05 \mathrm{mg} / 1$ & 1 & \\
\hline
\end{tabular}




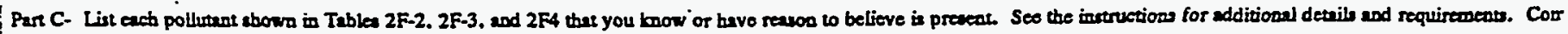
oso tablo for cach ourfall.

\begin{tabular}{|c|c|c|c|c|c|c|}
\hline \multirow{2}{*}{$\begin{array}{l}\text { Polhuint } \\
\text { and } \\
\text { CAS Number } \\
\text { (if availible) }\end{array}$} & \multicolumn{2}{|c|}{$\begin{array}{l}\text { Maximum Values } \\
\text { (inctudo unita) }\end{array}$} & \multicolumn{2}{|c|}{$\begin{array}{l}\text { Averngo Valuea } \\
\text { (inchude unitu) }\end{array}$} & \multirow{2}{*}{$\begin{array}{l}\text { Nitomber } \\
\text { of } \\
\text { Storm } \\
\text { Eveotn } \\
\text { Sumpled }\end{array}$} & \multirow[b]{2}{*}{ Source of Pollutuats } \\
\hline & $\begin{array}{c}\text { Grab Sample } \\
\text { Takea During } \\
\text { Fint } 30 \\
\text { Mintes }\end{array}$ & $\begin{array}{l}\text { Flow } \\
\text { Comouponited }\end{array}$ & $\begin{array}{l}\text { Grab Samplo } \\
\text { Taken During } \\
\text { Fint } 30 \\
\text { Minute }\end{array}$ & $\begin{array}{l}\text { Flow-weighied } \\
\text { Coconporito }\end{array}$ & & \\
\hline 1.1.1.etrictboroutheos 7i-55-6 & $<0.010 \mathrm{mg} / \mathrm{l}$ & & $<0.010 \mathrm{mg} / \mathrm{l}$ & & 1 & \\
\hline 1.1.2.2-tetrectionoutane $77-345$ & $<0.010 \mathrm{mg} / \mathrm{l}$ & & $<0.010 \mathrm{mg} / 1$ & & 1 & \\
\hline $1,1.2-$ trictionoctanes $7900-5$ & $<0.010 \mathrm{mg} / \mathrm{l}$ & & $<0.010 \mathrm{mg} / \mathrm{l}$ & & 1 & \\
\hline 1.1-dictorothen 75-36-3 & $<0.010 \mathrm{mg} / 1$ & & $<0.010 \mathrm{mg} / 1$ & & 1 & \\
\hline 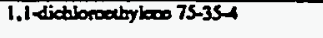 & $<0.010 \mathrm{mg} / \mathrm{l}$ & & $<0.010 \mathrm{mg} / \mathrm{l}$ & & 1 & \\
\hline 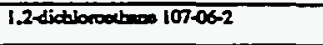 & $<0.010 \mathrm{mg} / \mathrm{l}$ & & $<0.010 \mathrm{mg} / 1$ & & 1 & \\
\hline 1.2-dictioropropenes 78.87.5 & $<0.010 \mathrm{mg} / \mathrm{l}$ & & $<0.010 \mathrm{mg} / 1$ & & 1 & \\
\hline 2-ctboroctbylvioglecter $110-75-8$ & $<0.010 \mathrm{mg} / 1$ & & $<0.010 \mathrm{mg} / \mathrm{l}$ & & 1 & \\
\hline$-001-43-2$ & $<0.010 \mathrm{mg} / \mathrm{l}$ & & $<0.010 \mathrm{mg} / \mathrm{l}$ & & 1 & \\
\hline 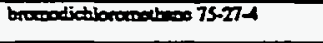 & $<0.010 \mathrm{mg} / \mathrm{l}$ & & $<0.010 \mathrm{mg} / 1$ & & 1 & \\
\hline bromolomeg $75-25-2$ & $<0.010 \mathrm{mg} / 1$ & & $<0.010 \mathrm{mg} / \mathrm{l}$ & & 1 & \\
\hline 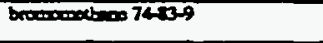 & $<0.010 \mathrm{mg} / \mathrm{l}$ & & $<0.010 \mathrm{mg} / 1$ & & 1 & \\
\hline arboo vetactibrits 56-23-5 & $<0.010 \mathrm{mg} / 1$ & & $<0.010 \mathrm{mg} / \mathrm{l}$ & & 1 & \\
\hline 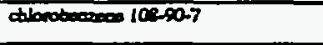 & $<0.010 \mathrm{mg} / \mathrm{l}$ & & $<0.010 \mathrm{mg} / \mathrm{l}$ & & 1 & \\
\hline chloractiox $75-00-3$ & $<0.010 \mathrm{mg} / 1$ & & $<0.010 \mathrm{mg} / \mathrm{l}$ & & 1 & \\
\hline chlocolom 67-66-3 & $<0.010 \mathrm{mg} / 1$ & & $<0.010 \mathrm{mg} / \mathrm{l}$ & & 1 & . \\
\hline chboranosbone 7487-3 & $<0.010 \mathrm{mg} / \mathrm{l}$ & & $<0.010 \mathrm{mg} / \mathrm{l}$ & & 1 & \\
\hline 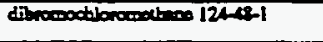 & $<0.010 \mathrm{mg} / \mathrm{l}$ & & $<0.010 \mathrm{mg} / \mathrm{l}$ & & 1 & \\
\hline 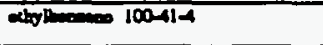 & $<0.010 \mathrm{mg} / \mathrm{l}$ & & $<0.010 \mathrm{mg} / \mathrm{l}$ & & 1 & \\
\hline cotsos loses-3 & $<0.010 \mathrm{mg} / 1$ & & $<0.010 \mathrm{mg} / 1$ & & 1 & \\
\hline vingl ctionite 75-014 & $<0.010 \mathrm{mg} / 1$ & & $<0.010 \mathrm{mg} / 1$ & & 1 & \\
\hline 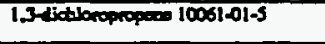 & $<0.010 \mathrm{mg} / \mathrm{l}$ & & $<0.010 \mathrm{mg} / 1$ & & 1 & \\
\hline tho-1.2-dictbroetheos 15660.5 & $<0.010 \mathrm{mg} / \mathrm{l}$ & & $<0.010 \mathrm{mg} / \mathrm{l}$ & & 1 & $\dot{1}$ \\
\hline apto exivity $12557-46-1$ & $14.4 \mathrm{poill}$ & $12.6 \mathrm{pci} / 1$ & $14.4 \mathrm{pci} / \mathrm{l}$ & $12.6 \mathrm{pei} / \mathrm{l}$ & 1 & \\
\hline beles saivity $1258746-1$ & 10.3 peill & $16.8 \mathrm{pci} / \mathrm{l}$ & $10.3 \mathrm{pci} / \mathrm{l}$ & $16.8 \mathrm{pei} / 1$ & 1 & \\
\hline recal ontilom & $29,000 \mathrm{col} .1 \mathrm{~L}$ & $35,000 \mathrm{cod} .1 \mathrm{~L}$ & $29,000 \mathrm{col} .1 \mathrm{~L}$ & $35,000 \mathrm{col} .1 \mathrm{~L}$ & 1 & \\
\hline Thellition 7440-285-0 & $<0.001 \mathrm{mg} / 1$ & $<0.001 \mathrm{mg} / \mathrm{l}$ & $<0.001 \mathrm{mg} / \mathrm{l}$ & $<0.001 \mathrm{mg} / 1$ & 1 & \\
\hline$U \sin 740-61+1$ & $0.041 \mathrm{mg} / \mathrm{l}$ & $0.038 \mathrm{mg} / \mathrm{l}$ & $0.041 \mathrm{mg} / 1$ & $0.038 \mathrm{mg} / \mathrm{l}$ & 1 & \\
\hline Carmentrity & $150 \mathrm{pei} / 1$ & $91 \mathrm{pci} / 1$ & 150 pei/l & 91 peill & 1 & \\
\hline
\end{tabular}

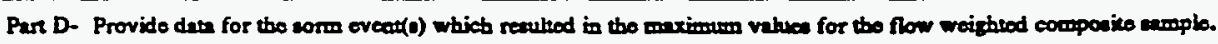

\begin{tabular}{|c|c|c|c|c|c|c|c|}
\hline $\begin{array}{l}1 . \\
\text { Dato of } \\
\text { Storm } \\
\text { Eveost }\end{array}$ & $\begin{array}{l}2 . \\
\text { Durition } \\
\text { of Storm } \\
\text { (in } \\
\text { minines) }\end{array}$ & $\begin{array}{l}3 . \\
\text { Total nimfall } \\
\text { durings storm oveat } \\
\text { (in inches) }\end{array}$ & 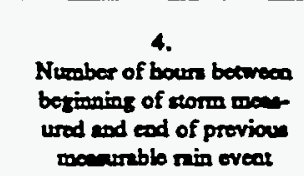 & 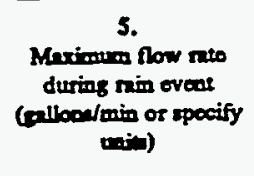 & $\begin{array}{l}6 . \\
\text { Total flow from } \\
\text { nin oveat } \\
\text { (Gallow or } \\
\text { spocify univ) }\end{array}$ & $\begin{array}{c}7 . \\
\text { Seavon } \\
\text { sumplo was } \\
\text { tikeo }\end{array}$ & $\begin{array}{l}8 . \\
\text { Form of } \\
\text { Procipintion } \\
\text { (rniafill, } \\
\text { mooweneti) }\end{array}$ \\
\hline $\begin{array}{l}10 / 14 / 91-1 \\
0 / 15 / 91\end{array}$ & 650 & 1.5 & 226.25 & 228 & 115,182 & Fall & Rninfall \\
\hline
\end{tabular}

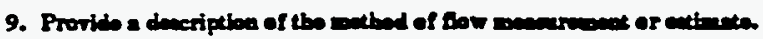

An ISCO 3230 Flow meter was med to obtin water leved reading. This instrument was calibrated with the normal dry weather flow, if present, as lev. zero. The flow meter was programmed with the characteritics of the converance so that flow rates and total flows conld be calenlated by the meter. 
Outfoll S11

i Parta B ad C Continued

1

\begin{tabular}{|c|c|c|c|c|c|c|}
\hline \multirow{2}{*}{$\begin{array}{l}\text { Pollutans } \\
\text { and } \\
\text { CAS Number } \\
\text { (if availiblo) }\end{array}$} & \multicolumn{2}{|c|}{$\begin{array}{l}\text { Mexionum Valuea } \\
\text { (inctudo uniti) }\end{array}$} & \multicolumn{2}{|c|}{$\begin{array}{l}\text { Average Valued } \\
\text { (ibeludo vaid) }\end{array}$} & \multirow{2}{*}{$\begin{array}{l}\text { Nurober } \\
\text { of } \\
\text { Storm } \\
\text { Eveoss } \\
\text { Samplod }\end{array}$} & \multirow[b]{2}{*}{ Sources of Polverants } \\
\hline & $\begin{array}{l}\text { Grab Sumple } \\
\text { Taken During } \\
\text { First } 30 \\
\text { Minuted }\end{array}$ & $\begin{array}{l}\text { Flow-weightiod } \\
\text { Comporite }\end{array}$ & $\begin{array}{l}\text { Grab Sumplo } \\
\text { Takea Drurias } \\
\text { Finat } 30 \\
\text { Minures }\end{array}$ & $\begin{array}{c}\text { Flow-weighted } \\
\text { Comporise } \\
\text {. }\end{array}$ & & \\
\hline Beriver 74a-37-3 & $0.0637 \mathrm{mg} / \mathrm{h}$ & $0.0635 \mathrm{mg} / \mathrm{h}$ & $0.0637 \mathrm{mg} / 1$ & $0.0635 \mathrm{mg} / \mathrm{l}$ & 1 & \\
\hline 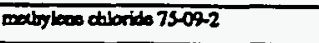 & $<0.010 \mathrm{mg} / \mathrm{l}$ & & $<0.010 \mathrm{mg} / 1$ & & 1 & \\
\hline 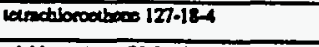 & $<0.010 \mathrm{mg} / \mathrm{l}$ & - & $<0.010 \mathrm{mg} / \mathrm{l}$ & & 1 & \\
\hline 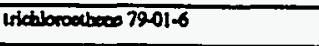 & $<0.010 \mathrm{mg} / \mathrm{l}$ & & $<0.010 \mathrm{mg} / \mathrm{l}$ & & 1 & \\
\hline Xyloosen & $<0.010 \mathrm{mg} / \mathrm{l}$ & & $<0.010 \mathrm{mg} / \mathrm{l}$ & & 1 & \\
\hline 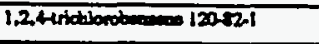 & $<0.010 \mathrm{mg} / \mathrm{l}$ & $<0.010 \mathrm{mg} / \mathrm{l}$ & $<0.010 \mathrm{mg} / \mathrm{l}$ & $<0.010 \mathrm{mg} / 1$ & 1 & \\
\hline 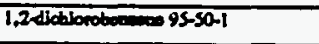 & $<0.010 \mathrm{mg} / 1$ & $<0.010 \mathrm{mg} / \mathrm{I}$ & $<0.010 \mathrm{mg} / \mathrm{l}$ & $<0.010 \mathrm{mg} / \mathrm{l}$ & 1 & \\
\hline 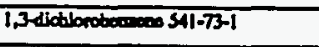 & $<0.010 \mathrm{mg} / \mathrm{l}$ & $<0.010 \mathrm{mg} / \mathrm{l}$ & $<0.010 \mathrm{mg} / \mathrm{l}$ & $<0.010 \mathrm{mg} / \mathrm{l}$ & 1 & \\
\hline 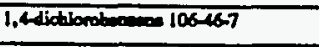 & $<0.010 \mathrm{mg} / \mathrm{l}$ & $<0.010 \mathrm{mg} / \mathrm{l}$ & $<0.010 \mathrm{mg} / \mathrm{l}$ & $<0.010 \mathrm{mg} / \mathrm{l}$ & 1 & \\
\hline 24 dhitrochenen $121-142$ & $<0.010 \mathrm{mg} / \mathrm{l}$ & $<0.010 \mathrm{mg} / 1$ & $<0.010 \mathrm{mg} / \mathrm{l}$ & $<0.010 \mathrm{mg} / \mathrm{l}$ & 1 & \\
\hline 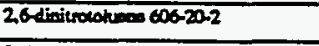 & $<0.010 \mathrm{mg} / \mathrm{l}$ & $<0.010 \mathrm{mg} / \mathrm{l}$ & $<0.010 \mathrm{mg} / \mathrm{l}$ & $<0.010 \mathrm{mg} / \mathrm{l}$ & 1 & \\
\hline 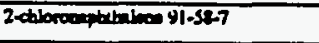 & $<0.010 \mathrm{mg} / \mathrm{l}$ & $<0.010 \mathrm{mg} / \mathrm{l}$ & $<0.010 \mathrm{mg} / 1$ & $<0.010 \mathrm{mg} / \mathrm{l}$ & 1 & \\
\hline 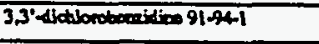 & $<0.010 \mathrm{mg} /$ & $<0.010 \mathrm{mg} / \mathrm{l}$ & $<0.010 \mathrm{mg} / \mathrm{l}$ & $<0.010 \mathrm{mg} / \mathrm{l}$ & 1 & \\
\hline 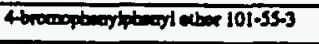 & $<0.010 \mathrm{mg} / \mathrm{l}$ & $<0.010 \mathrm{mg} / \mathrm{l}$ & $<0.010 \mathrm{mg} / 1$ & $<0.010 \mathrm{mg} / 1$ & 1 & \\
\hline 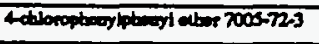 & $<0.010 \mathrm{mg} / 1$ & $<0.010 \mathrm{mg} / 1$ & $<0.010 \mathrm{mg} / 1$ & $<0.010 \mathrm{mg} / 1$ & 1 & \\
\hline 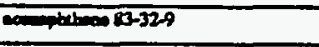 & $<0.010 \mathrm{mg} / \mathrm{l}$ & $<0.010 \mathrm{mg} / \mathrm{l}$ & $<0.010 \mathrm{mg} / 1$ & $<0.010 \mathrm{mg} / \mathrm{l}$ & 1 & \\
\hline 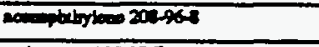 & $<0.010 \mathrm{mg} / 1$ & $<0.010 \mathrm{mg} / \mathrm{l}$ & $<0.010 \mathrm{mg} / 1$ & $<0.010 \mathrm{mg} / \mathrm{l}$ & 1 & \\
\hline $120-127$ & $<0.010 \mathrm{mg} / \mathrm{l}$ & $<0.010 \mathrm{mg} / \mathrm{l}$ & $<0.010 \mathrm{mg} / \mathrm{l}$ & $<0.010 \mathrm{mg} / \mathrm{l}$ & 1 & \\
\hline Wheng.5 & $<0.030 \mathrm{mg} / \mathrm{l}$ & $<0.030 \mathrm{mg} / \mathrm{l}$ & $<0.030 \mathrm{mg} / 1$ & $<0.030 \mathrm{mg} / \mathrm{l}$ & 1 & \\
\hline 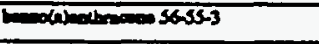 & $<0.010 \mathrm{mg} / \mathrm{l}$ & $<0.010 \mathrm{mg} / \mathrm{l}$ & $<0.010 \mathrm{mg} / \mathrm{l}$ & $<0.010 \mathrm{mg} / \mathrm{l}$ & 1 & \\
\hline 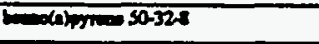 & $<0.010 \mathrm{mg} / \mathrm{l}$ & $<0.010 \mathrm{mg} / \mathrm{h}$ & $<0.010 \mathrm{mg} / 1$ & $<0.010 \mathrm{mg} / \mathrm{l}$ & 1 & \\
\hline 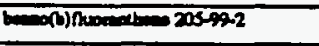 & $<0.010 \mathrm{mg} / \mathrm{l}$ & $<0.010 \mathrm{mg} / \mathrm{l}$ & $<0.010 \mathrm{mg} / 1$ & $<0.010 \mathrm{mg} / \mathrm{l}$ & 1 & \\
\hline 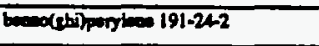 & $<0.010 \mathrm{mg} / \mathrm{l}$ & $<0.010 \mathrm{mg} / \mathrm{l}$ & $<0.010 \mathrm{mg} / 1$ & $<0.010 \mathrm{mg} / \mathrm{l}$ & 1 & \\
\hline 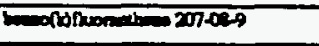 & $<0.010 \mathrm{mg} / \mathrm{l}$ & $<0.010 \mathrm{mg} / \mathrm{l}$ & $<0.010 \mathrm{mg} / \mathrm{l}$ & $<0.010 \mathrm{mg} / \mathrm{h}$ & 1 & \\
\hline 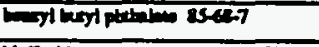 & $<0.010 \mathrm{mg} / \mathrm{l}$ & $<0.010 \mathrm{mg} / \mathrm{l}$ & $<0.010 \mathrm{mg} / \mathrm{l}$ & $<0.010 \mathrm{mg} / 1$ & 1 & \\
\hline 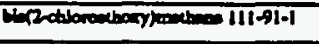 & $<0.010 \mathrm{mg} / 1$ & $<0.010 \mathrm{mg} / \mathrm{l}$ & $<0.010 \mathrm{mg} / 1$ & $<0.010 \mathrm{mg} / \mathrm{l}$ & 1 & \\
\hline 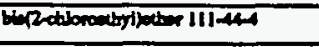 & $<0.010 \mathrm{mg} / \mathrm{l}$ & $<0.010 \mathrm{mg} / \mathrm{l}$ & $<0.010 \mathrm{mg} / \mathrm{l}$ & $<0.010 \mathrm{mg} / \mathrm{l}$ & 1 & \\
\hline 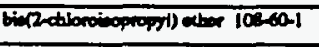 & $<0.010 \mathrm{mg} / \mathrm{l}$ & $<0.010 \mathrm{mg} / \mathrm{l}$ & $<0.010 \mathrm{mg} / 1$ & $<0.010 \mathrm{mg} / \mathrm{l}$ & 1 & \\
\hline 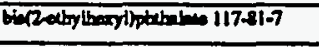 & $<0.010 \mathrm{mg} / \mathrm{l}$ & $<0.010 \mathrm{mg} / \mathrm{l}$ & $<0.010 \mathrm{mg} / 1$ & $<0.010 \mathrm{mg} / \mathrm{l}$ & 1 & \\
\hline 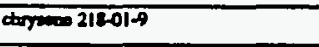 & $<0.010 \mathrm{mg} / \mathrm{l}$ & $<0.010 \mathrm{mg} / 1$ & $<0.010 \mathrm{mg} / \mathrm{l}$ & $<0.010 \mathrm{mg} / \mathrm{l}$ & 1 & \\
\hline 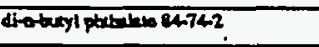 & $<0.010 \mathrm{mg} / \mathrm{l}$ & $<0.010 \mathrm{mg} / \mathrm{l}$ & $<0.010 \mathrm{mg} / \mathrm{l}$ & $<0.010 \mathrm{mg} / 1$ & 1 & \\
\hline 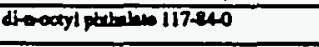 & $<0.010 \mathrm{mg} / \mathrm{l}$ & $<0.010 \mathrm{mg} / \mathrm{l}$ & $<0.010 \mathrm{mg} / 1$ & $<0.010 \mathrm{mg} / 1$ & 1 & \\
\hline 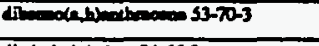 & $<0.010 \mathrm{mg} / 1$ & $<0.010 \mathrm{mg} / \mathrm{l}$ & $<0.010 \mathrm{mg} / 1$ & $<0.010 \mathrm{mg} / 1$ & 1 & \\
\hline 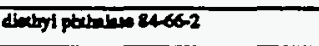 & $<0.010 \mathrm{mg} / \mathrm{l}$ & $<0.010 \mathrm{mg} / \mathrm{l}$ & $<0.010 \mathrm{mg} / \mathrm{l}$ & $<0.010 \mathrm{mg} / \mathrm{l}$ & 1 & \\
\hline 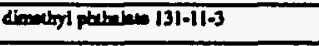 & $<0.010 \mathrm{mg} / \mathrm{l}$ & $<0.010 \mathrm{mg} / 1$ & $<0.010 \mathrm{mg} / \mathrm{l}$ & $<0.010 \mathrm{mg} / 1$ & 1 & \\
\hline 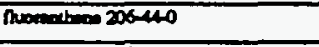 & $<0.010 \mathrm{mg} / \mathrm{l}$ & $<0.010 \mathrm{mg} / \mathrm{l}$ & $<0.010 \mathrm{mg} / \mathrm{l}$ & $<0.010 \mathrm{mg} / \mathrm{l}$ & 1 & \\
\hline Duomesos 26.73-7 & $<0.010 \mathrm{mg} / \mathrm{l}$ & $<0.010 \mathrm{mg} / \mathrm{l}$ & $<0.010 \mathrm{mg} / 1$ & $<0.010 \mathrm{mg} / 1$ & 1 & \\
\hline 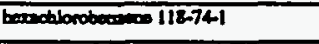 & $<0.010 \mathrm{mg} / 1$ & $<0.010 \mathrm{mg} / \mathrm{l}$ & $<0.010 \mathrm{mg} / \mathrm{l}$ & $<0.010 \mathrm{mg} / \mathrm{l}$ & 1 & \\
\hline 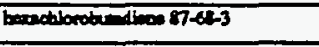 & $<0.010 \mathrm{mg} / \mathrm{l}$ & $<0.010 \mathrm{mg} / \mathrm{l}$ & $<0.010 \mathrm{mg} / \mathrm{l}$ & $<0.010 \mathrm{mg} / \mathrm{l}$ & 1 & \\
\hline 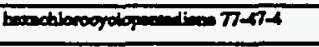 & $<0.010 \mathrm{mg} / \mathrm{l}$ & $<0.010 \mathrm{mg} / \mathrm{l}$ & $<0.010^{\circ} \mathrm{mg} / \mathrm{l}$ & $<0.010 \mathrm{mg} / \mathrm{l}$ & 1 & \\
\hline 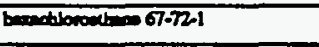 & $<0.010 \mathrm{mg} / \mathrm{l}$ & $<0.010 \mathrm{mg} / \mathrm{l}$ & $<0.010 \mathrm{mg} / \mathrm{l}$ & $<0.010 \mathrm{mg} / 1$ & 1 & \\
\hline $193,32.5$ & $<0.010 \mathrm{mg} / \mathrm{l}$ & $<0.010 \mathrm{mg} / \mathrm{l}$ & $<0.010 \mathrm{mg} / \mathrm{l}$ & $<0.010 \mathrm{mg} / \mathrm{l}$ & 1 & \\
\hline moptenowe $72-59-1$ & $<0.010 \mathrm{mg} / 1$ & $<0.010 \mathrm{mg} / \mathrm{l}$ & $<0.010 \mathrm{mg} / \mathrm{l}$ & $<0.010 \mathrm{mg} / \mathrm{l}$ & 1 & \\
\hline 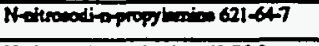 & $<0.010 \mathrm{mg} / \mathrm{l}$ & $<0.010 \mathrm{mg} / \mathrm{l}$ & $<0.010 \mathrm{mg} / 1$ & $<0.010 \mathrm{mg} / 1$ & 1 & \\
\hline 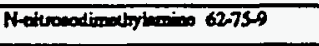 & $<0.010 \mathrm{mg} / \mathrm{l}$ & $<0.010 \mathrm{mg} / \mathrm{l}$ & $<0.010 \mathrm{mg} / \mathrm{l}$ & $<0.010 \mathrm{mg} / \mathrm{l}$ & 1 & \\
\hline 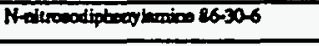 & $<0.010 \mathrm{mg} / \mathrm{l}$ & $<0.010 \mathrm{mg} / \mathrm{l}$ & $<0.010 \mathrm{mg} / 1$ & $<0.010 \mathrm{mg} / 1$ & 1 & \\
\hline 20 tethateon $91.20-3$ & $<0.010 \mathrm{mg} / 1$ & $<0.010 \mathrm{mg} / 1$ & $<0.010 \mathrm{mg} / \mathrm{l}$ & $<0.010 \mathrm{mg} / 1$ & 1 & \\
\hline 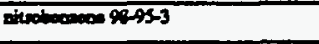 & $<0.010 \mathrm{mg} / 1$ & $<0.010 \mathrm{mg} / 1$ & $<0.010 \mathrm{mg} / \mathrm{l}$ & $<0.010 \mathrm{mg} / 1$ & 1 & \\
\hline thenthessols & $<0.010 \mathrm{mg} / \mathrm{l}$ & $<0.010 \mathrm{mg} / \mathrm{I}$ & $<0.010 \mathrm{mg} / \mathrm{l}$ & $<0.010 \mathrm{mg} / \mathrm{l}$ & 1 & \\
\hline mpines 129000 & $<0.010 \mathrm{mg} / \mathrm{l}$ & $<0.010 \mathrm{mg} / \mathrm{l}$ & $<0.010 \mathrm{mg} / \mathrm{l}$ & $<0.010 \mathrm{mg} / \mathrm{l}$ & 1 & \\
\hline 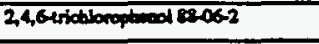 & $<0.030 \mathrm{mg} / 1$ & $<0.030 \mathrm{mg} / \mathrm{l}$ & $<0.030 \mathrm{mg} / \mathrm{l}$ & $<0.030 \mathrm{mg} / 1$ & 1 & \\
\hline 24dictionopheod 120-13-2 & $<0.030 \mathrm{mg} / \mathrm{l}$ & $<0.030 \mathrm{mg} / \mathrm{l}$ & $<0.030 \mathrm{mg} / \mathrm{l}$ & $<0.030 \mathrm{mg} / 1$ & 1 & \\
\hline 24dinectyphad 105-67-9 & $<0.030 \mathrm{mg} / \mathrm{l}$ & $<0.030 \mathrm{mg} / \mathrm{l}$ & $<0.030 \mathrm{mg} / \mathrm{l}$ & $<0.030 \mathrm{mg} / 1$ & 1 & \\
\hline 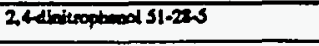 & $<0.030 \mathrm{mg} / \mathrm{l}$ & $<0.030 \mathrm{mg} / 1$ & $<0.030 \mathrm{mg} / 1$ & $<0.030 \mathrm{mg} / 1$ & 1 & \\
\hline
\end{tabular}


Outfall S11

: Part B and C Continued

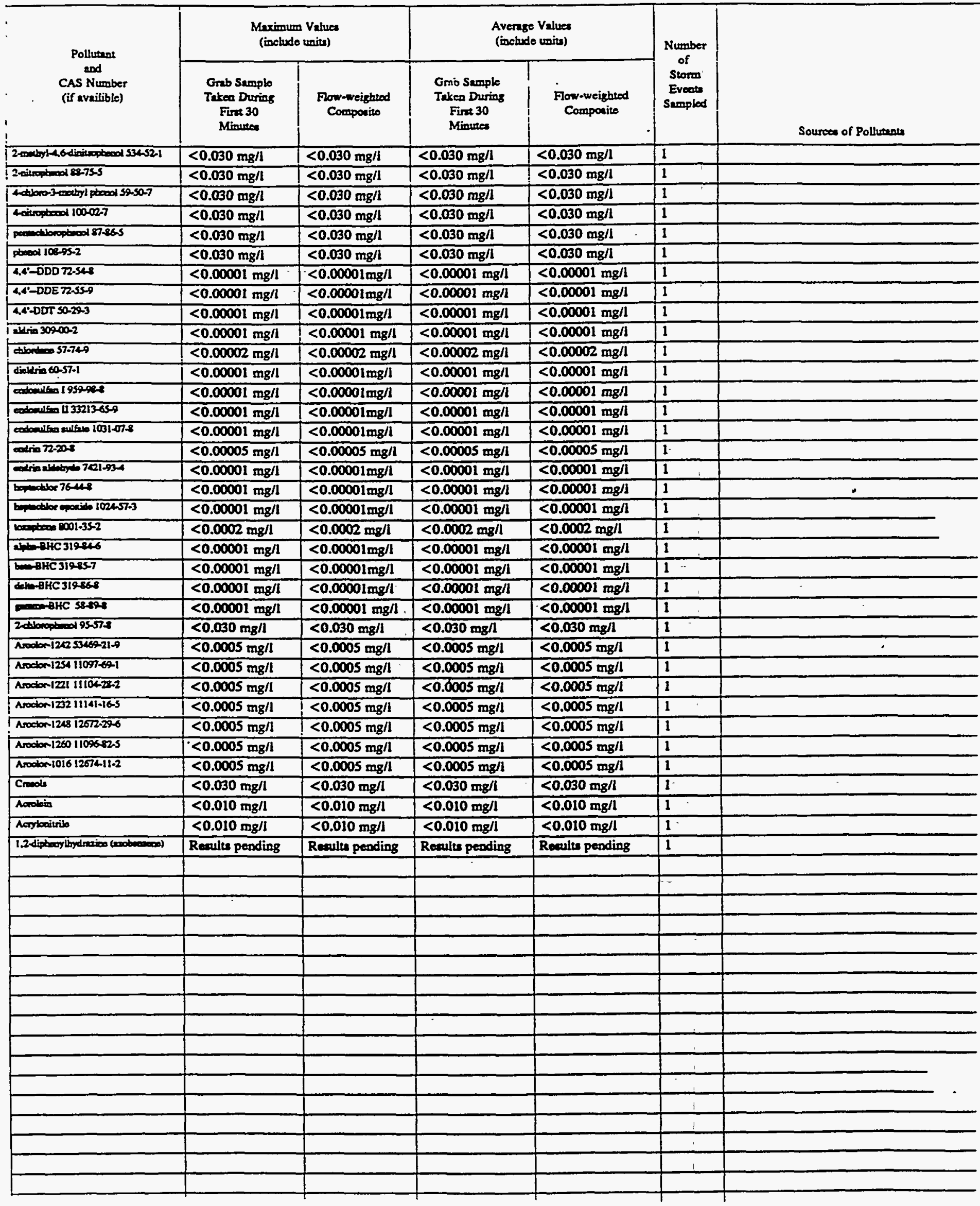


Vu. Dicherge Informetion (continued from page 3 of Form 2F)

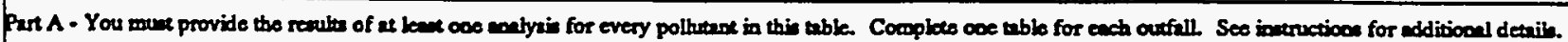

\begin{tabular}{|c|c|c|c|c|c|c|}
\hline \multirow{2}{*}{$\begin{array}{l}\text { Pollutant } \\
\text { and } \\
\text { CAs Number } \\
\text { (if nvilible) }\end{array}$} & \multicolumn{2}{|c|}{$\begin{array}{l}\text { Marimum Vahes } \\
\text { (inchude unios) }\end{array}$} & \multicolumn{2}{|c|}{$\begin{array}{l}\text { Avenge Vahue } \\
\text { (iochude unies) }\end{array}$} & \multirow{2}{*}{$\begin{array}{l}\text { Nurober } \\
\text { of } \\
\text { Siorm } \\
\text { Evente } \\
\text { Simpled }\end{array}$} & \multirow[b]{2}{*}{ Sources of Pollheran } \\
\hline & $\begin{array}{c}\text { Gnb Sumple } \\
\text { Taken During } \\
\text { Fint } 30 \\
\text { Mrowees }\end{array}$ & $\begin{array}{l}\text { Flow-weighted } \\
\text { Compotive }\end{array}$ & $\begin{array}{c}\text { Grab Semple } \\
\text { Tuken During } \\
\text { Firs } 30 \\
\text { Mivenes }\end{array}$ & $\begin{array}{l}\text { Fon-weighted } \\
\text { Compoites }\end{array}$ & & \\
\hline pil and Gomen & $<2 m g / 1$ & & $<2 \mathrm{mg} / \mathrm{l}$ & & 1 & \\
\hline 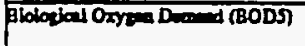 & $7.9 \mathrm{mg} / \mathrm{l}$ & $<5 \mathrm{mg} / \mathrm{l}$ & $7.9 \mathrm{mg} / \mathrm{l}$ & $<5 \mathrm{mg} / \mathrm{l}$ & 1 & \\
\hline 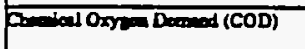 & $15 \mathrm{mg} / \mathrm{h}$ & $24 \mathrm{mg} / 1$ & $15 \mathrm{mg} / \mathrm{l}$ & $24 \mathrm{mg} / \mathrm{l}$ & 1 & \\
\hline Toel Simpoded Solthe (TSS) & $<5 \mathrm{mg} / \mathrm{l}$ & $<5 \mathrm{mg} / 1$ & $<5 \mathrm{mg} / \mathrm{l}$ & $<5 \mathrm{mg} / \mathrm{l}$ & 1 & \\
\hline Toul Kjellabl Nitrones & $0.38 \mathrm{mg} / \mathrm{l}$ & $0.22 \mathrm{mg} / \mathrm{l}$ & $0.38 \mathrm{mg} / \mathrm{l}$ & $0.22 \mathrm{mg} / \mathrm{l}$ & 1 & \\
\hline 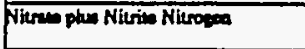 & $<0.1 \mathrm{mg} / 1$ & $0.27 \mathrm{mg} / 1$ & $<0.1 \mathrm{mg} / \mathrm{l}$ & $0.27 \mathrm{mg} / 1$ & 1 & \\
\hline Toel Propoptore & $<0.1 \mathrm{mg} / \mathrm{l}$ & $<0.1 \mathrm{mg} / \mathrm{l}$ & $<0.1 \mathrm{mg} / \mathrm{l}$ & $<0.1 \mathrm{mg} / \mathrm{l}$ & 1 & \\
\hline pH & Mine & Matele 7.6 & $\mathrm{M}=7.6$ & Modian 7.6 & & \\
\hline
\end{tabular}

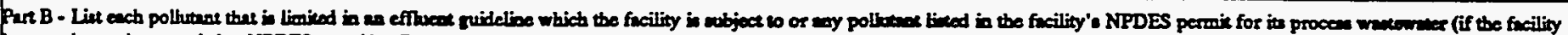

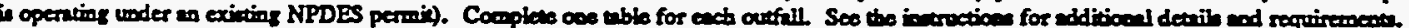

\begin{tabular}{|c|c|c|c|c|c|c|}
\hline \multirow{2}{*}{$\begin{array}{l}\text { Polluwass } \\
\text { nod } \\
\text { CAs Number } \\
\text { (if avnilible) }\end{array}$} & \multicolumn{2}{|c|}{ 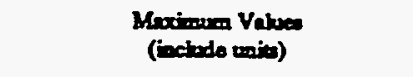 } & \multicolumn{2}{|c|}{ 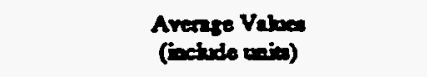 } & \multirow{2}{*}{$\begin{array}{l}\text { Nivober } \\
\text { of } \\
\text { Stromen } \\
\text { Evente } \\
\text { Semplod }\end{array}$} & \multirow{2}{*}{$\begin{array}{c}: \\
\text { Sources of Polluenon }\end{array}$} \\
\hline & 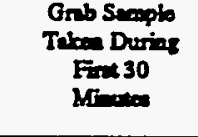 & $\begin{array}{l}\text { Frow-wrizhtod } \\
\text { Compocito }\end{array}$ & 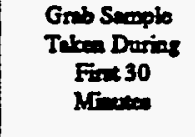 & $\begin{array}{c}\text { Fon-weighted } \\
\text { Compoive }\end{array}$ & & \\
\hline 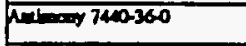 & $<0.003 \mathrm{mg} / \mathrm{l}$ & $<0.003 \mathrm{mg} / \mathrm{l}$ & $<0.003 \mathrm{mg} / \mathrm{l}$ & $<0.003 \mathrm{mg} / 1$ & 1 & \\
\hline pride & $<0.002 \mathrm{mg} / 1$ & & $<0.002 \mathrm{mg} / 1$ & & 1 & \\
\hline 7000179-6 & $<0.0002 \mathrm{mg} / 1$ & $<0.0002 \mathrm{mg} / \mathrm{l}$ & $<0.0002 \mathrm{mg} / 1$ & $<0.0002 \mathrm{mg} / \mathrm{l}$ & 1 & \\
\hline and & $0.002 \mathrm{mg} / \mathrm{l}$ & & $0.002 \mathrm{mg} / \mathrm{l}$ & & 1 & - \\
\hline 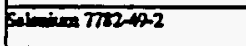 & $<0.002 \mathrm{mg} / \mathrm{h}$ & $<0.002 \mathrm{mg} / \mathrm{l}$ & $<0.002 \mathrm{mg} / \mathrm{l}$ & $<0.002 \mathrm{mg} /$ & 1 & \\
\hline sultanes & $<10 \mathrm{mg} / \mathrm{l}$ & $<10 \mathrm{mg} / \mathrm{l}$ & $<10 \mathrm{mg} / \mathrm{l}$ & $<10 \mathrm{mg} / 1$ & 1 & \\
\hline Surtinamenter & $<0.05 \mathrm{mg} / 1$ & $<0.05 \mathrm{mg} / \mathrm{l}$ & $<0.05 \mathrm{mg} / \mathrm{l}$ & $<0.05 \mathrm{mg} /$ & 1 & \\
\hline the:ming 7429-90-5 & $0.13 \mathrm{mg} / 1$ & $0.17 \mathrm{mg} / \mathrm{l}$ & $0.13 \mathrm{mg} / \mathrm{h}$ & $0.17 \mathrm{mg} / 1$ & 1 & \\
\hline 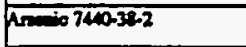 & $<0.04 \mathrm{mg} / \mathrm{l}$ & $<0.04 \mathrm{mg} / 1$ & $<0.04 \mathrm{mg} / \mathrm{l}$ & $<0.04 \mathrm{mg} / \mathrm{l}$ & 1 & \\
\hline $30000-740-129$ & $0.016 \mathrm{mg} / \mathrm{l}$ & $0.017 \mathrm{mg} / 1$ & $0.016 \mathrm{mg} / \mathrm{l}$ & $0.017 \mathrm{mg} / \mathrm{l}$ & 1 & \\
\hline Boryllien 740-1.-7 & $<0.0004 \mathrm{mg} / \mathrm{l}$ & $<0.0004 \mathrm{mg} / \mathrm{l}$ & $<0.0004 \mathrm{mg} /$ & $<0.0004 \mathrm{mg} / \mathrm{l}$ & 1 & \\
\hline $\cos \sin 740-13-9$ & $<0.004 \mathrm{mg} / \mathrm{l}$ & $<0.004 \mathrm{mg} / \mathrm{l}$ & $<0.004 \mathrm{mg} / \mathrm{l}$ & $<0.004 \mathrm{mg} / \mathrm{l}$ & 1 & \\
\hline Cakin 7400-70-2 & $66.0 \mathrm{mg} / \mathrm{h}$ & $49.7 \mathrm{mg} / \mathrm{l}$ & $66.0 \mathrm{mg} / \mathrm{l}$ & $49.7 \mathrm{mg} / \mathrm{l}$ & 1 & \\
\hline Curcaicon $7460-47.3$ & $<0.006 \mathrm{mg} / \mathrm{l}$ & $<0.006 \mathrm{mg} / \mathrm{l}$ & $<0.006 \mathrm{mg} / \mathrm{l}$ & $<0.006 \mathrm{mg} / 1$ & 1 & \\
\hline Cobeh 7400-124 & $<0.002 \mathrm{mg} / \mathrm{l}$ & $<0.002 \mathrm{mg} / \mathrm{l}$ & $<0.002 \mathrm{mg} / \mathrm{l}$ & $<0.002 \mathrm{mg} / \mathrm{l}$ & 1 & \\
\hline $60 \mathrm{mon} 740-509$ & $<0.006 \mathrm{mg} / \mathrm{l}$ & $<0.006 \mathrm{mg} / \mathrm{l}$ & $<0.006 \mathrm{mgh}$ & $<0.006 \mathrm{mg} / \mathrm{h}$ & 1 & \\
\hline row 7432596 & $0.21 \mathrm{mg} / \mathrm{h}$ & $0.25 \mathrm{mg} / \mathrm{l}$ & $0.21 \mathrm{mg} / \mathrm{l}$ & $0.25 \mathrm{mg} / \mathrm{n}$ & 1 & \\
\hline mand 709-921 & $<0.02 \mathrm{mg} / \mathrm{l}$ & $<0.02 \mathrm{mg} / \mathrm{l}$ & $<0.02 \mathrm{mg} / \mathrm{h}$ & $<0.02 \mathrm{mg} / \mathrm{l}$ & 1 & \\
\hline 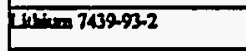 & $<0.02 \mathrm{mg} / \mathrm{l}$ & $<0.02 \mathrm{mg} / \mathrm{l}$ & $<0.02 \mathrm{mg} / \mathrm{l}$ & $<0.02 \mathrm{mg} / \mathrm{l}$ & 1 & \\
\hline 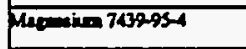 & $5.67 \mathrm{mg} / \mathrm{l}$ & $4.55 \mathrm{mg} / 1$ & $5.67 \mathrm{mg} / 1$ & $4.55 \mathrm{mg} / 1$ & 1 & \\
\hline M-moven $7439 x-5$ & $0.053 \mathrm{mg} / \mathrm{l}$ & $0.046 \mathrm{mg} / 1$ & $0.053 \mathrm{mg} / \mathrm{l}$ & $0.046 \mathrm{mg} / \mathrm{h}$ & 1 & \\
\hline Matydean 7039-7 & $<0.006 \mathrm{mg} / \mathrm{l}$ & $<0.006 \mathrm{mg} / 1$ & $<0.006 \mathrm{mg} / \mathrm{l}$ & $<0.006 \mathrm{mg} / 1$ & 1 & \\
\hline Niodeal 7400020 & $<0.008 \mathrm{mg} / \mathrm{l}$ & $<0.008 \mathrm{mg} / \mathrm{l}$ & $<0.008 \mathrm{mg} / \mathrm{h}$ & $<0.008 \mathrm{mg} / 1$ & 1 & \\
\hline Pomingm 740-027 & $1.6 \mathrm{mg} / \mathrm{l}$ & $2.1 \mathrm{mg} / 1$ & $1.6 \mathrm{mg} / \mathrm{l}$ & $2.1 \mathrm{mg} / \mathrm{l}$ & 1 & \\
\hline 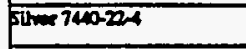 & $<0.006 \mathrm{mg} / \mathrm{h}$ & $<0.006 \mathrm{mg} / \mathrm{l}$ & $<0.006 \mathrm{mg} / \mathrm{l}$ & $<0.006 \mathrm{mg} / \mathrm{l}$ & 1 & \\
\hline sodk= $740-22.5$ & $2.6 \mathrm{mgh}$ & $1.54 \mathrm{mg} /$ & $2.6 \mathrm{mg} / 1$ & $1.54 \mathrm{mg} /$ & 1 & \\
\hline Thentan $7400-3256$ & $<0.01 \mathrm{mg} / 1$ & $<0.01 \mathrm{mg} / \mathrm{l}$ & $<0.01 \mathrm{mg} / \mathrm{h}$ & $<0.01 \mathrm{mg} / 1$ & 1 & \\
\hline $2 \sin 740-060$ & $<0.01 \mathrm{mg} / 1$ & $<0.01 \mathrm{mg} / \mathrm{l}$ & $<0.01 \mathrm{mg} / \mathrm{l}$ & $<0.01 \mathrm{mg} / \mathrm{l}$ & 1 & \\
\hline
\end{tabular}




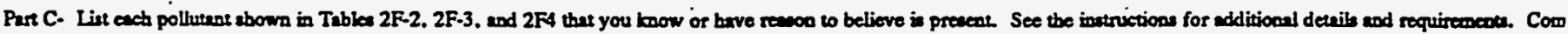
coc tuble for cach ourfll

\begin{tabular}{|c|c|c|c|c|c|c|}
\hline \multirow{2}{*}{$\begin{array}{l}\text { Pollunase } \\
\text { and } \\
\text { CAS Number } \\
\text { (if avilible) }\end{array}$} & \multicolumn{2}{|c|}{$\begin{array}{l}\text { Meximum Vahuea } \\
\text { (include unitu) }\end{array}$} & \multicolumn{2}{|c|}{$\begin{array}{l}\text { Averige Value } \\
\text { (inclesde imits) }\end{array}$} & \multirow{2}{*}{$\begin{array}{l}\text { Number } \\
\text { of } \\
\text { Storm } \\
\text { Evear } \\
\text { Sempled }\end{array}$} & \multirow[b]{2}{*}{ Sources of Polbutanta } \\
\hline & $\begin{array}{c}\text { Grab Surople } \\
\text { Telben Duriag } \\
\text { First } 30 \\
\text { Minutes }\end{array}$ & $\begin{array}{l}\text { Flow-weighted } \\
\text { Comporite }\end{array}$ & $\begin{array}{l}\text { Grab Sample } \\
\text { Tainen During } \\
\text { Fint } 30 \\
\text { Mide }\end{array}$ & $\begin{array}{c}\text { Flow-weighted } \\
\text { Componite }\end{array}$ & & \\
\hline 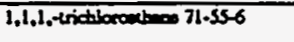 & $<0.010 \mathrm{mg} / \mathrm{l}$ & & $<0.010 \mathrm{mg} / \mathrm{l}$ & & 1 & \\
\hline 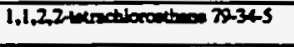 & $<0.010 \mathrm{mg} / 1$ & & $<0.010 \mathrm{mg} / 1$ & & 1 & \\
\hline 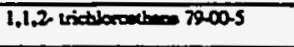 & $<0.010 \mathrm{mg} / \mathrm{l}$ & & $<0.010 \mathrm{mg} / 1$ & & 1 & \\
\hline 1,1-dictlonotiones 75-34,3 & $<0.010 \mathrm{mg} / 1$ & & $<0.010 \mathrm{mg} / \mathrm{l}$ & & 1 & \\
\hline 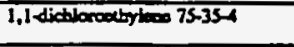 & $<0.010 \mathrm{mg} / \mathrm{l}$ & & $<0.010 \mathrm{mg} / \mathrm{l}$ & & 1 & \\
\hline 1.2didiborouthe $10706-2$ & $<0.010 \mathrm{mg} / 1$ & & $<0.010 \mathrm{mg} / \mathrm{l}$ & & 1 & \\
\hline 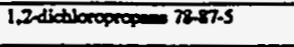 & $<0.010 \mathrm{mg} / \mathrm{l}$ & & $<0.010 \mathrm{mg} / \mathrm{l}$ & & 1 & \\
\hline 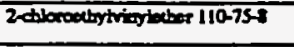 & $<0.010 \mathrm{mg} / \mathrm{l}$ & & $<0.010 \mathrm{mg} / 1$ & & 1 & \\
\hline $71-43-2$ & $<0.010 \mathrm{mg} / 1$ & & $<0.010 \mathrm{mg} / 1$ & & 1 & \\
\hline 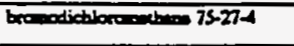 & $<0.010 \mathrm{mg} / \mathrm{l}$ & & $<0.010 \mathrm{mg} / \mathrm{l}$ & & 1 & \\
\hline bromotom $75-25-2$ & $<0.010 \mathrm{mg} / \mathrm{l}$ & & $<0.010 \mathrm{mg} / \mathrm{l}$ & & 1 & \\
\hline brodecthes 7483-9 & $<0.010 \mathrm{mg} / 1$ & & $<0.010 \mathrm{mg} / 1$ & & 1 & \\
\hline atcan wenctarits $56-23-5$ & $<0.010 \mathrm{mg} / \mathrm{h}$ & & $<0.010 \mathrm{mg} / \mathrm{l}$ & & 1 & \\
\hline 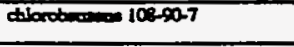 & $<0.010 \mathrm{mg} / \mathrm{l}$ & & $<0.010 \mathrm{mg} / \mathrm{l}$ & & 1 & \\
\hline chloromben 7500.3 & $<0.010 \mathrm{mg} / \mathrm{l}$ & & $<0.010 \mathrm{mg} / 1$ & & 1 & \\
\hline cblordform 67.66-3 & $<0.010 \mathrm{mg} / \mathrm{l}$ & & $<0.010 \mathrm{mg} / 1$ & & 1 & $\therefore$ \\
\hline 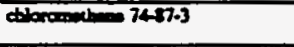 & $<0.010 \mathrm{mg} / 1$ & & $<0.010 \mathrm{mg} / 1$ & & 1 & \\
\hline 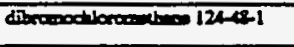 & $<0.010 \mathrm{mg} / \mathrm{l}$ & & $<0.010 \mathrm{mg} / 1$ & & 1 & \\
\hline 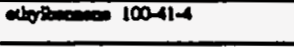 & $<0.010 \mathrm{mg} / 1$ & & $<0.010 \mathrm{mg} / 1$ & & 1 & \\
\hline tohem $1002 x-3$ & $<0.010 \mathrm{mg} / 1$ & & $<0.010 \mathrm{mg} / 1$ & & 1 & \\
\hline ind chibite 75-014 & $<0.010 \mathrm{mg} / 1$ & & $<0.010 \mathrm{mg} / \mathrm{l}$ & & 1 & \\
\hline 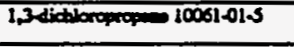 & $<0.010 \mathrm{mg} / 1$ & & $<0.010 \mathrm{mg} / 1$ & - & 1 & \\
\hline 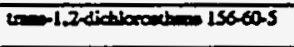 & $<0.010 \mathrm{mg} / \mathrm{l}$ & & $<0.010 \mathrm{mg} / \mathrm{l}$ & & 1 & $\cdot$ \\
\hline 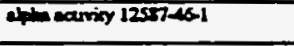 & $4.6 \mathrm{pci} / 1$ & $6.8 \mathrm{pci} / 1$ & $4.6 \mathrm{pei} / \mathrm{l}$ & $6.8 \mathrm{psi} / \mathrm{l}$ & 1 & \\
\hline bxeseamxy $12587-461$ & $6.8 \mathrm{pci} / \mathrm{l}$ & $7.6 \mathrm{pci} / 1$ & $6.8 \mathrm{pci} / \mathrm{l}$ & $7.6 \mathrm{pei} / \mathrm{l}$ & 1 & \\
\hline ind od form & $145 \mathrm{co} .1 \mathrm{~L}$ & $950 \mathrm{co} / .1 \mathrm{~L}$ & $145 \mathrm{col} .1 \mathrm{~L}$ & $950 \mathrm{col} .1 \mathrm{~L}$ & 1 & \\
\hline Thellien 240280 & $<0.001 \mathrm{mg} / \mathrm{l}$ & $<0.001 \mathrm{mg} / \mathrm{l}$ & $<0.001 \mathrm{mg} / \mathrm{l}$ & $<0.001 \mathrm{mg} / \mathrm{l}$ & 1 & \\
\hline Unasing $740061-1$ & $0.003 \mathrm{mg} / 1$ & $0.002 \mathrm{mg} / 1$ & $0.003 \mathrm{mg} / 1$ & $0.002 \mathrm{mg} / \mathrm{h}$ & 1 & \\
\hline Genesing & $7.0 \mathrm{pei} / \mathrm{l}$ & $5.6 \mathrm{pci} / 1$ & $7.0 \mathrm{pci} / \mathrm{l}$ & $5.6 \mathrm{pei} / \mathrm{l}$ & 1 & \\
\hline
\end{tabular}

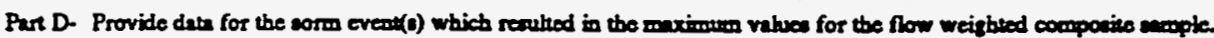

\begin{tabular}{|c|c|c|c|c|c|c|c|}
\hline $\begin{array}{l}1 . \\
\text { Date of } \\
\text { Storm } \\
\text { Event }\end{array}$ & $\begin{array}{l}2 . \\
\text { Durion } \\
\text { of Stomm } \\
\text { (in } \\
\text { minutea) }\end{array}$ & 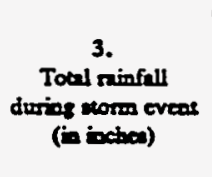 & 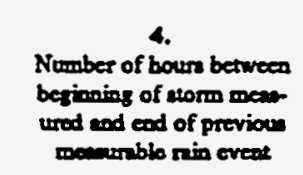 & $\begin{array}{l}\text { S. } \\
\text { Meximum flow mite } \\
\text { during min event } \\
\text { (allocesimin or epecify } \\
\text { uniw) }\end{array}$ & $\begin{array}{l}6 . \\
\text { Total flow from } \\
\text { rin eveot } \\
\text { (ralloos or } \\
\text { pecify unity) }\end{array}$ & $\begin{array}{c}7 . \\
\text { Season } \\
\text { exmple wase } \\
\text { tuken }\end{array}$ & 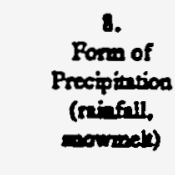 \\
\hline $6 / 4 / 92$ & 210 & 1 & 128.8 & 15.1 & 2,867 & Spring & Rainfall \\
\hline
\end{tabular}

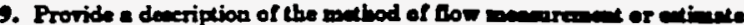

An ISCO 3230 Flow meter was wed to obtain water level reatings. This instrument was calibrated with the narmal dry weather flow, if present, as bere zero. The flow meter was programmed with the characteristics of the conveyance so that fow rates and total flows could be calculated by the metter. 


\begin{tabular}{|c|c|c|c|c|c|c|}
\hline \multirow{2}{*}{$\begin{array}{l}\text { Pollumase } \\
\text { and } \\
\text { CAs Number } \\
\text { (if avilible) }\end{array}$} & \multicolumn{2}{|c|}{$\begin{array}{l}\text { Meximum Values } \\
\text { (ibehuto uniru) }\end{array}$} & \multicolumn{2}{|c|}{$\begin{array}{l}\text { Averngo Valuce } \\
\text { (inchude unita) }\end{array}$} & \multirow{2}{*}{$\begin{array}{l}\text { Number } \\
\text { of } \\
\text { Storm } \\
\text { Evecos } \\
\text { Susoplod }\end{array}$} & \multirow[b]{2}{*}{ Sources of Polbuents } \\
\hline & $\begin{array}{c}\text { Grab Semplo } \\
\text { Tuken During } \\
\text { Finst } 30 \\
\text { Minutea }\end{array}$ & $\begin{array}{l}\text { Flow-woighlod } \\
\text { Compocite }\end{array}$ & $\begin{array}{l}\text { Grab Sumplo } \\
\text { Tikeo Duriag } \\
\text { First } 30 \\
\text { Minuzea }\end{array}$ & $\begin{array}{c}\text { Fiont-weighted } \\
\text { Compocite } \\
.\end{array}$ & & \\
\hline Beriver 7440-39-3 & $0.0485 \mathrm{mg} / \mathrm{l}$ & $0.0362 \mathrm{mg} / \mathrm{h}$ & $0.0485 \mathrm{mg} / \mathrm{l}$ & $0.0362 \mathrm{mg} / \mathrm{l}$ & 1 & \\
\hline methylacos chloride 75092 & $<0.010 \mathrm{mg} / \mathrm{l}$ & & $<0.010 \mathrm{mg} / \mathrm{l}$ & & 1 & \\
\hline 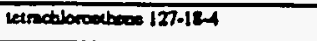 & $<0.010 \mathrm{mg} / \mathrm{l}$ & & $<0.010 \mathrm{mg} / \mathrm{l}$ & & 1 & \\
\hline Irietionsochono 7901.6 & $<0.010 \mathrm{mg} / 1$ & & $<0.010 \mathrm{mg} / \mathrm{l}$ & & 1 & \\
\hline Xyleosed & $<0.010 \mathrm{mg} / \mathrm{l}$ & & $<0.010 \mathrm{mg} / \mathrm{l}$ & & 1 & \\
\hline 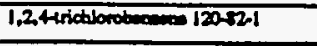 & $<0.010 \mathrm{mg} / \mathrm{h}$ & $<0.010 \mathrm{mg} / \mathrm{l}$ & $<0.010 \mathrm{mg} / \mathrm{l}$ & $<0.010 \mathrm{mg} / 1$ & 1 & \\
\hline 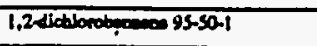 & $<0.010 \mathrm{mg} / 1$ & $<0.010 \mathrm{mg} / \mathrm{l}$ & $<0.010 \mathrm{mg} / \mathrm{l}$ & $<0.010 \mathrm{mg} / 1$ & 1 & \\
\hline 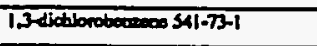 & $<0.010 \mathrm{mg} / \mathrm{l}$ & $<0.010 \mathrm{mg} / \mathrm{l}$ & $<0.010 \mathrm{mg} / \mathrm{l}$ & $<0.010 \mathrm{mg} / \mathrm{l}$ & 1 & \\
\hline 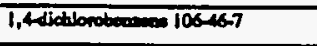 & $<0.010 \mathrm{mg} / \mathrm{l}$ & $<0.010 \mathrm{mg} / \mathrm{l}$ & $<0.010 \mathrm{mg} / 1$ & $<0.010 \mathrm{mg} / 1$ & 1 & \\
\hline 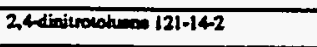 & $<0.010 \mathrm{mg} / \mathrm{l}$ & $<0.010 \mathrm{mg} / \mathrm{l}$ & $<0.010 \mathrm{mg} / \mathrm{l}$ & $<0.010 \mathrm{mg} / 1$ & 1 & \\
\hline 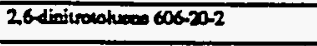 & $<0.010 \mathrm{mg} / \mathrm{l}$ & $<0.010 \mathrm{mg} / \mathrm{l}$ & $<0.010 \mathrm{mg} / 1$ & $<0.010 \mathrm{mg} / \mathrm{l}$ & 1 & \\
\hline 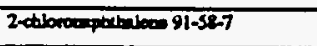 & $<0.010 \mathrm{mg} / \mathrm{l}$ & $<0.010 \mathrm{mg} / \mathrm{l}$ & $<0.010 \mathrm{mg} / \mathrm{l}$ & $<0.010 \mathrm{mg} / \mathrm{l}$ & 1 & \\
\hline 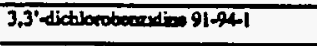 & $<0.010 \mathrm{mg} / \mathrm{I}$ & $<0.010 \mathrm{mg} / \mathrm{l}$ & $<0.010 \mathrm{mg} / \mathrm{l}$ & $<0.010 \mathrm{mg} / \mathrm{l}$ & 1 & \\
\hline 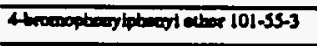 & $<0.010 \mathrm{mg} / \mathrm{I}$ & $<0.010 \mathrm{mg} / \mathrm{l}$ & $<0.010 \mathrm{mg} / \mathrm{l}$ & $<0.010 \mathrm{mg} / !$ & 1 & \\
\hline 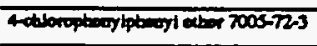 & $<0.010 \mathrm{mg} / \mathrm{l}$ & $<0.010 \mathrm{mg} / \mathrm{l}$ & $<0.010 \mathrm{mg} / \mathrm{l}$ & $<0.010 \mathrm{mg} / 1$ & 1 & \\
\hline 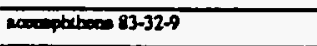 & $<0.010 \mathrm{mg} / \mathrm{l}$ & $<0.010 \mathrm{mg} / \mathrm{l}$ & $<0.010 \mathrm{mg} / \mathrm{l}$ & $<0.010 \mathrm{mg} / \mathrm{l}$ & 1 & \\
\hline 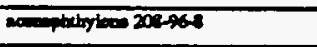 & $<0.010 \mathrm{mg} / \mathrm{l}$ & $<0.010 \mathrm{mg} / \mathrm{l}$ & $<0.010 \mathrm{mg} / \mathrm{l}$ & $<0.010 \mathrm{mg} / \mathrm{l}$ & 1 & \\
\hline $120-127$ & $<0.010 \mathrm{mg} / \mathrm{l}$ & $<0.010 \mathrm{mg} / \mathrm{l}$ & $<0.010 \mathrm{mg} / \mathrm{l}$ & $<0.010 \mathrm{mg} / \mathrm{l}$ & 1 & . \\
\hline 2. $92 \pi .5$ & $<0.030 \mathrm{mg} / \mathrm{l}$ & $<0.030 \mathrm{mg} / \mathrm{l}$ & $<0.030 \mathrm{mg} / \mathrm{l}$ & $<0.030 \mathrm{mg} / 1$ & 1 & \\
\hline 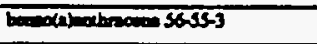 & $<0.010 \mathrm{mg} / \mathrm{I}$ & $<0.010 \mathrm{mg} / \mathrm{l}$ & $<0.010 \mathrm{mg} / \mathrm{l}$ & $<0.010 \mathrm{mg} / \mathrm{l}$ & 1 & \\
\hline 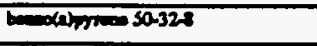 & $<0.010 \mathrm{mg} / \mathrm{l}$ & $<0.010 \mathrm{mg} / \mathrm{l}$ & $<0.010 \mathrm{mg} / 1$ & $<0.010 \mathrm{mg} / \mathrm{l}$ & 1 & \\
\hline 205092 & $<0.010 \mathrm{mg} / \mathrm{l}$ & $<0.010 \mathrm{mg} / \mathrm{l}$ & $<0.010 \mathrm{mg} / \mathrm{l}$ & $<0.010 \mathrm{mg} / \mathrm{l}$ & 1 & \\
\hline beosodehiporglose 191-24-2 & $<0.010 \mathrm{mg} / 1$ & $<0.010 \mathrm{mg} / \mathrm{l}$ & $<0.010 \mathrm{mg} / \mathrm{l}$ & $<0.010 \mathrm{mg} / \mathrm{l}$ & 1 & \\
\hline 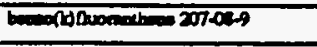 & $<0.010 \mathrm{mg} / 1$ & $<0.010 \mathrm{mg} / \mathrm{l}$ & $<0.010 \mathrm{mg} / \mathrm{l}$ & $<0.010 \mathrm{mg} / \mathrm{l}$ & 1 & \\
\hline 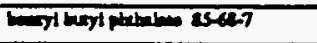 & $<0.010 \mathrm{ug} / \mathrm{l}$ & $<0.010 \mathrm{mg} / \mathrm{l}$ & $<0.010 \mathrm{mg} / \mathrm{l}$ & $<0.010 \mathrm{mg} / 1$ & 1 & \\
\hline 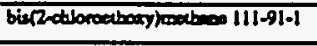 & $<0.010 \mathrm{mg} / \mathrm{l}$ & $<0.010 \mathrm{mg} / 1$ & $<0.010 \mathrm{mg} / 1$ & $<0.010 \mathrm{mg} / \mathrm{l}$ & 1 & 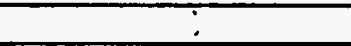 \\
\hline 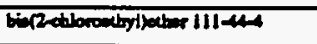 & $<0.010 \mathrm{mg} / \mathrm{l}$ & $<0.010 \mathrm{mg} / 1$ & $<0.010 \mathrm{mg} / \mathrm{l}$ & $<0.010 \mathrm{mg} / \mathrm{l}$ & 1 & \\
\hline 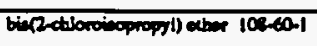 & $<0.010 \mathrm{mg} / \mathrm{l}$ & $<0.010 \mathrm{mg} / \mathrm{l}$ & $<0.010 \mathrm{mg} / \mathrm{l}$ & $<0.010 \mathrm{mg} / \mathrm{l}$ & 1 & \\
\hline 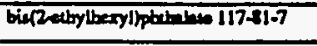 & $<0.010 \mathrm{mg} / \mathrm{l}$ & $<0.010 \mathrm{mg} / \mathrm{l}$ & $<0.010 \mathrm{mg} / 1$ & $<0.010 \mathrm{mg} / \mathrm{l}$ & 1 & \\
\hline ctryous 21801-9 & $<0.010 \mathrm{mg} / 1$ & $<0.010 \mathrm{mg} / \mathrm{ll}$ & $<0.010 \mathrm{mg} / \mathrm{l}$ & $<0.010 \mathrm{mg} / \mathrm{l}$ & 1 & \\
\hline 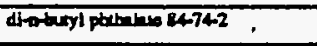 & $<0.010 \mathrm{mg} / \mathrm{l}$ & $<0.010 \mathrm{mg} / \mathrm{l}$ & $<0.010 \mathrm{mg} / \mathrm{l}$ & $<0.010 \mathrm{mg} / 1$ & 1 & \\
\hline 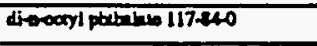 & $<0.010 \mathrm{mg} / \mathrm{l}$ & $<0.010 \mathrm{mg} / \mathrm{l}$ & $<0.010 \mathrm{mg} / 1$ & $<0.010 \mathrm{mg} / \mathrm{l}$ & 1 & \\
\hline 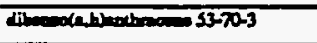 & $<0.010 \mathrm{mg} / \mathrm{l}$ & $<0.010 \mathrm{mg} / \mathrm{l}$ & $<0.010 \mathrm{mg} / 1$ & $<0.010 \mathrm{mg} / \mathrm{l}$ & 1 & \\
\hline dintyl pthathe 8466-2 & $<0.010 \mathrm{mg} / \mathrm{l}$ & $<0.010 \mathrm{mg} / \mathrm{l}$ & $<0.010 \mathrm{mg} / \mathrm{l}$ & $<0.010 \mathrm{mg} / \mathrm{l}$ & 1 & \\
\hline divectyl pothelab $|3|-1 \mid-3$ & $<0.010 \mathrm{mg} / \mathrm{l}$ & $<0.010 \mathrm{mg} / \mathrm{l}$ & $<0.010 \mathrm{mg} / 1$ & $<0.010 \mathrm{mg} / \mathrm{l}$ & 1 & \\
\hline Theorocheoe 2064140 & $<0.010 \mathrm{mg} / \mathrm{l}$ & $<0.010 \mathrm{mg} / \mathrm{l}$ & $<0.010 \mathrm{mg} / 1$ & $<0.010 \mathrm{mg} / 1$ & 1 & \\
\hline Theosuce $86-73-7$ & $<0.010 \mathrm{mg} / \mathrm{l}$ & $<0.010 \mathrm{mg} / \mathrm{t}$ & $<0.010 \mathrm{mg} / \mathrm{l}$ & $<0.010 \mathrm{mg} / 1$ & 1 & \\
\hline berectlorotion $118-74$ & $<0.010 \mathrm{mg} / \mathrm{l}$ & $<0.010 \mathrm{mg} / \mathrm{t}$ & $<0.010 \mathrm{mg} / \mathrm{l}$ & $<0.010 \mathrm{mg} / 1$ & 1 & \\
\hline berachborobendione 87-68-3 & $<0.010 \mathrm{mg} / \mathrm{l}$ & $<0.010 \mathrm{mg} / 1$ & $<0.010 \mathrm{mg} / 1$ & $<0.010 \mathrm{mg} / 1$ & 1 & \\
\hline 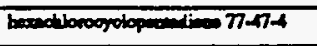 & $<0.010 \mathrm{mg} / 1$ & $<0.010 \mathrm{mg} / 1$ & $<0.010 \mathrm{mg} / \mathrm{l}$ & $<0.010 \mathrm{mg} / 1$ & 1 & \\
\hline beactionodheos 67.72.1 & $<0.010 \mathrm{mg} / \mathrm{l}$ & $<0.010 \mathrm{mg} / 1$ & $<0.010 \mathrm{mg} / 1$ & $<0.010 \mathrm{mg} / \mathrm{l}$ & 1 & \\
\hline 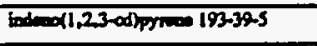 & $<0.010 \mathrm{mg} / 1$ & $<0.010 \mathrm{mg} / \mathrm{l}$ & $<0.010 \mathrm{mg} / \mathrm{l}$ & $<0.010 \mathrm{mg} / \mathrm{s}$ & 1 & \\
\hline inephoreses 72-59-1 & $<0.010 \mathrm{mg} / \mathrm{l}$ & $<0.010 \mathrm{mg} / 1$ & $<0.010 \mathrm{mg} / \mathrm{l}$ & $<0.010 \mathrm{mg} / 1$ & 1 & \\
\hline 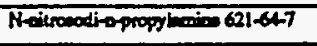 & $<0.010 \mathrm{mg} / \mathrm{l}$ & $<0.010 \mathrm{mg} / 1$ & $<0.010 \mathrm{mg} / \mathrm{l}$ & $<0.010 \mathrm{mg} / 1$ & 1 & \\
\hline Natronodimobylarion 62.75-9 & $<0.010 \mathrm{mg} / \mathrm{l}$ & $<0.010 \mathrm{mg} / \mathrm{l}$ & $<0.010 \mathrm{mg} / \mathrm{l}$ & $<0.010 \mathrm{mg} / 1$ & 1 & \\
\hline 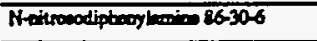 & $<0.010 \mathrm{mg} / \mathrm{l}$ & $<0.010 \mathrm{mg} / \mathrm{l}$ & $<0.010 \mathrm{mg} / \mathrm{l}$ & $<0.010 \mathrm{mg} / \mathrm{l}$ & 1 & \\
\hline 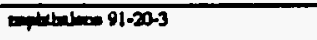 & $<0.010 \mathrm{mg} / \mathrm{l}$ & $<0.010 \mathrm{mg} / \mathrm{l}$ & $<0.010 \mathrm{mg} / \mathrm{l}$ & $<0.010 \mathrm{mg} / 1$ & 1 & \\
\hline astrobecteoso $9-95-3$ & $<0.010 \mathrm{mg} / 1$ & $<0.010 \mathrm{mg} / \mathrm{l}$ & $<0.010 \mathrm{mg} / \mathrm{l}$ & $<0.010 \mathrm{mg} / \mathrm{l}$ & 1 & \\
\hline 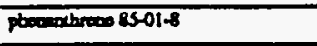 & $<0.010 \mathrm{mg} / \mathrm{l}$ & $<0.010 \mathrm{mg} / \mathrm{l}$ & $<0.010 \mathrm{mg} / \mathrm{l}$ & $<0.010 \mathrm{mg} / \mathrm{l}$ & 1 & \\
\hline 87900129000 & $<0.010 \mathrm{mg} / \mathrm{l}$ & $<0.010 \mathrm{mg} / 1$ & $<0.010 \mathrm{mg} / \mathrm{l}$ & $<0.010 \mathrm{mg} / 1$ & 1 & \\
\hline 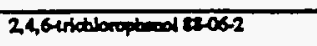 & $<0.030 \mathrm{mg} / \mathrm{l}$ & $<0.030 \mathrm{mg} / \mathrm{l}$ & $<0.030 \mathrm{mg} / \mathrm{l}$ & $<0.030 \mathrm{mg} / \mathrm{l}$ & 1 & \\
\hline 2,4dictiboroptered 1200-30-2 & $<0.030 \mathrm{mg} / \mathrm{l}$ & $<0.030 \mathrm{mg} / 1$ & $<0.030 \mathrm{mg} / \mathrm{l}$ & $<0.030 \mathrm{mg} / 1$ & 1 & \\
\hline 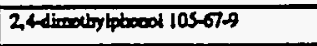 & $<0.030 \mathrm{mg} / \mathrm{l}$ & $<0.030 \mathrm{mg} / \mathrm{l}$ & $<0.030 \mathrm{mg} / 1$ & $<0.030 \mathrm{mg} / \mathrm{l}$ & 1 & \\
\hline 24dinitropbeod 51-225 & $<0.030 \mathrm{mg} / \mathrm{l}$ & $<0.030 \mathrm{mg} / \mathrm{l}$ & $<0.030 \mathrm{mg} / \mathrm{l}$ & $<0.030 \mathrm{mg} / \mathrm{l}$ & 1 & \\
\hline
\end{tabular}


Outfall S12

Parta B and C Contiaived

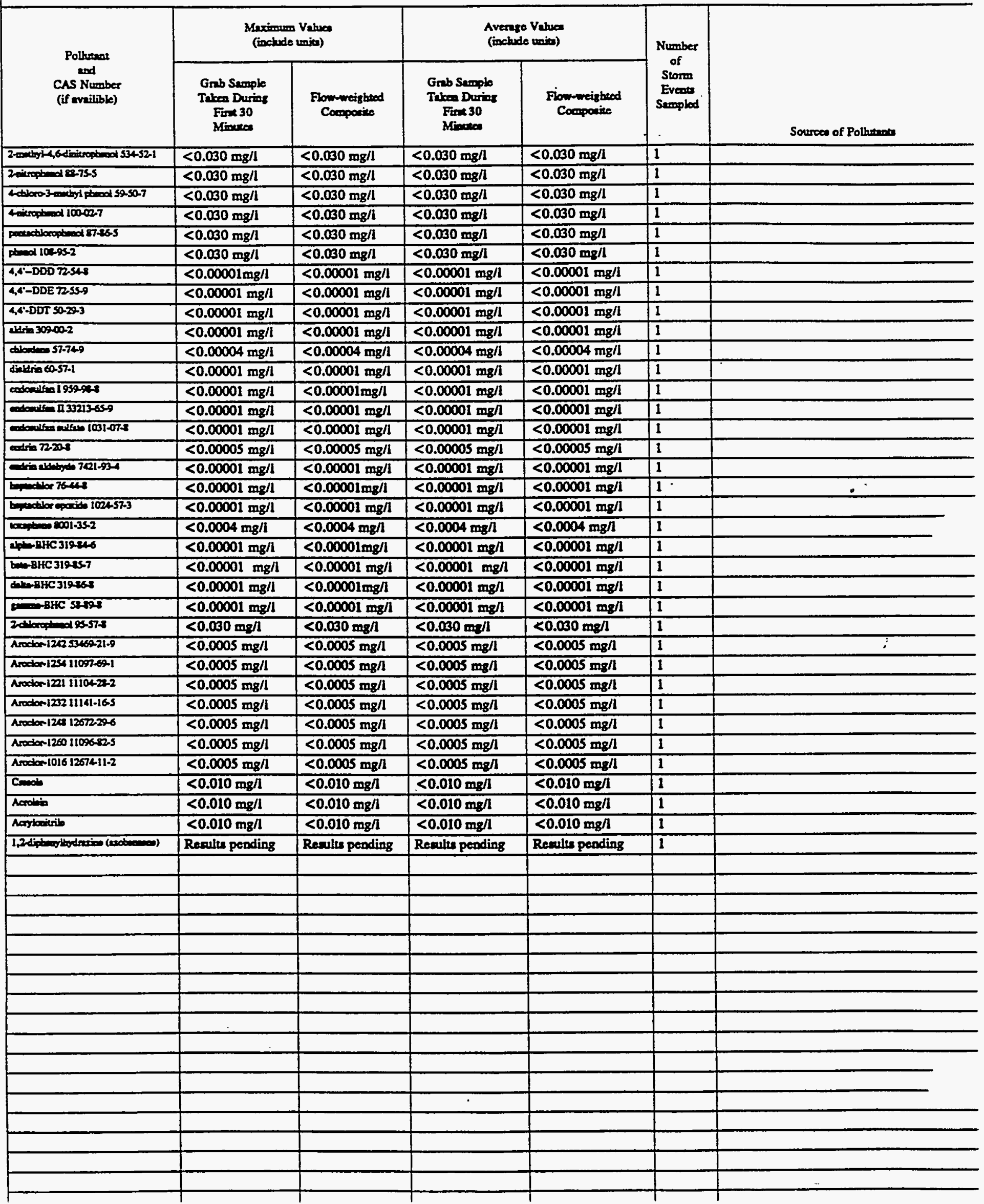


VI. Dischare information (contiound froon page 3 of Form 2 )

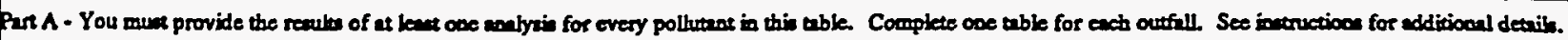

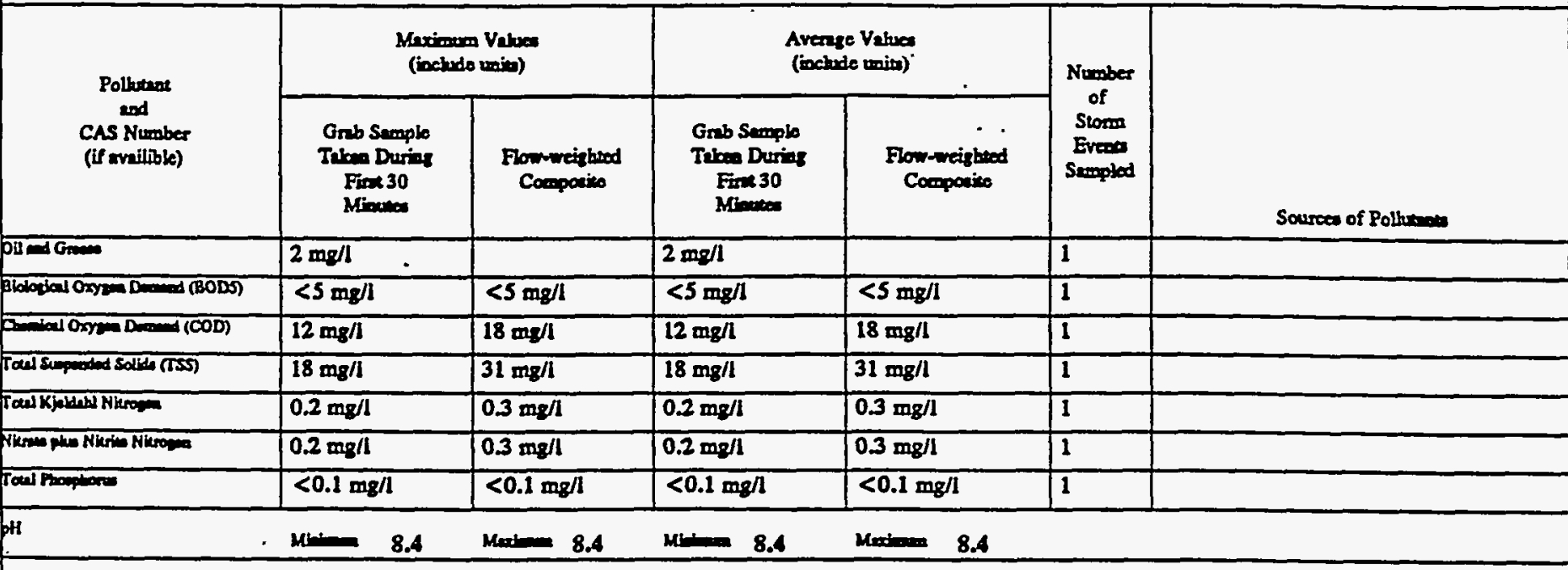

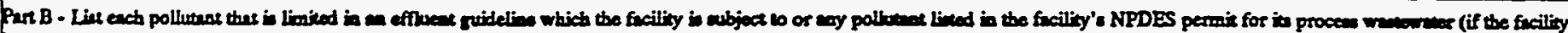

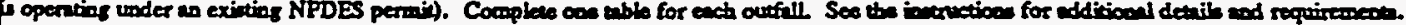

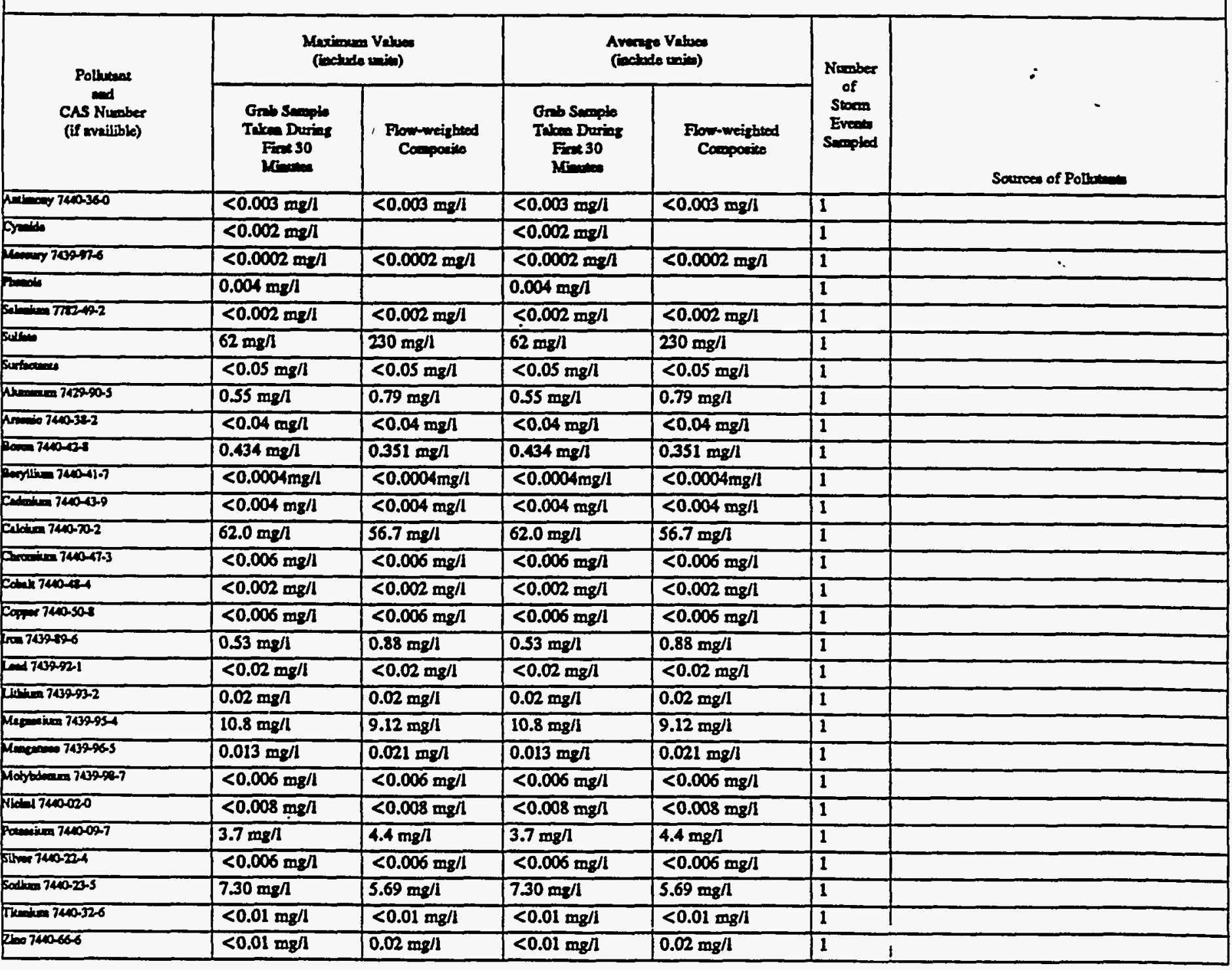


Continend from tront.

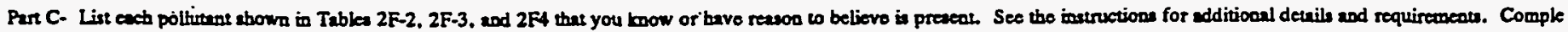
ono tublo for each outfall.

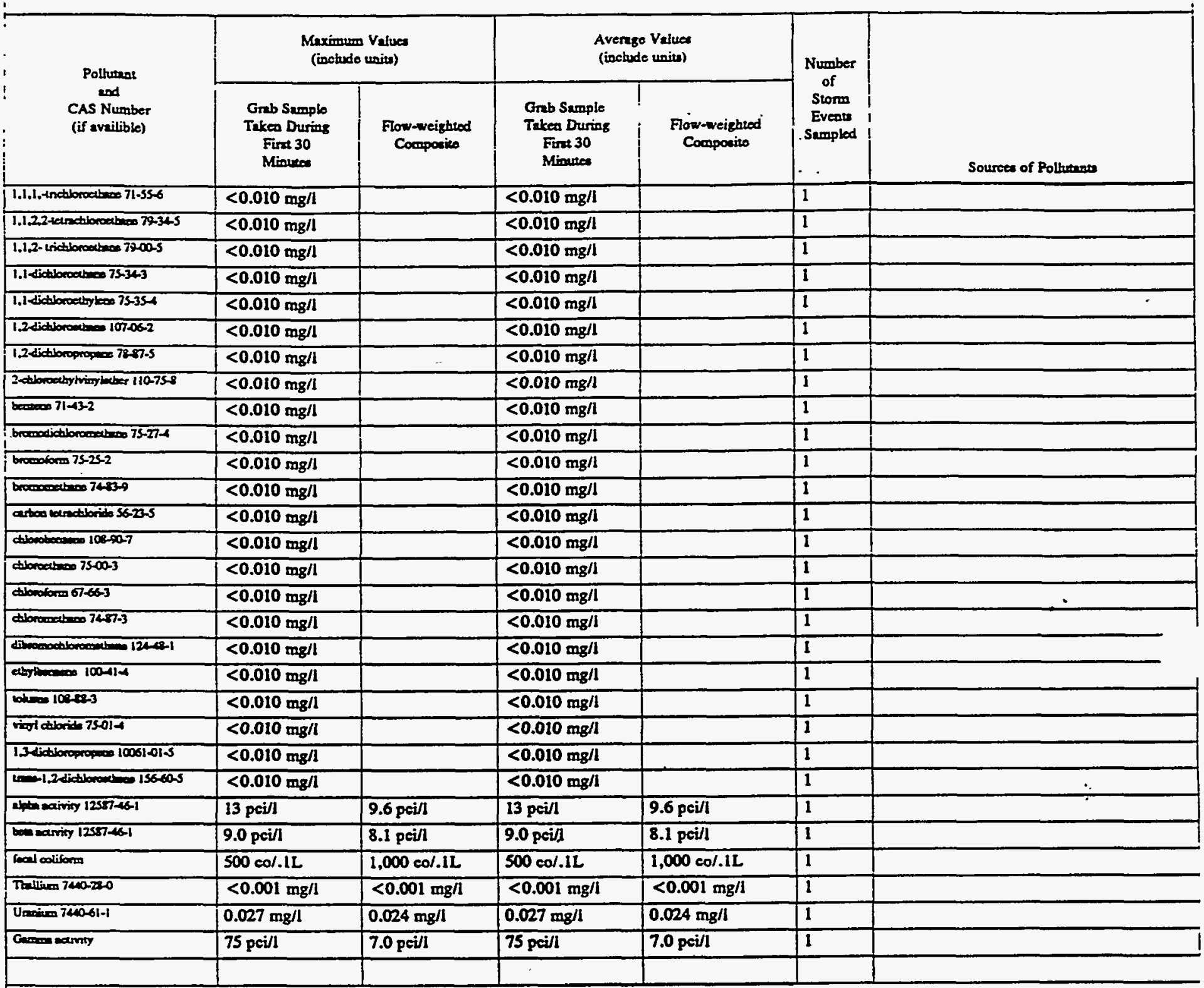

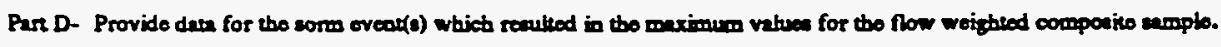

\begin{tabular}{|c|c|c|c|c|c|c|c|}
\hline $\begin{array}{l}1 . \\
\text { Dite of } \\
\text { Sworm } \\
\text { Eveas }\end{array}$ & $\begin{array}{l}2 . \\
\text { Duntion } \\
\text { of Stom } \\
\text { (in } \\
\text { minurtes) }\end{array}$ & $\begin{array}{l}3 . \\
\text { Toual rinfall } \\
\text { during storm oveos } \\
\text { (in iochea) }\end{array}$ & 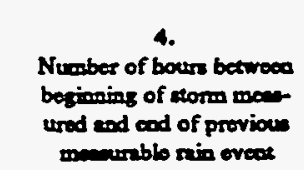 & $\begin{array}{l}\text { S. } \\
\text { Mexim flow rate } \\
\text { during nin event } \\
\text { (allocedmin or specify } \\
\text { unie) }\end{array}$ & $\begin{array}{l}6 . \\
\text { Total fiow from } \\
\text { rain oveos } \\
\text { (enllows or } \\
\text { aproify unia) }\end{array}$ & $\begin{array}{c}7 . \\
\text { Seacon } \\
\text { sample wes } \\
\text { tiken }\end{array}$ & $\begin{array}{l}\text { 8. } \\
\text { Form of } \\
\text { Procipitusion } \\
\text { (rivfill. } \\
\text { sommench) }\end{array}$ \\
\hline $\begin{array}{l}10 / 14 / 91-1 \\
0 / 15 / 91\end{array}$ & 650 & 1.4 & 226.25 & 1,010 & 302,103 & Fall & Ruinfall \\
\hline
\end{tabular}

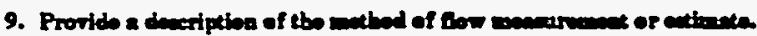

An ISCO 3230 Fow meter was med to obtein water level readings. This intrument was calibrated with tbo aormal dry weather flow, if present, as leved zero. The flow meter was programmed with the characteristics of the convegance so that flow rates and total llows conld be calculated by the meter. 
Outiall S13

Pasta B and C Continued

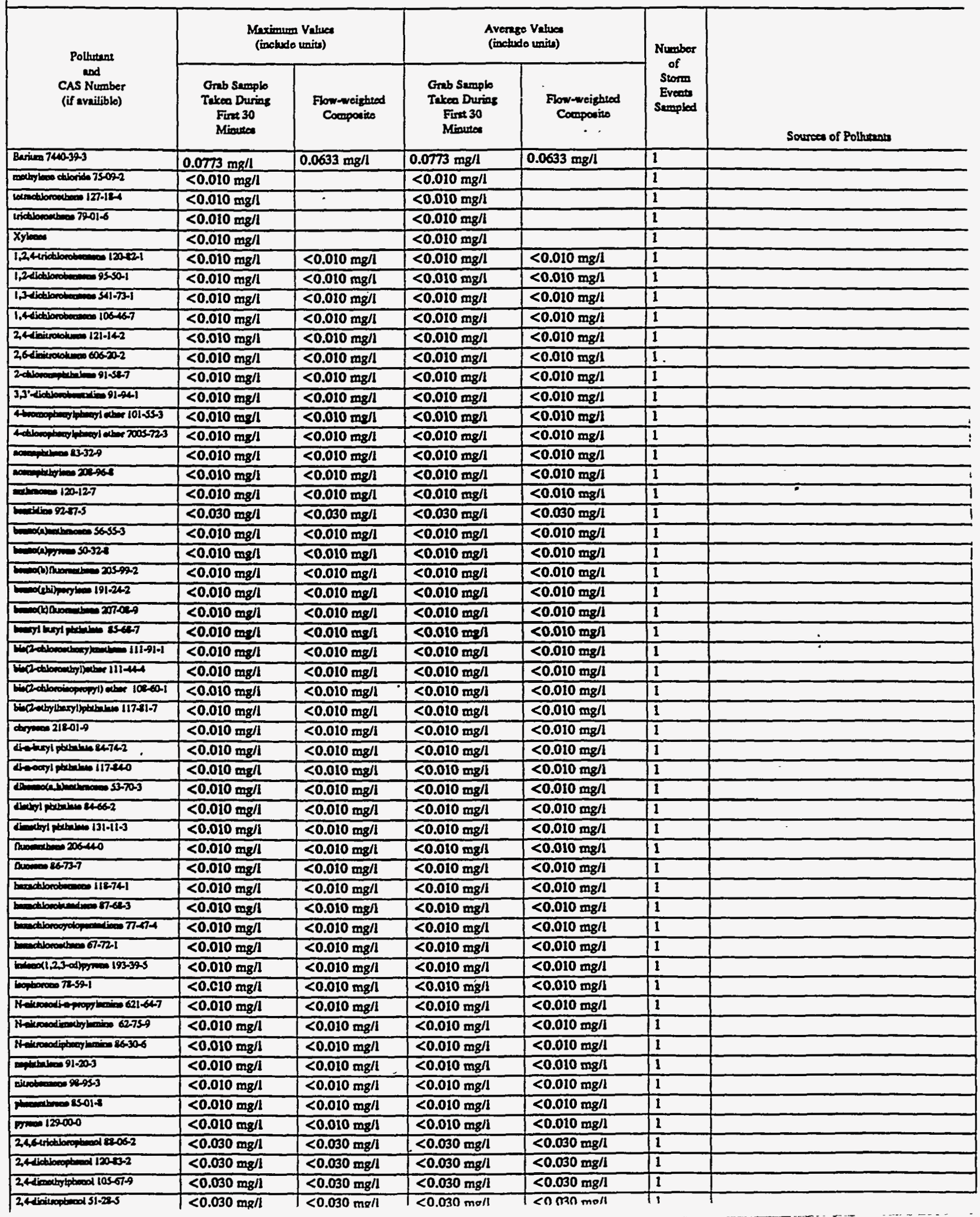


Outfall $\$ 13$

Parte B and C Contioued

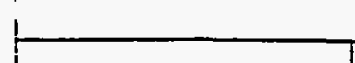

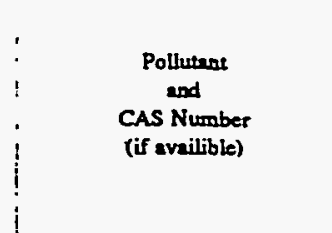

2-anby-4,6-dinitrobeol 53452-1

2-aitrophenol 88-75-5

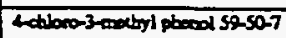

Laitroptiond 100-027

pacectibropbeool 87 \&6-5

phecol los-95-2

4.4-DDD 72-548

4,4'-DDE 72-55-9

4.4:-00T 50-29-3

âtrio $30900-2$

ctbonder-749

dituria 60-57-1

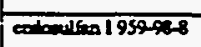

colonilan II 33213-65

cedouten oulan 1031-078

codrin 72-20-8

eotrin altabydo 7621-93-4

bepector 76-418

bepection apocido IOS4-57-3

contreest-35-2

arb-8HC 319546

bDC 319-85-7

Wm-BHC 31946-8

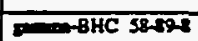

2diboipteol 95-574

Aledor 122 53469-21-9

Arobor-1254 11097691

Aratorizl 11104-22-2

Aroolom-1232 11161-16-S

Aroden 1248 126722966

Alober1260 1100605

Arodor-1016 12674-11-2

Crnols

Aonole:

Aartocititb

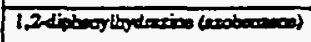

\section{Merimum Valuse \\ (inctude units)}

(ivolude units)

Goub Smole

Trken During

Firet 30

Minite:

$<0.030 \mathrm{mg} / \mathrm{l}$

$<0.030 \mathrm{mg} / \mathrm{l}$

$<0.030 \mathrm{mg} / 1$

$<0.030 \mathrm{mg} / \mathrm{l}$

$<0.030 \mathrm{mg} / 1$

$<0.030 \mathrm{mg} / \mathrm{l}$

$<0.00001 \mathrm{mg} / \mathrm{l}$

$<0.00001 \mathrm{mg} / \mathrm{t}$

$<0.00001 \mathrm{mg} / \mathrm{l}$

$<0.00001 \mathrm{mg} / 1$

$<0.00002 \mathrm{mg} / 1$

$<0.00001 \mathrm{mg} / 1$

$<0.00001 \mathrm{mg} / 1$

$<0.00001 \mathrm{mg} / \mathrm{l}$

$<0.00001 \mathrm{mg} / \mathrm{l}$

$<0.00005 \mathrm{mg} / 1$

$<0.00001 \mathrm{mg} / 1$

$<0.00001 \mathrm{mg} / 1$

$<0.00001 \mathrm{mg} / \mathrm{l}$

$<0.0002 \mathrm{mg} / 1$

$<0.00001 \mathrm{mg} / \mathrm{l}$

$<0.00001 \mathrm{mg} / 1$

$<0.00001 \mathrm{mg} / 1$

$<0.00001 \mathrm{mg} / 1$

$<0.030 \mathrm{mg} / \mathrm{l}$

$<0.0005 \mathrm{mg} / \mathrm{h}$

$<0.0005 \mathrm{mg} / 1$

$<0.0005 \mathrm{mg} / \mathrm{l}$

$<0.0005 \mathrm{mg} / \mathrm{l}$

$<0.0005 \mathrm{mg} / \mathrm{l}$

$<0.0005 \mathrm{mg} / 1$

$<0.0005 \mathrm{mg} / 1$

$<0.030 \mathrm{mg} / \mathrm{l}$

$<0.010 \mathrm{mg} / \mathrm{l}$

$<0.010 \mathrm{mg} / \mathrm{l}$

Rearles peoding

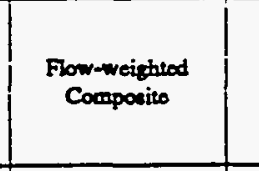

$<0.030 \mathrm{mg} / \mathrm{l}$

$<0.030 \mathrm{mg} / \mathrm{l}$

$<0.030 \mathrm{mg} / \mathrm{l}$

$<0.030 \mathrm{mg} / \mathrm{l}$

$<0.030 \mathrm{mg} / \mathrm{l}$

$<0.030 \mathrm{mg} / \mathrm{l}$

$<0.00001 \mathrm{mg} / 1$

$<0.00001 \mathrm{mg} / \mathrm{l}$

$<0.00001 \mathrm{mg} / 1$

$<0.00001 \mathrm{mg} / \mathrm{l}$

$<0.00002 \mathrm{mg} / \mathrm{l}$

$<0.00001 \mathrm{mg} / 1$

$<0.00001 \mathrm{mg} / \mathrm{l}$

$<0.00001 \mathrm{mg} / 1$

$<0.00001 \mathrm{mg} / \mathrm{l}$

$<0.00005 \mathrm{mg} / \mathrm{l}$

$<0.00001 \mathrm{mg} / \mathrm{l}$

$<0.00001 \mathrm{mg} / \mathrm{l}$

$<0.00001 \mathrm{mg} / \mathrm{l}$

$<0.0002 \mathrm{mg} / \mathrm{l}$

$<0.00001 \mathrm{mg} / \mathrm{l}$

$<0.00001 \mathrm{mg} / \mathrm{l}$

$<0.00001 \mathrm{mg} / \mathrm{l}$

$<0.00001 \mathrm{mg} / \mathrm{l}$

$<0.030 \mathrm{mg} / \mathrm{l}$

$<0.0005 \mathrm{mg} / \mathrm{l}$

$<0.0005 \mathrm{mg} / \mathrm{l}$

$<0.0005 \mathrm{mg} / \mathrm{l}$

$<0.0005 \mathrm{mg} / \mathrm{l}$

$<0.0005 \mathrm{mg} / \mathrm{l}$

$<0.0005 \mathrm{mg} / \mathrm{l}$

$<0.0005 \mathrm{mg} / \mathrm{l}$

$<0.030 \mathrm{mg} / \mathrm{l}$

$<0.010 \mathrm{mg} / \mathrm{l}$

$<0.010 \mathrm{mg} / 1$

Reaultes peading
Avengo Values

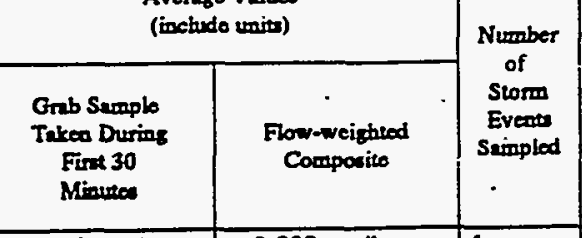

$<0.030 \mathrm{mg} / \mathrm{l}$

$<0.030 \mathrm{mg} / \mathrm{l}$

$<0.030 \mathrm{mg} / \mathrm{l}$

$<0.030 \mathrm{mg} / \mathrm{l}$

$<0.030 \mathrm{mg} / \mathrm{l}$

$<0.030 \mathrm{mg} / \mathrm{h}$

$<0.00001 \mathrm{mg} / \mathrm{l}$

$<0.00001 \mathrm{mg} / \mathrm{l}$

$<0.00001 \mathrm{mg} / \mathrm{l}$

$<0.00001 \mathrm{mg} / \mathrm{l}$

$<0.00002 \mathrm{mg} / \mathrm{l}$

$<0.00001 \mathrm{mg} / \mathrm{l}$

$<0.00001 \mathrm{mg} / \mathrm{l}$

$<0.00001 \mathrm{mg} / 1$

$<0.00001 \mathrm{mg} / \mathrm{l}$

$<0.00005 \mathrm{mg} / \mathrm{l}$

$<0.00001 \mathrm{mg} / \mathrm{h}$

$<0.00001 \mathrm{mg} / \mathrm{l}$

$<0.00001 \mathrm{mg} / \mathrm{h}$

$<0.0002 \mathrm{mg} /$

$<0.00001 \mathrm{mg} / \mathrm{l}$

$<0.00001 \mathrm{mg} /$

$<0.00001 \mathrm{mg} / 1$

$<0.00001 \mathrm{mg} / \mathrm{l}$

$<0.030 \mathrm{mg} / \mathrm{l}$

$<0.0005 \mathrm{mg} / \mathrm{l}$

$<0.0005 . \mathrm{mg} / 1$

$<0.0005 \mathrm{mg} / \mathrm{l}$

$<0.0005 \mathrm{mg} / \mathrm{l}$

$<0.0005 \mathrm{mg} / \mathrm{l}$

$<0.0005 \mathrm{mg} / 1$

$<0.0005 \mathrm{mg} / \mathrm{l}$

$<0.030 \mathrm{mg} / \mathrm{l}$

$<0.010 \mathrm{mg} / \mathrm{l}$

$<0.010 \mathrm{mg} / \mathrm{l}$

Recults peoding
$<0.030 \mathrm{mg} / \mathrm{l}$

$<0.030 \mathrm{mg} / \mathrm{l}$

$<0.030 \mathrm{mg} / \mathrm{l}$

$<0.030 \mathrm{mg} / \mathrm{l}$

$<0.030 \mathrm{mg} / \mathrm{l}$

$<0.030 \mathrm{mg} / \mathrm{l}$

$<0.00001 \mathrm{mg} / \mathrm{l}$

$<0.00001 \mathrm{mg} / \mathrm{l}$

$<0.00001 \mathrm{mg} / \mathrm{l}$

$<0.00001 \mathrm{mg} / \mathrm{l}$

$<0.00002 \mathrm{mg} / \mathrm{l}$

$<0.00001 \mathrm{mg} / \mathrm{l}$

$<0.00001 \mathrm{mg} / \mathrm{l}$

$<0.00001 \mathrm{mg} / \mathrm{l}$

$<0.00001 \mathrm{mg} / \mathrm{l}$

$<0.00005 \mathrm{mg} / \mathrm{l}$

$<0.00001 \mathrm{mg} / \mathrm{l}$

$<0.00001 \mathrm{mg} /$

$<0.00001 \mathrm{mg} / \mathrm{l}$

$<0.0002 \mathrm{mg} / \mathrm{l}$

$<0.00001 \mathrm{mg} / 1$

$<0.00001 \mathrm{mg} / \mathrm{l}$

$<0.00001 \mathrm{mg} / \mathrm{l}$

$<0.00001 \mathrm{mg} / \mathrm{l}$

$<0.030 \mathrm{mg} / \mathrm{l}$

$<0.0005 \mathrm{mg} / \mathrm{l}$

$<0.0005 \mathrm{mg} / \mathrm{l}$

$<0.0005 \mathrm{mg} / \mathrm{l}$

$<0.0005 \mathrm{mg} / \mathrm{l}$

$<0.0005 \mathrm{mg} / \mathrm{l}$

$<0.0005 \mathrm{mg} / \mathrm{l}$

$<0.0005 \mathrm{mg} / \mathrm{l}$

$<0.030 \mathrm{mg} / \mathrm{l}$

$<0.010 \mathrm{mg} / 1$

$<0.010 \mathrm{mg} / \mathrm{l}$

Reculls pending
Sources of Polhonnts

\begin{tabular}{|c|c|c|c|c|c|c|}
\hline & 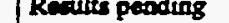 & & & & & \\
\hline & & & & & & \\
\hline & & & & & & \\
\hline & & & & & & \\
\hline & & & & & & \\
\hline & & & & & & \\
\hline & & & & & - & \\
\hline & & $\rightarrow$ & & & & \\
\hline & & & & & & \\
\hline & & & & & & 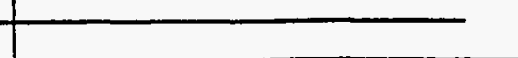 \\
\hline & & & & & & \\
\hline & & & & & & 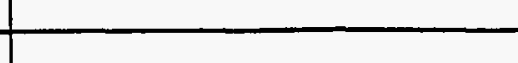 \\
\hline & & & & & & \\
\hline+2 & - & 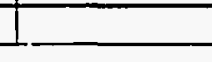 & - & 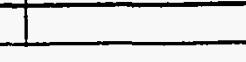 & 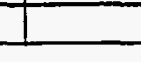 & 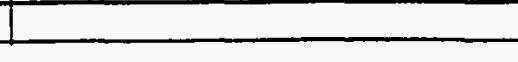 \\
\hline
\end{tabular}


VII. Discherge Lnformation (continued from page 3 of Form 2 P

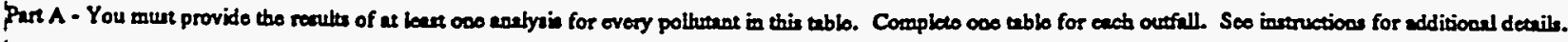

\begin{tabular}{|c|c|c|c|c|c|}
\hline \multirow{2}{*}{$\begin{array}{l}\text { Pollutant } \\
\text { and } \\
\text { CAS Number } \\
\text { (if aviliblo) }\end{array}$} & \multicolumn{2}{|c|}{$\begin{array}{l}\text { Maximum Values } \\
\text { (inchude units) }\end{array}$} & \multicolumn{2}{|c|}{$\begin{array}{l}\text { Avernge Vatues } \\
\text { (inchude units) }\end{array}$} & \multirow{2}{*}{$\begin{array}{l}\text { Number } \\
\text { of } \\
\text { Stom } \\
\text { Eveate } \\
\text { Sempled }\end{array}$} \\
\hline & $\begin{array}{c}\text { Gab Sumple } \\
\text { Takeo During } \\
\text { Fint } 30 \\
\text { Minetee }\end{array}$ & $\begin{array}{l}\text { Flow-weightiod } \\
\text { Compotive }\end{array}$ & $\begin{array}{c}\text { Grab Semple } \\
\text { Taken Durias } \\
\text { First } 30 \\
\text { Minuteo }\end{array}$ & $\begin{array}{l}\text { Flow-wëighted } \\
\text { Compotive }\end{array}$ & \\
\hline pil mod Growe & $<2 \mathrm{mg} / \mathrm{l}$ & & $<2 \mathrm{mg} / \mathrm{l}$ & & 1 \\
\hline 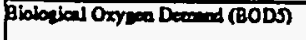 & $8 \mathrm{mg} / \mathrm{l}$ & $8.25 \mathrm{mg} / \mathrm{l}$ & $8 \mathrm{mg} / \mathrm{l}$ & $8.25 \mathrm{mg} / 1$ & 1 \\
\hline 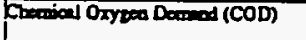 & $30 \mathrm{mg} / \mathrm{l}$ & $33 \mathrm{mg} / \mathrm{l}$ & $30 \mathrm{mg} / \mathrm{l}$ & $33 \mathrm{mg} / \mathrm{l}$ & 1 \\
\hline Troul Surpoender Solide (TSS) & $<5 \mathrm{mg} / 1$ & $57 \mathrm{mg} / \mathrm{l}$ & $<5 \mathrm{mg} / \mathrm{l}$ & $57 \mathrm{mg} / \mathrm{l}$ & 1 \\
\hline 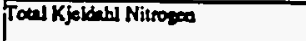 & $0.7 \mathrm{mg} / \mathrm{l}$ & $0.8 \mathrm{mg} / \mathrm{l}$ & $0.7 \mathrm{mg} / \mathrm{l}$ & $0.8 \mathrm{mg} / \mathrm{l}$ & 1 \\
\hline Firne plue Nitrite Nitrooses & $0.1 \mathrm{mg} / 1$ & $1.0 \mathrm{mg} / \mathrm{l}$ & $0.1 \mathrm{mg} / 1$ & $1.0 \mathrm{mg} / \mathrm{l}$ & 1 \\
\hline Toeal Prosiphors & $<0.1 \mathrm{mg} / \mathrm{l}$ & $0.14 \mathrm{mg} / \mathrm{l}$ & $<0.1 \mathrm{mg} / \mathrm{l}$ & $0.14 \mathrm{mg} / \mathrm{l}$ & 1 \\
\hline $\mathrm{BH}$ & Minimm 85 & Meximan 8.5 & Minimm 8.5 & Mraimers 8.5 & \\
\hline
\end{tabular}

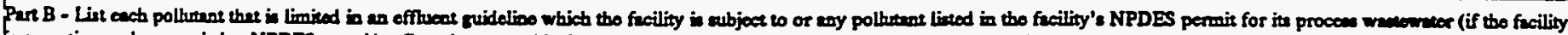

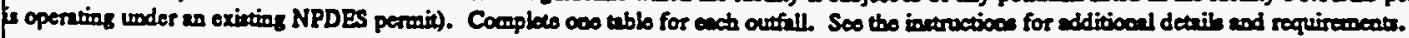

\begin{tabular}{|c|c|c|c|c|c|c|}
\hline \multirow{2}{*}{$\begin{array}{l}\text { Pollutinat } \\
\text { and } \\
\text { CAs Number } \\
\text { (if availiblo) }\end{array}$} & \multicolumn{2}{|c|}{$\begin{array}{l}\text { Meximus Values } \\
\text { (iboludo unia) }\end{array}$} & \multicolumn{2}{|c|}{$\begin{array}{l}\text { Averese Vahuea } \\
\text { (ibotudo unim) }\end{array}$} & \multirow{2}{*}{$\begin{array}{l}\text { Numbor } \\
\text { of } \\
\text { Siorm } \\
\text { Ereote } \\
\text { Simplod }\end{array}$} & \multirow{2}{*}{ Sources of Polkenes } \\
\hline & $\begin{array}{l}\text { Orab Samplo } \\
\text { Taken Duriog } \\
\text { Firs } 30 \\
\text { Minusen }\end{array}$ & $\begin{array}{l}\text { Flow-mrighed } \\
\text { Compocito }\end{array}$ & 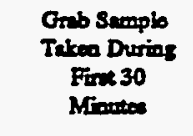 & $\begin{array}{l}\text { Fow-wrighteod } \\
\text { Componito }\end{array}$ & & \\
\hline Artimany $740-360$ & $<0.003 \mathrm{mg} / \mathrm{l}$ & $<0.003 \mathrm{mg} / \mathrm{l}$ & $<0.003 \mathrm{mg} / \mathrm{l}$ & $<0.003 \mathrm{mg} / \mathrm{l}$ & 1 & \\
\hline onim & $<0.002 \mathrm{mg} / 1$ & & $<0.002 \mathrm{mg} / 1$ & & 1 & \\
\hline 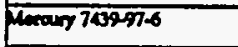 & $<0.0002 \mathrm{mg} / 1$ & $<0.0002 \mathrm{mg} / 1$ & $<0.0002 \mathrm{mg} / \mathrm{l}$ & $<0.0002 \mathrm{mg} / 1$ & 1 & \\
\hline pande & $0.002 \mathrm{mg} / \mathrm{l}$ & & $0.002 \mathrm{mg} / \mathrm{l}$ & & 1 & $\because$ \\
\hline 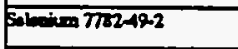 & $<0.002 \mathrm{mg} / \mathrm{l}$ & $<0.002 \mathrm{mg} / \mathrm{l}$ & $<0.002 \mathrm{mg} / \mathrm{l}$ & $<0.002 \mathrm{mg} / \mathrm{l}$ & 1 & \\
\hline Pulfore & $100 \mathrm{mg} / \mathrm{l}$ & $110 \mathrm{mg} / 1$ & $100 \mathrm{mg} / \mathrm{l}$ & $110 \mathrm{mg} / 1$ & 1 & \\
\hline Purtaneses & $<0.05 \mathrm{mg} / \mathrm{l}$ & $<0.05 \mathrm{mg} / \mathrm{l}$ & $<0.05 \mathrm{mg} / \mathrm{l}$ & $<0.05 \mathrm{mg} / 1$ & 1 & \\
\hline 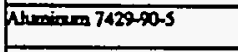 & $0.08 \mathrm{mg} / \mathrm{l}$ & $1.90 \mathrm{mg} / \mathrm{L}$ & $0.08 \mathrm{mg} / \mathrm{l}$ & $1.90 \mathrm{mg} / \mathrm{l}$ & 1 & \\
\hline $4=10 \mathrm{i}$ 740-38-2 & $<0.04 \mathrm{mg} / \mathrm{l}$ & $<0.04 \mathrm{mg} / \mathrm{l}$ & $<0.04 \mathrm{mg} / \mathrm{l}$ & $<0.04 \mathrm{mg} / \mathrm{l}$ & 1 & \\
\hline $30-740-268$ & $41.7 \mathrm{mg} / 1$ & $7.42 \mathrm{mg} / \mathrm{l}$ & $41.7 \mathrm{mg} / 1$ & $7.42 \mathrm{mg} / \mathrm{l}$ & 1 & \\
\hline 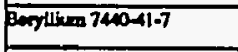 & $<0.0004 \mathrm{mg} / \mathrm{l}$ & $0.0013 \mathrm{mg} / \mathrm{l}$ & $<0.0004 \mathrm{mg} / \mathrm{l}$ & $0.0013 \mathrm{mg} / \mathrm{I}$ & 1 & \\
\hline Paxim 740-33-9 & $<0.004 \mathrm{mg} / \mathrm{l}$ & $<0.004 \mathrm{mg} / \mathrm{l}$ & $<0.004 \mathrm{mg} / \mathrm{I}$ & $<0.004 \mathrm{mg} / \mathrm{l}$ & 1 & \\
\hline Celoiden 7460-70-2 & $106 \mathrm{mg} / \mathrm{l}$ & $54.8 \mathrm{mg} / \mathrm{l}$ & $106 \mathrm{mg} / \mathrm{l}$ & $54.8 \mathrm{mg} / \mathrm{l}$ & 1 & \\
\hline Chencien 740-7.3 & $<0.006 \mathrm{mg} / \mathrm{l}$ & $<0.006 \mathrm{mg} / \mathrm{l}$ & $<0.006 \mathrm{mg} / 1$ & $<0.006 \mathrm{mg} / \mathrm{l}$ & 1 & \\
\hline Cotak 740-18-4 & $<0.002 \mathrm{mg} / \mathrm{l}$ & $0.003 \mathrm{mg} / \mathrm{l}$ & $<0.002 \mathrm{mg} / \mathrm{l}$ & $0.003 \mathrm{mg} / \mathrm{l}$ & 1 & \\
\hline 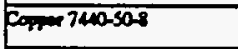 & $<0.006 \mathrm{mg} / \mathrm{l}$ & $<0.006 \mathrm{mg} / 1$ & $<0.006 \mathrm{mg} / 1$ & $<0.006 \mathrm{mg} / \mathrm{l}$ & 1 & \\
\hline 20007432496 & $1.31 \mathrm{mg} / 1$ & $4.43 \mathrm{mg} / 1$ & $1.31 \mathrm{mg} / 1$ & $4.43 \mathrm{mg} / 1$ & 1 & \\
\hline 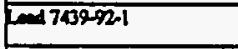 & $<0.02 \mathrm{mg} / \mathrm{l}$ & $<0.02 \mathrm{mg} / \mathrm{l}$ & $<0.02 \mathrm{mg} / \mathrm{l}$ & $<0.02 \mathrm{mg} / \mathrm{l}$ & 1 & \\
\hline futhem 7439-93.2 & $10.5 \mathrm{mg} / 1$ & $2.31 \mathrm{mg} / 1$ & $10.5 \mathrm{mg} / 1$ & $2.31 \mathrm{mg} / \mathrm{l}$ & 1 & \\
\hline 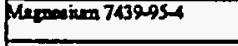 & $17.6 \mathrm{mg} / \mathrm{l}$ & $7.73 \mathrm{mg} / 1$ & $17.6 \mathrm{mg} / 1$ & $7.73 \mathrm{mg} / 1$ & 1 & \\
\hline 743996.5 & $1.98 \mathrm{mg} / \mathrm{l}$ & $1.04 \mathrm{mg} / \mathrm{l}$ & $1.98 \mathrm{mg} / \mathrm{h}$ & $1.04 \mathrm{mg} / \mathrm{l}$ & 1 & \\
\hline 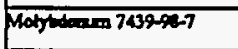 & $<0.006 \mathrm{mg} / \mathrm{l}$ & $<0.006 \mathrm{mg} / \mathrm{l}$ & $<0.006 \mathrm{mg} / 1$ & $<0.006 \mathrm{mg} / \mathrm{l}$ & 1 & \\
\hline$\sqrt{501+17400020}$ & $<0.008 \mathrm{mg} / \mathrm{l}$ & $<0.008 \mathrm{mg} / \mathrm{l}$ & $<0.008 \mathrm{mg} / 1$ & $<0.008 \mathrm{mg} / \mathrm{l}$ & 1 & \\
\hline Pomixn 74009-7 & $8.7 \mathrm{mg} / \mathrm{l}$ & $5.7 \mathrm{mg} / 1$ & $8.7 \mathrm{mg} / \mathrm{l}$ & $5.7 \mathrm{mg} / \mathrm{l}$ & 1 & \\
\hline sithor 746a-2at & $<0.006 \mathrm{mg} / \mathrm{l}$ & $<0.006 \mathrm{mg} / 1$ & $<0.006 \mathrm{mg} / \mathrm{l}$ & $<0.006 \mathrm{mg} / \mathrm{l}$ & 1 & \\
\hline sodiven 7400-23-5 & $17.1 \mathrm{mg} / 1$ & $3.36 \mathrm{mg} / \mathrm{l}$ & $17.1 \mathrm{mg} / \mathrm{l}$ & $3.36 \mathrm{mg} / 1$ & 1 & \\
\hline Tivanium 7400-32-6 & $<0.01 \mathrm{mg} / 1$ & $0.02 \mathrm{mg} / 1$ & $<0.01 \mathrm{mg} / \mathrm{l}$ & $0.02 \mathrm{mg} / 1$ & 1 & \\
\hline $2 \log 7400606$ & $<0.01 \mathrm{mg} / \mathrm{l}$ & $0.02 \mathrm{mg} / \mathrm{l}$ & $<0.01 \mathrm{mg} / 1$ & $0.02 \mathrm{mg} / 1$ & 1 & \\
\hline
\end{tabular}


Corkinead from froes.

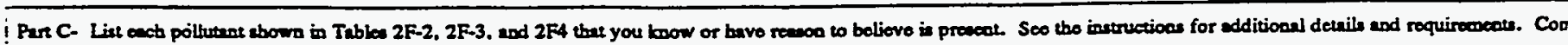
oos tuble for cach outfill.

\begin{tabular}{|c|c|c|c|c|c|c|}
\hline \multirow{2}{*}{ 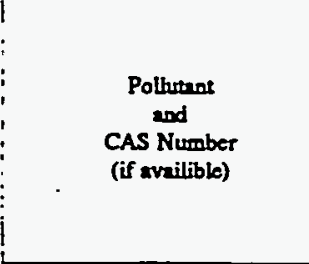 } & \multicolumn{2}{|c|}{$\begin{array}{l}\text { Mexionum Valuca } \\
\text { (inchude units) }\end{array}$} & \multicolumn{2}{|c|}{$\begin{array}{l}\text { Average Valuea } \\
\text { (inchude units) }\end{array}$} & \multirow{2}{*}{$\begin{array}{l}\text { Number } \\
\text { of } \\
\text { Storm } \\
\text { Eveav } \\
\text { Sempled }\end{array}$} & \multirow[b]{2}{*}{ Sources of Polhutans } \\
\hline & $\begin{array}{l}\text { Grib Sumplo } \\
\text { Taken During } \\
\text { Funt } 30 \\
\text { Minutes }\end{array}$ & $\begin{array}{l}\text { Flow-weighted } \\
\text { Comporito }\end{array}$ & $\begin{array}{l}\text { Grab Sumplo } \\
\text { Taken During } \\
\text { Find } 30 \\
\text { Minues }\end{array}$ & $\begin{array}{l}\text { Flow-weightied } \\
\text { Comporite }\end{array}$ & & \\
\hline 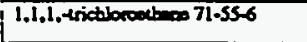 & $<0.010 \mathrm{mg} / \mathrm{l}$ & & $<0.010 \mathrm{mg} / \mathrm{l}$ & & 1 & \\
\hline 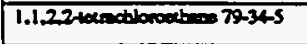 & $<0.010 \mathrm{mg} / \mathrm{l}$ & & $<0.010 \mathrm{mg} / 1$ & & $\mathrm{I}^{\prime}$ & \\
\hline 1.1.2-trictibroutheos 79-00.5 & $<0.010 \mathrm{mg} / 1$ & & $<0.010 \mathrm{mg} / \mathrm{l}$ & & 1 & \\
\hline 1.1 dictiopoutense 75-34-3 & $<0.010 \mathrm{mg} / 1$ & & $<0.010 \mathrm{mg} / \mathrm{l}$ & & 1 & \\
\hline 1.1-didblorcedtyloes 75-35-4 & $<0.010 \mathrm{mg} / !$ & & $<0.010 \mathrm{mg} / 1$ & & 1 & \\
\hline 1.2-dictibroctane $107.06-2$ & $<0.010 \mathrm{mg} / 1$ & & $<0.010 \mathrm{mg} / \mathrm{l}$ & & 1 & \\
\hline 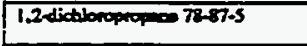 & $<0.010 \mathrm{mg} / \mathrm{l}$ & & $<0.010 \mathrm{mg} / \mathrm{l}$ & & 1 & \\
\hline 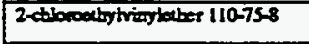 & $<0.010 \mathrm{mg} / \mathrm{l}$ & & $<0.010 \mathrm{mg} / \mathrm{l}$ & & 1 & \\
\hline $600000071-13-2$ & $<0.010 \mathrm{mg} / 1$ & & $<0.010 \mathrm{mg} / 1$ & & 1 & \\
\hline 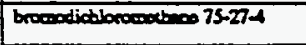 & $<0.010 \mathrm{mg} / 1$ & & $<0.010 \mathrm{mg} / \mathrm{l}$ & & 1 & \\
\hline brocolofore 7S-2S-2 & $<0.010 \mathrm{mg} / \mathrm{l}$ & & $<0.010 \mathrm{mg} / \mathrm{l}$ & & 1 & \\
\hline 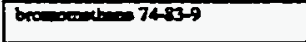 & $<0.010 \mathrm{mg} / \mathrm{l}$ & & $<0.010 \mathrm{mg} / 1$ & & 1 & \\
\hline certeon reanchibrito 56-23-5 & $<0.010 \mathrm{mg} / 1$ & & $<0.010 \mathrm{mg} / 1$ & & 1 & \\
\hline chlopobous000 10890.7 & $<0.010 \mathrm{mg} / 1$ & & $<0.010 \mathrm{mg} / \mathrm{l}$ & & 1 & \\
\hline chloceaberes $75-00-3$ & $0.014 \mathrm{mg} / 1$ & & $0.014 \mathrm{mg} / 1$ & & 1 & \\
\hline Cblarofocom 67-66-3 & $<0.010 \mathrm{mg} / 1$ & & $<0.010 \mathrm{mg} / 1$ & & 1 & . \\
\hline 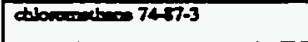 & $<0.010 \mathrm{mg} / 1$ & & $<0.010 \mathrm{mg} / \mathrm{l}$ & & 1 & \\
\hline 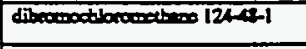 & $<0.010 \mathrm{mg} / 1$ & . & $<0.010 \mathrm{mg} / 1$ & & 1 & \\
\hline 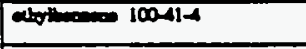 & $<0.010 \mathrm{mg} / 1$ & & $<0.010 \mathrm{mg} / 1$ & & 1 & \\
\hline noter $100-4-3$ & $<0.010 \mathrm{mg} / \mathrm{l}$ & & $<0.010 \mathrm{mg} / \mathrm{l}$ & . & 1 & \\
\hline 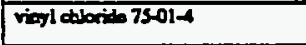 & $<0.010 \mathrm{mg} / 1$ & & $<0.010 \mathrm{mg} / \mathrm{l}$ & & 1 & \\
\hline 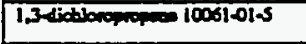 & $<0.010 \mathrm{mg} / 1$ & & $<0.010 \mathrm{mg} / 1$ & & 1 & \\
\hline 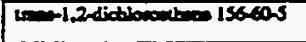 & $<0.010 \mathrm{mg} / \mathrm{l}$ & & $<0.010 \mathrm{mg} / 1$ & & 1 & $\therefore$ \\
\hline Alpton exivity $12587-46-1$ & $53 \mathrm{pci} / 1$ & 47 peill & 53 pcill & 47 peill & 1 & $\cdot$ \\
\hline beciving $12577-46-1$ & $42 \mathrm{pci} / \mathrm{l}$ & 33 peill & $42 \mathrm{pqi} / 1$ & 33 pcill & 1 & \\
\hline boal coliform & $100 \mathrm{co} / .1 \mathrm{~L}$ & $9,000 \mathrm{col} .1 \mathrm{~L}$ & $100 \mathrm{co} / .1 \mathrm{~L}$ & $9,000 \mathrm{co} / .1 \mathrm{~L}$ & 1 & \\
\hline Trallien 740-200 & $<0.001 \mathrm{mg} / \mathrm{l}$ & $<0.001 \mathrm{mg} / \mathrm{l}$ & $<0.001 \mathrm{mg} / 1$ & $<0.001 \mathrm{mg} / \mathrm{l}$ & 1 & \\
\hline Unaive 7400-61-1 & $0.103 \mathrm{mg} / \mathrm{l}$ & $0.125 \mathrm{mg} / \mathrm{l}$ & $0.103 \mathrm{mg} / 1$ & $0.125 \mathrm{mg} / \mathrm{l}$ & 1 & \\
\hline Conexivity & $6.4 \mathrm{pci} / \mathrm{l}$ & 2.3 pcill & $6.4 \mathrm{pei} / 1$ & $2.3 \mathrm{pei} / 1$ & 1 & \\
\hline
\end{tabular}

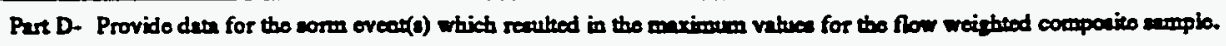

\begin{tabular}{|c|c|c|c|c|c|c|c|}
\hline $\begin{array}{l}1 . \\
\text { Dats of } \\
\text { Storm } \\
\text { Event }\end{array}$ & $\begin{array}{l}2 . \\
\text { Durition } \\
\text { of Storm } \\
\text { (in } \\
\text { minitea) }\end{array}$ & 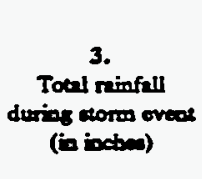 & 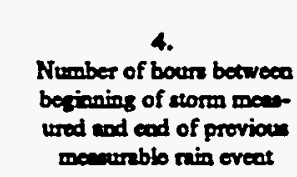 & $\begin{array}{l}\text { S. } \\
\text { Maxion flow noto } \\
\text { during min eveat } \\
\text { (alliocu/min or specify } \\
\text { univ) }\end{array}$ & $\begin{array}{c}6 . \\
\text { Total flow from } \\
\text { rim event } \\
\text { (allow or } \\
\text { spocify unito) }\end{array}$ & $\begin{array}{c}7 . \\
\text { Seacos } \\
\text { semplo wat } \\
\text { taken }\end{array}$ & $\begin{array}{l}8 . \\
\text { Form of } \\
\text { Procipitation } \\
\text { (ninfill, } \\
\text { commels) }\end{array}$ \\
\hline $\begin{array}{l}10 / 14 / 91-1 \\
0 / 15 / 91\end{array}$ & 650 & 1.6 & 226.25 & 229 & 31,800 & Fall & Reinfall \\
\hline
\end{tabular}

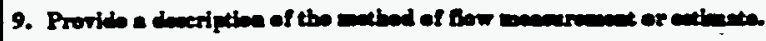

An ISCO 3230 Flow meter was ased to obtain water level readings. This instrment was calibrated with the normal dry weather flow, if present, as lere zero. The flow meter was programmed with the characteristics of the conveyance so that flow rates and total flows conld be calculated by the meter. 
Outfall S14

Parts, B and C Continued

\begin{tabular}{|c|c|c|c|c|c|c|}
\hline \multirow{2}{*}{$\begin{array}{l}\text { Pollutent } \\
\text { and } \\
\text { CAS Number } \\
\text { (if availible) }\end{array}$} & \multicolumn{2}{|c|}{$\begin{array}{l}\text { Maximum Valuea } \\
\text { (includo unites) }\end{array}$} & \multicolumn{2}{|c|}{$\begin{array}{l}\text { Average Values } \\
\text { (inchude unite) }\end{array}$} & \multirow{2}{*}{$\begin{array}{l}\text { Number } \\
\text { of } \\
\text { Storm } \\
\text { Events } \\
\text { Semplod }\end{array}$} & \multirow[b]{2}{*}{ Sources of Polturants } \\
\hline & $\begin{array}{l}\text { Gab Sample } \\
\text { Taken During } \\
\text { First } 30 \\
\text { Minutea }\end{array}$ & $\begin{array}{l}\text { Flow-weizhted } \\
\text { Composite }\end{array}$ & $\begin{array}{l}\text { Grab Sumple } \\
\text { Takea Durims } \\
\text { Fint } 30 \\
\text { Minutea }\end{array}$ & $\begin{array}{l}\text { Flow-weighted } \\
\text { Componite }\end{array}$ & & \\
\hline Bariven 740.39-3 & $0.186 \mathrm{mg} / \mathrm{l}$ & $0.110 \mathrm{mg} / \mathrm{l}$ & $0.186 \mathrm{mg} / \mathrm{l}$ & $0.110 \mathrm{mg} / \mathrm{l}$ & 1 & \\
\hline 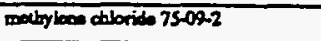 & $<0.010 \mathrm{mg} / \mathrm{l}$ & & $<0.010 \mathrm{mg} / 1$ & & 1 & \\
\hline Letroctibreathoos $127-184$ & $<0.010 \mathrm{mg} / \mathrm{l}$ & & $<0.010 \mathrm{mg} / \mathrm{l}$ & & 1 & \\
\hline trichlow $7901-6$ & $<0.010 \mathrm{mg} / 1$ & & $<0.010 \mathrm{mg} / \mathrm{l}$ & & 1 & \\
\hline Xylooden & $<0.010 \mathrm{mg} / \mathrm{l}$ & & $<0.010 \mathrm{mg} / \mathrm{l}$ & & 1 & \\
\hline 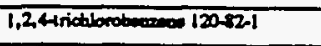 & $<0.010 \mathrm{mg} / 1$ & $<0.010 \mathrm{mg} / \mathrm{l}$ & $<0.010 \mathrm{mg} / !$ & $<0.010 \mathrm{mg} / 1$ & 1 & \\
\hline 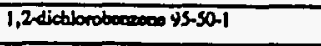 & $<0.010 \mathrm{mg} / \mathrm{l}$ & $<0.010 \mathrm{mg} / \mathrm{l}$ & $<0.010 \mathrm{mg} / \mathrm{l}$ & $<0.010 \mathrm{mg} / 1$ & 1 & \\
\hline 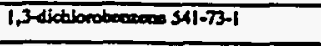 & $<0.010 \mathrm{mg} / 1$ & $<0.010 \mathrm{mg} / 1$ & $<0.010 \mathrm{mg} / \mathrm{l}$ & $<0.010 \mathrm{mg} / 1$ & 1 & \\
\hline 1.4dictsoroberemeno $106-16-7$ & $<0.010 \mathrm{mg} / \mathrm{l}$ & $<0.010 \mathrm{mg} / \mathrm{l}$ & $<0.010 \mathrm{mg} / \mathrm{t}$ & $<0.010 \mathrm{mg} / \mathrm{l}$ & 1 & \\
\hline 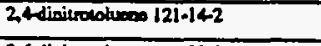 & $<0.010 \mathrm{mg} / \mathrm{l}$ & $<0.010 \mathrm{mg} / 1$ & $<0.010 \mathrm{mg} / \mathrm{I}$ & $<0.010 \mathrm{mg} / 1$ & 1 & \\
\hline 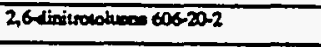 & $<0.010 \mathrm{mg} / \mathrm{l}$ & $<0.010 \mathrm{mg} / 1$ & $<0.010 \mathrm{mg} / \mathrm{l}$ & $<0.010 \mathrm{mg} / 1$ & 1 & \\
\hline 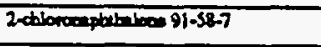 & $<0.010 \mathrm{mg} / \mathrm{l}$ & $<0.010 \mathrm{mg} / 1$ & $<0.010 \mathrm{mg} / \mathrm{l}$ & $<0.010 \mathrm{mg} / 1$ & 1 & \\
\hline 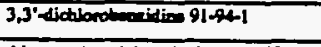 & $<0.010 \mathrm{mg} / \mathrm{l}$ & $<0.010 \mathrm{mg} / 1$ & $<0.010 \mathrm{mg} / \mathrm{l}$ & $<0.010 \mathrm{mg} / \mathrm{l}$ & 1 & \\
\hline 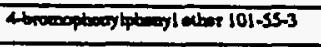 & $<0.010 \mathrm{mg} / \mathrm{l}$ & $<0.010 \mathrm{mg} / \mathrm{l}$ & $<0.010 \mathrm{mg} / \mathrm{l}$ & $<0.010 \mathrm{mg} / \mathrm{l}$ & 1 & \\
\hline 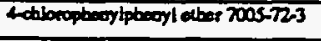 & $<0.010 \mathrm{mg} / \mathrm{l}$ & $<0.010 \mathrm{mg} / 1$ & $<0.010 \mathrm{mg} / \mathrm{l}$ & $<0.010 \mathrm{mg} / \mathrm{l}$ & 1 & \\
\hline omenteces 83-32-9 & $<0.010 \mathrm{mg} / \mathrm{l}$ & $<0.010 \mathrm{mg} / \mathrm{l}$ & $<0.010 \mathrm{mg} / \mathrm{l}$ & $<0.010 \mathrm{mg} / 1$ & 1 & \\
\hline 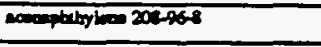 & $<0.010 \mathrm{mg} / \mathrm{l}$ & $<0.010 \mathrm{mg} / 1$ & $<0.010 \mathrm{mg} / 1$ & $<0.010 \mathrm{mg} / 1$ & 1 & \\
\hline $120-12.7$ & $<0.010 \mathrm{mg} / 1$ & $<0.010 \mathrm{mg} / 1$ & $<0.010 \mathrm{mg}^{\prime \prime}$ & $<0.010 \mathrm{mg} / \mathrm{l}$ & 1 & \\
\hline 9207.5 & $<0.030 \mathrm{mg} / 1$ & $<0.030 \mathrm{mg} / 1$ & $<0.030 \mathrm{mg} / \mathrm{l}$ & $<0.030 \mathrm{mg} / 1$ & 1 & \\
\hline 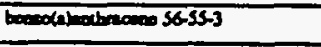 & $<0.010 \mathrm{mg} / \mathrm{l}$ & $<0.010 \mathrm{mg} / \mathrm{l}$ & $<0.010 \mathrm{mg} / \mathrm{l}$ & $<0.010 \mathrm{mg} / \mathrm{l}$ & 1 & \\
\hline 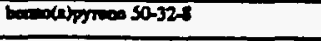 & $<0.010 \mathrm{mg} / 1$ & $<0.010 \mathrm{mg} / 1$ & $<0.010 \mathrm{mg} / \mathrm{l}$ & $<0.010 \mathrm{mg} / \mathrm{l}$ & 1 & \\
\hline 205922 & $<0.010 \mathrm{mg} / 1$ & $<0.010 \mathrm{mg} / \mathrm{l}$ & $<0.010 \mathrm{mg} / \mathrm{l}$ & $<0.010 \mathrm{mg} / 1$ & 1 & \\
\hline 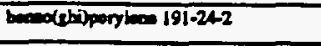 & $<0.010 \mathrm{mg} / \mathrm{l}$ & $<0.010 \mathrm{mg} / \mathrm{l}$ & $<0.010 \mathrm{mg} / \mathrm{l}$ & $<0.010 \mathrm{mg} / \mathrm{l}$ & 1 & \\
\hline 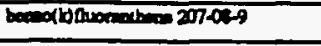 & $<0.010 \mathrm{mg} / 1$ & $<0.010 \mathrm{mg} / 1$ & $<0.010 \mathrm{mg} / \mathrm{l}$ & $<0.010 \mathrm{mg} / \mathrm{l}$ & 1 & \\
\hline 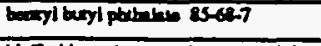 & $<0.010 \mathrm{mg} / \mathrm{l}$ & $<0.010 \mathrm{mg} / \mathrm{l}$ & $<0.010 \mathrm{mg} / \mathrm{l}$ & $<0.010 \mathrm{mg} / 1$ & $I$ & \\
\hline 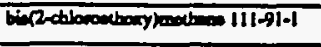 & $<0.010 \mathrm{mg} / \mathrm{l}$ & $<0.010 \mathrm{mg} / 1$ & $<0.010 \mathrm{mg} / \mathrm{l}$ & $<0.010 \mathrm{mg} / \mathrm{l}$ & 1 & $:$ \\
\hline 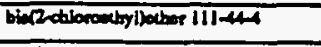 & $<0.010 \mathrm{mg} / \mathrm{l}$ & $<0.010 \mathrm{mg} / \mathrm{l}$ & $<0.010 \mathrm{mg} / \mathrm{l}$ & $<0.010 \mathrm{mg} / 1$ & 1 & \\
\hline 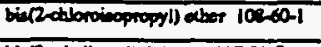 & $<0.010 \mathrm{mg} / \mathrm{I}$ & $<0.010 \mathrm{mg} / \mathrm{l}$ & $<0.010 \mathrm{mg} / \mathrm{l}$ & $<0.010 \mathrm{mg} / \mathrm{l}$ & 1 & \\
\hline 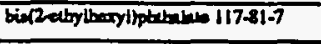 & $<0.010 \mathrm{mg} / 1$ & $<0.010 \mathrm{mg} / \mathrm{l}$ & $<0.010 \mathrm{mg} / \mathrm{l}$ & $<0.010 \mathrm{mg} / 1$ & 1 & \\
\hline काplocos 21201-9 & $<0.010 \mathrm{mg} / \mathrm{l}$ & $<0.010 \mathrm{mg} / \mathrm{l}$ & $<0.010 \mathrm{mg} / 1$ & $<0.010 \mathrm{mg} / 1$ & 1 & \\
\hline 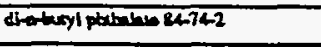 & $<0.010 \mathrm{mg} / \mathrm{l}$ & $<0.010 \mathrm{mg} / \mathrm{l}$ & $<0.010 \mathrm{mg} / \mathrm{l}$ & $<0.010 \mathrm{mg} / 1$ & 1 & \\
\hline 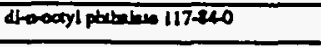 & $<0.010 \mathrm{mg} / \mathrm{l}$ & $<0.010 \mathrm{mg} / \mathrm{l}$ & $<0.010 \mathrm{mg} / \mathrm{l}$ & $<0.010 \mathrm{mg} / \mathrm{l}$ & 1 & \\
\hline 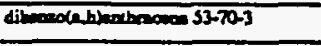 & $<0.010 \mathrm{mg} / \mathrm{l}$ & $<0.010 \mathrm{mg} / \mathrm{l}$ & $<0.010 \mathrm{mg} / 1$ & $<0.010 \mathrm{mg} / 1$ & 1 & \\
\hline 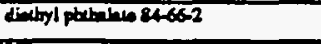 & $<0.010 \mathrm{mg} / \mathrm{l}$ & $<0.010 \mathrm{mg} / 1$ & $<0.010 \mathrm{mg} / \mathrm{l}$ & $<0.010 \mathrm{mg} / \mathrm{l}$ & 1 & \\
\hline 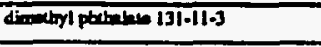 & $<0.010 \mathrm{mg} / 1$ & $<0.010 \mathrm{mg} / \mathrm{l}$ & $<0.010 \mathrm{mg} / 1$ & $<0.010 \mathrm{mg} / 1$ & 1 & \\
\hline Areonethenes 206410 & $<0.010 \mathrm{mg} / \mathrm{l}$ & $<0.010 \mathrm{mg} / \mathrm{l}$ & $<0.010 \mathrm{mg} / \mathrm{l}$ & $<0.010 \mathrm{mg} / 1$ & 1 & \\
\hline 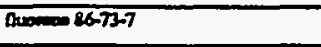 & $<0.010 \mathrm{mg} / 1$ & $<0.010 \mathrm{mg} / \mathrm{l}$ & $<0.010 \mathrm{mg} / \mathrm{l}$ & $<0.010 \mathrm{mg} / 1$ & 1 & \\
\hline $118-74$ & $<0.010 \mathrm{mg} / 1$ & $<0.010 \mathrm{mg} / \mathrm{l}$ & $<0.010 \mathrm{mg} / \mathrm{l}$ & $<0.010 \mathrm{mg} / 1$ & 1 & \\
\hline 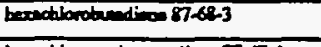 & $<0.010 \mathrm{mg} / \mathrm{l}$ & $<0.010 \mathrm{mg} / \mathrm{l}$ & $<0.010 \mathrm{mg} / \mathrm{l}$ & $<0.010 \mathrm{mg} / \mathrm{l}$ & 1 & \\
\hline 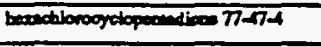 & $<0.010 \mathrm{mg} / \mathrm{l}$ & $<0.010 \mathrm{mg} / \mathrm{l}$ & $<0.010 \mathrm{mg} / \mathrm{l}$ & $<0.010 \mathrm{mg} / 1$ & 1 & \\
\hline 67.72 .1 & $<0.010 \mathrm{mg} / \mathrm{l}$ & $<0.010 \mathrm{mg} / \mathrm{l}$ & $<0.010 \mathrm{mg} / 1$ & $<0.010 \mathrm{mg} / \mathrm{l}$ & 1 & \\
\hline$-(1,2,3-\alpha)$ prianos $193-39,-5$ & $<0.010 \mathrm{mg} / \mathrm{l}$ & $<0.010 \mathrm{mg} / \mathrm{l}$ & $<0.010 \mathrm{mg} / \mathrm{l}$ & $<0.010 \mathrm{mg} / \mathrm{l}$ & 1 & \\
\hline Wophonowe $72-59-1$ & $<0.010 \mathrm{mg} / \mathrm{l}$ & $<0.010 \mathrm{mg} / \mathrm{l}$ & $<0.010 \mathrm{mg} / \mathrm{l}$ & $<0.010 \mathrm{mg} / \mathrm{l}$ & 1 & 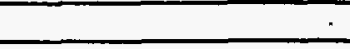 \\
\hline 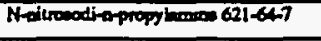 & $<0.010 \mathrm{mg} / \mathrm{l}$ & $<0.010 \mathrm{mg} / \mathrm{l}$ & $<0.010 \mathrm{mg} / 1$ & $<0.010 \mathrm{mg} / \mathrm{l}$ & 1 & \\
\hline 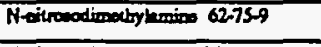 & $<0.010 \mathrm{mg} / \mathrm{l}$ & $<0.010 \mathrm{mg} / \mathrm{l}$ & $<0.010 \mathrm{mg} / \mathrm{l}$ & $<0.010 \mathrm{mg} / \mathrm{l}$ & 1 & \\
\hline 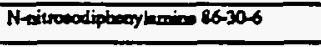 & $<0.010 \mathrm{mg} / 1$ & $<0.010 \mathrm{mg} / \mathrm{l}$ & $<0.010 \mathrm{mg} / \mathrm{l}$ & $<0.010 \mathrm{mg} / \mathrm{l}$ & 1 & \\
\hline 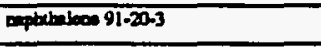 & $<0.010 \mathrm{mg} / \mathrm{l}$ & $<0.010 \mathrm{mg} / \mathrm{l}$ & $<0.010 \mathrm{mg} / \mathrm{l}$ & $<0.010 \mathrm{mg} / \mathrm{l}$ & 1 & \\
\hline 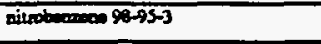 & $<0.010 \mathrm{mg} / \mathrm{l}$ & $<0.010 \mathrm{mg} / \mathrm{l}$ & $<0.010 \mathrm{mg} / \mathrm{l}$ & $<0.010 \mathrm{mg} /$ & $I$ & \\
\hline phantheose $2501-8$ & $<0.010 \mathrm{mg} / \mathrm{l}$ & $<0.010 \mathrm{mg} / \mathrm{l}$ & $<0.010 \mathrm{mg} / \mathrm{l}$ & $<0.010 \mathrm{mg} / \mathrm{l}$ & 1 & \\
\hline pyonos 129000 & $<0.010 \mathrm{mg} / \mathrm{l}$ & $<0.010 \mathrm{mg} / \mathrm{l}$ & $<0.010 \mathrm{mg} / \mathrm{l}$ & $<0.010 \mathrm{mg} / \mathrm{l}$ & 1 & \\
\hline 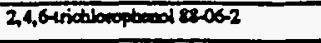 & $<0.030 \mathrm{mg} / \mathrm{I}$ & $<0.030 \mathrm{mg} / \mathrm{l}$ & $<0.030 \mathrm{mg} / \mathrm{L}$ & $<0.030 \mathrm{mg} / \mathrm{l}$ & 1 & \\
\hline 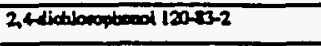 & $<0.030 \mathrm{mg} / 1$ & $<0.030 \mathrm{mg} / \mathrm{l}$ & $<0.030 \mathrm{mg} / \mathrm{l}$ & $<0.030 \mathrm{mg} / \mathrm{l}$ & 1 & \\
\hline 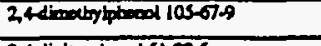 & $<0.030 \mathrm{mg} / \mathrm{l}$ & $<0.030 \mathrm{mg} / \mathrm{l}$ & $<0.030 \mathrm{mg} / 1$ & $<0.030 \mathrm{mg} / \mathrm{l}$ & 1 & \\
\hline 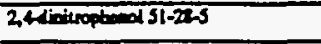 & $<0.030 \mathrm{mg} / 1$ & $<0.030 \mathrm{mg} / 1$ & $<0.030 \mathrm{mg} / 1$ & $<0.030 \mathrm{mg} / 1$ & 1 & \\
\hline
\end{tabular}


Outfall S14

Parte B and C Continued

\begin{tabular}{|c|c|c|c|c|c|c|}
\hline \multirow{2}{*}{$\begin{array}{l}\text { Polturant } \\
\text { and } \\
\text { CAS Number } \\
\text { (if availible) }\end{array}$} & \multicolumn{2}{|c|}{$\begin{array}{l}\text { Meximum Values } \\
\text { (inctude units) }\end{array}$} & \multicolumn{2}{|c|}{$\begin{array}{l}\text { Avernge Values } \\
\text { (inctude units) }\end{array}$} & \multirow{2}{*}{$\begin{array}{c}\text { Number } \\
\text { of } \\
\text { Storm } \\
\text { Evente } \\
\text { Sampled }\end{array}$} & \multirow[b]{2}{*}{ Sources of Pollutents } \\
\hline & $\begin{array}{c}\text { Grab Semople } \\
\text { Takeo During } \\
\text { Firnt } 30 \\
\text { Mirites }\end{array}$ & $\begin{array}{l}\text { Flow-weightod } \\
\text { Compocite }\end{array}$ & $\begin{array}{l}\text { Grab Sample } \\
\text { Teken During } \\
\text { Fira } 30 \\
\text { Misuceo }\end{array}$ & $\begin{array}{l}\text { Flow-weightod } \\
\text { Componite }\end{array}$ & & \\
\hline 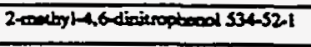 & $<0.030 \mathrm{mg} / 1$ & $<0.030 \mathrm{mg} / \mathrm{l}$ & $<0.030 \mathrm{mg} / \mathrm{l}$ & $<0.030 \mathrm{mg} / \mathrm{l}$ & 1 & \\
\hline 2-sitropbood 28-75-5 & $<0.030 \mathrm{mg} / 1$ & $<0.030 \mathrm{mg} / \mathrm{l}$ & $<0.030 \mathrm{mg} / 1$ & $<0.030 \mathrm{mg} / \mathrm{l}$ & 1 & \\
\hline 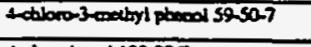 & $<0.030 \mathrm{mg} / \mathrm{l}$ & $<0.030 \mathrm{mg} / \mathrm{l}$ & $<0.030 \mathrm{mg} / \mathrm{l}$ & $<0.030 \mathrm{mg} / \mathrm{l}$ & 1 & \\
\hline Antropbood 10002.7 & $<0.030 \mathrm{mg} / \mathrm{l}$ & $<0.030 \mathrm{mg} / 1$ & $<0.030 \mathrm{mg} / \mathrm{l}$ & $<0.030 \mathrm{mg} / \mathrm{l}$ & 1 & \\
\hline 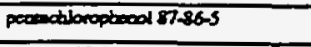 & $<0.030 \mathrm{mg} / \mathrm{l}$ & $<0.030 \mathrm{mg} / \mathrm{l}$ & $<0.030 \mathrm{mg} / \mathrm{l}$ & $<0.030 \mathrm{mg} / \mathrm{l}$ & 1 & \\
\hline pteced 100-95-2 & $<0.030 \mathrm{mg} / \mathrm{l}$ & $<0.030 \mathrm{mg} / \mathrm{l}$ & $<0.030 \mathrm{mg} / \mathrm{l}$ & $<0.030 \mathrm{mg} / \mathrm{l}$ & 1 & \\
\hline $4,4^{\prime}-\mathrm{DDD} 72-548$ & $<0.00001 \mathrm{mg} / \mathrm{l}$ & $<0.00001 \mathrm{mg} / \mathrm{l}$ & $<0.00001 \mathrm{mg} / \mathrm{l}$ & $<0.00001 \mathrm{mg} / \mathrm{l}$ & 1 & \\
\hline $4.4^{\circ}-D D E 72-55-9$ & $<0.00001 \mathrm{mg} / \mathrm{l}$ & $<0.00001 \mathrm{mg} / 1$ & $<0.00001 \mathrm{mg} / \mathrm{l}$ & $<0.00001 \mathrm{mg} / \mathrm{l}$ & 1 & \\
\hline 4,4-DDT 50-20.3 & $<0.00001 \mathrm{mg} / \mathrm{l}$ & $<0.00001 \mathrm{mg} / \mathrm{l}$ & $<0.00001 \mathrm{mg} / \mathrm{l}$ & $<0.00001 \mathrm{mg} / \mathrm{l}$ & 1 & \\
\hline $2 \mathrm{drin} 30900-2$ & $<0.00001 \mathrm{mg} / \mathrm{l}$ & $<0.00001 \mathrm{mg} / \mathrm{l}$ & $<0.00001 \mathrm{mg} / \mathrm{l}$ & $<0.00001 \mathrm{mg} / 1$ & 1 & \\
\hline chlorater & $<0.00002 \mathrm{mg} / \mathrm{l}$ & $<0.00002 \mathrm{mg} / \mathrm{l}$ & $<0.00002 \mathrm{mg} / \mathrm{l}$ & $<0.00002 \mathrm{mg} / 1$ & 1 & \\
\hline dietdin 60-57.1 & $<0.00001 \mathrm{mg} / \mathrm{l}$ & $<0.00001 \mathrm{mg} / \mathrm{l}$ & $<0.00001 \mathrm{mg} / \mathrm{l}$ & $<0.00001 \mathrm{mg} / \mathrm{l}$ & 1 & \\
\hline 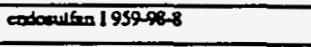 & $<0.00001 \mathrm{mg} / \mathrm{l}$ & $<0.00001 \mathrm{mg} / \mathrm{l}$ & $<0.00001 \mathrm{mg} / \mathrm{l}$ & $<0.00001 \mathrm{mg} / \mathrm{I}$ & 1 & \\
\hline odoull6en 1133213659 & $<0.00001 \mathrm{mg} / \mathrm{l}$ & $<0.00001 \mathrm{mg} / \mathrm{l}$ & $<0.00001 \mathrm{mg} / \mathrm{l}$ & $<0.00001 \mathrm{mg} / \mathrm{l}$ & 1 & \\
\hline 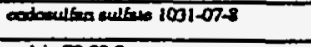 & $<0.00001 \mathrm{mg} / \mathrm{l}$ & $<0.00001 \mathrm{mg} / 1$ & $<0.00001 \mathrm{mg} / \mathrm{l}$ & $<0.00001 \mathrm{mg} / \mathrm{l}$ & 1 & \\
\hline codrio $72-20-8$ & $<0.00005 \mathrm{mg} / \mathrm{l}$ & $<0.00005 \mathrm{mg} / \mathrm{l}$ & $<0.00005 \mathrm{mg} / \mathrm{l}$ & $<0.00005 \mathrm{mg} / \mathrm{l}$ & 1 & \\
\hline Codrin abdotyde 7421-93-4 & $<0.00001 \mathrm{mg} / \mathrm{h}$ & $<0.00001 \mathrm{mg} / 1$ & $<0.00001 \mathrm{mg} / \mathrm{h}$ & $<0.00001 \mathrm{mg} / \mathrm{l}$ & 1 & \\
\hline 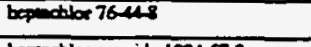 & $<0.00001 \mathrm{mg} / \mathrm{l}$ & $<0.00001 \mathrm{mg} / \mathrm{i}$ & $<0.00001 \mathrm{mg} / 1$ & $<0.00001 \mathrm{mg} / \mathrm{l}$ & 1 & \\
\hline bepectior opecide 100657.3 & $<0.00001 \mathrm{mg} / \mathrm{l}$ & $<0.00001 \mathrm{mg} / 1$ & $<0.00001 \mathrm{mg} / \mathrm{l}$ & $<0.00001 \mathrm{mg} / \mathrm{l}$ & 1 & \\
\hline $100000000001-35-2$ & $<0.0002 \mathrm{mg} / 1$ & $<0.0002 \mathrm{mg} / 1$ & $<0.0002 \mathrm{mg} / 1$ & $<0.0002 \mathrm{mg} / 1$ & 1 & \\
\hline apb-BiसC 319846 & $<0.00001 \mathrm{mg} / 1$ & $<0.00001 \mathrm{mg} / \mathrm{l}$ & $<0.00001 \mathrm{mg} / \mathrm{l}$ & $<0.00001 \mathrm{mg} / \mathrm{l}$ & 1 & \\
\hline 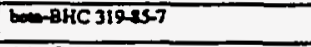 & $<0.00001 \mathrm{mg} / \mathrm{l}$ & $<0.00001 \mathrm{mg} / 1$ & $<0.00001 \mathrm{mg} / \mathrm{l}$ & $<0.00001 \mathrm{mg} / \mathrm{l}$ & 1 & \\
\hline d-8HC $31906-$ & $<0.00001 \mathrm{mg} / \mathrm{l}$ & $<0.00001 \mathrm{mg} / \mathrm{l}$ & $<0.00001 \mathrm{mg} / \mathrm{l}$ & $<0.00001 \mathrm{mg} / \mathrm{l}$ & 1 & \\
\hline$-8 H C 58528$ & $<0.00001 \mathrm{mg} / \mathrm{l}$ & $<0.00001 \mathrm{mg} / 1$ & $<0.00001 \mathrm{mg} / 1$ & $<0.00001 \mathrm{mg} / 1$ & 1 & \\
\hline $20+1000000000195-57-3$ & $<0.030 \mathrm{mg} / 1$ & $<0.030 \mathrm{mg} / \mathrm{l}$ & $<0.030 \mathrm{mg} / \mathrm{l}$ & $<0.030 \mathrm{mg} / \mathrm{l}$ & 1 & \\
\hline Arodond $226253462-21-9$ & $<0.0005 \mathrm{mg} / \mathrm{l}$ & $<0.0005 \mathrm{mg} / \mathrm{l}$ & $<0.0005 \mathrm{mg} / \mathrm{l}$ & $<0.0005 \mathrm{mg} / 1$ & 1 & \\
\hline Arodor-123+11000-69-1 & $<0.0005 \mathrm{mg} / \mathrm{l}$ & $<0.0005 \mathrm{mg} / 1$ & $<0.0005 \mathrm{mg} / 1$ & $<0.0005 \mathrm{mg} / \mathrm{l}$ & 1 & \\
\hline Nocton-1221 11104-28-2 & $<0.0005 \mathrm{mg} / \mathrm{l}$ & $<0.0005 \mathrm{mg} / 1$ & $<0.0005 \mathrm{mg} / \mathrm{l}$ & $<0.0005 \mathrm{mg} / 1$ & 1 & \\
\hline Aroctar-1232 11141-16-5 & $<0.0005 \mathrm{mg} / \mathrm{l}$ & $<0.0005 \mathrm{mg} / \mathrm{i}$ & $<0.0005 \mathrm{mg} / \mathrm{l}$ & $<0.0005 \mathrm{mg} / \mathrm{l}$ & 1 & \\
\hline Aroctor $121212672-296$ & $<0.0005 \mathrm{mg} / 1$ & $<0.0005 \mathrm{mg} / \mathrm{s}$ & $<0.0005 \mathrm{mg} / \mathrm{l}$ & $<0.0005 \mathrm{mg} / 1$ & 1 & \\
\hline Aroctar-12600 11006682-5 & $<0.0005 \mathrm{mg} / \mathrm{l}$ & $<0.0005 \mathrm{mg} / \mathrm{l}$ & $<0.0005 \mathrm{mg} / \mathrm{l}$ & $<0.0005 \mathrm{mg} / \mathrm{l}$ & 1 & \\
\hline Anochar 10161207411.2 & $<0.0005 \mathrm{mg} / 1$ & $<0.0005 \mathrm{mg} / \mathrm{l}$ & $<0.0005 \mathrm{mg} / \mathrm{h}$ & $<0.0005 \mathrm{mg} / \mathrm{l}$ & 1 & \\
\hline Crate & $<0.030 \mathrm{mg} / \mathrm{t}$ & $<0.030 \mathrm{mg} / \mathrm{l}$ & $<0.030 \mathrm{mg} / \mathrm{l}$ & $<0.030 \mathrm{mg} / \mathrm{l}$ & 1 & \\
\hline Acrobin & $<0.010 \mathrm{mg} / \mathrm{l}$ & $<0.010 \mathrm{mg} / \mathrm{l}$ & $<0.010 \mathrm{mg} / \mathrm{l}$ & $<0.010 \mathrm{mg} / \mathrm{l}$ & 1 & \\
\hline Acoglasitrib & $<0.010 \mathrm{mg} / \mathrm{l}$ & $<0.010 \mathrm{mg} / \mathrm{l}$ & $<0.010 \mathrm{mg} / 1$ & $<0.010 \mathrm{mg} / \mathrm{l}$ & 1 & \\
\hline 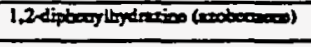 & Results pending & Reaults pending & Reanults pending & Renulte pending & 1 & \\
\hline & & & & & & \\
\hline & & & & & & \\
\hline & & & & & & \\
\hline & & & & & & \\
\hline & & & & & & \\
\hline & & & & & & \\
\hline & & & & & & \\
\hline & & & & & & \\
\hline & & & & & & \\
\hline & & & & & & \\
\hline & & & & & & \\
\hline & & & & & & \\
\hline & & & & & & \\
\hline & & & & & & \\
\hline
\end{tabular}



Coskined from frove.

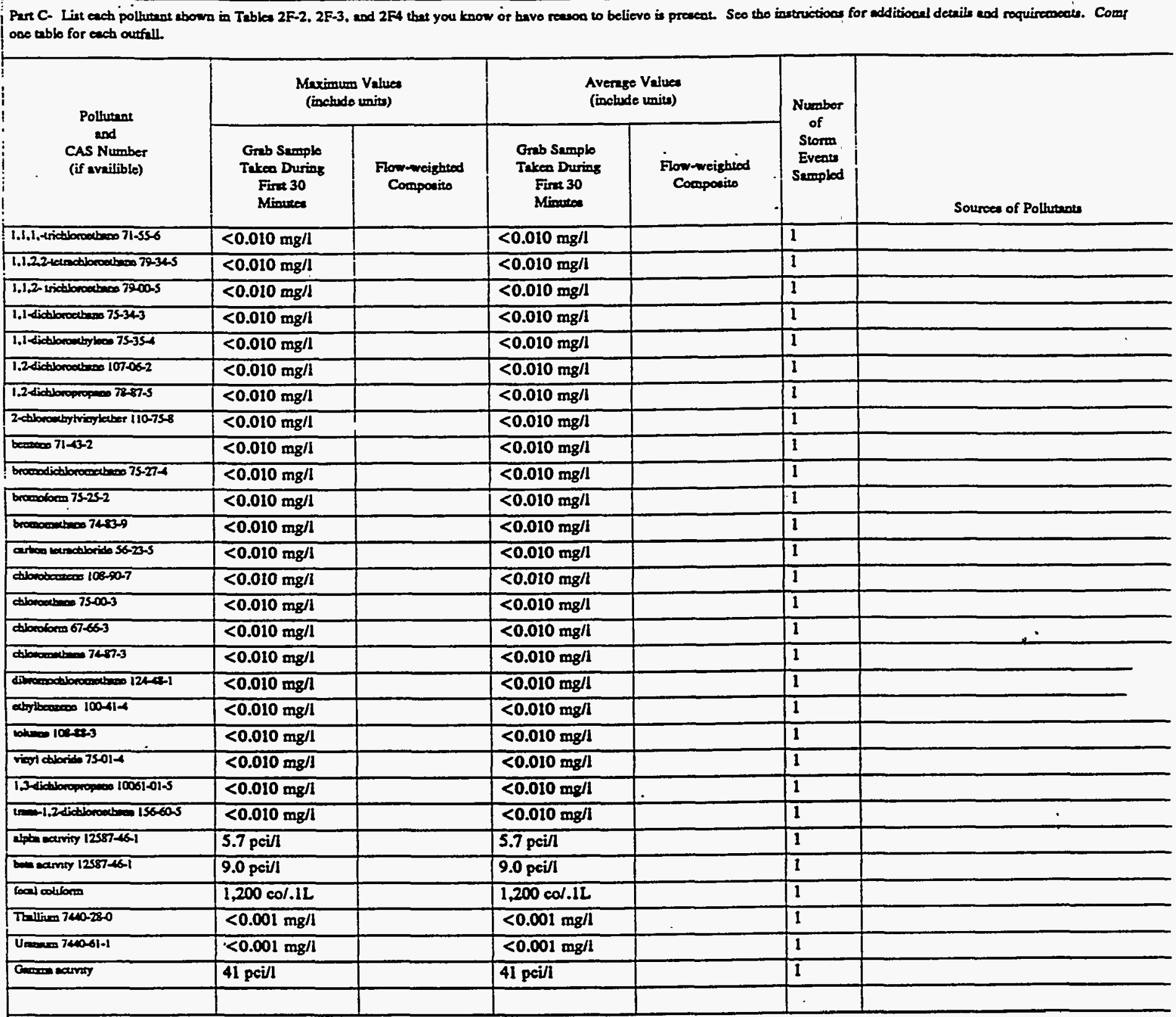

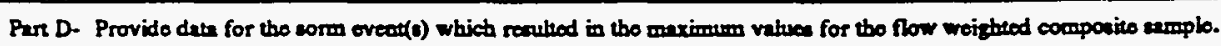

\begin{tabular}{|c|c|c|c|c|c|c|c|}
\hline $\begin{array}{l}1 . \\
\text { Dite of } \\
\text { Storm } \\
\text { Evede }\end{array}$ & $\begin{array}{l}2 . \\
\text { Durtion } \\
\text { of Stom } \\
\text { (in } \\
\text { minuria) }\end{array}$ & $\begin{array}{l}3 . \\
\text { Toul minfill } \\
\text { during torm eveot } \\
\text { (in iocbea) }\end{array}$ & $\begin{array}{l}4 \text {. } \\
\text { Number of bours between } \\
\text { beginning of storm mear } \\
\text { ured and ead of provious } \\
\text { menumble min eveas }\end{array}$ & $\begin{array}{l}\text { S. } \\
\text { Meximum flow mes } \\
\text { during nin eveat } \\
\text { (alloce/min or epocify } \\
\text { unitu) }\end{array}$ & $\begin{array}{l}6 . \\
\text { Total flow from } \\
\text { sin oveas } \\
\text { (allow or } \\
\text { specify units) }\end{array}$ & $\begin{array}{c}7 . \\
\text { Searon } \\
\text { nample wes } \\
\text { tuken }\end{array}$ & 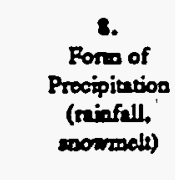 \\
\hline $\begin{array}{l}10 / 14 / 91-1 \\
0 / 15 / 91\end{array}$ & 650 & 1.5 & 226.5 & & & Fall & Ruinfall \\
\hline
\end{tabular}

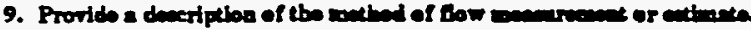

An ISCO 3230 Flow meter was used to obtain water leved readings. This instrument was celibrated with the normal dry weather flow, if present, as leen zero. The flow meter wes programmed with the characteristics of the convegance so that flow rates and total fows coubd be calculnted by the meter. 
Uutfall S15

Partu B and C Continued

\begin{tabular}{|c|c|c|c|c|c|c|}
\hline \multirow{2}{*}{$\begin{array}{l}\text { Polturant } \\
\text { and } \\
\text { CAS Number } \\
\text { (if availible) }\end{array}$} & \multicolumn{2}{|c|}{$\begin{array}{l}\text { Meximimom Values } \\
\text { (includo units) }\end{array}$} & \multicolumn{2}{|c|}{$\begin{array}{l}\text { Avengo Values } \\
\text { (inchude units) }\end{array}$} & \multirow{2}{*}{$\begin{array}{l}\text { Number } \\
\text { of } \\
\text { SLorm } \\
\text { Evects } \\
\text { Serropled }\end{array}$} & \multirow[b]{2}{*}{ Sources of Polbureats } \\
\hline & $\begin{array}{c}\text { Grab Sample } \\
\text { Trikea Duriog } \\
\text { Firnt } 30 \\
\text { Minutee }\end{array}$ & $\begin{array}{l}\text { Fow-weighted } \\
\text { Compotite }\end{array}$ & $\begin{array}{l}\text { Grab Semple } \\
\text { Tekeo During } \\
\text { Fint } 30 \\
\text { Minutea }\end{array}$ & $\begin{array}{l}\text { Flow-weighed } \\
\text { Composino }\end{array}$ & & \\
\hline Baviven 740.3963 & $0.0069 \mathrm{mg} / 1$ & & $0.0069 \mathrm{mg} / \mathrm{l}$ & & 1 & \\
\hline molyloos diboride 75-02-2 & $<0.010 \mathrm{mg} / \mathrm{l}$ & & $<0.010 \mathrm{mg} / \mathrm{l}$ & & 1 & \\
\hline Leinctionouberos $127 \cdot 18-4$ & $<0.010 \mathrm{mg} / \mathrm{l}$ & & $<0.010 \mathrm{mg} / \mathrm{l}$ & & 1 & \\
\hline Irictionoulbes $7901-6$ & $<0.010 \mathrm{mg} / \mathrm{l}$ & & $<0.010 \mathrm{mg} / \mathrm{l}$ & & 1 & \\
\hline Xybeosen & $<0.010 \mathrm{mg} / \mathrm{l}$ & & $<0.010 \mathrm{mg} / \mathrm{l}$ & & 1 & \\
\hline 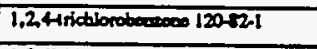 & $<0.010 \mathrm{mg} / \mathrm{l}$ & & $<0.010 \mathrm{mg} / \mathrm{l}$ & & 1 & \\
\hline 1.2. dictionobosoceos 95-50-1 & $<0.010 \mathrm{mg} / \mathrm{l}$ & & $<0.010 \mathrm{mg} / \mathrm{l}$ & & 1 & \\
\hline 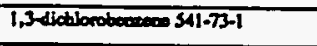 & $<0.010 \mathrm{mg} / \mathrm{l}$ & & $<0.010 \mathrm{mg} / \mathrm{l}$ & & 1 & \\
\hline 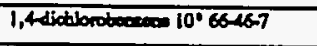 & $<0.010 \mathrm{mg} / \mathrm{l}$ & & $<0.010 \mathrm{mg} / \mathrm{l}$ & & 1 & \\
\hline 2,4 dinitrochatume $121-142$ & $<0.010 \mathrm{mg} / \mathrm{l}$ & & $<0.010 \mathrm{mg} / \mathrm{l}$ & & 1 & \\
\hline 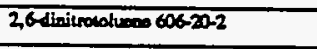 & $<0.010 \mathrm{mg} / \mathrm{l}$ & & $<0.010 \mathrm{mg} / \mathrm{l}$ & & 1 & \\
\hline 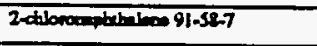 & $<0.010 \mathrm{mg} / \mathrm{l}$ & & $<0.010 \mathrm{mg} / 1$ & & 1 . & . \\
\hline 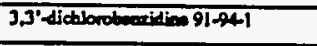 & $<0.010 \mathrm{mg} / \mathrm{l}$ & & $<0.010 \mathrm{mg} / \mathrm{l}$ & & 1 & \\
\hline 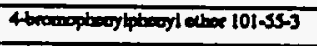 & $<0.010 \mathrm{mg} / \mathrm{l}$ & & $<0.010 \mathrm{mg} / 1$ & & 1 & \\
\hline 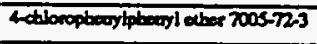 & $<0.010 \mathrm{mg} / 1$ & & $<0.010 \mathrm{mp} / \mathrm{l}$ & & 1 & \\
\hline 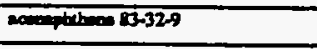 & $<0.010 \mathrm{mg} / \mathrm{l}$ & & $<0.010 \mathrm{mg} / 1$ & & 1 & \\
\hline 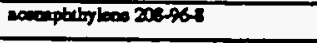 & $<0.010 \mathrm{mg} / \mathrm{l}$ & & $<0.010 \mathrm{mg} / \mathrm{l}$ & & 1 & \\
\hline whenosos $120-127$ & $<0.010 \mathrm{mg} / 1$ & & $<0.010 \mathrm{mg} / \mathrm{l}$ & & 1 & \\
\hline madidin 927.5 & $<0.030 \mathrm{mg} / \mathrm{l}$ & & $<0.030 \mathrm{mg} / \mathrm{l}$ & & 1 & \\
\hline 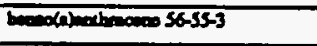 & $<0.010 \mathrm{mg} / \mathrm{l}$ & & $<0.010 \mathrm{mg} / \mathrm{l}$ & & 1 & \\
\hline 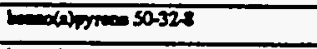 & $<0.010 \mathrm{mg} / \mathrm{l}$ & & $<0.010 \mathrm{mg} / \mathrm{l}$ & & 1 & \\
\hline 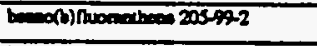 & $<0.010 \mathrm{mg} / \mathrm{l}$ & & $<0.010 \mathrm{mg} / \mathrm{l}$ & & 1 & \\
\hline 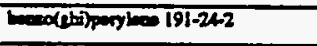 & $<0.010 \mathrm{mg} / \mathrm{l}$ & & $<0.010 \mathrm{mg} / \mathrm{l}$ & & 1 & \\
\hline $20100-9$ & $<0.010 \mathrm{mg} / \mathrm{l}$ & & $<0.010 \mathrm{mg} / \mathrm{l}$ & & 1 & \\
\hline 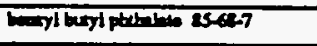 & $<0.010 \mathrm{mg} / \mathrm{l}$ & & $<0.010 \mathrm{mg} / \mathrm{l}$ & & 1 & \\
\hline 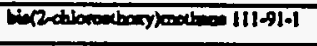 & $<0.010 \mathrm{mg} / \mathrm{l}$ & & $<0.010 \mathrm{mg} / \mathrm{l}$ & & 1 & $\because$ \\
\hline 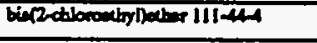 & $<0.010 \mathrm{mg} / \mathrm{f}$ & & $<0.010 \mathrm{mg} / \mathrm{l}$ & & 1 & \\
\hline 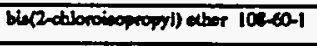 & $<0.010 \mathrm{mg} / \mathrm{l}$ & . & $<0.010 \mathrm{mg} / \mathrm{l}$ & & 1 & \\
\hline 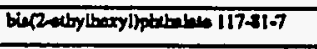 & $<0.010 \mathrm{mg} / \mathrm{l}$ & & $<0.010 \mathrm{mg} / \mathrm{l}$ & & 1 & \\
\hline chrosen 21501-9 & $<0.010 \mathrm{mg} / 1$ & & $<0.010 \mathrm{mg} / \mathrm{l}$ & & 1 & \\
\hline 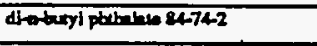 & $<0.010 \mathrm{mg} / \mathrm{l}$ & & $<0.010 \mathrm{mg} / \mathrm{l}$ & & 1 & \\
\hline 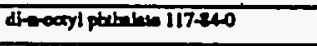 & $<0.010 \mathrm{mg} / 1$ & & $<0.010 \mathrm{mg} / \mathrm{l}$ & & 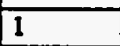 & \\
\hline 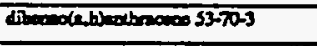 & $<0.010 \mathrm{mg} / 1$ & & $<0.010 \mathrm{mg} / \mathrm{l}$ & & 1 & \\
\hline 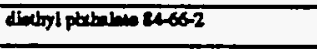 & $<0.010 \mathrm{mg} / \mathrm{l}$ & & $<0.010 \mathrm{mg} / \mathrm{l}$ & & 1 & \\
\hline denotogl pathins 131-11-3 & $<0.010 \mathrm{mg} / \mathrm{l}$ & & $<0.010 \mathrm{mg} / \mathrm{l}$ & & 1 & \\
\hline 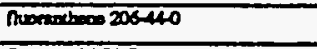 & $<0.010 \mathrm{mg} / 1$ & & $<0.010 \mathrm{mg} / \mathrm{l}$ & & 1 & \\
\hline Dowomise $16-73-7$ & $<0.010 \mathrm{mg} / \mathrm{l}$ & & $<0.010 \mathrm{mg} / \mathrm{l}$ & & 1 & \\
\hline terecthonobecoceos $118-74-1$ & $<0.010 \mathrm{mg} / \mathrm{l}$ & & $<0.010 \mathrm{mg} / \mathrm{l}$ & & 1 & \\
\hline 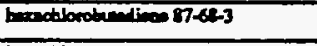 & $<0.010 \mathrm{mg} / \mathrm{l}$ & & $<0.010 \mathrm{mg} / \mathrm{l}$ & & 1 & \\
\hline 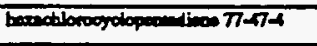 & $<0.010 \mathrm{mg} / \mathrm{l}$ & & $<0.010 \mathrm{mg} / \mathrm{l}$ & & 1 & \\
\hline 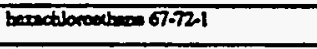 & $<0.010 \mathrm{mg} / \mathrm{l}$ & & $<0.010 \mathrm{mg} / \mathrm{l}$ & & 1 & \\
\hline 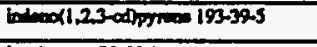 & $<0.010 \mathrm{mg} / 1$ & & $<0.010 \mathrm{mg} / \mathrm{l}$ & & 1 & \\
\hline 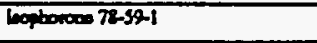 & $<0.010 \mathrm{mg} / 1$ & & $<0.010 \mathrm{mg} / \mathrm{l}$ & & 1 & \\
\hline N-milcosodis-propylemino 621647 & $<0.010 \mathrm{mg} / \mathrm{l}$ & & $<0.010 \mathrm{mg} / \mathrm{l}$ & & 1 & \\
\hline 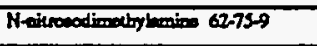 & $<0.010 \mathrm{mg} / \mathrm{l}$ & & $<0.010 \mathrm{mg} / \mathrm{l}$ & & 1 & \\
\hline 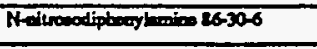 & $<0.010 \mathrm{mg} / \mathrm{l}$ & & $<0.010 \mathrm{mg} / \mathrm{l}$ & & 1 & \\
\hline $91-20-3$ & $<0.010 \mathrm{mg} / \mathrm{l}$ & & $<0.010 \mathrm{mg} / \mathrm{l}$ & & 1 & \\
\hline 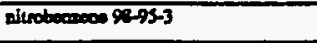 & $<0.010 \mathrm{mg} / \mathrm{l}$ & & $<0.010 \mathrm{mg} / \mathrm{l}$ & & 1 & \\
\hline phonetion 25018 & $<0.010 \mathrm{mg} / \mathrm{l}$ & & $<0.010 \mathrm{mg} / \mathrm{l}$ & & 1 & \\
\hline proos 122000 & $<0.010 \mathrm{mg} / \mathrm{l}$ & & $<0.010 \mathrm{mg} / \mathrm{l}$ & & 1 & \\
\hline 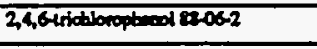 & $<0.030 \mathrm{mg} / \mathrm{l}$ & & $<0.030 \mathrm{mg} / \mathrm{l}$ & & 1 & \\
\hline 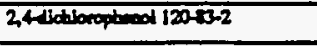 & $<0.030 \mathrm{mg} / 1$ & & $<0.030 \mathrm{mg} / \mathrm{l}$ & & 1 & \\
\hline 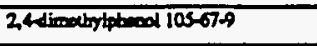 & $<0.030 \mathrm{mg} / \mathrm{t}$ & & $<0.030 \mathrm{mg} / \mathrm{l}$ & & 1 & \\
\hline 24-4initrophood S1-2253 & $<0.030 \mathrm{mg} / \mathrm{h}$ & & $<0.030 \mathrm{mg} / \mathrm{l}$ & & 1 & \\
\hline
\end{tabular}


Outfall S15

Parta B and C Continued

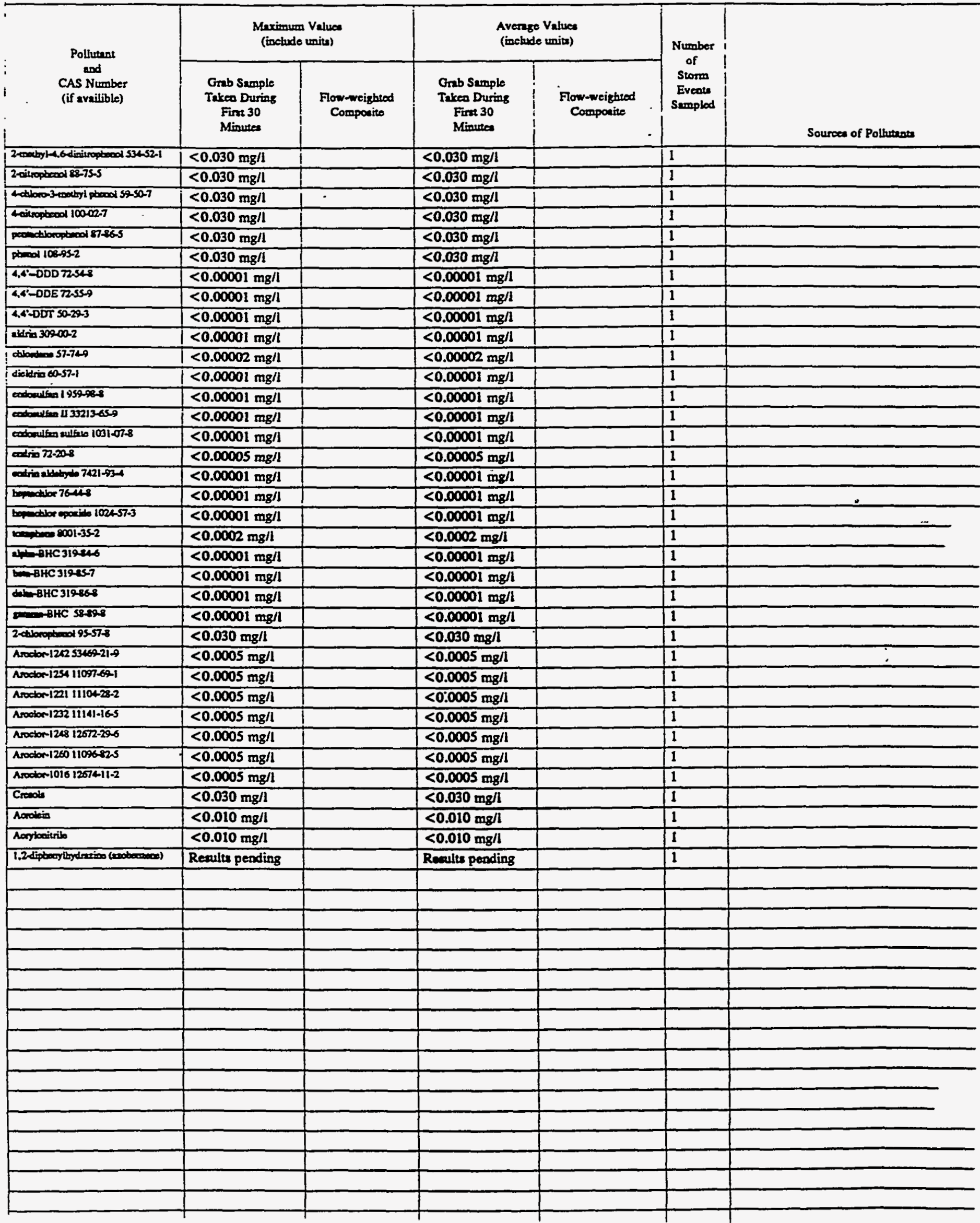

(........ 
VII. Diechargo Lnformution (contiaund from page 3 of Form 2F)

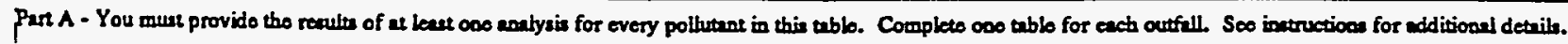

\begin{tabular}{|c|c|c|c|c|c|c|}
\hline \multirow{2}{*}{$\begin{array}{l}\text { Pollutunt } \\
\text { and } \\
\text { CAS Number } \\
\text { (if aviliblo) }\end{array}$} & \multicolumn{2}{|c|}{$\begin{array}{l}\text { Maximum Valuee } \\
\text { (include units) }\end{array}$} & \multicolumn{2}{|c|}{$\begin{array}{l}\text { Averngo Values } \\
\text { (inchude unitu) }\end{array}$} & \multirow{2}{*}{$\begin{array}{l}\text { Number } \\
\text { of } \\
\text { Storm } \\
\text { Evertar } \\
\text { Suropled }\end{array}$} & \multirow[b]{2}{*}{ Sourcose of Polkenen } \\
\hline & $\begin{array}{c}\text { Grab Sample } \\
\text { Takeo During } \\
\text { Fint } 30 \\
\text { Minuma }\end{array}$ & $\begin{array}{l}\text { Frow-woighed } \\
\text { Compotins }\end{array}$ & $\begin{array}{l}\text { Grab Sumplo } \\
\text { Takeo During } \\
\text { Fira } 30 \\
\text { Mineres }\end{array}$ & $\begin{array}{l}\text { Flowiwloighed } \\
\text { Coosposico }\end{array}$ & & \\
\hline pil nod Oromen & <2 mg/l & & $<2 \mathrm{mg} / \mathrm{l}$ & & I & \\
\hline 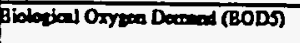 & $6.44 \mathrm{mg} / \mathrm{l}$ & & $6.44 \mathrm{mg} / \mathrm{l}$ & & T & \\
\hline Dricil Oxyseos Dormed (COD) & $20 \mathrm{mg} / \mathrm{l}$ & & $20 \mathrm{mg} / \mathrm{l}$ & & 1 & \\
\hline Toul Sapooded Solide (TSO) & $15 \mathrm{mg} / 1$ & & $15 \mathrm{mg} / \mathrm{l}$ & & I & \\
\hline Toul Kjethedl Nitropon & $0.7 \mathrm{mg} / \mathrm{l}$ & & $0.7 \mathrm{mg} / \mathrm{l}$ & & 1 & \\
\hline Nitrate phen Nitrive Nitroper & $<0.1 \mathrm{mg} / \mathrm{l}$ & & $<0.1 \mathrm{mg} / \mathrm{l}$ & & 1 & \\
\hline Toul Praphone & $<0.1 \mathrm{mg} / 1$ & & $<0.1 \mathrm{mg} / \mathrm{l}$ & & 1 & \\
\hline $\mathrm{H}$ & Minimes 7.5 & Mrimen 7.5 & Minimin 75 & Merimim 7.5 & & \\
\hline
\end{tabular}

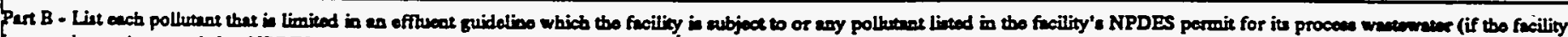

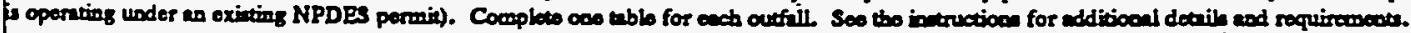

\begin{tabular}{|c|c|c|c|c|c|c|}
\hline \multirow{2}{*}{$\begin{array}{l}\text { Poltumane } \\
\text { and } \\
\text { CAs Numbor } \\
\text { (if availible) }\end{array}$} & \multicolumn{2}{|c|}{$\begin{array}{l}\text { Maximurm Valuven } \\
\text { (inoludo unitu) }\end{array}$} & \multicolumn{2}{|c|}{$\begin{array}{l}\text { Averese Values } \\
\text { (Eoludo unite) }\end{array}$} & \multirow{2}{*}{$\begin{array}{l}\text { Number } \\
\text { of } \\
\text { Storem } \\
\text { Eveoce } \\
\text { Sumplod }\end{array}$} & \multirow{2}{*}{ Sourcen of Polberome } \\
\hline & 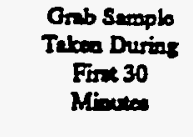 & $\begin{array}{l}\text { Fow-weighted } \\
\text { Comporive }\end{array}$ & 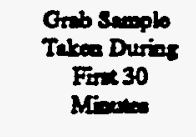 & $\begin{array}{l}\text { Fow-wreifted } \\
\text { Compotice }\end{array}$ & & \\
\hline teiminosy $740-360$ & $<0.003 \mathrm{mg} / \mathrm{l}$ & & $<0.003 \mathrm{mg} / \mathrm{l}$ & & 1 & \\
\hline Ondente & $<0.002 \mathrm{mg} / 1$ & & $<0.002 \mathrm{mg} / \mathrm{l}$ & & 1 & \\
\hline Himang 743907.6 & $<0.0002 \mathrm{mg} / 1$ & & $<0.0002 \mathrm{mg} / \mathrm{l}$ & & $I$ & \\
\hline Theode & $0.001 \mathrm{mg} / \mathrm{l}$ & & $0.001 \mathrm{mg} / \mathrm{l}$ & & 1 & $\bar{?}$ \\
\hline 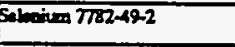 & $<0.002 \mathrm{mg} / 1$ & & $<0.002 \mathrm{mg} / \mathrm{l}$ & & $I$ & \\
\hline sulfis & $50 \mathrm{mg} / \mathrm{l}$ & " & $50 \mathrm{mg} / \mathrm{l}$ & & 1 & \\
\hline perfmoveres & $<0.05 \mathrm{mg} / \mathrm{l}$ & & $<0.05 \mathrm{mg} / 1$ & & 1 & \\
\hline Themenom 7429-90-5 & $0.23 \mathrm{mg} / \mathrm{l}$ & & $0.23 \mathrm{mg} / \mathrm{l}$ & & 1 & \\
\hline $7400-38-2$ & $<0.04 \mathrm{mg} / \mathrm{l}$ & & $<0.04 \mathrm{mg} / \mathrm{l}$ & & 1 & \\
\hline $3000007400-125$ & $0.016 \mathrm{mg} / \mathrm{l}$ & & $0.016 \mathrm{mg} / \mathrm{l}$ & & 1 & \\
\hline Berpllinen 740-11-7 & $<0.0004 \mathrm{mg} / \mathrm{l}$ & & $<0.0004 \mathrm{mg} / \mathrm{l}$ & & $i$ & \\
\hline 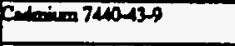 & $<0.004 \mathrm{mg} / 1$ & & $<0.004 \mathrm{mg} / \mathrm{l}$ & & $I$ & \\
\hline Feldom 7 ina-xa-2 & $11.8 \mathrm{mg} / \mathrm{l}$ & & $11.8 \mathrm{mg} / 1$ & & 1 & \\
\hline Dranion 740-17.3 & $<0.006 \mathrm{mg} / 1$ & & $<0.006 \mathrm{mg} / \mathrm{l}$ & & 1 & \\
\hline 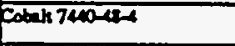 & $<0.002 \mathrm{mg} / 1$ & & $<0.002 \mathrm{mg} / 1$ & & 1 & \\
\hline Eopper 7440-50-8 & $<0.006 \mathrm{mg} / \mathrm{l}$ & & $<0.006 \mathrm{mg} / \mathrm{l}$ & & 1 & \\
\hline 8007439896 & $0.49 \mathrm{mg} / \mathrm{h}$ & & $0.49 \mathrm{mg} / \mathrm{l}$ & & 1 & \\
\hline $\cos 7439-2-1$ & $<0.02 \mathrm{mg} / \mathrm{l}$ & & $<0.02 \mathrm{mg} / \mathrm{l}$ & & 1 & \\
\hline Tistiven 7439-93-2 & $<0.02 \mathrm{mg} / \mathrm{l}$ & & $<0.02 \mathrm{mg} / \mathrm{l}$ & & 1 & \\
\hline Mepomikn 7439-95-4 & $1.99 \mathrm{mg} / \mathrm{l}$ & & $1.99 \mathrm{mg} / \mathrm{l}$ & & 1 & \\
\hline 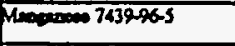 & $0.463 \mathrm{mg} / \mathrm{l}$ & & $0.463 \mathrm{mg} / \mathrm{l}$ & & 1 & \\
\hline 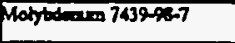 & $<0.006 \mathrm{mg} / \mathrm{l}$ & & $<0.006 \mathrm{mg} / \mathrm{l}$ & & 1 & \\
\hline 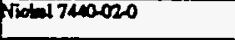 & $<0.008 \mathrm{mg} / \mathrm{l}$ & & $<0.008 \mathrm{mg} / \mathrm{l}$ & & 1 & \\
\hline 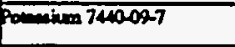 & $4.2 \mathrm{mg} / 1$ & & $4.2 \mathrm{mg} / 1$ & & $I$ & \\
\hline sinem $740-2 a 4$ & $<0.006 \mathrm{mg} / \mathrm{l}$ & & $<0.006 \mathrm{mg} / \mathrm{l}$ & & 1 & \\
\hline Botien 740-20.5 & $0.33 \mathrm{mg} / \mathrm{l}$ & & $0.33 \mathrm{mg} / \mathrm{l}$ & & 1 & \\
\hline 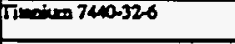 & $<0.01 \mathrm{mg} / \mathrm{l}$ & & $<0.01 \mathrm{mg} / \mathrm{l}$ & & 1 & \\
\hline 21007440666 & $0.07 \mathrm{mg} / \mathrm{l}$ & & $0.07 \mathrm{mg} / \mathrm{l}$ & & 1 & \\
\hline
\end{tabular}


Conlinued from front.

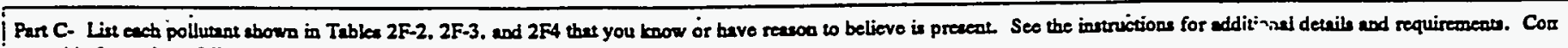
one table for cach outfall.

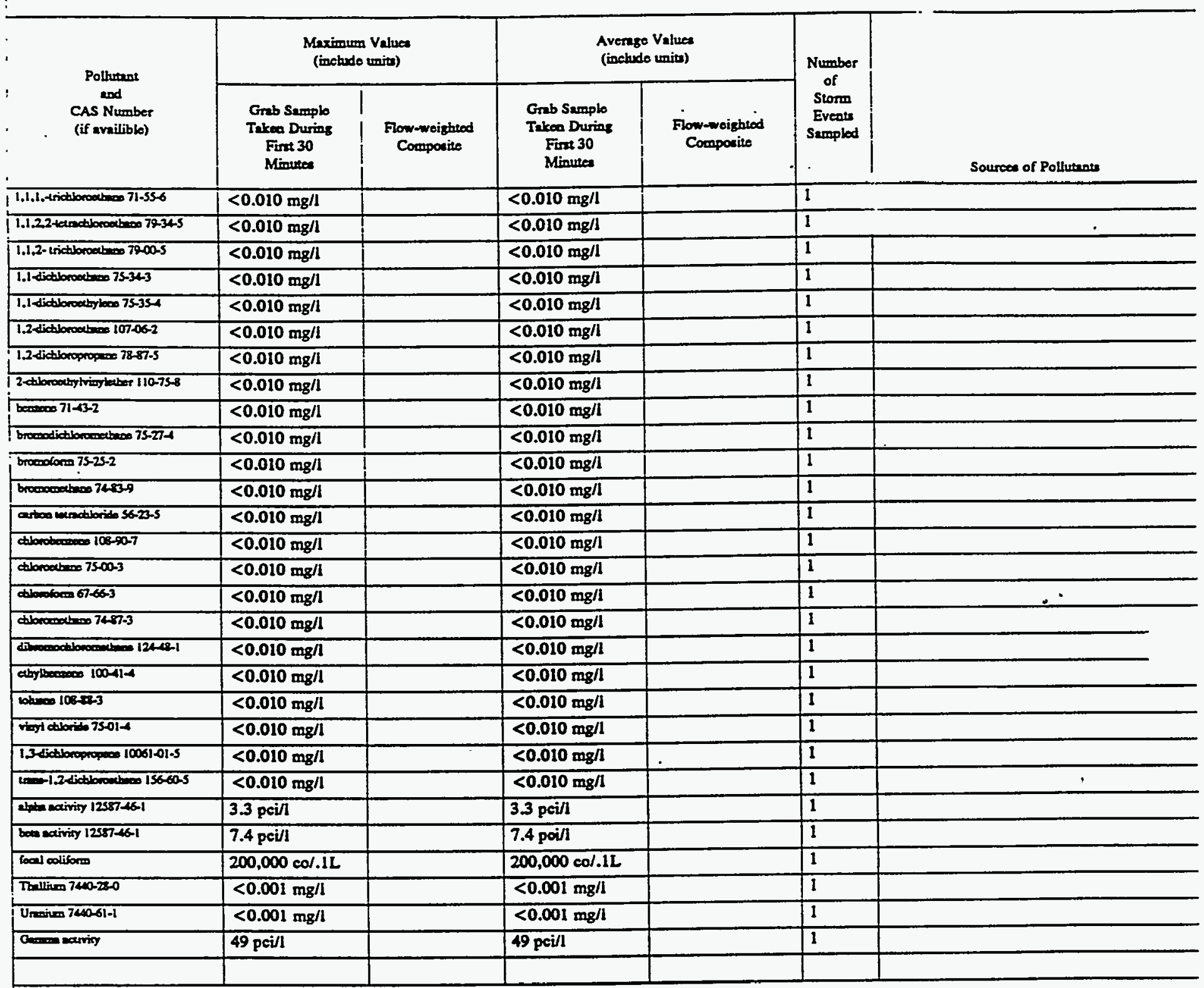

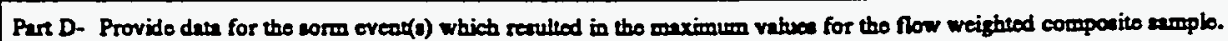

\begin{tabular}{|c|c|c|c|c|c|c|c|}
\hline $\begin{array}{l}1 . \\
\text { Dato of } \\
\text { Storm } \\
\text { Event }\end{array}$ & $\begin{array}{l}2 . \\
\text { Durition } \\
\text { of Stomm } \\
\text { (in } \\
\text { minutea) }\end{array}$ & $\begin{array}{c}3 . \\
\text { Total minfall } \\
\text { during storm eveat } \\
\text { (in incow) }\end{array}$ & 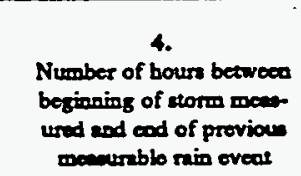 & $\begin{array}{l}\text { S. } \\
\text { Meximus flow now } \\
\text { durins nim eveot } \\
\text { (alloos/min or specify } \\
\text { usitu) }\end{array}$ & $\begin{array}{l}6 . \\
\text { Total flow from } \\
\text { rin eveat } \\
\text { (pallow or } \\
\text { ppecify unita) }\end{array}$ & $\begin{array}{c}7 . \\
\text { Season } \\
\text { ample was } \\
\text { tokes }\end{array}$ & $\begin{array}{l}8 . \\
\text { I Form of } \\
\text { Procipitation } \\
\text { (nimfall. } \\
\text { comment) }\end{array}$ \\
\hline $\begin{array}{l}10 / 14 / 91-1 \\
0 / 15 / 91\end{array}$ & 650 & 1.5 & 226.5 & & & Fall & Rainfull \\
\hline
\end{tabular}

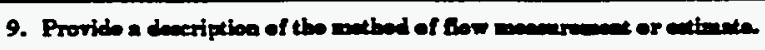

An ISCO 3230 Flow meter wes used to obtain water level readings. This instrument was calibrated with the normal dry weather flow, if present, as leved zero. The flow meter was programmed with the characteristics of the conveyane so that flow rates and total llows could be calculated by the meter. 
Outfall S16

Partu B end C Continued

\begin{tabular}{|c|c|c|c|c|c|c|}
\hline \multirow{2}{*}{$\begin{array}{l}\text { Pollutans } \\
\text { ond } \\
\text { CAS Number } \\
\text { (if availible) }\end{array}$} & \multicolumn{2}{|c|}{$\begin{array}{l}\text { Maximum Valueas } \\
\text { (inciude tunita) }\end{array}$} & \multicolumn{2}{|c|}{$\begin{array}{l}\text { Avorago Values } \\
\text { (inchudo univ) }\end{array}$} & \multirow{2}{*}{$\begin{array}{l}\text { Number } \\
\text { of } \\
\text { Siorm } \\
\text { Events } \\
\text { Seropled }\end{array}$} & \multirow[b]{2}{*}{ Sources of Pollutuats } \\
\hline & $\begin{array}{l}\text { Grab Semple } \\
\text { Taken During } \\
\text { Fint } 30 \\
\text { Mroutes }\end{array}$ & $\begin{array}{l}\text { Flow-weighted } \\
\text { Composite }\end{array}$ & $\begin{array}{c}\text { Greb Ssmplo } \\
\text { Taken During } \\
\text { Fint } 30 \\
\text { Minuteo }\end{array}$ & $\begin{array}{l}\text { Fow-weighrod } \\
\text { Composite }\end{array}$ & & \\
\hline Bariven 7400-39-3 & $0.0178 \mathrm{mg} / \mathrm{l}$ & & $0.0178 \mathrm{mg} / \mathrm{l}$ & & 1 & \\
\hline 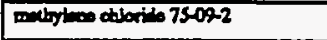 & $<0.010 \mathrm{mg} / \mathrm{l}$ & & $<0.010 \mathrm{mg} / \mathrm{l}$ & & 1 & \\
\hline 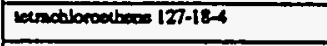 & $<0.010 \mathrm{mg} / \mathrm{l}$ & & $<0.010 \mathrm{mg} / \mathrm{l}$ & & 1 & \\
\hline inditionocabeos 7901.6 & $<0.010 \mathrm{mg} / \mathrm{l}$ & & $<0.010 \mathrm{mg} / \mathrm{l}$ & & 1 & \\
\hline \begin{tabular}{|l|} 
Xyloomen \\
\end{tabular} & $<0.010 \mathrm{mg} / \mathrm{l}$ & & $<0.010 \mathrm{mg} / \mathrm{l}$ & & 1 & \\
\hline 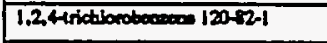 & $<0.010 \mathrm{mg} / \mathrm{h}$ & & $<0.010 \mathrm{mg} / \mathrm{l}$ & & 1 & \\
\hline 1,2 dictbonoberimose $95-50-1$ & $<0.010 \mathrm{mg} / \mathrm{l}$ & & $<0.010 \mathrm{mg} / 1$ & & 1 & \\
\hline 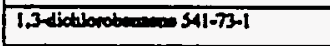 & $<0.010 \mathrm{mg} / \mathrm{I}$ & & $<0.010 \mathrm{mg} / 1$ & & 1 & \\
\hline 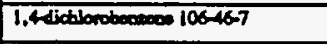 & $<0.010 \mathrm{mg} / \mathrm{l}$ & & $<0.010 \mathrm{mg} / 1$ & & 1 & \\
\hline 2,4dinisolotures $121-14-2$ & $<0.010 \mathrm{mg} / \mathrm{l}$ & & $<0.010 \mathrm{mg} / 1$ & & 1 & \\
\hline 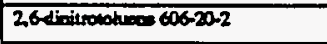 & $<0.010 \mathrm{mg} / \mathrm{h}$ & & $<0.010 \mathrm{mg} / 1$ & & 1 & \\
\hline 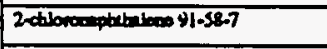 & $<0.010 \mathrm{mg} / \mathrm{l}$ & & $<0.010 \mathrm{mg} / \mathrm{l}$ & & 1 & \\
\hline 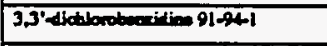 & $<0.010 \mathrm{mg} / 1$ & & $<0.010 \mathrm{mg} / \mathrm{l}$ & & 1 & \\
\hline 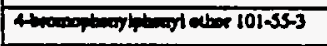 & $<0.010 \mathrm{mg} / \mathrm{l}$ & & $<0.010 \mathrm{mg} / \mathrm{l}$ & & 1 & \\
\hline 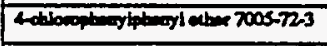 & $<0.010 \mathrm{mg} / 1$ & & $<0.010 \mathrm{mg} / \mathrm{l}$ & & 1 & \\
\hline 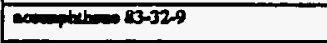 & $<0.010 \mathrm{mg} / \mathrm{ll}$ & & $<0.010 \mathrm{mg} / 1$ & & 1 & \\
\hline 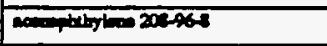 & $<0.010 \mathrm{mg} / 1$ & & $<0.010 \mathrm{mg} / \mathrm{l}$ & & 1 & \\
\hline 120.127 & $<0.010 \mathrm{mg} / \mathrm{l}$ & & $<0.010 \mathrm{mg} / \mathrm{l}$ & & 1 & \\
\hline rileg27.5 & $<0.030 \mathrm{mg} / \mathrm{l}$ & & $<0.030 \mathrm{mg} / \mathrm{l}$ & & 1 & \\
\hline 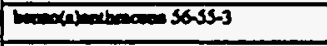 & $<0.010 \mathrm{mg} / \mathrm{l}$ & & $<0.010 \mathrm{mg} / 1$ & & 1 & \\
\hline 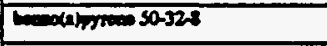 & $<0.010 \mathrm{mg} / \mathrm{l}$ & & $<0.010 \mathrm{mg} / \mathrm{l}$ & & 1 & \\
\hline 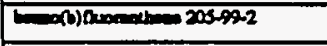 & $<0.010 \mathrm{mg} / \mathrm{l}$ & & $<0.010 \mathrm{mg} / 1$ & & 1 & \\
\hline 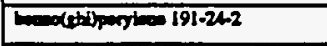 & $<0.010 \mathrm{mg} / \mathrm{l}$ & & $<0.010 \mathrm{mg} / \mathrm{l}$ & & 1 & \\
\hline $207-0 \mathrm{ag}$ & $<0.010 \mathrm{mg} / \mathrm{l}$ & & $<0.010 \mathrm{mg} / 1$ & & 1 & \\
\hline 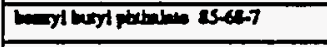 & $<0.010 \mathrm{mg} / 1$ & & $<0.010 \mathrm{mg} / \mathrm{h}$ & & 1 & \\
\hline 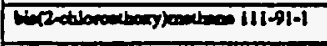 & $<0.010 \mathrm{mg} / \mathrm{l}$ & & $<0.010 \mathrm{mg} / \mathrm{l}$ & & 1 & $\cdot$ \\
\hline 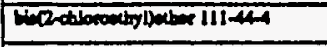 & $<0.010 \mathrm{mg} / \mathrm{l}$ & & $<0.010 \mathrm{mg} / 1$ & & 1 & \\
\hline 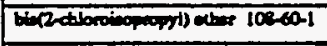 & $<0.010 \mathrm{mg} / \mathrm{l}$ & $\dot{.}$ & $<0.010 \mathrm{mg} / 1$ & & 1 & \\
\hline 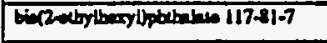 & $<0.010 \mathrm{mg} / \mathrm{l}$ & & $<0.010 \mathrm{mg} / \mathrm{l}$ & & 1 & \\
\hline cromes 21201A & $<0.010 \mathrm{mg} / \mathrm{l}$ & & $<0.010 \mathrm{mg} / 1$ & & 1 & \\
\hline draturyl pectelne 8474-2 & $<0.010 \mathrm{mg} / \mathrm{l}$ & & $<0.010 \mathrm{mg} / 1$ & & 1 & \\
\hline 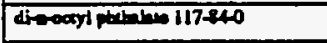 & $<0.010 \mathrm{mg} / \mathrm{l}$ & & $<0.010 \mathrm{mg} / 1$ & & 1 & \\
\hline 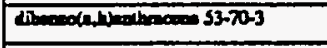 & $<0.010 \mathrm{mg} / \mathrm{l}$ & & $<0.010 \mathrm{mg} / \mathrm{l}$ & & 1 & \\
\hline 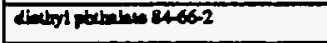 & $<0.010 \mathrm{mg} / \mathrm{l}$ & & $<0.010 \mathrm{mg} / 1$ & & 1 & \\
\hline 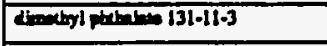 & $<0.010 \mathrm{mg} / \mathrm{l}$ & & $<0.010 \mathrm{mg} / \mathrm{l}$ & & 1 & \\
\hline Theocentheon $206-140$ & $<0.010 \mathrm{mg} / \mathrm{l}$ & & $<0.010 \mathrm{mg} / 1$ & & 1 & \\
\hline $26-73-7$ & $<0.010 \mathrm{mg} / \mathrm{t}$ & & $<0.010 \mathrm{mg} / 1$ & & 1 & \\
\hline 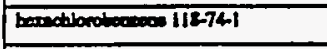 & $<0.010 \mathrm{mg} / \mathrm{l}$ & & $<0.010 \mathrm{mg} / 1$ & & 1 & \\
\hline 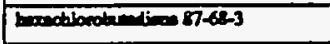 & $<0.010 \mathrm{mg} / \mathrm{l}$ & & $<0.010 \mathrm{mg} / 1$ & & 1 & \\
\hline 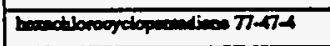 & $<0.010 \mathrm{mg} / 1$ & & $<0.010 \mathrm{mg} / 1$ & & 1 & \\
\hline 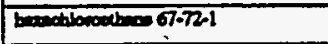 & $<0.010 \mathrm{mg} / 1$ & & $<0.010 \mathrm{mg} / 1$ & & 1 & \\
\hline 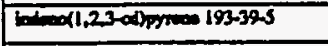 & $<0.010 \mathrm{mg} / \mathrm{l}$ & & $<0.010 \mathrm{mg} / 1$ & & 1 & \\
\hline Eoplowoen $7259-1$ & $<0.010 \mathrm{mg} / \mathrm{l}$ & & $<0.010 \mathrm{mg} / \mathrm{l}$ & & 1 & \\
\hline 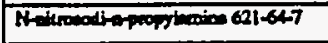 & $<0.010 \mathrm{mg} / 1$ & & $<0.010 \mathrm{mg} / \mathrm{l}$ & & 1 & \\
\hline 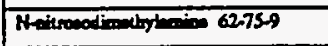 & $<0.010 \mathrm{mg} / 1$ & & $<0.010 \mathrm{mg} / 1$ & & 1 & \\
\hline 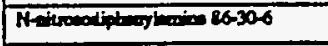 & $<0.010 \mathrm{mg} / \mathrm{l}$ & & $<0.010 \mathrm{mg} / \mathrm{l}$ & & 1 & \\
\hline $2-1$ theos $91-20-3$ & $<0.010 \mathrm{mg} / \mathrm{l}$ & & $<0.010 \mathrm{mg} / 1$ & & 1 & \\
\hline 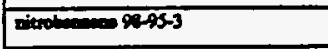 & $<0.010 \mathrm{mg} / \mathrm{l}$ & & $<0.010 \mathrm{mg} / \mathrm{l}$ & & 1 & \\
\hline 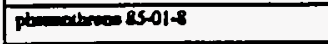 & $<0.010 \mathrm{mg} / 1$ & & $<0.010 \mathrm{mg} / \mathrm{h}$ & & 1 & \\
\hline 129000000 & $<0.010 \mathrm{mg} / \mathrm{I}$ & & $<0.010 \mathrm{mg} / \mathrm{l}$ & & 1 & \\
\hline 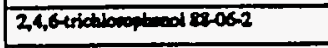 & $<0.030 \mathrm{mg} / \mathrm{l}$ & & $<0.030 \mathrm{mg} / 1$ & & 1 & \\
\hline 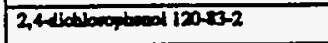 & $<0.030 \mathrm{mg} / \mathrm{l}$ & & $<0.030 \mathrm{mg} / \mathrm{l}$ & & 1 & \\
\hline 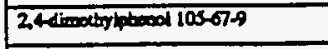 & $<0.030 \mathrm{mg} / \mathrm{l}$ & & $<0.030 \mathrm{mg} / \mathrm{l}$ & & 1 & \\
\hline 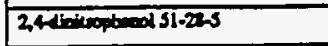 & $<0.030 \mathrm{mg} / \mathrm{l}$ & & $<0.030 \mathrm{mg} / 1$ & & 1 & \\
\hline
\end{tabular}


Outfall S16

Perta B and C Continuod

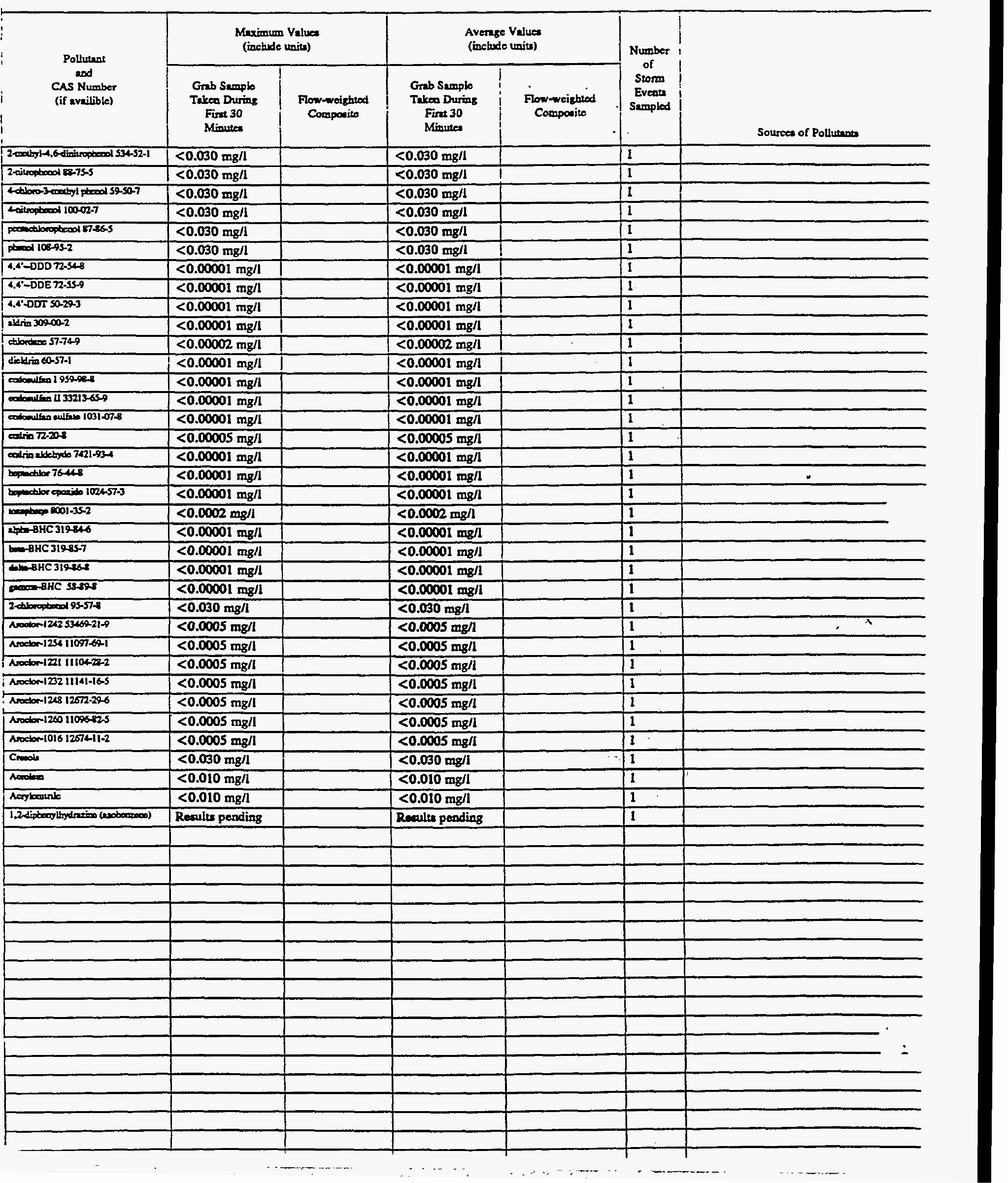




\begin{tabular}{|c|c|c|c|c|c|c|}
\hline \multirow{2}{*}{$\begin{array}{l}\text { Pollutent } \\
\text { and } \\
\text { CAS Number } \\
\text { (if availible) }\end{array}$} & \multicolumn{2}{|c|}{$\begin{array}{l}\text { Meximum Values } \\
\text { (isectude unite) }\end{array}$} & \multicolumn{2}{|c|}{$\begin{array}{l}\text { Average Valuea } \\
\text { (include units) }\end{array}$} & \multirow{2}{*}{$\begin{array}{c}\text { Number } \\
\text { of } \\
\text { Storm } \\
\text { Event } \\
\text { Sempled }\end{array}$} & \multirow[b]{2}{*}{ Sources of Polturentes } \\
\hline & $\begin{array}{l}\text { Grab Semple } \\
\text { Taken During } \\
\text { Firn } 30 \\
\text { Minuteo }\end{array}$ & $\begin{array}{l}\text { Flow weighted } \\
\text { Comporite }\end{array}$ & $\begin{array}{l}\text { Grab Semple } \\
\text { Trken During } \\
\text { Fint } 30 \\
\text { Minurea }\end{array}$ & $\begin{array}{l}\text { Fow-weightod } \\
\text { Componito }\end{array}$ & & \\
\hline oil and Greane & $<2 \mathrm{mg} / \mathrm{l}$ & & $<2 \mathrm{mg} / \mathrm{l}$ & & 1 & \\
\hline Pioberical Oxy & $<5 \mathrm{mg} / \mathrm{l}$ & $<5 m g / l$ & $<5 \mathrm{mg} / \mathrm{l}$ & $<5 \mathrm{mg} / 1$ & 1 & \\
\hline 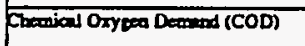 & $6.1 \mathrm{mg} / 1$ & $7.9 \mathrm{mg} / \mathrm{l}$ & $6.1 \mathrm{mg} / 1$ & $7.9 \mathrm{mg} / \mathrm{l}$ & 1 & \\
\hline Toeal Suppoeded Solide (TSS) & $<5 \mathrm{mg} / \mathrm{l}$ & $5 \mathrm{mg} / 1$ & $<5 \mathrm{mg} / \mathrm{l}$ & $5 \mathrm{mg} / \mathrm{l}$ & 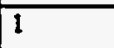 & \\
\hline Toul Kjeldeal Nitrosen & $0.2 \mathrm{mg} / \mathrm{l}$ & $0.21 \mathrm{mg} / \mathrm{l}$ & $0.2 \mathrm{mg} / \mathrm{l}$ & $0.21 \mathrm{mg} / \mathrm{l}$ & 1 & \\
\hline Nitnes plue Nitrive Nitrogea & $1.6 \mathrm{mg} / \mathrm{l}$ & $0.36 \mathrm{mg} / \mathrm{l}$ & $1.6 \mathrm{mg} / \mathrm{l}$ & $0.36 \mathrm{mg} / \mathrm{l}$ & 1 & \\
\hline Toual Prosphorne & $<0.1 \mathrm{mg} / \mathrm{l}$ & $<0.1 \mathrm{mg} / \mathrm{l}$ & $<0.1 \mathrm{mg} / \mathrm{l}$ & $<0.1 \mathrm{mg} / \mathrm{l}$ & 1 & \\
\hline pH & Mishers 82 & Merimen 8.2 & Minims 8.2 & Meximen 8.2 & - & \\
\hline
\end{tabular}

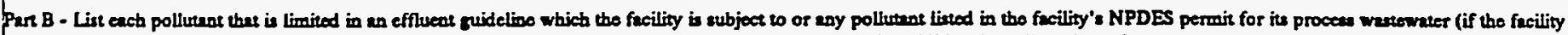

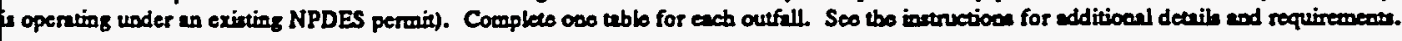

\begin{tabular}{|c|c|c|c|c|c|c|}
\hline \multirow{2}{*}{$\begin{array}{l}\text { Polletenat } \\
\text { and } \\
\text { CAs Number } \\
\text { (if availible) }\end{array}$} & \multicolumn{2}{|c|}{$\begin{array}{l}\text { Maximum Valuce } \\
\text { (inchedo univa) }\end{array}$} & \multicolumn{2}{|c|}{$\begin{array}{l}\text { Average Values } \\
\text { (inchede univs) }\end{array}$} & \multirow{2}{*}{$\begin{array}{l}\text { Numbers } \\
\text { of } \\
\text { Storm } \\
\text { Eveoti } \\
\text { Sempled }\end{array}$} & \multirow{2}{*}{$\begin{array}{c}: \\
\text { Sourees of Pollutunt }\end{array}$} \\
\hline & $\begin{array}{l}\text { Grab Semplo } \\
\text { Takes During } \\
\text { First } 30 \\
\text { Misutea }\end{array}$ & $\begin{array}{l}\text { Flow-weightod } \\
\text { Compotive }\end{array}$ & $\begin{array}{l}\text { Grab Semplo } \\
\text { Takes During } \\
\text { Fint } 30 \\
\text { Minued }\end{array}$ & $\begin{array}{l}\text { Flow-weighted } \\
\text { Comporits }\end{array}$ & & \\
\hline Trianod 7400.360 & $<0.04 \mathrm{mg} / \mathrm{l}$ & $<0.01 \mathrm{mg} / \mathrm{l}$ & $<0.04 \mathrm{mg} / \mathrm{l}$ & $<0.01 \mathrm{mg} / \mathrm{l}$ & 1 & \\
\hline pyando & $<0.002 \mathrm{mg} / \mathrm{l}$ & & $<0.002 \mathrm{mg} / \mathrm{l}$ & & 1 & \\
\hline Matary 743907.6 & $<0.0002 \mathrm{mg} / \mathrm{l}$ & $<0.0002 \mathrm{mg} / \mathrm{l}$ & $<0.0002 \mathrm{mg} / 1^{\circ}$ & $<0.0002 \mathrm{mg} / \mathrm{l}$ & 1 & \\
\hline phen & $0.002 \mathrm{mg} / \mathrm{l}$ & & $0.002 \mathrm{mg} / \mathrm{l}$ & & 1 & . \\
\hline Solenthen $7782-492$ & $<0.002 \mathrm{mg} / \mathrm{l}$ & $<0.002 \mathrm{mg} / \mathrm{l}$ & $<0.002 \mathrm{mg} / \mathrm{l}$ & $<0.002 \mathrm{mg} / \mathrm{l}$ & 1 & \\
\hline Pulfete & $<10 \mathrm{mg} / \mathrm{l}$ & $<10 \mathrm{mg} / \mathrm{l}$ & $<10 \mathrm{mg} / \mathrm{l}$ & $<10 \mathrm{mg} / \mathrm{l}$ & 1 & \\
\hline artrowentes & $<0.05 \mathrm{mg} / \mathrm{l}$ & $<0.05 \mathrm{mg} / \mathrm{l}$ & $<0.05 \mathrm{mg} / \mathrm{l}$ & $<0.05 \mathrm{mg} / \mathrm{l}$ & 1 & \\
\hline Alenisen 742900-5 & $0.08 \mathrm{mg} / \mathrm{l}$ & $0.14 \mathrm{mg} / \mathrm{l}$ & $0.08 \mathrm{mg} / \mathrm{l}$ & $0.14 \mathrm{mg} / \mathrm{l}$ & 1 & \\
\hline $4 \pi 00007440-38.2$ & $<0.04 \mathrm{mg} / \mathrm{l}$ & $<0.04 \mathrm{mg} / \mathrm{l}$ & $<0.04 \mathrm{mg} / \mathrm{l}$ & $<0.04 \mathrm{mg} / 1$ & 1 & \\
\hline Bow00 7400-28 & $0.020 \mathrm{mg} / \mathrm{l}$ & $0.016 \mathrm{mg} / \mathrm{l}$ & $0.020 \mathrm{mg} / 1$ & $0.016 \mathrm{mg} / \mathrm{l}$ & 1 & \\
\hline Berpllium 740-1.7 & $<0.0004 \mathrm{mg} / \mathrm{l}$ & $<0.0004 \mathrm{mg} / \mathrm{l}$ & $<0.0004 \mathrm{mg} / \mathrm{l}$ & $<0.0004 \mathrm{mg} / \mathrm{l}$ & 1 & \\
\hline admium 740-139-9 & $<0.004 \mathrm{mg} / \mathrm{l}$ & $<0.004 \mathrm{mg} / \mathrm{l}$ & $<0.004 \mathrm{mg} / \mathrm{l}$ & $<0.004 \mathrm{mg} / \mathrm{l}$ & 1 & \\
\hline Calcium $740-702-2$ & $34.7 \mathrm{mg} / \mathrm{l}$ & $41.6 \mathrm{mg} / \mathrm{l}$ & $34.7 \mathrm{mg} / \mathrm{l}$ & $41.6 \mathrm{mg} / \mathrm{l}$ & 1 & \\
\hline Chromiven 744a-7.3 & $<0.006 \mathrm{mg} / \mathrm{l}$ & $0.007 \mathrm{mg} / \mathrm{l}$ & $<0.006 \mathrm{mg} / \mathrm{l}$ & $0.007 \mathrm{mg} / \mathrm{l}$ & 1 & \\
\hline Cobalt 740-18-4 & $<0.002 \mathrm{mg} / \mathrm{l}$ & $<0.002 \mathrm{mg} / \mathrm{l}$ & $<0.002 \mathrm{mg} / \mathrm{l}$ & $<0.002 \mathrm{mg} / \mathrm{l}$ & 1 & \\
\hline Coppor 7400-50-8 & $<0.006 \mathrm{mg} / \mathrm{l}$ & $<0.006 \mathrm{mg} / \mathrm{l}$ & $<0.006 \mathrm{mg} / \mathrm{l}$ & $<0.006 \mathrm{mg} / \mathrm{l}$ & 1 & \\
\hline 10007439896 & $0.09 \mathrm{mg} / \mathrm{l}$ & $0.26 \mathrm{mg} / \mathrm{l}$ & $0.09 \mathrm{mg} / 1$ & $0.26 \mathrm{mg} / \mathrm{l}$ & 1 & \\
\hline Fead 7439-92-1 & $<0.02 \mathrm{mg} / 1$ & $<0.02 \mathrm{mg} / \mathrm{l}$ & $<0.02 \mathrm{mg} / 1$ & $<0.02 \mathrm{mg} / \mathrm{l}$ & 1 & \\
\hline Fibium 7439-93-2 & $0.09 \mathrm{mg} / \mathrm{l}$ & $0.41 \mathrm{mg} / \mathrm{l}$ & $0.09 \mathrm{mg} / \mathrm{l}$ & $0.41 \mathrm{mg} / \mathrm{l}$ & 1 & \\
\hline 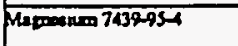 & $13.5 \mathrm{mg} / \mathrm{l}$ & $15.3 \mathrm{mg} / \mathrm{l}$ & $13.5 \mathrm{mg} /$ & $15.3 \mathrm{mg} / \mathrm{l}$ & 1 & \\
\hline Menomone $743996-5$ & $0.027 \mathrm{mg} / 1$ & $0.086 \mathrm{mg} / \mathrm{l}$ & $0.027 \mathrm{mg} / 1$ & $0.086 \mathrm{mg} / \mathrm{l}$ & 1 & \\
\hline Molybdeaur 7439-98-7 & $<0.006 \mathrm{mg} / \mathrm{l}$ & $<0.006 \mathrm{mg} / \mathrm{l}$ & $<0.006 \mathrm{mg} / \mathrm{l}$ & $<0.006 \mathrm{mg} / \mathrm{l}$ & 1 & \\
\hline Nibelel 7400000 & $<0.008 \mathrm{mg} / \mathrm{l}$ & $<0.008 \mathrm{mg} / \mathrm{l}$ & $<0.008 \mathrm{mg} / \mathrm{l}$ & $<0.008 \mathrm{mg} / \mathrm{l}$ & 1 & \\
\hline Pomenorus 7400097 & $1.3 \mathrm{mg} / \mathrm{l}$ & $1.3 \mathrm{mg} / 1$ & $1.3 \mathrm{mg} / 1$ & $1.3 \mathrm{mg} / 1$ & 1 & \\
\hline Sither 740-22-4 & $<0.006 \mathrm{mg} / \mathrm{l}$ & $<0.006 \mathrm{mg} / \mathrm{l}$ & $<0.006 \mathrm{mg} / \mathrm{l}$ & $<0.006 \mathrm{mg} / \mathrm{l}$ & 1 & \\
\hline sodinan 740-23-3 & $0.95 \mathrm{mg} / \mathrm{l}$ & $0.94 \mathrm{mg} / \mathrm{l}$ & $0.95 \mathrm{mg} / \mathrm{l}$ & $0.94 \mathrm{mg} / \mathrm{l}$ & 1 & \\
\hline Titeminas 7440-32-6 & $<0.01 \mathrm{mg} / \mathrm{l}$ & $<0.02 \mathrm{mg} / \mathrm{l}$ & $<0.01 \mathrm{mg} / 1$ & $<0.02 \mathrm{mg} / 1$ & 1 & \\
\hline 2100740666 & $<0.01 \mathrm{mg} / \mathrm{h}$ & $0.01 \mathrm{mg} / \mathrm{l}$ & $<0.01 \mathrm{mg} / \mathrm{l}$ & $0.01 \mathrm{mg} / \mathrm{l}$ & 1 & \\
\hline
\end{tabular}


Continued from frove.

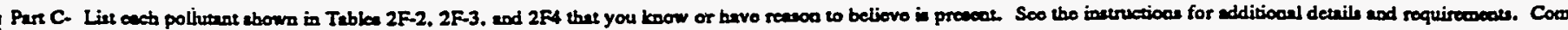
ono teble for each outrall.

\begin{tabular}{|c|c|c|c|c|c|c|}
\hline \multirow{2}{*}{$\begin{array}{c}\text { Pollutunt } \\
\text { and } \\
\text { CAS Number } \\
\text { (if availible) }\end{array}$} & \multicolumn{2}{|c|}{$\begin{array}{l}\text { Muximum Values } \\
\text { (inchade univ) }\end{array}$} & \multicolumn{2}{|c|}{$\begin{array}{l}\text { Average Values } \\
\text { (include units) }\end{array}$} & \multirow{2}{*}{$\begin{array}{l}\text { Number } \\
\text { of } \\
\text { Storm } \\
\text { Evente } \\
\text { Sampled }\end{array}$} & \multirow[b]{2}{*}{ Sources of Poltutents } \\
\hline & $\begin{array}{c}\text { Grab Sumple } \\
\text { Taken Durims } \\
\text { Fint } 30 \\
\text { Minitea }\end{array}$ & $\begin{array}{l}\text { Flow-weighted } \\
\text { Componite }\end{array}$ & $\begin{array}{c}\text { Grab Sample } \\
\text { Taken During } \\
\text { Firat } 30 \\
\text { Minuted }\end{array}$ & $\begin{array}{l}\text { Flow-weighted } \\
\text { Comporite }\end{array}$ & & \\
\hline 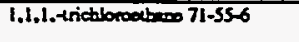 & $<0.010 \mathrm{mg} / \mathrm{l}$ & & $<0.010 \mathrm{mg} / 1$ & & 1 & \\
\hline 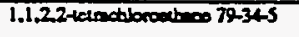 & $<0.010 \mathrm{mg} / \mathrm{l}$ & & $<0.010 \mathrm{mg} / \mathrm{l}$ & & 1 & \\
\hline 1.1 .2 - trithbreocheos $7900-5$ & $<0.010 \mathrm{mg} / \mathrm{l}$ & & $<0.010 \mathrm{mg} / \mathrm{l}$ & & 1 & \\
\hline 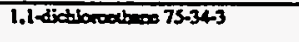 & $<0.010 \mathrm{mg} / \mathrm{l}$ & & $<0.010 \mathrm{mg} / 1$ & & 1 & \\
\hline 1.1 dictibroothyloces 75-35-4 & $<0.010 \mathrm{mg} / \mathrm{l}$ & & $<0.010 \mathrm{mg} / \mathrm{l}$ & & $I$ & \\
\hline 1.2 dieblorecthen $10706-2$ & $<0.010 \mathrm{mg} / \mathrm{l}$ & & $<0.010 \mathrm{mg} / \mathrm{l}$ & & 1 & \\
\hline 1.2 dictioropropenos 78.87 .5 & $<0.010 \mathrm{mg} / 1$ & & $<0.010 \mathrm{mg} / 1$ & & 1 & \\
\hline 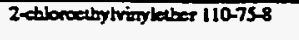 & $<0.010 \mathrm{mg} / 1$ & & $<0.010 \mathrm{mg} / 1$ & & 1 & \\
\hline bestecos $71-43-2$ & $<0.010 \mathrm{mg} / \mathrm{l}$ & & $<0.010 \mathrm{mg} / 1$ & & 1 & \\
\hline 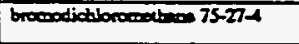 & $<0.010 \mathrm{mg} / \mathrm{l}$ & & $<0.010 \mathrm{mg} / \mathrm{l}$ & & 1 & \\
\hline brocolocem 75-25-2 & $<0.010 \mathrm{mg} / \mathrm{l}$ & & $<0.010 \mathrm{mg} / 1$ & & 1 & \\
\hline bromodectione 7483-9 & $<0.010 \mathrm{mg} / 1$ & & $<0.010 \mathrm{mg} / 1$ & & 1 & \\
\hline artwo menciaboide 56-23-5 & $<0.010 \mathrm{mg} / \mathrm{l}$ & & $<0.010 \mathrm{mg} / \mathrm{l}$ & & 1 & \\
\hline dibobecosecos $108-9$ & $<0.010 \mathrm{mg} / 1$ & & $<0.010 \mathrm{mg} / \mathrm{l}$ & & 1 & \\
\hline ctibroetheseo $75-00-3$ & $<0.010 \mathrm{mg} / 1$ & & $<0.010 \mathrm{mg} / \mathrm{l}$ & · & 1 & \\
\hline chlaroforen 67-66-3 & $<0.010 \mathrm{mg} / 1$ & & $<0.010 \mathrm{mg} / \mathrm{l}$ & & 1 & $\therefore$ \\
\hline chibounetheres 74-57-3 & $<0.010 \mathrm{mg} / \mathrm{l}$ & & $<0.010 \mathrm{mg} / 1$ & & 1 & \\
\hline dibceonoctionocothen $12408-1$ & $<0.010 \mathrm{mg} / \mathrm{l}$ & & $<0.010 \mathrm{mg} / \mathrm{l}$ & & 1 & \\
\hline atylteonecos 100414 & $<0.010 \mathrm{mg} / \mathrm{l}$ & & $<0.010 \mathrm{mg} / 1$ & & 1 & \\
\hline botuseos 108-58-3 & $<0.010 \mathrm{mg} / \mathrm{l}$ & & $<0.010 \mathrm{mg} / 1$ & & 1 & \\
\hline vingl chloride 75014 & $<0.010 \mathrm{mg} / \mathrm{l}$ & & $<0.010 \mathrm{mg} / \mathrm{l}$ & & 1 & \\
\hline 1,3-dictionopicopoce 10061-01-5 & $<0.010 \mathrm{mg} / 1$ & - & $<0.010 \mathrm{mg} / 1$ & - & 1 & \\
\hline 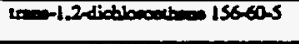 & $<0.010 \mathrm{mg} / 1$ & & $<0.010 \mathrm{mg} / \mathrm{l}$ & & 1 & $\dot{-}$ \\
\hline alpke eainty $12587-46-1$ & $3.8 \mathrm{pci} / \mathrm{l}$ & 10 pcill & $3.8 \mathrm{pci} / 1$ & $10 \mathrm{pci} / \mathrm{l}$ & 1 & \\
\hline betmearinty $12587-46-1$ & $1.0 \mathrm{pci} / 1$ & $7.0 \mathrm{pci} / \mathrm{l}$ & $1.0 \mathrm{pei} / 1$ & 7.0 pcill & 1 & \\
\hline Focal oulifores & $15 \mathrm{co} / .1 \mathrm{~L}$ & $234 \mathrm{co} / .1 \mathrm{~L}$ & $15 \mathrm{co} / .1 \mathrm{~L}$ & $234 \mathrm{co} / .1 \mathrm{~L}$ & 1 & \\
\hline Thellinen 7440-280 & $<0.03 \mathrm{mg} / 1$ & $<0.001 \mathrm{mg} / \mathrm{l}$ & $<0.03 \mathrm{mg} / \mathrm{l}$ & $<0.001 \mathrm{mg} / 1$ & 1 & \\
\hline Unantom 7440-61-1 & $0.004 \mathrm{mg} / \mathrm{l}$ & $0.005 \mathrm{mg} / \mathrm{l}$ & $0.004 \mathrm{mg} / \mathrm{l}$ & $0.005 \mathrm{mg} / \mathrm{l}$ & 1 & \\
\hline Comenexivisy & $-0.78 \mathrm{pci} / \mathrm{l}$ & 60 peill & $-0.78 \mathrm{pci} / \mathrm{l}$ & $60 \mathrm{pci} / \mathrm{l}$ & 1 & \\
\hline
\end{tabular}

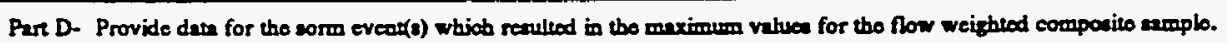

\begin{tabular}{|c|c|c|c|c|c|c|c|}
\hline $\begin{array}{l}1 . \\
\text { Date of } \\
\text { Storm } \\
\text { Event }\end{array}$ & $\begin{array}{l}2 . \\
\text { Durution } \\
\text { of Stom } \\
\text { (in } \\
\text { minutes) }\end{array}$ & $\begin{array}{l}3 . \\
\text { Total minfiall } \\
\text { during storm oveat } \\
\text { (in incton) }\end{array}$ & $\begin{array}{l}4 . \\
\text { Number of bours betweed } \\
\text { begioning of storm meti- } \\
\text { ured and end of provious } \\
\text { ocererable rain oveat }\end{array}$ & $\begin{array}{l}\text { S. } \\
\text { Meximum flow rato } \\
\text { during ruin oveat } \\
\text { (alloow/min or specify } \\
\text { unita) }\end{array}$ & $\begin{array}{l}6 . \\
\text { Toul flow from } \\
\text { nim event } \\
\text { (eallons or } \\
\text { spocify unios) }\end{array}$ & $\begin{array}{c}7 . \\
\text { Scosod } \\
\text { taken }\end{array}$ & $\begin{array}{l}8 . \\
\text { Porm of } \\
\text { Preoipitition } \\
\text { (rainfull, } \\
\text { nommela) }\end{array}$ \\
\hline $\begin{array}{l}12 / 13 / 91 \\
8 / 28 / 92\end{array}$ & $\begin{array}{l}250 \\
535\end{array}$ & $\begin{array}{l}0.25 \\
1.15\end{array}$ & $\begin{array}{l}88.2 \\
124.3\end{array}$ & $\begin{array}{l}\text { NA } \\
405.2\end{array}$ & $\begin{array}{l}\text { NA } \\
61,400\end{array}$ & $\begin{array}{l}\text { Fall (grab) } \\
\text { Summer } \\
\text { (composite) }\end{array}$ & $\begin{array}{l}\text { Rainfall } \\
\text { Rainfall }\end{array}$ \\
\hline
\end{tabular}

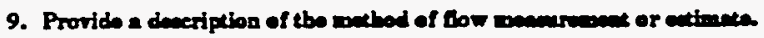

An ISCO 3230 Flow meter was used to obtain water levd readings. This instrument was calibrated with the normal dry veather flow, if present, as leve zero. The flow meter was programmed with the characteristics of the conreyance so that flow rates and total bows could be calculated by the meter. 
Outfall S17

Parre a, and C Continued

\begin{tabular}{|c|c|c|c|c|c|c|}
\hline \multirow{2}{*}{$\begin{array}{l}\text { Pollutuat } \\
\text { and } \\
\text { CAS Number } \\
\text { (if availible) }\end{array}$} & \multicolumn{2}{|c|}{$\begin{array}{l}\text { Meximum Valuea } \\
\text { (include unite) }\end{array}$} & \multicolumn{2}{|c|}{$\begin{array}{l}\text { Average Valuea } \\
\text { (include units) }\end{array}$} & \multirow{2}{*}{$\begin{array}{c}\text { Number } \\
\text { of } \\
\text { Stom } \\
\text { Everess } \\
\text { Samopled }\end{array}$} & \multirow[b]{2}{*}{ Sources of Polluranes } \\
\hline & $\begin{array}{c}\text { Grib Semplo } \\
\text { Tuken During } \\
\text { Fint } 30 \\
\text { Minutea }\end{array}$ & $\begin{array}{l}\text { Flow-weighted } \\
\text { Compotice }\end{array}$ & $\begin{array}{c}\text { Grab Semple } \\
\text { Taken Duriog } \\
\text { Firn } 30 \\
\text { Minutes }\end{array}$ & $\begin{array}{l}\text { Fow-weightod } \\
\text { Composite }\end{array}$ & & \\
\hline Bariven 74a-39-3 & $0.0499 \mathrm{mg} / \mathrm{l}$ & $0.0625 \mathrm{mg} / \mathrm{l}$ & $0.0499 \mathrm{mg} / \mathrm{l}$ & $0.0625 \mathrm{mg} / 1$ & 1 & \\
\hline 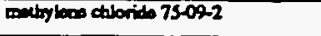 & $<0.010 \mathrm{mg} / \mathrm{l}$ & & $<0.010 \mathrm{mg} / \mathrm{l}$ & & 1 & \\
\hline Letrectibroubseoce $127.18-4$ & $<0.010 \mathrm{mg} / \mathrm{l}$ & & $<0.010 \mathrm{mg} / \mathrm{I}$ & & 1 & \\
\hline inctubrodheses $7901-6$ & $<0.010 \mathrm{mg} / \mathrm{l}$ & & $<0.010 \mathrm{mg} / \mathrm{l}$ & & 1 & \\
\hline xylowen & $<0.010 \mathrm{mg} / \mathrm{l}$ & & $<0.010 \mathrm{mg} / \mathrm{l}$ & & 1 & \\
\hline 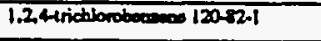 & $<0.010 \mathrm{mg} / 1$ & $<0.010 \mathrm{mg} / \mathrm{l}$ & $<0.010 \mathrm{mg} / \mathrm{l}$ & $<0.010 \mathrm{mg} / \mathrm{l}$ & 1 & \\
\hline 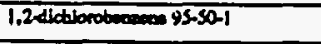 & $<0.010 \mathrm{mg} / 1$ & $<0.010 \mathrm{mg} / \mathrm{l}$ & $<0.010 \mathrm{mg} / \mathrm{l}$ & $<0.010 \mathrm{mg} / \mathrm{l}$ & 1 & \\
\hline 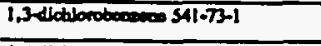 & $<0.010 \mathrm{mg} / 1$ & $<0.010 \mathrm{mg} / 1$ & $<0.010 \mathrm{mg} / \mathrm{l}$ & $<0.010 \mathrm{mg} / \mathrm{l}$ & 1 & \\
\hline 1.4dictionobocescose $100-18-7$ & $<0.010 \mathrm{mg} / \mathrm{l}$ & $<0.010 \mathrm{mg} / \mathrm{l}$ & $<0.010 \mathrm{mg} / \mathrm{l}$ & $<0.010 \mathrm{mg} / \mathrm{l}$ & 1 & \\
\hline 24dinitrochesuos $|2|-14-2$ & $<0.010 \mathrm{mg} / \mathrm{I}$ & $<0.010 \mathrm{mg} / \mathrm{l}$ & $<0.010 \mathrm{mg} / \mathrm{l}$ & $<0.010 \mathrm{mg} / \mathrm{l}$ & 1 & \\
\hline 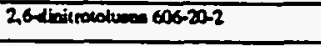 & $<0.010 \mathrm{mg} / \mathrm{l}$ & $<0.010 \mathrm{mg} / \mathrm{l}$ & $<0.010 \mathrm{mg} / \mathrm{l}$ & $<0.010 \mathrm{mg} / \mathrm{L}$ & 1 & \\
\hline 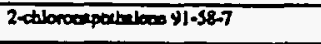 & $<0.010 \mathrm{mg} / \mathrm{l}$ & $<0.010 \mathrm{mg} / \mathrm{l}$ & $<0.010 \mathrm{mg} / \mathrm{l}$ & $<0.010 \mathrm{mg} / 1$ & 1 . & \\
\hline 3.3.-dictionoturadine $91-941$ & $<0.020 \mathrm{mg} / \mathrm{l}$ & $<0.010 \mathrm{mg} / \mathrm{l}$ & $<0.020 \mathrm{mg} / \mathrm{l}$ & $<0.010 \mathrm{mg} / \mathrm{l}$ & 1 & \\
\hline 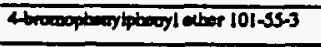 & $<0.010 \mathrm{mg} / \mathrm{l}$ & $<0.010 \mathrm{mg} / 1$ & $<0.010 \mathrm{mg} / \mathrm{l}$ & $<0.010 \mathrm{mg} / \mathrm{L}$ & 1 & \\
\hline 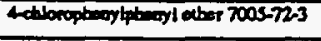 & $<0.010 \mathrm{mg} / \mathrm{h}$ & $<0.010 \mathrm{mg} / \mathrm{l}$ & $<0.010 \mathrm{mg} / \mathrm{l}$ & $<0.010 \mathrm{mg} / 1$ & 1 & \\
\hline $20=83-32-9$ & $<0.010 \mathrm{mg} / \mathrm{l}$ & $<0.010 \mathrm{mg} / \mathrm{l}$ & $<0.010 \mathrm{mg} / \mathrm{l}$ & $<0.010 \mathrm{mg} / 1$ & 1 & \\
\hline 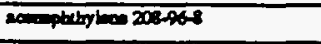 & $<0.010 \mathrm{mg} / \mathrm{l}$ & $<0.010 \mathrm{mg} / 1$ & $<0.010 \mathrm{mg} / \mathrm{l}$ & $<0.010 \mathrm{mg} / \mathrm{l}$ & 1 & \\
\hline $120-127$ & $<0.010 \mathrm{mg} / \mathrm{l}$ & $<0.010 \mathrm{mg} / \mathrm{I}$ & $<0.010 \mathrm{mg} / 1$ & $<0.010 \mathrm{mg} / \mathrm{h}$ & 1 & \\
\hline idimon 97.5 & $<0.050 \mathrm{mg} / \mathrm{t}$ & $<0.030 \mathrm{mg} / 1$ & $<0.050 \mathrm{mg} / \mathrm{l}$ & $<0.030 \mathrm{mg} / \mathrm{l}$ & 1 & \\
\hline 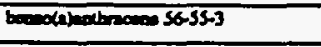 & $<0.010 \mathrm{mg} / 1$ & $<0.010 \mathrm{mg} / 1$ & $<0.010 \mathrm{mg} / \mathrm{l}$ & $<0.010 \mathrm{mg} / \mathrm{l}$ & 1 & \\
\hline 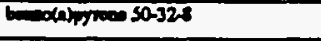 & $<0.010 \mathrm{mg} / \mathrm{l}$ & $<0.010 \mathrm{mg} / \mathrm{l}$ & $<0.010 \mathrm{mg} / \mathrm{l}$ & $<0.010 \mathrm{mg} / \mathrm{l}$ & 1 & \\
\hline 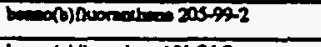 & $<0.010 \mathrm{mg} / \mathrm{l}$ & $<0.010 \mathrm{mg} / 1$ & $<0.010 \mathrm{mg} / \mathrm{l}$ & $<0.010 \mathrm{mg} / 1$ & 1 & \\
\hline 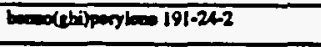 & $<0.010 \mathrm{mg} / \mathrm{l}$ & $<0.010 \mathrm{mg} / \mathrm{l}$ & $<0.010 \mathrm{mg} / \mathrm{l}$ & $<0.010 \mathrm{mg} / \mathrm{l}$ & 1 & \\
\hline 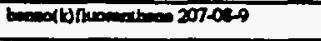 & $<0.010 \mathrm{mg} / \mathrm{l}$ & $<0.010 \mathrm{mg} / \mathrm{l}$ & $<0.010 \mathrm{mg} / \mathrm{h}$ & $<0.010 \mathrm{mg} / 1$ & 1 & \\
\hline 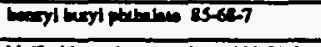 & $<0.010 \mathrm{mg} / \mathrm{l}$ & $<0.010 \mathrm{mg} / 1$ & $<0.010 \mathrm{mg} / \mathrm{l}$ & $<0.010 \mathrm{mg} / 1$ & 1 & \\
\hline 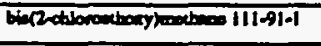 & $<0.010 \mathrm{mg} / \mathrm{l}$ & $<0.010 \mathrm{mg} / \mathrm{l}$ & $<0.010 \mathrm{mg} / \mathrm{l}$ & $<0.010 \mathrm{mg} / \mathrm{l}$ & 1 & $\because$ \\
\hline 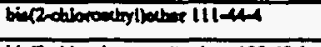 & $<0.010 \mathrm{mg} / \mathrm{l}$ & $<0.010 \mathrm{mg} / \mathrm{l}$ & $<0.010 \mathrm{mg} / \mathrm{l}$ & $<0.010 \mathrm{mg} / \mathrm{l}$ & 1 & \\
\hline 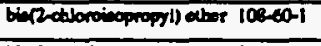 & $<0.010 \mathrm{mg} / \mathrm{l}$ & $<0.010 \mathrm{mg} / \mathrm{l}$ & $<0.010 \mathrm{mg} / \mathrm{l}$ & $<0.010 \mathrm{mg} / \mathrm{l}$ & 1 & \\
\hline 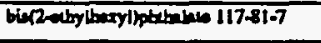 & $<0.010 \mathrm{mg} / 1$ & $<0.010 \mathrm{mg} / 1$ & $<0.010 \mathrm{mg} / 1$ & $<0.010 \mathrm{mg} / \mathrm{l}$ & 1 & \\
\hline ctoprasos $218-01-9$ & $<0.010 \mathrm{mg} / \mathrm{l}$ & $<0.010 \mathrm{mg} / \mathrm{l}$ & $<0.010 \mathrm{mg} / 1$ & $<0.010 \mathrm{mg} / 1$ & 1 & \\
\hline 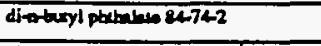 & $<0.010 \mathrm{mg} / \mathrm{l}$ & $<0.010 \mathrm{mg} / \mathrm{l}$ & $<0.010 \mathrm{mg} / \mathrm{l}$ & $<0.010 \mathrm{mg} / 1$ & 1 & \\
\hline 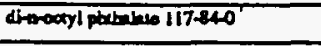 & $<0.010 \mathrm{mg} / \mathrm{l}$ & $<0.010 \mathrm{mg} / \mathrm{l}$ & $<0.010 \mathrm{mg} / \mathrm{l}$ & $<0.010 \mathrm{mg} / 1$ & 1 & \\
\hline 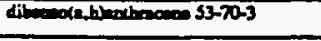 & $<0.010 \mathrm{mg} / \mathrm{l}$ & $<0.010 \mathrm{mg} / \mathrm{l}$ & $<0.010 \mathrm{mg} / \mathrm{l}$ & $<0.010 \mathrm{mg} / 1$ & 1 & \\
\hline dieblyl pataine 8466-2 & $<0.010 \mathrm{mg} / \mathrm{L}$ & $<0.010 \mathrm{mg} / 1$ & $<0.010 \mathrm{mg} / \mathrm{l}$ & $<0.010 \mathrm{mg} / \mathrm{l}$ & 1 & \\
\hline 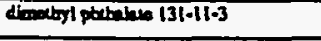 & $<0.010 \mathrm{mg} /$ & $<0.010 \mathrm{mg} / \mathrm{l}$ & $<0.010 \mathrm{mg} / \mathrm{l}$ & $<0.010 \mathrm{mg} / \mathrm{l}$ & 1 & \\
\hline 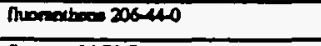 & $<0.010 \mathrm{mg} / \mathrm{l}$ & $<0.010 \mathrm{mg} / \mathrm{l}$ & $<0.010 \mathrm{mg} / \mathrm{l}$ & $<0.010 \mathrm{mg} / \mathrm{l}$ & 1 & \\
\hline nowne- $86-73-7$ & $<0.010 \mathrm{mg} / \mathrm{l}$ & $<0.010 \mathrm{mg} / 1$ & $<0.010 \mathrm{mg} / 1$ & $<0.010 \mathrm{mg} / \mathrm{l}$ & 1 & \\
\hline 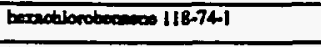 & $<0.010 \mathrm{mg} / \mathrm{l}$ & $<0.010 \mathrm{mg} / \mathrm{l}$ & $<0.010 \mathrm{mg} / \mathrm{l}$ & $<0.010 \mathrm{mg} / \mathrm{l}$ & 1 & \\
\hline 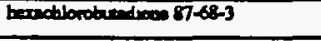 & $<0.010 \mathrm{mg} / \mathrm{h}$ & $<0.010 \mathrm{mg} / \mathrm{l}$ & $<0.010 \mathrm{mg} / \mathrm{l}$ & $<0.010 \mathrm{mg} / 1$ & 1 & \\
\hline 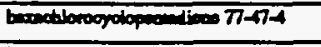 & $<0.010 \mathrm{mg} / \mathrm{l}$ & $<0.010 \mathrm{mg} / \mathrm{l}$ & $<0.010 \mathrm{mg} / \mathrm{l}$ & $<0.010 \mathrm{mg} / \mathrm{l}$ & 1 & \\
\hline $67 \cdot 72.1$ & $<0.010 \mathrm{mg} / 1$ & $<0.010 \mathrm{mg} / \mathrm{l}$ & $<0.010 \mathrm{mg} / \mathrm{l}$ & $<0.010 \mathrm{mg} / \mathrm{I}$ & 1 & \\
\hline $193-39.5$ & $<0.010 \mathrm{mg} / \mathrm{l}$ & $<0.010 \mathrm{mg} / \mathrm{l}$ & $<0.010 \mathrm{mg} / \mathrm{l}$ & $<0.010 \mathrm{mg} / \mathrm{l}$ & 1 & \\
\hline 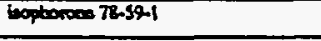 & $<0.010 \mathrm{mg} / 1$ & $<0.010 \mathrm{mg} / \mathrm{l}$ & $<0.010 \mathrm{mg} / 1$ & $<0.010 \mathrm{mg} / \mathrm{l}$ & 1 & \\
\hline Whainocoditoppopy lemino $621-647$ & $<0.010 \mathrm{mg} / \mathrm{l}$ & $<0.010 \mathrm{mg} / \mathrm{I}$ & $<0.010 \mathrm{mg} / \mathrm{l}$ & $<0.010 \mathrm{mg} / \mathrm{I}$ & 1 & \\
\hline 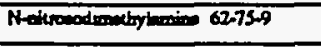 & $<0.010 \mathrm{mg} / \mathrm{l}$ & $<0.010 \mathrm{mg} / \mathrm{l}$ & $<0.010 \mathrm{mg} / 1$ & $<0.010 \mathrm{mg} / \mathrm{l}$ & 1 & \\
\hline 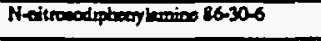 & $<0.010 \mathrm{mg} / \mathrm{l}$ & $<0.010 \mathrm{mg} / \mathrm{l}$ & $<0.010 \mathrm{mg} / \mathrm{l}$ & $<0.010 \mathrm{mg} / \mathrm{I}$ & 1 & \\
\hline Daptetheloce $91-20-3$ & $<0.010 \mathrm{mg} / \mathrm{l}$ & $<0.010 \mathrm{mg} / \mathrm{l}$ & $<0.010 \mathrm{mg} / \mathrm{l}$ & $<0.010 \mathrm{mg} / \mathrm{l}$ & 1 & \\
\hline 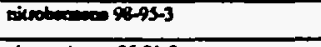 & $<0.010 \mathrm{mg} / \mathrm{l}$ & $<0.010 \mathrm{mg} / \mathrm{l}$ & $<0.010 \mathrm{mg} / 1$ & $<0.010 \mathrm{mg} / 1$ & 1 & \\
\hline 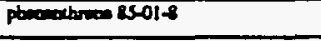 & $<0.010 \mathrm{mg} / \mathrm{l}$ & $<0.010 \mathrm{mg} / \mathrm{l}$ & $<0.010 \mathrm{mg} / \mathrm{l}$ & $<0.010 \mathrm{mg} / \mathrm{l}$ & 1 & \\
\hline p91000 122,000 & $<0.010 \mathrm{mg} / 1$ & $<0.010 \mathrm{mg} / \mathrm{l}$ & $<0.010 \mathrm{mg} / \mathrm{l}$ & $<0.010 \mathrm{mg} / \mathrm{l}$ & 1 & \\
\hline 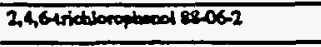 & $<0.030 \mathrm{mg} / \mathrm{h}$ & $<0.030 \mathrm{mg} / \mathrm{l}$ & $<0.030 \mathrm{mg} / \mathrm{l}$ & $<0.030 \mathrm{mg} / \mathrm{l}$ & 1 & \\
\hline 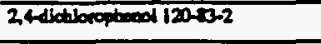 & $<0.030 \mathrm{mg} / \mathrm{l}$ & $<0.030 \mathrm{mg} / 1$ & $<0.030 \mathrm{mg} / \mathrm{l}$ & $<0.030 \mathrm{mg} / \mathrm{l}$ & 1 & \\
\hline 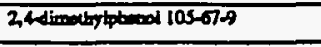 & $<0.030 \mathrm{mg} / \mathrm{l}$ & $<0.030 \mathrm{mg} / \mathrm{l}$ & $<0.030 \mathrm{mg} / \mathrm{I}$ & $<0.030 \mathrm{mg} / \mathrm{l}$ & 1 & \\
\hline 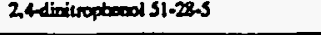 & $<0.050 \mathrm{mg} / \mathrm{l}$ & $<0.030 \mathrm{mg} / \mathrm{l}$ & $<0.050 \mathrm{mg} / \mathrm{l}$ & $<0.030 \mathrm{mg} / \mathrm{l}$ & 1 & \\
\hline
\end{tabular}


Outfall 517

; Pasta B and C Continived

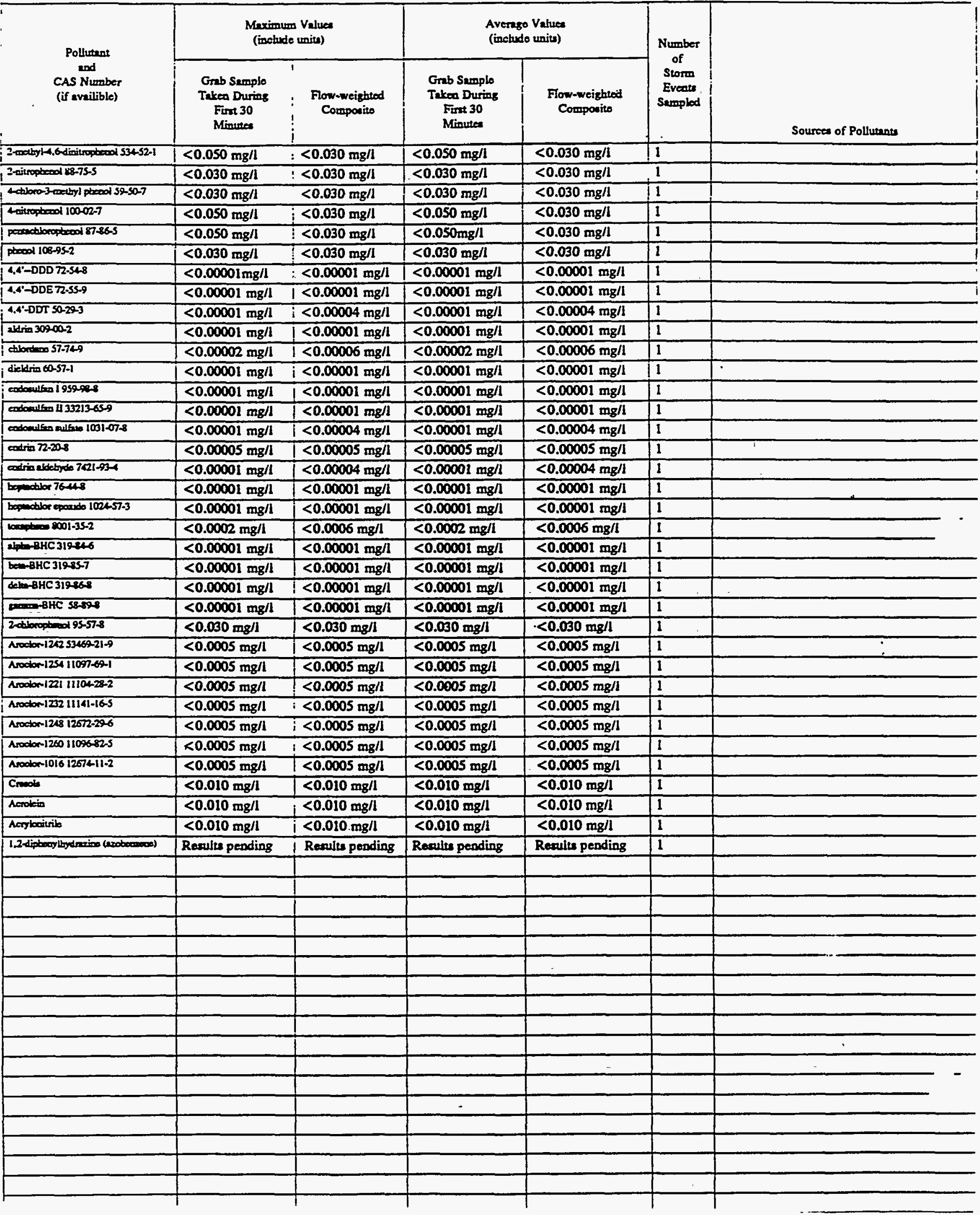


MII. Discharge Information (continuod from page 3 of Form 2F)

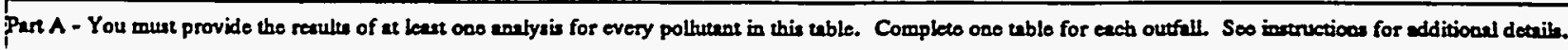

\begin{tabular}{|c|c|c|c|c|c|c|}
\hline \multirow{2}{*}{$\begin{array}{l}\text { Pollutust } \\
\text { nad } \\
\text { CAS Number } \\
\text { (if avnilible) }\end{array}$} & \multicolumn{2}{|c|}{$\begin{array}{l}\text { Merimum Values } \\
\text { (include units) }\end{array}$} & \multicolumn{2}{|c|}{$\begin{array}{l}\text { Average Valuea } \\
\text { (include unitu) }\end{array}$} & \multirow{2}{*}{$\begin{array}{l}\text { Number } \\
\text { of } \\
\text { Storm } \\
\text { Eveols } \\
\text { Stroplod }\end{array}$} & \multirow[b]{2}{*}{ Sourcee of Pollutente } \\
\hline & $\begin{array}{l}\text { Grab Semplo } \\
\text { Taken During } \\
\text { Firat } 30 \\
\text { Minuted }\end{array}$ & $\begin{array}{l}\text { Flow-weigtied } \\
\text { Compotito }\end{array}$ & $\begin{array}{c}\text { Grab Samplo } \\
\text { Tekee Durims } \\
\text { Firnt } 30 \\
\text { Minutec }\end{array}$ & $\begin{array}{l}\text { Flow-weighted } \\
\text { Componite }\end{array}$ & & \\
\hline pil exed Greace & $<2 \mathrm{mg} / \mathrm{ml}$ & & $<2 \mathrm{~m} / \mathrm{g} / \mathrm{l}$ & & 1 & \\
\hline Diological Oxype Deted (BODS) & $37.1 \mathrm{mg} / \mathrm{l}$ & $7.8 \mathrm{mg} / 1$ & $37.1 \mathrm{mg} / \mathrm{l}$ & $7.8 \mathrm{mg} / 1$ & 1 & \\
\hline Chemical Oxy dea Deand (COD) & $7.7 \mathrm{mg} / 1$ & $54.0 \mathrm{mg} / 1$ & $7.7 \mathrm{mg} / 1$ & $54.0 \mathrm{mg} / \mathrm{l}$ & 1 & \\
\hline Toul Suppoded Solide (TSS) & $11 \mathrm{mg} / \mathrm{l}$ & $70 \mathrm{mg} / \mathrm{l}$ & $11 \mathrm{mg} / \mathrm{l}$ & $70 \mathrm{mg} / \mathrm{l}$ & 1 & \\
\hline Total Kjethat Nitroyen & $0.3 \mathrm{mg} / \mathrm{l}$ & $1.3 \mathrm{mg} / \mathrm{l}$ & $0.3 \mathrm{mg} / \mathrm{l}$ & $1.3 \mathrm{mg} .1$ & 1 & \\
\hline 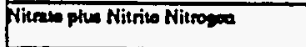 & $3.3 \mathrm{mg} / \mathrm{l}$ & $<0.1 \mathrm{mg} / \mathrm{l}$ & $3.3 \mathrm{mg} / \mathrm{l}$ & $<0.1 \mathrm{mg} / \mathrm{l}$ & 1 & \\
\hline Toul Ptooptorn & $<0.1 \mathrm{mg} / \mathrm{l}$ & $0.60 \mathrm{mg} / \mathrm{l}$ & $<0.1 \mathrm{mg} / \mathrm{l}$ & $0.60 \mathrm{gm} / \mathrm{l}$ & 1 & \\
\hline pH & Minimen 8.3 & Meximes 8.3 & Mirimen 8.3 & Maxim 8.3 & & \\
\hline
\end{tabular}

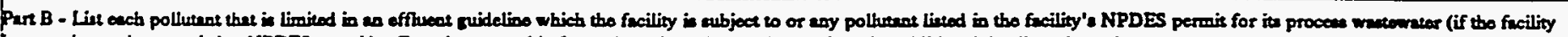

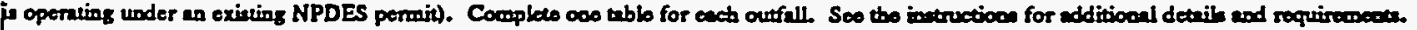

\begin{tabular}{|c|c|c|c|c|c|c|}
\hline \multirow{2}{*}{$\begin{array}{l}\text { Polbureat } \\
\text { and } \\
\text { CAs Nuraber } \\
\text { (if availible) }\end{array}$} & \multicolumn{2}{|c|}{$\begin{array}{l}\text { Marimum Valuce } \\
\text { (Dotedo unito) }\end{array}$} & \multicolumn{2}{|c|}{$\begin{array}{l}\text { Arense Veluese } \\
\text { (inoludo unitr) }\end{array}$} & \multirow{2}{*}{$\begin{array}{l}\text { Number } \\
\text { of } \\
\text { Storm } \\
\text { Everes } \\
\text { Sempled }\end{array}$} & \multirow[b]{2}{*}{ Sources of Polbutenter } \\
\hline & $\begin{array}{c}\text { Orab Samplo } \\
\text { Takeo During } \\
\text { Fins } 30 \\
\text { Minusece }\end{array}$ & $\begin{array}{l}\text { Fow-weighted } \\
\text { Compoino }\end{array}$ & $\begin{array}{l}\text { Grab Semple } \\
\text { Tuken During } \\
\text { Fure } 30 \\
\text { Mfoces }\end{array}$ & $\begin{array}{l}\text { Flow-weighed } \\
\text { Componito }\end{array}$ & & \\
\hline Aximod 7440-360 & $<0.04 \mathrm{mg} / \mathrm{l}$ & $<0.01 \mathrm{mg} / \mathrm{l}$ & $<0.04 \mathrm{mg} / 1$ & $<0.01 \mathrm{mg} / \mathrm{l}$ & $\bar{I}$ & \\
\hline Fraith & $<0.002 \mathrm{mg} / \mathrm{l}$ & & $<0.002 \mathrm{mg} / \mathrm{l}$ & & 1 & \\
\hline Morwy 7439 & $<0.0002 \mathrm{mg} / \mathrm{l}$ & $<0.0002 \mathrm{mg} / \mathrm{l}$ & $<0.0002 \mathrm{mg} / \mathrm{l}$ & $<0.0002 \mathrm{mg} / \mathrm{l}$ & 1 & \\
\hline Sheode & $0.002 \mathrm{mg} / \mathrm{l}$ & & $0.002 \mathrm{mg} / \mathrm{l}$ & & 1 & $\div$ \\
\hline $32 \sin 7782-292$ & $<0.002 \mathrm{mg} / \mathrm{l}$ & $<0.002 \mathrm{mg} / \mathrm{l}$ & $<0.002 \mathrm{mg} / \mathrm{l}$ & $<0.002 \mathrm{mg} / \mathrm{l}$ & 1 & \\
\hline Pellen & $<10 \mathrm{mg} / \mathrm{l}$ & $<10 \mathrm{mg} / \mathrm{l}$ & $<10 \mathrm{mg} / \mathrm{l}$ & $10 \mathrm{mg} / 1$ & 1 & \\
\hline sirtactenses & $<0.05 \mathrm{mg} / \mathrm{l}$ & $<0.05 \mathrm{mg} / \mathrm{l}$ & $<0.05 \mathrm{mg} / \mathrm{l}$ & $<0.05 \mathrm{mg} / \mathrm{l}$ & 1 & \\
\hline Numinam 742900-5 & $0.38 \mathrm{mg} / \mathrm{l}$ & $1.50 \mathrm{mg} / 1$ & $0.38 \mathrm{mg} / \mathrm{l}$ & $1.50 \mathrm{mg} / \mathrm{l}$ & 1 & \\
\hline Ansois 740-38-2 & $<0.04 \mathrm{mg} / \mathrm{l}$ & $<0.04 \mathrm{mg} / \mathrm{l}$ & $<0.04 \mathrm{mg} / \mathrm{l}$ & $<0.04 \mathrm{mg} / \mathrm{l}$ & 1 & \\
\hline 300007400025 & $0.017 \mathrm{mg} / \mathrm{l}$ & $0.044 \mathrm{mg} / \mathrm{l}$ & $0.017 \mathrm{mg} / \mathrm{l}$ & $0.044 \mathrm{mg} / \mathrm{l}$ & 1 & \\
\hline Boryllinem 7400-41-7 & $<0.0004 \mathrm{mg} / \mathrm{l}$ & $<0.0004 \mathrm{mg} / \mathrm{l}$ & $<0.0004 \mathrm{mg} / \mathrm{l}$ & $<0.0004 \mathrm{mg} / \mathrm{l}$ & 1 & \\
\hline Condmiven $7440-13-9$ & $<0.004 \mathrm{mg} / \mathrm{l}$ & $<0.004 \mathrm{mg} / \mathrm{l}$ & $<0.004 \mathrm{mg} / 1$ & $<0.004 \mathrm{mg} / \mathrm{l}$ & 1 & \\
\hline Caloingon 74a-20-2 & $13.5 \mathrm{mg} / \mathrm{h}$ & $31.0 \mathrm{mg} / \mathrm{t}$ & $13.5 \mathrm{mg} / 1$ & $31.0 \mathrm{mg} / 1$ & 1 & \\
\hline puroming $740-77-3$ & $<0.006 \mathrm{mg} / \mathrm{l}$ & $0.009 \mathrm{mg} / \mathrm{l}$ & $<0.006 \mathrm{mg} / 1$ & $0.009 \mathrm{mg} / \mathrm{l}$ & 1 & \\
\hline Cotalitimases & $<0.002 \mathrm{mg} / \mathrm{l}$ & $<0.002 \mathrm{mg} / \mathrm{l}$ & $<0.002 \mathrm{mg} / \mathrm{l}$ & $<0.002 \mathrm{mg} / \mathrm{l}$ & 1 & \\
\hline Coprom 740-50s & $<0.006 \mathrm{mg} / \mathrm{l}$ & $<0.006 \mathrm{mg} / 1$ & $<0.006 \mathrm{mg} / \mathrm{l}$ & $<0.006 \mathrm{mg} / \mathrm{l}$ & 1 & \\
\hline 50007439096 & $0.36 \mathrm{mg} / \mathrm{l}$ & $1.8 \mathrm{mg} / \mathrm{l}$ & $0.36 \mathrm{mg} / \mathrm{l}$ & $1.8 \mathrm{mg} / \mathrm{l}$ & 1 & \\
\hline $\ln 17432921$ & $<0.02 \mathrm{mg} / \mathrm{l}$ & $<0.02 \mathrm{mg} / \mathrm{l}$ & $<0.02 \mathrm{mg} / \mathrm{l}$ & $<0.02 \mathrm{mg} / \mathrm{l}$ & 1 & \\
\hline Ilbiven 7439-93-2 & $<0.02 \mathrm{mg} / \mathrm{h}$ & $<0.02 \mathrm{mg} / \mathrm{l}$ & $<0.02 \mathrm{mg} / 1$ & $<0.02 \mathrm{mg} / 1$ & 1 & \\
\hline Mraposiven 7439-954 & $3.03 \mathrm{mg} / \mathrm{l}$ & $3.54 \mathrm{mg} / 1$ & $3.03 \mathrm{mg} / 1$ & $3.54 \mathrm{mg} / 1$ & 1 & \\
\hline Mroposomen 7439-96-5 & $0.027 \mathrm{mg} / \mathrm{l}$ & $0.097 \mathrm{mg} / \mathrm{l}$ & $0.027 \mathrm{mg} / \mathrm{l}$ & $0.097 \mathrm{mg} / \mathrm{l}$ & 1 & \\
\hline 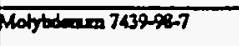 & $<0.006 \mathrm{mg} / \mathrm{l}$ & $<0.006 \mathrm{mg} / 1$ & $<0.006 \mathrm{mg} / 1$ & $<0.006 \mathrm{mg} / 1$ & 1 & \\
\hline Nionel 7440020 & $<0.008 \mathrm{mg} / \mathrm{l}$ & $<0.008 \mathrm{mg} / \mathrm{l}$ & $<0.008 \mathrm{mg} / \mathrm{l}$ & $<0.008 \mathrm{mg} / \mathrm{l}$ & 1 & \\
\hline Poesonken 740.097 & $1.0 \mathrm{mg} / \mathrm{l}$ & $8.8 \mathrm{mg} / \mathrm{l}$ & $1.0 \mathrm{mg} . \mathrm{l}$ & $8.8 \mathrm{mg} / 1$ & 1 & \\
\hline silve 744a-2at & $<0.006 \mathrm{mg} / \mathrm{l}$ & $<0.006 \mathrm{mg} / \mathrm{l}$ & $<0.006 \mathrm{mg} / \mathrm{l}$ & $<0.006 \mathrm{mg} / \mathrm{l}$ & 1 & \\
\hline sothen 7410-22-5 & $0.92 \mathrm{mg} / \mathrm{l}$ & $0.17 \mathrm{mg} / 1$ & $0.92 \mathrm{mg} / 1$ & $0.17 \mathrm{mg} / 1$ & 1 & \\
\hline Tianinon 7400-32-6 & $<0.01 \mathrm{mg} / \mathrm{l}$ & $0.02 \mathrm{mg} / \mathrm{l}$ & $<0.01 \mathrm{mg} / \mathrm{l}$ & $0.02 \mathrm{mg} / \mathrm{l}$ & 1 & \\
\hline $2 \operatorname{lix} 7440-606$ & $<0.01 \mathrm{mg} / 1$ & $0.02 \mathrm{mg} / \mathrm{l}$ & $<0.01 \mathrm{mg} / \mathrm{l}$ & $0.02 \mathrm{mg} / \mathrm{l}$ & 1 & \\
\hline
\end{tabular}


Contluoud from frone.

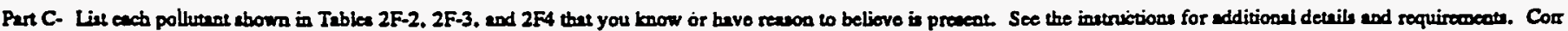
ono teble for each outfill.

\begin{tabular}{|c|c|c|c|c|c|c|}
\hline \multirow{2}{*}{$\begin{array}{l}\text { Polbutant } \\
\text { and } \\
\text { CAS Number } \\
\text { (if availible) }\end{array}$} & \multicolumn{2}{|c|}{$\begin{array}{l}\text { Maximum Values } \\
\text { (includo units) }\end{array}$} & \multicolumn{2}{|c|}{$\begin{array}{l}\text { Average Valuoa } \\
\text { (include units) }\end{array}$} & \multirow{2}{*}{$\begin{array}{l}\text { Number } \\
\text { of } \\
\text { Storm } \\
\text { Evente } \\
\text { Sumplod }\end{array}$} & \multirow[b]{2}{*}{ Sources of Polhutants } \\
\hline & $\begin{array}{l}\text { Grab Sumple } \\
\text { Taken During } \\
\text { Fint } 30 \\
\text { Minutes }\end{array}$ & $\begin{array}{l}\text { Flow-weightod } \\
\text { Componito }\end{array}$ & $\begin{array}{c}\text { Grab Sample } \\
\text { Taken Duriog } \\
\text { Firnt } 30 \\
\text { Minutes }\end{array}$ & $\begin{array}{l}\text { Flow-weighted } \\
\text { Componite }\end{array}$ & & \\
\hline 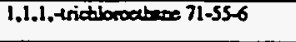 & $<0.010 \mathrm{mg} / \mathrm{l}$ & & $<0.010 \mathrm{mg} / \mathrm{l}$ & & 1 & \\
\hline 1.12 .2 telnodibroul 1000 79-34-5 & $<0.010 \mathrm{mg} / 1$ & & $<0.010 \mathrm{mg} / 1$ & & 1 & \\
\hline 1,1,2-2rictionedinen 7900-5 & $<0.010 \mathrm{mg} / 1$ & & $<0.010 \mathrm{mg} / 1$ & & 1 & \\
\hline 1.1 dictbocotbos $75-343$ & $<0.010 \mathrm{mg} / 1$ & & $<0.010 \mathrm{mg} / 1$ & & 1 & \\
\hline 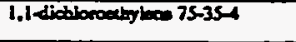 & $<0.010 \mathrm{mg} / 1$ & & $<0.010 \mathrm{mg} / \mathrm{l}$ & & 1 & \\
\hline 1.2dictbreothen $10708-2$ & $<0.010 \mathrm{mg} / \mathrm{l}$ & & $<0.010 \mathrm{mg} / 1$ & & 1 & \\
\hline 1.2-dictoroprop 7000.787 .5 & $<0.010 \mathrm{mg} / \mathrm{l}$ & & $<0.010 \mathrm{mg} / 1$ & & 1 & \\
\hline 2-ationoctbylvinglather $110-75-8$ & $<0.010 \mathrm{mg} / 1$ & & $<0.010 \mathrm{mg} / \mathrm{l}$ & & 1 & \\
\hline$b=0=0000071-43-2$ & $<0.010 \mathrm{mg} / 1$ & & $<0.010 \mathrm{mg} / \mathrm{l}$ & & 1 & \\
\hline browodichlonomechapo 75-274 & $<0.010 \mathrm{mg} / \mathrm{l}$ & & $<0.010 \mathrm{mg} / \mathrm{l}$ & & 1 & \\
\hline brocolionem 75-25-2 & $<0.010 \mathrm{mg} / \mathrm{l}$ & & $<0.010 \mathrm{mg} / \mathrm{l}$ & & 1 & \\
\hline broenconobers 7483-9 & $<0.010 \mathrm{mg} / \mathrm{l}$ & & $<0.010 \mathrm{mg} / \mathrm{l}$ & & 1 & \\
\hline arten recuctibride S6-23-S & $<0.010 \mathrm{mg} / 1$ & & $<0.010 \mathrm{mg} / \mathrm{l}$ & & 1 & \\
\hline 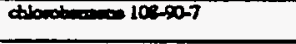 & $<0.010 \mathrm{mg} / 1$ & & $<0.010 \mathrm{mg} / \mathrm{l}$ & & 1 & \\
\hline ctocicothen $7500-3$ & $<0.010 \mathrm{mg} / \mathrm{l}$ & & $<0.010 \mathrm{mg} / 1$ & & 1 & \\
\hline ctibroform 67-66-3 & $<0.010 \mathrm{mg} / \mathrm{l}$ & & $<0.010 \mathrm{mg} / 1$ & & 1 & - \\
\hline 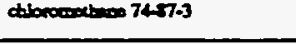 & $<0.010 \mathrm{mg} / \mathrm{l}$ & & $<0.010 \mathrm{mg} / 1$ & & 1 & \\
\hline 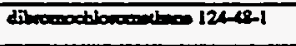 & $<0.010 \mathrm{mg} / \mathrm{l}$ & & $<0.010 \mathrm{mg} / 1$ & & 1 & \\
\hline abyloweon 100414 & $<0.010 \mathrm{mg} / \mathrm{l}$ & & $<0.010 \mathrm{mg} / 1$ & & 1 & \\
\hline bhem 100203 & $<0.010 \mathrm{mg} / \mathrm{l}$ & & $<0.010 \mathrm{mg} / \mathrm{l}$ & & 1 & \\
\hline vispl chloride 75014 & $<0.010 \mathrm{mg} / \mathrm{l}$ & & $<0.010 \mathrm{mg} / \mathrm{l}$ & & 1 & \\
\hline 1.3-dictibropioperos $10001-01-5$ & $<0.010 \mathrm{mg} / \mathrm{l}$ & & $<0.010 \mathrm{mg} / \mathrm{l}$ & & 1 & \\
\hline 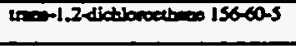 & $<0.010 \mathrm{mg} / \mathrm{l}$ & & $<0.010 \mathrm{mg} / 1$ & & 1 & $\dot{1}$ \\
\hline alpte naivity $12587-16-1$ & 3.4 pcill & $0.80 \mathrm{pci} / \mathrm{l}$ & $3.4 \mathrm{pei} / 1$ & $0.08 \mathrm{pci} / 1$ & 1 & \\
\hline ben eavity $12657-46-1$ & $1.5 \mathrm{pei} / 1$ & $13 \mathrm{pci} / \mathrm{s}$ & $1.5 \mathrm{psi} / 1$ & $13 \mathrm{pci} / 1$ & 1 & \\
\hline fool odifores & $28 \mathrm{co} / .1 \mathrm{~L}$ & $1226 \mathrm{co} / .1 \mathrm{~L}$ & $28 \mathrm{co} / . \mathrm{IL}$ & $1226 \mathrm{cl} / .1 \mathrm{~L}$ & 1 & \\
\hline Thallien 746-28-0 & $<0.03 \mathrm{mg} / 1$ & $<0.001 \mathrm{mg} / \mathrm{l}$ & $<0.03 \mathrm{mg} / 1$ & $<0.002 \mathrm{mg} / 1$ & 1 & \\
\hline Unamim 746061-1 & $<0.001 \mathrm{mg} / \mathrm{l}$ & $<0.001 \mathrm{mg} / \mathrm{l}$ & $0.001 \mathrm{mg} / \mathrm{l}$ & $<0.001 \mathrm{mg} / \mathrm{l}$ & 1 & \\
\hline Gromexivity & $164 \mathrm{pci} / \mathrm{l}$ & $-10 \mathrm{pci} / \mathrm{l}$ & 164 poill & -10 pcill & 1 & \\
\hline
\end{tabular}

Part D- Provide deta for the corm oveat(s) which resultod in tho maximum values for the flow weighted compocito amplo.

\begin{tabular}{|c|c|c|c|c|c|c|c|}
\hline $\begin{array}{l}1 . \\
\text { Dats of } \\
\text { Storm } \\
\text { Eveat }\end{array}$ & $\begin{array}{l}2 . \\
\text { Durntion } \\
\text { of Storm } \\
\text { (in } \\
\text { miouten) }\end{array}$ & $\begin{array}{l}3 . \\
\text { Toul minfiall } \\
\text { during storm ovent } \\
\text { (in incteos) }\end{array}$ & 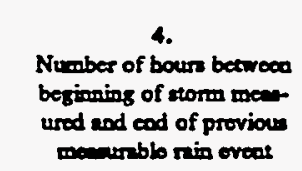 & $\begin{array}{l}\text { S. } \\
\text { Mrximum flow rate } \\
\text { during min eveos } \\
\text { (balloos/min or specify } \\
\text { unitu) }\end{array}$ & $\begin{array}{c}6 . \\
\text { Total flow from } \\
\text { nim oveat } \\
\text { (plloow or } \\
\text { epocify unia) }\end{array}$ & $\begin{array}{c}7 . \\
\text { Seacon } \\
\text { semplo was } \\
\text { tukea }\end{array}$ & $\begin{array}{l}\text { Boing of } \\
\text { Procipitution } \\
\text { (rinfull. } \\
\text { nom }\end{array}$ \\
\hline $\begin{array}{l}12 / 13 / 91 \\
8 / 28 / 92\end{array}$ & $\begin{array}{l}250 \\
535\end{array}$ & $\begin{array}{l}0.25 \\
0.9\end{array}$ & $\begin{array}{l}88.2 \\
124.3\end{array}$ & $\begin{array}{l}\text { NA } \\
31.8\end{array}$ & $\begin{array}{l}\text { NA } \\
2,250\end{array}$ & $\begin{array}{l}\text { Fall (grab) } \\
\text { Summer } \\
\text { (composito) }\end{array}$ & $\begin{array}{l}\text { Ruinfoll } \\
\text { Rainfall }\end{array}$ \\
\hline
\end{tabular}

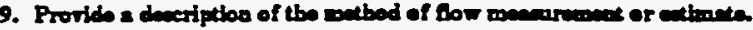

An ISCO 3230 Fow meter mas used to obtain water level readings. This instrument was calibrated with the normal dry weather flow, if present, as lere zero. The $10 \mathrm{w}$ meter was programmed with the characteristics of the conveyance so that flow rates and total flow conld be calculated by the meter. 


\begin{tabular}{|c|c|c|c|c|c|c|}
\hline \multirow{2}{*}{$\begin{array}{l}\text { Pollutant } \\
\text { end } \\
\text { CAS Number } \\
\text { (if aveilible) }\end{array}$} & \multicolumn{2}{|c|}{$\begin{array}{l}\text { Mnximum Valuea } \\
\text { (include units) }\end{array}$} & \multicolumn{2}{|c|}{$\begin{array}{l}\text { Avonge Velues } \\
\text { (include unita) }\end{array}$} & \multirow{2}{*}{$\begin{array}{c}\text { Number } \\
\text { of } \\
\text { Storm } \\
\text { Events } \\
\text { Sempled }\end{array}$} & \multirow[b]{2}{*}{ Sources of Pollimats } \\
\hline & $\begin{array}{l}\text { Grab Sumple } \\
\text { Tnken During } \\
\text { Fint } 30 \\
\text { Minutec }\end{array}$ & $\begin{array}{l}\text { Fow-weighted } \\
\text { Componitio }\end{array}$ & $\begin{array}{c}\text { Grab Sample } \\
\text { Takeo During } \\
\text { Firn } 30 \\
\text { Minuteo }\end{array}$ & $\begin{array}{c}\text { Filow-weightod } \\
\text { Composito } \\
.\end{array}$ & & \\
\hline Buriven 740-3Q-3 & $0.0358 \mathrm{mg} / \mathrm{l}$ & $0.413 \mathrm{mg} / \mathrm{l}$ & $0.0358 \mathrm{mg} / \mathrm{h}$ & $0.413 \mathrm{mg} / 1$ & 1 & \\
\hline mothyloos chloride $7509-2$ & $<0.010 \mathrm{mg} / \mathrm{l}$ & & $<0.010 \mathrm{mg} / \mathrm{l}$ & & 1 & \\
\hline 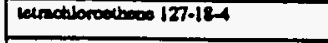 & $<0.010 \mathrm{mg} / \mathrm{I}$ & & $<0.010 \mathrm{mg} / \mathrm{l}$ & & 1 & \\
\hline trictibroucheres 79016 & $<0.010 \mathrm{mg} / \mathrm{l}$ & & $<0.010 \mathrm{mg} / \mathrm{l}$ & & 1 & \\
\hline Xyloos & $<0.010 \mathrm{mg} / \mathrm{l}$ & & $<0.010 \mathrm{mg} / \mathrm{l}$ & & 1 & \\
\hline 1.24 trichlow $120,82.1$ & $<0.010 \mathrm{mg} / \mathrm{l}$ & $<0.010 \mathrm{mg} / \mathrm{I}$ & $<0.010 \mathrm{mg} / \mathrm{l}$ & $<0.010 \mathrm{mg} / 1$ & 1 & \\
\hline 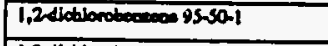 & $<0.010 \mathrm{mg} / \mathrm{l}$ & $<0.010 \mathrm{mg} / \mathrm{l}$ & $<0.010 \mathrm{mg} / \mathrm{l}$ & $<0.010 \mathrm{mg} / \mathrm{l}$ & 1 & \\
\hline 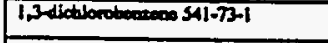 & $<0.010 \mathrm{mg} / \mathrm{l}$ & $<0.010 \mathrm{mg} / \mathrm{l}$ & $<0.010 \mathrm{mg} / \mathrm{l}$ & $<0.010 \mathrm{mg} / 1$ & 1 & \\
\hline 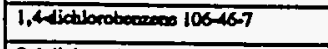 & $<0.010 \mathrm{mg} / \mathrm{l}$ & $<0.010 \mathrm{mg} / 1$ & $<0.010 \mathrm{mg} / \mathrm{l}$ & $<0.010 \mathrm{mg} / 1$ & 1 & \\
\hline 24 -disiderotiven $121.14-2$ & $<0.010 \mathrm{mg} / \mathrm{l}$ & $<0.010 \mathrm{mg} / 1$ & $<0.010 \mathrm{mg} / \mathrm{l}$ & $<0.010 \mathrm{mg} / \mathrm{l}$ & 1 & \\
\hline 20-dinitroludus $606-20-2$ & $<0.010 \mathrm{mg} / \mathrm{l}$ & $<0.010 \mathrm{mg} / 1$ & $<0.010 \mathrm{mg} / \mathrm{l}$ & $<0.010 \mathrm{mg} / 1$ & 1 & \\
\hline 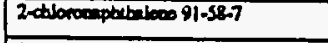 & $<0.010 \mathrm{mg} / \mathrm{l}$ & $<0.010 \mathrm{mg} / 1$ & $<0.010 \mathrm{mg} / \mathrm{l}$ & $<0.010 \mathrm{mg} / 1$ & 1 & \\
\hline 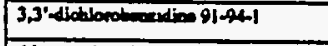 & $<0.020 \mathrm{mg} / \mathrm{l}$ & $<0.010 \mathrm{mg} / \mathrm{l}$ & $<0.020 \mathrm{mg} / \mathrm{l}$ & $<0.010 \mathrm{mg} / \mathrm{l}$ & 1 & \\
\hline 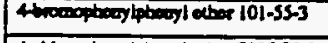 & $<0.010 \mathrm{mg} / \mathrm{l}$ & $<0.010 \mathrm{mg} / 1$ & $<0.010 \mathrm{mg} / \mathrm{l}$ & $<0.010 \mathrm{mg} / \mathrm{l}$ & 1 & \\
\hline 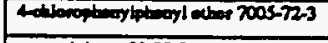 & $<0.010 \mathrm{mg} / 1$ & $<0.010 \mathrm{mg} / \mathrm{l}$ & $<0.010 \mathrm{mg} / \mathrm{l}$ & $<0.010 \mathrm{mg} / 1$ & 1 & \\
\hline 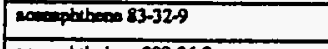 & $<0.010 \mathrm{mg} / \mathrm{l}$ & $<0.010 \mathrm{mg} / \mathrm{l}$ & $<0.010 \mathrm{mg} / 1$ & $<0.010 \mathrm{mg} / \mathrm{l}$ & 1 & \\
\hline 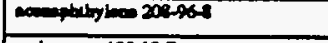 & $<0.010 \mathrm{mg} / 1$ & $<0.010 \mathrm{mg} / 1$ & $<0.010 \mathrm{mg} / 1$ & $<0.010 \mathrm{mg} / \mathrm{l}$ & 1 & \\
\hline manowese 120-12.7 & $<0.010 \mathrm{mg} / \mathrm{l}$ & $<0.010 \mathrm{mg} / 1$ & $<0.010 \mathrm{mg} / \mathrm{I}$ & $<0.010 \mathrm{mg} / \mathrm{l}$ & 1 & - \\
\hline 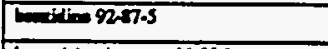 & $<0.050 \mathrm{mg} / \mathrm{l}$ & $<0.030 \mathrm{mg} / \mathrm{l}$ & $<0.050 \mathrm{mg} / \mathrm{l}$ & $<0.030 \mathrm{mg} / \mathrm{l}$ & 1 & \\
\hline 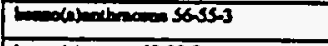 & $<0.010 \mathrm{mg} / \mathrm{l}$ & $<0.010 \mathrm{mg} / 1$ & $<0.010 \mathrm{mg} / \mathrm{l}$ & $<0.010 \mathrm{mg} / 1$ & 1 & \\
\hline 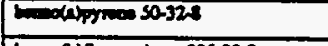 & $<0.010 \mathrm{mg} / \mathrm{l}$ & $<0.010 \mathrm{mg} / \mathrm{l}$ & $<0.010 \mathrm{mg} / \mathrm{l}$ & $<0.010 \mathrm{mg} / \mathrm{l}$ & 1 & \\
\hline 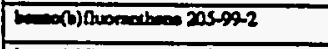 & $<0.010 \mathrm{mg} / \mathrm{l}$ & $<0.010 \mathrm{mg} / \mathrm{l}$ & $<0.010 \mathrm{mg} / 1$ & $<0.010 \mathrm{mg} / \mathrm{l}$ & 1 & \\
\hline 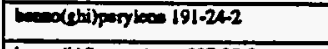 & $<0.010 \mathrm{mg} / \mathrm{l}$ & $<0.010 \mathrm{mg} / \mathrm{l}$ & $<0.010 \mathrm{mg} / \mathrm{l}$ & $<0.010 \mathrm{mg} / \mathrm{l}$ & 1 & \\
\hline 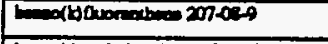 & $<0.010 \mathrm{mg} / \mathrm{l}$ & $<0.010 \mathrm{mg} / \mathrm{l}$ & $<0.010 \mathrm{mg} / \mathrm{l}$ & $<0.010 \mathrm{mg} / \mathrm{l}$ & 1 & \\
\hline 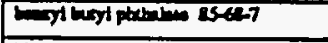 & $<0.010 \mathrm{mg} / 1$ & $<0.010 \mathrm{mg} / \mathrm{l}$ & $<0.010 \mathrm{mg} / \mathrm{t}$ & $<0.010 \mathrm{mg} / \mathrm{l}$ & 1 & \\
\hline 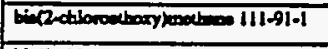 & $<0.010 \mathrm{mg} / \mathrm{l}$ & $<0.010 \mathrm{mg} / \mathrm{l}$ & $<0.010 \mathrm{mg} / \mathrm{l}$ & $<0.010 \mathrm{mg} / \mathrm{l}$ & 1 & $\therefore$ \\
\hline 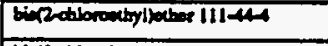 & $<0.010 \mathrm{mg} / \mathrm{l}$ & $<0.010 \mathrm{mg} / \mathrm{l}$ & $<0.010 \mathrm{mg} / \mathrm{l}$ & $<0.010 \mathrm{mg} / \mathrm{l}$ & 1 & \\
\hline 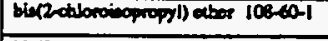 & $<0.010 \mathrm{mg} / \mathrm{l}$ & $<0.010 \mathrm{mg} / \mathrm{l}$ & $<0.010 \mathrm{mg} / \mathrm{l}$ & $<0.010 \mathrm{mg} / \mathrm{l}$ & 1 & \\
\hline 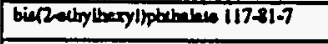 & $<0.010 \mathrm{mg} / \mathrm{l}$ & $<0.010 \mathrm{mg} / \mathrm{l}$ & $<0.010 \mathrm{mg} / \mathrm{l}$ & $<0.010 \mathrm{mg} / 1$ & 1 & \\
\hline chromen 21801-9 & $<0.010 \mathrm{mg} / 1$ & $<0.010 \mathrm{mg} / \mathrm{l}$ & $<0.010 \mathrm{mg} / \mathrm{l}$ & $<0.010 \mathrm{mg} / \mathrm{l}$ & 1 & \\
\hline drotaryi patbatan 2474-2 & $<0.010 \mathrm{mg} / \mathrm{l}$ & $<0.010 \mathrm{mg} / \mathrm{l}$ & $<0.010 \mathrm{mg} / \mathrm{I}$ & $<0.010 \mathrm{mg} / \mathrm{l}$ & 1 & \\
\hline 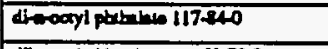 & $<0.010 \mathrm{mg} / 1$ & $<0.010 \mathrm{mg} / 1$ & $<0.010 \mathrm{mg} / 1$ & $<0.010 \mathrm{mg} / \mathrm{l}$ & 1 & \\
\hline $53-70-3$ & $<0.010 \mathrm{mg} / \mathrm{l}$ & $<0.010 \mathrm{mg} / \mathrm{l}$ & $<0.010 \mathrm{mg} / 1$ & $<0.010 \mathrm{mg} / 1$ & 1 & \\
\hline 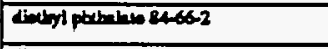 & $<0.010 \mathrm{mg} / \mathrm{l}$ & $<0.010 \mathrm{mg} / \mathrm{l}$ & $<0.010 \mathrm{mg} / \mathrm{l}$ & $<0.010 \mathrm{mg} / \mathrm{l}$ & 1 & \\
\hline 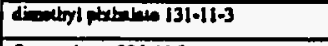 & $<0.010 \mathrm{mg} / \mathrm{l}$ & $<0.010 \mathrm{mg} / \mathrm{l}$ & $<0.010 \mathrm{mg} / \mathrm{I}$ & $<0.010 \mathrm{mg} / \mathrm{l}$ & 1 & \\
\hline 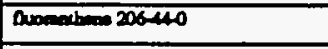 & $<0.010 \mathrm{mg} / \mathrm{l}$ & $<0.010 \mathrm{mg} / 1$ & $<0.010 \mathrm{mg} / \mathrm{l}$ & $<0.010 \mathrm{mg} / \mathrm{l}$ & 1 & \\
\hline Dowome 26-73-7 & $<0.010 \mathrm{mg} / \mathrm{l}$ & $<0.010 \mathrm{mg} / \mathrm{l}$ & $<0.010 \mathrm{mg} / 1$ & $<0.010 \mathrm{mg} / 1$ & 1 & \\
\hline 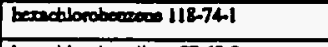 & $<0.010 \mathrm{mg} / 1$ & $<0.010 \mathrm{mg} / \mathrm{l}$ & $<0.010 \mathrm{mg} / \mathrm{l}$ & $<0.010 \mathrm{mg} / 1$ & 1 & - \\
\hline 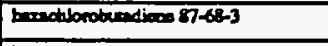 & $<0.010 \mathrm{mg} / \mathrm{l}$ & $<0.010 \mathrm{mg} / \mathrm{l}$ & $<0.010 \mathrm{mg} / \mathrm{l}$ & $<0.010 \mathrm{mg} / \mathrm{l}$ & 1 & \\
\hline 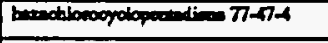 & $<0.010 \mathrm{mg} / \mathrm{l}$ & $<0.010 \mathrm{mg} / \mathrm{I}$ & $<0.010 \mathrm{mg} / 1$ & $<0.010 \mathrm{mg} / 1$ & 1 & \\
\hline 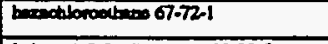 & $<0.010 \mathrm{mg} / \mathrm{l}$ & $<0.010 \mathrm{mg} / \mathrm{l}$ & $<0.010 \mathrm{mg} / \mathrm{l}$ & $<0.010 \mathrm{mg} / \mathrm{l}$ & 1 & \\
\hline 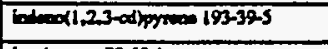 & $<0.010 \mathrm{mg} / \mathrm{l}$ & $<0.010 \mathrm{mg} / \mathrm{l}$ & $<0.010 \mathrm{mg} / \mathrm{l}$ & $<0.010 \mathrm{mg} / \mathrm{l}$ & 1 & \\
\hline inopbroweos 78-59-1 & $<0.010 \mathrm{mg} / \mathrm{l}$ & $<0.010 \mathrm{mg} / \mathrm{l}$ & $<0.010 \mathrm{mg} / \mathrm{l}$ & $<0.010 \mathrm{mg} / \mathrm{l}$ & 1 & \\
\hline 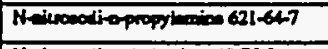 & $<0.010 \mathrm{mg} / \mathrm{l}$ & $<0.010 \mathrm{mg} / \mathrm{I}$ & $<0.010 \mathrm{mg} / 1$ & $<0.010 \mathrm{mg} / \mathrm{l}$ & 1 & \\
\hline 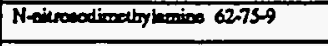 & $<0.010 \mathrm{mg} / \mathrm{l}$ & $<0.010 \mathrm{mg} / \mathrm{l}$ & $<0.010 \mathrm{mg} / \mathrm{l}$ & $<0.010 \mathrm{mg} / \mathrm{l}$ & 1 & \\
\hline 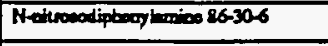 & $<0.010 \mathrm{mg} / \mathrm{l}$ & $<0.010 \mathrm{mg} / \mathrm{l}$ & $<0.010 \mathrm{mg} / 1$ & $<0.010 \mathrm{mg} / \mathrm{l}$ & 1 & \\
\hline 2 ateos $41-20-3$ & $<0.010 \mathrm{mg} / \mathrm{l}$ & $<0.010 \mathrm{mg} / 1$ & $<0.010 \mathrm{mg} / \mathrm{l}$ & $<0.010 \mathrm{mg} / \mathrm{l}$ & 1 & \\
\hline 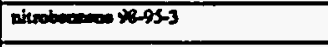 & $<0.010 \mathrm{mg} / \mathrm{l}$ & $<0.010 \mathrm{mg} / \mathrm{l}$ & $<0.010 \mathrm{mg} / \mathrm{l}$ & $<0.010 \mathrm{mg} / \mathrm{l}$ & 1 & \\
\hline 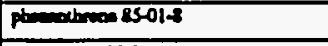 & $<0.010 \mathrm{mg} / \mathrm{l}$ & $<0.010 \mathrm{mg} / \mathrm{l}$ & $<0.010 \mathrm{mg} / 1$ & $<0.010 \mathrm{mg} / \mathrm{l}$ & 1 & \\
\hline Fnowe 129000 & $<0.010 \mathrm{mg} / \mathrm{l}$ & $<0.010 \mathrm{mg} / \mathrm{l}$ & $<0.010 \mathrm{mg} / 1$ & $<0.010 \mathrm{mg} / \mathrm{l}$ & 1 & \\
\hline 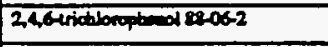 & $<0.030 \mathrm{mg} / \mathrm{l}$ & $<0.030 \mathrm{mg} / \mathrm{l}$ & $<0.030 \mathrm{mg} / 1$ & $<0.030 \mathrm{mg} / \mathrm{l}$ & 1 & \\
\hline 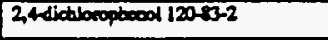 & $<0.030 \mathrm{mg} / \mathrm{l}$ & $<0.030 \mathrm{mg} / \mathrm{l}$ & $<0.030 \mathrm{mg} / \mathrm{l}$ & $<0.030 \mathrm{mg} / \mathrm{l}$ & 1 & \\
\hline 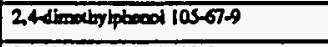 & $<0.030 \mathrm{mg} / \mathrm{I}$ & $<0.030 \mathrm{mg} / \mathrm{l}$ & $<0.030 \mathrm{mg} / \mathrm{l}$ & $<0.030 \mathrm{mg} / \mathrm{l}$ & 1 & \\
\hline 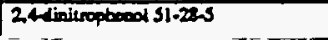 & $<0.050 \mathrm{mg} / \mathrm{I}$ & $<0.030 \mathrm{mg} / \mathrm{l}$ & $<0.050 \mathrm{mg} / 1$ & $<0.030 \mathrm{mg} / \mathrm{l}$ & 1 & \\
\hline
\end{tabular}


Outfall S18

: Pare B and C Continued

\begin{tabular}{|c|c|c|c|c|c|c|}
\hline \multirow{2}{*}{$\begin{array}{l}\text { Pollutunt } \\
\text { and } \\
\text { CAS Number } \\
\text { (if aveilible) }\end{array}$} & \multicolumn{2}{|c|}{$\begin{array}{l}\text { Maximusm Valuea } \\
\text { (inchudo unita) }\end{array}$} & \multicolumn{2}{|c|}{$\begin{array}{l}\text { Average Valuea } \\
\text { (inchudo units) }\end{array}$} & \multirow{2}{*}{$\begin{array}{l}\text { Number } \\
\text { of } \\
\text { Stom } \\
\text { Eveate } \\
\text { Sempled }\end{array}$} & \multirow[b]{2}{*}{ Sources of Pollumensests } \\
\hline & $\begin{array}{c}\text { Grab Sample } \\
\text { Taken During } \\
\text { Firt } 30 \\
\text { Minutet }\end{array}$ & $\begin{array}{l}\text { Flow-weighted } \\
\text { Componite }\end{array}$ & $\begin{array}{l}\text { Grab Sumple } \\
\text { Tiken During } \\
\text { First } 30 \\
\text { Minutea }\end{array}$ & $\begin{array}{l}\text { Flow-weighted } \\
\text { Comporite }\end{array}$ & & \\
\hline 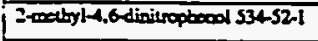 & $<0.050 \mathrm{mg} / \mathrm{l}$ & $<0.030 \mathrm{mg} / \mathrm{l}$ & $<0.050 \mathrm{mg} / \mathrm{l}$ & $<0.030 \mathrm{mg} / \mathrm{l}$ & 1 & 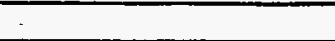 \\
\hline 2 -aikropheod 88.75.5 & $<0.030 \mathrm{mg} / \mathrm{l}$ & $<0.030 \mathrm{mg} / \mathrm{l}$ & $<0.030 \mathrm{mg} / \mathrm{l}$ & $<0.030 \mathrm{mg} / 1$ & 1 & \\
\hline 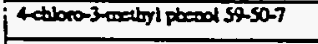 & $<0.030 \mathrm{mg} / \mathrm{l}$ & $<0.030 \mathrm{mg} / \mathrm{l}$ & $<0.030 \mathrm{mg} / \mathrm{l}$ & $<0.030 \mathrm{mg} / \mathrm{h}$ & 1 & \\
\hline sillopbeod 10002.7 & $<0.050 \mathrm{mg} / \mathrm{l}$ & $<0.030 \mathrm{mg} / \mathrm{l}$ & $<0.050 \mathrm{mg} / \mathrm{l}$ & $<0.030 \mathrm{mg} / \mathrm{l}$ & 1 & \\
\hline 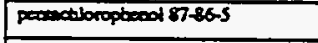 & $<0.050 \mathrm{mg} / 1$ & $<0.030 \mathrm{mg} / \mathrm{l}$ & $<0.050 \mathrm{mg} / 1$ & $<0.030 \mathrm{mg} / \mathrm{l}$ & 1 & \\
\hline$p b e 00$ l $100-95-2$ & $<0.030 \mathrm{mg} / \mathrm{l}$ & $<0.030 \mathrm{mg} / \mathrm{l}$ & $<0.030 \mathrm{mg} / \mathrm{l}$ & $<0.030 \mathrm{mg} / \mathrm{l}$ & 1 & \\
\hline $4,4-0 D D 72-548$ & $<0.00001 \mathrm{mg} / \mathrm{l}$ & $<0.00001 \mathrm{mg} / \mathrm{l}$ & $<0.00001 \mathrm{mg} / \mathrm{l}$ & $<0.00001 \mathrm{mg} / 1$ & 1 & \\
\hline 4,4"-DDE 72-55-9 & $<0.00001 \mathrm{mg} / \mathrm{l}$ & $<0.00001 \mathrm{mg} / \mathrm{l}$ & $<0.00001 \mathrm{mg} / \mathrm{l}$ & $<0.00001 \mathrm{mg} / 1$ & 1 & \\
\hline $4,4^{\prime}-\mathrm{DDT} 50-20-3$ & $<0.00001 \mathrm{mg} / \mathrm{l}$ & $<0.00004 \mathrm{mg} / \mathrm{l}$ & $<0.00001 \mathrm{mg} / \mathrm{l}$ & $<0.00004 \mathrm{mg} / \mathrm{l}$ & 1 & \\
\hline 2hin 30900.2 & $<0.00001 \mathrm{mg} / \mathrm{l}$ & $<0.00001 \mathrm{mg} / \mathrm{l}$ & $<0.00001 \mathrm{mg} / \mathrm{l}$ & $<0.00001 \mathrm{mg} / \mathrm{l}$ & 1 & \\
\hline thenteces $57-749$ & $<0.00002 \mathrm{mg} / \mathrm{l}$ & $<0.00006 \mathrm{mg} / \mathrm{I}$ & $<0.00002 \mathrm{mg} / \mathrm{l}$ & $<0.00006 \mathrm{mg} / 1$ & 1 & \\
\hline diedria 60-57-1 & $<0.00001 \mathrm{mg} / \mathrm{l}$ & $<0.00001 \mathrm{mg} / \mathrm{l}$ & $<0.00001 \mathrm{mg} / \mathrm{l}$ & $<0.00001 \mathrm{mg} / \mathrm{l}$ & 1 & \\
\hline 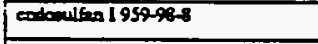 & $<0.00001 \mathrm{mg} / 1$ & $<0.00001 \mathrm{mg} / \mathrm{l}$ & $<0.00001 \mathrm{mg} / \mathrm{l}$ & $<0.00001 \mathrm{mg} / \mathrm{l}$ & 1 & \\
\hline 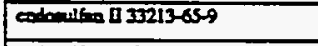 & $<0.00001 \mathrm{mg} / \mathrm{l}$ & $<0.00001 \mathrm{mg} / \mathrm{l}$ & $<0.00001 \mathrm{mg} / \mathrm{l}$ & $<0.00001 \mathrm{mg} / \mathrm{l}$ & 1 & \\
\hline 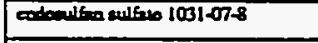 & $<0.00001 \mathrm{mg} / \mathrm{l}$ & $<0.00004 \mathrm{mg} / \mathrm{l}$ & $<0.00001 \mathrm{mg} / \mathrm{h}$ & $<0.00004 \mathrm{mg} / \mathrm{I}$ & 1 & \\
\hline andria $72-20-8$ & $<0.00005 \mathrm{mg} / \mathrm{l}$ & $<0.00005 \mathrm{mg} / 1$ & $<0.00005 \mathrm{mg} / \mathrm{l}$ & $<0.00005 \mathrm{mg} / \mathrm{I}$ & 1 & \\
\hline 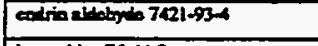 & $<0.00001 \mathrm{mg} / \mathrm{l}$ & $<0.00004 \mathrm{mg} / \mathrm{l}$ & $<0.00001 \mathrm{mg} / \mathrm{l}$ & $<0.00004 \mathrm{mg} / \mathrm{l}$ & 1 & \\
\hline bepectior 7648 & $<0.00001 \mathrm{mg} / \mathrm{l}$ & $<0.00001 \mathrm{mg} / \mathrm{l}$ & $<0.00001 \mathrm{mg} / \mathrm{l}$ & $<0.00001 \mathrm{mg} / \mathrm{l}$ & 1 & 2 \\
\hline pocotior apacido $1024-57-3$ & $<0.00001 \mathrm{mg} / \mathrm{l}$ & $<0.00001 \mathrm{mg} / \mathrm{h}$ & $<0.00001 \mathrm{mg} / \mathrm{l}$ & $<0.00001 \mathrm{mg} / 1$ & 1 & \\
\hline $001-35-2$ & $<0.0002 \mathrm{mg} / \mathrm{l}$ & $<0.0006 \mathrm{mg} / \mathrm{l}$ & $<0.0002 \mathrm{mg} / \mathrm{l}$ & $<0.0006 \mathrm{mg} / \mathrm{h}$ & 1 & \\
\hline $46-8 K C 31986$ & $<0.00001 \mathrm{mg} / \mathrm{l}$ & $<0.00001 \mathrm{mg} / \mathrm{l}$ & $<0.00001 \mathrm{mg} / \mathrm{h}$ & $<0.00001 \mathrm{mg} / \mathrm{l}$ & 1 & \\
\hline-8 - 31923.7 & $<0.00001 \mathrm{mg} / 1$ & $<0.00001 \mathrm{mg} / \mathrm{h}$ & $<0.00001 \mathrm{mg} / \mathrm{t}$ & $<0.00001 \mathrm{mg} / \mathrm{l}$ & 1 & \\
\hline DE-BHC 319268 & $<0.00001 \mathrm{mg} / \mathrm{h}$ & $<0.00001 \mathrm{mg} / \mathrm{l}$ & $<0.00001 \mathrm{mg} / 1$ & $<0.00001 \mathrm{mg} / \mathrm{l}$ & 1 & \\
\hline $50.94 C 58+94$ & $<0.00001 \mathrm{mg} / \mathrm{l}$ & $<0.00001 \mathrm{mg} / \mathrm{l}$ & $<0.00001 \mathrm{mg} / 1$ & $<0.00001 \mathrm{mg} / 1$ & 1 & \\
\hline 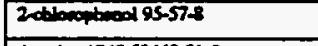 & $<0.030 \mathrm{mg} / \mathrm{l}$ & $<0.030 \mathrm{mg} / \mathrm{l}$ & $<0.030 \mathrm{mg} / \mathrm{l}$ & $<0.030 \mathrm{mg} / 1$ & 1 & $\therefore$ \\
\hline Arodon-121232536021-9 & $<0.0005 \mathrm{mg} / \mathrm{l}$ & $<0.0005 \mathrm{mg} / \mathrm{l}$ & $<0.0005 \mathrm{mg} / \mathrm{l}$ & $<0.0005 \mathrm{mg} / \mathrm{l}$ & 1 & - \\
\hline Arodoen-12se 11097691 & $<0.0005 \mathrm{mg} / \mathrm{l}$ & $<0.0005 \mathrm{mg} / \mathrm{l}$ & $<0.0005 \mathrm{mg} / \mathrm{l}$ & $<0.0005 \mathrm{mg} / \mathrm{l}$ & 1 & \\
\hline Arodor-121 11104-22.2 & $<0.0005 \mathrm{mg} / \mathrm{l}$ & $<0.0005 \mathrm{mg} / \mathrm{l}$ & $<0.0005 \mathrm{mg} / \mathrm{l}$ & $<0.0005 \mathrm{mg} / \mathrm{l}$ & 1 & \\
\hline Arodor-1232 II141-16-S & $<0.0005 \mathrm{mg} / \mathrm{l}$ & $<0.0005 \mathrm{mg} / \mathrm{l}$ & $<0.0005 \mathrm{mg} / \mathrm{l}$ & $<0.0005 \mathrm{mg} / \mathrm{l}$ & 1 & \\
\hline Arodor-1248 12072-29-6 & $<0.0005 \mathrm{mg} / \mathrm{l}$ & $<0.0005 \mathrm{mg} / \mathrm{l}$ & $<0.0005 \mathrm{mg} / \mathrm{l}$ & $<0.0005 \mathrm{mg} / \mathrm{l}$ & 1 & \\
\hline Arodon 12601100682.5 & $<0.0005 \mathrm{mg} / \mathrm{l}$ & $<0.0005 \mathrm{mg} / \mathrm{l}$ & $<0.0005 \mathrm{mg} / \mathrm{l}$ & $<0.0005 \mathrm{mg} / \mathrm{l}$ & 1 & \\
\hline Arodo-1016 12674-11-2 & $<0.0005 \mathrm{mg} / \mathrm{l}$ & $<0.0005 \mathrm{mg} / 1$ & $<0.0005 \mathrm{mg} / 1$ & $<0.0005 \mathrm{mg} / \mathrm{l}$ & 1 & \\
\hline Croosis & $<0.010 \mathrm{mg} / \mathrm{l}$ & $<0.010 \mathrm{mg} / \mathrm{l}$ & $<0.010 \mathrm{mg} / \mathrm{l}$ & $<0.010 \mathrm{mg} / \mathrm{l}$ & 1 & \\
\hline Acoutein & $<0.010 \mathrm{mg} / \mathrm{l}$ & $<0.010 \mathrm{mg} / \mathrm{l}$ & $<0.010 \mathrm{mg} / \mathrm{l}$ & $<0.010 \mathrm{mg} / \mathrm{l}$ & 1 & \\
\hline Aarykovitrite & $<0.010 \mathrm{mg} / \mathrm{l}$ & $<0.010 \mathrm{mg} / \mathrm{l}$ & $<0.010 \mathrm{mg} / \mathrm{l}$ & $<0.010 \mathrm{mg} / 1$ & 1 & \\
\hline 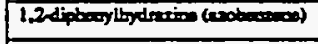 & Rewults pending & Recults pending & Resules pending & Reaulu pending & 1 & \\
\hline & & & & & & \\
\hline & & & & & & \\
\hline & & & & & & \\
\hline & & & & & & \\
\hline & & & & & & \\
\hline & & & & & & \\
\hline & & & & & & \\
\hline & & & & & & \\
\hline & & & & & & \\
\hline & & & & & & \\
\hline & & & & & & \\
\hline & & & & & & \\
\hline & & & & & & \\
\hline & & & & & & \\
\hline
\end{tabular}


VII. Discharge Information (contioued from page 3 of Form $2 F$

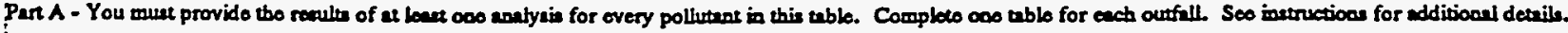

\begin{tabular}{|c|c|c|c|c|c|c|}
\hline \multirow{2}{*}{$\begin{array}{l}\text { Pollunent } \\
\text { and } \\
\text { CAS Number } \\
\text { (if avilible) }\end{array}$} & \multicolumn{2}{|c|}{$\begin{array}{l}\text { Maximum Valuea } \\
\text { (include units) }\end{array}$} & \multicolumn{2}{|c|}{$\begin{array}{l}\text { Average Vahues } \\
\text { (inchude unitu) }\end{array}$} & \multirow{2}{*}{$\begin{array}{l}\text { Number } \\
\text { of } \\
\text { Storm } \\
\text { Eveets } \\
\text { Srmplod }\end{array}$} & \multirow[b]{2}{*}{ Sources of Pollueneses } \\
\hline & $\begin{array}{c}\text { Grab Sumple } \\
\text { Takeo During } \\
\text { First } 30 \\
\text { Minurou }\end{array}$ & $\begin{array}{l}\text { Fow-weightod } \\
\text { Comporito }\end{array}$ & $\begin{array}{c}\text { Grab Semplo } \\
\text { Tekee During } \\
\text { Firm } 30 \\
\text { Minuteo }\end{array}$ & $\begin{array}{l}\text { Fow-weighted } \\
\text { Compocite }\end{array}$ & & \\
\hline pit and Oranes & $<2 \mathrm{mg} / 1$ & & $<2 \mathrm{mg} / \mathrm{l}$ & & 1 & \\
\hline Biolopial Oxyean Demend (BOD) & $<5 \mathrm{mg} / 1$ & & $<5 \mathrm{mg} / \mathrm{l}$ & & 1 & \\
\hline Cramial Oxy Danod (COD) & $8.4 \mathrm{mg} / \mathrm{l}$ & & $8.4 \mathrm{mg} / \mathrm{l}$ & & 1 & \\
\hline Polal Surpooded Solide (TSS) & $<5 \mathrm{mg} / \mathrm{l}$ & & $<5 \mathrm{mg} / \mathrm{l}$ & & 1 & \\
\hline 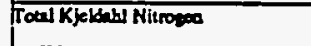 & $0.3 \mathrm{mg} / \mathrm{l}$ & & $0.3 \mathrm{mg} / \mathrm{l}$ & & 1 & \\
\hline Nitrene plue Nitrice Nitropon & $<0.1 \mathrm{mg} / 1$ & & $<0.1 \mathrm{mg} / \mathrm{l}$ & & 1 & \\
\hline Toul Phooptonse & $<0.1 \mathrm{mg} / \mathrm{l}$ & & $<0.1 \mathrm{mg} / 1$ & & 1 & \\
\hline
\end{tabular}

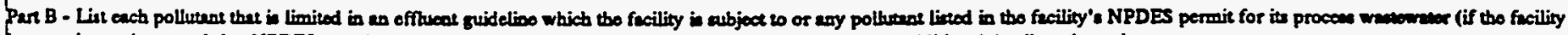

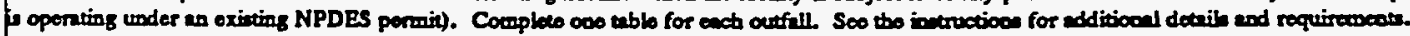

\begin{tabular}{|c|c|c|c|c|c|c|}
\hline \multirow{2}{*}{$\begin{array}{l}\text { Polbunane } \\
\text { and } \\
\text { CAs Number } \\
\text { (if availible) }\end{array}$} & \multicolumn{2}{|c|}{$\begin{array}{l}\text { Maximum Vabued } \\
\text { (inoludo unito) }\end{array}$} & \multicolumn{2}{|c|}{$\begin{array}{l}\text { Averuge Vahese } \\
\text { (inchedo unies) }\end{array}$} & \multirow{2}{*}{$\begin{array}{c}\text { Nuraber } \\
\text { of } \\
\text { Storem } \\
\text { Eveote } \\
\text { Seropled }\end{array}$} & \multirow{2}{*}{$\begin{array}{l}: \\
\text { Soursece of Polluteres }\end{array}$} \\
\hline & $\begin{array}{c}\text { Grab Semple } \\
\text { Takm During } \\
\text { First } 30 \\
\text { Minetee }\end{array}$ & $\begin{array}{l}\text { Flow-woizhrod } \\
\text { Compocite }\end{array}$ & $\begin{array}{c}\text { Grab Samplo } \\
\text { Triken Durins } \\
\text { Fint } 30 \\
\text { Mindes }\end{array}$ & $\begin{array}{l}\text { Flow-weigtrod } \\
\text { Componite }\end{array}$ & & \\
\hline Animoor 7400-360 & $<0.04 \mathrm{mg} / \mathrm{l}$ & & $<0.04 \mathrm{mg} / \mathrm{l}$ & & 1 & \\
\hline anoin & $<0.002 \mathrm{mg} / \mathrm{l}$ & & $<0.002 \mathrm{mg} / \mathrm{l}$ & & 1 & \\
\hline Mnemy 7439076 & $<0.0002 \mathrm{mg} / 1$ & & $<0.0002 \mathrm{mg} / \mathrm{l}$ & & 1 & \\
\hline Prodeds & $0.002 \mathrm{mg} / \mathrm{l}$ & & $0.002 \mathrm{mg} / \mathrm{l}$ & & 1 & $i^{\prime}$ \\
\hline$=6 \operatorname{lom} 782-192$ & $<0.002 \mathrm{mg} / 1$ & & $<0.002 \mathrm{mg} / \mathrm{l}$ & & 1 & \\
\hline Pulate & $16 \mathrm{mg} / \mathrm{l}$ & & $16 \mathrm{mg} / \mathrm{l}$ & & 1 & \\
\hline Purfactense & $<0.05 \mathrm{mg} / \mathrm{l}$ & & $<0.05 \mathrm{mg} / 1$ & & 1 & \\
\hline Thentave $7429-90-5$ & $0.06 \mathrm{mg} / \mathrm{l}$ & & $0.06 \mathrm{mg} / 1$ & & 1 & \\
\hline Arsodo 7400-38-2 & $<0.04 \mathrm{mg} / \mathrm{l}$ & & $<0.04 \mathrm{mg} / \mathrm{l}$ & & 1 & \\
\hline $800000740-125$ & $0.103 \mathrm{mg} / \mathrm{h}$ & & $0.103 \mathrm{mg} / \mathrm{l}$ & & 1 & \\
\hline Banglithen 740-1.7 & $<0.0004 \mathrm{mg} / \mathrm{l}$ & & $<0.0004 \mathrm{mg} / \mathrm{l}$ & & 1 & \\
\hline Cataing 7440-43-9 & $<0.004 \mathrm{mg} / \mathrm{l}$ & & $<0.004 \mathrm{mg} / \mathrm{l}$ & & 1 & \\
\hline Calotum 740-70-2 & $29.9 \mathrm{mg} / \mathrm{l}$ & & $29.9 \mathrm{mg} / \mathrm{l}$ & & 1 & \\
\hline Cbraming 740-47-3 & $<0.006 \mathrm{mg} / \mathrm{l}$ & & $<0.006 \mathrm{mg} / \mathrm{l}$ & & 1 & \\
\hline Cotall 74to-484 & $<0.002 \mathrm{mg} / \mathrm{l}$ & & $<0.002 \mathrm{mg} / \mathrm{l}$ & & 1 & \\
\hline Copper 74k0-50-8 & $<0.006 \mathrm{mg} / \mathrm{l}$ & & $<0.006 \mathrm{mg} / \mathrm{l}$ & & 1 & \\
\hline 5007439096 & $<0.06 \mathrm{mg} / \mathrm{l}$ & & $<0.06 \mathrm{mg} / \mathrm{l}$ & & 1 & \\
\hline 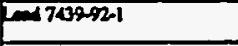 & $<0.02 \mathrm{mg} / 1$ & & $<0.02 \mathrm{mg} / 1$ & & 1 & \\
\hline Ththam 7/39-93-2 & $0.02 \mathrm{mg} / \mathrm{l}$ & & $0.02 \mathrm{mg} / \mathrm{l}$ & & 1 & \\
\hline Maposian 743995-4 & $9.91 \mathrm{mg} / \mathrm{l}$ & & $9.91 \mathrm{mg} / \mathrm{l}$ & & 1 & \\
\hline $7400000-5$ & $0.002 \mathrm{mg} / 1$ & & $0.002 \mathrm{mg} / 1$ & & 1 & \\
\hline 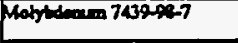 & $0.009 \mathrm{mg} / \mathrm{I}$ & & $0.009 \mathrm{mg} / \mathrm{l}$ & & 1 & \\
\hline Nibled 7400000 & $<0.008 \mathrm{mg} / \mathrm{l}$ & & $<0.008 \mathrm{mg} / 1$ & & 1 & \\
\hline 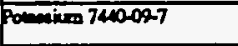 & $1.9 \mathrm{mg} / 1$ & & $1.9 \mathrm{mg} / \mathrm{l}$ & & 1 & \\
\hline $5 \sin =7400-224$ & $<0.006 \mathrm{mg} / \mathrm{l}$ & & $<0.006 \mathrm{mg} / 1$ & & 1 & \\
\hline soliven 74ta-22-3 & $2.09 \mathrm{mg} / \mathrm{l}$ & & $2.09 \mathrm{mg} / 1$ & & 1 & \\
\hline 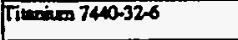 & $<0.01 \mathrm{mg} / 1$ & & $<0.01 \mathrm{mg} / \mathrm{l}$ & & 1 & \\
\hline 21007400666 & $<0.01 \mathrm{mg} / 1$ & & $<0.01 \mathrm{mg} / \mathrm{l}$ & & 1 & \\
\hline
\end{tabular}


Continued from froot.

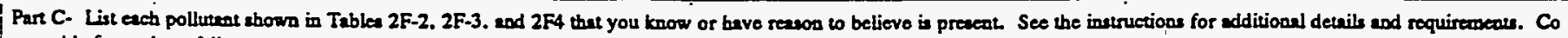
oso uabie for each outfall.

\begin{tabular}{|c|c|c|c|c|c|c|}
\hline \multirow{2}{*}{$\begin{array}{c}\text { Polturent } \\
\text { and } \\
\text { CAS Number } \\
\text { (if availible) }\end{array}$} & \multicolumn{2}{|c|}{$\begin{array}{l}\text { Meximum Values } \\
\text { (inchude unive) }\end{array}$} & \multicolumn{2}{|c|}{$\begin{array}{l}\text { Average Valuea } \\
\text { (inchude units) }\end{array}$} & \multirow{2}{*}{\begin{tabular}{|c|} 
\\
Number \\
of \\
Storm \\
Eveatu \\
Sempled \\
\end{tabular}} & \multirow[b]{2}{*}{ Souroces of Pollutents } \\
\hline & $\begin{array}{l}\text { Grab Semple } \\
\text { Taken During } \\
\text { Fint } 30 \\
\text { Minutea }\end{array}$ & $\begin{array}{l}\text { Flow-weightod } \\
\text { Comporite }\end{array}$ & $\begin{array}{l}\text { Grab Semple } \\
\text { Taken During } \\
\text { Firn } 30 \\
\text { Minutea }\end{array}$ & $\begin{array}{l}\text { Flow-weighted } \\
\text { Comporite }\end{array}$ & & \\
\hline 1.1.1.1.enictborodberes $71.55-6$ & $<0.010 \mathrm{mg} / \mathrm{l}$ & & $<0.010 \mathrm{mg} / \mathrm{l}$ & & 1 & \\
\hline 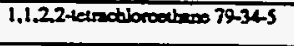 & $<0.010 \mathrm{mg} / \mathrm{l}$ & & $<0.010 \mathrm{mg} / 1$ & & 1 & \\
\hline 1.1.2- Irichborod beses 7900.5 & $<0.010 \mathrm{mg} / \mathrm{l}$ & & $<0.010 \mathrm{mg} / \mathrm{l}$ & & 1 & \\
\hline 1.1 dieblowodheres 75-34-3 & $<0.010 \mathrm{mg} / \mathrm{l}$ & & $<0.010 \mathrm{mg} / \mathrm{l}$ & & 1 & \\
\hline 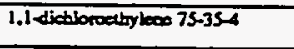 & $<0.010 \mathrm{mg} / \mathrm{l}$ & & $<0.010 \mathrm{mg} / \mathrm{l}$ & & 1 & \\
\hline 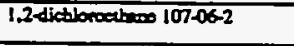 & $<0.010 \mathrm{mg} / \mathrm{l}$ & & $<0.010 \mathrm{mg} / 1$ & & 1 & \\
\hline 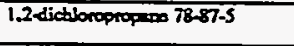 & $<0.010 \mathrm{mg} / \mathrm{l}$ & & $<0.010 \mathrm{mg} / \mathrm{l}$ & & 1 & \\
\hline 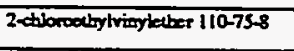 & $<0.010 \mathrm{mg} / \mathrm{l}$ & & $<0.010 \mathrm{mg} / \mathrm{l}$ & & 1 & \\
\hline $71-43-2$ & $<0.010 \mathrm{mg} / \mathrm{l}$ & & $<0.010 \mathrm{mg} / \mathrm{l}$ & & 1 & \\
\hline 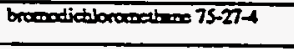 & $<0.010 \mathrm{mg} / \mathrm{l}$ & & $<0.010 \mathrm{mg} / \mathrm{l}$ & & 1 & \\
\hline broendorne $75-25-2$ & $<0.010 \mathrm{mg} / \mathrm{l}$ & & $<0.010 \mathrm{mg} / 1$ & & 1 & \\
\hline $7483-9$ & $<0.010 \mathrm{mg} / \mathrm{l}$ & & $<0.010 \mathrm{mg} / 1$ & & 1 & \\
\hline arteon verndibrido $56-22-5$ & $<0.010 \mathrm{mg} / \mathrm{l}$ & & $<0.010 \mathrm{mg} / \mathrm{l}$ & & 1 & \\
\hline $108-90-7$ & $<0.010 \mathrm{mg} / \mathrm{l}$ & & $<0.010 \mathrm{mg} / 1$ & & 1 & \\
\hline $75+00-3$ & $<0.010 \mathrm{mg} / \mathrm{l}$ & & $<0.010 \mathrm{mg} / \mathrm{l}$ & & 1 & \\
\hline dboroform 67-66-3 & $<0.010 \mathrm{mg} / \mathrm{l}$ & & $<0.010 \mathrm{mg} / \mathrm{l}$ & & 1 &. \\
\hline 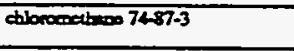 & $<0.010 \mathrm{mg} / \mathrm{l}$ & & $<0.010 \mathrm{mg} / \mathrm{l}$ & & 1 & \\
\hline 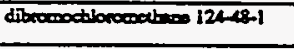 & $<0.010 \mathrm{mg} / \mathrm{l}$ & & $<0.010 \mathrm{mg} / 1$ & & 1 & \\
\hline $100-114$ & $<0.010 \mathrm{mg} / \mathrm{l}$ & & $-<0.010 \mathrm{mg} / 1$ & & 1 & \\
\hline when $108-3$ & $<0.010 \mathrm{mg} / \mathrm{l}$ & & $<0.010 \mathrm{mg} / \mathrm{l}$ & & 1 & \\
\hline ving chboribs 75-OIA & $<0.010 \mathrm{mg} / \mathrm{l}$ & & $<0.010 \mathrm{mg} / 1$ & & 1 & \\
\hline 1.3-dictbropopopen 10061.01-5 & $<0.010 \mathrm{mg} / \mathrm{l}$ & & $<0.010 \mathrm{mg} / 1$ & & 1 & \\
\hline 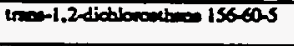 & $<0.010 \mathrm{mg} / \mathrm{l}$ & & $<0.010 \mathrm{mg} / 1$ & & 1 & : \\
\hline alphe sctivgy $1258746-1$ & -1.5 pcill & & $-1.5 \mathrm{pci} / 1$ & & 1 & \\
\hline bese samply $12587+46-1$ & $8.4 \mathrm{pci} / \mathrm{l}$ & & $8.4 \mathrm{pci} / \mathrm{l}$ & & 1 & \\
\hline rocal colitorm & $20 \mathrm{co} / .1 \mathrm{~L}$ & & $20 \mathrm{col} .1 \mathrm{~L}$ & & 1 & \\
\hline Tadlinem 7440-23-0 & $<0.005 \mathrm{mg} / \mathrm{l}$ & & $<0.005 \mathrm{mg} / \mathrm{l}$ & & 1 & \\
\hline Unanrun 7440-61-1 & $<0.001 \mathrm{mg} / \mathrm{l}$ & & $<0.001 \mathrm{mg} / 1$ & & 1 & \\
\hline Gemene savity & $5.0 \mathrm{pci} / \mathrm{l}$ & & $5.0 \mathrm{peill}$ & & 1 & \\
\hline . & & & & & & \\
\hline
\end{tabular}

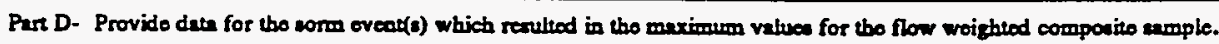

\begin{tabular}{|c|c|c|c|c|c|c|c|}
\hline $\begin{array}{l}1 . \\
\text { Date of } \\
\text { Storm } \\
\text { Evens }\end{array}$ & $\begin{array}{l}2 . \\
\text { Duration } \\
\text { of Storm } \\
\text { (in } \\
\text { mininea) }\end{array}$ & $\begin{array}{l}3 . \\
\text { Tooel ninfill } \\
\text { during etorm oveas } \\
\text { (in incbea) }\end{array}$ & 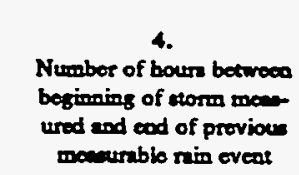 & $\begin{array}{l}\text { S. } \\
\text { Maximum flow rate } \\
\text { during nin eveat } \\
\text { (alloos/min or specify } \\
\text { unis) }\end{array}$ & $\begin{array}{c}6 . \\
\text { Total flow from } \\
\text { nim oveas } \\
\text { (oallonis or } \\
\text { spocify waits) }\end{array}$ & $\begin{array}{c}7 . \\
\text { Season } \\
\text { eample wase } \\
\text { tuken }\end{array}$ & $\begin{array}{l}8 . \\
\text { Eorm of } \\
\text { Procipintion } \\
\text { (niafill. } \\
\text { coommeth) }\end{array}$ \\
\hline $08 / 26 / 91$ & 410 & 1.3 & 160.2 & & & Summer & Rein \\
\hline
\end{tabular}

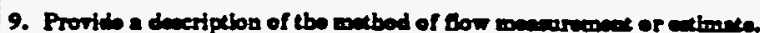

An ISCO 3230 Flow meter was used to obtain water level rendings. This instrument was calibrated with the normal dry weather flow, if present, as leves zero. The flow meter was programmed with the characteristics of the conveyance so that flow rates and total flows could be calculated by the meter. 
Outfall S19

- Parte B and C Continued

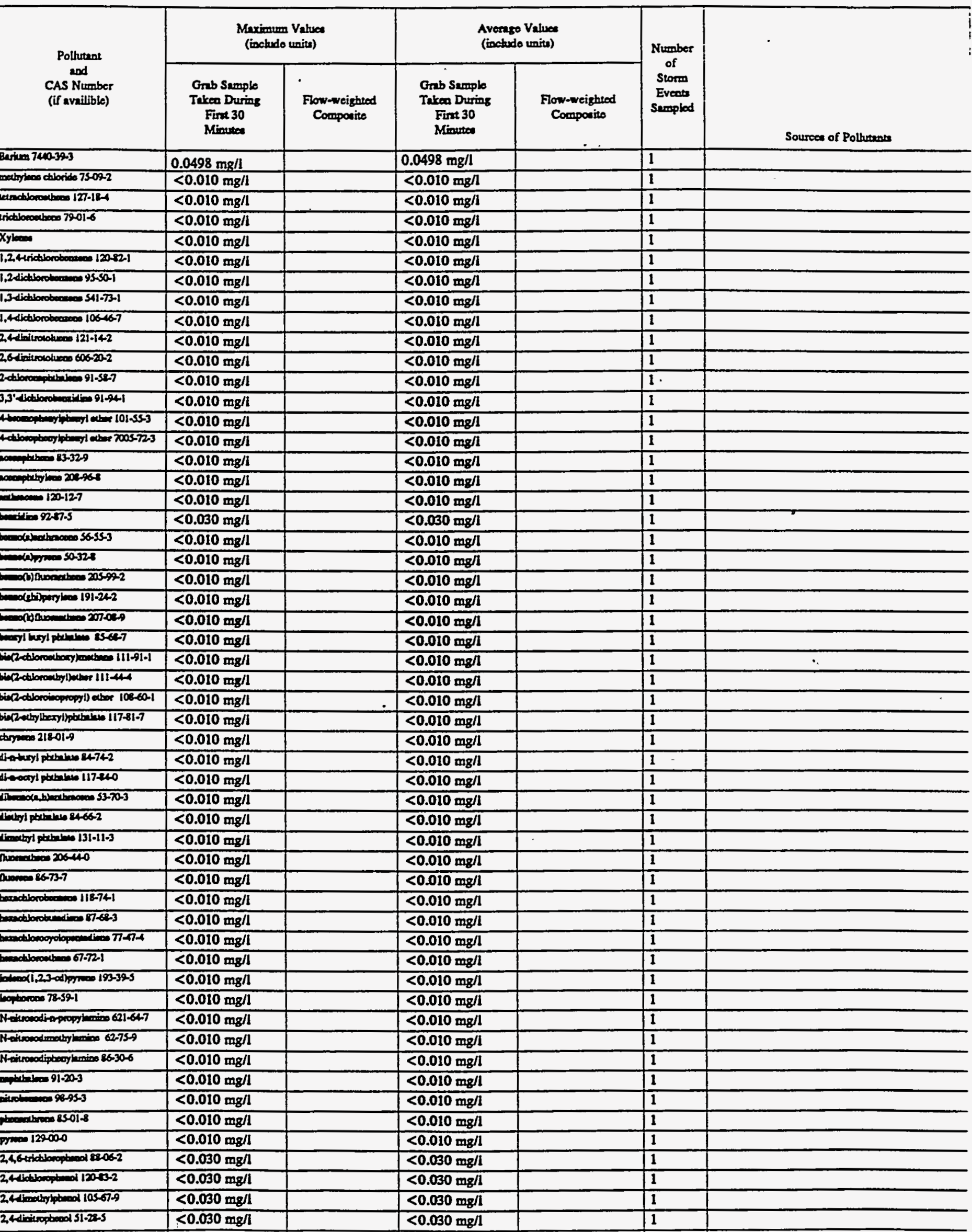


Outfall S19

Parts B and C Continuad

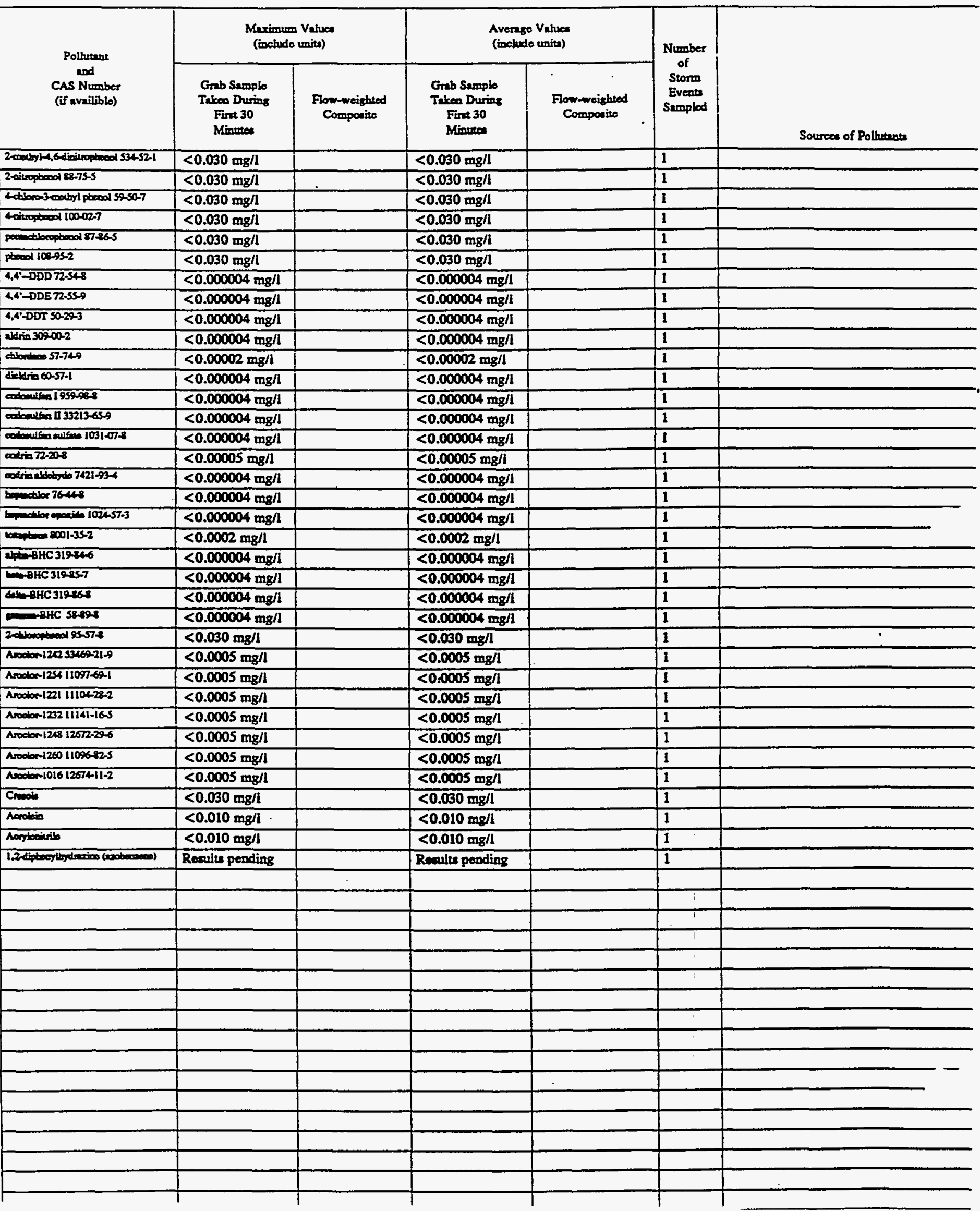


VII. Dischnrge Information (coatioued from page 3 of Form 2P

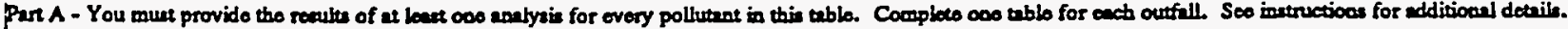

\begin{tabular}{|c|c|c|c|c|c|c|}
\hline \multirow{2}{*}{$\begin{array}{c}\text { Pollutant } \\
\text { and } \\
\text { CAs Number } \\
\text { (if availible) }\end{array}$} & \multicolumn{2}{|c|}{$\begin{array}{l}\text { Maximum Values } \\
\text { (include units) }\end{array}$} & \multicolumn{2}{|c|}{$\begin{array}{l}\text { Avernge Vahuea } \\
\text { (inchude unitu) }\end{array}$} & \multirow{2}{*}{$\begin{array}{l}\text { Numbet } \\
\text { of } \\
\text { Stomm } \\
\text { Eveots } \\
\text { Srmplad }\end{array}$} & \multirow[b]{2}{*}{ Sounces of Polthand } \\
\hline & $\begin{array}{c}\text { Grb Samplo } \\
\text { Takea During } \\
\text { Fint } 30 \\
\text { Minures }\end{array}$ & $\begin{array}{l}\text { Flow-weighted } \\
\text { Componito }\end{array}$ & $\begin{array}{c}\text { Greb Samplo } \\
\text { Tuken During } \\
\text { Firat } 30 \\
\text { Minures }\end{array}$ & $\begin{array}{l}\text { Flow-weithted } \\
\text { Compoits }\end{array}$ & & \\
\hline pil end Graves & $<2 \mathrm{mg} / \mathrm{l}$ & & $<2 \mathrm{mg} / 1$ & & 1 & \\
\hline Bialopial Oxyeson Deandin (BODS) & $<5 \mathrm{mg} / \mathrm{l}$ & $<5 \mathrm{mg} / 1$ & $<5 \mathrm{mg} / 1$ & $<5 \mathrm{mg} / \mathrm{l}$ & 1 & \\
\hline 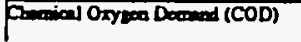 & $26 \mathrm{mg} / \mathrm{l}$ & $37 \mathrm{mg} / 1$ & $26 \mathrm{mg} / 1$ & $37 \mathrm{mg} / 1$ & 1 & \\
\hline Totel Sopendod Solde (TSG) & $<5 \mathrm{mg} / \mathrm{l}$ & $20 \mathrm{mg} / 1$ & $<5 \mathrm{mg} / 1$ & $20 \mathrm{mg} / 1$ & 1 & \\
\hline Total Kjeldebl Nitroped & $1 \mathrm{mg} / \mathrm{l}$ & $0.62 \mathrm{mg} / \mathrm{l}$ & $1 \mathrm{mg} / \mathrm{l}$ & $0.62 \mathrm{mg} / \mathrm{l}$ & 1 & \\
\hline Nitrie plus Nitrise Nitroged & $1.3 \mathrm{mg} / 1$ & $4.6 \mathrm{mg} / \mathrm{l}$ & $1.3 \mathrm{mg} / 1$ & $4.6 \mathrm{mg} / \mathrm{l}$ & 1 & \\
\hline Toeal Phoeptorsu & $<0.1 \mathrm{mg} / 1$ & $0.2 \mathrm{mg} / \mathrm{l}$ & $<0.1 \mathrm{mg} / 1$ & $0.2 \mathrm{mg} / 1$ & 1 & \\
\hline $\mathrm{PH}$ & Miningen & Meximen 8.0 & $M i=8.0$ & Maximan & & \\
\hline
\end{tabular}

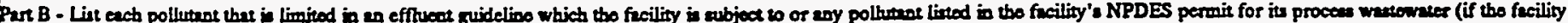

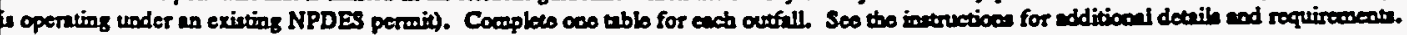

\begin{tabular}{|c|c|c|c|c|c|c|}
\hline \multirow{2}{*}{$\begin{array}{l}\text { Polhutane } \\
\text { eod } \\
\text { CAs Number } \\
\text { (if nviliblo) }\end{array}$} & \multicolumn{2}{|c|}{$\begin{array}{l}\text { Mexienum Valuce } \\
\text { (pl2budo unitu) }\end{array}$} & \multicolumn{2}{|c|}{$\begin{array}{l}\text { Averige Vahuen } \\
\text { (imabudo unita) }\end{array}$} & \multirow{2}{*}{$\begin{array}{l}\text { Number } \\
\text { of } \\
\text { Storm } \\
\text { Eveno } \\
\text { Semplod }\end{array}$} & \multirow{2}{*}{$\begin{array}{c}: \\
\text { Sources of Polkunass }\end{array}$} \\
\hline & $\begin{array}{c}\text { Orab Samplo } \\
\text { Tatua During } \\
\text { Fine } 30 \\
\text { Mrosece }\end{array}$ & $\begin{array}{l}\text { Flow-weighted } \\
\text { Compocito }\end{array}$ & 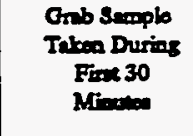 & $\begin{array}{l}\text { From-mrighed } \\
\text { Componiso }\end{array}$ & & \\
\hline Trim $=00740-360$ & $<0.04 \mathrm{mg} / 1$ & $<0.04 \mathrm{mg} / \mathrm{l}$ & $<0.04 \mathrm{mg} / \mathrm{l}$ & $<0.04 \mathrm{mg} / \mathrm{l}$ & 1 & \\
\hline xaming & $<0.002 \mathrm{mg} / \mathrm{h}$ & & $<0.002 \mathrm{mg} / \mathrm{l}$ & & 1 & \\
\hline Thenen $7439-976$ & $0.0003 \mathrm{mg} / \mathrm{l}$ & $0.0003 \mathrm{mg} / \mathrm{l}$ & $0.0003 \mathrm{mg} / \mathrm{l}$ & $0.0003 \mathrm{mg} / \mathrm{l}$ & 1 & \\
\hline then & $0.010 \mathrm{mg} / \mathrm{l}$ & & $0.010 \mathrm{mg} / 1$ & & 1 & $\because$ \\
\hline Aleiven $7782-49-2$ & $<0.002 \mathrm{mg} / \mathrm{l}$ & $<0.002 \mathrm{mg} / \mathrm{l}$ & $<0.002 \mathrm{mg} / \mathrm{l}$ & $<0.002 \mathrm{mg} / \mathrm{l}$ & 1 & \\
\hline Pulfor & $12 \mathrm{mg} / 1$ & $40 \mathrm{mg} / \mathrm{l}$ & $12 \mathrm{mg} / \mathrm{l}$ & $40 \mathrm{mg} / \mathrm{I}$ & 1 & \\
\hline 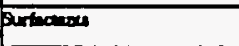 & $0.06 \mathrm{mg} / \mathrm{l}$ & $<0.05 \mathrm{mg} / 1$ & $0.06 \mathrm{mg} / \mathrm{l}$ & $<0.05 \mathrm{mg} / \mathrm{l}$ & 1 & \\
\hline chaminam 7420-90-5 & $0.15 \mathrm{mg} / \mathrm{l}$ & $1.54 \mathrm{mg} / \mathrm{l}$ & $0.15 \mathrm{mg} / 1$ & $1.54 \mathrm{mg} / 1$ & 1 & \\
\hline 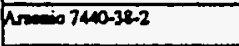 & $<0.04 \mathrm{mg} / \mathrm{l}$ & $<0.04 \mathrm{mg} / \mathrm{l}$ & $<0.04 \mathrm{mg} / 1$ & $<0.04 \mathrm{mg} / \mathrm{l}$ & 1 & \\
\hline $80000740-128$ & $0.012 \mathrm{mg} / 1$ & $0.014 \mathrm{mg} / \mathrm{l}$ & $0.012 \mathrm{mg} / 1$ & $0.014 \mathrm{mg} / \mathrm{l}$ & 1 & \\
\hline Derplliven 7400-11-7 & $<0.0004 \mathrm{mg} / \mathrm{l}$ & $<0.0004 \mathrm{mg} / 1$ & $<0.0004 \mathrm{mg} / 1$ & $<0.0004 \mathrm{mg} / \mathrm{l}$ & 1 & \\
\hline admine 740-43-9 & $<0.004 \mathrm{mg} / \mathrm{l}$ & $<0.004 \mathrm{mg} / \mathrm{l}$ & $<0.004 \mathrm{mg} / \mathrm{h}$ & $<0.004 \mathrm{mg} / \mathrm{l}$ & 1 & \\
\hline Celcter 7410-70-2 & $7.1 \mathrm{mg} / 1$ & $15.4 \mathrm{mg} / \mathrm{l}$ & $7.1 \mathrm{mg} / \mathrm{l}$ & $15.4 \mathrm{mg} / \mathrm{l}$ & 1 & \\
\hline Corouningen 7400-7.3 & $<0.006 \mathrm{mg} / \mathrm{l}$ & $<0.006 \mathrm{mg} / \mathrm{l}$ & $<0.006 \mathrm{mg} / \mathrm{l}$ & $<0.006 \mathrm{mg} / 1$ & 1 & \\
\hline Colatis 7400-48-4 & $<0.002 \mathrm{mg} / \mathrm{l}$ & $<0.002 \mathrm{mg} / \mathrm{l}$ & $<0.002 \mathrm{mg} / 1$ & $<0.002 \mathrm{mg} / 1$ & 1 & \\
\hline Coppos 7440-50-8 & $<0.006 \mathrm{mg} / \mathrm{l}$ & $<0.006 \mathrm{mg} / \mathrm{l}$ & $<0.006 \mathrm{mg} / \mathrm{l}$ & $<0.006 \mathrm{mg} / \mathrm{l}$ & 1 & \\
\hline $50007439-696$ & $0.20 \mathrm{mg} / \mathrm{I}$ & $1.84 \mathrm{mg} / \mathrm{l}$ & $0.20 \mathrm{mg} / \mathrm{l}$ & $1.84 \mathrm{mg} / 1$ & 1 & \\
\hline 7nd 70992-1 & $<0.02$ mg/1 & $<0.02 \mathrm{mg} / \mathrm{l}$ & $<0.02 \mathrm{mg} / \mathrm{I}$ & $<0.02 \mathrm{mg} / 1$ & 1 & \\
\hline Intinen 7439-93-2 & $<0.02 \mathrm{mg} / \mathrm{l}$ & $<0.02 \mathrm{mg} / 1$ & $<0.02 \mathrm{mg} / \mathrm{l}$ & $<0.02 \mathrm{mg} / \mathrm{l}$ & 1 & \\
\hline 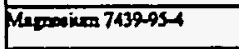 & $1.55 \mathrm{mg} / \mathrm{l}$ & $3.00 \mathrm{mg} / 1$ & $1.55 \mathrm{mg} / \mathrm{l}$ & $3.00 \mathrm{mg} / \mathrm{l}$ & 1 & \\
\hline 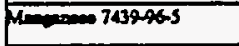 & $0.010 \mathrm{mg} / \mathrm{l}$ & $0.052 \mathrm{mg} / \mathrm{l}$ & $0.010 \mathrm{mg} / \mathrm{l}$ & $0.052 \mathrm{mg} / \mathrm{l}$ & 1 & \\
\hline 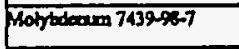 & $<0.006 \mathrm{mg} / \mathrm{l}$ & $<0.006 \mathrm{mg} / \mathrm{l}$ & $<0.006 \mathrm{mg} / \mathrm{l}$ & $<0.006 \mathrm{mg} / \mathrm{l}$ & 1 & \\
\hline Nobel 7400-020 & $<0.008 \mathrm{mg} / \mathrm{l}$ & $<0.008 \mathrm{mg} / \mathrm{l}$ & $<0.008 \mathrm{mg} / 1$ & $<0.008 \mathrm{mg} / \mathrm{l}$ & 1 & \\
\hline Promingm 7400997 & $1.1 \mathrm{mg} / 1$ & $3.4 \mathrm{mg} / 1$ & $1.1 \mathrm{mg} / \mathrm{l}$ & $3.4 \mathrm{mg} / \mathrm{l}$ & 1 & \\
\hline $901 \mathrm{~m}=740-204$ & $<0.006 \mathrm{mg} / 1$ & $<0.006 \mathrm{mg} / 1$ & $<0.006 \mathrm{mg} / 1$ & $<0.006 \mathrm{mg} / \mathrm{l}$ & 1 & \\
\hline 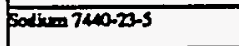 & $0.30 \mathrm{mg} / 1$ & $0.64 \mathrm{mg} / \mathrm{l}$ & $0.30 \mathrm{mg} / \mathrm{l}$ & $0.64 \mathrm{mg} / \mathrm{l}$ & 1 & \\
\hline 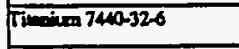 & $<0.01 \mathrm{mg} / \mathrm{l}$ & $0.02 \mathrm{mg} / 1$ & $<0.01 \mathrm{mg} / \mathrm{l}$ & $0.02 \mathrm{mg} / 1$ & 1 & \\
\hline 200740066 & $0.10 \mathrm{mg} / 1$ & $0.08 \mathrm{mg} / \mathrm{l}$ & $0.10 \mathrm{mg} / \mathrm{l}$ & $0.08 \mathrm{mg} / \mathrm{l}$ & 1 & \\
\hline
\end{tabular}


Contineed from front.

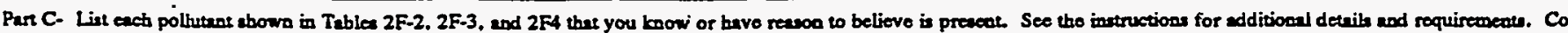
ose tablo for each ourfill.

\begin{tabular}{|c|c|c|c|c|c|c|}
\hline \multirow{2}{*}{$\begin{array}{l}\text { Pollutent } \\
\text { and } \\
\text { CAS Number } \\
\text { (if availible) }\end{array}$} & \multicolumn{2}{|c|}{$\begin{array}{l}\text { Maximum Values } \\
\text { (inchudo units) }\end{array}$} & \multicolumn{2}{|c|}{$\begin{array}{l}\text { Average Values } \\
\text { (inctude unitu) }\end{array}$} & \multirow{2}{*}{$\begin{array}{c}\text { Number } \\
\text { of } \\
\text { Storm } \\
\text { Everts } \\
\text { Sampled }\end{array}$} & \multirow[b]{2}{*}{ Sources of Pollutuns } \\
\hline & $\begin{array}{l}\text { Grub Sample } \\
\text { Takeo During } \\
\text { First } 30 \\
\text { Minute }\end{array}$ & $\begin{array}{l}\text { Flow-weighted } \\
\text { Composite }\end{array}$ & $\begin{array}{c}\text { Gab Sample } \\
\text { Taken During } \\
\text { First } 30 \\
\text { Minute }\end{array}$ & $\begin{array}{l}\text { Flow-weighiod } \\
\text { Comporite }\end{array}$ & & \\
\hline 1.1.1.trictionectione 71-55-6 & $<0.010 \mathrm{mg} / \mathrm{l}$ & & $<0.010 \mathrm{mg} / 1$ & & 1 & \\
\hline 1.1.2.2-vetroctionoethene 79-34-5 & $<0.010 \mathrm{mg} / \mathrm{l}$ & & $<0.010 \mathrm{mg} / \mathrm{l}$ & & 1 & \\
\hline 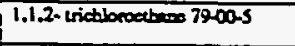 & $<0.010 \mathrm{mg} / 1$ & & $<0.010 \mathrm{mg} / \mathrm{l}$ & & 1 & \\
\hline 1.1 didtoroctareso $75-34-3$ & $<0.010 \mathrm{mg} / \mathrm{l}$ & & $<0.010 \mathrm{mg} / \mathrm{l}$ & & 1 & \\
\hline 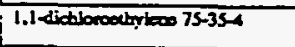 & $<0.010 \mathrm{mg} / \mathrm{l}$ & & $<0.010 \mathrm{mg} / \mathrm{l}$ & & 1 & \\
\hline 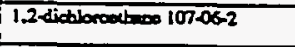 & $<0.010 \mathrm{mg} / \mathrm{l}$ & & $<0.010 \mathrm{mg} / \mathrm{l}$ & & 1 & \\
\hline 1.2-dichloropopopese $78-87-5$ & $<0.010 \mathrm{mg} / \mathrm{l}$ & & $<0.010 \mathrm{mg} / \mathrm{l}$ & & 1 & \\
\hline 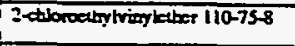 & $<0.010 \mathrm{mg} / \mathrm{l}$ & & $<0.010 \mathrm{mg} / \mathrm{l}$ & & 1 & \\
\hline $7=000007-43-2$ & $<0.010 \mathrm{mg} / \mathrm{l}$ & & $<0.010 \mathrm{mg} / 1$ & & 1 & \\
\hline 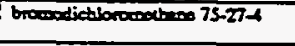 & $<0.010 \mathrm{mg} / \mathrm{l}$ & & $<0.010 \mathrm{mg} / \mathrm{l}$ & & 1 & \\
\hline $75-25-2$ & $<0.010 \mathrm{mg} / 1$ & & $<0.010 \mathrm{mg} / \mathrm{l}$ & & 1 & \\
\hline browecolomes 7483-9 & $<0.010 \mathrm{mg} / \mathrm{l}$ & & $<0.010 \mathrm{mg} / \mathrm{l}$ & & 1 & \\
\hline artwoo merectibrido $56-23-5$ & $<0.010 \mathrm{mg} / \mathrm{l}$ & & $<0.010 \mathrm{mg} / \mathrm{l}$ & & 1 & \\
\hline chborataneos $108-90.7$ & $<0.010 \mathrm{mg} / \mathrm{l}$ & & $<0.010 \mathrm{mg} / \mathrm{l}$ & & 1 & \\
\hline chibroetheres $75-00-3$ & $<0.010 \mathrm{mg} / 1$ & & $<0.010 \mathrm{mg} / \mathrm{l}$ & & 1 & \\
\hline chborofores 67-66-3 & $<0.010 \mathrm{mg} / \mathrm{l}$ & & $<0.010 \mathrm{mg} / \mathrm{l}$ & & 1 & $\therefore$ \\
\hline chbractolation $7487-3$ & $<0.010 \mathrm{mg} / \mathrm{l}$ & & $<0.010 \mathrm{mg} / \mathrm{l}$ & & 1 & \\
\hline 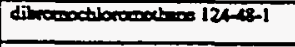 & $<0.010 \mathrm{mg} / \mathrm{l}$ & & $<0.010 \mathrm{mg} / \mathrm{l}$ & & $I$ & \\
\hline alighocesecos 100414 & $<0.010 \mathrm{mg} / \mathrm{l}$ & & $<0.010 \mathrm{mg} / \mathrm{l}$ & & 1 & \\
\hline wheres $108-58-3$ & $<0.010 \mathrm{mg} / \mathrm{l}$ & & $<0.010 \mathrm{mg} / \mathrm{l}$ & & 1 & \\
\hline vingl chlaride 73-014 & $<0.010 \mathrm{mg} / \mathrm{l}$ & & $<0.010 \mathrm{mg} / \mathrm{l}$ & & 1 & \\
\hline 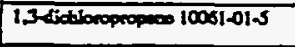 & $<0.010 \mathrm{mg} / \mathrm{l}$ & & $<0.010 \mathrm{mg} / \mathrm{l}$ & & I & \\
\hline $4=1.2$ dictioned on is6-60-S & $<0.010 \mathrm{mg} / \mathrm{l}$ & & $<0.010 \mathrm{mg} / \mathrm{l}$ & & 1 & $\therefore$ \\
\hline atpleativity $12587-46-1$ & $0.46 \mathrm{pci} / 1$ & $3.1 \mathrm{pei} / 1$ & $0.46 \mathrm{pci} / \mathrm{l}$ & $3.1 \mathrm{pci} / 1$ & 1 & \\
\hline betestiving $12557-46-1$ & $4.6 \mathrm{pci} / \mathrm{l}$ & 8.5 peill & $4.6 \mathrm{peill}$ & 8.5 pcill & 1 & \\
\hline$\sqrt{\text { rocol cotiform }}$ & $30,000 \mathrm{co} / .1 \mathrm{~L}$ & $68,000 \mathrm{co} / .1 \mathrm{~L}$ & $30,000 \mathrm{co} / .1 \mathrm{~L}$ & $68,000 \mathrm{co} / .1 \mathrm{~L}$ & 1 & \\
\hline Thilling 746-280 & $<0.001 \mathrm{mg} / \mathrm{l}$ & $<0.001 \mathrm{mg} / \mathrm{l}$ & $<0.001 \mathrm{mg} / \mathrm{l}$ & $<0.001 \mathrm{mg} / \mathrm{l}$ & 1 & \\
\hline Unomim 74a-61-1 & $<0.001 \mathrm{mg} / \mathrm{l}$ & $0.001 \mathrm{mg} / \mathrm{l}$ & $<0.001 \mathrm{mg} / \mathrm{l}$ & $0.001 \mathrm{mg} / 1$ & 1 & \\
\hline Germen sarvity & $38 \mathrm{pci} / \mathrm{l}$ & $6.8 \mathrm{pci} / \mathrm{l}$ & 38 pcill & 6.8 pcill & 1 & \\
\hline
\end{tabular}

Part D- Provide deta for the sorm eveat(b) which renuled in the maximum values for the flow weighted comporito aempla.

\begin{tabular}{|c|c|c|c|c|c|c|c|}
\hline $\begin{array}{l}1 . \\
\text { Dale of } \\
\text { Stom } \\
\text { Eveat }\end{array}$ & $\begin{array}{l}2 . \\
\text { Duration } \\
\text { of Storm } \\
\text { (in } \\
\text { minemes) }\end{array}$ & $\begin{array}{l}3 . \\
\text { Toow nimfill } \\
\text { during stom eveast } \\
\text { (in inches) }\end{array}$ & $\begin{array}{l}4 \text {. } \\
\text { Nuaber of hour between } \\
\text { beginning of utorm mean- } \\
\text { ured and cod of proviour } \\
\text { meanblo nin ovent }\end{array}$ & $\begin{array}{l}\text { S. } \\
\text { Meximum flow nto } \\
\text { during min oveot } \\
\text { (ellowedmin or epooify } \\
\text { univ) }\end{array}$ & $\begin{array}{l}6 . \\
\text { Total flow from } \\
\text { min eveat } \\
\text { (Galioas or } \\
\text { epecify units) }\end{array}$ & $\begin{array}{c}7 . \\
\text { Secion } \\
\text { enmplo was } \\
\text { tukeo }\end{array}$ & 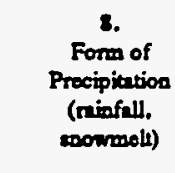 \\
\hline $9 / 24 / 91$ & 365 & 1.6 & 133.5 & 69.6 & 14,000 & Fall & Rninfall \\
\hline
\end{tabular}

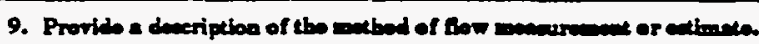

An ISCO 3230 Fow meter was used to obtain water level readings. This intrument was cahibrated with the normal dry weather flow, if present, as les zero. The flow meter was programmed with the characteristics of the coavegance so that flow rates and total flows conld be calculated by the meter. 
Outrall S2O

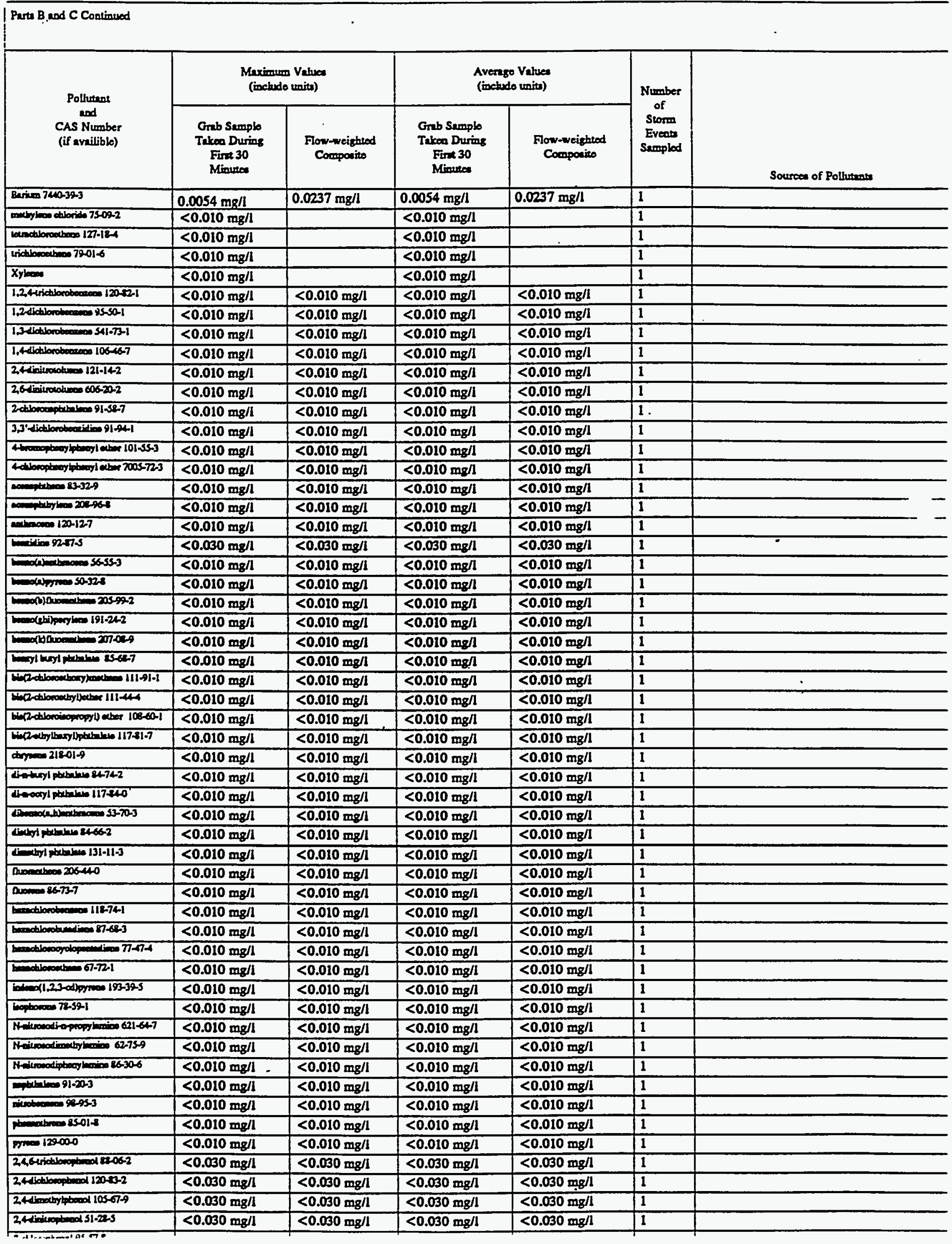


Outfall 520

Parts B and C Continued

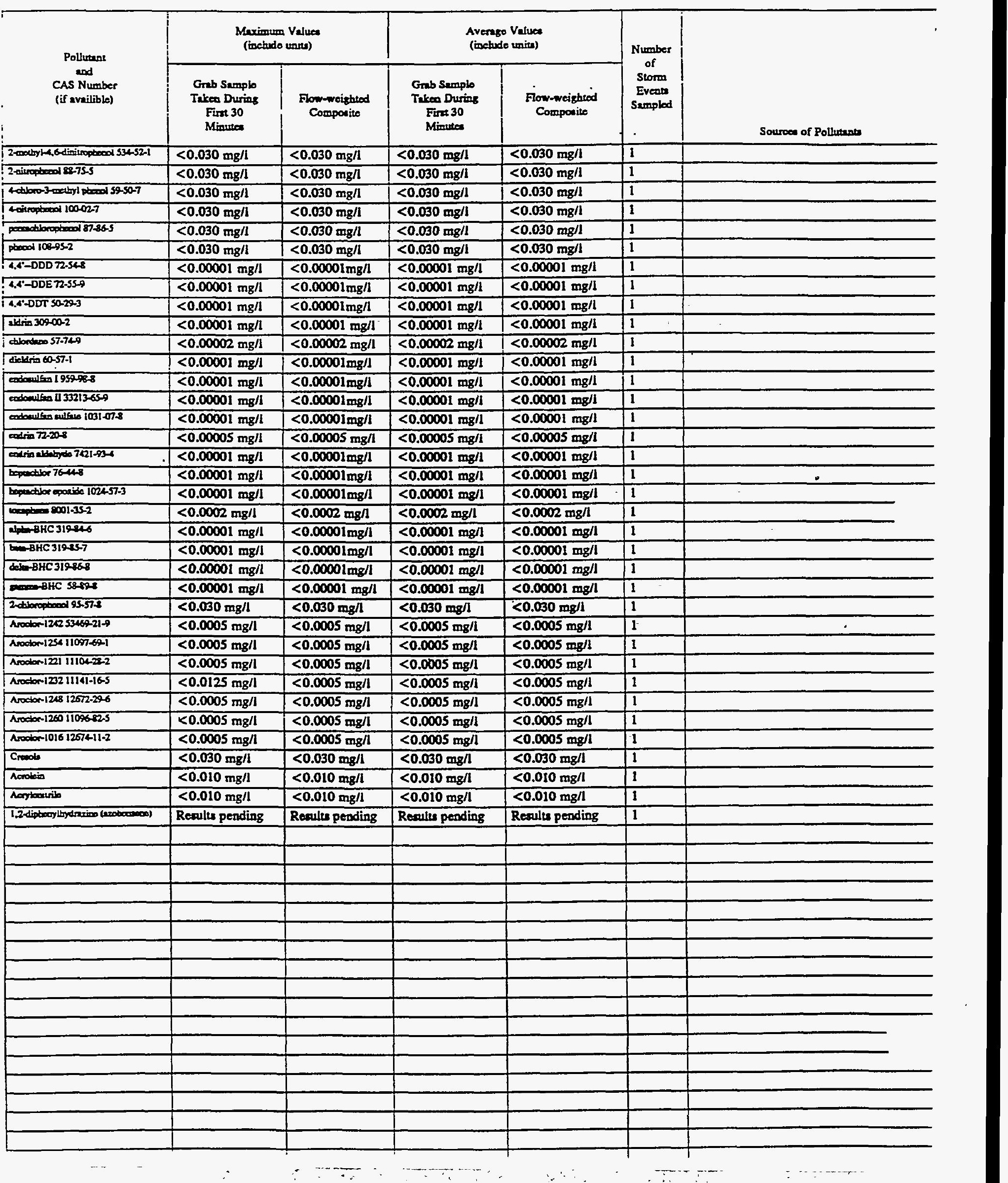


VII. Diecharge Informutioa (continued from page 3 of Form 2F)

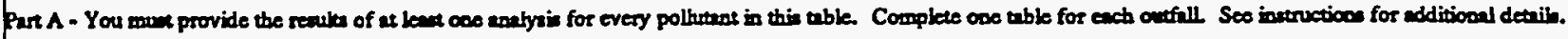

\begin{tabular}{|c|c|c|c|c|c|c|}
\hline \multirow{2}{*}{$\begin{array}{l}\text { Pollumast } \\
\text { and } \\
\text { CAS Number } \\
\text { (if enilible) }\end{array}$} & \multicolumn{2}{|c|}{$\begin{array}{l}\text { Moxionum Valuea } \\
\text { (include tritu) }\end{array}$} & \multicolumn{2}{|c|}{$\begin{array}{l}\text { Avenige Value } \\
\text { (ibelude inia) }\end{array}$} & \multirow{2}{*}{$\begin{array}{l}\text { Number } \\
\text { of } \\
\text { Siorm } \\
\text { Evenen } \\
\text { Sempled }\end{array}$} & \multirow[b]{2}{*}{ Sources of Pollhwores } \\
\hline & $\begin{array}{c}\text { Grab Semple } \\
\text { Takén During } \\
\text { Firk } 30 \\
\text { Minesed }\end{array}$ & $\begin{array}{l}\text { Flow-weigbted } \\
\text { Componite }\end{array}$ & $\begin{array}{c}\text { Grab Semple } \\
\text { Teten During } \\
\text { Firse } 30 \\
\text { Minoted }\end{array}$ & $\begin{array}{l}\text { Four-weifibed } \\
\text { Compoties }\end{array}$ & & \\
\hline Pil & $<2 \mathrm{mg} / \mathrm{l}$ & & $<2 \mathrm{mg} / \mathrm{l}$ & & 1 & \\
\hline Blolopial Oxy Dened (BOD) & $<5 \mathrm{mg} / \mathrm{l}$ & & $<5 \mathrm{mg} / 1$ & & 1 & \\
\hline Chical Oxy $D$ Dand (COD) & $29 \mathrm{mg} / \mathrm{l}$ & . & $29 \mathrm{mg} / \mathrm{l}$ & & 1 & \\
\hline Toul suppoded solden (TSS) & $7 \mathrm{mg} / \mathrm{l}$ & & $7 \mathrm{mg} / 1$ & & 1 & \\
\hline Toul Kfoblad Nieroger & $0.70 \mathrm{mg} / \mathrm{l}$ & & $0.70 \mathrm{mg} / 1$ & & 1 & \\
\hline 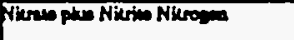 & $1.0 \mathrm{mg} / \mathrm{l}$ & & $1.0 \mathrm{mg} / \mathrm{l}$ & & 1 & \\
\hline Toul Phoophones & $<0.1 \mathrm{mg} / \mathrm{l}$ & & $<0.1 \mathrm{mg} / 1$ & & 1 & \\
\hline & Minim & Merian 8.0 & Mirimen 8.0 & 8.0 & & \\
\hline
\end{tabular}

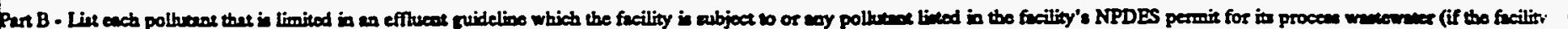

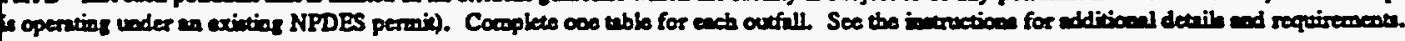

\begin{tabular}{|c|c|c|c|c|c|c|}
\hline \multirow{2}{*}{$\begin{array}{l}\text { Pollwast } \\
\text { CAs Number } \\
\text { (if evilible) }\end{array}$} & \multicolumn{2}{|c|}{$\begin{array}{l}\text { Maximunas Valuea } \\
\text { (ixchede vaine) }\end{array}$} & \multicolumn{2}{|c|}{$\begin{array}{l}\text { Averaze Vabues } \\
\text { (iochede unio) }\end{array}$} & \multirow{2}{*}{$\begin{array}{l}\text { Number } \\
\text { of } \\
\text { Stoom } \\
\text { Eveosed } \\
\text { Simpled }\end{array}$} & \multirow{2}{*}{$\begin{array}{c}: \\
\text { Sources of Pollution }\end{array}$} \\
\hline & 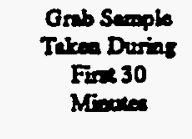 & $\begin{array}{l}\text { Fow-weithed } \\
\text { Componiss }\end{array}$ & 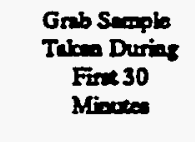 & $\begin{array}{l}\text { Fom-weighed } \\
\text { Compocize }\end{array}$ & & \\
\hline $1=1-0.1740 .360$ & $<0.04 \mathrm{mg} / \mathrm{l}$ & & $<0.04 \mathrm{mg} / \mathrm{l}$ & & 1 & \\
\hline andith & $<0.002 \mathrm{mg} / \mathrm{l}$ & & $<0.002 \mathrm{mq} / \mathrm{l}$ & & 1 & \\
\hline 160000, 7439-97-6 & $<0.0002 \mathrm{mg} / \mathrm{l}$ & & $<0.0002 \mathrm{mg}^{\circ}$ & & 1 & \\
\hline nans & $0.003 \mathrm{mg} / \mathrm{l}$ & & $0.003 \mathrm{mg} / \mathrm{l}$ & & 1 & $?$ \\
\hline $54 \sin 7 \sin 22$ & $<0.002 \mathrm{mg} / 1$ & & $<0.002 \mathrm{mg} / \mathrm{l}$ & & 1 & \\
\hline solfisen & $56 \mathrm{mg} / 1$ & & $36 \mathrm{mg} / \mathrm{l}$ & & 1 & \\
\hline surficameses & $<0.05 \mathrm{mg} / \mathrm{l}$ & & $<0.05 \mathrm{mg} / \mathrm{l}$ & & 1 & \\
\hline Ah-ian $762290-5$ & $0.29 \mathrm{mg} / 1$ & & $0.29 \mathrm{mg} / 1$ & & 1 & \\
\hline $2 \sin 2440-32-2$ & $<0.04 \mathrm{mg} / \mathrm{l}$ & & $<0.04 \mathrm{mg} / \mathrm{l}$ & & 1 & \\
\hline $300000740-124$ & $0.608 \mathrm{mg} / \mathrm{l}$ & & $0.608 \mathrm{mg} / 1$ & & 1 & \\
\hline 3 anding 740-41.7 & $<0.0004 \mathrm{mg} / \mathrm{l}$ & & $<0.0004 \mathrm{mg} / \mathrm{l}$ & & 1 & \\
\hline $2 x+14=740-13-9$ & $<0.004 \mathrm{mg} / \mathrm{l}$ & & $<0.004 \mathrm{mg} / \mathrm{l}$ & & 1 & \\
\hline 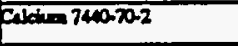 & $32.0 \mathrm{mg} / \mathrm{h}$ & & $32.0 \mathrm{mg} / \mathrm{h}$ & & 1 & \\
\hline 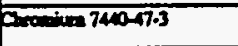 & $<0.006 \mathrm{mg} / \mathrm{l}$ & & $<0.006 \mathrm{mg} / \mathrm{l}$ & & 1 & \\
\hline Codack 740048-4 & $<0.002 \mathrm{mg} / \mathrm{l}$ & & $<0.002 \mathrm{mg} / \mathrm{l}$ & & 1 & \\
\hline Fomp 7400-sos & $<0.006 \mathrm{mg} / \mathrm{h}$ & & $<0.006 \mathrm{mg} / \mathrm{l}$ & & 1 & \\
\hline ron 709098 & $0.38 \mathrm{mg} / \mathrm{l}$ & & $0.38 \mathrm{mg} / \mathrm{h}$ & & 1 & \\
\hline 7.109291 & $<0.02 \mathrm{mg} / \mathrm{l}$ & & $<0.02 \mathrm{mg} / \mathrm{i}$ & & 1 & \\
\hline Whand 713993-2 & $0.02 \mathrm{mg} / 1$ & & $0.02 \mathrm{mg} / \mathrm{l}$ & & 1 & \\
\hline Mrapaina 709954 & $8.54 \mathrm{mg} / \mathrm{l}$ & & $8.54 \mathrm{mg} / \mathrm{l}$ & & 1 & \\
\hline$M=0000=739695$ & $0.040 \mathrm{mg} / \mathrm{l}$ & & $0.040 \mathrm{mg} / \mathrm{l}$ & & 1 & \\
\hline 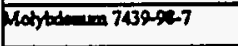 & $<0.006 \mathrm{mg} / \mathrm{l}$ & & $<0.006 \mathrm{mg} / \mathrm{h}$ & & 1 & \\
\hline N16al 740000 & $<0.008 \mathrm{mg} / \mathrm{l}$ & & $<0.008 \mathrm{mg} / \mathrm{l}$ & & 1 & \\
\hline pominom 740-027 & $5.5 \mathrm{mg} / \mathrm{l}$ & & $5.5 \mathrm{mg} / \mathrm{l}$ & & 1 & \\
\hline sitwan 740-24 & $<0.006 \mathrm{mg} / \mathrm{l}$ & & $<0.006 \mathrm{mg} / \mathrm{l}$ & & 1 & \\
\hline 30 d. $740-22-5$ & $20.1 \mathrm{mg} / \mathrm{l}$ & & $20.1 \mathrm{mg} / 1$ & & 1 & \\
\hline Tremisen 740-326 & $<0.01 \mathrm{mg} / \mathrm{l}$ & & $<0.01 \mathrm{mg} / \mathrm{l}$ & & 1 & \\
\hline $270240066-6$ & $0.05 \mathrm{mg} / \mathrm{l}$ & & $0.05 \mathrm{mg} / 1$ & & 1 & \\
\hline
\end{tabular}


Conthoed from front.

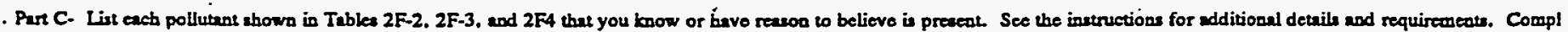
- one table for cach outfall.

\begin{tabular}{|c|c|c|c|c|c|c|}
\hline \multirow{2}{*}{$\begin{array}{l}\text { Pollutene } \\
\text { and } \\
\text { CAS Number } \\
\text { (if availible) }\end{array}$} & \multicolumn{2}{|c|}{$\begin{array}{l}\text { Maximum Valuea } \\
\text { (inchude units) }\end{array}$} & \multicolumn{2}{|c|}{$\begin{array}{l}\text { Avernge Values } \\
\text { (include units) }\end{array}$} & \multirow{2}{*}{$\begin{array}{l}\text { Number } \\
\text { of } \\
\text { Storm } \\
\text { Eveots } \\
\text { Sumplod } \\
\text {. . }\end{array}$} & \multirow[b]{2}{*}{ Sources of Pollutents } \\
\hline & $\begin{array}{l}\text { Grab Sample } \\
\text { Taken During } \\
\text { Firs } 30 \\
\text { Minuter }\end{array}$ & $\begin{array}{l}\text { Flow-wcighed } \\
\text { Componito }\end{array}$ & $\begin{array}{c}\text { Grab Sample } \\
\text { Taken During } \\
\text { First } 30 \\
\text { Minute }\end{array}$ & $\begin{array}{l}\text { Flow-weighted } \\
\text { Composite }\end{array}$ & & \\
\hline & $<0.010 \mathrm{mg} / \mathrm{l}$ & & $<0.010 \mathrm{mg} / \mathrm{l}$ & & 1 & \\
\hline 1.1 .22 -eanctionsetbeso $79-34-5$ & $<0.010 \mathrm{mg} / \mathrm{l}$ & & $<0.010 \mathrm{mg} / \mathrm{l}$ & & 1 & \\
\hline 1.1.2-trictibrectatere 7900-5 & $<0.010 \mathrm{mg} / \mathrm{l}$ & & $<0.010 \mathrm{mg} / \mathrm{l}$ & & 1 & \\
\hline 1.1 dictorocthese $75-34-3$ & $<0.010 \mathrm{mg} / 1$ & & $<0.010 \mathrm{mg} / \mathrm{l}$ & & 1 & \\
\hline 1.1 dictborcetbyleos 75-35-4 & $<0.010 \mathrm{mg} / \mathrm{l}$ & & $<0.010 \mathrm{mg} / \mathrm{l}$ & & 1 & \\
\hline$: .2$-dichbroction $107-06-2$ & $<0.010 \mathrm{mg} / \mathrm{l}$ & & $<0.010 \mathrm{mg} / \mathrm{l}$ & & 1 & \\
\hline 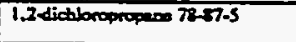 & $<0.010 \mathrm{mg} / \mathrm{l}$ & & $<0.010 \mathrm{mg} / \mathrm{l}$ & & 1 & \\
\hline 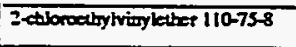 & $<0.010 \mathrm{mg} / \mathrm{l}$ & & $<0.010 \mathrm{mg} / \mathrm{l}$ & & 1 & \\
\hline besenese $71<3-2$ & $<0.010 \mathrm{mg} / \mathrm{l}$ & & $<0.010 \mathrm{mg} / \mathrm{l}$ & & 1 & \\
\hline 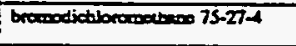 & $<0.010 \mathrm{mg} / \mathrm{l}$ & & $<0.010 \mathrm{mg} / \mathrm{l}$ & & 1 & \\
\hline browolonem 75-25-2 & $<0.010 \mathrm{mg} / \mathrm{l}$ & & $<0.010 \mathrm{mg} / \mathrm{l}$ & & 1 & \\
\hline browancethene 7483-9 & $<0.010 \mathrm{mg} / \mathrm{l}$ & & $<0.010 \mathrm{mg} / \mathrm{l}$ & & 1 & \\
\hline 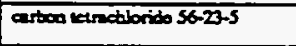 & $<0.010 \mathrm{mg} / 1$ & & $<0.010 \mathrm{mg} / \mathrm{l}$ & & 1 & \\
\hline ctorobenecos $108-907$ & $<0.010 \mathrm{mg} / \mathrm{l}$ & . & $<0.010 \mathrm{mg} / \mathrm{l}$ & & 1 & \\
\hline 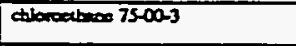 & $<0.010 \mathrm{mg} / \mathrm{l}$ & & $<0.010 \mathrm{mg} / \mathrm{l}$ & & 1 & \\
\hline chlorofores 67-66-3 & $<0.010 \mathrm{mg} / \mathrm{l}$ & & $<0.010 \mathrm{mg} / \mathrm{l}$ & & 1 &. \\
\hline 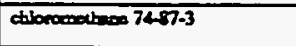 & $<0.010 \mathrm{mg} / \mathrm{l}$ & & $<0.010 \mathrm{mg} / 1$ & & 1 & \\
\hline 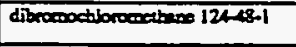 & $<0.010 \mathrm{mg} / \mathrm{l}$ & & $<0.010 \mathrm{mg} / \mathrm{l}$ & & 1 & \\
\hline 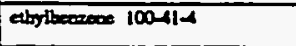 & $<0.010 \mathrm{mg} / \mathrm{l}$ & & $<0.010 \mathrm{mg} / 1$ & & 1 & \\
\hline lolece $108-2+3$ & $<0.010 \mathrm{mg} / \mathrm{l}$ & & $<0.010 \mathrm{mg} / 1$ & & 1 & \\
\hline videfl chloride 75014 & $<0.010 \mathrm{mg} / 1$ & & $<0.010 \mathrm{mg} / 1$ & & 1 & \\
\hline 1,3-dictionapiopeces $10061-01-5$ & $<0.010 \mathrm{mg} / \mathrm{l}$ & & $<0.010 \mathrm{mg} / 1$ & - & 1 & \\
\hline 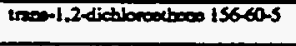 & $<0.010 \mathrm{mg} / \mathrm{l}$ & & $<0.010 \mathrm{mg} / \mathrm{l}$ & & 1 & - \\
\hline Liphectivity $12587-16-1$ & -0.89 pcill & & -0.89 pcill & & 1 & \\
\hline betenaviny $12587-16-1$ & $8.4 \mathrm{pei} / 1$ & & $8.4 \mathrm{pci} / \mathrm{l}$ & & 1 & \\
\hline focal coliform & $260 \mathrm{co} / .1 \mathrm{~L}$ & & $260 \mathrm{co} / .1 \mathrm{~L}$ & & 1 & \\
\hline Thelling 740-28-0 & $<0.001 \mathrm{mg} / \mathrm{l}$ & & $<0.001 \mathrm{mg} / \mathrm{l}$ & & 1 & \\
\hline Unaivon 740-61-1 & $<0.001 \mathrm{mg} / 1$ & & $<0.001 \mathrm{mg} / \mathrm{l}$ & & 1 & \\
\hline Conom activity & 1.4 pcill & & $1.4 \mathrm{pci} / \mathrm{l}$ & & 1 & \\
\hline
\end{tabular}

Part D- Provide dan for the sorm evenu(u) which reaulted in the maximim value for the flow weighted componite ample.

\begin{tabular}{|c|c|c|c|c|c|c|c|}
\hline $\begin{array}{l}1 . \\
\text { Date of } \\
\text { Storm } \\
\text { Evens }\end{array}$ & $\begin{array}{l}2 . \\
\text { Durtion } \\
\text { of Storm } \\
\text { (in } \\
\text { minimes) }\end{array}$ & $\begin{array}{l}3 . \\
\text { Tokel ninfall } \\
\text { durings stomm oveat } \\
\text { (in incboe) }\end{array}$ & $\begin{array}{l}\text { N. } \\
\text { Number of hours between } \\
\text { boginning of torm meas- } \\
\text { ured and cod of previous } \\
\text { meanublo min eveot }\end{array}$ & $\begin{array}{l}\text { S. } \\
\text { Maximum flow rate } \\
\text { duriag min eveat } \\
\text { (olloow/min or apocify } \\
\text { unity) }\end{array}$ & $\begin{array}{l}6 . \\
\text { Total flow from } \\
\text { min eveat } \\
\text { (olloos or } \\
\text { pecify unita) }\end{array}$ & $\begin{array}{c}7 . \\
\text { Season } \\
\text { expolo was } \\
\text { taten }\end{array}$ & $\begin{array}{l}8 . \\
\text { Form of } \\
\text { Procipitabion } \\
\text { (rinfill. } \\
\text { encomoly) }\end{array}$ \\
\hline $9 / 24 / 91$ & 365 & 1.6 & 133.5 & & & Fall & Rainfall \\
\hline
\end{tabular}

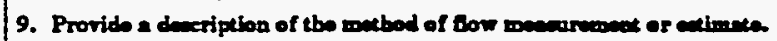

An ISCO 3230 Flow meter was used to obtain water level readings. This instrument was colibrated with the normal dry weather flow, if present, as level zero. The flow meter was programmed with the characteristics of the conveyance so that flow rates and total flows could be calenlated by the meter. 
Outfall S21

Parta B ind C Continued

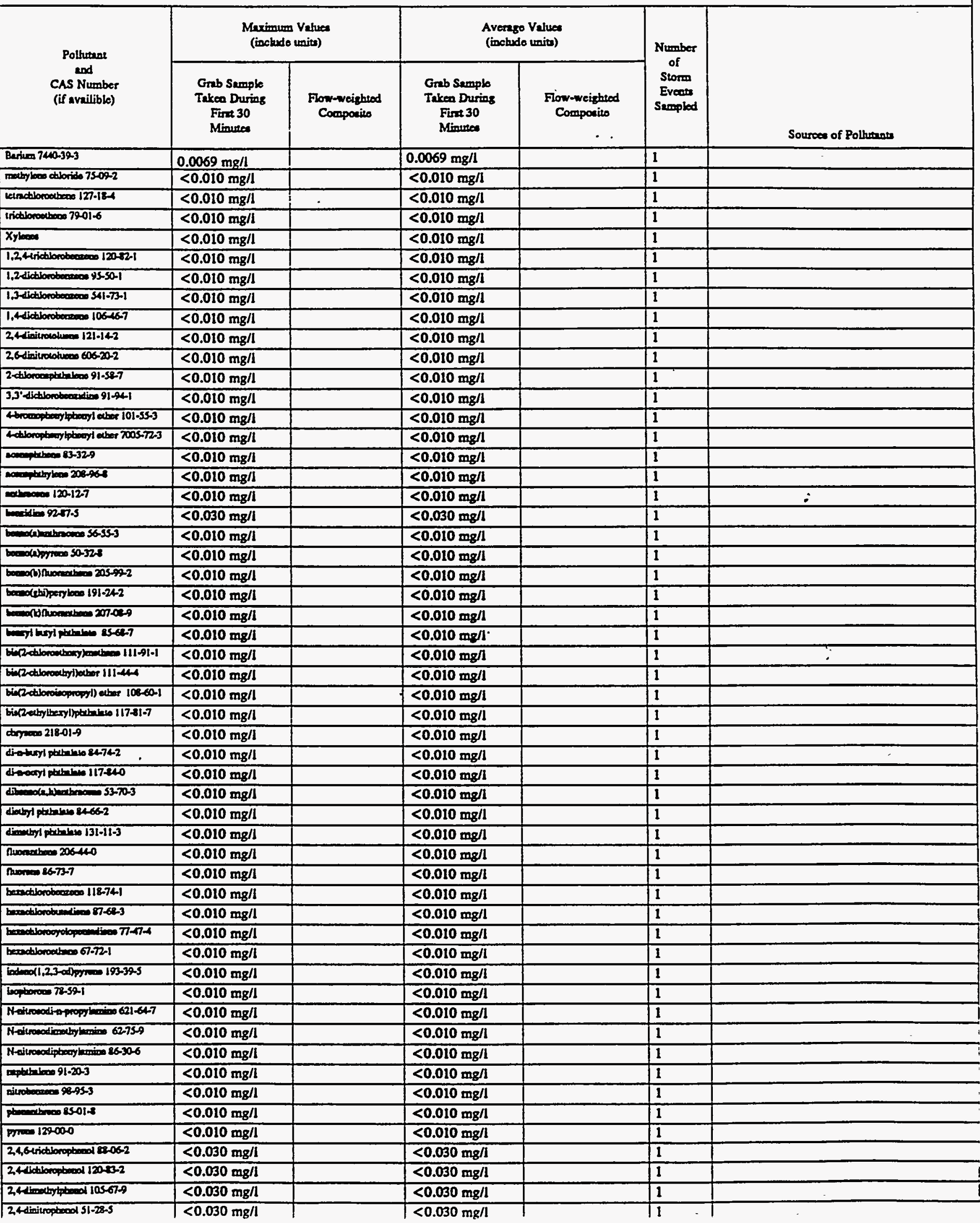


Outfoll S21

P Part B and C Continuod

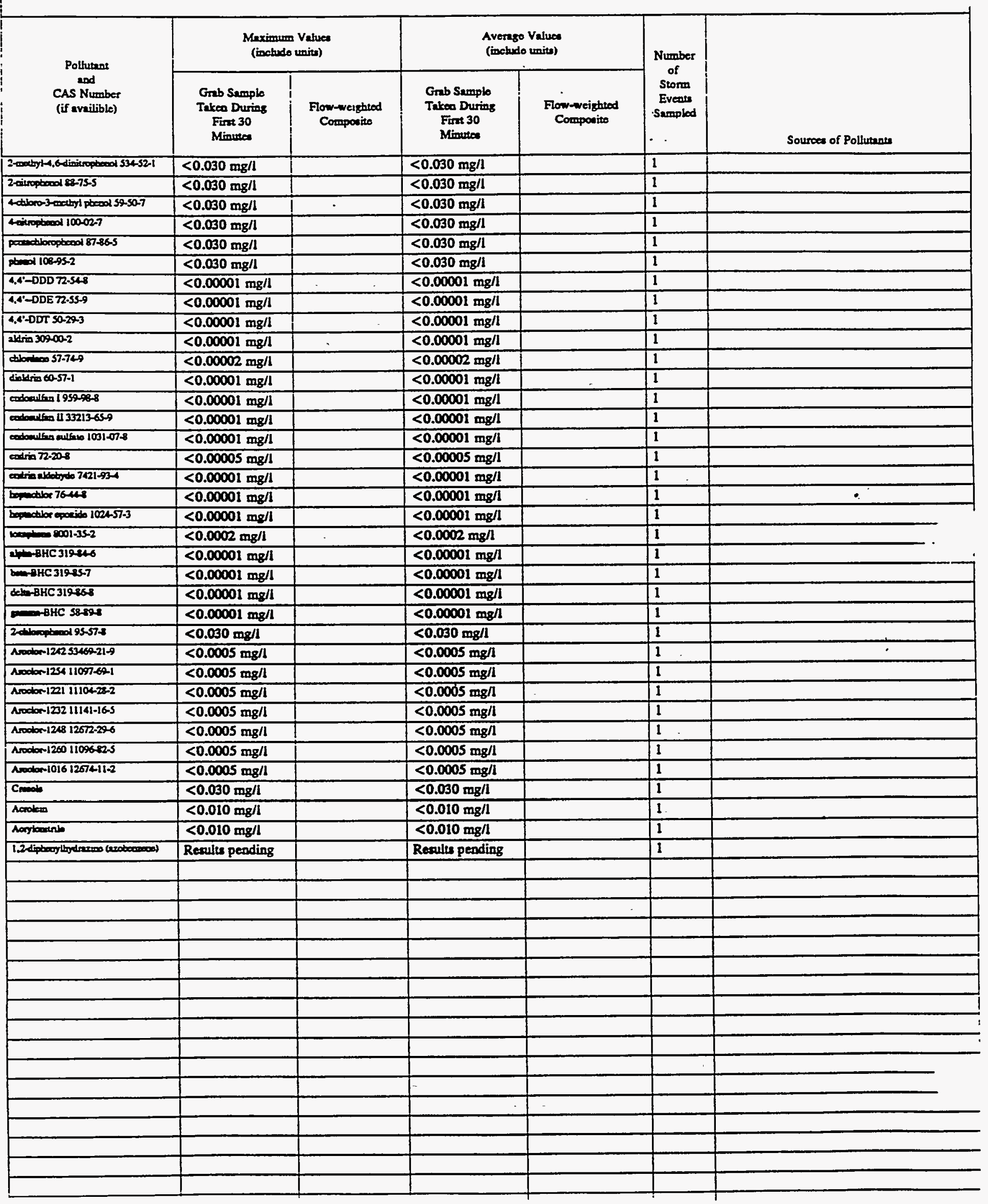


VII. Discharge Information (continued from page 3 of Form 2 F)

Pan A - You must provide the results of at least one analynis for every pollutant in this table. Complete one table for eseh outfall. See instruetions for additional detuils.

\begin{tabular}{|c|c|c|c|c|c|c|}
\hline \multirow{2}{*}{$\begin{array}{l}\text { Pollutant } \\
\text { and } \\
\text { CAS Number } \\
\text { (if availible) }\end{array}$} & \multicolumn{2}{|c|}{$\begin{array}{l}\text { Maximum Values } \\
\text { (include units) }\end{array}$} & \multicolumn{2}{|c|}{$\begin{array}{l}\text { Average Valuei } \\
\text { (include unitu) }\end{array}$} & \multirow{2}{*}{$\begin{array}{l}\text { Number } \\
\text { of } \\
\text { Stomn } \\
\text { Eveats } \\
\text { Sumpled }\end{array}$} & \multirow[b]{2}{*}{ Sources of Pollutunts } \\
\hline & $\begin{array}{l}\text { Grab Sample } \\
\text { Takes During } \\
\text { Finst } 30 \\
\text { Minurea }\end{array}$ & $\begin{array}{l}\text { - Flow-weighted } \\
\text { Composite } \\
\text { : }\end{array}$ & $\begin{array}{cc}\text { Grub Sumple } \\
\text { Takes During } \\
\text { Firt } 30 \\
\text { - Minures }\end{array}$ & $\begin{array}{l}\text { Flow-weightod } \\
;\end{array}$ & & \\
\hline pil and Groeser & $<2 \mathrm{mg} / \mathrm{l}$ & & $<2 \mathrm{mg} / \mathrm{l}$ & $i$ & 1 & i \\
\hline Blolopial Oxyber Dewaed (BOD) & $<5 \mathrm{mg} / \mathrm{l}$ & & $<S \mathrm{mg} / \mathrm{l}$ & ! & 1 & \\
\hline Chamical Oxypeo Derned (COD) & $<5 \mathrm{mg} / \mathrm{l}$ & & $<5 \mathrm{mg} / \mathrm{l}$ & $\bar{i}$ & 1 & \\
\hline Toul Sumpended Solide $\pi S S$ & $10 \mathrm{mg} / \mathrm{l}$ & & $10 \mathrm{mg} / \mathrm{l}$ & $i$ & 1 & \\
\hline Toul Kjoldehl Nitrogea & $0.3 \mathrm{mg} / \mathrm{l}$ & $i$ & $0.3 \mathrm{mg} / \mathrm{l}$ & $i$ & 1 & ! \\
\hline Nitrates plus Nitrive Nitrogea & $0.47 \mathrm{mg} / \mathrm{l}$ & $\mathrm{i}$ & $0.47 \mathrm{mg} / \mathrm{l}$ & 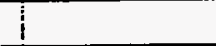 & 1 & i \\
\hline Toul Phosphore & $<0.1 \mathrm{mg} / \mathrm{l}$ & $!$ & $\mid<0.1 \mathrm{mg} / \mathrm{l}$ & $T$ & 11 & 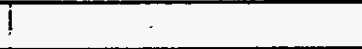 \\
\hline & Minimen & Maximem 7.9 & Minimen 7.9 & Maximam 7.9 & & \\
\hline
\end{tabular}

Part B - List each pollutent that is limirod in an effluent guideline which the fucility is subjoct to or any pollutust listod in the facility's NPDES permit for its procens wartewnter (if the facility is operating under en exinting NPDES pernits. Complete one tuble for each outsall. See the inutruetions for additional details and requirements.

\begin{tabular}{|c|c|c|c|c|c|c|}
\hline \multirow{2}{*}{$\begin{array}{l}\text { Pollutunt } \\
\text { and } \\
\text { CAs Number } \\
\text { (if aveilible) }\end{array}$} & \multicolumn{2}{|c|}{$\begin{array}{l}\text { Maximum Valuea } \\
\text { (includo units) }\end{array}$} & \multicolumn{2}{|c|}{$\begin{array}{l}\text { Average Valuea } \\
\text { (include units) }\end{array}$} & \multirow{2}{*}{$\begin{array}{l}\text { Number } \\
\text { of } \\
\text { Storm } \\
\text { Events } \\
\text { Samplod }\end{array}$} & \multirow[b]{2}{*}{ Sources of Pollutunes } \\
\hline & $\begin{array}{c}\text { Grab Sumple } \\
\text { Takeo During } \\
\text { Fint } 30 \\
\text { Minutes }\end{array}$ & $\begin{array}{l}\text { Flow-weighted } \\
\text { Comporite }\end{array}$ & $\begin{array}{l}\text { Grab Sumple } \\
\text { Tukea Duriog } \\
\text { Firat } 30 \\
\text { Minutea }\end{array}$ & $\begin{array}{l}\text { Flow-weighted } \\
\text { Componite }\end{array}$ & & \\
\hline Animooy 7400.360 & $<0.04 \mathrm{mg} / \mathrm{l}$ & & $<0.04 \mathrm{mg} / \mathrm{l}$ & & 1 & \\
\hline Fynde & $<0.002 \mathrm{mg} / \mathrm{l}$ & & $<0.002 \mathrm{mg} / \mathrm{l}$ & & 1 & \\
\hline Metary 7439-97-6 & $<0.0002 \mathrm{mg} / \mathrm{l}$ & & $<0.0002 \mathrm{mg} / \mathrm{l}$ & & 1 & ? \\
\hline proode & $0.001 \mathrm{mg} / \mathrm{h}$ & & $0.001 \mathrm{mg} / \mathrm{l}$ & & 1 & \\
\hline Feloiven $7782-69-2$ & $<0.002 \mathrm{mg} / \mathrm{l}$ & & $<0.002 \mathrm{mg} / \mathrm{l}$ & & 1 & \\
\hline pillane & $21 \mathrm{mg} / \mathrm{l}$ & & $21 \mathrm{mg} / \mathrm{l}$ & & $I$ & \\
\hline Purfectenes & $0.14 \mathrm{mg} / \mathrm{l}$ & & $0.14 \mathrm{mg} / \mathrm{l}$ & & 1 & \\
\hline phenen $7429-90-5$ & $1.06 \mathrm{mg} / \mathrm{l}$ & & $1.06 \mathrm{mg} / \mathrm{l}$ & & 1 & \\
\hline 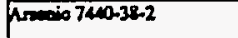 & $<0.04 \mathrm{mg} / 1$ & & $<0.04 \mathrm{mg} / \mathrm{l}$ & & 1 & \\
\hline $800007400-28$ & $0.652 \mathrm{mg} / 1$ & & $0.652 \mathrm{mg} / 1$ & & 1 & \\
\hline Benfliner 740-11-7 & $<0.0004 \mathrm{mg} / \mathrm{l}$ & & $<0.0004 \mathrm{mg} / \mathrm{l}$ & & 1 & ; \\
\hline Cedasion 7400-43-9 & $<0.004 \mathrm{mg} / \mathrm{l}$ & & $<0.004 \mathrm{mg} / \mathrm{l}$ & & 1 & \\
\hline calaim 740-70-2 & $21.2 \mathrm{mg} / 1$ & & $212 \mathrm{mg} / \mathrm{l}$ & & 1 & \\
\hline Ctroeming $7440-17.3$ & $<0.006 \mathrm{mg} / \mathrm{l}$ & & $<0.006 \mathrm{mg} / 1$ & & 1 & \\
\hline Cobak 7400-18-4 & $<0.002 \mathrm{mg} / \mathrm{l}$ & & $<0.002 \mathrm{mg} / \mathrm{l}$ & & 1 & \\
\hline Fopper 7440-50-8 & $<0.006 \mathrm{mg} / \mathrm{l}$ & & $<0.006 \mathrm{mg} / 1$ & & 1 & \\
\hline $\operatorname{ros} 743982-6$ & $1.57 \mathrm{mg} / 1$ & & $1.57 \mathrm{mg} / \mathrm{l}$ & & 1 & \\
\hline $20001439-92-1$ & $<0.02 \mathrm{mg} / 1$ & & $<0.02 \mathrm{mg} / \mathrm{l}$ & & 1 & \\
\hline Sillow 7439-93-2 & $0.10 \mathrm{mg} / \mathrm{l}$ & & $0.10 \mathrm{mg} / \mathrm{l}$ & & 1 & \\
\hline Megroencon 7439-95-4 & $2.51 \mathrm{mg} / 1$ & & $2.51 \mathrm{mg} / 1$ & & 1 & \\
\hline $4=000000007439-96-5$ & $0.089 \mathrm{mg} / \mathrm{l}$ & & $0.089 \mathrm{mg} / \mathrm{l}$ & & 1 & \\
\hline Mohpdoed 7439-96-7 & $<0.006 \mathrm{mg} / \mathrm{l}$ & & $<0.006 \mathrm{mg} / \mathrm{l}$ & $=$ & 1 & \\
\hline 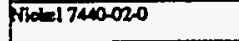 & $<0.008 \mathrm{mg} / \mathrm{l}$ & & $<0.008 \mathrm{mg} / \mathrm{l}$ & & 1 & \\
\hline Poteminem 7400-09-7 & $1.3 \mathrm{mg} / 1$ & & $1.3 \mathrm{mg} / \mathrm{l}$ & & 1 & \\
\hline Silver 74ta-22-1 & $<0.006 \mathrm{mg} / \mathrm{l}$ & & $<0.006 \mathrm{mg} / \mathrm{l}$ & & 1 & \\
\hline podium 740-22-5 & $0.37 \mathrm{mg} / \mathrm{l}$ & & $0.37 \mathrm{mg} / \mathrm{l}$ & & 1 & \\
\hline Tikenium 7460-32-6 & $0.02 \mathrm{mg} / \mathrm{l}$ & & $0.02 \mathrm{mg} / \mathrm{l}$ & & 1 & \\
\hline $\lim 7400606$ & $0.02 \mathrm{mg} / \mathrm{l}$ & & $0.02 \mathrm{mg} / \mathrm{l}$ & & 1 & \\
\hline
\end{tabular}


Continued from frote.

Part C. List each pollutant shown in Tables 2F-2. 2F-3, and 2F4 that you know or tave reason to believo is present. See the instructions for additional detaile and requireoneots. Corr one table for each outfall.

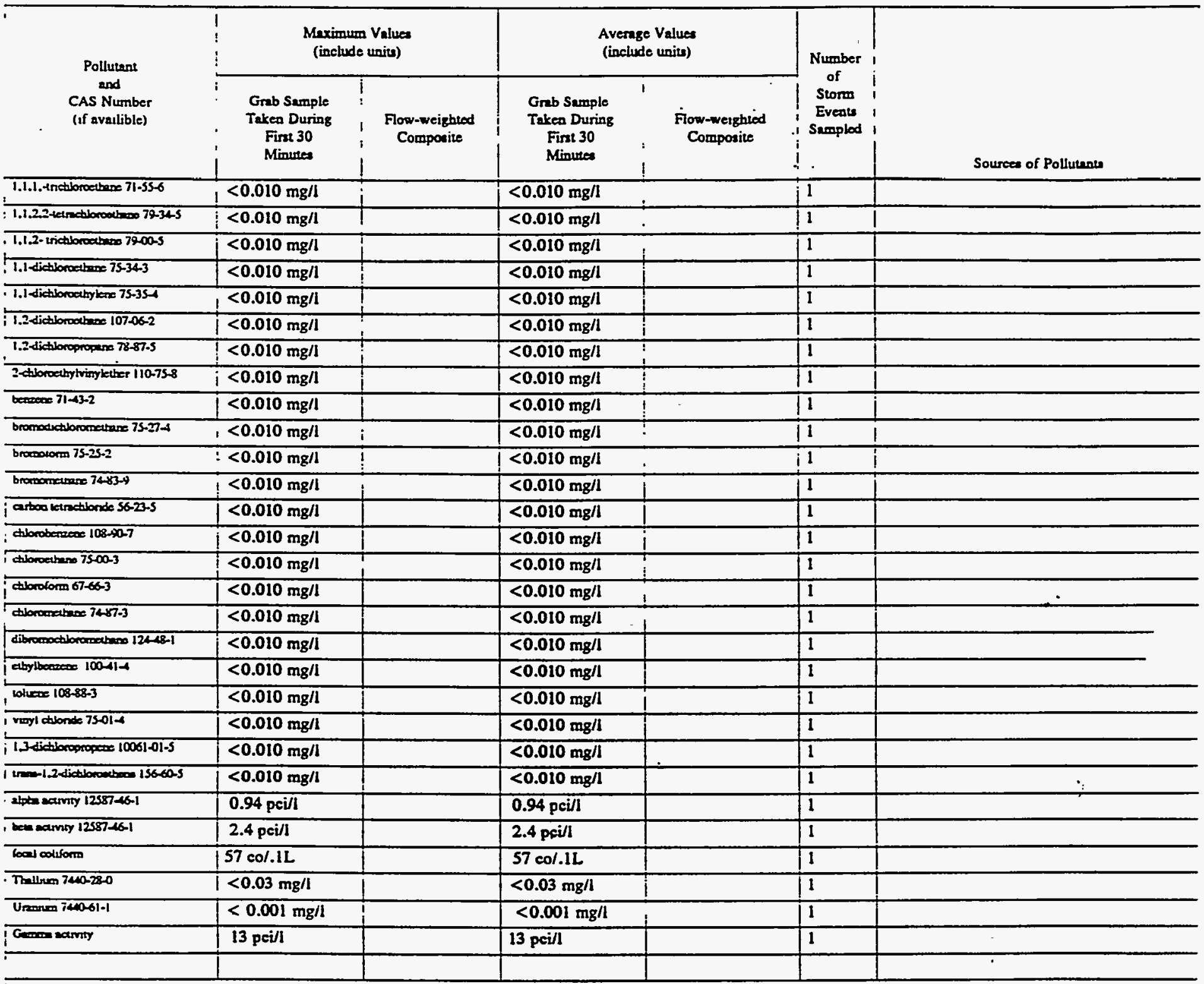

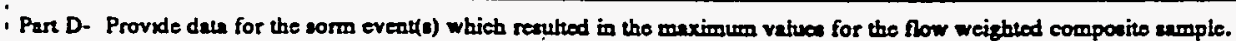

\begin{tabular}{|c|c|c|c|c|c|c|c|}
\hline $\begin{array}{l}\text { 1. } \\
\text { Dete of } \\
\text { Storm } \\
\text { Event }\end{array}$ & $\begin{array}{l}2 . \\
\text { Duration } \\
\text { of Storm } \\
\text { (in } \\
\text { minutes) }\end{array}$ & $\begin{array}{l}\text { 3. } \\
\text { Total ninfall } \\
\text { during storm event } \\
\text { (is inctoes) }\end{array}$ & $\begin{array}{l}4 . \\
\text { Number of hours between } \\
\text { beginains of stom meas. } \\
\text { ured and ead of previous } \\
\text { mewarable min event }\end{array}$ & $\begin{array}{l}\text { S. } \\
\text { Maximum flow rate } \\
\text { duriog min eveal } \\
\text { Galloos/min or specify } \\
\text { unity) }\end{array}$ & $\begin{array}{l}6 . \\
\text { Total flow from } \\
\text { rain event } \\
\text { (gallons or } \\
\text { specify units) }\end{array}$ & $\begin{array}{c}7 . \\
\text { Season } \\
\text { sumple was } \\
\text { tukea }\end{array}$ & $\begin{array}{l}8 . \\
\text { Form of } \\
\text { Precipization } \\
\text { (nimfall. } \\
\text { coowmelt) }\end{array}$ \\
\hline $12 / 13 / 91$ & 250 & 0.25 & 88.2 & NA & NA & Fall & Rainfall \\
\hline
\end{tabular}

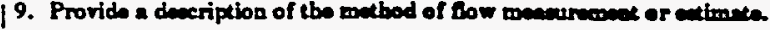

An ISCO 3230 Flow meter was used to obtain water level readings. This instrument was calibrated with the normal dry weather flow, if present, as levt. ' zero. The flow meter was programmed with the characteristics of the conveyance so that flow rates and total flows could be calculated by the meter. 


\begin{tabular}{|c|c|c|c|c|c|c|}
\hline \multirow{2}{*}{$\begin{array}{l}\text { Polluteat } \\
\text { and } \\
\text { CAs Number } \\
\text { (if availible) }\end{array}$} & \multicolumn{2}{|c|}{$\begin{array}{l}\text { Maximum Values } \\
\text { (inchude unitu) }\end{array}$} & \multicolumn{2}{|c|}{$\begin{array}{l}\text { Average Values } \\
\text { (Liclude uniss) }\end{array}$} & \multirow{2}{*}{$\begin{array}{l}\text { Number } \\
\text { of } \\
\text { Siorm } \\
\text { Eveats } \\
\text { Sxmpiod }\end{array}$} & \multirow[b]{2}{*}{ Sources of Pollutants } \\
\hline & $\begin{array}{l}\text { Grab Sumple } \\
\text { Taken During } \\
\text { First } 30 \\
\text { Minutee }\end{array}$ & $\begin{array}{l}\text { Flow-weighted } \\
\text { Componito }\end{array}$ & $\begin{array}{l}\text { Grab Semple } \\
\text { Taken During } \\
\text { Fint 30 } \\
\text { Minutea }\end{array}$ & $\begin{array}{c}\text { Fow-weightod } \\
\text { i } \\
\text { Composite }\end{array}$ & & \\
\hline 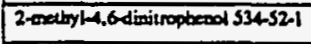 & $<0.050 \mathrm{mg} / 1$ & & $<0.050 \mathrm{mg} / \mathrm{h}$ & : & 1 & $i$ \\
\hline 2-nitrophenol 88-75-5 & $<0.030 \mathrm{mg} / \mathrm{l}$ & & $<0.030 \mathrm{mg} / \mathrm{l}$ & & 1 & \\
\hline tatsoro-3-3ethyl pheod s9-5a-7 & $<0.030 \mathrm{mg} / \mathrm{l}$ & & $<0.030 \mathrm{mg} / \mathrm{l}$ & & 1 & ! \\
\hline 4 dilroptoond $100-02-7$ & $<0.050 \mathrm{mg} / \mathrm{l}$ & & $<0.050 \mathrm{mg} / \mathrm{l}$ & $!$ & 1 & \\
\hline 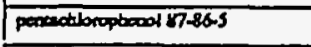 & $<0.050 \mathrm{mg} / \mathrm{l}$ & & $<0.050 \mathrm{mg} / 1$ & $!$ & 1 & \\
\hline phoond 108-92-2 & $<0.030 \mathrm{mg} / \mathrm{l}$ & & $<0.030 \mathrm{mg} / \mathrm{l}$ & 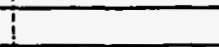 & 1 & \\
\hline 4,4'-DDD 72-548 & $<0.00001 \mathrm{mg} / \mathrm{l}$ & & $<0.00001 \mathrm{mg} / \mathrm{l}$ & T & 1 & \\
\hline $4,4^{4}-\mathrm{DDE} 72.55-9$ & $<0.00001 \mathrm{mg} / \mathrm{l}$ & & $<0.00001 \mathrm{mg} / \mathrm{l}$ & $\overrightarrow{1}$ & 1 & \\
\hline 4,4-DOT 50-29-3 & $<0,00001 \mathrm{mg} / \mathrm{l}$ & & $<0.00001 \mathrm{mg} / \mathrm{l}$ & ! & 1 & \\
\hline Adsin 30200-2 & $<0.00001 \mathrm{mg} / 1$ & & $<0.00001 \mathrm{mg} / \mathrm{l}$ & i & 1 & \\
\hline ctiondares 57.74-9 & $<0.00002 \mathrm{mg} / \mathrm{l}$ & & $<0.00002 \mathrm{mg} / \mathrm{l}$ & I & 1. & \\
\hline diseldrin 60-57-1 & $<0.00001 \mathrm{mg} / 1$ & & $<0.00001 \mathrm{mg} / \mathrm{l}$ & T & 1 & \\
\hline codoculathan $1959-98-8$ & $<0.00001 \mathrm{mg} / \mathrm{l}$ & & $<0.00001 \mathrm{mg} / 1$ & & 1 & \\
\hline codsoultan I1 33213-65-9 & $<0.00001 \mathrm{mg} / 1$ & & $<0.00001 \mathrm{mg} / \mathrm{l}$ & & 1 & \\
\hline 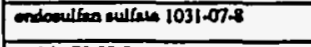 & $<0.00001 \mathrm{mg} / \mathrm{l}$ & & $<0.00001 \mathrm{mg} / \mathrm{l}$ & 7 & 1 & \\
\hline codrin 72.20.8 & $<0.00005 \mathrm{mg} / \mathrm{l}$ & & $<0.00005 \mathrm{mg} / \mathrm{l}$ & & 1 & \\
\hline codrin aldebodo 7421-934 & $<0.00001 \mathrm{mg} / \mathrm{l}$ & & $<0.00001 \mathrm{mg} / \mathrm{l}$ & & 1 & \\
\hline hepectibe $76-448$ & $<0.00001 \mathrm{mg} / \mathrm{l}$ & & $<0.00001 \mathrm{mg} / \mathrm{l}$ & & 1 & 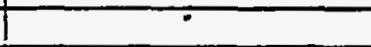 \\
\hline repectibor epouide 102457.3 & $<0.00001 \mathrm{mg} / \mathrm{l}$ & & $<0.00001 \mathrm{mg} / \mathrm{l}$ & & 1 & \\
\hline 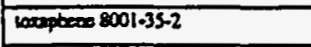 & $<0.0002 \mathrm{mg} / \mathrm{l}$ & & $<0.0002 \mathrm{mg} / \mathrm{l}$ & & 1 & \\
\hline 4\% & $<0.00001 \mathrm{mg} / \mathrm{l}$ & & $<0.00001 \mathrm{mg} / \mathrm{l}$ & & 1 & \\
\hline W0.-8HC $319-85.7$ & $<0.00001 \mathrm{mg} / \mathrm{l}$ & & $<0.00001 \mathrm{mg} / \mathrm{l}$ & & 1 & \\
\hline d)-BHC 31286 & $<0.00001 \mathrm{mg} / \mathrm{l}$ & & $<0.00001 \mathrm{mg} / \mathrm{l}$ & & 1 & \\
\hline anm-BHC $58-898$ & $<0.00001 \mathrm{mg} / \mathrm{l}$ & & $<0.00001 \mathrm{mg} / \mathrm{l}$ & & 1 & \\
\hline 2-ctionowbonol 95-57-5 & $<0.030 \mathrm{mg} / \mathrm{l}$ & & $<0.030 \mathrm{mg} / \mathrm{l}$ & i & 1 & $\dot{.}$ \\
\hline Arocion $12 \sqrt{2}$ 53469-21-9 & $<0.0005 \mathrm{mg} / \mathrm{l}$ & . & $<0.0005 \mathrm{mg} / 1$ & & 1 & \\
\hline Aroctor-1256 1109769-1 & $<0.0005 \mathrm{mg} / \mathrm{l}$ & & $<0.0005 \mathrm{mg} / \mathrm{l}$ & $T$ & 1 & \\
\hline Arocior-1221 11104-22-2 & $<0.0005 \mathrm{mg} / \mathrm{l}$ & & $<0.0005 \mathrm{mg} / 1$ & & 1 & \\
\hline Aroctom 123211141.16 .5 & $<0.0005 \mathrm{mg} / \mathrm{l}$ & & $<0.0005 \mathrm{mg} / \mathrm{l}$ & 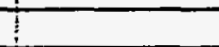 & 1 & \\
\hline Aroctor 124812672.296 & $<0.0005 \mathrm{mg} / \mathrm{l}$ & 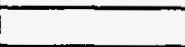 & $<0.0005 \mathrm{mg} / \mathrm{l}$ & $i$ & 1 & \\
\hline Arocton-1200 1100682.5 & $<0.0005 \mathrm{mg} / \mathrm{l}$ & & $<0.0005 \mathrm{mg} / 1$ & $i$ & 1 & \\
\hline Aroctom 10161207411.2 & $<0.0005 \mathrm{mg} / \mathrm{l}$ & & $<0.0005 \mathrm{mg} / \mathrm{l}$ & & 1 & \\
\hline Croose & $<0.010 \mathrm{mg} / \mathrm{l}$ & & $<0.010 \mathrm{mg} / \mathrm{ll}$ & & 1 & \\
\hline Actoleter & $<0.010 \mathrm{mg} / \mathrm{l}$ & & $<0.010 \mathrm{mg} / \mathrm{l}$ & & 1 & \\
\hline Aaylowitnk & $<0.010 \mathrm{mg} / \mathrm{l}$ & & $<0.010 \mathrm{mg} / 1$ & & 1 & \\
\hline 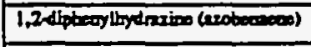 & Rexultes pending & & Rewults pending & & 1 & \\
\hline & & & & & & - \\
\hline & & & & & & \\
\hline & & & & & & \\
\hline & & & & & & \\
\hline & & & & & & \\
\hline & & & & & & \\
\hline & & & & & & \\
\hline & & & & & & \\
\hline & & & & & & \\
\hline & & & & & & \\
\hline & & & & & & \\
\hline & & & & & & \\
\hline & & & & & & \\
\hline & & & & & & \\
\hline & $i$ & & & & & \\
\hline & 1 & & & & & \\
\hline
\end{tabular}


Outfall S22

Para B and C Continued

\begin{tabular}{|c|c|c|c|c|c|c|}
\hline \multirow{2}{*}{$\begin{array}{l}\text { Polthunat } \\
\text { and } \\
\text { CAS Number } \\
\text { (if availible) }\end{array}$} & \multicolumn{2}{|c|}{$\begin{array}{l}\text { Maximum Value } \\
\text { (include uniu) }\end{array}$} & \multicolumn{2}{|c|}{$\begin{array}{l}\text { Avernge Values } \\
\text { (inelode units) }\end{array}$} & \multirow{2}{*}{$\begin{array}{c}\text { Nuraber } \\
\text { of } \\
\text { Stomm } \\
\text { Eveas } \\
\text { Sempiod }\end{array}$} & \multirow[b]{2}{*}{ Sources of Polluenses } \\
\hline & $\begin{array}{l}\text { Grab Sample } \\
\text { Takea During } \\
\text { Firnt } 30 \\
\text { Minutes }\end{array}$ & $\begin{array}{cc}\vdots & \\
\vdots & \text { Flow-weightod } \\
i & \text { Composite } \\
\vdots & \end{array}$ & $\begin{array}{cc}\vdots & \text { Gnbb Sample } \\
\text { Taken During } \\
\text { Fint } 30 \\
\text { Minutes }\end{array}$ & $\begin{array}{c}\text { Fow-weightod } \\
\text { Componite }\end{array}$ & & \\
\hline Beriven 740-39-3 & $0.0196 \mathrm{mg} / 1$ & i & $0.0196 \mathrm{mg} / \mathrm{l}$ & $!$ & 1 & 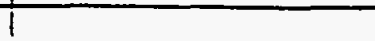 \\
\hline meloyleno ctibride 75-09-2 & $<0.010 \mathrm{mg} / \mathrm{l}$ & $T$ & $<0.010 \mathrm{mg} / \mathrm{l}$ & $\bar{\vdots}$ & 1 & \\
\hline 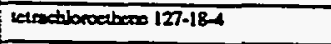 & $<0.010 \mathrm{mg} / \mathrm{l}$ & 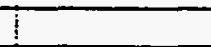 & $<0.010 \mathrm{mg} / \mathrm{l}$ & 1 & 1 & \\
\hline inctionocheos 79016 & $<0.010 \mathrm{mg} / 1$ & 1 & $<0.010 \mathrm{mg} / \mathrm{l}$ & 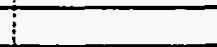 & 1 & \\
\hline$x y=0$ & $<0.010 \mathrm{mg} / \mathrm{l}$ & 1 & $<0.010 \mathrm{mg} / \mathrm{l}$ & $\mathrm{i}$ & 1 & \\
\hline 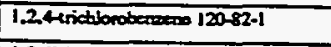 & $<0.010 \mathrm{mg} / \mathrm{l}$ & $i$ & $1<0.010 \mathrm{mg} / \mathrm{l}$ & $!$ & 1 & \\
\hline 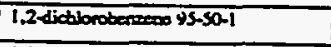 & $<0.010 \mathrm{mg} / \mathrm{t}$ & 1 & $<0.010 \mathrm{mg} / \mathrm{l}$ & $!$ & 1 & \\
\hline 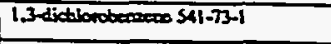 & $<0.010 \mathrm{mg} / \mathrm{l}$ & $!$ & $<0.010 \mathrm{mg} / \mathrm{l}$ & $i$ & 1 & \\
\hline 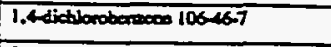 & $<0.010 \mathrm{mg} / \mathrm{l}$ & + & $<0.010 \mathrm{mg} / \mathrm{l}$ & 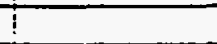 & 1 & \\
\hline 24disingedvenos $121-142$ & $<0.010 \mathrm{mg} / \mathrm{l}$ & $i$ & $<0.010 \mathrm{mg} / \mathrm{l}$ & $!$ & 1 & \\
\hline 2.6-dinitrodokesos 606-20-2 & $<0.010 \mathrm{mg} / \mathrm{l}$ & 1 & $\mid<0.010 \mathrm{mg} / \mathrm{h}$ & ! & 1 & \\
\hline 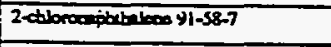 & $<0.010 \mathrm{mg} / \mathrm{l}$ & & $<0.010 \mathrm{mg} / 1$ & $i$ & 1 & \\
\hline 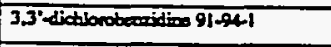 & $<0.020 \mathrm{mg} / \mathrm{l}$ & & $<0.020 \mathrm{mg} / 1$ & & 1 & \\
\hline 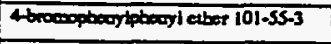 & $<0.010 \mathrm{mg} / \mathrm{l}$ & & $<0.010 \mathrm{mg} / \mathrm{l}$ & & 1 & \\
\hline 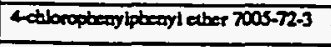 & $<0.010 \mathrm{mg} / \mathrm{l}$ & & $<0.010 \mathrm{mg} / \mathrm{l}$ & & 1 & \\
\hline 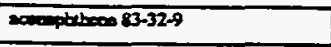 & $<0.010 \mathrm{mg} / \mathrm{l}$ & & $<0.010 \mathrm{mg} / \mathrm{l}$ & 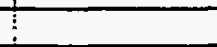 & 1 & \\
\hline 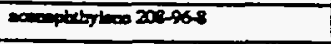 & $<0.010 \mathrm{mg} / \mathrm{l}$ & & $<0.010 \mathrm{mg} / \mathrm{l}$ & & 1 & $\div$ \\
\hline 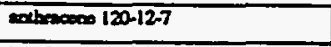 & $<0.010 \mathrm{mg} / \mathrm{l}$ & & $<0.010 \mathrm{mg} / 1$ & & 1 & \\
\hline 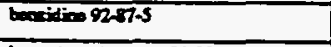 & $<0.050 \mathrm{mg} / \mathrm{l}$ & & $<0.050 \mathrm{mg} / \mathrm{l}$ & & 1 & \\
\hline 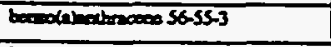 & $<0.010 \mathrm{mg} / \mathrm{l}$ & & $<0.010 \mathrm{mg} / \mathrm{l}$ & & 1 & \\
\hline bociodatoryous $50-32-8$ & $<0.010 \mathrm{mg} / \mathrm{l}$ & & $<0.010 \mathrm{mg} / \mathrm{l}$ & & 1 & \\
\hline 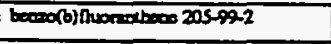 & $<0.010 \mathrm{mg} / \mathrm{l}$ & & $<0.010 \mathrm{mg} / \mathrm{l}$ & & 1 & \\
\hline beneodechiperykeo 191-24-2 & $<0.010 \mathrm{mg} / 1$ & & $<0.010 \mathrm{mg} / 1$ & & 1 & \\
\hline 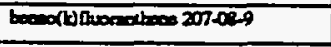 & $<0.010 \mathrm{mg} / 1$ & & $<0.010 \mathrm{mg} / \mathrm{l}$ & - & 1 & \\
\hline 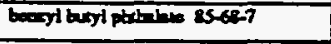 & $<0.010 \mathrm{mg} / \mathrm{l}$ & & $<0.010 \mathrm{mg} / 1$ & & 1 & - \\
\hline 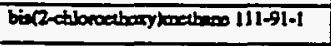 & $<0.010 \mathrm{mg} / 1$ & & $<0.010 \mathrm{mg} / \mathrm{l}$ & & 1 & \\
\hline 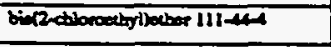 & $<0.010 \mathrm{mg} / \mathrm{l}$ & & $<0.010 \mathrm{mg} / \mathrm{l}$ & & 1 & \\
\hline 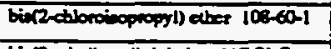 & $<0.010 \mathrm{mg} / \mathrm{l}$ & & $<0.010 \mathrm{mg} / \mathrm{l}$ & & 1 & \\
\hline 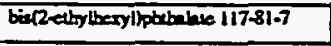 & $<0.010 \mathrm{mg} / \mathrm{l}$ & $i$ & $<0.010 \mathrm{mg} / \mathrm{l}$ & & 1 & \\
\hline 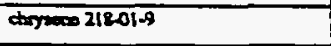 & $<0.010 \mathrm{mg} / \mathrm{l}$ & $i$ & $<0.010 \mathrm{mg} / \mathrm{l}$ & $\frac{1}{1}$ & 1 & \\
\hline dioturyl ptenche 84-74-2 & $<0.010 \mathrm{mg} / \mathrm{l}$ & & $<0.010 \mathrm{mg} / \mathrm{l}$ & & 1 & \\
\hline 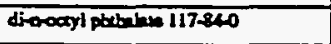 & $<0.010 \mathrm{mg} / \mathrm{l}$ & & $<0.010 \mathrm{mg} / 1$ & & 1 & \\
\hline 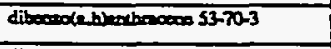 & $<0.010 \mathrm{mg} / \mathrm{l}$ & & $<0.010 \mathrm{mg} / 1$ & & 1 & \\
\hline 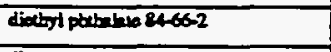 & $<0.010 \mathrm{mg} / \mathrm{l}$ & & $<0.010 \mathrm{mg} / \mathrm{i}$ & & 1 & \\
\hline dinctoyl pothelab $131-11-3$ & $<0.010 \mathrm{mg} / \mathrm{l}$ & & $<0.010 \mathrm{mg} / \mathrm{l}$ & & 1 & \\
\hline 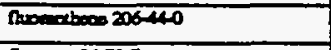 & $<0.010 \mathrm{mg} / \mathrm{l}$ & & $<0.010 \mathrm{mg} / \mathrm{l}$ & & 1 & \\
\hline 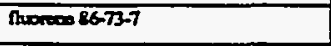 & $<0.010 \mathrm{mg} / \mathrm{l}$ & & $<0.010 \mathrm{mg} / 1$ & & 1 & \\
\hline 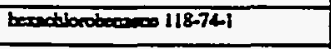 & $<0.010 \mathrm{mg} / \mathrm{l}$ & & $<0.010 \mathrm{mg} / \mathrm{I}$ & & 1 & \\
\hline 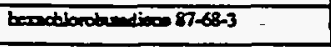 & $<0.010 \mathrm{mg} / \mathrm{l}$ & & $<0.010 \mathrm{mg} / \mathrm{l}$ & & 1 & \\
\hline 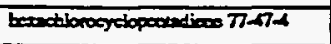 & $<0.010 \mathrm{mg} / 1$ & & $<0.010 \mathrm{mg} / 1$ & & 1 & \\
\hline 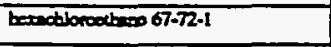 & $<0.010 \mathrm{mg} / \mathrm{l}$ & & $<0.010 \mathrm{mg} / 1$ & & 1 & \\
\hline $193-39.5$ & $<0.010 \mathrm{mg} / 1$ & & $<0.010 \mathrm{mg} / \mathrm{l}$ & & 1 & \\
\hline sophorowe 78-59-1 & $<0.010 \mathrm{mg} / \mathrm{l}$ & & $<0.010 \mathrm{mg} / \mathrm{l}$ & & 1 & \\
\hline 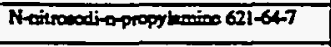 & $<0.010 \mathrm{mg} / \mathrm{l}$ & & $<0.010 \mathrm{mg} / \mathrm{l}$ & & 1 & \\
\hline 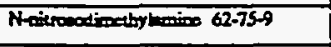 & $<0.010 \mathrm{mg} / \mathrm{l}$ & & $<0.010 \mathrm{mg} / \mathrm{l}$ & & 1 & \\
\hline 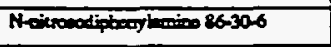 & $<0.010 \mathrm{mg} / \mathrm{l}$ & & $<0.010 \mathrm{mg} / \mathrm{l}$ & & 1 & \\
\hline $90.21-20-3$ & $<0.010 \mathrm{mg} / \mathrm{l}$ & & $<0.010 \mathrm{mg} / 1$ & & 1 & \\
\hline 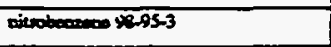 & $<0.010 \mathrm{mg} / \mathrm{l}$ & & $<0.010 \mathrm{mg} / \mathrm{l}$ & & 1 & \\
\hline poodrocos 85018 & $<0.010 \mathrm{mg} / \mathrm{l}$ & & $<0.010 \mathrm{mg} / \mathrm{l}$ & & 1 & \\
\hline 870001229000 & $<0.010 \mathrm{mg} / 1$ & & $<0.010 \mathrm{mg} / \mathrm{l}$ & $\cdot$ & 1 & \\
\hline 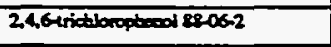 & $<0.030 \mathrm{mg} / \mathrm{l}$ & & $<0.030 \mathrm{mg} / \mathrm{l}$ & & 1 & \\
\hline 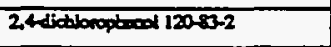 & $<0.030 \mathrm{mg} / \mathrm{l}$ & & $<0.030 \mathrm{mg} / \mathrm{l}$ & & 1 & \\
\hline 24dimabybocod 105-67-9 & $<0.030 \mathrm{mg} / \mathrm{l}$ & & $<0.030 \mathrm{mg} / \mathrm{l}$ & & 1 & \\
\hline
\end{tabular}

24dinabyboed 105-67-9

$<0.030 \mathrm{mg} / \mathrm{l}$

$<0.030 \mathrm{mg} / \mathrm{l}$ 


\section{APPENDIX B \\ Y-12 SITE MAPS}

: 


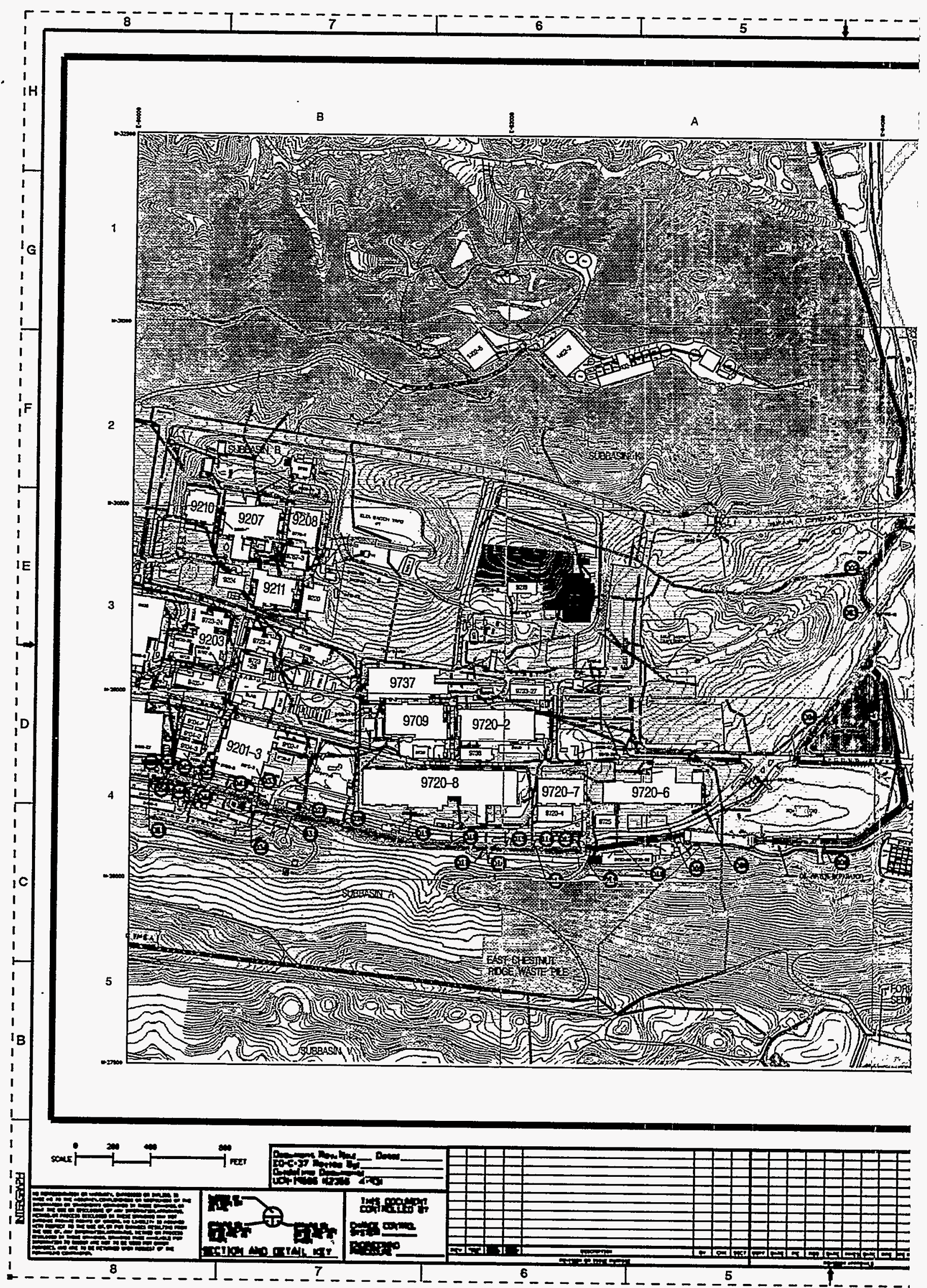


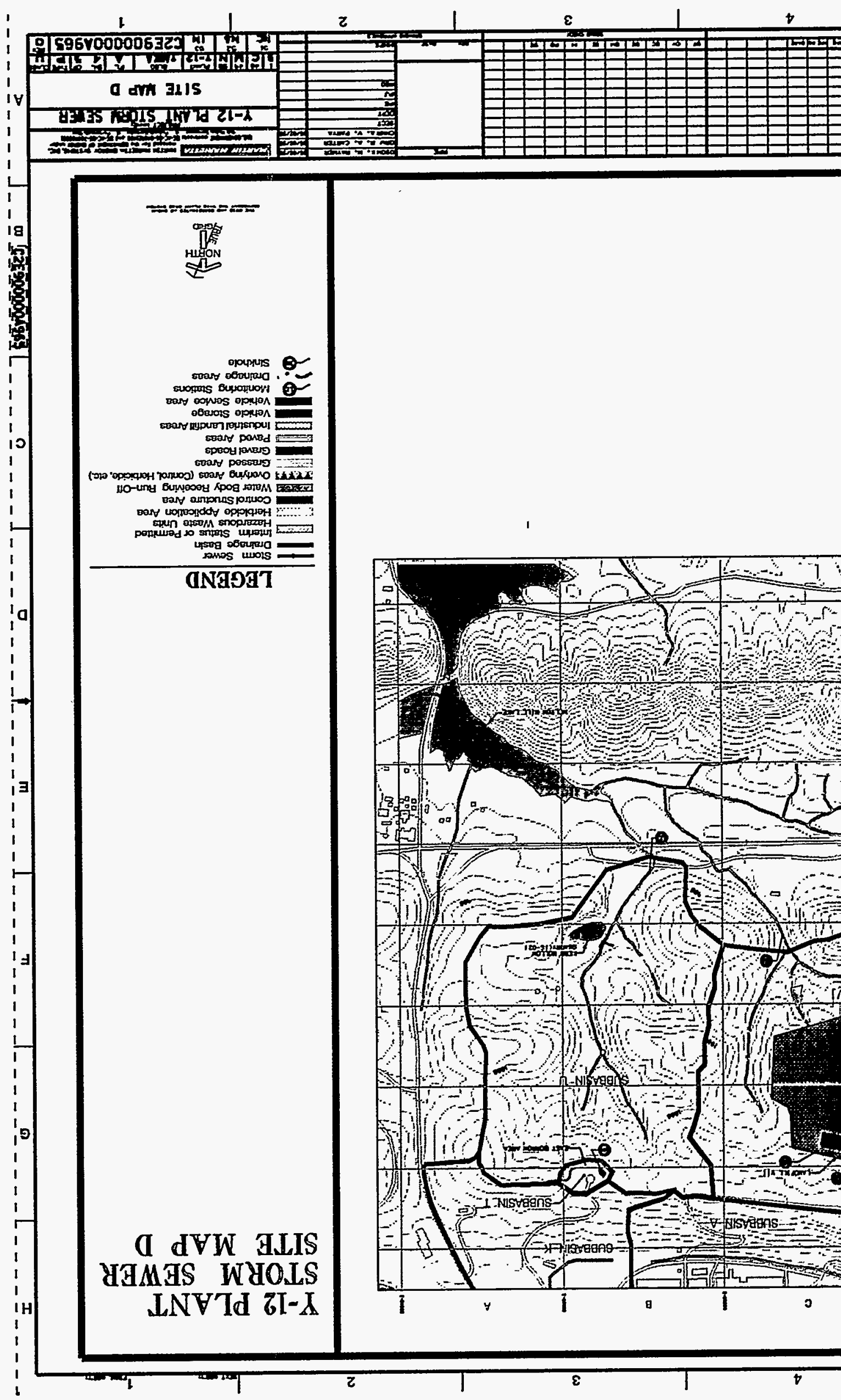




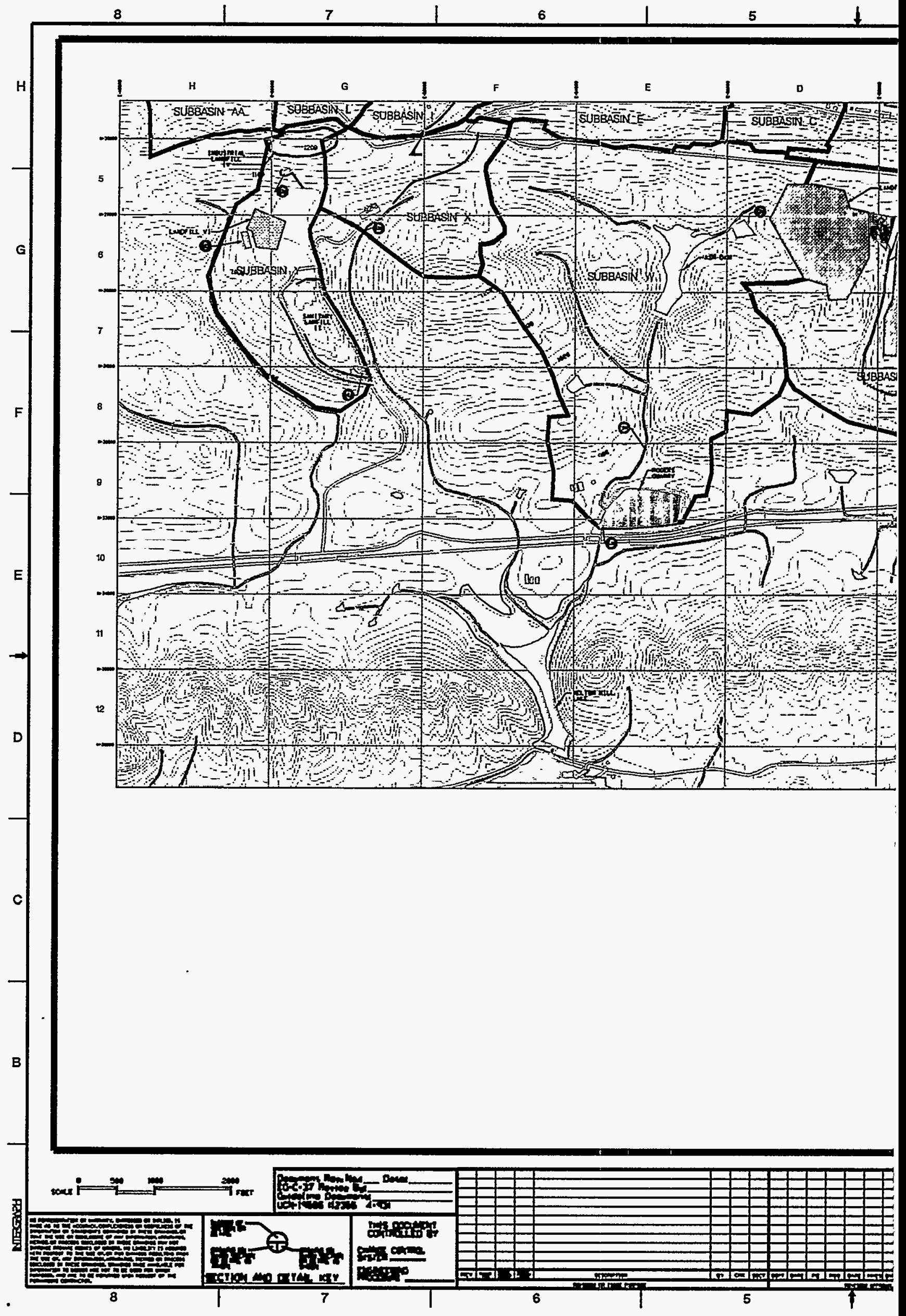




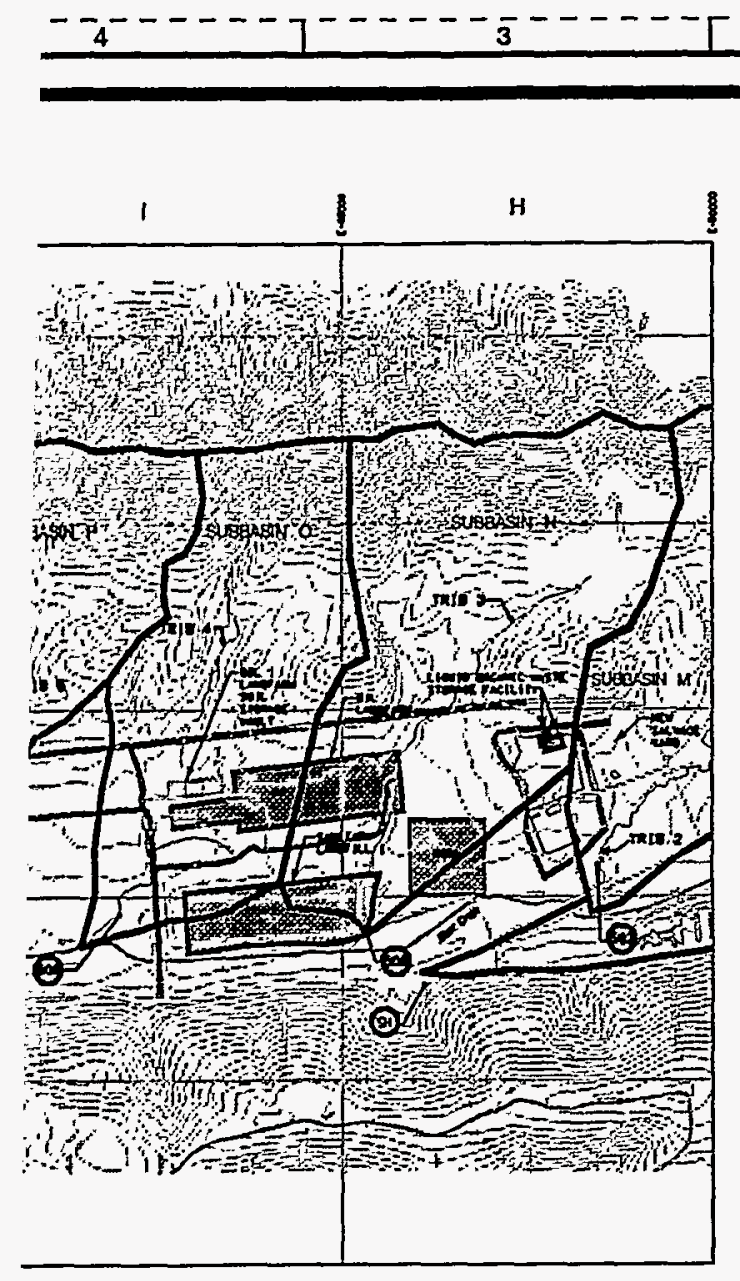

Y-12 PLANT STORM SEWER SITE MAP $\mathbb{E}$

\section{LEGEND}
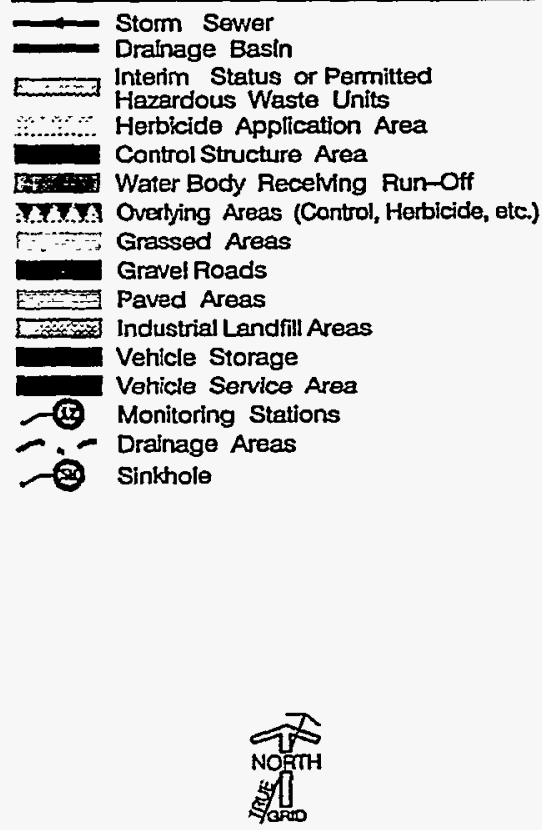


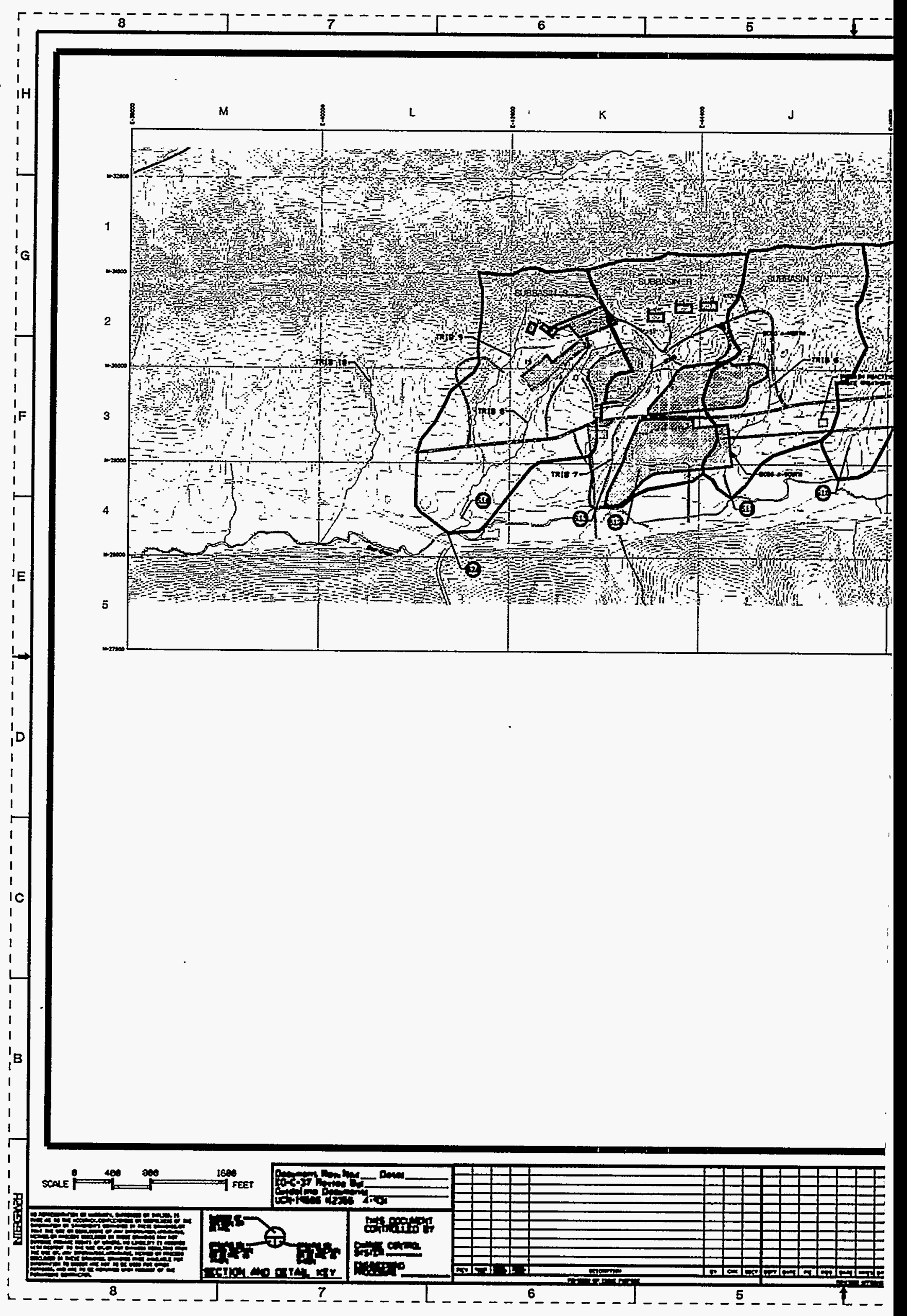




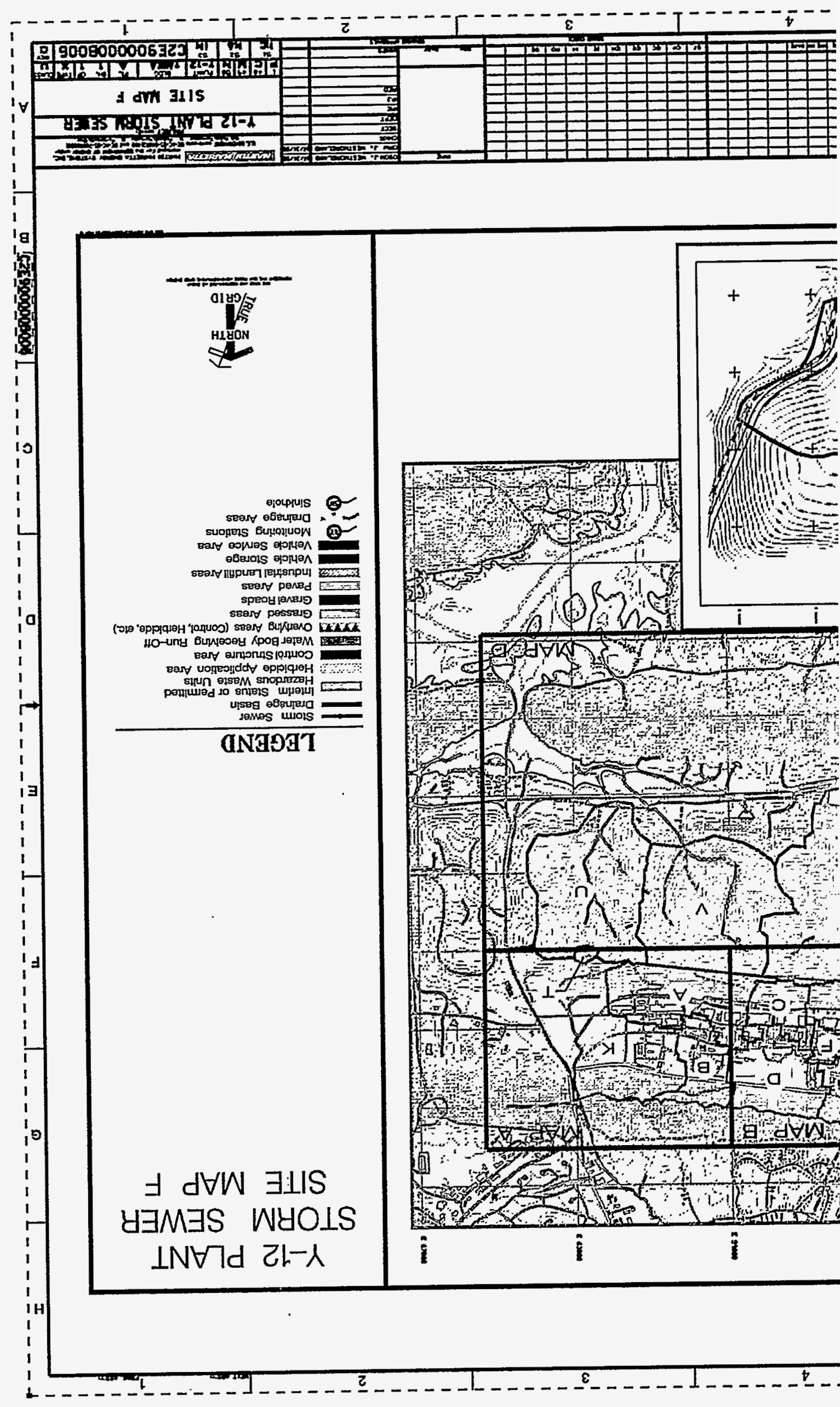




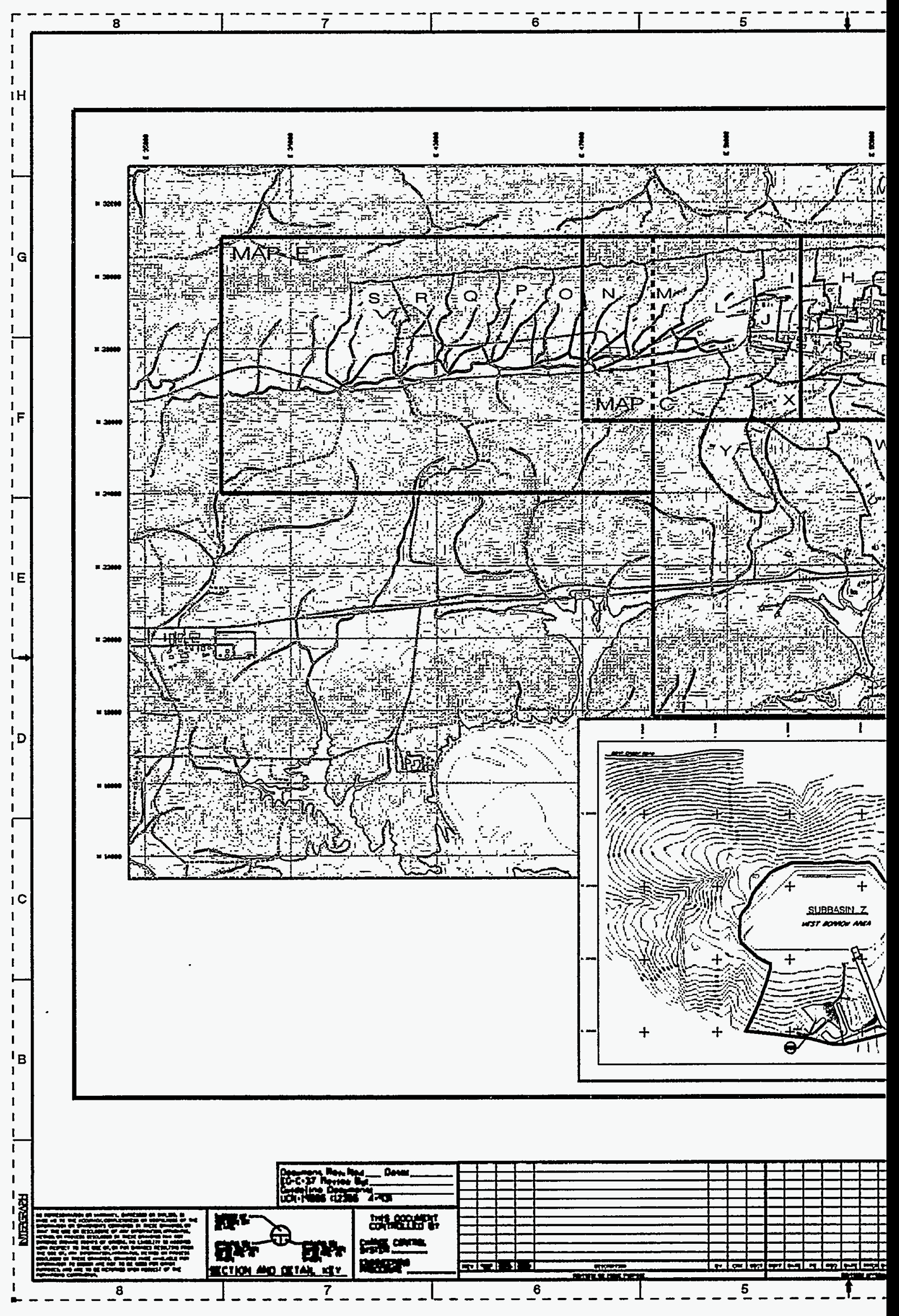


APPENDIX C

Y-12 PLANT NATIONAL POLLUTANT DISCHARGE ELIMINATION SYSTEM PERMIT 
PART I 2

EFFLLUENT LIMITATIONS AND MONITORING REQUIREMENTS 2

Minor Outfalls without Storm Water 066, 068, 117 2

Minor Outfalls 073, 077, 122, 133 3

Minor Outfall 125 4

Minor Outfalls used for potabie water line fushing 013, 031 5

Storm Water Category I Outfalls 003, 006, 007, 008, 009, 011, 015, 018, 032, 033, 045, 046, 058, 062, 086, 087, 098, 110, 134, 213, S01, S03, S04, S06, S07, S09, S15, S16, and S18

Storm Water Category II Outfalls 004, 010, 014, 016, 019, 020, 041, 044, 057, 063, 064, 067, 083, 088, 099, 126, S02, S08, S10, S11, S12, S13, S17, S20, S24

Storm Water Category n Outfalls S21, S22, S25, S26, S27, S28 \& S29 8

Storm Water Category II Outfall S19 9

Storm Water Category III Outfalls 002, 034, 042, 047, 048, 054, 071, 109, 113, 114, 505, \& S14 10

Outfall 201 11

Outfall 200 $+$ 12

Outfall 135 13

Outfall 021 14

Outfall 017 15

Outfall 055 16

Outfall 55A 16

Outfall 550

Outfall 551 17

Outfall 051 18

Outfall 501 19

Outfall 502 21

Outfall 503 23

Outfall 512 24 
Outfall 520

Narrative Conditiona/Requirements for Discharges

MONITORING PROCEDURES 26

DEFINITIONS

REPORTING 28

SCHEDULE OF COMPLIANCE 29

Mercury Treatmeat and Remediation

Addition of Clinch River Raw Water

Ammonia Reduction at Outfall 017

PART II

GENERAL PROVISIONS

Duty to Reapply

Right of Entri:

Availability of Reports

Proper Operation and Maintenance

Treatment Facility Failure (Industrial Sources)

Property Rights

Sererability

Other Information

CAANGES AFFECTING THE PERMIT _ 33

Planned Changes ___ 33

Permil Modification, Revocation, or Termination __ 33

Change of Ornership

Change of Mailing Address___ 34

NONCONLPLLNCE ___ 34

Effect of Noncompliance ___ 34

Reponing of Noncompliance ___ 34

Bypassing __ 35

Diversion — 35

Upset__ 35

Washour __ 36

Adverse Impact ___ 36

LUABITIES _ 36

Civil and Criminal Liability______ 36

Liability Under State Law ___ 37

PART III _ 38

TOXIC POLLUTANTS___ 38

COOLNG TOWER REQUIREMENTS ___ 38 
Perait Table of Contents, Page it

BIOMONITORING REQUIREMENTS, CHRONIC 39

PLACEMENT OF SIGNS

40

REÖPENER CLAUSE FOR PRLMARY INDÜSTRIES

41

WASTEWATER CONTROL

41

BIOLOGICAL MONITORING AND ABATEMENT PROGRAM 42

RADIOLOGICAL MONITORNG 42

MERCURY REMEDIATION 43

ADDITION OF RAW WATER TO EAST FORK POPLAR CREEK____ 43

CHANGES TO STORM WATER MONITORING AND OUTFALLS 43

BEST MANAGEMENT PRACTICES, FLUSHING WATER LINES 44

TEMPERATURE PROFHE OF EAST FORK POPLAR CREEK 44 FECAL COLFORM ANALYSES INVESTIGATION 44 SUBMISSION OF FORM 2C 45

PART IV 48

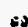

STORM WATER POLLUTION PREVENTION PLAN 46 POLLUTANT SOURCES AND PATEWAYS 46 STORM WATER MANAGEMENT CONTROLS 46

FACILITY INSPECTION 37 SPILL PREVENTION CONTROL AND COUNTERMEASURES 47

PLAN REVIEW AND UPDATE 47

PLAN IMPLEMENTATION 47

PLAN AVAILABIITY 47

PLAN MODIFICATION 48 MONITORING PLAN 48

SARA TITLE II, SECTION 313 PRIORITY CEEMICALS 48 


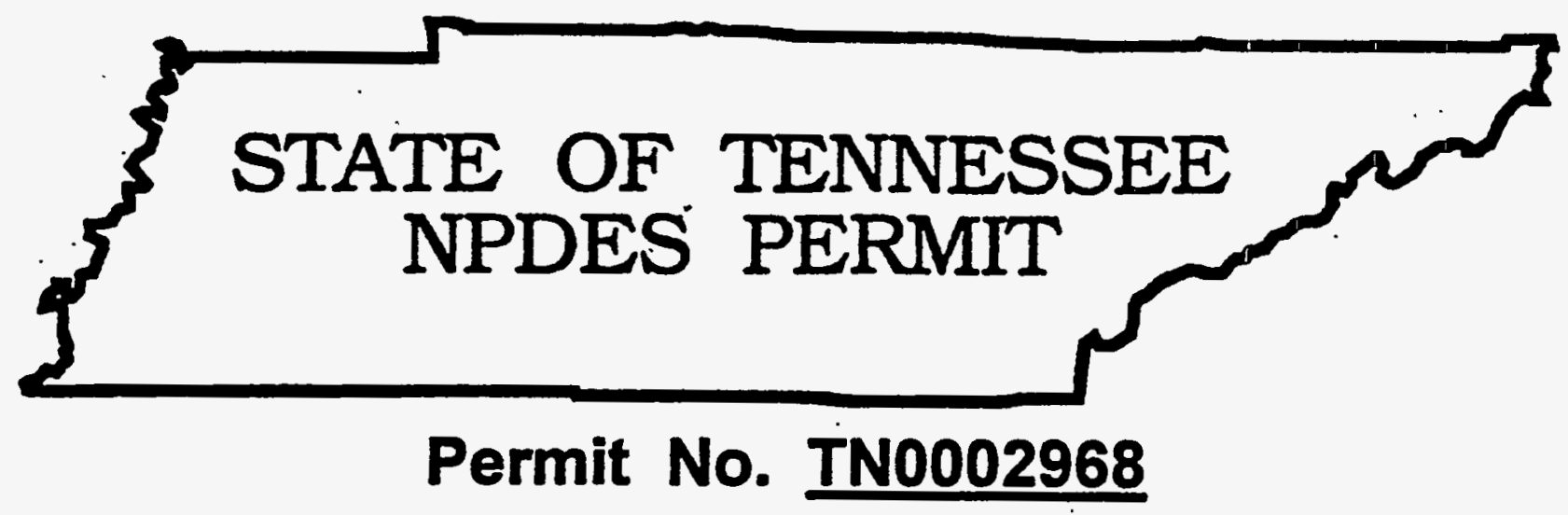

Authorization to discharge under the

National Pollutant Discharge Elimination System

Issued By

Tennessee Department of Environment and Conservation

Division of Water Pollution Control

401 Church Street, L \& C Annex - 6th Floor

Nashville, Tennessee $37243-1534$

Under authority of the Tennessee Water Quality Control Act of 1977 (T.C.A. 69-3-101, et seg.) and the delegation of authority from the United States Environmental Protection Agency under the Federal Water Pollution Control Act, as amended by the Clean Water Act of 1977 (33 U.S.C. 1251, et seg.)

\section{Discharger: United States Department of Energy Y-12 SITE}

is authorized to discharge process wastewaters and other wastewaters which have been accepted for treatment via waste acceptance procedures, cooling tower blowdown, cooling waters, condensate, sump waters, storm water runoff and ground water

from a facility located at Oak Ridge, Andeŕson County, Tennessee

to receiving waters named: East Fork Poplar Creek, Bear Creek, McCoy Branch, unnamed tributary to Bear Creek and unnamed tributaries to the Clinch River

in accordance with effluent limitations, monitoring requirements and other conditions set forth herein.

This permit shall become effective on: July 1, 1995

This permit shall expire on: April 27, 2000

Issuance date: April 28, 1995

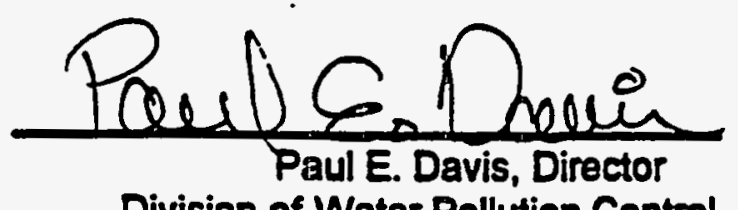


DOE Y-12 PLANT, TNO002968, ANDERSON COLNT:

PAGE 2 OF 50

\section{PART I}

A EFFLUENT LIMITATIONS AND MONITORING REQUIREMENTS

United States Department of Energy, Y-12 Site, is authorized to discharge steam condensate through Outfalls 066, 068 and 117 to East Fork Poplar Creek. The discharge may also contain infiltrated ground waters. Discharges 066, 068 \& 117 shall be limited and monitored by the permittee as specified below:

\section{Outfalls 066, $068 \& 117$}

Elluent characteristics Efnuent limitations Monitoring Requirements

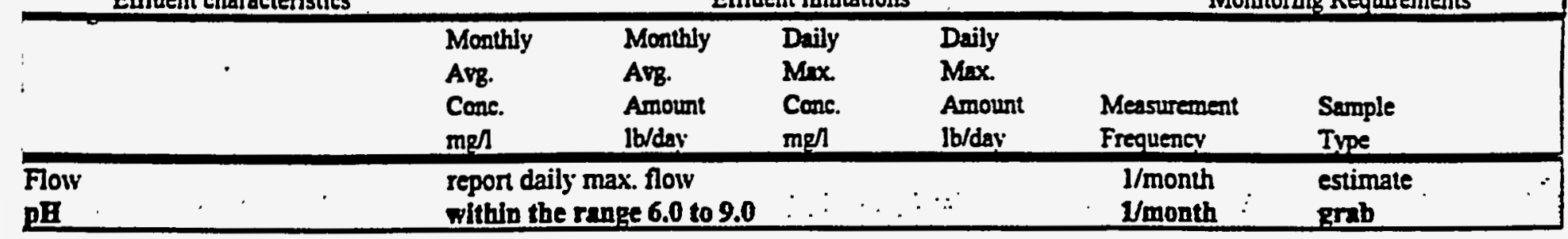

Daily maximum flow measured for the month shall be reported as million gallons per day. 
United States Depanment of Energy, Y-12 Site, is authorized to discharge steam condensate and cooling waters through Outfalls 073, 077, 122 and 133 to East Fork Poplar Creek. The discharge may have some infiltrated ground nater in it. Discharges.073, 077. 122 and 133 shall be limited and monitored by the permittee as specified below:

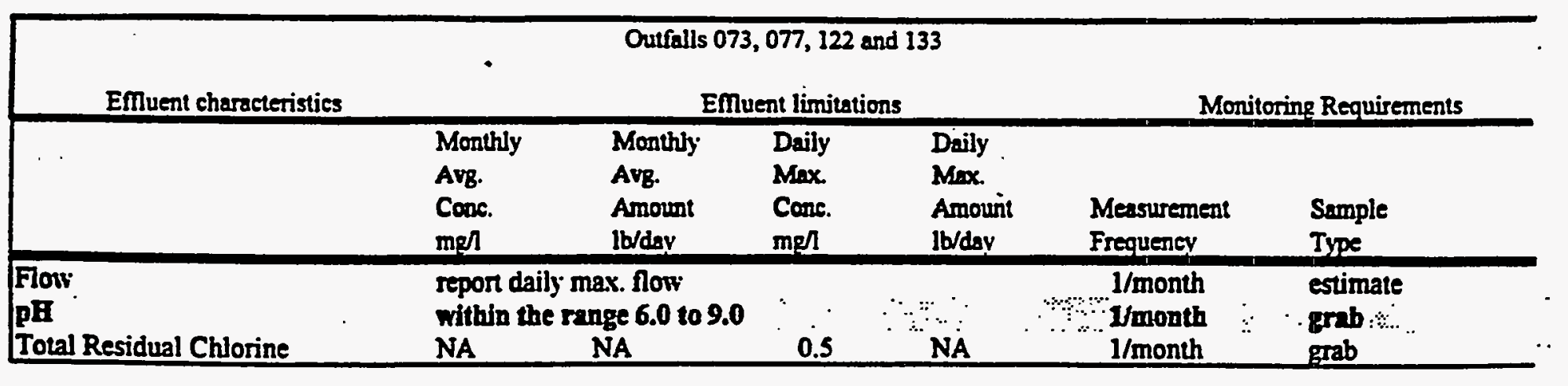

Daily mavimum flow measured for the month shall be reported as million gallons per day. 
United States Department of Energy. Y-12 Site, is authorized to discharge potable water when flushing out water lines as required by maintenance activity for the water system through Outfalls 013 and 031 to East Fork Poplar Creek without limitation. Discharges 013 and 031 shall be operated according to best mariagement practices to minimize impacts instream as described in the plan to be submitted under a Best Mapagement Practices Plan, see Permit Part III-L.

Waters discharged through other outfalls from fire hydrants or sprinklers or via vahes at the extremities of the potable water distribution lines shall be perminted with no other requirement other than best management practices be followed as reflected in the plan developed in Permit Part III-L. Best management practices will not be required of emergency situations such as fire-fighting. 
United States Deparment of Energy, Y-12 Site, is authorized to discharge storm waters, steam condensate, cooling tower blowdown and groundwater through Outfalls 003,006,007, 008, 009, 011, 015, 018, 032, 033, 045, 046, 058, 062, 086, 087, 098, 110, 134, and 213 to East Fork Poplar Creek; to discharge storm water runoff through Outfalls Sól, S03, SO4, and S15 which discharge to Bear Creek. The United States Deparment of Energy, Y-12 Site shall conduet instream monitoring and storm water monitoring at points in tributaries of Bear Creek which for tracking purposes in this permit are designated as S06, S07, and S09; and shall monitor discharge from the East Borrow area pond, designated as Outfall S16, which goes to the same tributary that received discharge from the Kerr Hollow Quarry; and shall monitor an instream monitoring point, S18, in an unnamed tributary. Flows from both S16 and S18 will eventually enter the Clinch River at about mile 41.1.

Discharges or monitoring points 003, 006, 007, 008, 009, 011, 015, 016, 018, 032, 033, 045, 046, 058, 062 , 086, 087, 098, 110, 134, 213, S01, S03, S04, S06, S07, S09, and S18 shall be limited and monitored by the permittee as follows.

Category I Storm Water Outfalls and Monitoring Points

Emuen! characteristics Emuent limitations Monitoring Requirements

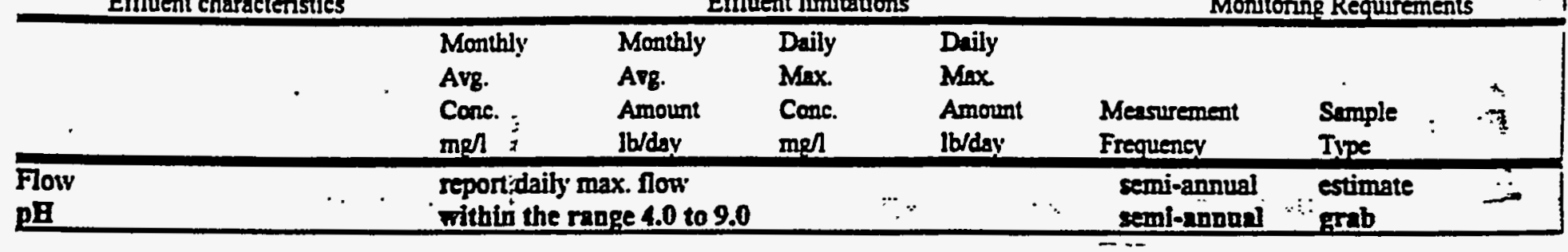

Outfalls S15 and S16 shall be limited and monitored by the permittee as follows.

Category I Storm Water Outfalls and Monitoring Points

Emuent characteristics Emvent limitations Monitoring Requirements

\begin{tabular}{|c|c|c|c|c|c|c|}
\hline 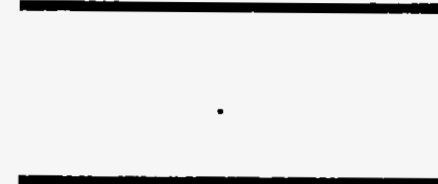 & $\begin{array}{l}\text { Monthly } \\
\text { Avg. } \\
\text { Conc. } \\
\text { mgn }\end{array}$ & $\begin{array}{l}\text { Monthly } \\
\text { Avg. } \\
\text { Amount } \\
\text { Jb/duy }\end{array}$ & $\begin{array}{l}\text { Daily } \\
\text { Max. } \\
\text { Conc. } \\
\text { mel }\end{array}$ & $\begin{array}{l}\text { Daily } \\
\text { Minx. } \\
\text { Amount } \\
\text { Ib/day }\end{array}$ & $\begin{array}{l}\text { Mearurement } \\
\text { Frequency }\end{array}$ & $\begin{array}{l}\text { Semple } \\
\text { Type }\end{array}$ \\
\hline $\begin{array}{l}\text { Flow } \\
\text { pR }\end{array}$ & $\begin{array}{l}\text { report da } \\
\text { within th }\end{array}$ & $\begin{array}{l}\text { 2x. flowi } \\
\text { uge } 6.0 \text { to } 10.0\end{array}$ & $\therefore \because$ & $\because$ & cemi-annual & $\begin{array}{l}\text { estimate } \\
\text { mab. }\end{array}$ \\
\hline
\end{tabular}

Daily maximum measured flow will be reported as million gallons per day.

In addition to the above mionitoring, the stom runoff shall be monitored by a characterization at least twice during the permit period using flow-weighted or time-weighted composites as part of the Storm Water Pollution Prevention Plan for the site. Characterization of a single outfall may be made where several outfalls are very similar rather than charecterization of each of the outfalls in this group of similar outfalls. However, such groupings must be listed in the Storm Water Pollution Prevention Plan and be approved by the Division of Water Pollution Control.

Storm water characterizations conducted the first year of the permit shall inchude characterization of Outfalls 007, 032 and 033. 
United States Department of Energy, Y-12 Site, is authorized to discharge cooling kater, steam condensate, storm waters and groundwater through Outfalls 004, 010, 014, 016, 019, 020, 041, 044, 057 063, 064, 067, 083, 088, 099, and 126 which discharge into East Fork Poplar Creek; and storm nater through Outfalls S02, S08, S10, S11, S12, S13 which discharge to Bear Creek. United States Department of Energy, Y-12 Site, shall monitor instream monitoring point designated as S17 which goes to the Clinch River at mile 41.1; instream monitoring point designated as S20 which goes to the Clinch River at mile 37.5 and instream monitoring point designated as Outall S24 which is in Bear Creek downstream of the burial grounds. Discharges 004, 010, 014, 019, 020, 041, 044, 057, 063, 064, 067, 083, 088, 099, 126, S02, S08, S10, S11, S12, S13, and instream monitoring points S17, S20, \& S24 shall be limited and monitored by the permittee as specified below:

Storm Water Category II Outfalls \& Monitoring Points

\begin{tabular}{|c|c|c|c|c|c|c|}
\hline \multicolumn{7}{|c|}{ Stom Water Category II Outfalls \& Monitoring Points } \\
\hline \multirow[t]{2}{*}{ Emuent characteristics } & \multicolumn{4}{|c|}{ Emuent limitations } & \multicolumn{2}{|c|}{ Monitoring Requirements } \\
\hline & $\begin{array}{l}\text { Monthly } \\
\text { Avg. } \\
\text { Conc. } \\
\text { men }\end{array}$ & $\begin{array}{l}\text { Monthly } \\
\text { Avg. } \\
\text { Amount } \\
\text { lb/day }\end{array}$ & $\begin{array}{l}\text { Daily } \\
\text { Max. } \\
\text { Conc. } \\
\text { men }\end{array}$ & $\begin{array}{l}\text { Daily } \\
\text { Max. } \\
\text { Amount } \\
\text { lb/day }\end{array}$ & $\begin{array}{l}\text { Measurement } \\
\text { Frequency }\end{array}$ & $\begin{array}{l}\text { Sample } \\
\text { Type }\end{array}$ \\
\hline $\begin{array}{l}\text { Fow } \\
\text { p8 } \\
\text { Total Residual Chlorine }\end{array}$ & $\begin{array}{l}\text { report de } \\
\text { within t } \\
\text { NA }\end{array}$ & $\begin{array}{l}\text { lax. flow } \\
\text { nge } 4.0 \text { to } 9.0 \\
\text { NA }\end{array}$ & 0.5 & NA & $\begin{array}{l}\text { 1/quarter } \\
\text { 1/quarter } \\
\text { 1/quarer }\end{array}$ & $\begin{array}{l}\text { estimate } \\
\text { grab } \\
\text { grab }\end{array}$ \\
\hline
\end{tabular}

Daily maximum measured or estimated flow will be reported as million gallons per day.

In addition to the above monitoring, the Category II outfalls shall have representative storm flow characterizations made according to the Storm Water Pollution Prevention Plan at least once per year. Storm water composite samples may be either flow-wreighted or time-weighted. Characterization of one outfall may be made where several outfalls are very similar rather than characterization of each of the outfalls in this group of similar outfalls. However, such groupings must be listed in the Storm Water Pollution Prevention Plan and be approved by the Division of Water Pollution Control.

Storm water sampling that is made of ponds or quarries where runoff collects may be sampled by other than an initial grab sample and a flow weighted composite where an alternate sampling scherne seems justified. Such alternative sampling for these sirutions may be made if the Division of Water Pollution Control' approves of the sampling methods.

1 Chlorine shall be tested only for the outfalls that are found to have some chlorinated uaters as pan of the discharge. Sampling for chlorinated discharges need not be conducted when waters are not chlorinated. 
DOE Y-12 PLAVT, TNO002968, ANDERSON COLNTY

PAOE 8 OF 50

United States Department of Energy, Y-12 Site, is authorized to discharge storm waters and groundwater through Outralls S21, S22, and S25 which discharge into an unnamed tributary which goes 10 the Clinch River at mile 33.2; and through Outfall S26 which goes to the headwaters of McCoy Branch and then to the Clinch River at mile 37.5, and through Outfalls S27, S28 and S29 which discharge into an unnamed tributary going to Clinch River at mile 41.1. Discharges S21, S22, S25, S26, S27, S28, \& S29 shall be limited and monitored by the permittee as specified below:

Storm Water Category II Outfalls \& Monitoring Points

Emuent characteristics Emuent limitations

Monitoring Requirements

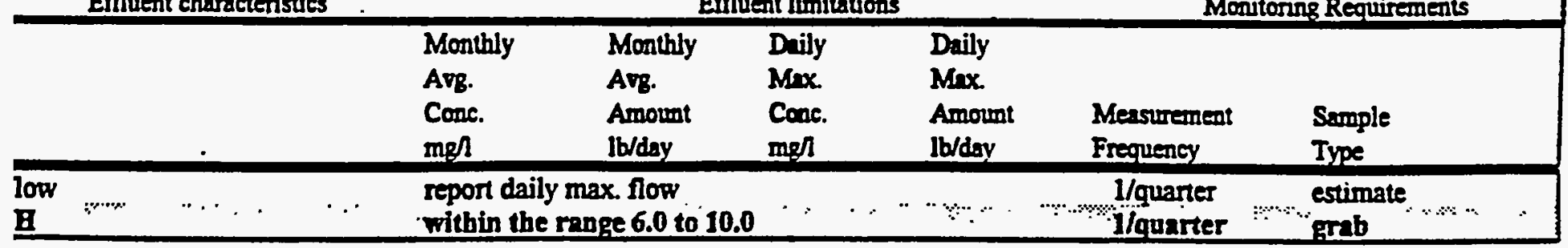

Daily maximum measured or estimated flow will be reported as million gallons per day.

In addition to the above monitoring, the Category II outfalls shall have representative storm flow characterizations made according to the Storm Water Pollution Prevention Plan at least once per year. Storm water composite samples may be either flow-urighted or time-neighted. Characterization of one outfall may be made where several outfalls are very similar rather than characterization of each of the outfalls in this group of similar outfalls. However, such groupings must be listed in the Storm Water Pollution Prevention Plan and be approved by the Division of Water Pollution Control.

Storm water sampling that is made of ponds or quarries where runoff collects may be sampled by other than an initial grab sample and a flow neighted composite where an alternate sampling scheme seems justified. Such altemative sampling for these situations may be made if the Division of Water Pollution Control approves of the sampling methods. 
United States Department of Energy, Y-12 Site, is authorized to discharge storm waters and groundwater through Outfall S19 which discharges from Rogers Quarry into McCoy Branch going to the Clinch River at mile 37.5. Discharge $S 19$ shall be limited and monitored by the permittee as specified below:

Storm Water Category II Outrall S19

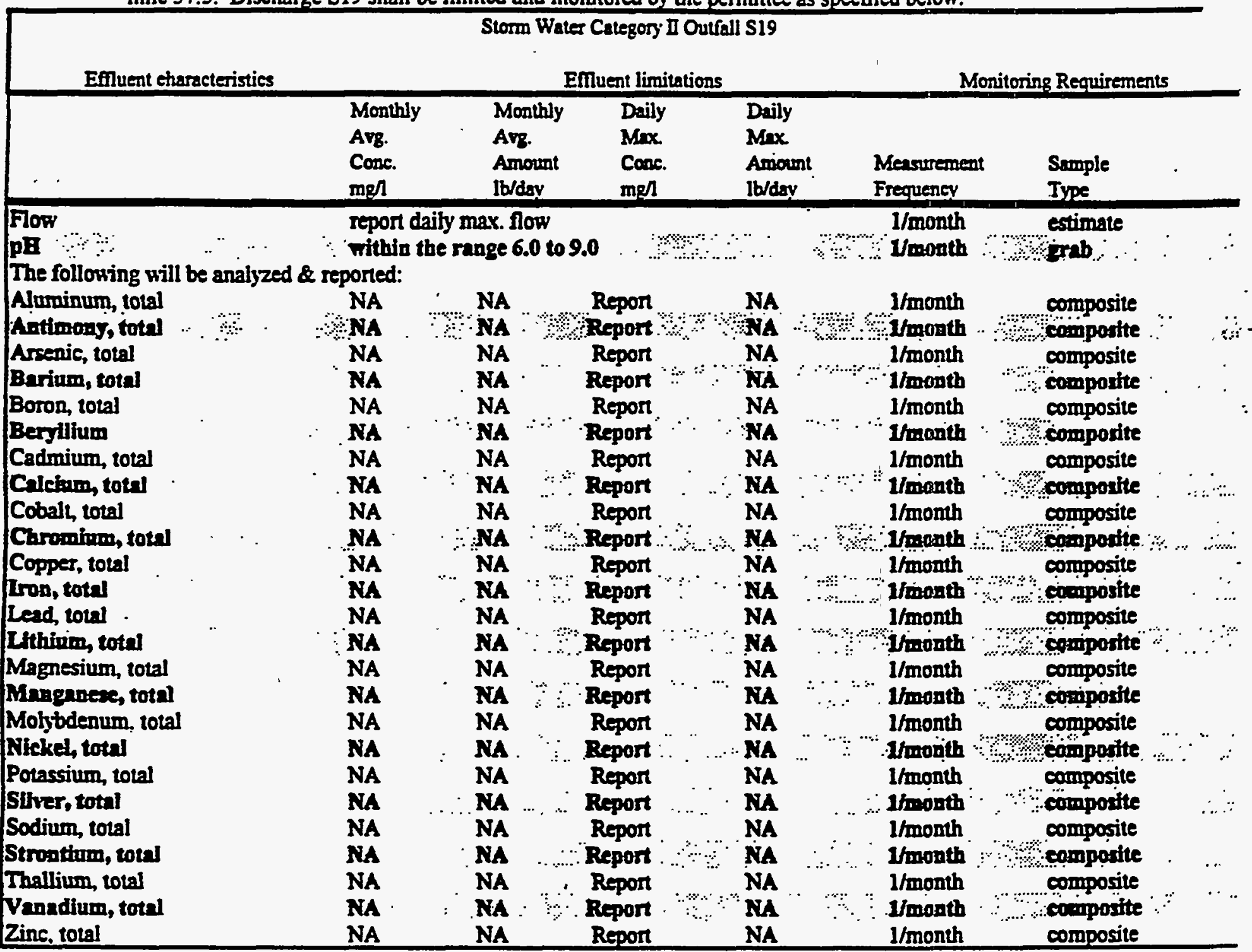

Daily maximum measured or estimated flow will be reported as million gallons per day.

In addition to the monthy monitoring Outfall S19 will also be considered as a Storm Water Category II monitoring point and shall have representative storm flow chareterizations made according to the Storm Water Pollution Prevention Plan at least once per year. Storm pater composite samples may be either flowweighted or time-prighted. Characterization of one outfall mry be made where ceveral outfalls are very similar rather than characterization of each of the outfalls in this group of similar outfalls. However, such groupings must be listed in the Storm Water Pollution Prevention Plan and be approved by the Division of Water Pollution Control. Storm water sampling that is made of this quarry may be sampled by other than an initial grab sample and a flow weighted composite if an alternate sampling scheme seerns justified. Such alternative sampling for these situations may be made if the Division of Water Pollution Control approves of the sampling methods. 
DOE Y-12 PLUT, TNO002968, ANDERSON COUNTY

PAGE 10 OF 50

United States Department of Energy, Y-12 Site, is authorized to discharge storm waters, cooling waters, cooling tower blowdown, steam condensate and groundwater, through Outfalls 002, 034, 042, 047, 048, 054, 071. 109 113, and 114 which discharge into East Fork Poplar Creak and an instream monitoring points, SOS \& S14, in tributaries which discharge to Bear Creek. Discharges and monitoring points 002, 034, 042, 047, 048, 054, 071, 109, 113, 114, S05 \& S14 shall be limited and monitored by the permittee as specified below:

Storm Water Category III Outfalls \& Monitoring Points

Emuent characteristics Emuent limitations

Monitoring Requirements

\begin{tabular}{|c|c|c|c|c|c|c|}
\hline & $\begin{array}{l}\text { Monthly } \\
\text { Avg. } \\
\text { Conc. } \\
\text { mp/1 }\end{array}$ & $\begin{array}{l}\text { Monthly } \\
\text { Avg. } \\
\text { Amount } \\
\text { lb/day }\end{array}$ & $\begin{array}{l}\text { Daily } \\
\text { Mro. } \\
\text { Cone. } \\
\text { men }\end{array}$ & $\begin{array}{l}\text { Daily } \\
\text { Max. } \\
\text { Amomt } \\
\text { Ib/day }\end{array}$ & $\begin{array}{l}\text { Measurement } \\
\text { Frequency }\end{array}$ & $\begin{array}{l}\text { Srmple } \\
\text { Type }\end{array}$ \\
\hline $\begin{array}{l}\text { Flow } \\
\text { PB }: \because \\
\text { Total Residual Chlorine } 1\end{array}$ & $\begin{array}{l}\text { report de } \\
\text { within } t \\
\text { NA }\end{array}$ & $\begin{array}{l}\text { lax. flow } \\
\text { nge } 4.0 \text { to: } \\
\text { NA }\end{array}$ & $\begin{array}{c}0.5 \\
0.5\end{array}$ & $\begin{array}{l}\because \because \because: \\
\text { NA }\end{array}$ & $\begin{array}{l}\text { 1/month } \\
\text { I/month } \\
\text { 1/month }\end{array}$ & $\begin{array}{l}\text { stimate } \\
\text { srab } \cdots \cdots \\
\text { grab }\end{array}$ \\
\hline
\end{tabular}

1 Chlorine monitoring may be omitted if paters being discharged are not chlorinated. Sampling for chlorinated discharges need not be conducted when waters are not chlorinated.

Daily maximum measured or estimated flow will be reported as million gallons per day.

In addition to the above monitoring. the Categony III outfalls shall have representative storm flow characterizations made according to the Storm Water Pollution Prevention Plan at least once per year. Storm water composite samples may be either flow-weighted or time-weighted. Characterization of one outfall may be made where several outfalls are very similar rather than characterization of each "of the outfalls in this group of similar outfalls. Howerer, such groupings must be listed in the Storm Water Pollution Prevention Plan and be approved by the Division of Water Pollution Control. 


\section{Outfall 201}

United States Department of Energy, Y-12 Site, shall monitor discharge to the headwaters of East Fork Poplar Creek at Outfall 201, a monitoring point in Eart Fork Poplar Creek below the North/South pipes (Outfall 200). Outfall 201 is at a point dounstream of Outfalls 135 and 200 . Outfall 201 shall be limited and monitored by the permittee as specified below:

Monitoring Point designated and Limited as Outfall 201

Enluent charecteristies

Emuent limitations

mell

lb/day men

Total Residual Chlorine

Temperature

pa

Total Suspended Solids

96 hour LC so, survival in $100 \%$ effluent

report

within the range 6.5 to 8.5

report

NA

report lb/day

.

NA

...

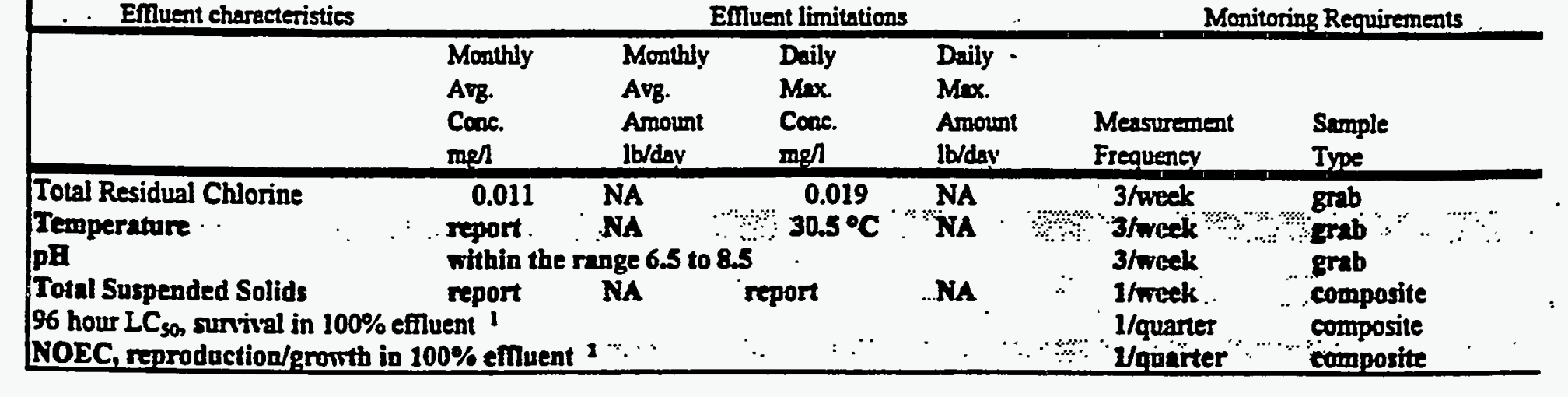

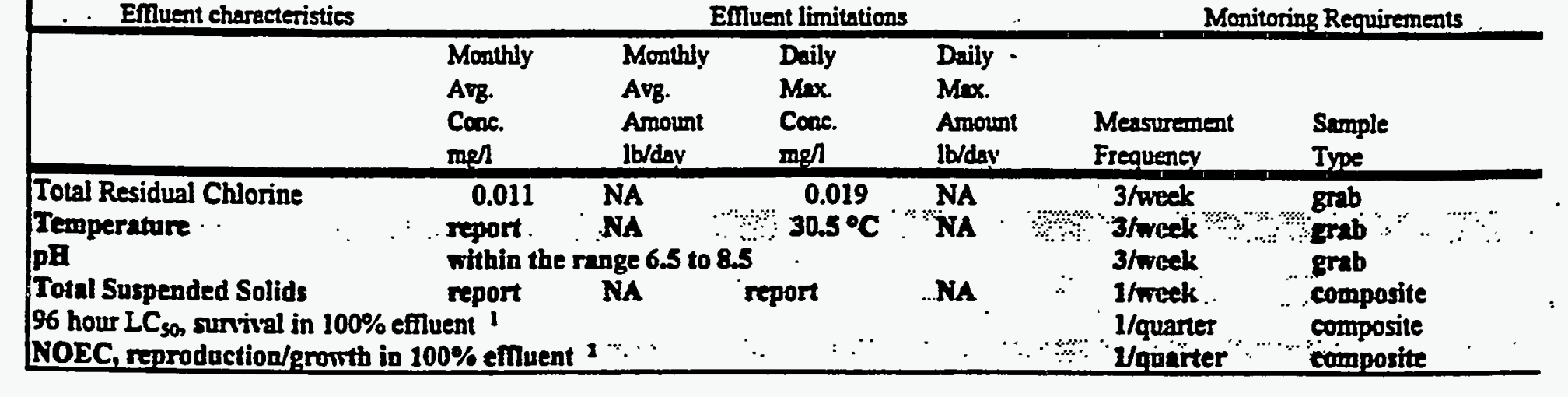

Measurement Frequency Monitoring Requirements

NOEC, reprodaction/gronth in $100 \%$ emuent

$1 \%$ 3/week

3 wreej

3/wieek

1 rreek.

1/quarter

Uquaiter

1 See Permit Part III-C for deseription of monitoring for toxicity testing. 
DOE Y-12 PLANT, TNOO02968, ANDERSON COUNTY

PAGE 12 OF 50

Outfall 200

United States Deparment of Energy, Y-12 Site, is authorized to discharge treated process wasterraters and Other wastewaters which have been accepted for treatment via waste acceptance procedures through Outfall 200 as well as treated groundwater, groundwates that infiltrates into the storm sewer system, cooling waters, condensate water, sump water, cooling tower blowdown, and storm waters. Outfall 200 discharges waters at the head of the stream through a set of pipes that have been called the North/South pipes (three parallel pipes and a fourth pipe that angles into the group from the southwest side). These pipes are not individualty listed as separate outfalls, but are considered a single outfall. Discharge 200 shall be limited and monitored by the permittee as specified below:

\section{Ouffall 200}

Emuent characteristics

Emuent limitations

Monitosing Requirements

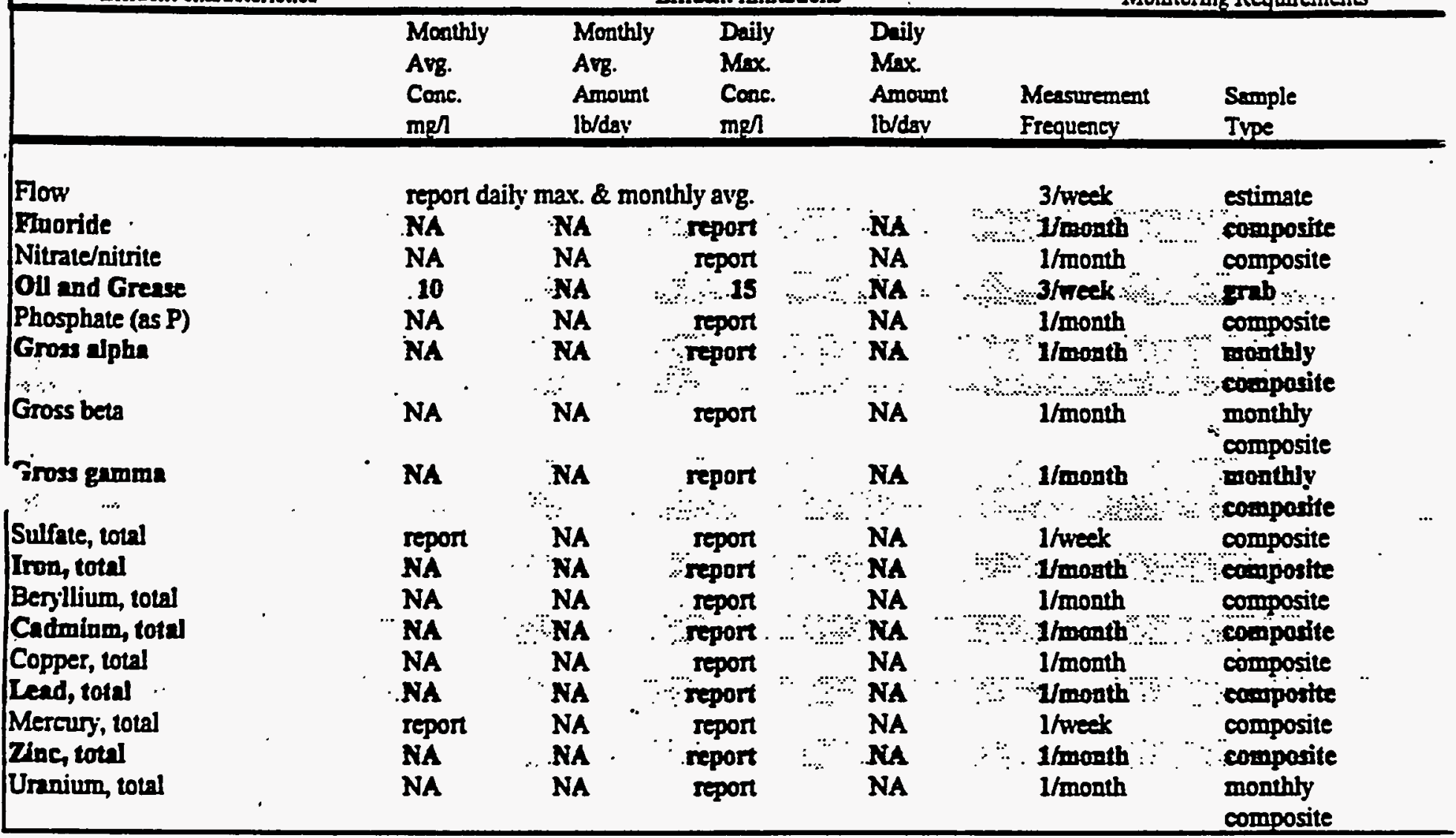

The average of the measured daily flows for the month and the maximum daily flow for the month will be reported as million gallons per day.

Radioactivity results pill be.reported in pCi/L. The monitoring for the radioactivity and total uranium will be reported with the Discharge Monitoring Data for the following month. For instance, radiosctivity monitoring results for the month of June would be reported with the July diseharge monitoring data. A report of the isolope specific data will be submitted to the Division each quarter if further investigation of isotope-specific data have been triggered as part of the Radiological Monitoring Plan (see Permit Part IIIH). Gross gamma shall be reported on the DMR, but other gamma may be required as specified in the Radiological Monitoring Plan. 


\section{Outfall 135}

United States Department of Energy, Y-12 Site, is authorized to discharge cooling waters, cooling tower blowdown, condensate, and storm waters through Outfall 135 which discharges to the headwaters of East Fork Poplar Creek. The discharge may contain infiltrated ground water. Discharge 135 shall be limited and monitored by the permittee as specified below:

\begin{tabular}{|c|c|c|c|c|c|c|}
\hline & & & fall 13 & & & \\
\hline Emuent characteristics & & & nt limi & & Mo & 18 Requirements \\
\hline & $\begin{array}{l}\text { Monthly } \\
\text { Avg. } \\
\text { Conc. } \\
\text { mp/1 }\end{array}$ & $\begin{array}{l}\text { Monthly } \\
\text { Avg. } \\
\text { Amount } \\
\text { lb/day }\end{array}$ & $\begin{array}{l}\text { Daily } \\
\text { Mox. } \\
\text { Conc. } \\
\text { mg/ }\end{array}$ & $\begin{array}{l}\text { Daily. } \\
\text { Max. } \\
\text { Amount } \\
\text { Jb/day }\end{array}$ & $\begin{array}{l}\text { Measurement } \\
\text { Frequency }\end{array}$ & $\begin{array}{l}\text { Sumple } \\
\text { Type }\end{array}$ \\
\hline Flow & report de & X. \& mor & avg. & & 3/week & estimate \\
\hline
\end{tabular}

The arerage of the measured daily flows for the month and the maximum daily flow for the month will be reported as million gallons per day. 
DOE Y-12 PLANT, TNOO02968, ANDERSON COUNTY

PAOE 14 OF 50

\section{Outfall 021}

United States Department of Energy, Y-12 Site, is authorized to discharge cooling water, cooling torer blowdown, steam condensate, groundwater and storm water through Outfall 021 to East Fork Poplar Creek. Outfall 021 shall be limited and monitored by the permittee as specified below:

\section{Outroll 021}

Emuent characteristics Emuent limitations

Monitoring Requirements

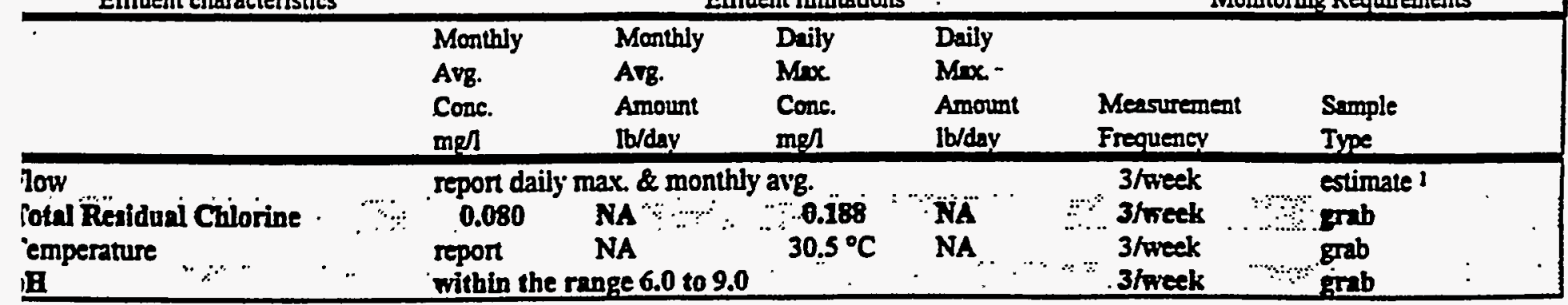

1 NOTE: Outfall 021 is to be sampled at a point which combines points designated as Outfalls 021022 and 023 in the application materials. The Outfall 021 for this permit will reflect the combined discharge of these effluents. Flow from the cooling water pipe formerty designated as Outfall 021 shall be maintained and used to double check estimates of the combined flow since this pipe will constitute almost all the flow where storm uater is not present.

The average of the measured daily flows for the month and the maximum daib flow for the month will be reported as million gallons per day. 


\section{Outfall 017}

United States Department of Energy, Y-12 Site, is authorized to discharge storm uaters, and groundwaters through Outfall 017 which discharges to East Fork Poplar Creek. Discharge 017 shall be limited and monitored by the permittee as specified below:

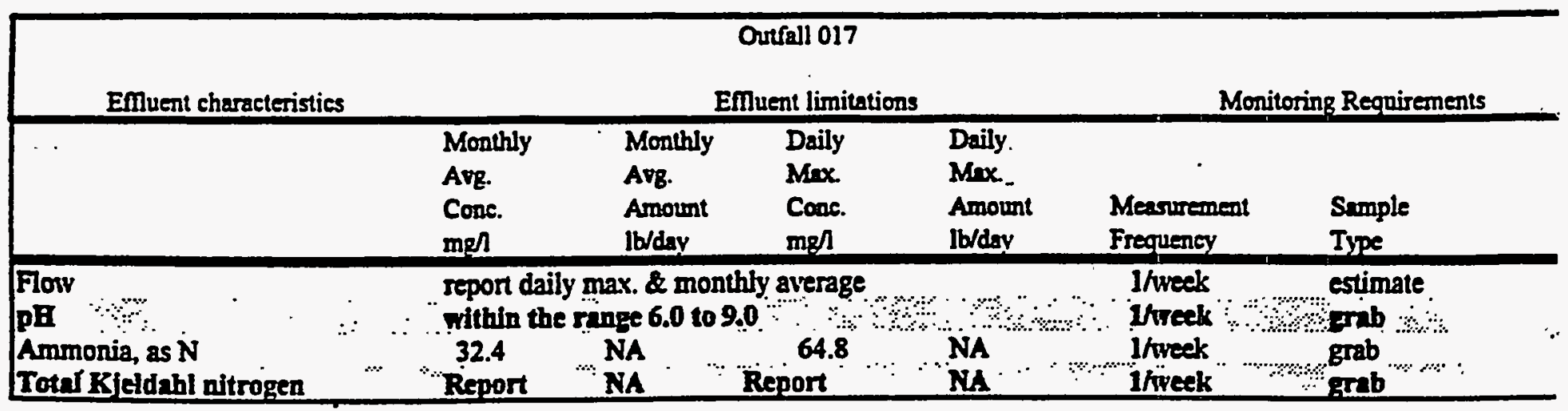

The average of the measured daily flows for the month and the maximum daily flow for the month will be reporred as million gallons per day.

A compliance schedule (Permit Part I-E) will be used to require interim treatment or otherwise prevent instream toxicity to aquatic organisms and for submission of an action plan for a treatment alternative and its implementation. Permittee must comply with the above ammonia limits by March 1, 1997. 
Outfall 055

United States Depanment of Energy, Y-12 Site, is authorized to discharge Building 9201-2 sump waters, : cooling waters, rain runoff through Outfall 055 to East Fork Poplar Creek. Discharge 055 shall be limited and monitored by the permittee as specified below:

\section{Outrall 055}

Emuent characteristics Efluent limitations Monitoring Requirements

\begin{tabular}{|c|c|c|c|c|c|c|}
\hline . & $\begin{array}{l}\text { Monthly } \\
\text { Arg. } \\
\text { Conc. } \\
\text { mell }\end{array}$ & $\begin{array}{l}\text { Monthly } \\
\text { An: } \\
\text { Amount } \\
\text { Ib/day }\end{array}$ & $\begin{array}{l}\text { Daily } \\
\text { Mix. } \\
\text { Conc. } \\
\text { man }\end{array}$ & $\begin{array}{l}\text { Daily } \\
\text { Mrx. } \\
\text { Amouni } \\
\text { Ib/day }\end{array}$ & $\begin{array}{l}\text { Measurement } \\
\text { Frequency }\end{array}$ & $\begin{array}{l}\text { Seruple } \\
\text { Type }\end{array}$ \\
\hline $\begin{array}{l}\text { Flow } \\
\text { pR } \cdots ; \\
\text { Mercury } \\
\text { Total Residual Chlorine }\end{array}$ & $\begin{array}{l}\text { report do } \\
\text { within } \\
\text { NA } \\
\text { NA }\end{array}$ & $\begin{array}{l}\text { nax. \& mon } \\
6.0-9.0 \% \\
\text { NA } \\
\text { NA }\end{array}$ & $\begin{array}{l}\text { avg. } \\
0.004 \\
0.5\end{array}$ & $\begin{array}{l}\text { NA } \\
\text { NA }\end{array}$ & $\begin{array}{l}\text { 2hweek } \\
\text { 2freel : } \\
\text { 2fweek } \\
\text { 2/week }\end{array}$ & $\begin{array}{l}\text { instantaneous } \\
\text { grab } \\
\text { grab } \\
\text { grab }\end{array}$ \\
\hline
\end{tabular}

The average of the measured daily flows for the month and the maximum daily flow for the month will be reponed as million gallons per day.

\section{Outfall 55A}

United States Deparment of Energj, Y-12 Site, shall monitor treated sump water efluent at a monitoring point after the treatment unit and before mixing with the cooling and other waters discharged through Outfall 055 at a point designated as Outfall 55A. Outfall 55A shall be discharged through Outfall 0.55 to East Fork Poplar Creek until Outfall 550 is completed and monitoring of the mercury treatment system is conducted sia the monitoring for Outfall 550. Discharge 55A shall be limited and monitored by the permittee as specified below:

\section{Outrall 5SA}

Emuent characteristics
Enthent linitations

Monitoring Requirements

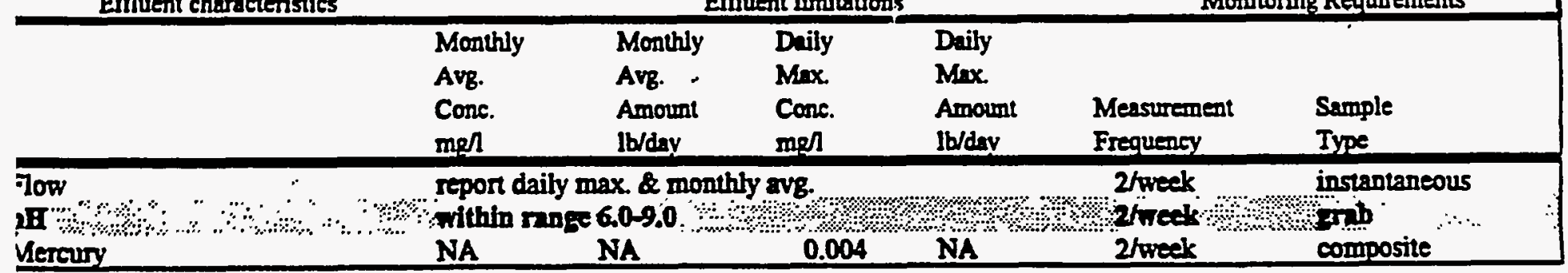

The average of the measured daily flows for the month and the maximum daily flow for the month will be reported as million gallons per day. 


\section{Oitfall 550}

United States Department of Energy, Y-12 Site, is authorized to discharge mercury contaminated wastewaters which have been treated with a mercury treatment system through Outfall 550 . Outfall 550 will discharge to East Fork Poplar Creek adjacent to Building 9201-2. Discharge 550 shall be limited and monitored by the permittee as specified below:

\section{Outrill sso}

\begin{tabular}{|c|c|c|c|c|c|c|}
\hline \multirow[t]{2}{*}{ Emuent characteristics } & & \multicolumn{2}{|c|}{ Emuent limitations } & & \multicolumn{2}{|c|}{ Monitorins Requirements } \\
\hline & $\begin{array}{l}\text { Monthy } \\
\text { Avg. } \\
\text { Conc. } \\
\text { mol }\end{array}$ & $\begin{array}{l}\text { Monthly } \\
\text { Avg. } \\
\text { Amount } \\
\mathrm{lb} / \mathrm{day}\end{array}$ & $\begin{array}{l}\text { Daily } \\
\text { Mox. } \\
\text { Conc. } \\
\text { me! }\end{array}$ & $\begin{array}{l}\text { Daily } \\
\text { Max. } \\
\text { Amount } \\
\text { lb/day }\end{array}$ & $\begin{array}{l}\text { Measurement } \\
\text { Frequency }\end{array}$ & $\begin{array}{l}\text { Sample } \\
\text { Type }\end{array}$ \\
\hline $\begin{array}{l}\text { Flow } \\
\text { pB } \\
\text { Mercury } \\
\end{array}$ & $\begin{array}{l}\text { report da } \\
\text { within } r \\
0.002\end{array}$ & $\begin{array}{l}\text { 2x. \& mon } \\
0.0-9.0\end{array}$ & $\begin{array}{l}\text { avg. } \\
0.004 \\
\end{array}$ & & $\begin{array}{l}\text { 1/week } \\
\text { 1/week } \\
\text { 1/week }\end{array}$ & $\begin{array}{l}\text { instantaneous } \\
\text { zrab } \therefore . . . \\
\text { composite }\end{array}$ \\
\hline
\end{tabular}

The average of the measured daily flows for the month and the maximum daily flow for the month will be reported as million gallons per day:

Outfall 551

United States Department of Energy, Y-12 Site, is authorized to discharge mercury contaminated wastewaters which have been treated sith a mercury treatment system. This treatment system and Outfall (which is yet to be constructed at the time of permit issuance) will discharge to the storm sewer system that enters East Fork Poplar Creek at Outfall 200. This monitoring point for the treatment system that discharges to the storm sever system will be designated as Outfall 551. Discharge 551 shall be limited and monitored by the permittee as specified below:

\section{Ourill 551}

Emuent characteristics Emuent limitations Monitoring Requirements

\begin{tabular}{|c|c|c|c|c|c|c|}
\hline & $\begin{array}{l}\text { Monthly } \\
\text { Avg. } \\
\text { Conc. } \\
\text { men }\end{array}$ & $\begin{array}{l}\text { Monthly } \\
\text { Avg. } \\
\text { Amouni } \\
\text { lb/day }\end{array}$ & $\begin{array}{l}\text { Daily } \\
\text { Mix. } \\
\text { Conce. } \\
\text { menl }\end{array}$ & $\begin{array}{l}\text { Daily } \\
\text { Max. } \\
\text { Amount } \\
\text { lb/day }\end{array}$ & $\begin{array}{l}\text { Measurement } \\
\text { Frequener: }\end{array}$ & $\begin{array}{l}\text { Sample } \\
\text { Type }\end{array}$ \\
\hline $\begin{array}{l}\text { Flow } \\
\text { pE } \\
\text { Mercun }\end{array}$ & \multicolumn{6}{|c|}{$\begin{array}{l}\text { report daily max. \& monthly ayg } \\
\text { within range 6.0-9.0 } \\
0.002\end{array}$} \\
\hline
\end{tabular}

The average of the measured daily flows for the month and the maximum daily flow for the month will be reported as million gallons per day.

NOTE: Once constneted and operational, a Form 2C will be submitted for Outfall 550, and, if construeted, Outfall 551 (see Pan III-O). The Form 2C shall be submitued nithin two years of the stan of discharges from the system so that the characterization of the wastewater may be re-evaluated. 
Outfall 051

United States Department of Energy, Y-12 Site. is authorized to discharge ground water through Outfall OS1 to East Fork Poplar Creek. Discharge 051 shall be limited and monitored by the permittee as specified below:

\section{Outfall 051}

Emuent characteristics Emuent limitations. Monitoring Requirements

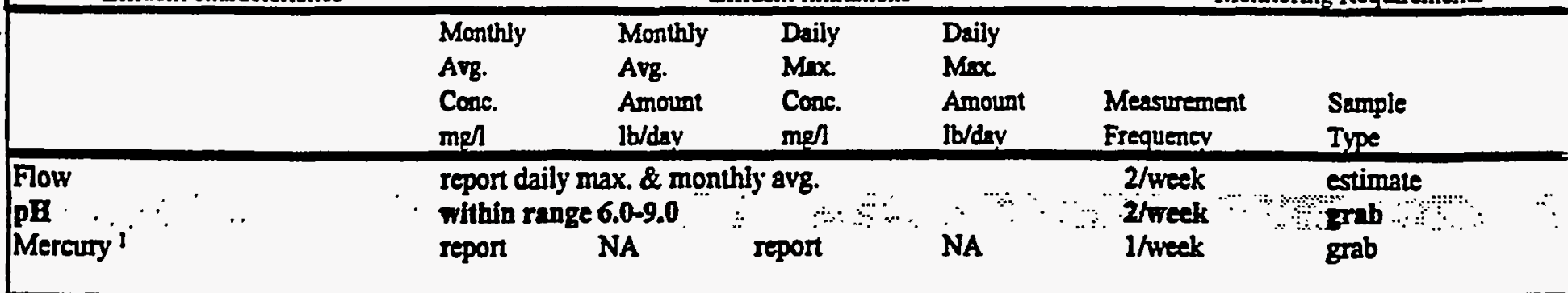

1 Mercury is to be addressed through an overall site effort. After mereury remediation and treatment projects required under this permit are completed, the mercury loading to the creek from this outfall shall not exceed a level which is detrimental to pater quality (see compliance schedule Permit Part I-E and Permit Part III-l).

The average of the measured daily flows for the month and the maximum daily flow for the month will be repored as million gallons per day. 


\section{Outfall 501}

United States Department of Energ:, Y-12 Site, is authorized to discharge process and other wastewaters which have been accepted ins treatment at the Central Pollution Control Facility (CPCF) via waste acceptance procedures as dessribed wastewater control procedures (see Permit Pan III-F), through Outfall 501, an internal monitoring point for the Central Pollution Control Facility, which discharges to the storm sewer system going to the North/South pipe (Outfall 200) which discharges into the headwaters of East Fork Poplar Creek. Discharge 501 shall be limited and monitored by the permittee as specified below:

Outrall 501

Emuent characteristics

Emuent limitations

Monitoring Requirements

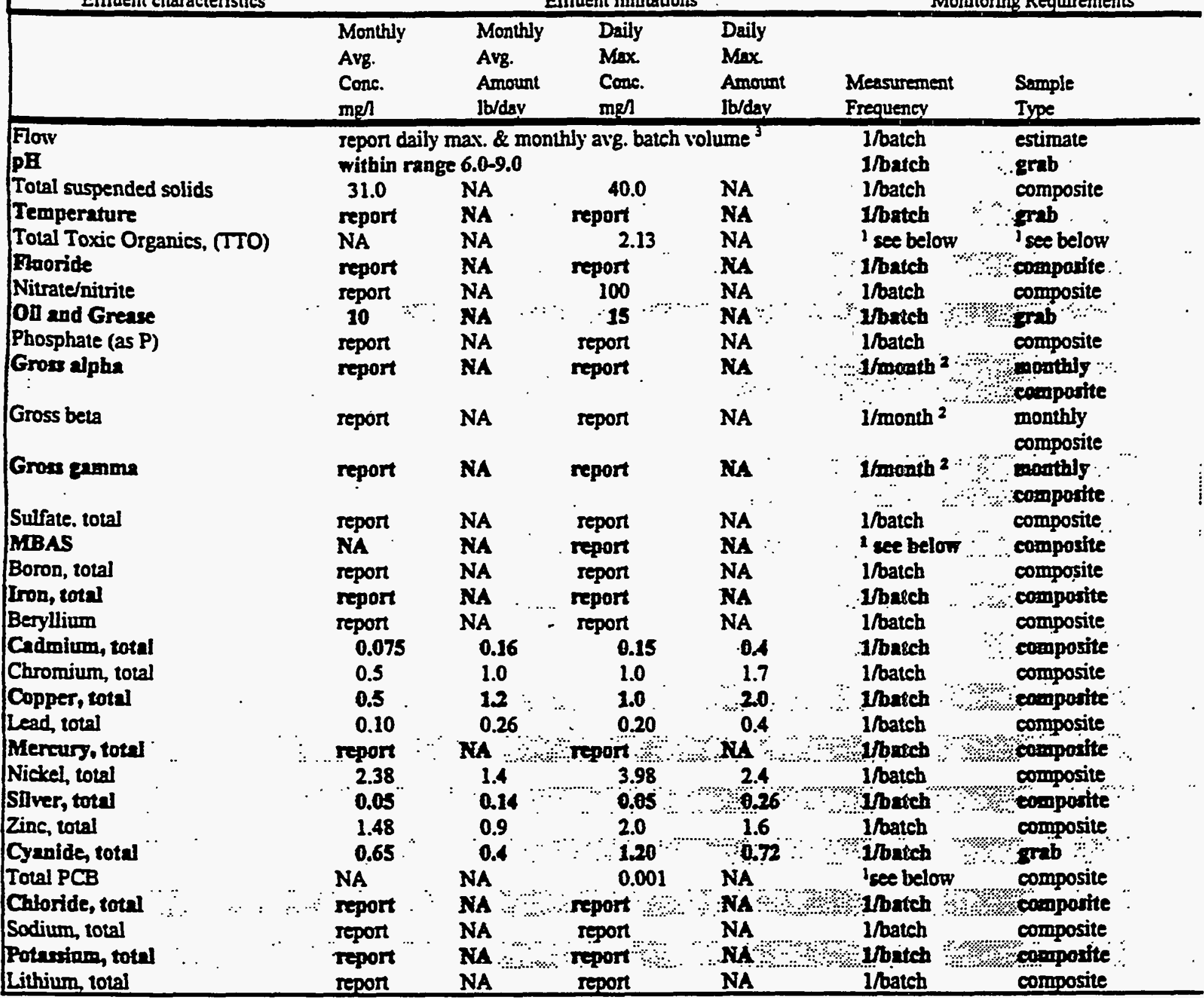


DOE Y-12 PLANT, TNO002968, ANDERSON COLBTT

PAGE 20 OF 50

(continued)

United States Department of Energr, Y-12 Site, Outfall 501, which discharges from CPCF to storm sewer going to the North/Sou Pipes to the headivaters of East Fork Poplar Creek.

Outfall 501 (continued)

Efluent characteristics Enuent limitations

Monitoring Requirements

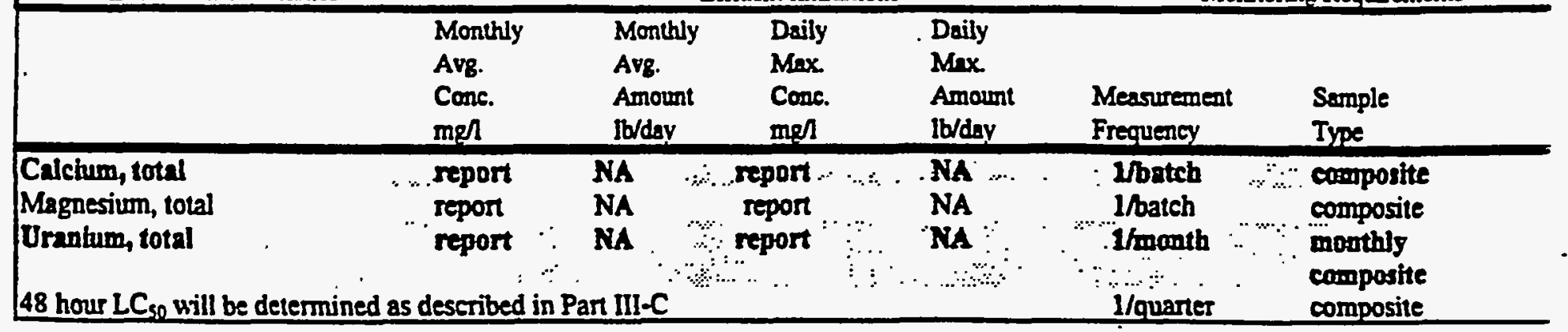

The average of the measured daily flows for the month and the maximum daily flow for the month will be reponed as million gallons per day.

1 Analyses for TTO, MBAS and PCBs shall be condueted on a sample immediately prior to a carbon column replacement. The volatile organics part of the TTO shall be collected by grab sample. An analyses must be made at least once per year whether the carbon column is replaced or not.

2 Radioactivity results will be reported in $\mathrm{pCi} / \mathrm{L}$. The monitoring for the radioactivity and total uranium will be reported with the Discharge Monitoring Data for the following month. For instance, radioactivity monitoring results for the month of June would be reported with the July discharge monitoring data. A report of the isotope specific data kill be submitted to the Division each quarter if further investigation of isotope-specific data have been triggered as part of the Radiological Monitoring Plan (see Permit Part IIIH). Gross gamma shall be reported on the DMR, but other gamma may be required as specified in the Radiological Monitoring Plan.

3 The number of batches discharged shall be included as a note on the DMR for the month. 
Outfall 502

United States Department of Energy, Y-12 Site, is authorized to discharge process and other pastewaters which have been accepted for treatment via waste acceptance procedures for the West End Treatment Facility (WETF) through Outfall 502, an internal monitoring point for the West End Treatment Facility, which discharges to the storm sewer system going to the North/South pipe which discharges into the headkaters of East Fork Poplar Creek (Outfall 200). Discharge 502 shall be limited and monitored by the permitzee as specified below:

Outfall 502

Emuent characteristics

Effluent limitations

Monitoring Reguirements

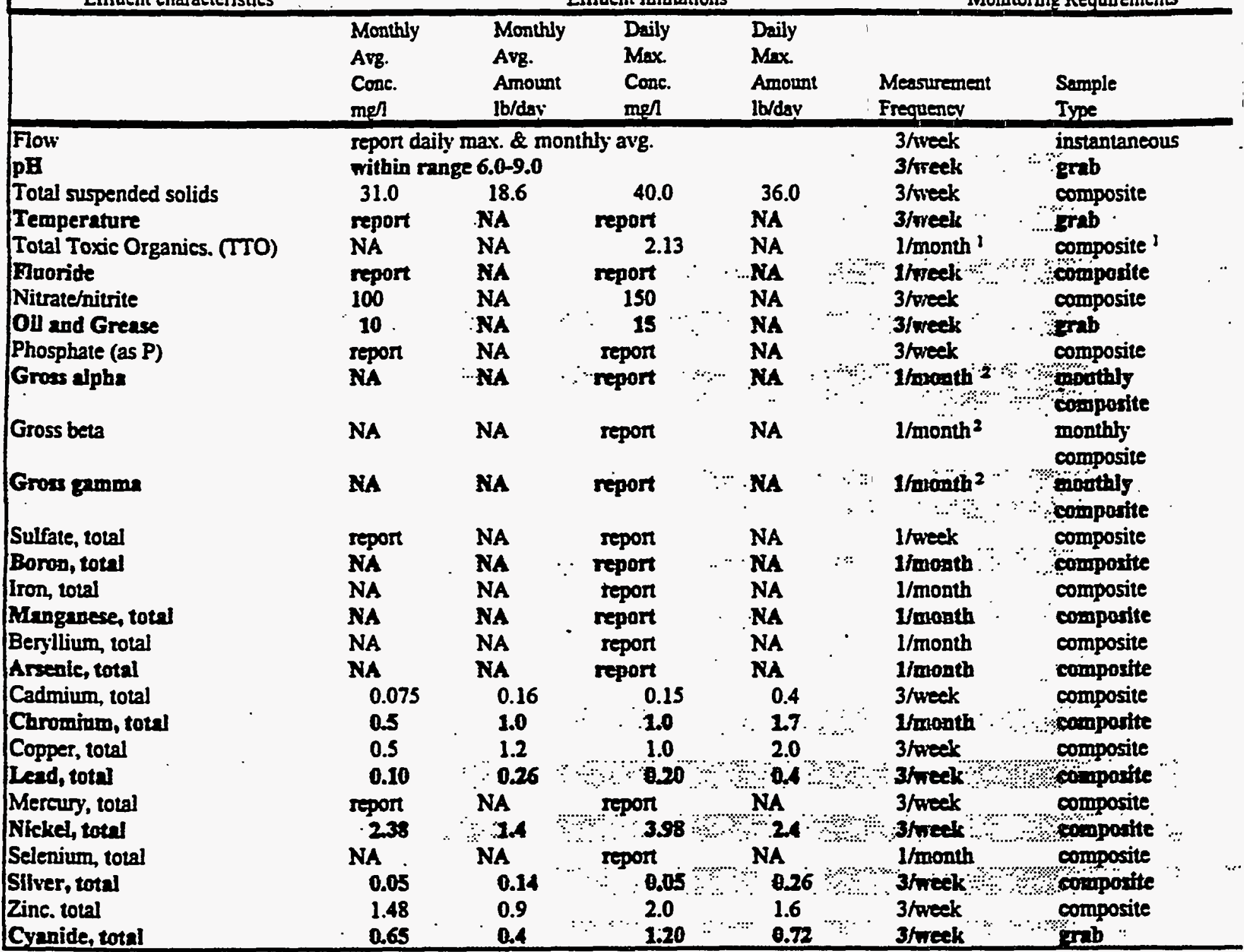


DOE Y-12 PLANT, TNO002968, ATDERSON COLATT

- PAGE 22 OF 50

(continued)

United States Deparment of Energy, Y-12 Site, Outfall 502, which discharges from WEIF to storm setrer " going to the North/South Pipes to the headwaters of East Fork Poplar Creek."

\begin{tabular}{|c|c|c|c|c|c|c|c|c|c|}
\hline \multicolumn{2}{|c|}{ Emuent characteristics } & & \multicolumn{3}{|c|}{ Emuent limitations } & & \multicolumn{2}{|c|}{ Monitoring Requirements } & \\
\hline & & $\begin{array}{l}\text { Monthly } \\
\text { Avg. } \\
\text { Conc. } \\
\text { mg/l }\end{array}$ & $\begin{array}{l}\text { Monthly } \\
\text { Avg. } \\
\text { Amount } \\
\text { lb/day }\end{array}$ & $\begin{array}{l}\text { Daily } \\
\text { Max. } \\
\text { Conc. } \\
\text { mo/ }\end{array}$ & $\begin{array}{l}\text { Daily } \\
\text { Max. } \\
\text { Amount } \\
\text { lb/day }\end{array}$ & $\begin{array}{l}\text { Measurement } \\
\text { Frequency }\end{array}$ & & $\begin{array}{l}\text { Semple } \\
\text { Type }\end{array}$ & \\
\hline $\begin{array}{l}\text { Total PCB } \\
\text { Chloride, total } \\
\text { Sodium, total } \\
\text { Potassium, total } \\
\text { Lithium, total } \\
\text { Calchum, total } \\
\text { Magnesium, total } \\
\text { Oranium, total } \\
40 \\
48 \text { hour LC }\end{array}$ & as d & $\begin{array}{l}\text { NA } \\
\text { report } \\
\text { report } \\
\text { report } \\
\text { report } \\
\text { report } \\
\text { report } \\
\text { NA } \\
\text { bed in Par }\end{array}$ & $\begin{array}{ll}\text { NA } & \\
\text { NA } & \\
\text { NA } & \\
\text { NA } & \ldots \\
\text { NA } & \\
\text { NA } & \ldots \\
\text { NA } & \\
\text { NA } & \end{array}$ & $\begin{array}{l}0.001 \\
\text { report } \\
\text { report } \\
\text { report } \\
\text { seport } \\
\text { report } \\
\text { report } \\
\text { report }\end{array}$ & $\begin{array}{l}\text { NA } \\
\text { NA } \\
\text { NA } \\
\text { NA } \\
\text { NA } \\
\text { NA } \\
\text { NA } \\
\text { NA }\end{array}$ & 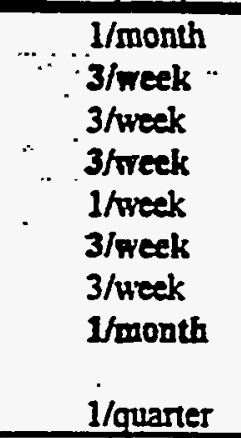 & $\begin{array}{l}\cdot . . \\
. . \\
. \cdot \\
\cdots \cdots \\
\cdots \cdot\end{array}$ & $\begin{array}{l}\text { composite } \\
\text { composite } \\
\text { composite } \\
\text { comporite } \\
\text { composite } \\
\text { composite } \\
\text { composite } \\
\text { costhily. } \\
\text { comporite } \\
\text { composite }\end{array}$ & \\
\hline
\end{tabular}

The average of the measured daily flows for the month and the maximum daily flow for the month will be reported as million gallons per day.

'Analyses for TTO shall be conducted on a composited sample, but the volatiles portion of the TTO shall be collected by a grab sample.

2 Radioactivity results will be reponed in pCil. The monitoring for the radioactivity and total uranium will be reported with the Discharge Monitoring Data for the following month. For instance, radioactivity monitoring results for the month of June would be reported with the July discharge monitoring data. A report of the isotope specific data will be submitted to the Division each quarter if further investigation of isotope-specific data have been triggered as par of the Radiological Monitoring Plan (see Permit Pan IIIH). Gross gamma shall be reported on the DMR, but other gamma may be required as specified in the Radiological Monitoring Plan. 
Outfall 503

United States Department of Energj;, Y-12 Site, is authorized to discharge storm waters. coal pile runoff, softener regeneration wastewaters. cooling waters and wastewaters which have been accepted for treatment via waste acceptance procedures for the Steam Plant Wastewater Treatment Facility (SPWTF) and any infiltrated groundwater. through Outfall 503, an internal monitoring point for the SPWTF, which discharges to the storm sewer system going to the North/South pipe which discharges into the headwaters of East Fork Poplar Creek. Discharge 503 shall be limited and monitored by the perminee as specified below:

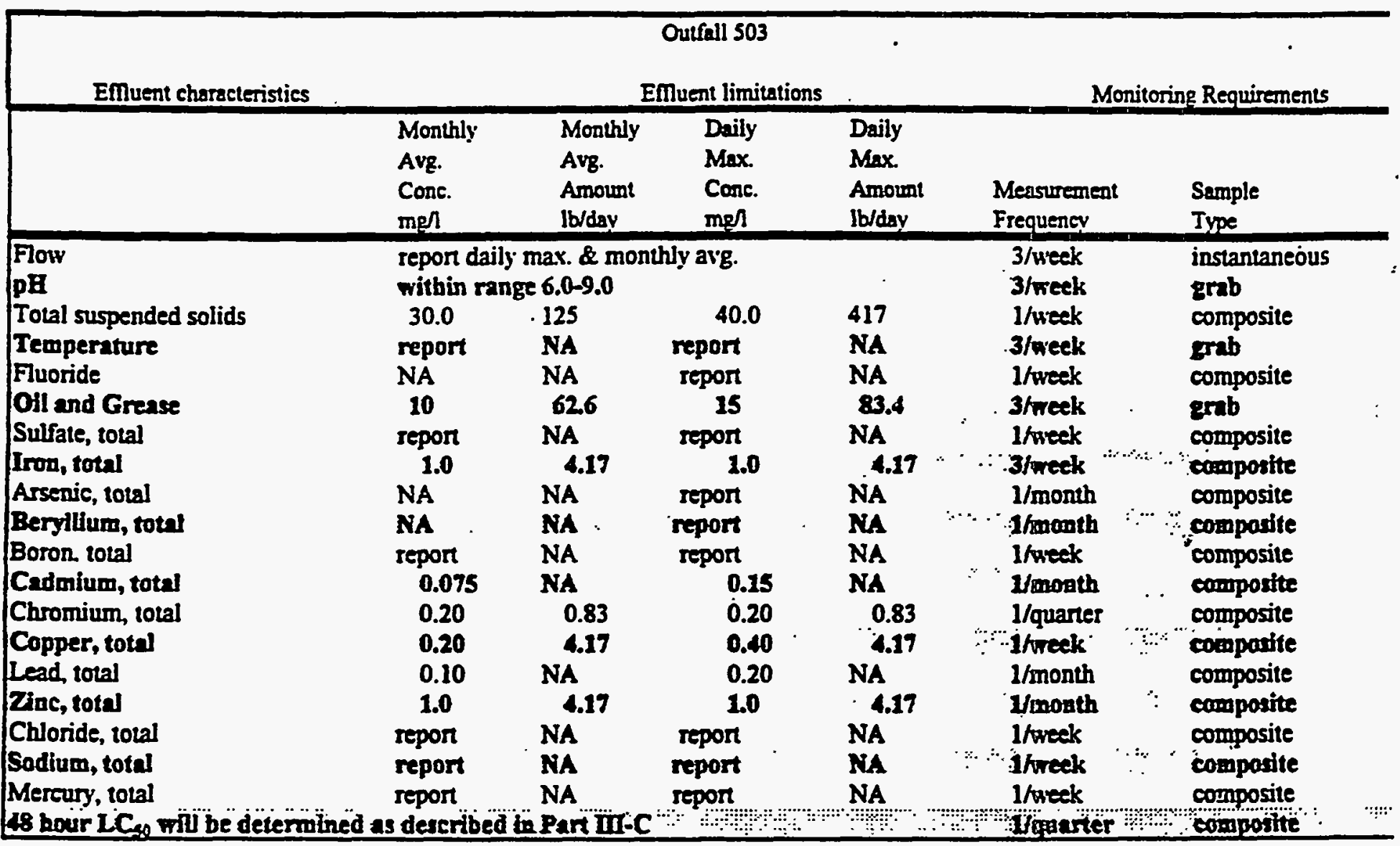

There shall be no discharge of PCB through Outfall 503.

The average of the measured daily flows for the month and the maximum daily flow for the month will be reported as million gallons per day. 
DOE Y-12 PLANT, TN0002968, ANDERSON COLNTY

PAGE 24 OF 50

\section{Outrall 512}

'United States Department of Energj; Y-12 Site, is authorized to discharge treated groundwaters and wastewaters which have been accepted for treatment via waste acceptance procedures for the Groundwater Treatment Facility (GWTF); through Outfall 512, an internal monitoring point for the GWTF, which discharges to the storm sevver system going to the North/South pipe which discharges into the headwaters of East Fork Poplar Creek. Discharge 512 shall be limited and monitored by the permittee as specified below:

Outfall 512

Emuent characteristics Emuent limitations Monitoring Requirements

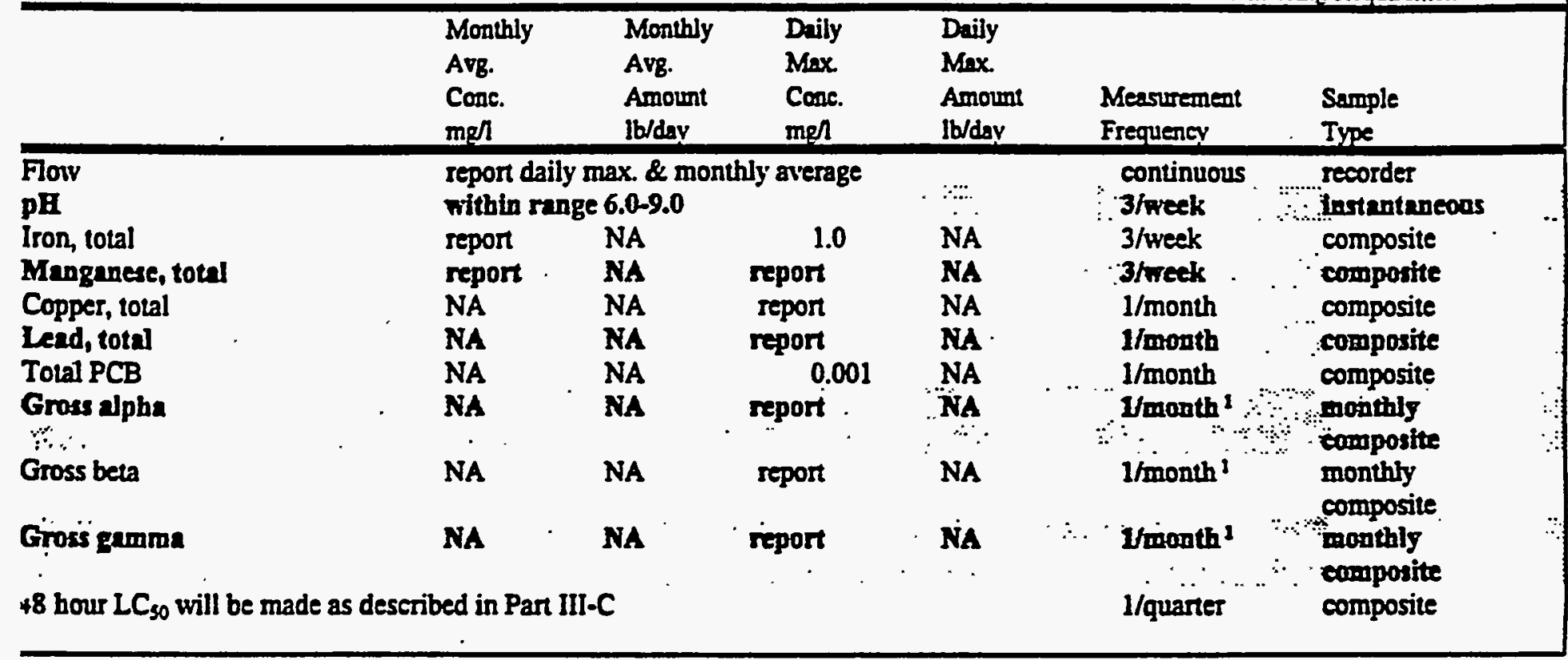

The average of the measured daily flows for the month and the maximum daily flow for the month will be reported as million gallons per day.

1 Radioactivity results will be reported in $\mathrm{pCi} / \mathrm{L}$. The monitoring for the radioactivity and total uranium will be reported with the Discharge Monitoring Data for the following month. For instance, radioactivity monitoring results for the month of June would be reported pith the July discharge monitoring data. A report of the isotope specific data will be submitted to the Division each quarter if further investigation of isotope-specific data have been triggered as part of the Radiological Monitoring Plan (see Permit Part IIIH). Gross gamma shall be ireported on the DMR, but other gamma may be required as specified in the Radiological Monitoring Plan. 
United States Deparment of Energy, Y.12 Site, is authorized to discharge steam condensate through Outfall 520, an internal monitoring point, which discharges to the storm sewer system going to the Norh/South pipe which discharges into the headwaters of Eart Fork Poplar Creek. Discharge 520 shall be limited and monitored by the permittee as specified below:

\begin{tabular}{|c|c|c|c|c|c|c|}
\hline \multirow[t]{2}{*}{ Emuent characteristics } & & & uttall 520 & - & \multicolumn{2}{|c|}{ Monitoring Requirements } \\
\hline & $\begin{array}{l}\text { Monthly } \\
\text { Avg. } \\
\text { Cone. } \\
\text { mgn }\end{array}$ & $\begin{array}{l}\text { Monthly } \\
\text { Avg. } \\
\text { Amount } \\
\text { Ib/day }\end{array}$ & $\begin{array}{l}\text { Daily } \\
\text { Max. } \\
\text { Conc. } \\
\text { men }\end{array}$ & $\begin{array}{l}\text { Daily } \\
\text { Max. } \\
\text { Amount } \\
\text { Jb/day }\end{array}$ & $\begin{array}{l}\text { Measurement } \\
\text { Frequency. }\end{array}$ & $\begin{array}{l}\text { Sample } \\
\text { Type }\end{array}$ \\
\hline $\begin{array}{l}\text { Fow } \\
\text { pB } \\
\text { Total Dissolied Solids }\end{array}$ & $\begin{array}{l}\text { monitor } \\
\text { ritbin } r \\
\text { NA }\end{array}$ & $\begin{array}{l}\text { laintain re } \\
6.0-9.0 \text {. } \\
\text { NA }\end{array}$ & depon & NA & $\begin{array}{l}\text { 3/wreek } \\
\text { 1/rreek } \\
\text { 1/wreek }\end{array}$ & $\begin{array}{l}\text { estimate } \\
\text { grab } \\
\text { grab }\end{array}$ \\
\hline
\end{tabular}

1 Flow records shall be made available for review by State and Federal regulatory personnel with an appropriate level of clearance.

Outfall $\mathbf{S 2 0}$ shall be included in the Radiological Monitoring Plan (see Permit Part III-F). A suminary report of chemical, radiological and biological data from the discharges related to this outfall will be made once per year. 
Narrative Conditions/Requirements for Discharges

There shall be no distinetly visible solids, scum, foam, oily slick, or the formation of slimes, bottom

- deposits or sludge banks in the receiving waters from the discharges.

The wastewater discharge must result in no other materials in concentrations sufficient to be hazardous or othersise detrimental to humans, livestock, ,hildlife, plant life, or fish and aquatic life in the receiving stream.

Sludge or any other material removed by any treatment works must be disposed of in a manner which prevents its entrance into or pollution of any surface or subsurface paters. Additionally, the disposal of such sludge or other material must be in compliance rith the Tennessee Solid Waste Disposal Act, TCA 68-31-101 et seq. and the Tennessee Hazardous Waste Management Act, TCA. 68-46-101 et seq.

The wastewater discharge must not cause an objectionable color contrast in the receiving stream.

It is recognized that the temperature of the cooling water discharges will be greater than the temperature of the water prior to its use for cooling or other purposes. This discharge must not cause the temperature change in East Fork Poplar Creek to exceed $3 \mathrm{C}^{\circ}$ relative to an upstream control point. Also, this discharge must not cause the temperature of East Fork Poplar Creek to exceed $30.5^{\circ} \mathrm{C}$ (except as a result of natural causes), and this discharge must not cause the maximum rate of temperature change in East Fork Poplar Creek to exceed $2 \mathrm{C}^{\circ}$ per hour (except as a result of natural causes). Compliance with this requirement will be evaluated through a temperature profile of the creek as described in permit Part III-M.

\section{B. MONITORING PROCEDURES}

1. Representative Sampling

Samples and measurements taken in compliance with the monitoring requirements specified above shall be representative of the volume and nature of the monitored discharges. Treated wasteuaters, cooling waters and other non-storm water effluents from outfalls shall be sampled after treatment and prior to mixing with uncontaminated storm sunoff or the receiving haters.

Storm water sampling shall be made as designated in the Storm Water Pollution Prevention Plan which is approved by the Division. Monitoring in waters of the State shall be representative of the waters being monitored.

Where parameters are below detection limits, determination of compliance with daify maximum limitations will be assumed whenever anabyes are below detection. Determination of compliance with monthly average limits will use a zero in place of all values that are below the detection level. Anslyses used to show compliance with permit conditions must either use a method with a detection level that demonstrates compliance with permit limits or be anatyzed with methodology having a detection level recognized by the Division of Water Pollution Control arch as the levels listed in TN Rule 1200-4-3-.05(8) or detection level recognized by EPA for those chemicals not listed in the Tennessee Rules.

\section{Tert Procedures}


a. Test procedures for the analysis of pollutants shall conform to regulations published pursuant to Section $30+(\mathrm{h})$ of the Clean Water Act, as amended, under which such procedures may be required.

b. Unless otherwise noted in c. below, all pollutant parameters shall be determined according to methods prescribed in Title 40, CFR, Part 136, as amended, promulgated pursuant to Section 304 (h) of the Act.

c. Radiological monitoring shall be made according 10 EPA methods 900.0 for alpha and beta scans, 901.1 for gamma scans, 905.0 for total radioactive strontium ("95 + 905r), and 906.0 for tritium and 908.0 where radiological analyses are made for uranium isotopes. Changes or modifications to these methodologies may be made as needed when scientifically justified for a particular sample. Results of radiological monitoring shall be reporied as pCi/L.

d. Because of the low total residual chlorine permit limits, acceptable methodologies for sampling total residual chlorine shall be restricted to amperometric titration, the DPD (n.n-diethyl-p -phenylenediamine) colorimetric method or use of a specific ion electrade.

3. Recording of Results

For each measurement or sample taken pursuant to the requirements of this permit, the permittee shall record the following information:

a. The exact place, date, and time of sampling;

b. The exact person(s) collecting samples;

c. The dates and times the analyses were performed;

d. The person(s) or laboratory who performed the anaiyses;

c. The anabtical techniques or methods used, and;

f. The results of all required analyses.

4. Records Retention

All records and information resulting from the monitoring activities required by this permit including all records of analyses performed and calibration and maintenance of instrumentation shall be retained for a minimum of three (3) years, or longer if requested by the Division of Water Pollution Control.

\section{DEFINITIONS}

The "daily maximum concentration" is a limitation on the average concentration, in milligrams per liter, of the discharge during any calendar day. When a proportional-to-flow composite sampling device is used, the daily concentration is the concentration of that 24-hour composite.

The "daily marimum amount" is a limitation on the total amount of any pollutant in the discharge by weight during any calendar day.

The "monthly average concentration", a limitation on the discharge concentration in milligrams per liter, is the arithmetic mean of all individual concentrations determined in a one-month period. The Division may, by letter, increase the monitoring frequency and/or establish a monthly and/or neekly average limit. For purposes of determining compliance with this permit, all analyses which are below the Division's recognized detection limits will be assigned a value of zero when the monthly average concentration is computed. 
The "monthly average smount",; a discharge limitation, means the total discharge by weight during a calendar month divided by the number of days in the month that the production or commercial facility was operating. Where less than daity sampling is required by a Permit, the monthly average amount shall be determined by the summation of all the measured daity discharges by weight divided by the number of days during the calendar month when the measurements were made. For purposes of determining compliance with this permith all analyses which are below the Division's recognized detection limits will be assigned a value of zero when the monthly average amount is computed.

A "composite sample", for the purposes of this permit, is said to be a sample collected continuously over a period of 24 hours at a rate proportional to the flow except for flow-weighted or time-weighted composite storm water monitoring samples associated with the Storm Water Pollution Prevention Plan.

A "monthly composite sample" for the purposes of this permit is a sample made by combining at least 4 separate 24 hour composite samples collected in separate weeks of the month at a rate proportional to the flow. Individual composites may be analyzed separately and the results averaged and reported as the monthly composite sample. If sampling cannot be made for four separate weeks of the month, the monthly composite samples shall be composited (or the anatyses averaged) for those reek(s) of the month in which sampling could be made.

For the purpose of this permit a "quarter" is defined as any one of the following three month periods: January 1 through March 31, April 1 through June 30, July 1 through September 30, October 1 through December 31.

For the purpose of this permit a "caleadar day" is defined as any 24-hour period.

For the purpose of this permit a "year" is defined as any twelve month period. For the purposes of compliance with the permit's monitoring requirements for outfalls and monitoring points such as a frequeney of once per year or twice per year (annual or semi-annual monitoring) a year shall refer to a twelve month period that starts with the effective date of the permit.

\section{REPORTING}

\section{Monitoring Results}

Monitoring results shall be recorded monthly and submitted monthly using Discharge Monitoring Repor Forms supplied by the Division of Water Pollution Control. The top two copies of each report are to be submitted, one to the IN Division of DOE Oversight and one to central office. A copy should be retained for the permitiee's files.

As an alternative to the submission of Diseharge Monitoring Reports (DMR) mentioned above, monitoring results may be submitted using both computer input media and paper forms. Submission of data with a computer format in addition to the paper DMR forms shall be made only with the authorization of the Director. The permittee may submit its dats in a format which allows direct input into the EPA Permit Compliance System.

Submittals shall be made or postmarked no later than 15 days after the completion of the reporting period for DMRs submitted only on paper. Submittals of paper copies along with electronic media shall be made no later than the last working day of the month folloning completion of the reporting period Discharge Monitoring Reports or other 
communications regarding data submissions or compliance with the terms of the permit shall be sent to:

Division of Water Pollution Control

Attention: Compliance Review

401 Church Street, 6th Floor, L \& C Annex

Deparment of Environment \& Conservation

Nashville, Tennessee 37243-1534

The first Discharge Monitoring Report or data submission is due

Discharge Monitoring Reports must be submitted under cover letter signed and certified by a responsible corporate officer, as defined at 40 CFR 122.22, or a general partner or proprietor, or a principal municipal executive officer or ranking elected official, or a duty authorized representative. Such authorization must be submitted in writing and must explain the duties and responsibilities of the authorized representative. Individual DMR reports must be initialed by the person reviewing the document and may be stamped with the signature of the responsible officer.

Computerized submittals of data must be made under authority of the permittee's authorized representative through the methods agreed upon in advance by the permittee and the Division.

2. Additional Monitoring by Permittee

If the permittee monitors any pollutant at the location(s) designated herein more frequently than required by this permit, using approved anahtical methods as specified above, the results of such monitoring shall be included in the calculation and reporting of the values required in the Discharge Monitoring Repon Form or in the computerized data submission. Such increased frequency shall also be indieated. If data are related to the Storm Water Pollution Prevention Plan, the data may be submitted in a repor for Storm water data rather than the DMR

\section{Falsifying Reports}

Knowingly making any false statement on any report required by this permit may result in the imposition of eriminal penalties as provided for in Section 309 of the Federal Water Pollution Control Act, as amended, and in Section 69-3-115 of the Tennessee Water Quality Control Act.

\section{E. SCHEDULES OF COMPLIANCE}

The following schedules shall be used for achieving compliance with permit limits or for accomplishing projects required in the permit. The permittee shall provide a brief summary of the status of these projects once every six months after the effective date of the permit.

\section{Mercury Treatment and Remediation}

Within the dates listed below, the permittee shall comply with the requirements listed. This compliance schedule shall begin on the effective date of this permit and shall continue until the dates listed. The permittee shall be in compliance with the limits set forth for total mercury after the dates listed below. 
By December 1, 1995:

The permittee shall have completed the source elimination project to replace/clean piping in Building $9201-2$ by December 1, 1995.

\section{By June 1, 1996:}

The permittee shall have completed the source elimination project to replace/clean piping in Building $9201-5$ by June 1, 1996.

\section{By September 1, 1996:}

The permittee shall have completed the source elimination project to replace/clean piping in Building $9201-4$ by September 1, 1996.

\section{By January 1, 1997:}

The permittee shall have completed the source elimination project to replace/clean piping in Building 9204-4 by January 1, 1997.

\section{By January 1, 1998:}

The permittee shall have a permanent mercury treatment system(s) installed and shall be treating sump waters from Buildings 9201-2, 9201-5, 9201-4, \& 9204-4.

By December 31, 1998:

The permittee shall have reduced mercury loading to the creck, calculated as an average of daily values over a three month period, to less than 5 grams per day as measured in the water column at Station 17 for flows less than 15 Million Gallons per Day. The monitoring of mercury at Station 17 shall consist of at least 60 daity analyses per quarter. Mercury loading data at Station 17 shall be calculated and reported for all days for which data are collected. However, for purposes of determining compliance with the 5 gram per day loading, the calculation of mercury loading may omit days during which flow volume exceeded 15 million gallons due to precipitation nunoff.

\section{By April 27, 2000:}

The permittee shall demonstrate compliance with the state's chronic water quality criteria for fish and aquatic life by the close of the permit period. Thus, the permittec shall demonstrate complianee with a total mercury concentration corresponding to the chronic criteria of 0.012 ugh as an instream value measured at Station 17 by the close of the permit period. This value equals 0.32 grams mercury per day for a flow of $7 \mathrm{MGD}$. For the purposes of this permit if the chronic criteria for total mercury is changed before the close of this permit's period, that criteria revision may be used to evaluate compliance with this requirement to meet the state's chronic water quality criteria.

\section{Addition of Clinch River Raw Water}

By March 1, 1997, the permittee shall comply with the Permit Part III-K requirement that a minimum flow of 7 million gallons per day be maintained at Station 17 for East Fork Poplar Creek as it leaves the Y-12 site (determined as an averaged low throughout the day, not as an instantaneous value). There shall be an accurate way to measure flows going past Station 17.

\section{Ammonia Reduction at Outfall 017}

By March 1, 1997, the permittee shall attain compliance with the permit limits of $32.4 \mathrm{mg} /$ ammonia (reported as Nitrogen) for a monthly average concentration and $64.8 \mathrm{mg} / \mathrm{ls}$ a daily maximum. This compliance schedule shall begin on the effective date of this permit 
and shall conunue until the date listed. The permittee shall be in compliance with the limit set forth for ammonia after February 1997.

During this time frame the permittee shall provide interim treatment for the discharge or implement management practices which will prevent instream toxicity to aquatic organisms. Further, the permittee shall submit a proposed action plan and preliminary engineering report to the Division for review and comment within 180 days of the effective date of this permit. This plan and report shall inciude the proposal for treating the ammonia contamination at Outfall 017 and/or proposal for remediation of ground water contamination which is contaminating the discharge. The plan may also include any proposals that could be used to mitigate instream toxicity from the ammonia going to the creek.

The permittee shall submit plans and specifications, if appropriate, for the remedial actions set forth in the approved action plan and preliminary engineering report within 180 days of receipt of the approval for the action plan and preliminary engineering report by the Division.

The permittee shall begin all remedial activities set forth in the approved action plan and engineering report as soon as possible, but compliance with permit limits must be obtained by March 1, 1997. 
DÓE Y-12 PZANT, TNO002968, ANDERSON COUNTY

PAGE 32 of 50

\section{PART II}

\section{GENERAL PROVISIONS}

1. Duty to Reapply

Permittee is not authorized to discharge after the expiration date of this permit. In order to receive authorization to discharge beyond the expiration date, the permittee shall submit such information and forms as are reguired to the Director no later than 180 days prior to the expiration date.

\section{Right of Entry}

The permittee shall allow the Director, the Regional Administrator of the U.S. Emvironmental Protection Agency, or their authorized representatives, upon the presentation of credentials:

a. To enter upon the permittee's premises where an effluent source is located or where records are required to be kept under the terms and conditions of this permit, and at reasonable times to copy these records;

b. To inspect at reasonable times any monitoring equipment or method or any collection, treaument, pollution management, or discharge facilities required under this permit; and

c. To sample at reasonable times any discharge of pollutants.

3. Availability of Reports

Except for data determined to be confidential under Section 308 of the Federal Water Pollution Control Act, as amended, all repors prepared in accordance with the terms of this permit shall be arailable for public inspection at the offices of the Division of Water Pollution Control. As required by the Federal Act, operational data shall not be considered confidential.

4. Proper Operation and Maintenance

a. The permittee shall at all times properiy operate and maintain all facilities and systems (and related appurtenances) for collection and treatment which are installed or used by the permittee to achieve compliance with the terms and conditions of this permit. Proper operation and maintenance also includes adequate laboratory and process controls and appropriate quality assurance procedures. This provision requires the operation of backup or auxiliary facilities or similar systems which are installed by a permittee only when the operation is necessary to achieve compliance with the conditions of the permit.

b. Where a backup power source is provided for the treament system or a pumping station, the power source must be tested onse per momth to prove reliability. The date of this test shall be recorded and records shall be kept on site.

c. Dilution water shall not be added to comply with requirements for effluents which are based on effluent guidelines for treatment facilities. 
5. Treatment Facility Failure (Indusrial Sources)

The permittee, in order to maintain compliance with this permit, shall control production, all discharges or both, upon reduction, loss, or failure of the treatment facility, until the facility is restored or an altemative method of treatment is provided. This requirement applies in such situations as the reduction, loss, or failure of the primary source of power.

6. Property Rights

The issuance of this permit does not convey any property rights in either real or personal property, or any exclusive privileges, nor does it authorize any injury to private property or any invasion of personal rights, nor any infringement of Federal, State, or local laws or regulations.

7. Sererability

The provisions of this permit are severable. If any provision of this permit due to any circumstance, is held invalid, then the application of such provision to other circumstances and to the remainder of this permit shall not be affected thereby.

8. Other Information

If the permittee becomes aware that he failed to submit any relevant facts in a permit application, or submitted incorrect information in a permit application or in any report to the Director, then he shall promptly submit such facts or information.

\section{B. Changes AFFECTING THE PERMIT}

\section{Planned Changes}

The permittee shall give notice to the Director as soon as possible if amy planned physical alterations or additions to the permitted facility. Notice is required onily when:

a. The alteration or addition to a permitted facility may meet one of the criteria for determining whether a facility is a new source in 40 CFR 122.29(b); or

b. The alteration or addition could significantly change the nature or inerease the quantity of pollutants discharged. This notification applies to pollutants which are subject neither to effluent limitations in the permit, nor to notification requirements under 40 CFR 122.42(a)(1).

2. Permit Modification, Revocation, or Termination

2. This permit may be modified, revoked and reissued, or terminated foi cause as described in 40 CFR 122.62 and 122.64, Federal Regiser, Volume 49, No. 188 (Wednesday, September 26, 1984), as amended.

b. The permittee shall furnish to the Director, within a reasonable time, any information which the Director may request to determine whether cause exists for modifying, revoking and reissuing, or terminating this permit or to determine compliance with this permit. The permittee shall also furnish to the Director, upon request, copies of records required to be kept by this permit.

c. If any applicable effluent standard or prohibition (inciuding any schedule of compliance specified in such effluent standard or prohibition) is established for any toxic pollutant 
PAGE 34 OF 50

under Section 307(a) of the Federal Water Pollution Control Act, as amended, the Director shall modify or revoke and reissue the permit to conform to the prohibition or to the effluent standard, providing that the eflluent standard is more stringent than the limitation in the permit on the toxic pollutant. The permittee shall comply sith these effluent standards or prohibitions within the time provided in the regulations that establish these standards or prohibitions, even if the permit has not yet been modified or revoked and reissued to incorporate the requirement.

3. Change of Ownership

This permit may be transferred to another person by the permittee if:

a. The permittee notifies the Director of the proposed transfer at least 30 days in advance of the proposed transfer date;

b. The notice includes a written agreement between the existing and the new permittees containing a specified date for transfer of permit responsibility, coverage, and liability between them; and

c. The Director, within 30 days, does not notify the current permittee and the new permittee of his intent to modify. revoke and reissue, or terminate the permit and to require that a new application be filed rather than agreeing to the transfer of the permit.

4. Change of Mailing Address

The permittee shall promptly provide to the Director written notice of any change of mailing address. In the absence of such notice the original address of the permittee will be assumed to be correct.

\section{NONCOMPLIANCE}

1. Effect of Noneompliance

All discharges shall be consistent with the terms and conditions of this permit. Any permit noncompliance constitutes a violation of applicable State and Federal laws and is grounds for enforcement action, permit termination, permit modification, or denial of permit reissuance.

2. Reporting of Noncompliance

a. 24-Hour Reporting

In the case of any noncompliance which would cause a threat to public drinking supplies, or any other discharge which could constime a threat to human health of the emvironment, the required notice of noncompliance shall be provided to the Tennessee Division of DOE Oversight or the Knoxville Field Office within 24 hours from the time the permittee becomes aware of the circumstances. (These offices should be contacted for names and phone numbers of emergency response personnel.)

A written submission must be provided within ten (10) days of the time the permittee becomes aware of the circumstances uniess this requirement is waived by the Director on a case-by-case basis. The permittee shall provide the Director with the following information:

i. A description of the discharge and cause of noncompliance; 
ii. The period of noncompliance, including exact dates and times or, if not corrected, the anticipated time the noncomplianee is expected to continue; and

iii. The steps being taken to reduce, eliminate, and prevent recurrence of the noncomplying discharge.

b. Scheduled Reporting

For instances of noneompliance which are not reported under subparagraph 2. a. above, the permittee shall report the noncompliance on the Discharge Monitoring Report. The report shall contain all information coneerning the steps taken, or planned, to reduce, eliminate, and prevent recurrence of the violation and the anticipated time the violation is expected to continue.

3. Bypassing

a. "Bypass" means the discharge of wastes from any portion of the collection or treatment system other than through the permined outfalls.

b. Bypassing is prohibited except where necessary to prevent loss of life or severe property damage, or where excessive storm drainage or runoff hould damage treatment or containment facilities.

c. No new or additional flows shall be added upstream of any point in the collection system which experiences chronic bypassing (greater than 5 events per year). Unless there is specific enforcement action to the contrary, the permittee is relieved of this requirement after the State has revierred and approved a plan for correction of the problem arid the correction work is undernay.

\section{Diversion}

a. "Diversion" is the intentional rerouting of wastewater within a treatment facility away from a biological portion of the treatment facility:

b. A diversion is permissible only when necessary to protect the active biomass from a rashout due to peak flow events anid when this action does not cause effluent limitations to be exceeded.

\section{Upset}

2. "Opset" means an exceptional incident in which there is unintentional and temporary noneompliance with technology based effluent limitations because of factors beyond the reasonable control of the permittee. An upset does not include noncompliance to the extent caused by operational error, improperly designed treatment facilities, inadequate treatment facilities, lack of preventive mainienance, or careless or improper operation.

b. An upset shall constitute an affirmative defense to an action brought for noncompliance with such technology - based permit effluent limitations if the permittee demonstrates, through properly signed, contemporaneous operating logs, or other relevant evidence that:

i. An upset occurred and that the permittee can identify the cause(s) of the upset;

ii. The permitted facility was at the time being operated in a prodent and workman-like manner and in compliance with proper operation and maintenance procectures; 
iii The permittee submitted information required under "Reporing of Noncompliance" within 24 hours of becoming aware of the upset (if this information is provided orally, a written submission must be provided nithin five days); and

iv. The permittee complied with any' remedial measures required under "Adverse Impact."

6. Washout

a. For domestic wastewater plants only, a "wahout" shall be defined as loss of Mixed Liquor Suspended Solids (MLSS) of $30 \%$ or more. This refers to the MLSS in the aeration basin(s) only. This does not include MLSS decrease due to solids wasting to the sludge disposal system. A washout can be caused by improper operation or from peak flows due to infiltration and inflow.

b. A washout is prohibited. If a washout occurs the permittee must report the incident to the appropriate field office within 24 hours by telephone. A mritten submission must be provided within five days. The washout must be noted on the discharge monitoring report. Each day of a washout is a separate violation.

7. Adverse Impact

The permittee shall take all reasonable steps to minimize any adverse impact to the waters of Tennessee resulting from noncompliance with this permit, including such accelerated or additional monitoring as necessary to determine the nature and impact of the noncomplying discharge. If shall not be a defense for the permittee in an enforcement action that it would have been necessary to halt or reduce the permitted activity in order to maintain compliance with the conditions of this permit.

\section{LIABLITIES}

1. Civil and Criminal Liability

Except as provided in permit conditions or "Bypassing", "Diversion", "Upset", and "Treatment Facility Failures", nothing in this permit shall be construed to relieve the permittee from crivil or criminal penalties for noneompliance. Notwithstanding this permit, the perminee shall remain liable for any damages sustained by the State of Tennessee, including but not limited to fish kills and losses of aquatic life and/or wildlife, as a result of the discharge of wastewater to any surface or subsurface waters. Additionally, notwithstanding this Permit, it shall be the responsibility of the permittee to conduct its wastewater treatment and/or discharge setivities in a manner such that public or private nuisances or health hazards will not be created. 
2. Liability Under State Law

Nothing in this permit shalt be construed to preciude the institution of any legal action or relieve the permittee from any responsibilities, liabilities, or penalties established pursuani to any applicable State law or the Federal Water Pollution Control Act, as amended. 
'DOE Y-12 PLANT, TNO002968, ANDERSON COLNT'

PAGE 38 OF 50

\section{PART III \\ OTHER REQUIREMENTS}

\section{A. TOXIC POLLUTANTS}

The permittee shall notify the Division of Water Pollution Control as soon as it knows or has reason to believe:

a. That any activity has occurred or will occur which would result in the discharge on a routine or frequent basis, of any toxic substance(s) (listed at 40 CFR 122, Appendix D, Table II and II) which is not limited in the permit, if that discharge will exceed the highest of the folloring "notification levels":

(1) One hundred mierograms per liter (100 ug/);

(2) Tro hundred micrograms per liter (200 ug/l) for acrolein and acrylonitrile; five hundred micrograms per liter (500 ug/) for 2,4-dinitrophenol and for 2-methyl-4,6-dinitrophenol; and one milligram per liter ( $1 \mathrm{mg} / \mathrm{h})$ for antimom;

(3) Five (5) times the maximum concentration value reported for that pollutant(s) in the permit application in accordance with $122.21(\mathrm{~g})(7)$; or

(4) The level established by the Director in accordance with 122.44(f).

b. That any activity has occurred or will occur which pould result in amy discharge, on a non-routine or infrequent basis, of a toxic pollutant which is not limited in the permit, if that discharge will exceed the highest of the following "notification levels":

(1) Five hundred micrograms per liter ( $500 \mathrm{ug} /)$;

(2) One milligram per liter ( $1 \mathrm{mg} /$ ) for antimons;

(3) Ten (10) times the maximum concentration value reported for that pollutant in the permit application in accordance rith $122.21(\mathrm{~g})(7)$; or

(4) The level established by the Director in accordance with 122.44(f).

\section{B. COOLING TOWER REQUIREMENTS}

The permittee shall notify the Division of Water Pollution control not less than three months prior to application of rood preservative ehemicals in any cooling tower and the expected maximum concentration of toxicants that are expected in the cooling tower blowdown. During the first week of cooling torier operation following a reapplication of nood preservatives, the permitter shall begin an acute toxicity testing program as described in Part III of the permit unless directed otherwise by the Director. Notification and testing also shall be required at the time of future treatments.

If the cooling tower or cooling syrtem contains components composed of asbertos-containing materials which may come in contact with the water, the permittee shall notify the Division of Water Pollution Control and shall test the tower blowdown for asbestos fibers on a quarterty basis. Results of asbestos testing shall be submitted as an attachment to the Discharge Monitoring Report for the period during which the tests were condueted. 


\section{BIOMONITORING REQUIREMENTS, CHRONIC}

The permittee shall conduct a 3-Brood Ceriodaphnia dubia Surival and Reproduction Test and a 7.Day Fathead Minnow (Pinephales promelos) Larval Survival and Grouth Test on samples of final effluent from Outfall 201. All tests should include concentrations at the LCso level, NOEC level, at $4 / 5$ of the LC50 and at $4 / 5$ of the NOEC. Toxicity will be demonstrated if more than $50 \%$ lethality of the test organisms occurs in 96 hours in $100 \%$ effluent or the no observable effect concentration (NOEC) for survival, reproduction, and growth is less than $100 \%$. All tests for Outfall 201 will use a minimum of three 24-hour composite samples of final effluent. If in any control, more than $10 \%$ of the test organisms die in 96 hours or more than $20 \%$ of the test organisms die in 7 days, the test (control end effluent) is considered invalid and the test shall be repeated within two (2) weeks. Toxicity demonstrated by the tests specified above constitutes a violation of this permit. Data developed during the chronic evaluation for the NOEC shall be used to determine a 96-hour LC50 for both Ceriodaphnia dubia and Pimephales promelas for the purposes of this permit.

The toxicity tests specified above shall be condueted quarterly and begin no later than ninety (90) days from the effective date of this permit. In the event of a test failure, the permintee must conduct a confirmation test within thirty (30) days and submit results from a confirmation test within sixty (60) days. Confirmation tests must include coneentrations equal to the LC50 limith NOEC limit, 4/5 of the LC50 limit, and 4/5 of the NOEC limit. All subsequent tests (including follow-up and quarterly tests) shall be conducted using serial dilutions until two (2) consecutive tests demonstrate compliance. The confimation test will not negate the initial failed test.

A "significant test failure" is defined as a result that is less than or equal to 4/5 of the permit limit. In the event of two (2) consecutive significant test failures or three (3) significant test failures nithin a twelve (12) month period, the permittee must initiate a Toxicity Identification Evaluation/Toxicity Reduction Evaluation (IIE/TRE) study within thitty (30) days and so rotify the Division by letter. This notification shall inciude a schedule of activities for the initial investigation. Additionally, the permittee shall submit quarterby progress reports throughout the term of the TIE/TRE study. Such reports shall inciude the data developed in the study. The toxicity must be reduced to allowable limits pithin two (2) years of initiation of the TIE/TRE study. Based on results obtained, the permittee may request an ertension of the TIE/TRE study period to conduct further analyses, but any extension of the two year period will be at the discretion of the Division. The TIE/TRE study may be terminated at any time upon the completion and submission of two (2) consecutive quarterly tests demonstrating compliance. During the course of the TIE/TRE study, the permintee will continue to conduct toxicity testing of Outfall 201 at the quarerly frequency prescribed herein but will not be required to perform the confirmation tests while the TIE/TRE is in progress.

Test procedures, quality assurance practices, and determination of eflluent lethality values will be made in accordance with Methods for Measuring the Acute Toxicity of Effluents to Freshwater and Marine Organisms, EPA/600/4-90/027 or the most current edition. Test procectures, quality assurance practices, determinations of eflluent chronic survival/reproduction and survival/growth values, and report formats will be made in accordance with Short-term Methods For Estimating the Chronic Toxicity of Efluents end Receiving Waters to Freshrater Organisms, EPA/600/4-89/001 or the most current edition.

Results of all tests, reference toxicant information, copies of raw data sheets, statisical analyzis and chemical analyses shall be compiled in a repor. The report will be writter in accordance with Short-term Methods For Estimating the Chronic Toxicity of Eflluents and Receiving Waters to Freshwater Organisms, EPA/600/4-89/001 or the most current edition. One copy of the report shall be submitted to the Division and one copy shall be submitted to the TN Division of DOE Oversight along with the discharge monitoring report (DMR). The lower value of the NOEC 
determined for survival/reproduction and sunvizal/growth shall be reported if these two values are different. Any tert initiated but terminated before completion must also be reported along with a complete explanation for termination of the test.

Toxicity Testing of Treatment Systems and Storm Sewer Points:

At the time of the toxicity testing for Outfall 201 , the treatment system effluents will be monitored for toxicity of their effluents. In addition to treatment plant effluents, testing will also include at least four separate places in the storm sewer gystem above the North/South pipes. Toxicity tests will be made using static definitive tests that determine a 48 hour LC50. Sampling for these static definitive terts may be either composite or grab eample (whichever is appropriate for the situation being sampled) and will be made during the period that chronic toxicity testing at Outfall 201 is being conducted. The tests for a particular treatment system or storm sewer monitoring point may use a single tert species if such is approved by the Division. While an on-going TIEIRE is being pursued, sampling associated rith the TIE/RE effors may be used as a substitute for campling sites within the storm setrer system. Results associated with the IIE/TRE, including tabulation of the raw data will be reported to the Division on a quarterly basis as pan of the IIE/RE progress repor.

If a sample cannot be collected from a treatment system at the time of the toxicity testing for Outfall 201, it mas be tested later in the quarter if there is a later opportunity to collect a sample from that system. If a test for the treatment systems or storm sewer monitoring points is invalid, the test that is invalid shall be repeated at a later time; hovever, an invalid tert shall not require that all treament systems and monitoring points be re-sampled and tested.

Data from such testing will be retained and made available to the Division upon request. These static toxicity tests have no permit limits but will serve information gathering purposes to:reflect what is happening in the storm sewer system at the time of toxicity testing at Outfall 201.

\section{PlaCEMENT OF SIGNS}

The permittee shall place and maintain a sign(s) to identify outfalls and stream monitoring points. The sign(s) should be clearly visible from the bank and the receiving waters. The signs should be made of durable material and have a white background with black letters.

Signs along Upper East Fork Poplar. Creek (within plant boundaries) that are clearty visible from the bank may be used to identify the fact that multiple discharges are made to the nearby stretch of the creek and shall note the types of discharges in that area of the creek. Individual outfalls in Upper East Fork Poplar Creek shall have a amall sign or label placed at the outfall that gives the outall number and the type of discharge made through that outfall.

The sign(s) are to provide notice that the discharge is regulated by the Temessee Department of Emvironment and Conservation, Division of Water Pollution Control. The following is given as an example of the minimal amount of information that must be ineluded on the sign: 


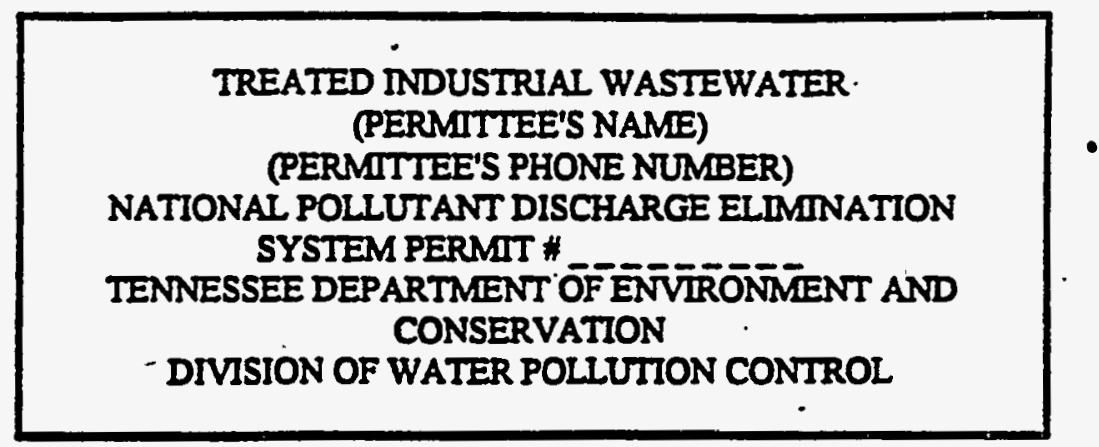

No later than sixty (60) days from permit issuance, the permittee shall have the above sign(s) on display in the location specified.

\section{E. REOPENER CLAUSE FOR PERMITS ISSUED TO SOURCES IN PRIMARY INDUSTRIES}

If an applicable standard or limitation is promulgated under Sections $301(\mathrm{~b})(2)(C)$ and (D), $304(B)(2)$, and $307(a)(2)$ and that effluent standard or limitation is more stringent than any effluent limitation in the permit or controls a pollutant not limited in the permit, permit shall be promptly modified or revoked and reissued to conform to that effluent standard or limitation.

\section{F. WASTEWATER CONTROL}

The permittee shall provide the Division a description of the procedures and criteria ised to determine which wastewaters are routed to which treatment system. The report shall deseribe, what vastewater acceptance criteria are used to determine which wastewaters are sent for treatment and the procedures used to control influents introduced to the treatment ijstems.

The report describing these procedures shall inelude whatever safeguards are in place to prevent introduction of hastewaters into a treatment system which are not appropriate for treatment. The report should also describe how a wastewater would be evaluated if it is of unusual character or different than what has been historically handled by the treatment systems. This deseription shall include a description of record-keeping and documentation of this process.

The report shall be submitted to the Division within one year of the permit effective date. Documentation of ruch decisions and operational records for the pastevater systems shall be maintained for at least three years and shall be made available to Department personnel within 15 days if requested.

The permittee may not add signifieant warteloads to the existing treatment systems without the knowvledge and approval of the Division. Signifieant prasteloads will only be added after review of such wasteloads based on the Wastewater Treatability and review by acceptable wastewates control procedures which are in place. Significant revisions to the Wastewater Control procedures for a treatment facility shall be sent to the Division.

The permittee shall keep its site survey of building and area drains up-to-date as part of the Wastewater Control program. If additional drains are added to a building or if drains are removed from service, the survey documentation shall be updated to reflect such changes. Where additional buildings or process areas are constructed and put in service at the site which connect to either the storm sewer system or the sewage collection system, it shall be included in the drain survey documentation. 


\section{G. BIOLOGICAL MONITORING AND ABATEMENT PROGRAM}

The program begun under the läst permit will be continued under this permit. Within 90 days from the effective date of the permit, the permittee shall review the biological monitoring and abatement program pertaining to the Y-12 site. The permittee shall submit to the Division of Water Pollution Control any revisions that shall be made to the program rithin 180 days of the permit effective date.

The plan should include studies to evaluate the receiving streams' biological communities throughout the duration of the permit. The permittee's representatives shall contact the Division's representatives for consultation during the review of the plan for needed revisions to the monitoring plan. Any revisions to the program must have the Division's approval prior to the implementing significant changes to the program. As knowiledge is gained about the environment and effects of the Y-12 site, the program may be modified with the approval of the Division.

Upon the Division's review of data from the Biological Monitoring Plan and Abatement Program, this permit may be modified, or alternatively, revoked or reissued, in order to reflect appropriate permit conditions.

In addition to the biological monitoring, the permittee shall continue sampling chemical and physical parameters at Stations 8 and 17 which can be used to assess biological information in Upper East Fork Poplar Creek.

The Biological Monitoring and Abatement Program shall inelude a study to determine the effect of mercury in East Fork Poplar Creek and its biota. Such information uill be collected in cooperation with EPA and Tennessee and will be intended to reach a site alternate criteris for chronic exposure to the mercury on the Oak Ridge Reservation. Data for East Fork Poplar Creek will be presented in a report kithin four years of the permit issuance date. The report will include both the analysis of the mercury situation as well as the raw data used to support the analysis of mercury in the creek.

\section{H. RADIOLOGICAL MONITORING}

Current radiological monitoring according to the Y-12 site radiological plan for the radioactive constituents of its liquid effluents will be continued under this permit until the radiological monitoring plan is modified. The permittee shall expand the radiological monitoring plan to include storm water monitoring. Radiological monitoring and reporting will be made for Station 17 and reported as part of this plan. The radiological plan must be expanded to include assessment of alpha, beta and gamma emitters. The perminee shall make sufficient data collection 20 allow determination of the appropriate parameters to be analyzed and reported for the radiological monitoring program. Data must be at least as precise as is necessary to evaluate results relative to the Derived Concentration Guide (DOE Order 5400.5). Where reasonable, the Division may require greater analytical precision. The permittee shall present a report summarizing the data to the Division of Water Pollution Control. If requested, the perminee shall make reconds of the raw data available to the Division of Water Pollution Control. Initial data collection for re-evaluation of the radiological monitoring plan shall be completed and reported to the Division by August 1 , 1995.

Once assessment and re-evaluation of the radiological monitoring plan is made, the plan may be modified to reflect appropriate monitoring. The radiological monitoring plan may be modified at any time with the approval of the Division and shall not require modification of this permit. The radiological monitoring plan shall be approved by the Division and analytical results of the monitoring shall be submitted to the Division in regular reports as specified in the plan. 


\section{MERCURY REMEDLATION}

The permittee shall reduce mereury loading to East Fork Poplar Creek to less than 5 grams mereury per day for waters of East Fork Poplar Creek leaving the plant site (excluding the loadings

- from storm generated events which cause the flow to exceed 15 million gallons per day). The loading shall be calculated each month by averaging daily values over the past three month period. Permit compliance sehedules in Permit Part I-E are set to require completion of projects which will help with reaching this requirement. Reduction of the mercury loading to less than 5 grams per day shall be accomplished by December 31, 1998. The permittee shall demonstrate compliance with the state's chronic water quality criteria for fish and aquatic life by the close of the permit period. Thus, the permittee shall demonstrate compliance with a total mercury concentration corresponding to the chronic criteria of $0.012 \mathrm{ug} / \mathrm{L}$ as an instream value measured at Station 17 by the close of the permit period. This value equals 0.32 grams mercury per day for a flow of 7 MGD. For the purposes of this permit if the chronic criteria for total mercury is changed before the close of this permit's period, that criteria revision may be used to evaluate compliance with this requirement to meet the state's chronic water quality criteria.

The permittee shall submit a yearly report summarizing the status of mercury concerns for the plant site. The report shall include a summary of the various schedules and activities that are ongoing for mereury treatment and remediation and make note of any changes. that have been necessary to scheduled activities. The report shall summarize monitoring data for mercury in the site effluents, storm water, and data from instream monitoring. The repor shall briefly summarize the status end results of biological monitoring and data collection for East Fork Poplar Creek which are available from the biological monitoring and abatement program: This report will be supplied to the Division with the DMR submittals for the October monitoring data.

\section{J. ADDITION OF RAW WATER TO EAST FORK POPLAR CREEK}

The permittee shall maintain a flow in East Fork Poplar Creek at Station 17 of 7 million gallons per day through the permit period. The natural flow in East Fork Poplar Creek has been augmented for many years by $Y-12$ plant flows. The plant flow needs to be managed to stabilize at a value which will protect the stream water quality and the aquatic life now in recovery. Raw water pumped from the Clinch River may be used to maintain this level of flow. Raw water pumped from the Clinch River may be discontinued whenever flows at Station 17 are greater than 7 MGD without this additional water being added to the creek or during emergency situations such as described in the next paragraph.

The permittee shall have a spill/accident plan ready such that base flow of raw water from the Clinch River can be shut off in the case of spills or other incidents in which Lake Reality will be used to capture contaminated plumes moving down the creek. The raw water vill not be added to the creek in such circumstances until it is safe to do so.

Until flow of 7 MGD can be assured at Station 17 by a capability of pumping raw water from the Clinch River, the permittee shall make reasonable effors to maintain flows as close to 7 MGD as possible. The capability to maintain flows at .7 MGD shall be in place by March 1, 1997.

\section{R. CHANGES TO STORM WATER MONITORING AND OUTFALIS}


The Division may request that any outfall that has been categorized in one storm kater category be sampled at the frequency of a higher classification storm water category. For example, if data indicate that a Storm Water Category I outfall is of greater concern for pollutant loads than previously believed, the Division may request that outfall be sampled as a Category II outfall.

If additional storm water monitoring points are appropriate, the permittee may include sampling of these in its Storm Water Pollution Prevention Plan. Such additional monitoring points can include instream monitoring, setting up temporary outfalls for storm water sampling of drainage sub-areas, and internal monitoring points in stom setwers and drainages. .

For storm water discharges associated nith industrial activity, inchuding storm water discharges associated with construction activity, that are not specificalty monitored or limited in this permit, the permittee shall meet conditions of the General Storm Water Rule (TCA 1200-4-10). A notification of intent (NOI) to comply with general storm water requirements bill be sent to the Division for such outfalls that are not specifically listed in this permit.

The permittee shall maintain one Storm Water Pollution Prevention Plan that includes all outfalls and activities at the site relating to storm nater regulation under this permit and the General Storm Water Rule. Storm Water Pollution Prevention Plan shall identify outfalls and monitoring points under this permit and the outfalls, monitoring points and activities that fall under the general permit rule.

If the Division determines that a storm water outfall should be specifically included under this permit's limitations or monitoring conditions, the Division nill require that form $2 \mathrm{~F}$ be filled out for the outfall and this permit will be modified to include that storm water outfall.

\section{BEST MANAGEMENT PRACTICES, FLUSENG WATER LINES}

The permittee shall develop best management practices for discharging water from the potable mater system such as flushing out the water supply lines. The plan shall also cover similar situations such as draining the potable pater lines in the plant, releases from fire hydrants or sprinkler systems for maintenance or testing purposes, etc. The plan shall be approved by the Division of Water Pollution Control. The plan must be submitted by August 1, 1995.

\section{TEMPERATURE PROFILE OF EAST FORK POPLAR CREEK}

The permittee shall develop a temperature profile during hot summer conditions from Ouffall 200 to Lake Reality at least once per year. The purpose of this profile is to see if thermal loadings from cooling waters are in compliance with this permit's limitations in the creek. Sampling points must include the North/South pipes, a point below Outfall 109 a point below Outfall 047 and a point below Outfall 021 . Additional sampling points mxy be chosen as appropriate. Results of the profile will be briefly summarized and reported to the Division with the DMR submittal for the period.

\section{N. INVESTIGATION OF FECAL COLTFORM ANALYSES}

The permittee shall imvestigate the high values of fecal coliform in the anabses reported for the application. The facility will determine if the high values reported for the application appear to be the result of sampling etror or if the presence of fecal coliforms is as widespread at the site as indieated by the application materials. If the fecal coliforms are verified as present in the storm sewer discharges and storm related runoff, the facility will submit a report indieating the possible causes for the presence of fecal coliforms and propose what action is needed to address the fecal coliforms present at the site. The repon will be due by August 1, 1995. 


\section{O. SUBMISSION OF FORM 2C FOR NEW TREATMENT SYSTEM OUTFALLS}

The permittee shall submit a Form $2 \mathrm{C}$ for the new mercury treatment systems once these outfalls are operational and data ceas be collected. Outfall data for Outfall 550 and 551 (if constructed) rill be evaluated to see if permit conditions and parameter monitoring should be changed in light of the actual discharge data. The Form $2 \mathrm{C}$ shall be submitted within 2 years of the start of the discharges from the outfall. 


\section{STORM WATER POLLUTION PREVENTIONPLAN}

The permittee shall develop, document and maintain a storm rater pollution control plan, which shall contain at a minimum the following items. The plan shall be signed by a principal executive officer in the case of a corporation, the owner or proprietor for a sole proprietorship, or a partner or general partner for a partnership. This plan may include provisions/conditions for storm water outfalls under this permit as well as the information for compliance with the general permit for storm water (TCA 1200-4-10) for construction activities or for industrial activity not covered under this individual permit.

\section{A. POLLUTANT SOURCES AND PATHWAYS}

1. A site map outlining the individual storm rater drainage areas, existing structural control measures, surface water bodies, and sinkholes.

2. A narrative description of significant materials ( 40 CFR 122.26) that are currently or in the past have been treated, stored, or disposed outside; materials management practices; existing structural and non-stnictural control measures to reduce pollutanis; and a description of any storm water treatment.

3. A list of significant spills and leaks of toxic or hazardous pollutants at the facility that have token place after the effective date of the permit.

4. A prediction of direction of flow and the possible pollutants associsted with each area of the plant that generates storm water.

5. A record of available sampling data describing pollutants in storm water discharges.

\section{B. STORM WATER MANAGEMENT CONTROLS}

1. Formulate a pollution prevention team with named individuals who shall develop the storm mater prevention plan and assist plant manager in its implementation.

2. Imentory tspes of materials handled and associated potential of release to storm water. Evaluate the following for potential pollutant contribution: loading and unloading operations, outdoor sorage and manufacturing activities, dust or particulate generating processes, and on site waste disposal practices. Consider toxicity of chemicals, quantity of chemicals, and history of leaks or spills of toxic or hazardous pollutants.

3. Design a preventive maintenance program inciuding inspection and maintenance of storm water management devices and testing plant equipment and systems to moover conditions which could cause failures.

4. The permittee shall maintain a clean, orderby facility.

5. The permittee shall have spill prevention and response procectures. Identify potential spill areas and drainage points. Specify material handling procectures and storage requirements. Identify spill cleanup procedures and provide to responsible perionnel. Responsible personnel and the neceseary equipment to implement cleanup must be available at all times phen the facility is in operation. 
6. The plan shall contain a narrative consideration of traditional storm water management practices (practices other than those which control the source of pollutants).

7. Identify areas of potentially high soil erosion and measures to limit erosion.

8. Employees at all levels of responsibility shall be trained in the components of the storm water prevention plan.

9. Qualified personnel shall be identified to inspect equipment, plant areas, and material handling areas. A tracking system should be developed to ensure corrective action and records of inspections shall be maintained.

10. Records shall be maintained of spills or other discharges, inspections and maintenance activities, and information deseribing the quality and quantity of storm water discharges.

11. The plan shall contain a certification that the discharge has been tested for the presence of non-storm vater discharges. The certification shall include a description of the results of any test for the presence of non-storm water discharges, the method used, the date of any testing, and the on-site drainage points that were directly obsened during the test.

\section{FACIITTY INSPECTION}

Inspections of the facility shall be conducted at least semi-annually to check the accuracy of the plan, maps, and whether measures in the plan to reduce pollutants in storm water rumoff are adequate and properby implemented or whether additional controls are needed. Records of these inspections shall be maintained for a period of three years.

\section{SPILL PREVENTION CONTROL AND COUNTERMEASURES}

Storm water management programs may refleet requirements for spill prevention control and countermeasures (SPCC) plans under section 311 of the CWA.

The permittee shall develop diking and containment as appropriate in waste and pasterater transfer areas.

\section{E. PLAN REVIEW AND UPDATE .}

The plan shall be reviewved and updated, if necessary, by the facility at leart anmually. The plan and all records shall be retained for at least three years after expiration of this permit.

\section{F. PLAN IMPLEMENTATION}

The plan should be developed and available for review within 180 days after permit coverage. Facilities should implement management practices as coon as possible but not later than one year after permit coverage. Where new construetion is specified, this should be accomplished as soon as possible. A schedule for new construction should be included in the plan.

\section{G. PLAN AVATIBILITY}

The plan shall be maintained by the permittee on the site or at a nearty office. Copies of the plan shall be submitted to the Division of Water Pollution Control within ten working days of a request. 
DOE Y-12 PLANT, TNO002968, ANDERSON COLNTY

PAOE 48 OF 50

\section{H. PLAN MOdIfICATION}

The plan shall be modified as required by the Director of the Division of Water Pollution Control.

\section{i MONTTORING PLAN}

The storm water discharges shall be monitored as outlined in the individual "Permit Limits" applicable to the storm mater ouffalls. All outfalls that comvey storm water associated with industrial activity must be identified. For each outfall monitored, the surface area and type of corer shall be identified.

Although storm water outfalls may be grouped together for storm water characterizations where outfalls are similar, the permittee shall make a minimum of 25 storm water characterizations per year under its monitoring plan, including both grab and composited sampling in each characterization. The 25 characterizations represents the minimum effort required by the permit. If additional monitoring is called for, the permittee should be prepared to do the additional monitoring.

The permittee shall develop monitoring to the extent possible that will provide for better, systematic investigation of pollutants that show up at the North/South pipes (Outfall 200). The goal is that pollutants at Outfall 200 be traced to a definite source that can be addressed.

Storm water characterizations conducted the first year of the permit shall include characterization of Outfalls 007, 032 and 033. The results of the characterization will be compared with results dereloped during the permit applieation for these outfalls.

The Storm Water Pollution Prevention Plan shall proride for follow up investigation of pollutant levels which are indicative of materials getting into the storm pater runoff. Such pollutant levels do not have to be a threat to water quality before invertigation is begun. For example, elevated chloride, sulfate, or feeal coliforms may serve as indicators of other pollutants without constituting a threat to water quality in and of themsehves.

\section{J. SARA TITLE II, SECTION 313 PRIORTY CHEMICALS}

The storm water pollution prevention plan shall include the following for those facilities subject to reporting requirements under SARA Title III, Section 313 for chemieals which are classified as Section 313 uater priority chemicals:

1. In areas where Section 313 priority chemicals are stored, processed or otherwise handled, appropriate containment, drainage control and/or diversionary structures shall be provided. At a minimum, one of the following preventive gystems or its equivalent shall be used:

2. curbing, culverting, gutters, sewers or other forms of drainge comtrol shall be provided.

b. Roofs, covers or other forms or protection to prevent storage piles from exposure to storm water and wind.

2. The plan shall include a diseussion of measures taken to conform with the following applicable guidelines:

2. Liquid storage areas where stoim water comes into contact with any equipment, tank container, or other veseel used for Section 313 water priority chemicals. 
i. Tank or container must be compatible vith. Section 313 water priority chemical which it stores.

ii. Liquid storage areas shall be operated to minimize discharges of Section 313 chemicals.

b. Material storage areas for Section 313 water priority chemicals other than liquids shall incorposate features which will minimize the discharge of Section 313 chemicals by reducing storm water contact.

c. Truek and rail car loading and unloading areas for Section 313 liquid chemicals shall be operated to minimize discharges of chemicals. Appropriate measures may include placement and maintenance of drip pans for use when making and breaking hose connections; a spill contingency plan; and/or other equivalent measures.

d. In plant areas where Section 313 chemicals are transferred, processed or handled, piping, processing equipment, and materials handling equipment shall be operated so as to minimize discharges of chemicals. Piping and equipment must be compatible with chemicals handled. Additional protection including covers and guards to prevent exposure to wind and pressure relief vents, and overhangs or door skirts to enclose trailer ends at truck loading docks shall be implemented. Visual inspections or leak tests shall be conducted on overhead piping that conveys Section 313 chemicals.

c. Discharges from areas covered by parts $2 a, 2 b, 2 c$, or $2 d$ :

i. Drainage from these areas should be restrained by valves or other positive means to prevent the discharge of a spill or excessive leakage. Containment units shall be drained manually.

ii. Flapper-type drain vahes shall not be used for drainage of containment units.

iii. If freility is not engineered as specified above, the final discharge of in:facility storm sewers should be equipped with a diversion system that could, in the event of an uncontrolled spill of a Section 313 chemical, return the spilled material to the facility.

iv. Records shall be kept of the frequency and estimated volume (in gallons) of discharges from containment area.

f. Facility site runoff other than from areas covered by parts $2 a, 2 b, 2 c$, and $2 d$ from which runoff could contain Section 313 chemicals shall incorporate the necessary drainage or other control features to prevent discharge of spilled or improperly disposed material and ensure the mitigation of pollutants in runoff or leachate.

8. All areas of the facility shall be inspected at specific intervals for leaks or conditions that could lead to discharges of Section 313 water priority chemicals or direct contact of storm water with raw materials, intermediate materials, praste materials or products. Inspection intervals shall be specified in the plan and shall be based on design and operational experience. Corrective action shall be taken promptly when a leak or condition which could cause significant releases of chemicals is discovered. If corrective action can not be taken immediately, the unit or process shall be shru down until the sinuation is corrected. When a leak or spill has ocourred, the 
contaminated material(s) mur be promptly remored and disposed in accordance with Federal, State, and local requirements and as deseribed in the plan:

h. Facilities shall hre the necessury security systems to prevent sccidental of intentional entry phich could cuuse a discharge.

i. Facility employees and contract personnel that work in areas where SARA Title III, Section 313 water priority chemicals are used or stored shall be trained in and informed or preventive mearures at the facility. Employee training shall be conducted at least ence per year in matters of pollution control laws and regulations, and in the storm wrter prevention plan. The plan chall designate a person who is accountable for spill prevention at the facility and indicate who will set up the necessary spill emergency procedures and reporting requirements.

j. The storm water prevemion plan for a freility subject to SARA Title III, Section 313 requirements shall be revieried by a registered professional engineer and certified to by such professional engineer. The registered professional engineer shall certify the plan every three years thereafter or as soon as practical after significant modifieations are made to the freility. Certification shall in no way relieve the owner or operator of a facility covered by the plan of their duty' to prepare and fully implement vich plan.

3. "Section 313 nater priority chemicals" means the following chemieals or chemieal categories:

a. lined at 40 CFR 372.65 purmant to Section 313 of Title III of the Superfind Amendments and Reauthorization Act (SARA) of 1986, aleo titled the Emerigency Plamning and Commmity Right-to-Know Aet of 1986;

b. present at or above threshold levels at a freility subject to SARA Tille III, Section 313 reporting requirement; and,

c. that meet at least one of the following criteria:

i. are listed in Appendix D in 40 CFR 122 on either Table II (organic priority pollumants). Table III (cerrin metals, gganides, and phenois) or Table V (cernin toxic pollutants and hazardous subarunces);

ii. . are listed as a herardous abrance purannt to section $311(\mathrm{~b})(2)(A)$ of the CWA at 40 CFR 116.4; or,

iii. are polluthnts for which EPA hus published acure of chronic toxicity criteril. 


\section{Distribution}

S. D. Easterling

K. G. Hanzelka

R. H. Harden III

E ElHouthore

C. C. Hill

A. K. Lee/DOE-OSTI (2)

M. Leslie, CDM-Federal Programs

S. D. Morris, DOE-ORO (3)

L. A. Muhs

E. M. Murrill

S. E. Poligone

R. M. Shedden

B. E. Skaggs

L. O. Vaughan

M. C. Wiest, Jr.

M. L. Willoughby

Y-12 Central Files

File-EMD-RC 